\title{
PHYSICAL PAPERS
}

HENRY A. ROWLAND

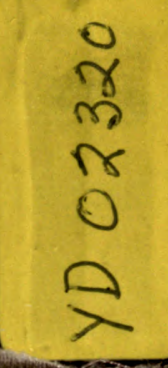




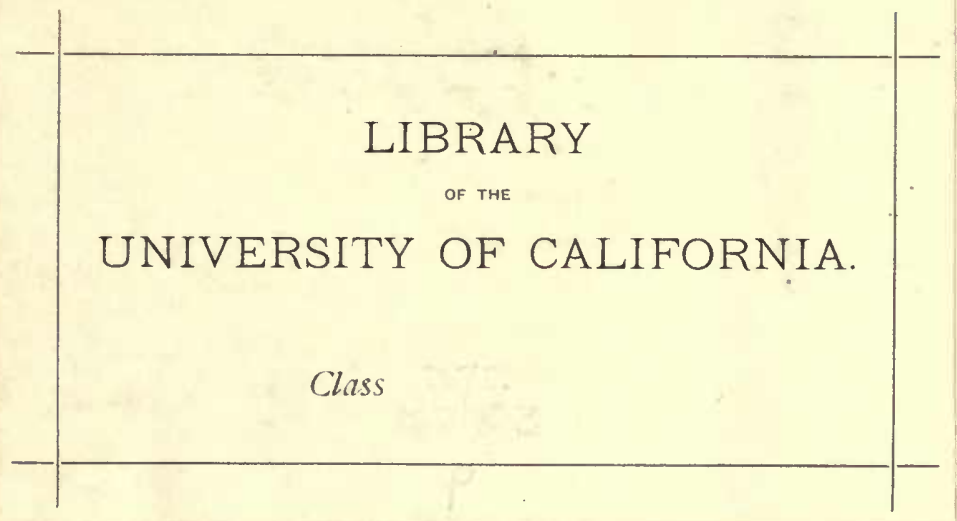

GENERAL 

THE

PHYSICAL PAPERS

OF

HENRY AUGUSTUS ROWLAND 




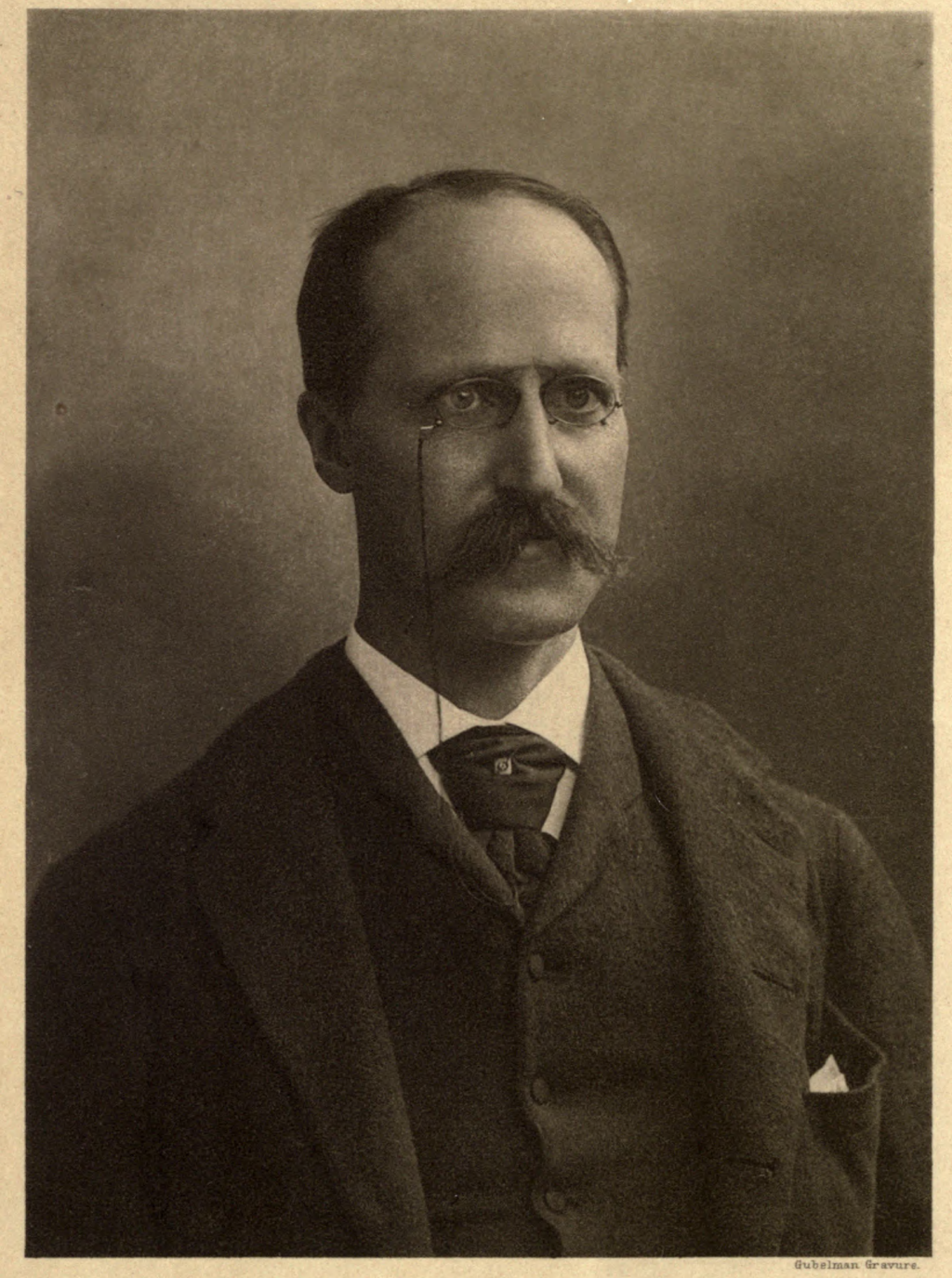

Gubelman Gravure. 


\section{THE}

\section{PHYSICAL PAPERS}

$\mathrm{OF}$

\section{HENRY AUGUSTUS ROWLAND \\ PH. D., LL. D.}

Professor of Physics and Director of the Physical Laboratory in The Johns Hopkins University $1876-1901$

COLLECTED FOR PUBLICATION BÝ A COMMITTEE OF THE FACULTY OF THE UNIVERSITY

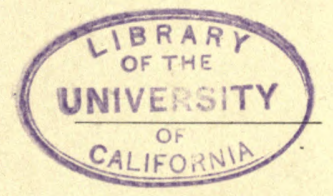

BALTIMORE

The Johns Hopkins Press 1902 


\section{GENERAL}

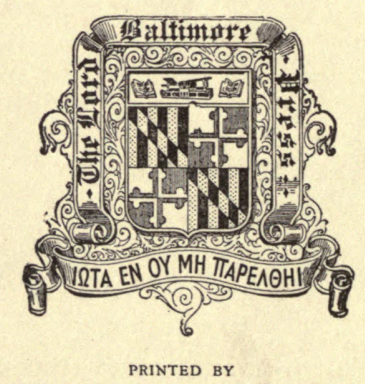

Ebe Jriedenmald Eompany BALTIMORE, MD., U.S. A. 


\section{HENRY AUGUSTUS ROWLAND}

Born, Honesdale, Pennsylvania, November 27, 1848

Died, Baltimore, Maryland, April 16, 1901

Doctor of Philosophy (Ph. D.), Johns Hopkins University, 1880. (Honoris Causa.)

1)octor of Laws (LL. D.), Yale University, 1895.

Doctor of Laws (LL. D.), Princeton University, 1896.

Fellow or Member of

The British Association for the Advancement of Science.

The Physical Society of London.

The Philosophical Society of Cambridge, England.

The Royal Society of London.

The Royal Society of Göttingen.

The Gioenian Academy of Natural Sciences, Catania, Sicily.

The French Physical Society.

The French Academy of Sciences.

The Literary and Philosophical Society of Manchester.

The Royal Lyncean Academy, Rome.

The Academy of Sciences, Stockholm.

The Italian Society of Spectroscopists.

The Royal Society of Edinburgh.

The Society of Arts, London.

The Royal Astronomical Society of England.

The Royal Society of Lombardy.

The Royal Physiographic Society of Lund.

The Royal Academy of Sciences, Berlin.

The Royal Academy of Sciences and Letters, Copenhagen.

The American Philosophical Society, Philadelphia.

The American Academy of Arts and Sciences, Boston.

The National Academy of Sciences, Washington.

The American Physical Society,-its first President.

The Astronomical and Astrophysical Society of America.

Delegate of the United States Government to the

International Congress of Electricians, Paris, 1881.

International Congress for the Determination of Electrical Units, Paris, 1882. Appointed Officer of the Legion of Honor of France.

Electrical Congress, Philadelphia, 1884,--President.

International Chamber of Delegates for the Determination of Electrical Units, Chicago, 1893,-President.

\section{Prizes and Medals.}

Rumford Medal, American Academy of Arts and Sciences.

Draper Medal, National Academy of Sciences.

Matteucci Medal.

Prize awarded by the Venetian Institute in competition for a critical paper on the Mechanical Equivalent of Heat. 



\section{PREFACE}

Shortly after the death of Professor Rowland in April, 1901, a committee of the Faculty of The'Johns Hopkins University was appointed by President Gilman to suggest to the Trustees of the University a plan for a memorial of their colleague. The committee, consisting of Professors Remsen, Welch and Ames decided to recommend that a volume be prepared containing the physical papers and addresses of Professor Rowland, and also a detailed description of the dividing engines which had been designed and constructed by him for the purpose of ruling diffraction gratings, and that this volume be published by the University Press. This recommendation was approved by the Trustees of the University; and the same committee, with the addition of Professor R. W. Wood, was empowered to prepare the volume for publication. The editorial supervision has been mainly undertaken by Professor Joseph S. Ames.

In deciding upon the scope of the proposed volume, it was thought best to include only the distinctly physical papers, inasmuch as Professor Rowland himself on several occasions when the question of the collection of his scienfific papers was raised, had expressed himself as opposed to the republication of the purely mathematical ones. It was also decided to omit tables of wave-lengths, as these are extremely bulky, and copies can be easily obtained. Professor Rowland left many thousand pages of manuscript notes and outlines of lectures, but none of this material was ready for publication, and the committee were not in a position to undertake the task of its preparation. No attempt has been made to include a biography of Professor Rowland, for this would properly form a volume by itself, and would require much time for its preparation. There was at hand, moreover, the memorial address of Dr. Mendenhall, which tells so well, though briefly, the story of his life. 
It was with difficulty, and only after a careful examination of many hundred volumes of scientific journals and transactions, that the committee were able to obtain copies of all of Professor Rowland's numerous and scattered articles; but they are convinced that no paper of importance has escaped their notice. In preparing for publication these memoirs and addresses, no alterations other than typographical have been made.

For permission to reprint some of the most valuable papers, thanks are due to various publishers. The committee wish especially to express their appreciation of the kindness of Messrs. A. and C. Black, and of The Times (London) for permission to reprint from the Encyclopædia Britannica the articles on "The Screw" and on "Diffraction Gratings," and of the Engineering Magazine Company, of New York, for permission to reprint the article on "Modern Theories as to Electricity."

The committee acknowledge their indebtedness also to Mr. N. Murray, Librarian of The Johns Hopkins University, who has personally superintended the details of publication, and whose advice has been often needed. The proofs have been revised by Mr. E. P. Hyde, Fellow in The Johns Hopkins University, who has thus been of the greatest assistance to the committee.

THE JOHNS HOPKINS UNIVERSTTY,

Baltimore, Maryland, DeCember 1, 1902. 


\section{CONTENTS}

PAGE

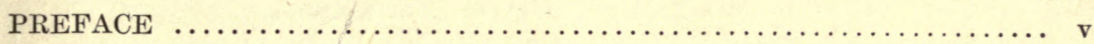

ADDRESS BY DR. T. C. MENDENHALL .................... 1

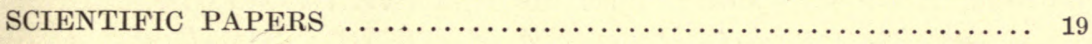

Part I. Early Papers. 21

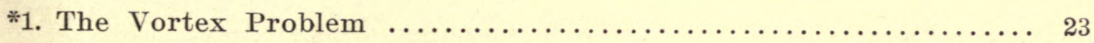

Scientific American XII, 308, 1865.

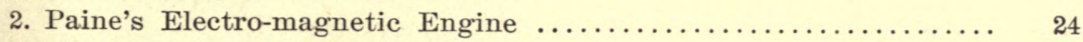

Scientific American XXV, 21, 1871.

3. Illustration of Resonances and Actions of a Similar Nature ....... 28 Journal of the Franklin Institute XCIV, 275-278, 1872.

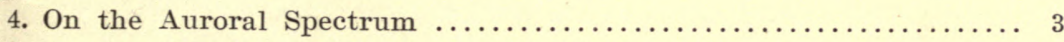

American Journal of Science (3), V, 320, 1873.

Part II. Magnetism and Electricity.

5. On Magnetic Permeability, and the Maximum of Magnetism of Iron,

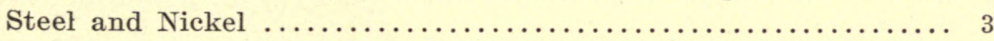

Philosophical Magazine (4), XLVI, 140-159, 1873.

6. On the Magnetic Permeability and Maximum of Magnetism of Nickel

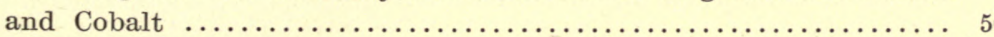

Philosophical Magazine (4), XLVIII, 321-340, 1874.

7. On a new Diamagnetic Attachment to the Lantern, with a Note on the Theory of the Oscillations of Inductively Magnetized Bodies.. 75 American Journal of Science (3), IX, 357-361, 1875.

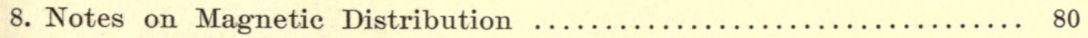

Proceedings of the American Academy of Arts and Sciences, XI, 191, 192, 1876.

9. Note on Kohlrausch's Determination of the Absolute Value of the

Siemens Mercury Unit of Electrical Resistance ............. 82 Philosophical Magazine (4), L, 161-163, 1875.

10. Preliminary Note on a Magnetic Proof Plane $\ldots \ldots \ldots \ldots \ldots \ldots \ldots . . \ldots 5$ American Journal of Science (3), X, 14-17, 1875.

* The numbers refer to corresponding ones in the Bibliography, page 681. 
11. Studies on Magnetic Distribution ........................ 89

American Journal of Scicnce (3), X, 325-335, 451-450, 1575 .

Ibid., XI, 17-29, 103-10s, 1876.

Philosophical Magazine (4), L, 25\%-2\%\%, 348-36\%, 18\%.

12. On the Magnetic Effect of Electric Conrection

American Journal of Science (3), XV, 30-38, 1878 .

13. Note on the Magnetic Effect of Electric Convection Philosophical Magazine (5), VII, 442-443, 1879.

14. Note on the Theory of Electric Absorption American Journal of Mathematics, I, 53-58, 1878.

15. Research on the Absolute Unit of Electrical Resistance American Journal of Sclence (3), IV, 281-291, 325-336, 430-439, 1878.

17. On Professors Ayrton and Perry's New Theory of the Earth's Magnetism, with a Note on a New Theory of the Aurora .......... 179 Philosophical Magazlue (5), VIII, 102-106, 1879. Proceedings of the Physical Society, III, 03-9S, 1879.

18. On the Diamagnetic Constants of Bismuth and Calc-spar in Absolute

Measure. By H. A. Rowland and W. W. Jacques ............ 184 American Journal of Sclence (3), XVIII, 360-371, 1879.

19. Preliminary Notes on Mr. Hall's recent Discovery 197 American Journal of Mathematics, II, 354-356, 1879. Philosophical Magazine (5), IX, 432-434, 1850. Proceedings of the Physical Society, IV, 10-13, 1850.

22. On the Efficiency of Edison's Electric Light. By H. A. Rowland and

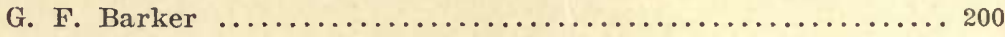
American Journal of Sclence (3), XIX, 337-339, 1850.

27. Electric Absorption of Crystals. By H. A. Rowland and E. L.

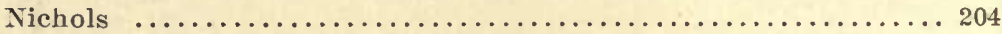
Philosophical Magazine (5), XI, 414-419, 1851. Proceedings of the Physical Society, IV, 215-221, 1 S81.

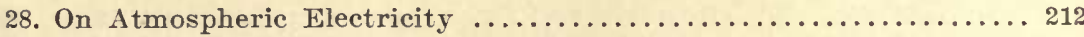
Johns Hopkins Unirersity Circuiars No. 19, pp. 4, 5, 1882.

34. The Determination of the Ohm. Extract from a letter to the Inter-

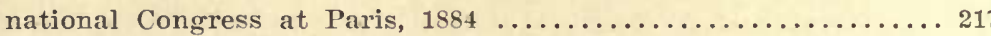
Procès-Verbaux, Deuxième Session, p. 37. Paris, 1854.

35. The Theory of the Dynamo ................................. 219 Report of the Electrical Conference at Philadelphia in November, 1884, pp. $72-83,90,91,104,107$. Washington, 1856.

36. On Lightning Protection .............................. 236 Report of the Electrical Conference at Philadelphia in November, 1884, pp. 172-174.

37. On the Value of the $\mathrm{Ohm}$

La Lumière Électrique, XXVI, pp. 1S8, $4 \pi i, 188 \pi$. 
PAag

38. On a Simple and Convenient Form of Water-battery ............ 241

American Journal of Science (3), XXXIII, 147, 1857.

Phllosophical Magazine (5), XXIII, 303, 1887.

Johns Hopkins University Circulars No. 57, p. 80, 1887.

40. On an Explanation of the Action of a Magnet on Chemical Action.

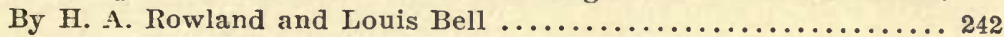

American Journal of Sclence (3), XxxvI, 39-47, 1888.

Philosophical Magazine (5), XXVI, 105-114, 1888.

43. On the Electromagnetic Effect of Convection-Currents. By H. A.

Rowland and C. T. Hutehinson ...............................

Phllosophical Magazine (5), XxVII, 445-460, 1889.

44. On the Ratio of the Electro-static to the Electro-magnetic Unit of

Electricity. By H. A. Rowland, E. H. Hall, and L. B. Fletcher... 266 American Journal of Sclence (3), XXXVIII, 289-298, 1889.

Philosophical Magazine (5), XXVIII, 304-315, 1889.

47. Notes on the Theory of the Transformer ................ 276

Philosophical Magazine (5), XXXIV, 54-57, 1892.

Electrical World, Xx, 20, 1832.

Johns Hopkins University Circulars No. 99, pp. 104, 105, 1892.

48. Notes on the Effect of Harmonics in the Transmission of Power by

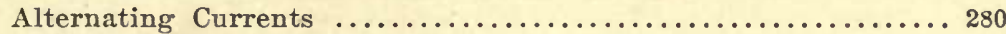

Electrical World, XX, 368, 1892.

La Lumière Électrique, XLVII, 42-44, 1893.

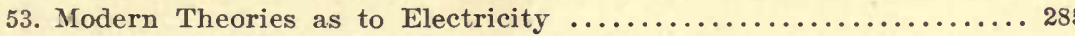

The Engineering Magazine, VIII, 589-596, 1895.

60. Electrical Measurement by Alternating Currents .............. 294 American Journal of Science (4), IV, 429-448, 1897.

Plillosophical Magazine (5), XLV, 66-85, 1898.

62. Electrical Measurements. By H. A. Rowland and T. D. Penniman.. 314 American Journal of Sclence (4), VIII, 35-57, 1899.

63. Resistance to Ethereal Motion. By H. A. Rowland, N. E. Gilbert and

P. C. McJunckin ............................... 338

Johus Hopkins University Cireulars No. 146, p. 60, 1900.

Part III. Heat.

16. On the Mechanical Equivalent of Heat, with Subsidiary Researches on the Variation of the Mercurial from the Air-Thermometer and on the Variation of the Specific Heat of Water ............... 343

Proceedings of the American Academy of Arts and Sciences, XV, 75-200, 1880.

21. Appendix to Paper on the Mechanical Equivalent of Heat, Containing the Comparison with Dr. Joule's Thermometer ........... 469 Proceedings of the American Academy of Arts and Sclences, XVI, 38.45, 1881.

20. Physical Laboratory; Comparison of Standards $\ldots \ldots \ldots \ldots \ldots \ldots .477$ Johns Hopkins Unirersity CIrcuiars No. 3, p. 31, 1880. 
29. Preliminary Notice of the Results Accomplished in the Manufacture and Theory of Gratings for Optical Purposes .............. 487 Johns Hopkins University Cireulars No. 17, pp. 248, 249, 1882. Phllosophical Magazine (4), XIII, 469-474, 1882. Nature, 26, 211-213, 1882.

30. On Concave Gratings for Optical Purposes .................. American Journal of Science (3), XxVI, 87-98, 1883. Philosophical Magazine (5), XVI, 197-210, 1883.

31. On Mr. Glazebrook's Paper on the Aberration of Concave Gratings. 505 American Journal of Science (3), XXVI, 214, 1883. Philosophical Magazine (5), XVI, 210, 1883.

33. Screw Encyclopaedia Britannica, Ninth Edition, Vol. 21.

39. On the Relative Wave-lengths of the Lines of the Solar Spectrum ... 512 American Journal of Science (3), XXXIII, 182-190, 1887. Phliosophical Magazine (5), XXIII, 257-265, 1897.

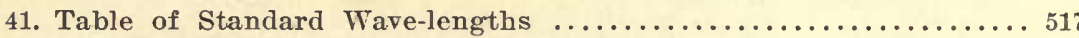
Philosophical Magazine (5), XXVII, 479-494, 1889.

42. A Few Notes on the Use of Gratings ..................... 519 Johns Hopkins University Circulars No. 73, pp. 73, 74, 1889.

46. Report of Progress in Spectrum Work The Chemical News, LxIII, 133, 1891.

Johns Hopkins University Circulars No. 85, pp. 41, 42, 1891. American Journal of Sclence (3), XLI, 243, 244, 1891.

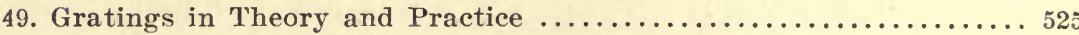
Philosophical Magazine (5), XxxV, 397-419, 1893. Astronomy and Astro-Physics, XII, 129-149, 1893.

50. A New Table of Standard Wave-lengths Philosophlcai Magazine (5), XxxvI, 49-75, 1893. Astronomy and Astro-Physics, XII, 321-347, 1893.

51. On a Table of Standard Wave-lengths of the Spectral Lines ....... 548 Memoirs of the American Academy of Arts and Sciences, XII, 101-186, 1896.

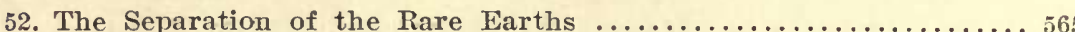
Johns Hopkins University Circulars No. 112, pp. 73, 74, 1894.

57. Notes of Observation on the Röntgen Rays. By H. A. Rowland, N.

R. Carmichael and L. J. Briggs $\ldots \ldots \ldots \ldots \ldots \ldots \ldots \ldots \ldots \ldots \ldots \ldots \ldots \ldots \ldots \ldots \ldots \ldots \ldots$ American Journal of Science (4), I, 247, 248, 1896. Philosophical Magazine (5), XLI, 381-382, 1896. 
58. Notes on Röntgen Rays. By H. A. Rowland, N. R. Carmichael and L. J. Briggs . .................................. 573 Electrical World, XxVII, 452, 1896.

59. The Röntgen Ray and its Relation to Physics ............... 576

Transactions of the American Institute of Electricai Engineers, XIII, $403-410,430,431,1896$.

64. Diffraction Gratings $\ldots \ldots \ldots \ldots \ldots \ldots \ldots \ldots \ldots \ldots \ldots \ldots \ldots \ldots \ldots \ldots$

Encyclopaedia Británnica, New Volumes, III, 458, 459, 1902.

ADDRESSES

1. A Plea for Pure Science. Address as Vice-President of Section B of the American Association for the Advancement of Science, Minneapolis, August 15, 1883 ........................... 593

Proceedings of the American Association for the Advancement of Science, XXXII, 105-126, 1883.

Science, II, 242-250, 1883.

Journal of the Frankiln Institute, CXVI, 279-299, 1883.

2. The Physical Laboratory in Modern Education. Address for Commemoration Day of the Johns Hopkins University, February 22,

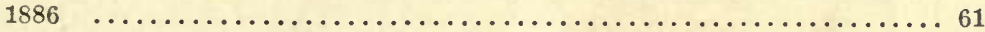

Johns Hopkins University Circulars No. 50, pp. 103-105, 1886.

3. Address as President of the Electrical Conference at Philadelphia,

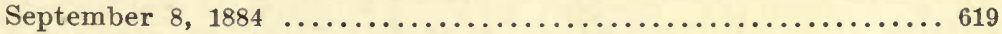

Report of the Electrlcal Conference at Philadelphia In September, 1884, Washington, 1886.

4. The Electrical and Magnetic Discoveries of Faraday. Address at The Opening of the Electrical Club House of New York City, 1888. 638 Electrical Review, Feb. 4, 1888.

5. On Modern Views with Respect to Electric Currents. Address before the American Institute of Electrical Engineers, New York,

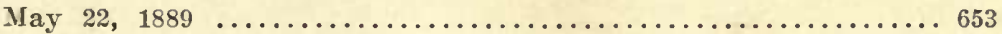
Transactions of the American Institute of Electrical Engineers, VI, 342$35 \overline{7}, 1889$.

6. The Highest Aim of the Physicist. Address as President of the

American Physical Society, New York, October 28, 1899 ...... 668 Sclence, X, 825-833, 1899.

American Journai of Science (4), VIII, 401-411, 1899.

Johns Hopkins Unlversity Circulars No. 143, pp. 17-20, 1900.

BIBLIOGRAPHY

DESCRIPTION OF THE DIVIDING ENGINES DESIGNED BY PROFESSOR ROWLAND

INDEX. 


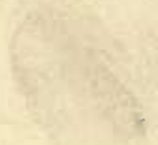




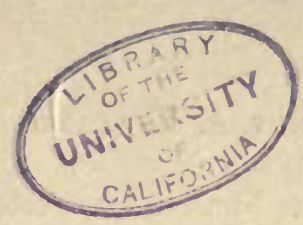

\title{
HENRY A. ROWLAND
}

\section{COMMEMORATIVE ADDRESS}

$\mathrm{BY}$

\author{
DR. THOMAS C. MENDENHALL
}

[Delivered before an assembly of friends, Baltimore, October 26, 1901.]

In reviewing the scientific work of Professor Rowland one is most impressed by its originality. In quantity, as measured by printed page or catalogue of titles, it has been exceeded by many of his contemporaries; in quality it is equalled by that of only a very, very small group. The entire collection of his important papers does not exceed thirty or forty in number and his unimportant papers were few. When, at the unprecedentedly early age of thirty-three years, he was elected to membership in the National Academy of Sciences, the list of his published contributions to science did not contain over a dozen titles, but any one of not less than a half-dozen of these, including what may properly be called his very first original investigation, was of such quality as to fully entitle him to the distinction then conferred.

Fortunately for him, and for science as well, he liked during a period of almost unparalleled intellectual activity, and his work was done during the last quarter of that century to which we shall long turn with admiration and wonder. During these twenty-five years the number of industrious cultivators of his own favorite field increased enormously, due in large measure to the stimulating effect of his own enthusiasm, and while there was only here and there one possessed of the divine afflatus of true genius, there were many ready to labor most assiduously in fostering the growth, development, and final fruition of germs which genius stopped only to plant. A proper estimate of the magnitude and extent of Rowland's work would require, therefore, a careful examination, analytical and historical, of the entire mass of contributions to physical science during the past twenty-five years, many of his own being fundamental in character and far-reaching in their influence upon the trend of thought, in theory and in practice. But it was 
quality, not quantity, that he himself most esteemed in any performance; it was quality that always commanded his admiration or excited him to keenest criticism; no one recognized more quickly than he a real gem, however minute or fragmentary it might be, and by quality rather than by quantity we prefer to judge his work to-day, as he would himself have chosen.

Rowland's first contribution to the literature of science took the form of a letter to The Scientific American, written in the early Autumn of 1865 , when he was not yet seventeen years old. Much to his surprise this letter was printed, for he says of it, "I wrote it as a kind of joke and did not expect them to publish it." Neither its humor nor its sense, in which it was not lacking, seems to have been appreciated by the editor, for by the admission of certain typographical errors he practically destroyed both. The embryo physicist got nothing but a little quiet amusement out of this, but in a letter of that day he declares his intention of some time writing a sensible article for the journal that so unexpectedly printed what he meant to be otherwise. This resolution he seems not to have forgotten, for nearly six years later there appeared in its columns what was, as far as is known, his second printed paper and his first serious public discussion of a scientific question. It was a keen criticism of an invention which necessarily involved the idea of perpetual motion, in direct conflict with the great law of the Conservation of Energy which Rowland had already grasped. It was, as might be expected, thoroughly well done, and received not a little complimentary notice in other journals. This was in 1871, the year following that in which he was graduated as a Civil Engineer from the Rensselaer Polytechnic Institute, and the article was written while in the field at work on a preliminary railroad survey. A year later, having returned to the Institute as instructor in physics, he published in the Journal of the Franklin Institute an article entitled "Illustrations of Resonances and Actions of a Similar Nature," in which he described and discussed various examples of resonance or "sympathetic" vibration. 'This paper, in a way, marks his admission to the ranks of professional students of science and may be properly considered as his first formal contribution to scientific literature; his last was an exhaustive article on spectroscopy, a subject of which he, above all others, was master, prepared for a new edition of the Encyclopædia Britannica, not yet published. Early in 1873 the American Journal of Science printed a brief note by Rowland on the spectrum of the Aurora, sent in response to a kindly and always appreciated letter from Pro- 
fessor George F. Barker, one of the editors of that journal. It is interesting as marking the beginning of his optical work. For a year, or perhaps for several years previous to this time, however, he had been busily engaged on what proved to be, in its influence upon his future career, the most important work of his life. To climb the ladder of reputation and success by simple, easy steps might have contented Rowland, but it would have been quite out of harmony with his bold spirit, his extraordinary power of analysis and his quick recognition of the relation of things. By the aid of apparatus entirely of his own construction and by methods of his own devising, he had made an investigation both theoretical and experimental of the magnetic permeability and the maximum magnetization of iron, steel and nickel, a subject in which he had been interested in his boyhood. On June 9, 1873 , in a letter to his sister, he says: "I have just sent off the results of my experiments to the publisher and expect considerable from it; not, however, filthy lucre, but good, substantial reputation." What he did get from it, at first, was only disappointment and discouragement. It was more than once rejected because it was not understood, and finally he ventured to send it to Clerk Maxwell, in England, by whose keen insight and profound knowledge of the subject it was instantly recognized and appraised at its full value. Regretting that the temporary suspension of meetings made it impossible for him to present the paper at once to the Royal Society, Maxwell said he would do the next best thing, which was to send it to the Philosophical Magazine for immediate publication, and in that journal it appeared in August, 1873, Maxwell himself having corrected the proofs to avoid delay. The importance of the paper was promptly recognized by European physicists, and abroad, if not at home, Rowland at once took high rank as an investigator.

In this research he unquestionably anticipated all others in the discovery and announcement of the beautifully simple law of the magnetic circuit, the magnetio analogue of $\mathrm{Ohm}$ 's law, and thus laid the foundation for the accurate measurement and study of magnetic permeability, the importance of which, both in theory and practice during recent years, it is difficult to overestimate. It has always seemed to me that when consideration is given to his age, his training, and the conditions under which his work was done, this early paper gives a better measure of Rowland's genius than almost any performance of his riper years. During the next year or two he continued to work along the same lines in Troy, publishing not many, but occasional, 
additions to and developments of his first magnetic research. There was also a paper in which he discussed Kohlrausch's determination of the absolute value of the Siemens unit of electrical resistance, foreshadowing the important part which he was to play in later years in the final establishment of standards for electrical measurement.

In 1875, having been appointed to the professorship of physics in the Johns Hopkins University, the faculty of which was just then being organized, he visited Europe, spending the better part of a year in the various centres of scientific activity, including several months at Berlin in the laboratory of the greatest Continental physicist of his time, von Helmholtz. While there he made a very important investigation of the magnetic effect of moving electrostatic charges, a question of first rank in theoretical interest and significance. His manner of planning and executing this research made a marked impression upon the distinguished Director of the laboratory in which it was done, and, indeed, upon all who had any relations with Rowland during its progress. He found what von Helmholtz himself had sought for in vain, and when the investigation was finished in a time which seemed incredibly short to his more deliberate and painstaking associates, the Director not only paid it the compliment of an immediate presentation to the Berlin Academy, but voluntarily met all expenses connected with its execution.

The publication of this research added much to Rowland's rapidlygrowing reputation, and because of that fact, as well as on account of its intrinsic value, it is important to note that his conclusions have been held in question, with varying degrees of confidence, from the day of their announcement to the present. The experiment is one of great difficulty and the effect to be looked for is very small and therefore likely to be lost among unrecognized instrumental and observational errors. It was characteristic of Rowland's genius that with comparatively crude apparatus he got at the truth of the thing in the very start. Others who have attempted to repeat his work have not been uniformly successful, some of them obtaining a wholly negative result, even when using apparatus apparently more complete and effective than that first employed by Rowland. Such was the experience of Lecher in 1884, but in 1888 Roentgen confirmed Rowland's experiments, detecting the existence of the alleged effect. The result seeming to be in doubt, Rowland himself, assisted by Hutchinson, in 1889 took it up again, using essentially his original method but employing more elaborate and sensitive apparatus. They not only confirmed the early experiments, 
but were able to show that the results were in tolerably close agreement with computed values. The repetition of the experiment by Himstedt in the same year resulted in the same way, but in 1897 the genuineness of the phenomenon was again called in question by a series of experiments made at the suggestion of Lippmann, who had proposed a study of the reciprocal of the Rowland effect, according to which variations of a magnetic field should produce a movement of an electrostatically charged body. This investigation, carried out by Crémieu, gave an absolutely negative result, and because the method was entirely different from that employed by Rowland and, therefore, unlikely to be subject to the same systematic errors, it naturally had much weight with those who doubted his original conclusions. Realizing the necessity for additional evidence in corroboration of his riews, in the Fall of the year 1900, the problem was again attacked in his own laboratory and he had the satisfaction, only a short time before his death, of seeing a complete confirmation of the results he had announced a quarter of a century earlier, concerning which, however, there had never been the slightest doubt in his own mind. It is a further satisfaction to his friends to know that a very recent investigation at the Tefferson Physical Laboratory of Harvard University, in which Rowland's methods were modified so as to meet effectively the objections made by his critics, has resulted in a complete verification of his conclusions.

On his return from Europe, in 1876, his time was much occupied with the beginning of the active duties of his professorship, and especially in putting in order the equipment of the laboratory over which he was to preside, much of which he had ordered while in Europe. In its arrangement great, many of his friends thought undue, prominence was given to the workshop, its machinery, tools, and especially the men who were to be employed in it. He planned wisely, however, for he meant to see to it that much, perhaps most, of the work under his direction should be in the nature of original investigation, for the successful execution of which a well-manned and equipped workshop is worth more than a storehouse of apparatus already designed and used by others.

He shortly found leisure, however, to plan an elaborate research upon the Mechanical Equivalent of Heat, and to design and supervise the construction of the necessary apparatus for a determination of the numerical value of this most important physical constant, which he determined should be exhaustive in character and, for some time to 
come, at least, definitive. While this work lacked the elements of originality and boldness of inception by which many of his principal researches are characterized, it was none the less important. While doing over again what others had done before him, he meant to do it, and did do it, on a scale and in a way not before attempted. It was one of the great constants of nature, and, besides, the experiment was one surrounded by difficulties so many and so great that few possessed the courage to undertake it with the deliberate expectation of greatly excelling anything before accomplished. These things made it attractive to Rowland.

The overthrow of the materialistic theory of heat, accompanied as it was by the experimental proof of its real nature, namely, that it is essentially molecular energy, laid the foundation for one of those two great generalizations in science which will ever constitute the glory of the nineteenth century. The mechanical equivalent of heat, the number of units of work necessary to raise one pound of water one degree in temperature, has, with much reason, been called the Golden Number of that century. Its determination was begun by an American, Count Rumford, and finished by Rowland nearly a hundred years later. In principle the method of Rowland was essentially that of Rumford. The first determination was, as we now know, in error by nearly 40 per cent; the last is probably accurate within a small fraction of 1 per cent. Rumford began the work in the ordnance foundry of the Elector of Bavaria at Munich, converting mechanical energy into heat by means of a blunt boring tool in a cannon surrounded by a definite quantity of water, the rise in temperature of which could be measured. Rowland finished it in an establishment founded for and dedicated to the increase and diffusion of knowledge, aided by all the resources and refinements in measurement which a hundred years of exact science had made possible. As the mechanical theory of heat was the germ out of which grew the principle of the conservation of energy, an exact determination of the relation of work and heat was necessary to a rigorous proof of that principle, and Joule, of Manchester, to whom belongs more of the credit for this proof than to any other one man or, perhaps, to all others put together, experimented on the mechanical equivalent of heat for more than forty years. He employed various methods, finally recurring to the early method of heating water by friction, improving on Rumford's device by creating friction in the water itself. Joule's last experiments were made in 1878, and most of Rowland's work was done in the year following. It excelled that of 
Joule, not only in the magnitude of the quantities to be observed, but especially in the greater attention given to the matter of thermometry. In common with Joule and other previous investigators, he made use of mercury thermometers, but this was only for convenience, and they were constantly compared with an air thermometer, the results being finally reduced to the absolute scale. By experimenting with water at different initial temperatures he obtained slightly different values for the mechanical equivalent of heat, thus establishing beyond question the variability of the specific heat of water. Indeed, so carefully and accurately was the experiment worked out that he was able to draw the variation curve and to show the existence of a minimum value at 30 degrees $\mathrm{C}$.

This elaborate and painstaking research, which is now classical, was everywhere awarded high praise. It was published in full by the American Academy of Arts and Sciences with the aid of a fund originally established by Count Rumford, and in 1881 it was crowned as a prize essay by the Venetian Institute. Its conclusions have stood the test of twenty years of comparison and criticism.

In the meantime, Rowland's interest had been drawn, largely perhaps through his association with his then colleague, Professor Hastings, toward the study of light. He was an early and able exponent of Maxwell's Magnetic Theory and he published important theoretical discussions of electro-magnetic action. Recognizing the paramount importance of the spectrum as a key to the solution of problems in ether physics, he set about improving the methods by which it was produced and studied, and was thus led into what will probably always be regarded as his highest scientific achievement.

At that time, the almost universally prevailing method of studying the spectrum was by means of a prism or a train of prisms. But the prismatic spectrum is abnormal, depending for its character largely upon the material made use of. The normal spectrum as produced by a grating of fine wires or a close ruling of fine lines on a plane reflecting or transparent surface had been known for nearly a hundred years, and the colors produced by scratches on polished surfaces were noted by Robert Boyle, more than two hundred years ago. Thomas Young had correctly explained the phenomenon according to the undulatory theory of light, and gratings of fine wire and, later, of rulings on glass were used by Fraunhofer who made the first great study of the dark lines of the solar spectrum. Imperfect as these gratings were, Fraunhofer succeeded in making with them some remarkably good measures 
of the length of light waves, and it was everywhere admitted that for the most precise spectrum measurements they were indispensable. In their construction, however, there were certain mechanical difficulties which seemed for a time to be insuperable. There was no special trouble in ruling lines as close together as need be; indeed, Nobert, who was long the most successful maker of ruled gratings, had succeeded in putting as many as a hundred thousand in the space of a single inch. The real difficulty was in the lack of uniformity of spacing, and on uniformity depended the perfection and purity of the spectrum produced. Nobert jealously guarded his machine and method of ruling gratings as a trade secret, a precaution hardly worth taking, for before many years the best gratings in the world were made in the United States. More than thirty years ago an amateur astronomer, in New York City, a lawyer by profession, Lewis M. Rutherfurd, became interested in the subject and built a ruling engine of his own design. In this machine the motion of the plate on which the lines were ruled was produced at first by a somewhat complicated set of levers, for which a carefully made screw was afterwards substituted. Aided by the skill and patience of his mechanician, Chapman, Rutherfurd continued to improve the construction of his machine until he was able to produce gratings on glass and on speculum metal far superior to any made in Europe. The best of them, however, were still faulty in respect to uniformity of spacing, and it was impossible to cover a space exceeding two or three square inches in a satisfactory manner. When Rowland took up the problem, he saw, as, indeed, others had seen before him, that the dominating element of a ruling machine was the screw by means of which the plate or cutting tool was moved along. The ruled grating would repeat all of the irregularities of this screw and would be good or bad just as these were few or many. The problem was, then, to make a screw which would be practically free from periodic and other errors, and upon this problem a vast amount of thought and experiment had already been expended. Rowland's solution of it was characteristic of his genius; there were no easy advances through a series of experiments in which success and failure mingled in varying proportions; "fire and fall back" was an order which he neither gave nor obeyed, capture by storm being more to his mind. He was by nature a mechanician of the highest type, and he was not long in devising a method for removing the irregularities of a screw, which astonished everybody by its simplicity and by the all but absolute perfection of its results. Indeed, the rery first screw made by this process ranks 
to-day as the most perfect in the world. But such an engine as this might only be worked up to its highest efficiency under the most favorable physical conditions, and in its installation and use the most careful attention was given to the elimination of errors due to variation of temperature, earth tremors, and other disturbances. Not content, however, with perfecting the macbinery by which gratings were ruled, Rowland proceeded to improve the form of the grating itself, making the capital discovery of the concave grating, by means of which a large part of the complex and otherwise troublesome optical accessories to the diffraction spectroscope might be dispensed with. Calling to his aid the wonderful skill of Brashear in making and polishing plane and concave surfaces, as well as the ingenuity and patience of Schneider, for so many years his intelligent and loyal assistant at the lathe and workbench, he began the manufacture and distribution, all too slowly for the anxious demands of the scientific world, of those beautifully simple instruments of precision which have contributed so much to the advance of physical science during the past twenty years. While willing and anxious to give the widest possible distribution to these gratings, thus giving everywhere a new impetus to optical research, Rowland meant that the principal spoils of the victory should be his, and to this end he constructed a diffraction spectrometer of extraordinary dimensions and began his classical researches on the Solar Spectrum. Finding photography to be the best means of reproducing the delicate spectral lines shown by the concave grating, he became at once an ardent student and, shortly, a master of that art. The outcome of this was that wonderful "Photographic Map of the Normal Solar Spectrum," prepared by the use of concave gratings six inches in diameter and twenty-one and a half feet radius, which is recognized as a standard everywhere in the world. As a natural supplement to this he directed an elaborate investigation of absolute wave-lengths, undertaking to give, finally, the wave-length of not only every line of the solar spectrum, but also of the bright lines of the principal elements, and a large part of this monumental task is already completed, mostly by Rowland's pupils and in his laboratory.

Time will not allow further expositions of the important consequences of his invention of the ruling engine and the concave grating.

Indeed, the limitations to which I must submit compel the omission of even brief mention of many interesting and valuable investigations relating to other subjects begun and finished during these years of activity in optical research, many of them by Rowland himself and 
many of them by his pupils, working out his suggestions and constantly stimulated by his enthusiasm. A list of titles of papers emanating from the physical laboratory of the Johns Hopkins University during this period would show somewhat of the great intellectual fertility which its director inspired, and would show, especially, his continued interest in magnetism and electricity, leading to his important investigations relating to electric units and to his appointment as one of the United States Delegates at important International Conventions for the better determination and definition of these units. In 1883 a committee appointed by the Electrical Congress of 1881, of which Rowland was a member, adopted 106 centimetres as the length of the mercury column equivalent to the absolute ohm, but this was done against his protest, for his own measurements showed that this was too small by about three-tenths of one per cent. His judgment was confirmed by the Chamber of Delegates of the International Congress of 1893, of which Rowland was himself President, and by which definitive values were given to a system of international units.

Rowland's interest in applied science cannot be passed over, for it was constantly showing itself, often, perhaps, unbidden, an unconscious bursting forth of that strong engineering instinct which was born in him, to which he often referred in familiar discourse, and which would unquestionably have brought him great success and distinction had he allowed it to direct the course of his life. Although everywhere looked upon as one of the foremost exponents of pure science, his ability as an engineer received frequent recognition in his appointment as expert and counsel in some of the most important engineering operations in the latter part of the century. He was an inventor, and might easily have taken first rank as such had he chosen to devote himself to that sort of work. During the last few years of his life he was much occupied with the study of alternating electric currents and their application to a system of rapid telegraphy of his own invention. A year ago his system received the award of a grand prix at the Paris Exposition, and only a few weeks after his death the daily papers published cablegrams from Berlin announcing its complete success as tested between Berlin and Hamburg, and also the intention of the German Postal Department to make extensive use of it.

But behind Rowland, the profound scholar and original investigator, the engineer, mechanician and inventor, was Rowland the man, and any estimate of his influence in promoting the interests of physical science during the last quarter of the nineteenth century would be 
quite inadequate if not made from that point of view. Born at Honesdale, Pennsylvania, on November 2\%, 1848, he had the misfortune, at the age of 11 years, to lose his father by death. This loss was made good, as far as it is possible to do so, by the loving care of mother and sisters during the years of his boyhood and youthful manhood. From his father he inherited his love for scientific study, which from the very' first seems to have dominated all of his aspirations, directing and controlling most of his thoughts. His father, grandfather, and greatgrandfather were all clergymen and graduates of Yale College. His father, who is described as one "interested in chemistry and natural philosophy, a lover of nature and a successful trout-fisherman," had felt, in his early youth, some of the desires and ambitions that afterward determined the career of his distinguished son, but yielding, no doubt, to the influence of family tradition and desire, he followed the lead of his ancestors. It is not unlikely, and it would not have been unreasonable, that similar hopes were entertained in regard to the future of young Henry, and his preparatory school work was arranged with this in view. Before being sent away from home, however, he had quite given himself up to chemical experiments, glass-blowing and other similar occupations, and the members of his family were often summoned by the enthusiastic boy to listen to lectures which were fully illustrated by experiments, not always free from prospective danger. His spare change was invested in copper wire and the like, and his first five-dollar bill brought him, to his infinite delight, a small galvanic battery. The sheets of the New York Observer, a treasured family newspaper, he converted into a huge hot-air balloon, which, to the astonishment of his family and friends, made a brilliant ascent and flight, coming to rest, at last, and in flames, on the roof of a neighboring house, and resulting in the calling out of the entire fire department of the town. When urged by his boy friends to hide himself from the rather threatening consequences of his first experiment in aeronautics, he courageously marched himself to the place where his balloon had fallen, saying, "No! I will go and see what damage I have done." When a little more than sixteen years old, in the spring of 1865 , he was sent to Phillips Academy at Andover, to be fitted for entering the academic course at Yale. His time there was given entirely to the study of Latin and Greek, and he was in every way out of harmony with his environment. He seems to have quickly and thoroughly appreciated this fact, and his very first letter from Andover is a cry for relief. "Oh, take me home!" is the boyish scrawl covering the last 
page of that letter, on another of which he says, "It is simply horrible; I can never get on here." It was not that he could not learn Latin and Greek if he was so minded, but that he had long ago become wholly absorbed in the love of nature and in the study of nature's laws, and the whole situation was to his ambitious spirit most artificial and irksome. Time did not soften his feelings or lessen his desire to escape from such uncongenial surroundings, and, at his own request, Dr. Farrand, Principal of the Academy at Newark, New Jersey, to which city the family had recently removed, was consulted as to what ought to. be done. Fortunately for everybody, his advice was that the boy ought to be allowed to follow his bent, and, at his own suggestion, he was sent, in the autumn of that year, to the Rensselaer Polytechnic Institute at Troy, where he remained five years, and from which he was graduated as a Civil Engineer in 1870.

It is unnecessary to say that this change was joyfully welcomed by young Rowland. At Andover the only opportunity that had offered for the exercise of his skill as a. mechanic was in the construction of a somewhat complicated device by means of which he outwitted some of his schoolmates in an early attempt to haze him and in this he took no little pride. At Troy he gave loose rein to his ardent desires, and his career in science may almost be said to begin with his entrance upon his work there and before he was seventeen years old.

He made immediate use of the opportunities afforded in Troy and its neighborhood for the examination of machinery and manufacturing processes, and one of his earliest letters to his friends contained a clear and detailed description of the operation of making railroad iron, the rolls, shears, saws, and other special machines being represented in uncommonly well executed pen drawings. One can easily see in this letter a full confirmation of a statement that he occasionally made later in life, namely, that he had never seen a machine, however complicated it might be, whose working he could not at once comprehend. In another letter, written within a few weeks of his arrival in Troy, he shows in a remarkable way his power of going to the root of things which even at that early age was sufficiently in evidence to mark him for future distinction as a natural philosopher. On the river he saw two boats equipped with steam pumps, engaged in trying to raise a half-sunken canal boat by pumping the water out of it. He described engines, pumps, etc., in much detail, and adds, "But there was one thing that I did not like about it; they had the end of their discharge pipe about ten feet above the water so that they had to overcome a 
pressure of about five pounds to the square inch to raise the water so ligh, and yet they let it go after they got it there, whereas if they had attached a pipe to the end of the discharge pipe and let it hang down into the water, the pressure of water on that pipe would just have balanced the five pounds to the square inch in the other, so that they could have used larger pumps with the same engines and thus have got more water out in a given time."

The facilities for learning physics, in his day, at the Rensselaer Polytechnic Institute were none of the best, a fact which is made the subject of keen criticism in his home correspondence, but he made the most of whatever was available and created opportunity where it was lacking. The use of a turning lathe and a few tools being allowed, he spent all of his leisure in designing and constructing physical apparatus of various kinds with which he experimented continually. All of his spare money goes into this and he is always wishing he had more. While he pays without grumbling his share of the expense of a class supper, he cannot help declaring that "it is an awful price for one night's pleasure; why, it would buy another galvanic battery." During these early years his pastime was the study of magnetism and electricity, and his lack of money for the purchase of insulated wire for electro-magnetic apparatus led him to the invention of a method of winding naked copper wire, which was later patented by some one else and made much of. Within six months of his entering the Institute he had made a delicate balance, a galvanometer, and an electrometer, besides a small induction coil and several minor pieces. A few weeks later he announces the finishing of a Ruhmkorff coil of considerable power, a source of much delight to him and to his friends. In December, 1866, he began the construction of a small but elaborately designed steam engine which ran perfectly when completed and furnished power for his experiments. A year later he is full of enthusiasm over an investigation which he wishes to undertake to explain the production of electricity when water comes in contact with red-hot iron, which he attributes to the decomposition of a part of the water. Along with all of this and much more he maintains a good standing in his regular work in the Institute, in some of which he is naturally the leader. He occasionally writes:- "I am head of my class in mathematics," - or "I lead the class in Natural Philosophy," but official records show that he was now and then "conditioned" in subjects in which he had no special interest. As early as 1868, before his twentieth birthday, he decided that he must devote his life to science. While not doubting his ability 
"to make an excellent engineer" as he declares, he decides against engineering, saying, "You know that from a child I have been extremely fond of experiment; this liking instead of decreasing has gradually grown upon me until it has become a part of my nature, and it would be folly for me to attempt to give it up; and I don't see any reason why I should wish it, unless it be avarice, for I never expect to be a rich man. I intend to devote myself hereafter to science. If she gives me wealth, I wili receive it as coming from a friend, but if not, I will not murmur."

He realized that his opportunity for the pursuit of science was in becoming a teacher, but no opening in this direction presenting itself he spent the first year after graduation in the field as a civil engineer. This was followed by a not very inspiring experience as instructor in natural science in a Western college, where he acquired, however, experience and useful discipline.

In the spring of 1872 he returned to Troy as instructor in physics, on a salary the amount of which he made conditional on the purchase by the Institute of a certain number of hundreds of dollars' worth of physical apparatus. If they failed in this, as afterward happened, his pay was to be greater, and he strictly held them to the contract. His three years at Troy as instructor and assistant professor were busy, fruitful years. In addition to his regular work he did an enormous amount of study, purchasing for that purpose the most recent and most advanced books on mathematics and physics. He built his electrodynamometer and carried out his first great research. As already stated, this quickly brought him reputation in Europe and what he prized quite as highly, the personal friendship of Maxwell, whose ardent admirer and champion he remained to the end of his life. In April, 1875 , he wrote, "It will not be very long before my reputation reaches this country," and he hoped that this would bring him opportunity to devote more of his time and energy to original research.

This opportunity for which he so much longed was nearer at hand than he imagined. Among the members of the Visiting Board at the West Point Military Academy in June, 1875, was one to whom had come the splendid conception of what was to be at once a revelation and a revolution in methods of higher education. In selecting the first faculty for an institution of learning which, within a single decade, was to set the pace for real unirersity work in America, and whose influence was to be felt in every school and college of the land before the end of the first quarter of a century, Dr. Gilman was guided by an instinct 
which more than all else insured the success of the new enterprise. A few words about Rowland from Professor Michie, of the Military Academy, led to his being called to West Point by telegraph, and on the banks of the Hudson these two walked and talked, "he telling me," Dr. Gilman has said, "his dreams for science and I telling him my dreams for higher education." Rowland, with characteristic frankness, writes of this interview, "Professor Gilman was very much pleased with me," which, indeed, was the simple truth. The engagement was quickly made. Rowland was sent to Europe to study laboratories and purchase apparatus, and the rest is history, already told and everywhere known.

Rowland's personality was in many respects remarkable. Tall, erect and lithe in figure, fond of athletic sports, there was upon his face a certain look of severity which was, in a way, an index of the exacting standard he set for himself and others. It did not conceal, however, what was, after all, his most striking characteristic, namely, a perfectly frank, open and simple straightforwardness in thought, in speech and in action. His love of truth held him in supreme control, and, like Galileo, he had no patience with those who try to make things appear otherwise than as they actually are. His criticisms of the work of others were keen and merciless, and sometimes there remained a sting of which he himself had not the slightest suspicion. "I would not have done it for the world," he once said to me after being told that his pitiless criticism of a scientific paper had wounded the feelings of its author. As a matter of fact he was warm-hearted and generous, and his occasionally seeming otherwise was due to the complete separation, in his own mind, of the product and the personality of the author. $\mathrm{He}$ possessed that rare power, habit in his case, of seeing himself, not as others see him, but as he saw others. He looked at himself and his own work exactly as if he had been another person, and this gave rise to a frankness of expression regarding his own performance which sometimes impressed strangers unpleasantly, but which, to his friends, was one of his most charming qualities. Much of his success as an investigator was due to a firm confidence in his own powers, and in the unerring course of the logic of science which inspired him to cling tenaciously to an idea when once he had given it a place in his mind. At a meeting of the National Academy of Science in the early days of our knowledge of electric generators, he read a paper relating to the fundamental principles of the dynamo. A gentleman who had had large experience with the practical working of dynamos listened to the paper, and at the 
end said to the Academy that unfortunately practice directly contradicted Professor Rowland's theory, to which instantly replied Rowland, "So much the worse for the practice," which, indeed, turned out to be the case.

Like all men of real genius, he had phenomenal capacity for concentration of thought and effort. Of this, one who was long and intimately associated with him remarks, "I can remember cases when he appeared as if drugged from mere inability to recall his mind from the pursuit of all-absorbing problems, and he had a triumphant joy in intellectual achievement such as we would look for in other men only from the gratification of an elemental passion." So completely consumed was he by fires of his own kindling that he often failed to give due attention to the work of others, and some of his public utterances give evidence of this curious neglect of the historic side of his subject.

As a teacher his position was quite unique. Unfit for the ordinary routine work of the class room he taught as more men ought to teach, by example rather than by precept. Says one of his most eminent pupils, "Even of the more advanced students only those who were able to brook severe and searching criticism reaped the full benefit of being under him, but he contributed that which, in a University, is above all teaching of routine, the spectacle of scientific work thoroughly done and the example of a lofty ideal."

Returning home about twenty years ago after an expatriation of several years, and wishing to put myself in touch with the development of methods of instruction in physics and especially in the equipment of physical laboratories, I visited Rowland very soon after, as it happened, the making of his first successful negative of the solar spectrum. That he was completely absorbed in his success was quite evident, but he also seemed anxious to give me such information as I sought. I questioned him as to the number of men who were to work in his laboratory, and although the college year had already begun he appeared to be unable to give even an approximate answer. "And what will you do with them?" I said. "Do with them?" he replied, raising the still dripping negative so as to get a better light through its delicate tracings, "Do with them?-I shall neglect them." The whole situation was intensely characteristic, revealing him as one to whom the work of a drillmaster was impossible, but ready to lead those who would be led and could follow. To be neglected by Rowland was often, indeed, more stimulating and inspiring than the closest personal supervision of men lacking his genius and magnetic fervor. 
In the fulness of his powers, recognized as America's greatest physicist, and one of a very small group of the world's most eminent, he died on April 16,1901, from a disease the relentless progress of which he had realized for several years and opposed with a splendid but quiet courage.

It was Rowland's good fortune to receive recognition during his life in the bestowal of degrees by higher institutions of learning; in election to membership in nearly all scientific societies worthy of note in Europe and America; in being made the recipient of medals of honor awarded by these societies; and in the generously expressed words of his distinguished contemporaries. It will be many years, however, before full measure can be had of his influence in promoting the interests of physical science, for with his own brilliant career, sufficient of itself to excite our profound admiration, must be considered that of a host of other, younger, men who lighted their torches at his flame and who will reflect honor upon him whose loss they now mourn by passing on something of his unquenchable enthusiasm, something of his high regard for pure intellectuality, something of his love of truth and his sweetness of character and disposition. 

SCIENTIFIC PAPERS 



\section{PART I}

EARLY PAPERS 



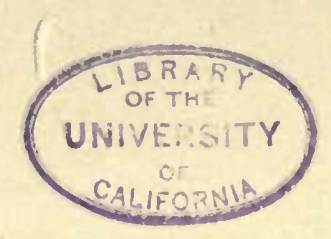

\section{THE VORTEX PROBLEM}

[Scientific American, XIII, 308, 1865]

Messrs. Editors:-In a late number of your paper an inquiry was made why a vortex was formed over the orifice of an outlet ${ }^{1}$ pipe; as, for instance, in a bath tub, when the water is running out. If the water be first started, the explanation will be on the same principle that a ball and string will, if started, wind itself up upon the hand; the ball being attached to the string will, as the string winds up, get nearer the hand, and, consequently, will have less far to go to make one revolution, and thus the momentum, though perhaps not great enough to carry it around in the great circle, is still sufficient to make it revolve in the smaller one.

Therefore, as the string is continually winding up, and the ball continually nearing the hand, it will, if the resistance of the air is not too great, continue to revolve until the string is wound up. Now, in the case of the water, each particle of it will represent the ball, the force of the water rushing toward the outlet will be the string, and, the water running out, and thus causing the particles to come nearer the center at every revolution, will represent the winding-up process. Thus, we see this case is analogous to the preceding, and the same reason that will apply to one will apply to the other. I suppose that some slight motion existing among the particles of the water, united to the motion produced by the outlet, causes the vortex to begin, and, once begun, it will continue until the water is exhausted.

Such motion could either previously exist, or might be produced by the form ${ }^{2}$ of the vessel, which would cause the water, in running to the outlet, to assume a certain direction.

H. A. R.

Troy, N. Y., October, 1865.

'[In the original article this reads "ontlet of an orifice," an obvious misprint.]

"[In the original article this word is "power," an obvious misprint.] 


\section{PAINE'S ELECTRO-MAGNETIC ENGINE}

[Scientific American, $X X V, 21,1871]$

\section{To the Editor of the Scientific American:}

Having noticed several articles in your paper with reference to Paine's electro-magnetic machine, I believe I cannot do better than describe a visit which I paid it about three months ago.

Entering the office in company with a friend, at about twelve o'clock one day, I was told that the machine was not running then, but would be in operation at one. Proceeding there alone, at about that time, I was, after the formality of sending up my name, conducted by a small boy, through numerous by-ways and passages, to the second story of a back building, where I was met by the illustrious inventor and a few select friends. Mr. Paine began by showing the small model machines, which he set in motion by a battery of four cups, of about a gallon capacity each. These models revolved very well, but apparently with no power, for they could be stopped easily. I then began to reason with him on the absurdity of his position, and adduced in my support the experiments of Joule, Mayer, Faraday and others. He, evidently, had no very high opinion of these, and pronounced the conservation of force an old fashioned idea, which had been overthrown in these enlightened days by his "experiments," though what the latter were I have never determined.

After conversing some time, to no purpose, he prepared to overthrow me and my authority at one blow, by an exhibition of The Machine. This was standing in front of a chimney, on one side of the room, with the axis of its wheels parallel to the wall. The wheel to which the magnets were attached was, unlike the models, inclosed in a cast iron case, which enveloped it closely above, but spread out into a rectangular base below. The latter rested directly on the floor. The axis of the wheel projected on each side, and, to one end, a pulley was attached, and to the other, the brake for operating the magnets. The machine had the general appearance of a fan blower with an enlarged pulley. The battery was attached to two binding screws, fixed to a 
standard on the chimney, and the current was supposed to pass from these, along wires, to the break piece, and thence to the magnets. A belt on the pulley connected with a shaft overhead, whence another belt proceeded to the pulley of a small circular saw.

As soon as the connection was made with the battery, the whole apparatus began to move, and soon the saw attained great velocity, shaking the building with violence. The latter effect was caused by a heavy fly wheel on the saw arbor, which probably was not well balanced. When well in motion, boards were applied and sawed with the greatest ease. To show the excess of power, they were sometimes placed on edge and passed over the saw, so as wholly to envelop it, and the cut made from end to end, without the velocity being at all diminished. On throwing off the belt from the saw, the machine still proceeded at the same velocity, with entire indifference to external resistance. On mentioning this to Mr. Paine, he informed me that when the saw was attached, and the resistance greater, the increased pull on the magnets brought them nearer together, by bending the heavy iron frame; and, as magnetic attraction varies inversely as the square of the distance, it only required a small change of distance to account for the increased power. I clearly indicated that I was skeptical on this point, and suggested that it would also work without variation if the power proceeded from some well governed steam engine in the neighborhood. On this he intimated that, if I were not careful, a force might proceed from his body which would act in conjunction with gravitation in causing me to be projected through the window, and strike with violence on the ground below.

The exhibition being over, on going down stairs in company with the rest, I tried the door of the room below, but found it locked, and the windows covered with papers. I desired to get in, but was met with the assurance that the room was rented by a man who was then absent.

This, I believe, is the last visit paid by an outsider to this wonderful invention. I have been there several times since, but there has been no admittance to me, or to any one else. I have since been to the owner of the building, and find that Mr. Paine rents the room to which I sought admittance, and also rents power in that same room, which is directly below that containing his machine. The engine from which the power comes generally stops work at twelve and starts again at one, but sometimes works all day.

My visits there have established the following facts: First, That my friend and I were denied admittance at twelve o'clock, but were 
invited to come at one. Second, That the shaft in the room below does not revolve between the hours of twelve and one. Third, That the room below, containing power, was rented by Mr. Paine, but that he kept it carefully locked, and misguided me as to the tenant. Fourth, That the working parts are concealed in an unnecessarily strong case, well adapted to the concealment of another source of power. Fifth, That part of the apparatus is attached to the wall, so that the machine must always occupy the same position on the floor. Sixth, That the models have not a power proportionate to their size. Seventh, That the machine runs at the same velocity, whether producing one horse power or a fraction of a horse power, and this without a governor.

These are the facts of the case. Where the power of the machine comes from I am unable to say. Is there some secret connection between this machine and the shaft below, and does the battery serve only to make this connection? Or does the battery, when applied, connect the apparatus with a larger battery? I leave these questions to others; but, unless the reasoning and experiments of a host of our greatest men be false, and unless the greatest development of modern science be overthrown, this machine cannot but derive its power from some extraneous source.

In a late communication to your paper, Mr. Paine sets himself up as the peer of Faraday, Tyndall and others, and gives as the reason, his long devotion to science. He evidently does not consider that to be ranked with such men requires something more than devotion; it requires brains; brains to discriminate between true science and quackish nonsense; brains to discover and originate. And pray what fact, among the thousands of science, does Mr. Paine pretend to have proved beyond doubt? Let him answer. As to Mr. Paine's "science," I assert that it is a tissue of error and ignorance, from beginning to end. Even his vaunted invention of metallic foil, wherewith to envelop his magnets or wire, can operate in no other manner than to the detriment of his machine, as any such metallic coating lengthens the demagnetization, which is the very thing to be guarded against. This is due to an induced current, which forms in the coating, and, being in the same direction as the primary current, operates in the same manner to keep up the magnetism. His reason for the machine's keeping at the same velocity also shows great ignorance of the subject. In the first place, the law of magnetic force, under these circumstances, is stated entirely wrong. For this case, the true law is complex, but most nearly approaches to that of inversely as the distance, instead of as the square of 
the distance. (See Joule, and also Tyndall, in the London, Edinburgk and Dublin Philosophical Magazine for 1850.) And, in the second place, approach of the poles would not necessarily increase the efficiency; in this kind of machine there is a distance of maximum efficiency; and if the magnets revolve at a distance greater than this, the attraction becomes too small; and if at a less distance, the times of magnetizing and demagnetizing the magnets become too great, and the machine goes too slowly. The distance in this machine is, undoubtedly, within the limit, for Mr. Paine prides himself upon its smallness, and so further reduction, could it take place, can act in no other manner than the opposite of that claimed. But it is my opinion that all the force brought to bear on the magnets could not move them one twohundredth of an inch, when attached to such a frame.

As to Mr. Paine's disregard for the conservation of force, I have little to say. His assertions are made directly in the face of this principle, and yet he has never adduced one experiment, or even a plausible reason, to prove what he says. He takes you into a building where shafts are revolving by the vulgar power of steam, and directs you to look while he evokes power from nothing. You must not tonch anything; you must not enter the room below; you must not be there while the engine next door is at rest; but you must simply look, and by that renowned maxim of fools, that "seeing is believing," you must believe that the whole structure of science has fallen, and that above its ruins nothing remains but Mr. Paine and his wonderful electro-magnetic machine.

HeNry A. Rowland, C. E.

Newark, N. J. 


\section{ILLUSTRATION OF RESONANCES AND ACTIONS OF A SIMILAR NATURE}

[Journal of the Franklin Institute, XCIV, 275-278, 1872]

At the present day, when scientific education is beginning to take its proper place in the public estimation, anything which can help toward imparting a clear idea of any physical phenomenon becomes important. There are a number of these phenomena, of which resonance is one, which play quite an important part in nature, but which as yet have not been illustrated with sufficient clearness in the lecture-room. Among these are the following: A person carrying water may so time his steps as to produce waves which shall rise and fall in unison with the motion of his body; soldiers in crossing a bridge must not keep step, or they may transmit such a vibration to it as to break it down; window-panes are sometimes cracked by sounding a powerful organpipe to which they can vibrate; a tuning-fork will respond to another of equal pitch sounded near it; and others will readily suggest themselves to the reader. In all these cases we have two bodies which can vibrate in equal times, connected together either directly or by some medium which transmits the motion from one to the other. We can, then, readily reproduce the circumstances in the lecture-room.

The vibrating bodies which I have found most convenient are pendulums; they are easily made, are seen well at a distance, and their time of vibration can be easily and quickly regulated. The apparatus can be prepared in the following manner: Fix a board, about a foot long, in a horizontal position; suspend a piece cf small stiff wire, of equal length, beneath its edge, parallel to it, and an inch or two distant, by means of threads. To one end of the board suspend a pendulum, consisting of a thread about ten or twenty inches long, to which is attached a ball weighing two or three ounces; join the thread of this pendulum to the horizontal wire by taking a turn of it around the wire, so that when the pendulum oscillates, it causes the wire to move back and forth in unison with it. To complete the apparatus, prepare a number of small pendulums by susperding bullets to threads, and let them have small hooks of wire to hang by. 
Having then set the heavy pendulum in motion, hang some of the light ones on the horizontal wire, and note the result: those which are shorter or longer than the heavy one will not be affected, but if any of them are nearly of the same length, they will begin to vibrate to a small extent, but will soon come to rest, after which they will commence again, but stop as before; but if any one happens to be of exactly the proper length, its motion will soon become very great, and immensely surpass in amplitude that of the heavy one, although the motion is derived from it. Of course the heavy pendulum must be retarded in giving motion to the light one, but it is hardly perceptible when there is great difference in the weight. In the same manner a tuning-fork will undoubtedly come to rest sooner when producing resonance than when vibrating freely. To show this retardation more clearly, suspend two pendulums, equal in weight and length, to the edge of a horizontal board, and connect their two threads together by a horizontal thread tied to each at a point an inch or two from the top, and drawn so tight as to pull each of the pendulums a little out of plumb. On starting one of these pendulums the other will gradually move, and finally absorb all the motion from the first, and bring it entirely, to rest; the action will then begin anew, and the motion will be entirely given back to the first ball. This experiment differs from that of resonance, inasmuch as in the case of the pendulums all the motion of the first ball is finally stored up in the second; but in the case of resonance the confined air is constantly giving out its motion to the atmosphere in waves of sound. To imitate this to some extent we must attach a rather large piece of paper to the second pendulum, so that it will meet with resistance, and then both balls will come to rest sooner than otherwise. If one of the balls is only two or three times heavier than the other, they will then also interchange motions; but when the heavy ball has the motion, the arc of its vibration will not be so great as that of the other when it vibrates.

To illustrate the use of Helmholtz resonance globes, or Koenig's apparatus for the analysis of sounds, we can enlarge and modify the first apparatus somewhat. Make the board six or eight feet long, and suspend at one end four or five of the heavy pendulums, and at the other the same number of light ones, each of which corresponds in time of vibration with one of the heavy ones. On now causing any of the heavy pendulums to vibrate, as No. 3, we shall meet with no response from any of the light ones except No. 7. If Nos. 1, 2 and 4 are set going at one time, the wire $A$ will be drawn hither and thither by the 
conflicting pulls with no seeming regularity, but each of the balls 5 , 6 and 8 will pick out from the confused motion the vibration due to itself, and will move in unison, but No. 7 will remain quiet. The short pendulums always produce the effect sooner than the long ones. To remedy this to some extent it is well to bend the wire $A$ into the shape shown in the figure. It is not well to make the pendulum more than twenty inches long, if a quick response is wished. There seems to be no limit to the number of pendulums which can be used or the distance to which the effect can be transmitted, though it is more decided when there are but few pendulums and they are near together. It may sometimes be more convenient to suspend the pendulums from a wire,

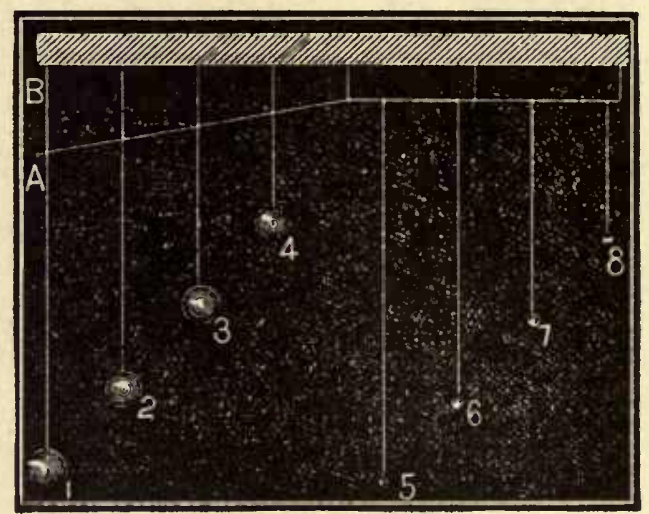

tightly stretched, than from a board. To make the balls visible at a distance, it may be well in some cases to make them of polished steel, and illuminate them by a beam from the electric lamp.

These experiments have many advantages which recommend them to teachers; they can be performed without purchased apparatus, and can be made to illustrate resonance and the kindred phenomena in all their details. Indeed, any one will be well repaid for spending an hour in performing them, simply for their own beauty. 


\section{ON THE AURORAL SPECTRUM}

[American Journal of Science [3], V, 320, 1873]

A letter from Henry A. Rowland, at present Instructor in Physics in the Rensselaer Polytechnic Institute at Troy, informs us that he observed the line of wave-length 431 in the auroral spectrum of last October. He says: "The observations were made with an ordinary chemical spectroscope of one prism, in which the scale was read by means of a lamp. Great care was taken in the readings, and after completing them the spectroscope was set aside until morning, when the readings were taken on the lines of comparison without altering the instrument in any way or even regulating the slit. The wave-lengths of the known lines were taken from Watts's 'Index of Spectra,' but as he does not give the wave-lengths of lines in the flame spectrum I am not quite certain that they are correct." On the scale of his instrument, $\mathrm{Li} \alpha$ was at $13.5^{\circ}, \mathrm{Ca} \alpha 21^{\circ}, \mathrm{Na} \alpha 27.5^{\circ}, \mathrm{Ca} \beta 36^{\circ}, \mathrm{Ca} \gamma 95.5^{\circ}$, and $\mathrm{K} \beta 110^{\circ}$. The aurora lines were as follows:

$\begin{array}{lcc} & \text { Seale-reading. } & \text { Wave-lengths. } \\ 1 & 19 & 628.3 \\ 2 & 35.5 & 554.3 \\ 3 & 95 & 425\end{array}$

"The wave-lengths of the auroral lines were obtained by graphical interpolation on such a large scale as to introduce little or no error." 



\section{PART II}

MAGNETISM AND ELECTRICITY 



\section{ON MAGNETIC PERMEABILITY, ${ }^{2}$ AND THE MAXIMUM OF MAGNETISM OF IRON, STEEL, AND NICKEL}

[Philosophical Magazine [4], XLVI, 140-159, 1873]

More than three years ago I commenced the series of experiments the results of which I now publish for the first time. Many of the facts which I now give were obtained then; but, for satisfactory reasons, they were not published at that time. The investigations were commenced with a view to determine the distribution of magnetism on iron bars and steel magnets; but it was soon found that little could be done without new experiments on the magnetic permeability of substances.

Few observations have been made as yet for determining the magnetic permeability of iron, and none, I believe, of nickel and cobalt, in absolute measure. The subject is important, because in all theories of induced magnetism a quantity is introduced depending upon the magnetic properties of the substance, and without a knowledge of which the problem is of little but theoretical interest; this quantity has always been treated as a constant, although the experiments on the maximum of magnetism show that it is a variable. However, the form of the function has never been determined, except so far as we may deduce it from the equation of Müller,

$$
I=220 d^{3} \tan \frac{m}{.00005 d^{2}},
$$

which, as will be shown, leads to wrong results. The quantities used by different persons are as follows:-

$\kappa$, Neumann's coefficient, or magnetic susceptibility (Thomson).

$k$, Poisson's coefficient.

$\mu$, coefficient of magnetization (Maxwell), or magnetic permeability (Thomson).

$\lambda$, introduced for convenience in the following paper.

IThe word "permeability" has been proposed by Thomson, and has the same meaning as "conductivity" as used by Faraday ("Papers on Electricity and Magnetism,' Thomson, p. 484; Maxwell's 'Electricity and Magnetism,' vol. ii, p. 51.) 
The relations of these quantities are given by the following equations:-

$$
\begin{aligned}
& k=\frac{4 \pi \kappa}{4 \pi \kappa+3}=\frac{\mu-1}{\mu+2}=\frac{\lambda-4 \pi}{\lambda+8 \pi}, \\
& \kappa=\frac{\mu-1}{4 \pi}=\frac{3 k}{4 \pi(1-k)}=\frac{\lambda-4 \pi}{16 \pi^{2}} \\
& \mu=\frac{1+2 k}{1-k}=4 \pi \kappa+1=\frac{\lambda}{4 \pi} .
\end{aligned}
$$

The first determination of the value of any of these quantities was made by Thalén. But more important experiments have been made by Weber, Von Quintus Icilius, and more recently by M. Reicke and Dr. A. Stoletow. ${ }^{2}$ The first three of these in their experiments used long cylindrical rods, or ellipsoids of great length; the last, who has made by far the most important experiments on this subject, has used an iron ring. The method of the ring was first used by Dr. Stoletow in September, 18\%1; but more than eight months before that, in January, 18\%1, I had used the same method, but with different apparatus, to measure the magnetism. He plots a curve showing the variation of $\kappa$; but he plots it with reference to $R$ as abscissa instead of $R \kappa$, and thus fails to determine the law. His method of experiment is much more complicated than mine, so that he could only obtain results for one ring; while by my method I have experimented on about a dozen rings and on numerous bars, so that I believe I have been enabled to find the true form of the function according to which $\mu$ varies with the magnetism of the bar or the magnetizing-force.

Many experiments have been made on the magnetism of iron without giving the results in absolute measure. Among these are the experiments of Müller, Joule, Lenz and Jacobi, Dub, and others. The experiments have been made by the attraction of electromagnets, by the deflection of a compass-needle, or, in one case, by measuring the induced current in a helix extending the whole length of the bar. By the last two methods the change in the distribution of magnetism over the bar when the magnetism of the bar varies is disregarded, if indeed it was thought of at all: even in a recent memoir of M. Cazin ${ }^{3}$ we have the statement made that the position of the poles is independent of the strength of the current. He does not give the experiment from which he deduces this result. Now it is very easy to show, from the formula

2 Phil. Mag., January, 1873.

${ }^{3}$ Annales de Chimie et de Physique, Feb., 1873, p. 171. 
of Green for the distribution of magnetism on a bar-magnet combined with the known variation of $\kappa$, that this can only be true for short and thick bars; and it has also been remarked by Thomson that this should be the case." An experiment made in 1870 places this beyond doubt. A small iron wire (No. 16), 8 inches long, was wound with two layers of fine insulated wire; a small hard steel magnet $\frac{1}{4}$ inch long suspended by a fibre of silk was rendered entirely astatic by a large magnet placed about 2 feet distant; the wire electromagnet was then placed near it, so that the needle hung $1 \frac{1}{2}$ inch from it and about 2 inches back from the end. On now exciting the magnet with a weak current, the needle took up a certain definite position, indicating the direction of the line of force at that point. When the current was very much increased, the needle instantly moved into a position more nearly parallel to the magnet, thus showing that the magnetism was now distributed more nearly at the ends than before. This shows that nearly all the experiments hitherto made on bar-magnets contain an error; but, owing to its small amount, we can accept the results as approximately true.

I believe mine are the first experiments hitherto made on this subject in which the results are expressed and the reasoning carried out in the language of Faraday's theory of lines of magnetic force; and the utility of this method of thinking is shown in the method of experimenting adopted for measuring magnetism. in absolute measure, for which I claim that it is the simplest and most accurate of any yet devised. Whether Faraday's theory is correct or not, it is well known that its use will give correct results; at the present time the tendency of the most advanced thought is toward the theory ${ }^{5}$; and indeed it has been pointed out by Sir William Thomson that it follows, from dynamical reasoning upon the magnetic rotation of the plane of polarization of light, that the medium in which this takes place must itself be in rotation, the axis of rotation being in the direction of the lines of force. Some substances must of necessity be more capable of assuming this rotary motion than others; and hence arises the notion of magnetic "conductivity" and "permeability."

Thomson has pointed out several analogies which may be used in calculating the distribution and direction of the lines of force under rarious circumstances. He has shown that the mathematical treatment

"Papers on Electricity and Magnetism, p. 512.

B “On Action at a Distance," Maxwell, 'Nature,' Feb. 27 and March 6 and 13, 1873.

'Thomson's 'Papers on Electricity and Magnetism,' p. 419, note; and Maxwell's 'Treatise on Electricity and Magnetism,' vol. ii, chap. xxi. 
of magnetism is the same as that of the flow of heat in a solid, as the static induction of electricity, and as the flow of a frictionless incompressible liquid through a porous solid. It is evident that to these analogies we may add that of the conduction of electricity. We readily see that the reason of the treatment being the same in each case is that the elementary law of each is similar to Ohm's law. Mr. Webb ${ }^{8}$ has shown that this law is useful in electrostatics; and I hope, in a sequel to this paper, to apply it to the distribution of magnetism: I give two equations derived in this way further on.

The absolute units to which $I$ have reduced my results are those in which the metre, gramme, and second are the fundamental units. The unit of magnetizing-force of helix I have taken as that of one turn of wire carrying the unit current per metre of length of helix, and is $4 \pi$ times the unit magnetic field. This is convenient in practice, and also because in the mathematical solution of problems in electrodynamics the magnetizing-force of a solenoid naturally comes out in this unit. The magnetizing-force of any helix is reduced to this unit by multiplying the strength of current in absolute units by the number of coils in the helix per metre of length. These remarks apply only to endless solenoids, and to those which are very long compared with their diameter. The unit of number of lines of force $I$ have taken as the number in one square metre of a unit field measured perpendicular to their direction. As my data for reducing my results to these units, I have taken the horizontal force of the earth's magnetism at Troy as 1.641, and the total force as $6 \cdot 2 \%$.

The total force, which will most seriously affect my results, is well "known to be nearly constant at any one place for long periods of time.

From the analogy of a magnet to a voltaic battery immersed in water I have obtained the following, on the assumption that " is constant, and that the resistance to the lines of force passing out into the medium is the same at every point of the bar.

Let $R=$ resistance to lines of force of one metre of length of bar. $R^{\prime}=$ resistance of medium along 1 metre of length of bar.

$Q^{\prime}=$ lines of force in bar at any point.

$Q_{\epsilon}=$ lines of force passing from bar along small distance $I$.

$\varepsilon=$ base of Napierian system of logarithms.

$x=$ distance from one end of helix.

'Maxwell's 'Treatise on Electricity and Magnetism,' arts. 243, 244 and 245.

8 "Application of Obm's Law to Problems in Electrostatics," Phil. Mag. S. 4, vol. $\mathrm{x} \times \mathrm{xv}, \mathrm{p} .325$ (1868). 
$b=$ total length of helix.

$s^{\prime}=$ resistance at end of helix of the rest of bar and medium.

$M=$ magnetizing-force of helix.

We then obtain

$$
\begin{aligned}
& Q_{\epsilon}=\frac{M I}{2 \sqrt{R R^{\prime}}} \frac{1-A}{\left(A \epsilon^{r b}-1\right)}\left(\epsilon^{r x}-\epsilon^{r(b-x)}\right), \ldots . . . . . ~(1) \\
& Q^{\prime}=\frac{\varepsilon^{r b}-1}{\left(A \varepsilon^{r b}-1\right)\left(\sqrt{ } R R^{\prime}-s^{\prime}\right)} \frac{M}{r}-\frac{M}{2 R} \frac{1-A}{A \varepsilon^{r b}-1}\left(\varepsilon^{r b}+1-\varepsilon^{r x}-\varepsilon^{r(b-x)}\right),
\end{aligned}
$$

in which

and

$$
r=\sqrt{\frac{R}{R^{\prime}}}
$$

$$
A=\frac{\sqrt{R R^{\prime}+s^{\prime}}}{\sqrt{R R^{\prime}-s^{\prime}}}
$$

for near the centre of an infinitely long bar, where $x>0$ and $<b$, and $b=\infty$, we have

$$
Q_{\mathrm{e}}=0 \text {, and } Q^{\prime}=\frac{M}{R} \cdots \cdots \cdot .
$$

For a ring-magnet, $s^{\prime}=0$;

$$
\therefore Q_{\varepsilon}=0 \text {, and } Q^{\prime}=\frac{M}{R} . \quad . . . .
$$

And if $a$ is the area of the bar or ring,

$$
a \lambda=\frac{1}{R}=\stackrel{Q^{\prime}}{M}, \text { or } \lambda=\frac{Q^{\prime}}{a M}, \quad . \quad . .
$$

in which $\lambda$ is the same as in the equations previously given. These equations show that we may find the value of $\lambda$, and hence the permeability, by experimenting either on an infinitely long bar or on a ringmagnet. Equations (4) evidently apply to the case where the diameter of the ring is large as compared with its section. The fact given by these equations can be demonstrated in another and, to some persons, more satisfactory manner. If $n$ is the number of coils per metre of helix and $n^{\prime}$ the number on a ring-magnet, $i$ the strength of current, and $\rho$ the distance from the axis of the ring to a given point in the

- Formulæ giving the same distribntion as this have been obtained by Biot and also by Green. See Biot's Traité de Physique, vol. iii, p. 7\%,10 and 'Essay on the Application of Mathematical Analysis to the Theories of Electricity and Magnetism,' by Green, $17 \mathrm{th}$ section.

10[In the original paper this was "vol. iv, p. 669." The correction was made later by Professor Rowland.] 
interior of the ring-solenoid, the magnetic field at that point will, as is well known, be

$$
2 n^{\prime} i \frac{1}{\rho},
$$

and at a point within an infinitely long solenoid

$$
4 \pi i n \text {. }
$$

If the solenoid contain any magnetic material, the field will be for the ring

and for the infinite solenoid

$$
2 n^{\prime} i \frac{\mu}{\rho}
$$

\section{$4 \pi \mu i n$.}

Therefore the number of lines of force in the whole section of a ringmagnet of circular section will be, if $a$ is the mean radius of the ring,

$$
Q^{\prime}=4 n^{\prime} i \mu \int_{-R}^{+R} \frac{\sqrt{R^{2}-x^{2}}}{a-x} d x=4 \pi n^{\prime} i \mu\left(a-\sqrt{a^{2}-R^{2}}\right) ;
$$

or, since $n^{\prime}=2 \pi$ an and $M=i n$, we have, by developing,

$$
Q^{\prime}=4 \pi M_{\mu}\left(\pi R^{2}\right)\left(1+\frac{1}{4} \frac{R^{2}}{a^{2}}+\frac{1}{8} \frac{R^{4}}{a^{4}}+\& c .\right) . .
$$

For the infinite electromagnet we have in the same way for a circular section,

$$
Q^{\prime}=4 \pi M \mu\left(\pi R^{2}\right) . \quad . \quad . \quad . \quad . \quad . \quad . \quad .
$$

When the section of the ring is thin, equation (6) becomes the same as equation (7), and either of them will give

which is the same as equation (5).

$$
\lambda=4 \pi \mu=\frac{Q^{\prime}}{\left(\pi R^{2}\right) M}, \quad . \quad . \quad . \quad . \quad .
$$

In all the rings used the last parenthesis of (6) is so nearly unity that the difference has in most cases been neglected, the slightest change in the quality of the iron producing many times more effect on the permeability than this. Whenever the difference amounted to more than $\frac{1}{200}$ it was not rejected.

The apparatus used to measure $Q^{\prime}$ was based upon the fact discovered by Faraday, that the current induced in a closed circuit is proportional to the number of lines of force cut by the wire, and that the deflection of the galvanometer-needle is also, for small deflections, proportional to that number. In the experiments of $1870-71$ an ordinary astatic galvanometer was used; but in those made this year a galvanometer was 
specially constructed for the purpose. It was on the principle of Thomson's reflecting instrument, but was modified to suit the case by increasing the size of the mirror to $\frac{5}{8}$ of an inch, by adding an astatic needle just above the coil without adding another coil, by loading the needle to make it vibrate slowly, and, lastly, by looking at the reflected image of the scale through a telescope instead of observing the reflection of a lamp on the scale. The galvanometer rested on a firm bracket attached to the wall of the laboratory near its foundation. In most of the experiments the needle made about five single vibrations per minute. The astatic needle was added to prevent any external magnetic force from deflecting the needle; and directive force was given by the magnet above. Each division of the scale was $\cdot 075$ inch long; and the extremities of the scale were reached by a deflection of $7^{\circ}$ in the needle from 0 . The scale was bent to a radius of 4 feet, and was 3 feet from the instrument. At first a correction was made for the resistance of the air, \&c.; but it was afterwards found by experiment that the correction was very exactly proportional to the deflection, and hence could be dispensed with. This instrument gave almost perfect satisfaction; and its accuracy will be shown presently.

The tangent-galvanometer was also a very fine instrument, and was constructed expressly for this series of experiments. The needle was $1 \cdot 1$ inch long, of hardened steel; and its deflections were read on a circle graduated to half degrees, and 5 inches in diameter. The average diameter of the ring was $16 \frac{1}{2}$ inches nearly, and was wound with several coils; so that the sensibility could be increased or diminished at pleasure, and so give the instrument a very wide range. The value of each coil in producing deflection was experimentally determined to within at least $\frac{1}{3}$ of 1 per cent by a method which I shall soon publish. The numbers to multiply the tangent of the deflection by, in order to reduce the current to absolute measure, were as follows:-

\begin{tabular}{|c|c|c|c|c|c|}
\hline Number of & oils. & & & & Multiplier. \\
\hline 1 & & & . & • & $\cdot 05377$ \\
\hline 3 & . & & . & . & $\cdot 01800$ \\
\hline 9 & . & & . & . & $\cdot 006007$ \\
\hline 27 & . & & . & . & $\cdot 002018$ \\
\hline 48 & & & & & $\cdot 001143$ \\
\hline
\end{tabular}

By this instrument I had the means of measuring currents which raried in strength several hundred times with the same accuracy for a large as for a small current. For greater accuracy a correction was . 
applied according to the formula of Blanchet and De ia Prevostaye for the length of the needle, the position of the poles being estimated; this correction in the deflections used was always less than 6 per cent. To eliminate any error in the position of the zero-point, two readings were always taken with the currents in opposite directions, each one being estimated with considerable accuracy to $\frac{1}{10}$ of a degree.

The experiments were carried on in the assay laboratory of the Institute, which was not being used at that time; and precautions were taken that the different parts of the apparatus should not interfere with each other. The disposition of the apparatus is represented in Plate II.

The current from the batiery $A$, of from two to six large Chester's "electropoion" cells No. 2, joined according to circumstances, passed to the commutator $B$, thence to the tangent-galvanometer $C$, thence to another commutator $D$, thence around the magnet $E$ (in this case a ring), and then back through the resistance-coils $K$ to the battery. To measure the magnetism excited in $E$, a small coil of wire $F$ was placed around it, ${ }^{11}$ which connected with the galvanometer $H$, so that, when the magnetism was reversed by the commutator $D$, the current induced in the coil $F$, due to twice cutting the lines of force of the ring, produced a sudden swing of the needle of $H$. As the needle swung very freely and would not of itself come to rest in ten or fifteen minutes, the little apparatus $I$ was added: this consisted of a small horseshoe magnet, on one branch of which was a coil of wire; and by sliding this back and forth, induced currents could be sent through the wire, which, when properly timed, soon brought the needle to rest. This arrangement was very efficient; and without it this form of galvanometer could hardly have been used. To compare the magnetism of the ring with the known magnetism of the earth, and thus reduce it to absolute measure, a ring $G$ supported upon a horizontal surface was included in the circuit; when this was suddenly turned over, it produced an induced current, due to twice cutting the lines of magnetic force which pass through the ring from the earth's magnetism. The induced current in the case of either coil, $F$ or $G$, is proportional to the number of the lines of force cut by the coils ${ }^{12}$ and to the number of wires in the coil, which latter is self erident, but may be deduced from the law of Gaugain. ${ }^{18}$ It is evident, then, that if $c$ is the deflection from coil $G$,

11 If a bar was used, this coil was placed at its centre.

${ }^{12}$ Faraday's Experimental Researches, vol. iii, series 29.

13 Dagnin's Traité de Physique, vol. iii, p. 691. 
and $h$ that from helix $F$, the number of lines of force passing through the magnet $E$, expressed in the unit we have chosen, will be

$$
Q^{\prime}=2 n^{\prime}\left(6 \cdot 27 \sin 74^{\circ} 50^{\prime}\right) \pi R^{2} \frac{1}{c} \frac{h}{2 n}, \ldots . .
$$

where $n^{\prime}$ is the number of coils in the ring $G, n$ the number in the helix $F, R$ the radius of $G, 6 \cdot 27$ the total magnetism of the earth, and $74^{\circ} 50^{\prime}$ the dip. The quantity $2 n^{\prime}\left(6 \cdot 27 \sin 74^{\circ} 50^{\prime}\right) \pi R^{2}$ is constant for the coil, and had the valne $14 \cdot 15$. This is the number of square metres of a unit field which, when cut once by a wire from the galvanometer, would produce the same deflection as the coil when turned over.

The experiments being made by reversing the magnetism of the bars, a rough experiment was made to see whether they had time to change in half a single vibration of the needle; it was found that this varied from sensibly 0 to nearly 1 second, so that there was ample time. It was also proved that the sudden impulse given to the needle by the change of current produced the same deflection as when the change was more gradual, which has also been remarked by Faraday, though he did not use such sudden induced currents. As a test of the method, the horizontal force of the earth's magnetism was determined by means of a vertical coil; it was found to be $1 \cdot 634$, while the true quantity is $1 \cdot 641$.

It is sometimes assumed that some of the action in a case like the present is due to the direct induction of the helix around the magnet on the coil $F$. I think that this is not correct; for when the helix is of fine wire closely surrounding the bar or ring, all the lines of force which affect $F$ must pass through the bar, and so no correction should be made. However, the correction is so small that it will hardly affect the result. If it were to be made, $\frac{Q^{\prime}}{a}$ (equation 5) should be diminished by $4 \pi M$; but, for the above reasons, it has not been subtracted. As a test of the whole arrangement, I have obtained the number of lines of force in a very long solenoid: the mean of two solenoids gave me

$$
Q^{\prime}=12.6 \% M\left(\pi R^{2}\right)
$$

while from theory we obtain, by equation $(7)(\mu=1)$,

$$
Q^{\prime}=12.5 \% M\left(\pi R^{2}\right)
$$

which is within the limits of error in measuring the diameter of the tubes, \&c.

All the rings and bars with which $I$ have experimented have had a circular section. In selecting the iron, care must be used to obtain a 
homogeneous bar; in the case of a ring I believe it is better to have it welded than forged solid; it should then be well annealed, and afterwards have the outside taken off all round to about $\frac{1}{8}$ of an inch deep in a lathe. This is necessary, because the iron is "burnt" to a considerable depth by heating even for a moment to a red heat, and a sort of tail appears on the curve showing the permeability, as seen on plotting Table III. To get the normal curve of permeability, the ring must only be used once; and then no more current must be allowed to pass through the helix than that with which we are experimenting at the time. If by accident a stronger current passes, permanent magnetism is given to the ring, which entirely changes the first part of the curve, as seen on comparing Table I with Table II. The areas of the bars and rings were always obtained by measuring their length or diameter across, and then calculating the area from the loss of weight in water. The following is a list of a few of the rings and bars used, the dimensions being given in metres and grammes. In the fourth column "annealed" means heated to a red heat and cooled in open air, " $\mathrm{C}$ annealed " means placed in a large crucible covered with sand, and placed in a furnace, where, after being heated to redness, the fire was allowed to die out; " natural " means that its temper was not altered from that it had when bought.

\begin{tabular}{|c|c|c|c|c|c|c|c|c|}
\hline $\begin{array}{l}\text { Results } \\
\text { given in } \\
\text { Table. }\end{array}$ & $\begin{array}{l}\text { Quality of } \\
\text { substance. }\end{array}$ & How made. & Temper. & $\begin{array}{l}\text { Spec. } \\
\text { grav. }\end{array}$ & Weight. & $\begin{array}{l}\text { Mean } \\
\text { diam. }\end{array}$ & A rea. & State. \\
\hline I. \{ & $\begin{array}{l}\text { "Burden } \\
\text { best"iron. }\end{array}$ & $\begin{array}{l}\text { Welded and } \\
\text { turned. }\end{array}$ & Annealed. & $7 \cdot 63$ & $148 \cdot 61$ & .0677 & $\begin{array}{c}\cdot 0000 \\
916\end{array}$ & Normal. \\
\hline II. & " " & " " & “" & $7 \cdot 63$ & $148 \cdot 61$ & .0677 & 916 & Magnetic. \\
\hline III. & 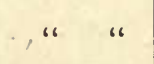 & “ “ \{ & $\begin{array}{l}\text { C an- } \\
\text { nealed. }\end{array}$ & \} $7 \cdot 63$ & $148 \cdot 01$ & .0677 & 912 & Burnt. \\
\hline IV. \{ & $\begin{array}{l}\text { Bessemer } \\
\text { steel. }\end{array}$ & $\begin{array}{l}\text { Turned from } \\
\text { large bar. }\end{array}$ & Natural. & $7 \cdot 84$ & $38 \cdot 34$ & .0420 & 371 & Normal. \\
\hline v. \{ & $\begin{array}{l}\text { Norway } \\
\text { iron }\end{array}$ & $\begin{array}{l}\text { Welded and } \\
\text { turned. }\end{array}$ & $\begin{array}{c}\mathrm{C} \text { an- } \\
\text { nealed. }\end{array}$ & \} $7 \cdot 83$ & $39 \cdot 78$ & .0656 & r695 & Magnetic. \\
\hline VI. \{ & $\begin{array}{c}\text { Cast } \\
\text { nickel. }{ }^{14}\end{array}$ & $\begin{array}{l}\text { Turned from } \\
\text { button. }\end{array}$ & $\ldots$ & $8 \cdot 83$ & $4 \cdot 806$ & .0200 & 0869 & Normal. \\
\hline VII. \{ & $\begin{array}{l}\text { Stubs' } \\
\text { steel. }\end{array}$ & $\begin{array}{c}\text { Hard-drawn } \\
\text { wire. }\end{array}$ & Natural. & $7 \cdot 73$ & $\ldots$ & $\cdots$ & 0969 & Normal. \\
\hline
\end{tabular}

The first three Tables are from the same ring.

Besides these I have used very many other bars and rings; but most of them were made before I had discovered the effect of burning upon

14 Almost chemically pure before melting. 
the iron, and hence did not give a normal curve for high magnetizingpowers. However, I have collected in Table VIII some of the results of these experiments; but I have many more which are not worked up yet.

In the following Tables $Q=\frac{Q^{\prime}}{\pi R^{2}}$ has been measured as previously described. It is evident that if, instead of reversing the current, we simply break it, we shall obtain a deflection due to the temporary magnetism alone. In this manner the temporary magnetism has been measured; and on subtracting this from $Q$, we can obtain the permanent magnetism.

The following abbreviations are made use of in the Tables, the other quantities being the same as previously described.

C.T.G. Number of coils of tangent-galvanometer used.

D.T.G. Deflection of tangent-galvanometer.

D.C. Deflection from coil $G$.

D.F. Deflection from helix $F$ on reversing the current.

Q. Magnetic field in interior of bar (total).

$D . B$. Deflection from $F$ on breaking current.

$T$. Magnetic field of bar due to temporary magnetism.

$P$. Magnetic field of bar due to permanent magnetism.

$n$. Number of coils in helix $F$.

$$
Q=T+P \text {. }
$$

Each observation given is almost always the mean of several. D.T.G. is the mean of four readings, two before and two after the observations on the magnetism; D.C. is the mean of from four to ten readings; D.F. mean of three; D.B. mean of two, except in Table I, where the deflection was read only once. In all these Tables the column containing the temporary magnetism $T$ can only be accepted as approximate, the experiments having been made more to determine $Q$ than $T$.

The value of $n$ was generally varied by coiling a wire more or less around the ring, but leaving its length the same.

The change in the value of D.C. is due to the change in the resistance of the galvanometer from change of temperature, copper wire increasing in resistance about 1 per cent for every $2^{\circ} \cdot 6 \mathrm{C}$. rise. In Table I the temperature first increased slowly, and then, after remaining stationary for a while, fell very fast. 
15 TABLE I.

"Burden Best" Iron, Normal.

\begin{tabular}{|c|c|c|c|c|c|c|c|c|c|c|c|c|c|c|c|}
\hline$\frac{\mathrm{T}}{\mathrm{M}}$ & C.T.G. & D.T.G. & M. & D.C. & $n$. & D.F. & $\frac{\text { D.F. }}{2 n .}$ & $\frac{\text { D. B. }}{n .}$ & Q. & $\lambda$ & $\begin{array}{c}\lambda \\
\text { Calcu- } \\
\text { lated. }\end{array}$ & $\mu=\frac{\lambda}{4 \pi}$ & T. & P. & $\frac{P}{\bar{M}}$ \\
\hline $3627^{\circ}$ & 48 & $4 \cdot 5$ & •1456 & $23 \cdot 4$ & 130 & $6 \cdot 5$ & $\cdot 1083$ & $\cdot 08$ & 715 & 4910 & 5845 & $390 \cdot 7$ & 528 & $187^{\circ}$ & $1284^{\circ}$ \\
\hline $7080^{\circ}$ & .. & $16 \cdot 45$ & $\cdot 5501$ & .. & .. & $54 \cdot 6$ & .910 & $\cdot 59$ & 6005 & 10920 & 10885 & $868 \cdot 7$ & 3894 & 2111. & 3838 . \\
\hline $7746^{\circ}$ & . & $20 \cdot 2$ & . 6815 & & & $87 \cdot 9$ & $1 \cdot 465$ & $\cdot 80$ & 9667 & 14180 & 14074 & 1129 & 5280 & $4387^{\circ}$ & $6437^{\circ}$ \\
\hline 8786. & . & $28 \cdot 6$ & $1 \cdot 011$ & $23 \cdot 3$ & 310 & $74 \cdot 2$ & $3 \cdot 71$ & $1 \cdot 34$ & 24600 & 24330 & 24000 & 1936 & 8882 & 15718 . & $15550^{\circ}$ \\
\hline $8766^{\circ}$ & .. & $31 \cdot 1$ & $1 \cdot 119$ & .. & .. & $88 \cdot 2$ & 4.41 & $1 \cdot 48$ & 29230 & 26120 & 26050 & 2078 & 9811 & $19419^{\circ}$ & $1735^{\circ}$ \\
\hline $8819^{\circ}$ & . & $1 \cdot 9$ & 1.155 & .. & . & $92 \cdot 6$ & $4 \cdot 63$ & $1 \cdot 53$ & 30820 & 26690 & 26660 & 2124 & 10180 & $20640^{\circ}$ & $1780^{\circ}$ \\
\hline ?8205. & . & $41 \cdot 12$ & $1 \cdot 623$ & & 2 & $29 \cdot 8$ & $\because \cdot 45$ & $2 \cdot 0$ & 49590 & $305 \% 0$ & 30740 & 2433 & 13310 & $36280^{\circ}$ & $22370^{\circ}$ \\
\hline $9460^{\circ}$ & 27 & $28 \cdot 35$ & $1 \cdot 766$ & $23 \cdot 1$ & ... & $32 \cdot 8$ & $8 \cdot 20$ & $2 \cdot 5$ & 54820 & 31030 & 31050 & 2470 & 16710 & $38110^{\circ}$ & $21570^{\circ}$ \\
\hline $9517^{\circ}$ & . & $29 \cdot 6$ & $1 \cdot 861$ & $\ddot{0}$ & .. & $34 \cdot 6$ & $8 \cdot 65$ & $2 \cdot 65$ & 57820 & 31070 & 31100 & $24 \% 2$ & 17710 & $40110^{\circ}$ & $21550^{\circ}$ \\
\hline $8812 \cdot$ & - & $3 \cdot 4$ & $2 \cdot 162$ & $23 \cdot 1$ & $\ldots$ & $39 \cdot 8$ & $9 \cdot 95$ & $2 \cdot 85$ & 66510 & $307 \% 0$ & 30756 & 2448 & 19050 & $47460^{\circ}$ & $21950^{\circ}$ \\
\hline $8115^{\circ}$ & . & $37 \cdot 45$ & $2 \cdot 512$ & .. & .. & $44 \cdot 7$ & $11 \cdot 18$ & $3 \cdot 05$ & 74730 & 29750 & 29930 & 2367 & 20390 & $54340^{\circ}$ & $21630^{\circ}$ \\
\hline $7985^{\circ}$ & . & $44 \cdot 45$ & $3 \cdot 223$ & * & . & $53 \cdot 5$ & $13 \cdot 38$ & $3 \cdot 85$ & 89430 & 27750 & 27390 & 2208 & 25740 & $63690^{\circ}$ & $19760^{\circ}$ \\
\hline $6674^{\circ}$ & 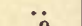 & $52 \cdot 1$ & 4.225 & $\cdots$ & $\therefore$ & $60 \cdot 3$ & $15 \cdot 08$ & $4 \cdot 85$ & 100800 & 23860 & 24730 & 1899 & 32420 & $67380^{\circ}$ & $15950^{\circ}$ \\
\hline 5070 & 9 & $34 \cdot 65$ & $6 \cdot 744$ & & .. & $73 \cdot 1$ & $18 \cdot 28$ & $7 \cdot 10$ & 122700 & 18210 & 18410 & 1448 & 47680 & $75020^{\circ}$ & $11130^{\circ}$ \\
\hline $19^{\circ}$ & . & $39 \cdot 8$ & $8 \cdot 136$ & $23 \cdot 0$ & & $77 \cdot 3$ & $19 \cdot 32$ & $7 \cdot 90$ & 129700 & 15940 & 16130 & 1269 & 53040 & $76660^{\circ}$ & $9423^{\circ}$ \\
\hline 6403 & 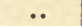 & $44 \cdot 3$ & $9 \cdot 542$ & .. & 1 & $40 \cdot 6$ & $20 \cdot 30$ & $9 \cdot 1$ & 136300 & 14280 & 13920 & 1137 & 61100 & $75200^{\circ}$ & $7881^{\circ}$ \\
\hline $4666^{\circ}$ & .. & $55 \cdot 1$ & $14 \cdot 04$ & . & . & $43 \cdot 5$ & $21 \cdot 75$ & $9 \cdot 8$ & 145400 & 10360 & 10760 & $824^{\cdot 1}$ & 65510 & 79890. & $5690^{\circ}$ \\
\hline $2816^{\circ}$ & 3 & $42 \cdot 95$ & $27 \cdot 18$ & .. & .. & $47 \cdot 4$ & $23 \cdot 70$ & $11 \cdot 5$ & 157700 & 5803 & 6350 & $461 \cdot 8$ & 76540 & $81160^{\circ}$ & $2985^{\circ}$ \\
\hline $2300^{\circ}$ & .. & $51 \cdot 3$ & $36 \cdot 6$ ) & & .. & $49 \cdot 1$ & $24 \cdot 55$ & $12 \cdot 7$ & 162700 & 4445 & 4523 & 353.8 & 84180 & $785 \% 0$ & 2145. \\
\hline 1702 & . & $60 \cdot 15$ & $\begin{array}{r}51 \cdot 18 \\
\infty\end{array}$ & $23 \cdot 4$ & $\cdots$ & $50 \cdot 3$ & $25 \cdot 15$ & $13 \cdot 2$ & $\begin{array}{l}166000 \\
175000\end{array}$ & 3243 & $\begin{array}{c}3310 \\
0\end{array}$ & 258.0 & 87120 & $78880^{\circ}$ & $1541^{\circ}$ \\
\hline
\end{tabular}

TABLE II.

"Burden Best" Iron, Magnetic.

\begin{tabular}{|c|c|c|c|c|c|c|c|}
\hline M. & Q. & $\lambda$. & $\mu$. & M. & Q. & $\lambda$. & $\mu$. \\
\hline$\cdot 1456$ & 426 & 2920 & 232 & $2 \cdot 930$ & 82720 & 28240 & 2247 \\
\hline .5699 & 3346 & 5987 & 476 & $4 \cdot 210$ & 100900 & 23950 & 1906 \\
\hline .6962 & 5700 & 8189 & 652 & $6 \cdot 769$ & 122800 & 18140 & 1444 \\
\hline $1 \cdot 080$ & 24350 & 22550 & 1795 & 7.273 & 124300 & 17090 & 1360 \\
\hline $1 \cdot 191$ & 29280 & 24580 & 1956 & $7 \cdot 626$ & 127100 & 16670 & 1326 \\
\hline 1.537 & 46150 & 30020 & 2389 & $11 \cdot 10$ & 139500 & 12570 & 1000 \\
\hline 1.590 & 49070 & 30260 & 2408 & $13 \cdot 61$ & 144700 & 10630 & 846 \\
\hline 1.933 & 59680 & 30860 & 2456 & $22 \cdot 10$ & 154600 & 6965 & 554 \\
\hline $2 \cdot 377$ & 71660 & 30150 & 2399 & & & & \\
\hline
\end{tabular}

16 TABLE III.

"Burden Best" Iron, Burnt.

\begin{tabular}{|c|c|c|c|c|c|c|c|c|c|c|c|}
\hline M. & Q. & $\lambda$. & $\mu$. & T. & M. & Q. & $\lambda$. & $\mu$. & T. & $P$. & P. \\
\hline$\cdot 143$ & 1001 & 7039 & 560 & 1.020 & 3.810 & 116900 & 30730 & 2446 & & 8 & \\
\hline .5อ3 & 9395 & 16980 & 1351 & 5115 & $4 \cdot 283$ & 120200 & 28060 & 2233 & & $4280^{\circ}$ & \\
\hline$\cdot 682$ & 16550 & $24: 40$ & 1929 & 6835 & $4 \cdot 722$ & 123900 & 26240 & 2088 & 30830 & 9715 & \\
\hline .962 & 37330 & 38780 & 3086 & 94.54 & 6.565 & 133100 & 20270 & 1613 & & 27876 & \\
\hline 1.070 & 42920 & 40130 & 3194 & 10300 & $9 \cdot 326$ & 141200 & 15140 & 1200 & 39810 : & 32620 . & \\
\hline $1 \cdot 153$ & 48830 & 42340 & 3369 & 10530 & 11.00 & 144400 & 13120 & 1045 & & 38300 & \\
\hline $1 \cdot 317$ & 59490 & 45 I 80 & 3595 & 11650 & $13 \cdot 44$ & 147500 & 10970 & 873 & 44070 & 47840 & 103430. \\
\hline $1 \cdot 340$ & 59580 & 44450 & 3538 & $13 \% 00$ & $23 \cdot 41$ & 155500 & 6642 & 529 & 51030 & 45880. & $10 \pm 470$. \\
\hline $2 \cdot 127$ & 90180 & 42400 & 3374 & 18470 & $32 \cdot 73$ & 159400 & 4870 & 387 & & 71710 & \\
\hline $2 \cdot 501$ & 98560 & 39400 & 3136 & 19920 & $32 \cdot 56$ & 158400 & 4864 & 387 & & 78640 & \\
\hline $2 \cdot 864$ & 104000 & 36310 & 2890 & 24600 & $51 \cdot 03$ & 165800 & 3250 & 259 & 56100 & 79400 & 109700 . \\
\hline $3 \cdot 151$ & 108200 & 34330 & 2732 & 24610 & & & & & & 83590 . & \\
\hline
\end{tabular}

${ }^{15}$ [Columans 1, 15, 16 were added to the original paper by Professor Rowland, after its publication.]

${ }^{16}$ [The last two columns of Tables III, IV, V, VII were added by Professor Rowland after the paper was published.] 
16 TABLE IV.

Bessemer Steel, Normal.

\begin{tabular}{|c|c|c|c|c|c|c|c|c|c|c|c|}
\hline M. & Q. & $\lambda$. & $\mu$. & T. & M. & Q. & $\lambda$. & $\mu$. & $\mathrm{T}$. & P. & P. \\
\hline$\cdot 1356$ & 327 & 2412 & 192 & 309 & $2 \cdot 756$ & 39960 & 14500 & 1154 & 13080 & 18. & 26880 . \\
\hline .2793 & 817 & 2995 & 238 & 797 & $3 \cdot 219$ & 950550 & 15700 & 1250 & 16350 & 90. & 34200 . \\
\hline$\cdot 5287$ & 1726 & 3264 & 260 & 1471 & $3 \cdot 551$ & $\mathbf{5 6 3 1 0}$ & 15860 & 1262 & 15980 & 255 . & 40330 . \\
\hline .9398 & 3833 & 4079 & 325 & 3106 & $4 \cdot 469$ & 71380 & 15970 & 1271 & 18340 & 727. & 53040 . \\
\hline $1 \cdot 421$ & 7702 & 5421 & 431 & 5576 & 5.698 & 85530 & 15010 & 1195 & 23610 & 2126 . & 61920 . \\
\hline $1 . \$ 80$ & 14080 & 7487 & 596 & 8972 & $11 \cdot 44$ & 119550 & 10450 & 832 & 28020 & 5108. & 91530 . \\
\hline 1.947 & 15420 & 7920 & 630 & 8938 & $20 \cdot 69$ & 138300 & 6685 & 532 & 41360 & 6482 & 96940. \\
\hline $2 \cdot 300$ & 24830 & 10800 & 859 & 11320 & 38.99 & 153700 & 3942 & 314 & 529301 & $13510^{\circ}$ & 100770 . \\
\hline
\end{tabular}

16 TABLE V.

Norway Iron, Magnetic.

\begin{tabular}{|c|c|c|c|c|c|c|c|c|c|c|c|}
\hline M. & Q. & $\lambda$. & $\mu$. & T. & M. & Q. & $\lambda$. & $\mu$. & T. & P. & P. \\
\hline$\cdot 1344$ & 865 & 6439 & 512 & & $2 \cdot 290$ & 105900 & 46240 & 3680 & 35240 & & $70660^{\circ}$ \\
\hline$\cdot 2673$ & 2550 & 9910 & 759 & 1892 & $4 \cdot 393$ & 134100 & 30520 & 2429 & $\mathbf{5 4 9 7 0}$ & 658. & - 79130 . \\
\hline$\cdot 5161$ & 13000 & 25200 & 2005 & 5857 & $5 \cdot 910$ & 142400 & 24090 & 1917 & 62810 & 7143 & . 79590 . \\
\hline .5572 & 15310 & 27480 & 2187 & 8110 & $7 \cdot 8 \pi 4$ & 149100 & 18940 & 1507 & 68490 & 7200 & . $80610^{\circ}$ \\
\hline$\cdot 6725$ & 30140 & 44820 & 3567 & 8921 & $13 \cdot 77$ & 156800 & 11390 & 906 & 77060 & $21220^{\circ}$ & . 79740 \\
\hline .9305 & 53800 & 57820 & 4602 & 13970 & $26 \cdot 84$ & 165800 & 6038 & 480 & 84710 & 39830 . & . $81090^{\circ}$ \\
\hline $1 \cdot 362$ & 77700 & 57110 & 4545 & 21630 & $36 \cdot 86$ & 168500 & 4572 & 364 & 87860 & 56070 . & . 80740 . \\
\hline $1 \cdot 788$ & 93000 & 52020 & 4140 & 28200 & & & & & & 64800 . & \\
\hline
\end{tabular}

TABLE VI.

Cast Nickel, Normal.

\begin{tabular}{|c|c|c|c|c|c|c|c|c|c|}
\hline M. & Q. & $\lambda$. & $\mu$. & T. & M. & Q. & $\lambda$. & $\mu$. & T. \\
\hline $1 \cdot 433$ & 852 & 595 & $47 \cdot 4$ & & $13 \cdot 43$ & 27100 & 2018 & $160 \cdot 6$ & 11260 \\
\hline 2.904 & 2377 & 819 & $65 \cdot 1$ & & 16.53 & 31050 & 1878 & $149 \cdot 5$ & 13530 \\
\hline $3 \cdot 527$ & 3685 & 1070 & $85 \cdot 1$ & & $21 \cdot 02$ & 34950 & 1663 & $132 \cdot 3$ & 16480 \\
\hline $5 \cdot 555$ & 10080 & 1815 & $144 \cdot 4$ & & $32 \cdot 17$ & 41980 & 1305 & $103 \cdot 8$ & 22300 \\
\hline $6 \cdot 783$ & 13680 & 2017 & 160.5 & 5120 & $33 \cdot 92$ & 42650 & 1257 & $100 \cdot 0$ & 23360 \\
\hline $7 \cdot 401$ & 15270 & 2063 & $164 \cdot 2$ & 5614 & $60 \cdot 91$ & 50860 & 855 & $66 \cdot 4$ & 29540 \\
\hline $9 \cdot 273$ & 19600 & 2114 & $168 \cdot 2$ & 7644 & $82 \cdot 36$ & 53650 & 651 & 51.8 & 33460 \\
\hline 11.78 & 24720 & 2098 & $167 \cdot 0$ & 9902 & $105 \cdot 2$ & 55230 & 525 & 41.8 & 35120 \\
\hline
\end{tabular}

16 TABLE VII.

Stubs' Steel Wire, Normal.

\begin{tabular}{|c|c|c|c|c|c|c|c|c|c|c|c|}
\hline M. & Q. & $\lambda$. & $\mu$. & T. & M. & Q. & $\lambda$. & $\mu$. & T. & P. & P. \\
\hline$\cdot 1673$ & 159 & 953 & $75 \cdot 9$ & & $13 \cdot 65$ & 54300 & 3978 & $316 \cdot 6$ & 20900 & & 33400 . \\
\hline .6237 & 7. 678 & 1087 & $86 \cdot 5$ & 598 & $19 \cdot 35$ & 77770 & 4020 & $319 \cdot 9$ & 29480 & 80 . & 48290 \\
\hline 1.084 & 1197 & 1104 & $87 \cdot 9$ & 1101 & $27 \cdot 431$ & 100800 & 3676 & $292 \cdot 6$ & 38590 & 96 . & 62210 \\
\hline $2 \cdot 043$ & 2448 & 1199 & $95 \cdot 4$ & 2257 & $33 \cdot 391$ & 111300 & 3335 & $265 \cdot 4$ & 45110 & 191. & 66190 \\
\hline $2 \cdot 714$ & 3446 & 1270 & $101 \cdot 0$ & 3095 & 35.581 & 115000 & 3228 & $256 \cdot 9$ & 45950 & 351. & 69050 \\
\hline $4 \cdot 221$ & 6278 & 1487 & $118 \cdot 4$ & 5145 & $38 \cdot 641$ & 119400 & 3092 & $246 \cdot 0$ & 48060 & 1133. & 71340 \\
\hline $10 \cdot 26$ & 33700 & 3286 & $261 \cdot 5$ & 16170 & & & & & & $17530^{\circ}$ & \\
\hline
\end{tabular}


The best method of studying these Tables is to plot them: one method of doing this is to take the value of the magnetizing-force as the abscissa, and that of the permeability as the ordinate; this is the method used by Dr. Stoletow; but, besides making the complete curve infinitely long, it forms a rery irregular curve, and it is impossible to get the maximum of magnetism from it. Another method is to employ the same abscissas, but to use the magnetism of the bar as ordinates; this gives a regular curve, but has the other two disadvantages of the first method; however, it is often employed, and gives a pretty good idea of the action. In Plate II, I have given a plot of Table V with the addition of the residual or permanent magnetism, which shows the general features of these curves as drawn from any of the Tables. It is observed that the total magnetism of the iron at first increases very fast as the magnetizing-force increases, but afterwards more and more slowly until near the maximum of magnetism, where the curve is parallel to the axis of $Q$. The concavity of the curve at its commencement, which indicates a rapid increase of permeability, has been noticed by several physicists, and was remarked by myself in my experiments of January, 1871; it has now been brought most forcibly before the public by Dr. Stoletow, whose paper refers principally to this point. ${ }^{17}$ M. Müller has given an equation of the form

$$
I=220 d^{\frac{3}{2}} \tan \frac{m}{\cdot 00005 d^{2}}
$$

to represent this curve; but it fails to give any concavity to the first part of the curve. A formula of the same form has been used by M. Cazin $;^{18}$ but his experiments carry little weight with them, on account of the small variation of the current which he used, this being only about five times, while $I$ have used a variation in many cases of more than three hundred times.

Weber has obtained, from the theory that the particles of the iron are always magnetic and merely turn round when the magnetizingforce is applied, an equation which would make the first part of the curve coincide with the dotted line in Plate II $;{ }^{10}$ and Maxwell, by addition to the theory, has obtained an equation which replaces the first

17 On the Magnetizing Function of Soft Iron, especially with the weaker decomposing powers. By Dr. A. Stoletow, of the University of Moscow. Translated in the Phil. Mag., January, 1873. See particularly p. 43.

18 Annales de Chimie et de Physique, February 1873, p. 182.

19 This is according to Maxwell's integration of Weber's equation, Weber having made some mistake in the integration. 
part of the curve by the broken line..$^{20}$ I believe that I have obtained at the least a very close approximation to the true equation of the curve, and will show further on that $Q$ and $M$ must satisfy the equation

$$
\frac{Q}{M}=A \sin \left(\frac{Q+a \frac{Q}{M}+H}{D}\right) \ldots . .
$$

It is very probable that Weber's theory may be so modified as to give a similar equation.

Space will not permit me to discuss the curves of temporary and permanent magnetism; but I will call attention to the following facts which the Tables seem to establish.

1. Nearly or quite all the magnetism of a bar is, with weak magnetizingforces, temporary; and this is more apparent in steel than in soft iron.

2. The temporary magnetism increases continually with the current.

3. The permanent magnetism at first increases very fast with the current, but afterwards diminishes as the current increases, when the iron is near its maximum of magnetism. ${ }^{2 t}$

I have now described the methods of plotting the Tables hitherto used; and I will now describe the third, which is, I believe, new. This is by using the values of the magnetism of the bar as abscissas, and those of the permeability as ordinates. In this way we obtain a perfectly regular curve, which is of finite dimensions, and from which the maximum of magnetism can be readily obtained. Plate III shows this method of plotting as applied to Table I. If we draw straight lines across the curve parallel to the axis of $Q$ and mark their centres, we find that they always fall very exactly upon a straight line, which is therefore a diameter of the curve. The curve of nickel shown upon the same Plate has this property in common with iron. I have made several attempts to get a ring of cobalt; but the button has always been too porous to use. However, I hope soon to obtain one, and thus make the law general for all the magnetic metals. There are two equations which may be used to express the curve: one is the equation of an inclined parabola; but this fails for the two ends of the curve; the other is an equation of the general form

$$
\lambda=A \sin \left(\frac{Q+a \lambda+H}{D}\right), . . . .
$$

20 Treatise on Electricity and Magnetism, Maxwell, vol. ii, chap. vi.

${ }^{21}$ The last clanse of this sentence cannot be considered yet as entirely settled, though I have other curves than those shown bere which show it well. [This note was added to the original paper by Professor Rowland.] 
in which $A, H, D$, and $a$ are constants depending upon the kind and quality of the metal used. $A$ is the maximum value of $\lambda$, and gives the height of the curve $E D$, Plate III; $a$ establishes the inclination of the diameter; $H$ is the line $A O$; and $D$ depends upon the line $A C$. The following equation, adapted to degrees and fractions of a degree, is the equation from which the values of $\lambda$ were found, as given in Table I:

$$
\lambda=31 \cdot 100 \sin \left(\frac{Q+\cdot 87 \lambda+5000}{1000}\right) .
$$

The large curve in Plate III was also drawn from this, and the dots added to show the coincidence with observation; it is seen that this is almost perfect. As $\lambda$ enters both sides of the equation, the calculation can only be made by successive approximations. We might indeed solve with reference to $Q$; but in this case some values of $\lambda$ as obtained from experiment may be accidentally greater than $A$, and so give an imaginary value to $Q$.

By plotting any Table in this way and measuring the distance $O C$, we have the maximum of magnetism.

I have given in the same Plate the curve drawn from the observations on the nickel ring with $Q$ on the same scale, but $\lambda$ on a scale four times as large as the other. The curve of nickel satisfies the equation

$$
\lambda=2120 \sin \left(\frac{Q+5 \cdot 35 \lambda+2000}{360}\right)
$$

quite well, but not so exactly as in the case of iron. This ring, when closely examined, was found to be slightly porous, which must have changed the curve slightly, and perhaps made it depart from the equation.

In Table VIII, I have collected some of the values of the constants in the formula when it is applied to the different rings and bars, and have also given some columns showing the maximum of magnetism. When any blank occurs, it is eaused by the fact that for some reason or other the observations were not sufficient to determine it. The values of $a, H, D$, and the value of $\lambda$, when $Q=0$, can in most cases only be considered approximate; for as they all vary so much, I did not think it necessary to calculate them exactly. For comparison, I have plotted Dr. Stoletow's curve and deduced the results given in the Table, of course reducing them to the same units as mine.

It will be observed that the columns headed "maximum of magnetism" contain, besides the maximum magnetic field, two columns 


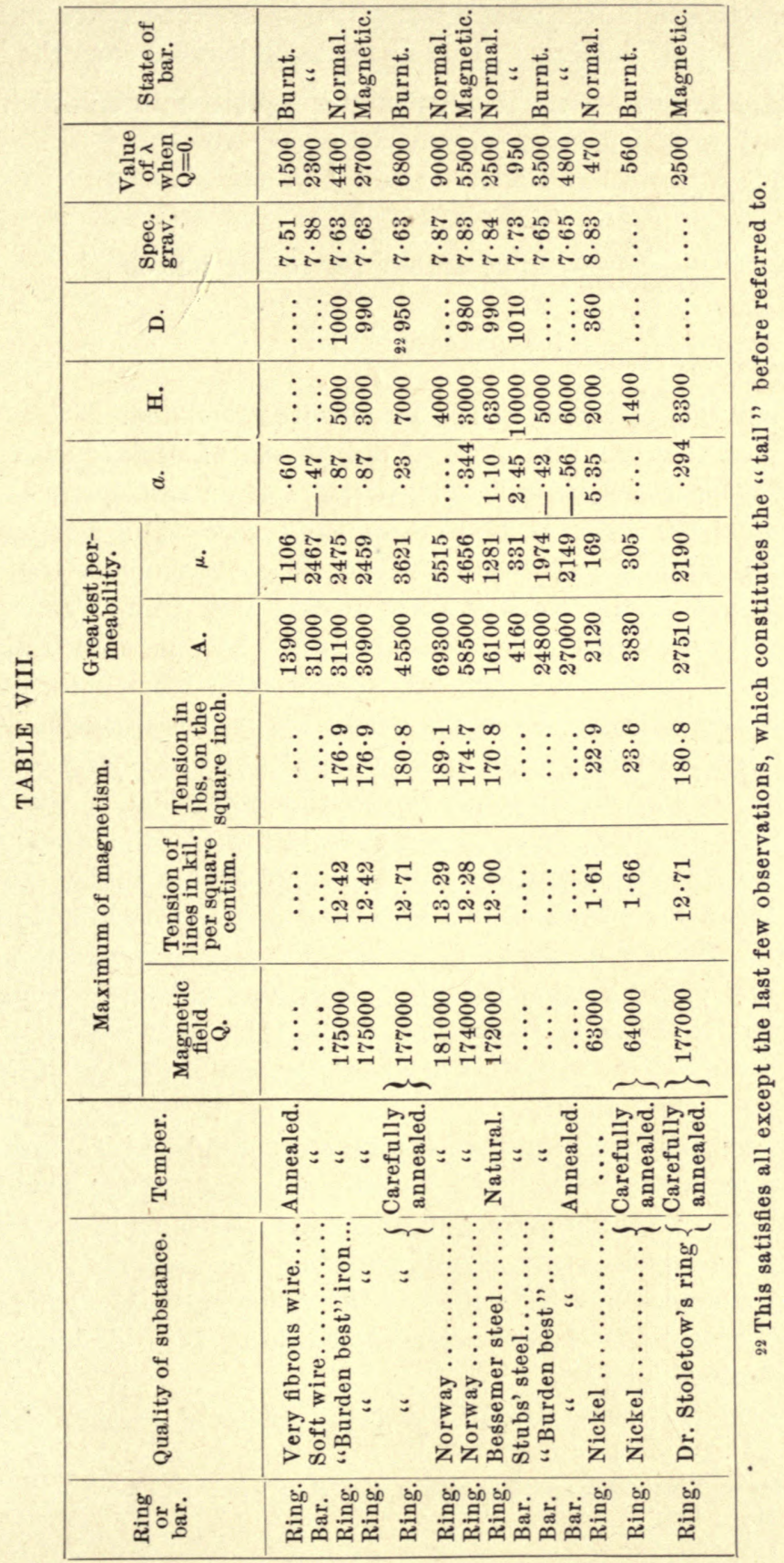


giving the tension of the lines of force per square centimetre and square inch of section of the lines. These have been deduced from the formula given by Maxwell ${ }^{23}$ for the tension per square metre, which is $\frac{Q^{2}}{\delta \pi}$ absolute units of force.

This becomes

$$
\left.\begin{array}{l}
\frac{Q^{2}}{2465500000} \text { kilogrammes per square centin, } \\
\frac{Q^{2}}{173240000} \text { lbs. per square inch, }
\end{array}\right\} \text {, }
$$

from which the quantities in the Table were calculated.

It is seen that the maximum of magnetism of ordinary bar iron is about 175,000 times the unit field, or $17 \% \mathrm{lbs}$. on the square inch, and for nickel 63,000 times, or $22.9 \mathrm{lbs}$. on the square inch. For pure iron, however, I think it may reach 180,000 , or go even above that. It is seen that one of the Norway rings gave a very high result; this is explained by the following considerations. All the iron rings were welded except this one, which was forged solid from a bar 2 inches wide and then turned. Even the purest bar iron is somewhat fibrous; and between the fibres we often find streaks of scale lying lengthwise in the bar and so diminishing the section somewhat if the ring be welded from the bar; when, however, it is forged solid, these streaks are thoroughly disintegrated; and hence we find a higher maximum of magnetism for a ring of this kind, and one approaching to that of pure iron. But a ring made in this way has to be exposed to so much heating and pounding that the iron is rendered unhomogeneous, and a tail appears to the curve like that in Table III. It is evident that this tail must always show itself whenever the section of the ring is not homogeneous throughout.

Hence we may conclude that the greatest weight which can be sustained by an electromagnet, with an infinite current is, for good but not pure iron, $354 \mathrm{lbs}$. per square inch of section, and for nickel $46 \mathrm{lbs}$.

Joule ${ }^{24}$ has made many experiments on the maximum sustainingpower of magnets, and has collected the following Table, which I give complete, except that $\mathrm{I}$ have replaced the result with his large magnet by one obtained later.

It is seen that these are all below my estimate, as they should be.

${ }^{23}$ Treatise on Electricity and Magnetism, vol. ii, p. 256.

24 Phil. Mag., 1851. 
For comparison, I have added a column giving the values of $Q$ which would give the sustaining-power observed; some of these are as high as any I have actually obtained, thus giving an experimental proof that my estimate of $354 \mathrm{lbs}$. cannot be far from correct, and illustrating the beauty of the absolute system of electrical measurement by which, from the simple deflection of a galvanometer-needle, we are able to predict how much an electromagnet will sustain without actually trying the experiment.

TABLE IX.

\begin{tabular}{|c|c|c|c|c|}
\hline Magnet belonging to & $\begin{array}{l}\text { Least area of } \\
\text { section, square } \\
\text { inch. }\end{array}$ & $\begin{array}{l}\text { Weight } \\
\text { sustained. }\end{array}$ & $\begin{array}{l}\text { Weight sus- } \\
\text { tained } \div \\
\text { least area. }\end{array}$ & Q. \\
\hline $\begin{array}{l}\text { Mr. Joule. }\left\{\begin{array}{l}1 \ldots \ldots \\
2 \ldots \ldots \\
3 \ldots \ldots \\
4 \ldots \ldots\end{array}\right. \\
\text { Mr. Nesbit. } \ldots \ldots \ldots\end{array}$ & $\begin{array}{l}10 . \\
\cdot 196 \\
\cdot 0436 \\
.0012 \\
4 \cdot 5 \\
3 \cdot 94 \\
\cdot 196\end{array}$ & $\begin{array}{c}2775 \\
49 \\
12 \\
\quad \cdot 202 \\
1428 \\
750 \\
50\end{array}$ & $\begin{array}{l}277 \\
250 \\
275 \\
162 \\
317 \\
190 \\
255\end{array}$ & $\begin{array}{l}154700 \\
147000 \\
154100 \\
118300 \\
165500 \\
128200 \\
148500\end{array}$ \\
\hline
\end{tabular}

In looking over the columns of Table VIII, which contain the values of the constants in the formula, we see how futile it is to attempt to give any fixed value to the permeability of iron or nickel; and we also see of how little value experiments on any one kind of iron are. Iron differs as much in magnetic permeability as copper does in electric conductivity.

It is seen that in the three cases when iron bars have been used, the value of $a$ is negative; we might consider this to be a general law, if I did not possess a ring which also gives this negative. All these bars had a length of at least 120 times their diameter.

The mathematical theory of magnetism has always been considered one of the most difficult of subjects, even when, as heretofore, $\mu$ is considered to be a constant; but now, when it must be taken as a function of the magnetism, the difficulty is increased many fold. There are certain cases, however, where the magnetism of the body is uniform, which will not be affected.

Troy, June 2, 1873. 


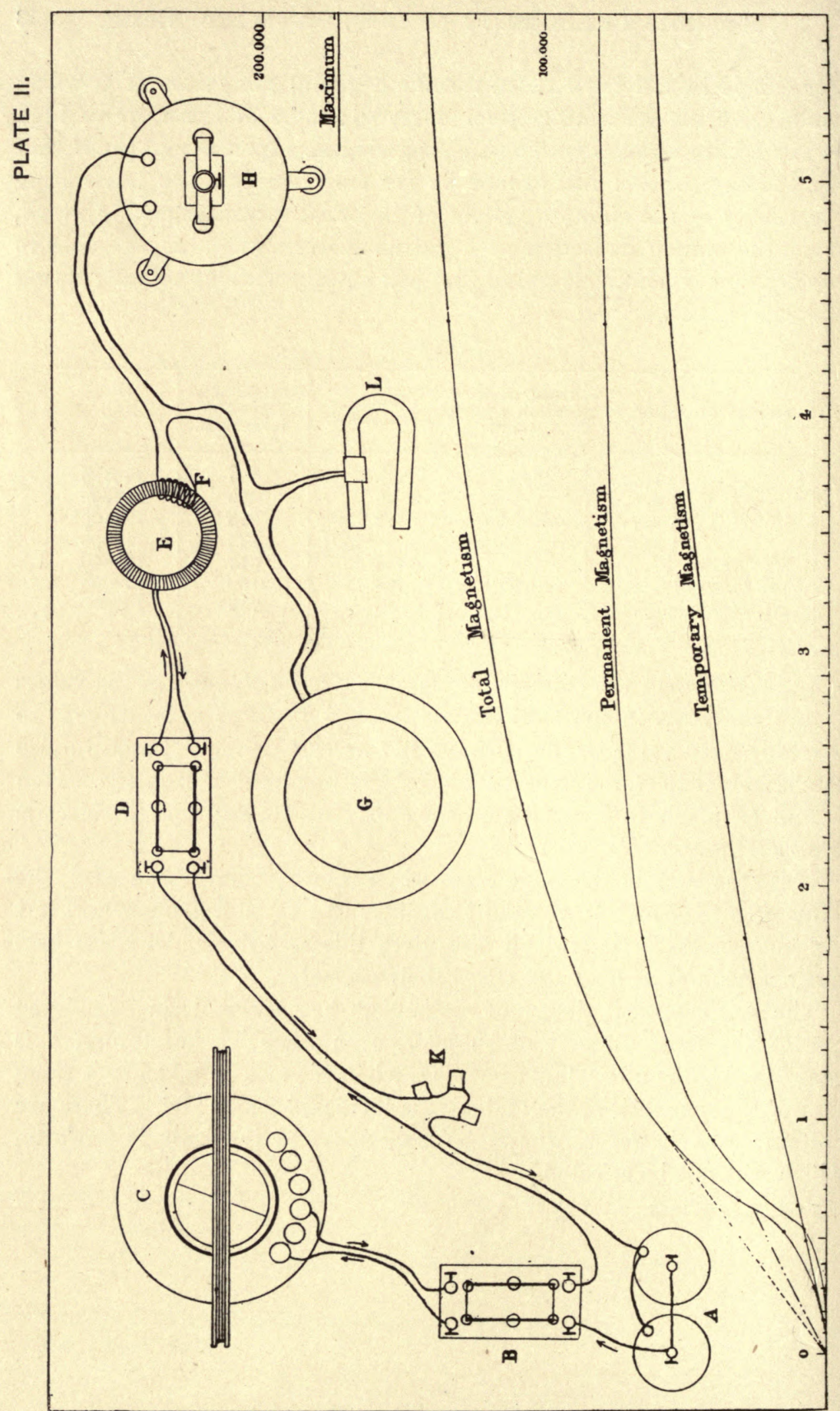

(54) 


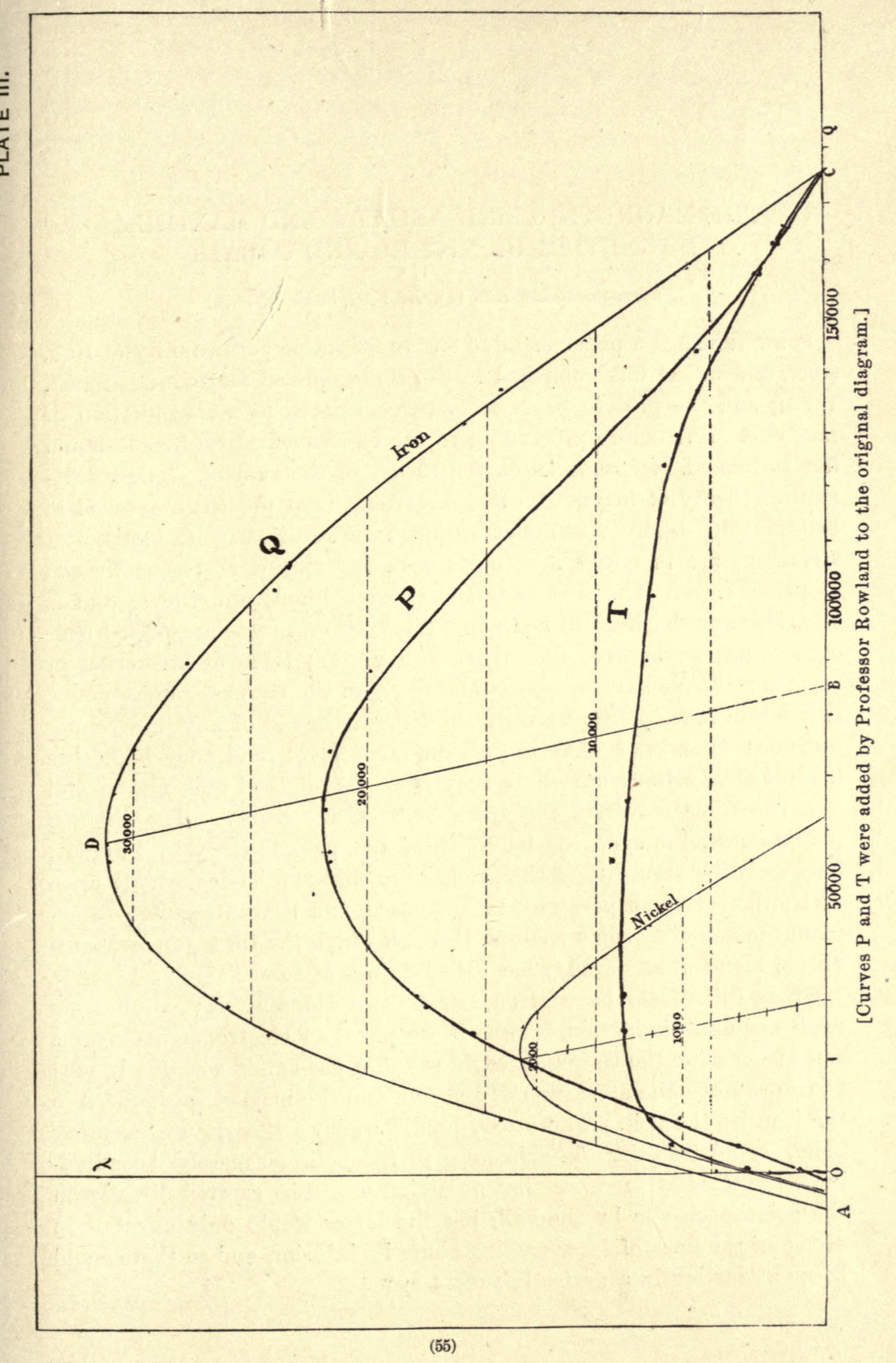




\section{ON THE MAGNETIC PERMEABILITY AND MAXIMUM OF MAGNE'TISM OF NICKEL AND COBALT}

[Philosophical Magazine [4], XLVIII, 321-340, 1874]

Some time ago a paper of mine on the magnetic permeability of iron, steel, and nickel was published in the Philosophical Magazine (August, 1873); and the present paper is to be considered as a continuation of that one. But before proceeding to the experimental results, I should like to make a few remarks on the theory of the subject. The mathematical theory of magnetism and electricity is at present developed in two radically different manners, although the results of both methods of treatment are in entire agreement with experiment as far as we can at present see. The first is the German method; and the second is Faraday's, or the English method. When two magnets are placed near each other, we observe that there is a mutual force of attraction or repulsion between them. Now, according to the German philosophers, this action takes place at a distance without the aid of any intervening medium: they know that the action takes place, and they know the laws of that action; but there they rest content, and seek not to find how the force traverses the space between the bodies. The English philosophers, however, led by Newton, and preeminently by Faraday, have seen the absurdity of the proposition that two bodies can act upon each other across a perfectly vacant space, and have attempted to explain the action by some medium through which the force can be transmitted along what Faraday has called "lines of force."

These differences have given rise to two different ways of looking upon magnetic induction. Thus if we place an electromagnet near a compass-needle, the Germans would say that the action was due in part to two causes-the attraction of the coil, and the magnetism induced in the iron by the coil. Those who hold Faraday's theory, on the other hand, would consider the substance in the helix as merely "conducting" the lines of force, so that no action would be exerted directly on the compass-needle by the coil, but the latter would only affect it in virtue of the lines of force passing along its interior, and so there could be no attraction in a perfectly vacant space. 
According to the first theory, the magnetization of the iron is represented by the excess of the action of the electromagnet over that of the coil alone; while by the second, when the coil is very close around the iron, the whole action is due to the magnetization of the iron. The natural unit of magnetism to be used in the first theory is that quantity which will repel an equal quantity at a unit's distance with a unit of force; on the second it is the number of lines of force which pass through a unit of surface when that surface is placed in a unit field perpendicular to the lines of force. The first unit is $4 \pi$ times the second. Now when a magnetic force of intensity $\mathfrak{S}^{1}$ acts upon a magnetic substance, we shall have $\mathfrak{B}=\mathfrak{H}+4 \pi \mathfrak{J}$, in, which $\mathfrak{B}$ is the magnetization of the substance according to Faraday's theory, and is what I formerly called the magnetic field, but which I shall hereafter call, after Professor Maxwell, the magnetic induction. $\mathfrak{\Im}$ is the intensity of magnetization according to the German theory, expressed in terms of the magnetic moment of the unit of volume. Now, when the substance is in the shape of an infinitely long rod placed in a magnetic field parallel to the lines of force, the ratio $\frac{\mathfrak{F}}{\mathfrak{H}}=\mu$ is called the magnetic permeability of the substance, and the ratio $\frac{\mathfrak{S}}{\mathfrak{h}}={ }_{\kappa}$ is Neumann's coefficient of magnetization by induction. Now experiment shows that for large values of $\mathfrak{K}$ the values of both $\mu$ and $x$ decrease, so that we may expect either $\mathfrak{J}$ or both $\mathfrak{B}$ and $\mathfrak{J}$ to attain a maximum value. In my former paper $I$ assumed that $\mathfrak{B}$ as well as $\mathfrak{J}$ attain a maximum; but on further considering the subject I see that we have no data for determining which it is at present. If it were possible for $\mathfrak{B}$ to attain a maximum value so that $\mu$ should approach to $0, \kappa$ would be negative, and the substance would then become diamagnetic for very high magnetizing forces. ${ }^{2}$ This is not contrary to observation; for at present we lack the means of producing a sufficiently intense magnetic field to test this experimentally, at least in the case of iron. To produce this effect at ordinary temperatures, we must have a magnetic field greater than the following-for iron 175,000 , for nickel 63,500 , and for

${ }^{1}$ I shall hereafter in all my papers use the notation as given in Professor Maxwell's 'Treatise on Electricity and Magnetism;' for comparison with my former paper I give the following:

$$
\begin{aligned}
& \mathfrak{B} \text { in this paper }=Q \text { in former one. } \\
& 5 \text { " } 5=4 \pi \mathrm{M} \text { " } \\
& \mathfrak{s} \text { " }=\frac{\mathrm{Q}}{4 \pi}-\mathrm{M} \text { " }
\end{aligned}
$$

'See Maxwell's 'Treatise on Electricity and Magnetism,' art. 844.-J. C. M. 
cobalt about 100,000 (?). These quantities are entirely beyond our reach at present, at least with any arrangement of solenoids. Thus, if we had a helix 6 inches in diameter and 3 feet long with an aperture of 1 inch diameter in the centre, a rough calculation shows that, with a battery of 350 large Bunsen cells, the magnetic field in the interior would only be 15,000 or 20,000 when the coils were arranged for the best effect. We might obtain a field of greater intensity by means of electromagnets, and one which might be sufficient for nickel; but we cannot be certain of its amount, as I know of no measurement of the field produced in this way. But our principal hope lies in heating some body and then subjecting it to a very intense magnetizing-force; for I have recently found, and will show presently, that the maximum of magnetization of nickel and iron decreases as the temperature rises, at least for the two temperatures $0^{\circ} \mathrm{C}$. and $220^{\circ} \mathrm{C}$. I am aware that iron and nickel have been proved to retain their magnetic properties at high temperatures, but whether they were in a field of sufficient intensity at the time cannot be determined. The experiment is at least worth trying by some one who has a magnet of great power, and who will take the trouble to measure the magnetic field of the magnet at the point where the heated nickel is placed. 'This could best be done by a small coil of wire, as used by Verdet.

But even if it should be proved that $\mathfrak{B}$ does not attain a maximum, but only $\mathfrak{\Im}$, it could still be explained by Faraday's theory; for we should simply have to suppose that the magnetic induction $\mathfrak{B}$ was composed of two parts-the first part, $4 \pi \mathfrak{\jmath}$, being due to the magnetic atoms alone, and the second, $\mathfrak{F}$, to those lines of force which traversed the æther between the atoms. To determine whether either of these quantities has a maximum value can probably never be done by experiment; we may be able to approach the point very nearly, but can never arrive at it, seeing that we should need an infinite magnetizing-force to do so. Hence its existence and magnitude must always be inferred from the experiments by some such process as was used in my first paper, where the curve of permeability was continued beyond the point to which the experiments were carried. Neither does experiment up to the present time furnish any clue as to whether it is $\mathfrak{B}$ or $\mathfrak{S}$ which attains a maximum.

As the matter is in this undecided state, I shall hereafter in most cases calculate both $\mathfrak{I}$ and $\kappa$ as well as $\mathfrak{B}$ and $\mu$, as I am willing to admit that $\mathfrak{\Im}$ may have a physical significance as well as $\mathfrak{B}$, even on Faraday's theory. 
There is a difficulty in obtaining a good series of experiments on nickel and cobalt which does not exist in the case of iron. It is principally owing to the great change in magnetic permeability of these substances by heat, and also to their small permeability. 'To obtain sufficient magnetizing-force to trace out the curve of permeability to a reasonable distance, we require at least two layers of wire on the rings, and have to send through that wire a very strong current. In this way great heat is developed; and on account of there being two layers of wire it cannot escape; and the ring being thus heated, its permeability is changed. So much is this the case, that when the rings are in the air, and the strongest current circulating, the silk is soon burned off the wire; and to obviate this I have in these experiments always immersed the rings in some non-conducting liquid, such as alcohol for low temperatures and melted paraffin for high temperatures, the rings being suspended midway in the liquid to allow free circulation. But I have now reason to suspect the efficacy of this arrangement, especially in the case of the paraffin. The experiments described in this paper were made at such odd times as I could command, and the first ones were not thoroughly discussed until the series was almost completed; hence I have not been so careful to guard against this error as I shall be in the future. This can be done in the following manner-namely, by letting the current pass through the ring for only a shirt time. But there is a difficulty in this method, because if the current is stopped the battery will recruit, and the moment it is joined to the ring a large and rapidly decreasing current will pass which it is impossible to measure accurately. I have, however, devised the following method, which I will apply in future experiments. It is to introduce into the circuit between the tangent-galvanometer and the ring a current-changer, by which the current can be switched off from the ring into another wire of the same resistance, so that the current from the battery shall always be constant. Just before making an observation the current is turned back into the ring, a reading is taken of the tangent-galvanometer by an assistant, and immediately afterward the current is reversed and the reading taken for the induced current; the tangent-galvanometer is then again read with the needle on the other side of the zero-point. The pressure of outside duties at present precludes me from putting this in practice. But the results which I have obtained, though probably influenced in the higher magnetizing-forces by this heating, are still so novel that they must possess value notwithstanding this defect; for they contain the only experiments yet made on the permeability of 
cobalt at ordinary temperatures, and of iron, nickel, and cobalt at high temperatures.

The rings of nickel and cobalt which I have used in the experiments of this paper were all turned from buttons of metal obtained by fusing under glass in a French crucible, it having been found that a Hessian crucible was very much attacked by the metal. The crucibles were in the fire three or four hours, and when taken out were very soft from the intense heat. As soon as taken out, the outside of the crucible was wet with water, so as to cool the metal rapidly and prevent crystallization; but even then the cooling inside went on very slowly. As the physical and chemical properties of these metals exercise great influence on their magnetic properties, I will give them briefly. A piece of nickel before melting was dissolved in $\mathrm{HCl}$; it gave no precipitate with $\mathrm{H}^{2} \mathrm{~S}$, and there were no indications of either iron or cobalt. A solution of the cobalt gave no precipitate with $\mathrm{H}^{2} \mathrm{~S}$, but contained small traces of iron and nickel. After melting the metals no tests have been made up to the present time; but it is to be expected that the metals absorbed some impurities from the crucibles. They probably did not contain any carbon. One button of each metal was obtained, from each of which two rings were turned. The cobalt was quite hard, but turned well in the lathe, long shavings of metal coming off and leaving the metal beautifully polished. The metal was slightly malleable, but finally broke with a fine granular fracture. The rings when made were slightly sonorous when struck; and the color was of a brilliant white slightly inclined to steel-color, but a little more red than steel. The nickel was about as hard as wrought iron, and was tough and difficult to turn in the lathe, a constant application of oil being necessary, and the turned surface was left very rough; the metal was quite malleable, but would become hard, and finally fly apart when pounded down thin if not annealed. When the rings were struck, they gave a dead sound as if made of copper. In both cases the specific gravity was considerably higher than that generally given for cast metal; but it may be that the metal to which they refer contained carbon, in which case it would be more easily melted. There is great liability to error in taking the specific gravity of these metals, because they contract so much on cooling, and unless this is carried on rapidly crystals may form, between which, as the metal contracts, vacant spaces may be left. As the specific gravity of my rings approaches to that of the pure metals precipitated by hydrogen, I consider it evidence of their purity. The dimensions of the rings and their other constants are as follows:- 


\begin{tabular}{|c|c|c|c|c|}
\hline Ring. & $\begin{array}{l}\text { Weight in } \\
\text { vacuo, in } \\
\text { grammes. }\end{array}$ & $\begin{array}{l}\text { Loss in water } \\
\text { at } 4^{\circ} \mathrm{C.,} \text { in } \\
\text { grammes. }\end{array}$ & $\begin{array}{l}\text { Specific } \\
\text { gravity. }\end{array}$ & $\begin{array}{l}\text { Moan dia- } \\
\text { meter, in } \\
\text { centimetres. }\end{array}$ \\
\hline $\begin{array}{l}\text { Nickel, No. I. } \quad \ldots \ldots \ldots \\
\text { Nickel, No. II. } \ldots \ldots \ldots \\
\text { Cobalt, No. I. } \ldots \ldots \ldots \\
\text { Cobalt, No. II. } \ldots \ldots \ldots\end{array}$ & $\begin{array}{c}21 \cdot 823 \\
10 \cdot 011 \\
4 \cdot 681\end{array}$ & $\begin{array}{c}2 \cdot 4560 \\
\cdots \\
1 \cdot 1435 \\
\cdot 5346\end{array}$ & $\begin{array}{l}8 \cdot 886 \\
8 \cdot 887 \\
8 \cdot 7553 \\
8 \cdot 7550\end{array}$ & $\begin{array}{l}3 \cdot 28 \\
2 \cdot 3 \\
1 \cdot 48\end{array}$ \\
\hline Ring. & $\begin{array}{l}\text { Mean circum- } \\
\text { ference, in } \\
\text { centimetres. }\end{array}$ & $\begin{array}{l}\text { Number of } \\
\text { coils of wire } \\
\text { on ring. }\end{array}$ & $\begin{array}{l}\text { Coils per } \\
\text { metre of cir- } \\
\text { cumference. }\end{array}$ & $\begin{array}{l}\text { Area of sec- } \\
\text { tion, in square } \\
\text { centimetres. }\end{array}$ \\
\hline $\begin{array}{l}\text { Nickel, No. I. } \quad \ldots \ldots \ldots \\
\text { Nickel, No. II. } \ldots \ldots \ldots \\
\text { Cobalt, No. I. } \quad \ldots \ldots \ldots \\
\text { Cobalt, No. II. } \ldots \ldots \ldots\end{array}$ & $\begin{array}{c}10 \cdot 304 \\
\ddot{7} \cdot \overrightarrow{791} \\
5 \cdot 686\end{array}$ & $\begin{array}{l}318 \\
\dddot{243} \\
158\end{array}$ & $\begin{array}{l}3086 \\
3119 \\
2779\end{array}$ & $\begin{array}{l}\cdot 2384 \\
\cdots \cdots \\
\cdot 1467 \\
.09403\end{array}$ \\
\hline
\end{tabular}

Up to the present time cnly the rings whose dimensions are given have been used.

The following Tables from the nickel ring No. I leave little to be desired in point of regularity, and confirm the fact proved in my first paper, that the laws deduced for iron hold also for nickel, and also confirm the value given in my other paper for the maximum value of magnetization of nickel. But the most important thing that they show is the effect of heat upon the magnetization of nickel; and Table III contains the first numerical data yet obtained on the effect of heat on the magnetic properties of any substance.

As all the rings were wound with two layers of wire, a slight correction was marde in the value of $\mathscr{B}$ for the lines of inductive force which passed through the air and not through the metal. In all the experiments of this paper greater care was used to obtain $T$ than in the first paper. Each value of $\mathfrak{H}, \mathfrak{B}$, and $T$ is the mean of four readings. In all the Tables I have left the order of the observations the same as that in which they were made, and have also put down the date, as I now have reason to suspect that the leaving of a ring in the inagnetized state in which it is after an experiment will in time affect its properties to a small extent. Let me here remark that the time necessary to simply make the observations is only a very small fraction of that required to prepare for them and to afterwards discuss them. And this, with the small amount of time at my disposal, will account for the late day at which I publish my results.

The following is the notation used, the measurements being made on that absolute system in which the metre, gramme, and second are the fundamental units. 
$\mathfrak{F}$ is the magnetizing-force acting on the metal.

$\mathfrak{B}$ is the magnetic induction within the metal (see Maxwell's 'Treatise on Electricity and Magnetism,' arts. 400, 592, and 604).

$\mu$ is the magnetic permeability of the metal $=\stackrel{\mathfrak{B}}{\mathfrak{K}}=4 \pi \kappa+1$.

$T$ is the portion of $\mathfrak{B}$ which disappears when the current is broken.

$P$ is the portion of $\mathfrak{B}$ which remains when the current is broken.

$\mathfrak{\Im}$ is the intensity of magnetization $=\frac{\mathfrak{B}-\mathfrak{S}}{4 \pi}$.

$\kappa$ is Neumann's coefficient of induced magnetization $=\frac{\mathfrak{J}}{\mathfrak{S}}$.

TABLE I.

Cast Nickel, Normal, at $15^{\circ} \mathrm{C}$.

Experiments made November 29, 1873.

\begin{tabular}{|c|c|c|c|c|c|c|c|c|c|c|}
\hline 5 & $\mathscr{B}$ & $\begin{array}{c}\mu . \\
\text { Ob- } \\
\text { served. }\end{array}$ & $\begin{array}{l}\text { Calcu- } \\
\text { lated. }\end{array}$ & Error. & T. & P. & গ. & $\begin{array}{c}\text { K. } \\
\text { Ob- } \\
\text { served. }\end{array}$ & $\begin{array}{l}\text { Caleu- } \\
\text { lated. }\end{array}$ & Error. \\
\hline $12 \cdot 84$ & 675 & $52 \cdot 6$ & $46 \cdot 4$ & $-6 \cdot 2$ & & & $52 \cdot 7$ & $4 \cdot 10$ & $3 \cdot 65$ & -.45 \\
\hline & 216 & & $80 \cdot 6$ & -.2 & 1263 & 906 & 170.5 & & $6 \cdot 27$ & -.08 \\
\hline $45 \cdot 1$ & 7451 & $165 \cdot 1$ & $166 \cdot 8$ & $1 \cdot 7$ & 2894 & 4557 & $589 \cdot 3$ & $13 \cdot 06$ & 13.08 & .02 \\
\hline $56 \cdot 1$ & 11140 & $198 \cdot 5$ & 99. & $\cdot 6$ & 378 & & $882 \cdot 0$ & 15. & & -.02 \\
\hline $70 \cdot 7$ & 15410 & 217 . & $217 \cdot 5$ & $-\cdot 3$ & 5018 & 103 & 1221 & 17. & $17 \cdot 21$ & -.04 \\
\hline $77 \cdot 52$ & 17100 & $220 \cdot 6$ & $20 \cdot 6$ & .0 & 5454 & 1. & 1355 & 17. & & 0 \\
\hline $90 \cdot 76$ & 20180 & $222 \cdot 3$ & $2 \cdot 0$ & $-\cdot 3$ & 648 & 3697 & 1599 & & $17 \cdot 60$ & -.01 \\
\hline $115 \cdot 4$ & 25170 & $218 \cdot 2$ & $214 \cdot 3$ & $-3 \cdot 9$ & 8313 & 168 & 1994 & 17. & & -.30 \\
\hline $139 \cdot 4$ & 28540 & $204 \cdot 7$ & $204 \cdot 3$ & -.4 & 10100 & 18440 & 2260 & $16 \cdot 21$ & $16 \cdot 18$ & -.03 \\
\hline & 324 & 18 & $6 \cdot 6$ & $-1 \cdot 2$ & & & 256 & & & .07 \\
\hline $195 \cdot 3$ & 346 & $177 \cdot 3$ & $179 \cdot 1$ & 1.8 & 320 & 213 & 2740 & $14 \cdot 03$ & 14 & .09 \\
\hline $229 \cdot 5$ & & & $165 \cdot 5$ & $2 \cdot 7$ & & & & & & $\cdot 15$ \\
\hline 275.9 & 40860 & 148 & $146 \cdot 3$ & $-1 \cdot 8$ & 17960 & 22900 & 3230 & $11 \cdot 71$ & $11 \cdot 46$ & -.25 \\
\hline $415 \cdot 2$ & & 111 & $112 \cdot 8$ & .9 & & & 3665 & & 8.77 & -.05 \\
\hline $727 \cdot 0$ & & & $72 \cdot 8$ & $\cdot 3$ & & & 4135 & & $5 \cdot 64$ & -.05 \\
\hline 1042 & & $53 \cdot 4$ & $52 \cdot 8$ & -.6 & 30680 & 25000 & 4344 & $4 \cdot 17$ & $4 \cdot 17$ & 0 \\
\hline & 63420 & $\ldots$ & 0 & & & 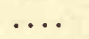 & 4940 & & & \\
\hline
\end{tabular}

$\mu=222 \sin \left(\frac{\mathfrak{B}+53 \mu+1200}{359}\right) . \quad \quad \kappa=176 \sin \left(\frac{\mathfrak{S}+50 \kappa+100}{28}\right)$.

TABLE II.

Cast Nickel, Magnetic, at $12^{\circ} \mathrm{C}$.

Experiments made December 6, 1873.

\begin{tabular}{|c|c|c|c|c|c|c|}
\hline 5 . & B. & $\mu$. & T. & P. & Э. & $\kappa$. \\
\hline $23 \cdot 25$ & 1245 & 53.55 & & & $97 \cdot 2$ & $4 \cdot 18$ \\
\hline $47 \cdot 69$ & 7786 & $163 \cdot 3$ & 3095 & 4691 & $615 \cdot 8$ & $12 \cdot 91$ \\
\hline $57 \cdot 78$ & 11460 & $198 \cdot 3$ & 3740 & 7720 & $907 \cdot 3$ & $15 \cdot 70$ \\
\hline $73 \cdot 43$ & 16040 & $218 \cdot 5$ & 5032 & 11008 & $1270 \cdot 6$ & $17 \cdot 30$ \\
\hline $88 \cdot 23$ & 19790 & $224 \cdot 3$ & 6554 & 13236 & 1568 & $17 \cdot 77$ \\
\hline $107 \cdot 3$ & 23530 & $219 \cdot 2$ & 7620 & 15910 & 1864 & $17 \cdot 36$ \\
\hline $153 \cdot 8$ & 30160 & $196 \cdot 1$ & 10940 & $19: 20$ & 2388 & $15 \cdot 52$ \\
\hline $206 \cdot 3$ & 35880 & $174 \cdot 0$ & 14030 & 21850 & 2839 & $13 \cdot 76$ \\
\hline $296 \cdot 4$ & 41310 & 139.4 & 18390 & 22920 & 3264 & 11.01 \\
\hline $421 \cdot \overline{8}$ & 46520 & $110 \cdot 3$ & 22520 & 24000 & 3668 & $8 \cdot 70$ \\
\hline
\end{tabular}


TABLE III.

Cast Nickel, Magnetic, at $220^{\circ} \mathrm{C}$.

Experiments made December 6, 1873.

\begin{tabular}{|c|c|c|c|c|c|c|}
\hline 5. & $\mathfrak{B}$. & $\mu$. & T. & P. & ঐ. & $\kappa$. \\
\hline $22 \cdot 60$ & 4502 & 199.2 & 2671 & 1831 & $356 \cdot 4$ & $15 \cdot 77$ \\
\hline $45 \cdot 06$ & 14000 & $310 \cdot 8$ & 5470 & 8530 & 1111 & $24 \cdot 65$ \\
\hline 52.96 & 16660 & $314 \cdot 6$ & 6350 & 10310 & 1322 & $24 \cdot 96$ \\
\hline $67 \cdot 42$ & 20300 & $301 \cdot 1$ & 7722 & 12578 & 1602 & $23 \cdot 88$ \\
\hline $80 \cdot 69$ & 22540 & $279 \cdot 3$ & 8914 & 13626 & 1787 & $22 \cdot 15$ \\
\hline $106 \cdot 4$ & 26420 & $248 \cdot 3$ & 11140 & 15280 & 2094 & $19 \cdot 68$ \\
\hline $150 \cdot 8$ & 30740 & $203 \cdot 8$ & 14040 & 16700 & 2434 & $16 \cdot 14$ \\
\hline $191 \cdot 0$ & 33530 & $175 \cdot 6$ & 15940 & 17590 & 2653 & $13 \cdot 89$ \\
\hline $294 \cdot 8$ & 38300 & $129 \cdot 9$ & 20240 & 18060 & 3024 & $10 \cdot 26$ \\
\hline $553 \cdot 6$ & 42630 & $77 \cdot 0$ & 24360 & 18270 & 3348 & $6 \cdot 05$ \\
\hline $789 \cdot 8$ & 43900 & $55 \cdot 6$ & 26060 & 17840 & 3431 & $4 \cdot 345$ \\
\hline \multicolumn{7}{|c|}{ Experiments made December 10,1873 . } \\
\hline 13.00 & 1537 & $118 \cdot 2$ & & & $109 \cdot 2$ & $9 \cdot 33$ \\
\hline $22 \cdot 37$ & 4262 & $190 \cdot 5$ & $\ldots$ & $\ldots$ & $337 \cdot 4$ & $15 \cdot 08$ \\
\hline $25 \cdot 15$ & 5337 & $212 \cdot 2$ & & & $422 \cdot 7$ & $16 \cdot 81$ \\
\hline $33 \cdot 19$ & 9486 & $285 \cdot 8$ & 4055 & 5431 & 752.3 & $22 \cdot 15$ \\
\hline $43 \cdot 28$ & 13570 & $313 \cdot 6$ & 5357 & 8213 & 1076 & $24 \cdot 88$ \\
\hline
\end{tabular}

In Table $I$ are given the results for nickel at about $15^{\circ} \mathrm{C}$., together with the values of $\mu$ and $\kappa$ calculated from the formulæ given below the Table. We see that the coincidence is almost perfect in both cases, which thus shows that the formula which we have hitherto used for $\lambda$ and $\mu$ can also be applied to $k$, at least within the limit of experiments hitherto made, although it must at last depart from one or the other of the curves. The greatest relative error is seen to be in the first line, where $\mathfrak{H}$ is small: this does not indicate any departure from the curve, but is only due to the too small deflections of the galvanometer; and the error indicates that of only a small fraction of a division at the galvanometer.

In the calculation of $\mu$ and $\kappa$ a method was used which may be of use to others in like circumstances, who have to calculate a large number of values of one variable from a function which cannot be solved with reference to that variable, but can be solved with reference to the other. Thus we have

$$
\mu=\beta \sin \left(\frac{B+b \mu+\pi}{D}\right), \ldots . . . .
$$

which can be solved with reference to $\mathfrak{B}$ but not to $\mu$; for we have

$$
\mathfrak{B}=D \sin ^{-1}\left(\frac{\mu}{\beta}\right)-b_{\mu}-\pi . . . \cdot . .
$$


Suppose we have values of $\mathfrak{B}$, and wish to find the corresponding values of $\mu$. We first calculate a few values of $\mathcal{B}$ from (2) so that we can plot the curve connecting $\mathfrak{B}$ and $\mu$. We then from the plot select a value of $\mu$ which we shall call $\mu^{\prime}$, as near the proper value as possible, and calculate the corresponding value of $\mathfrak{B}$, which we shall call $\mathfrak{B}^{\prime}$. Our problem then is, knowing $\mathfrak{B}^{\prime}$ and $\mu^{\prime}$, to find the value of $\mu$ corresponding to $\mathfrak{B}$ when this is nearly equal to $\mathfrak{B}^{\prime}$. Let $\mathfrak{B}^{\prime}$ receive a small increment $\Delta \mathfrak{B}^{\prime}$, so that $\mathfrak{B}=\mathfrak{B}^{\prime}+\Delta \mathfrak{B}^{\prime}$; then we have, from Taylor's theorem, since $\mu=\varphi\left(\mathfrak{B}^{\prime}+\triangle \mathfrak{B}^{\prime}\right)$ and $\mu^{\prime}=\varphi\left(\mathfrak{B}^{\prime}\right)$,

$$
\mu=\mu^{\prime}+\frac{d \mu^{\prime}}{d \mathfrak{B}^{\prime}}\left(\Delta \mathfrak{B}^{\prime}\right)+\frac{1}{2} \frac{d^{2} \mu^{\prime}}{d \mathfrak{B}^{\prime 2}}\left(\Delta \mathfrak{B}^{\prime}\right)^{2}+\& \mathrm{c} .
$$

Remembering that the constants in (1) refer to degrees of arc and not to the absolute value of the arc, we have

$$
\mu=\mu^{\prime}+\frac{\mathfrak{B}-\mathfrak{B}^{\prime}}{57 \cdot 3 \frac{D}{\beta} \frac{1}{\sqrt{1-\frac{\mu^{2}}{\xi^{2}}}}-b}+\& c .,
$$

which is in the most convenient form for calculation by means of Barlow's Tables of squares, \&c., and is very easy to apply, being far easier than the method of successive approximation.

On comparing the magnetic curve Table II with the normal curve Table I, we see that the magnetic curve of nickel bears the same relation to the normal curve as we have already found for iron; that is, the magnetic curve falls below the normal curve for all points before the vertex, but afterwards the two coincide.

Hence we see that at ordinary temperatures the magnetic properties of nickel are a complete reproduction of those of iron on a smaller scale. But when we come to study the effect of temperature we shall find a remarkable difference, and shall find nickel to be much more susceptible than iron to the influence of heat.

In Table III we have experiments on the permeability of nickel at a high temperature, the ring being maintained at $220^{\circ} \mathrm{C}$. by being placed in a bath of melted paraffin: in this bath the silk covering of the wire remained quite perfect, but after many hours became somewhat weak. After completing the experiments on this and the cobalt rings, on unwinding some of them I found the outside layer quite perfect; but, especially in the smallest ring, the silk on the inside layer was much weaker, although the insulation was still perfect when the wire was in place. I can only account for this by the electric current generating heat in the wire, which was unable to pass outward because 
of the outside layer and also of the pieces of paper which were used to separate the layers of wire; hence the ring at high magnetizing-powers must have been at a somewhat higher temperature than the bath, to an amount which it is impossible to estimate. It is probable that it was not very great, however; for at this high temperature continued for hours it requires but little increase of heat to finally destroy the silk. We can, however, tell the direction of the error.

We see, on comparing Tables I and II with Table III, the great effect of heat on the magnetic properties of nickel. We see that for low magnetization the permeability is greatly increased, which is just opposite to what we might expect; but on plotting the curve we also notice the equally remarkable fact, that the maximum of magnetization

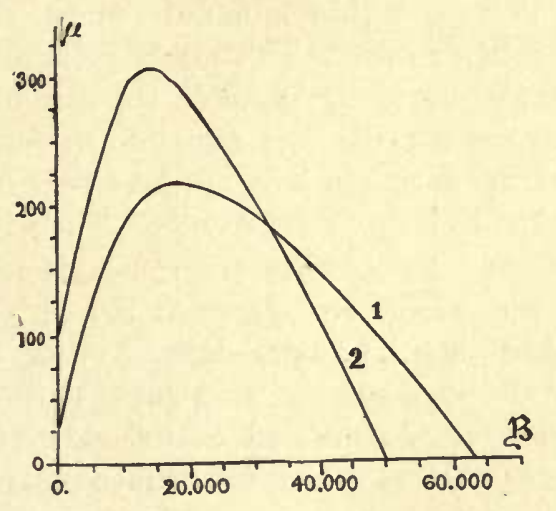
1. Curve at $15^{\circ} \mathrm{C}$.
2. Curve at $220^{\circ} \mathrm{C}$.

is decreased from $\mathfrak{B}=63,400$ or $\mathfrak{F}=4940$ to $\mathfrak{B}=49,000$ or $\mathfrak{F}=3800$. This curious result is shown in the annexed figure, where we see that for low magnetizing-forces $\mu$ is increased to about three or four times its value at $15^{\circ} \mathrm{C}$., and the maximum value of $\mu$ is increased from 222 to 315 . When $\mathfrak{B}$ has a value of $32,000, \mu$ is not affected by this change of temperature, seeing that the two curves coincide; but above that point $\mu$ is less at $220^{\circ} \mathrm{C}$. than at $15^{\circ} \mathrm{C}$. In other words, if nickel is heated from $15^{\circ} \mathrm{C}$. to $220^{\circ} \mathrm{C}$., the magnetization of nickel will increase if the magnetizing-force is small, but will decrease if it is large. It is impossible to say at present whether increase of temperature above $220^{\circ}$ will always produce effects in the same direction as below it or not.

These remarkable effects of heat, it seems to me, will, when followed out, lead to the discovery of most important connections between heat and magnetism, and will finally result in giving us much more light upon the nature of heat and magnetism, and that equally important 
question of what is a molecule. 'T'o accomplish this we must obtain a series of eurves for the same ring between as wide limits of temperature as possible. We must then plot our results in a suitable manner; and from the curves thus formed we can find what would probably happen if the temperature were lowered to the absolute zəro, or were increased to the point at which nickel is said to lose its magnetism. In such inquiries as these the graphical method is almost invaluable, and little can be expected without its aid.

In applying the formula to this eurve, we do not find so good an agreement as at the lower temperature. I do not consider this conclusive that the formula will not agree with observation at this temperature; for I have noticed that the curves of different specimens of iron and nickel seem to vary within a minute range, not only in their elements but also in their form. This might perhaps be accounted for by some small want of homogeneity, as in the case of burning in iron and nickel; but at present the fact remains without an explanation. But the amount of the deviation is in all eases very small when all the precautions are taken to insure good results. The nature of the deviation is in this case as follows: when the constants in the formula are chosen to agree with the observed curve at the vertex and at the two ends, then the observed curve falls slightly below the eurve of the formula at nearly all other points. In a curve plotted about 5 inches high and broad, the greatest distance between the two eurves is only about $\frac{1}{12}$ of an inch, and could be much reduced by changing the constants. For the benefit of those who wish to study this deviation, I have calculated the following values, which will give the curve touching the vertex and the two ends of the observed curve of Table III. They are to be used by plotting in connection with that Table.

\begin{tabular}{|c|c|c|}
\hline$\kappa$. & \multicolumn{2}{|c|}{ Э. } \\
\hline $\begin{array}{l}0 \\
12.75 \\
18 \cdot 75 \\
22.5 \\
25\end{array}$ & $\begin{array}{r}-140 \\
205 \\
455 \\
703\end{array}$ & $\begin{array}{l}3802 \\
2833 \\
2269 \\
1835\end{array}$ \\
\hline
\end{tabular}

I have not as yet obtained a complete curve of iron at a high temperature; but as far as I have tried, it does not seem to be affected much, at least for high magnetizing-powers. I have, however, found that the maximum of magnetization of iron decreases about 2 per cent by a 
rise of temperature from $15^{\circ} \mathrm{C}$. to $222^{\circ} \mathrm{C}$, while that of nickel decreases $22 \cdot 7$ per cent.

The experiments which I have made with cobalt do not seem to be so satisfactory as those made with nickel and iron. There are some things about them which I cannot yet explain; but as they are the only exact experiments yet made on cobalt, they must possess at least a transient value. The difficulties of getting a good cobalt-curve are manifold, and are due to the following properties-(1) its small permeability, (2) its sensitiveness to temperature, and (3) its property of having its permeability increased by rise of temperature at all magnetizingpowers within the limits of experiment. The following are the results with No. I:-

TABLE IV.

Cast Cobalt, Normal, at $5^{\circ} \mathrm{C}$.

Experiments made November 27, 1873.

\begin{tabular}{|c|c|c|c|c|c|c|c|c|}
\hline $\mathfrak{b}$. & B. & $\mu$. & T. & P. & Э. & $\begin{array}{c}\mathrm{Ob}^{\mathrm{b}}- \\
\text { served. }\end{array}$ & $\begin{array}{l}\text { K. } \\
\text { lated. } \\
\text { lated. }\end{array}$ & Error. \\
\hline $49 \cdot 33$ & 4303 & $87 \cdot 24$ & 3702 & 601 & $338 \cdot 5$ & $6 \cdot 86$ & $6 \cdot 75$ & $-\cdot 11$ \\
\hline $58 \cdot 83$ & 5608 & $95 \cdot 32$ & 4526 & 1082 & $441 \cdot 6$ & $7 \cdot 51$ & $7 \cdot 44$ & -.07 \\
\hline $76 \cdot 47$ & 8409 & $109 \cdot 95$ & 6175 & 2234 & $663 \cdot 1$ & $8 \cdot 67$ & $8 \cdot 79$ & $\cdot 12$ \\
\hline $93 \cdot 15$ & 11623 & $124 \cdot 8$ & 7826 & 3797 & $917 \cdot 5$ & $9 \cdot 85$ & $9 \cdot 81$ & -.04 \\
\hline $113 \cdot 0$ & 14993 & $132 \cdot 7$ & 9805 & 5188 & $1193 \cdot 1$ & $10 \cdot 48$ & $10 \cdot 44$ & -.04 \\
\hline $129 \cdot 3$ & 17439 & $134 \cdot 9$ & 10580 & 6859 & $1387 \cdot 8$ & $10 \cdot 66$ & $10 \cdot 72$ & .06 \\
\hline $159 \cdot 4$ & 22309 & $140 \cdot 0$ & 14090 & 8219 & $1775 \cdot 3$ & $11 \cdot 06$ & $11 \cdot 00$ & -.06 \\
\hline $189 \cdot 0$ & 26769 & $141 \cdot 6$ & 16260 & 10509 & $2130 \cdot 3$ & $11 \cdot 19$ & 10.97 & -.22 \\
\hline $219 \cdot 6$ & 30580 & $139 \cdot 3$ & 18200 & 12380 & $2433 \cdot 5$ & $11 \cdot 01$ & $10 \cdot 83$ & -18 \\
\hline $264 \cdot 7$ & 35525 & $134 \cdot 2$ & 21120 & 14405 & $2827 \cdot 0$ & $10 \cdot 60$ & $10 \cdot 50$ & $-\cdot 10$ \\
\hline $351 \cdot 1$ & 43421 & $123 \cdot 7$ & 25670 & 17751 & $3455 \cdot 0$ & $9 \cdot 76$ & $9 \cdot 73$ & -.03 \\
\hline $400 \cdot 0$ & 46640 & $116 \cdot 6$ & 27830 & 18810 & $3711 \cdot 5$ & $9 \cdot 20$ & $9 \cdot 34$ & $\cdot 14$ \\
\hline $552 \cdot 1$ & 55410 & $100 \cdot 4$ & 34090 & 21320 & $4409 \cdot 0$ & $7 \cdot 91$ & $8 \cdot 16$ & $\cdot 25$ \\
\hline $732 \cdot 1$ & 63400 & $86 \cdot 6$ & 39850 & 23550 & $5045 \cdot 0$ & $6 \cdot 81$ & $6 \cdot 93$ & $\cdot 12$ \\
\hline $999 \cdot 8$ & 71800 & $71 \cdot 8$ & 47310 & 24490 & $5714 \cdot 0$ & $5 \cdot 63$ & $5 \cdot 55$ & -.08 \\
\hline 1471 & 80770 & $54 \cdot 9$ & 55870 & 24900 & $6430 \cdot 0$ & $4 \cdot 29$ & $3 \cdot 98$ & $-\cdot 31$ \\
\hline$\ldots$ & $\cdots \cdots$ & $\cdots \cdots$ & $\ldots \ldots$ & $\ldots \ldots$ & 8160 & $\ldots$ & 0 & $\cdots \cdots$ \\
\hline
\end{tabular}

$$
\kappa=11 \sin \frac{\Im+190 \kappa+120}{46} \text {. }
$$

TABLE V.

Cast Cobalt, Magnetic, at - $5^{\circ} \mathrm{C}$.

Experiments made November 28, 1873.

\begin{tabular}{|c|c|c|c|c|c|c|}
\hline $\mathfrak{b}$. & B. & $\mu$. & T. & P. & \$. & $\kappa$. \\
\hline $48 \cdot 47$ & 3702 & $76 \cdot 37$ & 3287 & 415 & $290 \cdot 8$ & $6 \cdot 00$ \\
\hline $76 \cdot 74$ & 7254 & $94 \cdot 34$ & 5760 & 1494 & $571 \cdot 1$ & $7 \cdot 44$ \\
\hline $112 \cdot 8$ & 14370 & $127 \cdot 5$ & 9388 & 4982 & $1134 \cdot 5$ & $10 \cdot 06$ \\
\hline $167 \cdot 6$ & 24130 & $144 \cdot 0$ & 14490 & 9640 & 1907 & $11 \cdot 38$ \\
\hline $264 \cdot 2$ & 35860 & $135 \cdot 7$ & 20420 & 15440 & 2833 & $10 \cdot 72$ \\
\hline $539 \cdot 9$ & 53940 & $99 \cdot 91$ & 33010 & 20930 & 4249 & $7 \cdot 87$ \\
\hline 1473 & 80760 & $54 \cdot 84$ & 55920 & 24840 & 6310 & $4 \cdot 28$ \\
\hline
\end{tabular}


TABLE VI.

Cast Cobalt, Magnetic, at $230^{\circ} \mathrm{C}$.

Experiments made February 3, 1874.

\begin{tabular}{|c|c|c|c|c|c|c|}
\hline $\mathfrak{k}$ & 9. & $\mu$. & T. & P. & ঐ. & $\kappa$. \\
\hline $13 \cdot 34$ & 1357 & $101 \cdot 8$ & 1165 & 192 & 107 & 8.02 \\
\hline $25 \cdot 67$ & 2916 & $113 \cdot 6$ & 2662 & 254 & 230 & $8 \cdot 96$ \\
\hline $38 \cdot 55$ & 4940 & $128 \cdot 2$ & 4397 & $\mathbf{5 4 3}$ & 390 & $10 \cdot 12$ \\
\hline $55 \cdot 56$ & 9400 & $169 \cdot 1$ & 7440 & 1960 & $743 \cdot 5$ & $13 \cdot 38$ \\
\hline $75 \cdot 16$ & 15800 & $210 \cdot 2$ & 10050 & 5750 & 1143 & $16 \cdot 65$ \\
\hline $101 \cdot 4$ & 23920 & $235 \cdot 9$ & 14260 & 9660 & 1895 & $18 \cdot 70$ \\
\hline $132 \cdot 7$ & 31260 & $235 \cdot 5$ & 17710 & 13550 & 2475 & $18 \cdot 66$ \\
\hline $172 \cdot 9$ & 38060 & $220 \cdot 2$ & 21820 & 16240 & 3015 & $17 \cdot 44$ \\
\hline $281 \cdot 8$ & 52520 & $186 \cdot 4$ & 31160 & 21360 & 4174 & $14 \cdot 76$ \\
\hline $393 \cdot 6$ & 63430 & $161 \cdot 2$ & 39070 & 24360 & 5039 & $12 \cdot 75$ \\
\hline 702.9 & 82070 & $117 \cdot 0$ & 54920 & 27150 & 6515 & $9 \cdot 27$ \\
\hline $989 \cdot 3$ & 95600 & $96 \cdot 63$ & 66750 & 28850 & 7584 & $7 \cdot 67$ \\
\hline 1282 & 106200 & $82 \cdot 87$ & 75820 & 30380 & 8422 & $6 \cdot 57$ \\
\hline
\end{tabular}

From Table IV we see that at ordinary temperatures cobalt does not offer any exception to the general law for the other magnetic metalsthat as the magnetization increases, the magnetic permeability first increases and then decreases. We also see that the results satisfy to a considerable degree of accuracy the equation which $I$ have used for the other magnetic metals. The departure from the equation is of exactly the nature that can be accounted for in either of two ways-either by the heating of the ring by the current for the higher magnetizingforces, or by some want of homogeneity in the ring. According to the first explanation, the maximum of magnetization at $0^{\circ} \mathrm{C}$. will be somewhat lower than the curve indicates; but by the second it must be higher. I, however, incline to the first, that it is due to heating, for two reasons: first, it is sufficient; and secondly, the smaller cobalt ring gives about the same maximum as this. Hence we may take as the provisional value of the maximum of magnetization of cobalt in round numbers $\mathfrak{\Im}=8000$, or $\mathfrak{B}=100,000$.

We also see from Table IV that, at least in this case, the permeability of cobalt is less than that of nickel, though we could without doubt select specimens of cobalt which should have this quality higher than a given specimen of nickel. The formula at the foot of the Table also shows, by the increased value of the coefficient of $\kappa$ in the right-hand member, that the diameter of the curve is much less inclined to the axis of $\mathfrak{\Im}$ in this case than in the case of nickel or iron. In this respect the three metals at present stand in the following order-cobalt, nickel, iron. This is the inverse order also of their permeability; but 
at present I have not found any law connecting these two, and doubt if any exact relation exists, though as a general rule the value of the constant is greater in those curves where the permeability is least.

In a short abstract in the 'Telegraphic Journal,' April 1, 18\%4, of a memoir by M. Stefan, it is stated "that the resistance of iron and nickel to magnetization is at first very great, then decreases to a minimum value, which is reached when the induced magnetic moment is become a third of its maximum." This will do for a very rough approximation, but is not accurate, as will be seen from the following Table of this ratio from my own experiments:-

\begin{tabular}{|c|c|c|c|c|c|}
\hline \multicolumn{6}{|c|}{ Experiments published in August, 1873.} \\
\hline $\begin{array}{l}\text { Iron. } \\
\text { Tables I } \\
\text { and II. }\end{array}$ & $\begin{array}{l}\text { Iron. } \\
\text { Table III. }\end{array}$ & $\begin{array}{l}\text { Bessemer } \\
\text { steel. } \\
\text { Table IV. }\end{array}$ & $\begin{array}{l}\text { Iron. } \\
\text { Table } \mathrm{V} \text {. }\end{array}$ & $\begin{array}{l}\text { Nickel. } \\
\text { Table VI. }\end{array}$ & $\begin{array}{c}\text { Steel. } \\
\text { Table VII. }\end{array}$ \\
\hline 1 & 1 & 1 & 1 & 1 & 1 \\
\hline $3 \cdot 02$ & $2 \cdot 64$ & $2 \cdot 65$ & $2 \cdot 68$ & $3 \cdot 15$ & $2 \cdot 46$ \\
\hline \multicolumn{6}{|c|}{ Experiments of present paper. } \\
\hline \multicolumn{2}{|c|}{$\begin{array}{l}\text { Nickel. } \\
\text { Tables I and II. }\end{array}$} & \multicolumn{2}{|c|}{$\begin{array}{l}\text { Nickel. } \\
\text { Table III. }\end{array}$} & \multicolumn{2}{|c|}{$\begin{array}{l}\text { Cobalt. } \\
\text { Tables IV and V. }\end{array}$} \\
\hline \multicolumn{2}{|c|}{1} & \multicolumn{2}{|c|}{1} & \multicolumn{2}{|c|}{1} \\
\hline \multicolumn{2}{|c|}{$\overline{3 \cdot 23}$} & \multicolumn{2}{|c|}{$\overline{3 \cdot 14}$} & \multicolumn{2}{|c|}{$4 \cdot 2$} \\
\hline
\end{tabular}

The average of these is, if we include Bessemer steel with the iron, as it is more iron than steel:-

$$
\text { Iron, } \frac{1}{2 \cdot 75}=\frac{4}{11} ; \quad \text { Nickel, } \frac{1}{3 \cdot 17} ; \quad \text { Cobalt, } \frac{1}{4 \cdot 2} \text {. }
$$

Hence the place of greatest permeability will vary with the kind of metal. From these, however, we can approximate to the value of $b$ in the formula; for we have

$$
\begin{gathered}
\text { for Iron, } b=\frac{27,000}{\beta} ; \text { for Nickel, } b=\frac{11,000}{\beta} ; \\
\text { for Cobalt, } b=26,000 .
\end{gathered}
$$

In Table $\mathrm{V}$ we have the results for cobalt in the magnetic state. We here find the same effect of magnetization as we have before found for iron and nickel. 
In Table VI we have results for cobalt at a high temperature, and see how greatly the permeability is increased by rise of temperature, this being for the vertex of the curve about 70 per cent. But on plotting the curve I was much surprised to find an entire departure from that regularity which $I$ had before found in all curves taken from iron and nickel when the metal was homogeneous. At present $I$ am not able to account for this, and especially for the fact that one of the measurements of $\mathfrak{B}$ is higher than that which we have taken for the maximum of magnetization, at, however, a lower temperature. The curve is exactly of the same nature as that which I have before found for a piece of nickel which had been rendered unhomogeneous by heating red-hot, and thus burning the outside. The smaller cobalt ring gives a curve of the same general shape as this, but has the top more rounded. I will not attempt without fresh experiments to explain these facts, but will simply offer the following explanations, some one of which may be true. First, it may be due to want of homogeneity in the ring; but it seems as if this should have affected the curve of Table IV more. Secondly, it may be at least partly due to the rise in temperature of the ring at high magnetizing-powers; and indeed we know that this must be greater in paraffin than in alcohol for several reasons: there is about twice as much heat generated in copper wire at $230^{\circ} \mathrm{C}$. as at $0^{\circ}$ with the same current; and this heat will not be conducted off so fast in paraffin as in alcohol, on account of its circulating with less freedom; it probably has less specific heat also. Thirdly, it may be due to some property of cobalt, by which its permeability and maximum of magnetization are increased by heat and the curve changed.

The experiments made with the small ring confirm those made with the large one as far as they go; but as it was so small, they do not possess the weight due to those with the larger one. But, curious as it may seem, although they were turned from the same button side by side, yet the permeability of the larger is about 45 per cent greater than that of the smaller. I have satisfied myself that this is due to no error in experiment, but illustrates what extremely small changes will affect the permeability of any metal.

We have now completed the discussion of the results as far as they refer to the magnetic permeability, leaving the discussion of the temporary and permanent or residual magnetism to the future, although these latter, when discussed, will throw great light upon the nature of the coercive force in steel and other metals. The whole subject seems to be a most fruitful one, and I can hardly understand why it has 
been so much neglected. It may have been that a simple method of experiment was not known; but if so, I believe that my method will be found both accurate and simple, though it may be modified to suit the circumstances. Professor Maxwell has suggested to me that it would be better to use rods of great length than rings, because that in a ring we can never determine its actual magnetization, but must always content ourselves with measuring the change on reversing or breaking the current. This is an important remark, because it has been found by MM. Marianini and Jamin, and was noticed independently by myself in some unpublished experiments of 1870 , that a bar of steel which has lain for some time magnetized in one direction will afterwards be more easily magnetized in that direction than in the other. This fact could not have been discovered from a ring; and indeed if a ring got a onesided magnetism in any way we might never know it, and yet it might affect our results, as indeed we have already seen in the case of the magnetic curve. But at the same time I think that greater errors would result from using long bars. I have tried one of iron 3 feet long and $\frac{1}{4}$ inch diameter; and the effect of the length was still apparent, although the ratio of length to diameter was 144 . To get exact results it would probably have to be several times this for the given specimen of iron, and would of course have to be greater for a piece of iron having greater permeability. This rod must be turned and must be homogeneous throughout-conditions which it would be very difficult to fulfil, and which would be impossible in the case of nickel and cobalt. We might indeed use ellipsoids of very elongated form; and this would probably be the best of all, as the mathematical theory of this case is complete, and it is one of the few where the magnetization is uniform, and which consequently will still hold, although the permeability may vary with the amount of magnetization. This form will, of course, satisfy Professor Maxwell's objection.

The method of the ring introduces a small error which has never yet been considered, and which will affect Dr. Stoletow's results as well as mine. The number of lines of induction passing across the circular section of a ring-magnet we have seen to be

$$
Q_{1}=4 n^{\prime} i \int_{-R}^{+R} \mu \frac{\sqrt{ } R^{2}-x^{2}}{a-x} d x,
$$

in which $a$ is the mean radius of the ring, $R$ the radius of the section, $n^{\prime}$ the number of coils in the helix, and $i$ the intensity of the current. Now in integrating this before, I assumed that $\mu$ was a constant throughout the section of the ring: now we have found that $\mu$ is a 
function of the magnetization, and hence a function of the magnetizingforce; but the latter varies in different parts of the section, and hence $\mu$ must vary. But the correction will be small, because the average value will be nearly the same as if it were a constant. We may estimate the correction in the following manner. Let $\mu$ and $\mathfrak{F}$ be the values of those quantities at any point in the section of the ring, $\mu^{\prime}$ and $\mathfrak{F E}^{\prime}$ the

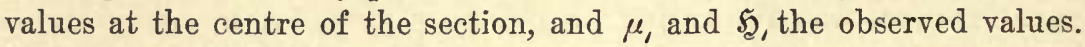
Then, by Taylor's theorem,

$$
\mu=\mu^{\prime}+\frac{d \mu^{\prime}}{d \mathfrak{H}^{\prime}}\left(\mathfrak{T}-\mathfrak{S}^{\prime}\right)+\frac{1}{2} \frac{d^{2} \mu^{\prime}}{d \mathcal{S}^{\prime 2}}\left(\mathfrak{S}-\mathfrak{S}^{\prime}\right)^{2}+\& \mathrm{c} .
$$

But $\mathfrak{S}=\frac{2 n^{\prime} i}{a-x}$ and $\mathfrak{H}^{\prime}=\frac{2 n^{\prime} i}{a}$, and so we have

$$
\begin{aligned}
Q_{1}=\pi R^{2} \mathfrak{S}^{\prime} \mu^{\prime}\left\{1+\frac{1}{4} \frac{R^{2}}{a^{2}}+\right. & \& \mathrm{c} .+\frac{\mathfrak{S}^{\prime}}{2 \mu^{\prime}} \frac{d \mu^{\prime}}{d \mathfrak{S}^{\prime}}\left(\frac{R^{2}}{a^{2}}+\frac{R^{4}}{a^{4}}+\& \mathrm{c} .\right) \\
& \left.+\frac{\mathfrak{S}^{\prime 2}}{8 \mu^{\prime}} \frac{d^{2} \mu^{\prime}}{d \mathfrak{S}^{\prime 2}}\left(\frac{R^{2}}{a^{2}}+3 \frac{R^{4}}{a^{4}}+\& \mathrm{c} .\right)+\& \mathrm{c} .\right\} .
\end{aligned}
$$

But in my Tables I have already calculated

$$
\mu_{1}=\frac{Q^{1}}{\pi R^{2} \mathfrak{H}^{\prime}\left(1+\frac{1}{4} \frac{R^{2}}{a^{2}}+\& \mathrm{c} .\right)} ;
$$

and as $\mu$, is very nearly equal to $\mu^{\prime}$, and $\mathfrak{H}_{1}$, to $\mathfrak{S}^{\prime}$, we have approximately

$$
\begin{aligned}
\mu^{\prime}=\mu_{1}\left\{1-\frac{\mathfrak{H}_{1}}{2 \mu_{1}} \frac{d \mu_{1}}{d \mathfrak{S}_{1}}\left(\frac{R^{2}}{a^{2}}\right.\right. & \left.+\frac{3}{4} \frac{R^{4}}{a^{4}}+\& \mathrm{c} .\right) \\
& \left.-\frac{\mathfrak{S}_{i}}{8 \mu_{1}} \frac{d^{2} \mu_{1}}{d \mathfrak{S}_{1}^{2}}\left(\frac{R^{2}}{a^{2}}+\frac{11}{4} \frac{R^{4}}{a^{4}}+\& \mathrm{c} .\right)-\& \mathrm{c} .\right\},
\end{aligned}
$$

which will give the value of $\mu^{\prime}$ corresponding to $Q^{\prime}$ and $\mathfrak{H}^{\prime}$. Hence the correct values of the quantities will be $\mu^{\prime}, \mathfrak{S}^{\prime}$, and $\mathfrak{B}^{\prime}=\mathfrak{T}^{\prime} \mu^{\prime}$.

The quantities $\frac{d \mu_{1}}{d \mathfrak{S}_{1}}$ and $\frac{d^{2} \mu_{1}}{d \mathfrak{F}_{1}^{2}}$ can be obtained either by measuring a plot of the curve, or from the empirical equation

$$
\mu=B \sin \frac{\mu \mathfrak{Y}+b \mu+\pi}{D},
$$

when we know the values of the constants. In this case

in which

$$
\begin{aligned}
& \frac{d \mu}{d \mathfrak{S}_{1}}= \pm \frac{\mu_{1} \sqrt{B^{2}-\mu_{l}^{2}}}{C}, \\
& \frac{d^{2} \mu_{1}}{d \mathscr{S}_{1}^{2}}=\frac{C_{\mu_{1}}\left(2 B^{2}-3 \mu_{1}^{2}\right) \mp \mu_{1}^{3}\left(\mathfrak{F}_{1}+b\right) \sqrt{B^{2}-\mu_{1}^{2}}}{\sigma^{3}},
\end{aligned}
$$

$$
C=5 \% \cdot 3 D \mp(\mathfrak{g}+b) \sqrt{B^{2}-\mu_{i}^{2}}
$$


In all these the upper signs are to be taken for all values of $\mathfrak{H}_{\text {, less than }}$ $\frac{90 D-b B-\pi}{B}$, and the lower signs for greater values.

On applying these formulæ to the observations, I have found that the corrections will in no way influence my conclusions, being always very small; but at the same time the calculation shows that it would be well to diminish the ratio $\frac{R}{a}$ as much as possible. In all my rings this ratio did not depart very much from $\frac{1}{6 \cdot 3}$; but I would advise future experimenters to take it at least as small as $\frac{1}{10}$ : the amount of correction will be very nearly proportional to the square of $\frac{R}{a}$.

\section{Summary.}

The following laws have been established entirely by my own experiments, though in that part of (2) which refers to iron I have been anticipated in the publication by Dr. Stoletow (Phil. Mag. Jan. 1873). When any measurements are given, they are on the metre, gramme, second system.

(1) Iron, nickel, and cobalt, in their magnetic properties at ordinary temperatures, differ from each other only in the quantity of those properties and not in the quality.

(2) As the magnetizing-force is increased from 0 upwards, the resistance of iron, nickel, and cobalt to magnetization decreases until a minimum is reached, and after that increases indefinitely. This minimum is reached when the metal has attained a magnetization of from .24 to $\cdot 38$ of the maximum of magnetization of the given metal.

(3) The curve showing the relation between the magnetization and the magnetic permeability, or Neumann's coefficient, is of such a form that a diameter can be drawn bisecting chords parallel to the axis of $\mathfrak{B}$, and is of very nearly the form given by the equation

$$
\mu=B \sin \frac{\mathfrak{B}+b_{\mu}+\pi}{D},
$$

where $B, b$, and $D$ are constants, $\mu$ is the ratio of the magnetization to the magnetizing-force in an infinitely long bar, and $\mathfrak{B}$ is the amount of magnetization.

(4) If a metal is permanently magnetized, its resistance to change of magnetism is greater for low magnetizing-powers than when it is in the normal state, but is the same for high magnetizing-powers. This 
applies to the permanent state finally attained after several reversals of magnetizing-force; but if we strongly magnetize a bar in one direction and then afterwards apply a weak magnetizing-force in the opposite direction, the change of magnetization will be very great.

(5) The resistances of nickel and cobalt to magnetization vary with the temperature; but whether it is increased or not in nickel depends upon the amount of magnetization: for a moderate amount of magnetization it decreases with rise of temperature very rapidly; but if the magnetization is high the resistance is increased. In cobalt it apparently always decreased, whatever the magnetization. The resistance of iron to magnetization is not much affected by the temperature.

(6) The resistance of any specimen of metal to magnetization depends on the kind of metal, on the quality 'of the metal, on the amount of permanent magnetization, on the temperature, and on the total amount of magnetization, and, in at least iron and nickel, decreases very much on careful annealing. The maximum of magnetization depends on the kind of metal and on the temperature.

(7) Iron, nickel, and cobalt all probably have a maximum of magnetization, though its existence can never be entirely established by experiment, and must always be a matter of inference; but if one exists, the values must be nearly as follows at ordinary temperatures. Iron when $\mathfrak{B}=175,000$ or when $\mathfrak{\Im}=13,900$; nickel when $\mathfrak{B}=63,000$ or when $\mathfrak{I}=4940$; cobalt when $\mathfrak{B}=100,000$ (?) or when $\mathfrak{\Im}=8000$ (?).

(8) The maximum of magnetization of iron and nickel decreases with rise of temperature, at least between $10^{\circ} \mathrm{C}$. and $220^{\circ} \mathrm{C}$., the first very slowly and the second very rapidly. At $220^{\circ} \mathrm{C}$. the maximum for iron is when $\mathfrak{B}=172,000$ and $\mathfrak{S}=13,600$, and for nickel when $\mathfrak{B}=49,000$ and $\mathfrak{J}=3800$.

The laws which govern temporary and residual magnetism, except so far as they have been hitherto given, I leave for the future, when I shall have time for further experiment on the subject to develop some points which are not yet quite clear.

Troy, Newo York, U. S. A., April, 1874. 
ON A NEW DIAMAGNETIC ATTACHMENT TO THE LANTERN, WITH A NOTE ON THE THEORY OF THE OSCILLATIONS OF INDUCTIVELY MAGNETIZED BODIES

[American Journal of Science [3], IX, 357-361, 1875]

\section{Description of Apparatus}

Some time ago, in thinking of the theory of diamagnetism, I came to the conclusion that apparatus of large size was by no means necessary in diamagnetic experiments, and on testing my conjectures experimentally, I was much pleased to find that they were true. So that for more than a year I have been in the habit of illustrating this subject to my classes by means of a small apparatus weighing only about a pound or two, which I place in my lantern and magnify to a large size on the screen.

The effects obtained in this way are very fine and are not surpassed by those with the largest magnets; and we are by no means confined. to strongly diamagnetic substances, but, with proper care, can use anything, even the most feeble. The apparatus which I used consisted of a horseshoe electro-magnet, made of an iron bar half an inch in diameter and about ten inches long, bent into the proper form, and surrounded with four or five layers of No. 16 wire. But the following apparatus will, without doubt, be found much more convenient. It can be made of any size, though the dimensions given will probably be found convenient.

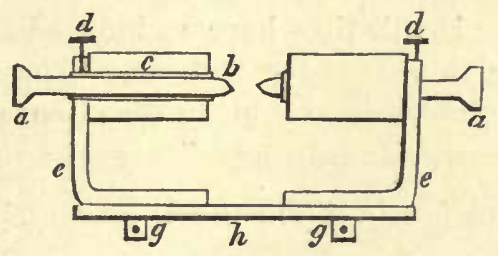

FIGURE 1.

The apparatus is represented in Fig. 1. To a straight bar of iron $h$, 7 in. long, $\frac{1}{4}$ in. thick, and $\frac{3}{4}$ in. wide, are attached two pieces $e e$ of the same kind of iron by two set screws $g g$, which move in slots in the 
piece $h$. Into these pieces are screwed two tubes $c$ made of iron and having an internal diameter of about $\frac{7}{16} \mathrm{in}$. and a thickness not to exceed $\frac{1}{16}$ in. Through these tubes the iron rods $a b$ slide and are held at any point by the screws $d$. One end $b$ of this rod is rounded off for diamagnetic experiments and the other enlarged and flattened at the end for magnecrystallic experiments. On the tube $c$ a helix of No. 16 or No. 18 wire is wound so as to make up a thickness of $\cdot 4$ or $\cdot 5$ of an inch and having a length of $2 \frac{1}{4}$ in. The object of the screws $g$ is principally to allow the rods $a b$ to be reversed quickly and to adjust the position of the helices. When the apparatus is to be used for only one kind of work it can be much simplified by doing away with many of the moving parts.

This instrument can be used either with the ordinary magic lantern, or better, with one having. a vertical attachment. In the latter case the plane of the instrument is horizontal and the substances are suspended from a wire made quite small, so as not to cut off too much light.

The suspending thread in the case of bismuth can be quite large but for other bodies a single fibre of silk is best; these in the shape of bars half an inch long can be each attached to a fibre having a little . wire hook at its upper end and hung in a cabinet until required.

The theory of feebly magnetic or diamagnetic bodies oscillating in a magnetic field is very simple and yet the results are of the greatest interest, especially the effect of the size of the apparatus, which is here given for the first time.

\section{THEORY}

Let a very small particle of a body whose coefficient of magnetization $\kappa$ is very small, and either positive or negative, be placed in a magnetic field of intensity $R$; it will then have an induced magnetic moment of $\kappa v R$, where $v$ is the volume of the element. The force acting on this particle to cause it to go in any given direction will be equal to the product of the magnetic moment into the rate of variation of $R$ in that direction, ${ }^{1}$ and hence is $\kappa v R \frac{d R}{d x}$ in the direction of $x$. The total force acting on the body in the direction of $x$ is therefore

$$
X=\frac{1}{2} \iiint \kappa \frac{d\left(R^{2}\right)}{d x} d x d y d z
$$

Thomson, Reprint of Papers, art. 6r9, Prob. vii. 
and the other components of the force are

and

$$
Y=\frac{1}{2} \iiint \kappa \frac{d\left(R^{2}\right)}{d y} d x d y d z
$$

$$
Z=\frac{1}{2} \iint^{\bullet} \int \kappa \frac{d\left(R^{2}\right)}{d z} d x d y d z
$$

Let, now, the axis of $z$ be vertical, the axis of $x$ in the line of the magnetic poles of the magnet, and $y$ at right angles to both. Then the moment of the forces acting on the body to turn it about the axis of $z$ is

$$
M=\frac{1}{2} \iiint\left(\kappa \frac{d\left(R^{2}\right)}{d x} y-\kappa \frac{d\left(R^{2}\right)}{d y} x\right) d x d y d z
$$

where the integration extends throughout the volume of the body.

If the body is suspended so as to turn freely about the axis of $z$ it will vibrate about the position for which $M$ is a minimum or else will remain at rest at that point. The number of single oscillations made when the angular elongation $\vartheta$ is very small, is

$$
n=\frac{1}{\pi} \sqrt{\frac{M}{\vartheta I}}
$$

in which $M$ and $\vartheta$ must be measured simultaneously, and $I$ is the moment of inertia of the body.

$$
\therefore n=\frac{1}{\pi \sqrt{2 y I}} \sqrt{\iiint \kappa\left(y \frac{d\left(R^{2}\right)}{d x}-x \frac{d\left(R^{2}\right)}{d y}\right) d x d y d z} .
$$

Now let us suppose that the whole apparatus changes size, the relation between the parts remaining constant, so that the apparatus becomes $m$ times as great as before. Then $x, y, d x, d y$, and $d z$ will increase $m$ times and $I, m^{5}$ times. To determine the changes in $\frac{d\left(R^{2}\right)}{d x}$ and $\frac{d\left(R^{2}\right)}{d y}$ we make use of the theorem of Sir Wm. Thomson, that "similar bars of different dimensions, sinilarly rolled, with lengths of wire proportional to the squares of their linear dimensions, and carrying equal currents, cause equal forces at points similarly situated with reference to them." But as the above only applies to equal currents, I have generalized it in the following: In any two magnetic systems whatever, similar in all their parts and composed of any number of permanent or electro-magnets, wires carrying currents, or bodies under magnetic induction, the magnetic force at similar points of each will be the same when the following conditions are complied with: 1st, the magnetic materials at similar points in the two systems must be exactly the same in quality and 
temper; $2 d$, the permanent magnets must be magnetized to the same degree at similar points of the systems; 3d, the coils of the electro-magnets and other wires or bundles of wires carrying the current must have similar external dimensions in the two systems and must have the product of the current by the number of wires passing through similar sections of the two systems proportional to the linear dimensions of the systems.

This will apply to the case we are considering when the product of the current by the number of the turns of wire varies in direct proportion to the size of the apparatus. Hence in this case $\frac{d\left(R^{2}\right)}{d x}$ and $\frac{d\left(R^{2}\right)}{d y}$ will vary inversely as $m$. Hence we see that $n$ will be inversely proportional to the size of the apparatus; and although we have only proved this for the case when $\kappa$ is small, it is easy to see that it is perfectly general. The advantage of small diamagnetic apparatus is thus apparent, for the smaller we make it the more vibrations the bar will make in a given time and the more promptly will the results be shown.

It might be thought that by hanging a very small bar in the field of a large magnet, we might obtain just as many vibrations as by the use of a small apparatus; but this is not so, for Sir Wm. Thomson has shown ${ }^{2}$ that the number of oscillations of a feebly magnetic or diamagnetic body of elongated form in a magnetic field is nearly independent of the length when that is short. So that the only way of increasing the number of vibrations is to decrease the size of the whole apparatus, or to increase the power of the magnets; the latter has a limit and hence we become dependent on the former.

The theory of the effect of the size of the body is very simple, and we may proceed as follows. Let the body be in the form of a small bar whose sectional area, $a$, is very small compared with its length, and let $\gamma$ be the angle of the axis of the bar with the line joining the poles, and $v$ the radius vector from the origin. Developing $R^{2}$ as a function of $x$ and $y$ by Taylor's theorem, and noting that as $R$ is symmetrical with reference to the planes $X Z$ and $Y Z$, only the even powers of $x$ and $y$ can enter into the development, we have, calling $R_{0}$ the value of $R$ at the origin,

$$
\begin{aligned}
R^{2}=R_{0}^{2}+\frac{1}{2}\left(\frac{d^{2}\left(R_{0}^{2}\right)}{d x^{2}} x^{2}+\frac{d\left(R_{0}^{2}\right)}{d y} y^{2}\right) \\
\quad+\frac{1}{2.3 .4}\left(\frac{d^{4}\left(R_{0}^{2}\right)}{d x^{4}} x^{4}+6 \frac{d^{4}\left(R_{0}^{2}\right)}{d x^{2} d y^{2}} x^{2} y^{2}+\frac{d^{4}\left(R_{0}^{2}\right)}{d y^{4}} y^{4}\right)+\mathbb{B} .
\end{aligned}
$$

${ }^{2}$ Reprint of Papers, art. 670. Remarques sur les oscillations d'aiguilles non crystallisées. 
When the vibrating body is very small the first two terms will suffice: hence we have

$$
M=\frac{1}{2} a_{\kappa}\left(\frac{d^{2}\left(R_{0}^{2}\right)}{d x^{2}}-\frac{d^{2}\left(R_{0}^{2}\right)}{d y^{2}} \sin \gamma \cos \gamma \int_{-\frac{1}{2} l}^{+\frac{1}{2} l} r^{2} d r\right.
$$

in which $l$ is the length of the bar. If $\delta$ is the density of the body (weight of a unit of volume), $I=\frac{a l^{3} \delta}{12}$ and $n$ becomes

$$
n=\frac{1}{\pi} \sqrt{\frac{\kappa}{2 \delta}\left(\frac{d^{2}\left(R_{0}^{2}\right)}{d x^{2}}-\frac{d^{2}\left(R_{0}^{2}\right)}{d y^{2}}\right),}
$$

in which, however, it is to be noted that $\frac{d^{2}\left(R_{0}^{2}\right)}{d y^{2}}$ is essentially negative and so the sign of the term containing it will be positive in the actual development.

This equation is independent of the dimensions of the body, and hence we conclude that when the body is small and very long as compared with its other dimensions, the number of vibrations which it will make in a given field is dependent merely on its coefficient of magnetization and on its density; a result first given by Sir Wm. Thomson, in the paper referred to. I have given it once more and put it in its present form merely to call attention to the facility with which $\kappa$ can be obtained from it when we have measured $R$ in different parts of the field by known methods. This could be done by means of a rotating coil as used by Verdet, or by my magnetic proof plane which I will soon describe, combined with my method of using the earth inductor. This will give the best method that I know of for obtaining $\kappa$ for diamagnetic or weak paramagnetic substances.

Troy, January 15, 1875. 


\section{NOTES ON MAGNETIC DISTRIBUTION}

[Proceedings of the American Academy of Arts and Sciences, $X I, 191,192,18 \pi 6$. Presented June 9, 1875]

In two papers which have recently appeared on this subject, by Mr. Sears (Amer. Jour. of Science, July, 18\%4), and Mr. Jacques (Proc. Amer. Acad. of Sciences, 1875, p. 445), a method is used for determining magnetic distribution, founded on induced currents, in which results contrary to those published by M. Jamin have been found. It does not seem to have been noticed that the method then used does not give what we ordinarily mean by magnetic distribution. In mathematical language, they have measured the surface integral of magnetic induction across the section of the bar instead of along a given length of its surface. ${ }^{1}$ M. Jamin's method gives a result depending on the so-called surface density of the magnetism, which is nearly proportional to the surface integral of the magnetic induction along a given length of the bar. Hence the discrepancy between the different results. Had the experiments of Mr. Sears and Mr. Jacques been made by sliding the helix inch by inch along the bars, their results would have confirmed those of M. Jamin. Four or five years ago, I made a large number of experiments in this way, which I am now rewriting for publication, and where the whole matter will be made clear. At present, I will give the following method of converting one into the other. Let $Q$ be the surface integral of magnetic induction across the section of the rod, and let $Q e$ be that along one inch of the rod: then $Q e \propto \frac{d Q}{\| x}, x$ being the distance along the rod. Hence, M. Jamin's results depend on the rate of variation of the magnetization of the rod, while those of Mr. Sears and Mr. Jacques depend on the magnetization. In conclusion, let me heartily agree with Mr. Jacques's remàrks about M. Jamin's conclusions from his experiments. Such experiments as those give no data whatever for a physical theory of magnetism, and can all be deduced from the ordinary mathematical theory, which is independent of physical

\footnotetext{
${ }^{1}$ Maxwell's Electricity and Magnetism, art. 402.
} 
hypothesis, combined with what is known with regard to the magnetizing function of iron. This will be shown in the paper I am rewriting. It seems to me that M. Jamin's method is very defective; and I know of no method of experimenting, which is theoretically without objection except that of induced currents, and this I have used in all my experiments on magnetic distribution for the last four or five years, and have developed into a system capable of giving results in absolute measure. Mr. Jacques is to be congratulated on pointing out these errors in M. Jamin's conclusions.

Troy, June 7, 1875. 


\title{
NOTE ON KOHLRAUSCH'S DETERMINATION OF THE ABSO- LUTE VALUE OF THE SIEMENS MERCURY UNIT OF ELECTRICAL RESISTANCE
}

\author{
[Philosophical Magazine [4], L, 161-163, 1875]
}

In looking over Kohlrausch's paper ${ }^{1}$ upon the determination of a resistance in absolute measure, with a view to undertaking something of the kind myself, and also, if possible, to discover the reason of the difference from the results of the Committee of the British Association, I think I have come across an error of sufficient magnitude and in the proper direction to account for the 2 per cent difference. Kohlrausch's experiments were made with such great care and by so experienced a person that it is only after due thought and careful consideration that I take it upon me to offer a few critical remarks.

We observe, then, first of all, that the principal peculiarity of his method consists in doing away with all measurements of the coils of the galvanometer, and in its place making accurate determinations of the logarithmic decrement both with the circuit closed and open, together with various absolute determinations rendered necessary by this change. In this way the logarithmic decrement is raised from being a small correction to a most important factor in the equation. Hence it is that we should carefully scrntinize the theory and see whether it be correct enough for this purpose; for only an approximation is needed for the first method.

The resistances to a bar magnet swinging within a coil may be divided into two principal parts-first, that due to the resistance of air and viscosity of suspending fibre, and, second, that due to the induced current in the coils. The first resistance is usually taken as proportional to the velocity, and thus assumes the viscosity of the air to be the most important element. This is probably true in most cases where the motion is slow. This factor is quite small compared with the second when the magnet is large and heavy and the coils wound close to it, as

${ }^{1}$ Poggendorff's Annalen, Ergänzungsband vi, p. 1; translated in Phil. Mag., S. 4, vol. xIrii, pp. 294, 342. 
in Kohlrausch's instrument. Kohlrausch's principal error lies in the omission of the coefficient of self-induction from his equations.

For the sake of clearness, and because the subject is quite often misapprehended, I shall commence at the beginning and deduce nearly all equations.

Let us proceed at first in the method of Helmholtz, using the notation of Maxwell's ' Electricity.'

Let a current of strength $I$ be passing in a circuit whose resistance is $R$, and coefficient of self-induction $L$. Also let a magnet be near the circuit whose potential energy with respect to the circuit is $I V$. Let $A$ be the electromotive force of ihe battery in the circuit.

The work done by the battery in the time $d t$ is equal to the sum of the work done in heating the wire, in moving the magnet, and in increasing the mutual potential of the circuit on itself." Hence we have

$$
A I d t=I^{2} R d t+1 \frac{d V}{d t} d t+\frac{1}{2} L \frac{d\left(I^{2}\right)}{d t} d t
$$

and if $A$ is equal to zero, we find

$$
I=-\frac{1}{R}\left(\frac{d V}{d t}+L \frac{d l}{d t}\right)
$$

If we apply this to the case of a magnet swinging within a coil the angle of the magnet from a fixed position being $x$, we have since $\frac{d V}{d x}$ is the moment of the force acting on the magnet with unit current and may be denoted by $q$,

$$
I=-\frac{1}{R}\left(q \frac{d x}{d t}+L \frac{d I}{d t}\right), \ldots . . . .
$$

where my $R$ is Kohlrausch's $w$.

This expression differs from that used by Kohlrausch in the addition of the last term, which is the correction due to self-induction. The last term vanishes whenever the magnet moves with such velocity as to keep the induced current constant; but in the swinging of a galvanometer-needle it has a value.

To form the equation of motion of the needle, we can proceed the rest of the way as Maxwell has done (Electricity, art. 762). Assuming that all frictional resistances to the needle are proportional to the relocity of the needle, we have

$$
B \frac{d^{2} x}{d t^{2}}+C \frac{d x}{d t}+D x=q I, \ldots . . . .
$$

where $B, C$, and $D$ are constants. 
Eliminating $I$ between this equation and (1), we find

$$
\left(R+L \frac{d}{d t}\right)\left(B \frac{d^{2} x}{d t^{2}}+C \frac{d x}{d t}+D x\right)+q^{2} \frac{a x}{c t}=0 . \quad .
$$

At first sight this equation will appear to be the same as that of Maxwell; but on further examination we see that it is more general in the value of $q$.

Equation (3) is the correct equation to use in this case, and reduces to that of Kohlrausch when $L=0$.

To see how this error will affect Kohlrausch's results, we must remember that he uses this equation to find the constant of his galvanometer, on which his whole experiment depends; and the error is so interwoven with all his results that an entire recomputation is necessary, provided the data for calculating the coefficient of self-induction of the galvanometer coils and earth inductor can be obtained.

The equation

$$
\frac{t^{2}}{\pi^{2}+\lambda^{2}}=\frac{t_{0}^{2}}{\pi^{2}+\lambda_{0}^{2}}
$$

does not hold when self-induction is considered; and so his fundamental equation (1) is not correct, containing a twofold error.

The linear differential equation (3) is easily solved; but as the results are complicated, it is hardly worth while at present, until a recalculation can be made. I prefer to solve it on the supposition that $L$ is small, and thus merely obtain a correction to Kohlrausch's equation connecting $t$ and $t_{0}$, after which equation (15) or (17) (Maxwell's 'Electricity,' art. 762) can be used when made more general by substituting $q$ for $G m$.

As far as I have had time to go at present, the correction seems to be in the direction of making Kohlrausch's determination more nearly coincide with that of the Committee on Electrical Standards of the British Association. Other engagements occupy my attention at present; but I hope to see these corrections made to an otherwise excellent determination of this most important unit.

London, August 4, 1875. 


\section{PRELIMINARY NOTE ON A MAGNETIC PROOF PLANE}

[American Journal of Science [3], $X, 14-17,1875]$

About four years ago I made a large number of experiments on the distribution of magnetism on iron and steel bars by means of a coil of wire sliding along the bar; the induced current in the coil as measured by a galvanometer was a measure of the number of lines of force cut by the coil and can be found in absolute measure by my method of using the earth inductor. These researches have never yet been published owing to circumstances beyond my control, but are known to quite a number of persons in this country, and will soon be published. The method there used is the only correct one that I know of for experimenting on magnetic distribution, and my purpose in this note is to extend it to bodies of all shapes, so that experiments on magnetic distribution may become as simple and easy to perform as those on electrical distribution. And so well has my magnetic proof plane accomplished this that I can illustrate the subject to my classes with the greatest ease.

The apparatus required is merely a small coil of wire $\frac{1}{4}$ to $\frac{1}{2}$ inch in diameter, containing from 10 to 50 turns, and a Thomson galvanometer. When we require to reduce to absolute measure, another coil about a foot in diameter and containing 20 or 30 turns is required. Having attached the small coil (or, as I call it, the magnetic proof plane) to the galvanometer, we have merely to lay it on the required spot, and when everything is ready, to pull it away suddenly and carry it to a distance, and the momentary deflection of the galvanometer needle will be proportional to that component of the lines of force at that point which is perpendicular to the plane of the coil. And if we apply it to the surface of a permanent magnet the so-called surface density of the magnetism at that point will be nearly proportional to the deflection. In the case of an electro-magnet the surface density will be nearly proportional to the defiection minus the deflection which would be produced by the helix alone, though the last is generally small and may be neglected. I use the words nearly proportional in the above statement because they are only exactly true in the cases where the lines of force 
proceed from the surface in a perpendicular direction; otherwise the deflections must be multiplied by the secant of the angle made by the lines of force with the surface of the magnet. In the case of an electromagnet made of very soft iron, theory shows that the lines pass out nearly perpendicular to the surface and so no correction is needed.

We can also, by a coil of this kind, determine the intensity of the magnetic field at any point and thus be able to make a complete map of it. Having done this, we have all the data necessary to substitute in the formula which I have given in this Journal, ${ }^{1}$ and by a simple experiment can thus determine the coefficient of magnetization of any diamagnetic or weak paramagnetic body probably in a more accurate manner than any Weber used. Only the largest-sized magnets could of course be used for this purpose with any accuracy, and indeed they are always to be preferred in obtaining the distribution by this method.

Having obtained the distribution for any given magnet, the distribution for any similar magnet of the same material but of different size becomes known by a well-known law of Sir William Thomson.

As, in the present state of our knowledge, magnetic measurements are of small value unless made on the absolute scale, we require to reduce our results to this system. There are several methods of doing this, but the simplest is that which I have used in my experiments on magnetic permeability, and consists in including an earth inductor in the circuit. A coil laid on a perfectly level surface is sufficient for this: when this is turned over, the induced current will be equal to $C=$ $\frac{2 n V A}{R}$ where $n$ is the number of turns in the coil, $A$ its mean area, $V$ the vertical component of the earth's magnetism, and $R$ the resistance of the circuit. When the small coil is pulled suddenly away the current will be $C^{\prime}=\frac{n^{\prime} Q a}{R}$, and so we have $Q=2 V \frac{A n C^{\prime}}{a n^{\prime} C^{\prime}}$, in which when a Thomson galvanometer is used $C^{\prime}$ and $C$ can be replaced by the corresponding deflections; hence $Q=2 V^{\circ} \frac{A n D}{a n^{\prime} D}$, in which $a$ and $n^{\prime}$ are the area and number of turns in the small coil and $Q$ is that component of the magnetic field we are measuring in the direction of the axis of the small coil.

As an illustration of this method I will give a few experiments made with the magnets of a Ruhmkorff diamagnetic apparatus, which was altogether about $2 \mathrm{ft}$. long and had its magnets $2 \mathrm{in}$. in diameter, with

I On a new diamagnetic attachment to the lantern, \&c., this Journal, May, 1875. 
a hole $\frac{1}{2}$ in. in diameter through them for experiments on the rotation of the plane of polarization of light, but which in these experiments were closed by the solid poles which were screwed on. The first experiments were with two dises of iron, $4 \cdot 6$ in. in diameter and $1 \frac{3}{8}$ in. thick, screwed on to the poles. In the first place the poles were turned away from one another, the current being sent through only one magnet, and the values of the magnetic field obtained at different points close to the surface of the disc. These may be numbered as follows: No. 1, at centre of face of disc; No. 2, on face of disc half an inch from the edge; No. 3, on centre of edge of disc. The measures are on the metre, gram, second system.

1st. Strength of current, 4.4 farads per second.
1. 2220 .
2. 3550 .
3. 4440.

2nd. Strength of current 8.3 farads per second.
1. 3600 .
2. 5300 .
3. 7500 .

Next the poles were turned toward each other and the current sent through both magnets, so as to make the poles of the same name. Current $4 \cdot 6$ farads per second.

1st. Distance of poles, 3 in.
1. 1300 .
3. 3800 .

2nd. Distance of poles, $1 \frac{1}{2} \mathrm{in}$.
1. 600 .
3. 4000 .

Here we see an approach to one of Faraday's places of no magnetic action.

After this the current in one of the magnets was reversed so as to make the poles opposite. Current the same.

1st. Distance of poles, 3 in.
1. 5800 .
2. 8200 .
3. 6700 .

2nd. Distance of poles, $1 \frac{1}{2}$ in.
1. 9800 .
2. 7500 .
3. 5800.

It is curious to note how the distribution changes with the distance of the dises; thus, on one disc free from the other, the edge of the disc has the greatest magnetic surface density, but when the two dises form opposite poles and are 3 in. apart, position 2 gives the greatest effect, while, when they are $1 \frac{1}{2} \mathrm{in}$. apart, the field is greatest at the centre. This entirely agrees with theory.

The conical poles for diamagnetic experiments were then screwed on. These were portions of cones with an angle at vertex of about $60^{\circ}$, with the vertex considerably rounded off. They were one inch apart and the poles were opposite. Current $4 \cdot 4$ farads per second. 
At centre of field between the poles ........... 12500

On the axis near one pole .................. 32100

On cone one inch from vertex .............. 11000

On cylindrical portion of magnet $2 \frac{1}{2}$ inches from the vertex of the cone ................... 5800

These poles were now replaced by frustums of cones with flat ends, the original diameter of the iron, 2 inches, being reduced at the end to $1 \frac{1}{2}$ inches, and they were placed $\frac{1}{4}$ inch apart. The field in this case between them was 61000 , or nearly up to the maximum of magnetization of nickel at common temperatures, and above that at high temperatures.

Troy, April 1, 1875. 


\section{STUDIES ON MAGNETIC DISTRIBUTION}

[Philosophical Magazine [4], L, 257-277, 348-367, 1875]

[American Journal of Science [3], X, 325-335, 451-459, 1875; XI, 17-29, 103-108, 1876]

\section{Part I.-Linear Distribution}

\section{Contents}

I. Preliminary remarks.

II. Mathematical theory.

III. Experimental methods for measuring linear distribution.

IV. Iron rods magnetized by induction.

V. Straight electro-magnets and permanent steel magnets.

VI. Miscellaneous applications.

I.

In a paper of mine published about two years ago, I alluded to some investigations which I had made in $18 \% 0$ and 1871 on the distribution of magnetism. It is with diffidence that I approach this subject, being aware of the great mathematical difficulties with which it is surrounded. But as the facts are still in advance of what is known on the subject, and as I see that other investigators ${ }^{1}$ are following hard upon my footsteps, I thought it would be well to publish them, particularly as it is no fault of mine that they did not appear some years ago. ${ }^{2}$ The mathematical theory which I give, although not particularly elegant, will at least be found to present the matter in a new and more simple light, and may be considered simply as a development of Faraday's idea of the analogy between a magnet and a voltaic battery immersed in water. I shall throughout speak of the conduction of, and resistance to, lines of magnetic force, and shall otherwise treat them as similar to lines of conducted electricity or heat, it now being well established from the researches of Professor Maxwell and others that this method gives exactly the same results as the other method of considering the action to take place at a distance.

In arranging this paper I have thought best to give the theory of

1 Particularly M. Jamin.

${ }^{2}$ All the experiments referred to in this paper were made in the winter of 1870-71. 
the distribution first, and then afterwards to see how the results agree with experiment; in this way we can find out the defects of the theory, and what changes should be made in it to adapt it to experiment.

At present I am acquainted with two formulæ giving the distribution of magnetism on bar magnets: the first was given by Biot, in his Traité de Physique Expérimentale et Mathématique, vol. iii, p. $7 \%$, and was obtained by him from the analogy of the magnet to a dry electric pile, or to a crystal of tourmaline electrified by heat. He compared his formula with Coulomb's observations, and showed it to represent the distribution with considerable accuracy. Green, in his 'Essay,' has obtained a formula which gives the same distribution; but he obtains it by a series of mathematical approximations which it is almost impossible to interpret physically. M. Jamin has recently used a formula of the same form; but I have as yet been unable to find how he obtained it. My own formulæ are also quite similar to these, but have the advantage of being obtained in a more simple manner than Green's; and, what is of more consequence, all the limitations are made at once, after which the solution is exact; so that although they are only approximate, yet we know just where they should differ from experiment.

II.

If we take an iron bar and magnetize one end of it either by a magnet or helix, we cause lines of magnetic induction ${ }^{3}$ to enter that end of the bar, and, after passing down it to a certain distance, to pass out into the air and so round to the bar again to complete their circuit. At every part of their circuit they encounter some resistance, and always tend to pass in that direction where it is the least: throughout their whole course they obey a law similar to Ohm's law; and the number of lines passing in any direction between two points is equal to the difference of magnetic potential of those points divided by the resistance to the lines.

The complete solution of the problem before us being impossible, let us limit it by two hypotheses. First, let us assume that the permeability of the bar is a constant quantity; and secondly, that the resistance to the lines of induction is composed of two parts, the first being that of the bar, and the second that of escaping from the bar into the

\footnotetext{
${ }^{3}$ For difference between lines of magnetic force and lines of magnetic induction see Maxwell's 'Treatise on Electricity and Magnetism,' arts. 400, 592, and 604.
} 
medium - and that the latter is the same at every part of the bar. The first of these assumptions is the one usually made in the mathematical theory of magnetic induction; but, as has been shown by the experiments of Müller, and more recently by those of Dr. Stoletow and myself, this is not true; and we shall see this when we come to compare the formula with experiment. The second assumption is more exact than the first for all portions of the bar except the ends.

Let us first take the case of a rod of iron with a short helix placed on any portion of it, through which a current of electricity is sent. The lines of magnetic induction stream down the bar on either side: at every point of the bar two paths are open to them, either to pass further down the rod, or to pass out into the air. We can then apply the ordinary equations for a derived circuit in electricity to this case.

Let $\mu$ be the magnetic permeability of the iron,

$R$ be the resistance of unit of length of the rod,

$R^{\prime}$ be the resistance of medium along unit of length of rod,

$\rho$ be the resistance at a given point to passing down the rod,

$s$ be the resistance at the end of the rod,

$Q^{\prime 4}$ be the number of lines of induction passing along the rod at a given point,

$Q^{\prime}{ }^{5}$ be the number of lines of induction passing from the rod into the medium along a small length of the rod $\Delta L$,

$L$ be the distance from the end of the rod to a given point,

$$
\begin{aligned}
& r=\sqrt{\frac{R}{R^{\prime}}}, \\
& A=\frac{\sqrt{R R^{\prime}}+s}{\sqrt{R R^{\prime}}-s} .
\end{aligned}
$$

To find $\rho$, the ordinary equation for the resistance of a derived circuit gives

whence

$$
\rho+d \rho=\frac{(\rho+R d L) \frac{R^{\prime}}{d L}}{\rho+R d L+\frac{R^{\prime}}{d L}},
$$

$$
\frac{d \rho}{d L}=\frac{1}{R^{\prime}}\left(R R^{\prime}-\rho^{2}\right)
$$

+ These are the surface-1ntegralsof magnetic induction (see Maxwell's 'Electricity,' art. 402)--the first across the section of the bar, and the second along a length $\Delta L$ of the surface of the bar.

It is to be noted that $Q^{\prime}{ }_{e}$ when $\Delta L$ is constant, is nearly proportional to the socalled surface-density of magnetism at the given point. 
and

$$
\rho=\sqrt{R R^{\prime}} \frac{A \varepsilon^{2 r L}-1}{A \varepsilon^{2 r L}+1} \quad \text {. . . . . }
$$

To find $Q^{\prime}$, we have

$$
d Q^{\prime}=\frac{Q^{\prime} \rho}{R^{\prime}} d L
$$

whence

$$
Q^{\prime}=\frac{C}{A+1}\left(A \varepsilon^{r L}+\varepsilon^{-r L}\right),{ }^{6} \quad . \quad . \quad . \quad .
$$

and

$$
Q_{\mathrm{\epsilon}}^{\prime}=\frac{Q^{\prime} \rho \Delta L}{R^{\prime}}=r \frac{C \Delta L}{A+1}\left(A \varepsilon^{r L}-\varepsilon^{-r L}\right) . \quad . \quad .
$$

When $L$ is very large, or $s=\sqrt{R R^{\prime}}$, we have

$$
Q^{\prime}=C_{l} \varepsilon^{r L_{e}} \text { and } Q_{\varepsilon}^{\prime}=C_{l} r \Delta L^{r L_{i}},
$$

in which $L$, is reckoned from an origin at any point of the rod.

These equations give the distribution on the part outside the helix; and we have now to consider the part covered by the helix. Let us

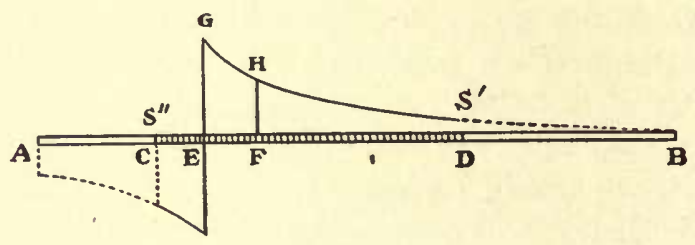

FIG. 1.

limit ourselves to the case where the helix is long and thin, so that the field in its interior is nearly uniform.

As we pass along the helix, the change of magnetic potential due to the helix is equal to the product of the intensity of the field multiplied by the distance passed over; so that in passing over an elementary distance $d y$ the difference of potential will be $\mathfrak{S} d y$. The number of lines of force which this difference of potential causes in the rod will be equal to $\mathfrak{5} d y$ divided by the sum of the resistances of the rod in both directions from the given point. These lines of force stream down the rod on either side of the point, creating everywhere a magnetic potential which can be calculated by equation (2), and which is represented by the curves in Fig. 1. In that figure $A B$ is the rod, $C D$ the helix, and

${ }^{6}$ This could have been obtained directly from the equation $\frac{d^{2} Q^{\prime}}{d L^{2}}=Q^{\prime} r^{2}$, and $Q^{\prime}{ }_{\epsilon}$ from the equation $Q_{\epsilon}^{\prime}=\frac{d Q^{\prime}}{d L} \Delta L$. 
$E$ the element of length $d y$. Now, if we take all the elements of the rod in the same way and consider the effect at $H F$, the total magnetic potential at this point will, by hypothesis No. 1, be equal to the sum of the potentials due to all the elements $d y$.

Let $\Delta Q^{\prime}$ be the number of lines of force produced in the bar at the point $E$ due to the elementary difference of potential at that point, $\mathfrak{S} d y$,

$\angle Q$ " be the number of lines of force arriving at the point $F$ due to the same element,

$Q_{\epsilon}^{\prime \prime}$ be the number of lines passing from bar along length $\Delta L$,

$\rho_{y}$ be the sum of the resistances of the bar in both directions from $E$,

$\rho_{x}$ be resistance at $F$ in direction of $D$,

$y$ be the distance $D E$,

$x$ be the distance $D F$,

$b$ be the distance $C D$,

$s^{\prime \prime}$ and $s^{\prime}$ be the resistance of the bar, \&c., respectively at $C$ in the direction of $A$, and at $D$ in direction of $B$,

$\mathfrak{S}$ be the magnetizing-force of helix in its interior.

Let

$$
\begin{aligned}
& A^{\prime}=\frac{\sqrt{R R^{\prime}}+s^{\prime}}{\sqrt{R R^{\prime}}-s^{\prime}}, \quad A^{\prime \prime}=\frac{\sqrt{R R^{\prime}}+s^{\prime \prime}}{\sqrt{R R^{\prime}}-s^{\prime \prime}}, \quad r=\sqrt{\frac{R}{R^{\prime}}} \\
& \rho_{y}=\frac{2\left(A^{\prime} A^{\prime \prime} \varepsilon^{2 r b}-1\right)}{\left.\left(A^{\prime \prime} \varepsilon^{2 r y}+1\right)\left(A^{\prime} \varepsilon^{2 r(b}-y\right)+1\right)} \sqrt{R R^{\prime}}, \\
& \Delta Q^{\prime}=\frac{\mathfrak{S} d y}{\rho_{y}}, \\
& \Delta Q^{\prime \prime}=\Delta Q^{\prime}\left(A^{\prime \prime} \varepsilon^{r x}+\varepsilon^{-r x}\right) \frac{\varepsilon^{r y}}{A^{\prime \prime} \varepsilon^{2 r y}+1} \\
&=\frac{\mathfrak{H}}{2 \sqrt{R R^{\prime}}} \frac{A^{\prime \prime} \varepsilon^{r x}+\varepsilon^{-r x}}{A^{\prime} A^{\prime \prime} \varepsilon^{2 r b}-1}\left(A^{\prime} \varepsilon^{2 r(b-y)}+1\right) \varepsilon^{r v} d y, \\
& Q_{\varepsilon}^{\prime \prime}=\frac{\Delta L}{R^{\prime}} \int \Delta Q^{\prime \prime} \rho_{x}=\frac{\mathfrak{S} \Delta L}{2 R^{\prime}} \frac{A^{\prime \prime} \varepsilon^{r x}-\varepsilon^{-r x}}{A^{\prime} A^{\prime \prime} \varepsilon^{2 r b}-1} \int_{x}^{b}\left(A^{\prime} \varepsilon^{2 r b} \varepsilon^{-r y}+\varepsilon^{r y}\right) d y \\
&=\frac{\mathfrak{S} \Delta L}{2 R^{\prime} r} \frac{A^{\prime \prime} \varepsilon^{r x}-\varepsilon^{-r x}}{A^{\prime} A^{\prime \prime} \varepsilon^{2 r b}-1}\left[A^{\prime} \varepsilon^{2 r b}\left(\varepsilon^{-r x}-\varepsilon^{-r b}\right)+\varepsilon^{r b}-\varepsilon^{r x}\right]
\end{aligned}
$$

This gives the positive part of $Q_{e}^{\prime \prime}$. To find the negative part, change $x$ into $b-x, A^{\prime}$ into $A^{\prime \prime}$, and $A^{\prime \prime}$ into $A^{\prime}$, and then change the sign of the whole.

When the helix is symmetrically placed on the bar, we have $s^{\prime}=s^{\prime \prime}$, $A^{\prime}=A^{\prime \prime}$; whence, adding the positive and negative parts together, we have 


$$
Q_{\varepsilon}^{\prime \prime}=\frac{\mathfrak{S} \Delta L}{2 \sqrt{R R^{\prime}}} \frac{1-A^{\prime}}{A^{\prime} \varepsilon^{r b}-1}\left(\varepsilon^{r(b-x)}-\varepsilon^{r x}\right), \quad . \quad . .
$$

which gives the number of lines of induction passing out from the rod along the length $\Delta L$ when the helix is symmetrically placed on the rod.

To get the number of lines of induction passing along the rod at a given point, we have

$$
Q^{\prime \prime}=\int_{0}^{x} Q_{\varepsilon}^{\prime \prime} d x=\frac{\mathfrak{g}}{2 R} \frac{1-A^{\prime}}{A^{\prime} \varepsilon^{r b}-1}\left(\varepsilon^{r b}+1-\varepsilon^{r x}-\varepsilon^{r(b-x)}+C^{\prime \prime \prime}, .\right.
$$

where

$$
C^{\prime \prime \prime}=-\frac{\mathfrak{H}}{r} \frac{\varepsilon^{r b}-1}{\left(A^{\prime} \varepsilon^{r b}-1\right)\left(\sqrt{R R^{\prime}}-s^{\prime}\right)} .
$$

When the bar extends a distance $L^{\prime}$ out of both ends of the helix, so that

we have

$$
s^{\prime}=\sqrt{R R^{\prime}} \frac{\varepsilon^{2 r L^{\prime}}+1}{\varepsilon^{2 r L^{\prime}}-1} \text { and } A^{\prime}=-\varepsilon^{2 r L^{\prime}}
$$

$$
C^{\prime \prime \prime \prime}=-\frac{\mathfrak{H}}{r} \frac{\left(\varepsilon^{r b}-1\right)\left(\varepsilon^{2 r L^{\prime}}-1\right)}{\left(\varepsilon^{r b} \varepsilon^{2 r L^{\prime}}+1\right) 2 \sqrt{R R^{\prime}}} .
$$

It may be well, before proceeding, to define what is meant by magnetic resistance, and the units in which it is measured. If $\mu$ is the magnetic permeability of the rod, we can get an idea of the meaning of magnetic resistance in the following manner. Suppose we have a rod infinitely long placed in a magnetic field of intensity $\mathfrak{S}$ parallel to the lines of force. Let $Q^{\prime}$ be the number of lines of inductive force passing through the rod, or the surface-integral of the magnetic induction across its section; also let $a$ be the area of the rod. Then by definition $\mu=\frac{Q^{\prime}}{a S^{5}}$. If $L$ is the length of the rod, the difference of potential at the ends will be $L \mathfrak{S}$; hence

and $R$ in the formulæ becomes

$$
Q^{\prime}=\frac{L \mathfrak{S}}{R_{\imath}} \text { and } R_{\imath}=\frac{L \mathfrak{S}_{\mathfrak{Y}}}{Q^{\prime}}=\frac{L}{a \mu},
$$

$$
R=\frac{R_{i}}{L}=\frac{1}{a \mu} .
$$

It is almost impossible to estimate $R^{\prime}$ theoretically, seeing that it will vary with the circumstances. We can get some idea of its nature, however, by considering that the principal part of it is due to the cylindric envelope of medium immediately surrounding the rod. The resistance of such an envelope per unit of length of rod is 


$$
\frac{1}{2 \pi \mu_{1}} \text { hyp. } \log \frac{D}{d},
$$

where $D$ is the diameter of the envelope, $d$ of the rod, and $\mu_{1}$ the permeability of the medium. But we are not able to estimate $D$. If, however, we have two magnetic systems similar in all their parts, it is evident that beyond a certain point similarly situated in each system we may neglect the resistance of the medium, and $\frac{D}{d}$ will be the same for the two systems. Hence $R^{\prime}$ is approximately constant for rods of all diameters in the same medium, and $r$ takes the form

$$
r=\frac{2}{d} \sqrt{\frac{1}{\pi \mu_{1} R}},
$$

It is evident that the reasoning would apply to rods of any section as well as circular.

In Green's splendid essay (Reprint, p. 111, or Maxwell's 'Treatise on Electricity and Magnetism,' art. 439) we find a formula similar to equation (5), but obtained in an entirely different manner, and applying only to rods not extending beyond the helix. In the 'Reprint,' $\beta$ corresponds to my $r$; and its value, using my notation, is obtained from the equation

where $p=\frac{r d}{2}$.

$$
\cdot 231863-2 \text { hyp. } \log p+2 p=\frac{4}{(\mu-1) p^{2}}, \ldots . .
$$

If we make $\mu$ a constant in this formula, we must have $p=\frac{r d}{2}=$ constant; hence

$$
r \propto \frac{1}{d},
$$

which is the same result for this case as from equation (7).

When $\mu$ in the two formulæ is made to vary, the results are not exactly the same; but still they give approximately the same results for the cases we shall consider; and since the formula is at the best only approximate, we shall not spend time in discussing the merits of the two.

\section{III.}

Among the various methods of measuring linear magnetic distribution, we find few up to the present time that are satisfactory. Coulomb used the method of counting the number of vibrations made by a magnetic needle when near various points of the magnet. Thus, in 
the curve of distribution most often reproduced from his work, he used a magnetized steel bar 27 French inches long and 2 lines in diameter placed vertically; opposite to it, and at a distance of 8 lines, he hung a magnetic needle 3 lines in diameter and 6 lines long, tempered very hard; and the number of oscillations made by it was determined. The square of this number is proportional to the magnetic field at that point, supposing the magnetism of the needle to be unchanged; and this, corrected for the magnetism of the earth, gives the magnetic field due to the magnet alone. This for points near the magnet and distant from the ends is nearly proportional to the so-called magnetic surface-density opposite the point. At the end Coulomb doubled the quantity thus found, seeing that the bar extended only on one side of the needle.

It will be seen that this method is only approximate, and almost incapable of giving results in absolute measure. The effect on the needle depends not only on that part of the bar opposite the needle, but on portions to either side, and gives, as it were, the average value for some distance; in the next place, the correction at the end, by multiplying by 2 , seems to be inadequate, and gives too small a result compared with other parts. For at points distant from the end the average surface-density at any point will be nearly equal to the average for a short distance on both sides, while at the end it will be greater than the average of a short distance measured back from the end. To these errors must be added those due to the mutual induction of the two magnets.

The next method we come to is that which has been recently used by M. Jamin, and consists in measuring the attraction of a piece of soft iron applied at different points of the magnet. In this case it does not seem to have been considered that the attraction depends not only on the magnetic density at the given point, but also on that around it, and that a piece of soft iron applied to a magnet changes the distribution immediately at all points, but especially at that where the iron is applied. The change is of course less when the magnet is of very hard steel and the piece of soft iron small. Where, however, we wish to get the distribution on soft iron, it becomes a quite serious difficulty. Another source of error arises from the fact that the coefficient of magnetization of soft iron is a function of the magnetization: this source of error is greatest when the contact-piece is long and thin, and is a minimum when it is short and thick and not in contact with the magnet. Hence this method will give the best results when the contact-piece is small and in the shape of a sphere and not in contact with 
the magnet, and when the method is applied to steel magnets. But after taking all these precautions, the question next arises as to how to obtain the magnetic surface-density from the experiments. Theory indicates, and M. Jamin has assumed, that the attractive force is nearly proportional to the square of the surface-density. But experiment does not seem to confirm this, except where there is some distance between the two bodies, at least in the case of a sphere and a plane surface, as in Tyndall's experiments (Phil. Mag., April, 1851). It is not necessary at present to consider the cause of this apparent discrepancy between theory and experiment; suffice it to say that the explanation of the phenomenon is without doubt to be sought for in the variable character of the magnetizing-function of iron. All I wish to show is that the attraction of iron to a magnet, especially when the two are in contact, is a very complicated phenomenon, whose laws in general are unknown, and hence is entirely unsuitable for experiments on magnetic distribution.

A third method is that used in determining the correction for the distribution on the magnets in finding the intensity of the earth's magnetism. Usually the distribution is not explicitly found in this case; but it is easy to see how it might be. Thus, one way would be as follows:-Take the origin of coordinates at the centre of the magnet. Develop the distribution in an ascending series of powers of $x$ with unknown constant coefficients. Calculate the magnetic force due to this distribution for any points along the axis, or else on a line perpendicular to the magnet at its centre. Determine the force at a series of points extending through as great a range and as near the magnet as possible. These experiments give a series of equations from which the coefficients in the expansion can be determined. Other and better methods of expansion might be found, except for short magnets, where the method suggested is very good.

The similarity of this method to that used by Gauss in determining the distribution on the earth is apparent.

A fourth method is similar to the above, except that the lines of force around the magnet are measured and calculated instead of the force.

The last two methods are very exact, but are also very laborious, and therefore only adapted to special investigations. Thus, by the change in direction of the lines of force around the magnet, we have a delicate means of showing the change in distribution, as, for instance, when the current around an electro-magnet varies. 
The fifth method is that used lately in some experiments of Mr. Sears (American Journal of Science, July, 1874), but only adapted to temporary magnetization. At a given point on the bar a small coil of wire is placed, and the current induced in it measured by the swing of the galvanometer-needle when the bar is demagnetized. It does not seem to have been noticed that what we ordinarily consider the magnetic distribution is not directly measured in this way; and indeed, to get correct results, the magnetization should have been reversed, seeing that a large portion of the magnetization will not disappear, on taking away the magnetizing-force, where the bar is long. The quantity which is directly measured is the surface-integral of the temporary magnetic induction across the section of the bar, while the magnetic surfacedensity is proportional to the surface-integral of magnetic induction along a given portion of the bar. In other words, the quantity measured is $Q$ instead of $\frac{Q_{\varepsilon}}{\Delta L}$. We can, however, derive one from the other very easily.

The sixth and last method is that which I used first in 1870 , and by which most of my experiments have been performed. This consists in sliding a small coil of wire, which just fits the bar and is also very narrow, along the bar inch by inch, and noting the induced current over each inch by the deflection of a galvanometer-needle. This measures $Q_{e}$, except for some corrections which I now wish to note. In the first case, to give exact results, the lines of force should pass out perpendicular to the bar, or the coil must be very small. But even when the last condition is fulfilled errors will be introduced at certain portions of the bar. The error is vanishingly small in most cases, except near the ends; and even there it is not large, except in special cases; for at this part the lines of force pass forward toward the end of the bar, and so the observation next to the end may be too small, while that at the end is too large. The correction can be made by finding where the lines of force through the centre of the section of the coil in its two positions meet the bar. The error from this source is not large, and may be avoided to a great extent.

One very great advantage in the method of induced currents is the facility with which the results can be reduced to absolute measure by including an earth-inductor in the circuit as I have before described (Phil. Mag., August, 1873). There is also no reaction (except a temporary one) between the magnet and current; so that the distribution remains unchanged. Hence it seems to me that this method is the only one capable of giving exact results directly. 
The coils of wire which I used consisted of from twenty to one hundred turns of fine wire wound on thin paper tubes which just fitted the bar and extended considerably beyond the coils. The coils were mostly from $\cdot 1$ to $\cdot 25$ of an inch wide and from $\cdot 1$ to $\cdot 2$ inch thick. A measure being laid by the side of the given bar under experiment, the coil was moved from one division of the rule to the next very quickly, and the deflection produced on an ordinary astatic galvanometer noted. After experience this could be done with great accuracy. It might be better in some cases to have the coil slide over a limited distance on the tube, though for the use to which I intend to put the results the other is best.

Up to $35^{\circ} Q_{\epsilon}$ is nearly proportional to the deflection; and when any larger value is put down in the Tables, it is the sum of two or more deflections. I have not the data in most cases to reduce my results to absolute measure, but took pains to ensure that certain series of experiments should be comparable among themselves.

Having measured $Q_{\epsilon}$ at all points of a rod, we may find $Q$ by adding up the values of $Q_{\mathrm{e}}$ from the end of the rod.

The magnetizing force to which the bar was subjected was in all cases a helix placed at some part of the bar. The iron bars were of course demagnetized thoroughly before use by placing them in the proper position with reference to the magnetic meridian and striking them.

In the Tables $L$ is the distance in inches from the zero-point, $Q_{\epsilon}$ is the deflection of the galvanometer when the helix is passed between the points indicated in the first column. Thus, in Table II, 34.7 is the deflection on the galvanometer when the helix was moved from the tenth to the eleventh inch from the zero-point; and so we may consider it as the value of $Q_{\epsilon}$ at $10 \frac{1}{2}$ inches; so that the values of $Q_{\epsilon}$ refer to the half inches, but $Q$ to the even inches.

In all the calculations the constants in the formulæ were taken to represent $Q$ most nearly, and then the corresponding formulæ for $Q_{\text {c }}$ taken with the same constants.

For ease in calculating by ordinary logarithmic Tables, we may put $\varepsilon^{r L}=10^{434 s r L}$.

IV.

Table $I$ is from a bar $17 \frac{1}{2}$ inches long with a magnetizing helix $1 \frac{1}{2}$ inch long at one end, the zero-point being at the other. Table II is from a bar 9 feet long with a helix $4 \frac{1}{2}$ inches long quite near one end, the zero-point being at 1 inch from the helix toward the long end. 
Table III is from a bar 2 feet long with a helix $4 \frac{1}{2}$ inches long near one end, so that its centre was $19 \frac{3}{4}$ inches from the end on which the experiments were made, the zero-point being at the end.

In adapting the formula to apply to the case of Table I, we may assume that at the end of the bar $s=\infty$ and $C=0$, which is equivalent to assuming that the number of lines of induction which pass out at the end of the rod are too small to be appreciated.

TABLE I.

Bar - 18 Inch Diameter. 0 at ENd of Bar.

\begin{tabular}{|c|c|c|c|c|c|c|}
\hline L. & $\begin{array}{c}\text { Q'́. } \\
\text { obb- } \\
\text { served. }\end{array}$ & $\begin{array}{l}\text { Qé. } \\
\text { Calcu- } \\
\text { lated. }\end{array}$ & $\begin{array}{c}\text { Error of } \\
Q_{e}^{\prime} .\end{array}$ & $\begin{array}{c}Q^{\prime} \\
\text { ob- } \\
\text { served. }\end{array}$ & $\begin{array}{l}\mathrm{Q}^{\prime} . \\
\text { calcu- } \\
\text { lated. }\end{array}$ & $\begin{array}{c}\text { Error of } \\
Q^{\prime} .\end{array}$ \\
\hline $\begin{array}{r}0 \\
3 \\
5 \\
6 \\
7 \\
8 \\
9 \\
10 \\
11 \\
12 \\
13 \\
14\end{array}$ & $\begin{array}{l}2.7 \\
3 \cdot 2 \\
2 \cdot 0 \\
2 \cdot 5 \\
3 \cdot 2 \\
3 \cdot 7 \\
4 \cdot 3 \\
5 \cdot 3 \\
6 \cdot 5 \\
7 \cdot 7 \\
9 \cdot 5\end{array}$ & $\begin{array}{l}\cdots \cdots \\
2 \cdots \\
2 \cdot 0 \\
2 \cdot 4 \\
3 \cdot 5 \\
4 \cdot 3 \\
5 \cdot 2 \\
6 \cdot 5 \\
8 \cdot 0 \\
9 \cdot 9\end{array}$ & $\begin{array}{c}\cdots \\
\cdots \\
-\quad \cdot 1 \\
-\cdot 4 \\
-\cdot .2 \\
0 \\
-\quad \cdot 1 \\
0 \\
+\quad \cdot 3 \\
+\quad \cdot 4\end{array}$ & $\begin{array}{l}0 \\
2 \cdot 7 \\
5 \cdot 9 \\
7 \cdot 9 \\
10 \cdot 4 \\
13 \cdot 6 \\
17 \cdot 3 \\
21 \cdot 6 \\
26 \cdot 9 \\
33 \cdot 4 \\
41 \cdot 1 \\
50 \cdot 6\end{array}$ & $\begin{array}{r}0 \\
3 \cdot 5 \\
6 \cdot 6 \\
8 \cdot 6 \\
11 \cdot 0 \\
13 \cdot 8 \\
17 \cdot 3 \\
21 \cdot 6 \\
26 \cdot 8 \\
33 \cdot 3 \\
41 \cdot 3 \\
51 \cdot 2\end{array}$ & $\begin{array}{c}0 \\
+\quad \cdot 8 \\
+\quad \cdot 7 \\
+\quad \cdot 7 \\
+\quad \cdot 6 \\
+\quad \cdot 2 \\
0 \\
0 \\
-\quad \cdot 1 \\
-\cdot 1 \\
+\quad \cdot 2 \\
+\quad \cdot 6\end{array}$ \\
\hline \multicolumn{7}{|c|}{$\begin{array}{l}Q^{\prime}=2 \cdot 60\left(\varepsilon^{\cdot 213 L}-\varepsilon^{-213 L}\right) . \\
Q_{\varepsilon}=r 2 \cdot 60\left(\varepsilon^{\cdot 213 L}+\varepsilon^{-213 L}\right)=\cdot 554\left(\varepsilon^{\cdot 213 L}+\varepsilon^{-212 L}\right) .\end{array}$} \\
\hline
\end{tabular}

In Table II observations were not made over the whole length of the rod, and the zero-point was not at the end of the bar. It is evident, however, that by giving a proper value to $s$ we may suppose the bar to end at any point. As the rod is very long, expressions of the form

$$
Q^{\prime}-C^{\prime \prime}=C^{\prime} \varepsilon^{-L}-C^{\prime \prime} \text { and } Q_{\epsilon}^{\prime}=r C^{\prime} \varepsilon^{-r L}
$$

will apply.

In Table II the observations were near the end of the rod, and were repeated several times. Neglecting the end of the rod, we have $s=\infty$.

In these Tables we see quite a good agreement between theory and observation; but on more careful examination we observe a certain law in the distribution of errors. Thus in Table $I$ the errors of $Q^{\prime}$ are all positive between 0 and 8 inches; and this has always been found to be the case at this part of the bar in all my experiments.

The explanation of this is very simple. In obtaining the formulæ, we assumed that the magnetic permeability of the bar $\mu$ was a constant 
TABLE II.

Bar -39 Inch Diameter. 0 at 1 inch from helix.

\begin{tabular}{|c|c|c|c|c|c|c|}
\hline L. & $\begin{array}{l}\text { Q'́. } \\
\text { Ob- } \\
\text { served. }\end{array}$ & $\begin{array}{l}\text { Qe. } \\
\text { Calcu- } \\
\text { lated. }\end{array}$ & $\begin{array}{c}\text { Error of } \\
Q_{\text {'. }}\end{array}$ & $\begin{array}{l}Q^{\prime}-C^{\prime \prime} . \\
\text { ob- } \\
\text { served. }\end{array}$ & $\begin{array}{l}\text { Q'-C"C.' } \\
\text { Calcu- } \\
\text { lated. }\end{array}$ & $\begin{array}{l}\text { Error of } \\
Q^{\prime} \text {. }\end{array}$ \\
\hline $\begin{array}{r}0 \\
1 \\
2 \\
3 \\
4 \\
5 \\
6 \\
7 \\
8 \\
9 \\
10 \\
11 \\
12 \\
13 \\
14 \\
15 \\
16 \\
17 \\
18 \\
19 \\
21 \\
23 \\
25 \\
27 \\
29 \\
31\end{array}$ & $\begin{array}{l}\not 31 \cdot 7 \\
65 \cdot 2 \\
59 \cdot 5 \\
53 \cdot 5 \\
51 \cdot 2 \\
46 \cdot 7 \\
43 \cdot 2 \\
40 \cdot 0 \\
37 \cdot 2 \\
34 \cdot 7 \\
31 \cdot 7 \\
29 \cdot 5 \\
25 \cdot 7 \\
25 \cdot 5 \\
22 \cdot 0 \\
21 \cdot 5 \\
20 \cdot 0 \\
19 \cdot 1 \\
32 \cdot 5 \\
27 \cdot 5 \\
23 \cdot 0 \\
18 \cdot 5 \\
14 \cdot 5 \\
11 \cdot 3\end{array}$ & $\begin{array}{l}70 \cdot 8 \\
65 \cdot 3 \\
60 \cdot 2 \\
55 \cdot 5 \\
51 \cdot 2 \\
47 \cdot 2 \\
43 \cdot 5 \\
40 \cdot 1 \\
37 \cdot 0 \\
34 \cdot 1 \\
31 \cdot 4 \\
28 \cdot 9 \\
26 \cdot 6 \\
24 \cdot 6 \\
22 \cdot 7 \\
20 \cdot 9 \\
19 \cdot 3 \\
17 \cdot 8 \\
31 \cdot 5 \\
26 \cdot 7 \\
22 \cdot 8 \\
19 \cdot 4 \\
16 \cdot 5 \\
14 \cdot 0\end{array}$ & 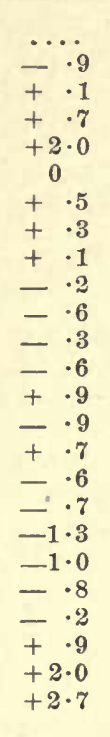 & $\begin{array}{r}825 \cdot 2 \\
753 \cdot 5 \\
688 \cdot 3 \\
628 \cdot 8 \\
575 \cdot 3 \\
524 \cdot 1 \\
477 \cdot 4 \\
434 \cdot 2 \\
394 \cdot 2 \\
357 \cdot 0 \\
322 \cdot 3 \\
290 \cdot 6 \\
261 \cdot 1 \\
235 \cdot 4 \\
209 \cdot 9 \\
187 \cdot 9 \\
166 \cdot 4 \\
146 \cdot 4 \\
127 \cdot 3 \\
94 \cdot 8 \\
67 \cdot 3 \\
44 \cdot 3 \\
25 \cdot 8 \\
11 \cdot 3 \\
0\end{array}$ & $\begin{array}{r}902 \cdot 5 \\
825 \cdot 9 \\
755 \cdot 1 \\
689 \cdot 8 \\
629 \cdot 5 \\
574 \cdot 3 \\
523 \cdot 1 \\
476 \cdot 0 \\
432 \cdot 5 \\
392 \cdot 5 \\
355 \cdot 6 \\
321 \cdot 5 \\
290 \cdot 1 \\
261 \cdot 2 \\
234 \cdot 5 \\
210 \cdot 0 \\
187 \cdot 3 \\
166 \cdot 4 \\
147 \cdot 1 \\
129 \cdot 4 \\
97 \cdot 8 \\
71 \cdot 1 \\
48 \cdot 6 \\
29 \cdot 0 \\
12 \cdot 6 \\
-1 \cdot 2\end{array}$ & $\begin{array}{l}+\cdot 7 \\
+1 \cdot 6 \\
+1 \cdot 5 \\
+\cdot \cdot 7 \\
-1 \cdot 0 \\
-1 \cdot 0 \\
-1 \cdot 4 \\
-1 \cdot 7 \\
-1 \cdot 7 \\
-1 \cdot 4 \\
-\cdot 8 \\
-\cdot 5 \\
+\cdot 1 \\
-\cdot \cdot 9 \\
+\cdot 1 \\
-0 \cdot 6 \\
+\quad \cdot 7 \\
+2 \cdot 1 \\
+3 \cdot 0 \\
+3 \cdot 8 \\
+4 \cdot 3 \\
+3 \cdot 2 \\
+1 \cdot 3 \\
-1 \cdot 2\end{array}$ \\
\hline \multicolumn{7}{|c|}{$\begin{array}{l}Q^{\prime}-C^{\prime \prime}=983 \varepsilon^{-.08135 L}-80 \cdot 5=983 \cdot(10)-0353 L-80 \cdot 5 . \\
Q_{e}^{\prime}=r 983 \varepsilon^{-08135 L} \Delta L=80 \cdot(10)-0353 L \Delta L .\end{array}$} \\
\hline
\end{tabular}

quantity; but it has been shown by Dr. Stoletow and myself, independently of each other, that $\mu$ increases as the magnetism of the bar increases when the latter is not great. Hence between 0 and 8 inches the resistance of the bar, $R$, is greater than at succeeding points, and hence a less number of lines of induction pass down the bar from 8 towards 0 than would be given by the formula, which has been adapted to the average value of $R$ at from 9 to 14 inches. In Table II this same fact shows itself towards the end of the Table, and would probably be more prominent had the Table been carried further. However, in this Table all things have combined to satisfy the formula with great accuracy.

In Table III we come across a fact of an entirely different nature from the above. Fig. 2 is the plot of this Table, and gives the values of $Q_{\epsilon}^{\prime}$ at different parts of the rod. 
TABLE III.

Bar 39 INGh Diameter. 0 at END of Bar.

\begin{tabular}{|c|c|c|c|c|c|c|}
\hline L. & $\begin{array}{c}\mathrm{Q}_{6}^{\prime} \\
\text { Ob- } \\
\text { served. }\end{array}$ & $\begin{array}{l}\text { Q'́. } \\
\text { Caleu- } \\
\text { lated. }\end{array}$ & $\begin{array}{c}\text { Error of } \\
\mathbf{Q}_{e^{\prime}}\end{array}$ & $\begin{array}{c}\mathrm{Q}^{\prime} \\
\text { Ob- } \\
\text { served. }\end{array}$ & $\begin{array}{l}\text { Q'. } \\
\text { Calcu- } \\
\text { lated. }\end{array}$ & $\begin{array}{c}\text { Error of } \\
Q^{\prime} \text {. }\end{array}$ \\
\hline $\begin{array}{r}0 \\
1 \\
2 \\
3 \\
4 \\
4 \\
5 \\
6 \\
7 \\
8 \\
9 \\
10 \\
11 \\
12 \\
13 \\
14 \\
15 \\
16\end{array}$ & $\begin{array}{l}19 \cdot 7 \\
16 \cdot 3 \\
16 \cdot 0 \\
15 \cdot 8 \\
16 \cdot 5 \\
17 \cdot 0 \\
17 \cdot 6 \\
18 \cdot 4 \\
19 \cdot 2 \\
20 \cdot 3 \\
21 \cdot 8 \\
22 \cdot 8 \\
24 \cdot 8 \\
26 \cdot 8 \\
28 \cdot 8 \\
31 \cdot 8\end{array}$ & $\begin{array}{l}15 \cdot 2 \\
15 \cdot 3 \\
15 \cdot 5 \\
15 \cdot 9 \\
16 \cdot 3 \\
16 \cdot 9 \\
17 \cdot 6 \\
18 \cdot 4 \\
19 \cdot 4 \\
20 \cdot 5 \\
21 \cdot 7 \\
23 \cdot 1 \\
24 \cdot 7 \\
26 \cdot 5 \\
28 \cdot 4 \\
30 \cdot 5\end{array}$ & 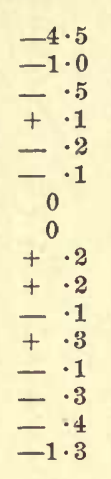 & $\begin{array}{r}0 . \\
19 \cdot 7 \\
36 \cdot 0 \\
52 \cdot 0 \\
67 \cdot 8 \\
84 \cdot 3 \\
101 \cdot 3 \\
118 \cdot 9 \\
137 \cdot 3 \\
156.5 \\
176 \cdot 8 \\
198 \cdot 6 \\
221 \cdot 4 \\
246 \cdot 2 \\
273 \cdot 0 \\
301 \cdot 8 \\
333 \cdot 6\end{array}$ & $\begin{array}{c}0 \\
15 \cdot 2 \\
30 \cdot 5 \\
46 \cdot 0 \\
61 \cdot 8 \\
78 \cdot 1 \\
95 \cdot 0 \\
112 \cdot 6 \\
130 \cdot 9 \\
150 \cdot 3 \\
170 \cdot 7 \\
192 \cdot 2 \\
215 \cdot 3 \\
239 \cdot 9 \\
266 \cdot 4 \\
294 \cdot 6 \\
325 \cdot 1\end{array}$ & $\begin{array}{c}0 \\
-4 \cdot 5 \\
-5 \cdot 5 \\
-6 \cdot 0 \\
-6 \cdot 0 \\
-6 \cdot 2 \\
-6 \cdot 3 \\
-6 \cdot 3 \\
-6 \cdot 4 \\
-6 \cdot 2 \\
-6 \cdot 1 \\
-6 \cdot 4 \\
-6 \cdot 1 \\
-6 \cdot 3 \\
-6 \cdot 6 \\
-7 \cdot 2 \\
-8 \cdot 5\end{array}$ \\
\hline
\end{tabular}

The horizontal line in the figure represents values of $L$, and the vertical ordinates are values of $Q_{e}^{\prime}$. The full line gives the observed distribution, and the dotted line that according to the formula.

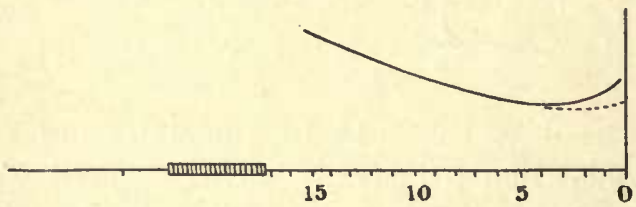

FIG. 2.-Distribution at end of bar.

The formula gives the distribution very nearly for all points except those near the end. The formula indicates that $Q_{\epsilon}^{\prime}$ decreases continually toward the end; but by experiment we see that it increases near this point. On first seeing this, I thought that it was due to some residual magnetism in the bar; but after repeating the experiment several times with proper care, I soon found that this was always the case. I give the following explanation of it:- In the formulæ we have assumed $R^{\prime}$, the resistance of the medium, to be a constant; now this resistance includes that of the lines of force as they pass from the rod through the medium and thus back to the other end of the rod; and of 
this whole quantity the part which affects the relative distribution at any part of the rod most is that of the medium immediately surrounding that part; and so the parts near the end have the advantage over those further back, inasmuch as the lines can pass forward as well as outward into the medium. The same thing takes place in the case of the distribution of electricity, where the "density" is inversely proportional to the resistance which the lines of inductive force experience from the medium; and here we find that the "density" is greatest on the projections of the body, showing that the resistance to the lines of induction is less in such situations, and by analogy showing that this must also be the case for lines of magnetic force. But this effect is not very great in cylinders until quite near the end; for Coulomb, in a long electrified cylinder, has found the density at one diameter back from the end only 1.25 times that at the centre; and so there is probably a long distance in the centre where the density is sensibly constant. Hence we may suppose that our second hypothesis, that $R^{\prime}$ is a constant, will be approximately correct for all parts of a bar except the ends, though of course this will vary to some extent with the distribution of the lines in the medium; at least the change in $R^{\prime}$ will be gradual except near the end, and so may be partially allowed for by giving a mean value to $r$.

Hence we see that could the formula be so changed as to include both the variation of $R$ and of $R^{\prime}$, it would probably agree with the three Tables given.

To study the effect of variation in the permeability more carefully, we can proceed in another manner, and use the formulæ only to get the value of $r$ at different parts of the rods.

No matter how $r$ may vary, equations (2) and (3) will apply to a very small distance $l$ along the rod; and as the orgin of coordinates may be at any point on the rod, if $Q^{\prime}$ and $Q_{\epsilon}^{\prime}$ are taken at one point and $Q$ and $Q_{e}$ at another point whose distance from the first is $l$, we shall have the four equations

$$
\begin{array}{ll}
Q=O, & Q^{\prime}=\frac{C}{A+1}\left(A \varepsilon^{r l}+\varepsilon^{-r l}\right), \\
Q_{\varepsilon}=\frac{A-1}{A+1} C r l, & Q_{\varepsilon}^{\prime}=r l \frac{C}{A+1}\left(A \varepsilon^{r l}+\varepsilon^{-r l}\right) .
\end{array}
$$

Calling $\frac{Q_{\epsilon}^{\prime}}{Q}=H$ and $\frac{Q^{\prime}}{Q}=G$, we shall find, on eliminating $C$ and $A$ and developing $\varepsilon^{r l}$ and $\varepsilon^{-r l}$, 


$$
r^{2}=\frac{2}{l^{2}}\left(\frac{G H+1}{G+H}-1\right), . . . . . .
$$

or, to a greater degree of approximation,

$$
r^{2}=\frac{1}{l^{2}}\left(\sqrt{12\left(2 \frac{G H+1}{G+H}+1\right)}-6\right) . . . .
$$

Before applying these formulæ to any series of observations, the latter should be freed from most of the irregularities due to accidental causes. For this purpose the following Tables have been plotted and a regular curve drawn to represent as nearly as possible the observations; in other cases a column of differences was formed and plotted. In either case the ordinates of the curves were accepted as the true quantities. But, for fear that some might accuse me of tampering with my observations, I have in all cases added these as they were obtained.

TABLE IV.

\begin{tabular}{|c|c|c|c|c|c|}
\hline L. & $\begin{array}{c}Q_{e}^{\prime} \\
\text { Observed. }\end{array}$ & $\begin{array}{c}Q_{e}^{\prime} . \\
\text { Corrected. }\end{array}$ & $\begin{array}{c}Q^{\prime} \\
\text { Corrected. }\end{array}$ & $r^{2}=\frac{\mathrm{R}}{\mathrm{R}^{\prime}}$ & $\frac{1}{r^{2}}=\frac{\mathbf{R}^{\prime}}{\mathbf{R}}$ \\
\hline $\begin{array}{c}1 \\
2 \\
3 \\
4 \\
5 \\
6 \\
7 \\
8 \\
9 \\
10 \\
11 \\
12 \\
13 \\
14 \\
15 \\
281 / 2\end{array}$ & $\begin{array}{r}24 \cdot 0 \\
17 \cdot 0 \\
13 \cdot 7 \\
11 \cdot 6 \\
10 \cdot 2 \\
9 \cdot 0 \\
8 \cdot 0 \\
7 \cdot 1 \\
6 \cdot 4 \\
5 \cdot 7 \\
4 \cdot 9 \\
4 \cdot 4 \\
3 \cdot 6 \\
3 \cdot 3 \\
22 \cdot 4\end{array}$ & $\begin{array}{c}24 \cdot 0 \\
17 \cdot 0 \\
13 \cdot 7 \\
11 \cdot 65 \\
10 \cdot 15 \\
9 \cdot 0 \\
8.0 \\
7 \cdot 15 \\
6 \cdot 35 \\
5 \cdot 65 \\
5 \cdot 0 \\
4 \cdot 4 \\
3.9 \\
3 \cdot 4 \\
22 \cdot 4\end{array}$ & $\begin{array}{r}151 \cdot 7 \\
127 \cdot 7 \\
110 \cdot 7 \\
97.0 \\
85 \cdot 4 \\
75 \cdot 2 \\
66 \cdot 2 \\
58 \cdot 2 \\
51 \cdot 1 \\
44 \cdot 7 \\
39 \cdot 1 \\
34 \cdot 1 \\
29 \cdot 7 \\
25 \cdot 8 \\
22 \cdot 4\end{array}$ & $\begin{array}{l}.041 \\
.0256 \\
.0192 \\
.0168 \\
.0150 \\
.0142 \\
.0150 \\
.0159 \\
.0160 \\
.0167 \\
.0180 \\
.0184 \\
.0184\end{array}$ & $\begin{array}{l}24.4 \\
39 \cdot 1 \\
52 \cdot 1 \\
59 \cdot 5 \\
66 \cdot 7 \\
70 \cdot 4 \\
66 \cdot 7 \\
62 \cdot 9 \\
52 \cdot 5 \\
59 \cdot 9 \\
55 \cdot 6 \\
54 \cdot 3 \\
54 \cdot 3\end{array}$ \\
\hline
\end{tabular}

Bar -19 Inch Diameter. 0 at Centre of Bar.

The correction is necessary, because small irregularities in the observations will produce immense changes in $r^{2}$.

Table IV contains some of the best observations I have obtained. It is from a bar 57 inches long with a helix $1 \frac{1}{2}$ inch long in the centre to magnetize it. Each quantity is the mean of six observations, these being made on both ends of the bar and with the current in opposite directions.

In this Table a source of error was guarded against which I have not 
seen mentioned elsewhere. When a bar of iron is magnetized at any part and the distribution over the rest quickly measured, on being then allowed to stand some time and the distribution again taken, it will have changed somewhat, the magnetism having, as it were, crept down the bar further. Hence in this Table time was allowed for the bar to reach its permanent state.

On looking over column 6 , which contains the values of $\frac{1}{\eta^{2}}=\frac{R^{\prime}}{R}=R^{\prime} a \mu$ (equation 7), we observe that as $Q^{\prime}$ decreases, the value of $R^{\prime} a \mu$ first increases and then decreases. Now it is not probable that $R^{\prime}$ undergoes any sudden change of this sort; and so it is probably due to change in the permeability of the rod. Hence by this method we arrive at the same results as by a more direct and exact method. ${ }^{7}$ But by this means we are able to prove in the most unequivocal manner that magnetic permeability is a function of the magnetization of the iron and not of the magnetizing force. Hence it is that I have preferred, in my papers on Magnetic Permeability, to consider it in this way in the formulæ and also in the plots, while Dr. Stoletow (in his paper, Phil. Mag., January, 1873) plots the magnetizing-function as a function of the magnetizing force.

When we plot the results in this Table with reference to $Q^{\prime}$ and $R^{\prime} a \mu$, the effect of the variation of $R^{\prime}$ is apparent; and we see, on comparing the curve with those given in my paper above referred to, that $R^{\prime}$ increases as $L$ increases, at least between $L=2$ and $L=8$, which is as we should suppose from the arrangement of the apparatus. For this Table I happen to have data for determining $Q$ in absolute measure; and these show that the maximum value of $\mu$ should be about where the Table shows it to be.

This method of finding the variation of $\mu$ is analogous to that of finding conductivity for heat by raising the temperature of one end of a bar and noting the distribution of heat over the bar; indeed the curves of distribution are nearly the same in the two cases.

If it were thought worth while, it would be very easy to obtain a curve of magnetic distribution for a rod and then enclose the whole rod in a helix and determine its curve of permeability. This would give data for determining $R^{\prime}$ in absolute measure at every point of the rod.

To complete the argument that the variation of $r^{2}$ is in great measure due to that of $\mu$, I have caused the magnetizing force on a bar to vary.

'Phil. Mag., August, 1873. 
Tables V, VI, and VII are from a bar 9 feet long and $\cdot 25$ inch in diameter. At the centre a single layer of fine wire was wound for a distance of 1 foot; and the current for magnetizing the bar was sent through this. The zero-point was at the centre of this helix and at the centre of the bar; so that the observations on the first 6 inches include the part of the bar covered by the helix.

The values of $Q_{\epsilon}^{\prime}$ are the sum of four observations on each end of the bar and with the current reversed. The three Tables are comparable with each other, the same arbitrary unit being used for all.

TABLE V.

Magnetizing CURRent $\cdot \mathbf{1 7 6}$.

\begin{tabular}{|c|c|c|c|c|c|c|}
\hline L. & $\begin{array}{c}\mathbf{Q}_{\varepsilon} \\
\text { Ob- } \\
\text { served. }\end{array}$ & $\begin{array}{c}Q_{e .} \\
\text { Cor- } \\
\text { rected. }\end{array}$ & $\begin{array}{c}\mathrm{Q}^{\prime} \cdot \\
\text { Cor- } \\
\text { rected. }\end{array}$ & $r^{2}=\frac{\mathbf{R}}{\mathbf{R}^{\prime}}$ & $\frac{1}{r^{2}}=\frac{\mathrm{R}^{\prime}}{\mathrm{R}}$ & $\begin{array}{l}\text { Q⿱⺈'.'- } \\
\text { Calcu- } \\
\text { lated. }\end{array}$ \\
\hline $\left.\begin{array}{r}0 \\
1 \\
2 \\
3 \\
4 \\
5 \\
6 \\
7 \\
8 \\
9 \\
10 \\
11 \\
12 \\
13 \\
14 \\
15 \\
16 \\
17 \\
18\end{array}\right\}$ & $\begin{array}{r}2 \cdot 7 \\
6 \cdot 9 \\
12 \cdot 7 \\
18 \cdot 2 \\
24 \cdot 4 \\
32 \cdot 4 \\
31 \cdot 5 \\
28 \cdot 2 \\
24 \cdot 9 \\
21 \cdot 4 \\
18 \cdot 6 \\
16 \cdot 8 \\
14 \cdot 2 \\
12 \cdot 0 \\
17 \cdot 7\end{array}$ & $\begin{array}{r}\ldots \ldots \\
\ldots \ldots \\
\ldots \ldots \\
\ldots \ldots \\
31 \cdot 7 \\
32 \cdot 0 \\
28 \cdot 2 \\
24 \cdot 7 \\
21 \cdot 7 \\
19 \cdot 0 \\
16 \cdot 4 \\
14 \cdot 2 \\
12 \cdot 0 \\
10 \cdot 0 \\
8 \cdot 2 \\
6 \cdot 6 \\
5 \cdot 1 \\
22 \cdot 4\end{array}$ & $\begin{array}{c}\ldots \ldots \\
\ldots \ldots \\
\ldots \ldots \\
\ldots \ldots \\
\cdots \cdots \\
220 \cdot 5 \\
188 \cdot 5 \\
160 \cdot 3 \\
135 \cdot 6 \\
113 \cdot 9 \\
94 \cdot 9 \\
78 \cdot 5 \\
64 \cdot 3 \\
52 \cdot 3 \\
42 \cdot 3 \\
34 \cdot 1 \\
27 \cdot 5 \\
22 \cdot 4\end{array}$ & $\begin{array}{l}\ldots \ldots \\
\ldots \ldots \\
\ldots \ldots \\
\ldots \ldots \\
\cdots \\
.0190 \\
.0212 \\
.0218 \\
.0236 \\
.0252 \\
.0278 \\
.0311 \\
.0367 \\
.0404 \\
.0440 \\
.0445 \\
.0570\end{array}$ & $\begin{array}{l}\ldots \\
\ldots \ldots \\
\ldots \ldots \\
\ldots \ldots \\
\ldots \ldots \\
52 \cdot 4 \\
47 \cdot 2 \\
45 \cdot 9 \\
42 \cdot 4 \\
39 \cdot 7 \\
36 \cdot 0 \\
32 \cdot 2 \\
27 \cdot 2 \\
24 \cdot 8 \\
22 \cdot 7 \\
22 \cdot 5 \\
17 \cdot 5\end{array}$ & 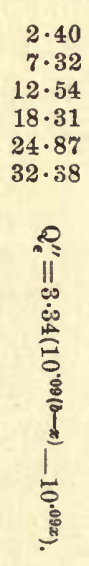 \\
\hline
\end{tabular}

Here we see an excellent confirmation of the results deduced from Table IV. In Table V, where the magnetizing force is very small, and where, consequently, no part of the iron has yet reached its minimum resistance, the value of $\frac{1}{r^{2}}=\frac{R^{\prime}}{R}=R^{\prime} a \mu$ decreases continually as the value of $Q^{\prime}$ decreases, as it should do. In Table VI, with a higher magnetizing power, which was sufficient to bring a portion of the bar to about the minimum resistance, we see that $\frac{1}{r^{2}}$ remains nearly stationary for a short distance from the helix and then decreases in value. In Table VII, where the bar is highly magnetized and the portion near the zero- 
TABLE VI.

Magnetizing Current $\cdot 31$.

\begin{tabular}{|c|c|c|c|c|c|c|}
\hline L. & $\begin{array}{c}\text { Q'. } \\
\text { Ob- } \\
\text { served. }\end{array}$ & $\begin{array}{c}\text { Q'. } \\
\text { Cor- } \\
\text { rected. }\end{array}$ & $\underset{\text { rected. }}{Q^{Q^{\prime}} .}$ & $r^{2}$ & $\frac{1}{r^{2}}$ & $\begin{array}{l}\text { Q'E. } \\
\text { Calcu- } \\
\text { lated. }\end{array}$ \\
\hline $\left.\begin{array}{r}0 \\
2 \\
3 \\
4 \\
5 \\
6 \\
7 \\
8 \\
9 \\
10 \\
11 \\
12 \\
13 \\
14 \\
15 \\
16 \\
17 \\
18\end{array}\right\}$ & $\begin{array}{l}16 \cdot 3 \\
22 \cdot 0 \\
32 \cdot 4 \\
43 \cdot 8 \\
55 \cdot 9 \\
55 \cdot 2 \\
46 \cdot 8 \\
81 \cdot 3 \\
61 \cdot 8 \\
46 \cdot 4 \\
35 \cdot 4 \\
\\
22 \cdot 0 \\
43 \cdot 0\end{array}$ & $\begin{array}{r}\ldots \\
\ldots \ldots \\
\ldots \ldots \\
\ldots \ldots \\
55 \cdot 1 \\
48 \cdot 1 \\
42 \cdot 3 \\
37 \cdot 4 \\
33 \cdot 0 \\
29 \cdot 0 \\
25 \cdot 3 \\
21 \cdot 9 \\
18 \cdot 7 \\
15 \cdot 6 \\
12 \cdot 7 \\
9 \cdot 8\end{array}$ & $\begin{array}{c}\ldots \ldots \\
\ldots \ldots \\
\ldots \ldots \\
\ldots \ldots \\
391 \cdot 9 \\
336 \cdot 8 \\
288 \cdot 7 \\
246 \cdot 4 \\
2090 \\
176 \cdot 0 \\
147 \cdot 0 \\
121 \cdot 7 \\
99 \cdot 8 \\
81 \cdot 1 \\
65 \cdot 5 \\
52 \cdot 8\end{array}$ & $\begin{array}{l}\ldots \ldots \\
\ldots \ldots \\
\ldots \ldots \\
\ldots \ldots \\
\ldots \\
.0204 \\
.0201 \\
.0202 \\
.0220 \\
.0243 \\
.0262 \\
.0300 \\
.0352 \\
.0405 \\
.0479\end{array}$ & $\begin{array}{l}\ldots \ldots \\
\ldots \ldots \\
\ldots \ldots \\
\ldots \ldots \\
49 \cdot 0 \\
49 \cdot 7 \\
49 \cdot 5 \\
45 \cdot 5 \\
41 \cdot 2 \\
38 \cdot 2 \\
33 \cdot 3 \\
28 \cdot 4 \\
24 \cdot 7 \\
20 \cdot 9\end{array}$ & 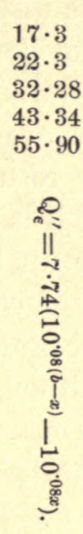 \\
\hline
\end{tabular}

TABLE VII.

Magnetizing CURRent $1 \cdot 12$.

\begin{tabular}{|c|c|c|c|c|c|c|}
\hline L. & $\begin{array}{c}\text { Q'́. } \\
\text { Ob- } \\
\text { served. }\end{array}$ & 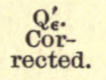 & $\begin{array}{c}\mathrm{Q}^{\mathbf{Q}^{\prime}} \\
\text { Cor- } \\
\text { rected. }\end{array}$ & $r^{2}$ & $\frac{1}{r^{2}}$ & $\begin{array}{l}\text { Q'́. } \\
\text { Calcu- } \\
\text { lated. }\end{array}$ \\
\hline $\left.\begin{array}{r}0 \\
1 \\
2 \\
3 \\
4 \\
5 \\
6 \\
7 \\
8 \\
9 \\
10 \\
11 \\
12 \\
13 \\
14 \\
15 \\
16 \\
17 \\
18 \\
19 \\
20\end{array}\right\}$ & $\begin{array}{r}3 \cdot 5 \\
9 \cdot 4 \\
15 \cdot 4 \\
27 \cdot 5 \\
44 \cdot 3 \\
66 \cdot 6 \\
71 \cdot 2 \\
59 \cdot 5 \\
51 \cdot 0 \\
45 \cdot 2 \\
40 \cdot 3 \\
36 \cdot 3 \\
33 \cdot 3 \\
30 \cdot 6 \\
28 \cdot 1 \\
25 \cdot 6 \\
23 \cdot 4 \\
20 \cdot 0 \\
34 \cdot 0 \\
96 \cdot 8\end{array}$ & $\begin{array}{l}\ldots \ldots \\
\ldots \ldots \\
\ldots \ldots \\
\ldots \ldots \\
\ldots \ldots \\
71 \cdot 2 \\
59 \cdot 7 \\
51 \cdot 2 \\
45 \cdot 2 \\
40 \cdot 3 \\
36 \cdot 8 \\
33 \cdot 5 \\
30 \cdot 5 \\
28 \cdot 0 \\
25 \cdot 4 \\
22 \cdot 7 \\
20 \cdot 3 \\
18 \cdot 1 \\
16 \cdot 0\end{array}$ & $\begin{array}{r}762 \cdot 4 \\
758 \cdot 9 \\
749 \cdot 5 \\
734 \cdot 1 \\
706 \cdot 6 \\
662 \cdot 3 \\
595 \cdot 7 \\
524 \cdot 5 \\
464 \cdot 8 \\
413 \cdot 6 \\
368 \cdot 4 \\
328 \cdot 1 \\
291 \cdot 3 \\
257 \cdot 8 \\
227 \cdot 3 \\
199 \cdot 3 \\
173 \cdot 9 \\
151 \cdot 2 \\
130 \cdot 2 \\
112 \cdot 8 \\
96 \cdot 8\end{array}$ & $\begin{array}{l}\ldots \\
\ldots \ldots \\
\ldots \ldots \\
\ldots \ldots \\
\ldots \ldots \\
\ldots . \\
.0239 \\
.0200 \\
.0162 \\
.0141 \\
.0120 \\
.0107 \\
.0110 \\
.0116 \\
.0118 \\
.0140 \\
.0147 \\
.0161 \\
.0180\end{array}$ & $\begin{array}{c}\ldots \ldots \\
\ldots \ldots \\
\ldots \ldots \\
\ldots \ldots \\
\ldots 1 \cdot \\
41 \cdot 8 \\
50 \cdot 0 \\
61 \cdot 7 \\
70 \cdot 9 \\
83 \cdot 3 \\
93 \cdot 5 \\
90 \cdot 9 \\
86 \cdot 2 \\
84 \cdot 7 \\
71 \cdot 4 \\
68 \cdot 0 \\
62 \cdot 1 \\
55 \cdot 6\end{array}$ & 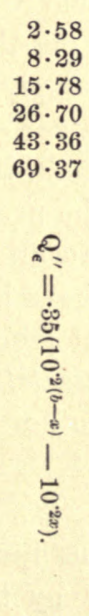 \\
\hline
\end{tabular}


points approaches the maximum of magnetization, $\frac{1}{r^{2}}$ increases in value as we pass down the bar; and having reached its maximum at $L=11 \frac{3}{4}$ nearly, it decreases. These Tables, then, show in the most striking manner the effect of the variation of the magnetic permeability of iron upon the distribution of magnetism.

It is evident that these Tables also give the data for obtaining the relative values of $R^{\prime}$ at different parts of the bar; but the results thus obtained are conflicting, and will need further experiment to obtain accurate results. Where such a small magnetizing force is used as in Table $\mathrm{V}$ it is almost impossible to attain accuracy; and allowance should be made for this in deducing results from it. The greatest liability to error is of course where the magnetization is small; for any small residual magnetism which the bar may contain will be more apparent here-although great care was taken to remove all residual magnetism before use. Besides this there are many other disturbances from which the higher magnetizing powers are free.

If we accept Green's formula as correct, these observations give us data for determining the magnetizing-function of iron in a unique manner, for nearly all other methods depend on absolute measurements of some kind. Thus the least value of $r^{2}$ in Table IV for a rod $\cdot 19$ inch diameter is $\cdot 0142$, which gives $p=\cdot 01132$, which in Green's formula (equation 8) gives $\mu=3388$ for the greatest permeability of this iron; and this is as nearly right as we can judge for this kind of iron. It is to be noted that Green's formula has been found for the portion of the bar covered by the helix; but, as seen from my formulæ, it will approximately apply to all portions, though it would be better to find a new formula for each case.

We shall, toward the last, resume this subject again; and so will leave it for the present.

The results which I have now given, and indeed all the results of this paper, have been deduced not only from the observations which I publish, but from very many others; so that my Taoles may be considered to represent the average of a very extended series of researches, though they are not really so.

\section{V.}

Let us now consider the case of that portion of the bar which is covered by the helix. First of all, when the helix is symmetrically placed on the rod, equations (5) and (6) will apply. As $Q^{\prime \prime}$ is the 
quantity which is usually taken to represent the distribution of magnetism, being ncarly proportional to the "surface-density" of magnetism, I shall principally discuss it.

In the first place, then, this equation (5) shows that the distribution of magnetism in a very elongated electromagnet, and indeed in a steel magnet, does not change when pieces of soft iron bars of the same diameter as the magnet are placed against the poles, provided that equal pieces are applied to both ends; otherwise there is a change. This result would be modified by taking into account the variation of the permeability, \&c.

Let us first consider the case where the rod projects out of the end of the helix, as in Tables V, VI, and VII. By giving proper values to the constants, we obtain the results given in the last column of the

TABLE VIII.

\begin{tabular}{|c|c|c|c|c|}
\hline \multirow{2}{*}{$x$. } & \multicolumn{4}{|c|}{ Strength of magnetizing current. } \\
\hline & $\cdot 108$ & $\cdot 194$. & $\cdot 378$. & $\cdot 600$ \\
\hline $\begin{array}{l}0 \\
1 \\
2 \\
3 \\
4 \\
5 \\
6\end{array}$ & $\begin{array}{l}2 \cdot 7 \\
2 \cdot 4 \\
3 \cdot 3 \\
4 \cdot 0 \\
5 \cdot 7\end{array}$ & $\begin{array}{l}3 \cdot 2 \\
2 \cdot 7 \\
3 \cdot 9 \\
6 \cdot 0 \\
8 \cdot 7\end{array}$ & $\begin{array}{r}\cdot 7 \\
.9 \\
.9 \\
1 \cdot 7 \\
4 \cdot 0 \\
9 \cdot 3\end{array}$ & $\begin{array}{r}.6 \\
.6 \\
.8 \\
.8 \\
3 \cdot 2 \\
14.7\end{array}$ \\
\hline
\end{tabular}

Tables. The agreement with observation is in most cases very perfect. We also see the same variation of $r$ that we before noticed in the rest of the curves, and we see that it is in just the direction theory would indicate from the change of $\mu$.

In these Tables we come to a very important subject, and one to which I called attention some years back-namely, the change in the distribution when the magnetizing force varies, and which is due to change of permeability. The following Tables and figures show this extremely well, and are from very long rods with a helix a foot long at their centre, as in the last three Tables. The bar in both these Tables was $\cdot 19$ inch in diameter and 5 feet long. The zero-point was at the centre of the bar and of the helix. The Tables give values of $Q_{\mathrm{E}}^{\prime}$ for the magnetizing forces which appear at the head of each column, and which are the tangents of the angles of deflection of the needles of a tangentgalvanometer. Table VIII only gives the part covered by the helix. Both Tables are from the mean of both ends of the bar. 
These experiments show in the most positive manner the effect we are considering; and we are impressed by them with the great complication introduced into magnetic distribution by the variable character of magnetic permeability.

In Fig. 3 I have represented the distribution on half the bar, as given in Table IX, the other half being of course similar. Here the greatest

TABLE IX.

\begin{tabular}{|c|c|c|c|}
\hline$x$ & $\begin{array}{c}\text { C. } \\
257 .\end{array}$ & $\begin{array}{c}\text { B. } \\
\cdot 363 .\end{array}$ & $\begin{array}{c}\text { A. } \\
1 \cdot 303 .\end{array}$ \\
\hline $\begin{array}{r}0 \\
1 \\
2 \\
3 \\
4 \\
5 \\
6 \\
7 \\
8 \\
10 \\
12 \\
15 \\
18 \\
30\end{array}$ & $\begin{array}{r}2 \cdot 5 \\
7 \cdot 2 \\
6 \cdot 1 \\
7 \cdot 7 \\
7 \cdot 9 \\
6 \cdot 5 \\
10 \cdot 0 \\
6 \cdot 2 \\
5 \cdot 0 \\
2 \cdot 0 \\
2 \cdot 0\end{array}$ & $\begin{array}{r}3 \cdot 1 \\
4 \cdot 1 \\
5 \cdot 9 \\
8 \cdot 2 \\
10 \cdot 9 \\
11 \cdot 5 \\
9 \cdot 0 \\
15 \cdot 0 \\
10 \cdot 9 \\
9 \cdot 8 \\
4 \cdot 7 \\
3 \cdot 6\end{array}$ & $\begin{array}{r}1 \cdot 1 \\
1 \cdot 3 \\
2 \cdot 1 \\
4 \cdot 0 \\
9 \cdot 6 \\
18 \cdot 6 \\
21 \cdot 3 \\
16 \cdot 8 \\
27 \cdot 4 \\
20 \cdot 9 \\
21 \cdot 5 \\
14 \cdot 8 \\
16 \cdot 5\end{array}$ \\
\hline
\end{tabular}

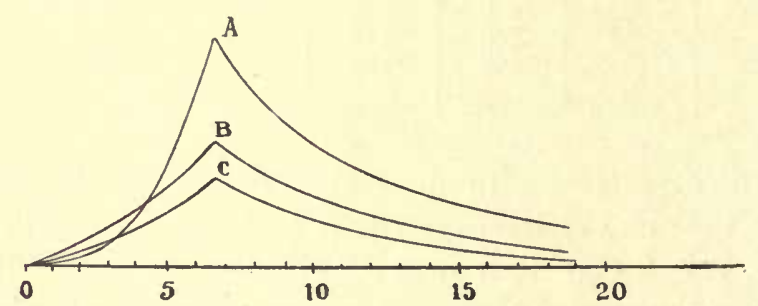

FIG. 3.-Plot of Table IX, showing surface-density for different values of the magnetizing force.

change is observed in the part covered by the helix, though there is also a great change in the other part. These Tables show that, as the magnetization of the bars increases, at least beyond a certain point, the curves on the part covered by the helix increase in steepness; and the figure even shows that near the middle of the helix an increase of magnetizing force may cause the surface-density to decrease; and Table VIII shows this even better. Should we calculate $Q^{\prime \prime}$, however, we should always find it to increase with the magnetizing force in all cases. These effects can be shown also in the case where the bar does not 
extend beyond the helix, but not nearly so well as in this case, seeing that here $Q^{\prime \prime}$ can obtain a greater value.

Assuming that $\mu$ is variable, the formula indicates the same change that we observe; for as $Q^{\prime \prime}$ increases from zero upwards, $\mu$ will first increase and then decrease; so that as we increase the magnetizing force from zero upwards, the curve should first decrease in steepness and then increase indefinitely in steepness. In these Tables the decrease of steepness is not very apparent, because the magnetization is always too great; and indeed on this account it is difficult to show it; but in Tables V, VI, and VII this action is shown to some extent by the

TABLE X.

\begin{tabular}{|c|c|c|c|c|}
\hline$x$ and $\mathrm{L}$. & $\begin{array}{c}\text { A. } \\
-245 .\end{array}$ & $\begin{array}{c}\text { B. } \\
-360 \text {. }\end{array}$ & $\begin{array}{c}\text { C. } \\
600 \text {. }\end{array}$ & $\begin{array}{c}\text { D. } \\
1 \bullet 09 .\end{array}$ \\
\hline $\begin{array}{r}0 \\
1 \\
2 \\
3 \\
4 \\
5 \\
6 \\
7 \\
8 \\
9 \\
10 \\
11 \\
12 \\
13 \\
14 \\
15 \\
16 \\
18 \\
20 \\
24 \\
36 \\
48\end{array}$ & 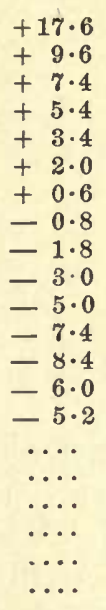 & $\begin{array}{l}+29.4 \\
+16.8 \\
+13.1 \\
+\quad 9.8 \\
+7.2 \\
+4.6 \\
+2.4 \\
+0.3 \\
=1.6 \\
-3.6 \\
-6.3 \\
-10.0 \\
-10.0 \\
-7.9 \\
-7.0 \\
-5.3 \\
-9.4 \\
=5.3 \\
-6.5 \\
-5.6 \\
-0.7\end{array}$ & 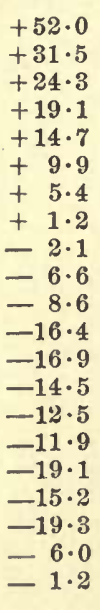 & 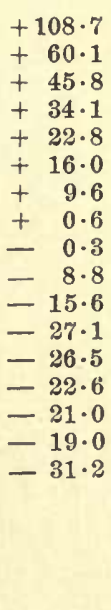 \\
\hline
\end{tabular}

values of $r$ in the formulæ. The change of distribution with the helix arranged in this way at the centre of the bar is greater than in almost every other case, because the magnetism of the bar, $Q^{\prime \prime}$, can change greatly throughout the whole length of the helix, and thus the value of $r$ be changed, and so the distribution become different.

The next case of distribution which I shall consider is that of a very long rod having a helix wound closely round it for some distance at one end.

Table $\mathrm{X}$ is from a bar 9 feet long with a helix wound for one foot along one end. The bar was 25 inch in diameter. All except the first 
column is the sum of two results with the current in opposite directions, and after letting the bar stand for some time, as indeed was done in nearly every case. 'The first column contains twice the quantities observed, so as to compare with the others. The zero-point was at the end of the bar covered by the helix.

The value of $Q_{\epsilon}^{\prime \prime}$ between 0 and 1 includes the lines of force passing out at the end of the bar, and is therefore too large.

In Fig. 4 we have a plot of the results found for this bar. The curves are such as we should expect from our theory, except for the variations introduced by the causes which we have hitherto considered. Thus the sharp rise in the curve when near the end of the bar has already been explained in connection with Table III. A small portion

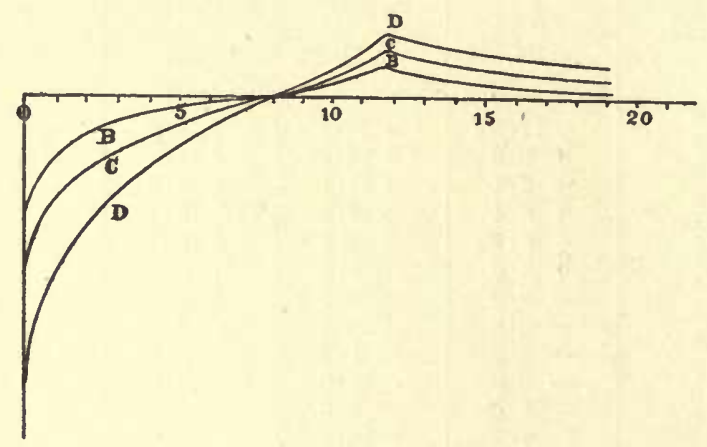

Fig. 4.-Plot of Table X.

of it, however, is due to those lines of induction which pass out through the end section of the bar; and in future experiments these should be estimated and allowed for.

To estimate the shape of the curve theoretically in this case, let us take equation (4) once more, and in it make $s^{\prime}=\infty$ and $s^{\prime \prime}=\sqrt{ } R R^{\prime}$, which will make it apply to this case. We shall then have $A^{\prime}=-1$, and $A^{\prime \prime}=\infty$, whence for the positive part of $Q_{\epsilon}^{\prime \prime}$ we have

$$
Q_{e}^{\prime \prime}=-\frac{\mathfrak{H} \Delta L}{2 R^{\prime} r}\left\{-1+2 \varepsilon^{r(x-b)}-\varepsilon^{2 r(x-b)}\right\}=\frac{\mathfrak{H} \Delta I}{2 R^{\prime} r}\left\{1-\varepsilon^{r(x-b)}\right\}^{2},
$$

and for the negative part

$$
Q_{\epsilon}^{\prime \prime}=-\frac{\mathfrak{S} \Delta L}{2 R^{\prime} r}\left(1+\varepsilon^{2 r(x-b)}\right)\left(1-\varepsilon^{-r x}\right) ;
$$

\footnotetext{
${ }^{8}$ When considering surface-density, we should also allow for the direct action of the helix, though this is always found too small to be worth taking into account except in very accurate experiments.
} 
therefore the real value is

$$
Q_{\epsilon}^{\prime \prime}=-\frac{\mathfrak{H} \Delta L}{2 R^{\prime} r}\left(\varepsilon^{(x-b)}\left(\varepsilon^{-r b}-2\right)+\varepsilon^{-r x}\right) .
$$

And if $x$ is reckoned from the end of the rod, we have

$$
Q_{\epsilon}^{\prime \prime}=\frac{\mathfrak{S}_{\mathrm{g}} \Delta L}{2 R^{\prime} r} \varepsilon^{-r(b+x)}\left\{1-2 \varepsilon^{r b}+\varepsilon^{2 r x}\right\} . \quad . \quad . \quad .
$$

When $x=0$, this becomes

$$
\frac{\mathfrak{S} \Delta L}{2 R^{\prime} r} \varepsilon^{-r b}\left(2-2 \varepsilon^{r b}\right) ;
$$

and when $x=b$, it becomes

$$
\frac{\mathfrak{g} \Delta L}{2 R^{\prime} r} \varepsilon^{-2 r b}\left(1-2 \varepsilon^{r b}+\varepsilon^{2 r b}\right),
$$

the ratio of which is

$$
\frac{1}{2}\left(\varepsilon^{-r b}-1\right) ;
$$

\begin{tabular}{|c|c|c|c|c|c|c|}
\hline$r=$ & 05. & $\cdot 1$. & $\cdot 15$. & $\cdot 20$. & 30. & $\infty$. \\
\hline$-\frac{1}{2}\left(\varepsilon^{-r b}-1\right)=$ & $\cdot 2256$ & $\cdot 3494$ & $\cdot 4173$ & .4546 & $\cdot 4863$ & $\cdot 500$ \\
\hline$\frac{-2}{\varepsilon^{-r b}-1}=$ & $4 \cdot 43$ & $2 \cdot 86$ & $2 \cdot 40$ & $2 \cdot 20$ & $2 \cdot 06$ & $2 \cdot 00$ \\
\hline
\end{tabular}

and this is the ratio of the values of $Q_{\epsilon}^{\prime \prime}$ at the ends of the helix. When $b$ is 12 inches, as in this case, we get the following values of this ratio:-

To compare this with our experiments, let us plot Table $\mathrm{X}$ once more, rejecting, however, the end observations and completing the curve by the eye, thus getting rid of the error introduced at this point. We then find for this ratio, according to the different curves,

$\begin{array}{lll}\text { B. } & \text { C. } & \text { D. } \\ 2 \cdot 1 & 2 \cdot 3 & 3 \cdot 2\end{array}$

It is seen that these are all above the limit 2, as they should bethough it is possible that it may fall below in some cases, owing to the variation of the permeability. As the magnetization increases, the values of the above ratio show that $r$ decreases, as we should expect it to do from the variation of $\mu$.

To find the neutral point in this case, we must have in formula (10)

$$
\varepsilon^{2 r}=2 \varepsilon^{r b}-1 \text {, }
$$


where $x$ is the distance of the neutral point from the end. Making $b=12$, we have from this:-

\begin{tabular}{|c|c|c|c|c|c|c|}
\hline$r=$ & 05. & $\cdot 10$. & 15. & $\cdot 20$. & 30. & $\infty$. \\
\hline \multirow{2}{*}{$x=$} & $10 \cdot 1$ & $8 \cdot 96$ & $8 \cdot 31$ & $7 \cdot 89$ & $\mathbf{7 \cdot 3 9}$ & $\mathbf{6 \cdot 0 0}$ \\
\hline
\end{tabular}

By experiment we find that the neutral point is, in all the cases we have given in Table $X$, between $\% \cdot 5$ and 8.1 inches, which are quite near the points indicated by theory for the proper values of $r$, though we might expect curve $D$ to pass through the point $x=9$, except for the disturbing causes we have all along considered.

Our formulæ, then, express the general facts of the distribution in this case with considerable accuracy.

These experiments and calculations show the change in distribution in an electromagnet when we place a piece of iron against one pole only. In an ordinary straight electromagnet the neutral point is at the centre. When a paramagnetic substance is placed against or near one end, the neutral point moves toward it; but if the substance is diamagnetic it moves from it.

The same thing will happen, though in a less degree, in the case of a steel magnet; so that its neutral point depends on external conditions as well as on internal.

We now come to practically the most interesting case of distribution, namely that of a straight bar magnetized longitudinally either by a helix around it, or by placing it in a magnetic field parallel to the lines of force; we shall also see that this is the case of a steel magnet magnetized permanently. This case is the one considered by Biot (Traité de Phys., tome iii, p. 77) and Green (Mathematical Papers of the late George Green, p. 111, or Maxwell's 'Treatise,' art. 439), though they apply their formulæ more particularly to the case of steel magnets. Biot obtained his formula from the analogy of the magnet to a Zamboni pile or a tourmaline electrified by heat. Green obtained his for the case of a very long rod placed in a magnetic field parallel to the lines of force, and, in obtaining it, used a series of mathematical approximations whose physical meaning it is almost impossible to follow. Prof. Maxwell has criticised his method in the following terms ('Treatise,' art. 439):- "Though some of the steps of this investigation are not rigorous, it is probable that the result represents roughly the actual magnetization in this most important case." From the theory which 
I have given in the first part of this paper we can deduce the physical meaning of Green's approximations; and these are included in the hypotheses there given, seeing that, when my formula is applied to the special case considered by Green, it agrees with it where the permeability of the material is great. My formula, however, is far more general than Green's.

It is to Green that we owe the important remark that the distribution in a steel magnet may be nearly represented by the same formula that applies to electromagnets.

As Green uses what is known as the surface-density of magnetization, let us first see how this quantity compares with those I have used.

Suppose that a long thin steel wire is so magnetized in the direction of its length that when broken up the pieces will have the same magnetic moment. While the rod is together, if we calculate its effect on exterior bodies, we shall see that the ends are the only portions which seem to act. Hence we may mathematically eonsider the whole action of the rod to be due to the distribution of an imaginary magnetic fluid over the ends of the rod. As any ease of magnetism can be represented by a proper combination of these rods, we see that all cases of this sort can be calculated on the supposition of there being two magnetic fluids distributed over the surfaces of the bodies, a unit quantity of which will repel another unit of like nature at a unit's distance with a unit of force. The surface-density at any point will then be the quantity of this fluid on a unit surface at the given point; and the linear density along a rod will be the quantity along a unit of length, supposing the density the same as at the given point.

Where we use induced currents to measure magnetism we measure the number of lines of force, or rather induction, cut by the wire, and the natural unit used is the number of lines of a unit field which will pass through a unit surface placed perpendicular to the lines of force. The unit pole produces a unit field at a unit's distance; hence the number of lines of force coming from the unit pole is $4 \pi$, and the linear density is

and the surface-density

$$
\lambda=\frac{Q_{\Sigma}}{4 \pi \Delta L}, \ldots . . . . . .
$$

$$
\delta=\frac{Q_{\varepsilon}}{4 \pi^{2} u \Delta L} \text {. . . . . . . . . }
$$

These really apply only to steel magnets; but as in the case of electromagnets the action of the helix is very smali compared with that of the 
iron, especially when it is very long and the iron soft," we can apply these to the cases we consider.

Transforming Green's formula into my notation, it gives

$$
i=\left(\frac{\pi d^{2}}{4}\right) \mathfrak{S}_{\mathrm{T}} \kappa r \frac{\varepsilon^{r(b-x)}-\varepsilon^{r x}}{1+\varepsilon^{r b}}, \text {. }
$$

in which $\kappa$ is Neumann's coefficient of magnetization by induction, and is equal to

$$
\frac{\mu-1}{4 \pi}
$$

This equation then gives

$$
Q_{e}^{\prime \prime}=\Delta L\left(\frac{\pi d^{2}}{4}\right) \operatorname{Sg} r(\mu-1) \frac{\varepsilon^{r(b-x)}-\varepsilon^{r x}}{1+\varepsilon^{r b}}, \ldots . .
$$

Equation (5) can be approximately adapted to this case by making $s^{\prime}=\infty$, which is equivalent to neglecting those lines of force which pass out of the end section of the bar. This gives $A^{\prime}=-1$; hence

$$
Q_{\mathrm{e}}^{\prime \prime}=\Delta L \frac{\mathfrak{g}}{\sqrt{R R^{\prime}}} \frac{\varepsilon^{r(b-x)}-\mathrm{\varepsilon}^{r x}}{1+\varepsilon^{r b}} . . . . . .
$$

Now we have found (equation 7 ) that $r=\frac{2}{d} \sqrt{\frac{1}{\pi \mu R^{\prime}}}$ nearly; and this in Green's formula (equation 14) gives

$$
Q_{\varepsilon}^{\prime \prime}=\Delta L \frac{\mathfrak{S}}{\sqrt{ } R R^{\prime}} \frac{\mu-1}{\mu} \frac{\varepsilon^{r(b-x)}-\varepsilon^{r x}}{1+\varepsilon^{r b}}, \ldots . .
$$

which is identical with my own when $\mu$ is large, as it always is in the case of iron, nickel, or cobalt at ordinary temperatures.

When $x$ is measured from the centre of the bar, my equation becomes

$$
\lambda=\frac{\mathscr{H}}{4 \pi \sqrt{R R^{\prime}}} \frac{\varepsilon^{r x}-\varepsilon^{-r x}}{\varepsilon^{2}+\varepsilon^{-\frac{r b}{2}}} . \quad . . . . \cdot
$$

The constant part of Biot's formula is not the same as this; but for any given case it will give the same distribution.

Both Biot and Green have compared their formulæ with Coulomb's experiments, and found them to represent the distribution quite well. Hence it will not be necessary to consider the case of steel magnets very extensively, though I will give a few results for these further on.

\footnotetext{
9I take this occasion to correct an error in Jenkin's 'Textbook of Electricity,' where it is stated that by the introduction of the iron bar into the helix, the nnmber of lines of force is increased 32 times. The number should bave been from a quite small number for a short thick bar and hard iron to nearly 6000 for a long thin bar and softest iron.
} 
At present let us take the case of electromagnets.

For observing the effect of the permeability, I took two wires 12.8 inches long and 19 inch in diameter, one being of ordinary iron and the other of Stubs' steel of the same temper as when purchased. These were wound uniformly from end to end with one layer of quite fine wire, making 600 turns in that distance.

In finding $\lambda$ from $Q_{\epsilon}^{\prime \prime}$, the latter was divided by $4 \pi J L$, except at the end, where the end-section was included with $J L$ in the proper manner. $x$ was measured from the end of the bar in inches.

The observations in Table XI are the mean of four observations made on both ends of the bar and with the current in both directions.

TABLE XI.

IroN ELECTROMAGNT.

\begin{tabular}{|c|c|c|c|c|}
\hline $\begin{array}{l}x=\text { distance } \\
\text { from end. }\end{array}$ & $\begin{array}{c}\mathrm{Q}_{\boldsymbol{\varepsilon}^{\cdot}} \\
\text { Observed. }\end{array}$ & $\begin{array}{c}4 \pi \lambda . \\
\text { Observed. }\end{array}$ & $\begin{array}{c}4 \pi \lambda \text {. } \\
\text { Computed. }\end{array}$ & Error. \\
\hline $\begin{array}{l}0 \\
\frac{1}{2} \\
1 \\
2 \\
3 \\
4 \\
5 \\
6\end{array}$ & $\begin{array}{r}22 \cdot 5 \\
12 \cdot 6 \\
19 \cdot 3 \\
12 \cdot 0 \\
6 \cdot 6 \\
3 \cdot 9 \\
2 \cdot 9\end{array}$ & $\begin{array}{r}41 \cdot 1 \\
25 \cdot 1 \\
19 \cdot 3 \\
12 \cdot 0 \\
6 \cdot 6 \\
3 \cdot 9 \\
2 \cdot 9\end{array}$ & $\begin{array}{r}33 \cdot 9 \\
26 \cdot 9 \\
18 \cdot 9 \\
11 \cdot 7 \\
7 \cdot 1 \\
4 \cdot 0 \\
1.7\end{array}$ & $\begin{array}{l}-7.2 \\
+1.8 \\
-0.4 \\
-\quad .3 \\
+\quad .5 \\
+\quad .1 \\
-1.2\end{array}$ \\
\hline \multicolumn{5}{|c|}{$4 \pi \lambda=42$} \\
\hline
\end{tabular}

The agreement with the formula in this Table is quite good; but we still observe the excess of observation over the formula at the end, as we have done all along. Here, for the first time, we see the error introduced by the method of experiment which I have before referred to (p. 98) in the apparently small value of $4 \pi \lambda$ at $x=\cdot \% 5$.

On trying the steel bar, I came across a curious fact, which, however. I have since found has been noticed by others. It is, that when an iron or steel bar has been magnetized for a long time in one direction and is then demagnetized, it is easier to magnetize it again in the same direction than in the opposite direction. The rod which I used in this experiment had been used as a permanent magnet for about a month, but was demagnetized before use. From this rod five cases of distribution were observed:-first, when the bar was used as an electromagnet with the magnetization in the same direction as the original mag- 
netism; second, ditto with magnetization contrary to original magnetism; third, when used as a permanent magnet with magnetism the same as the original magnetism; fourth, ditto with magnetism opposite; and fifth, same as third, but curve taken after several days. The permanent magnetism was given by the current.

The observations in Tables XI and XII can be compared together, the quantities being expressed in the same unknown arbitrary unit. It is to be noted that the bars in Tables XI and XII were subjected to the same magnetizing force.

TABLE XII.

STUBS' STEEL.

\begin{tabular}{|c|c|c|c|c|c|c|c|c|c|c|}
\hline \multirow{3}{*}{$x$. } & \multicolumn{4}{|c|}{ Electromagnet. } & \multicolumn{6}{|c|}{ Permanent Magnet. } \\
\hline & \multicolumn{2}{|c|}{$\begin{array}{l}\text { Magnetism } \\
\text { same as } \\
\text { original. }\end{array}$} & \multicolumn{2}{|c|}{$\begin{array}{l}\text { Magnetism } \\
\text { opp site to } \\
\text { orlginal. }\end{array}$} & \multicolumn{2}{|c|}{$\begin{array}{l}\text { Magnetism } \\
\text { same as } \\
\text { original. }\end{array}$} & \multicolumn{2}{|c|}{$\begin{array}{l}\text { Magnetism } \\
\text { opposite to } \\
\text { original. }\end{array}$} & \multicolumn{2}{|c|}{$\begin{array}{l}\text { Same as third, } \\
\text { after three or } \\
\text { four days. }\end{array}$} \\
\hline & $\mathbf{Q}_{e^{*}}$ & $4 \pi \lambda$. & $\mathbf{Q}_{\epsilon^{\cdot}}$ & $4 \pi \lambda$ & $\mathbf{Q}_{\boldsymbol{\epsilon}^{\cdot}}$ & $4 \pi \lambda_{0}$ & $\mathbf{Q}_{e^{\cdot}}$ & $4 \pi \lambda$. & $\mathrm{Q}_{\mathrm{e}^{\cdot}}$ & $4 \pi \lambda$ \\
\hline $\begin{array}{l}0 \\
\frac{1}{2}\end{array}$ & $\begin{array}{l}23 \cdot 3 \\
11 \cdot 5\end{array}$ & $\begin{array}{l}42 \cdot 5 \\
23 \cdot 0\end{array}$ & $\begin{array}{r}15 \cdot 9 \\
7 \cdot 7\end{array}$ & $\begin{array}{l}29 \cdot 0 \\
15 \cdot 4\end{array}$ & $14 \cdot 4$ & $13 \cdot 7$ & $4 \cdot 8$ & $4 \cdot 6$ & $12 \cdot 8$ & $12 \cdot 2$ \\
\hline $\begin{array}{l}1 \\
1 \frac{1}{8}\end{array}$ & $\begin{array}{l}8 \cdot 2 \\
6 \cdot 1\end{array}$ & $\begin{array}{l}16 \cdot 4 \\
12 \cdot 2\end{array}$ & $\begin{array}{l}5 \cdot 9 \\
4 \cdot 3\end{array}$ & $\begin{array}{r}11 \cdot 8 \\
8.6\end{array}$ & $8 \cdot 2$ & $8 \cdot 2$ & $4 \cdot 0$ & $4 \cdot 0$ & $7 \cdot 3$ & $7 \cdot 3$ \\
\hline 2 & $7 \cdot 4$ & $7 \cdot 4$ & $5 \cdot 5$ & $5 \cdot 5$ & $5 \cdot 3$ & $5 \cdot 3$ & $2 \cdot 9$ & $2 \cdot 9$ & $4 \cdot 8$ & $4 \cdot 8$ \\
\hline $\begin{array}{l}3 \\
4\end{array}$ & $3 \cdot 6$ & $3 \cdot 6$ & $2 \cdot 7$ & $2 \cdot 5$ & $3 \cdot 0$ & $3 \cdot 0$ & $1 \cdot 6$ & $1 \cdot 6$ & $2 \cdot 9$ & $2 \cdot 9$ \\
\hline $\begin{array}{l}4 \\
6\end{array}$ & 1.7 & $\cdot 8$ & $1 \cdot 0$ & $\cdot 5$ & $2 \cdot 2$ & $1 \cdot 1$ & $\cdot 9$ & $\cdot 4$ & $2 \cdot 0$ & $1 \cdot 0$ \\
\hline
\end{tabular}

First of all, from these Tables and figures (p. 119) we notice the change in distribution due to the quality of the substance; thus in Fig. 5 we see that the curves for steel are much more steep than that of iron, and would thus give greater values to $r$ in the formula-a result to be expected. We also observe in both figures the great change in distribution due to the direction of magnetization. In the case of the electromagnet this amounts to little more than a change in scale; but in the permanent magnet there is a real change of form in the curve. It seems probable that this change of form would be done away with by using a sufficient magnetizing power or magnetizing by application of permanent magnets; for it is probable that the fall in the curve $E$ is due to the magnetizing force having been sufficient to change the polarity completely at the centre, but only partially at the ends.

On comparing the distribution on electromagnets with that on permanent magnets, we perceive that the curve is steeper toward the end in 
electromagnets than in permanent magnets. At first I thought it might be due to the direct action of the helix, but on trial found that the latter was almost inappreciable. I do not at present know the explanation of it.

As before mentioned, Coulomb has made many experiments on the distribution of magnetism on permanent magnets; and so I shall only consider this subject briefly. I have already given one or two results in Table XII.

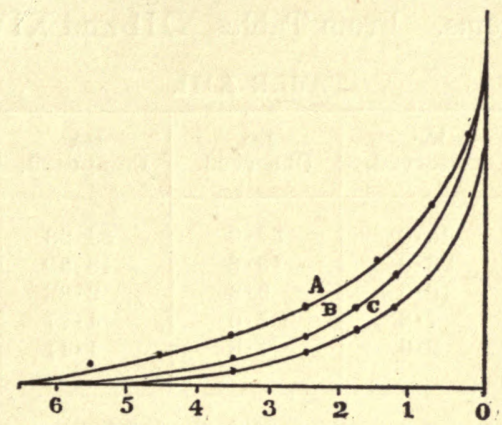

FIG. 5.-Results from electromagnets.

A. Iron, from Table XI.

B. Steel, from Table XII, magnetized same as originally.

C. Steel, from Table XII, magnetized opposite to its original magnetism.

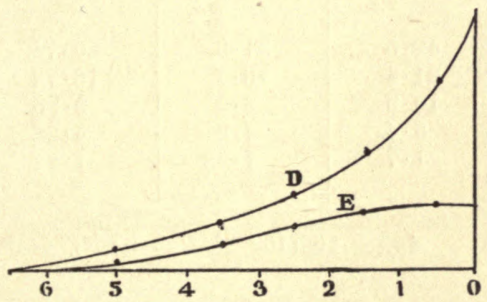

FiG. 6.-Results from steel permanent magnets.

D. Magnetized in its original direction, Table XII.

E. Magnetized opposite to its original direction, Table XII.

Scale four times that of Fig. 5 .

The following Tables were taken from two exactly similar Stubs' steel rods not hardened, one of which was subsequently used in the experiments of Table XII. They were 12.8 inches long and $\cdot 19$ inch in diameter.

The coincidence of these observations with the formula is very re- 
markable; but still we see a little tendency in the end observation to rise above the value given oy the formula.

In equation (7), and also from Green's formula, we have seen that for a given quality and temper of steel $p=\frac{r d}{2}$ is a constant. From Coulomb's experiments on a steel bar $\cdot 176$ inch in diameter (whose quality and temper is unknown, though it was probably hardened) Green has calculated the value of this constant, and obtained $\cdot 05482$, which was found from the French inch as the unit of length, but which is constant for all systems. From Tables XIII and XIV we find the value

TABLE XIII.

\begin{tabular}{|c|c|c|c|c|}
\hline$x$. & $\begin{array}{c}Q_{\epsilon^{*}} \\
\text { Observed. }\end{array}$ & $\begin{array}{c}4 \pi \lambda . \\
\text { Observed. }\end{array}$ & $\begin{array}{l}4 \pi \lambda . \\
\text { Computed. }\end{array}$ & Error. \\
\hline $\begin{array}{l}0 \\
1 \cdot 28 \\
2 \cdot 56 \\
3 \cdot 84 \\
5 \cdot 12 \\
6 \cdot 40\end{array}$ & $\begin{array}{r}46 \cdot 6 \\
23 \cdot 8 \\
12 \cdot 6 \\
7 \cdot 2 \\
2 \cdot 3\end{array}$ & $\begin{array}{r}34 \cdot 9 \\
18 \cdot 6 \\
9 \cdot 8 \\
5 \cdot 6 \\
1 \cdot 8\end{array}$ & $\begin{array}{r}34 \cdot 26 \\
18 \cdot 60 \\
9 \cdot 88 \\
4 \cdot 77 \\
1 \cdot 41\end{array}$ & $\begin{array}{c}-\cdot 6 \\
0 \cdot 1 \\
+\cdot 1 \\
-\cdot 8 \\
-\cdot 4\end{array}$ \\
\hline
\end{tabular}

TABLE XIV.

\begin{tabular}{|c|c|c|c|c|}
\hline$x$. & $\begin{array}{c}\mathbf{Q}_{\boldsymbol{\epsilon}^{-}} \\
\text {Observed. }\end{array}$ & $\begin{array}{c}4 \pi \lambda \text {. } \\
\text { Observed. }\end{array}$ & $\begin{array}{c}4 \pi \lambda \text {. } \\
\text { Computed. }\end{array}$ & Error. \\
\hline $\begin{array}{l}0 \\
1 \cdot 28 \\
2 \cdot 56 \\
3 \cdot 84 \\
5 \cdot 12 \\
6 \cdot 40\end{array}$ & $\begin{array}{r}42 \cdot 6 \\
21 \cdot 4 \\
10 \cdot 9 \\
5 \cdot 4 \\
1 \cdot 7\end{array}$ & $\begin{array}{c}31 \cdot 9 \\
16 \cdot 7 \\
8 \cdot 5 \\
4 \cdot 2 \\
1 \cdot 33\end{array}$ & $\begin{array}{r}30 \cdot 74 \\
16 \cdot 72 \\
8 \cdot 86 \\
4 \cdot 28 \\
1 \cdot 27\end{array}$ & $\begin{array}{c}-1 \cdot 2 \\
0 \\
+.4 \\
+.1 \\
-\cdot 1\end{array}$ \\
\hline
\end{tabular}

of $r$ to be $\cdot 4674$, whence $\frac{r d}{2}=\cdot 04440$ for steel not hardened. As the steel becomes harder this quantity increases, and can probably reach about twice this for very hard steel.

To show the effect of hardening; I broke the bar used in Table XIV at the centre, thus producing two bars $6 \cdot 4$ inches long. One of these halves was hardened till it could scarcely be scratched by a file; but the other half was left unaltered. The following Table gives the distribution, using the same unit as that of Tables XIII and XIV. The bars were so short that the results can hardly be relied on; but they will at least suffice to show the change. 
In Fig. 7 I have attempted to give the curve of distribution from Table XV, and have made the curves coincide with observation as nearly as possible, making a small allowance, however, for the errors introduced by the shortness of the bar. It is seen that the effect of hardening in a bar of these dimensions is to increase the quantity of magnetism, but especially that near the end. Had the bar been very long, no increase

TABLE XV.

\begin{tabular}{|c|r|r|r|r|}
\hline \multirow{2}{*}{$x}$. & \multicolumn{2}{|c|}{ Soft Steel, A. } & \multicolumn{2}{|c|}{ Hard Steel, B. } \\
\cline { 2 - 5 } & $Q_{\epsilon^{\cdot}}$ & $4 \pi \lambda$. & $Q_{\epsilon^{*}}$ & $4 \pi \lambda$. \\
\hline & & & & \\
\hline 0 & $20 \cdot 4$ & $29 \cdot 1$ & $47 \cdot 7$ & $68 \cdot 1$ \\
$\cdot 64$ & $9 \cdot 8$ & $15 \cdot 3$ & $13 \cdot 9$ & $21 \cdot 7$ \\
$1 \cdot 28$ & $6 \cdot 0$ & $9 \cdot 4$ & $7 \cdot 0$ & $11 \cdot 0$ \\
$1 \cdot 92$ & $3 \cdot 8$ & $3 \cdot 0$ & $2 \cdot 6$ & $2 \cdot 0$ \\
$3 \cdot 20$ & & & & \\
\hline
\end{tabular}

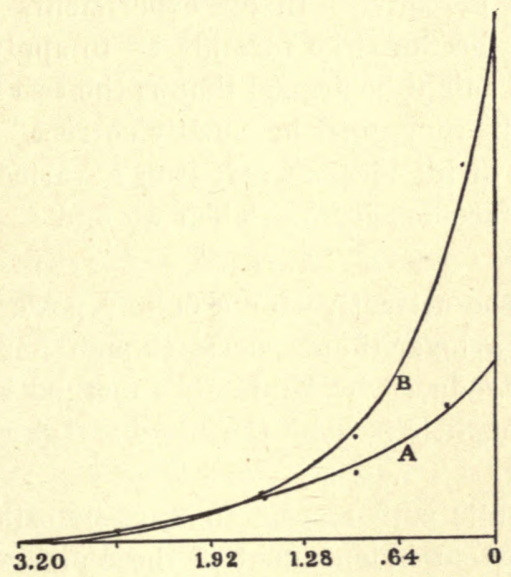

FIG. 7.-Results from permanent magnets.
A. Soft steel.
B. Hard steel.

in the total quantity of magnetism would have taken place; but the distribution would have been changed. From this we deduce the important fact that hardening is most useful for short magnets. And it would seem that almost the only use in hardening magnets at all is to concentrate the magnetism and to reduce the weight. Indeed I have made magnets from iron wire whose magnetization at the central section was just as intense as in a steel wire of the same size; but to all appearance it was less 
strongly magnetized than the stecl, because the magnetism was more diffused; and as the magnetism was not distributed so nearly at the end as in the steel, its magnctis moment and time of vibration were less.

It is for these reasons that many makers of surveyors' compasses find it unnecessary to harden the needles, seeing these are long and thin.

We might deduce all these facts from the formulæ on the assumption that $r$ is greater the harder the iron or steel.

Having now considered briefly the distribution on electromagnets and steel magnets, and found that the formulæ represent it in a general way, we may now use them for solving a few questions that we desire to solve, though only in an approximate manner.

VI.

M. Jamin, in his recent experiments on magnetic distribution, has obtained some very interesting results, although I have shown his method to be very defective. In his experiments on iron bars magnetized at one end, he finds the formula $\varepsilon^{r l}$ to apply to long ones as I have done. Now it might be argued that as the two methods apparently give the same result, they must be equally correct. But let us assume that the attraction of his piece of soft iron $F$ varied as some nnknown power $n$ of the surface-density $\delta$. Then we find

$$
F=C \varepsilon^{n r L} \text {, }
$$

which shows that the attractive force or any power of that force can be represented by a logarithmic curve, though not by the same one. Hence the error introduced by M. Jamin's method is insidious and not easily detected, though it is none the less hurtful and misleading, but rather the more so.

However, his results with respect to what he calls the normal magnet ${ }^{\text {to }}$ are to some extent independent of these errors; and we may now consider them.

Thus, in explaining the effect of placing hardened steel plates on one another, he says, "Quand on superpose deux lames aimantées pareilles, les courbes qui représentent les valeurs de $F$ [the attractive force on the piece of soft iron] s'élèvent, parce que le magnétisme quitte les faces que l'on met en contact pour se réfugier sur les parties extérieures. En même temps, les deux courbes se rapprochent l'une de l'autre et du milieu de l'aimant. Cet effet augmente arec une troisième

10 'On the Theory of the Normal Magnets,' Comptes Rendus, March 31, 1873; translated in Phil. Mag., June, 1873. 
lame et avec une quatrième. Finalement les deux courbes se joignent au milieu."

In applying the formula to this case of a compound magnet, we have only to remark that when the bars lie closely together they are theoretically the same as a solid magnet of the same section, but are practically found to be stronger, because thin bars can be tempered more uniformly hard than thick ones. The addition of the bars to each other is similar, then, to an increase in the area of the rod, and should produce nearly the same effect on a rod of rectangular section as the increase of diameter in a rod of circular section. Now the quantity $p=\frac{r d}{2}$ is nearly constant in these rods for the same quality of steel, whence $r$ decreases as $d$ increases; and this in equation (17) shows that as the diameter is increased, the length being constant, the curves become less and less steep, until they finally become straight lines. This is exactly the meaning of M. Jamin's remark.

Where the ratio of the diameter to the length is small, the curves of distribution are apparently separated from each other and are given by the equation

$$
\lambda=\frac{\mathfrak{g}}{4 \pi \sqrt{R \bar{R}^{\prime}}} \varepsilon^{-r x}, \ldots . . . \quad . \quad . .
$$

which is not dependent on the length of the rod. This is exactly the result found by Coulomb (Biot's Physique, vol. iii, pp. 74, 75). M. Jamin has also remarked this. He states that as he increases the number of plates the curves approach each other and finally unite; this he calls the "normal magnet;" and he supposes it to be the magnet of greatest power in proportion to its weight. "From this moment," says he, "the combination is at its maximum." The normal magnet, as thus defined, is very indefinite, as M. Jamin himself admits.

By our equations we can find the condition for a maximum, and can give the greatest values to the following, supposing the weight of the bar to be a fixed quantity in the first three.

1st. The magnetic moment.

2nd. The attractive force at the end.

3rd. The total number of lines of magnetic force passing from the bar.

4th. The magnetic moment, the length being constant and diameter variable.

Either of these may be regarded as a measure of the power of the bar, according to the view we take. The magnetic moment of a bar is easily found to be 


$$
M=\frac{\mathfrak{S}}{4 \pi r^{2} R^{\prime}}\left\{\begin{array}{l}
b \\
2-\frac{1}{r} \frac{1-\varepsilon^{-r b}}{1+\varepsilon^{-r b}}
\end{array}\right\}
$$

and if $r$ is the weight of a unit of volume of the steel and $W$ is the weight of the magnet, we have finaliy

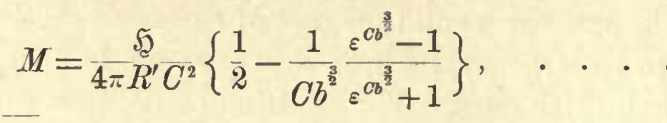

where $C=\frac{r}{\sqrt{ } b}=p \sqrt{\frac{\pi r}{W}}$.

This only attains a maximum when $\frac{b}{d}=\infty$, or the rod is infinitely long compared with its diameter.

- The second case is rather indefinite, seeing it will depend upon whether the body attracted is large or small. When it is small, we require to make the surface-density a maximum, the weight being constant. We find

$$
\delta_{0}=\frac{\mathfrak{S}}{\gamma \pi^{2} R^{\prime} p} \frac{\varepsilon^{o b^{\frac{3}{2}}}-1}{\varepsilon^{c b^{\frac{3}{2}}}+1}, \ldots . . . .
$$

which attains a maximum as before when $\frac{b}{d}=\infty$. When the attracted body is large, the attraction will depend more nearly upon the linear density,

$$
\lambda_{0}=\frac{\mathfrak{F}}{4 C \pi R^{\prime} \sqrt{b}} \frac{\varepsilon_{\varepsilon}^{c b^{\frac{3}{2}}}-1}{c^{c^{\frac{3}{2}}}+1}, \ldots . . .
$$

which is a maximum when $\frac{b}{d}=\frac{1.42}{p}$.

For the third case we have the value of $Q^{\prime \prime}$ at the centre of the bar from equation (6),

$$
Q^{\prime \prime}=\frac{\mathfrak{S}}{2 C^{2} R^{\prime} b}{ }_{\varepsilon^{c b^{\frac{3}{2}}}+1}^{\left(\varepsilon^{\frac{1}{2} c^{\frac{3}{2}}}-1\right)^{2}} . . . . . .
$$

The condition for a maximum gives in this case

$$
\frac{b}{d}=\frac{1 \cdot 65}{p} \text {. }
$$

For the last case, in which the magnetic moment for a given length is to be made a maximum, we find

$$
\frac{b}{d}=\frac{1}{p} \text {. }
$$

This last result is useful in preparing magnets for determining the 
intensity of the earth's magnetism, and shows that the magnets should be made short, thick, and hard for the best effect."

But for all ordinary purposes the results for the second and third cases seem most important, and lead to nearly the same result; taking the mean we find for the maximum magnet

$$
\frac{b}{d}=\frac{1 \cdot 5}{p} \text {. . . . . . . . . . }
$$

We see from all our results that the ratio of the length of a magnet to its diameter in all cases is inversely as the constant $p$. This constant increases with the hardness of the steel; and hence the harder the steel the shorter we can make our magnets. It would seem from this that the temper of a steel magnet should not be drawn at all, but the hardest steel used, or at least that in which $p$ was greatest. The only disadvantage in using very hard steel seerns to be the difficulty in imparting the magnetism at first; and this may have led to the practice of drawing the temper; but now, when we have such powerful electromagnets, it seems as if magnets might be made shorter, thicker, and harder than is the custom. With the relative dimensions of magnets now used, however, hardening might be of little value.

We can also see from all these facts, that if we make a compound magnet of hardened steel plates there will be an advantage in filing more of them together, thus making a thicker magnet than when they are softer. We also observe that as we pile them up the distribution changes in just the way indicated by M. Jamin, the curve becoming less and less steep.

Sybstituting in the formula the value of $p$ which we have found for Stub's steel not hardened, but still so hard as to rapidly dull a file, we find the best ratio of length to diameter to be 33.8-and for the same steel hardened, about $1 \%$, though this last is only a rough approximation. This gives what M. Jamin has called the normal magnet. The ratio should be less for a U-magnet than for a straight one.

For all magnets of the same kind of steel in which the ratio of length to diameter is constant the relative distribution is the same; and this is not only true for cur approximate formula, but would be found so for the exact one.

Thus for the "normal magnet" the distribution becomes

$$
\lambda=C^{y}\left(\varepsilon^{3} \frac{x}{b}-\varepsilon^{-8 \frac{x}{b}}\right),
$$

11 Weber recommends square bars eight times as long as they are broad, and tempered very hard. (Taylor's Scientific Memoirs, vol. ii, p. 86.) 
where $C$ is a constant, and $x$ is measured from the centre. The distribution will then be as follows:-

\begin{tabular}{|c|c|c|c|c|c|c|}
\hline${ }_{b}^{x}=$ & 0. & 1. & 2. & 3. & 4. & 5. \\
\hline$\frac{\bar{\lambda}}{\bar{C}}=$ & 0 & .609 & 1.27 & $2 \cdot 05$ & 3.02 & $4 \cdot 26$ \\
\hline
\end{tabular}

This distribution is not the same as that given by M. Jamin; but as his method is so defective, and his "normal magnet" so indefinite, the agreement is sufficiently near.

The surface-density at any point of a magnet is

$$
\delta=\frac{\mathfrak{S}}{8 \pi^{2} p R^{\prime}} \frac{\varepsilon^{2 p \frac{x}{d}}-\varepsilon^{-2 p \frac{x}{d}}}{\varepsilon^{p \frac{b}{d}}-\varepsilon^{-p \frac{b}{d}}}, \ldots . . . . .
$$

which, for the same kind of steel, is dependent only on $\frac{x}{d}$ and $\frac{b}{d}$. Hence in two similar magnets the surface-density is the same at similar

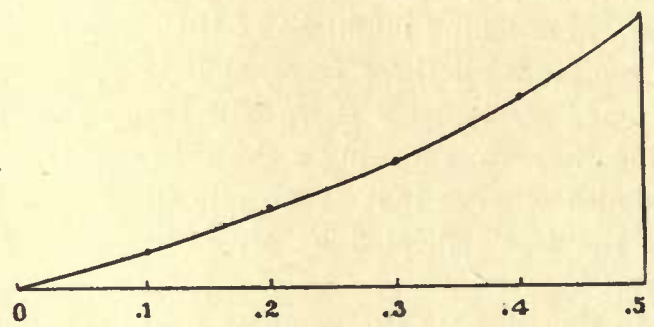

FrG. 8. -Distribution on "normal magnet."

points, the linear density is proportional to the linear dimensions, the surface integral of magnetic induction over half the magnet or across the section is proportional to the surface dimensions of the magnets, and the magnetic moments to the volumes of the magnets. The forces at similar points with regard to the two magnets will then be the same. All these remarks apply to soft iron under induction, provided the inducing force is the same-and hence include Sir William Thomson's well-known law with regard to similar electromagnets; and they are accurately true notwithstanding the approximate nature of the formula from which they have here been deduced.

Our theory gives us the means of determining what effect the boring of a hole through the centre of a magnet would have. In this case $R^{\prime}$ 
is not much affected, but $R$ is increased. Where the magnet is used merely to affect a compass-needie, we should then see that the hole through the centre has little effect where the magnet is short and thick; but where it is long, the attraction on the compass-needle is much diminished. Where the magnet is of the U-form, and is to be used for sustaining weights, the practice is detrimental, and the sustaining-power is diminished in the same proportion as the sectional area of the magnet. The only case that I know of where the hole through the centre is an advantage, is that of the deflecting magnets for determining the intensity of the earth's magnetism, which may be thus made lighter without much diminishing their magnetic moment.

In conclusion, let me express my regret at the imperfection of the theory given in this paper; for although the equations are more general than any yet given, yet still they rest upon two quite incorrect hypotheses; and so, although we have found these formulæ of great use in pursuing our studies on magnetic distribution, yet much remains to be done. A nearer approximation to the true distribution could readily be obtained; but the result would, without doubt, be very complicated, and would not repay us for the trouble.

In this paper, as well as in all others which I have published on the subject of magnetism, my object has not only been to bring forth new 'results, but also to illustrate Faraday's method of lines of magnetic force, and to show how readily calculations can be made on this system. For this reason many points have been developed at greater length than would otherwise be desirable. 


\section{ON THE MAGNETIC EFFECT OF ELECTRIC CONVECTION ${ }^{2}$}

[American Journal of Science [3], $X V, 30-38,1878$ ]

The experiments described in this paper were made with a view of determining whether or not an electrified body in motion produces magnetic effects. There seems to be no theoretical ground upon which we can settle the question, seeing that the magnetic action of a conducted electric current may be ascribed to some mutual action between the conductor and the current. Hence an experiment is of value. Professor Maxwell, in his 'Treatise on Electricity' Art. 770, has computed the magnetic action of a moving electrified surface, but that the action exists has not yet been proved experimentally or theoretically.

The apparatus employed consisted of a vulcanite disc 21.1 centimetres in diameter and .5 centimetre thick which could be made to revolve around a vertical axis with a velocity of 61 . turns per second. On either side of the disc at a distance of $\cdot 6 \mathrm{~cm}$. were fixed glass plates having a diameter of $38.9 \mathrm{~cm}$. and a hole in the centre of $7.8 \mathrm{~cm}$. The vulcanite disc was gilded on both sides and the glass plates had an annular ring of gilt on one side, the outside and inside diameters being $24.0 \mathrm{~cm}$. and $8.9 \mathrm{~cm}$. respectively. The gilt sides could be turned toward or from the revolving disc but were usually turned toward it so that the problem might be calculated more readily and there should be no uncertainty as to the electrification. The outside plates were usually connected with the earth; and the inside disc with an electric battery, by means of a point which approached within one-third of a millimetre of the edge and turned toward it. As the edge was broad, the point would not discharge unless there was a difference of potential between it and the edge. Between the electric battery and the disc,

\footnotetext{
1 The experiments described were made in the laboratory of the Berlin University through the kindness of Professor Helmholtz, to whose advice they are greatly in. debted for their completeness. The idea of the experiment first occurred to me in 1868 and was recorded in a note book of that date.
} 
a commutator was placed, so that the potential of the latter could be made plus or minus at will. All parts of the apparatus were of nonmagnetic material.

Over the surface of the disc was suspended, from a bracket in the wall, an extremely delicate astatic needle, protected from electric action and currents of air by a brass tube. The two needles were 1.5 $\mathrm{cm}$. long and their centres $17.98 \mathrm{~cm}$. distant from each other. The readings were by a telescope and scale. The opening in the tube for observing the mirror was protected from electrical action by a metallic cone, the mirror being at its vertex. So perfectly was this accomplished that no effect of electrical action was apparent either on charging the battery or reversing the electrification of the disc. The needles were so far apart that any action of the disc would be many fold greater on the lower needle than the upper. The direction of the needles was that of the motion of the disc directly below them, that is, perpendicular to the radius drawn from the axis to the needle. As the support of the needle was the wall of the laboratory and the revolving disc was on a table beneath it, the needle was reasonably free from vibration.

In the first experiments with this apparatus no effect was observed other than a constant deflection which was reversed with the direction of the motion. This was finally traced to the magnetism of rotation of the axis and was afterward greatly reduced by turning down the axis to $.9 \mathrm{~cm}$. diameter. On now rendering the needle more sensitive and taking several other precautions a distinct effect was observed of several millimetres on reversing the electrification and it was separated from the effect of magnetism of rotation by keeping the motion constant and reversing the electrification. As the effect of the magnetism of rotation was several times that of the moving electricity, and the needle was so extremely sensitive, numerical results were extremely hard to be obtained, and it is only after weeks of trial that reasonably accurate results have been obtained. But the qualitative effect, after once being obtained, never failed. In hundreds of observations extending over many weeks, the needle always answered to a change of electrification of the disc. Also on raising the potential above zero the action was the reverse of that when it was lowered below. The swing of the needle on reversing the electrification was about $10 \cdot$ or $15 \cdot$ millimetres and therefore the point of equilibrium was altered 5 or $7 \frac{1}{2}$ millimetres. This quantity varied with the electrification, the velocity of motion, the sensitiveness of the needle, etc. 
The direction of the action may be thus defined. Calling the motion of the disc + when it moved like the hands of a watch laid on the table with its face up, we have the following, the needles being over one side of the disc with the north pole pointing in the direction of positive motion. The motion being + , on electrifying the disc + the north pole moved toward the axis, and on changing the electrification, the north pole moved away from the axis. With - motion and + electrification, the north pole moved away from the axis, and with electrification, it moved toward the axis. The direction is therefore that in which we should expect it to be.

To prevent any suspicion of currents in the gilded surfaces, the latter, in many experiments, were divided into small portions by radial scratches, so that no tangential currents could take place without sufficient difference of potential to produce sparks. But to be perfectly certain, the gilded disc was replaced by a plane thin glass plate which could be electrified by points on one side, a gilder induction plate at zero potential being on the other. With this arrangement, effects in the same direction as before were obtained, but smaller in quantity, seeing that only one side of the plate could be electrified.

The inductor plates were now removed, leaving the disc perfectly free, and the latter was once more gilded with a continuous gold surface, having only an opening around the axis of $3.5 \mathrm{~cm}$. The gilding of the disc was connected with the axis and so was at a potential of zero. On one side of the plate, two small inductors formed of pieces of tinfoil on glass plates, were supported, having the disc between them. On electrifying these, the disc at the points opposite them was electrified by induction but there could be no electrification except at points near the inductors. On now revolving the disc, if the inductors were very small, the electricity would remain nearly at rest and the plate would as it were revolve through it. Hence in this case we should have conduction without motion of electricity, while in the first experiment we had motion without conduction. I have used the term "nearly at rest" in the above, for the following reasons. As the disc revolves the electricity is being constantly conducted in the plate so as to retain its position. Now the function which expresses the potential producing these currents and its differential coefficients must be continuous throughout the disc, and so these currents must pervade the whole disc. 
To calculate these currents we have two ways. Either we can consider the electricity at rest and the motion of the disc through it to produce an electromotive force in the direction of motion and proportional to the velocity of motion, to the electrification, and to the surface resistance; or, as Professor Helmholtz has suggested, we can consider the electricity to move with the disc and as it comes to the edge of the inductor to be set free to return by conduction currents to the other edge of the inductor so as to supply the loss there. The problem is capable of solution in the case of a disc without a hole in the centre but the results are too complicated to be of much use. Hence scratches were made on the disc in concentric circles about $\cdot 6 \mathrm{~cm}$. apart by which the radial component of the currents was destroyed and the problem became easily calculable.

For, let the inductor cover $\frac{1}{n}$ th part of the circumference of any one of the conducting circles; then, if $C$ is a constant, the current in the circle outside the inductor will be $+\frac{C}{n}$, and inside the area of the inductor $-C \frac{(n-1)}{n}$. On the latter is superposed the convection current equal to $+C$. Hence the motion of electricity throughout the whole circle is $\frac{1}{n}$ what it would have been had the inductor covered the whole circle.

In one experiment $n$ was about 8 . By comparison with the other experiments we know that had electric conduction alone produced effect we should have observed at the telescope $-5 \cdot \mathrm{mm}$. Had electric convection alone produced magnetic effect we should have had $+5 \cdot 7 \mathrm{~mm}$. And if they both had effect it would have been $+.7 \mathrm{~mm}$., which is practically zero in the presence of so many disturbing causes. No effect was discovered, or at least no certain effect, though every care was used. Hence we may conclude with reasonable certainty that electricity produces nearly if not quite the same magnetic effect in the case of convection as of conduction, provided the same quantity of electricity passes a given point in the convection stream as in the conduction stream.

The currents in the disc were actually detected by using inductors covering half the plate and placing the needle over the uncovered portion; but the effect was too small to be measured accurately. To prove 
this more thoroughly numerical results were attempted, and, after weeks of labor, obtained. I give below the last results which, from the precautions taken and the increase of experience, have the greatest weight.

The magnetizing force of the dise was obtained from the deflection of the astatic needle as follows. Turning the two needles with poles in the same direction and observing the number $n$ of vibrations, and then turning them opposite and finding the number $n^{\prime}$ of vibrations in that position, we shall find, when the lower needle is the strongest,

$$
X-X^{\prime} \frac{n^{2}-n^{\prime 2}}{n^{2}+n^{\prime 2}}=\frac{n^{\prime 2}}{n^{2}+n^{\prime 2}} \frac{\Delta}{D} H, \quad . \quad . .
$$

where $X^{\prime}$ and $X$ are the forces on the upper and lower needle respectively, $\Delta$ the deflection, $D$ the distance of the scale and $H$ the horizontal component of the earth's magnetism. As $X^{\prime}$ and $n^{\prime}$ are very small the first term is nearly $X-X^{\prime}$. The torsion of the silk fibre was too small to affect the result, or at least was almost eliminated by the method of experiment.

The eléctricity was in the first experiment distributed nearly uniformly over the disc with the exception of the opening in the centre and the excess of distribution on the edge. The surface density on either side was

$$
\sigma=\frac{V-V^{\prime}}{2 \pi(B-\beta)}, \quad . \quad . \quad . \quad . \quad . \quad .
$$

$V-V^{\prime}$ being the difference of potential between the disc and the outside plates, $\beta$ the thickness of the dise and $B$ the whole distance apart of the outside plates. The excess on the edge was (Maxwell's Electricity, Art. 196, Eq. 18),

$$
E=2\left(V-V^{\prime}\right) \frac{B C}{\pi(B-\beta)} \log _{e}\left(2 \cos \frac{\pi \beta}{2 B}\right), \quad . .
$$

where $C$ is the radius of the disc.

We may calculate the magnetic effect on the supposition that, as in the conducted current, the magnetizing force due to any element of surface is proportional to the quantity of electricity passing that element in a unit of time. The magnetic effect due to the uniform distribution has the greatest effect. With an error of only a small 
fraction of a per cent, we may consider the two sides of the disc to coincide in the centre. Taking the origin of coördinates at the point of the disc under the needle and the centre of the disc on the axis of $X$ we find for both sides of the disc, the radial component of the force parallel to the disc,

$$
\begin{aligned}
X=\frac{8 \pi N \sigma a}{v} \int_{-(c+b)}^{c-b} \int_{0}^{y^{\prime}} & \frac{(b+x) d x d y}{\left(a^{2}+x^{2}+y^{2}\right)^{\frac{3}{2}}} \\
& =\frac{8 \pi N \sigma a}{v} \int_{-(c+b)}^{c-b} \frac{(b+x) \sqrt{C^{2}-(b+x)^{2}}}{\left(a^{2}+x^{2}\right) \sqrt{a^{2}+C^{2}-b^{2}-2 b x}},
\end{aligned}
$$

where $a$ is the distance of the needle from the disc and $b$ that from the axis; $N$ is the number of revolutions of the disc per second and $v=28,800,000,000$ centimetres per second according to Maxwell's determination. The above integral can be obtained exactly by elliptic integrals, but as it introduces a great variety of complete and incomplete elliptic integrals of all three orders, we shall do best by expanding as follows:

$$
X=\frac{4 \pi N \sigma}{v} P=\frac{4 \pi N \sigma}{v}\left(A_{1}+A_{2}+A_{3}+\& \mathrm{c} .\right), . .
$$

$A_{1}=2 b\left(\arctan \frac{C-b}{a}+\arctan \frac{C+b}{a}\right)-a \log _{\epsilon} \frac{M}{N}$,

$A_{2}=-\frac{a}{2 b}\left((s+b) \log _{\mathrm{e}} \frac{M}{N}-2 C\right)$,

$A_{3}=\frac{a}{16 b^{2}}\left\{-4 C s+\left(3 s^{2}+2 s b+a^{2}\right) \log _{\epsilon} \frac{M}{N}\right.$

$$
\left.+\left(5 s^{3}+3 s^{2} b+a^{2}(s+b)\right) \frac{4 C b}{M N}\right\} \& c ., \& c .
$$

where

$$
s=\frac{a^{2}+C^{2}-b^{2}}{2 b}, \quad M=a^{2}+(C+b)^{2}, \quad N=a^{2}+(C-b)^{2} .
$$

From this must be subtracted the effect of the opening in the centre, for which the same formula will apply.

The magnetic action of the excess at the edge may be calculated on the supposition that that excess is concentrated in a circle of a little smaller diameter, $C^{\prime}$, than the disc; therefore,

$$
X_{1}=\frac{N E}{v} \frac{a k}{2 b \sqrt{C^{\prime} b}}\left\{\frac{2-k^{2}}{1-k^{2}} E(k)-F(k)\right\}, \ldots
$$


where $k=\frac{2 \sqrt{ } C^{\prime} b}{\sqrt{a^{2}+\left(C^{\prime}+b\right)^{2}}}$, and $F(k)$ and $E(k)$ are complete elliptic integrals of the second and first orders respectively.

The determination of the potential was by means of the spark which Thomson has experimented on in absolute measure. For sparks of length $l$ between two surfaces nearly plane, we have on the centimetre, gram, second system, from Thomson's experiments,

$$
V-V^{\prime}=117 \cdot 5(l+.0135),
$$

and for two balls of finite radius, we find, by considering the distribution on the two sheets of an hyperboloid of revolution,

$$
V-V^{\prime}=11 \% \cdot 5(l+\cdot 0135) \frac{r}{2 \sqrt{r+1}} \log _{e} \frac{\sqrt{r+1+1}}{\sqrt{r+1-1}},
$$

where $r$ is the ratio of the length of spark to diameter of balls and had in these experiments a value of about 8 . In this case

$$
V-V^{\prime}=109 \cdot 6(l+\cdot 0135) \text {. }
$$

A battery of nine large jars, each $48 . \mathrm{cm}$. high, contained the store of electricity supplied to the disc, and the difference of potential was determined before and after the experiment by charging a small jar and testing its length of spark. Two determinations were made before and two after each experiment, and the mean taken as representing the potential during the experiment.

The velocity of the disc was kept constant by observing a governor. The number of revolutions was the same, nearly, as determined by the sizes of the pulleys or the sound of: a Seebeck siren attached to the axis of the disc; the secret of this agreement was that the driving cords were well supplied with rosin. The number of revolutions was $61 \cdot$ per second.

In such a delicate experiment, the disturbing causes, such as the changes of the earth's magnetism, the changing temperature of the room, \&c., were so numerous that only on few days could numerical results be obtained, and even then the accuracy could not be great. The centimetre, gram, second system, was used.

First Series. $a=2 \cdot 05, b=9 \cdot 08, n=\cdot 697, D=110 \cdot, H-\cdot 182$ nearly, $B=1 \cdot 68, \beta=\cdot 50, C=10 \cdot 55, N-61 \cdot, v=28,800,000,000 \cdot$, $n^{\prime}=.0533, C^{\prime}=10$. 


\begin{tabular}{|c|c|c|c|c|}
\hline $\begin{array}{l}\text { Direction of } \\
\text { motion. }\end{array}$ & $\begin{array}{l}\text { Electrifica- } \\
\text { tion of disc. }\end{array}$ & $\begin{array}{l}\text { Scale reading } \\
\text { in mm. }\end{array}$ & $\begin{array}{l}\text { Deflection on } \\
\text { reversing } \\
\text { electrificat'n } \\
\text { in mm. }\end{array}$ & $\begin{array}{l}\text { Length of } \\
\text { spark. }\end{array}$ \\
\hline+ & \pm & $\begin{array}{c}99 \cdot \\
107 \cdot 5 \\
101 \cdot 5\end{array}$ & $7 \cdot 25$ & $\cdot 295$ \\
\hline - & $\frac{+}{+}$ & $\begin{array}{l}68 \cdot 5 \\
76 \cdot 5 \\
68 \cdot 0\end{array}$ & $8 \cdot 25$ & $\cdot 290$ \\
\hline+ & $\frac{+}{+}$ & $\begin{array}{l}97 . \\
91 \cdot 5 \\
100 .\end{array}$ & $7 \cdot 00$ & $\cdot 282$ \\
\hline - & $\frac{+}{+}$ & $\begin{array}{l}59 \cdot \\
65 \cdot 5 \\
58 \cdot 5\end{array}$ & $6 \cdot 75$ & $\cdot 265$ \\
\hline+ & $\frac{t}{t}$ & $\begin{array}{l}92 \cdot 5 \\
85 \cdot \\
91 \cdot 0\end{array}$ & $6 \cdot 75$ & $\cdot 290$ \\
\hline- & $\frac{+}{+}$ & $\begin{array}{l}52 \cdot 5 \\
57 \cdot 5 \\
51 \cdot 5\end{array}$ & $5 \cdot 50$ & $\cdot 285$ \\
\hline+ & $\frac{+}{+}$ & $\begin{array}{l}82 \cdot 0 \\
76 \cdot 0 \\
81 \cdot 7\end{array}$ & $5 \cdot 85$ & $\cdot 285$ \\
\hline - & $\frac{+}{+}$ & $\begin{array}{l}36 \cdot 5 \\
43 \cdot 0 \\
36 \cdot 5\end{array}$ & $6 \cdot 50$ & $\cdot 275$ \\
\hline+ & $\frac{+}{+}$ & $\begin{array}{l}68 \cdot 0 \\
61 \cdot 0 \\
68 \cdot 0\end{array}$ & $7 \cdot 00$ & $\cdot 290$ \\
\hline - & $\frac{+}{+}$ & $\begin{array}{l}27 \cdot 5 \\
33 \cdot 5 \\
26 \cdot 5\end{array}$ & $6 \cdot 50$ & $\cdot 288$ \\
\hline \multicolumn{3}{|c|}{ Mean values. } & $6 \cdot 735$ & $\cdot 2845$ \\
\hline
\end{tabular}

Hence

$$
\Delta=\frac{\cdot 6735}{2}=\cdot 337 \text { and } l=\cdot 2845 .
$$

From equation (1),

$$
X-\cdot 99 X^{\prime}=\frac{1}{305700} .=\cdot 00000327 .
$$

By calculation from the electrification we find 


$$
X-\cdot 99 X^{\prime}=\frac{1}{296800}=\cdot 0000033 \%
$$

The effect on the upper needle, $X^{\prime}$, was about $\frac{1}{50}$ of that on the lower $X$.

Second Series. Everything the same as before except the following. $b=7 \cdot 65, n^{\prime}=\cdot 0525$.

\begin{tabular}{|c|c|c|c|c|}
\hline $\begin{array}{l}\text { Direction of } \\
\text { motion. }\end{array}$ & $\begin{array}{l}\text { Electrifica- } \\
\text { tion of disc. }\end{array}$ & $\begin{array}{l}\text { Scale reading } \\
\text { in } \mathrm{mm} .\end{array}$ & $\begin{array}{l}\text { Deflection on } \\
\text { reversing } \\
\text { electrificat'n } \\
\text { in mm. }\end{array}$ & $\begin{array}{l}\text { Length of } \\
\text { spark. }\end{array}$ \\
\hline+ & $\frac{+}{+}$ & $\begin{array}{l}172 \cdot 5 \\
165 \cdot 5 \\
172 \cdot 5\end{array}$ & $7 \cdot 0$ & $\cdot 300$ \\
\hline- & $\frac{+}{+}$ & $\begin{array}{l}120 \cdot 0 \\
127 \cdot 5 \\
121 \cdot 5 \\
129 \cdot 0\end{array}$ & $7 \cdot 5$ & .295 \\
\hline+ & $\frac{\bar{t}}{+}$ & $\begin{array}{l}163 \cdot 5 \\
170 \cdot 5 \\
163 \cdot 0 \\
170 \cdot 5\end{array}$ & $7 \cdot 25$ & .297 \\
\hline- & $\frac{+}{+}$ & $\begin{array}{l}118 \cdot 0 \\
127 \cdot 0 \\
120 \cdot 0 \\
127 \cdot 5\end{array}$ & $8 \cdot 25$ & $\cdot 270$ \\
\hline \multicolumn{3}{|c|}{ Mean values. } & $7 \cdot 50$ & $\cdot 2955$ \\
\hline
\end{tabular}

$$
\therefore \quad \Delta=\cdot 375, \quad l=\cdot 2955 .
$$

Hence for this case we have from equation (1),

$$
X-\cdot 99 X^{\prime}=\frac{1}{315000^{\circ}}=\cdot 0000031 \% \text {. }
$$

And from the electrification,

$$
X-\cdot 99 X^{\prime}=\frac{1}{286000}=\cdot 00000349 .
$$

Third Series. Everything the same as in the first series, except $b=8 \cdot 1, n^{\prime}=\cdot 0521, D=114$. 


\begin{tabular}{|c|c|c|c|c|}
\hline $\begin{array}{l}\text { Direction of } \\
\text { motion. }\end{array}$ & $\begin{array}{l}\text { Electrifica- } \\
\text { tion of disc. }\end{array}$ & $\begin{array}{l}\text { Scale reading } \\
\text { in mm. }\end{array}$ & $\begin{array}{c}\text { Deflection on } \\
\text { reversing } \\
\text { electrificat' } \\
\text { in mm. }\end{array}$ & $\begin{array}{l}\text { Length of } \\
\text { spark. }\end{array}$ \\
\hline- & $\frac{+}{+}$ & $\begin{array}{l}151 \cdot 0 \\
158 \cdot 5 \\
151 \cdot 0\end{array}$ & 7.50 & $\cdot 287$ \\
\hline+ & $\frac{+}{+}$ & $\begin{array}{l}192 \cdot 0 \\
185 \cdot 5 \\
193 \cdot 5\end{array}$ & $7 \cdot 25$ & $\cdot 292$ \\
\hline- & $\frac{\bar{t}}{+}$ & $\begin{array}{l}157 \cdot 5 \\
148 \cdot 5 \\
157 \cdot 5 \\
150 \cdot 0\end{array}$ & $8 \cdot 25$ & .295 \\
\hline+ & $\frac{\bar{t}}{+}$ & $\begin{array}{l}185 \cdot 0 \\
192 \cdot 5 \\
185 \cdot 5 \\
193 \cdot 5\end{array}$ & $7 \cdot 75$ & $\cdot 302$ \\
\hline - & $\underline{+}$ & $\begin{array}{l}151 \cdot 0 \\
143 \cdot 5 \\
150 \cdot 5\end{array}$ & $7 \cdot 25$ & $\cdot 287$ \\
\hline \multicolumn{3}{|c|}{ Mean values. } & $7 \cdot 60$ & $\cdot 2926$ \\
\hline
\end{tabular}

$\therefore \quad \Delta=\cdot 380, l=\cdot 2926$.

For this case from equation (1)

$$
X-.99 X^{\prime}=\frac{1}{295000^{\circ}}=\cdot 00000339,
$$

and from the electrification

$$
X=\cdot 99 X^{\prime}=\frac{1}{281500^{\circ}}=\cdot 00000355 .
$$

The error amounts to 3,10 and 4 per cent respectively in the three series. Had we taken Weber's value of $v$ the agreement would have been still nearer. Considering the difficulty of the experiment and the many sources of error, we may consider the agreement very satisfactory. The force measured is, we observe, about $\frac{1}{50000}$ of the horizontal force of the earth's magnetism.

The difference of readings with + and - motion is due to the magnetism of rotation of the brass axis. This action is eliminated from the result.

It will be observed that this method gives a determination of $v$, the ratio of the electromagnetic to the electrostatic system of units, and if carried out on a large scale with perfect instruments might give good results. The value $v=300,000,000$. metres per second satisfies the first and last series of the experiments the best.

Berlin, February 15, 1876. 


\section{NOTE ON THE MAGNETIC EFFECT OF ELECTRIC CONVECTION}

[Philosophical Magazine [5], VII, 442, 443, 1879]

Johns Hopkins University, Baltimore, April 8, 1878.

To the Editors of the Philosophical Magazine and Journal.

Gentlemen:-Some three years since, while in Berlin, I made some experiments on the magnetic effect of electric convection, which have since been published in the 'American Journal of Science' for January, 1878. But previous to that, in 1876, Professor Helmholtz had presented to the Berlin Academy an abstract of my paper, which has been widely translated into many languages. But, although Helmholtz distinctly says, "Ich bemerke dabei, das derselbe den Plan für seine (Rowland's) Versuche schon gefasst und vollständig überlegt hatte, als er in Berlin ankam, ohne vorausgehende Einwirkung von meiner Seite," yet nevertheless I now find that the experiment is being constantly referred to as Helmholtz's experiment-and that if I get any credit for it whatever, it is merely in the way of carrying out Helmholtz's ideas, instead of all the credit for ideas, design of apparatus, the carrying out of the experiment, the calculation of results, and everything which gives the experiment its value.

Unfortunately for me, Helmholtz had already experimented on the subject with negative results; and I found, in travelling through Germany that others had done the same. The idea occurred in nearly the same form to me eleven years ago; but as I recognized that the experiment would be an extremely delicate one, I did not attempt it until I could have every facility, which Helmholtz kindly gave me.

Helmholtz kindly suggested a more simple form of commutator than I was about to use, and also that I should extend my experiments so as to include an uncoated glass disk as well as my gilded vulcanite ones; but all else I claim as my own,-the method of experiment in all its details, the laboratory work, the method of calculation-indeed everything connected with the experiment in any way, as completely as if it had been carried out in my own laboratory 4000 miles from the Berlin laboratory.

Yours truly,

H. A. Rowland. 


\section{NOTE ON THE THEORY OF ELECTRIC ABSORPTION}

[American Journal of Mathematics, I, 53-58, 1878]

In experimenting with Leyden jars, telegraph cables and condensers of other forms in which there is a solid dielectric, we observe that after complete discharge a portion of the charge reappears and forms what is known as the residual charge. This has generally been explained by supposing that a portion of the charge was conducted below the surface of the dielectric, and that this was afterwards conducted back again to its former position. But from the ordinary mathematical theory of the subject, no such consequence can be deduced, and we must conclude that this explanation is false. Maxwell, in his "Treatise on Electricity and Magnetism,' vol. 2, chap X, has shown that a substance composed of layers of different substances can have this property. But the theory of the whole subject does not yet seem to have been given.

Indeed, the general theory would involve us in very complicated mathematics, and our equations would have to apply to non-homogeneous, crystalline bodies in which $\mathrm{Ohm}$ 's law was departed from and the specific inductive eapacity was not constant; we should, moreover, have to take account of thermo-electric currents, electrolysis, and electro-magnetic induction. Hence in this paper I do not propose to do more than to slightly extend the subject beyond its present state and to give the general method of still further extending it.

Let us at first, then, take the case of an isotropic body in general, in which thermo-electric currents and electrolysis do not exist, and on and in which the changes of currents are so slow that we can omit electro-magnetic induction. The equations then become ${ }^{2}$

$$
\begin{aligned}
& \frac{d}{d x}\left(\% \frac{d V}{d x}\right)+\frac{d}{d y}\left(\% \frac{d V}{d y}\right)+\frac{d}{d z}\left(\% \frac{d V}{d z}\right)+4 \pi \rho=0, \\
& \frac{d}{d x}\left(k \frac{d V}{d x}\right)+\frac{d}{d y}\left(k \frac{d V}{d y}\right)+\frac{d}{d z}\left(k \frac{d V}{d z}\right)-\frac{d \rho}{d t}=0,
\end{aligned}
$$

in which $\chi$ is the specific inductive capacity of the substance, $k$ the

1 Maxwell's Treatise, Art. 325. 
electric conductivity, $V$ the potential, $\rho$ the volume density of the electricity, and $t$ the time.

The subtraction of one equation from the other gives

$$
\begin{aligned}
\frac{d D}{d x} \frac{d}{d x}\left(\log \frac{k}{\chi}\right)+\frac{d V}{d y} \frac{d}{d y} & \left(\log \frac{k}{\chi}\right) \\
+ & \frac{d V}{d z} \frac{d}{d z}\left(\log \frac{k}{\chi}\right)-\frac{1}{k} \frac{d \rho}{d t}-\frac{4 \pi \rho}{\chi}=0 .
\end{aligned}
$$

To introduce the condition that there shall be no electric absorption, we must observe that when that phenomenon exists, a charge of electricity appears at a point where there was no charge before; in other words, the relative distribution has been changed. Hence, if the relative distribution remains the same, no electric absorption can take place. Our condition is, then,

$$
\frac{\rho}{\rho^{\prime}}=c,
$$

where $c$ is independent of $t$, and $\rho^{\prime}$ and $\rho^{\prime}$ are the densities at the points $x, y, z$, and $x^{\prime}, y^{\prime} z^{\prime}$. This gives

or

$$
\begin{gathered}
\frac{d}{d t}\left(\log \frac{\rho}{\rho^{\prime}}\right)=0, \\
\frac{1}{\rho} \frac{d \rho}{d t}=\frac{d}{d t}\left(\log \frac{\rho}{\rho_{0}}\right)=-c, \ldots . . .
\end{gathered}
$$

where $c$ is a function of $t$ only and not of $x, y, z$, and $\rho_{0}$ is the value of $\rho$ at the time $t=0$. As we have

$$
\frac{1}{m} \frac{d V}{d n} \frac{d m}{d n}=\frac{d V}{d x} \frac{d}{d x}\left(\log \frac{k}{\chi}\right)+\frac{d V}{d y} \frac{d}{d y}\left(\log \frac{k}{\chi}\right)+\frac{d V}{d z} \frac{d}{d z}\left(\log \frac{k}{\%}\right),
$$

where $m=\frac{k}{\chi}$ and $n$ is a line in the direction of the current at the given point, equation (1) becomes

From equation (2)

$$
\frac{1}{m} \frac{d V}{d n} \frac{d m}{d n}-\frac{1}{k} \frac{d \rho}{d t}-\frac{4 \pi \rho}{\chi}=0
$$

and hence

$$
\rho=\rho_{0} \varepsilon^{-\int_{0}^{t} t d}
$$

$$
\frac{1}{m} \frac{d V}{d n} \frac{d m}{d n}+\rho_{0} \varepsilon \mathcal{f}_{t}^{0}\left(\frac{c}{k}-\frac{4 \pi}{\chi}\right)=0
$$

If we denote the strength of current at the point by $S$, we have 


$$
S=-k \frac{d V}{d u}
$$

and

$$
\frac{1}{c m-4 \pi m^{2}} \frac{d m}{d n}=+\frac{\rho_{0}}{S^{t}} \varepsilon_{t}^{f_{c}^{0}} ; \quad . . . . .
$$

this equation (3) gives the value of $\frac{k}{\%}=m$ at all points of the body and at all times so that the phenomenon of electric absorption shall not take place. As this equation makes $m$ a function of $x, y, z, S$ and $t$, the relation in general is entirely too complicated to ever apply to physical phenomena, without some limitation. Firstly then, as $c$ is only an arbitrary function of $t$, we shall assume that it is constant;

$$
\therefore \frac{1}{c m-4 \pi m^{2}} \frac{d m}{d n}=+\frac{\rho_{0} \varepsilon^{-e t}}{S} \text {. . . . . . }
$$

The most important case is where $m$ is a constant. Then

and

$$
\frac{d m}{d n}=0 \text {, }
$$

$$
c=4 \pi m, \quad S=S_{0} \varepsilon^{-e t}, \quad \rho=\rho_{0} \varepsilon^{-e} .
$$

In this case, therefore, we see that both the electrification and the currents die away at the rate $c$. The case where Ohm's law is true and the specific inductive capacity is constant is included in this case, seeing that when $k$ and $\chi$ are both constants their ratio, $m$, is constant. But it also includes the cases where $k$ and $\chi$ are both the same functions of $V, S$, or $x, y, z$, seeing that their ratio, $m$, would be constant in this case also.

When $m$ is not constant, the chances are very small against its satisfying equation (4).

Hence, we may in general conclude, that electric absorption will almost certainly take place unless the ratio of conductivity to the specific inductive capacity is constant throughout the body.

This ratio, $m$, may become a variable in several manners, as follows:

1st manner.-The body may not be homogeneous. This includes the case, which Maxwell has given, where the dielectric was composed of layers of different substances.

$2 d$ manner.-The body may not obey $0 \mathrm{hm}$ 's law; in this case $k$ would be variable.

$3 d$ manner. The specific inductive capacity, $\chi$, may vary with the electric force. 
It is to be noted that the cases of electric absorption which we observe are mostly those of condensers formed of two planes, or of one cylinder inside another, as in a telegraph cable. Our theory shows that different explanations can be given of these two cases.

The case of parallel plates does not admit of being explained, except on the supposition that $m$ varies in the first manner above given, or in this manner in combination with the others, for we can only conceive of the conductivity and the specific inductive capacity as being functions of the ordinate or of the electric force. As the latter is constant for all points between the plates, $m$ would still be constant although it were a function of the electric force, and thus electric absorption would not take place.

We may then conclude that in the case of parallel plates, omitting explanations based on electrolysis or thermo-electric currents, the only explanation that we can give at present is that which depends on the non-homogeneity of the body, and is the case which Maxwell has given in the form of two different materials. Our equations show that the form of layers is not necessary, but that any departure from homogeneity is sufficient. It is to be noted that the homogeneity, which we speak of, is electrical homogeneity, and that a mass of crystals with their axes in different directions would evidently not be electrically homogeneous and would thus possess the property in question. In the case of glass it is very possible that this may be the case and it would certainly be so for ice or any other crystalline substance which had been melted and cooled.

In the case of hard india rubber, the black color is due to the particles of carbon, and as other materials are incorporated into it during the process of manufacture, it is certainly not electrically homogeneous.

As to the ordinary explanation that the electricity penetrates a little below the surface and then reappears again to form the residual charge, we see that it is in general entirely false. We could, indeed, form a condenser in which the surface of the dielectric would be a better conductor than the interior and which would act thus. But in general, the theory shows that the action takes place throughout the mass of the dielectric, where that is of a fine grained structure and apparently homogeneous, as in the case of glass, and consists of a polarization of every part of the dielectric.

To consider more fully the case of a condenser made of parallel plates, let us resume our original equations. Without much loss of generality we can assume a laminated structure of the substance in 
the direction of the plane $Y Z$, so that $m$ and $V$ will be only functions of the ordinate $x$. Our equations then become

$$
\begin{aligned}
& \frac{d}{d x}\left(\chi \frac{d V}{d x}\right)+4 \pi \rho=0, \\
& \frac{d}{d x}\left(k \frac{d V}{d x}\right)-\frac{d \rho}{d t}=0 .
\end{aligned}
$$

Eliminating $\rho$ we find

$$
\frac{1}{4 \pi} \frac{d}{d t} \frac{d}{d x}\left(x \frac{d V}{d x}\right)+\frac{d}{d x}\left(k \frac{d V}{d x}\right)=0 .
$$

Now let us make $p=\chi \frac{d V}{d x}$ and as $t$ and $x$ are independent, we find on integration,

$$
\frac{d}{d t}\left(p-p_{0}\right)+4 \pi\left(p m-p_{0} m_{0}\right)=0,
$$

where $p_{0}$ is the value of $p$ for some initial value of $x$, say at the surface of the condenser, and is an arbitrary function of $t$, seeing that we may vary the charge at the surface of the body in any arbitrary manner. This equation establishes $p$ as a function of $m$ and $t$ only, and as we have

$$
\rho=-\frac{1}{4 \pi} \frac{d p}{d x}
$$

$\rho$ will also be a function of these only.

Let us now suppose that at the time $t=0$, the condenser is charged, having had no charge before, and let us also suppose that the different strata of the dielectric are infinitely thin and are placed in the same order and are of the same thickness at every part of the substance, so that a finite portion of the substance will have the same properties at every part.

In this case $m$ will be a periodic function of $x$, returning to the same value again and again. As $\rho$ is a function of this and of $t$ only, at a given time $t$, it must return again and again to the same value as we pass through the substance, indicating a uniform polarized structure throughout the body.

This conclusion would have been the same had we not assumed a laminated structure of the dielectric. In all other cases, except that of two planes, electric absorption can take place, as we have before remarked, even in perfectly homogeneous bodies, provided that Ohm's law is departed from or that the electric induction is not proportional to the electric force, as well as in non-homogeneous bodies. But where the body is thus homogeneous, electric absorption is not due to a uni- 
form polarization, but to distinct regions of positive and negative electrification.

In the whole of the investigation thus far we have sought for the means of explaining the phenomenon solely by means of the known laws of electric induction and conduction. But many of the phenomena of electric absorption indicate electrolytic action, and it is possible that in many cases this is the cause of the phenomenon. The only object of this note is to partially generalize Maxwell's explanation, leaving the electrolytic and other theories for the future. 


\section{RESEARCH ON THE ABSOLUTE UNIT OF ELECTRICAL RESISTANCE ${ }^{1}$}

[American Journal of Science [3], XV, 281-291, 325-336, 430-439, 1878]

\section{Preliminary Remarks}

Since the classical determination of the absolute unit of electrical resistance by the Committee on Electrical Standards of the British Association, two re-determinations have been made, one in Germany and the other in Denmark, which each differ two per cent from the British Association determination, the one on one side and the other on the other side, making a total difference of four per cent between the two. Such a great difference in experiments which are capable of considerable exactness, seems so strange that I decided to make a new determination by a method different from any yet used, and which seemed capable of the greatest exactness; and to guard against all error, it was decided to determine all the important factors in at least two different ways, and to eliminate most of the corrections by the method of experiment, rather than by calculation. The method of experiment depended upon the induction of a current on a closed circuit, and in this respect, resembled that of Kirchhoff, but it differed from his inasmuch as, in my experiment, the induction current was produced by reversing the main current, and in Kirchhoff's by removing the circuits to a distance from each other. And it seems to me that this method is capable of greater exactness than any other, and it certainly possessed the greatest simplicity in theory and facility in experiment.

In the carrying out of the experiment I have partly availed myself of my own instruments and have partly drawn on the collection of the University, which possesses many unique and accurate instruments for electric and magnetic measurements. To insure uniformity and accuracy, the coils of all these instruments have been wound with my own hands and the measurements reduced to a standard rule which was

${ }^{1}$ I am greatly indebted to Mr. Jacques, Fellow of the University, who is an excellent observer, for his assistance during the experiment, particularly in reading the tangent galranometer. 
again compared with the standard at Washington. Unlike many German instruments, quite fine wire has always been used and the number of coils multiplied, for in this way the constants of the coils can be more exactly determined, there is less relative action from the wire connecting the coils, and above all we know exactly where the current passes.

The experiment was performed in the back room of a small house near the University, which was reasonably free from magnetic and other physical disturbances. As the magnetic disturbance was eliminated in the experiment, it was not necessary to select a region entirely free from such disturbance. The small probable error proves that sufficient precaution was taken in this respect.

The result of the experiment that the British Association unit is too great by about .88 per cent, agrees well with Joule's experiment on the heat generated in a wire by a current, and makes the mechanical equivalent as thus obtained very nearly that which he found from friction: it is intermediate between the result of Lorenz and the British Association Committee; and it agrees almost exactly with the British Association Committee's experiments, if we accept the correction which I have applied below.

The difference of nearly three per cent which remains between my result and that of Kohlrausch is difficult to explain, but it is thought that something has been done in this direction in the criticism of his method and results which are entered into below. My value, when introduced into Thomson's and Maxwell's values of the ratio of the electromagnetic to the electrostatic units of electricity, caused a yet further deviation from its value as given in Maxwell's electromagnetic theory of light: but experiments on this ratio have not yet attained the highest accuracy.

\section{History}

The first determination of the resistance of a wire in absolute measure was made by Kirchhoff ${ }^{2}$ in 1849 in answer to a question propounded by Neumann, in whose theory of electrodynamic induction a constant appeared whose numerical value was unknown until that time. His method, like that of this paper, depended on induction from currents: only one galvanometer was used and the primary current was measured by allowing only a small proportion of it to pass through the galvano-

\footnotetext{
${ }^{2}$ Bestimmung der Constanten von welcher dle Intensität inducirter elektrischer Ströme abhängt. Pogg. Ann., Bd. 76, S. 412.
} 
meter by means of a shunt, while all the induced current passed through it. But, owing to the heating of the wires, the shunt ratio cannot be relied upon as constant, and hence the defect of the method. At present this experiment has only historical value, seeing that no exact record was kept of it in a standard resistance. However, we know that the wire was of copper and the temperature $0^{\circ} \mathrm{R}$. and that the result obtained gave the resistance of the wire $\frac{1}{7}$ smaller than Weber found for the same wire at $50^{\circ} \mathrm{R}$. in 1851 .

In 1851, Weber published experiments by two methods, first by means of an earth inductor, and second by observing the damping of a swinging needle. Three experiments gave for the resistance of the circuit $1903 \cdot 10^{\mathrm{s}}, 1898 \cdot 10^{8}$, and $1900 \cdot 10^{\mathrm{s}}, \frac{\mathrm{mm}}{\mathrm{sec}}$, but it is to be noted that a correction of five-eighths per cent was made on account of the time, two seconds, which it took to turn the earth-inductor, and that no account was taken of the temperature, although the material was copper. He finds for the value of the Jacobi unit, $598 \cdot 10^{7} \frac{\mathrm{mm}}{\mathrm{sec}}$. Three years after that, in 1853, Weber made another determination of the specific resistance of copper." But these determinations were more to develope the method than for exact measurement, and it was not until $1862^{5}$ that Weber made an exact determination which he expected to be standard. In this last determination he used a method compounded of his first two methods by which the constant of the galvanometer was eliminated, and the same method has since been used by Kohlrausch in his experiments of 1870 . The results of these experiments were embodied in a determination of the value of the Siemens unit and of a standard which was sent by Sir Wm. Thomson. As the old Siemens units seem to vary among themselves one or two per cent, and as the result from Thomson's coil differs more than one per cent from that which would be obtained with any known value of the Siemens unit, we cannot be said to know the exact result of these experiments at the present time. Beside which, it was not until the experiments of Dr. Matthiessen on the electric permanence of metals and alloys, that a suitable material could be selected for the standard resistance.

The matter was in this state when a committee was appointed by the

3 Elektrodynamische Maasbestimmangen; or Pogg. Ann., Bd. 82, S. 337.

4 Abh. d. Kön. Ges. d. Wissenchaften zu Göttingen, Bd. 5.

${ }^{3} \mathrm{Zur}$ Galvanometrie, Göttingen, 186:. Also Abb. d. K. Ges. d. Wis. zu Göttingen, Bd. 10 . 
British Association in 1861, who, by their experiments which have extended through eight years, have done so much for the absolute system of electrical measurements. But the actual determination of the unit was made in 1863-4. The method used was that of the revolving coil of Sir William Thomson, the principal advantage of which was its simplicity and the fact that the local variation of the earth's magnetism was entirely eliminated and only entered into the calculation as a small correction. The principle of the method is of extreme beauty, seeing that the same earth's magnetism which causes the needle at the centre of the coil to point in the magnetic meridian also causes the current in the revolving coil which deflects the needle from that meridian. Whenever a conducting body moves in a magnetic field, currents are generated in it in such direction that the total resultant action is such that the lines of force are apparently dragged after the body as though they met with resistance in passing through it: and so we may regard Thomson's method as a means of measuring the amount of this dragging action.

But, however beautiful and apparently simple the method may appear in theory, yet when we come to the details we find many reasons for not expecting the finest results from it. Nearly all these reasons have been stated by Kohlrausch, and I can do barely more in this direction than review his objections, point out the direction in which each would affect the result, and perhaps in some cases estimate the amount.

In the first place, as the needle also induced currents in the coil which tended in turn to deflect the needle, the needle must have a very small magnetic moment in order that this term may be small enough to be treated as a correction. For this reason the magnetic needle was a small steel sphere $8 \mathrm{~mm}$. diameter, and not magnetized to saturation. It is evident that in a quiescent magnetic field such a magnet would give the direction of the lines of force as accurately as the large magnets of Gauss and Weber, weighing many pounds. But the magnetic force due to the revolving coil is intermittent and the needle must show as it were the average force, together with the action due to induced magnetization. Whether the magnet shows the average force acting on it or not, depends upon the constancy of the magnetic axis, and there seems to be no reason to suppose that this would change in the slightest, though it would have been better to have made the form of the magnet such that it would have been impossible. The induced magnetism of the sphere would not affect the result, were it not for the time taken in magnetization: on this account the needle is dragged 
with the coil, and hence makes the deflection greater than it should be, and the absolute value of the $\mathrm{Ohm}$ too small by a very small quantity. The currents induced in the suspended parts also act in the same direction. Neither of these can be estimated, but they are evidently very minute.

The mere fact that this small magnet was attached to a comparatively large mirror which was exposed to air currents could hardly have affected the results, seeing that the disturbances would have been all eliminated except those due to air currents from the revolving coil, and which we are assured did not exist from the fact that no deflection took place when the coil was revolved with the circuit broken. In revolving the coil in opposite directions very different results were obtained, and the explanation of this has caused considerable discussion. As this is of fundamental importance I shall consider it in detail.

The magnet was suspended by a single fibre seven feet long, and the deflection was diminished by its torsion -00132. No mention is made of the method used for untwisting the fibre, and we see that it would require only $2 \cdot 11$ turns to deflect the needle $1^{\circ}$ from the meridian. To estimate the approximate effect of this, we may omit from Maxwell's equation "all the other minor corrections and we have

$$
R=\frac{1}{2} \frac{G K w \cos \varphi}{\sin \varphi+t(\varphi-\beta)}=\frac{G K w}{\frac{1}{2}} \frac{1}{\tan \varphi(1+t)} \frac{1}{\left(1-\frac{\beta t}{\sin \varphi(1+t)}\right)} \text { nearly, }
$$

where we have substituted $\varphi-\beta$ for $\varphi$ in Maxwell's equation in the term involving $t$. In this equation $\varphi$ is measured from the magnetic meridian; but let us take $\psi$ as the angle from the point of equilibrium. Then $\psi^{\prime}=\varphi^{\prime}+\alpha$ and $\psi^{\prime \prime}=\varphi^{\prime \prime}-\alpha$, where $\psi^{\prime}$ and $\varphi^{\prime}$ are for negative: rotation and $\psi^{\prime \prime}$ and $\varphi^{\prime \prime}$ for positive rotation and $\alpha=\arcsin \frac{\beta t}{1+t}$.

Let

$$
C=\frac{2}{G K w} .
$$

Then

$$
\begin{aligned}
& C R^{\prime}=\frac{1}{\tan \psi^{\prime}(1+t)} \\
& C R^{\prime \prime}=\frac{1}{\tan \psi^{\prime \prime}(1+t)} . \\
& R,=\frac{1}{2}\left(R^{\prime}+R^{\prime \prime}\right) .
\end{aligned}
$$

Where $R^{\prime}$ and $R^{\prime \prime}$ are the apparent values of the resistance as calculated from the negative and positive rotations, and $R$, is the mean of the

6 'Reports on Electrical Standards,' p. 103. 
two as taken from the table published by the British Association Committee. If $R$ is the true resistance,

$$
C R=\frac{1}{\tan \varphi^{\prime}(1+t)\left(1+\frac{\sin \alpha}{\sin \varphi^{\prime}}\right)}=\frac{1}{\tan \varphi^{\prime \prime}(1+t)\left(1-\frac{\sin \alpha}{\sin \varphi^{\prime \prime}}\right)} .
$$

We shall then find approximately

$$
R=\frac{1+\tan \psi^{\prime} \tan \alpha}{R^{\prime}\left(1+\frac{\sin \alpha}{\sin \varphi^{\prime}}\right)\left(1-\frac{\tan \alpha}{\tan \psi^{\prime \prime}}\right)}=\frac{1-\tan \psi^{\prime \prime} \tan \alpha}{R^{\prime \prime}\left(1-\frac{\sin \alpha}{\sin \varphi^{\prime \prime}}\right)\left(1+\frac{\tan \alpha}{\tan \psi^{\prime \prime}}\right)} .
$$

When $\boldsymbol{\alpha}$ is small compared with $\psi^{\prime \prime}$ or $\psi^{\prime \prime}$, and when these are also small, we have

$$
R=R \cdot\left(1+u^{2}\left(\alpha^{2}-\frac{1}{2} \psi^{2}\right)+\& c .\right) .
$$

So that by taking the mean of positive and negative rotations, the effect of torsion is almost entirely eliminated. Now $\alpha$ is the angle by which the needle is deflected from the magnetic meridian by the torsion and its value is $\frac{1}{2 \psi}\left(1-\frac{R^{\prime}}{R^{\prime \prime}}\right)$ nearly, when $\alpha$ is small, and this, in one or two of their experiments, exceeds unity or $\alpha$ exceeds $28^{\circ} .6$, which is absurd. Taking even one of the ordinary cases where $\frac{R^{\prime}}{R^{\prime \prime}}=1.02$ and $\psi$ is about $\frac{1}{20}$, we have $a=12^{\circ}$. nearly, which is a value so large that it would surely have been noticed. Hence we may conclude that no reasonable amount of torsion in the silk fibre could have produced the difference in the results from positive and negative rotation, as has been stated by Mr. Fleming Jenkin in his 'Report on the New Unit of Electrical Resistance."

The greatest value which we can possibly assign to $\alpha$ which might have remained unnoticed is $\frac{1}{10}$, which would not have affected the the experiment to any appreciable extent.

Another source of error which may produce the difference we are discussing is connected with the heavy metal frame of the apparatus, in which currents can be induced by the revolving coil. The coil passes so near the frame-work that the currents in it must be quite strong and produce considerable magnetic effect. Kohlrausch has pointed out the existence of these currents, but has failed to consider the theory of them. Now, from the fact that after any number of revolutions the number of lines of force passing through any part of the apparatus is the same as before, we immediately deduce the

? 'Reports on Electrical Standards,' London, 1873, p. 191. 
fact that, if Ohm's law be correct, the algebraical sum of the currents at every point in the frame is zero, and hence the average magnetic action on the needle zero. But although these currents can have no direct action, they can still act by modifying the current in the coil; for while the coil is nearing one of the supports the current in the coil is less than the normal amount, and while it is leaving it is greater; and although the total current in the coil is the normal amount, yet it acts on the needle at a different angle. By changing the direction of rotation, the effect is nearly but not quite eliminated. The amount of the effect is evidently dependent upon the velocity of rotation and increases with it in some unknown proportion, and the residual effect is evidently in the direction of making the action on the needle too small and thus of increasing $R$. If these currents are the cause of the different values of $R$ obtained with positive and negative rotation, we should find that if we picked out those experiments in which this difference was the greatest, they should give a larger value of $R$ than the others. Taking the mean of all the results $^{8}$ in which this difference is greater than one per cent, we find for the Ohm $1.0033 \frac{\text { earth quad. }}{\text { sec. }}$, and when it is less than one per cent, .9966 earth quad., which is in accordance with the theory, the average velocities being $\frac{100}{26}$ and $\frac{100}{43}$ nearly. But the individual observations have too great a probable error for an exact comparison.

But whatever the cause of the effect we are considering, the following method of correction must apply. The experiments show that $R$ is a function of the velocity of rotation, and hence, by Taylor's theorem, the true resistance $R_{0}$ must be

$$
R_{0}=R\left(1+A w+B w^{2}+\& c .\right),
$$

and when $R$ is the mean of results with positive and negative rotations,

$$
R_{0}=R\left(1+B w^{2}+D w^{4}+\& c .\right) .
$$

Supposing that all the terms can be omitted except the first two, and using the above results for large and small velocities, we find $R_{0}$ $=.9926 \frac{\text { earth quad. }}{\text { sec. }}$ But if we reject the two results in which the

${ }^{8}$ In the table published by the Committee the different columns do not agree, and I have thought it probable that the last two numbers in the next to the last column should read 1.0032 and 1.0065 instead of 1.0040 and .9981 , and in my discussion I have considered them to read thus. 
difference of positive and negative rotations is over seven per cent, we find

$$
R_{0}=\cdot 9934 \frac{\text { earth quad }}{\text { sec. }} .
$$

The rejection of all the higher powers of $w$ renders the correction uncertain, but it at least shows that the $\mathrm{Ohm}$ is somewhat smaller than it was meant to be, which agrees with my experiments.

It is to be regretted that the details of these experiments have never been published, and so an exact estimate of their value can never be made. Indeed we have no data for determining the value of the $\mathrm{Ohm}$ from the experiments of 1863. All we know is that, in the final result, the 1864 experiments had five times the weight of those of 1863 , and that the two results differed 16 per cent, but which was the larger is not stated. Now the table of results published in the report of the 1864 experiments contains many errors, some of which we can find out by comparison of the columns. The following corrections seem probable in the eleven experiments: No. 4, second column, read $4.63 \% 5$ for $4 \cdot 6275$. No. 10 , fourth and fifth columns, read 1.0032 and +0.32 in place of 1.0040 and +0.40 . No. 11, fourth and fifth columns, read 1.0065 and +0.65 in place of 0.9981 and $-0 \cdot 19$. Whether we make these corrections or not the mean value is entirely incompatible with the statement with respect to the 1863 experiments. With the corrections the mean value of the 1864 experiments is $1 \mathrm{Ohm}=1.000 \% 1 \frac{\text { earth quad. }}{\text { sec. }}$, and without them, using the fourth column, it is 1.00014 . With the corrections the difference between fast and slow rotation is $\cdot 6$ per cent.

In the year 1870 Professor F. Kohlrausch made a new determination of Siemen's unit in absolute measure, the method being one formed out of a combination of Weber's two methods of the earth inductor and of damping, by which the constant of the galvanometer was eliminated, and is the same as Weber used in his experiments of 1862. His formula for the resistance of the circuit, omitting small corrections, is

$$
w=\frac{32 S^{2} T^{2} t_{0}}{\pi^{2} K} \frac{\left(\lambda-\lambda_{0}\right) A B}{\left(A^{2}+B^{2}\right)^{2}} \text { approximately, }
$$

where $S$ is the surface of the earth inductor, $T$ is the horizontal intensity of the earth's magnetism, $K$ the moment of inertia of the magnet, $t_{0}$ the time of vibration of the magnet, $\lambda$ the logarithmic decrement, and $A$ and $B$ are the arcs in the method of recoil. 
One of the principal criticisms I have to offer with respect to this method is the great number of quantities difficult to observe, which enter the equation as squares, cubes, or even fourth powers. Thus $S^{2}$ depends upon the fourth power of the radius of the earth inductor. Now this earth inductor was wound years before by W. Weber, and the mean radius determined from the length of wire and controlled by measuring the circumference of the layers. Now the wire was nearly $3.2 \mathrm{~mm}$. diameter with its coating, and the outer and inner radii were $115 . \mathrm{mm}$. and $142 \mathrm{~mm}$. Hence the diameter of the wire occupied two per cent of the radius of the coil, making it uncertain to what point the radius should be measured. As the coil is wound, each winding sinks into the space between the two wires beneath, except at one spot where it must pass over the tops of the lower wires. The wire must also be wound in a helix. All these facts tend to diminish $S$ and make its value as deduced from the length of the wire too large; and any kinks or irregularities in the wire tend in the same direction. And these errors must be large in an earth-inductor of such dimensions, where the wire is so large and many layers are piled on each other. If we admit an error of one-half a millimetre in the radius as determined in this way, it would diminish the value of $S^{2} 1 \cdot 4$ per cent, and make Kohlrausch's result only $\cdot 6$ per cent greater than the result of the British Association Committee.

Three other quantities, $T, \lambda$ and $K$, are very hard to determine with accuracy, and yet $T$ enters as a square. It is to be noted that this earth-inductor is the same as that used by Weber in his experiment of 1862, and which also gave a larger value to the $\mathrm{Ohm}$ than those of the British Association Committee. Indeed, the results with this inductor and by this method form the only cases where the absolute resistance of the Ohm has been found greater than that from the experiments of the British Association Committee.

There seems to be a small one-sided error in $A$ and $B$ which Kohlrausch does not mention, but which Weber, in his old experiments of 1851 , considered worthy of $a \cdot 6$ per cent correction, and which would diminish $\frac{A B}{\left(A^{2}+B^{2}\right)^{2}}$ by 1.2 per cent. This is the error due to loss of time in turning the earth-inductor. As Kohlrausch's needle had a longer time of vibration than Weber's, the correction will be much snaller. In Weber's estimate the damping was not taken into account, and indeed it is impossible to do so with exactness. To get some idea of the value of the correction, however, we can assume that the current 
from the earth-inductor is uniform through a time $t^{\prime \prime \prime}$, and the complete solution then depends on the elimination of nine quantities from ten complicated equations, and which can only be accomplished approximately. If $\gamma$ is the true value of the angular velocity, as given to the needle by the earth-inductor, and $\gamma$ is the velocity as deduced from the ordinary equation for the method of recoil, I find

$$
\frac{\gamma}{\gamma_{1}}=1+\frac{1}{24}\left(\pi \frac{t}{T}\right) \frac{\left(1-9\left(\frac{\lambda}{\pi}\right)^{2}\right)\left(1+\varepsilon^{4 \lambda}\right)}{\left(1+\varepsilon^{2 \lambda}\right)^{2}}+\& c .
$$

where $\lambda$ is the logarithmic decrement, $\varepsilon$ the base of the natural system of logarithms, $T$ the time of vibration of the needle, and $t$ the time during which the uniform current from the earth-inductor flows. In the actual case, the current from the earth-inductor is nearly proportional to $\sin t$, and hence it will be more exact to substitute

$$
4\left(\frac{t}{\pi}\right)^{2} \int_{0}^{\frac{1}{t} t} t \sin t d t=4\left(\frac{t}{\pi}\right)^{2}
$$

in the place of $t^{2}$. The formula then becomes

$$
\frac{\gamma}{\gamma_{1}}=1+\frac{1}{6}\left(\frac{t}{T}\right)^{2}\left(1-9\left(\frac{\lambda}{\pi}\right)^{2}\right) \frac{1+\varepsilon^{4 \lambda}}{\left(1+\varepsilon^{2 \lambda}\right)^{2}}+\& c .
$$

This modification is more exact when $\lambda$ is small than when it is large, but it is sufficiently exact in all cases to give some idea of the magnitude of the error to be feared from this source. Kohlrausch does not state how long it took him to turn his earth-inductor, but as $T=34$ seconds, we shall assume $\frac{t}{T}=\frac{1}{10}$ and as $\lambda=\frac{1}{2}$ nearly, we have

$$
\frac{\gamma}{\gamma_{1}}=1.0008 \text {, }
$$

which would diminish the value of the resistance by $\cdot 16$ per cent.

As the time we have allowed for turning the earth-inductor is probably greater than it actually was, the actual correction will be less than this.

The correction for the extra current induced in the inductor and galvanometer, as given by Maxwell's equation,' has been shown by Stoletow to be too small to affect the result appreciably.

We may sum up our criticism of this experiment in a few words. The method is defective because, although absolute resistance has the dimensions of $\frac{\text { space }}{\text { time }}$, yet in this method the fourth power of space and

9 'Electricity and Magnetism,' art. 762. 
the square of time enter, besides other quantities which are difficult to determine. The instruments are defective, because the earth-inductor was of such poor proportion and made of such large wire that its average radius was difficult to determine, and was undoubtedly overestimated.

It seems probable that a paper scale, which expands and contracts with the weather was used. And lastly, the results with this inductor and by this method have twice given greater results than anybody else has ever found, and greater than the known values of the mechanical equivalent of heat would indicate.

The latest experiments on resistance have been made by Lorenz of Copenhagen, ${ }^{10}$ by a new method of his own, or rather by an application of an experiment of Faraday's. It consists in measuring the difference of potential between the centre and edge of a disc in rapid rotation in a field of known magnetic intensity.

A lengthy criticism of this experiment is not needed, seeing that it was made more to illustrate the method than to give a new value to the $\mathrm{Ohm}$. The quantity primarily determined by the experiment was the absolute resistance of mercury, and the $\mathrm{Ohm}$ will have various values according to the different values which we assume for the resistance of mercury in $\mathrm{Ohms}$.

One of the principal defects of the experiment is the large ratio between the radius of the revolving disc and the coil in which it revolved.

In conclusion I give the following table of results, reduced as nearly as possible to the absolute value of the $\mathrm{Ohm}$ in $\frac{\text { earth quad. }}{\text { sec. }}$."

10 Pogg. Ann., Bd. cxlix, (1873), p. 251.

II Since this was written, a new determination has been made by $H$. F. Weber, of Znrich, in which the different results agree with great accuracy. The result has been expressed in Siemen's units, and the comparison seems to have been made simply with a set of resistance coils and not with standards. The modern Siemen's units seem to be reasonably exact, but from the table published by the British Association Committee in 1864 , it seems that at that time there was uncertainty as to its value. He obtains 1 S. U. $=.9550 \frac{\text { earth quad. }}{\text { sec. }}$, which is greater or less than the British Association determination, according as we take the different ratios of the Siemen's to the British Association unit, ranging from 14 per cent above to 1.92 per cent below. In any case the result agrees reasonably well with my own. The apparatus used does not seem to have been of the best, and the exact details are not given. But wooden coils to wind the wire on seem to have been used, which should immediately condemn the experiment where a pair of coils is used, seeing that in that case the constant, both of magnetic effect and of indaction, depend on the dis. tance of the coils. It is unfortunate that sufficient details are not given for me to enter into a criticism of the experiment. 


\begin{tabular}{|c|c|c|c|}
\hline Date. & Observer. & Value of Ohm. & Remarks. \\
\hline 1849 & Kirchhoff & $\cdot 88$ to $\cdot 90$ & Approximately. \\
\hline 1851 & Weber... & .95 to .97 & " \\
\hline 1862 & Weber.. & $\left\{\begin{array}{l}1 \cdot 088 \\
1.075\end{array}\right.$ & $\begin{array}{l}\text { From Thomson's unit. } \\
\text { From Weber's valne of Siemen's unit. }\end{array}$ \\
\hline $1863-4$ & B. A. Committee. & $\left\{\begin{array}{c}1 \cdot 0000 \\
.993\end{array}\right.$ & $\begin{array}{l}\text { Mean of all results. } \\
\text { Corrected to a zero velocity of coil. }\end{array}$ \\
\hline 1870 & Kohlrausch & 1.0196 & \\
\hline 1873 & Lorenz & $\left\{\begin{array}{l}\cdot 970 \\
.980\end{array}\right.$ & $\begin{array}{l}\text { Taking ratio of quicksilver nnit to } \mathrm{Ohm}= \\
.962 \text {. } \\
\text { Taking ratio of quicksilver nnit to } \mathrm{Ohm}= \\
.953 \text {. }\end{array}$ \\
\hline 1876 & Rowland........ & .9912 & $\begin{array}{l}\text { From a preliminary comparison with the } B \text {. } \\
\text { A. unit. }\end{array}$ \\
\hline
\end{tabular}

\section{TheORY OF THE Method}

When a current is induced in a circuit by magnetic action of any kind, Faraday has shown that the induced current is proportional to the number of lines of force cut by the circuit and inversely as the resistance of the circuit. If we have two circuits near each other, the first of which carries a current, and the second is then removed to an infinite distance, there will be a current in it proportional to the number of lines of force cut. Let now a unit current be sent through the second circuit and one of strength $E$ through the first; then, on removing the second circuit, work will be performed which we easily see is also proportional to the number of lines of force cut. Hence, if $E M$ is the work done, $Q$ is the induced current, and $R$ is the resistance of the second circuit,

$$
Q=C E \frac{M}{R},
$$

where $C$ is a constant whose value is unity on the absolute system.

When the current in the first circuit is broken, the lines of force contract on themselves, and the induced current is the same as if the second circuit had been removed to an infinite distance. If the current is reversed the induced clirrent is twice as great; hence in this case

$$
Q=2 E \frac{M}{R} \text { or } R=2 M \frac{E}{Q} .
$$

Hence, to measure the absolute resistance of a circuit on this method, we must calculate $M$ and measure the ratio of $Q$ to $E$. $M$ is known as the mutual potential of the two circuits with unit currents, and mathematical methods are known for its calculation.

The simplest and best form in which the wire can be wound for the 
calculation of $M$ is in parallel circular coils of equal size and of as small sectional area as possible. For measuring $E$ a tangent galvanometer is needed, and we shall then have

$$
\bar{E}=\frac{H}{G} \tan \theta,
$$

where $H$ is the horizontal intensity of the earth's magnetism at the place of the tangent galvanometer, and $G$ the constant of the galvanometer.

For measuring $Q$ we must use the ballistic method, and we have

$$
Q=\frac{H^{\prime}}{G^{\prime}} \frac{T}{\sqrt{\pi^{2}+\lambda^{2}}} E^{\frac{\lambda}{\pi} \tan -1 \frac{\pi}{\lambda}} 2 \sin \frac{1}{2} \theta^{\prime},
$$

which for very small values of $\lambda$ becomes

$$
\begin{gathered}
Q=\frac{H^{\prime}}{G^{\prime}} \frac{T}{\pi} 2 \sin \frac{1}{2} \theta^{\prime}\left(1+\frac{1}{2} \lambda-\frac{1}{6} \lambda^{2}\right), \\
\therefore \quad R=M \frac{H}{H^{\prime}} \frac{G^{\prime}}{G^{\prime}} \frac{\pi \tan \theta}{T^{\prime} \sin \frac{1}{2} \theta^{\prime}} \frac{1}{1+\frac{1}{2} \lambda-\frac{1}{6} \lambda^{2}},
\end{gathered}
$$

where $H^{\prime}$ is the horizontal component of the earth's magnetism at the place of the small galvanometer, $G^{\prime}$ its constant, $T$ the time of vibration of the needle, and $\lambda$ the logarithmic decrement.

The ratio of $H^{\prime}$ to $H$ can be determined by allowing a needle to vibrate in the two positions. But this introduces error, and by the following method we can eliminate both this and the distance of the mirror from the scale by which we find $\theta^{\prime}$ and the error of tangent galvanometer due to length of needle. The method merely consists in placing a circle around the small galvanometer and then taking simultaneous readings with the current passing through it and the tangent galvanometer, before and after each experiment. Let $\alpha$ and $\alpha^{\prime}$ be the deflections of the tangent galvanometer and the other galvanometer respectively, and let $G^{\prime \prime}$ be the constant of the circle at the point where the needle hangs, then

and we have finally

$$
\frac{H}{G} \tan \alpha=\frac{H^{\prime}}{G^{\prime \prime}} \tan \alpha^{\prime},
$$

$$
R=M \frac{\pi}{T} \frac{G}{G^{\prime \prime}} \frac{\tan \alpha^{\prime}}{\tan \alpha} \frac{\tan \theta}{\sin \frac{1}{2} \theta^{\prime}} \frac{1}{1+\frac{1}{2} \lambda-\frac{1}{6} \lambda^{2}},
$$

which does not contain $H$ or $H^{\prime}$, and the distance of the mirror from the scale does not enter except as a correction in the ratio of $\sin \frac{1}{2} \theta$ and $\tan \alpha^{\prime}$; and, as $\alpha$ and $\theta$ can be made nearly equal, the correction 
of the tangent galvanometer for the length of needle is almost eliminated. When the method of recoil is used, we must substitute $\frac{1}{1+\frac{1}{2}\left(\frac{\lambda}{\pi}\right)}$ for the term involving $\lambda$, and $\sin \frac{1}{2} A^{\prime}+\sin \frac{1}{2} B^{\prime}$ in the place of $\sin \frac{1}{2} \theta^{\prime}$ $A^{\prime}$ and $B^{\prime}$ being the greater and smaller ares in that method. This is on the supposition that $\lambda$ is small.

The ratio of $G^{\prime \prime}$ to $G$ must be so large, say 12,000 , that it is difficult to determine it by direct experiment, but it is found readily by measurement or indirect comparison.

It is seen that in this equation the quantities only enter as the first powers, and that the only constants to be determined which enter the equation are $M, G$ and $G^{\prime \prime}$, which all vary in simple proportion to the linear measurement. It is to be noted also that the only quantities which require to be reduced to standard measure are $M$ and $T$, and that the others may all be made on any arbitrary scale. No correction is needed for temperature except to $M$. Indeed, I believe that this method exceeds all others in simplicity and probable accuracy and its freedom from constant errors, secing that every quantity was varied except $G^{\prime \prime}$ and $G$, whose ratio was determined within probably one in three thousand by two methods.

Having obtained the resistance of the circuit by this method, we have next to measure it in ohms. For this purpose the resistance of the circuit was always adjusted until it was equal to a certain German silver standard, which was afterward carefully compared with the ohm. This standard was about thirty-five ohms.

By this method, the following data are needed.

1. Ratio of constants of galvanometer and circle.

2. Ratio of the tangents of the two deflections of tangent galvanometer.

3. Ratio of the deflection to the swing of the other galvanometer.

4. Mutual potential of induction coils on each other.

5. Time of vibration of the needle.

6. Resistance of standard in ohms.

For correction we need the following:

1. The logarithmic decrement.

2. Distance of mirror from scale.

3. Coefficient of torsion of suspending fibre.

4. Rate of chronometer.

5. Correction to reduce to standard metre. 
6. Variation of the resistance of German silver with the temperature.

\%. Temperature of standard resistance.

8. Arc of swing when the time of vibration is determined.

9. Length of needle in tangent and other galvanometer (nearly compensated by the method).

10. The variation of resistance of circuit during the experiment.

The following errors are compensated by the method of experiment.

1. The local and daily variation of the earth's magnetism.

2. The variation of the magnetism of the needle.

3. The magnetic and inductive action of the parts of the apparatus on each other.

4. The correction for length of needle in the tangent galvanometer (nearly).

5. The axial displacement of the wires in the coils for induction.

6. The error due to not having the coils of the galvanometer and the circle parallel to the needle.

\%. Scale error (partly).

8. The zero error of galvanometers.

\section{Calculation of Constaxts}

Circle.-For obtaining the ratio of $G$ to $G^{\prime \prime}$, it is best to calculate them separately and then take their ratio, though it might be found by Maxwell's method ('Electricity' article 753). But as the ratio is great, the heating of the resistances would produce error in this latter method.

For the simple circle,

$$
G^{\prime \prime}=2 \pi \frac{A^{2}}{\left(A^{2}+B^{2}\right)^{\frac{3}{2}}}=\frac{2 \pi}{A}\left(1-\frac{3}{2}\left(\frac{B}{A}\right)^{2}+\& c .\right),
$$

where $A$ is its radius and $B$ the distance of the plane of the circle to the needle on its axis.

Galvanometer for Induction Current.-For the more sensitive galvanometer, we must first assume some form which will produce a nearly uniform field in its interior, without impairing its sensitiveness. If we make the galvanometer of two circular coils of rectangular section whose depth is to its width as 108 to 100 , and whose centres of sections are at a radius apart from each other, we shall have Maxwell's modification of Helmholtz's arrangement. The constant can then be found by calculation or comparison with another coil. 
Maxwell's formulæ are only adapted to coils of small section. Hence we must investigate a new formula. ${ }^{12}$

Let $N$ be the total number of windings in the galvanometer.

Let $R$ and $r$ be the outer and inner radii of the coils.

Let $X$ and $x$ be the distances of the planes of the edges of the coils from the centre.

Let $\alpha$ be the angle subteuded by the radius of any winding at the centre. Let $b$ be the length of the radius vector drawn from the centre to the point where we measure the force.

I et $\theta$ be the angle between this line and the axis.

Let $c$ be the distance from the centre to any winding.

Let $w$ be the potential of the coil at the given point.

Then (Maxwell's 'Electricity,' Art. 695), for one winding,

$$
\begin{aligned}
w=-2 \pi\left\{1-\cos \alpha+\sin ^{2} \alpha\left(\frac{b}{c} Q_{1}^{\prime}(\alpha)\right.\right. & Q_{1}(\theta) \\
& \left.\left.+\frac{1}{2}\left(\frac{b}{c}\right)^{2} Q_{2}^{\prime}(\alpha) Q_{2}(\theta)+\& c .\right)\right\},
\end{aligned}
$$

and for two coils symmetrically placed on each side of the origin,

$$
\begin{aligned}
w=4 \pi\left\{\cos \alpha-\sin ^{2} \alpha\left(\frac{1}{2}\left(\frac{b}{c}\right)^{2} Q_{2}^{\prime}(\alpha) Q_{2}(\theta)\right.\right. & \\
& \left.\left.+\frac{1}{4}\left(\frac{b}{c}\right)^{4} Q_{4}^{\prime}(\alpha) Q_{4}(\theta)+\& c .\right)\right\},
\end{aligned}
$$

where $Q_{2}(\theta), Q_{4}(\theta)$, \&c., denote zonal spherical harmonics, and $Q_{2}^{\prime}(\alpha)$, $Q_{4}^{\prime}(\alpha) \& c$., denote the differential coefficients of spherical harmonics with respect to $\cos \alpha$.

As the needle never makes a large angle with the plane of the coils, it will be sufficient to compute only the axial component of the force, which we shall call $F$. Let us make the first computation without substitution of the limits of integration, and then afterward substitute these:

and we can write

$$
\begin{aligned}
& F=\frac{1}{2} \frac{N}{(R-r)(X-x)} \iint \frac{d w}{d x} d x d r, \\
& F=\frac{1}{2} \frac{N}{(R-r)(X-x)} \int w d r
\end{aligned}
$$

$$
F=\frac{2 \pi N}{(R-r)(X-x)}\left\{H_{0}+H_{2} b^{2} Q_{2}(\theta)+H_{4} b^{4} Q_{4}+\& c .\right\}
$$

12 A formula involving the first two terms of my series, but applying only to the special case of a needle in the centre of a single circle of rectangular section, is given by Weber in his 'Elektrodynamische Maasbestimmungen inbesondere Widerstandsmessungen,' S. 372 . 
where

$$
H_{0}=x \log _{\epsilon}\left(r+\sqrt{\left.x^{2}+r^{2}\right)},\right.
$$

$$
\begin{aligned}
& H_{i}=-\frac{1.3 .5 \ldots(2 i-1) \sin ^{3} \alpha}{i(1.2 .3 \ldots i) x^{i-1}}\left\{A_{,} \frac{\cos ^{2 i-4} \alpha}{2 i-1}+B, \frac{\cos ^{2 i-6} \alpha}{2 i-3}+\& \mathrm{c} .\right\} \\
& A_{1}=i \text {, } \\
& B_{1}=A_{،} \frac{2 i-4}{2 i-1}-\frac{i(i-1)(i-2)}{(2 i-1) 2}, \\
& C_{1}=B_{1} \frac{2 i-6}{2 i-3}+\frac{i(i-1)(i-2) \ldots(i-4)}{(2 i-1)(2 i-3) 2.4}, \\
& D_{1}=C_{1} \frac{2 i-8}{2 i-5}-\frac{i(i-1) \ldots(i-6)}{(2 i-i)(2 i-3)(2 i-5) 2.4 .6}, \\
& E_{1}=\& c ., \& c \text {. }
\end{aligned}
$$

Substituting the limits for $x, r$ and $\alpha$, we find

$$
\begin{gathered}
H_{0}=X \log _{\epsilon} \frac{R+\sqrt{X^{2}+R^{2}}}{r+\sqrt{X^{2}+r^{2}}}-x \log _{\epsilon} \frac{R+\sqrt{x^{2}+R^{2}}}{r+\sqrt{x^{2}+r^{2}}} \\
H_{2}=-\frac{1}{2}\left\{\frac{1}{X}\left(\frac{R^{3}}{\left(R^{2}+X^{2}\right)^{\frac{3}{2}}}-\frac{r^{3}}{\left(r^{2}+X^{2}\right)^{\frac{3}{2}}}-\frac{1}{x}\left(\frac{R^{3}}{\left.R^{2}+x^{2}\right)^{\frac{3}{2}}}-\frac{r^{3}}{\left(r^{2}+x^{2}\right)^{\frac{3}{2}}}\right)\right\}\right. \\
H_{4}=-\frac{1}{24}\left\{\frac{R^{3}}{X^{3}\left(X^{2}+R^{2}\right)^{\frac{3}{2}}}\left(20 X^{4}+7 X^{2} R^{2}+2 R^{4}\right)\right. \\
-\frac{r^{3}}{X^{3}\left(X^{2}+r^{2}\right)^{\frac{3}{2}}}\left(20 X^{4}+7 X^{2} r^{2}+2 r^{4}\right) \\
-\frac{R^{3}}{x^{3}\left(x^{2}+R^{2}\right)^{\frac{3}{2}}}\left(20 x^{4}+7 x^{2} R^{2}+2 R^{4}\right) \\
\left.+\frac{r^{3}}{x^{3}\left(x^{2}+r^{2}\right)^{\frac{3}{2}}}\left(20 x^{4}+7 x^{2} r^{2}+2 r^{4}\right)\right\} .
\end{gathered}
$$

The needle consisted of two parallel laminæ of steel of length, $l$, and a distance, $W$, from each other. As the correction for length is small, we may assume that the magnetism of each lamina is concentrated in two points at a distance $n l$ from each other, where $n$ is a quantity to be determined.

Hence

$$
G=\frac{2 \pi N}{(R-r)(X-x)}\left\{H_{0}+H_{2} \frac{l^{2} n^{2}}{4} Q_{2}\left(\theta^{\prime}\right)+H_{4} \frac{l^{4} n^{4}}{16} Q_{4}\left(\theta^{\prime}\right)+\text { etc. }\right\},
$$

where $\cos \theta^{\prime}=\frac{W}{\sqrt{\left(\frac{1}{2} n l\right)^{2}+W^{2}}}$, seeing that the needle hangs parallel to the coils. In short thick magnets, the polar distance is about $\frac{2}{3} l$ and the value of $n$ will be about $\frac{3}{4}$. For all other magnets it will be between this and unity. In the present case $n=\frac{3}{4}$ nearly.

As all the terms after the first are very minute, this approximation is sufficient, and will at least give us an idea of the amount of this source of error. 


\section{INDUCTION CoILS}

The induction coils were in the shape of two parallel coils of nearly equal size and of nearly square section.

Let $A$ and $a$ be the mean radii of the coils. Let $b$ be the mean distance apart of the coils.

Let

$$
c=\frac{2 \sqrt{A a}}{\sqrt{(A+a)^{2}+b^{2}}} .
$$

Supposing the coils concentrated at their centre of section we know that

$$
M_{0}=4 \pi \sqrt{A a}\left\{\left(\frac{2}{c}-c\right) F(c)-\frac{2}{c} E(c)\right\}
$$

where $F(c)$ and $E(c)$ are elliptic integrals.

If $\zeta$ and $\eta$ are the depth and width of each coil, the total value of $M$ will be, when $A=a$ nearly,

and we find

$$
M=M_{0}+\frac{1}{1^{2}}\left\{\frac{d^{2} M_{0}}{d \bar{A}^{2}} \eta^{2}+\frac{d^{2} M_{0}}{d b^{2}} \iota^{2}\right\}+\text { etc. }
$$

$$
\begin{aligned}
& \frac{d^{2} M_{0}}{d A^{2}}=-\frac{\pi}{A}\left\{\frac{E(c)}{2 A\left(1-c^{2}\right)}\left(2 A c+\frac{4 b^{2} c^{3}}{8 A\left(1-c^{2}\right)}\left(1-3 c^{2}+2 c^{4}\right)\right)\right. \\
& \left.-F(c)\left(c+\frac{b^{2} c^{3}}{4 A^{2}}\right)\right\} \\
& \frac{d^{2} M_{0}}{d b^{2}}=\frac{\pi c}{A\left(1-c^{2}\right)}\left\{F^{\prime}(c)\left(2\left(1-c^{2}\right)-\frac{b^{2} c^{2}}{4 A^{2}}\left(2-c^{2}\right)\right)\right. \\
& -E(c)\left(2-c^{2}-t^{2} c^{2} \frac{1-c^{2}+c^{4}}{2 A^{2}\left(1-c^{2}\right)}\right\} \text {. }
\end{aligned}
$$

\section{CoRRECTIONS}

Calling $\beta$ and $\delta$ the scale deflections corresponding to $\tan \alpha^{\prime}$ and sin $\frac{1}{2} \theta^{\prime}$, we may write our equation for the value of the resistance

$$
R^{\prime}=\frac{K}{T} \frac{\tan \theta}{\tan \alpha} \frac{\beta}{\delta} \frac{1-\frac{1}{4}\left(\frac{\beta}{D}\right)^{2}+\frac{1}{8}\left(\frac{\beta}{D}\right)^{4}}{1-\cdot 35\left(\frac{\delta}{D}\right)^{2}+\cdot 22\left(\frac{\delta}{D}\right)^{4}}(1+A+\text { etc. }),
$$

where $R^{\prime}$ is the resistance of the circuit at a given temperature $17 \cdot 0^{\circ} \mathrm{C}$, and $K=2 \pi M \frac{G}{G^{\prime \prime \prime}}(1+a+b+$ etc.), in which $A, B$, etc. and $a, b$, etc. are the variable and constant corrections respectively.

a. Correction for damping.

$$
a=-\frac{1}{2} \lambda+\frac{5}{12} \lambda^{2} .
$$




\section{b. Torsion of fibre.}

The needle of the tangent galvanometer was sustained on a point and so required no correction. The correction for the torsion in the other galvanometer is the same for $\beta$ and $\delta$ and hence only affects $T$. Therefore, if $t$ is the coefficient of torsion,

c. Rate of chronometer.

$$
b=-\frac{1}{2} t \text {. }
$$

Let $p$ be the number of seconds gained in a day above the normal time

$$
c=-\frac{p}{86400}
$$

d. Reduction to normal metre. The portion of this reduction which depends on temperature must be treated under the variable corrections. Let $m$ be the excess of the metre used above the normal metre, expressed in metres; then

$$
d=+m \text {. }
$$

e. Correction of $T$ for the arc of vibration. This arc was always the same, starting at $c_{1}$ and being reduced by damping to about $c_{n}$,

$$
c=+\frac{1}{128 n \dot{\lambda}}\left(c_{1}^{2}-c_{n}^{2}\right)
$$

where $c_{1}$ and $c_{\mathrm{n}}$ are the total arcs of oscillation.

$f$. Correction for length of needles. For the tangent galvanometer, the correction is variable. For the circle it is

$$
f=+\frac{3}{4}\left(\frac{l}{A}\right)^{2}
$$

where $l$ is half the distance between the poles of the needle and $A$ the radius of circle. For the other galvanometer it is included in the formula for $G$.

A. Reduction to normal metre. As the dimension of $R$ is a velocity and the induction coils were wound on brass, the correction is

$$
A=+r\left(t^{\prime}-t^{\prime \prime}\right) \text {. }
$$

where $\gamma$ is the coefficient of expansion of brass or copper, $t^{\prime}$ the actual and $t^{\prime \prime}$ the normal temperature.

B. Correction of standard resistance for temperature. Let $\mu$ be the variation of the resistance for $1^{\circ} \mathrm{C}$., $t^{\prime \prime \prime}$ be the actual and $t^{\prime \prime}$ the normal temperature $1 \% \cdot{ }^{\circ} 0 \mathrm{C}$; then

$$
B=-\mu\left(t^{\prime \prime \prime}-t^{\mathrm{lv}}\right)
$$


C. Correction for length of needle in tangent galvanometer,

$$
C=+\frac{15}{4} \sin \left(\alpha+\alpha^{\prime}\right)\left(\frac{l^{\prime}}{A^{\prime}}\right)^{2}\left(\alpha^{\prime}-\alpha\right),
$$

where $l^{\prime}$ is half the distance between the poles of the needle and $A^{\prime}$ is the radius of the coil.

D. The resistance of the circuit was constantly adjusted to the standard, but during the time of the experiment the change of temperature of the room altered the resistance slightly; this change was measured and the correction will be plus or minus one-half this. The resistance was adjusted several times during each experiment. The correction is $\pm D$.

Some of the errors which are compensated by the experiment need no remark and I need speak only of the following.

No. 3. By the introduction of commutators at various points all mutual disturbance of instruments could be compensated.

No. 5. In winding wire in a groove, it may be one side or the other of the centre. By winding the coils on the centre of cylinders which set end to end, on reversing them and taking the mean result, this error is avoided.

No. 6. The circle was always adjusted parallel to the coils of the galvanometer. Should they not be parallel to the needle, $G$ and $G^{\prime \prime}$ will be altered in exactly the same ratios and will thus not affect the result. The same may be said of the deflection of the magnet from the magnetic meridian due to torsion.

No. \%. $\beta$ and $\delta$ both ranged over the same portion of the scale and so scale error is partly compensated.

No. 8. The zero-point of all galvanometers was eliminated by equal deflections on opposite sides of the zero-point.

\section{INSTRUMENTS}

Wire and coils.-The wire used in all instruments was quite small silk-covered copper wire, and was always wound in accurately turned ${ }^{13}$ brass grooves in which a single layer of wire just fitted. The separate layers always had the same number of windings, and the wire was wound so carefully that the coils preserved their proper shape through-

${ }^{13} \mathrm{~T}$ 'o obtain an accurate coil an accurate groove is necessary, seeing that otherwise the wire will be heaped up in certain places. The circle of the tangent galvanometer, which was made to order in Germany, had to be returned in this country before use, and much time was lost before finding out the source of the difficulty. 
out. No paper was used between the layers. As the wire was small, very little distortion was produced at the point where one layer had to rise over the tops of the wires below. Corrections were made for the thickness of the steel tape used to measure the circumference of each layer; also for the sinking of each layer into the spaces between the wires below, seeing that the tape measures the circumference of the tops of the wires. The steel tape was then compared with the standard.

The advantages of small wire over large are many; we know exactly where the current passes; it adapts itself readily to the groove without kinks; it fills up the grooves more uniformly; the connecting wires hare less proportional magnetic effect; and lastly, we can get the dimensions more exactly. The size of wire adopted was about No. 22 for most of the instruments.

The mean radius having been computed, the exterior and interior radii are found by addition and substraction of half the depth of the coil. The sides of the coil were taken as those of the brass groove.

All coils were wound by myself personally to insure uniformity and exactness.

Tangent galvanometer.-This was entirely of brass or bronze, and had a circle about $50 \mathrm{~cm}$. diameter. The needle was $2.7 \mathrm{~cm}$. long and its position was read on a circle $20 . \mathrm{cm}$. diameter, graduated to $15^{\prime}$. The graduated circle was raised so that the aluminium pointer was on a level with it, thus avoiding parallax. The needle and pointer only weighed a gram or two, and rested on a point at the centre which was so nicely made that it wonld make several oscillations within $1^{\circ}$ and would come to rest within $1^{\prime}$ or $2^{\prime}$ of the same point every time. I much prefer a point with a light needle carefully made to any suspended needle for the tangent galvanometer, especially as a raised circle can then alone be used. The needle was suspended at a distance from any brass which might have been magnetic. There were a series of coils ascending nearly as the numbers $1,3,9,27,81,243$, whose constants were all known, but only one was used in this experiment. The probable error of a single reading was about $\pm 1^{\prime}$.

Galvanometer for induction current.-This was a galvanometer on a new plan, especially adapted for the absolute measurement of weak currents. It was entirely of brass, except the wooden base, and was large and heavy, weighing twenty or twenty-five pounds. It could be used with a mirror and scale or as a sine galvanometer. It will be 
necessary to describe here only those portions which affect the accuracy of the present experiment.

The coils were of the form described above in the theoretical portion, and were wound on a brass cylinder about $8.2 \mathrm{~cm}$. long and $11.6 \mathrm{~cm}$. diameter in two deep grooves about $3 \cdot \mathrm{cm}$. deep and $2 \cdot 5 \mathrm{~cm}$. wide. The opening in the centre for the needle was about $5.5 \mathrm{~cm}$. diameter and the cylinder was split by a saw-cut so as to diminish the damping effect. This coil was mounted on a brass column rising from a graduated circle by which the azimuth of the coil could be determined by two verniers reading to $30^{\prime \prime}$. Through the opening in the coil beneath the needle passed a brass bar $95 \mathrm{~cm}$. long and $2 \mathrm{~cm}$. broad, carrying a small telescope at one end. In the present experiment, this bar was merely used in the comparison of the constant of the instrument with that of another instrument. For this purpose the instrument is used as a sine galvanometer by which a great range can be secured, and it could be compared with a coil having a constant twenty-three times less and which was used with telescope and scale.

The coils contained about five pounds of No. 22 silk-covered copper wire in 1790 turns.

Two needles were used in this galvanometer, each constructed so that its magnetic axis should be invariable; this was accomplished by affixing two thin laminæ of glass-hard steel, to the two sides of a square piece of wood, with their planes vertical. This made a sort of compound magnet very strong for its length, and with a constant magnetic axis. The first needle had a nearly rectangular mirror 2.4 by $1.8 \mathrm{~cm}$. on the sides and $.22 \mathrm{~cm}$. thick. The other needle had a circular mirror $2.05 \mathrm{~cm}$. diameter and about $1 \mathrm{~mm}$. thick. The needle of the first was $1.27 \mathrm{~cm}$. and of the second $1.20 \mathrm{~cm}$. long, and the pieces of wood were about $\cdot 45 \mathrm{~cm}$. and $\cdot 6 \mathrm{~cm}$. square respectively. The moment of inertia of both was much increased by two small brass weights attached to wires in extension of the magnetic axis, thus extending the needles to a length of $4.9 \mathrm{~cm}$. and $4.2 \mathrm{~cm}$. respectively. The total weights were $5 \cdot 1$ and 5.6 grams and the times of vibration about $7 \cdot 8$ and 11.5 seconds. They were suspended by three single fibres of silk about 43 $\mathrm{cm}$. long.

In front of the needle was a piece of plane-parallel glass. This and the mirrors were made by Steinheil of Munich, and were most perfect in every way.

In the winding of the coils every care was taken, seeing that a small error in so small a coil would produce great relative error. And for 
this reason the constant was also found by comparison with another coil. The following were the dimensions:

$$
\begin{array}{rlrl} 
& \text { Mean radius } 4 \cdot 3212 \mathrm{~cm} . \\
R & =5 \cdot 6212 & r & =3 \cdot 0212 \\
X & =3 \cdot 475565 & x & =.935565 \\
R-r & =2 \cdot 6000 & X-x & =2.54000 \\
N=1790 .
\end{array}
$$

whence

$$
F=1832 \cdot 25-1 \cdot 70 b^{2} Q_{2}(\theta)-4 \cdot 50 b^{4} Q_{4}(\theta)+\cdot 90 b^{6} Q_{6}(\theta)-\& c .
$$

Taking the mean dimensions of the two needles, we have

$$
\begin{aligned}
l & =1 \cdot 23, \quad w=\cdot 52, \quad n=\frac{3}{4}, \quad \cos \theta^{\prime}=\cdot 748 . \\
Q_{2}\left(\theta^{\prime}\right) & =+339, \quad Q_{4}\left(\theta^{\prime}\right)=-\cdot 354, \quad Q_{6}\left(\theta^{\prime}\right)=-\cdot 275 . \\
\therefore \quad G & =1832 \cdot 25-\cdot 083+\cdot 071-\cdot 002+\& \mathrm{c} .=1832 \cdot 24 .
\end{aligned}
$$

The coil with which this galvanometer was compared was the large coil of an electro-dynamometer similar to that described in Maxwell's 'Electricity,' Art. 725, but smaller. The coil was on Helmholtz's principle with a diameter of $27.5 \mathrm{~cm}$., and was very accurately wound on the brass cylinder. There was a total of 240 windings in the coil. The constant of this coil was $78 \cdot 371$ by calculation.

To eliminate the difference of intensity of the earth's magnetism, an observation was first made and then the positions of the instruments were changed so that each occupied exactly the position of the other: the square root of the product of the two results was the true result free from error.

The coils of the galvanometer could be separated so that an outer and inner pair could be used together. By comparing these parts separately and adding the constants together we find $G$. Hence two comparisons are possible, one with the coils together and the other with them separate. The results were for the ratio of the constants

which give

$$
23 \cdot 3931 \text { and } 23 \cdot 4008 \text {, }
$$

The mean result is

$$
G=1833 \cdot 37 \text { and } 1833 \cdot 98 \text {. }
$$

$$
1833 \cdot 67 \pm \cdot 09 \text {, }
$$

and this includes seven determinations with two reversals of instruments. This result is one part in thirteen hundred greater than found by direct calculation, which is to be accounted for by the small size of the galvanometer coils and the consequent difficulty of their accurate measurement. As comparison with the electro-dynamometer has such 
a small probable error, and as it is a much larger coil, it seems best to give this number twice the weight of that found by calculation: we thus obtain

as the final result.

$$
G=1833 \cdot 19
$$

It does not seem probable that this can be in error more than one part in two or three thousand.

Telescope, scate, \&c.-The telescope, mirrors and plane-parallel glass were all from Steinheil in Munich, and left nothing to be desired in this direction, the image of the scale being so perfect that fine scratches on it could be distinguished. The telescope had an aperture of $4 \mathrm{~cm}$. and a magnifying power of 20 was used. The scale was of silvered brass, one metre long and graduated to millimetres.

Induction coils.-A coil was wound in a groove in the centre of each of three accurately turned brass cylinders of different lengths. Two of them only were used at a time, by placing them end to end, the ends being ground so that they laid on each other nicely. The two coils could be placed in four positions with respect to each other, in each of which they were very exactly the same distance apart. This distance for each of the four positions, was determined at three parts of the circumference by means of a cathetometer, with microscopic objective, reading to $\frac{1}{20} \mathrm{~mm}$. The mean of all twelve determinations was the mean distance. In using the coils they were always used in all four positions. The probable error of each set of twelve readings was $\pm .001 \mathrm{~mm}$. The data are as follows, naming the coils, $A, B$ and $C$ :

Mean radius of $A=13 \cdot 710$, of $B=13 \cdot 690$, of $C=13 \cdot 720$.

Mean distance apart of $A$ and $B=6.534$, of $A$ and $C=9 \cdot 574$, of $B$ and $C=11 \cdot 471$.

$N=154$ for each coil, $\xi=\cdot 90, \eta=\cdot 84$.

For $A$ and $B$ we have

$$
M=3774860 \cdot+\frac{1}{12}(74250 \cdot-66510 \cdot)=3775500 \cdot
$$

The remaining terms of the series are practically zero, as was found by dividing one of the coils into parts and calculating the parts separately and adding them.

For $A$ and $C$

For $B$ and $C$

$$
M=2561410 \cdot+\frac{1}{12}(34000 \cdot-27230 \cdot)=2561974 .
$$

$$
M=2050600 \cdot+\frac{1}{12}\left(27500 \cdot-19800^{\circ}\right)=2051320 .
$$

The calculation of the elliptic integrals was made by aid of the tables of the Jacobi function, $q$, given in Bertrand's 'Traité de Calcul Inte- 
grale' as well as by the expansions in terms of the modulus after transforming them by the Landen substitution.

The Circle.-The circle whose constant we have called $G^{\prime \prime}$ and which was around the galvanometer whose constant was $G$, was a large wooden one containing a single coil of No. 22 wire. $^{14}$ To prevent warping, it was laid up out of small pieces of wood with the grain in the direction of the circumference, and was carefully turned with a minute groove near one edge in which the wire could just lie. It was about $5 . \mathrm{cm}$. broad, 1.8 thick and $82.7 \mathrm{~cm}$. diameter. As the room had no fire in it, the circle remained perfect throughout the experiment. The wire was straightened by stretching and measured before placing on the circle, which last was done with great care to prevent stretching; after the experiment it was measured and found exact to $\frac{1}{10} \mathrm{~mm}$.

The circle was adjusted parallel and concentric with the coils of the galvanometer, but at a distance of $1.1 \mathrm{~cm}$. to one side, in order to allow the glass tube with the suspending fibre to pass. The length of wire was $259.58 \mathrm{~cm}$. which gives a mean radius of $41.31344 \mathrm{~cm}$. These data give $G^{\prime \prime}=\cdot 151925$. Preliminary results were also obtained by use of another circle.

Chronometer.-To obtain the time of vibration, a marine chronometer giving mean solar time was used. The rate was only half a second per day.

Wheatstone bridge.-To compare the resistance of the circuit with the arbitrary German silver standard, a bridge on Jenkin's plan, made by Elliott of London, was used. A Thomson galvanometer with a single battery cell gave the means of accurately adjusting the resistance, one division of the scale representing one part in fifty thousand.

- Thermometers.-Accurate thermometers graduated to half degrees were used for finding the temperature of the standard.

The arbitrary standard. - This was made of about seventy feet of German silver wire, mounted in the same way as the British Association Standard. Immediately after usc, two copies, one in German silver and the other in platinum-silver alloy, were made. It had a resistance of about 35 ohms. The temperature was taken as $17^{\circ} \mathrm{C}$.

To obtain the accurate resistance of this standard in ohms, I had two standards of $10 \mathrm{ohms}$ and one of 1,100 , and 1,000 ohms. The $1-\mathrm{ohm}$, and one of the 10-ohm standards, were made by Elliott of London, and

\footnotetext{
${ }^{14}$ In another part of my paper I have criticlsed the use of wooden circles for coil, but it is unobjectionable in the case of a single wire, especially when the needle is suspended near its centre.
} 
the others by Messrs. Warden, Muirhead and Clark of the same place. But on careful comparison I found that Warden, Muirhead and Clark's 10-ohm standard was 1.00171 times that of Messrs. Elliott Bros. On stating these facts to the two firms I met no response from the first firm, but the second kindly undertook to make me a standard which should be true by the standards in charge of Professor Maxwell at Cambridge. ${ }^{16}$ At present I give the result of the comparison with these standards, as well as some others, and also with a set of resistance coils by Messrs. Elliott Bros.

Commutators.- No commutators except those having mercury connections were used, and those in the circuit whose resistance was determined were so constructed as to offer no appreciable resistance. The commutator by which the main current was reversed, could be operated in a fraction of a second, so as to cause no delay in the reversal.

Connecting wires.-These were of No. 22 or No. 16 wire and were all carefully twisted together. The insulation was tested and found to be excellent.

Inductor for damping.-This has already been described in my first paper on 'Magnetic Permeability,' and merely consisted of a small horse-shoe magnet with a sliding coil, which was introduced into the secondary circuit. By moving it back and forth, the induced current could be used to stop the vibrations of the needle and make it stationary at the zero point. This is necessary in the method where the first throw of the galvanometer needle constitutes the observation, but in the method of recoil it is not necessary to use it very often. I prefer the method of the first throw as a general rule, but I have used both methods.

This method of damping will be found much more efficient than that of the damping magnet as taught by Weber, and after practice a single movement will often bring the needle exactly to rest at the zero point.

Arrangement of apparatus.- Two rooms on the ground floor of a small building near the University were set aside for the experiment, making a space $8 \mathrm{~m}$. long by $3.7 \mathrm{~m}$. wide. The plan of the arrangement is seen at Fig. 1. The current from the battery, in the University, entered at $A$, the battery being eighteen one-gallon cells of a chromate battery, arranged two abreast and eight for tension. The

${ }^{15}$ As this is nearly a year since, and as I cannot tell when the standard will arrive, I now publish the results as so far obtained, hoping to make a more exact comparison in future. 
resistance of the circuit was about $20 \mathrm{ohms}$, and of the whole battery about $\frac{1}{2} \mathrm{ohm}$, thus insuring a reasonably constant current.

At $B$ some resistance could be inserted by withdrawing plugs so as to vary the current.

At $C$ is the tangent galvanometer with commutator on a brick pier. The nearness of the commutator produces no error, seeing that we only wish to determine the ratio of two currents. The effect of currents in the commutator was, however, vanishingly small in any case.

At $D$ is the principal commutator which reversed the current in the induction coils, $L$, or in the circle, $F$, when it was in the circuit.

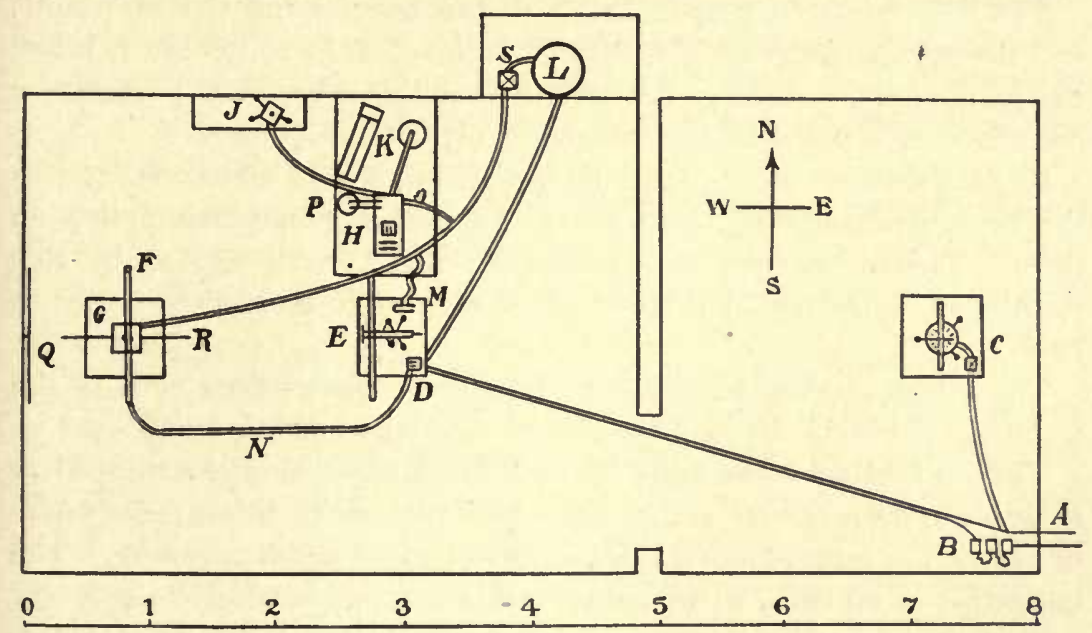

Fig. 1.

The secondary circuit included the induction coil, $L$, the damping inductor, $M$, and the galvanometer $G$.

At $H$ was the Jenkin's bridge, with standard at $P$, in a beaker of water, and a Thomson galvanometer at $J K$. The secondary circuit could be joined to the bridge by raising a $U$-shaped piece of wire out of the mercury cups.

The telescope and scale, $E$, were on a heavy wooden table, and the two galvanometers on brick piers with marble tops.

A row of gas-burners at $Q$ illuminated the silvered scale in the most perfect manner.

Adjustments and tests. - The circle, $F$, must be parallel to coils of galvanometer, $G$. The circle and coils of galvanometer were first adjusted with their planes vertical and then adjusted in azimuth by 
measurement from the end of the bar, $R$, to the sides of the circle, $F$. The adjustment was always within $30^{\prime}$, which would only cause an error of one part in 25000 .

The needle must hang in the magnetic meridian by a fibre without torsion, and the coils must be parallel to it. These adjustments were carefully made, but, as has been shown, the error from this source is compensated.

The needle must hang in the centre of the galvanometer coils and on the axis of the circle. The error from this source is vanishingly small.

The scale must be perpendicular to the line joining the zero point. and the galvanometer needle, it must be level and not too much below the galvanometer needle. All errors from this source are partially or entirely compensated by the method of experiment.

The induction coils, $L$, must be horizontal, and at the same level as the two galvanometers, so as not to produce any magnetic action on them. The error from this source is exactly compensated by this method of experiment, but could never amount to more than 1 part in 2000.

The tangent galvanometer should have the plane of its coils in the magnetic meridian, but all errors are compensated.

The connecting wires must be so twisted together and arranged as to produce no magnetic action, but tests were made in all cases where the error was not compensated, and found to be practically zero. The insulation of all coils, wires and commutators was carefully tested.

Method of experiment.-As has been stated before, the method generally used was that of the first throw of the needle, though the method of recoil was also used. For the successful use of the first method a quickly vibrating needle and the damping inductor are indispensable, seeing that with a slow moving needle we can never be certain of its being at rest. By this method it is not necessary to have the needle at rest at the zero point, but, if it vibrates in an arc of only a millimetre or two, we have only to wait till it comes to rest at its point of greatest elongation on either side of the zero point and then reverse the commutator. The error by this method is in the direction of making the throw greater in proportion of the cosine of the phase to unity. The smallest throw used was $100 \mathrm{~mm}$. Hence, if the needle vibrated through a total arc of $2 \mathrm{~mm}$., the error would be 1 in 17,000 . In reality the needle was always brought to rest much more nearly than this.

The method of recoil was used once with the needle vibrating in $\% \cdot 8$ 
seconds, but the time of vibration was too short and another needle was constructed vibrating in 11.5 seconds, which was a sufficiently long period to be used successfully after practice.

There seems to be no error introduced by the time taken to reverse the commutator in the method of recoil, seeing that the breaking of the current stops the needle and the making starts it in the opposite direction. As the time was only a fraction of a second the error is minute in any case.

While the current is broken in the reversal, the battery may recuperate a little and there is also some action from the extra current, but there seems to be no doubt that long before the four or six seconds which the needle takes to reach its greatest elongation everything has again settled to its normal condition and the current resumes its original strength. Hence the error from these sources may be considered as vanishingly small.

Some experiments were made by simply breaking the current and they gave the same result as by reversal.

The following is the order of observations corresponding to each experiment.

1st. The time of vibration of needle was observed.

$2 \mathrm{~d}$. The current was passed around the circle, $F$, so as to observe $\beta$ and $\alpha$. Simultaneous readings were taken at the two galvanometers. The commutator at the tangent galvanometer was then reversed and readings again taken. After that the commutator to the circle was reversed and the operation repeated. This gave four readings for the circle and eight for the tangent galvanometer, as both ends of the needle were read. In some cases these were increased to six and twelve respectively. This operation was repeated three times with currents of different strengths, constituting three observations each of $\alpha$ and $\beta$. To eliminate any action due to the induction coils, they were sometimes connected in one way and sometimes in the opposite way.

$3 \mathrm{~d}$. The resistance of the circuit was adjusted equal to the arbitrary standard.

4th. The circle, $F$, was thrown out of the circuit and the observations of $\theta$ and $\delta$ begun. Two throws, $\delta$, one on either side of zero were observed and one reading of $\theta$ taken. The commutators at $s$ and $C$ were then reversed, and the operation repeated. This whole operation was then repeated with currents of three different strengths. The position of the two induction coils was now reversed and observations again made with the three currents. The resistance was now com- 
pared with the standard, the difference noted, and the resistance again adjusted. The observations were completed by turning the induction coils into the two other positions which they could occupy with respect to each other, followed by another comparison of resistance with standard.

5th. Observations of $\alpha$ and $\beta$ were again made as before.

6 th. The time of vibration was again determined.

The observations as here explained furnished data for three computations of the resistance of the circuit, one with each of the three currents. In each of these three computations, $\alpha$ was the mean of 16 readings, $\beta$ of 8 or sometimes $12, \theta$ of 16 and $\delta$ of 16 . In using the method of recoil nearly the same order was observed.

The time of vibration was determined by allowing the needle to vibrate for about ten seconds and making ten observations of transits before and after that period. During the experiment, I usually observed at the telescope and Mr. Jacques at the tangent galvanometer.

The methods of obtaining the corrections require no explanation.

\section{RESULTS}

The constant corrections are as follows for the first needle.

$$
\begin{aligned}
& a=-\frac{1}{2} \lambda+\frac{5}{12} \lambda^{2}=-\cdot 00711 . \\
& b=-\frac{1}{2} t=-00020 \\
& c=-\cdot 000006, \\
& d=+\cdot 000074 \text { at } 20^{\circ} \mathrm{C} . \\
& e=\frac{1}{128_{n} \lambda}\left(c_{1}^{2}-c_{n}^{2}\right)=+\cdot 00003, \\
& f=+\cdot 00003, \\
& a+b+c+d+e+f=-\cdot 00718 .
\end{aligned}
$$

For method of recoil it becomes - $\cdot 00016$.

Hence for $A$ and $B, \log K=11 \cdot 4536030$

Hence for $A$ and $C, \log K=11 \cdot 2852033$

Hence for $B$ and $C, \log K=11 \cdot 1886619$

For method of recoil using $A$ and $B, \log K=11.4566630$.

For second needle and method of recoil,

$$
\begin{aligned}
& a=-\frac{1}{2}\left(\frac{\lambda}{\pi}\right)^{2}=-\cdot 000050, \\
& b=-\frac{1}{2} t=-\cdot 00025, \\
& c=-000006 \\
& d=+\cdot 000074
\end{aligned}
$$




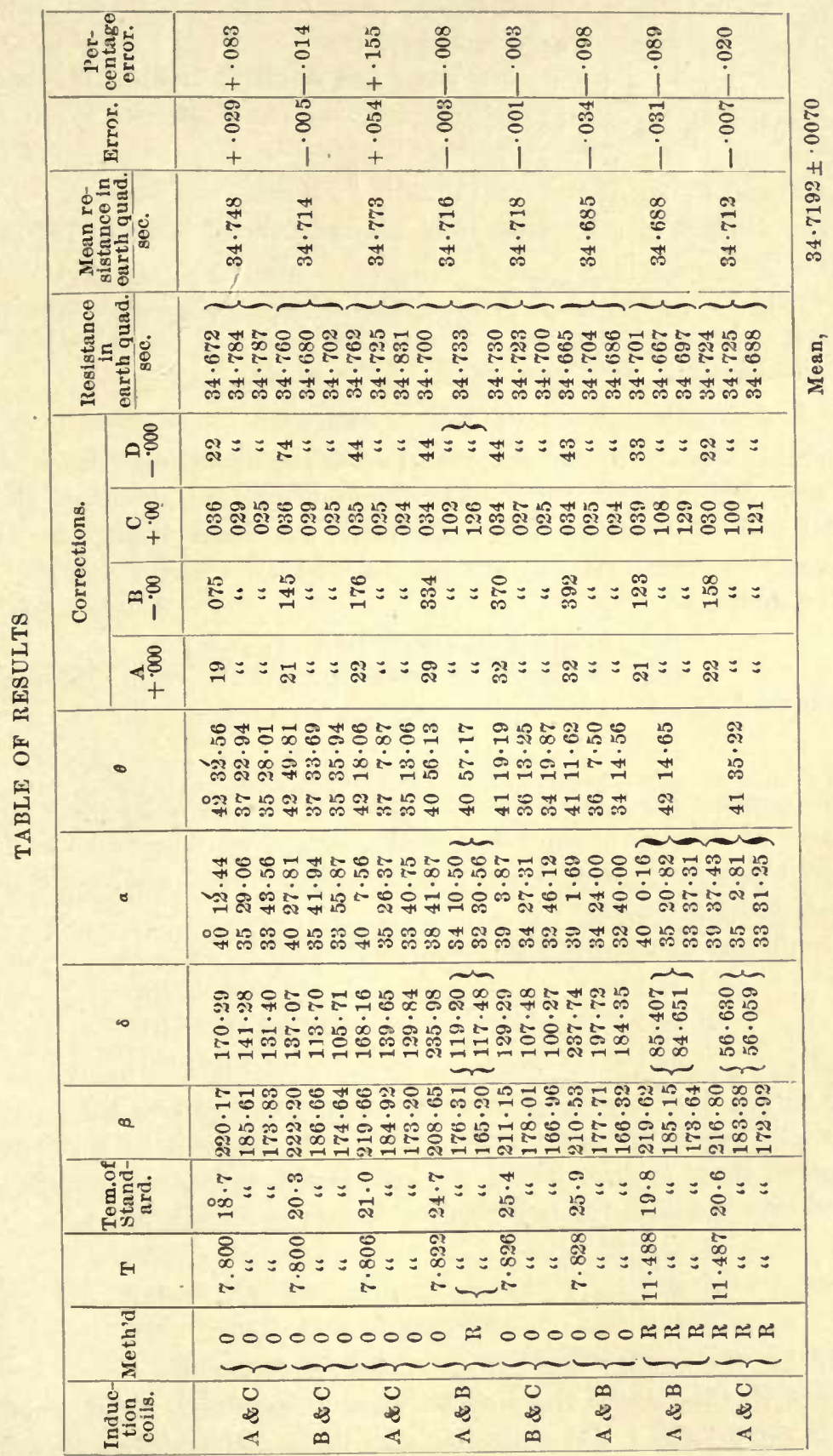




$$
\begin{gathered}
e=+\cdot 00003 \\
f=+\cdot 00003 \\
a+b+c+d+e+f=-\cdot 00017
\end{gathered}
$$

For $A$ and $B, \log K=11.4566587$

For $A$ and $C, \log K=11.2882590$

For $B$ and $C, \log K=11 \cdot 1917176$

The distance of the mirror from the scale varied between 192.3 and $193.5 \mathrm{~cm}$.

Should we reject the quantity $34 \cdot 831$ in the third experiment so as to make the mean result of that experiment $34 \cdot 744$ instead of $34 \cdot 773$, we should obtain as a mean result of the whole

$$
34 \cdot 7156 \pm \cdot 0053
$$

which has a less probable error than when the above observation is retained. The number of plus and minus errors are also more nearly equal and the greatest difference from the mean 1 part in 1100. However the two results do not differ more than 1 part in 10,000 .

We shall take

$$
R=34 \cdot \% 19 \pm \cdot 00 \% \frac{\text { earth quad. }}{\text { second. }} 1 \% \cdot{ }^{\circ} \mathrm{C} .
$$

as the final result.

\section{Discussion}

On glancing over the table we see that the number of negative errors greatly exceed the number of positive, but, if we take only the four errors which are greater than 1 part in 5,000, we shall find two of them negative and two positive.

Combining the results with the different coils we have

$$
\begin{aligned}
& A \text { and } B \ldots \ldots \ldots \ldots \ldots . . .634 \cdot 696 \pm \cdot 005
\end{aligned}
$$

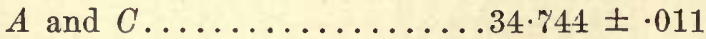

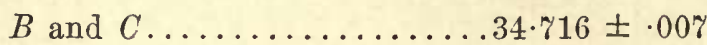

Had we no other results to go by, we might suppose that the value of $M$ might not have been found as exactly for these coils as we have supposed them to be. But if we include the preliminary results rejected on account of the imperfect circle used, we shall find

$$
\begin{aligned}
& A \text { and } B \ldots \ldots \ldots \ldots \ldots \ldots \ldots . .34 \cdot 704 \pm .006
\end{aligned}
$$

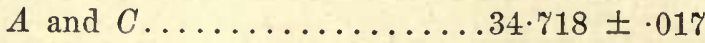

$$
\begin{aligned}
& B \text { and } C \ldots \ldots \ldots \ldots \ldots \ldots \ldots+34 \cdot 758 \pm \cdot 016
\end{aligned}
$$

which has the greatest error in an entirely different place.

From the first series the probable error of each determination of $M$ is 1 in about 2,000. But as this includes the experimental errors which 
are about equal to $\pm_{\frac{1}{400}}$, the real probable error of $M$ must be about 1 part in 2,500. The number of observations is however too small for an exact estimate of the probable errors.

Taking the results with currents of different strengths, we find

For strongest current ................34.716

For medium current .................. $34 \cdot 715$

For weakest current ...............34.727

which are almost perfectly accordant. Taking the results from the method of recoil and the ordinary method, we find

For ordinary method $\ldots \ldots \ldots \ldots \ldots .34 \cdot 726 \pm .010$

For method of recoil ...........34.705 $\pm \cdot 006$

If the probable error is subtracted from the first and added to the second they will very nearly equal each other. Hence the difference is probably accidental. Indeed, by the combination of the results it does not seem possible to find any constant source of error, and therefore the errors should be eliminated by the combination of the results.

In the final result

$$
R=34 \cdot 7192 \pm \cdot 00 \% 0
$$

the probable error, $\pm \cdot 00 \%$, includes all errors except the ratio of $G$ to $G^{\prime \prime}$. We may estimate the probable error of $G$ at $\pm \frac{1}{3000}$ and of $G^{\prime \prime}$ at $\pm \frac{1}{5000}$.

Hence the final probable error of $R$, including all variables, is $\pm \frac{1}{2300}$ or $\pm \cdot 04$ per cent,

$$
\text { or } R=34 \cdot 719 \pm \cdot 015 \text {. }
$$

The probable error of the British Association determination was $\pm \cdot 08$ per cent, not including the probable error of the constants; and of Kohlrausch's determination $\pm \cdot 33$ per cent, including constant errors.

\section{Comparison with the OHM}

The difficulty in obtaining proper standards for comparison has been explained above and I shall have to wait until the arrival of the new standard before making the exact comparison. At present I give the following results, which seem to warrant the rejection of Messrs. Elliott Bros'. 10-ohm standard and to make that of Messrs. Warden, Muirhead and Clark correct. I shall designate the coils by the letter of the firm and by the number of ohms. Experiment gave the following results:

$\mathrm{W}(10)=1.00171 \times \mathrm{E}(10)$, experiment of June 8, $187 \%$.

$W(10)=1.00166 \times \mathrm{E}(10)$, experiment of Feb. 23, 1878.

W $(1,000): W(100):: W(10): \cdot 999876$ E (1), experiment of February $23,1878$.

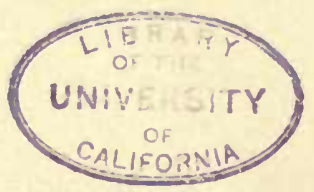


Now the greatest source of error in making coils is in passing from the unit to the higher numbers. As the reproduction of single units is a very simple process the single ohm is without much doubt correct, and as the above proportion is correct within one part in 8,000 of what it should be, it seems to point to the great exactness of the standards then used, seeing that the exactness of the proportion could hardly have been accidental. It is also to be noted that Messrs. Warden, Muirhead \& Clark's 10 -ohm standard agreed more exactly with a set of coils by Messrs. Elliott Bros. than their own unit E (10).

The resistance of my coil as derived from the different standards is as follows:

From Elliott Bros. resistance coils............ $34 \cdot 979$ ohıns.

From Elliott Bros. 10-ohm standard............ $35.083 \mathrm{ghms.}$

From W., M. \& C.'s 10-ohm standard .........35.024 ohms.

From W., M. \& C.'s 100-ohm standard ..........35.035 ohms. These give for my determination the values of the ohm as follows:

From Elliott Bros. resistance coils ........99257 $\frac{\text { earth quad. }}{\text { sec. }}$

From Elliott Bros. 10-ohm standard.........98963 "

From W., M. \& C.'s 10-ohm standard .......99129 “

From W., M. \& C.'s 100-ohm standard.......999098 “ For the reasons given above I accept the mean of the last two results as the value of the ohm.

To preserve my standard I have made two extra copies of it, the one in German silver and the other in platinum silver alloy. The comparisons are given below. No. 1 is in German silver and the other in platinum silver alloy. The temperature is $1 \% \cdot{ }^{\circ} \mathrm{C}$.

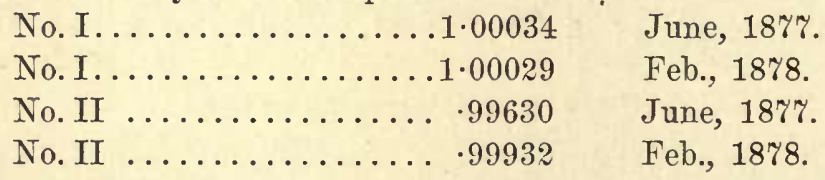

These are the values of the copies in terms of the original standard whose resistance is $34 \cdot 719 \frac{\text { earth quad. }}{\text { sec. }}$.

From these results it would seem that the German silver of which the standard and No. I were composed was perfectly constant in resistance. The wire has been in my possession for several years and seems to have reached its constant state.

The final result of the experiment is

$$
1 \mathrm{ohm}=.9911 \frac{\text { earth quad. }}{\text { sec. }}
$$


ON PROFESSORS AYRTON AND PERRY'S NEW THEORY OF THE EARTH'S MAGNETISM, WITH A NOTE ON A NEW THEORY OF THE AURORA ${ }^{1}$

\section{[Philosophical Magazine, [5], VIII, 102-106, 1879. Proceedings of the Physical Society, III, 93-98, 18\%9]}

Some years ago, while in Berlin, I proved by direct experiment that electric convection produced magnetic action; and I then suggested to Professor Helmholtz that a theory of the earth's magnetism might be based upon the experiment. But upon calculating the potential of the earth required to produce the effect, I found that it was entirely too great to exist without producing violent perturbations in the planetary movements, and other violent actions.

I have lately read Professors Ayrton and Perry's publication of the same theory; and as they seem to have arrived at a result for the potential much less than I did, I have thought it worth while to publish my reasons for the rejection of the theory.

The first objection to the theory that struck me was, that not only the relative motion but also the absolute motion through space of the earth around the sun might also produce action. And to this end I instituted an experiment as soon as I came home from Berlin.

I made a condenser of two parallel plates with a magnetic needle enclosed in a minute metal box between them; for I reasoned that, when the plates were charged and were moved forward by the motion of the carth around the sun, they would then act in opposite directions on the enclosed needle, and so cause a deflection when the electrification of the condenser was reversed. On trying the experiment in the most careful manner, there was not the slightest trace of action after all sources of error had been eliminated.

But the experiment did not satisfy me, as I saw there was some electricity on the metal case surrounding the needle. And so I attacked the problem analytically, and arrived at the curious result that if an electrified system moves forward without rotation through space, the

\footnotetext{
${ }^{1}$ Read before the Physical Society, June 29th.
} 
magnetic force at any point is dependent on the electrical force at that same point-or, in other words, that all the equipotential surfaces have the same magnetic action. Hence, when we shield a needle from electrostatic action, we also shield it from magnetic action.

This theorem only applies to irrotational motion, and assumes that the elementary law for the magnetic action of electric convection is the same as the most simple elementary law for closed circuits. Hence we see that, provided the earth were uniformly electrified on the exterior of the atmosphere, there would be no magnetic action on the earth's surface due to mere motion of translation through space.

In calculating the magnetic action due to the rotation, I have taken the most favorable case, and so have assumed the earth to be a sphere of magnetic material of great permeability, $\mu$. It does not seem probable that it would make much difference whether the inside sphere rotated or was stationary; or at least the magnetic action would be greatest in the latter case; and hence by considering it stationary we should get the superior limit to the amount of magnetism.

Let $a$ be the radius of the sphere moving with angular velocity $w$, and let $\sigma$ be its surface-density in electrostatic measure, and $n$ the ratio of the electromagnetic to the electrostatic unit of electricity. Then the current-function will be

$$
\varphi=\frac{\sigma}{n} w a^{2} \int \sin \theta d \theta=-\frac{\sigma}{n} w a^{2} \cos \theta .
$$

Hence (Maxwell's 'Treatise,' $\S 672$ ) the magnetic potential inside the sphere is

$$
\Omega=-\frac{8 \pi}{3} \frac{\sigma}{n} w a r \cos \theta,
$$

and outside the sphere

$$
\Omega^{\prime}=\frac{4}{3} \pi \frac{\sigma}{n} w a^{4} \frac{\cos \theta}{r^{2}} .
$$

The magnetic force in the interior of the sphere is thus

$$
F=\frac{8}{8} \pi \frac{\sigma}{n} w a,
$$

or the field is uniform. If the electric potential of the sphere on the electrostatic system is $\nabla$, we may write

$$
F=\frac{2}{3} \frac{w}{n} V,
$$

which is independent of the dimensions of the sphere. 
In this uniform field in the interior of the sphere, let a smaller sphere of radius $a^{\prime}$ be situated; the potential of its induced magnetization will be

$$
\Omega_{1}=\frac{\mu-1}{\mu+2} a^{\prime 3} F \frac{\cos \theta}{r^{2}} .
$$

Hence the expression for the potential for the space between the two spheres will be

$$
U=\frac{2}{3} \frac{w}{n} V\left\{-r \cos \theta+\frac{\mu-1}{\mu+2} a^{\prime 3} \frac{\cos \theta}{r^{2}}\right\} ;
$$

and outside the electrified sphere it will be

$$
U^{\prime}=\frac{w}{n} V\left(a^{3}+2 a^{\prime 3} \frac{\mu-1}{\mu+2}\right) \frac{\cos \theta}{r^{2}} .
$$

Let us now take the most favorable case for the production of magnetism that we can conceive, making $\alpha^{\prime}=a$ and $\mu=\infty$; we then have

$$
U^{\prime}=\frac{w}{n} V a^{3} \frac{\cos \theta}{r^{2}},
$$

which is the potential of an elementary magnet of magnetic moment

$$
\frac{w}{n} V a^{3} \text {. }
$$

But Gauss ${ }^{2}$ has estimated the magnetic moment of the earth to be

$$
3 \cdot 3092 a^{3} \text {. }
$$

on the millimetre mg. second system. Hence we have

$$
V=3.3092 \frac{n}{w}
$$

for the potential in electrostatic units on the mm. mg. second system. In electromagnetic units it is thus

$$
V_{1}=3 \cdot 3092 \frac{n^{2}}{w} ;
$$

and hence in volts it is this quantity divided by $10^{11}$.

As the earth makes one revolution in $23^{\mathrm{h}} 56^{\prime} 4^{\prime \prime}$, or in 86164 seconds, we have

$$
w=\frac{2 \pi}{86164}
$$

and

$$
n=299,000,000,000^{3} \text { millims. per second. }
$$

2 Taylor's Scient. Mem., vol. ii, p. 225.

${ }^{3}$ From a preliminary calculation of a new determination made with the greatest care, and having a probable error of 1 in 1300 . 
Hence the earth must be electrified to a potential of about

$$
41 \times 10^{15} \text { volts }
$$

in order, under the most favorable circumstances, to account for the earth's magnetism. This would be sufficient to produce a spark in atmospheric air of ordinary density of about

$$
6,000,000 \text { miles! }
$$

Professors Ayrton and Perry have only found the potential $10^{8}$ volts, or $400,000,000$ times less than I find it.

It was this large quantity which caused me to reject the theory; for I saw what an immense effect it would have in planetary perturbations; and $I$ even imagined to myself the atmosphere flying away, and the lighter bodies on the earth carried away into space by the repulsion. And, doubtless, had not Professors Ayrton and Perry made some mistake in their calculation by which the force was diminished $16 \times 10^{16}$ times, they would have feared like results.

For according to Thomson's formula, the force would be equal to a pressure outwards of

which amounts to no less than

$$
r=\frac{V^{2}}{8 \pi a^{2}}
$$

$$
1,800,000 \text { grms. }
$$

per square centimetre! or 10,000 kil. per square inch! Such an electrostatic force as this would undoubtedly tear the earth to pieces, and distribute its fragments to the uttermost parts of the universe. If the moon were electrified to a like potential, the force of repulsion would be greater than the gravitation attraction to the earth, and it would fly off through space.

For these reasons I rejected the theory, and now believe that the magnetism of the earth still remains, as before, one of the great mysteries of the universe, toward the solution of which we have not yet made the most distant approach.

${ }^{4}$ That this is not too great may be estimated from my Berlin experiment, where a disk moving 5,000,000 times as fast as the earth with a potential of 10,000 volts, prodnced a magnetic force of $5 \frac{1}{500}$ of the earth's magnetism,

$$
5,000,000 \times 10,000 \times 50,000=2,500,000,000,000,000,
$$

which is of the same order of magnitude as the quantity calculated, namely $61 \times$ 1015. It can be seen that this reasoning is correct, because the formulre show that two spheres of unequal size, rotating with equal angular velocity and charged to the same potential, produce the same magnetic force at similar points in the two systems. 
In connection with the theory of the earth's magnetism, I had also framed a theory of the Aurora which may still hold. It is that the earth is electrified, and naturally that the electricity resides for the most part on the exterior of the atmosphere-and that the air-currents thus carry the electricity toward the poles, where the air descending leaves it-and that the condensation so produced is finally relieved by discharge.

The total effect would thus be to cause a difference of potential between the earth and the upper regions of the air both at the poles and the equator. At the poles the discharge of the aurora takes place in the dry atmosphere. At the equator the electrostatic attraction of the earth for the upper atmospheric layers causes the atmosphere to be in unstable equilibrium. At some spot of least resistance the upper atmosphere rushes toward the earth, moisture is condensed, and a conductor thus formed on which electricity can collect; and so the whole forms a conducting system by which the electric potential of the upper air and the earth become more nearly equal. This is the phenomenon known as the thunderstorm.

Hence, were the earth electrified, the electricity would be carried to the higher latitudes by convection, would there discharge to the earth as an aurora, and passing back to the equator would get to the upper regions as a lightning discharge, once more to go on its unending cycle. I leave the details of this theory to the future.

Baltimore, May 30, 1879.

Appendix.-Since writing the above, Professors Ayrton and Perry's paper has appeared in full; and I am thus able to point out their error more exactly. Their formula at the foot of page 406 is almost the same as mine; but on page $40 \%$, in the fourth equation, the exponent of $n$ should be $+\frac{1}{2}$ instead of $-\frac{1}{2}$, which increases their result by about $600,000,000$, and makes it practically the same as my own.

Rotterdam, July 13. 


\section{ON THE DIAMAGNETIC CONSTANTS OF BISMUTH AND CALC-SPAR IN ABSOLUTE MEASURE}

[American Journal of Science [3], XVII], 360-371, 1879]

\section{PART I.-By H. A. Rowland}

Since my experiments on the magnetic constants of iron, nickel and cobalt, I have sought the means of determining those of some diamagnetic substances, and to that end have described a method in this Journal for May, 1875 (vol. ix, page 35\%). As Mr. Jacques, Fellow of the University, was willing to take up the experimental portion, I have here worked up the subject more in detail and brought the formulæ into practical shape. No experiments have been made on this subject so far, but some rough comparisons with iron have been made by Becquerel, Plucker and Weber. But as iron varies so greatly, and as the methods of experiment are inexact, we cannot be said to know much about the subject. As, however, the relative results of these experiments and those of Faraday can be accepted as reasonably exact for diamagnetic substances and weak paramagnetic ones, it is only necessary to make a determination of one substance such as bismuth, and then the rest can be readily found. But as bismuth is very crystalline it is necessary to make our formulæ general, unless we use bismuth in a powder, which would introduce error.

The general method of experiment has been indicated in the paper before referred to, but I may here state that it consists in counting the number of vibrations made by a bar hung in the usual manner between the poles of an electromagnet. The distribution of the magnetic force in the field being known, we can then calculate the force acting on the body, and the comparison of this with the time of vibration gives us the means of determining the constant sought. But I will leave the more exact description to be given by Mr. Jacques in the experimental part. 


\section{Exploration of Field}

The first operation to be performed is to find a formula to express the force of the field at any point, and an experimental means of determining it in absolute measure. The magnet used was one on the method of Ruhmkorff, and hence the field was nearly symmetrical around the axis of the two branches, and also with respect to a plane perpendicular to the axis at a point midway between its poles. Should any want of symmetry exist by accident, it will be nearly neutralized in its effect on the final result, seeing that the diamagnetic bar hangs symmetrically.

The proper expansion of the magnetic potential for this case is therefore a series of zonal spherical harmonics, including only the uneven powers. Hence, if $V$ is the potential,

$$
V=A_{1} Q_{v} r+A_{1} Q_{u} r^{r^{3}}+A_{v} Q_{v} r^{5}+\text { etc., . . . . }
$$

where $r$ is the distance from the centre of symmetry, $Q, Q_{\text {u, }}$, etc., are the spherical harmonics with respect to the angle between $r$ and the axis, and $A_{1}, A_{\text {, }}, A_{v}$, etc., are constants to be found by experiment. The only method known of measuring a strong magnetic field with accuracy is by means of induced currents, and in this case I have used a modification of the method of the proof plane as I have described it in this Journal, III, vol. x, p. 14. In the method there described the coil was to be drawn rapidly away from the given point: in the present case the coil was moved along the axis, thus measuring the difference of the field at several points; on then placing it at the centre and drawing it away, the field was measured at that point. The field at the other points along this axis could then be found by adding the measured difference to this quantity. This method is far more accurate than the direct measurement at the different points.

When a wire is moved in a magnetic field the current induced in it is equal to the change of its potential energy, supposing it to transmit a unit current, divided by the resistance of the circuit. The potential energy of a wire in a magnetic field is (Maxwell's Elec., Art. 410),

$$
P=\int\left(l \frac{d V}{d x}+m \frac{d V}{d y}+n \frac{d V}{d z}\right) d S
$$

which is simply the surface integral of $V$ over any surface whose edge is in the wire.

In the present case, take the axis of $x$ in the direction of the axis of the poles and the surface, $S$, parallel to the plane $Y Z$, and let $\rho$ be the 
distance in this plane from the centre of the coil we are calculating. Then

$$
P=2 \pi \int \frac{d V}{d x} \rho d \rho=\pi \int \frac{d V}{d x} d\left(\rho^{2}\right)
$$

for a single circle.

From (1)

and

$$
\begin{gathered}
\frac{d V}{d x}=\Sigma_{-\infty}^{0}(i+1) A_{i+1} r^{i} Q_{i} \\
\rho^{2}=x^{2}\left(\frac{1}{\mu^{2}}-1\right) ; \quad r^{i}=\frac{x^{i}}{\mu^{i}}
\end{gathered}
$$

where $\mu=\cos \theta$,

$$
\begin{aligned}
\therefore \quad P & =-2 \pi x^{i+2} \Sigma(i+1) A_{i+1} \int_{1}^{\mu} \frac{Q_{i} d_{i \mu}}{\mu^{i+3}}, \\
P & =2 \pi \rho^{2} \sum_{\infty}^{0} A_{i+1} \frac{r^{i} Q_{i+1}}{i+2} .
\end{aligned}
$$

For a circle of rectangular section we must obtain the mean value of this quantity throughout the section of the coil.

$$
\therefore M=\frac{1}{\eta \xi} \int_{x_{0}-\frac{1}{b} \eta}^{x_{0}+\frac{1 \eta}{\rho_{0}}} \int_{\rho_{0}-\frac{1}{2} \xi}^{\rho_{0}+\frac{1}{\xi} \xi} P d x d \rho,
$$

where $x_{0}$ and $\rho_{0}$ are the values of $x$ and $\rho$ at the centre of section and $\eta$ and $\xi$ are the width and depth of the groove in which the coil is wound. We can calculate this quantity best by the formula of Maxwell (Electricity, Art. 700),

$$
M=P_{0}+\frac{1}{24}\left(\frac{d^{2} p_{0}}{d \rho^{2}} \xi^{2}+\frac{d^{2} p_{0}}{d x^{2}} \eta^{9}\right)+\text { etc. }
$$

Thus we finally find

$$
\begin{aligned}
M= & \pi \rho^{2}\left\{A,\left(1+\frac{1}{12} \frac{\xi^{2}}{\rho^{2}}\right)+\frac{1}{2} A_{\ldots \mu_{0}^{2}}\left(Q^{\prime} \ldots+\frac{1}{2}\left(5 \mu^{2}-3\right) \frac{\xi^{2}}{l^{2}}+\frac{1}{2} \frac{r^{2}}{\mu^{2}}\left(1-\mu^{2}\right)\right)\right. \\
& \left.+\frac{1}{3} A_{v} Q_{v}^{\prime} r_{0}^{4}+\text { etc. }\right\} \text {. . . . . . . . . . . . . . (2) }
\end{aligned}
$$

It is by aid of this equation that we find the coefficients $A_{\text {, }} A_{\text {, }}$, etc. in the expansion of the magnetic potential, $V$. For, let the coil be moved in the field from a position where $M$ has the value $M^{\prime}$ to where it has the value $M^{\prime \prime}$ : then if the coil be joined to a galvanometer the current induced will be equal to

$$
\frac{M^{\prime}-M^{\prime \prime}}{R}
$$

where $R$ is the resistance of the circuit. If an earth inductor is included in the circuit whose integral area is $E$, when it is reversed the current is $\frac{2 H E}{R}$ where $H$ is the component of the earth's magnetism 
perpendicular to the plane of the inductor. The current as measured by the galvanometer in the first case will be $C \sin \frac{1}{2} \delta\left(1+\frac{1}{2} \lambda\right)$ and in the second $C \sin \frac{1}{2} D\left(1+\frac{1}{2} \lambda\right)$, where $C$ is the constant of the galvanometer and $\lambda$ is the logarithmic decrement.

Hence

$$
\begin{aligned}
\frac{M^{\prime}-M^{\prime \prime}}{R} & =C \sin \frac{1}{2} \delta\left(1+\frac{1}{2} \lambda\right), \\
\frac{2 H E}{R} & =C \sin \frac{1}{2} D\left(1+\frac{1}{2} \lambda\right), \\
\therefore M^{\prime}-M^{\prime \prime} & =2 H E \frac{\sin \frac{1}{2} \delta}{\sin \frac{1}{2} D} .
\end{aligned}
$$

In this way we can obtain a series of equations containing $A_{\iota}, A_{\prime \prime}$, etc., and ean thus find these by elimination.

This completes the exploration, and we have as a result a formula giving the magnetic potential of the field in absolute measure throughout a certain small region in which we can experiment.

The next process is to consider the action of this field upon any body which we may hang in it.

\section{Crystalline Body in Magnetic Field}

Let the body have such feeble magnetic action that the magnetic field is not very much influenced by its presence. In all crystalline substances we know there exist in general three axes at right angles to each other, along which the magnetic induction is in the direction of the magnetic force. Let $k_{1}, k_{2}$ and $k_{3}$ be the coefficients of magnetization in the directions of these axes and let a set of coördinate axes be drawn parallel to these crystalline axes, the coördinates referred to which are designated by $x^{\prime}, y^{\prime}$ and $z^{\prime}$, and the magnetic components of the force parallel to which are $X^{\prime}, Y^{\prime}$ and $Z^{\prime}$.

The energy of the crystalline body will then be

$$
E=-\frac{1}{2} \iint f\left(k_{1} X^{\prime 2}+k_{2} Y^{\prime 2}+k_{3} Z^{\prime 2}\right) d x^{\prime} d y^{\prime} d z^{\prime}
$$

In most cases it is more convenient to refer the equation to axes in some other direction through the crystal. Let these axes be $X, Y, Z$.

Then

$$
\begin{aligned}
& x=x^{\prime} \alpha+y^{\prime} \beta+z^{\prime} \gamma \\
& y=x^{\prime} a^{\prime}+y^{\prime} \xi^{\prime}+z^{\prime} \gamma^{\prime} \\
& z=x^{\prime} a^{\prime \prime}+y^{\prime} \beta^{\prime \prime}+z^{\prime} \gamma^{\prime \prime} \\
& X^{\prime}=\frac{d V}{d x^{\prime}}=\frac{d V}{d x} a+\frac{d V}{d y} a^{\prime}+\frac{d V}{d z} a^{\prime \prime} \\
& Y^{\prime}=\text { etc. }
\end{aligned}
$$


Hence

$$
\begin{aligned}
& X^{\prime}=X \alpha+Y u^{\prime}+Z \alpha^{\prime \prime} \\
& Y^{\prime}=X \beta+Y \beta^{\prime}+Z \beta^{\prime \prime} \\
& Z^{\prime}=X_{\gamma}+Y_{\gamma^{\prime}}+Z_{\gamma^{\prime \prime}}
\end{aligned}
$$

where $\alpha, \beta, \gamma ; \alpha^{\prime}, \beta^{\prime}, \gamma^{\prime}$; and $\alpha^{\prime \prime}, \beta^{\prime \prime}, \gamma^{\prime \prime}$ are the direction cosines of the new axes with reference to the old.

We then find

$$
\begin{aligned}
E & =-\frac{1}{2} \iiint\left\{X^{2}\left(k_{1} \alpha^{2}+k_{2} \beta^{2}+k_{3} \gamma^{2}\right)+Y^{2}\left(k_{1} \alpha^{\prime 2}+k_{2} \beta^{\prime 2}+k_{3} \gamma^{\prime 2}\right)+Z^{2}\left(k_{1} \alpha^{\prime \prime 2}+k_{2} \beta^{\prime \prime 2}\right.\right. \\
& \left.+k_{3} \gamma^{\prime \prime 2}\right)+2 X Y\left(k_{1} \alpha \alpha^{\prime}+k_{2} \beta \xi^{\prime}+k_{3} \gamma^{\prime}\right)+2 X Z\left(k_{1} \alpha \alpha^{\prime \prime}+k^{2} \beta \xi^{\prime \prime}+k_{3} \gamma \gamma^{\prime \prime}\right)+2 Y Z \\
& \left.\left(k_{1} \alpha^{\prime} \alpha^{\prime \prime}+k_{2} \xi^{\prime} \xi^{\prime \prime}+k_{3} \gamma^{\prime} \gamma^{\prime \prime}\right)\right\} d x d y d z
\end{aligned}
$$

The most simple and in many respects the most interesting cases are when the crystal has only one optic or magnetic axis. In this case $k_{2}=k_{3}$.

Hence

$$
E=-\frac{1}{2} \iiint\left\{\left(X^{2}+Y^{2}+Z^{2}\right) k_{2}+\left(X \alpha+Y \alpha^{\prime}+Z u^{\prime \prime}\right)^{2}\left(k_{1}-k_{2}\right)\right\} d x d y d z
$$

where $\alpha, \alpha^{\prime}$ and $\alpha^{\prime \prime}$ are the direction cosines of the magnetic axis with respect to the coördinate axes.

The first case to consider is that of a mass of crystal in a uniform magnetic field. The magnetic forces which enter the equation are those due to the magnetic action of the body as well as to the field in which the body is placed. In the case of very weak magnetic or diamagnetic bodies the forces are almost entirely those of the field alone. Hence in the case under consideration we may put $Y=0$ and $Z=0$.

Hence

$$
E=-\frac{1}{2} \iiint X^{2}\left(\left(k_{1}-k_{2}\right) \alpha^{2}+k_{2}\right) d x d y d z,
$$

and if $v$ is the volume of the body

$$
E=-\frac{1}{2} X^{2}\left(\left(k_{1}-k_{2}\right) \alpha^{2}+k_{2}\right) v .
$$

As this expression is the same at all points of the field there is no force acting to translate the body from one part of the field to another. The moment of the force tending to increase $\varphi$, where $\varphi=\cos ^{-1} \alpha$, is

$$
-\frac{d E}{d \varphi}=v X^{2}\left(k_{1}-k_{2}\right) \sin \varphi \cos \varphi
$$

By observing the moment of the force which acts on a crystal placed in a uniform magnetic field we can thus find the value of $k_{1}-k_{2}$ or the difference of the magnetic constant along the axis and at right angles to it. The differences of the constants can also be found in the case of crystals with three axes by a similar process.

The next case which $I$ shall consider is that of a bar hanging in a 
magnetic field. Let the field be symmetrical around an horizontal axis, and also with reference to a plane perpendicular to that axis at the centre. If the bar is very long with reference to its section and a plane can be passed through it and the axis we must have $Z=0$, and the equation becomes

$$
E=-\frac{1}{2} \iiint\left\{\left(X^{2}+Y^{2}\right) k_{2}+\left(X \alpha+Y \alpha^{\prime}\right)^{2}\left(k_{1}-k_{2}\right)\right\} d x d y d z .
$$

Let the axis of $X$ coincide with the long axis of the bar, as this will in the end lead to the most simple result, seeing that we have to integrate along the length of the bar.

Let $r$ be the length along the bar from the centre to any point, and let $\theta$ be the angle made by the bar with the axis of symmetry: then

$$
X=-\frac{d V}{d r} \quad Y=-\frac{1 d V}{r d \theta}
$$

also let the section of the bar be

$$
a=d y d z
$$

and let the axis of the bar pass through the origin from which we have developed the potential in terms of spherical harmonics. We can then write as before

$$
V=A_{1} Q_{v} r+A_{1 /} Q_{u} r^{3}+A_{v} Q_{v} r^{3}+\text { etc. }
$$

where $Q_{1}, Q_{\text {، }}$, etc., are zonal spherical harmonics with reference to the angle $\theta$,

$$
\begin{aligned}
& X=-\left\{A_{1} Q_{\imath}+3 A_{\ldots} Q_{\ldots} r^{2}+5 A_{v} Q_{r} r^{4}+\text { etc. }\right\} \\
& Y=+\left\{A_{1} Q_{\imath}^{1}+A_{\ldots} Q_{\ldots{ }^{\prime}} r^{2}+A_{v} Q_{r}^{\prime} r^{4}+\text { etc. }\right\} \sin \theta
\end{aligned}
$$

from which we have the following:

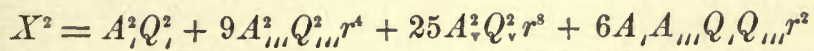

$$
\begin{aligned}
& +10 A_{1} A_{v} Q_{1} Q_{v} r^{4}+30 A_{u} A_{v} Q_{u} Q_{v} r^{6}+\text { etc., }
\end{aligned}
$$

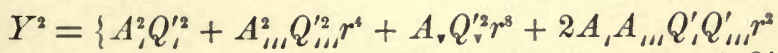

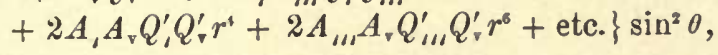

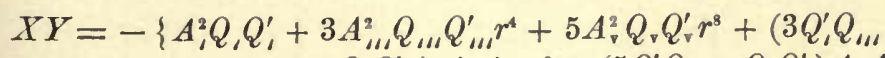

$$
\begin{aligned}
& \left.+Q_{1} Q_{u}^{\prime}\right) A_{1} A_{11} r^{2}+\left(5 Q_{1}^{\prime} Q_{v}+Q_{1} Q_{v}^{\prime}\right) A_{1} A_{v} r^{4}+\left(5 Q_{\prime \prime \prime}^{\prime} Q_{v}\right. \\
& \left.\left.+3 Q_{u} Q_{v}^{\prime}\right) A_{11} A_{v} r^{8}+\text { etc }\right\} \sin \theta \text {. }
\end{aligned}
$$

The moment of the force tending to increase $\theta$ is

$$
\theta=-\frac{d E}{d \theta}
$$

whence we may write, $\theta=-\frac{1}{2} a\left\{A\left(\left(k_{1}-k_{2}\right) a^{2}+k_{2}\right)+B\left(\left(k_{1}-k_{2}\right) \alpha^{\prime 2}+k_{2}\right)-C\left(k_{1}-k_{2}\right) \alpha u^{\prime}\right\}$, 
where

$$
\begin{aligned}
& A=-\frac{d}{d \theta} \int_{-l}^{+l} X^{2} d r=\sin \theta \frac{d}{d \mu} \int_{-l}^{+\imath} X^{2} d r, \\
& B=-\frac{d}{d \theta} \int_{-l}^{+l} Y^{2} d r=\sin \theta \frac{d}{d \mu} \int_{-l}^{+l} Y^{2} d r \\
& C=-\frac{d}{d \theta} \int_{-l}^{+l} 2 X Y d r=\sin \theta \frac{d}{d \mu} \int_{-l}^{+l} 2 X Y d r,
\end{aligned}
$$

where $l$ is half the length of the bar and $\mu=\cos \theta$.

$$
\begin{aligned}
& A=4 l \sin \theta\left\{A_{3}^{2} Q_{1} Q_{1}^{\prime}+\frac{9}{5} A_{11}^{2} Q_{1 \ldots} Q_{1,}^{\prime} l^{4}+\frac{25}{9} A_{\mathrm{v}}^{2} Q_{\mathrm{v}} Q_{v}^{\prime} l^{8}+A_{1} A_{\ldots \prime}\left(Q_{1}^{\prime} Q_{\ldots}\right.\right. \\
& \left.\left.+Q_{1} Q_{\ldots 1}^{\prime}\right) l^{2}+A_{1} A_{\mathrm{v}}\left(Q_{1}^{\prime} Q_{\mathrm{v}}+Q_{\mathrm{v}} Q_{\mathrm{v}}^{\prime}\right) l^{4}+\frac{15}{7} A_{11} A_{\mathrm{v}}\left(Q_{11}^{\prime} Q_{\mathrm{v}}+Q_{11} Q_{\mathrm{v}}^{\prime}\right) l^{6}\right\} \\
& B=4 l \sin \theta\left\{A_{1}^{2}\left(Q_{1}^{\prime} Q_{1}^{\prime \prime} \sin ^{2} \theta-Q_{1}^{\prime 2} \cos \theta\right)+A_{11}^{2}\left(Q_{11}^{\prime} Q_{\ldots,}^{\prime \prime} \sin ^{2} \theta\right.\right. \\
& \left.-Q_{i \prime \prime}^{\prime \prime} \cos \theta\right) \frac{l^{4}}{5}+A_{\mathrm{v}}\left(Q_{\mathrm{v}}^{\prime} Q_{v}^{\prime \prime} \sin ^{2} \theta-Q_{v}^{\prime} \cos \theta\right) \frac{l^{8}}{9}+A_{1} A_{11}\left(\left(Q_{,}^{\prime} Q_{1 \prime \prime}^{\prime \prime}\right.\right.
\end{aligned}
$$

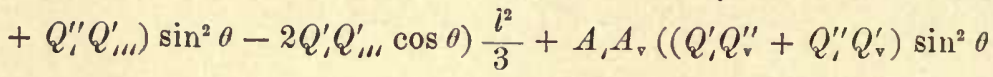

$$
\begin{aligned}
& \left.-2 Q^{\prime} Q_{v}^{\prime} \cos \theta\right) \frac{l^{4}}{5}+A_{11} A_{v}\left(\left(Q_{u \prime}^{\prime} Q_{v}^{\prime \prime}+Q_{\ldots 1}^{\prime \prime} Q_{v}^{\prime}\right) \sin ^{2} \theta\right. \\
& \left.\left.-2 Q^{\prime}{ }_{11} Q_{r}^{\prime} \cos \theta\right) \frac{i^{6}}{7}\right\} \text {, }
\end{aligned}
$$

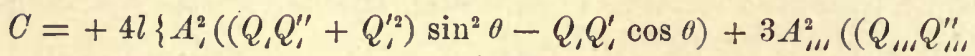

$$
\begin{aligned}
& \left.\left.+Q_{u 1}^{\prime 2}\right) \sin ^{2} \theta-Q_{1,} Q_{11}^{\prime} \cos \theta\right) \frac{l^{4}}{5}+5 A_{v}^{2}\left(\left(Q_{v} Q_{v}^{\prime \prime}-Q_{v}^{\prime 2}\right) \sin ^{2} \theta\right.
\end{aligned}
$$

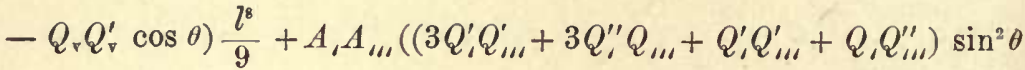

$$
\begin{aligned}
& \left.-\left(3 Q_{1}^{\prime} Q_{\text {u }}+Q_{1} Q_{u}^{\prime}\right) \cos \theta\right) \frac{l^{2}}{3}+A_{1} A_{\mathrm{v}}\left(\left(5 Q_{,}^{\prime} Q_{\mathrm{v}}^{\prime}+5 Q_{\mathrm{\prime}}^{\prime \prime} Q_{\mathrm{v}}+Q_{\mathrm{v}}^{\prime} Q_{\mathrm{v}}^{\prime}\right.\right. \\
& \left.\left.+Q_{1} Q_{\mathrm{v}}^{\prime \prime}\right) \sin ^{2} \theta-\left(5 Q_{1}^{\prime} Q_{\mathrm{v}}+Q_{1} Q_{\mathrm{v}}^{\prime}\right) \cos \theta\right) \frac{l^{4}}{5}+A_{11} A_{\mathrm{v}}\left(\left(5 Q_{11}^{\prime \prime} Q_{\mathrm{v}}\right.\right.
\end{aligned}
$$

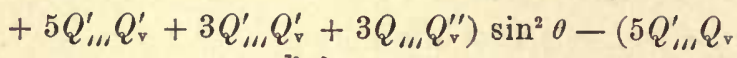

$$
\begin{aligned}
& \left.\left.+3 Q_{\ldots}\left(Q_{v}^{\prime}\right) \cos \theta\right) \frac{l^{8}}{9}\right\} \text {. }
\end{aligned}
$$

Where

$$
\begin{aligned}
& Q_{1}=\cos \theta, \\
& Q_{\prime \prime \prime}=\frac{1}{2}\left(5 \cos ^{3} \theta-3 \cos \theta\right), \\
& Q_{v}=\frac{1}{8}\left(63 \cos ^{5} \theta-70 \cos ^{3} \theta+15 \cos \theta\right), \\
& Q_{,}^{\prime}=1 \\
& Q_{\prime \prime \prime}^{\prime}=\frac{3}{2}\left(5 \cos ^{2} \theta-1\right), \\
& Q_{v}^{\prime}=\frac{15}{8}\left(21 \cos ^{4} \theta-14 \cos ^{2} \theta+1\right), \\
& Q_{\prime}^{\prime \prime}=0 \\
& Q_{\prime \prime \prime}^{\prime \prime}=15 \cos \theta \\
& Q_{v}^{\prime \prime}=\frac{15}{2}\left(21 \cos ^{3} \theta-7 \cos \theta\right), \\
& \mu=\cos \theta .
\end{aligned}
$$




$$
\begin{aligned}
& A=4 l \sin \theta\left\{\left(A_{1}^{2}+\frac{81}{20} A_{11 l^{2}} l^{4}+\frac{1875}{192} A_{v}^{2} l^{8}-3 A_{1} A_{11} l^{2}+\frac{15}{4} A_{1} A_{\mathrm{v}} l^{4}\right.\right. \\
& \left.-\frac{675}{56} A_{11} A_{\nabla} l^{6}\right) \mu+\left(-27 A_{11}^{2} l^{4}-\frac{4375}{24} A_{v}^{2} l^{8}+10 A_{1} A_{11} l^{2}\right. \\
& \left.-35 A_{1} A_{v} l^{4}+\frac{4275}{28} A_{11} A_{v} l^{6}\right){ }_{14}^{8}+\left(\frac{135}{4} A_{11}^{2} l^{4}+\frac{56625}{64} A_{v}^{2} l^{8}\right. \\
& \left.+\frac{199}{4} A_{1} A_{\mathrm{v}} l^{4}-\frac{3465}{8} A_{11} A_{\mathrm{v}} l^{6}\right) \mu^{6}+\left(-\frac{6125}{4} A_{\mathrm{v}}^{2} l^{8}+\frac{675}{2} A_{11} A_{\mathrm{v}} l^{6}\right) \mu^{7} \\
& \left.+\frac{55125}{64} A_{v}^{2} 7^{8} \mu^{9}\right\} \\
& B=4 l \sin \theta\left\{\left(-A_{1}^{2}-\frac{99}{20} A_{11 l^{2}} l^{4}-\frac{725}{64} A_{\mathrm{v}}^{2} l^{8}+6 A_{1} A_{111} l^{2}-\frac{45}{4} A_{1} A_{\mathrm{v}} l^{4}\right.\right. \\
& \left.+\frac{225}{14} A_{11} A_{\mathrm{v}} l^{6}\right) \mu+\left(\frac{63}{2} A_{1 .}^{2} l^{4}-\frac{1575}{16} A_{\mathrm{v}} l^{8}-10 A_{1} A_{\mu} l^{2}+\frac{171}{4} A_{1} A_{\mathrm{v}} l^{4}\right. \\
& \left.-\frac{2475}{14} A_{11} A_{\mathrm{v}} l^{6}\right) \mu^{3}+\left(-\frac{13}{4} \underline{5} A_{11}^{2} l^{4}-\frac{162}{32}{ }^{2} A_{\mathrm{v}}^{2} l^{8}-1 \frac{8}{4} 9 A_{1} A_{\mathrm{v}} l^{4}\right. \\
& \left.\left.+\frac{945}{2} A_{11} A_{\nabla} l^{6}\right) \mu^{6}+\left(\frac{77425}{48} A_{\mathrm{v}}^{2} l^{8}-\frac{675}{2} A_{1,1} A_{\nabla} l^{6}\right) \mu^{7}+\frac{55125}{64} A_{\nabla} l^{8} \mu^{9}\right\} \\
& C=4 l\left\{\left(-A_{1}^{2}-\frac{125}{64} A_{\mathrm{v}}^{2} l^{8}-\frac{1}{2} A_{1} A_{11} l^{2}+{ }_{4}^{3} A_{1} A_{\mathrm{v}} l^{4}-\frac{5}{2} A_{11} A_{\mathrm{v}} l^{8}\right)\right. \\
& +\left(-\frac{3}{2} A_{1} A_{11} l^{2}\right) \mu+\left(-\frac{29 \eta}{2}{ }^{2} A_{11}^{2} l^{4}-6 A_{1} A_{1} l^{2}-\frac{7}{2} A_{1} A_{\nabla} l^{4}\right.
\end{aligned}
$$

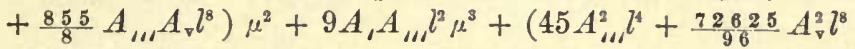

$$
\begin{aligned}
& \left.+\frac{5}{2} A_{1} A_{11} l^{2}+\frac{511}{4} A_{1} A_{\mathrm{v}} l^{4}-\frac{6485}{12} A_{11} A_{\mathrm{v}} l^{8}\right) \mu^{4}-\frac{15}{2} A_{1} A_{1 / l^{2} l^{2} \mu^{5}} \\
& +\left(-\frac{99}{4} A_{11 l^{2}} l^{4}-\frac{17975}{24} A_{v}^{2} l^{8}-\frac{441}{5} A_{1} A_{v} l^{4}+\frac{24815}{48} A_{11} A_{\nabla} l^{8}\right) \mu^{6}
\end{aligned}
$$

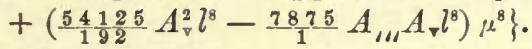

Or we can write

$$
\begin{aligned}
& A=4 l \sin \theta\left\{L_{\mu}+L^{\prime} \mu^{3}+L^{\prime \prime} \mu^{6}+\text { etc. }\right\} \\
& B=4 l \sin \theta\left\{M_{\mu}+M^{\prime} \mu^{3}+\text { etc. }\right\} \\
& C=\nu l\left\{N+N^{\prime} \mu+N^{\prime \prime} \mu^{2}+\text { etc. }\right\}
\end{aligned}
$$

where the values of $L, M$, etc., are apparent.

To sum up we may then write as before

$$
\theta=-\frac{1}{2} a\left\{A\left[\left(k_{1}-k_{2}\right) \alpha^{2}+k_{2}\right]+B\left[\left(k_{1}-k_{2}\right) \alpha^{\prime 2}+k_{2}\right]-C\left(k_{1}-k_{2}\right) \alpha \alpha^{\prime}\right\}
$$

where $A, B$ and $C$ are the quantities we have found, $\alpha$ is the cosine of the angle made by the axis of the crystal with the axis of the bar, and $\iota^{\prime}$ is the cosine of the angle made by the same axis with a horizontal line at right angles to the bar.

The equation

$$
\theta=0
$$

gives equilibrium at some angle depending on $\alpha$ and $\alpha^{\prime}$, and if either of these is zero the angle can be either $\theta=0$ or $\frac{1}{2} \pi$, one of which will be stable and the other unstable according as the body is para- or diamagnetic.

For a diamagnetic crystal like bismuth with the axis at right angles to the bar we can put

$$
\mu=\cos \theta=\sin \psi \text { and } \alpha=0,
$$

and we can write 


$$
\begin{aligned}
\theta=-\frac{1}{2} a\left\{4 l k _ { 2 } \left(L \mu+L_{\mu \mu^{\prime 2}}\right.\right. & + \text { etc. }) \\
& \left.+4 l\left[\left(k_{1}-k_{2}\right) \alpha^{\prime 2}+k_{2}\right]\left[M \mu+M^{\prime} \mu^{3}+\text { etc. }\right]\right\}
\end{aligned}
$$

or for very small values of $\mu$ we can write in terms of $\psi$

$$
\theta=-2 a l \psi^{\prime}\left\{k_{2} L+\left(\left(k_{1}-k_{2}\right) \alpha^{\prime 2}+k_{2}\right) M \xi\right. \text {. }
$$

If $I$ is the moment of inertia of the bar and $t$ is the time of a single vibration, we may write

$$
\theta=I \frac{\pi^{2}}{t^{2}} \psi
$$

If we hang up the bar so that $\alpha^{\prime}=0$ we have

$$
k_{2}(L+M)=-\frac{\pi^{2} I}{2 a l t^{2}},
$$

and if we hang it up so that $\alpha^{\prime}=\frac{1}{2} \pi$ we have again

whence

$$
k_{2} L+k_{1} M=-\frac{\pi^{2} I^{\prime}}{2 a l t^{\prime 2}},
$$

where

$$
\begin{gathered}
k_{2}=-\frac{\pi^{2} I}{2 a l t^{2}} \frac{1}{L+M}, \\
k_{1}=-\frac{1}{M}\left(\frac{\pi^{2} I^{\prime}}{2 a l t^{\prime 2}}+k_{2} L\right),
\end{gathered}
$$

$$
\begin{aligned}
& L=A_{1}^{2}-3 A_{1} A_{11} l^{2}+\left(\frac{81}{20} A_{31}^{2}+\frac{15}{4} A_{1} A_{v}\right) l^{4}-\frac{675}{56} A_{11} A_{v} l^{6}+\frac{1875}{192} A_{v}^{2} l^{8} \\
& M=-A_{1}^{2}+6 A_{1} A_{11} l^{2}-\left(\frac{99}{20} A_{u \prime}^{2}+\frac{45}{4} A_{1} A_{v}\right) l^{4}+\frac{225}{14} A_{11} A_{v} l^{6}-\frac{725}{64} A_{v}^{2} l^{8} \\
& \left.L+M=3 A_{1} A_{1, l^{\prime}} l^{2}-{ }_{10} \frac{9}{10} A_{1 \prime}^{2}+\frac{15}{2} A_{1} A_{\mathrm{v}}\right) l^{4}+\frac{225}{56} A_{11} A_{\mathrm{v}} l^{6}-\frac{75}{48} A_{\mathrm{v}}^{2} l^{8} \text {. }
\end{aligned}
$$

For a cleavage bar of calc spar we must use the general equation. For equilibrium we have

$$
k_{1}\left\{A \alpha^{2}+B u^{\prime 2}-C \alpha u^{\prime}\right\}+k_{2}\left\{A\left(1-\alpha^{2}\right)+B\left(1-a^{\prime 2}\right)+C a \alpha^{\prime}\right\}=0,
$$

which gives us the ratio of $k_{1}$ to $k_{2}$. For this experiment it is best to hang up the bar so that the axis is in the horizontal plane and we should then have

$$
a^{2}=1-a^{\prime 2} .
$$

For obtaining another relation it is best to suspend the bar with $\alpha^{\prime}=0$ and we then have the position of stable equilibrium at the point $\theta=\frac{1}{2} \pi$ which gives

$$
\theta=-2 a l \psi^{\prime}\left\{L\left[\left(k_{1}-k_{2}\right) a^{2}+k_{2}\right]+M k_{2}\right\}=\frac{\pi^{2} I}{t^{2}} \psi,
$$

whence 


$$
k_{1}=-\frac{\pi^{2} I}{2 a l l^{2}} \frac{1}{I \cdot\left[\left(1-\frac{k_{2}}{k_{1}}\right) u^{2}+\frac{k_{2}}{k_{1}}\right]+M \frac{k_{2}}{k_{1}}} ;
$$

these various equations give the complete solution of the problem of finding the various coefficients of magnetization.

PART II.-BY W. W. JACQUes

In the foregoing part of this paper there have been deduced mathematical expressions for the constants $k$ and $k^{\prime}$ both for bismuth and for calc-spar crystals. In these expressions it is necessary to substitute certain quantities obtained by a series of experiments, and it is the purpose of the remaining portion of the paper to describe briefly the way in which these quantities were obtained.

These experiments are naturally divided into two parts. First, the exploration of the small magnetic field between the two poles of the electromagnet, and second, the determination of the time of swing and certain other constants relating to little bars of the substances experimented upon when suspended in this field.

In order to insure the constancy of the magnetic field, a galvanometer and variable resistance were inserted in the circuit through which the magnetizing current circulated. This space between the poles of the electromagnet in which the experiments were performed was a little larger than a hen's egg.

The method of exploring this field was as follows: In the line joining the centre of the two poles was placed a little brass rod, along which a very small coil of fine wire was made to slide. To this rod were fixed two little set-screws to regulate the distance through which the coil could be moved. Starting now always from the centre, the coil was moved successively through distances $a, b$ and $c$, and the corresponding deflections of a delicate mirror galvanometer contained in the circuit were noted. To each of these deflections was added the deflection due to quickly pulling the coil away from the centre to a distance such that the magnetic potential was negligibly small. Of course, experiments were made on both sides of the centre of the field in order to eliminate any want of symmetry, and the distances through which the coil moved were all carefully measured with a dividing engine.

In order to reduce the deflections of the galvanometer to absolute 
measure, an earth inductor was included in the circuit with the little coil and galvanometer and the deflections produced by this were compared with those produced by moving the little coil. These deflections were taken between every two observations with the little coil.

The deflections due to moving the little coil, those due to the earth inductor and that due to pulling the coil away from the centre are given in the following table:

\begin{tabular}{|c|c|c|}
\hline Coil & $\begin{array}{l}\text { Distance } a \text {. } \\
4.407 \mathrm{~cm} \text {. }\end{array}$ & $\begin{array}{l}\text { Distance } b . \\
9.655 \mathrm{~cm} .\end{array}$ \\
\hline Earth inductor & ... $33.138 \mathrm{~cm}$. & $33 \cdot 137 \mathrm{~cm}$. \\
\hline
\end{tabular}

In order to determine the proper quantities for substitution in the expression for the magnetic potential of the field, it was necessary to measure, besides, the deflections due to the little coil when moved through various distances and those due to the earth inductor.

The mean radius of the small coil........ $=3912 \mathrm{~cm}$.

Number of turns . ...............

Width if coil .....................

Depth of coil ....................

Integral area of earth inductor.......... $=20716.2 \mathrm{~cm}$.

Horizontal intensity of earth's magnetism.... $=\cdot 1984 \mathrm{cgs}$.

The quotient of the mean radius of the coil by the distance moved gave $\tan \theta$.

The linear measurements were made with a dividing engine.

The horizontal intensity of the earth's magnetism was determined by measuring the time of swing of a bar magnet and its effect upon a smaller galvanometer needle. The proper substitution of these quantities in the formula given gave the expression in absolute measure for the magnetic potential at any part of the field.

The remaining part of the experiment and the part that was attended with greatest difficulty, was to prepare little bars of the substances and to determine the times of vibration of these when suspended, first with the axis vertical and then with it horizontal in the magnetic field. Besides this, the dimensions and the moment of inertia of each bar had to be determined, and, in the case of the calc-spar, the angle the bar made with the equatorial line of the poles when in its position of equilibrium, had to be measured.

Bismuth and calc-spar were the two crystals experimented upon; quite a number of other substances were tried but failed to give good 
results because of the iron contained in them as an impurity. The bars were each about $15 \mathrm{~mm}$. long and about $2 \mathrm{~mm}$. in cross section. The force to be measured being only about 00000001 of that exerted in the case of iron it was necessary to carry out the experiments with the very greatest care.

In order to obtain bars free from iron, very fine crystals of chemically pure substances were selected and the bars cleaved from them. They were then polished with their various sides parallel to the cleavage planes by rubbing on clean plates of steatite with oil. In order to remove any particles of iron that might have collected upon them during these processes, they were carefully washed with boiling hydrochloric acid and with distilled water and then wrapped in clean papers, and never touched except after washing the hands with hydrochloric acid and distilled water.

In order to reduce to a minimum the causes that might interfere with the accurate determination of the times of vibration of these bars the poles of the magnet were encased by a box of glass. From the top of this a tube four feet long extended up toward the ceiling, and inside this was hung a single fibre of silk so small as to be barely visible to the naked eye. The bars were placed in little slings of coarser silk fibre and suspended by this. Outside the glass case was a microscope placed horizontally and having a focus of about six inches. This was directed toward the suspended bar, and when the latter was at rest the cross hairs of the microscope fell upon a little scratch in one end of the bar. Near by was a telegraph sounder arranged to tick seconds. The bar was set swinging through a small are by making and breaking the current, and the interval between two successive transits of the little scratch on the bar by the cross hairs of the microscope was measured in seconds and tenths of a second by the ear. By keeping count through a large number of successive transits the time of a single swing could be determined with very great accuracy. The bar was caused to swing only through a few degrees of arc and such small correction for amplitude as was found necessary was applied. The time of swing was determined first with the axis vertical and then with it horizontal. But besides the time of swing of each bar it was necessary to measure: the length; area of section; moment of incrtia in each position; and for the calc-spar bar the angle it made with the equatorial plane of the magnet when in its position of equilibrium. This was not necessary in the case of bismuth, because its position of equilibrium lay in the equatorial plane. 


\section{Bismuth.}

$\begin{array}{cccc}\begin{array}{c}\text { Time of } \\ \text { swing. }\end{array} & \begin{array}{c}\text { Moment of } \\ \text { inertia. }\end{array} & \begin{array}{c}\text { Half } \\ \text { length. }\end{array} & \begin{array}{c}\text { Area of } \\ \text { section. }\end{array}\end{array}$

Axis, vertical

' $7 \cdot 18 \mathrm{sec} . \quad \cdot 10976 \mathrm{cgs}$.

Axis, horizontal $5 \cdot 76$ sec. $\quad \cdot 10943 \mathrm{cgs}$.

$.7709 \mathrm{~cm} . \quad \cdot 03778 \mathrm{~cm}$.

\section{Calc-Spar.}

$\begin{array}{lcrcc}\text { Time of } & \text { Moment of } & \text { Half } & \text { Area of } \\ \text { swing. } & \text { inertia. } & \text { length. } & \text { section. }\end{array}$

Axis, vertical

$46 \cdot 35 \mathrm{sec} \cdot \cdot 0303 \mathrm{cgs}$.

Axis, horizontal $43 \cdot 39$ sec. $\cdot 0300 \mathrm{cgs}$.

$.8015 \mathrm{~cm} . \quad \cdot 0300 \mathrm{~cm} .50^{\circ} 30^{\prime}$

The linear measurements were made with a dividing engine, the moments of inertia were calculated from the dimensions of the bars. The angle at which the calc-spar stood was measured by projecting the linear axis on a scale placed at a distance.

The above quantities being all determined and properly substituted, the solution of the equations gave for

Bismuth ...................... $=-.000000012554$

$k_{\text {، }}=-.000000014324$

Calc-spar

$k_{1}=-.00000003 \% 930$

$k_{\text {॥ }}=-.000000040330$ 


\section{PRELIMINARY NOTES ON MR. HALL'S RECENT DISCOVERY ${ }^{1}$}

[Philosophical Magazine [5], IX, 432-434, 1880; Proceedings of the Physical Society, IV, $10-13,1880$; American Journal of Mathematics, II, 354-356, 1879]

The recent discovery by Mr. Hall ${ }^{2}$ of a new action of magnetism on electric currents opens a wide field for the mathematician, seeing that we must now regard most of the equations which we have hitherto used in electromagnetism as only approximate, and as applying only to some ideal substance which may or may not exist in nature, but which certainly does not include the ordinary metals. But as the effect is very small, probably it will always be treated as a correction to the ordinary equations.

The facts of the case seem to be as follows, as nearly as they have yet been determined:-Whenever a substance transmitting an electric current is placed in a magnetic field, besides the ordinary electromotive force in the medium, we now have another acting at right angles to the current and to the magnetic lines of force. Whether there may not be also an electromotive force in the direction of the current has not yet been determined with accuracy; but it has been proved, within the limits of accuracy of the experiment, that no electromotive force exists in the direction of the lines of magnetic force. This electromotive force in a given medium is proportional to the strength of the current and to the magnetic intensity, and is reversed when either the primary current or the magnetism is reversed. It has also been lately found that the direction is different in iron from what it is in gold or silver.

To analyze the phenomenon in gold, let us suppose that the line $A B$ represents the original current at the point $A$, and that $B C$ is the new effect. The magnetic pole is supposed to be either above or below the paper, as the case may be. The line $A C$ will represent the final resultant electromotive force at the point $A$. The circle with arrow represents the direction in which the current is rotated by the magnetism.

1 From the American Journal of Mathematics. Communicated by the Physical Society.

${ }^{2}$ Phil. Mag. [5], vol. ix, p. 225. 
It is seen that all these effects are such as would happen were the electric current to be rotated in a fixed direction with respect to the lines of magnetic force, and to an amount depending only on the magnetic force and not on the current. This fact seems to point immediately to that other very important case of rotation, namely the rotation of the plane of polarization of light. For, by Maxwell's theory, light is an electrical phenomenon, and consists of waves of electrical displacement, the currents of displacement being at right angles to the direction of propagation of the light. If the action we are now considering takes place in dielectrics, which point Mr. Hall is now investigating, the rotation of the plane of polarization of light is explained.

I give the following very imperfect theory at this stage of the paper, hoping to finally give a more perfect one either in this paper or a later one.

North Pole above.

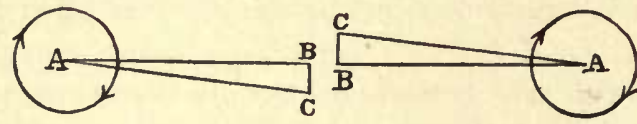

North Pole below.

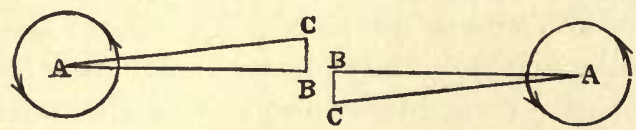

Let $\mathfrak{S}$ be the intensity of the magnetic field, and let $E$ be the original electromotive force at any point, and let $c$ be a constant for the given medium. Then the new electromotive force $E^{\prime}$ will be

$$
E^{\prime}=\mathrm{c} \mathfrak{g} E,
$$

and the final electromotive force will be rotated through an angle which will be very nearly equal to $\mathfrak{c} \mathfrak{c}$. As the wave progresses through the medium, each time it (the electromotive force) is reversed it will be rotated through this angle; so that the total rotation will be this quantity multiplied by the number of waves. If $\lambda$ is the wave-length in air, and $i$ is the index of refraction, and $c$ is the length of medium, then the number of waves will be $\frac{c i}{\lambda}$, and the total rotation

$$
\theta=c \mathrm{cS} \frac{i}{\lambda} \text {. }
$$

The direction of rotation is the same in diamagnetic and ferromagnetic bodies as we find by experiment, being different in the two; for it 
is well known that the rotation of the plane of polarization is opposite in the two media, and Mr. Hall now finds his effect to be opposite in the two media. This result I anticipated from this theory of the magnetic rotation of light.

But the formula makes the rotation inversely proportional to the wave-length, whereas we find it more nearly as the square or cube. This I consider to be a defect due to the imperfect theory; and it would possibly disappear from the complete dynamical theory. But the formula at least makes the rotation increase as the wave-length decreases, which is according to experiment. Should an exact formula be finally obtained, it seems to me that it would constitute a very important link in the proof of Maxwell's theory of light, and, together with a very exact measure of the ratio of the electromagnetic to the electrostatic units of electricity which we made here last year, will raise the theory almost to a demonstrated fact. The determination of the ratio will be published shortly; but I may say here that the final result will not vary much, when all the corrections have been applied, from $299,700,000$ metres per second; and this is almost exactly the velocity of light. We cannot but lament that the great author of this modern theory of light is not now here to work up this new confirmation of his theory, and that it is left for so much weaker hands.

But before we can say definitely that this action explains the rotation of the plane of polarization of light, the action must be extended to dielectrics, and it must be proved that the lines of electrostatic action are rotated around the lines of force as well as the electric currents. Mr. Hall is about to try an experiment of this nature.

I am now writing the full mathematical theory of the new action, and hope to there consider the full consequences of the new discovery.

Addition.-I have now worked out the complete theory of the rotation of the plane of polarization of light, on the assumption that the displacement currents are rotated as well as the conducted currents. The result is very satisfactory, and makes the rotation proportional to $\frac{i^{3}}{\lambda^{2}}$, which agrees very perfectly with observation. The amount of rotation calculated for gold is also very nearly what is found in some of the substances which rotate the light the least. Hence it seems to me that we have very strong ground for supposing the two phenomena to be the same. 


\section{ON THE EFFICIENCY OF EDISON'S ELECTRIC LIGH'T}

By H. A. Rowland and George F. Barker

|American Journal of Science, [3], XIX, 337-339, 1880]

The great interest which is now being felt throughout the civilized world in the success of the various attempts to light houses by electricity, together with the contradictory statements made with respect to Mr. Edison's method, have induced us to attempt a brief examination of the efficiency of his light. We deemed this the more important because most of the information on the subject has not been given to the public in a trustworthy form. We have endeavored to make a brief but conclusive test of the efficiency of the light, that is, the amount of light which could be obtained from one horse power of work given out by the steam engine. For if the light be economical, the minor points, such as making the carbon strips last, can undoubtedly be put into practical shape.

Three methods of testing the efficiency presented themselves to us. The first was by means of measuring the horse power required to drive the machine, together with the number of lights which it would give. But the dynamometer was not in very wood working order, and it was difficult to determine the number of lights and their photometric power, as they were scattered throughout a long distance, and so this method was abandoned. Another method was by measuring the resistance of, and amount of, current passing through a single lamp. But the instruments available for this purpose were very rough, and so this method was abandoned for the third one. This method consisted in putting the lamp under water and observing the total amount of heat generated in the water per minute. For this purpose, a calorimeter, holding about $1 \frac{1}{4} \mathrm{kil}$. of water, was made out of very thin copper: the lamp was held firmly in the centre, so that a stirrer could work around it. The temperature was noted on a delicate Baudin thermometer graduated to $0 \cdot 1^{\circ} \mathrm{C}$.

As the experiment was only meant to give a rough idea of the efficiency within two or three per cent, no correction was made for 
radiation, but the error was avoided as much as possible by having the mean temperature of the calorimeter as near that of the air as possible, and the rise of temperature small. The error would then be much less than one per cent. A small portion of the light escaped through the apertures in the cover, but the amount of energy must have been very minute.

In order to obtain the amount of light and eliminate all changes of the engine and machine, two lamps of nearly equal power were generally used, one being in the calorimeter while the other was being measured. They were then reversed and the mean of the results taken. The apparatus for measuring the light was one of the ordinary Bunsen instruments used for determining gas-lights, with a single candle at ten inches distance. The candles used were the ordinary standards, burning 120 grains per hour. They were weighed before and after each experiment, but as the amount burned did not vary more than one per cent from 120 grains per hour, no correction was made.

As the strips of carbonized paper were flat, very much more light was given out in a direction perpendicular to the surface than in the plane of the edge. Two observations were taken of the photometric power, one in a direction perpendicular to the paper, and the other in the direction of the edge, and we are required to obtain the average light from these. If $L$ is the photometric power perpendicular to the paper, and $l$ that of the edge, then the average, $\lambda$, will evidently be very nearly

$$
\begin{aligned}
& \lambda=L \int_{\frac{1 \pi}{4}}^{0} \cos \alpha \sin \alpha d \alpha+l \int_{1 \pi \pi}^{00} \sin ^{2} \alpha d \alpha, \\
& \lambda=\frac{3}{2} L+\frac{\pi}{4} l .
\end{aligned}
$$

In the paper lamps we found $l=\frac{1}{3} L$ nearly; hence $\lambda=\frac{3}{4} L$ nearly.

\begin{tabular}{|c|c|c|c|}
\hline No. & Kind of Carbon. & Size of Carbon. & $\begin{array}{l}\text { A pproximate } \\
\text { resistance when cold. }\end{array}$ \\
\hline 580 & Paper. & Large. & 147 ohms. \\
\hline 201 & " & "، & $147 \quad 6$ \\
\hline 850 & " & Small. & 170 " \\
\hline 809 & "، & "، & 154 \\
\hline 817 & Fibre. & Large. & 87 \\
\hline
\end{tabular}
The lamps used were as follows:

The capacity of the calorimeter was obtained by adding to the capacity of the water, the copper of the calorimeter and the glass of the 
lamp and thermometer. The calorimeter and cover weighed 0.103 kil. and the lamps about 0.035 kil.

First experiment, No. 201 in calorimeter and No. 580 in photometer; capacity of calorimeter $=1.153+.009+\cdot 00 \%=1.169$ kil. The temperature rose from $18^{\circ} \cdot 28 \mathrm{C}$. to $23^{\circ} \cdot 11 \mathrm{C}$. in five minutes, or $1^{\circ} \cdot 75$ $\mathrm{F}$. in one minute. Taking the mechanical equivalent as $775 \cdot$, which is about right for the degrees of this thermometer, this corresponds to an expenditure of 3486 foot pounds per minute. The photometric power of No. 580 was $17 \cdot 5$ candles maximum, or $13 \cdot 1$ mean, $\lambda$.

When the lamps were reversed, the result was 3540 foot pounds for No. 580, and a power of 13.5 or 10.1 candles mean. The mean of these two gives, therefore, a power of 3513 foot pounds per minute for 11.6 candles, or $109 \cdot 0$ candles to the horse power.

To test the change of efficiency when the temperature varied, we tried another experiment with the same pair of lamps, and also used some others where the radiating area was smaller, and, consequently, the temperature had to be higher to give out an equal light.

We combine the results in the following table, having calculated the number of candles per indicated horse power by taking 70 per cent of the calculated value, thus allowing about 30 per cent for the friction of the engine, and the loss of energy in the magneto-electric machine, heating of wires, etc. As Mr. Edison's machine is undoubtedly one of the most efficient now made, it is believed that this estimate will be found practically correct. The experiment on No. 817 was made by observing the photometric power before and after the calorimeter experiment, as two equal lamps could not be found. As the fibre was round, it gave a nearly equal light in all directions as was found by experiment.

\begin{tabular}{|c|c|c|c|c|c|c|c|c|c|}
\hline \multicolumn{2}{|c|}{$\underset{\text { in }}{\text { Lamps used }}$} & \multicolumn{2}{|c|}{ Photometric Power. } & \multirow{2}{*}{ 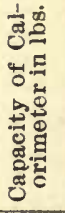 } & \multirow{2}{*}{ 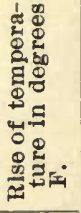 } & \multirow{2}{*}{ 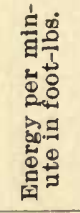 } & \multirow{2}{*}{ 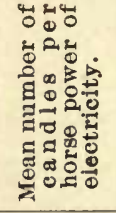 } & \multirow{2}{*}{ 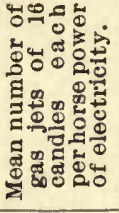 } & \multirow{2}{*}{ 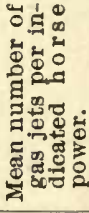 } \\
\hline $\begin{array}{l}\text { Calori- } \\
\text { meter. }\end{array}$ & $\begin{array}{l}\text { Photo- } \\
\text { meter. }\end{array}$ & $\begin{array}{l}\text { Measured } \\
\text { perpen- } \\
\text { dicular to } \\
\text { paper, L. }\end{array}$ & $\begin{array}{c}\text { Average, } \\
\lambda_{.}\end{array}$ & & & & & & \\
\hline 201 & 580 & $17 \cdot 5$ & $13 \cdot 1$ & $2 \cdot 57$ & 10.75 & 3486 . & $109 \cdot 0$ & 6.8 & $4 \cdot 8$ \\
\hline $\mathbf{5 8 0}$ & 201 & $13 \cdot 5$ & $10 \cdot 1$ & 2.82 & $1^{\circ} \cdot 62$ & 3540 . & 109.0 & 0.0 & 70 \\
\hline 580 & 201 & $3 S \cdot 5$ & $28 \cdot 9$ & 2.74 & $2^{\circ} \cdot 44$ & 5181 . & 2043 & 12. & \\
\hline 201 & 580 & $44 \cdot 6$ & $33 \cdot 5$ & 276 & $2^{\circ} \cdot 29$ & 4898 . & 2040 & & \\
\hline 850 & 809 & $19 \cdot 0$ & $14 \cdot 3$ & 2.81 & $1^{\circ} \cdot 14$ & $2483 \cdot$ & & & \\
\hline 809 & 850 & $12 \cdot 2$ & $9 \cdot 2$ & 2.79 & $1^{\circ} \cdot 54$ & $3330^{\circ}$ & $\int$ & $0^{\circ}$ & 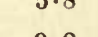 \\
\hline \multicolumn{2}{|c|}{817} & & $17 \cdot 2$ & 2.73 & $1^{\circ} \cdot 28$ & 2708 . & $209 \cdot 6$ & $13 \cdot 1$ & $9 \cdot 2$ \\
\hline
\end{tabular}


The increased efficiency, with rise of temperature, is clearly shown by the table, and there is no reason, provided the carbons can be made to stand, why the number of candles per horse power might not be greatly increased, seeing that the amount which can be obtained from the are is from 1000 to 1500 candles per horse power. Provided the lamp can be made either cheap enough or durable enough, there is no reasonable doubt of the practical success of the light, but this point will evidently require much further experiment before the light can be pronounced practicable.

In conclusion, we must thank Mr. Edison for placing his entire establishment at our disposal in order that we might form a just and unbiased estimate of the economy of his light. 


\section{ELECTRIC ABSORPTION OF CRYSTALS}

By H. A. Rowland and E. L. Nichols 1

[Philosophical Magazine [5], XI, 414-419, 1881; Proceedings of the Physical Society, IV, 215-221, 1851]

\section{I}

The theory of electric absorption does not seem to have as yet attracted the general attention which its importance demands; and from the writings of many physicists we should gather the impression that the subject is not thoroughly understood. Nevertheless the subject has been reduced to mathematics; and a more or less complete theory of it has been in existence for many years. Clausius seems to have been the first to give what is now considered the best theory. His memoir, 'On the Mechanical Equivalent of an Electric Discharge,' \&c., was read at the Berlin Academy in $1852 .{ }^{2}$ In an addition to this memoir in 1866 he shows that a dielectric medium having in its mass particles imperfectly conducting would have the property of electric absorption. Maxwell, in his 'Electricity,' art. 325, gives this theory in a somewhat different form, and shows that a body composed of layers of different substances would possess the property in question. One of us, in a note in the 'American Journal of Mathematics,' No. 1, 1878 , put the matter in a somewhat different form, and investigated the conditions for there being no electric absorption.

All these theories agree in showing that there should be no electric absorption in a perfectly homogeneous medium. A mass of glass can hardly be regarded as homogeneous, seeing that when we keep it melted for a long time a portion separates out in crystals. Glass can thus be roughly regarded as a mass of crystals with their axes in different directions in a medium of a different nature. It should thus have electric absorption. Among all solid bodies, we can select

${ }^{1}$ Commnnicated by the Physical Society, having been read May 14th, 1881.

${ }^{2}$ I have obtained my knowledge of this memoir from the French translation, entitled Théorie Mécanique de la Chaleur, par R. Clausins, translated into French by F. Folie: Paris, 1869. The 'Addition' does not appear in the memoir published in Pogg. Ann., vol. Ixxxvi, p. 337, but was added in 1866 to the collection of memoirs. 
none which we can regard as perfectly homogeneous along any given line through them, except crystals. The theory would then indicate that crystals should have no electric absorption; and it is the object of this paper to test this point. The theory of both Clausius and Maxwell refers only to the case of a condenser made of two parallel planes. In the 'Note' referred to, one of us has shown that in other forms of condenser there can be electric absorption even in the case of homogeneous bodies. Hence the problem was to test the electric absorption of a crystal, in the case of an infinite plate of crystal with parallel sides. The considerations with regard to the infinite plate were avoided by using the guard-ring principle of Thomson.

The crystals which could be obtained in large and perfect plates were quartz and calcite. These were of a rather irregular form, about 35 millim. across and ${ }^{3}$ millim. thick, and perfectly ground to plane parallel faces. There were two quartz plates cut from the same crystal perpendicular to the axis, and two cleavage-plates of Iceland spar. There were also several specimens of glass ground to the same thickness; the plates were all perfectly transparent, with polished faces. Examined by polarized light, the quartz plates seemed perfectly homogeneous at all points except near the edge of one of them. This one showed traces of amethystine structure at that point; and a portion of one edge had a piece of quartz of opposite rotation set in; but the portion which was used in the experiment was apparently perfectly regular in structure. The fact that there are two species of quartz, right- and left-handed, with only a slight change in their crystalline structure, and that, as in amethyst, they often occur together, makes it not improbable that most pieces of right-handed quartz contain some molecules of left-handed quartz, and vice versa. In this case quartz might possess the property of electric absorption to some degree. But Iceland spar should evidently more nearly satisfy the conditions. It is unfortunate that the two pieces of quartz were not cut from different crystals.

This reasoning was confirmed by the experiments, which showed that the quartz had about one-ninth the absorption of glass; but that the Iceland spar had none whatever, and is thus the first solid so far found having no electric absorption. Some crystals of mica, \&c., were tried; but calc spar is the only one which we can say, à priori, is per-

${ }^{3}$ [There is a gap in the printed article. On examination of the various plates if the Physical Laboratory of the Johns Hopkins University, some have been found on abont $2 \mathrm{~mm}$. thickness, which are probably those used in this research.] 
fectly homogeneous. Thus mica and selenite are so very lamellar in their character, that few specimens ever appear in which the laminæ are not more or less separated from one another; and thus they should have electric absorption.

\section{II}

In the ordinary method of experimenting with the various forms of Leyden jar, there are, besides the residual discharge due to electric absorption in the substance of the insulator, two other sources of a return charge. The surface of the glass being more or less conducting, an electric charge creeps over the surface from the edges of the tinfoil. In discharging the jar in the usual way by a connecting wire, this surface remains charged, and the electricity is gradually conducted back to the coatings, and thus recharges them. If, furthermore, the coatings be fastened to the glass with shellac or other cement, the return charge may be partly due to it; for we have between the coatings not merely glass, but layers of glass, cement, \&c., which the theory shows to give a residual discharge. Besides the coatings are not planes; and hence, as one of us has shown, there may be a return charge, even if the glass gave none between infinite planes. If the plates were merely laid on the glass without cementing, the same result would follow, since the insulator would then consist of air and glass in layers.

In the present research these were sources of error to be avoided, since the residual discharge due to the insulating plates themselves were to be compared. The condenser-plates were copper disks. These were amalgamated, so that there was a layer of mercury between them and the dielectric, which excluded the air and conducted the electricity directly to the surface of the dielectric: thus the condition of a single substance between the plates was fulfilled. The errors due to the creeping of the charge over the surface of the dielectric and that due to the plates not being infinite were avoided, the first entirely and the second partially, by the use of the guard-ring principle of Sir Wm. Thomson.

Plate IV represents this apparatus. The plate of crystal, $c$, was placed between two amalgamated plates of copper, $a$ and $b$, over the upper one of which the guard-ring, $d$, was carefully fitted; this ring, when down, served to charge and discharge the surface around the plate, $a$; and so the errors above referred to from the creeping of the charge along the plate, and from the plate not being infinite, were avoided. 
PLATE IV.

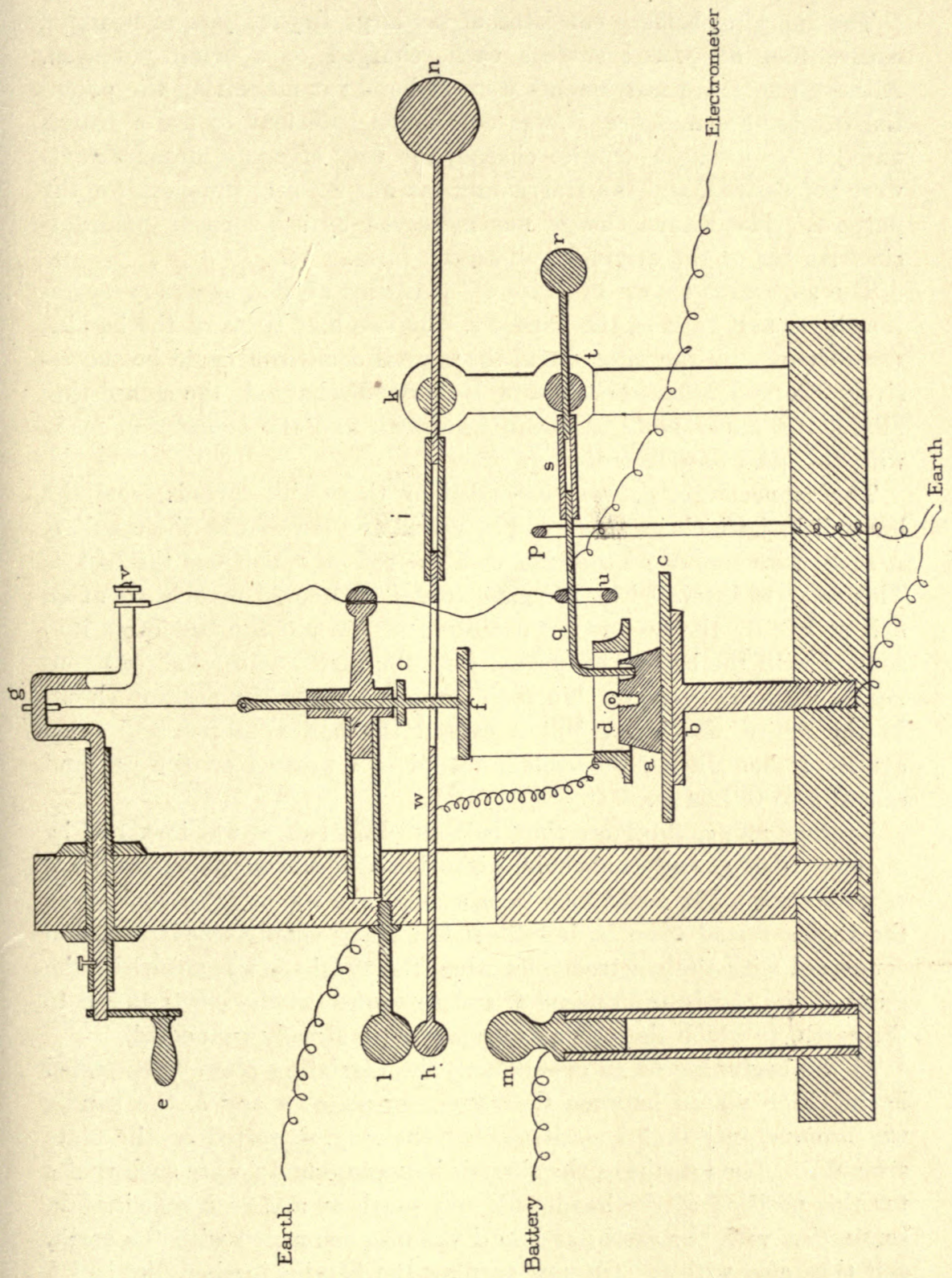


The charging battery consisted of six large Leyden jars of nearly a square foot of coated surface each, charged to a small potential. Although accurate instruments were at hand for measuring the potential in absolute measure, it was considered sufficient to use a Harris unit-jar for giving a definite charge; for very accurate measurements were not desired, and the Harris unit-jar was entirely sufficient for the purpose. The return charge was measured by a Thomson quadrantelectrometer of the original well-known form.

The apparatus shown in Plate IV performs all the necessary operations by a half turn of the handle $e$. By two half turns of the handle, one forward and the other back, the crystal condenser could be successively charged from the Leyden battery, "discharged, the guard-ring raised, the upper plate, a, again insulated, and the connection made with the quadrant-electrometer.

The copper ring, $d$, was suspended by three silk threads from the brass disk, $f$, which in turn could be raised and lowered by the crank, $g$. A small wire connected the ring with the rod on which was the ball, $h$. This rod was insulated by the glass tube $i$, and could revolve about an axis at $k$. By the up-and-down motion of the rod the ball came into contact with the ball $(l)$ connected with the earth, or the ball $(m)$ connected with the battery. When the cranks were in the position shown in the figure, the heavy ball $n$ caused the ball $h$ to rise and press against $l$; but when $f$ descended, the piece $o$ pressed on the rod and caused $h$ to fall on $m$.

Another rod, $q$, also more than balanced by a ball, $r$, was insulated by a glass tube, $s$, and connected with the quadrant-electrometer by a very fine wire. It could also turn around a pivot at $t$; so that when the ring $u$ rested upon it, it fell on the upper condenser-plate $a$, and connected with the electrometer; when the weight $u$ was raised by the crank $v$, the rod rested against $f$, and so connected the electrometer to the earth, to which the other quadrants were already connected.

At the beginning of an experiment, the insulating plate to be tested having been placed between the condenser-plates $a$ and $b$, the handle was brought into such a position that the ring, $d$, rested on the plate around $a$. The lengths of the threads between $d$ and $f$ were such that $o$ for this position of the handle did not touch $w$, and so $h$ remained in connection with the earth; and so $d$ was also connected with the earth, and thus also with $b$. On now turning the handle further, the ball $h$ descended to the ball $m$, and thus charged the condenser for any time desired. On now reversing the motion, the following operations took place: 
First, the ball $h$ rose and discharged the condenser.

Second, the guard-ring $d$ ascended.

Third, the $\operatorname{rod} q$, which had been previously in contact with $p$, thus bringing the quadrant-electrometer to zero, now moved down and rested on the upper condenser-plate $a$. Thus any return charge quickly showed itself on the electrometer. The amount of deflection of the instrument depends upon the character of the dielectric, its thickness, the charge of the battery, the time of contact with the battery, and upon the length of time of discharging.

\section{III}

In comparing the glass with the crystal plates, the electrometer was rendered as little sensitive as the ordinary arrangement of the instrument without the inductor-plate would allow. The electric absorption of the glass plates for a charge in the battery of two or three sparks from the Harris unit-jar then sufficed, after 20 or 30 seconds contact with the battery and 5 seconds discharging time, to give a deflection of about 200 scale-divisions, which were millimetres. The quartz and calcite plates were then alternately substituted for the glass, the same charge and the same intervals of contact being used, and the resulting deflections noted-two plates of each substance of the same thickness being used.

The results of the measurements are given in the following Tables, the effect of the glass being called 100 .

TABLE I.

(a)

April 12, 1880.

Charge of battery, 2 sparks. Contact, 30 seconds.

Glass (1st plate).............100.0

Quartz (1st plate).............17·1

" (2nd plate)...........20.0

Calcite (1st plate)........... 0.0

" (2nd plate).......... 0.0

(b)

April 13, 1880.

Charge of battery, 3 sparks. Contact, 20 seconds.

Glass (1st plate)............100.0

Quartz (1st plate)............. 19.3

Calcite (1st plate)........... 0.0 (c)

April 14, 1980.

Charge, 3 sparks.

Contact, 10 seconds.

Plates carefully dried by being in desiccator over night.

Glass (1st plate)............100.0 Quartz (1st plate)............. 10.7

Calcite (1st plate)........... 0.0

(d)

April 22, 1880.

Charge, 2 sparks.

Contact, 30 seconds.

Plate in desiccator since April 14.

Glass (2nd plute).............100.0

" (1st plate)............96.3

Quartz (1st plate)........... 13.4

" (2nd plate)........... 12.1

Calcite (1st plate)............ $0 \cdot 0$

" (2nd plate)........... 0.0 
TABLE II.

May 1.-Relative Effects for Different Intensities of Charge and TIME OF CONTACT

\begin{tabular}{|c|c|c|c|c|}
\hline \multirow{2}{*}{$\begin{array}{l}\text { Charge of } \\
\text { Battery. }\end{array}$} & \multirow{2}{*}{ Material. } & \multicolumn{3}{|c|}{ Deflections, in millimetres. } \\
\hline & & $\begin{array}{c}\text { Contact, } \\
5 \text { seconds. }\end{array}$ & $\begin{array}{l}\text { Contact, } \\
10 \text { seconds. }\end{array}$ & $\begin{array}{l}\text { Contact, } \\
20 \text { seconds. }\end{array}$ \\
\hline One spark... & $\begin{array}{l}\text { Glass (1st)..... } \\
\text { Quartz (1st)... } \\
\text { Calcite (1st).. }\end{array}$ & $\begin{array}{r}133 \cdot 0 \\
13 \cdot 0 \\
0 \cdot 0\end{array}$ & $\begin{array}{r}189 \cdot 3 \\
22 \cdot 7 \\
0 \cdot 0\end{array}$ & $\begin{array}{r}225 \cdot 0 \\
34 \cdot 3 \\
0 \cdot 0\end{array}$ \\
\hline Two sparks.. & $\begin{array}{l}\text { Glass (1st).... } \\
\text { Quartz (1st).. } \\
\text { Calcite (1st).. }\end{array}$ & $\begin{array}{c}\text { Off the scale } \\
24 \cdot 0 \\
0.0\end{array}$ & $\begin{array}{c}\text { Off the scale } \\
35 \cdot 0 \\
0.0\end{array}$ & $\begin{array}{c}\text { Off the scale } \\
50.0 \\
0.0\end{array}$ \\
\hline
\end{tabular}

These Tables seem to prove beyond question that calcite in clear crystal has no electric absorption. Quartz seems to have about $\frac{1}{9}$ that of glass; but we have remarked that quartz is not a good substance to test the theory upon.

Some experiments were made with cleavage-plates of selenite, which are always more or less imperfect, as the laminæ are very apt to separate. These gave, however, effects about $\frac{1}{3}$ or $\frac{1}{4}$ those of glass.

In order to test still further the absence of electric absorption in calcite, the electrometer was rendered very sensitive, and the calcite plates were tested with gradually increasing charges, from that which in glass gave 200 millim. after 1 second contact, up to the maximum charge (ten sparks of the unit-jar) which the condensers were capable of carrying. In these trials, the calcite still showed no effect, even with 30 seconds contact. During these experiments glass was frequently substituted for the calcite, to leave no question but that the apparatus was in working order.

It is to be noted that the relative effects of the quartz and the glass were different for dried plates and plates exposed to the atmosphere. This was possibly due to the glass being a better insulator, and thus retaining its charge better when dry than in its ordinary condition.

\section{IV}

Thus we have found, for the first time, a solid which has no electric absorption; and it is a body which, above all others, the theory of Clausius and Maxwell would indicate. The small amount of the effect 
in quartz and selenite also confirms the theory, provided that we can show that in the given piece of quartz some molecules of right-handed quartz were mixed with the left; for we know that the theoretical conditions for the absence of electric absorption are rarely satisfied by laminated substances like selenite or mica. If the theory is confirmed, the apparatus here described should give the only test we yet have of the perfect homogeneity of insulating bodies; for any optical test cannot penetrate, as this does, to the very structure of the molecule. 


\section{ON ATMOSPHERIC ELECTRICITY}

[Presented to the Congress of Electricians, Paris, September 17, 1881, and here translated from their Proceedings]

[Johns Hopkins University Circulars, No. 19, pp. 4, 5, 1882]

Among the subjects to be discussed by this Congress is that of atmospheric electricity, and I should like, at this point, to urge the importance of a series of general and accurate experiments performed simultaneously on a portion of the earth's surface as extended as possible. Here and there on the globe, it is true, an observer has occasionally performed a series of experiments, extending even over several years: but the different observers have not worked in accordance with any preconcerted plan, it has not been possible to compare their instruments, and even where absolute measurements have been obtained, the exact meaning of the quantity measured has not been perceived. Let us take, for instance, Sir William 'Thomson's water dropping apparatus, which is used at the Kew Observatory. This apparatus is composed of one tube rising a few feet above the building and of another tube near the ground, so that it is in the angle made by the house and the ground. This apparatus indicates a daily variation in the electricity of the atmosphere, but the result is evidently influenced by the conditions of the experiment. Another observer who should fit up an apparatus in another country might obtain entirely different conditions, so that it would be impossible to compare the results. Hence the necessity of having a system.

The principal aim of scientific investigation is to be able to understand more completely the laws of nature, and we generally succeed in doing this by bringing together observation and theory. In science proper, observations and experiments are valuable only in so far as they rest on a theory either in the present or in the future. We can as yet present only a plausible theory of atmospheric electricity, but the real way of arriving at the truth in this case is to let ourselves be guided in our future experiments by those which have hitherto been made on this subject. 
The principal facts which have been discovered can be stated in a few words. In clear weather, the potential increases as we go higher, at least for certain parts of Europe, and there is a diurnal and annual variation of this quantity which the presence of fogs causes also to vary. The first observers were inclined to attribute the electricity of the atmosphere to the evaporation of water, and an old experiment which consisted in dropping a ball of red-hot platinum into water placed on a gold leaf electrometer, was supposed to confirm this view. Even recently a distinguished physicist held this opinion in the case of electric storms. Now when a ball of platinum is thus dropped into water, the excessive commotion thus produced will certainly give rise to electricity; but to assert that this electricity is due to evaporation may very well be an error. It is true that occasionally a red-hot meteorite may fall into the sea, reproducing thus the laboratory experiment; but most of the water is evaporated quietly. Recently one of my students used under my direction a Thomson quadrant electrometer in order to investigate this question, and although he evaporated large quantities of different liquids, he did not find any trace of electrization. I hope to prove thus conclusively that the electricity of the atmosphere cannot be the result of evaporation.

Sir William Thomson thinks that the experiments which have been made hitherto indicate that the earth is charged negatively. This conclusion would certainly explain all the experiments hitherto performed in Europe; but the only method of reaching certainty on this point is to execute a series of experiments on the whole surface of the globe, and it is this method that I propose to-day. This series of experiments would furnish data for determining not only the fact of terrestrial magnetism, but also by the aid of Gauss's theorem the amount of the charge on the solid portion of the earth; however, this amount cannot be determined for the upper atmosphere. What we want to know is the law according to which the electric potential varies as we ascend on the whole surface of the globe and at the same instant of time, so that it may be possible to obtain the surface integral of the rate of variation of the potential over the whole globe. If the earth were ever to receive an increase of charge coming either from the exterior or from the upper atmosphere, this increase would be known. When, in the London Physical Society, I criticized the theory of Profs. Ayrton and Perry on terrestrial magnetism, I gave at the end of my paper a brief outline of a recent theory on auroras and storms, which was built on the hypothesis of the electrization of the earth. After mature reflec- 
tion I still wish to present to you this theory, which deserves to be thought of in mapping out a system of international experiments on atmospheric electricity.

Suppose Sir William Thomson's explanation is correct and that the earth is charged with electricity, let us examine what would then happen. If the earth were not exposed to disturbing causes, a portion of the electricity of the globe would discharge itself into the atmosphere and would distribute itself nearly as uniformly as the resistance of the air would allow. The exterior atmosphere thus charged would set itself in motion, and we should have winds produced by the electric repulsions, and this would last until the electricity had been distributed in a uniform manner on the earth and in the exterior strata of the atmosphere; when all would be still once more. An observer stationed on the earth would have no idea of the charge of the exterior atmosphere; but he would discover the charge of the earth by means of the ordinary instruments used in experiments on the electricity of the atmosphere, such as Becquerel's arrows and Thomson's water dropping apparatus. There would be another result which however could not be measured by observers situated on the earth, namely, the extension of the atmosphere beyond the limits determined by calculation. The rarefied air being electrified would repel itself, and possibly there would be then in the exterior atmosphere a region in which the pressure would vary very slightly for a great difference of elevation. We have learned from auroras and meteors that the atmosphere extends to a much greater distance than that indicated by Newton's logarithmic formula, but I think that what $I$ have said is the first rational explanation of this fact.

Observe now what would happen if the earth of which we speak were subject to the disturbing causes which exist on our globe; the most important of these disturbing factors are the winds and the general atmospheric circulation. This circulation constantly carries the atmosphere from the equator to the two poles, but with very little uniformity. However, near the poles there must be many points at which the air comes down towards the earth and thus shapes its course towards the equator. Now a body which is a bad conductor, like air, when it is charged tends to earry its charge along with it wherever it goes, and thus the air carries its charge until the moment when it descends towards the earth; then it will leave it behind in the exterior atmosphere, in accordance with the tendency of electricity to remain at the surface of charged bodies. The charge will therefore accumulate in the exterior atmosphere, until there is a great tension; the atmosphere 
will then discharge itself either towards the earth or through the rarefied air in the shape of an aurora. At these points the rarefied air probably heaps itself up to a greater height than elsewhere, which would explain the great height at which auroras are sometimes observed.

The equilibrium which existed previously at the equator would also be destroyed by the absence, at this point, of the primitive charge in the exterior atmosphere, and the earth would have a tendency to discharge itself towards the exterior atmosphere. Owing to the difference in the conditions at this point, this tendency will be apt to show itself by the storms which arise oftenest in the equatorial region. Thus the electricity of the earth would tend to circulate in the same way as the air from the equator to the poles and conversely.

But I do not intend to insist upon this theory here; I wish simply through it to bring out the importance of establishing on the whole surface of the globe a system of general observations on atmospheric electricity. Even if the theory is false, it is only by observation that the truth can be attained. In my opinion, it is almost unworthy of the advanced state of our sciences to-day, that it should be at present impossible for us to indicate accurately the origin of the energy which manifests itself in auroras and storms. For I have pointed out above that it is necessary to give up explaining these phenomena by the hypothesis of the production of electricity by evaporation.

I propose therefore that from this section of the Congress a committee be formed to examine what is to be done in order to establish on the whole earth, and especially in the polar regions, a systematic series of observations on atmospheric electricity.

\section{Editorial Note.-International Commission of Electricians}

[Professor Rowland sailed from New York, October 14, to attend an international commission of electricians, then about to assemble in Paris. Professor John Trowbridge of Cambridge sailed about the same date. These two gentlemen were selected to represent the United States government by the Department of State-Congress having made provision for the appointment of two civilian commissioners.

This official commission is the outgrowth of the congress of electricians which was held a year ago in Paris. That body requested the French government to invite other nations to unite in constituting three international commissions for the study of certain specified problems, namely:

I. A re-determination of the value of the ohm. 
II. (a) atmospheric electricity.

(b) protection against damage from telegraphic and telephonic wires-(paratonnerres).

(c) terrestrial currents on telegraphic lines.

(d) the establishment of an international telemeteorographic line.

III. Determination of a standard of light.

The study of atmospheric electricity was proposed to the congress by Mr. Rowland. After hearing his paper on this subject, the section to which he belonged adopted on his motion the following resolution which was subsequently approved by the entire congress.

Resolved that an international commission be charged with determining the precise methods of observation for atmospheric electricity, in order to generalize this study on the surface of the globe.

As Mr. Rowland did not retain his manuscript, the foregoing translation of the paper as it is printed in the Comptes Rendus of the congress has been made by Mr. P. B. Marcou and is printed here with the author's consent.] 


\title{
THE DETERMINATION OF THE OHM
}

\author{
Extrait D'une Lettre de M. Hexky A. Rowland
}

[Conférence Internationale pour la Detérmination des Unités Électriques. Procès-Verbaux, Deuxième Session, p. 37, Paris, 1884]

Les expériences relatives à la détermination de l'ohm ont été préparées à Baltimore au moyen d'une partie du crédit de 12,500 dollars alloué daus ce but, l'année dernière, par le Congrès des États-Unis.

Après une étude préliminaire, les appareils destinés à ces expériences ont été mis en construction en juin 1883. Les autorités de l'Université Johns Hopkins ont bien voulu mettre à ma disposition une construction qui est située en dehors de la ville, à l'endroit appelé Clifton, et qui a été transformée en laboratoire.

La source d'électricité qui servira aux expériences est une pile secondaire du système Planté, chargée par une machine dynamo-électrique actionnée par une machine à vapeur d'environ 5 chevaux de force.

Trois méthodes au moins seront employées pour la determination de l'ohm. La première repose sur l'induction mutuelle de deux circuits; j'ai dejà fait usage de cette méthode en 1878, mais dans les nouvelles expériences les dimensions des appareils seront considérablement augmentées; les bobines auront un mètre de diamètre.

La deuxième méthode est basée sur l'échauffement d'un conducteur par le courant électrique, le même fil étant échauffé successivement par le courant et par des moyens mécaniques. Les appareils employés seront ceux qui m'ont servi, en $18 \% 9$, pour déterminer l'équivalent mécanique de la chaleur. Afin d'éviter les pertes, le calorimètre sera rempli d'un liquide non conducteur au lieu d'eau. Pour mesurer l'énergie électrique, on a construit un électrodynamomètre ayant des bobines d'un mètre de diamétre.

La troisième méthode est celle de Lorenz. Pour determiner la vitesse du disque, il sera fait usage d'un diapason mû par un mécanisme d'horlogerie, construit par líönig, de Paris.

La comparison de l'unité de l'Association Britannique avec l'unité mercurielle est près d'être terminée; en dehors de cela, aucun résultat 
n’a été obtenu jusqu'à présent, mais je crois pourvoir donner mes résultats définitifs en novembre.

Comme ces expériences seront faites avec les précautions les plus grandes et dans des conditions très favorables, grâce à la générosité du Congrès, il est à espérer qu'aucune décision concernant la valeur définitive de l'ohm ne sera prise avant cette époque; de cette manière, les États-Unis et d'autres pays pourront accepter l'étalon arrêté.

Henry A. Rowland. 


\section{5}

\section{THE THEORY OF THE DYNAMO}

[Report of the Electrical Conference at Philadelphia in November, 1884, pp. 72-83, 90, 91, 104-107, Washington, $188^{\circ}$; Electrical Review (N. Y.), November 1, 8, 15, 22, 1884]

I will now proceed with the discussion of 'The Theory of the Dynamo-Electric Machine.' I only claim in the skeleton of the theory which I have here prepared to give a few points which may be of interest and possibly of value to those who are constructing these machines.

The principal losses of the machine I put down under the following heads: (1) Mechanical friction; (2) Foucault currents in the armature; (3) energy of the current used in sustaining the magnet; (4) self-induction of the coils; (5) heating of the armature.

Of course the efficiency of the machine would be equal to the whole work of the machine minus the different losses divided by the work, namely:

$$
E=\frac{w-l_{1}-l_{2}-e t c .}{w}
$$

Thus, when the losses are known, the efficiency of the machine is known.

The mechanical friction I shall not discuss.

With respect to Foucault currents in the armature, by dividing up the armature in the proper way, we can get rid of most of these. It is very often effected in the Siemens armature by dividing up the armature into discs.

I have purposely omitted the loss due to change of magnetism in the armature as the armature revolves. I drew attention to this fact several years ago. It has been recently experimented upon and found that, although there is some heating effect, it is very small indeed.

With respect to the energy used in sustaining the magnet, if the magnet were of steel there would, of course, be no loss. The only reason for not using a steel magnet is that the field is comparatively weak. The field of a steel magnet is, I suppose, less than one-third of the field due to a good electro-magnet; the two could not be made equal by any possible means. Therefore, in most dynamo machines, the magnet is produced by the current. 
It is a question what the form of the magnet and the position of these coils should be in order to get the greatest field with the least expenditure of energy. I have one or two propositions to make on this subject which I think are of some interest.

The first proposition I have to make is that a round magnet is better than one of elongated cross-section. If the coils are long, and they are usually long enough for the purpose, although the theory assumes an infinite length, the magnetic force at any time acting on a round iron core is exactly the same as on an elongated core. But the area of a circular section is much greater than that of an elongated section of the same circumference, and therefore the same amount of wire which would be used to go around the elongated magnet, would, if extended on a circular section of the same circumference, surround much more iron.

The principal object of making an elongated magnet is that it may include the whole length of the armature. Most makers who adopt this form think it better to elongate the cross-section than to have a long pole piece. But we have seen that the round form is more efficient in general than the elongated form, and the only question is whether it will be more efficient in this particular case. I shall proceed in this theory upon the known fact that we can consider lines of force as if they were conducted by the iron and the air outside. The conductivity of the iron for the lines of force is very great, much greater than that of air. I experimented on it many years ago, and my idea is that it varies (according to the degree of magnetization) from several hundred up to 5,000 times that of air. The conductivity for iron is very great, especially for wrought iron; for cast iron it is probably less. Therefore the lines of force will be conducted down through the iron from any point over a circular cross-section very nearly as easily as they are from an elongated cross-section, and the saving in the wire will be considerable.

I have another proposition to make with respect to the magnet, and that is that one circuit of the lines of force is better than a number. There is a loss from having a number of electro-magnets, even if they are round. For this reason, that the same magnetic force is acting in each of thesc coils provided there is the same number of wires per unit of length; and the same wire will go more times around the same iron concentrated in one magnet than when subdivided into several, and will, therefore, act upon it with more magnetizing force.

That proposition not only applies to this form of magnet (Fig. 1), 
but it also applies to the form where we have the armature revolving between two magnets like this (Fig. 2), because we can turn this lower magnet over and bring the two together. The circuits of the lines of force are around in this direction and in this (arrows, Fig. 2). So that there are two circuits of the lines of force instead of one. The energy expended for a given amount of work will be less with this form (Fig. 1) than with this (Fig. 2). That is of very great value to makers of machines.

The theorem applies to a number of those old machines where there

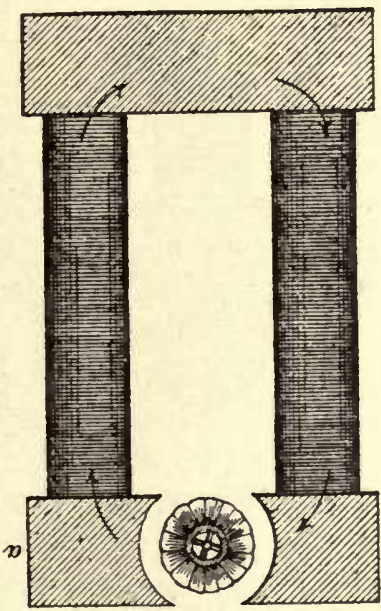

Fig. 1.

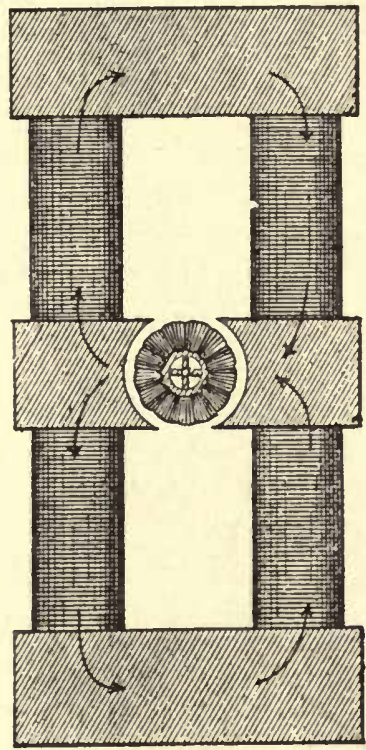

Fio.2.

was a very large number of little magnets revolving around other little magnets. More work is used in sustaining the magnets in that form of machine than in the more modern form where we have only a few circuits.

I had a number of drawings made of magnets in the Electrical Exhibition, and I find very great difference in this respect; more difference where Siemens armatures are used than in any other kind. In discussing these drawings I do not give any names, nor say whether one machine as a whole is better or worse than another.

First, I will discuss the general forms of the magnet, and then I wish to say something in respect to the form of the pole pieces that inclose 
the armature. Of course this form belongs both to the Gramme ring and the Siemens armature. Most modern machines are of this nature, either Gramme or Siemens, and we may consider them both one if we wish.

We will now proceed with respect to the field in this form of magnet (Fig. 3). The lines of force proceed down the magnet, and are supposed to go across here $(a b)$, where wires wound around the revolving armature cut them, and so produce a current. It is evident that any lines which escape across this open space (arrows) are lost. If there

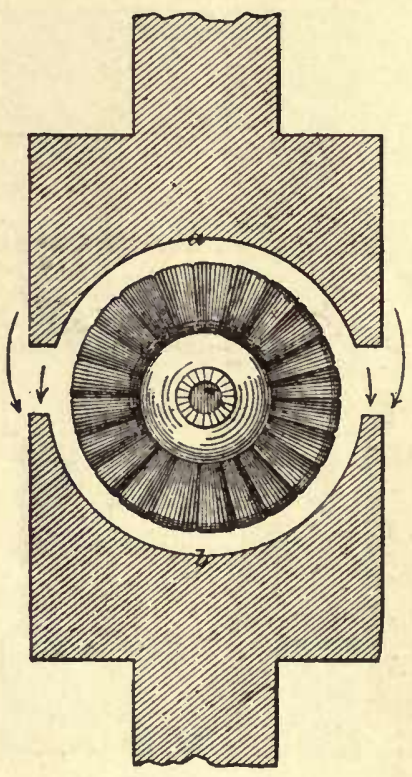

FIG. 3.

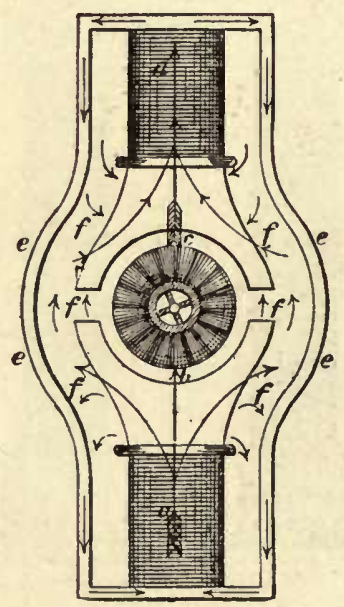

Fig. 4.

was any leakage of the wire around the magnet, the current, instead of going around the magnet, would go off somewhere else, and we should consider the machine defective because there was a loss of the current. So if any of these lines of force, instead of going directly across there $(a b)$, go across the open space (arrows), as they naturally would do, all those lines of force are lost, and we would have to add so much more current in order to make up for this outside loss. I have an illustration of such losses of lines of force from a drawing, which I will give you (Fig. 4).

This machine has two magnets-one above and one below. The lines 
of force pass up through here $(a b c d)$ and then out and around through here (ee), \&c., to complete the circuit. As I saw the machine in the exhibition these outside pieces $(e e)$ were closer to the poles of the magnets than I have drawn them. If they are put too near, some lines of force, instead of passing across the field of force, where the wires revolve, as they ought to do, pass off at these openings, the circuits going around in this way (arrows $f f$ ). In this case there is a loss due to leakage of the lines of force, and we shall therefore have to expend

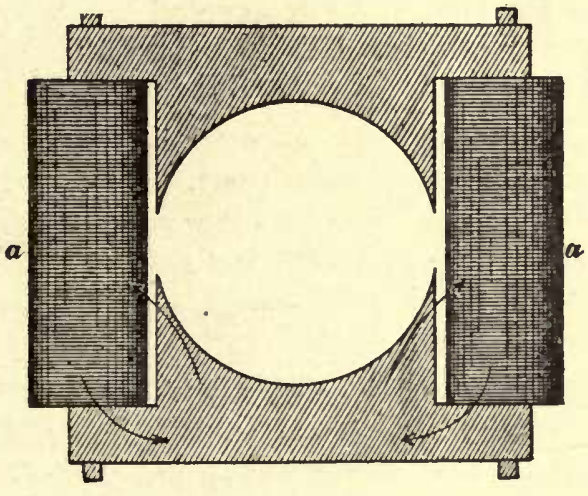

F1G. 5.

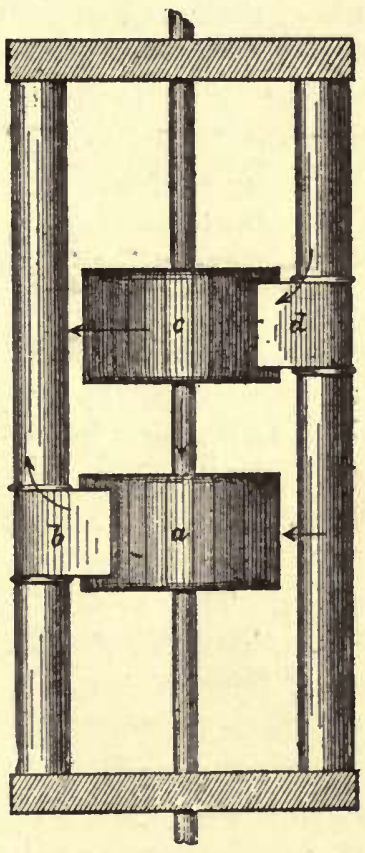

Fig. 6.

more energy in keeping up the magnet. There is energy expended in keeping up the field outside as well as in keeping up the field through the armature. It is important that this point should be considered. These questions, ' How many lines of force go across this opening and are effective in producing the current, and how many escape off without passing through the opening and are lost?' are just as important as the question of the leakage of the current in the wire. There are defects in many of these machines in that respect. In this form of machine (Fig. 1), where there is a simple circuit, this magnet has to be 
attached somewhere. Very often the magnet is turned vertically, poles downward, and attached to a cast-iron bench. I have no doubt that some lines of force are lost (not much perhaps) in passing across from the magnet to this iron bench. The makers of the machine, I suppose, considered this to some extent, but what is needed is measurement on that point.

Here is another form of magnet (Fig. 5). That machine would be defective. It has two magnets and two magnetic circuits in the place of one, and many of the lines of force probably make little private eircuits of their own around in that way (arrows). Those lines of force are of course lost, and it is more or less defective in that respect. It would be better to diminish the number of magnetic circuits to one.

(I am only giving a general idea of the principle of these machines, and I do not refer to any in particular.)

It is also important that these lines of magnetic induction shall find easy passage around in order to produce the most intense field. Thus the opening between the armature and pole pieces must be made as small as possible, in order that the lines of force may find easy passage across it. Everybody recognizes that. Suppose we had a machine made in the following manner (Fig. 6), in which there is a magnet with a Gramme ring here $(a)$, and pole piece here $(b)$, a ring here $(c)$, and pole piece here $(d)$, but no pole pieces opposite these. How are the lines of force to pass around? I do not know that it would be easy to see how. They evidently go around here (arrows) and get to the other side the best way they can. There is no easy passage around for the lines of force in this case.

A Member. May they not to some extent follow the shaft?

Professor Rowland. It is evident that if the shaft is made large enough some go along the shaft in that way (arrows), but there is no easy way for them to get around.

I have here a formula for the amount of work which one has to expend upon a magnet in order to produce a certain effect. I will take the case which I have considered most efficient, where there is one magnetic circuit. It is an original idea of Faraday that these lines of force are conducted. We suppose the lines of force to pass through the iron and across the opening in this way (arrows, Fig. 1), and they are caused to do that by what may be called the magneto-motive force of the helix.

I will just obtain an expression for the number of lines of force $B$. This is not the quantity which Maxwell considers, but it includes the 
whole number of lines of force which pass through the magnet. We may write $B$, proportional to $N$, the number of turns of the wire around the magnet, and $C$, the current; and inversely proportional to the resistance to these lines of force in going around the circuit. The resistance to the lines of force is proportional to $L$, the length of the iron of the system, divided by $S$, the cross-section of the magnet, supposing it to be uniform, into $\mu$, the magnetic permeability of the iron (or the conductivity of the iron for the lines of force). This quantity $\mu$ varies with the current, and can readily be obtained. Some years ago I gave a formula for it. It can be expressed simply as dependent upon the magnetization of the iron and a constant depending upon the iron alone. We have something more to add:

Let $l$ be twice the width of the opening between armature and pole piece, and $A$ the area across which the lines of force flow; then we have to add $\frac{l}{A}$ and another quantity, which we can call $p$, which depends upon the resistance of these lines of force which escape in all directions and represents the loss due to that escapement. Thus we have the final value for the number of lines of force (or rather induction) in the magnet

$$
B=\frac{N C}{\frac{L}{S \mu}+\frac{l}{A+p}} .
$$

This gives us an equation which may be solved with respect to $\mu$. The curve for the magnetic permeability is of this nature (Fig. 7). It will be of a more or less flat form, according to the value of $l$ and $p$. Therefore, in increasing the magnetic force upon the magnet, it becomes easier and easier to magnetize it until a certain point is reached, and after that it becomes harder and harder. In practice the core should have sufficient cross-section to produce a very strong magnetic field, but not so great as to require too much wire to wind it. The two must be balanced, which can only be done by calculation or, better, by experiments on the machine. By examining the force of the magnet at each point, and in that way getting an idea of how these lines of force go, we can see whether the cross-section of the core is large enough to produce all the lines of force necessary for our purpose or not. Of course, in order to have sufficient magneto-motive force to send lines of force across the opening in sufficient quantity, we must have sufficient wire. As the thickness of the coil is increased, we have to use more wire in proportion for a certain diameter of core, which is a disadvan- 
tage, since each coil acts very nearly the same as every other in producing force. But if the core is very short indeed, wire must be piled on it to a very great extent in order to get sufficient magneto-motive force, and as iron is cheaper than copper it might be better to lengthen out the core. I do not know where the lengthening should end, but I should suppose when the requisite wire on the magnet makes a moderately thin layer. Of course, as we lengthen out the magnet, the resistance of the circuit to magnetization becomes greater; but that is a very small quantity. I do not suppose the increase is very much for a considerable lengthening of the magnet. As I said before, the magnetic conductivity of iron is many times greater than that of air, and we can lengthen out the cores without producing much loss on account of that lengthening.

Some persons have suggested that there might be a slight gain from

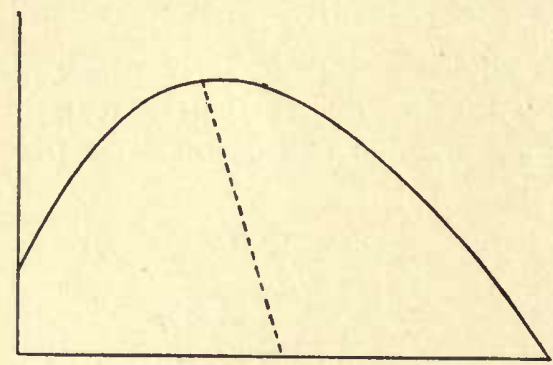

FIG. 7.

the fact that iron, after it has been magnetized a great number of times in the same direction, rather likes to be magnetized in the same direction afterwards. If the core is made of any material similar to steel, such as wrought iron or anything of that sort, it might be possible to have some gain from the coercive power of the magnet. There would be loss from that cause at first; but from the continual use of the machine I think it very likely the iron might get a set in the direction of the force. If the core were of steel, for instance, it might be that one could send a strong current through at first and magnetize the steel, and then be able to diminish the current considerably and still keep up a very large magneto-motive force. I do not know how practical that would be, but it seems to me that one could produce a very strong field in that way. In the commencement of the operation of the machine, we would have to send a powerful current to magnetize the steel, and then, without stopping the current, to diminish it. Then the set of 
the steel would be in the same direction with the current and produce the field with less expenditure of energy than if it were simply iron.

There is no difference between a shunt and a series machine. The magnetizing force on the magnet $I$ have set down as proportional to the number of turns multiplied by the current; that is, proportional to the cross-section of the coils multiplied by the current per unit of crosssection, so that the magnetizing action can be the same either from a strong current or a weak current. Therefore, if the exterior dimensions of the coils are the same in both cases, the same energy is expended in each in order to produce the same force, so that there is no

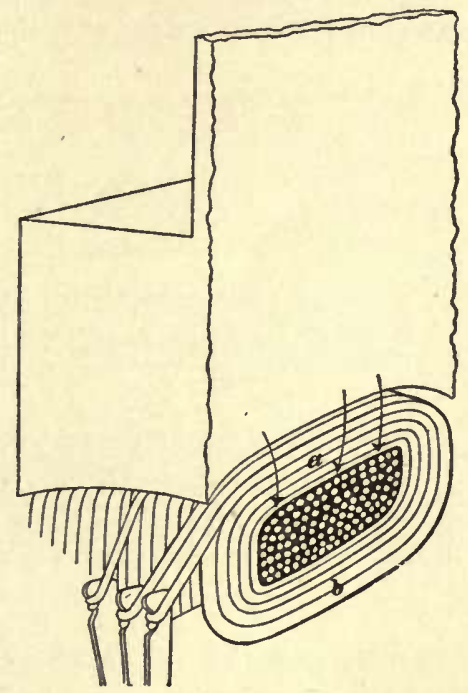

FIg. 8.

difference between a shunt machine and a series machine as far as the economy of the magnet is concerned.

I do not wish to take up too much of your time, and will go on to the heating of the armature. Of course the amount of energy expended in the heating of the armature will be dependent on the resistance of the armature. It is well known that the efficiency of the circuit will merely depend upon the relation between the resistance of the armature and the exterior circuit.

There is one other point in regard to losses; ' dead wire,' I think, is the technical term for it; I mean that portion of the wire which does not cut the lines of force. In the Gramme pattern the armature is 
inside of the rings. In the Siemens pattern the coils are around the ends of the armature. In a section of the Gramme ring (Fig. 8), the outside portion of the wire $(a)$ is active, since the lines of force follow the core and the outside of the ring around; but the lines of force do not go through the core of the ring, so that the inside portion (b) is dead, so that we can say nearly half the wire is dead wire. In the Siemens armature one cannot see immediately how much dead wire there will be, because it depends upon the length of the armature. The wire is wound around in that way (Fig. 9), and this portion ( $a a)$ is active, and this portion $(b b)$ is dead. If the armature is very thick we would have more dead wire than when it is simply long. I cannot say which has the more dead wire, but I dare say the Gramme has more

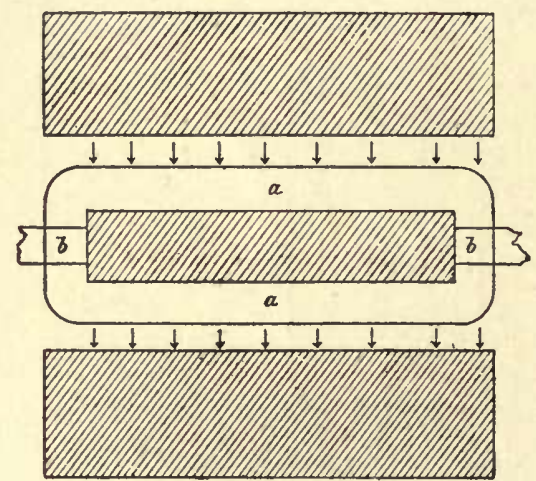

Fig. 9.

than the Siemens. Furthermore, either in the Gramme ring or the Siemens armature (Fig. 10) we have the lines of force running across here (arrows); that portion is active; but these portions ( $a a$ ) in between the poles are dead, and when the armature revolves we have the lines of force turning around, and I think that would add more dead wire. I believe an attempt has been made to throw out these coils.

There is no necessity to go further. As I have said, the efficiency of the circuit depends upon the ratio of the resistance of the armature to the resistance of the wires, and therefore, as far as this point is concerned, any machine can be made as efficient as one pleases by putting in greater and greater external resistance. But as the magnet remains the same, we would find a point where the efficiency as a whole would not increase for an increase of external resistance, but would actually diminish. There are other things to be taken account of, such as losses 
due to the self induction of the coils which produce sparks in them. I have requested Professor Fitzgerald to take up that point, and will leave it for him to consider.

There is another point with regard to the dynamo which can be treated in this simple manner with no use of the calculus. This is rery simple reasoning if you only know the principles. I shall consider two machines similar in all respects, except that one is larger than the other, or rather consider one machine, and see what the effect will be when that machine gradually changes in size.

The point from which we start shall be that the magnetic field is constant in the two machines. For, owing to the fact that there is a limit in the magnetization of a magnet, we cannot have a field with more

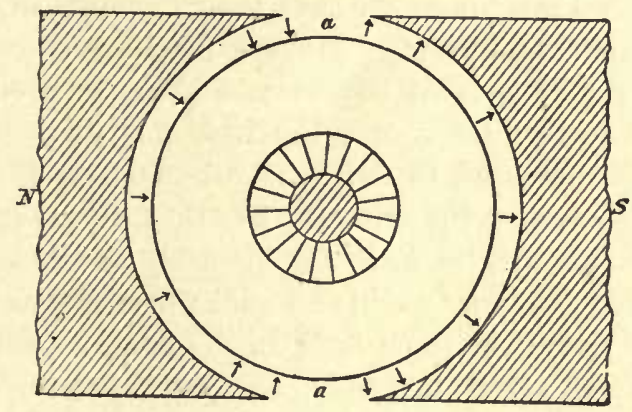

FIG. 10.

than certain strength produced by iron, and I will suppose that the strength is reasonably near that maximum for iron. It cannot be up to the maximum strength, of course, but somewhere near it. I made some experiments many years ago upon an ordinary magnet, the results of which were published in Silliman's Journal, by means of what I call the magnetic proof plane. (Am. J. Sci., vol. 10, 1875, p. 14.) It applies beautifully to dynamo machines, and I obtained everything with it that $I$ have referred to here. If I remember right, $I$ found in that magnet about one-third of the field that an iron magnet could possibly have.

It is theoretically possible to get a force equal to the magnetizability of the iron, but practically, I suppose that instance is about the case of the ordinary dynamo machine. We start, then, with the supposition that the field of force in the two machines, one of which is larger than 
the other, is constant. That is to say, the magnetizing force at any point of one machine is equal to that at a similar point in the other machine. In making a drawing of the machines, it would not matter about the scale of dimensions; the force at a certain point is a certain amount whatever the scale.

Next consider what must be the current through the wire in the two machines. There are the same numbers of turns of wire around the magnet, and everything is the same except the dimensions. Consider the current passing around the coil of a tangent galvanometer. If the galvanometer grow, in order to produce the same effect at the centre (and not only at the centre but at every point), the current must increase in direct proportion to the radius of the coil. When the coil is twice as large the current must be twice as large, in order to produce the same force at every point. Thus, if there is no difference in the material of the two machines, we have their currents in direct proportion to their linear dimensions. Make a machine twice as large and the current in the coils must be twice as great to produce the same magneto-motive force. Of course the wire has increased in size; if the machine has increased to twice its original size the cross-section of the wire has increased four times. In other words, from that cause the current per unit of area will vary inversely as the square of $l$, the linear dimensions; and since we have found the current to vary directly as $l$, in order to retain the same force in the field, by a combination of the two results, it varies inversely, as $l$. Therefore, so far as the magnets are concerned, the heating effect, which depends upon the current per unit of cross-section, will decrease with the size, while the surface will increase in proportion to the square of the size. There will, therefore, be less danger of heating in a large magnet than in a small magnet, but this is only with respect to the magnet.

The resistance of any part of the machine varies, of course, directly as the length of the wire, and inversely as the cross-section. The crosssection varies as $l^{2}$, so that resistance varies inversely as $l$. Therefore the larger the machine the less the resistance; one machine being twice as large as the other, the resistance will be half as great. This applies not only to the work of the magnets, but to the work of the armature.

I will now consider the electro-motive force. The electro-motive force is proportional to the product of the current and the resistance, or we may write $E=R C$. We have the current proportional to l, and the resistance inversely proportional to $l$; therefore the electro-motive force is constant. As we are running the machine, it turns out that 
the electro-motive force does not vary with the size, but we shall presently see how this is modified so as to get greater electro-motive force for the larger machine.

The work done is $C^{2} R$ in any part of the machine, or in the whole machine, just as you please. This varies directly as $l$. Therefore the one machine which is twice as large as the other requires twice as much power to run it, and twice as much electrical energy comes out of it. But it is to be remenbered that the weight of the machine varies as $l^{3}$, and we only get work proportional to $l$ out of it.

So far as results go, we have constructed two machines which differ only in size. The efficiency of these two machines is a constant quantity. That will be rather startling to some, who think a large machine is more efficient than a small one. As far as we have gone in any two machines, one of which is simply larger than the other, the efficiency is the same.

But if we calculate the angular velocity of the armature to keep the proper current we shall find that it varies inversely as the square of the linear dimensions. In other words, in one machine twice as large as another the velocity of the armature must be only one-fourth as great in order to produce the proper current in the wires. This takes account, I think, of every irregularity in the machine. The two machines are exactly the same in every respect. I have not added the loss for the self-induction of the coil. I have an idea that this also should be taken into account, but Mr. Fitzgerald will consider that point.

Now the question comes up, can we increase the velocity of the armature above that point? Is it practically necessary that we should run one machine at one-fourth of the angular velocity if it is twice as large? It is a practical question; but I should certainly think the velocity was not in that proportion. I should think it would be more nearly inversely as the size and not inversely as the square of the size. If so, then by so arranging the wire of the armature as to increase the proportion of external resistance we can have the same current per unit of section when running the armature faster and the same electromotive force. If we do that, this whole theory applies; but we shall have increased the external resistance of the machine in comparison with the resistance of the armature, and when we do that we increase the efficiency of the machine.

I think it is from this cause that we find large machines more efficient than smaller ones; but it is also evident that there is a limit to this, 
which can only be obtained, I suppose, from practically making the machines and seeing how much faster they may be run without flying to pieces. As far as this theory goes, the increase comes not from the size of the machine, but from the fact that we can get a greater electromotive force with the same angular velocity, and so can reduce the internal resistance in proportion. In very large machines we can make the wire with one turn, not several turns-simply bars on the machines. We thus decrease the resistance of the machine, and at the same time, if we run it above this proportion which I have pointed out, we obtain the proper electro-motive force. In other words, the proper electromotive force is more easily obtained from the large than the small machine, because it is not practically necessary to decrease the velocity so as to keep it inversely as the square of the size.

\section{[Discussion by Professor Elihu Thomson and others.]}

With respect to Mr. Thomson's remarks, I am very glad to see the matter taken up in this spirit and to have my principles intelligently criticised. However, there was one remark which I wish to state immediately as an error, of course, with regard to the steel. Steel can be magnetized to exactly the same degree as soft iron. There is no difference between soft iron and steel in that respect, except that we require an immensely greater force to magnetize steel to the same extent as iron. There are some old papers of mine, which were published in the 'Philosophical Magazine,' I believe, in 1873, relating to experiments where I took iron and steel and several other metals, and showed that the maximum magnetization was the same in all cases.

But with respect to a number of statements with regard to flat magnets and round magnets I am very glad to see my remarks criticised in the manner that they were, because it shows the need of exactly what I stated; and that is experiments upon this subject. The question is one of quantity. My reasoning gave results in one direction, and Mr. Thomson gave reasons for making the magnet in another way, and it is a quantitative question of course as to which is the best; and for that reason I want very much to see experiments made in the manner which I have described by means of this 'magnetic proof plane,' so as to find out what the escape of the lines of magnetic force in all cases is.

I think we can decide on one point that was brought up without any trouble, and that is with respect to the dynamo made with extended pole piece (Fig. 2), where it was assumed that the lines of force had a 
teudency to go in a particular direction, that it was a sort of gun shooting the lines of force through the armature. That is not true, because they do not have any tendency to go that way at all, and we would only add that much to the area of the end of the magnet. Very few lines of force will go out there, and by putting this additional magnet on we add to the area of the magnet. The lines of force will go out at the sides probably in greater numbers than they would at the end, so that I do not think that particular objection holds in that particular case. It is a question of quantity; the thing should be measured and found out. I see very plainly in my own mind that more lines of force would go out the side by adding this iron here (Fig. 2) than would go out at the end of it by leaving it vacant, as in Fig. 1. But it is a matter of mere opinion. Another reason for having fewer magnets is that the surface is greater in the case of the larger number than of the smaller number for the lines of force to escape from.

There was another point brought up here with respect to the machine which was made in this way (Fig. 4). It was stated that there was some gain from the magnetic action of this coil on the iron outside. There is undoubtedly a gain: the question is how much, and whether more lines do not escape than would make up for that. With no experiments to go on, it is a case of judgment. My own judgment would be that there would be very little gain; but, as I said before, the thing should be measured, and then we could find out about that point.

[Discussion by Professors Sylvanus Thompson and Anthony and others.]

I am very glad that that point of hollow magnets has been brought up, as I think that the question of hollow magnets, hollow lightning rods, and a great many similar things, causes more difficulty, especially to practical men, than almost anything else. It can be explained in a very few words. Take a hollow bar having the magnetizing coil around it acting to send lines of force along it. They have got to go out to make their complete circuit. They could only end at a certain point if we had free magnetism, that is, a separate magnetic fluid. I speak not from a physical sense but from a mathematical point of view. The principal resistance to the propagation of these lines of force is in the air and not in the magnet. If we take away a large portion of the interior of that magnet we will have the surface the same as it was before, and consequently the external resistances are the 
same. In such a case as that we leave the magnet about as strong as it was before. But that would not be the case if we compress magnetism until we get it up to the point of magnetization of the centre. In that case we should need the whole mass, and it is almost impossible to magnetize to any extent without the centre coming in. It depends on the length of the bar. If we bring the bar around, making a complete magnetic circuit of the thing, so that the lines of force do not have to pass out into the air at all when we put a wire around it so as to wind it like a ring at every point, in that case the whole cross-section becomes equally magnetized, if it is not bent too much. If it is a large ring of small cross-section, it is perfectly magnetized across from side to side. We know that perfectly well; it is a result of the law of conservation of energy. The case of dynamos is like that. We require the whole cross-section to transmit these lines around. The resistance to the magnetization comes partly from this opening and partly from the iron. We have no gain in making these cylinders hollow; indeed we rather increase the outside surface to let lines of force flow into the air. In the case of a dynamo machine, the solid form is not only desirable, but by far the most efficient.

I have thought of that matter a great deal, and experimented upon it. Indeed this closed circuit is the very idea from which the permeability of the iron is determined. All the calculations upon that subject are based upon that law. I think there can be no doubt that in the dynamo the solid form is the proper form, and that the whole crosssection is effective. The whole cross-section of a round piece is just as effective as the whole cross-section of a flat piece. The flat piece exposes more surface to the air, and there is more surface for the force to escape from. That is another reason for not making the magnets flat. The round form is that in which there is the least surface, and therefore the least liability of the lines of force to escape. You can conduct the lines of force by a round piece to any point you desire much better than by a flat piece.

\section{[Discussion by Professor Sylvanus Thompson.]}

I do not know that the theory bears upon the solidity of the core. of course, the more iron in there the better is the efficiency of the machine. I suppose there would be no objection to dividing that cylinder up into a number, so that the Foucault currents could not exist, if the exterior form was round; but I do have an objection to 
making it any other shape. Indeed, currents could be more thoroughly eliminated by dividing up the cross-section than by making it of a very elongated form.

\section{[Discussion by Professor Elihu Thomson.]}

I do not like to rise so often, but I think there is some misapprehension. I have not said anything about large masses of iron. There are the same masses of iron in my method as in any other. The only question is as to making them round or elongated. Of course by dividing this core up it becomes similar to a core of the Ruhmkorff coil, and the currents change very rapidly. From Professor Sylvanus Thompson's remarks, I thought that that was desirable. One cannot say that the current is transferred from the core to the wires outside. The same current might take place, and, if the resistances are the same, would take place in the wires outside in both cases. By lengthening the time of action one decreases the electro-motive force or decreases the external current. If the time is ten minutes one would have one electro-motive force for the external current; if it is five minutes, the electro-motive force would be somewhere near twice as great as before, the whole quantity of electricity passing being the same in both cases. 


\section{ON LIGHTNING PROTECTION}

[Report of the Electrical Conference at Philadelphia in November, 1884, pp. 172-174; Washington, 1886]

As this is an important question, especially in some of the Western States, I will say a few words.

In order to protect buildings from lightning we must have a space into which the lightning cannot come, and have the house situated in that space. What sort of a space do we know in electrical science into which electricity cannot enter from the outside? It is a closed spaceI mean a space inclosed by a very good conducting body. All the lightning in the world might play around a hollow copper globe and it would not affect in the slightest degree anything inside the globe; but the the walls of the vessel need not be solid metal. Of course, if solid, it is all the better; but if it is made of a net-work of very good conducting material it would protect the inside from lightning strokes. A spark striking on one side of such wire cage would find it easier to go around through the wire of the cage to the other side than it would to go through the centre. This is Maxwell's idea, with reference to protection of houses from lightning, viz., to enclose the house in a rough cage of conducting material. Suppose, for instance, this box is the house, and suppose we start from the roof and run a rod diagonally to each corner and thence down to the earth. We thus make a rough cage. Of course there are openings on the sides; and if we wished to make a better protection we could put rods down the sides wherever we wished. Now, there is ground underneath the house, and the lightning might, by jumping across the centre, find a good conductor through the middle of the house and go down to the earth in that way. How do we prevent that? By running the lightning-rods clear across underneath the house. Then the lightning would find it easier to go around the house than to jump across, even if there were a good conductor through the middle. A house inclosed in a cage of that sort would be perfectly protected, even if it were a powder magazine, or anything of that sort. Of course, in the case of petroleum storage reservoirs, where fumes are given off, there would be danger then, as the stroke might ignite the 
fumes of the petroleum. That would not be the case of a powder magazine. The protection in that case could be made perfect.

It is not necessary to have lightning-rods insulated. Indeed the question is, can we insulate a lightning-rod? We may insulate it for a small potential, but lightning coming from a mile or two to strike a house is not going to pay any attention to such an insulator; we may just as well nail the lightning-rod directly to the house as far as that goes.

The idea of having the lightning-rods inclose the bottom as well as the sides of the house is very important, because we do not know, and we hare no right to assume, that the earth is a good conductor. We are perfectly certain if the earth forms a good conductor that then the lightning could go down at the sides into the earth. By inclosing the house in a case both below and above we obviate all that difficulty, and it makes no difference whether the earth is a good conductor or not.

I am glad of this public opportunity to say something with regard to a peculiar form of lightning-rod; it is in reference to a form of a rod shaped like the letter $U$. I think the idea is that the lightning strikes on one side, and that it goes down and has inertia and flies up again. The company which advocated this idea had the impudence to bring a lawsuit against a scientific man who said it was a humbug. A company of course can make a great deal of trouble to one man; but when there is such a gross humbug as that around, one would like to undergo the danger of a lawsuit. There is nothing scientific about it; it will endanger life in any house in which it is placed.

Mr. Scotr. I would like to ask whether a building constructed of iron would not be completely protected from lightning?

Professor Rowland. Yes, if it has a floor of iron too. If a gas-pipe came up into the centre the lightning might find it easier to go across to the pipe than to go around. But if we made a floor of iron the lightning would find it easier to go around than across to the pipe. It must be an entirely inclosed house.

Mr. Sсотт. Then would not a petroleum tank entirely constructed of iron with an iron bottom be the safest inclosure possible for petroleum?

Professor Rowland. The peculiarity of that is that the fumes of petroleum are all the time coming out from the cracks. The whole outside is probably covered with petroleum. I suppose also the ground is saturated with petroleum. The petroleum as far as the inside goes would be perfectly safe. 
Lieutenant Fiske. I would like to ask how far lightning obeys the ordinary law of currents, whether it takes the path of least resistance or not. Do high potentials always do that? In general across a narrow space the resistance is greater than going around by the iron, and the question is, to what extent does the lightning obey the law of circuits?

Professor Rowland. I would like to say one word more with respect to petroleum. In the case of the tank you have a mixture of the petroleum vapor and air which probably would explode. Unless the tank was a very good conductor there might be also a little spark in the interior, not enough to hurt a man in there; but the smallest spark inside the tank would cause an explosion. I am not certain whether the iron of the tank is a good enough conductor to prevent every trace of spark in the interior. Indeed, suppose we had a tank with a cover upon it. That is supposed to be a closed ressel, yet the lightning would have to pass from top to bottom between the cover and the tank, and perhaps a little spark would take place in the interior; and possibly in going from one of the plates of the iron tank to the other it may find some resistance and jump over some small plate in the interior of the tank. It would be a most difficult thing to protect.

With regard to that other question, lightning in the air, of course, does not obey Ohm's law; it is entirely a discontinuous anomaly. It is like the breaking of a metal. A piece of metal is supposed to break at a certain strain; but it does not always break then; it pulls out in strings or something of that sort. One cannot measure the distance and say the lightning is going to jump across that distance. 


\section{THE VALUE OF THE OHM}

[La Lumiè̀re Électrique, $X X V I$, pp. 188, 189, 477, 1887]

La Valeur de l'Unité de Résistance de l'Association Britannique.

A la dernière réunion de l' Association britannique, le professeur $\mathrm{H}$. A. Rowland a donné la valeur définitive de l'unité de résistance électrique de l'Association, telle qu'elle a été determinée par la commission américaine. La valeur donnée en $18 \% 6$ était : unité $B . A=$ $0.9878 \mathrm{ohm}$.

Dans la dernière détermination, on s'est servi des méthodes de Kirchhoff et de celle de Lorenz.

La première a donné une valeur de $0.98646 \pm 40$ et la seconde 0.9864 \pm 18 ; son erreur probable est donc de moins de la moitié de celle de la première méthode.

Le professeur Rowland a également déterminé la résistance d'une colonne de mercure de $1 \mathrm{~mm} .^{2}$ de section et de 100 centimètres de longueur, et a trouvé 0.95349 unités $B . A$.

Valeur de l'Étalon $B$. A. de l'Ohm, d'après les Mesures de la Commission, Américaine, par Rowland.

Les observations ont été terminées en 1884 déjà, mais les calculs viennent d'être terminés et seront publiés prochainement. En 1786:

Rowland a trouvé 1 unité $B . A .=0.9878 \mathrm{ohm}$.

Kimball a trouvé 1 unité $B . A .=0.9870 \mathrm{ohm}$.

Maintenant Rowland trouve par la méthode de Kirchhoff et à l'aide de 73 observations

$$
1 \text { unité } B . A .=(0.98627 \pm 40) \text { ohms }
$$

et Kimball par la méthode de Lorenz et au moyen de 43 observations

$$
1 \text { unité } B . A .=(0.98642 \pm 18) \text { ohms. }
$$

En combinant les deux résultats, on trouve que l'unité mercurielle est égale à 0.95349 unités $B$. $A$., c'est-à-dire que l'ohm de mercure correspond à une colonne de mercure de $106 \cdot 32 \mathrm{~cm}$.

Rappelons ici les valeurs obtenues par différents physiciens et qui se rapprochent le plus du résultat ci-dessus: 
Lord Rayleigh $\ldots \ldots \ldots \ldots \ldots \ldots \ldots .106 \cdot 25 \mathrm{~cm}$. Glazebrook ...................106.29 cm. Wiedemann ..................106.19 cm. Mascart ..................... Weber ...................... $106 \cdot 16 \mathrm{~cm}$. 


\section{ON A SIMPLE AND CONVENIENT FORM OF WATER BATTERY}

[American Journal of Science [3], XXXIII, 147, 188\%; Philosophical Magazine [5], XXIII, 303, 1887; Johns Hopkins University Circular's, No. 57, p. 80, 1887]

For some time I have had in use in my laboratory a most simple, convenient and cheap form of water battery whose design has been in one of my note-books for at least fifteen years. It has proved so useful that I give below a description for the use of other physicists.

Strips of zinc and copper, each two inches wide, are soldered together along their edges so as to make a combined strip of a little less than four inches wide, allowing for the overlapping. It is then cut by shears into pieces about one-fourth of an inch wide, each composed of half zinc and half copper.

A plate of glass, very thick and a foot or less square, is heated and coated with shellac about an eighth of an inch thick. The strips of copper and zinc are bent into the shape of the letter $\mathrm{U}$, with the branches about one-fourth of an inch apart, and are heated and stuck to the shellac in rows, the soldered portion being fixed in the shellac, and the two branches standing up in the air, so that the zinc of one piece comes within one-sixteenth of an inch of the copper of the next one. A row of ten inches long will thus contain about thirty elements. The rows can be about one-eighth of an inch apart and therefore in a space ten inches square nearly 800 elements can be placed. The plate is then warmed carefully so as not to crack and a mixture of beeswax and resin, which melts more easily than shellac, is then poured on the plate to a depth of half an inch to hold the elements in place. A frame of wood is made around the back of the plate with a ring screwed to the centre so that the whole can be hung up with the zinc and copper elements below.

When required for use, lower so as to dip the tips of the elements into a pan of water and hang up again. The space between the elements being $\frac{1}{16}$ inch, will hold a drop of water which will not evaporate for possibly an hour. Thus the battery is in operation in a minute and is perfectly insulated by the glass and cement.

This is the form I have used, but the strips might better be soldered face to face along one edge, cut up and then opened. 


\title{
ON AN EXPLANATION OF THE ACTION OF A MAGNET ON CHEMICAL ACTION ${ }^{1}$
}

\author{
By Henry A. Rowland and Louis Bell
}

[American Journal of Science [3], XXXVI, 39-47, 1888; Philosophical Magazine [5]. $X X V I, 105-114,1888]$

In the year 1881 Prof. Remsen discovered that magnetism had a very remarkable action on the deposition of copper from one of its solutions on an iron plate, and he published an account in the American Chemical Journal for the year 1881. There were two distinct phenomena then described, the deposit of the copper in lines approximating to the equipotential lines of the magnet, and the protection of the iron from chemical action in lines around the edge of the poles. It seemed probable that the first effect was due to currents in the liquid produced by the action of the magnet on the electric currents set up in the liquid by the deposited copper in contact with the iron plate. The theory of the second kind of action was given by one of us, the action being ascribed to the actual attraction of the magnet for the iron and not to the magnetic state of the latter. It is well known since the time of Faraday that a particle of magnetic material in a magnetic field tends to pass from the weaker to the stronger portions of the field, and this is expressed mathematically by stating that the force acting on the particle in any direction is proportional to the rate of variation of the square of the magnetic force in that direction. This rate of variation is greatest near the edges and points of a magnetic pole, and more work will be required to tear away a particle of iron or steel from such an edge or point than from a hollow. This follows whether the tearing away is done mechanically or chemically. Hence the points and edges of a magnetic pole, either of a permanent or induced magnet, are protected from chemical action.

One of Prof. Remsen's experiments illustrates this most beautifully. He places pieces of iron wire in a strong magnetic field, with their axes along the lines of force. On attacking them with dilute nitric acid they are eaten away until they assume an hour-glass form, and are

${ }^{1}$ Read at the Manchester meeting of the British Association, September, 1887. 
furthermore pitted on the ends in a remarkable manner. On Prof. Remsen's signifying that he had abandoned the field for the present, we set to work to illustrate the matter in another manner by means of the electric currents produced from the change in the electrochemical nature of the points and hollows of the iron.

The first experiments were conducted as follows: Two bits of iron or steel wire about $1 \mathrm{~mm}$. in diameter and $10 \mathrm{~mm}$. long were imbedded side by side in insulating material, and each was attached to an insulated wire. One of them was filed to a sharp point, which was exposed by cutting away a little of the insulation, while the other was laid bare on a portion of the side. The connecting wires were laid to a reflecting galvanometer, and the whole arrangement was placed in a small beaker held closely between the poles of a large electromagnet, the iron wires being in the direction of the lines of force. When there was acid or any other substance acting upon iron in the beaker, there was always a deflection of the galvanometer due to the slightly different action on the two poles. When the magnet was excited the phenomena were various. When dilute nitric acid was placed in the beaker and the magnet excited, there was always a strong throw of the needle at the moment of making circuit, in the same direction as if the sharp pointed pole had been replaced by copper and the other by zinc. This throw did not usually result in a permanent deflection, but the needle slowly returned toward its starting point and nearly always passed it and produced a reversed deflection. This latter effect was disregarded for the time being, and attention was directed to the laws that governed the apparent 'protective throw' since the reversal was so long delayed as to be quite evidently due to after effects and not to the immediate action of the magnet.

With nitric acid this throw was always present in greater or less degree, and sometimes remained for some minutes as a temporary deflection, the time varying from this down to a few seconds. The throw was independent of direction of current through the magnet, and apparently varied in amount with the strength of acid and with the amount of deflection due to the original difference between the poles. This latter fact simply means that the effect produced by the magnet is more noticeable as the action on the iron becomes freer.

When a pair of little plates exposed in the middle were substituted for the wires, or when the exposed point of the latter was filed to a flat surface, the protective throw disappeared, though it is to be noted that the deflection often gradually reversed in direction when the cur- 
rent was sent through the magnet; i. e., only the latter part of the previous phenomenon appeared under tliese circumstances.

When the poles, instead of being placed in the field along the lines of force, were held firmly perpendicular to them, the protective throw disappeared completely, though as before there was a slight reverse after-effect.

Some of Professor Remsen's experiments on the corrosion of a wire in strong nitric acid were repeated with the same results as he obtained, viz.: the wire was eaten away to the general dumb-bell form, though the protected ends instead of being club-shaped were perceptibly holjowed. When the wire thus exposed was filed to a sharp point the extreme point was very perfectly protected, while there was a slight tendency to hollow the sides of the cone, and the remainder of the wire was as in the previous experiments. In both cases the bars were steel and showed near the ends curious corrugations, the metal being left here and there in sharp ridges and points. In one case the cylinder was eaten away on sides and ends so that a ridge of almost knife-like sharpness was left projecting from the periphery of the ends.

These were the principal phenomena observed with nitric acid. Since this acid is the only one which attacks iron freely in the cold, in Prof. Remsen's experiment, this was the one to which experiments were in the main confined. With the present method, however, it was possible to trace the effect of the magnet whenever there was the slightest action on the iron, and consequently a large number of substances, some of which hardly produce any action, could be used with not a little facility.

In thus extending the experiments some difficulties had to be encountered. In many cases the action on the iron was so irregular that it was only after numerous experiments under widely varying conditions that the effect of the magnet could be definitely determined. Frequently the direction of the original action would be reversed in the course of a series of experiments without any apparent cause, but in such case the direction of the effect due to the magnet remained always unchanged, uniformly showing protection of the point so long as the wires remained parallel to the lines of force. When, howerer, the original action and the magnetic effect coincided in direction, the repetition of the latter showed a decided tendency to increase the former.

When using solutions of various salts more or less freely precipitated by the iron, it frequently happened that the normal protective throw was nearly or quite absent, but showed itself when the magnet circuit was broken as a violent throw in the reverse direction, showing that the combination had been acting like a miniature storage battery which 
promptly discharged itself when the charging was discontinued by breaking the current through the magnet. The gradual reversal of the current some little time after exciting the magnet was noted frequently in these cases, as before. Owing to this peculiarity and their generally very irregular action, the various salts were disagreeable substances to experiment with, though as a rule they gave positive results.

Unless the poles were kept clean experimenting became difficult from the accumulation of Aecomposition products about them and oxidation of their surfaces. A few experiments showed how easily the original deflection could be modified, nearly annulled or even reversed in direction by slight differences in the condition of the poles. These difficulties of the method are, however, more than counterbalanced by its rapidity and delicacy when proper precautions are taken.

Nearly thirty substances were tested in the manner previously described; but comparatively fow of them gave very decided effects with the magnet, though, as later experiments have shown, the protective action is a general one. The substances first tried were as follows. The table shows the various acids and salts tried, and their effects as shown by the original apparatus:

\begin{tabular}{|c|c|c|}
\hline Substances. & $\begin{array}{l}\text { Effect due to } \\
\text { Magnet. }\end{array}$ & Notes. \\
\hline 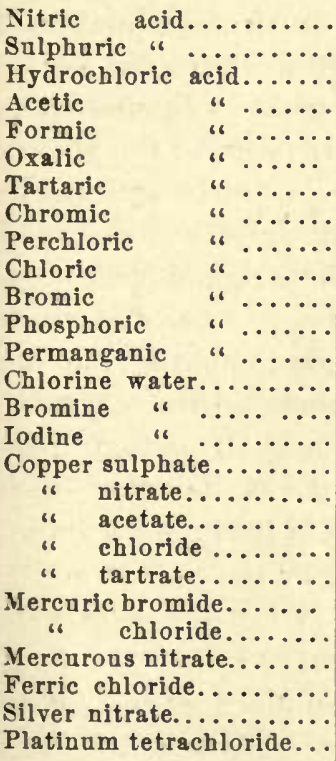 & $\begin{array}{c}\text { Strong. } \\
\text { Little or none. } \\
\text { " None. } \\
\text { "“ } \\
\text { " } \\
\text { Some effect. } \\
\text { "“ } \\
\text { None. } \\
\text { "“ } \\
\text { Slight effect. } \\
\text { Decided " } \\
\text { " } \\
\text { some. } \\
\text { " } \\
\text { slight. } \\
\text { Some. } \\
" ، \\
\text { Decided. } \\
\text { Some. } \\
\text { " }\end{array}$ & $\begin{array}{l}\text { Mainly showing as throw, on breaking. } \\
\text { “" } \\
\text { "“ } \\
\text { Throw, on breaking. } \\
\text { Very slight solution, weak. } \\
\text { Mainly as throw on breaking. [breaking. } \\
\text { Both protective throw, and sometimes on } \\
\text { Action very irregular. } \\
\text { " }\end{array}$ \\
\hline
\end{tabular}


Several things are worthy of note in this list. In the first place those solutions of metallic salts which are precipitated by iron all show distinct signs of protective action when the current is passed through the magnet. Of the various acids this is not generally true; only those show the magnetic effect, which act on iron without the evolution of hydrogen, and are powerful oxidizing agents. In general, substances which acted without the evolution of hydrogen gave an effect with the magnet.

From these experiments it was quite evident that the protective action, whatever its cause, was more general than at first appeared and steps were next taken to extend it to the other magnetic metals. Small bars were made of nickel and cobalt and tried in the same manner as before. These metals are acted on but very slightly by most acids, and the range of substances which could be used was therefore very small, but all the substances which gave the magnetic effects with iron poles gave a precisely similar, though much smaller effect, whenever they were capable of acting at all on the nickel and cobalt. This was notably the case with nitric acid, bromine water, chlorine water, and platinum tetrachloride, which were the substances acting readily on the metals in question. Even with these powerful agents, however, the magnetic action was very much less than with iron, and experimentation on metals even more weakly magnetic was evidently hopeless.

As a preliminary step toward ascertaining the cause of the magnetic action and its non-appearance where the active substance evolved hydrogen, it now became necessary to discover and if possible eliminate the cause of the reversal of the current which regularly followed the protective throw. Experiments soon showed that it could not be ascribed to accumulation of decomposition products around the electrodes, and polarization, while it could readily neutralize the original deflection, could not reverse its direction. Whatever the cause, it was one which did not act with any great regularity, and it was soon found that stirring the liquid while the magnet was on, uniformly produced the effect observed. Since one pole was simply exposed over a small portion of its side while the other had a sharp projecting point, it was the latter which was most freely attacked when there were currents in the liquid, whether these were stirred up artificially or were produced by the change in galvanic action due to the presence of the magnet. When the poles were placed in fine sand saturated with acid this reversing action was much diminished, and in fact anything which tended to hinder free circulation of the liquid produced the same effect. Several materials were tried and 
of these the most successful was an acidulated gelatine which was allowed to harden around the poles. In this case the protective throw was not nearly as large as in the free acid, since the electrodes tended to become polarized while the gelatine was hardening, and only weakly acid gelatine would harden at all; but the reversing action completely disappeared, so that, when the magnet was put on, a permanent deflection was produced instead of a transitory throw.

This point being cleared up attention was next turned to the negative results obtained with acids which attack iron with evolution of hydrogen. The galvanometer was made much more sensitive and removed from any possible disturbing action due to the magnet; and with these precautions the original experiments were repeated, it seeming probable that even if the magnetic effect were virtually annulled by the hydrogen evolred, some residual effect might be observed.

This residual effect was soon detected, first with hydrobromic acid, and then with hydrochloric, hydriodic, sulphuric and otlers. The strongest observed effect was with hydriodic acid, but as this may possibly have contained traces of free iodine it may be regarded as somewhat doubtful. The effect in all these cases was very small, and though now and then suspected in the previous work, could not have been definitely determined, much less measured.

Some rough measurements were made on the electromotive forces involved in this class of phenomena by getting the throw of the galvanometer for various small known values of the E. M. F. The values found raried greatly, ranging from less than 0.0001 volt in case of the acids evolving hydrogen, up to 0.02 or 0.03 volts with nitric acid and certain salts. These were the changes produced by the magnet, while the initial electromotive forces normally existing between the poles would be, roughly speaking, from 0.0001 to nearly 0.05 volts, never disappearing and rarely reaching the latter figure.

From these experiments it therefore appears that the protective action of the magnetic field is general, extending to all substances which act chemically on the magnetic metals. While this is so, the strongest effect is obtained with those substances which act without the evolution of hydrogen. But the series is really quite continuous, perchloric acid for instance producing but little more effect than hydrobromic, while this in turn differs less from perchloric than from an acid like acetic. It seems probable that the action of the hydrogen evolved is partially to shield the pole at which it is cvolved, and lessen the difference between the poles produced by the magnet. It probably acts merely 
mechanically, for it is to be noted that those acids which evolve a gas other than hydrogen (perchloric acid, for instance), which is not absorbed by the water, tend to produce little magnetic effect compared with those which act with gut the evolution of any gas.

As to the actual cause of the protective action exercised by the magnetic field, all these experiments go to show that it is quite independent of the substance acting, with the exception above noted, and is probably due to the attractive action of the magnet on the magnetic metals forming the poles subjected to chemical action, as we have before explained.

In the first place, whenever iron is acted upon chemically in a magnetic field those portions of it about which the magnetic force varies most rapidly are very noticeably protected, and this protection as nearly as can be judged varies very nearly with the above quantity. Wherever there is a point there is almost complete protection, and wherever there is a flat surface, no matter in how strong a field, it is attacked freely. Whenever in the course of the action there is a point formed, the above condition is satisfied and protection at once appears. Thus, in the steel bars experimented on, whenever the acid reached a spot slightly harder than the surrounding portions it produced a little elevation from which the lines of force diverged, and still further shielding it produced a ridge or point, sharp as if cut with a minute chisel. Nickel and cobalt tend to act like iron, though they are attacked with such difficulty that the phenomena are much less strongly marked. With the non-magnetic metals they are completely absent. Now, turning to the experiments with the wires connected with a galvanometer, the same facts appear in a slightly different form.

When the poles were placed perpendicular to the lines of force instead of parallel to them, the magnet produced no effect whatever, showing, first, that the effect previously observed depended not merely on the existence of magnetic force but on its relation to the poles, and, secondly, that when the poles were so placed as to produce little deflection of the lines of force the protective effect disappeared.

When the pointed pole was blunted the effect practically disappeared, the poles remaining parallel to the lines of force, and when plates were substituted for the wires no effect was produced in any position, showing that the phenomena were not due to the directions of magnetization but to the nature of the field at the exposed points. In short, whatever the shape or arrangement of the exposed surfaces, if at any point or points the rate of variation of the square of the magnetic force is 
greater than elsewhere, such points will be protected, while if the force is sensibly constant over the surfaces exposed there will be no protection at any point. With all the forms of experimentation tried this law held without exception. It therefore appears that the particles of magnetic material on which the chemical action could take place are governed by the general law of magnetic attraction and are held in place against chemical energy precisely as they would be held against purely mechanical force. To sum up:

When the magnetic metals arc exposed to chemical action in a magnetic field such action is decreased or arrested at any points where the rate of variation of the square of the magnetic force tends toward a maximum.

It is quite clear that the above law expresses the facts thus far obtained, and while in any given case the action of the magnet is often complicated by subsidiary effects due to currents or by-products, the mechanical laws of motion of particles in a magnetic field hold here as elsewhere and cause the chemical action to be confined to those points where the magnetic force is comparatively uniform.

The effect of currents set up in the liquid during the action of the magnet cannot be disregarded especially in such experiments as those of Nichols (this Journal, xxxi, 272, 1886) where the material acted on was powdered iron and the disturbances produced by the magnet would be particularly potent. The recent experiments of Colardeau (Journal de Physique, March, 1887) while perhaps neglecting the question of direct protection of the poles, have furnished additional proof of the purely mechanical action of the magnet by reproducing some of the characteristic phenomena where chemical action was eliminated and the only forces acting were the ordinary magnetic attractions.

An attempt was made to reverse the magnetic action, i. e. to deposit iron in a magnetic field and increase its deposition where there was a sharp pole immediately behind the plate on which the iron was being deposited. This attempt failed. The action was very irregular and the results not decisive. The question of stirring effect was also examined. Usually stirring the liquid about one pole increased the action on that pole, but sometimes produced little effect or even decreased it. This however is in entire agreement with the irregular action sometimes observed in the case of the after-effect in the original experiments.

An excellent method of experiment is to imbed an iron point in wax leaving the minute point exposed: imbed a flat plate also in wax and expose a point in its centre. Place the point opposite to the plate, but 
not too near and place in the liquid between the poles of a magnet and attach to the galvanometer as before.

There is a wide field for experiment in the direction indicated above, for it is certainly very curious that the effect varies so much. If hydrogen were as magnetic as iron, of course acids which liberated it would have no action. But it is useless to theorize blindly without further experiment; and we are drawn off by other fields of research.

In this Journal for 1886, (l. c.) Professor E. L. Nichols has investigated the action of acids on iron in a magnetic field. He remarks that the dissolving of iron in a magnetic field is the same as removing it to an infinite distance and hence the amount of heat generated by the reaction should differ when this takes place within or without the magnetic field. Had he calculated this amount of heat due to the work of withdrawing it from the field, he would probably have found his method of experiment entirely too rough to show the difference, for it must be very small. He has not given the data, however, for us to make the calculation. The results of the experiments were inconclusive as to whether there was greater or less heat generated in the field than without.

In the same Journal for December, 1887 , he describes experiments on the action of the magnet on the passive state of iron in the magnetic field. In a note to this paper and in another paper in this Journal for April, 1888, he describes an experiment similar to the one in this paper but without our theory with regard to the action of points. Indeed he states that the ends of his bars acted like zinc, while the middle was like platinum, a conclusion directly opposite to ours. The reason of this difference has been shown in this paper to be probably due to the currents set up in the liquid by the reaction of the magnet and the electric currents in the liquid.

In conclusion we may remark that our results differ from Professor Nichols in this: First, we have given the exact mathematical theory of the action and have confirmed it by our experiments, having studied and avoided many sources of error, while Professor Nichols gives no theory and does not notice the action of points. Secondly, our experiments give a protective action to the points and ends of bars, while Professor Nichols thinks the reverse holds and that these are more easily dissolved than unmagnetized iron. 


\title{
ON THE ELECTROMAGNETIC EFFECT OF CONVECTION- CURRENTS
}

\author{
By Henry a. Rowland and Cary T. Hutcuinson
}

[Philosophical Magazine [5], XXVII, 445-460, 1889]

The first to mention the probable existence of an effect of this kind was Faraday, "who says:- "If a ball be electrified positively in the middle of a room and then be moved in any direction, effects will be produced as if a current in the same direction had cxisted." He was led to this conclusion by reasoning from the lines of force.

Maxwell, writing presumably in 1872 or 1873 , outlines an experiment, similar to the one now used, for the proof of this effect.

The possibility of the magnetic action of convection-currents occurred to Professor Rowland in 1868, and is recorded in a note-book of that date.

In his first experiments, made in Berlin in 1876, Prof. Rowland used a horizontal hard rubber disk, coated on both sides with gold, and revolving between two glass condenser-plates. Each coating of the disk formed a condenser with the side of the glass nearer it; the two sides of the disk were charged to the same potential. The needle was placed perpendicular to a radius, above the upper condenser-plate, and nearly over the edge of the disk. The diameter of the hard rubber disk was $21 \mathrm{~cm}$., and the speed 61 per second.

The needle system was entirely protected from direct electrostatic effect. On reversing the electrification, deflexions of from 5 to $7 \cdot 5$ $\mathrm{mm}$. were obtained, after all precautions had been taken to guard against possible errors. Measurements were made, and the deflexions as calculated and observed agreed quite well; but it was not possible to make the measurements with as great accuracy as was desired, and hence the present experiment.

Helmholtz, ${ }^{2}$ in $18 \% 5$ and later, carried out some experiments bearing

1 Experimental Researches, vol. i, art. 1644.

2 Tiss. Abh. i, p. 778. 
on this subject. According to the "potential theory" of electrodynamics which he wished to test, unclosed circuits existed. The end of one of these open circuits would exert an action on a close magnetic or electric circuit. So the following experiment was made by M. Schiller, under his direction.

A closed steel ring was uniformly magnetized, the magnetic axis coinciding with the mean circle of the ring. This was hung by a long fibre and placed in a closed metal case. A point attached to a Holtz machine was fixed near the box, and a brush-discharge was kept up from thio point. If the point acted as a current-end, a deflexion would be ex pected, on the potential theory. No deflexion was observed, although the calculated deflexion was 23 scale-divisions. The inference is tha' either the potential theory is untrue, or else that there is no unclosed circuit in this case, $i$. $e$. that the convection-currents completing the circuit have an electromagnetic effect.

Schiller's further work, not bearing directly upon convection-currents, leads him to the conclusion that all circuits are closed, and that displacement-currents have an electromagnetic effect.

Dr. Lecher is reported to have repeated Professor Rowland's experiment, with negative results. His paper has not been found.

Röntgen " has discovered a similar action; he rotates a dielectric disk between the enlarged plates of a horizontal condenser and gets a deflexion of his needle. He apparently guards against the possibility of this being due to a charge on his disk. A calculation of the force he measures shows it to be almost one-eighth of that in the Berlin experiment. His apparatus is not symmetrically arranged, the disk being much closer to the upper condenser-plate; the distances from the upper and lower plates are 0.14 and $0.25 \mathrm{~cm}$. respectively. He uses a difference of potential corresponding to a spark-length of $0.3 \mathrm{~cm}$. in air between balls of $2 \mathrm{~cm}$. diameter, $i$. $e$. about 33 electrostatic units, equal to the sparking potential between plane surfaces : $t 0 \cdot 26$ $\mathrm{cm}$. The disk is an imperfect conductor, and altogether it does not seem elear, in spite of the precautions taken, that this is not due to convection-currents.

In the Berlin apparatus, as stated above, the needle is near the edge of the disk; the magnetic effect produced is assumed to be proportional to the surface-density multiplied by the linear velocity; hence the force will be much greater at the edge of the disk than near the centre; but 
Plate V

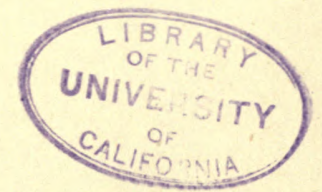




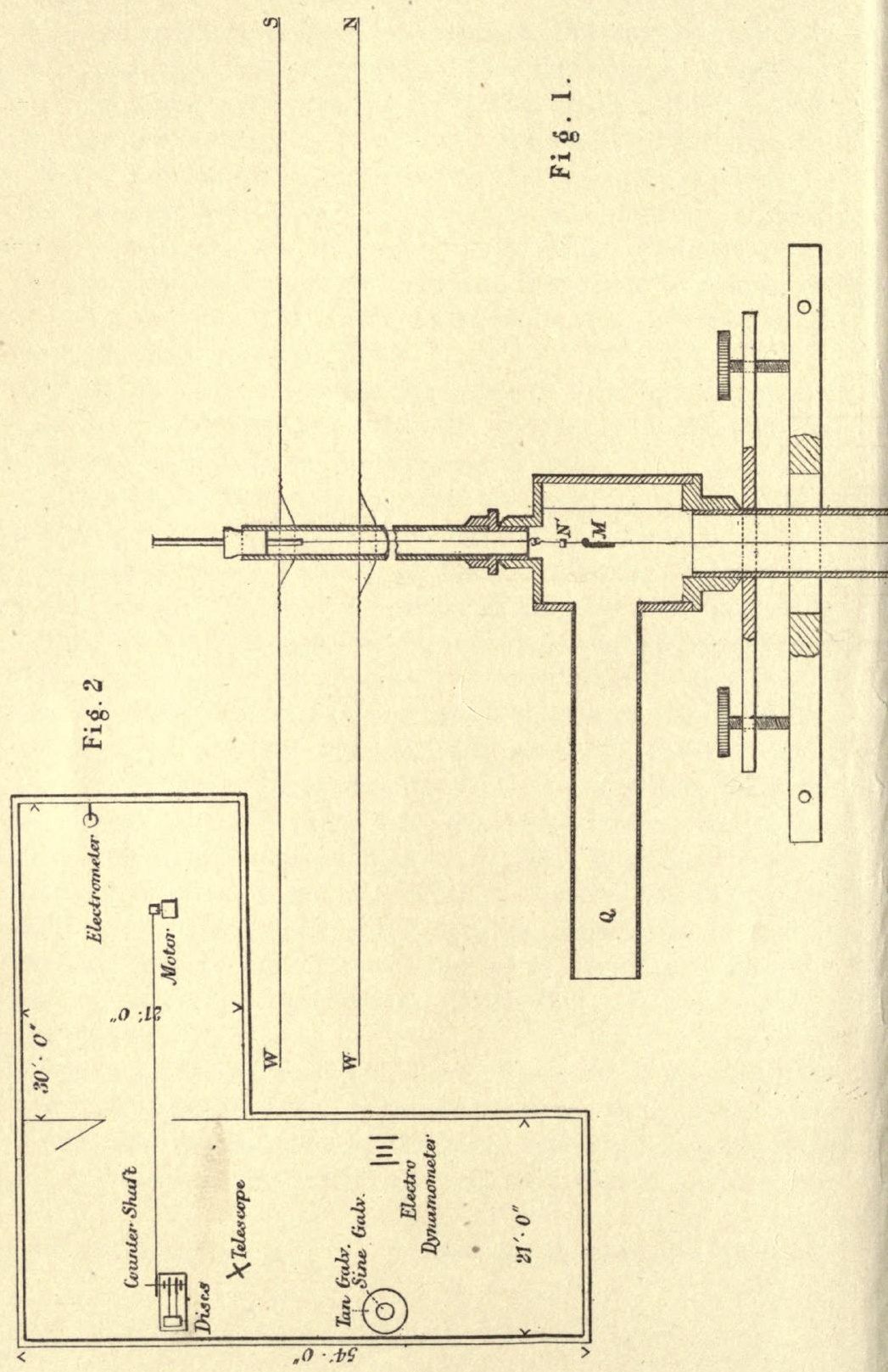



the field will be more irregular, and so make accurate measurements more difficult.

In the present apparatus a uniform field is secured by using two vertical disks rotating about horizontal axes in the same line; the needle system is placed between the disks, opposite their centres. The disks are in the meridian; they are gilded on the faces turned towards the needle. Between the disks are placed two glass condenser-plates gilded on the surfaces near the disk; and between these glasses is the needle. The whole apparatus is symmetrical about the lower needle of the astatic system.

Each disk is surrounded by a gilded hard rubber guard-plate in order to keep the density of the charge uniform at the edges. The guardplates are provided with adjusting-screws to enable them to be put accurately in the plane of the disks; and the glass plates in turn have adjusting-screws for securing parallelism with the guard-plates. The glass was carefully chosen as being nearly plane. Disks, glass plates, and guard-plates all have radial scratches, to prevent conduction-currents from circulating around the coatings.

In the periphery of the disk are set eight brass studs which penetrate radially for about 5 centim., then turning off at a right angle rum parallel to the axis until they come out on the surface of the disks. They there make contact with the gold foil. Metal brushes set in the gnard-plate bear on these studs, and in this way the disks are electrified.

The figure (Pl. V, Fig. 1) gives a vertical projection of the entire disk-apparatus: $-D D$ are the disks; $G G G G$ the guard-rings; $Y Y Y Y$ the condenser-plates; $R R R R$ hard rubber rings fitting on the shouldcrs $A A ; X X X X$ bearing-boxes for the axle; $P P P P$ supportingstandards; $E E$ metal bases sliding in the bed $B B$, and held in any position by screws $Z ; F F$ the bases carrying the glass plates, sliding in the same way as the others. $S S S S$ are the adjusting-screws for the guard-plates, and $t t$ for the glass plates. $L L L L$ are collars for catching the oil from the bearings; $C C, C^{\prime} C^{\prime}$ are speed-counters, $C C$ gear with the axle, and $C^{\prime} C^{\prime}$ with $C C$ in the manner shown; each has 200 teeth, and speed-reading is taken every 40,000 revolutions.

The needle system is enclosed in the brass tube $T$, ending in the larger cylindrical box in which are the mirror and upper needle. This is closed in by the conical mouth-piece $Q$, across the opening of which is placed a wire grating. The mirror is shown at $M$, the upper needle at $N^{\prime \prime}$ and the lower at $N$. The system is hung by a fibre-suspension about $30 \mathrm{~cm}$. in length, protected by a glass tube. The needle- 
system is made by fitting two small square blocks of wood on an aluminium wire; on two sides of each of the wooden blocks are cemented small scraps of highly magnetized watch-spring. The needle thus made is about $1 \times 1 \times 10 \mathrm{~mm}$.

The mirror is fixed just below the upper needle, and is read by a telescope $200 \mathrm{~cm}$. distant. The plane of the mirror is at an angle of $45^{\circ}$ with the plane of the disks for convenience. The whole is supported by the board $O O$ attached to a wall-bracket.

Two controlling magnets $(W W$ ) with their poles turned in opposite directions are used. By means of the up and down motion of either magnet, any change in the sensitiveness can be attained; and by the motion in azimuth, the zero point is controlled. The advantage of its use lies in the extremely delicate means it affords of changing the sensitiveness, much more delicate than with a single magnet.

The bed-plate $B$ is screwed to one end of a table, at the other end of which a countershaft is placed (Fig. 2). This is run by an electric motor in the next room, the belt running through the open doorway. The motor is 14 metres from the needle.

Although the disks and countershaft were carefully balanced when first set up, and the table braced and weighted by a heavy stone slab, yet at the speed used, 125 per second, the shaking of the entire apparatus was considerable; the needle was so unsteady that it could not be read. This was seen to be due to vibrations of the telescope itself and not to the needle. To prevent it, each leg of the table on which the telescope rested was set in a box about $30 \mathrm{~cm}$. deep filled with sawdust, and a heavy stone slab was placed on top of this table. This entirely did away with the trouble; the swing of the needle was as regular when the apparatus was revolving as when it was at rest.

The two hard rubber rings $(R R)$ mentioned above have grooves cut in their peripheries; in these grooves wires are wound. These serve as a galvanometer for determining the needle-constant. When not in use they are held in the position shown in the figure, but when it is desired to determine the needle-constant they are slipped on the shoulders $(A A A A)$ and pushed up in contact with the back of the disks. Each has two turns: this arrangement will be referred to as the diskgalvanometer.

If a known current is sent through the disk-galvanometer, and the geometrical constant be known, the part of the constant depending on the field and needle is determined.

The current is measured by a sine-galvanometer, placed in another 
part of the room. 'To determine $H$ at the sine-galvanometer a metre brass circle is put around the sine-galvanometer, and the needle of the latter used as the needle of the tangent-galvanometer thus made. Using this tangent-glavanometer in connection with a Weber electrodynamometer, $H$ at the sine-galvanometer is measured.

The charging was by a Holtz machine connected to a battery of six gallon Leyden jars. These latter are in circuit with a reversing-key, an electrostatic gauge, and the disks.

The potential was measured by a large absolute electrometer; all previous observers have used spark-length between balls, with Thomson's formula. Greater accuracy is claimed for this work, largely on this account.

In this instrument the movable plate is at one end of a balance-arm, from the other end of which hangs, on knife-edges, a balance-pan. This movable plate is surrounded by a guard-ring.

The lower plate is fixed by an insulating rod to a metal stem, which slides up and down in guides. The distances are read off on a scale on the metal stem. The zero reading is got by inserting a piece of plane parallel glass whose thickness has been measured. The lower plate and guard-ring have a diameter of $35 \mathrm{~cm}$., and the movable disk a diameter of $10 \mathrm{~cm}$.

The routine of the observations was as follows:-A determination of $H$ and the needle-constant $(\beta)$ was first made. The electrostatic gauge was then set at a certain point, and readings of difference of potential were taken. The disks were now started, electrified, and a series of three elongations of the needle taken; the electrification reversed and three more elongations taken, \&c.

About every five minutes speed-readings had to be noted, and at each reversal it was necessary to replenish the charge in order to keep the gauge-arm just at the mark. In this way a 'series' of readings consisting of about 25 reversals was made. After the series, electrometer readings were again taken; the conditions were then changed in some way, and another series begun.

The circumstances to be changed are:-distance of disks from needle; distance of glass plates from needle; electrification; and direction of rotation.

The calculation of the deflexion is based on the assumption that the magnetic effect of a rotating charge is proportional to the quantity of electricity passing any point per second, just as with a conductioncurrent. Below are the formulæ used. 
In the equations the letters have the following meanings. All quantities are given in terms of C. G. S. units.

$X=$ Distance from centre of disk to lower needle.

$r=$ Distance from centre of disk to upper needle.

$c=$ Radius of disk.

$l=$ Distance between needles.

$a=$ Radius of windings of disk-galvanometer.

$b=$ Distance, centre of disk-galvanometer to lower needle.

$\rho=$ Distance, centre of disk-galvanometer to upper needle.

$N=$ Number of revolutions per second.

$\boldsymbol{\sigma}=$ Surface-density of electrification in electrostatic measure.

$V=$ Ratio of the units.

$\alpha=$ Angle of torsion of the electro-dynamometer.

$\varphi=$ Angle of deflexion of sine-galvanometer.

$\delta=$ Angle of deflexion of tangent-galvanometer.

$\Delta=$ Change of zero-point on electrifying the disks $=$ half the charge on reversing.

$s=$ Scale-reading for disk-galvanometer.

$v=$ Weight on pan of electrometer.

$D=$ Distance of glass plates and disks.

$\boldsymbol{e}=$ Electrometer reading.

$x=$ Condenser distance.

Force, in the direction of the axis, due to a circular current of radius $c$, at a distance $x$ on the axis

Strength of convection-current

$$
=2 \pi I \frac{c^{2}}{\left(c^{2}+x^{2}\right)^{3}}
$$

$$
=2 \pi c d c \frac{N \pi}{V} \text {. }
$$

$\therefore$ total force due to the disk of radius $c$

$$
\begin{aligned}
& =4 \pi^{2} \frac{N_{\sigma}}{V} \int_{0}^{c} \frac{c^{3} d c}{\left(c^{2}+x^{2}\right)^{\frac{3}{3}}} . \\
& =4 \pi^{2} \frac{N_{\sigma}}{V}\left\{\frac{c^{2}+2 x^{2}}{\left(c^{2}+x^{2}\right)^{\frac{1}{2}}}-2 x\right\}=4 \pi^{2} \frac{N_{\sigma}}{V} A ;
\end{aligned}
$$

and for the two disks acting in the same direction, total force

$$
X=8 \pi^{2} \underset{V}{N} A \text {. }
$$

This gives the force on the lower needle. 
Correction for the upper needle:

Potential at any point due to a circular current,

$$
V^{\prime}=\int I d \varpi
$$

equals the solid angle subtended at the point by the circle

$$
=2 \pi\left\{\frac{1}{2} P_{1}\left(\begin{array}{c}
c^{2} \\
r
\end{array}\right)-\frac{1.3}{2,4} P_{3}\left(\begin{array}{l}
c \\
r
\end{array}\right)^{4} \ldots-(-)^{i} \frac{1.3 \ldots(2 i-1)}{2.4 \ldots 2 i} P_{2 i-1}\left(\begin{array}{l}
c \\
r
\end{array}\right)^{2 i}\right\}
$$

$(i=1.2 .3 \ldots)$

$$
\therefore V^{\prime}=2 \pi I \Sigma_{i}\left\{-(-)^{i} \frac{1.3 \ldots(2 i-1)}{2.4 \ldots 2 i} P_{2 i-1}\left(\frac{c}{r}\right)^{2 i}\right\} \text {. }
$$

Substituting the value of $I$, we have as the potential of the disk

$$
\begin{gathered}
2 \pi \Sigma_{i} \int_{0}^{c} 2 \pi c \frac{N \sigma}{V}\left\{-(-)^{i} \frac{1.3 \ldots(2 i-1)}{2.4 \ldots 2 i} P_{2 i-1}\left(\frac{c}{r}\right)^{2 i}\right\} d c, \\
V=4 \pi^{2} \frac{N \sigma}{V} c^{2} \Sigma_{i}\left\{-(-)^{i} \frac{1.3 \ldots(2 i-1)}{2.4 \ldots 2 i(2 i+2)} P_{2 i-1}\left(\frac{c}{r}\right)^{2 i}\right\} .
\end{gathered}
$$

But

and

$$
P_{i}=(-)^{i} \frac{r^{i+1}}{1.2 \ldots i} \frac{\partial^{i}}{\partial x^{i}}\left(\frac{1}{r}\right)
$$

$\therefore$ The force

$$
\frac{\partial}{\partial x} P_{i}=\frac{i+1}{r}\left\{\mu P_{i}-P_{i+1}\right\}-\mu=\frac{x}{r} .
$$

$$
\begin{aligned}
& =-\frac{\partial V}{\partial x} \\
& \begin{aligned}
=4 \pi^{2} \frac{N \sigma}{V} c^{2} \Sigma_{i}\left[( - ) ^ { i } \frac { 1 . 3 \ldots ( 2 i - 1 ) } { 2 . 4 \ldots 2 i \cdot ( 2 i + 2 ) } \left(P_{2 i-1}\left\{-\frac{2 i c^{2 i} \cdot x}{r^{2 i}{ }^{1} r}\right\}\right.\right. \\
\\
\left.\left.\left.\left.\quad+\left(\frac{c}{r}\right)^{2 i}\right\} \frac{2 i}{r}\left(\mu P_{2 i-1}-P_{2 i}\right)\right\}\right)\right]
\end{aligned} \\
& =4 \pi^{2} \frac{N \sigma}{V} c^{2} \Sigma_{-i}\left\{(-)^{i+1} \frac{1.3 \ldots(2 i-1)}{2.4 \ldots 2(i+1)} \frac{2 i}{r}\left(\frac{c}{r}\right)^{2 i} P_{2 i}\right\} ;
\end{aligned}
$$

and for the two,

$$
X_{1}=8 \pi^{2} \frac{N \sigma}{V} \frac{c^{2}}{r}\left[\frac{1}{4} P^{2}\left(\frac{c}{r}\right)^{2}-\frac{1.3}{2.6} P_{4}\left(\frac{c}{r}\right)^{4}+\ldots\right],
$$

where the sign of the entire expression has been changed, since the poles of the upper and lower needles are opposite.

Or

$$
X_{1}=8 \pi^{2} \frac{N \sigma}{V} B
$$


Needle-constant.

The disk-galvanometer windings have in the same way, for the lower needle, the force due to current $I$ in one turn

For the four turns,

$$
\begin{gathered}
=2 \pi I \frac{a^{2}}{\left(a^{2}+b^{2}\right)^{\frac{3}{2}}} \\
=2 \pi I C .
\end{gathered}
$$

$$
X^{\prime}=8 \pi I C .
$$

Upper needle.-The force is got in the same way as for the disk, omitting the integration, $i$. e. we must multiply the general term of $B$ by $\frac{2(i+1)}{a^{2}}$ and replace $2 \pi \frac{N \sigma}{V}$ by $I$. This gives

$$
2 \pi I \Sigma_{i}\left\{(-)^{i+1} \frac{1.3 \ldots(2 i-1)}{2.4 \ldots 2 i} \frac{2 i}{\rho}\left(\frac{a}{\rho}\right)^{2 i} P_{2 i}\right\}
$$

$a$ replacing $c$, and $\rho, r$.

For the total force,

or

$$
X_{1}^{\prime}=\frac{8 \pi I}{\rho}\left[P_{2}\left(\frac{a}{\rho}\right)^{2}-\frac{3}{2} P_{4}\left(\frac{a}{\rho}\right)^{4}+\ldots\right],
$$

$$
X_{1}^{\prime}=8 \pi I D \text {. }
$$

Forces acting on the needle system:-

Let $M=$ moment of lower needle,

then

Let $M^{\prime}=$ moment of upper needle,

Couple on lower needle due to field $=H M \sin \theta$,

Couple on upper needle due to field $=-H^{\prime} M^{\prime} \sin \theta$.

Total couple $=\left(H M-H^{\prime} M^{\prime}\right) \sin \theta$.

Due to disk-galvanometer:

Couple on lower needle $=M X^{\prime} \cos \theta$,

Couple on upper needle $=M^{\prime} X_{1}^{\prime} \cos \theta$.

Total couple $=\left\{M X^{\prime}+M^{\prime} X_{1}^{\prime}\right\} \cos \theta$, $=8 \pi I\left\{M C+M^{\prime} D\right\} \cos \theta$.

$\therefore$ for equilibrium,

$$
8 \pi I\left\{M O+M^{\prime} D\right\} \cos \theta=\left\{H M-H^{\prime} M^{\prime}\right\} \sin \theta,
$$

or

$$
I=\frac{\left(H M-H^{\prime} M^{\prime}\right) \tan \theta}{8 \pi(C+D) M\left\{1+\frac{D}{C}\left(\frac{M^{\prime}}{M}-1\right)\right\}}
$$


But $\frac{D}{C}=0.03$ nearly, and $\frac{M^{\prime}}{M}$ is approximately unity.

$$
\therefore I=\frac{\left(H M-H^{\prime} M^{\prime}\right)}{8 \pi M(C+D)} \tan \theta
$$

or

$$
\frac{\left(H M-H^{\prime} M^{\prime}\right)}{M}=\frac{8 \pi(C+D)}{\tan \theta} I=\beta \text { (say). }
$$

Similarly, for the revolving disks,

$$
\begin{aligned}
8 \pi^{2} \frac{N \sigma}{V}\{A+B\} & =\frac{\left(H M-H^{\prime} M^{\prime}\right)}{M} \tan \Delta . \\
& =\beta \tan \Delta . \\
\therefore V & =8 \pi^{2} \frac{N \sigma}{V} \frac{A+B}{\beta \cdot \Delta} .
\end{aligned}
$$

For the sine-galvanometer:

$$
\begin{gathered}
I=\frac{H}{\Gamma} \sin \varphi . \\
\Gamma=1831 . \\
\therefore I=10^{-4} 5.46 H \sin \varphi,
\end{gathered}
$$

and

$$
\beta=10^{-4} .5 \cdot 46 H \frac{8 \pi(C+D)}{\tan \theta} \sin \varphi .
$$

For measurement of $H$ :-

Electrodynamometer,

$$
\begin{aligned}
i & =g \frac{\sqrt{K}}{T} \sqrt{\sin \alpha .} \\
g & =\text { constant of windings }=10^{-3} \cdot 6 \cdot 454 . \\
K & =\text { moment of inertia }=10^{2} \cdot 8 \cdot 266 . \\
T & =\text { time of one swing }=2 \cdot 441 . \\
\therefore \quad i & =10^{-2} \cdot 7 \cdot 59 \sqrt{\sin \alpha .}
\end{aligned}
$$

Tangent galvanometer:-

$$
\begin{aligned}
i & =\frac{H}{G} \tan \delta=\frac{H . b}{2 \pi n} \tan \delta . \\
n & =\text { no. turns }=10 . \\
& b=\text { radius turns }=49.98 . \\
\therefore \quad i & =0.795 H \tan \delta
\end{aligned}
$$

and, substituting the value of $i$,

$$
H=10^{-2} \cdot 9 \cdot 55 \frac{\sqrt{\sin \alpha}}{\tan \delta} .
$$


Surface density $(\sigma)$ :-

$\sigma$ is obtained from electrometer-readings.

$$
\begin{aligned}
\sigma & =\frac{V}{4 \pi e} . \\
\nabla & =D \sqrt{\frac{8 \pi g w}{A}} . \\
A & =\text { corrected area of movable plate } \\
& =\frac{1}{2} \pi\left\{R^{2}+R_{1}^{2}+\ldots\right\}=\frac{1}{2} \pi\{51 \cdot 01\} . \\
\therefore \quad V & =10 \times 1 \cdot 756 D \sqrt{w}, \\
\sigma & =1.397 \frac{D}{e} \sqrt{w} .
\end{aligned}
$$

and

As soon as the attempt was made to electrify the apparatus, difficulties of insulation were met with. The charged system was quite extensive, and the opportunity for leakage was abundant; in addition, the winter here has been very damp. Most of the trouble of this kind has been due to the glass in the apparatus; in no case where glass was used as an insulator has it proved satisfactory, not even when the air was dry. First, the stand with glass legs, on which the Leyden-jar battery was placed, was found to furnish an excellent earth-connection.

Paraffin blocks interposed stopped this. The reversing-key had three glass rods in it, all of which were found to leak; six different specimens of glass, some bought particularly for this as insulating glass, were all found to allow great leakage. Shellacing had no effect. Hard rubber was finally substituted for glass; and after that the key insulated very well, even in damp weather.

On charging the glass plates, the disks being earthed, it seemed almost as if there was a direct earth-connection, so rapid was the fall of the charge. This was not regarded at the time, as the plates were always kept earthed; but later, when it became necessary to charge the plates, the insulation had to be made good.

Investigation showed that this was caused by leakage directly through the substance of the glass to the brass back-pieces $(H H)$. Hard rubber pieces were substituted, and the trouble was entirely removed.

There was at first a deflexion in reversing the electrification while the disks were at rest. This was of course due to direct electrostatic effect; but it was not for some time clear where the point of weakness in the electrostatic screen lay. It was found to be the faulty contact between the tinfoil covering of the glass tube and the brass collar; the brass had been lacquered. After this was corrected there was never 
again any deflexion on reversing the charge, although the precaution was taken of testing it every day or so.

The currents induced in the axle by the rotation caused no inconrenience; if the disks are rotated in the same direction their effect is added, while the effect of the axles is in opposite directions. Even when the disks were rotated oppositely, the deflexion due to the axles was only 3 or $4 \mathrm{~cm}$., and remained perfectly constant.

On running the disks, unelectrified, without the glass plates between them and the needle, a deflexion of 4 or $5 \mathrm{~cm}$. was noticed. This was perfectly steady deflexion, and could easily be shown to be due to the presence of the plate, as it ceased when the plates were replaced.

This was very troublesome for a time, especially as the presence of a brass plate in place of the glass was found to diminish the deflexion, but did not bring the needle back to zero as the glasses did. On looking at the figure (Plate V, Fig. 1) it will be seen that there is a brass plug $(I)$ closing the bottom of the tube in which the needle is placed. The rapid rotation of the disks caused a very appreciable exhaustion at the centre, and consequently a steady stream of air was sucked down the tube through the open mouthpiece, and out through the imperfect connection of the plug. Air-currents were not at first suspected, as the deflexion was so very steady. The brass plate used was smaller than the glass, and hence did not completely shield the tube.

After the brass back-pieces $(H H)$ had been taken out, and a hard rubber substituted, it was found that with one direction of rotation the needle was extremely unsteady; it would run up the scale for several centimetres, stop suddenly, \&c.-evidently a forced vibration. This was traced to air-currents also. Now, the air blew into the open mouth of the cone. The apparatus had been run for some months with this open, and not the slightest irregularity had been seen. But the hard rubber pieces were very much larger than the brass ones which were removed; they filled up the lower space to a greater extent, and deflected the air upwards more than before, causing the unsteadiness. With the opposite rotation the air was thrown down instead of up, and consequently did not affect the needle.

The first systematic observations were made in January, 1889, with the disks charged and plates earthed. The deflexion on reversing was got without difficulty, and it was in the direction to be expected; that is, with positive electrification, the effect was equivalent to a current in the direction of motion of the disk. A number of series were taken in the next two months; they agreed among themselves well enough, but 
did not follow the law assumed. The deviation can best be explained in this way:-The equations above show that for a fixed position of the disks $\Delta \propto \sigma \frac{N}{\beta} \frac{D}{e} \propto \frac{N}{\beta}$. If then, $N$ and $\beta$ being constant, the condenser plates are moved up to the disk, step by step, thus varying $e$, and $D$ be changed at the same time so as to keep $D / e \propto \sigma$, a constant, the deflexions should be constant.

Such was not found to be the case; the deflexions were directly proportioned to $e$ instead of being constant: that is, with greater difference of potential, the deflexions were greater, although the surface density remained constant. Finally this was found to be due to a charge on the back surface of the gold coating. The end of the axle comes nearly up to the surface of the disk and taken with all the brass work must form a condenser of a certain capacity with the inner face of the gold foil.

This made a change necessary in the method of working; the disks had to be earthed and the glasses charged. This was done; but now the deflexions were found always to be greater with positive rotation (Zenith, North, Nadir, South) then with negative.

It was considered possible that the brushes might have something to do with this, so they were taken off. Earth connection with the disk was made by drilling through to the surface of the disk in the line of the axle and setting in a screw, which came flush with the surface and also made contact with the axle; this, however, made no difference, the deflexions for negative rotation were always smaller.

Table I gives the results. of a number of observations. All were taken with the plates charged and the disks earthed by means of the axle.

The meaning of the letters has been given; $1 / \beta$ is directly proportional to the needle sensitiveness.

The sudden variations in the values of $1 / \beta$ are due to changes purposely made in the needle.

The last column gives the values of $V$. This work is not intended as a determination of $\nabla$, but the calculation is made merely to show to what degree of approximation the effect follows the assumed law.

The deflexions are about the same as those obtained in the Berlin experiments -5 to $8 \mathrm{~mm}$. on reversing. The force measured then was $1 / 50000 H$; now it is $1 / 125000 H$. The sensitiveness of the needle in the two cases was almost the same. In the former experiment a force of $3 \times 10^{-7}$ deflected the needle $1^{\prime}$ of arc; the corresponding num- 
ber now is $2.7 \times 10^{-7}$, slightly more sensitive. The scale distances were 110 and $200 \mathrm{~cm}$. respectively. So this experiment gives about

TABLE I.

\begin{tabular}{|c|c|c|c|c|c|c|c|c|}
\hline No. & Rotation. & $x$. & e. & N. & $\sigma$. & $1 / \beta$ & $2 \Delta$. & V. \\
\hline 1 & + & $2 \cdot 54$ & $1 \cdot 24$ & 122 & $1 \cdot 16$ & $1 \cdot 50.10^{5}$ & $\underset{5 \cdot 3}{\operatorname{mm}}$ & $2 \cdot 42.10^{10}$ \\
\hline 2 & + & $2 \cdot 57$ & 66 & 125 & $1 \cdot 30$ & $3 \cdot 11$ & $9 \cdot 0$ & $3 \cdot 38$ \\
\hline 3 & + & 66 & 66 & 129 & $1 \cdot 23$ & $2 \cdot 15$ & $6 \cdot 94$ & $3 \cdot 00$ \\
\hline 4 & - & 6 & 6 & 129 & $1 \cdot 23$ & "6 & $5 \cdot 58$ & $3 \cdot 68$ \\
\hline 5 & + & 16 & $1 \cdot 21$ & 127 & $1 \cdot 21$ & $2 \cdot 25$ & $5 \cdot 6$ & $3 \cdot 74$ \\
\hline 6 & - & 66 & 6 & 133 & $1 \cdot 21$ & 66 & $5 \cdot 7$ & $3 \cdot 74$ \\
\hline 7 & + & 6 & "6 & 130 & $1 \cdot 47$ & 6 & $8 \cdot 4$ & $3 \cdot 10$ \\
\hline 8 & - & 16 & 66 & 133 & $1 \cdot 47$ & 6 & $7 \cdot 3$ & $3 \cdot 64$ \\
\hline 9 & + & 16 & $1 \cdot 24$ & 121 & $1 \cdot 32$ & $2 \cdot 22$ & $9 \cdot 4$ & $2 \cdot 26$ \\
\hline 10 & - & " & 66 & 130 & $1 \cdot 32$ & 6 & $7 \cdot 2$ & $3 \cdot 16$ \\
\hline 11 & + & "6 & 6 & 125 & $1 \cdot 26$ & $2 \cdot 17$ & $7 \cdot 6$ & $2 \cdot 70$ \\
\hline 12 & - & 6 & 6 & 126 & $1 \cdot 26$ & 6 & $5 \cdot 7$ & $3 \cdot 64$ \\
\hline 13 & + & $2 \cdot 85$ & $1 \cdot 50$ & 125 & $1 \cdot 19$ & $2 \cdot 23$ & $6 \cdot 5$ & $2 \cdot 82$ \\
\hline 14 & - & 6 & 6 & 129 & $1 \cdot 19$ & 6 & $5 \cdot 0$ & $3 \cdot 78$ \\
\hline 15 & - & "6 & "6 & 125 & $1 \cdot 11$ & $2 \cdot 19$ & $5 \cdot 85$ & $2 \cdot 82$ \\
\hline 16 & + & 6 & $1 \cdot 43$ & 127 & $1 \cdot 08$ & $2 \cdot 35$ & $7 \cdot 3$ & $2 \cdot 46$ \\
\hline 17 & - & 66 & 66 & 128 & $1 \cdot 08$ & 16 & $5 \cdot 4$ & $3 \cdot 32$ \\
\hline 18 & - & 6 & 6 & 129 & $1 \cdot 08$ & 6 & $5 \cdot 3$ & $3 \cdot 42$ \\
\hline 19 & + & $3 \cdot 22$ & $1 \cdot 80$ & 123 & $1 \cdot 13$ & $2 \cdot 44$ & $5 \cdot 1$ & $3 \cdot 30$ \\
\hline 20 & - & "6 & 6 & 124 & $1 \cdot 13$ & "6 & $4 \cdot 9$ & $3 \cdot 48$ \\
\hline & & & & & & & & $3 \cdot 19 \times 10^{10}$ \\
\hline
\end{tabular}

TABLE II.

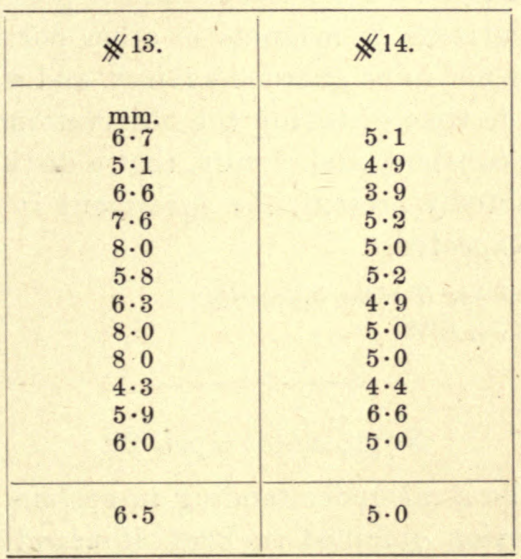

the same scale-deflexion at twice the distance with a force $\frac{4}{10}$ as great. The agreement between the two is seen to be quite good.

The observations, except Nos. 1, 2, 15, and 18 given above, were taken 
in pairs-first one direction of rotation and the other immediately afterwards, everything except the rotation being kept constant.

The table shows that, in every case except one, the deflexion for negative rotation is appreciably smaller than the corresponding positive.

The difference is too great to be due to accidental errors in the readings, as the following table, giving the successive deflexions in the case of $\$ 13$ and $\$ 14$ will show.

There is but one deflexion in $\$ 13$ as small as the mean of 14 , and but one in $\$ 14$ as large as the mean of 13 .

This is a fair example of the way the deflexions run. As a further illustration of this take 17 and 18 ; these two are identical in arrangement, but the direction of rotation is in one case got by crossing the belts from the countershaft to the disks and leaving the main bolt straight; in the other the main belt is crossed while the auxiliary belts are straight. The deflexions are the same. This, too, shows that the difference cannot be due to any effect of the countershaft. The cause of this has not yet been explained. The work is to be continued with this and also with new apparatus, made like the Berlin apparatus, but with the disk much larger, $30 \mathrm{~cm}$. in diameter; at least double the speed then obtained will be used. This ought to give deflexions on reversal of 1.5 to $1.7 \mathrm{~cm}$.

The values of $V$ do not agree so well as might be looked for; but when, in addition to the numerous difficulties already mentioned, the smallness of the deflexion is considered, and the possibility of the needle being affected by currents or magnets in other portions of the laboratory, so far away as not to be guarded against, and which might well be changed between the time of taking the observation and the determination of the needle-constant, and, finally, that a distubing cause of some kind is still undoubtedly present, the agreement is seen to be as good as could justly be expected.

Physical Laboratory, Johns Hopkins University,

April 22, 1889.

\section{Note, added April 29}

There seems to be a misunderstanding in certain quarters as to the nature of the deflexion obtained in Prof. Rowland's first experiment. The paper reads:- "The swing of the needle on reversing the electrification was about 10 to $15 \mathrm{~mm}$., and therefore the point of equilibrium was altered 5 to $7.5 \mathrm{~mm}$." This has been construed to mean that the 
deflexion was merely a throw, and that no continuous deflexion was obtained. This is entirely erroneous; there was always a continuous deflexion. The throw was read merely because the needle was always more or less unsteady, and better results could be got by seizing a favorable moment when the needle was quiet and reading the throw, than by attempting to take the successive elongations, or waiting for the needle to come to rest. In the experiment described above the needle was very steady and no such trouble was experienced. On electrifying, the needle would take up a certain position and would remain there as long as the charge was kept up; on reversal, it would move off to a new and perfectly definite position about 6 to $7 \mathrm{~mm}$. away, and remain there, \&c.

H. A. R.

C. T. H. 


\section{ON THE RATIO OF THE ELECTROMAGNETIC TO THE ELECTROSTATIC UNIT OF ELECTRICITY}

Br Hesgr A. Rowlasd, with the sasistance of $\mathrm{E}$. H. HALL and L. B. FLETCHEs

[Phitosophieal Magazime [5], XXVIIT, 304-315, 1859; Ameriean Journal of Beience [3], XXXVIII, 282-298, 1889]

The determination described below was made in the laboratory of the Johns Hopkins University about ten years ago, and was laid aside for further experiment before publication. The time never arrived to complete it, and I now seize the opportunity of the publication of a determination of the ratio by $\mathrm{Mr}$. Rosa in which the same standard condenser was used, to publish it. Mr. Rosa has used the method of getting the ratio in terms of a resistance. Ten years ago the absolute resistance of a wire was a very uncertain quantity and, therefore, I adopted the method of measuring a quantity of electricity electrostatically and then, by passing it through a galvanometer, measuring it electromagnetically.

The method consisted, then, in charging a standard condenser, whose geometrical form was accurately known, to a given potential as measured by a very accurate absolute electrometer, and then passing it through a galvanometer whose constant was accurately known, and measuring the swing of the needle.

\section{Descriptios OF Isstruments}

Electrometer.-This was a very fine instrument made partly according to my design by Edelmann, of Munich. As first made, it had many faults which were, however, corrected here. It is on Thomson's guard ring principle with the movable plate attached to the arm of a balance and capable of accurate adjustment. The dise is $10.18 \mathrm{~cm}$. diameter in an opening of $10.38 \mathrm{~cm}$. and the guard plates about $33.0 \mathrm{~cm}$. diameter. All the surfaces are nickel plated and ground and polished to optical surfaces and capable of accurate adjustment so that the distance between the plates can be very accurately determined. The balance is sensitive to a mg. or less and the exact position of the beam 
is read by a hair moring before a seale and observed by a lens in the manner of Sir Wm. Thomson. The instrument has been tested throughout itts entire range by varying the distances and weights to give the constant potential of a standard gange, and found to give relative readings to about 1 in 400 at least. It is constructed throughout in the most elaborate and careful manner and the working parts are enclosed in sheet brass to prevent exterior action.

As the balance carmot be in equilibrium by combined weights and electrostatic forces, it was found best to limit its swing to a $\frac{1}{10} \mathrm{~mm}$. on each side of its normal position. The mean of two readings of the distance, one to make the hair jump up and the other down, constituuted one reading of the instrument.

The adjustments of the plattes parallel to each other and of the movable plate in the plane of the guard ring could be made to almost $\frac{\mathrm{I}}{40} \mathrm{~mm}$.

The formula for the difference of potential of the two plattes is

$$
W^{2}=\frac{8 \pi d l^{2} w y}{4}
$$

where $d$ is the distance of the plattes, $w g$ the absolute force on the morable platte and $A$ its corrected area. According to Maxwell

$$
A=\frac{1}{2} \pi\left\{R^{e}+R^{v x}-\left(\left(R^{2 x}-R^{e}\right) \frac{a}{d+a}\right\}\right.
$$

where $R$ and $R^{\prime \prime}$ are the radiil of the dise and the opening for it and $a$ $=-221\left(R^{\prime \prime}-R\right)$. The last correction is only about 1 in 500 , and hence we have, finally,

$$
V=17-221 \sqrt{w} d\left\{1+\frac{-0002}{d}\right\} .
$$

Stcomdiond comdenser.-This very accuratte instrument was made from my designs by Mrr. Grumow, them of New York, and consisted of one hollow ball, wery accurattely turmed and nickel platted, in which two balls of different sizes could be hung by a sillk cord. The balls could be very accurattely adjustted in the centre of the hollow one. Contact was made by two waires aboutt $\frac{1}{100}$ inch diametter, one of which was protruded through the ortter ball until it tersehed the inmer one; by a suitable mechanism it was them withdrawn and the second one introduced at another place to effect the discharge. This could be effected five times exery second. The diametters of the balls have been aceurattely determined by weighing in wratter, and the electrostatic eapacities found to be

50-069 and $29-556$ e.g. s. units.

A further description is given in Mr. Rosa's paper. 
Galvanometer for Electrical Discharges.-'This was very carefully in. sulated by paper and then put in hot wax in a vacuum to extract the moisture and fill the spaces with wax. It had two coils, each of about 70 layers of 80 turns each of No. 36 silk covered copper wire. They were half again as large as the ordinary coils of a Thomson galvanometer. The two coils were fixed on the two sides of a piece of vulcanite and the needle was surrounded on all sides by a metal box to protect it from the electrostatic action of the coils. A metal cone was attached to view the mirror through. The insulation was perfect with the quickest discharge.

The constant was determined by comparison with the galvanometer described in this Journal, vol. xv, p. 334. The constant then given has recently been slightly altered. The values of its constant are

By measurement of its coils . . . . . . . . 1832.24

By comparison with coils of electrodynamometer... 1833.67

By comparison with single circle ............ 1832.56

Giving these all equal weights, we have

$1832 \cdot 82$

instead of $1833 \cdot 19$ as used before.

The ratio of the new galvanometer constant to this old one was found by two comparisons to be

Mean, $10 \cdot 4141$

Hence we have

$$
G=1908 \% \text {. }
$$

Electrodynamometer.-This was almost an exact copy of the instrument described in Maxwell's treatise on electricity except on a smaller scale. It was made very accurately of brass and was able to give very good results when carefully used. The strength of current is given by the formula

$$
S=\frac{C \sqrt{K}}{t} \sqrt{\sin \alpha}
$$

where $K$ is the moment of inertia of the suspended coil, $t$ its time of vibration, $\alpha$ the reading of the head, and $C$ a constant depending on the number of coils and their form. 
LARGE COILS.

Total number of windings $\ldots \ldots \ldots \ldots \ldots .240$

Depth of groove .................. $84 \mathrm{~cm}$.

Width of groove ................ $\cdot 76 \mathrm{~cm}$.

Mean radius of coils ............. $13.741 \mathrm{~cm}$.

Mean distance apart of coils ......... $13 \cdot 786 \mathrm{~cm}$.

SUSPENDED COILS.

Total number of windings $\ldots \ldots \ldots \ldots \ldots \quad 126$

Depth of groove ................. $\cdot 41 \mathrm{~cm}$.

Width of groove ................ $\cdot 38 \mathrm{~cm}$.

Mean radius $\ldots \ldots \ldots \ldots \ldots \ldots \ldots \ldots, 2 \cdot 760 \mathrm{~cm}$.

Mean distance apart ............. 2.707 cm.

These data give, by Maxwell's formulæ,

$$
C=0.00645 \% \text {. }
$$

In order to be sure of this constant, I constructed a large tangent galvanometer with a circle $80 \mathrm{~cm}$. diameter and the earth's magnetism was determined many times by passing the current from the electrodynamometer through this instrument and also by means of the ordinary method with magnets. In this way the following values were found.

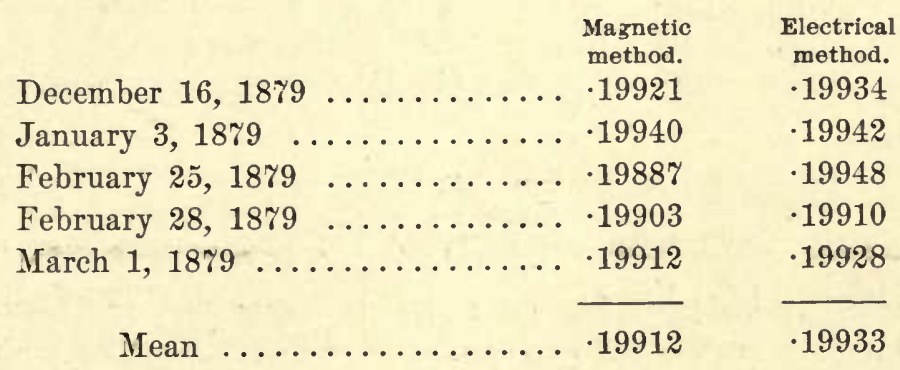

which differ only about 1 in 1000 from each other. Hence we have for $C$ :

From calculation from coils .......... 006457

From tangent galvanometer .......... 006451

Mean .................... 006454 c. g. s. units.

The suspension was bifilar and no correction was found necessary for the torsion of the wire at the small angles used. 
The method adopted for determining the moment of inertia of the suspended coil was that of passing a tube through its centre and placing weights at different distances along it. In this way was found

$$
K=826 \cdot 6 \text { c. g. s. units. }
$$

The use of the electrodynamometer in the experiment was to determine the horizontal intensity of the earth's magnetism at any instant in the position of the ballistic galvanometer. This method was necessary on account of the rapid changes of this quantity in an ordinary building ${ }^{2}$ and also because a damping magnet, reducing the earth's field to about $\frac{1}{3}$ its normal value, was used. For this purpose the ballistic galvanometer was set up inside the large circle of $80 \mathrm{~cm}$. diameter with one turn of wire and simultaneous readings of the electrodynamometer and needle of ballistic galvanometer were made.

\section{Theory of Experiment.}

We have for the potential

$$
V=\frac{8 \pi g}{A} d \sqrt{w}=e d \sqrt{w}\left[1+\frac{.0002}{d}\right]
$$

For the magnetic intensity acting on the needle

For the condenser charge

$$
H=\frac{2 \pi n p^{2} c \sqrt{K \sin \alpha}}{t\left(p^{2}+b^{2}\right)^{\frac{3}{2} \tan \varphi}}
$$

$$
Q=2 \frac{H T}{\pi G} \sin \frac{\theta}{2}\left(1+\frac{1}{2} \lambda\right)=N \frac{V C}{v}
$$

Whence

$$
v=\frac{e G C\left(p^{2}+b^{2}\right)^{\frac{3}{2}}}{2 n c \sqrt{K p^{2}}} \frac{N t \sqrt{w d}}{T \sqrt{\sin \alpha}} \frac{\tan \varphi}{2 \sin \frac{1}{2} \theta}\left[1-\frac{\lambda}{2}+\text { etc. }\right]
$$

but $\tan \varphi=\frac{1}{2} \frac{\beta}{D}\left[1-\frac{1}{4}\left(\frac{\beta}{D}\right)^{2}+\frac{1}{8}\left(\frac{\beta}{D}\right)^{4}\right]$

and $2 \sin \frac{1}{2} \theta=\frac{1}{2} \frac{\delta}{D}\left[1-\frac{1}{3}\left(\frac{\delta}{D}\right)^{2}\right]$ nearly.

So that finally

$$
v=\frac{e G C\left(p^{2}+b^{2}\right)^{\frac{3}{2}}}{2 n c \sqrt{K} K p^{2}} \frac{N+\beta \sqrt{ } w}{T \sqrt{\sin \alpha}} \frac{d}{\delta}[1-A-B-C+D+E-F+1]
$$

$A=0 ; \cdot 0011 ; \cdot 0030 ; \cdot 0056 ; \cdot 0090$ for $1,2,3,4,5$ discharges as investigated below.

${ }_{1}^{1}$ This experiment was completed before the new physical laboratory was finished. 


$$
\begin{aligned}
& B=\frac{1}{4}\left(\frac{\xi}{D}\right)^{2}-\frac{1}{8}\left(\frac{\beta}{D}\right)^{4} \\
& C=\frac{\lambda}{2} \\
& D=\frac{.0002}{d} \\
& E=\frac{1}{3}\left(\frac{\hat{\sigma}}{D}\right)^{2}
\end{aligned}
$$

$F=\cdot 0013$ for first ball of condenser and $\cdot 0008$ for other, as investigated below.

$I=$ correction for torsion of fibre $=0$ as it is eliminated.

$e=$ constant of electrometer $=17 \cdot 221$.

$G=$ constant of ballistic galvanometer $=1908 \%$.

$p=$ radius of large circle $=42 \cdot 105 \mathrm{~cm}$.

$n=$ number of coils on circle $=1$.

$c=$ constant of electrodynamometer $=\cdot 006454$.

$K=$ moment of inertia of coil of electrodynamometer $=826 \cdot 6$.

$b=$ distance of plane of large circle from needle $=1 \cdot 27$.

$C=$ capacity of condenser $=50 \cdot 069$ or $29 \cdot 556$.

$D=$ distance of mirror from scale $=170 \cdot 18 \mathrm{~cm}$.

$w=$ weight in pan of balance.

$t=$ time of vibration of suspended coil.

$T=$ time of vibration of needle of ballistic galvanometer.

$\beta=$ deflection of needle on scale when constant current is passed.

$\alpha=$ reading of head of electrodynamometer when constant current is passed.

$\grave{\delta}=$ swing caused by discharge of condenser.

$d=$ distance of plates of electrometer.

$N=$ number of discharges from condenser.

$\lambda=$ logarithmic decrement of needle.

$A=$ correction due to discharges not taking place in an instant.

The principal correction, requiring investigation is $A$. Let the position and velocity of the needle be represented by

$$
x=a_{0} \sin b t \text { and } v=a_{0} b \cos b t \text {, where } b=\frac{\pi}{T} \text {. }
$$

At equal periods of time $t, 2 t, .3 t$, etc., let new impulses be given to the needle so that the velocity is increased by $v_{0}$ at each of these times. The equations which will represent the position and velocity of the needle at any time are, then, 


$$
\begin{array}{clll}
\text { between } 0 \text { and } t, & x=a_{0} \sin b t & v=a_{0} b \cos b t \\
\text { " } \quad t \text {, and } 2 t, & x=a^{\prime} \sin b\left(t+t^{\prime}\right) & v=a^{\prime} b \cos b\left(t+t^{\prime}\right) \\
\text { “ } 2 t \text {, and } 3 t, & x=a^{\prime \prime} \sin b\left(t+t^{\prime \prime}\right) & v=a^{\prime \prime} b \cos b\left(t+t^{\prime \prime}\right)
\end{array}
$$

At the times $0, t, 2 t$, etc., we must have

$$
\begin{array}{cl}
x=0 & \multicolumn{1}{c}{v_{0}=a_{0} b} \\
a_{0} \sin b t_{1}=a^{\prime} \sin b\left(t_{1}+t^{\prime}\right) & v_{0}+a_{0} b \cos b t_{1}=a^{\prime} b \cos b \\
a^{\prime} \sin b\left(2 t_{1}+t^{\prime}\right)=a^{\prime \prime} \sin b\left(2 t,+t^{\prime \prime}\right) & v_{0} a^{\prime} b \cos b\left(2 t,+t^{\prime \prime}\right) \\
\text { etc. } & =a^{\prime \prime} b \cos b\left(2 t,+t^{\prime \prime}\right) \\
& \text { etc. }
\end{array}
$$

Whence we have the following series of equations to determine $a^{\prime}, a^{\prime \prime}$, etc., and $t^{\prime}, t^{\prime \prime}$, etc.

$$
\begin{aligned}
& a_{0}^{2} b^{2}=v_{0}^{2} \\
& a^{\prime 2} b^{2}=a_{0}^{2} b^{2}+v_{0}{ }^{2}+2 v_{0} a_{0} b \cos b t_{i} ; \sin b\left(t_{1}+t^{\prime}\right)=\frac{a_{0}}{a^{\prime}} \sin b t, \\
& a^{\prime \prime 2} b^{2}=a^{\prime 2} b^{2}+v_{0}{ }^{2}+2 v_{0} a^{\prime} b \cos b\left(2 t_{1}-t^{\prime}\right) ; \sin b\left(2 t_{1}+t^{\prime \prime}\right)=\frac{a_{0}^{\prime}}{a^{\prime \prime}} \sin b\left(2 t,+t^{\prime}\right) \\
& a^{\prime \prime \prime}{ }^{\prime \prime} b^{2}=a^{\prime \prime 2} b^{2}+v_{0}{ }^{2}+2 v_{0} a^{\prime \prime} b \cos b\left(3 t_{1}+t^{\prime \prime}\right) ; \sin b\left(3 t_{1}+t^{\prime \prime \prime}\right)=\frac{a^{\prime \prime}{ }_{0}}{a^{\prime \prime}} \sin b\left(3 t_{1}+t^{\prime \prime \prime}\right) \\
& \text { etc. } \\
& \text { etc. }
\end{aligned}
$$

When $t$, is small compared with the time of vibration of the magnet, we have very nearly $t^{\prime}=-\frac{1}{2} t_{1} ; t^{\prime \prime}=-t_{1}, t^{\prime \prime \prime}=-\frac{3}{2} t_{1}$, etc.

Whence

$$
\begin{aligned}
& a^{\prime 2}=2 a_{0}{ }^{2}\left(1+\cos b t_{0}\right)=4 a_{0}{ }^{2}\left(1-\frac{1}{4}\left(b t_{0}\right)^{2}\right) \\
& a^{\prime \prime 2}=9 a_{0}{ }^{2}\left(1-\frac{2}{3}\left(b t_{1}\right)^{2}\right) \\
& a^{\prime \prime \prime 2}=16 a_{0}{ }^{2}\left(1-\frac{4}{5}\left(b t_{1}\right)^{2}\right) \\
& a^{1 \mathrm{v2}}=25 a_{0}^{2}\left(1-2\left(b t_{0}\right)^{2}\right) \\
& a^{\text {v2 }}=
\end{aligned}
$$

$$
\begin{aligned}
& a^{\prime}=2 a_{0}\left(1-\frac{1}{8}\left(b t_{1}\right)^{2}\right) \\
& a^{\prime \prime}=3 r_{0}\left(1-\frac{1}{3}\left(b t_{1}\right)^{2}\right) \\
& a^{\prime \prime \prime}=4 a_{0}\left(1-\frac{5}{8}\left(b t_{1}\right)^{2}\right) \\
& a^{i v}=5 a_{0}\left(1-\left(b t_{1}\right)^{2}\right)
\end{aligned}
$$

Now $a_{0}, a^{\prime}, a^{\prime \prime}, a^{\prime \prime \prime}$ and $a^{15}$ are the values of $\delta$ with $1,2,3,4$ and 5 discharges and $a_{0}, 2 a_{0}, 3 a_{0}, 4 a_{0}$ and $5 a_{0}$ are the values provided the discharges were simultaneous.

This correction is quite uncertain as the time, $t$, is uncertain.

In assuming that the impulses were equal we have not taken account of the angle at which the needle stands at the second and subsequent discharges, nor the magnetism induced in the needle under the same circumstances. One would diminish and the other would increase the 
effect. I satisfied myself by suitable experiments that the error from this cause might be neglected.

The method of experiment was as follows: The store of electricity was contained in a large battery of Leyden jars. This was attached to the electrometer. The reading of the potential was taken, the handle of the discharger was turned and the momentary swing observed and the potential again measured. The mean of the potentials observed, with a slight correction, was taken as the potential during the time of discharge. This correction came from the fact that the first reading was taken before the connection with the condenser was made. The first reading is thus too high by the ratio of the capacities of the condenser and battery and the mean reading by half as much. Hence we must multiply $d$ by $1-F$ where $F=.0013$ for first ball of condenser and .0008 for other. This will be the same for 1 or 5 discharges. From 10 to 20 observations of this sort constituted a set, and the mean value of $\frac{d}{\delta}$, which was calculated for each observation separately, was taken as the result of the series.

Before and after each series the times of vibration, $t$ and $T$, and the readings, $\beta$ and $\alpha$, were taken. The logarithmic decrement was observed almost daily.

\section{Results}

The table on the following page gives the results of all the observations.

These results can be separated according to the number of discharges as follows:

1.

2.

3.

4.

5.

$300 \cdot 59$

$298 \cdot 37$

$295 \cdot 73$

$296 \cdot 43$

$296 \cdot 50$

$300 \cdot 17$

$298 \cdot 61$

$296 \cdot 40$

$297 \cdot 24$

$296 \cdot 37$

$296 \cdot 72$

$297 \cdot 43$

298.75

$301 \cdot 82$

$297 \cdot 38$

$297 \cdot 84$

297.78

298.66

295.02

296.87

$298 \cdot 90$

$300 \cdot 19$

296.75

$295 \cdot 22$

$296 \cdot 31$

298.57

299.05

$300 \cdot 80$

$296 \cdot 56$

$295 \cdot 80$

298.48

$297 \cdot 26$

$297 \cdot 15$

296.69 





In taking the mean, I have ignored the difference in the weights due to the number of observations, as other errors are so much greater than those due to estimating the swing of the needle incorrectly.

It will be seen that the series with one discharge is somewhat greater than with a larger number. This may arise from the uncertainty of the correction for the greater number of discharges, and I think it is best to weight them inversely as this number. As the first series has, also, nearly twice the number of any other, I have weighted them as follows:

\begin{tabular}{ll}
$\mathrm{Wt}$. & $v \times 10^{-8}$ \\
8 & $298 \cdot 80$ \\
4 & $298 \cdot 48$ \\
3 & $297 \cdot 26$ \\
2 & $297 \cdot 15$ \\
1 & $296 \cdot 69$ \\
\cline { 2 - 2 } Mean & $298 \cdot 15$
\end{tabular}

Or $v=29815000000 \mathrm{~cm}$. per second.

It is impossible to estimate the weight of this determination. It is slightly smaller than the velocity of light, but still so near to it that the difference may well be due to errors of experiment. Indeed the difference amounts to a little more than half of one per cent. It is seen that there is a systematic falling off in the value of the ratio. This is the reason of my delaying the publication for ten years.

Had the correction, $A$, for the number of discharges been omitted, this difference would have vanished; but the correction seems perfectly certain, and I see no cause for omitting it. Indeed I have failed to find any sufficient cause for this peculiarity which may, after all, be accidental.

As one of the most accurate determinations by the direct method and made with very elaborate apparatus, I think, however, it may possess some interest for the scientific world. 


\section{NOTES ON THE THEORY OF THE TRANSFORMER}

[Johns Hopkins University Circulars, No. 99, pp. 104, 105, 1892; Philosophical Magazine [5], XXXIV, 54-57, 1892 ; Electrical World, XX, 20, 1892]

As ordinarily treated the coefficient of self and mutual induction of transformers is assumed to be a constant and many false conclusions are thus drawn from it.

I propose to treat the theory in general, taking account of the hysteresis as well as the variation in the magnetic permeability of the iron. ${ }^{1}$

The quantity $p$ as used by Maxwell is the number of lines of magnetic induction enclosed by the given conductor. This will be equal to the number of turns of the wire into the electric current multiplied by the magnetic permeability and a constant. But the magnetic permeability is not a constant but a function of the magnetizing force, and hence we must write

$$
p=B n y+C(n y)^{3}+D(n y)^{5}+\text { ete. }
$$

Where $B, C$, etc., are constants, $n$ is the number of turns and $y$ the strength of current.

In this series only the odd powers of $y$ can enter in order to express the fact that reversal of the current produces a negative magnetization equal in amount to the direct magnetization produced by a direct current. This is only approximately true, however, and we shall presently correct it by the introduction of hysteresis. It is, however, very nearly true for a succession of electric waves.

To introduce hysteresis, first suppose the current to be alternating so that $y=c \sin (b t+e)$ where $t$ is the time and $e$ the phase. The introduction of a term $A \cos (b t+e)$ into the value of the number of lines of induction will then represent the effect very well. But the current is not in general a simple sine curve and so we must write

$$
y=a_{1} \sin \left(b t+e_{1}\right)+a_{2} \sin \left(2 b t+e_{2}\right)+a_{3} \sin \left(3 b t+e_{3}\right)+.
$$

1 The problem is treated by the method of magnetic circuit first applied by me to iron bars in my paper on 'Magnetic Distribution' (Phtl. Mag., 1875), and afterwards to the magnetic circuit of dynamos at the Electrical Conference at Philadelphia in 1884. I also used the samc method in my paper on magnetic permeability in 1873 . 
In this case it is much more difficult to express the hysteresis empirically. In most cases the first term in the value of $y$ is the largest. A term of the same nature as before will, in this case, suffice to express the hysteresis approximated. We can then write for the total flux of magnetic induction

$$
p=A \cos \left(b t+e_{1}\right)+B n y+C n^{3} y^{3}+D n^{5} y^{5}+\text { etc. }
$$

Problem 1.-To find the electromotive force necessary to make the electric current a sine curve in a transformer without secondary. Let the resistance be $R$, and make $y=c \sin (b t)$. Then Maxwell's equation becomes

$$
E=R y+n \frac{d p}{d t}
$$

Substituting the value of $y$ we have

$$
E=(R c-A b n) \sin (b t)+B u c b \cos (b t)+3 C n^{3} \sin ^{2}(b t) \cos b t+\text { etc. }
$$

But

$$
\begin{aligned}
& \operatorname{Sin}^{2} b t \cos b t=\frac{1}{4}(\cos b t-\cos 3 b t) \\
& \operatorname{Sin}^{4} b t \cos b t=\frac{1}{16}(\cos 5 b t-3 \cos 3 b t+2 \cos b t) \\
& \operatorname{Sin}^{6} b t \cos b t=\text { etc. }
\end{aligned}
$$

Hence the electromotive force that must be given to the circuit must contain not only the given frequency of the current but also frequencies of $3,5,7$, etc., times as many. In other words, the odd harmonics.

Problem 2.-Transformer without secondary, the electromotive force being a sine curve.

$$
E \sin b t=R y+n \frac{d p}{d t} .
$$

First it is to be noted that when we place in this equation the general value of $y$ and make the coefficients of like functions of $b t$ zero, all the even harmonics will strike out.

Hence the value of the electric current will be

$$
y=a_{1} \sin \left(b t+e_{1}\right)+a_{3} \sin \left(3 b t+e_{3}\right)+a_{6} \sin \left(5 b t+e_{5}\right)+.
$$

Substituting this value in the value for $p$, the equation is theoretically sufficient to determine $a_{1}, a_{3}$, etc., and $e_{1}, e_{3}$, etc. The equations are cubic or of higher order and the solution can only be approximate and I have not thought it worth while to go further with the calculation. However, it is easy to draw the following conclusion:

1. A simple harmonic current through an iron transformer will produce a secondary electromotive force and current, or both, which contain not only the fundamental period but the higher odd harmonics. 
2. This effect is not due to hysteresis but to the variation in the magnetic permeability.

3. The harmonics increase with the increase in magnetization of the iron and nearly vanish as the magnetization decreases, although it is doubtful if they ever quite vanish. Hence, an increase of resistance will decrease the harmonics.

4. In the method of introducing the hysteresis into the equations, it enters as an addition to the resistance in the term $R a_{1}+A n b$, where $R$ is the resistance, $a_{1}$ the maximum current, $A$ the coefficient of hysteresis, which is dependent upon the amount of magnetization of the iron, $n$ the number of turns of wire, and $b=\frac{2 \pi}{T}$ is $2 \pi$ divided by the time of a complete period.

The introduction of the hysteresis into the ordinary equations, therefore, presents little or no difficulty.

Many observers have noted that the current curve in a transformer was not a sine curve and Prof. Ayrton has shown the presence of the odd harmonics but gives no explanation. Mr. Fleming has attributed them to hysteresis, but I believe the present paper gives the first true explanation.

Problem 3.-To find the work of hysteresis. Let the resistance, $R$, be zero. The work done will then be the integral of the current times the electromotive force, or

$$
w=-\int y \frac{d p}{d t} d t
$$

the integral to be taken for one period of the current.

$$
\begin{gathered}
w=\int_{0}^{\frac{2 \pi}{5}}\left[b A \sin \left(b t+e_{1}\right) y+B n y \frac{d y}{d t}+C n^{3} 3 y^{2} \frac{d y}{d t}+\right] d t \\
w=A \frac{\pi}{b} a_{1}
\end{gathered}
$$

All the other terms are zero.

In a unit of time the energy absorbed is

$$
\frac{A a_{1}}{2} \text {. }
$$

Steinmetz has found by experiment that this varies as the 1.6 power of the magnetic induction. Of course the present theory gives nothing of this but only suggests a way of introducing the hysteresis into calculations of this nature. For this purpose replace $A$ by $A^{1} a_{1}{ }^{\cdot 6}$ and the 
work of hysteresis becomes $\frac{A^{1}}{2} a_{1}^{1.6}$ which is thus the formula of Steinmetz.

In the case where a secondary exists the number of turns of wire being $n^{1}$ and the current $y^{1}$, we have simply to replace $n y$ in the above formula by $n y+n^{1} y^{1}$ and change the phase of the hysteresis term so as to be $90^{\circ}$ from the combined magnetizing force, $n y+n^{1} y^{1}$. The equations of the currents will then be, by Maxwell's formula,

$$
\begin{aligned}
& E=R y+n \frac{d p}{d t} \\
& O=R^{1} y^{1}+n^{1} \frac{d p}{d t},
\end{aligned}
$$

which suffice to determine both $y$ and $y^{1}$. The result is too complicated to be attractive. The equations show, however, that the odd harmonics must appear in either the electromotive forces or the primary or secondary currents, if not in all of them at once. The exact distribution is only a case of complicated calculation.

It is to be specially noted that all formulæ by which self induction is balanced by a condenser will not be correct when applied to an iron transformer but only to an air transformer. They will, however, apply approximately to iron transformers in which the magnetization is small and thus probably will apply better to transformers with an open magnetic circuit than with a closed one.

Also an iron transformer should not be compared with an air transformer or two iron transformers with different magnetizations with each other.

In conclusion I may add that the mathematical difficulties might be overcome by another mode of attack but other work draws me in another direction and I leave the matter to be worked up further by others. 
NOTES ON THE EFFECT OF HARMONICS ON THE TRANSMISSION OF POWER BY ALTERNATING CURRENTS

[Electrical World, XX, 368, 1892; La Lumière Électrique, XL VII, 42-44, 1893]

In a recent number of The Johns Hopkins University Circular and the Phil. Mag. for July, 1892, ${ }^{1}$ I have shown that an iron transformer introduces harmonics of the periods $3,5,7$, etc., ${ }^{2}$ times the fundamental period into the currents and electromotive forces both primary and secondary of a transformer and that these increased in value as the iron was more and more magnetized.

It is my present object to call attention to the effect of these harmonics on the transmission of power and its measurement. For lighting purposes they are evidently of very little significance, as currents of all periods are equally efficient in producing heat. There is a loss, however, in the fact that they cause more loss of heat in the wires and the iron of the transformers. But for the transmission of power the case is very different. Here the motors are designed to run at speeds dependent on the period; if there is more than one period the adjustment fails, and there is a loss. The harmonics are thus useless in the transmission of power by synchronous motors, and are of very little use in motors with revolving fields. In these cases the harmonics travel around the circuits, heating the wires and the iron without producing valuable work. They then represent an almost complete loss in the transmission of power, and as they may contain 10,20 or even 30 or 40 per cent of the current, according to the magnetization of the transformer, they are probably responsible for some loss of efficiency in many cases, as will be shown further on.

Indeed, I believe they are the explanation of many seeming mysteries in the working of alternating current motors.

Special arrangements of condensers and coils can be made to pick out these harmonics so that they become more important than the

${ }^{1}$ See also the Electrical World of July 9, 1892.

${ }^{2}$ The periods $2,4,6$, etc., can evidently be introduced by magnetizing the iron of the transformer in one direction by a constant current, or having it originally with an asymmetrical magnetic set. 
original period. This may occur accidentally and cause many curious results in the working of motors.

It is, then, of the first importance in the transmission of power that the curves shall be pure sine curves, and dynamos, transformers and motors must be designed in the future with reference to this point. It would seem, also, that most calculations on the efficiency of power transmission by alternating currents must be at fault unless they include the action of the harmonics.

As to the amount of loss from this cause it is difficult to decide in general. With synchronous motors the harmonics simply flow around the wires without producing useful current of any kind. But this may not cause great loss if the resistance is small. Indeed, considerable distortion may represent small loss of power in certain cases and great loss in others, according to the difference of phase of the current and electromotive force in the harmonics.

In the case of motors with rotary fields the harmonics produce fields revolving with velocities $3,5,7$, etc., times the primary field. Now it is essential for the efficiency of these motors that the armature shall revolve nearly as fast as the field, and hence the efficiency for the harmonics must be very small indeed, and this must decrease the efficiency of the apparatus as a whole.

As to the heating of the wires by the harmonics, it is easy to see that the total heating due to all the currents of different periods will simply be the sum of the heatings due to each of the currents separately.

The effect of harmonics on the hysteresis is much more complicated and can hardly be calculated without further experiment. However, the following hypotheses may give some idea of the action. Let the primary electromotive force be considered unity, and let $a_{3}, a_{5}$, etc., be the electromotive forces of the harmonics. If these acted separately on the hysteresis the total would be:

$$
1+3\left(\frac{a_{3}}{3}\right)^{1 \cdot 6}+5\left(\frac{a_{5}}{5}\right)^{1 \cdot 6}+
$$

Again, if they all combined so that the maximum electromotive force is equal to the sum of them all, the hysteresis will be nearly:

$$
\left(1+\frac{a_{3}}{3}+\frac{a_{5}}{5}+\right)^{1 \cdot 6}
$$

${ }^{3}$ Dynamos and motors introduce the odd harmonics on acconnt of the variations of the self-induction of the machine, which becomes very apparent when a strong current is flowing. The armature reactions may also introduce the harmonics. 
However, it is hardly probable that this last condition would be often satisfied, in which case this formula would give too great a value. When the harmonics are small this last formula can be written nearly

$$
1+1 \cdot 6\left(\frac{a_{3}}{3}+\frac{a_{5}}{5}+\text { etc. }\right)
$$

As an example, suppose $a_{3}=\cdot 3$ and $a_{5}=\cdot 2$ and $a_{7}=1$, these two formulæ give an increase of 10 and 24 per cent in the loss due to hysteresis.

The current heating is only

Or, in the example,

$$
1+a_{3}^{2}+a_{b}^{2}+\text { etc. }^{4}
$$

$$
1+\cdot 09+\cdot 04+\cdot 01=1 \cdot 14
$$

It would seem, then, that the losses due to hysteresis and current heating may be much increased by the harmonics.

I believe the statement has been made that the form of the curve does not influence the hysteresis. This is evidently incorrect, unless we take the top of the curve to reckon from, in which case the statement agrees with the second hypothesis given above if the harmonics are of the proper phase.

To estimate the influence on the efficiency of a plant, assume the efficiency of the dynamo and synchronous motor with primary currents as each equal to 90 per cent, and of the two transformers equal to 93 per cent, and assume that all the currents have the same harmonics as given above. The total efficiency will be 70 per cent. If the harmonics are now added, the 30 per cent loss will become about 35 per cent, the efficiency will be decreased to 65 per cent nearly, a loss of 5 per cent. There is too much assumption about this calculation to warrant full belief, and the figures are given more as a challenge to further investigation than as facts. That there is a decrease of efficiency is certain, but the amount must be determined by further experiment and mathematical investigation. But, however small the loss, provided it occurs in the transformers or the dynamos and motors, it may be of great consequence on account of its heating effect, because the output of these is limited by the amount of the heat generated.

The practical conclusion seems to be that transformers and the armatures of dynamos to be used in the transmission of power must be designed for low magnetizations. By experiment with transformers,

4 This formula assumes that the resistance is the same for the harmonics, whereas it is greater on account of the 'skin' effect. 
made by Dr. Duncan in this laboratory, immense distortion of the curves has been found when the induction exceeds 12,000 lines per square centimetre, while the curves are comparatively smooth with only 5000; hence I scarcely think it advisable to use more than 5000 for transformers, even though low frequency were used. As to dynamos and motors the limit will depend on the variety of machine used and will not influence the better class very much.

The fixing of the limit of magnetization of transformers at 5000 causes the output with given current to vary inversely as the frequency. As the hysteresis with slow frequency will be less, we may increase the current somewhat to make up for it. As to the exact law, it depends on the relative dimensions of wire and iron. Practically we might estimate for an ordinary transformer that the output varied inversely as the eight-tenth power of the frequency.

The law that the output varies inversely as the four-tenth power of the frequency assumes that the magnetization increases with decrease of frequency and thus distorts the curves as shown above.

The immense increase of the size and cost of transformers when distortion of the curve is avoided precludes the use of very low frequencies even were it otherwise desirable.

It is to be noted that the action of the iron in producing harmonies is directly on the electromotive force, and the amount of current flowing will depend on the resistance and the self-induction of the circuit. The resistance, owing to so-called 'skin' effect, will be greater for the harmonics than for the fundamental period. Self-induction depending on the air will always diminish the harmonics, while if it is due to iron it may either increase or decrease them according to their phase.

The measurement of the energy supplied by an alternating current is also much complicated by the presence of harmonics.

Let the current be

$$
C=A_{1} \sin (b t+\varphi)+A_{3} \sin \left(3 b t+\varphi_{3}\right)+A_{5} \sin \left(5 b t+\varphi_{5}\right)+
$$

and electromotive force

$$
E=B_{1} \sin b t+B_{3} \sin \left(3 b t+\psi_{3}^{\prime}\right)+B_{5} \sin \left(5 b t+\psi_{6}^{\prime}\right)+
$$

The energy transmitted is, then, per unit of time

$$
\int_{0}^{31} C E d t=\frac{1}{2 \pi} \int_{0}^{2 \pi} C E d(b t)
$$

If $n$ is the number of complete periods in the primary term, then $b=$ $2 \pi n$ and the energy transmitted per second becomes

$$
\frac{1}{2}\left[A_{1} B_{1} \cos \varphi+A_{3} B_{3} \cos \left(\varphi_{3}-\psi_{3}\right)+A_{5} B_{5} \cos \left(\varphi_{5}-\psi_{3}\right)+\text { etc. }\right]
$$


An ordinary wattmeter in the form of an electrodynamometer with non-inductive coils would give the correct value of this quantity, but any attempt to multiply the mean electromotive force by the current and the cosine of the phase would lead to an incorrect result unless this was done for each harmonic separately.

It is to be noted that the introduction of condensers to balance selfinduction will only work for one period at a time.

Indeed very many of the results hitherto obtained by observers and theorists will require modification in the presence of these harmonics.

It would seem from the above that the transmission of a current for electric lighting is quite a different thing from the transmission of a suitable current for motors. It will be remembered that the transmission in the Frankfort-Lauffen experiment was one of a lighting current alone and that some mystery seems to hang over the motor tests. Can the presence of these harmonics have anything to do with this? 


\section{MODERN THEORIES AS TO ELECTRICITY}

[The Engineering Magazine, VIII, 589-596, January, 1895]

It is not uncommon for electricians to be asked whether modern science has yet determined the nature of electricity, and we often find difficulty in answering the question. When the latter comes from a person of small knowledge which we know to be of a vague and general nature, we naturally answer it in an equally vague and general manner; but when it comes from a student of science anxious and able to bear the truth, we can now answer with certainty that electricity no longer exists. Electrical phenomena, electrostatic actions, electromagnetic action, electrical waves,--these still exist and require explanation; but electricity, which, according to the old theory, is a viscous fluid throwing out little amœba-like arms that stick to neighboring light substances and, contracting, draw them to the electrified body, electricity as a self-repellent fluid or as two kinds of fluid, positive and negative, attracting each other and repelling themselves,-this electricity no longer exists. For the name electricity, as used up to the present time, signifies at once that a substance is meant, and there is nothing more certain to-day than that electricity is not a fluid.

This makes the task of one who attempts to explain modern electrical theory a very difficult one, for the idea of electricity as a fluid pervades the whole language of electrical science, and even the definitions of electrical units as adopted by all scientists suggest a fluid theory. No wonder, then, that some practical men have given up in despair and finally concluded that the easiest way to understand a telegraph line is to consider that the earth is a vast reservoir of electrical fluid, which is pumped up to the line wire by the battery and finally descends to its proper level at the distant end. Is not this the proper conclusion to draw from that unfortunate term 'electric current'? Remembering this fact,- that we cannot yet free ourselves from these old theories, and exactly suit our words to our meaning,-we shall now try to understand the modern progress in electrical theory.

This whole progress is based upon something in the human mind which warns us against the possibility of attraction at a distance 
through vacant space: Newton felt this impossibility in the case of gravitation, but it is to Faraday that we must look principally for the idea that electrical and magnetic actions must be carried on by means of a medium filling all space and usually called the ether. The development of this idea leads to the modern theory of electrical phenomena.

Take an ordinary steel magnet and, like Faraday, cover it with a sheet of paper, and upon this sprinkle iron filings. Mapped before us we see Faraday's lines of magnetic force extending from pole to pole. We can calculate the form of these lines on the supposition that a magnetic fluid is either distributed over the poles of the magnet or on its molecules, assuming that attraction takes place through space without an intervening medium. But at this idea the mind of Faraday revolted, and he conceived that these lines, drawn for us by the iron filings, actually exist in the ether surrounding the magnet; he even conceived of them as having a tension along their length and a repulsion for one another perpendicular to their length.

Two magnets, then, near each other, become connected by these lines, which, like little elastic bands always pulling along their length, strive to bring the magnets together. These so-called lines of force (now called tubes of force) were, by his theory, conducted better by iron and worse by bismuth than by the ether of space, and so gave the explanation of magnetic attraction and diamagnetic repulsion.

The same theory of lines of force was also applied by Faraday to electrified bodies, and thus all electrostatic attractions were explained. By this idea of lines of force it will be seen that Faraday did away with all action at a distance and with all magnetic and electrical fluids, and substituted, instead, a system in which the ether surrounding the magnet or the electrified body became the all-important factor and the magnet or electrified body became simply the place where the lines of force ended: where a line of magnetic force ended, there was a portion of imaginary magnetic fluid; where a line of electric force ended, there was a portion of imaginary electric fluid. As the quantities of socalled plus and minus electricity in any system are equal, we can thus imagine every charged electrical system to be composed of a group of tubes of electrical force (more strictly electric induction) which unite the plus and minus electrified bodies, each unit tube having one unit of plus electricity on one end and one unit of minus electricity on the other. The tension along the tube explains the reason why such an arrangement acts as if there were real plus and minus electrical fluids on the ends of the tube, attracting one another at a dis- 
tance. Consider a plus electrified sphere far away from other bodies. The lines of force radiate from it in all directions, and, being symmetrical around the sphere, they pull it equally in all directions. Now bring near it a minus electrified body, and the lines of force turn toward it and become concentrated on the side of the sphere toward such a body. Hence the lines pull more strongly in the direction of the negative body, and the sphere tends to approach it.

In the case of a conducting body the lines of force always pass outwards perpendicularly to the surface, and hence, if we know the distribution of the lines over the surface, or the so-called surface density of the electricity, we can always tell in which direction the body tends to move. It is not necessary to know whether there are any attracting bodies near the conductor, but only the distribution of the lines. These lines then do away with all necessity for considering action at a distance, for we only have to imagine a kind of ether in which lines of force with given properties can exist, and we have the explanation of electric attraction.

But the question now arises as to how the lines of electric force can be produced in the ether, or, in other words, how bodies can be charged.

In the first place we know that equal quantities of plus and minus electricity are always produced. As an illustration, suppose it is required to charge two balls with electricity. Pass a conducting wire between them with a galvanic battery in its circuit. The galvanic battery generates the lines of force; these crowd together around it and push each other sideways until their ends are pushed down the wire and many of them are pushed out upon the balls.

When the tension backwards along the lines of force just balances the forward push of the electromotive force of the battery, equilibrium is established. If the wire is a good conductor, there may be electrical oscillations before the lines come to rest in a given position, and this I shall consider below.

The motion of the ends of the lines of force over and in the wire constitutes what is called an electric current in the wire which is accompanied by magnetic action around it and also by waves of electromagnetic disturbance which pass outward into space.

If, after equilibrium is established, we remove the wire, we have simply two charged spheres connected by lines of electrostatic force and thereby attracted to each other. If we replace the battery by a dynamo or by an electric machine the effect is the same.

But there is another way by which bodies are often charged and 
that is by friction. In this case we can suppose the glass to take hold of one end of the lines of force and the rubber the other end and it is then only necessary to pull the bodies asunder to fill the space with lines. The friction is merely needed to bring the two bodies into intimate contact and remove them gently from each other.

The following considerations may guide us in understanding the details of the process. It is well known from Faraday's researches that a given quantity of electricity has a fixed relation to the chemical equivalents of substances. Thus it requires 10,000 absolute electromagnetic units of electricity to deposit 114 grams of silver, 68 grams of copper, 34 grams of zinc, etc.

Hence we can consider, for instance, in chloride of silver that the atoms of silver are joined to the atoms of chlorine by lines of electrostatic force which hold them to each other. If, by rubbing the chloride of silver, we could remove the chlorine on the rubber while leaving the silver, we could stretch them asunder and so fill space with the lines of electrostatic force. According to this theory, then, each atom has a number of lines of force attached to it, and it is only by stretching the atoms apart that we can fill an appreciable space with them and so cause electrostatic action at a distance.

We come to the conclusion, then, that all electrification is originally produced by separating the atoms of bodies from one another, which can be done by breaking contact, by friction, or by direct chemical action of one substance on another, or in some other manner not so common. The lines of electrostatic force in a case of electricity at rest must always begin and end on matter, and they can never have their ends in space free from matter. The ends can be carried along with the matter, constituting electric convection, or they can slide through a metallic conductor or an electrolyte or rarefied gas, making what we call an electric current; but, as they cannot end in a vacuum, they cannot pass through it. Thus we conclude that a vacuum is a perfect non-conductor of electricity.

The exact process by which the ends of the lines of force pass through and along a conductor can at present be only dimly imagined, and no existing theory can be considered as entirely satisfactory. In the case of an electrolyte, however, we can form a fairly perfect picture of what takes place as the decomposition goes on. Thus, in the case of zinc and copper in hydrochloric acid, we can imagine the zinc plate attracting the chlorine of the acid, thus stretching out the natural line of electric force connecting the chlorine atom and the first hydrogen 
atom; we can imagine the atoms of chlorine and hydrogen in the body of the liquid recombining with each other and their lines of force uniting until they form a complete line long enough to stretch from the zinc to the copper plate; and all without once making a line of force without its end upon matter. We can further imagine the ends of this line sliding along the copper and zinc plates to the conducting wires and down their length, thus making an electric current and carrying the energy of chemical action to a great distance.

If the ends of the lines should slide along the wire without any resistance, the wire would be a perfect conductor: but all substances present some resistance, and in this case heat is generated. This we always find where an electric current passes along a wire: as to the exact nature of this resistance or the nature of metallic conduction in general we know little, but I believe we are approaching the time when we can at least imagine what happens in this most interesting case.

Besides the heating due to the electric current, steadily flowing, we must now account for the magnetic lines of force surrounding the current and the magnetic induction of one current on the other.

If the current is produced by the ends of the tubes of electrostatic force moving along the wire, then we may imagine that the movement of the lines of electrostatic force in space produces the lines of magnetic force in a direction at right angles to the motion and to the direction of the lines of electrostatic force. At the same time we must be careful not to assume too readily that one is the cause and the other the effect: for we well know that a moving line of magnetic force (more properly induction) produces, as Faraday and Maxwell have shown, an electric force perpendicular to the magnetic line and to the direction of motion. Neither line can move without being accompanied by the other, and we can, for the moment, imagine either one as the cause of the other. However, for steady currents, it is simpler to take the moving lines of electrostatic force as the cause and the magnetic lines as the effect.

We have now to consider what happens when we have to deal with variable currents rather than steady ones.

In this case we know from the calculations of the great Maxwell and the demonstrations of Hertz that waves of electromagnetic disturbance are given out. To produce these waves, however, very violent disturbances are necessary. A fan waved gently in the air scarcely produces the mildest sort of waves, while a bee, with comparatively small wings moved quickly and vigorously, emits a loud sound. 
So, with electricity, we must have a very violent electrical vibration before waves carrying much energy are given out.

Such a vibration we find when a spark passes from one conductor to another. The electrical system may be small in size, but the immensely rapid vibrations of millions of times per second, like the quick vibration of a bee's wing, sends out a volume of waves that a slowly moving current is not capable of producing. The velocity of these waves is now known to be very nearly 300,000 kilometers per second. This is exactly the velocity of waves of light, or other radiation in general, and there is no doubt at present in the minds of physicists that these waves of radiation are electromagnetic waves.

By this great discovery, which almost equals in importance that of gravitation, Maxwell has connected the theories of electricity and of light, and no theory of one can be complete without the other. Indeed they must both rest upon the properties of the same medium which fills all space-the ether.

Not only must this ether account for all ordinary electrical and magnetic actions, and for light and other radiation, but it must also account for the earth's magnetism and for gravitation.

To account for the earth's magnetism, we must suppose the ether to have such properties that the rotation of ordinary matter in it produces magnetism. To account for gravitation it must have such properties that two masses of matter in it tend to move toward each other with the known law of force, and without any loss of time in the action of the force. We know that moving electrical or magnetic bodies require a time represented by the velocity of light before they can attract each other in the line joining them. But, for gravitation, no time is allowable for the propagation of the attraction.

But the problem is not so hopeless as it at first appears. Have we not in two hundred and fifty years ascended from the idea of a viscous fluid surrounding the electrified body and protruding arms outward to draw in the light surrounding bodies to the grand idea of a universal medium which shall account for electricity, magnetism, light, and gravitation?

The theory of electricity and magnetism reduces itself, then, to the theory of the ether and its connection with ordinary matter, which we imagine to be always immersed in it. The ether is the medium by which alone one portion of matter can act upon another portion at a distance through apparently racant space.

Let us then attempt to see in greater detail what the ether must explain in order that we may, if possible, imagine its nature. 
1st. It must be able to explain electrostatic attraction. These electrostatic forces are mostly rather feeble as we ordinarily see them. Air breaks down and a spark passes when the tension on the ether amounts to about $\frac{1}{100}$ pound to the square inch. It is the air, however, that causes the break-down. Take the air entirely away, and we then know no limit to this force. In a suitable liquid it may amount to 500 times that in air or 5 pounds to 1 square inch, and become a very strong force indeed. In a perfect vacuum the limit is unknown, but it cannot be less than in a liquid, and may thus possibly amount to hundreds, if not thousands, of pounds to the square inch.

2d. It must explain magnetic action. These actions are apparently stronger than electrostatic actions, but in reality they are not necessarily so. A tension on the ether of only a few hundred pounds on the square inch will account for all magnetic attraction that we know of, although we are able to fix no limit to the force the ether will sustain. No signs have ever been discovered of the ether breaking down.

Again, we must be able to account for the magnetic rotation of polarized light as it passes through the magnetic field; and it can only be accounted for by assuming a rotation around the lines of magnetic force. This action, however, takes place only while the lines of magnetic force pass through matter, and it has never been observed in the ether itself. The velocity of rotation, however, is immense, the plane of polarization rotating in some cases $300,000,000$ times per second.

The ether must also account for the earth's magnetism. If we assume that magnetic lines of force are simply vortex filaments in the ether, we have only to suppose that the ether is carried around by the rotation of the earth, and we have the explanation needed. The magnetism of the earth would then be simply a whirlpool in the ether.

$3 d$. The ether must be able to transmit to a distance an immense amount of energy either by means of electromagnetic waves as in light or by the similar action which takes place in the ether surrounding a wire carrying an electric current.

The amount of energy which can be transmitted by the ether in this manner is enormous, far exceeding that which can be carried by anything composed of ordinary matter. Thus take the case of sunlight: on the earth's surface illuminated by strong sunlight a horsepower of energy falls on every 7 square feet. At the surface of the sun the etherial waves carry energy outward at the rate of nearly 8000 horse-power per square foot! 
Again, an electric wire as large as a knitting needle, surrounded with a tube half an inch in diameter in which a perfect vacuum has been made to prevent the escape of electricity, may convey to a distance a thousand horse-power, indeed even ten thousand or more horsepower, there being apparently no limit to the amount the ether can carry.

Compare this with the steam-engine, where only a few hundred horse-power require an enormous and clumsy steam pipe. Or, again, the amount carried by a steel shaft, which, at ordinary rate of speed, would require to be about a foot in diameter to transmit 10,000 horsepower.

When we compare the energy transmitted through a square foot of ether in waves, as in the case of the sun, with the amount that can be conveyed by means of sound waves in air or even sound waves in steel, the comparison becomes simply ridiculous, the ether being so immensely superior. As quick as light, the ether sends its wave energy to the distance of a million miles while the sluggard air carries it one. Thus, with equal strain on each, the ether carries away a million times the energy that the air could do.

4th. The ether must account for gravitation. For this purpose we are allowed no time whatever to transmit the attraction. As soon as the position of two bodies is altered, just so soon must the line of action from one to the other be in the straight line between them.

If this were not so, the motion of the planets around the sun would be greatly altered. Toward the invention of such an ether, capable of carrying on all these actions at once, the minds of many scientific men are bent. Now and then we are able to give the ether such properties as to explain one or two of the phenomena, but we always come into conflict with other phenomena that equally demand explanation.

There is one trouble about the ether which is rather difficult to explain, and that is the fact that it does not seem to concentrate itself about the heavenly bodies. As far as we are able to test the point, light passes in a straight line through space even when near one of the larger planets, unless the latter possesses an atmosphere. This could hardly happen unless the ether was entirely incompressible or else possessed no weight.

If the ether is the cause of gravitation, however, it is placed outside the category of ordinary matter, and it may thus have no weight although still having inertia,-a thing impossible for ordinary matter where the weight is always exactly proportional to inertia. 
Ether, then, is not matter, but something on which many of the properties of matter depend.

It is curious to note that Newton conceived of a theory of gravitation based on the ether, which he supposed to be more rare around ordinary matter than in free space. But the above considerations would cause the rejection of such a theory. We have absolutely no adequate theory of gravitation as produced by ether.

To explain magnetism, physicists usually look to some rotation in the ether. The magnetic rotation of the plane of polarization of light together with the fact of the mere rotation of ordinary matter, as exemplified by the earth's magnetism, both point to rotation in the ether as the cause of magnetism. A smoke ring gives, to some extent, the modern idea of a magnetic line of force. It is a vortex filament in the ether.

Electrostatic action is more difficult to explain, and we have hardly got further than the vague idea that it is due to some sort of elastic yielding in the ether.

Light and radiation in general are explained when we understand clearly magnetic and electrostatic actions as the two are linked together with certainty by Maxwell's theory.

Where is the genius who will give us an ether that will reconcile all these phenomena with one another and show that they all come from the properties of one simple fluid filling all space, the life-blood of the universe-the ether? 


\section{ELECTRICAL MEASUREMENT BY ALTERNATING CURRENTS}

[American Journal of Science [4], IV, 429-448, 1847; Philosophical Magazine [5], XLV, $66-85,1898]$

The electrical quantities pertaining to an electric current which it is usually necessary to measure, outside of current, electromotive force, watts, etc., are resistances, self and mutual inductances and capacities. I propose to treat of the measurement of alternating currents, electromotive force and watts in a separate paper. Resistances are ordinarily best dealt with by continuous currents, except liquid resistances. I propose to treat in this paper, however, mainly of inductances, self and mutual, and of capacities together with their ratios and values in absolute measure as obtained by alternating currents. I also give a few methods of resistance measurement more accurate than usually given by means of telephones or electrodynamometers as usually used and specially suitable for resistances of electrolytic liquids.

I have introduced many new and some old methods, depending upon making the whole current through a given branch circuit equal to zero. These always require two adjustments and they must often be made simultaneously. However, some of them admit of the adjustments being made independently of each other, and these, of course, are the most convenient. But all these zero methods do not admit of any great accuracy unless very heavy currents are passed through the resistances. The reason of this is that an electrodynamometer cannot be made nearly as sensitive for small curfents as a magnetic galvanometer. The deflection of an electrodynamometer is as the square of the current. To make it doubly sensitive requires double the number of turns in both the coils. Hence we quickly reach a limit of sensitiveness. It is easy to measure an alternating current of $\cdot 0001$ ampere and difficult for 00001 ampere. A telephone is more sensitive and an instrument made by suspending a piece of soft iron at an angle of $45^{\circ}$, as invented by Lord Rayleigh, is also probably more sensitive.

For this reason I have introduced here many new methods, depending upon adjusting two currents to a phase-difference of $90^{\circ}$ which I believe to be a new principle. This I do by passing one current through 
the fixed and the other through the suspended coil of an electrodynamometer. By this means a heavy current can be passed through the fixed coils and a minute current through the movable coil, thus multiplying the sensitiveness possibly 1000 times over the zero current method.

I have also found that many of the methods become very simple if we use mutual inductances made of wires twisted together and wound into coils. In this way the self inductances of the coils are all practically equal and the mutual inductances of pairs of coils also equal. Hence we have only to measure the minute difference of these two to reduce the constants of the coil to one constant, and yet by proper connections we can vary the inductances in many ratios. Three wires is a good number to use. However, the electrostatic induction between the wires must be carefully allowed for or corrected if much greater accuracy than $\frac{1}{100}$ is desired.

By these various methods the measurement of capacities and inductances has been made as easy as the measurement of resistances, while the accuracy has been vastly improved and many sources of error suggested.

Relative results are more accurate than absolute as the period of an alternating current is difficult to determine, and its wave form may depart from a true sine curve.

Let self inductances, mutual inductances, capacities and resistances be designated by $L$ or $l, M$ or $m, C$ or $c, R$ or $r$ with the same suffixes when they apply to the same circuit, the mutual inductance having two suffixes. Let $b$ be $2 \pi$ times the number of complete periods per second, or $b=2 \pi n$. The quantities $b L, b M$ or $\frac{1}{b C}$ are of the dimensions of resistance and thus $\frac{L}{M}, b^{2} L C$ or $b^{2} M C$ have no dimensions. $b^{2} L M, \frac{L}{C}$ or $\frac{M}{C}$ have dimensions of the square of resistances.

Where we have a mutual inductance $\mathrm{M}_{12}$, we have also the two self inductances of the coils $L_{1}$ and $L_{2}$. When these coils are joined in the two possible manners, the self inductance of the whole is

$$
L_{1}+L_{2}+2 M_{12} \text { or } L_{1}+L_{2}-2 M_{12} \text {. }
$$

In case of a twisted wire coil the last is very small. Likewise $L_{1} L_{2}-M^{2}{ }_{12}$ will be very small for a twisted wire coil, as is found by multiplying the first two equations together.

If there are more coils we can write similar equations. For three coils we have 
$$
L_{1}+L_{2}+L_{3}+2 M_{12}+2 M_{13}+2 M_{23}
$$
1. $L_{1}+L_{2}+L_{3}-2 M_{12}-2 M_{13}+2 M_{23}$
2. $L_{1}+L_{2}+L_{3}-2 M_{12}+2 M_{13}-2 M_{23}$
3. $L_{1}+L_{2}+L_{3}+2 M_{12}-2 M_{13}-2 M_{23}$

Connecting them in pairs, we have the self inductances

$$
\begin{array}{lll}
L_{1}+L_{2}+2 M_{12} & L_{1}+L_{3}+2 M_{13} & L_{2}+L_{3}+2 M_{23} \\
L_{1}+L_{2}-2 M_{12} & L_{1}+L_{3}-2 M_{13} & L_{2}+L_{3}-2 M_{23}
\end{array}
$$

There are many advantages in twisting the wires of the standard inductance together, but it certainly increases the electrostatic action between the coils. This latter source of error must be constantly in mind, however, and, for great accuracy, calculated and corrected for. But by proper choice of method we may sometimes eliminate it.

For the most accurate standards, I do not recommend the use of twisted wire coils, at least without great caution. But for many purposes it certainly is a great convenience, especially where only an accuracy of one per cent is desired. In some calculations I have made, I have obtained corrections of from one to one-tenth per cent from this cause.

For twisted wires the above results reduce to $3 L+6 M, 3 L-2 M$. Similar equations can be obtained for a larger number of wires. For twisted wire coils, $n$ wires joined abreast, the self induction is $\frac{L+(n-1) M}{n}$, which is practically equal to $L$ or $M$. The resistance is $R / n$.

When we have $n=p+m$ wires twisted and wound in a coil and we connect them $p$ direct and $m$ reverse, the resistance and self induction will be

$$
\frac{n R^{3}+b^{2} R[A C+B C-n A B]}{(n R)^{2}+(b C)^{2}} \text { and } \frac{R^{2}[n(A+B)-C]+b^{2} A B C}{(n R)^{2}+(b C)^{2}},
$$

where $R$ is the resistance of one coil and

$$
\begin{aligned}
& A=L+(n-1) M \\
& B=L-M \\
& C=n L+(4 m p-n) M .
\end{aligned}
$$

This gives self inductances and resistances equal or less than $L$ and $R$. The correction for electrostatic induction remains to be put in. For the general case, the equation is very complicated for coils abreast, with mutual inductances.

The number of mutual inductances to be obtained is $M$ for two wires, $0, M, 2 M$ for three wires, $0, M, 2 M, 3 M$ for four wires, etc. From 
these results we see that we are always able to reduce mutual to self inductance. Measuring the self inductance of a coil connected in different ways, we can always determine the mutual inductances in terms of the self inductances.

Thus we need not search for methods of directly comparing mutual inductances with each other, although I have given two of these, but we can content ourselves with measuring self inductances and capacities. Fortunately most of the methods are specially adapted to the latter, the ratio of self inductance to capacity being capable of great exactness by many methods.

In the use of condensers I have met with great difficulty from the presence of electric absorption. I have found that this can be represented by a resistance placed in the circuit of the condenser, which resistance is a function of current period.

I have developed Maxwell's theory of electric absorption in this manner. Correcting his equations for a small error, I have developed the resistance and capacity of a condenser as follows:

Let a condenser be made of strata of thicknesses $a_{1} a_{2}$, etc., and specific induction capacities $k_{1} k_{2}$, etc., and resistances $p_{1} p_{2}$, etc. Then we have

$$
\begin{aligned}
& R=\frac{B_{0}}{b^{2}}-\frac{B_{2}}{b^{4}}+\frac{B_{4}}{b^{6}}-\text { etc. } \\
& \frac{1}{C}=A_{0}-\frac{A_{2}}{b^{2}}+\frac{A_{4}}{b^{4}}-\text { etc. }
\end{aligned}
$$

where

$$
\begin{aligned}
& B_{0}=\frac{a_{1}}{r_{1} k_{1}^{2}}+\frac{a_{2}}{r_{2} k_{2}^{2}}+\text { etc. } \\
& B_{2}=(4 \pi)^{2}\left\{\frac{a_{1}}{r_{1}^{3} k_{1}^{4}}+\frac{a_{2}}{r_{2}{ }^{3} k_{2}^{4}}+\text { etc. }\right\} \\
& B_{4}=(4 \pi)^{4}\left\{\frac{a_{1}}{r_{1}^{5} k_{1}^{6}}+\text { etc. }\right\}
\end{aligned}
$$

etc.

$$
\begin{aligned}
& A_{0}=4 \pi\left\{\begin{array}{l}
a_{1} \\
k_{1}
\end{array}+\frac{a_{2}}{k_{2}}+\text { etc. }\right\} \\
& A_{2}=(4 \pi)^{3}\left\{\frac{a_{1}}{r_{1}^{2} k_{1}^{3}}+\frac{a_{2}}{r_{2}^{2} k_{2}^{3}}+\text { etc. }\right\} .
\end{aligned}
$$

etc.

Mr. Penniman has experimented in the Johns Hopkins University laboratory with condensers by method 25 and found some interesting results. With a mica standard condenser of $\frac{1}{3}$ microfarad he was not 
able to detect any electric absorption, although I have no doubt one of the more accurate methods will show it.

With a condenser, probably of waxed paper, he found

Number of complete
periods per second.

$14 \cdot 0$

$32 \cdot 0$

$53 \cdot 3$

$131 \cdot 1$
Capacity in
microfarads.

$4 \cdot 64$

$4 \cdot 96$

$4 \cdot 96$

$4 \cdot 94$
Apparent resistance in ohms.

$139 \cdot 6$

$34 \cdot 1$

$20 \cdot 5$

$5 \cdot 2$

The first value of the capacity seems to be in error, possibly one of calculation. However, the result seems to show a nearly constant capacity but a resistance increasing rapidly with decrease of period, as Maxwell's formulæ show. The constant value of the capacity remains to be explained.

Mr. Penniman will continue the investigation with other condensers, liquid and solid, as well as plates in electrolytic liquids.

The results in the other measurements have been fairly satisfactory, but many of the better methods have only been recently discovered and are thus untried. But we must acknowledge at once that work of the nature here described is most liable to error. Every alternating current has, not only its fundamental period, but also its harmonics, so that very accurate absolute values are almost impossible to be obtained without great care. To eliminate them, I propose to use an arrangement of two parallel circuits, one containing a condenser and the other a self-inductance, each with very little resistance. The long period waves will pass through the second side and the short ones through the condenser side. By shunting off some of the current from the second side, it will be more free from harmonics than the first one.

However, in a multipolar dynamo, especially one containing iron, there is danger of long period waves also, which this method might intensify. A second arrangement, using the condenser side, might eliminate them. However, many dynamos without iron and without too many poles and properly wound produce a very good curve without harmonics, especially if the resistance in the circuit is replaced by a self inductance having no iron. These remarks apply only to absolute determinations. Ratios of inductance, self and mutual, and capacity are independent of the period, and thus it can always be eliminated. Measurements of resistances also are independent.

But there are other errors which one who has worked with continuous 
currents may fall into. Nearly all alternating currents generate electromagnetic waves which are so strong that currents exist in every closed circuit with any opening between conductors in the vicinity.

We eliminate this source of error by twisting wires together and other expedients. But in avoiding one error, we plunge into another. For, by twisting wires we introduce electrostatic capacity between them, which may vitiate our results. Thus, in methods 23 or 24 for comparing mutual inductances, if there is electrostatic capacity between the wires, a current will flow through the electrodynamometer in the testing circuit and destroy the balance.

Various expedients suggest themselves to eliminate this trouble, as, for instance, the variation of the resistance $A$ in the above, but I shall reserve them for a future paper. I may say, however, that it is sometimes possible, as in method 12 for instance, to choose a method in which the error does not exist.

However, with the best of methods, much rests with the experimenter, as errors from electromagnetic and electrostatic induction are added to errors from defective insulation when we use alternating currents.

These errors are generally less than one per cent, however, and intelligent and careful work reduces them to less than this.

The following methods generally refer by number to the plate on which the resistances, etc., are generally marked. One large circle with a small one inside represent an electrodynamometer. Of course the circuit of the small coil can be interchanged with the large one. Generally we make the smaller current go through the hanging coil.

By the methods 1 to 14, we adjust the electrodynamometer to zero by making the phase difference in the two coils $90^{\circ}$. For greatest sensitiveness, the currents through the two coils must be the greatest possible, heating being the limit. This current should be first calculated from the impedance of the circuit, as there is danger of making it too great.

In the second series of methods, 15-26, the branch circuit in which the current is to be 0 is indicated by 0 .

Resistances in the separate circuits are represented by $R, R^{\prime}, R$, etc., and $r, r^{\prime}, r_{\text {, }}$, etc. Corresponding self inductances and capacities in the same circuits are $L, L^{\prime}, L$, etc., and $l, l^{\prime}, l_{\text {, }}$, etc., or $C, C^{\prime}, C_{l}$, etc., and $c, c^{\prime}, c$, etc. $b=2 \pi n$ where $n$ is the number of complete current waves per second.

The currents must be as heavy as possible, $\frac{1}{10}$ ampere or more, and it is well to make those that require a current of more than $\frac{1}{100}$ ampere of 
larger wire freely suspended in oil. A larger current can, however, be passed through an ordinary resistance box for a second or two without danger. A few fixed coarse resistances of large wire in air or oil with ordinary resistance boxes for fine adjustment, are generally all that are required. Special boxes avoiding electrostatic induction are, however, the best, but are not now generally obtainable.

In some methods, such as $8,9,10$, etc., we can eliminate undesirable terms containing the current period by using a key which suddenly changes the connections before the period has time to change much.

In using twisted wire mutual inductances, methods 7 and 12 are about or entirely free from error due to electrostatic action between the wires. In all the methods this error is less when the resistance of the coils is least and in 23 and 24 when $A$ is least. In method 8 the error is very small when the coil resistances and $R$ are small and $r$ great. In this method with 1 henry and 1 microfarad the error need not exceed 1 in 1000 . Probably the same remarks apply to $9,10,11$, also. By suitable adjustment of resistances in the other method, the error may be reduced to a minimum. It can, of course, be calculated and corrected for.

An electrodynamometer can be made to detect :0001 ampere without making the self inductance of the suspended coil more than $.000 \%$ henrys or that of the stationary coils more than .0006 henrys, the latter coil readily sustaining a current of $\frac{1}{10}$ amperes without much heating.

An error may creep in by methods 1-14 if the current through the suspension is too great, thus heating it and possibly twisting it. This should be tested by short circuiting the suspended coil or varying the current. For the zero method it is eliminated by always adjusting until there is no motion on reversing the current through one coil.

Inductances containing iron introduce harmonics and vary with current strength. Thus they have no fixed value.

Closed circuits or masses of metal near a self inductance, diminish it, and increase the apparent resistance which effects vary with the period. Short circuits in coils are thus detected.

Electrolytic cells act as capacities which, as well as the apparent resistance, vary with the current period. They also introduce harmonics. The same may be said of an electric arc.

An incandescent lamp or hot wire introduces harmonics into the circuit.

Hysteresis in an iron inductance acts as an apparent resistance in 
the wire almost independent of the current period, and does not, of itself, introduce harmonics. The harmonics are due to the variation of the magnetic permeability with the amount of magnetization.

Electric absorption in a condenser acts as a resistance varying with the square of the period, the capacity also varying, as I have shown above.

In general any circuit containing resistances, inductances and capacities combined acts as / resistance and inductance or capacity, both of which vary with the current period, the square of the current period alone entering. For symmetry the square of the current period can alone enter in all these cases and those above.

Hence only inductances containing no iron or not near any closed metallic circuits have a fixed value. The same may be said of condensers, as they must be free from electric absorption or electrolytic action to have constants independent of the period. There is no apparent hysteresis in condensers and the constants do not apparently vary with the electrostatic force.

The following numbers indicate both the number of the method and the figures in the plate, p. 302.

\section{Method 1.}

$$
\frac{L^{\prime}}{c}=\frac{\left[r\left(R_{1}+R^{\prime \prime}\right)+R_{11}\left(r+R_{t}\right)\right]\left[R^{\prime}\left(R_{1}+R_{u}\right)+R^{\prime \prime}\left(R_{1}+R^{\prime}\right)\right]}{\left(R_{1}+R^{\prime \prime}+R_{u}\right)^{2}}
$$

\section{Method 2.}

$$
\begin{aligned}
& \frac{L^{\prime}}{c} \text { or } b^{2} L L^{\prime} \text { or }-\frac{1}{b^{2} c c^{\prime}}= \\
& \frac{\left[R_{\| \prime} R^{\prime}-R_{\imath} R^{\prime \prime}\right]\left[R_{,}\left(r+R^{\prime \prime}\right)+R_{\prime \prime}\left(r+R_{)}\right)\right]}{R_{\text {॥ }}\left(R_{\imath}+R_{\text {॥ }}\right)}
\end{aligned}
$$

\section{Method 3.}

In (1) make $R^{\prime}=R^{\prime \prime}=R_{u}=0$ or in (2) make $R^{\prime \prime}=R_{\iota}=0, R_{\|}=\infty$, $\frac{L^{\prime}}{c}=r R^{\prime}$

In case the circuit $r$ contains some self inductance, $l$, we can correct for it by the equation

$$
\frac{L^{\prime}}{c}=r R^{\prime}\left(\frac{1}{1-b^{2} l c}\right)
$$



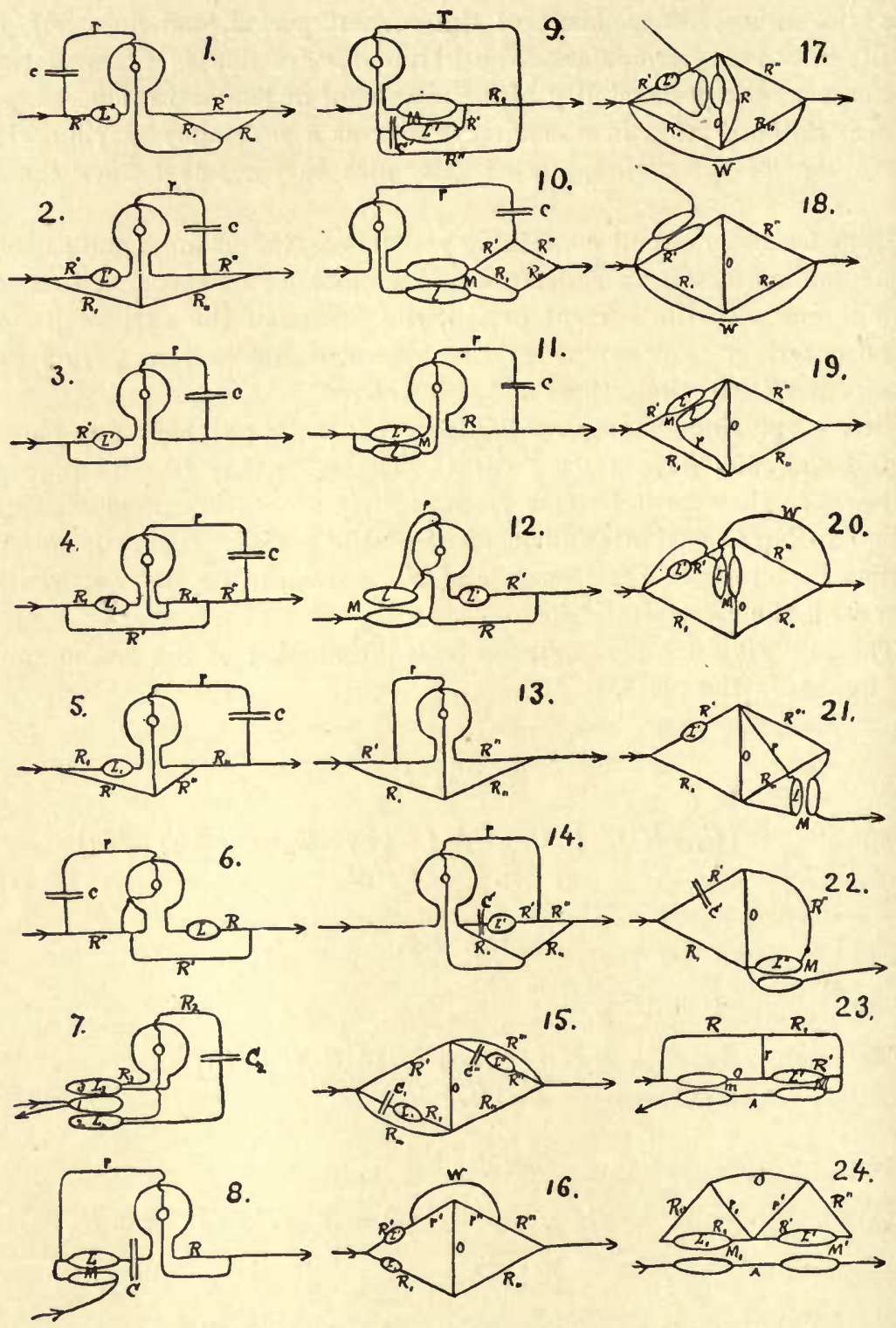

In methods 1 to 14 inclusive the concentric circles are the coils of the electrodynamometer. Either one is the fixed coil and the other the hanging coll. Oblong figures are inductances and when near each other, are mutual inductances. A pair of cross lines is a condenser. 


\section{Method 4.}

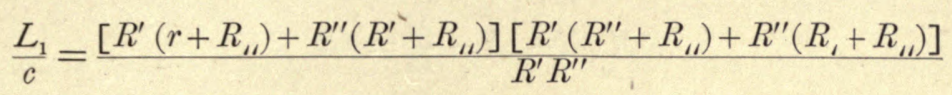

Method 5.

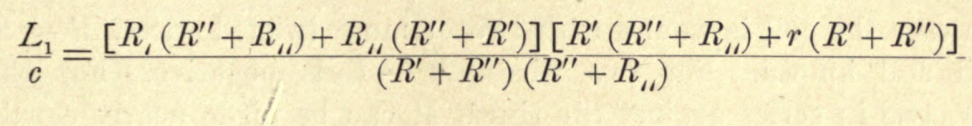

Method 6.

$$
\frac{L}{c} \text { or } \frac{l}{C}=\left(R+R^{\prime}\right)\left(R^{\prime \prime}+r\right)
$$

We can correct for self inductions, $L^{\prime}, L^{\prime \prime}$ in the circuits $R^{\prime}, R^{\prime \prime}$ by using the exact equation

$$
\begin{aligned}
& b^{2}\left[L^{\prime}\left(r+R^{\prime \prime}\right)+\left(L^{\prime \prime}-\frac{1}{v^{2} c}\right) R^{\prime}\right]\left[L^{\prime \prime}\left(R+R^{\prime}\right)+R^{\prime \prime}\left(L+L^{\prime}\right)\right]+ \\
& R^{\prime} R^{\prime \prime}\left(r+R^{\prime \prime}\right)\left(R+R^{\prime}\right)=0
\end{aligned}
$$

or approximately

$$
\begin{aligned}
\frac{L}{c}= & \left(R+R^{\prime}\right)\left(R^{\prime \prime}+r\right)-\frac{L^{\prime}}{c}-\frac{L^{\prime \prime}}{c} \frac{R+R^{\prime}}{R^{\prime \prime}}+b^{2} \frac{L\left[L^{\prime}\left(r+R^{\prime \prime}\right)+L^{\prime \prime} R^{\prime}\right]}{R^{\prime}} \\
& + \text { etc. }
\end{aligned}
$$

\section{Method \%.}

$$
R_{2} R_{3} M_{13} M_{12}+b^{2}\left[L_{3} M_{12}-M_{23} M_{13}\right]\left[L_{2} M_{13}-M_{23} M_{12}\right]=0
$$

For a coil containing three twisted wires, $M_{12}=M_{13}=M_{23}$ and the self inductions of the coils are also equal to each other and nearly equal to the mutual inductions. Put an extra self induction $L_{3}$ in $R_{3}$ and a capacity $C_{2}$ in $R_{2}$. Replace $L_{3}$ by $L+L_{3}$ and $L_{2}$ by $L-\frac{1}{b^{2} C_{2}}$ and we can write

$$
\frac{L_{3}+L-M}{C_{2}}=R_{2} R_{3}+b^{2}(L-M)\left(L_{3}+L-M\right) .
$$

As $L-M$ is very small and can be readily known, the formula will give $\frac{L_{3}}{C_{2}}$. When $L-M=0$ we have

$$
\frac{L_{2}}{C_{3}} \text { or } \frac{L_{3}}{C_{2}}=R_{2} R_{3}
$$

\section{Method 8.}

$$
\begin{aligned}
b^{2} M(M+r) & =r R & & 2 b^{2} M^{2}=r R+(r R)^{\prime} \\
\text { or } b^{2} M(M-L) & =(r R)^{\prime} & 2 b^{2} L M & =r R-(r R)^{\prime}
\end{aligned}
$$


Placing a capacity in the circuit $R$, we have also

$$
\begin{gathered}
b^{2} M(M+L)-\frac{M}{C}=r R \\
\text { or } b^{2} M(M-L)+\frac{M}{C}=r R
\end{gathered}
$$

In case the coil is wound with two or more twisted wires, $M-L$ is small and known. For two wires, $M-L$ is negative. For three wires, two in series against the third, $M$ can be made nearly equal to $2 L$. Hence $M, L$ and $C$ can be determined absolutely, or $C$ in terms of $M$ or vice versa.

To correct for the self induction, $l$, or $r$ we have the exact equations

$$
\begin{aligned}
& b^{2} M(M+L)=r R+b^{2} l(L+M) \\
& b^{2} M(M-L)=r R+b^{2} l(L-M) \\
& b^{2} M(M+L)-\frac{M}{C}=r R+b^{2} l\left(L+M-\frac{1}{b^{2} C}\right) \\
& b^{2} M(M-L)+\frac{M}{C}=r R+b^{2} l\left(L-M-\frac{1}{b^{2} C}\right)
\end{aligned}
$$

If the condenser is put in $r$, we have

$$
\begin{gathered}
\frac{L+M}{c}=r R-b^{2} M(L+M) \\
\text { or } \frac{L-M}{c}=r R+b^{2} M(L-M)
\end{gathered}
$$

Method 9.

$$
\begin{aligned}
b^{2} L^{\prime} M-\frac{M}{C^{\prime}} & =R_{\iota}\left[R^{\prime}+R_{\iota}+\frac{r R^{\prime \prime}}{r+R^{\prime \prime}}\right] \\
\text { or }-b^{2} L^{\prime} M+\frac{M}{C^{\prime}} & =R_{\iota}\left[R^{\prime}+R_{\iota}+\frac{r R^{\prime \prime}}{r+R^{\prime \prime}}\right]
\end{aligned}
$$

Making $R^{\prime \prime}=\infty$ and $r+R^{\prime}=r$ we have

$$
-b^{2} L^{\prime} M+\frac{M}{c^{\prime}} \text { or } b^{2} L^{\prime} M-\frac{M}{C^{\prime}}=R_{\imath}\left(r+R_{\imath}\right)
$$

Taking two observations we can eliminate $b^{2} L^{\prime} M$ and we have

$$
\frac{M}{C^{\prime}}=R_{,}\left\{r-(r)^{\prime}\right\}
$$

Knowing $L^{\prime} M$ we can find $C^{\prime}$. Throwing out $C^{\prime}$ (i. e., making it $\infty$ ) we can find $b^{2} L^{\prime} M$ in absolute measure: then put in $C^{\prime}$ and find its value as above.

To correct for self induction in $R_{\text {, }}$ we have for case $R^{\prime \prime}=\infty$, the exact equation 


$$
b^{2} L^{\prime} M-\frac{M}{C^{\prime}}=R_{\iota}\left(r+R_{\imath}\right)+b^{2}\left[L^{\prime}+L_{\imath}-M\right] L_{\imath}-\frac{L^{\prime}}{C^{\prime}}
$$

The correction, therefore, nearly vanishes for two twisted wires in a coil where $L^{\prime}-M=0$ and $C$ is taken out.

\section{Method 10.}

$-b^{2} l M+\frac{M}{c}$ or $b^{2} l M-\frac{M}{c}=$

$$
\frac{\left[R_{,} R^{\prime \prime}-R_{u} R^{\prime}\right]\left\{r\left[R^{\prime}+R^{\prime \prime}+R_{1}+R_{\|}\right]+\left(R^{\prime}+R_{1}\right)\left(R^{\prime \prime}+R_{u}\right)\right\}}{\left[R^{\prime}+R^{\prime \prime}+R_{\iota}+R_{u}\right]^{2}}
$$

This can be used in the same manner as 9 to which it readily reduces. But it is more general and always gives zero deflection when adjusted, however $M$ is connected. To throw out $C$ make it $\infty$.

\section{Method 11.}

$$
\begin{aligned}
& \frac{L-M}{c}=r R+b^{2}(l-M)(L-M) \\
& \frac{L+M}{c}=r R+b^{2}(l+M)(L+M)
\end{aligned}
$$

For the upper equation the last term may be made small and the method may be useful for determining $L-M$ when $c$ is known. Method 8, however, is better for this.

$$
\begin{aligned}
& \text { Method 12. } \\
& \frac{L^{\prime}}{l}=\frac{R+R^{\prime}}{r}
\end{aligned}
$$

Should the circuits $R$ and $r$ also have small self inductances, $L$ and $l$, we can use the exact equation

$$
L^{\prime}+L=\frac{R+R^{\prime}}{r} l \frac{1+\frac{L r}{l R}}{1-\frac{b^{2} L l}{r R}}=\frac{R+R^{\prime}}{r} l\left[1+\frac{L r}{l R}+\frac{b^{2} L l}{r R}+\text { etc. }\right]
$$

When $L^{\prime}$ and $l$ are approximately known, we can write the following, using the approximate value on the right side of the equation

$$
\frac{L^{\prime}}{l}=\frac{R+R^{\prime}}{r}\left[1+\frac{L r}{l R}-\frac{L}{l} \frac{r}{R+R^{\prime}}+\frac{b^{2} L l}{r R}+\text { etc. }\right]
$$

Taking out $L^{\prime}$ and putting a condenser, $C$, in $R$ we have

$$
\frac{l}{C}=r R^{\prime}-b^{2} l C R\left(R+R^{\prime}\right)
$$

For a condenser, $R$ can be small or zero. 
Method 13.

(A) $\left[b L^{\prime \prime}-\frac{1}{b C^{\prime \prime}}\right]^{2}=\frac{\left[R_{\prime \prime} R^{\prime}-R_{1} R^{\prime \prime}\right]\left[R_{\prime \prime}\left(r+R_{1}\right)+R_{1}\left(r+R^{\prime \prime}\right)\right]}{R_{1}^{\prime}}$

This determines capacities or self inductions in absolute value. As described above, mutual induction can also be determined by converting it into self induction.

$$
\begin{aligned}
& \text { (B) }\left[b L_{\|}-\frac{1}{b C_{\|}^{-}}\right]^{2}=\frac{\left[R^{\prime \prime} R_{1}-R^{\prime} R_{\| \prime}\right]\left[R_{\prime \prime}\left(r+R_{)}\right)+R_{1}\left(r+R^{\prime \prime}\right)\right]}{R^{\prime}\left(r+R_{\imath}\right)}
\end{aligned}
$$

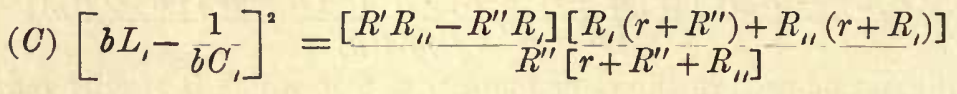

$$
\begin{aligned}
& {\left[b L^{\prime}-\frac{1}{b C^{\prime}}\right]^{2}=} \\
& \frac{\left[R_{6} R^{\prime \prime}-R_{\prime \prime} R^{\prime}\right]\left[r\left[R^{\prime}+R_{1}+R^{\prime \prime}+R_{\prime \prime}\right]+\left[R^{\prime}+R_{d}\right]\left[R^{\prime \prime}+R_{\prime \prime}\right]\right]}{R_{\prime \prime}\left[r+R^{\prime \prime}+R_{\prime \prime}\right]}
\end{aligned}
$$

Of course, in any of these equations, methods 13 or $14, L^{\prime \prime}$ is eliminated by making $L^{\prime \prime}=0$ or the condenser, $C$, is omitted by making $C=\infty$.

\section{Method 15.}

$$
\begin{aligned}
& \frac{1}{b^{2} C, C^{\prime \prime}} \text { or } b^{2} L, L^{\prime \prime} \text { or }-\frac{L_{1}^{\prime}}{C^{\prime \prime}}= \\
& \frac{R^{\prime} R_{11}\left(R_{1}+R_{1 \prime \prime}\right)\left(R^{\prime \prime}+R^{\prime \prime \prime}\right)-R^{\prime \prime} R_{\imath} R^{\prime \prime \prime} R_{t \prime \prime}}{R^{\prime} R_{1 \prime}-R^{\prime \prime \prime} R_{t \prime \prime}} \\
& \frac{C^{\prime \prime}}{C_{1}} \text { or } \frac{L_{1}}{L^{\prime \prime}} \text { or }-b^{2} L_{1} C^{\prime \prime \prime}=\frac{R_{1 \prime \prime} R^{\prime \prime \prime} R_{1}-R^{\prime} R_{\prime \prime}\left(R_{1}+R_{1 \prime \prime}\right)}{R^{\prime} R_{\prime \prime}\left(R^{\prime \prime}+R^{\prime \prime \prime}\right)-R^{\prime \prime} R_{\prime \prime} R^{\prime \prime \prime}}
\end{aligned}
$$

When $R_{m}=\infty$ we have

$$
\begin{gathered}
\frac{L_{\prime}}{U^{\prime \prime}}=\frac{R^{\prime} R_{\prime \prime}\left(R^{\prime \prime}+R^{\prime \prime \prime}\right)-R^{\prime \prime} R_{1} R^{\prime \prime \prime}}{R^{\prime \prime \prime}}=R^{\prime} R_{\prime \prime}-\frac{R^{\prime \prime}}{R^{\prime \prime \prime}}\left[R^{\prime \prime \prime} R_{,}-R^{\prime} R_{\prime \prime}\right] \\
b^{2} L_{,} C^{\prime \prime}=\frac{R^{\prime \prime \prime} R_{-}-R^{\prime} R_{\prime \prime}}{R^{\prime \prime} R^{\prime \prime \prime}}
\end{gathered}
$$

If we adjust by continuous current, we shall have $R^{\prime \prime \prime} R_{1}-R^{\prime} R_{11}=0$. For a condenser we can made $R^{\prime \prime}=0$ provided there is no electric absorption. In this case $b^{2} L, C^{\prime \prime}$ is indeterminate and we can adjust to find $\frac{L_{1}}{C^{\prime \prime}}$. However, two simultaneous adjustments are required.

But I have shown that the presence of electric absorption in a condenser causes the same effect as a resistance in its circuit, the resistance, however, varying with the period of the current. Hence $R^{\prime \prime}$ must 
include this resistance. However, the value of $R^{\prime \prime}$ will not affect the first adjustment much and so the method is easy to work. If it is sensitive enough it will be useful in measuring the electric absorption of condensers in terms of resistance.

It has the advantage of being practically independent of the current period for $\frac{L}{C}$ as it should be.

For comparison of capacities the same simplification does not occur.

Indeed the method is of very little value in this case, being surpassed by 16 .

\section{Method 16.}

(A) $\left[R_{d} R^{\prime \prime}-R_{\| \prime} R^{\prime}\right]\left[W+r^{\prime}+r^{\prime \prime}\right]+W\left[R, r^{\prime \prime}-r^{\prime} R_{u}\right]=0$

$$
L^{\prime}{ }^{\prime} \text { or } \frac{C^{\prime}}{C^{\prime}}=\frac{R^{\prime \prime}}{R_{u}}+\frac{\left(W r^{\prime \prime}\right)}{R_{u}\left(W+r^{\prime} r+{ }^{\prime \prime}\right)}
$$

The first equation is satisfied by adjusting the Wheatstone bridge so as to make

$$
\left(R_{\imath} R^{\prime \prime}-R_{\|} R^{\prime}\right)=0 \quad R_{\imath} r^{\prime \prime}-R_{\text {" }} r^{\prime}=0 \quad R_{,}\left(R_{u}+r^{\prime \prime}\right)-R_{\text {" }}\left(R^{\prime}+r^{\prime}\right)=0
$$

That is

$$
\frac{R_{\iota}}{R_{،}}=\frac{R^{\prime}}{R^{\prime \prime}}=\frac{r^{\prime}}{r^{\prime \prime}}
$$

We can then adjust $W$ with alternating currents. This is a very good method and easy of application but requires many resistances of known ratio. Many of these, however, may be equal without disadvantage. A well known case is given by making $r^{\prime}$ and $r^{\prime \prime}=0$.

$(B)$ By placing self inductions or condensers in $R$, and $r^{\prime \prime}$ instead of the above we have the following

$$
\begin{aligned}
& \frac{c^{\prime \prime}}{C^{\prime}} \text { or }-b^{2} L_{i} c^{\prime \prime} \text { or } \frac{L}{l^{\prime \prime}}=\frac{R^{\prime} R_{u}-R_{,}\left(W+R^{\prime \prime}\right)}{R^{\prime \prime}\left(W+r^{\prime}+r^{\prime \prime}\right)+W r^{\prime \prime}}, \\
& +\frac{1}{b^{2} C^{\prime}, c^{\prime \prime}} \text { or }-\frac{L}{c^{\prime \prime}} \text { or }+b^{2} L l^{\prime \prime}= \\
& \frac{\left(W+r^{\prime}+r^{\prime \prime}\right)\left(R, R^{\prime \prime}-R_{\prime \prime} R^{\prime}\right)+W\left(R_{\imath} r^{\prime \prime}-R_{\prime \prime} r^{\prime}\right)}{W+R^{\prime \prime}}
\end{aligned}
$$

Making $R^{\prime \prime}=0$ we have

$$
\frac{c^{\prime \prime}}{C^{\prime \prime}} \text { or }-b^{2} L, c^{\prime \prime} \text { or } \frac{L}{l^{\prime \prime}}=\frac{R^{\prime} R_{1 \prime}-R, W}{W r^{\prime \prime}}
$$

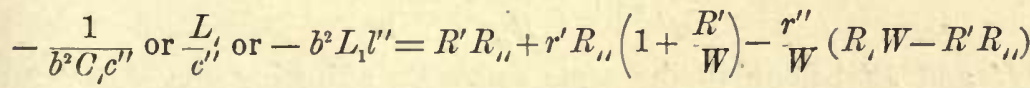

In case we adjust the bridge to $R, W-R^{\prime} R_{1}=0$ and a condenser 
is in $r^{\prime \prime}$ so that we can make $r^{\prime \prime}=0$, the value of $-b^{2} L_{c} c^{\prime \prime}$ will be indeterminate and we can find $\frac{L_{i}}{c^{\prime \prime}}$ by the adjustment of $W$ alone.

This is an excellent method, apparently, as only ore adjustment is required.

However, see the remarks on method 15. This present method $r^{\prime \prime}=0$ for $\frac{L}{c}$ is Anderson's with, however, alternating currents instead of direct as in his.

The other two values are imaginary in this case. Indeed the whole method, $B$, is only of special value for $\frac{L}{c}$, as two adjustments are needed for the others.

\section{Method 17.}

(A) $W=\infty \cdot R=\infty$

$$
\begin{aligned}
& b^{2} M L^{\prime}=R_{\iota} R^{\prime \prime}-R_{u} R^{\prime} \\
& \frac{L^{\prime}}{M}=\frac{R^{\prime}+R_{1}+R^{\prime \prime}+R_{u}}{R_{\text {" }}}
\end{aligned}
$$

By this method the self induction of the mutual induction coil is eliminated. But it is difficult to apply, as two resistances must be adjusted and the adjustment will only hold while the current period remains constant. The same remarks apply to $B$ and $C$ following.

(B) $R=\infty$.

$$
\begin{aligned}
& b^{2} M L^{\prime}=\frac{W\left[R_{1} R^{\prime \prime}-R^{\prime} R_{\|}\right]}{W+R_{1}+R_{1 "}} \\
& \frac{L^{\prime}}{M}=\frac{W\left[R^{\prime}+R_{1}+R^{\prime \prime}+R_{11}\right]+\left(R^{\prime}+R_{\imath}\right)\left(R^{\prime \prime}+R_{\| \prime}\right)}{R_{\text {" }} W}
\end{aligned}
$$

(C) $W=\infty$$$
b^{2} M L^{\prime}=\frac{R}{R+R^{\prime \prime}+R_{\text {" }}}\left(R_{\imath} R^{\prime \prime}-R^{\prime} R_{\text {" }}\right)
$$$$
\frac{L^{\prime}}{M}=\frac{R\left(R^{\prime}+R_{1}+R^{\prime \prime}+R_{1 \prime}\right)+\left(R^{\prime}+R_{\imath}\right)\left(R^{\prime \prime}+R_{\text {"I }}\right)}{R R_{\text {" }}}
$$

Method 18.

$$
\begin{gathered}
R_{,} R^{\prime \prime}-R^{\prime} R_{\text {" }}=0 \\
\frac{L^{\prime}}{M^{\prime}}=1+\frac{R^{\prime \prime}}{R_{\text {" }}}+\frac{R^{\prime}+R^{\prime \prime}}{W}
\end{gathered}
$$

$L^{\prime}$ and $M^{\prime}$ belong to the same coil. By adjusting the Wheatstone bridge first, $W$ can then be afterwards adjusted. 
To find the ratio for any other coil independent of the induction coil, we can first find $\frac{L^{\prime}}{M^{\prime}}$ as above. Then add $L$ to the same circuit and we can find $\frac{L+L^{\prime}}{M^{\prime}}$. Whence we can get $L$. This seems a convenient method if it is sensitive enough, as the value of $\frac{L^{\prime}}{M}$, should be accurately known for the inductance standard.

\section{Method 19.}

$$
\frac{L^{\prime}}{M}=\frac{R^{\prime}+R_{\prime}}{r}-b^{2} \frac{L^{\prime} l-M^{2}}{r^{2}}\left(\frac{l}{M}+1\right)=\frac{R^{\prime}+R_{\prime}}{r}-\frac{R^{\prime} R_{\prime \prime}-R^{\prime \prime} R_{\prime}}{r R_{\prime \prime}}\left(\frac{l}{M}+1\right)
$$

This is useful in obtaining the constants of an induction standard. For twisted wires $L^{\prime} l-M^{2}$ should be nearly 0, depending, as it does, on the magnetic leakage between the coils. $\frac{l}{M}$ is often known suffciently nearly for substitution in the right hand member. It can, however, be found by reversing the inductance standard.

\section{Method 20 .}

$$
R^{\prime} R_{\text {" }}-R^{\prime \prime} R_{1}=0
$$

$$
\begin{aligned}
& \frac{M}{L}=\frac{R_{1 \prime}}{R_{1}+R_{1 \prime}} ; \frac{M}{L^{\prime}}=\frac{W}{R^{\prime \prime}} \frac{R_{11}{ }^{2}}{\left(R_{\imath}+R_{\|}\right)^{2}} ; \frac{L}{L^{\prime}}=\frac{W}{R^{\prime}+R^{\prime \prime}} L>M \text {; } \\
& L^{\prime} \text { any value. }
\end{aligned}
$$

In case of a standard inductance, $M$ and $L$ are known, especially when the wires are twisted.

The method can then be used for determining any other inductance, $L^{\prime}$, and is very convenient for the purpose.

$R_{\|}$and $R_{1}+R_{u}$ are first calculated from the inductance standard. The Wheatstone bridge is then adjusted and $W$ varied until a balance is obtained. This balance is independent of the current period, as also in the next two methods.

Method 21.

$$
\begin{gathered}
R^{\prime} R_{\prime \prime}-R^{\prime \prime} R_{\imath}=0 \\
\frac{l}{M}=\frac{R^{\prime}+R_{l}}{R_{\imath}} ; \frac{L^{\prime}}{M}=\frac{\left(R^{\prime}+R_{\imath}\right)^{2}}{r R_{\imath}} ; \frac{L^{\prime}}{l}=\frac{R^{\prime}+R_{\imath}}{r} l>M .
\end{gathered}
$$

This is Niven's method adapted to alternating currents. See remarks to method 20 . 
Methods 20 and 21 are specially useful when one wishes to set up an apparatus for measuring self induction, as the resistances $R^{\prime}, R^{\prime \prime}$, $R_{\iota}, R_{\|}$can be adjusted once for all in case of a given induction standard and only $W$ or $r$ need be varied afterwards.

$$
\begin{aligned}
& \text { Method 22. } \\
& \frac{L^{\prime \prime}}{M}=\frac{R^{\prime}+R_{\iota}}{R_{\imath}} ; \stackrel{M}{C^{\prime}}=R_{\imath} R^{\prime \prime} ; \frac{L^{\prime \prime}}{C^{\prime}}=R^{\prime \prime}\left(R^{\prime}+R_{\imath}\right)
\end{aligned}
$$

This is Carey Foster's method adapted to alternating currents and changed by making $R^{\prime \prime}$ finite instead of zero.

The ratio of $R^{\prime}+R$, to $R$, is computed from the known value of the induction standard. $R^{\prime \prime}$ is then adjusted and $C^{\prime}$ obtained. In general the adjustment can be obtained by changing $R$, and $R^{\prime \prime}$. The adjustment is independent of the current period.

\section{Method 23.}

$$
\begin{gathered}
b^{2} m L^{\prime}=r R_{1}+R\left[r+R^{\prime}+R_{f}\right] \\
\frac{M r-L^{\prime} R}{m}=r+R^{\prime}+R^{\prime}
\end{gathered}
$$

If we make $R=0$ we have

$$
\begin{gathered}
b^{2} m L^{\prime}=r R, \\
\frac{M}{m}=\frac{r+R^{\prime}+R_{1}}{r}
\end{gathered}
$$

This method requires two simultaneous adjustments. $M$ must also be greater than $m$. As $M$ and $L^{\prime}$ belong to the same coil, we can consider this method as one for determining $m$ in terms of the $M$ and $L^{\prime}$ of some standard coil.

The resistance, $A$, can be varied to test for, or even correct, the error due to electrostatic action between the wires of the induction standard.

$$
\begin{gathered}
\text { Method 2.4. } \\
\frac{L_{i}^{\prime}}{M_{1}}=\frac{L^{\prime}}{M^{\prime}} \frac{r_{\prime}^{\prime}}{r^{\prime}} ; \frac{M}{M^{\prime}}=\frac{r^{\prime}\left(r_{1}+R_{1}+R_{s \prime}\right)}{r_{1}\left(r^{\prime}+R^{\prime}+R^{\prime \prime}\right)}
\end{gathered}
$$

This is a good method for comparing standards. We first determine $\frac{L_{11}}{M}$ for each coil by one of the previous methods. Then we can calculate $\frac{r_{1}}{r^{\prime}}$ and adjust the other resistances to balance.

It is independent of the period of the current and suitable for stand- 
ards of equal as well as of different values, as the mutual inductances can have any ratio to each other.

For twisted wire coils $r_{1}=r^{\prime}$ very nearly. See method 23 for the use of the resistance, $A$.

\section{Method 25.}

In Fig. 6 remove the shunt $R^{\prime}$ and self induction $L$.

This method thei depends upon the measurement of the angular deflection when a self induction or a capacity is put in the circuit of the small coil of the electrodynamometer and comparing this with the deflection, when the circuit only contains resistance.

The resistance of the circuit, $r$, is supposed to be so great compared with $R$ that the current in the main circuit remains practically unaltered during the change.

There is also an error due to the mutual induction of the electrodynamometer coils which vanishes when $r$ is great.

$$
\frac{1}{b^{2} c^{2}} \text { or } b^{2} l^{2}=R^{\prime \prime}\left[r+R^{\prime \prime}\right]\left[\frac{r_{1}+R_{1}^{\prime \prime}}{R_{\imath}^{\prime \prime}} \frac{\theta}{\theta}-\frac{r+R^{\prime \prime}}{R^{\prime \prime}}\right]
$$

These formulas assume that the deflection is proportional to $\theta$. This assumption can be obviated by adjusting $\theta=\theta^{\prime}$ when we have

$$
\frac{1}{b^{2} c^{2}} \text { or } b^{2} l^{2}=\frac{\left(r+R^{\prime \prime}\right)\left(r, R^{\prime \prime}-r R_{1}^{\prime \prime}\right)}{R^{\prime \prime}}
$$

These can be further simplified by making $R^{\prime \prime}=R_{\text {, }}$.

The method thus becomes very easy to apply and capable of considerable accuracy. As the absolute determination depends on the current period, however, no great accuracy can be expected for absolute values except where this period is known and constant, a condition almost impossible to be obtained. The comparison of condensers or of inductances is, however, independent of the period and can be carried out, however variable the period, by means of a key to make the change instantaneously.

\section{Method 26.}

Similar results can be obtained by putting the condenser or inductance in $R^{\prime \prime}$ instead of $r$, but the current through the electrodynamometer suspension is usually too great in this case unless $r$ is enormous. We have in this case for equal deflections,

$$
\frac{1}{b^{2} C^{\prime \prime 2}} \text { or } b^{2} L^{\prime \prime 2}=R^{\prime \prime}\left(R^{\prime \prime}+r\right)\left(\frac{r R_{1}^{\prime \prime}-r_{1} R^{\prime \prime}}{r_{1} R^{\prime \prime}}\right)
$$

where $r$, and $R$," are the resistances without condenser or self induction. 
This is a very good method in many respects.

For using 25 and 26, a key to make instantaneous change of connections is almost necessary.

To measure resistance by alternating currents, a Wheatstone bridge is often used with a telephone.

I propose to increase the sensitiveness of the method by using my method of passing a strong current through the fixed coils of an electrodynamometer while the weaker testing current goes through the suspended system.

Using non-inductive resistances, methods $10,13 A, B, C$, and 14 all reduce to proper ones. 10 or 14 is specially good and I have no doubt will be of great value for liquid resistances. The liquid resistances must, however, be properly designed to avoid polarization errors. The increase of accuracy over using the electrodynamometer in the usual manner is of the order of magnitude of 1000 times.

Since writing the above I have tried some of the methods, especially 6 and 12, with much satisfaction. By the method 12, results to 1 in 1000 can be obtained. Replacing $L^{\prime}$ by an equal coil, the ratio of the two, all other errors being eliminated, can be obtained to 1 in 10,000 , or even more accurately.

The main error to be guarded against in method 12 , or any other where large inductances or resistances are included, arises from twisting the wires leading to these. The electrostatic action of the leads, or the twisted wire coils of an ordinary resistance box, may cause errors of several per cent. Using short small wire leads far apart, the error becomes very small.

Method 6 is also very accurate, but the electric absorption of the condensers makes much accuracy impossible unless a series of experiments is made to determine the apparent resistance due to this cause.

In method $12 \mathrm{I}$ have not yet detected any error due to twisting the wires of coils $l$. However, the electrostatic action of twisted wire coils is immense and the warning against their use which I have given above has been well substantiated by experiment. . Only in case of low resistances and low inductances or in cases like that just mentioned is it to be tolerated for a moment. Connecting two twisted wires in a coil in series with a resistance between them, I have almost neutralized the self induction, which was one henry for each coil or four henrys for them in series!

Altogether the results of experiment justify me in claiming that 
these methods will take a prominent place in electrical measurement, especially where fluid resistances, inductances and capacities are to be measured. They also seem to me to settle the question as to standard inductances or capacities, as inductances have a real constant which can now be compared to 1 in 10,000 , at least.

The new method of measuring liquid resistances with alternating currents allows a tube of quite pure water a meter long and $6 \mathrm{~mm}$. diameter having a resistance of $10,000,000 \mathrm{ohms}$ to be determined to 1 in 1000 or even 1 in 10,000 . The current passing through the water is very small, being at least 500 times less than that required when the bridge is used in the ordinary way. Hence polarization scarcely enters at all.

It is to be noted that all the methods 15 to 24 can be modified by passing the main current through one coil of the electrodynamometer and the branch current through the other. The deflection will then be zero for a more complicated relation than the ones given. If, however, one adjustment is known and made, the method gives the other equation.

Thus method 18 requires $R, R^{\prime \prime}-R^{\prime} R_{\text {" }}=0$. Hence, when this is satisfied we must have the other condition alone to be satisfied. Also in method 22, when we know the ratio of the self and mutual inductances in the coil, the resistances can be adjusted to satisfy one equation while the experiment will give the other and hence the capacity in terms of the inductances.

Again, pass a current whose phase can be varied through one coil of the electrodynamometer, and the circuit to be tested through the other. Vary the adjustments of resistances until the deflection is zero, however the phase of current through the first coil may be varied.

The best methods to apply the first modification to are $15 \mathrm{~A}, 16 \mathrm{~A}$ and $B, 18,20,21,22$ and 24 . In these, either a Wheatstone bridge can be adjusted or the ratio of the self and mutual inductances in a given coil can be assumed as known and the resistances adjusted thereby.

The value of this addition is in the increased accuracy and sensitiveness of the method, an increase of more than one hundred fold being assured.

As a standard I recommend two or three coils laid together with their inductances determined and not a condenser, even an air condenser. 


\title{
ELECTRICAL MEASUREMENTS
}

\author{
Br Henry A. Rowland and Thomas Dobbin Penniman \\ [American Journal of Science [4], VIII, 35-57, 1899]
}

In a previous article ${ }^{1}$ mention was made of some work then being carried on at the Johns Hopkins University to test the methods for the measurement and comparison of self-inductance, mutual inductance, and capacity there described.

In the present paper, there will be given an account of the experiments performed with some of the methods described in the previous article, together with a method for the direct measurement of the effect of electric absorption in terms of resistance.

The methods that were tried were $25,26,9,3,12$ and 6 .

\section{Apparatus}

Description of the Electrodynamometer, Dynamos, Coils, Condensers, Resistances and Connections used in the Experiments

Electrodynamometer.-The electrodynamometer was one constructed at the University, having a sensitiveness, with the coils in series, of 1 scale division deflected for $\cdot 0007$ ampere.

The hanging coil was made up of 240 turns of No. 34 copper wire $B$ and $S$ gauge. The coil was suspended by a bronze wire connected with one terminal of the coil. The other terminal of the coil was a loop of wire hanging from the bottom of the coil and attached to the side of the case; both the suspension and the loop were brought out to binding posts. The resistance of the coil with suspension was $21.7 \mathrm{ohms}$.

The fixed coils were made up of 300 turns each of No. $30 \mathrm{~B}$ and $S$ gauge copper wire. The coils were wound on cup-shaped metal forms and soaked in a preparation of wax. The form was then removed and the coils placed a radius apart as in the arrangement of Helmholtz.

Dynamos.-There were two dynamos used, a Westinghouse alternator, and a small alternating dynamo constructed at the University.

1 This Journal, iv, p. 429, December, 1897; Philosophical Magazine, January, 1898. 
The Westinghouse dynamo was one having 10 poles so that each revolution of the armature produced 5 complete periods. The period of this dynamo was determined by taking the time of 1000 revolutions of the armature. This was accomplished by having the armature make an electric connection with a bell every 200 revolutions and taking the time of 5 of these. The taking of the speed during every experiment gave more regular results, as the speed was constantly changing, the dynamo being run by the engine in the University power-house when it was subject to great change of load. This dynamo had a period of about 132 complete periods per second.

For the production of a current of less period than that of the Westinghouse, the small alternator constructed at the University was used. This dynamo was run by a small continuous Sprague motor. The armature of the small alternator consisted of 8 coils, which coils were fastened flat on a German silver plate, the plate revolving between 8 field pieces producing 4 poles. The object of having the coils of the armature on a metal plate was to secure a nearly constant speed. The metal plate produced a load that varied as the velocity and due to induced currents in the plate. The varying load, depending on the velocity of the moving plate, produced a nearly constant speed, which rendered unnecessary the constant taking of the speed. When this dynamo was used, the speed was only determined two or three times during a series of readings or experiments. The average of these determinations was taken as the speed during the whole series of experiments under consideration.

Coils.-The coils whose inductances were determined were all made in the same way, being wound on a metal form and soaked in a preparation of wax. When the wax was hard the metal form was removed. This enabled the coils to be placed close together, as their sides were flat and smooth. The coils all had the same internal and external diameter, but their width varied, that being determined by the number of turns that were desired.

Coils. $P_{1}$. External diameter $35.46 \mathrm{~cm}$., internal diameter $23 \cdot 8$ cm., was made up of about 1200 turns of No. $16 B$ and $S$ gauge single covered cotton copper wire, roughly wound; the turns were not smooth; self-inductance as finally determined $\cdot 566$ henry.

$P_{2}$. Same dimensions. Turns were put on evenly. The number of turns was 1300 of No. $16 B$ and $S$ single covered cotton copper wire. Self-inductance $\cdot 724$ henry.

$A$. Same internal and external diameters as $P$, but the width was 
$4 \cdot 3 \mathrm{~cm}$. Number of turns $3 \% 00$ No. $20 B$ and $S$ gauge single covered cotton copper wire. Self-inductance as determined $5 \cdot 30$ henrys.

$B_{1} B_{2}$. This coil was made by winding two wires in parallel and all four of the terminals brought out to binding posts. Thus the coils could be used as two single coils, when the coils will be denoted by the symbols $B_{1}$ and $B_{2}$ as the case may be, or as a single coil, the coils $B_{1}$ and $B_{2}$ being joined up in series or in parallel. The dimensions of the coils $B_{1} B_{2}$ were the same as $A$. Each of the coils $B_{1}$ and $B_{2}$ were made up of 1600 turns of No. 22 $B$ and $S$ single covered cotton copper wire. The self-inductance of these coils taken separately when compared with $P$, which was determined absolutely, was nearly 1 henry. On this account $B$ was taken as being 1 henry, and the other coils were compared with it as a standard.

$C$. Same dimensions as $P_{2}$. Number of turns 1747 of No. $22 B$ and $S$ single covered cotton copper wire. Self-inductance as determined $1 \cdot 30$ henrys.

Condensers. -2 and 3. Two paraffined paper condensers that had a capacity of 2 and 3 microfarads respectively.

$\frac{1}{3} \mathrm{~d}$ Troy. A $\frac{1}{3} \mathrm{~d}$ microfarad standard mica condenser built by the Troy Electric Co.

$\frac{1}{3} \mathrm{~d}$ Elliott. A $\frac{1}{3} \mathrm{~d}$ microfarad standard mica condenser built by Elliott Bros.

Resistances. - The resistances used in the experiments were of two kinds, those wound with double wire so as to have no self-inductance, as the ordinary resistance box, and those wound on frames or cards which had some small self-inductance, but almost no electrostatic capacity. The resistances which had self-inductance are called open resistances to distinguish them from resistance boxes, and were of different kinds and dimensions.

\section{Sources of Error and Experimental Difficulties}

In all work with alternating currents there are two great sources of error that have to be guarded against. These are the errors that may arise from the inductance of one part of the apparatus on another, as, for example, the direct induction of a coil in the circuit on the coils of the electrodynamometer, and the effect of the electrostatic capacity of the leads and connections. In connecting the coils great care had to be taken to avoid the effect of electrostatic action of the leads and connections. For if there was a current of very considerable magni- 
tude, the difference of potential between the terminals of the coil might be great. If the connections under these circumstances were made with double wire, as is customary, a great error was introduced due to the electrostatic capacity of the leads. The error was sometimes as much as 7 per cent (see method 24). This error could be shown to be due to the electrostatic action of the leads by shifting a resistance in circuit with the coil in question from one end of the double wire to the other. The effect of this was to still further increase the difference of potential between the leads, and this increased the error. Experiments of this character showed the necessity of using open leads and open resistances having little or no capacity in all cases in which the coils experimented on and the resistance boxes used in their determination have a current of any considerable magnitude passing through them. In several of the following methods constancy of current was necessary. This was accomplished by various means that will be described in their actual application.

\section{METHODS}

The methods that were tried were $25,26,9,3,12$ and 6 described in this Journal, December, 189\%.2

Method 25.-Method of equal deflections. Absolute method for the determination of self-inductance or capacity in terms of electromagnetic units.

In this method the hanging coil is shunted off the fixed coils circuit, and this with a non-inductive resistance in circuit with the hanging coils is made the same as that of a certain inductive resistance in circuit with the hanging coil. The connections are made as in the Figs. 1, 2, where $C_{0} \varepsilon^{i b t}, C_{1} \varepsilon^{i\left(b t+\phi_{1}\right)}, C_{2} \varepsilon^{i\left(b t+\phi_{2}\right)}$ are currents. $R, R^{\prime}, r$, resistances. They represent the entire resistance of their respective branches. $L$ represents self-inductance of the coil by which it is placed. The outer circle in Fig. 1 represents the fixed coils and the small circle the hanging coil of the electrodynamometer. In Fig. 2 the terminals of the fixed and hanging coils are represented by $F$ and $H . \quad D$ is a reversing commutator. $K$ is a key to send the current first through the inductive and then through the non-inductive resistance. $b=2 \pi n$, $n=$ complete alternations per sec. This is the general notation adopted throughout the article.

${ }^{2}$ Phil. Mag., January, 1898. 
The quantity to be found is $C_{0} C_{1} \cos \varphi_{1}$, which is proportional to the deflection of the hanging coil in the two positions of $K$.

In one position

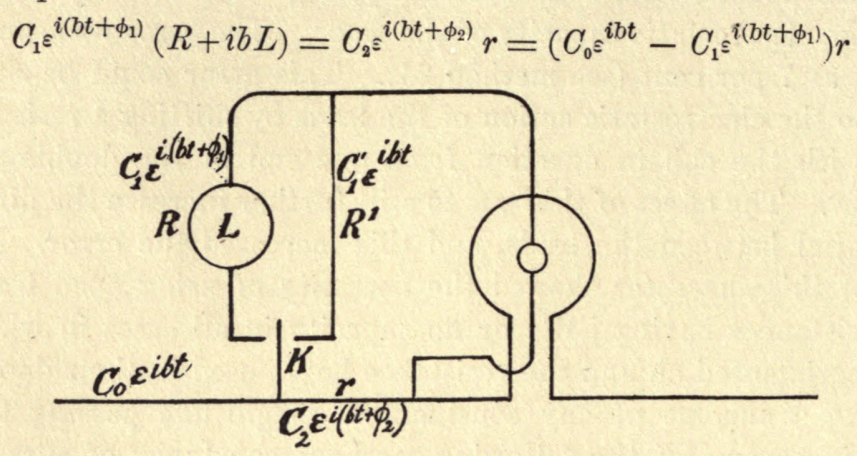

FIG. 1.

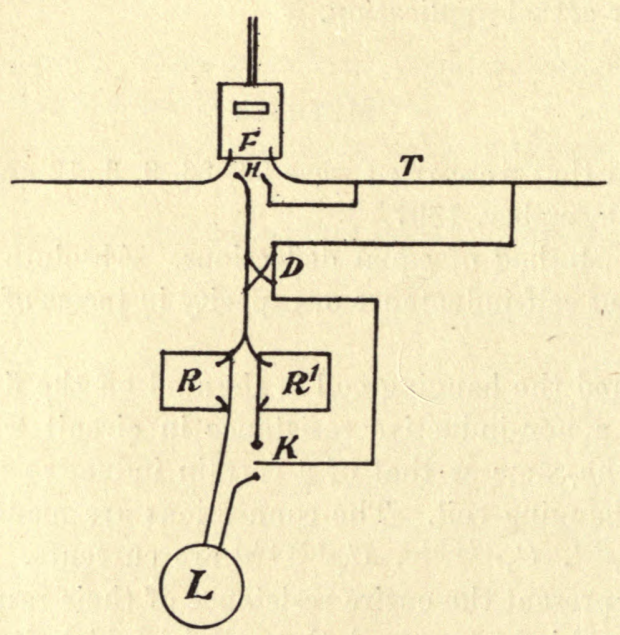

FIG. 2.

Therefore

$$
C_{0} C_{1} \cos \phi_{1}=C_{0} \frac{2 r(R+r)}{(R+r)^{2}+b^{2} L^{2}} \propto D
$$

In the other position of $K$

Therefore

$$
C_{1}^{\prime} \varepsilon^{i b t} R^{\prime}=C_{2}^{\prime} \varepsilon^{i b t} r=\left(C_{0} \varepsilon^{i b t}-C_{1}^{\prime} \varepsilon^{i b t}\right) r
$$

$$
C_{0} C_{1}^{\prime}=C_{0}^{2} \frac{r}{R^{\prime}+r} \propto D^{\prime}
$$


$\phi=0$, as $\phi$ is an angle whose tangent is $\frac{b l}{R^{\prime}}$ and $C=0$ nearly. In the case of equal deflection $D=D^{\prime}$ and therefore

$$
b^{2} L^{2}=\left(R^{\prime}-R\right)(R+r)
$$

If capacity had been used in the place of self-inductance the formula would be

$$
\frac{1}{b^{3} c^{2}}=\left(R^{\prime}-R\right)(R+r)
$$

If self-inductance and capacity were used in series

$$
b^{2}\left(L-\frac{1}{b^{2} c}\right)^{2}=\left(R^{\prime}-R\right)(R+r)
$$

The application of this formula to the measurement of self-inductance gave results that agreed to within the accuracy with which the period of the alternations could be determined. That is, the results agreed to within about 1 per cent. In the determination of $L$ the resistance in circuit $R$ was varied from the least possible resistance as determined by the coils up to $1000 \mathrm{ohms}$ and more, and the selfinductance was determined under these various conditions. These results agreed among themselves, and were apparently independent of the resistance in circuit with it. In the application of this method to the determination of capacity, however, great trouble was encountered, as the capacity apparently varied both with the resistance in circuit with it and with the period. This variation was regular for each period, the value derived depending on the resistance in circuit. This irregularity of derived value of the capacity led to the investigation and development of Maxwell's formula on the effect of absorption, a necessary characteristic of heterogeneous substances.

When the formula was deduced, as may be seen in the article already referred to, the absorption comes in as an added resistance, the resistance being constant for a given period. By an inspection of the results this was found to be the case. The finding of the resistance due to absorption in this method is one of approximation, but the values deduced compare very favorably with those determined by direct measurement, as will be seen later when various results are collected. In the actual experiments the condensers used were two paraffined paper condensers of about 2 and 3 microfarads. The currents used had different periods, as seen in the table following, where $n=133,53 \cdot 3$, $31 \cdot 9$ and 14.

The process was to place in the condenser circuit a resistance $R$, and 
then to move the key $K$ back and forth until $R^{\prime}$ was found that gave the same deflection. $D$, Fig. 2, was now reversed and the process repeated. This was repeated with different values of $R$ and $n$ and the apparent capacity. This gave great variation of apparent capacity with different values of $R$, which should not be the case, and, therefore, gave a means of finding the resistance due to absorption or absorption resistance, as we will designate, by approximation. As the effect of absorption is a resistance it is possible to find what resistance, if added to $R$, will make all the values of the capacity as determined for the different values of $R$ the same. Therefore it should be the same for any two values of $R$. Calling the two values of $R$ in the two cases $R_{1}$ and $R_{2}$ respectively and the two corresponding values of $R^{\prime}, R_{1}{ }^{\prime}$, and $R_{2}{ }^{\prime}$, and let $A$ be the added resistance due to absorption, the capacity should be the same in the two cases, or

$$
\begin{gathered}
\frac{1}{b^{2} c^{2}}=\left[R_{1}^{\prime}-\left(R_{1}+A\right)\right]\left[R_{1}+A+r\right]=\left[R_{2}^{\prime}-\left(R_{2}+A\right)\right]\left[-R_{2}+A+r\right] \\
\therefore A=\frac{\left(R_{2}^{\prime}-R_{2}\right)\left(R_{2}+r\right)-\left(R_{1}^{\prime}-R_{1}\right)\left(R_{1}+r\right)}{R_{1}^{\prime}-R_{2}^{\prime}-2\left(R_{1}+R_{2}\right)}
\end{gathered}
$$

From this $A$ is found for the period used. By doing this for a number of different values of $R$, the true value of $A$ is approximated. $A$ was thus found for the condensers 2 and 3 microfarads with different values of $n$. The calculations were again performed adding to the different values of $R$ a constant resistance $A$. The capacity that was found when $A$ is added to $R$ is called the corrected capacity. In the table below are collected the corrected values of the capacities together with $n$ and the resistance $A$.

\begin{tabular}{cccccl} 
Capacity & 4.94 & 4.96 & 4.96 & 4.64 & microfarads. \\
\hline$n$ & 131.1 & $53 \cdot 3$ & 31.98 & 14. & complete alternations. \\
\hline A & 5.19 & 20.5 & 34.09 & 139.62 & absorption resistance in ohms.
\end{tabular}

The last value of the capacity seems 'to be an error, possibly one of calculation. However, the results seem to show a nearly constant capacity, but a resistance increasing rapidly with decrease of period, as Maxwell's formula shows. The constant value of the capacity remains to be explained.

But in the above, determinations of absorption resistance are by approximation. Professor Rowland has, therefore, devised a method by which it can be measured directly. This method, with the results that have been derived by it, will now be given. 
Method for the Direct Measurement of Absorption Resistance

In a Wheatstone bridge (Fig. 3) let the resistance of the different arms be denoted by $R_{\imath}, R^{\prime}, R_{u}, R^{\prime \prime}$ and $r$. Let $R$, have in circuit a self-inductance $L$, and let $r$ have in circuit with it a self-inductance.

Let $C, \varepsilon^{i b t}$ be the current through $R$, and $C \varepsilon^{i(b t+\phi)}$ be the current through $r$ when a periodic electromotive force is applied to $a$ and $d$ in the figure.

Let $C^{\prime}$, be the current through $R_{i}$, and $C^{\prime}$ be the current through $r$ when there is a constant difference of potential between $a$ and $d$. The ratio of the current in this case is

$$
\frac{c^{\prime}}{C_{\prime}^{\prime}}=\frac{R^{\prime \prime} R_{1}-R^{\prime} R_{\Perp}}{R^{\prime}\left(R^{\prime \prime}+R_{\text {॥ }}\right)+r\left(R^{\prime}+R^{\prime \prime}\right)}
$$

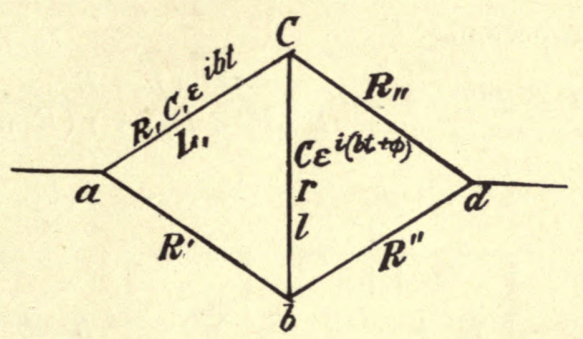

FIG. 3.

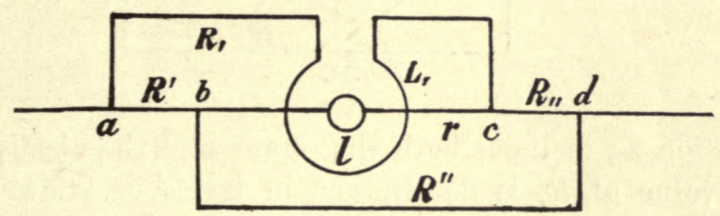

FIG. 4.

When a periodic electromotive force is applied to $a$ and $d$, the ratio of the currents in this case is

$$
\frac{c}{C^{\prime}} \varepsilon^{i \phi}=\frac{R^{\prime \prime} R_{1}-R^{\prime} R_{u}+i b R^{\prime \prime} L}{R^{\prime}\left(R^{\prime \prime}+R_{u}\right)+r\left(R^{\prime}+R^{\prime \prime}\right)+i b l\left(R^{\prime}+R^{\prime \prime}\right)}
$$

Separating the real and imaginary parts

$\frac{c}{O_{1}} \cos \phi=\frac{\left(R^{\prime \prime} R_{1}-R^{\prime} R^{\prime \prime}\right)\left[R^{\prime}\left(R^{\prime \prime}+R_{1}\right)+r\left(R^{\prime}+R_{u \prime}\right)\right]+b^{2} l L_{\imath} R^{\prime \prime}\left(R^{\prime}+R^{\prime \prime}\right)}{\left[R^{\prime}\left(R^{\prime \prime}+R_{u}\right)+r\left(R^{\prime}+R^{\prime \prime}\right)\right]^{2}+b^{2} l^{2}\left(R^{\prime}+R^{\prime \prime}\right)^{2}}$

If now the fixed coils of the electrodynamometer are placed in the $R$, arm of the bridge, and the hanging coil is placed in cross connection of the bridge, as in Fig. 4, the different resistances may be adjusted 21 
until there is no deflection, in which case $\phi=90^{\circ}$ or $\cos \phi=0$, therefore

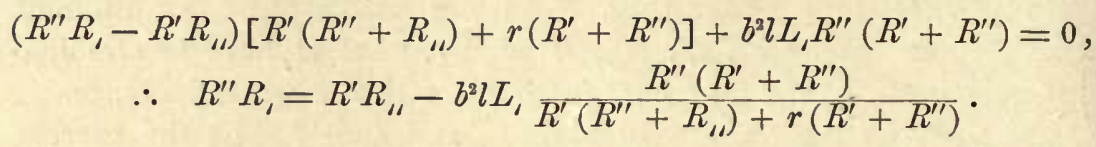

If in connection with $L^{\prime}$ a capacity $C$ is added, the formula becomes, substituting for $L_{\imath}, L_{\imath}-\frac{1}{\bar{b}^{2} c}$.

$$
R^{\prime \prime} R_{\iota}=R^{\prime} R_{\text {॥ }}-\left(b^{2} l L_{\iota}-\frac{l}{c}\right) \frac{\left(R^{\prime \prime} R^{\prime}+R^{\prime \prime}\right)}{R^{\prime}\left(R^{\prime \prime}+R_{\text {॥ }}\right)-r\left(R^{\prime}+R^{\prime \prime}\right)} .
$$

In most cases since $l$ and $L$, are generally the self-inductances of the instruments the term $b_{2} l L$, can be neglected in comparison with $\frac{l}{c}$ and the equation becomes

$$
R^{\prime \prime} R_{\iota}=R^{\prime} R_{\text {॥ }}+\frac{l}{c} \frac{R^{\prime \prime}\left(R^{\prime}+R^{\prime \prime}\right)}{R^{\prime}\left(R^{\prime \prime}+R_{\text {॥ }}\right)+r\left(R^{\prime}+R^{\prime \prime}\right)} .
$$

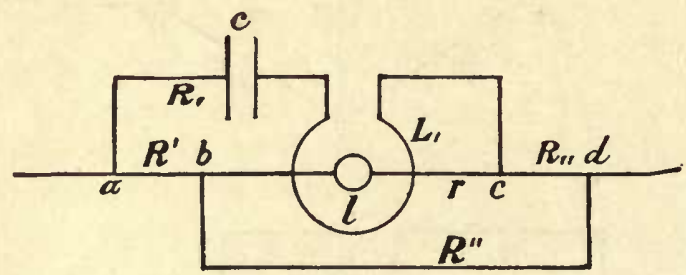

FIG. 5.

In this equation $R$, includes both the ohmic and the absorption resistance. The value of $R$, is determined in terms of known quantities, that is the resistance and $l$ and $C$. It was not necessary that $l$ and $C$ should be exactly known as the last term in the equation above plays the part of a correction term, and is in all cases below small and in some cases negligible. The capacities that were used in the experiments were the 2 and 3 microfarads, the $\frac{1}{3}$ microfarad Elliott condenser, and the $\frac{1}{3}$ microfarad Troy condenser.

Experiments.-The process of experimenting was to apply a periodic electromotive force to $a$ and $d$, and to adjust the different resistances until there was no deflection of the coil in the same way as in the ordinary measurement of resistance on a Wheatstone bridge. The different resistances $R^{\prime}, R^{\prime \prime}, R_{\text {" }}$ and $r$ being known, the apparent value of the resistance $R$, was found, and knowing the ohmic resistance of the $R$, circuit, the absorption resistance appears as the difference. 
Some interest lies not alone in that the method is applicable, but that it confirmed the supposition that absorption resistance acts as an ordinary ohmic resistance in series in the circuit. This was confirmed by the fact that when condensers were in series and in parallel, their absorption resistances acted under these conditions like ohmic resistances, being increased in the one case and decreased in the other, and in the right ratio. This agreement was not exact, as the absorption resistance was extremely sensitive both to change of period and change of temperature. The great sensitiveness to change of temperature was shown either by letting the current go through the condensers for a little time, or placing the condensers before a hot air flue; in either case after cooling, the absorption resistance returned to its original value. The cooling was very slow, as there was very little radiation from the condensers inclosed in wooden boxes.

The results are now given for the condensers 2 and 3 microfarads. In the calculation of the results the last term of the equation, that is $\frac{l R^{\prime \prime}\left(R^{\prime}+R^{\prime \prime}\right)}{c R^{\prime}\left(R^{\prime \prime}+R_{1 \prime}\right)+r\left(R^{\prime}+R^{\prime \prime}\right)}$ has been left out, as it was very small when condensers 2 and 3 microfarads were used.

Condengers 2 and 3 Microfarads in Parallel. $n=134, l=\cdot 0007 . \therefore$ last term negligible.

\begin{tabular}{|c|c|c|c|c|c|c|}
\hline $\mathrm{R}^{\prime \prime}$ & $\mathbf{R}_{\text {" }}$ & $r$ & $\mathbf{R}^{\prime}$ & $\mathbf{R}$ & $\begin{array}{l}\text { Resis. of } \\
\mathrm{R}^{\prime} \text { circuit } \\
\text { in ohms. }\end{array}$ & $\begin{array}{l}\text { Resistance } \\
\text { due to } \\
\text { absorption. }\end{array}$ \\
\hline $422 \cdot 6$ & $488 \cdot 6$ & $5457 \cdot 3$ & $347 \cdot 9$ & $39 \cdot 29$ & $33 \cdot 77$ & $5 \cdot 30$ \\
\hline $1488 \cdot 6$ & $488 \cdot 2$ & “ & $123 \cdot 4$ & $40 \cdot 50$ & "، & $6 \cdot 73$ \\
\hline $984 \cdot 1$ & “ & “ & $82 \cdot 1$ & $40 \cdot 72$ & $33 \cdot 81$ & $6 \cdot 91$ \\
\hline $2671 \cdot 6$ & “ & “ & $22 \cdot 5$ & $41 \cdot 116$ & “" & $7 \cdot 30$ \\
\hline $423 \cdot 0$ & “ & “ & $357 \cdot 3$ & $41 \cdot 237$ & “ & $7 \cdot 42$ \\
\hline $5474 \cdot 3$ & “ & “ & $464 \cdot 5$ & $41 \cdot 42$ & “ & $7 \cdot 61$ \\
\hline 6734 . & ، & "، & $374 \cdot 9$ & $41 \cdot 67$ & “ & $7 \cdot 86$ \\
\hline \multicolumn{5}{|c|}{$1 \mathrm{ohm}$ in $\mathrm{R}^{\prime \prime}=\frac{3}{4}$ scale division. } & “ & \\
\hline 7486 . & 4 & " & $638 \cdot 6$ & $41 \cdot 64$ & “ & $7 \cdot 83$ \\
\hline 9466 . & “ & “ & $81 \cdot 15$ & $41 \cdot 85$ & ، & $8 \cdot 04$ \\
\hline
\end{tabular}

Condensers 2 and 3 placed before the register and heated for 1 hour:

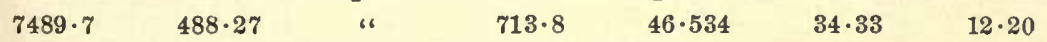

After standing $1 \frac{1}{2}$ hours in air at temperature of $12^{\circ} \cdot 2 \mathrm{C}$. condenser has been open so that resistances have been cooled:

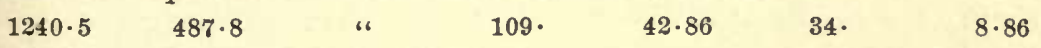

After standing some little time:

$\begin{array}{lllllll}7482.5 & 487.8 & \text { " } & 651 \cdot 6 & 42.47 & 34 & 8.49\end{array}$

The above table shows conclusively the heating of the condenser by the current, and the dependence of the absorption upon the temperature. 
Condensers 2 and 3 in Parallel. $\mathrm{N}=57 \cdot 6$.

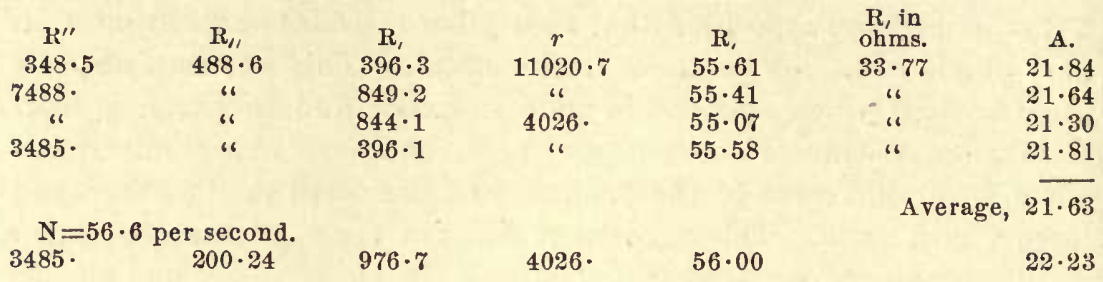

Comparing these values with those found in the use of method 25 the agreement is at once apparent.

\begin{tabular}{|c|c|c|c|c|c|}
\hline $\mathrm{N}=$ & $134^{\circ}$ & 131. & $57 \cdot 6$ & $56 \cdot 6$ & 53. \\
\hline Method 25 & \multicolumn{4}{|c|}{$5 \cdot 19$} & $20 \cdot 5$ \\
\hline $\begin{array}{l}\text { Direct measure- } \\
\text { ment. }\end{array}$ & $\begin{array}{l}5 \cdot 30 \\
7 \cdot 00\end{array}$ & & $21 \cdot 63$ & $22 \cdot 23$ & \\
\hline
\end{tabular}

It should be remembered, in comparing the results, that the values obtained by method 25 would naturally be smaller than those found by direct measurement, as in method 25 the current going through the condensers was extremely small; there was therefore practically no heating.

The experiments that confirm the mathematical theory that the absorption resistance could be treated as ordinary ohmic resistance were performed with the two condensers, $\frac{1}{3}$ Troy and $\frac{1}{3}$ Elliott microfarad condensers. These are next given.

In these results it was necessary to take into account, in the calculation of the apparent value of $R$, the last term of the equation, that is

$$
\frac{l}{c} \frac{R^{\prime \prime}\left(R^{\prime}+R^{\prime \prime}\right)}{R^{\prime}\left(R^{\prime \prime}+R_{\prime \prime}\right)+r\left(R^{\prime}+R^{\prime \prime}\right)}
$$

$\frac{1}{3}$ Troy and $\frac{1}{3}$ Elliott in series, 1 o'clock.

\begin{tabular}{|c|c|c|c|c|c|c|}
\hline $\begin{array}{c}R^{\prime \prime} \\
4751 \cdot 8\end{array}$ & $\begin{array}{c}\mathbf{R}_{/ \prime} \\
\mathbf{4 9 9} \cdot 9\end{array}$ & $\begin{array}{c}\mathbf{R}^{\prime} \\
404 \cdot 8\end{array}$ & $\stackrel{r}{4754}$. & $\begin{array}{c}\text { Apparent } \\
\text { value } \\
\text { of } R \\
43 \cdot 141\end{array}$ & $\begin{array}{c}\text { Ohmic resist- } \\
\text { ance } \\
\text { of } \mathbf{R} \\
34 \cdot 143\end{array}$ & $\begin{array}{c}\text { Absorption } \\
\text { resistance } \\
\text { A. } \\
8.998\end{array}$ \\
\hline \multicolumn{7}{|c|}{$\frac{1}{3}$ Troy, 2 o’clock. } \\
\hline \multicolumn{7}{|c|}{$\frac{1}{3}$ Elliott, 2.45 o'clock. } \\
\hline \multicolumn{7}{|c|}{$\frac{1}{3}$ Troy and $\frac{1}{3}$ Elliott in parallel, 4 o'clock. } \\
\hline $\begin{array}{r}4749 \cdot 3 \\
\quad \frac{1}{3} \mathrm{~T}\end{array}$ & $\begin{array}{l}497 \cdot 6 \\
\text { and } \frac{1}{3}\end{array}$ & $\begin{array}{l}350 \cdot 23 \\
\text { lott in }\end{array}$ & $\begin{array}{r}\text { "ries. } \\
\text { res. }\end{array}$ & $36 \cdot 94$ & $34 \cdot 15$ & $2 \cdot 79$ \\
\hline $4748 \cdot 5$ & $497 \cdot 55$ & $418 \cdot 15$ & “ & $44 \cdot 612$ & $34 \cdot 12$ & $10 \cdot 492$ \\
\hline
\end{tabular}


Calculating what the absorption resistance should be for $\frac{1}{3}$ Troy and $\frac{1}{3}$ Elliott in series, from the absorption resistances of the two condensers when determined separately, it is equal to $10 \cdot 26 \mathrm{ohms}$, which is greater than the first and less than the last value above, showing that the condensers were heating during the experiments. Calculating the absorption resistance of $\frac{1}{3}$ Troy and $\frac{1}{3}$ Elliott in parallel in the same way, it is equal to $2 \cdot 209 \mathrm{ohms}$, which is less than the value afterwards obtained by experinent for the same reason.

The method was shown not to be based on any false supposition, by substituting in place of the condenser a coil of known self-inductance. When this was done the value of $R_{1}$ as calculated from the other resistances and the self-inductances should be the same as the actual ohmic resistance of the circuit.

This was tried with two coils $P_{2}$ and $A$ and the agreement was remarkably elose, as seen in the next table.

Coil $P$ used in place of condenser in the $R$, circuit:

\begin{tabular}{|c|c|c|c|c|c|}
\hline $\mathbf{R}^{\prime \prime}$ & $\mathbf{R}_{\prime \prime}$ & $\mathbf{R}^{\prime}$ & $r$ & $\begin{array}{l}\text { Deduced value } \\
\text { of } \mathbf{R}_{l}\end{array}$ & $\begin{array}{c}\text { Actual value } \\
\text { of } \mathbf{R}_{\boldsymbol{s}} \\
77 \cdot 8\end{array}$ \\
\hline $474 \cdot 9$ & 487.8 & $758 \cdot 2$ & 5457 . & $77 \cdot 86$ & $77 \cdot 8$ \\
\hline
\end{tabular}

Coil $A$ in place of condenser in the $R$, circuit:

$\begin{array}{llllll}474.9 & 487.8 & 218 \cdot 3 & & & \\ 4 & & 224.12 & 223.9\end{array}$

In these experiments great care was taken that the measurements of the resistances were performed immediately after the adjustment. In this way the actual resistances at the time of the experiment were obtained, and so the effect of the heating by the current was somewhat eliminated.

Methods 26, 9 and 3 give good results, but the methods that gave the most satisfaction were methods 12 and 6 , method 12 being for the comparison of two self-inductances and method 6 for the comparison of a self-inductance with a capacity. These give some remarkable results, the theory and deductions of the methods being as follows:

\section{Method 12.-Zero Method for the Comparison of two Self-Inductances}

Let the connections be made as in the figure where the hanging coil and the fixed coils are in two distinct circuits.

Let $C_{0} \varepsilon^{i b t}$ etc. be the currents, $A^{\prime}$ and $A^{\prime \prime}$ reversing commutators, $R^{\prime \prime}, R$ and $r$ the resistance of the different circuits, $L^{\prime \prime}$ and $L$ the selfinductances, $M$ the mutual inductance of the coils $B_{1}$ and $B_{2}$ by which it is placed. When a periodic electromotive force $\alpha \varepsilon^{i b t}$ is applied to $A, B$ the quantity to be found is $C_{1} C_{3} \cos \left(\phi_{3}-\phi_{1}\right)$ where $\phi_{3}-\phi_{1}$ is the difference of phase. 
The current in the $R^{\prime \prime}$ circuit is then

$$
C_{,} \varepsilon^{i(b t+\phi)}=\frac{i b M C_{0} \varepsilon^{t b t}}{R^{\prime \prime}+i b L^{\prime \prime}} . \quad . \quad . . . .
$$

The current in the $R$ circuit is

$$
\begin{array}{r}
\quad C_{3} \varepsilon^{i\left(b t+\phi_{3}\right)}(R+i b L)=C_{2} \varepsilon^{i\left(b t+\phi_{2}\right) r} \\
\therefore \quad C_{3} \varepsilon^{i\left(b t+\phi_{3}\right)} \frac{R+r+i b L}{r}=C_{0} \varepsilon^{i b t} .
\end{array}
$$

Substituting the value of $C_{0} \varepsilon^{i b t}$ in equation (1) and simplifying, it becomes

$$
C_{,} \varepsilon^{i(b t+\phi)}=C_{s} \varepsilon^{i\left(b t+\phi_{s}\right)} \frac{-b^{2} L M+i b M(R+r)}{R^{\prime \prime} r-i b L^{\prime \prime} r} .
$$

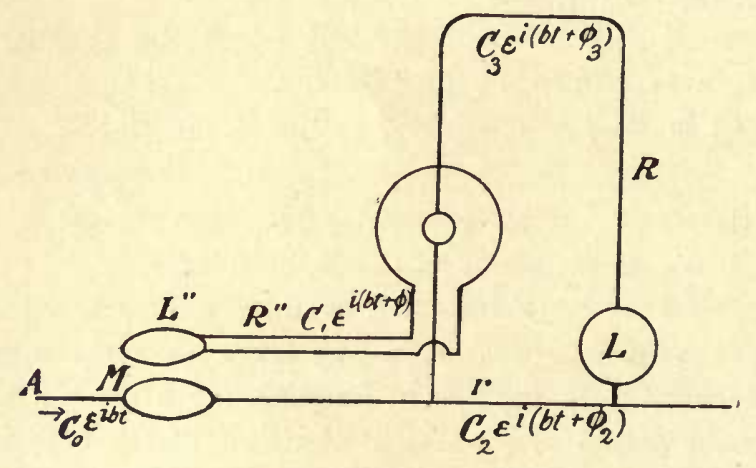

FiG. 6.

Therefore the deflection is proportional to

$$
C_{1} C_{3} \cos \left(\phi_{3}-\phi_{1}\right)=C_{s}^{2}-\frac{b^{2} L M R^{\prime \prime} r+b^{2} L^{\prime \prime} M r(R+r)}{R^{\prime \prime 2} r^{2}+b^{2} L^{2} r^{2}} ;
$$

and the condition for zero deflection is

$$
\begin{gathered}
-b^{2} L M R^{\prime \prime} r+b^{2} L^{\prime \prime} M r(R+r)=0, \\
\therefore \quad \frac{L}{L^{\prime \prime}}=\frac{R+r}{R^{\prime \prime}} .
\end{gathered}
$$

The condition therefore of zero deflection is independent of $M$. But $M$ is one of the factors of the electromotive force in the $R^{\prime \prime}$ circuit, and on it therefore depends the sensitiveness, as it determines the current through the $R^{\prime \prime}$ circuit. In the first figures of this method the fixed coils are in the $R^{\prime \prime}$ circuit, and the hanging coil in the $R$ circuit, but this is not necessary, as the fixed and hanging coils can be reversed. The choice of which of the above arrangements should be used depends 
on the impedances of the two circuits, as other things being equal the smaller current should go through the hanging coil.

Experiments. - The coils used in the experiments were coils $P_{1}, P_{2}$, $C, B_{1}, B_{2}$, and $A$, which coils are described on page 315 . From the dimensions of $P_{2}$ and its self-inductance as found by method 25, $B_{1}$ was designed to have a self-inductance of one henry. This will be shown to be nearly the case. For ease of comparison $B_{1}$ has been taken in the calculations of the results as being equal to one henry, and the other coils were compared with this coil as a standard.

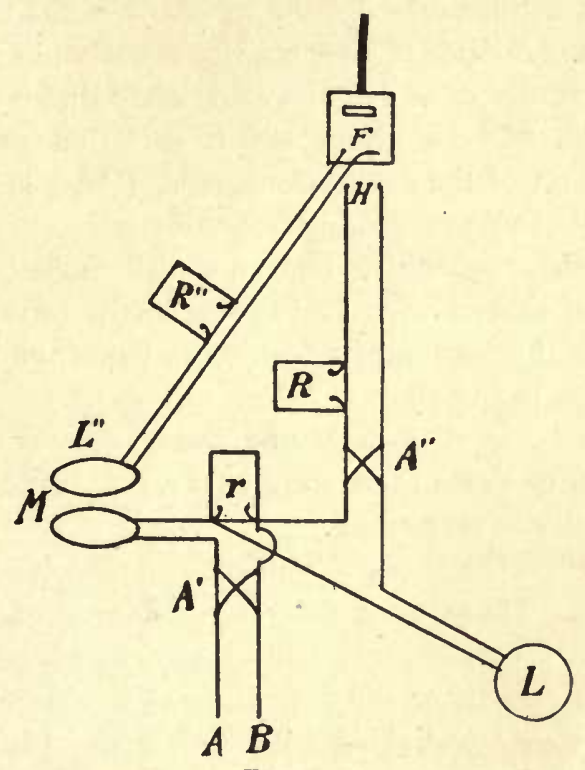

FIG. 7.

In these experiments the connections were made as in the figure $\%$, the coil $B_{1}$ that was taken as the standard being placed in circuit with the fixed coils of the electrodynamometer as $L^{\prime \prime}$ and the resistance of this circuit was unaltered during the experiments in any particular series. The coils whose self-inductances were to be determined were placed in the hanging coil circuit and the resistance $R$ was changed until there was no deflection. The resistance of the two circuits, $R^{\prime \prime}$ and $R+r$ were then measured by a Wheatstone bridge.

The resistance $r$ was in all cases small in order that $C_{0} \varepsilon^{i b t}$ should be large, and therefore by induction $C_{1} \varepsilon^{i(b t+\phi)}$ the current through the fixed coils was made large and the instrument sensitive. The method 
being very accurate, as will be seen later, great care had to be used to eliminate all sources of error, as for example, electrostatic action. In the first trial of the method small differences were noticed in the ratio of two self-inductances, depending both on the resistances used, and also on the connections of the coils, whether the leads were double, single, long or short. The same variation was noticed when several coils were joined in series and compared with another coil, and when these coils were compared separately and their sum taken.

This irregularity led to an investigation of the effects of various resistances and connections in one of the circuits, the other circuit being unaltered. A little farther on, the variation in the deduced value of the self-inductance of one of the coils, when different resistances and leads were used, will be given, which variation was caused by the electrostatic action of the connections, etc. (Page 316.)

The necessity of eliminating electrostatic action made obligatory the use of open resistances which had small self-inductances. These resistances were of three kinds-resistances in the form of spirals, resistances wound on thin strips of micanite or paper, and those wound on open frames; see page 316.

The self-inductance of the first and second classes of resistances was very small, as in one case there were only a few turns, and in the other the cross-section was very small.

The third class were those wound on frames whose self-inductances were calculated. There were several resistances of $2000 \mathrm{ohms}$ each, whose self-inductances were $\cdot 0000436$ henry, which would hardly affect the phase of the current or the impedance of the circuit.

These coils were subdivided into resistances of various amounts. Another frame resistance used was of $7463 \mathrm{ohms}$ divided into parts of about $250 \mathrm{ohms}$ each. The self-inductance of the entire $7463 \mathrm{ohms}$ was $\cdot 000105$ henry.

As the open resistances were not divided into small amounts it was necessary to use resistance boxes for adjustment; as few ohms as possible were used in each case.

From the fact that the coils of the electrodynamometer had selfinductance a correction was introduced in order that the ratio of the resistances should give the ratio of the self-inductances of the coils direct.

The value of this correction in ohms was calculated as follows: 
Calculation of Correction Due to Fixed and Hanging Coils

Self-inductance of fixed coils $=f=\cdot 0164$ henry

" " " hanging coil $=h=\cdot 0007$ "

Correction due to fixed coils. From an inspection of the tables it is seen that

$$
\frac{L}{B_{1}+f}=\frac{R+r}{R^{\prime \prime}} \text {, or } \frac{L}{1.0164}=\frac{R+r}{902},
$$

where $L$ is the self-inductance of some coil and $R+r$ is the corresponding resistance. $B$, is taken as equal to 1 henry

$$
\therefore \frac{L}{R+r}=\frac{1 \cdot 0164}{902} \text {. }
$$

But the comparison of $L$ with $B_{1}=1$ is wanted, therefore both numerator and denominator of $\frac{1.0164}{902}$ are divided by 1.0164 or

$$
\begin{aligned}
& \therefore \frac{L}{R+r}=\frac{1=B_{1}}{887 \cdot 45}, \\
& \therefore \quad \frac{L}{B}=\frac{R+r}{887 \cdot 45} .
\end{aligned}
$$

That is, the self-inductance of $\cdot 0164$ henry of the fixed coils produced a correction of $88 \% \cdot 45-902=-14.55 \mathrm{ohms}$, which must be applied to the $R^{\prime \prime}$ circuit if the self-inductance of that circuit is to be considered as 1 henry.

Correction due to hanging coil. The self-inductance $=\cdot 0164$ henry of the fixed coils gives a correction of $-14.55 \mathrm{ohms}$, therefore the selfinductance .0007 henry of the hanging coil gives a correction of -.62 ohms to the $R+r$ circuit. Applying these corrections, the results obtained for the several coils under various conditions are given below. The results are given in the following order.

First. The values are calculated using double leads in the circuits but open resistances as far as possible.

Second. The variation of the apparent value of the self-inductance of one of the coils with different positions of the coil, resistances, and different kinds of leads.

Third. Short leads separated about 6 inches and crossed, used with all the coils except $B_{1}$.

Fourth. Open leads and open resistances in the determinations. In the table $R^{\prime \prime}$ was open resistance plus the resistance of coil $B_{1}$ and fixed coils of instrument. $R+r$ was made up of the small coil and open resistance plus the amount in the Queen ordinary resistance box. 
After all the inductive effect of the leads was removed and the ordinary resistance box used as little as possible, there was a different value obtained for the ratio of the self-inductances dependent on the position of the reversing commutator $A^{\prime}$. With all the coils used the greater value occurred with the same position of $A^{\prime}$. This was due to the electrostatic action between the coils $B_{1}$ and $B_{2}$, for if the terminals of the coil $B_{2}$ and the commutator $A^{\prime}$ were reversed at the same time, there was no change in the value of the ratio of the inductances. This showed that it was dependent on the coil itself and not on the leads and it could therefore not be eliminated.

It is to be noticed that the values obtained for the lower number of alternations are always greater than those found with the higher number of alternations. This was caused by the electrostatic action of the turns of the coil on each other. In the case of the coil $P_{2}$ this effect would be caused by supposing a capacity of $\cdot 0007$ microfarads shunted across the terminals.

The results are now given comparing the different coils with $B_{1}$ as a standard and equal to 1 henry.

\section{Double Leads of Beli Wire and Open Resistaxce}

$r=106 \mathrm{ohms}, n=45$ complete periods per second.

\begin{tabular}{|c|c|c|c|c|c|c|c|c|c|}
\hline $\begin{array}{l}\text { Colls. } \\
\mathrm{P}_{1}+\mathrm{P}_{2}\end{array}$ & $\mathbf{R}^{\prime \prime}$ & Correc. & & Queen. & $\mathrm{R}+r$ & $\begin{array}{l}\text { Cor- } \\
\text { reo. }\end{array}$ & $\begin{array}{c}\text { Aver- } \\
\text { age. }\end{array}$ & $\begin{array}{c}\text { Com. } \\
\mathbf{A}^{\prime} \text {. }\end{array}$ & Ratio. \\
\hline$+C$ & $901 \cdot 6$ & $-14 \cdot 55$ & $887 \cdot 05$ & 292 & $2300 \cdot 2$ & -62 & $2304 \cdot 9$ & 1 & $2 \cdot 5983$ \\
\hline 6 & " & c6 & , & 310 & $2311 \cdot 0$ & " & & 2 & \\
\hline $\mathrm{C}$ & 11 & 66 & 16 & 19 & $1158 \cdot 3$ & "6 & $1159 \cdot 0$ & 1 & $1 \cdot 3099$ \\
\hline "6 & "6 & 66 & " & 22 & $1161 \cdot 2$ & " & & 2 & \\
\hline$C+P_{1}$ & 16 & $" 6$ & 16 & 103 & 1659 . & "6 & $1661 \cdot 2$ & 1 & $1 \cdot 8727$ \\
\hline 6 & " & 66 & c6 & 109 & $1664 \cdot 8$ & 66 & & 2 & \\
\hline$C+P_{2}$ & 6 & 16 & "6 & 92 & $1800 \cdot 2$ & " & $1802 \cdot 6$ & 1 & $2 \cdot 0288$ \\
\hline "6 & " & 66 & "6 & 99 & $1806 \cdot 5$ & "6 & & 2 & \\
\hline $\mathbf{A}$ & $901 \cdot 7$ & 66 & $887 \cdot 15$ & 149 & $4776 \cdot 5$ & " & $4786 \cdot 5$ & 1 & $5 \cdot 3956$ \\
\hline 16 & "6 & 16 & c6 & 196 & $4818 \cdot 0$ & 6 & & 2 & \\
\hline
\end{tabular}

Current increased about $2 \frac{1}{2}$ times.

\begin{tabular}{|c|c|c|c|c|c|c|c|c|c|}
\hline A & "6 & 16 & 66 & 141 & 4787.0 & 16 & $4781 \cdot 3$ & 1 & $5 \cdot 3898$ \\
\hline 66 & "، & 16 & " & 184 & 4807 . & "6 & & 2 & \\
\hline$A+C$ & $901 \cdot 6$ & 16 & $887 \cdot 05$ & 211 & 5936 . & 66 & $5958 \cdot 3$ & 1 & $6 \cdot 7170$ \\
\hline " & " & 16 & a & 264 & 5982 . & " & & 2 & \\
\hline \multirow{2}{*}{$A+\underset{\text { ( }}{C}+P_{2}$} & "6 & 66 & " & 51 & $6575 \cdot 5$ & " & $6602 \cdot 5$ & 1 & $7 \cdot 4430$ \\
\hline & ، & 66 & "6 & 104 & $6631 \cdot 0$ & "6 & & 2 & \\
\hline A & 902. & 6 & $887 \cdot 45$ & 158 & $4778 \cdot 9$ & 6 & $4795 \cdot 25$ & 1 & $5 \cdot 4036$ \\
\hline 66 & (6 & 66 & 6 & 192 & 4813 & "6 & & 2 & \\
\hline \multirow{2}{*}{$\mathrm{P}_{1}+\mathrm{P}_{2}$} & " & " & 6 & 183 & $1146 \cdot 5$ & 16 & $1146 \cdot 7$ & 1 & $1 \cdot 9922$ \\
\hline & 66 & 16 & 66 & 186 & $1148 \cdot 5$ & 6 & & 2 & \\
\hline \multirow[t]{2}{*}{$\mathrm{P}_{2}$} & "6 & 6 & 6 & 7 & $643 \cdot 15$ & 16 & $642 \cdot 67$ & 1 & $\cdot 7212$ \\
\hline & "6 & 6 & "6 & 8 & $643 \cdot 6$ & " & & 2 & \\
\hline \multirow[t]{2}{*}{$P_{1}$} & 66 & 66 & "6 & 91 & $502 \cdot 5$ & 6 & $502 \cdot 16$ & 1 & $\cdot 5658$ \\
\hline & 6 & 16 & "6 & & $503 \cdot 1$ & 16 & & 2 & \\
\hline
\end{tabular}


DOUbLe LeADs. $n=$ about 133 complete alternations per sec.

\begin{tabular}{|c|c|c|c|c|c|c|c|c|c|}
\hline $\begin{array}{c}\text { Coils. } \\
P_{1}\end{array}$ & $\begin{array}{c}\mathbf{R}^{\prime \prime} \\
901 \cdot 9\end{array}$ & $\begin{array}{l}\text { Correc. } \\
-14 \cdot 55\end{array}$ & $887 \cdot 35$ & $\begin{array}{l}\text { Queen. } \\
90+s\end{array}$ & $\begin{array}{c}\mathrm{R}+r \\
500 \cdot 4\end{array}$ & $\begin{array}{l}\text { Cor- } \\
\text { rec. } \\
+\cdot 62\end{array}$ & $\begin{array}{l}\text { Aver- } \\
\text { age. } \\
499 \cdot 69\end{array}$ & $\begin{array}{c}\text { Com. } \\
A^{\prime} \text {. } \\
1\end{array}$ & $\begin{array}{l}\text { Ratio. } \\
.5631\end{array}$ \\
\hline $\begin{array}{l}1 \\
6\end{array}$ & "، & "، & "، & "، & $500 \cdot 23$ & " & & 2 & \\
\hline $\mathrm{P}_{2}$ & “" & “" & “" & 3 & $639 \cdot 35$ & "“ & $638 \cdot 85$ & 1 & $\cdot 7198$ \\
\hline A & $901 \cdot 87$ & "، & $7 \cdot 32$ & & $\begin{array}{r}639 \cdot 6 \\
4742 \cdot 2\end{array}$ & "، & $4750 \cdot 48$ & 1 & $5 \cdot 3537$ \\
\hline “" & " & “" & "، & 133 & $4760 \cdot 0$ & “" & & 2 & \\
\hline C & $901 \cdot 9$ & “" & $887 \cdot 35$ & 44 & $1151 \cdot 4$ & “" & $1150 \cdot 94$ & 1 & $1 \cdot 2970$ \\
\hline “" & “" & " & "، & 44 & $1151 \cdot 4$ & " & & 2 & \\
\hline
\end{tabular}

In the above determinations the coils were arranged in the way as indicated in the figure having leads of double bell wire.

a Series of Determinations of a Under Various Conditions.

Open resistance $R$ on table (original position).

\begin{tabular}{|c|c|c|c|c|c|c|c|c|c|}
\hline $\begin{array}{c}\text { Coils. } \\
\text { A }\end{array}$ & $\begin{array}{c}\mathbf{R}^{\prime \prime} \\
902 \cdot 0\end{array}$ & & $87 \cdot 4$ & $\begin{array}{l}\text { Queen. } \\
149+s\end{array}$ & & $\begin{array}{l}\text { Cor- } \\
\text { rec. } \\
-\cdot 62\end{array}$ & $\begin{array}{l}\text { Aver- } \\
\text { age. } \\
4786 \cdot 58\end{array}$ & $\begin{array}{c}\text { Com. } \\
A^{\prime} \text {. } \\
1\end{array}$ & $\begin{array}{r}\text { Ratio. } \\
5 \cdot 3936\end{array}$ \\
\hline & 16 & “" & "“ & $196+8$ & 4818 . & " & & 2 & \\
\hline “ & $901 \cdot 95$ & “" & $887 \cdot 4$ & $?$ & & “" & $4795 \cdot 38$ & 1 & $5 \cdot 403$ \\
\hline
\end{tabular}

Open resistance $R$ moved up to coil $A\left(b_{1}\right)$.

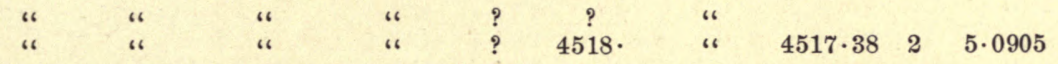

Open resistance $R$ moved to the other side of $A\left(b_{2}\right)$.

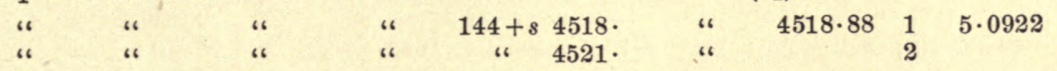

Coil $A$ placed in $P_{1}$ position and open resistance $R$ restored to its position, and $159^{\prime}$ of double wire added to the circuit.

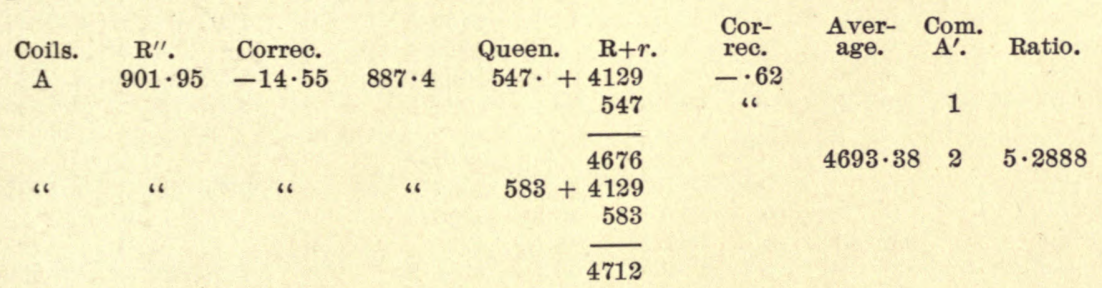

Coil $A$ at end of double wire $69^{\prime}+159^{\prime}=228^{\prime}$ long.

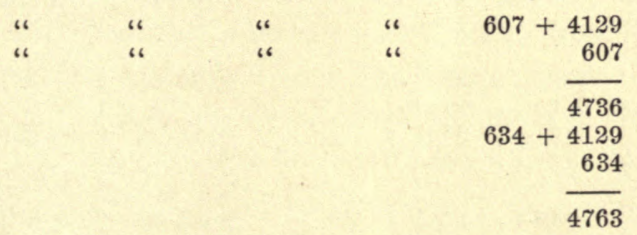

New leads placed in $B_{1}$ circuit, the wires were about 6 " from each other. 
Coils. $\mathbf{R}^{\prime \prime}$. Correc.

A $\quad 902.6 \quad-14.55$

"

Quen $569+4129$

$$
\begin{array}{r}
\frac{569}{4698} \\
594+4129 \\
594 \\
\hline 4723
\end{array}
$$

Open resistance placed next Coil $A$.

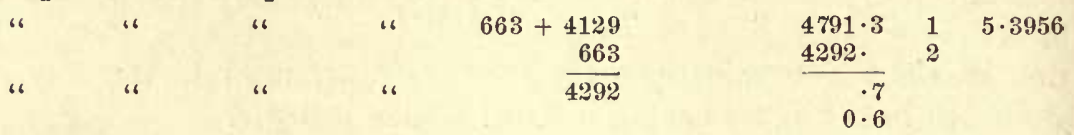

\begin{tabular}{|c|c|c|c|c|c|c|c|c|c|c|}
\hline $\begin{array}{l}\text { Pe- } \\
\text { riod. } \\
40\end{array}$ & $\begin{array}{c}\text { Coils. } \\
P_{1}\end{array}$ & $\begin{array}{l}\mathbf{R}^{\prime \prime} \\
902 .\end{array}$ & $\begin{array}{l}\text { Correc. } \\
-14 \cdot 55\end{array}$ & $887 \cdot 45$ & $\begin{array}{c}\text { Queen. } \\
90+s\end{array}$ & $\begin{array}{c}\mathbf{R}+r . \\
503 \cdot 07\end{array}$ & $\begin{array}{l}\text { Cor- } \\
\text { rec. } \\
-\cdot 62\end{array}$ & $\begin{array}{l}\text { Aver- } \\
\text { age. } \\
502 \cdot 71\end{array}$ & $\begin{array}{c}\text { Com. } \\
\mathbf{A}^{\prime} . \\
1\end{array}$ & $\begin{array}{l}\text { Iatio. } \\
\cdot 5664\end{array}$ \\
\hline 6 & 16 & 6 & "6 & w & $90+s$ & $503 \cdot 6$ & 6 & & 2 & \\
\hline 133 & 16 & 6 & 66 & 66 & $88+s$ & $522 \cdot 53$ & 66 & & 1 & \\
\hline “ & 6 & " & "6 & "6 & $88+s$ & $502 \cdot 15$ & 66 & $501 \cdot 72$ & 2 & $\cdot 5653$ \\
\hline 40 & $P_{2}$ & 90255 & 66 & 888 . & $17+s$ & $644 \cdot 3$ & "6 & & 1 & \\
\hline " & $66^{\circ}$ & " & 6 & "6 & $18+s$ & $644 \cdot 76$ & ، & $643 \cdot 91$ & 2 & .7251 \\
\hline 133 & "6 & 16 & 16 & 66 & $17+s$ & $643 \cdot 05$ & $"$ & & 1 & \\
\hline "6 & 66 & " & 66 & 6 & $17+s$ & $643 \cdot 1$ & 66 & $642 \cdot 45$ & 2 & $\cdot 7234$ \\
\hline 40 & $\mathrm{C}$ & $902 \cdot 4$ & 66 & $887 \cdot 85$ & $28+s$ & $1159 \cdot 6$ & " & & 1 & \\
\hline " & "6 & "6 & 66 & "6 & $28+s$ & $1159 \cdot 1$ & ، & $1158 \cdot 73$ & 2 & $1 \cdot 3050$ \\
\hline 133 & 6 & “" & "6 & " & $24+s$ & $1157 \cdot 0$ & "، & & 1 & \\
\hline "6 & "، & 16 & 6 & 6 & $26+s$ & $1158 \cdot 8$ & "6 & $1157 \cdot 28$ & 2 & $1 \cdot 3034$ \\
\hline 40 & $\mathrm{C}+\mathrm{P}_{1}$ & 902. & "6 & 88745 & $105+s$ & $1658 \cdot 8$ & 66 & & 1 & \\
\hline 6 & " & 6 & "6 & 6 & $110+s$ & $1664 \cdot 1$ & 6 & $1660 \cdot 77$ & 2 & $1 \cdot 8713$ \\
\hline 133 & 66 & 46 & 6 & 6 & $101+s$ & $1656 \cdot 7$ & 16 & & 1 & \\
\hline 6 & " & ": & 66 & " & $106+s$ & $1660 \cdot 3$ & "6 & $1657 \cdot 96$ & 2 & $1 \cdot 8682$ \\
\hline 40 & $\mathrm{C}+\mathrm{P}_{2}$ & $902 \cdot 5$ & 6 & $887 \cdot 95$ & $10+s$ & $1803 \cdot 0$ & 6 & & 1 & \\
\hline "، & 16 & " & "6 & 6 & $12+s$ & $1805 \cdot 0$ & ، & $1803 \cdot 3$ & 2 & $2 \cdot 0261$ \\
\hline 133 & 66 & “ & 6 & 66 & $8+s$ & $1800 \cdot 5$ & 16 & & 1 & \\
\hline "1 & 66 & 6 & 66 & 6 & $8+s$ & $1800 \cdot 2$ & 66 & $1799 \cdot 65$ & 2 & $2 \cdot 0221$ \\
\hline 40 & $\begin{aligned} P_{1} & +P_{2} \\
& +C\end{aligned}$ & $902 \cdot 4$ & 6 & $887 \cdot 85$ & $60+s$ & $2306 \cdot 3$ & 66 & $2307 \cdot 98$ & 1 & $2 \cdot 5995$ \\
\hline "، & " & "6 & 4 & 16 & $?$ & $2310 \cdot 9$ & “6 & & 2 & \\
\hline 133 & "، & " & " & 66 & $56+s$ & $2304 \cdot 1$ & 66 & $2304 \cdot 13$ & 1 & $2 \cdot 5951$ \\
\hline 6 & 66 & "6 & 6 & 6 & $57+s$ & $2305 \cdot 4$ & $" 6$ & & 2 & \\
\hline 40 & A & $902 \cdot 43$ & 66 & $887 \cdot 88$ & $85+s$ & 4703 & "6 & & 1 & \\
\hline 6 & 66 & 16 & 66 & 6 & $106+s$ & $4724 \cdot 2$ & "6 & $4712 \cdot 98$ & 2 & $5 \cdot 3080$ \\
\hline 133 & 66 & $902 \cdot 4$ & 66 & $887 \cdot 85$ & $82+s$ & $4704 \cdot 2$ & 66 & & 1 & \\
\hline " & ، & "6 & 6 & ، & $85+s$ & $4707 \cdot 0$ & "، & $4704 \cdot 98$ & 2 & $5 \cdot 2991$ \\
\hline 40 & $\begin{array}{l}A+C \\
-2 M I\end{array}$ & $902 \cdot 35$ & 6 & $887 \cdot 8$ & $1146+s$ & $9149 \cdot 5$ & 66 & & 1 & \\
\hline "6 & "، & " & 6 & 6 & $1227+s$ & $9233 \cdot 5$ & 16 & $9190 \cdot 88$ & 2 & $10 \cdot 3515$ \\
\hline 133 & "6 & $902 \cdot 4$ & 66 & $887 \cdot 85$ & $1170+s$ & $9171 \cdot 7$ & 16 & & 1 & \\
\hline " & "6 & 16 & 16 & 6 & $1194+s$ & $9191 \cdot 7$ & ، & $9181 \cdot 08$ & 2 & $10 \cdot 3395$ \\
\hline 40 & $\begin{array}{l}A+C \\
+2 M\end{array}$ & $902 \cdot 35$ & 16 & $887 \cdot 8$ & $111+8$ & $2550 \cdot 9$ & "6 & & 1 & \\
\hline 6 & " & 6 & 66 & 6 & $146+8$ & $2556 \cdot 4$ & 66 & $2553 \cdot 03$ & 2 & $2 \cdot 8716$ \\
\hline 133 & " & " & " & 16 & $38+s$ & $2548 \cdot 7$ & "6 & & 1 & \\
\hline " & "6 & "6 & "6 & "6 & $38+s$ & $2548 \cdot 7$ & "6 & $2548 \cdot 08$ & 2 & $2 \cdot 8701$ \\
\hline 40 & $A+C$ & $902 \cdot 6$ & .6 & $888 \cdot 05$ & 123 & 5852 . & 66 & & 1 & \\
\hline "6 & "6 & " & "، & 16 & 169 & 5898. & 6 & $5880 \cdot 13$ & 2 & $6 \cdot 6225$ \\
\hline 133 & " & " & "6 & "6 & 134 & $5863 \cdot 5$ & 16 & & 1 & \\
\hline 16 & 66 & " & "6 & 6 & 140 & 5869 & "6 & $5865 \cdot 63$ & 2 & $6 \cdot 6054$ \\
\hline
\end{tabular}

In the following all connections were made with open leads, and open resistances were used. 
The above results show to what accuracy self-inductances of different values can be compared to each other, or to one of the self-inductances taken as a standard. The reason that the agreement between the different determinations is not greater than it is, even though the electrodynamometer was sensitive to a change of 1 part in 10000 in $R+r$, is that there was always some little heating of the resistances, and although they were measured in each determination on a Wheatstone bridge, still it was impossible to determine the exact resistance at the time that the experiment was made. This slight effect of the heating of the resistance would not enter in the comparison of two nearly equal self-inductances, that is the comparison of a coil with a standard. The accuracy of this comparison can be made to depend on the accuracy with which $R+r$ can be determined for zero deflection, and this can be done to about 1 part in 10000 . To do this, first the standard coil and the coil to be compared are substituted in turn in place of $L$ in figure; they are thus compared separately to a third coil. But as the standard and the coil to be compared are nearly equal in self-inductance, the difference or self-inductance can be determined by the amount necessary to change $R+r$, and this change will be nearly independent of the slight heating of the resistances. To make a coil of the same self-inductance as the standard, the standard is placed in the $R+r$ circuit and the value of $R+r$ is found that produces no deflection. The coil to be compared is then substituted in place of the standard keeping $R+r$ fixed, and the self-inductance of this coil is changed until there is no deflection, as in the case of the standard. The accuracy with which this can be done depends on the accuracy with which $R+r$ can be set or 1 part in 10000 . The method therefore gives a means of comparing and constructing coils to agree in selfinductance to within 1 part in 10000 with a standard.

\section{Method 6.-Zero Method for the Comparison of Self-Inductance with Capacity}

This method resembles method 12 and the connections are made as in the figures when both the hanging coil and fixed coils of the electrodynamometer are shunted off the main circuit.

Let the currents he denoted by $C_{0} \varepsilon^{i b t}, C_{1} \varepsilon^{i\left(b t+\phi_{1}\right)}, C_{2} \varepsilon^{i\left(b t+\phi_{2}\right)}, C_{3} \varepsilon^{i\left(b t+\phi_{3}\right)}$, and $C_{\varepsilon^{i}} \varepsilon^{i\left(b t+\phi_{4}\right)}$. The resistance by $R^{\prime \prime}, r^{\prime}, R$ and $r$. The capacity by $C$. The self-inductance by $L . A^{\prime}$ and $A^{\prime \prime}$ are reversing commutators and $F$ the terminals of the fixed coils and $H$ the terminals of the hanging coil of the electrodynamometer. 
If now a periodic electromotive force is applied to the terminals $A$ and $B$ the equations connecting the different currents are as below, from which equations the quantity $C_{1} C_{3} \cos \left(\phi_{1}-\phi_{3}\right)$ is to be found, which is proportional to the deflection. From the figure

$$
\begin{gathered}
C_{4} \varepsilon^{i\left(b t+\phi_{4}\right)} r^{\prime}=C_{1} \varepsilon^{i\left(b t+\phi_{1}\right)}\left(R^{\prime \prime}+\frac{1}{i b c}\right), \\
C_{4} \varepsilon^{i\left(b t-\phi_{4}\right)}=C_{0} \varepsilon^{i b t}-C_{1} \varepsilon^{i\left(b t+\phi_{t}\right)}, \\
\therefore \quad C_{0} \varepsilon^{i b t}=C_{1} \varepsilon^{i\left(b t+\phi_{l}\right)} \frac{R^{\prime \prime}+r^{\prime} \frac{1}{i b c}}{r^{\prime}}
\end{gathered}
$$

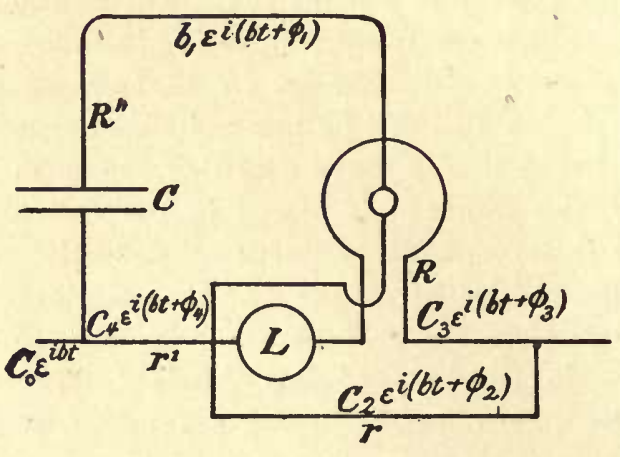

FIG. 8.

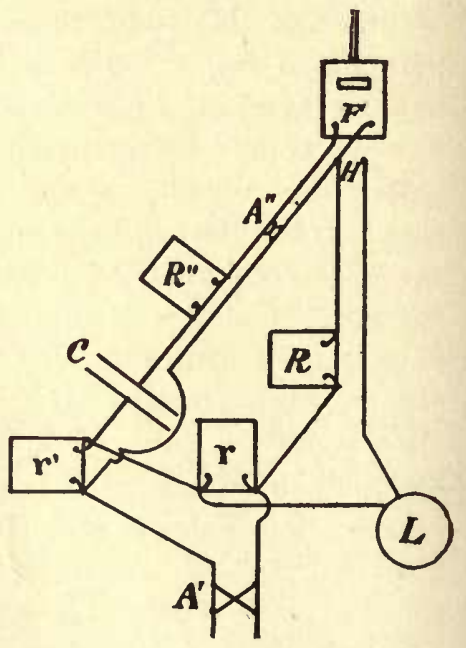

FIG. 9.

In the same way it is found that

$$
\begin{gathered}
C_{0} \varepsilon i b t=C_{3} \varepsilon^{i\left(b t-\phi_{8}\right)} \frac{R+r+i b L}{r}, \\
\therefore \quad C_{1} C_{8} \varepsilon^{i\left(\phi_{1}-\phi_{3}\right)}=C_{3}^{2} \frac{(R+r) r^{\prime}+i b L r^{\prime}}{\left(R^{\prime \prime}+r^{\prime}\right) r+\frac{r}{i b c}} .
\end{gathered}
$$

Therefore the real part is

$$
C_{1} C_{3} \cos \left(\phi_{1}-\phi_{3}\right)=C_{3}^{2} \frac{(R+r)\left(R^{\prime \prime}+r^{\prime}\right) r r^{\prime}-\frac{L}{c} r r^{\prime}}{\left(R^{\prime \prime}+r^{\prime}\right)^{2} r^{2}+\frac{r^{2}}{b^{2} c^{2}}} \times D,
$$


where $D$ is the deflection. When $D$ is equal to zero

or

$$
\begin{aligned}
& (R+r)\left(R^{\prime \prime}+r^{\prime}\right)-\frac{L}{c}=0 \\
& \frac{L}{c}=\left(R^{\prime \prime}+r^{\prime}\right)(R+r) .
\end{aligned}
$$

In the experiments by this method the $\frac{1}{3}$ microfarad Elliott condenser was used, and it was compared with the different coils $P_{1}, P_{2}, A$, and $C$. The connections were made with open leads and open resistances were used as far as possible, but it was necessary to use resistance boxes for the last adjustments. The connections having been made as in figure, the process of experimenting was to keep $r$ and $r^{\prime}$ constant and to adjust $R^{\prime \prime}$ and $R$ until there was no deflection of the hanging coil. The resistance of the circuits $R^{\prime \prime}+r^{\prime}$ and $R+r$ were then measured on a Wheatstone bridge. The commutator $A^{\prime}$ was reversed and the process was repeated. The condensor had absorption (see p. 323) which caused the resistance $R^{\prime \prime}+r^{\prime}$ to be increased by $7 \cdot 11 \mathrm{ohms}$. When the capacity is calculated, taking into account the absorption, it is called the corrected capacity, as in the other tables of the paper.

Collected Results.

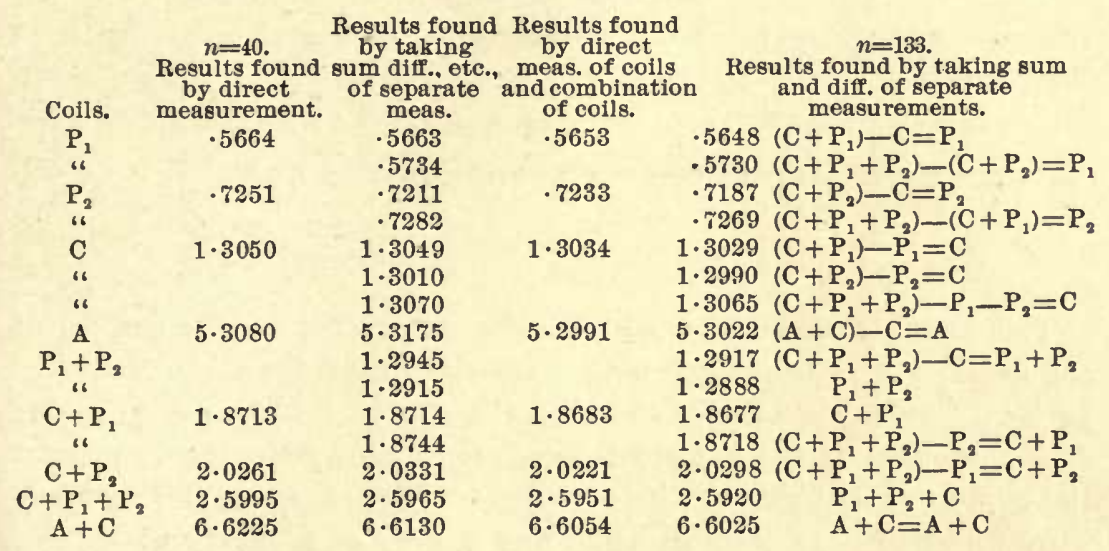

In method 12 corrections due to the hanging coil and fixed coils were calculated so that the ratio of the resistances would give the ratio of the self-inductances direct. In this method (6) since the capacity was in circuit with the hanging coil, the self-inductance was so small that it was neglected. The self-inductance of the coils $P$, etc., which were joined in circuit with the fixed coils, were increased by the self-inductance of the fixed coils, that is by $\cdot 0164$ henry. 
The table below gives the various results.

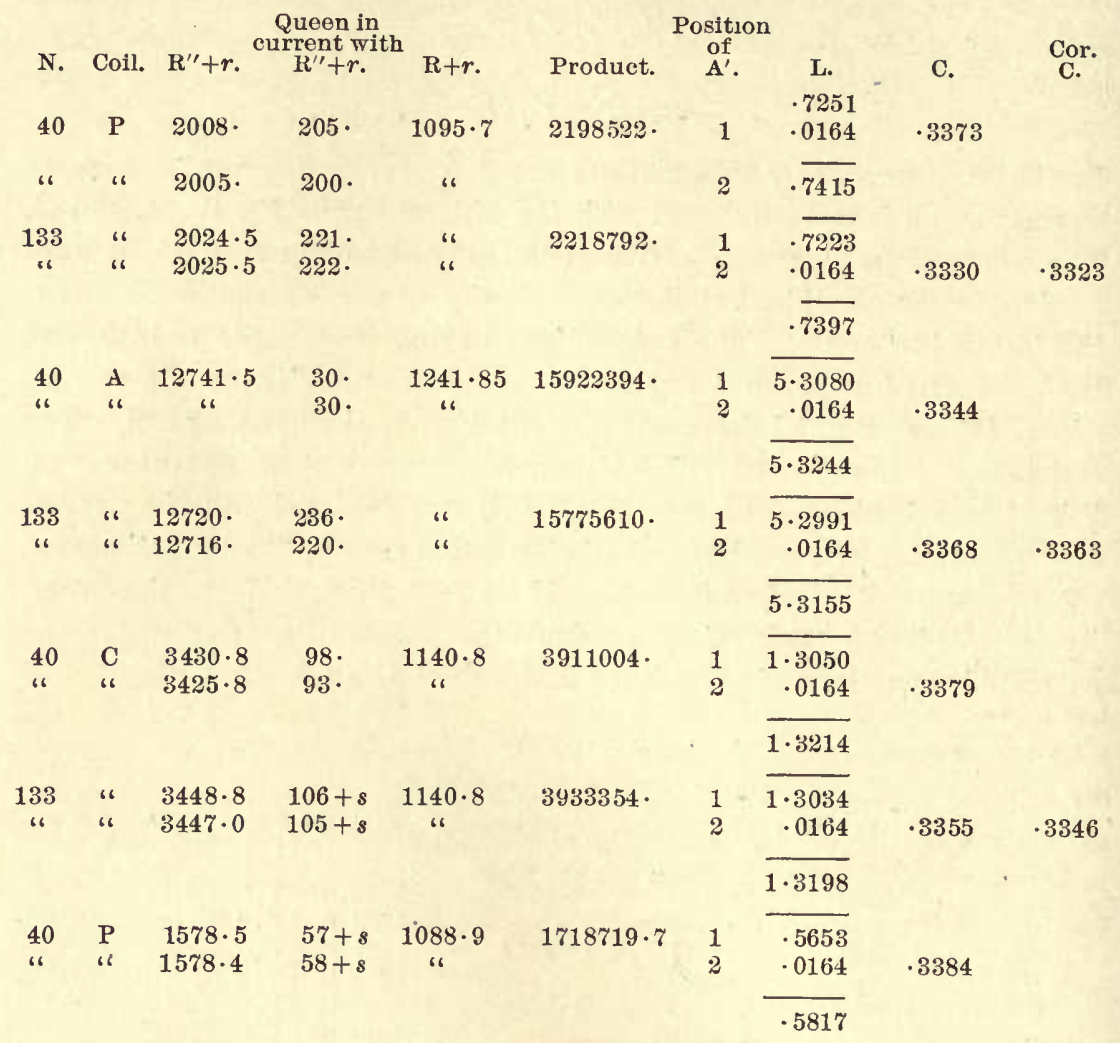

This method can be used with great accuracy for the comparison of the capacity of a condenser with a standard condenser. In the comparison, first one condenser and then the other would be placed in the $R+r$ circuit. If the two condensers are of nearly the same capacity, the degree of accuracy of the comparison depends upon the accuracy with which $R^{\prime \prime}+r^{\prime}$ can be set. The degree of accuracy of setting $R^{\prime \prime}+r^{\prime}$ varies with the value of the self-inductance with which the condensers are compared. In the experiments just given, using the different coils, the degree of accuracy with which two $\frac{1}{3}$ microfarad condensers could have been compared would vary from 1 part in 2000 to one part in 14000. The two condensers are supposed to be without absorption, as its presence would cause trouble unless the absorption resistances were known. 
Résumé.-Summing up the results deduced in this paper, it is seen that the methods for the absolute determination of self-inductance and capacity do not give as concordant results as could be wished. The irregularity of results was caused, in the most part, both in the determination of self-inductance and capacity by the variation of the periods of the currents used in the experiments. As the period enters directly into the determination of self-inductance and capacity, all variations of the period will appear in the results. The determination of capacity is complicated by the presence of electric absorption (p. 323 et seq.). The effect of electric absorption is shown to be that of an added resistance in series with the condenser, called absorption resistance. A direct method is given by which absorption resistance can be measured (p. 319), and experiments are given which show that when condensers possessing absorption are in series or in parallel, their absorption resistances act under these conditions as ohmic resistances in series with the separate condensers (p. 323). Absorption resistance is also found to be extremely sensitive to temperature.

The methods for the comparison of two self-inductances or a selfinductance and a capacity are independent of the period, and when the self-inductances are of different magnitudes the comparison can be made with an accuracy of 1 part in 10000. These methods, therefore, give a means of comparison of a self-inductance with a standard selfinductance, or a capacity with a standard capacity to an accuracy of 1 part in 10000 , or they allow the establishment of standards. 


\title{
RESISTANCE TO ETHEREAL MOTION
}

\author{
By H. A. Rowland, N. E. Gilbert and P. C. MCJunckin
}

[Johns Hopkins University Circulars, No. 146, p. 60, 1900]

An attempt has been made to determine within what limits it is possible to say that there is no frictional or viscous resistance in the ether of space. Modern theories of magnetism are based on some kind of rotary or vortical motion in the ether and if a piece of iron is magnetized we imagine that the molecules, or something about them, rotate also. The existence of permanent magnets shows that any retardation due to any kind of resistance must be very slight.

In the case of an electro-magnet, any energy used in overcoming such resistance, if it exists, must be derived from the exciting current and the disappearance of such energy will produce an apparent resistance added to that of the wire. An attempt was therefore made to determine whether a wire carrying a current had the same electrical resistance when producing a magnetic field that it had when not producing it.

The experiment consisted in winding two coils of wire together on an iron core and determining whether the resistance was the same in two cases:-

(1). When the current was so passed through the coils that both produced a field in the same direction.

(2). When the current was so passed that the fields produced counterbalanced each other.

The great difficulty in the experiment lay in the necessity of measuring the resistance of a coil in which a comparatively large current was flowing. In order to overcome the effect of changes in resistance due to changes in temperature, two coils were wound, as nearly as possible identical, and these double coils were used for the four arms of a Wheatstone's bridge so that the temperature would rise in all four arms equally. Each coil consisted of about 2500 turns of doubled No. 30 copper wire, the whole enclosed in an iron case, boiled in wax for five hours and cooled in a vacuum. The insulation resistance was then about eleven megohms. Iron cores were used and it was found that the cases effectually protected the coils against sudden changes in tem- 
perature due to air currents as well as serving for yokes to the magnets. A current of one-tenth ampere was used which insured a high state of magnetization in the iron when two coils were in series, giving 5000 turns.

The coils were connected in the bridge in such a way that the two coils in one case formed the opposite arms of the bridge. By means of a reversing switch the current in one of these coils could be reversed. This changed the field which might affect two opposite arms of the bridge and thus doubled the deflection. Another switch might have been inserted in the other pair of arms and thus doubled the deflection again but errors due to the switches would also have been doubled and no advantage gained. The switch was carefully constructed with large copper rods dipping into copper mercury cups but, at best, the inaccuracies of the switch limited the accuracy of the experiment.

The fine adjustments were made by resistance boxes shunted round one of the coils. About $15,000 \mathrm{ohms}$ in this shunt balanced the bridge. A change of one ohm in the shunt gave a deflection of two millimeters and indicated a change in the resistance of the arm of $\frac{5}{100000} \mathrm{ohm}$. The whole resistance being over $100 \mathrm{ohms}$ this would give a determination of one part in 2,000,000 or, since the deflection is doubled, one part in $4,000,000$ for each arm. The result of 30 readings each way was that the shunt resistance was about $3 \cdot 4$ ohms less with magnetic field than without. The shunt was so placed that this gives a less resistance by one part in 1,200,000 when producing a magnetic field.

The above result is in the wrong direction. The difficulty may lie in the fact that the galvanometer, though used at night, was unsteady at best, or it may be due to leakage. The resistance of the coils was 100 ohms while the insulation resistance was $11,000,000 \mathrm{ohms}$. If the leakage is symmetrical along the doubled wire it will not affect the galvanometer upon reversing the current in one coil. This assumption may not be justified. 

PART III

HEA T 

ON THE MECHANICAI, EQUIVALENT OF HEAT, WITH SUBSIDIARY RESEARCHES ON THE VARIATION OF THE MERCURIAL FROM THE AIR THERMOMETER, AND ON THE VARIATION OF THE SPECIFIC HEAT OF WATER ${ }^{2}$

[Proceedings of the American Academy of Arts and Sciences, $X \nabla, 75-200,1880]$

Investigations on Light and Heat, made and published wholly or in part with appropriation from the RUMFORD FUND

Presented June 11th, 1879

\section{CONTENTS}

I. Introductory remarks . . . 343

II. Thermometry . . . . . $\quad . \quad 345$

(a.) General view of Thermometry . . . . . 345

(b.) The Mercurial Thermometer 346

(c.) Relation of the Mercurial and Air Thermometers 352

1. General and Historical Remarks . . . 352

2. Description of Apparatus . . . 358

3. Results of Comparison 366 (d.) Reduction to the Absolnte Scale . . . . . 381 Appendix to Thermometry . 384

III. Calorimetry . . . . . . 387

(a.) Specific Heat of Water . 387

(b.) Heat Capacity of the Calorimeter . . . . . 399

IV. Determination of Equivalent . 404 (a.) Historical Remarks . . 404

1. General Review of Methods.... . 405

2. Results of Best Determinations . . . 409

(b.) Description of Apparatus 422

1. Preliminary Remarks . 422

2. General Description . 424

3. Detalls . . 426

(c) Theory of the Experiment 430

1. Estimation of Work Done . . . 430

2. Radiation . . . 435

3. Corrections to Thermometers, etc. . . 439

(d.) Results . . . . . . 441

1. Constant Data . . 441

2. Experimental Data and Tables of Results . 441

V. Concluding Remarks and Criticism of Results and Methods 465

\section{I.-INTRODUCTORY REMARKS}

Among the more important constants of nature, the ratio of the heat unit to the unit of mechanical work stands forth prominent, and

1 This research was originally to have been performed in connection with Professor Pickering, but the plan was frustrated by the great distance between onr residences. An appropriation for this experiment was made by the Amerlcan Academy of Arts and Sciences at Boston, from the fund which was instituted by Connt Rnmford, and liberal ald was also given by the Trustees of the Johns Hopkins University, who are desirous, as far as they can, to promote original scientific investigations. 
is used almost daily by the physicist. Yet, when we come to consider the history of the subject carefully, we find that the only experimenter who has made the determination with anything like the accuracy demanded by modern science, and by a method capable of giving good results, is Joule, whose determination of thirty years ago, confirmed by some recent results, to-day stands almost, if not quite, alone among accurate results on the subject.

But Joule experimented on water of one temperature only, and did not reduce his results to the air thermometer; so that we are still left in doubt, even to the extent of one per cent, as to the value of the equivalent on the air thermometer.

The reduction of the mercurial to the air thermometer, and thence to the absolute scale, has generally been neglected between $0^{\circ}$ and $100^{\circ}$ by most physicists, though it is known that they differ several tenths of a degree at the $45^{\circ}$ point. In calorimetric researches this may produce an error of over one, and even approaching two per cent, especially when a Geissler thermometer is used, which is the worst in this respect of any that I have experimented on; and small intervals on the mercurial thermometers differ among themselves more than one per cent from the difference of the glass used in them.

Again, as water is necessarily the liquid used in calorimeters, its variation of specific heat with the temperature is a very important factor in the determination of the equivalent. Strange as it may appear, we may be said to know almost nothing about the variation of the specific heat of water with the temperature between $0^{\circ}$ and $100^{\circ} \mathrm{C}$.

Regnault experimented only above $100^{\circ} \mathrm{C}$. The experiments of Hirn, and of Jamin and Amaury, are absurd, from the amount of variation which they give. Pfaundler and Platter confined themselves to points between $0^{\circ}$ and $13^{\circ}$. Münchausen seems to have made the best experiments, but they must be rejected because he did not reduce to the air thermometer.

In the present series of researches, I have sought, first, a method of measuring temperatures on the perfect gas thermometer with an accuracy scarcely hitherto attempted, and to this end have made an extended study of the deviation of ordinary thermometers from the air thermometer; and, secondly, I have sought a method of determining the mechanical equivalent of heat so accurate, and of so extended a range, that the variation of the specific heat of water should follow from the experiments alone. 
As to whether or not these have been accomplished, the following pages will show. The curious result that the specific heat of water on the air thermometer decreases from $0^{\circ}$ to about $30^{\circ}$ or $35^{\circ}$, after which it increases, seems to be an entirely unique fact in nature, seeing that there is apparently no other substance hitherto experimented upon whose specific heat decreases on rise of temperature without change of state. From a thermodynamic point of view, however, it is of the same nature as the decrease of specific heat which takes place after the vaporization of a liquid.

The close agreement of my result at $15^{\circ} .7 \mathrm{C}$. with the old result of Joule, after approximately reducing his to the air thermometer and latitude of Baltimore, and correcting the specific heat of copper, is very satisfactory to us both, as the difference is not greater than 1 in 400 , and is probably less.

I hope at some future time to make a comparison with Joule's thermometers, when the difference can be accurately stated.

\section{II.-THERMOMETRY}

\section{(a.) General View}

The science of thermometry, as ordinarily studied, is based upon the changes produced in bodies by heat. Among these we may mention change in volume, pressure, state of aggregation, dissociation, amount and color of light reflected, transmitted, or emitted, hardness, pyro-electric and thermo-electric properties, electric conductivity or specific induction capacity, magnetic properties, thermo-dynamic properties, \&c.; and on each of these may be based a system of thermometry, each one of which is perfect in itself, but which differs from all the others widely. Indeed, each method may be applied to nearly all the bodies in nature, and hundreds or thousands of thermometric scales may be produced, which may be made to agree at two fixed points, such as the freezing and boiling points of water, but which will in general differ at nearly, if not all, other points.

But from the way in which the science has advanced, it has come to pass that all methods of thermometry in general use to the present time have been reduced to two or three, based respectively on the apparent expansion of mercury in glass and on the absolute expansion of some gas, and more lately on the second law of thermodynamics.

Each of these systems is perfectly correct in itself, and we have no right to designate either of them as incorrect. We must decide a priori 
on some system, and then express all our results in that system: the accuracy of science demands that there should be no ambiguity on that subject. In deciding among the three systems, we should be guided by the following rules:-

1st. The system should be perfectly definite, so that the same temperature should be indicated, whatever the thermometer.

$2 d$. The system should lead to the most simple laws in nature.

Sir William Thomson's absolute system of thermometry, coinciding with that based on the expansion of a perfect gas, satisfies these most nearly. The mercurial thermometer is not definite unless the kind of glass is given, and even then it may vary according to the way the bulb is blown. The gas thermometer, unless the kind of gas is given, is not definite. And, further, if the temperature as given by either of these thermometers was introduced into the equations of thermo-dynamics, the simplest of them would immediately become complicated.

Throughout a small range of temperature, these systems agree more or less completely, and it is the habit even with many eminent physicists to regard them as coincident between the freezing and boiling points of water. We shall see, however, that the difference between them is of the highest importance in thermometry, especially where differences of temperature are to be used.

For these reasons I have reduced all my measures to the absolute system.

The relation between the absolute system and the system based on the expansion of gases has been determined by Joule and Thomson in their experiments on the flow of gases through porous plugs (Philosophical Transactions for 1862, p. 579). Air was one of the most important substances they experimented upon.

To measure temperature on the absolute scale, we have thus only to determine the temperature on the air thermometer, and then reduce to the absolute scale. But as the air thermometer is very inconvenient to use, it is generally more convenient to use a mercurial thermometer which has been compared with the air thermometer. Also, for small changes of temperature the air thermometer is not sufficiently sensitive, and a mercurial thermcmeter is necessary for interpolation. I shall occupy myself first with a careful study of the mercurial thermometer.

\section{(b.) The Mercurial Thermometer}

Of the two kinds of mercurial thermometers, the weight thermometer is of little importance to our subject. I shall therefore confine myself 
principally to that form having a graduated stem. For convenience in use and in calibration, the principal bulb should be elongated, and another small bulb should be blown at the top. This latter is also of the utmost importance to the accuracy of the instrument, and is placed there by nearly all makers of standards. ${ }^{2}$ It is used to place some of the mercury in while calibrating, as well as when a high temperature is to be measured; also, the mercury in the larger bulb can be made free from air-bubbles by its means.

Most standard thermometers are graduated to degrees; but Regnault preferred to have his thermometers graduated to parts of equal capacity whose value was arbitrary, and others have used a single millimeter division. As thermometers change with age, the last two methods are the best; and of the two I prefer the latter where the highest accuracy is desired, seeing that it leaves less to the maker and more to the scientist. The cross-section of the tube changes continuously from point to point, and therefore the distribution of marks on the tube should be continuous, which would involve a change of the dividing engine for each division. But as the maker divides his tube, he only changes the length of his divisions every now and then, so as to average his errors. This gives a sufficiently exact graduation for large ranges of temperature; but for small, great errors may be introduced. Where there is an arbitrary scale of millimeters, I believe it is possible to calibrate the tube so that the errors shall be less than can be seen with the naked eye, and that the table found shall represent very exactly the gradual variation of the tube.

In the calibration of my thermometers with the millimetric scale, I have used several methods, all of which are based upon some graphical method. The first, which gives all the irregularities of the tube with great exactness, is as follows:

A portion of the mercury having been put in the upper bulb, so as to leave the tube free, a column about $15 \mathrm{~mm}$. long is separated off. This is mored from point to point of the tube, and its length carefully measured on the dividing engine. It is not generally necessary to move the column its own length every time, but it may be moved $20 \mathrm{~mm}$. or $25 \mathrm{~mm}$., a record of the position of its centre being kept. To eliminate any errors of division or of the dividing engine, readings were then taken on the scale, and the lengths reduced to their value in scale divisions. The area of the tube at every point is inversely as

${ }^{2}$ Geissler and Casella omit it, which should condemn their thermometers. 
the length of the column. We shall thus have a series of figures nearly equal to each other, if the tube is good. By subtracting the smallest from each of the others, and plotting the results as ordinates, with the thermometer scale as abscissas, and drawing a curve through the points so found, we have means of finding the area at any point. The curve should not be drawn exactly through the points, but rather around them, seeing they are the average areas for some distance each side of the point. With good judgment, the curve can be drawn with great accuracy. I then draw ordinates every $10 \mathrm{~mm}$., and estimate the average area of the tube for that distance, which I set down in a table. As the lengths are uniform, the volume of the tube to any point is found by adding up the areas to that point.

But it would be unwise to trust such a method for very long tubes, seeing the mercury column is so short, and the columns are not end to end. Hence I use it only as supplementary to one where the column is about $50 \mathrm{~mm}$. long, and is always moved its own length. This establishes the volumes to a series of points about $50 \mathrm{~mm}$. apart, and the other table is only used to interpolate in this one. There seems to be no practical object in using columns longer than this.

Having finally constructed the arbitrary table of volumes, I then test it by reading with the eye the length of a long mercury column. No certain error was thus found at any point of any of the thermometers which I have used in these experiments.

While measuring the column, great care must be taken to preserve all parts of the tube at a uniform temperature, and only the extreme ends must be touched with the hands, which should be covered with cloth.

If $\nabla$ is the volume on this arbitrary scale, the temperature on the mercurial thermometer is found from the formula $T=C V-t_{0}$, where $C$ and $t_{0}$ are constants to be determined. If the thermometer contains the $0^{\circ}$ and $100^{\circ}$ points, we have simply

$$
C=\frac{100}{V_{100}-V_{0}}
$$

Otherwise $C$ is found by comparison with some other thermometer, which must be of the same kind of glass.

It is to be carefully noted that the temperature on the mercurial thermometer, as I have defined it, is proportional to the apparent expansion of mercury as measured on the stem. By defining it as proportional to the true volume of mercury in the stem, we have to introduce a correction to ordinary thermometers, as Poggendorff has shown. 
As I only use the mercurial thermometer to compare with the air thermometer, and as either definition is equally correct, I will not further discuss the matter, but will use the first definition, as being the simplest.

In the above formula I have implicitly assumed that the apparent expansion is only a function of the temperature; but in solid bodies like glass there seems to be a progressive change in the volume as time advances, and especially after it has been heated. And hence in mercurial and alcohol thermometers, and probably in general in all thermometers which depend more or less on the expansion of solid bodies, we find that the reading of the thermometer depends, not only on its present temperature, but also on that to which it has been subjected within a short time; so that, on heating a thermometer up to a certain temperature, it does not stand at the same point as if it had been cooled from a higher temperature to the given temperature. As these effects are without doubt due to the glass envelope, we might greatly diminish them by using thermometers filled with liquids which expand more than mercury: there are many of these which expand six or eight times as much, and so the irregularity might be diminished in this ratio. But in this case we should find that the correction for that part of the stem which was outside the vessel whose temperature we were determining would be increased in the same proportion; and besides, as all the liquids are quite volatile, or at least wet the glass, there would be an irregularity introduced on that account. A thermometer with liquid in the bulb and mercury in the stem would obviate these inconveniences; but even in this case the stem would have to be calibrated before the thermometer was made. By a comparison with the air-thermometer, a proper formula could be obtained for finding the temperature.

But I hardly believe that any thermometer superior to the mercurial can at present be made,--that is, any thermometer within the same compass as a mercurial thermometer,-and I think that the best result for small ranges of temperature can be obtained with it by studying and avoiding all its sources of error.

To judge somewhat of the laws of the change of zero within the limits of temperature which I wished to use, I took thermometer No. 6163, which had lain in its case during four months at an average temperature of about $20^{\circ}$ or $25^{\circ} \mathrm{C}$, , and observed the zero point, after heating to various temperatures, with the following result. The time of heating was only a few minutes, and the zero point was taken imme- 
diately after; some fifteen minutes, however, being necessary for the thermometer to entirely cool.

TABLE I.-Showing Change of Zero Point.

\begin{tabular}{|c|c|c|c|}
\hline $\begin{array}{c}\text { Temperature } \\
\text { of Bulb } \\
\text { before finding } \\
\text { the 0 Point. }\end{array}$ & $\begin{array}{c}\text { Change of } \\
\text { 0 Point. }\end{array}$ & $\begin{array}{c}\text { Temperature } \\
\text { of Bulb } \\
\text { before finding } \\
\text { the 0 Point. }\end{array}$ & $\begin{array}{c}\text { Change of } \\
\text { 0 Point. }\end{array}$ \\
\hline 22.5 & 0 & 70.0 & -.115 \\
30.0 & -.016 & 81.0 & -.170 \\
40.5 & -.033 & $90 \cdot 0$ & -.231 \\
51.0 & -.039 & $100 \cdot 0$ & -.313 \\
60.0 & -.105 & $100 \cdot 0$ & -.347 \\
\hline
\end{tabular}

The second $100^{\circ}$ reading was taken after boiling for some time.

It is seen that the zero point is always lower after heating, and that in the limits of the table the lowering of the zero is about proportional to the square of the increase of temperature above $25^{\circ} \mathrm{C}$. This law is not true much above $100^{\circ}$, and above a certain temperature the phenomenon is reversed, and the zero point is higher after heating; but for the given range it seems quite exact.

It is not my purpose to make a complete study of this phenomenon with a view to correcting the thermometer, although this has been undertaken by others. But we see from the table that the error cannot exceed certain limits. The range of temperature which $I$ have used in each experiment is from $20^{\circ}$ to $30^{\circ} \mathrm{C}$., and the temperature rarely rose above $40^{\circ} \mathrm{C}$. The change of zero in this range only amounts to $0^{\circ} .03 \mathrm{C}$.

The exact distribution of the error from this cause throughout the scale has never been determined, and it affects my results so little that I have not considered it worth investigating. It seems probable, however, that the error is distributed throughout the scale. If it were uniformly distributed, the value of each division would be less than before by the ratio of the lowering at zero to the temperature to which the thermometer was heated.

The maximum errors produced in my thermometers by this cause would thus amount to 1 in 1300 nearly for the $40^{\circ}$ thermometer, and to about 1 in 2000 for the others. Rather than allow for this, it is better to allow time for the thermometer to resume its original state.

Only a few observations were made upon the rapidity with which the zero returned to its original position. After heating to $81^{\circ}$, the 
zero returned from $-0^{\circ} \cdot 1 \% 0$ to $-0^{\circ} \cdot 148$ in two hours and a half. After heating to $100^{\circ}$, the zero returned from $-0^{\circ} \cdot 347$ to $-0^{\circ} \cdot 110$ in nine days, and to $-0^{\circ} .022$ in one month. Reasoning from this, I should say that in one week thermometers which had not been heated above $40^{\circ}$ should be ready for use again, the error being then supposed to be less than 1 in 4000 , and this would be partially eliminated by comparing with the air thermometer at the same intervals as the thermometer is used, or at least heating to $40^{\circ}$ one week before comparing with the air thermometer.

As stated before, when a thermometer is heated to a very high point, its zero point is raised instead of lowered, and it seems probable that at some higher point the direction of change is reversed again; for, after the instrument comes from the maker, the zero point constantly rises until it may be $0^{\circ} \cdot 6$ above the mark on the tube. This gradual change is of no importance in my experiments, as I only use differences of temperature, and also as it was almost inappreciable in my thermometers.

Another source of error in thermometers is that due to the pressure on the bulb. In determining the freezing point, large errors may be made, amounting to several hundredths of a degree, by the pressure of pieces of ice. In my experiments, the zero point was determined in ice, and then the thermometer was immersed in the water of the comparator at a depth of about $60 \mathrm{~cm}$. The pressure of this water affected the thermometer to the extent of about $0^{\circ} \cdot 01$, and a correction was accordingly made. As differences of temperature were only needed, no correction was made for variation in pressure of the air.

It does not seem to me well to use thermometers with too small a stem, as I have no doubt that they are subject to much greater irregularities than those with a coarse bore. For the capillary action always exerts a pressure on the bulb. Hence, when the mercury rises, the pressure is due to a rising meniscus which causes greater pressure than the falling meniscus. Hence, an apparent friction of the mercurial column. Also, the capillary constant of mercury seems to depend on the electric potential of its surface, which may not be constant, and would thus cause an irregularity.

My own thermometers did not show any apparent action of this kind, but Pfaundler and Platter mention such an action, though they give another reason for it. 


\section{(c.) Relation of the Mercurial and Air Thermometers}

\section{General and Historical Remarks}

Since the time of Dulong and Petit, many experiments have been made on the difference between the mercurial and the air thermometer, but unfortunately most of them have been at high temperatures. As weight thermometers have been used by some of the best experimenters, I shall commence by proving that the weight thermometer and stem thermometer give the same temperature; at the same time, however, obtaining a convenient formula for the comparison of the air thermometer with the mercurial.

For the expansion of mercury and of glass the following formulæ must hold:-

$$
\begin{array}{cl}
\text { For mercury, } & V=V_{0}\left(1+a t+b t^{2}+d c c_{0}\right) ; \\
\text { " glass. } & V^{\prime}=V_{0}^{\prime}\left(1+a t+\beta t^{2}+d c c_{0}\right) ;
\end{array}
$$

In both the weight and stem thermometers we must have $V=\nabla^{\prime}$.

$$
\therefore \quad V_{0}^{\prime}=V_{0} \frac{1+a t+b t^{2}+d c .}{1+a t+\beta t^{2}+\& c}=V_{0}\left(1+A t+B t^{2}+\& c .\right) .
$$

where $V_{0}^{\prime}$ and $\nabla_{0}$ are the volumes of the glass and of the mercury reduced to zero, and $t$ is the temperature on the air thermometer. The temperature by the weight thermometer is

$$
T=100 \frac{P_{0}-P_{\mathrm{t}}}{P_{0}-P_{100}} \frac{P_{100}}{P_{t}}=100 \frac{\frac{P_{0}}{P_{t}}-1}{\frac{P_{0}}{P_{100}}-1}
$$

where $P_{0}, P$, \&c., are the weights of mercury in the bulb at $0^{\circ} \mathrm{C}$., $t^{\circ}$ C., \&c.

Now these weights are directly as the volumes of the mercury at $0^{\circ}$.

$$
\therefore \quad \frac{P_{0}}{P_{t}}=1+A t+B t^{2}+\& c .
$$

seeing that $\nabla$ is constant.

$$
\therefore \quad T^{\prime}=100 \frac{A t+B t^{2}+\& c .}{100 A+(100)^{2} B+d c .} .
$$

In the stem thermometers we have $\nabla_{0}$, the volume of mercury at $0^{\circ}$, constant, and the volume of the glass that the mercury fills, reduced to $0^{\circ}$, variable. As the volume of the glass $V_{0}^{\prime}$ is the volume reduced to $0^{\circ}$, it will be proportional to the volume of bulb plus the volume of the tube as read off on the scale which should be on the tube. 


$$
\begin{aligned}
& \therefore T=100 \frac{\left(V_{0}^{\prime}\right)_{t}-\left(V_{0}^{\prime}\right)_{0}}{\left(V_{0}^{\prime}\right)_{100}-\left(V_{0}^{\prime}\right)_{0}}=100 \frac{\frac{\left(V^{\prime}\right)_{i}}{\left(V_{0}^{\prime}\right)_{0}}-1}{\frac{\left(V_{0}^{\prime}\right)_{100}}{\left(V_{0}^{\prime}\right)_{0}}-1} ; \\
& \therefore \quad T=100 \frac{A t+B t^{2}+\& c .}{100 A+(100)^{2} B+\& c .}
\end{aligned}
$$

which is the same as for the weight thermometer.

If the fixed points are $0^{\circ}$ and $t^{\circ}$ instead of $0^{\circ}$ and $100^{\circ}$, we can write

$$
\begin{aligned}
& T=t^{\prime} \frac{A t+B t^{2}+C t^{3}+\& c .}{A t^{\prime}+B t^{\prime 3}+C t^{\prime 3}+\& c .} \\
& T=t\left\{1+\left(t-t^{\prime}\right)\left[\frac{B}{A}+\frac{C}{A}+\frac{B^{2}}{A^{2}} t^{\prime}+\frac{C}{A} t\right]+\& c .\right\} \\
& T=t\left\{1+\left(t-t^{\prime}\right)\left[\frac{B}{A}+\frac{B^{2}}{A^{2}} t^{\prime}+\frac{C}{A}\left(t+t^{\prime}\right)\right]+\& c .\right\}
\end{aligned}
$$

As $T$ and $t$ are nearly equal, and as we shall determine the constants experimentally, we may write

$$
t=T-a t\left(t^{\prime}-t\right)(b-t)+\& c .
$$

where $t$ is the temperature on the air thermometer, and $T$ that on the mercurial thermometer, and $a$ and $b$ are constants to be determined for each thermometer.

The formula might be expanded still further, but I think there are few cases which it will not represent as it is. Considering $b$ as equal to 0 , a formula is obtained which has been used by others, and from which some very wrong conclusions have been drawn. In some kinds of glass there are three points which coincide with the air thermometer, and it requires at least an equation of the third degree to represent this.

The three points in which the two thermometers coincide are given by the roots of the equation

and are, therefore,

$$
t\left(t^{\prime}-t\right)(b-t)=0
$$

$$
\begin{aligned}
& t=0 \\
& t=t^{\prime} \\
& t=b .
\end{aligned}
$$

In the following discussion of the historical results, I shall take $0^{\circ}$ and $100^{\circ}$ as the fixed points. Hence, $t^{\prime}=100^{\circ}$. To obtain $a$ and $b$, two observations are needed at some points at a distance from $0^{\circ}$ and $100^{\circ}$. That we may get some idea of the values of the constants in the formula for different kinds of glass, I will discuss some of the experimental results of Regnault and others with this in view. 
Regnault's results are embodied, for the most part, in tables given on p. 239 of the first volume of his Relation des Expériences. The figures given there are obtained from curves drawn to represent the mean of his experiments, and do not contain any theoretical results. The direct application of my formula to his experiments could hardly be made without immense labor in finding the most probable value of the constants.

But the following seem to satisfy the experiments quite well:-

$\begin{array}{llrl}\text { Cristal de Choisy-le-Roi } & b=0, & a=.00000032 . \\ \text { Verre Ordinaire } & b=245^{\circ}, & a=.00000034 . \\ \text { Verre Vert } & b=270^{\circ}, & a=.000000095 \\ \text { Verre de Suède } & b=+10^{\circ}, & a=.00000014 .\end{array}$

From these values I have calculated the following:-

TABLE II.-Regnaul't's Results Compared with the Formula.

\begin{tabular}{|c|c|c|c|c|c|c|c|c|c|c|c|c|}
\hline \multirow{2}{*}{ 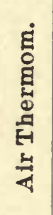 } & \multicolumn{3}{|c|}{ Choisy-le-Roi. } & \multicolumn{3}{|c|}{ Verre Ordinaire. } & \multicolumn{3}{|c|}{ Verre Vert. } & \multicolumn{3}{|c|}{ Verre de Suède. } \\
\hline & $\begin{array}{l}0 \\
0 \\
0 \\
0 \\
00 \\
0\end{array}$ & 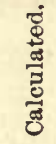 & 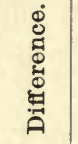 & 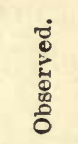 & 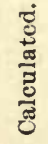 & 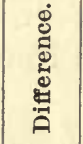 & $\begin{array}{l}\text { D } \\
\text { : } \\
0 \\
0 \\
0\end{array}$ & 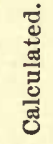 & 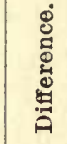 & $\begin{array}{l}\text { D. } \\
0 \\
0 \\
0 \\
00 \\
0\end{array}$ & 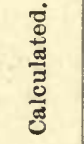 & 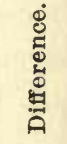 \\
\hline 100 & 0 & 0 & 0 & 0 & 0 & 0 & 0 & 0 & 0 & 0 & 0 & 0 \\
\hline \multicolumn{4}{|c|}{$120120 \cdot 12120.09+.03$} & & 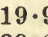 & $+\cdot 05$ & 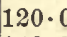 & $120 \cdot($ & -.0 & $120 \cdot 0$ & $120 \cdot 04$ & 0 \\
\hline \multicolumn{13}{|c|}{$140140 \cdot 29140 \cdot 25+.04139 \cdot 85139 \cdot 80+.05140 \cdot 21140 \cdot 22-.01140 \cdot 11140 \cdot 10+.01$} \\
\hline \multirow{2}{*}{\multicolumn{4}{|c|}{$160160 \cdot 52160.49+.03$}} & \multicolumn{2}{|c|}{$159 \cdot 74159 \cdot 7$} & +.02 & $160 \cdot 4$ & $160 \cdot 3$ & $+\cdot 0$ & & $160 \cdot 21$ & -.01 \\
\hline & & & & & 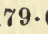 & -.05 & $180 \cdot 6$ & $180 \cdot 6$ & -0 & $180 \cdot 3$ & $180 \cdot 34$ & -.01 \\
\hline \multicolumn{4}{|c|}{$200201 \cdot 25201 \cdot 28-.03$} & \multicolumn{2}{|c|}{$199.70199 \cdot 6$} & +.01 & $200 \cdot 8$ & 200. & -.0 & $200 \cdot 5$ & 200 & -.03 \\
\hline \multicolumn{4}{|c|}{$220221 \cdot 82221 \cdot 86-\cdot 04$} & \multicolumn{2}{|c|}{$219 \cdot 80219 \cdot ?$} & +.02 & $221 \cdot 2$ & $221 \cdot 2$ & -.0 & $220 \cdot 7$ & $220 \cdot 78$ & -.03 \\
\hline \multirow{2}{*}{\multicolumn{4}{|c|}{$\begin{array}{l}240242 \cdot 55242 \cdot 56-.01 \\
260263 \cdot 44263 \cdot 46-.02\end{array}$}} & \multicolumn{2}{|c|}{$239 \cdot 90239 \cdot 9$} & -.06 & $241 \cdot 6$ & $241 \cdot 6$ & -0 & $241 \cdot 1$ & $241 \cdot 08$ & +.08 \\
\hline & & & & \multirow{2}{*}{\multicolumn{2}{|c|}{$\begin{array}{r}260 \cdot 20260 \cdot 2 \\
3280.58280 .0\end{array}$}} & -.01 & $262 \cdot 1$ & $262 \cdot($ & +0 & & & \\
\hline \multicolumn{4}{|c|}{$280284 \cdot 48284 \cdot 52-\cdot 04$} & & & -.02 & $282 \cdot 8$ & 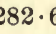 & $+\cdot 2$ & & & \\
\hline \multirow{2}{*}{\multicolumn{4}{|c|}{$\begin{array}{l}300305 \cdot 72305 \cdot 76-\cdot 04 \\
320327 \cdot 25327 \cdot 20-\cdot 05\end{array}$}} & \multirow{2}{*}{\multicolumn{2}{|c|}{$301 \cdot 08301 \cdot 1$}} & -.04 & & & & & & \\
\hline & & & & & & .00 & & & & & & \\
\hline \multicolumn{3}{|c|}{$340349 \cdot 30348 \cdot 8$} & $+\cdot 42$ & $434 \cdot 0$ & $342 \cdot$ & $+\cdot 36$ & & & & & & \\
\hline
\end{tabular}

The formula, as we see from the table, represents all Regnault's curves with great accuracy, and if we turn to his experimental results we shall find that the deviation is far within the limits of the experimental errors. The greatest deviation happens at $340^{\circ}$, and may be accounted for by an error in drawing the curve, as there are few experimental results so high as this, and the formula seems to agree with them almost as well as Regnault's own curve.

${ }^{3}$ Corrected from $280 \cdot 52$ in Regnault's table. 
The object of comparing the formula with Regnault's results at temperatures so much higher than I need, is simply to test the formula through as great a range of temperatures, and for as many kinds of glass, as possible. If it agrees reasonably well throughout a great range, it will probably be very accurate for a small range, provided we obtain the constants to represent that small range the best.

Having obtained a formula to represent any series of experiments, we can hardly expect it to hold for points outside our series, or even for interpolating between experiments too far apart, as, very often, a small change in one of the constants may affect the part we have not experimented on in a very marked manner. Thus in applying the formula to points between $0^{\circ}$ and $100^{\circ}$ the value of $b$ will affect the result very much. In the case of the glass Choisy-le-Roi many values of $b$ will satisfy the observations besides $b=0$. For the ordinary glass, however, $b$ is well determined, and the formula is of more value between $0^{\circ}$ and $100^{\circ}$.

The following table gives the results of the calculation.

TABLE III.-RegradLT's Results CoMpared with the Formula.

\begin{tabular}{|c|c|c|c|c|c|c|}
\hline \multirow{2}{*}{$\begin{array}{l}\text { Air } \\
\text { Thermom- } \\
\text { eter. }\end{array}$} & $\begin{array}{c}\text { Calculated } \\
a=\cdot 00000032 \\
b=0\end{array}$ & $\begin{array}{c}\text { Calculated } \\
a=00000034 \\
b=245\end{array}$ & Observed. & \multirow[t]{2}{*}{$d$} & $\begin{array}{c}\text { Calculated } \\
a=00000044 \\
b=260 .\end{array}$ & \multirow[t]{2}{*}{$\Delta$} \\
\hline & Choisy-le-Roi. & $\begin{array}{c}\text { Verre } \\
\text { Ordinaire. }\end{array}$ & $\begin{array}{c}\text { Verre } \\
\text { Ordinaire. }\end{array}$ & & $\begin{array}{c}\text { Verre } \\
\text { Ordinaire. }\end{array}$ & \\
\hline 0 & 0 & 0 & 0 & & 0 & \\
\hline 10 & $10 \cdot 00$ & $10 \cdot 07$ & $\ldots \ldots$ & & $10 \cdot 10$ & $\ldots$ \\
\hline 20 & $19 \cdot 99$ & $20 \cdot 12$ & $\ldots \ldots$ & $\ldots \ldots$ & $20 \cdot 17$ & $\ldots \ldots$ \\
\hline 30 & $29 \cdot 98$ & $30 \cdot 15$ & $30 \cdot 12$ & $+\cdot 03$ & $30 \cdot 21$ & $+\cdot 09$ \\
\hline 40 & $39 \cdot 97$ & $40 \cdot 17$ & $40 \cdot 23$ & -.06 & $40 \cdot 23$ & 0 \\
\hline 50 & $49 \cdot 96$ & $50 \cdot 17$ & $50 \cdot 23$ & -.06 & $50 \cdot 23$ & 0 \\
\hline 60 & $59 \cdot 95$ & $60 \cdot 15$ & $60 \cdot 24$ & -.09 & $60 \cdot 21$ & -.03 \\
\hline 70 & $69 \cdot 95$ & $70 \cdot 12$ & $70 \cdot 22$ & $-\cdot 10$ & $70 \cdot 18$ & -.04 \\
\hline 80 & $79 \cdot 96$ & $80 \cdot 09$ & $80 \cdot 10$ & -.01 & $80 \cdot 11$ & +.01 \\
\hline 90 & 89.97 & 90.05 & $\ldots \ldots$ & $\ldots \ldots$ & $90 \cdot 07$ & $\ldots \ldots$ \\
\hline 100 & 100 & 100 & 100 & $\ldots \ldots$ & 100 & $\ldots \ldots$ \\
\hline
\end{tabular}

Regnault does not seem to have published any experiments on Choisyle-Roi glass between $0^{\circ}$ and $100^{\circ}$, but in the table between pp. 226, 22\%, there are some results for ordinary glass. The separate observations do not seem to have been very good, but by combining the total number of observations I have found the results given above. The numbers in the fourth column are found by taking the mean of Regnault's results for points as near the given temperature as possible. The agreement 
is only fair, but we must remember that the same specimens of glass were not used in this experiment as in the others, and that for these specimens the agreement is also poor above $100^{\circ}$. The values $a=$ $.000,000,44$ and $b=260^{\circ}$ are much better for these specimens, and the seventh column contains the values calculated from these values. These values also satisfy the observations above $100^{\circ}$ for the given specimens.

The table seems to show that between $0^{\circ}$ and $100^{\circ}$ a thermometer of Choisy-le-Roi almost exactly agrees with the air thermometer. But this is not at all conclusive. Regnault, however, remarks," that between $0^{\circ}$ and $100^{\circ}$ thermometers of this glass agree more nearly with the air thermometer than those of ordinary glass, though he states the difference to amount to $\cdot 1$ to $\cdot 2$ of a degree, the mercurial thermometer standing below the air thermometer. With the exception of this remark of Regnault's, no experiments have ever been published in which the direction of the deviation was similar to this. All experiments have found the mercurial thermometer to stand above the air thermometer between $0^{\circ}$ and $100^{\circ}$, and my own experiments agree with this. However, no general rule for all kinds of glass can be laid down.

Boscha has given an excellent study of Regnault's results on this subject, though I cannot agree with all his conclusions on this subject. In discussing the difference between $0^{\circ}$ and $100^{\circ}$ he uses a formula of the form

$$
T-t=\frac{b}{a} t(100-t),
$$

and deduces from it the erroneous conclusion that the difference is greatest at $50^{\circ} \mathrm{C}$., instead of between $40^{\circ}$ and $50^{\circ}$. His results for $T-t$ at $50^{\circ}$ are

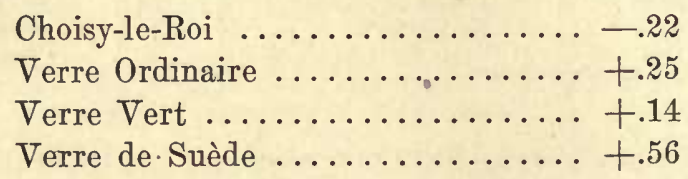

and these are probably somewhat nearly correct, except the negative value for Choisy-le-Roi.

With the exception of Regnault, very few observers have taken up this subject. Among these, however, we may mention Recknagel, who 
has made the determination for common glass between $0^{\circ}$ and $100^{\circ}$. I have found approximately the constants for my formula in this case, and have calculated the values in the fourth column of the following table.

TABLE IV.-ReckNagel's Results Compared with the Formula.

\begin{tabular}{|c|c|c|c|}
\hline \multirow{2}{*}{$\begin{array}{c}\text { Air } \\
\text { Thermometer. }\end{array}$} & \multicolumn{2}{|c|}{ Mercurial Thermometer. } & \multirow{2}{*}{ Difference. } \\
\cline { 2 - 4 } & Observed. & Calculated. & \\
\hline & & & \\
\hline 0 & 0 & 0 & 0 \\
10 & $10 \cdot 08$ & $10 \cdot 08$ & 0 \\
20 & $20 \cdot 14$ & $20 \cdot 14$ & 0 \\
30 & $30 \cdot 18$ & $30 \cdot 18$ & 0 \\
40 & $40 \cdot 20$ & $40 \cdot 20$ & 0 \\
50 & $50 \cdot 20$ & $50 \cdot 20$ & 0 \\
60 & $60 \cdot 18$ & $60 \cdot 18$ & $+\cdot 01$ \\
70 & $70 \cdot 14$ & $70 \cdot 15$ & $+\cdot 01$ \\
80 & $80 \cdot 10$ & $80 \cdot 11$ & $+\cdot 01$ \\
90 & $90 \cdot 05$ & $90 \cdot 06$ & 0 \\
100 & $100 \cdot 00$ & 0 & \\
\hline
\end{tabular}

$$
\begin{aligned}
& b=290^{\circ}, \quad a=.00000033, \\
& T=t+a t(100-t)(b-t) .
\end{aligned}
$$

It will be seen that the values of the constants are not very different from those which satisfy Regnault's experiments.

There seems to be no doubt, from all the experiments we have now discussed, that the point of maximum difference is not at $50^{\circ}$, but at some less temperature, as $40^{\circ}$ to $45^{\circ}$, and this agrees with my own experiments, and a recent statement by Ellis in the Philosophical Magazine. And I think the discussion has proved beyond doubt that the formula is sufficiently accurate to express the difference of the mercurial and air thermometers throughout at least a range of $200^{\circ}$, and hence is probably very accurate for the range of only $100^{\circ}$ between $0^{\circ}$ and $100^{\circ}$.

Hence it is only necessary to find the constants for my thermometers. But before doing this it will be well to see how exact the comparison must be. As the thermometers are to be used in a calorimetric research in which differences of temperature enter, the error of the mercurial compared with the air thermometer will be

$$
\frac{d T}{d t}-1=a\left\{b t^{\prime}-2\left(b+t^{\prime}\right) t+3 t^{2}\right\}
$$


which for the constants used in Recknagel's table becomes

$$
\text { Error }=\frac{d T}{d t}-1=.00000033\left\{29000-780 t+3 t^{2}\right\} .
$$

This amounts to nearly one per cent at $0^{\circ}$, and thence decreases to $45^{\circ}$, after which it increases again. As only $0^{\circ} \cdot 2$ at the $40^{\circ}$ point produces this large error at $0^{\circ}$, it follows that an error of only $0^{\circ} .02$ at $40^{\circ}$ will produce an error of $\frac{1}{1000}$ at $0^{\circ}$. At other points the errors will be less.

Hence extreme care must be taken in the comparison and the most accurate apparatus must be constructed for the purpose.

\section{Description of Apparates}

\section{The Air Thermometer}

In designing the apparatus, I have had in view the production of a uniform temperature combined with ease of reading the thermometers, which must be totally immersed in the water. The uniformity, however, needed only to apply to the air thermometer and to the bulbs of the mercurial thermometer, as a slight variation in the temperature of the stems is of no consequence. A uniform temperature for the air thermometer is important, because it must take time for a mass of air to heat up to a given temperature within $0^{\circ} .01$ or less.

Fig. 1 gives a section of the apparatus. This consists of a large copper vessel, nickel-plated on the outside, with double walls an inch apart, and made in two parts, so that it could be put together watertight along the line $a b$. As seen from the dimensions, it required about 28 kilogrammes of water to fill it. Inside of this was the vessel $m d e f g h k l n$, which could be separated along the line $d k$. In the upper part of this vessel, a piston, $q$, worked, and could draw the water from the vessel. The top was closed by a loose piece of metal, $o p$, which fell down and acted as a valve. The bottom of this inner vessel had a false bottom, $c l$, above which was a row of large holes; above these was a perforated diaphragm, $s$. The bulb of the air thermometer was at $t$, with the bulbs of the mercurial thermometers almost touching it. The air thermometer bulb was very much elongated, being about $18 \mathrm{~cm}$. long and 3 to $5 \mathrm{~cm}$. in diameter. Although the bulbs of the thermometers were in the inner vessel, the stems were in the outer one, and the reading was accomplished through the thick glass window $u v$. 
The change of the temperature was effected by means of a Bunsen burner under the vessel $w$.

The working of the apparatus was as follows: The temperature having been raised to the required point, the piston $q$ was worked to stir up the water; this it did by drawing the water through the holes

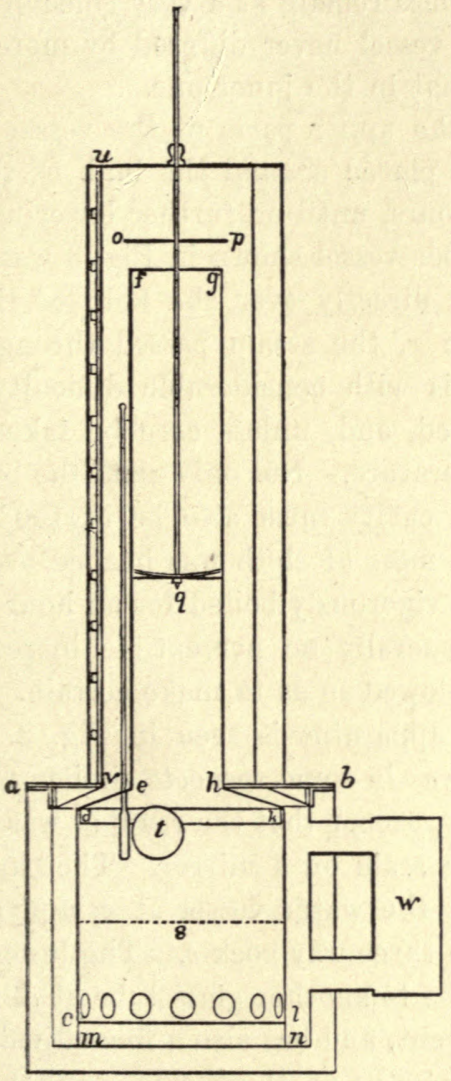

FIG. 1.

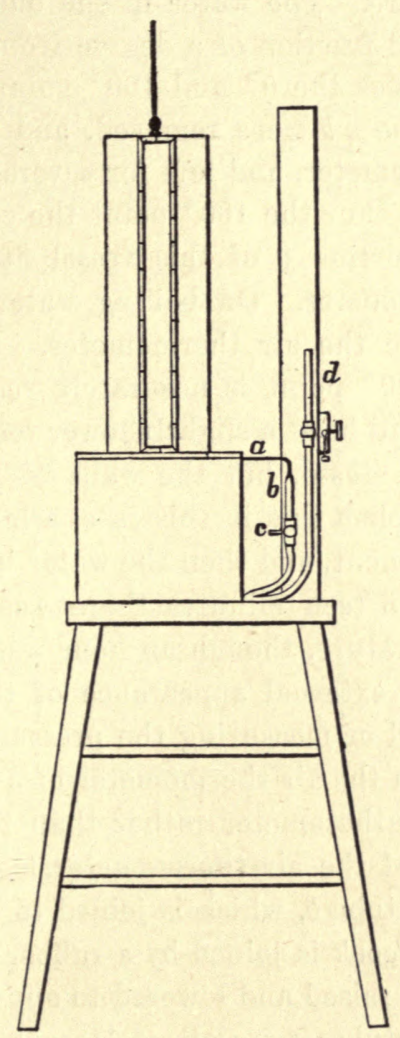

FIG. 2.

at $c l$ and the perforated diaphragm $s$, and thence up through the apparatus to return on the outside. When the whole of the water is at a nearly uniform temperature the stirring is stopped, the valve $o p$ falls into place, and the connection of the water in the outer and inner vessels is practically closed as far as currents are concerned, and before the water inside can cool a little the outer water must have cooled considerably. 
So effective was this arrangement that, although some of the thermometers read to $0^{\circ} .007 \mathrm{C}$., yet they would remain perfectly stationary for several minutes, even when at $40^{\circ} \mathrm{C}$. At very high temperatures, such as $80^{\circ}$ or $90^{\circ} \mathrm{C}$., the burner was kept under the vessel $w$ all the time, and supplied the loss of the outer vessel by radiation. The inner vessel would under these circumstances remain at a very constant temperature. The water in the outer vessel never differed by more than a small fraction of a degree from that in the inner one.

To get the $0^{\circ}$ and $100^{\circ}$ points the upper parts of the vessel above the line $a b$ were removed, and ice placed around the bulb of the air thermometer, and left for several hours, until no further lowering took place. For the $100^{\circ}$ point the copper vessel shown in Fig. 3 was used. The portion $y$ of this vessel fitted directly over the bulb of the air thermometer. On boiling water in $x$, the steam passed through the tube to the air thermometer. It is with considerable difficulty that the $100^{\circ}$ point is accurately reached, and, unless care be taken, the bulb will be at a slightly lower temperature. Not only must the bulb be in the steam, but the walls of the cavity must also be at $100^{\circ}$. To accomplish this in this case, a large mass of cloth was heaped over the instrument, and then the water in $x$ vigorously boiled for an hour or so. After fifteen minutes there was generally no perceptible increase of temperature, though an hour was allowed so as to make certain.

The external appearance of the apparatus is seen in Fig. 2. The method of measuring the pressure was in some respects similar to that used in the air thermometer of Jolly, except that the reading was taken by a cathetometer rather than by a scale on a mirror. The capillary stem of the air thermometer leaves the water vessel at $a$, and passes to the tube $b$, which is joined to the three-way cock $c$. The lower part of the cock is joined by a rubber tube to another glass tube at $d$, which can be raised and lowered to any extent, and has also a fine adjustment. These tubes were about $1.5 \mathrm{~cm}$. diameter on the inside, so that there should be little or no error from capillarity. 'Both tubes were exactly of the same size, and for a similar reason.

The three-way cock is used to fill the apparatus with dry air, and also to determine the capacity of the tube above a given mark. In filling the bulb, the air was pumped out about twenty times, and allowed to enter through tubes containing chloride of calcium, sulphuric acid, and caustic soda, so as to absorb the water and the carbonic acid. 


\section{The Cuthetometer}

The cathetometer was one made by Meyerstein, and was selected because of the form of slide used. The support was round, and the telescope was attached to a sleeve which exactly fitted the support. The greatest error of cathetometers arises from the upright support not being exactly true, so that the telescope will not remain in level at all heights. It is true that the level should be constantly adjusted, but it is also true that an instrument can be made where such an adjustment is not necessary. And where time is an element in the accuracy, such an instrument should be used. In the present case it was absolutely necessary to read as quickly as possible, so as not to

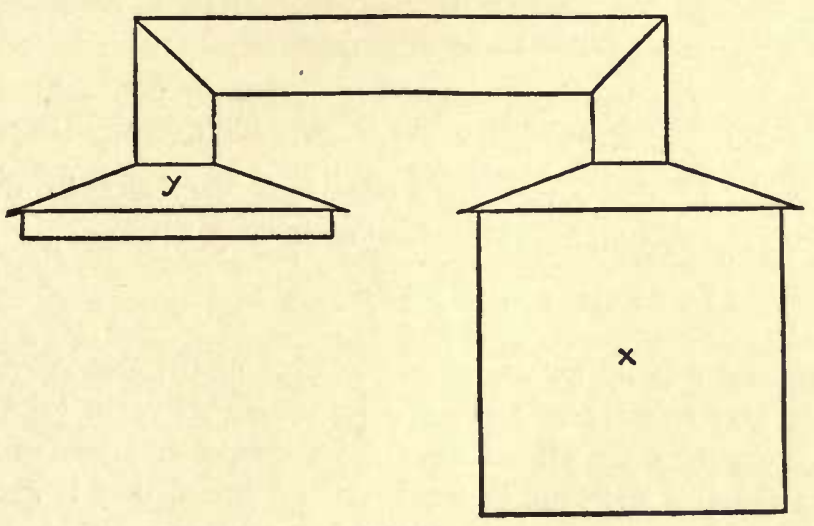

Fig. 3.

leave time for the column to change. In the first place the round column, when made, was turned in a lathe to nearly its final dimensions. The line joining the centres of the sections must then have been.very accurately straight. In the subsequent fitting some slight irregularities must have been introduced, but they could not have been great with good workmanship." The upright column was fixed, and the telescope moved around it by a sleeve on the other sleeve. Where the objects to be measured are not situated at a very wide angle from each other, this is a good arrangement, and has the advantage that any side of the column can be turned toward the object, and so, even if it

3 The change of level along the portion generally used did not amount to more than 1 of a division, or about $.01 \mathrm{~mm}$. at the mercury column, as this is about the smallest quantity which conld be observed on the level. 
were crooked, we could yet turn it into such a position as to nearly eliminate error.

It was used at a distance of about $110 \mathrm{~cm}$. from the object, and no difficulty was found after practice in setting it on the column to $\frac{1}{50} \mathrm{~mm}$. at least. The cross hairs made an angle of $45^{\circ}$ with the horizontal, as this was found to be the most sensitive arrangement.

The scale was carefully calibrated, and the relative errors ${ }^{\circ}$ for the portion used were determined for every centimeter, the portion of the scale between the $0^{\circ}$ and $100^{\circ}$ points of the air thermometer being assumed correct. There is no object in determining the absolute value of the scale, but it should agree reasonably well with that on the barometer; for let $H_{0}, H_{t}$, and $H_{100}$ be the readings of the barometer, and $h_{0}, h_{t}$, and $h_{100}$ the readings of the cathetometer at the temperatures denoted by the subscript. Then approximately

$$
t=\frac{\left(H_{t}+h_{t}\right)-\left(H_{0}+h_{0}\right)}{\left(H_{100}+h_{100}\right)-\left(H_{0}+h_{0}\right)}=\frac{H_{t}-H_{0}+h_{t}-h_{0}}{H_{100}-H_{0}+h_{100}-h_{0}} .
$$

As the height of the barometer varies only very slightly during an experiment, the value of this expression is very nearly

$$
\frac{h_{t}-h_{0}}{h_{100}-h_{0}}
$$

which does not depend on the absolute value of the scale divisions.

But the best manner of testing a cathetometer is to take readings upon an accurate scale placed near the mercury columns to be measured. I tried this with my instrument, and found that it agreed with the scale to within two or three one-hundredths of a millimeter, which was as near as I could read on such an object.

In conclusion, every care was taken to eliminate the errors of this instrument, as the possibility of such errors was constantly present in my mind; and it is supposed that the instrumental errors did not amount to more than one or two one-hundredths of a millimeter on the mercury column. The proof of this will be shown in the results obtained.

\section{The Burometer}

This was of the form designed by Fortin, and was made by James Green of New York. The tube was $2.0 \mathrm{~cm}$. diameter nearly on the outside, and about $1.7 \mathrm{~cm}$. on the inside. The correction for capillarity is therefore almost inappreciable, especially as, when it remains con- 
stant, it is exactly eliminated from the equation. The depression for this diameter is about $.08 \mathrm{~mm}$., but depends upon the height of the meniscus. The height of the meniscus was generally about $1.3 \mathrm{~mm}$.; but according as it was a rising or falling meniscus, it varied from 1.4 to $1.2 \mathrm{~mm}$. These are the practical values of the variation, and would have been greater if the barometer had not been attached to the wall a little loosely, so as to have a slight motion when handled. Also in use the instrument was slightly tapped before reading. The variation of the height of the meniscus from 1.2 to $1.4 \mathrm{~mm}$. would affect the reading only to the extent of .01 to $.02 \mathrm{~mm}$.

The only case where any correction for capillarity is needed is in finding the temperatures of the steam at the $100^{\circ}$ point, and will then affect that temperature only to the extent of about $0^{\circ} \cdot 005$.

The scale of the instrument was very nearly standard at $0^{\circ} \mathrm{C}$., and was on brass.

At the centre of the brass tube which surrounded the barometer, a thermometer was fixed, the bulb being surrounded by brass, and therefore indicating the temperature of the brass tube.

In order that it should also indicate the temperature of the barometer, the whole tube and thermometer were wrapped in cloth until a thickness of about 5 or $6 \mathrm{~cm}$. was laid over the tube, a portion being displaced to read the thermometers. This wrapping of the barometer was very important, and only poor results were obtained before its use; and this is seen from the fact that $1^{\circ}$ on the thermometer indicates a correction of $12 \mathrm{~mm}$. on the barometer, and hence makes a difference of $0^{\circ} .04$ on the air thermometer.

As this is one of the most important sources of error, I have now devised means of almost entirely eliminating it, and making continual reading of the barometer unnecessary. This $I$ intend doing by an artificial atmosphere, consisting of a large vessel of air in ice, and attached to the open tube of the manometer of the air thermometer.

The Thermometers

The standard thermometers used in my experiments are given in the following table on the next page.

The calibration of the first four thermometers has been described. The calibration of the Kew standard was almost perfect, and no correction was thought necessary. The scale divided on the tube was to half-degrees Fahrenheit; but as the $32^{\circ}$ and $212^{\circ}$ points were not correct, it was in practice used as a thermometer with arbitrary divisions. 


\begin{tabular}{|c|c|c|c|c|c|c|c|c|c|c|c|}
\hline 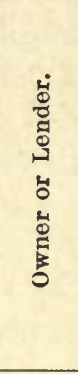 & 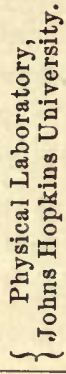 & $\begin{array}{l}= \\
=\end{array}$ & = & $\begin{array}{l}= \\
=\end{array}$ & 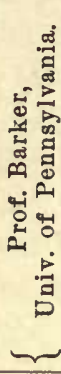 & 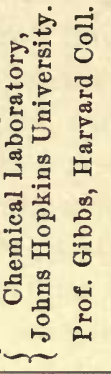 & $=$ & 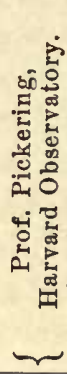 & 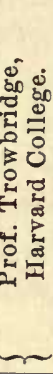 & 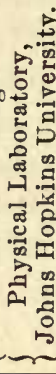 & 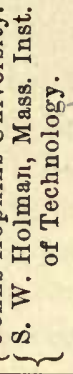 \\
\hline 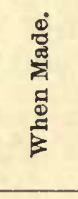 & $\begin{array}{c}5 \\
\substack{0 \\
0 \\
\infty \\
-1}\end{array}$ & $=$ & $=$ & $=$ & 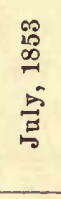 & ఏ & $=$ & a & 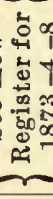 & 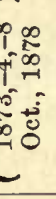 & $=$ \\
\hline $\begin{array}{l}\frac{\dot{0}}{0} \\
\frac{ \pm}{\leftrightarrows} \\
\Sigma\end{array}$ & 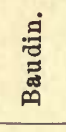 & $=$ & $=$ & $=$ & 递 & 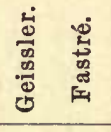 & $=$ & 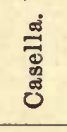 & $=$ & 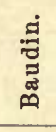 & $=$ \\
\hline 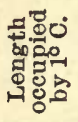 & $\vdots$ & & 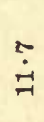 & $\stackrel{\stackrel{\leftrightarrow}{\leftrightarrow}}{\dot{\sigma}}$ & $\stackrel{\dot{\sigma}}{\dot{H}}$ & 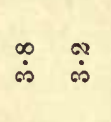 & $\dot{1}$ & $\stackrel{\varphi}{-}$ & $\stackrel{\mathscr{q}}{\dot{x}}$ & $\stackrel{0}{\dot{H}}$ & $\stackrel{9}{\dot{H}}$ \\
\hline 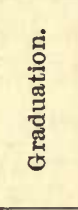 & 离 & $=$ & $=$ & $=$ & $\begin{array}{l}\text { si } \\
\stackrel{20}{0} \\
\dot{20}\end{array}$ & 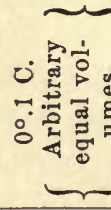 & & $\begin{array}{l}\text { s. } \\
\stackrel{1}{-1}\end{array}$ & $\begin{array}{l}0 \\
? \\
: \\
0\end{array}$ & $\begin{array}{l}0 \\
: \\
:\end{array}$ & $\begin{array}{l}0 \\
-1 \\
8\end{array}$ \\
\hline 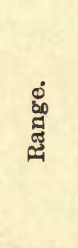 & $\begin{array}{l}\stackrel{\circ}{8} \\
\stackrel{-1}{+} \\
\circ\end{array}$ & $\begin{array}{l}\stackrel{8}{*} \\
\therefore \\
\vdots \\
1\end{array}$ & $\begin{array}{l}\stackrel{\circ}{\circ} \\
\stackrel{8}{\circ} \\
\stackrel{\infty}{1} \\
1\end{array}$ & $\begin{array}{l}\stackrel{\circ}{\circ} \\
\stackrel{\circ}{+} \\
\stackrel{\circ}{\circ} \\
1\end{array}$ & $\begin{array}{l}\stackrel{0}{\circ} \\
\stackrel{-1}{0} \\
\circ \\
\stackrel{0}{\stackrel{1}{1}}\end{array}$ & 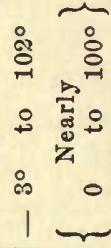 & & 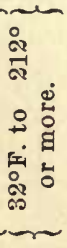 & 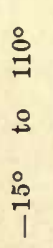 & $\begin{array}{l}0 \\
01 \\
0 \\
+ \\
0 \\
0 \\
\circ \\
\stackrel{1}{1} \\
1\end{array}$ & 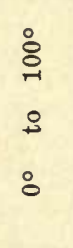 \\
\hline 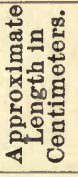 & 28 & ำ & 오 & 옹 & 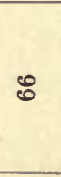 & if & $\stackrel{\infty}{\rightarrow+}$ & س & 우 & 50 & $\overrightarrow{0}$ \\
\hline 莣 & $\stackrel{5}{0}$ & $\stackrel{\bullet}{0}$ & $\frac{18}{60}$ & $\stackrel{8}{0}$ & 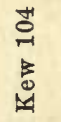 & 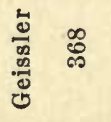 & શ્રે & 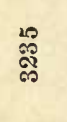 & 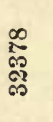 & $\stackrel{0}{\stackrel{0}{0}}$ & 苑 \\
\hline
\end{tabular}

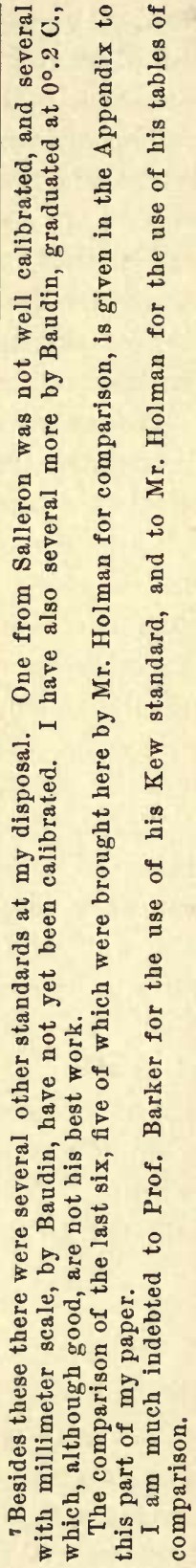


The interval between the $0^{\circ}$ and $100^{\circ}$ points, as Welsh found it, was $180^{\circ} \cdot 12$, using barometer at 30 inches, or $180^{\circ} .05$ as corrected to $760 \mathrm{~mm}$. of mercury. ${ }^{8}$ At the present time it is $179^{\circ} \cdot 68,^{\circ}$ showing a change of 1 part in 486 in twenty-five years. This fact shows that the ordinary method of correcting for change of zero is not correct, and that the coefficient of expansion of glass changes with time. ${ }^{10}$

I have not been able to find any reference to the kind of glass used in this thermometer. But in a report by Mr. Welsh we find a com-

TABLE VI.-COMPARISON BY WELSH, 1852.

\begin{tabular}{|c|c|c|c|c|}
\hline $\begin{array}{l}\text { Mean of } \\
\text { Kew Standards } \\
\text { Nos. } 4 \text { and } 14 .\end{array}$ & $\begin{array}{l}\text { Fastré 231, } \\
\text { Regnault. }\end{array}$ & $\begin{array}{c}\Delta \\
\text { Kew. }\end{array}$ & $\begin{array}{l}\text { Troughton and } \\
\text { simms } \\
\text { (Royal Society). }\end{array}$ & $\underset{\text { Kew. }}{\Delta}$ \\
\hline $32^{\circ} \cdot 00$ & $32^{\circ} \cdot 00$ & 0 & $32^{\circ} \cdot 00$ & 0 \\
\hline $38 \cdot 71$ & $38 \cdot 72$ & $+\cdot 01$ & $38 \cdot 70$ & -.01 \\
\hline $45 \cdot 04$ & $45 \cdot 03$ & -.01 & 45.03 & -.01 \\
\hline $49 \cdot 96$ & $49 \cdot 96$ & .00 & $49 \cdot 96$ & .00 \\
\hline $55 \cdot 34$ & $55 \cdot 37$ & $+\cdot 03$ & $55 \cdot 34$ & .00 \\
\hline 60.07 & $60 \cdot 05$ & -.02 & $60 \cdot 06$ & -.01 \\
\hline $65 \cdot 39$ & $65 \cdot 41$ & $+\cdot 02$ & $65 \cdot 36$ & -.03 \\
\hline $69 \cdot 93$ & 69.95 & $+\cdot 02$ & 69.93 & .00 \\
\hline $74 \cdot 69$ & $74 \cdot 69$ & .00 & $74 \cdot 72$ & $+\cdot 03$ \\
\hline 80.05 & $80 \cdot 06$ & $+\cdot 01$ & $80 \cdot 14$ & +.09 \\
\hline $85 \cdot 30$ & $85 \cdot 33$ & +.03 & $85 \cdot 44$ & $+\cdot 14$ \\
\hline $90 \cdot 50$ & $90 \cdot 51$ & +.01 & $90 \cdot 56$ & +.06 \\
\hline $95 \cdot 26$ & $95 \cdot 24$ & -.02 & 95.40 & $+\cdot 14$ \\
\hline $101 \cdot 77$ & 101.77 & .00 & $101 \cdot 94$ & $+\cdot 15$ \\
\hline $109 \cdot 16$ & $109 \cdot 15$ & -.01 & $109 \cdot 25$ & $+\cdot 08$ \\
\hline $212 \cdot 00$ & $212 \cdot 00$ & .00 & 212.00 & .00 \\
\hline
\end{tabular}

parison, made on March 19, 1852, of some of his thermometers with two other thermometers, - one by Fastré, examined and approved by Regnault, and the other by Troughton and Simms. The thermometer which I used was made a little more than a year after this; and it is

\footnotetext{
${ }^{8}$ Boiling point, Welsh, Ang. 17, 1853, 212 .17 ; barometer 30 in.

Freezing point, " " " $32^{\circ} .05$.

Boiling point, Rowland, June 22, 1878, 2120.46; barometer $760 \mathrm{~mm}$.

Freezing point, " " " $32^{\circ} .78$.
}

The freezing point was taken before the boiling point in either case.

$9179^{\circ} \cdot 70$, as determined again in January, 1879.

10 The increase shown here is 1 in 80 nearly! It is evidently connected with the change of zero; for when glass has been heated to $100^{\circ}$, the mean coefficient of ex. pansion between 0 and $100^{\circ}$ often changes as much as 1 in 50 . Hence it is not strange that it should change 1 in 80 in twenty-five years. I believe this fact has been noticed in the case of standards of length. 
reasonable to suppose that the glass was from the same source as the standards Nos. 4 and 14 there used. We also know that Regnault was consulted as to the methods, and that the apparatus for calibration was obtained under his direction.

I reproduce the table on preceding page with some alterations, the principal one of which is the correction of the Troughton and Simms thermometers, so as to read correctly at $32^{\circ}$ and $212^{\circ}$, the calibration being assumed correct,- but the divisions arbitrary.

It is seen that the Kew standards and the Fastré agree perfectly, but. that the Troughton and Simms standard stands above the Kew thermometers at $100^{\circ} \mathrm{F}$.

The Geissler standard was made by Geissler of Bonn, and its scale was on a piece of milk glass, enclosed in a tube with the stem. The calibration was fair, the greatest error being about $0^{\circ} .015 \mathrm{C}$., at $50^{\circ} \mathrm{C}$.; but no correction for calibration was made, as the instrument was only used as a check for the other thermometers.

\section{Results of CoMPaRISON}

\section{Calculation of Air Thermomater}

This has already been described, and it only remains to discuss the formula and constants, and the accuracy with which the different. quantities must be known.

The well-known formula for the air thermometer is

$$
T=\frac{1}{a} \frac{H-h+\frac{v}{V}\left\{H \frac{1+b t^{\prime}}{1+a t^{\prime}}-h \frac{1+b t}{1+a t}\right\}+b H^{\prime} T^{\prime}}{h-\frac{v}{V}\left\{H^{1+b t^{\prime}}-h \frac{1+b t}{1+a t^{\prime}}-a t\right.} .
$$

Solving with reference to $T$, and placing in a more convenient form, we have

where

$$
T=\frac{1}{\alpha} \frac{H-h^{\prime}+\frac{v}{V} H \frac{1}{1+\gamma t}}{h^{\prime}-\frac{v}{V} H \frac{1}{1+\gamma t}-H \frac{b}{\alpha}} \text { nearly, }
$$

and

For the first bulb,

$$
h^{\prime}=h\left(1+\frac{v}{V} \frac{1}{1+\gamma t^{\prime}}\right),
$$

For the second bulb,

$$
r=a-b=\cdot 00364 \text {. }
$$

$$
\frac{v}{V}=\cdot 005 \%
$$

$$
\frac{v}{V}=\cdot 0058
$$


To discuss the crror of $T$ due to errors in the constants, we must replace $\alpha$ by its experimental value, seeing that it was determined with the same apparatus as that by which $T$ was found. As it docs not change very much, we may write approximately

$$
T=100 \frac{H-h}{H_{100}-h}\left\{1-\frac{v}{V} \frac{1}{1+\gamma t}\left(\frac{H_{100}-H}{h}\right)-\frac{b_{100} H_{100}-b H}{\alpha h}\right\} .
$$

From this formula we can obtain by differentiation the error in each of the quantities, which would make an error of one-tenth of one per cent in $T$. The values are for $T=40^{\circ}$ nearly; $t=20^{\circ}$; $H_{100}-h=270 \mathrm{~mm}$. ; and $h=750 \mathrm{~mm}$. If $x$ is the variable,

$$
\Delta x=\frac{d x}{d T} \Delta T=\frac{d x}{d T^{\prime}} \frac{T}{1000}=.04 \frac{d x}{d T^{\prime}} .
$$

\begin{tabular}{|c|c|c|c|c|c|c|c|}
\hline & $H$ & $H_{100}$ or $h$. & $\frac{v}{V}$ & $\begin{array}{c}\frac{b_{10 n}}{a} \\
\frac{b}{a} \text { constant. }\end{array}$ & $\frac{\frac{b}{a}}{\frac{b_{100}}{a} \text { constant. }}$ & $\begin{array}{l}\frac{b_{100}}{a} \\
\frac{b_{10 m}-b}{a} \text { const'nt. }\end{array}$ & $\begin{array}{c}\frac{b_{100}-b}{a} \\
\frac{b_{100}}{a} \text { constant. }\end{array}$ \\
\hline $\begin{array}{c}\text { Absolute } \\
\text { value, } \\
\Delta x\end{array}$ & $.11 \mathrm{~mm}$ & $\cdot 27 \mathrm{~mm}$. & .005 & .00074 & .00087 & .0047 & .00087 \\
\hline $\begin{array}{l}\text { Relative } \\
\text { value, } \\
\frac{\Delta x}{x}\end{array}$ & $\cdots$ & $\ldots$ & $0 \cdot 9$ & $\cdot 10$ & $\cdot 12$ & .62 & $\cdots$ \\
\hline
\end{tabular}

TABLE VII.-Errors Prondcisg an Error in $T$ of 1 in 1000 at $40^{\circ}$ C.

From this table it would seem that there should be no difficulty in detêrmining the $40^{\circ}$ point on the air thermometer to at least 1 in 2000 ; and experience has justified this result. The principal difficulty is in the determination of $H$, sceing that this includes errors in reading the barometer as well as the cathetometer. For this reason, as mentioned before, I have designed another instrument for future use, in which the barometer is nearly dispensed with by use of an artificial atmosphere of constant pressure.

The value of $\frac{v}{V}$ does not seem to affect the result to any great extent; and if it was omitted altogether, the error would be only about 1 in 1000 , assuming that the temperature $t$ was the same at the determination of the zero point, the $40^{\circ}$ point, and the $100^{\circ}$ point. It seldom varied much.

The coefficient of expansion of the glass influences the result very slightly, especially if we know the difference of the mean coefficients 
between $0^{\circ}$ and $100^{\circ}$, and say $-10^{\circ}$ and $+10^{\circ}$. This difference $I$ at first determined from Regnault's tables, but afterwards made a determination of it, and have applied the correction."

The table given by Regnault is for one specimen of glass only; and I sought to better it by taking the expansion at $100^{\circ}$ from the mean of the five specimens given by Regnault on p. 231 of the first volume of his Relation des Experiences, and reducing the numbers on page $23 \%$ in the same proportion. I thus found the values given in the second column of the following table.

TABLE VIII.-CoEFFICIENT OF Expansion OF THE Glass of the AIR TherMometer, According to the Air Thermometer.

\begin{tabular}{|c|c|c|c|c|c|c|}
\hline \multirow{2}{*}{$\begin{array}{l}\text { Tempera- } \\
\text { ture ac- } \\
\text { cording to } \\
\text { Air Ther- } \\
\text { mometer. }\end{array}$} & \multirow{2}{*}{$\begin{array}{c}\text { Values of } b \\
\text { used for a first } \\
\text { Calculation. }\end{array}$} & \multirow{2}{*}{$\begin{array}{c}\text { b from } \\
\text { Regnault's } \\
\text { Table, } \\
\text { Glass No. } 5 .\end{array}$} & \multicolumn{4}{|c|}{ Experimental Results. } \\
\hline & & & $\begin{array}{l}\text { Apparent } \\
\text { Coefficient of } \\
\text { Expansion of } \\
\text { Mercury. }\end{array}$ & $\begin{array}{l}\text { b, using } \\
\text { Regnault's } \\
\text { Value for } \\
\text { Mercury. }{ }^{12}\end{array}$ & $\begin{array}{c}b, \text { using } \\
\text { Recknagel's } \\
\text { Value for } \\
\text { Mercury.13 }\end{array}$ & $\begin{array}{l}b, \text { using } \\
\text { Wullner's } \\
\text { Value for } \\
\text { Mercury. }\end{array}$ \\
\hline 0 & .0000252 & .0000263 & $\ldots \ldots$ & $\ldots \ldots$ & $\ldots \ldots$ & \\
\hline $20^{\circ}$ & .0000253 & .0000264 & .00015410 & .0000254 & .0000264 & .0000273 \\
\hline $40^{\circ}$ & .0000256 & .0000267 & .00015395 & .0000258 & .0000266 & .0000276 \\
\hline $60^{\circ}$ & .0000259 & .0000270 & .00015391 & .0000261 & .0000267 & .0000278 \\
\hline $80^{\circ}$ & - 0000262 & .0000273 & $\ldots \ldots$ & ..... & .... & . . . \\
\hline $100^{\circ}$ & .0000264 & $\cdot 0000276$ & .00015381 & .0000277 & .0000277 & .0000287 \\
\hline
\end{tabular}

The second column contains the values which I have used, and one of the last three columns contains my experimental results, the last being probably the best. The errors by the use of the second column compared with the last are as follows:-

$\frac{1}{10000}$ from using $b_{100}-b_{40}=\cdot 0000008$ instead of $\cdot 0000011$;

$\frac{1}{700}$ from using $b_{100}=.0000264$ instead of $\cdot 000028 \%$; or, $\frac{1}{4000}$ for both together.

As the error is so small, I have not thought it worth while to entirely recalculate the tables, but have calculated a table of corrections (see opposite page), and have so corrected them.

11 This was determined by means of a large weight thermometer in which the mercury had been carefully boiled. The glass was from the same tube as that of the air thermometer, and they were cut from it within a few inches of each other.

12 Relations des Expériences, i, 328.

${ }^{13}$ Pogg. Ann., cxiii, 135.

14 Experimental Physik, Wüllner, i, 67. 


$$
\begin{aligned}
& T^{\prime}=T^{\prime}\left\{1+373\left(b_{100}^{\prime}-b_{100}\right)-(273+T)\left(b^{\prime}-b\right)\right\}, \\
& T=T^{\prime \prime}\left\{1-.0008 .58+\left(273+T^{\prime}\right)\left(b-b^{\prime}\right)\right\},
\end{aligned}
$$

$T=.99975 T^{\prime}$ approximately between 0 and $40^{\circ}$. The last is true within less than $\frac{1}{1000}$ of a degree.

The two bulbs of the air thermometer used were from the same piece of glass tubing, and consequently had nearly, if not quite, the same coefficient of expansion.

In the reduction of the barometer and other mercurial columns to zero, the coefficient $\cdot 000162$ was used, seeing that all the scales were of brass.

In the tables the readings of the thermometers are reduced to volumes of the tube from the tables of calibration, and they are corrected for the pressure of water, which increased their reading, except at $0^{\circ}$, by about $0^{\circ} \cdot 01 \mathrm{C}$.

\begin{tabular}{|c|c|c|}
\hline$T^{\nu}$ & $T$ & \\
\hline $\begin{array}{l}\text { Calculated } \\
\text { Temperature. }\end{array}$ & $\begin{array}{c}\text { Corrected } \\
\text { Temperature. }\end{array}$ & Correction. \\
\hline $\begin{array}{r}0 \\
10 \\
20 \\
30 \\
40 \\
50 \\
60 \\
80 \\
100\end{array}$ & $\begin{array}{c}0 \\
0 \\
9 \cdot 9971 \\
19 \cdot 9946 \\
29 \cdot 9924 \\
39 \cdot 9907 \\
49 \cdot 9894 \\
59 \cdot 9865 \\
79 \cdot 9880 \\
100 \cdot\end{array}$ & $\begin{array}{c}0 \\
-.0029 \\
-.0054 \\
-.0076 \\
-.0093 \\
-.0106 \\
-.0135 \\
-.0120 \\
0\end{array}$ \\
\hline
\end{tabular}

TABLE IX.-TABLE OF CORRECTIONS.

The order of the readings was as follows in each observation:-1st, barometer; $2 \mathrm{~d}$, cathetometer; $3 \mathrm{~d}$, thermometers forward and backward; 4th, cathetometer; 5th, barometer, \&c.,-repeating the same once or twice at each temperature. In the later observations, two series like the above were taken, and the water stirred between them.

The following results were obtained at various times for the value of $\alpha$ with the first bulb:- 
obtained by using the coefficient of expansion of glass .000026t at $100^{\circ}$, or $\alpha=\cdot 0036698$, using the coefficient $\cdot 000028 \%$.

The thermometers Nos. $6163,6165,6166$, were always taken out of the bath when the temperature of $40^{\circ}$ was reached, except on November 14, when they remained in throughout the whole experiment.

The thermometer readings are reduced to volumes by the tables of calibration.

TABLE X.-1st Series, Nov. 14, $18 \% 7$.

\begin{tabular}{|c|c|c|c|c|c|c|}
\hline $\begin{array}{l}\text { Relative } \\
\text { Weight. }\end{array}$ & Thermometer. & $\underset{6163 .}{V}$ & $\underset{6166 .}{V}$ & $\begin{array}{c}V \\
6167 .\end{array}$ & $\begin{array}{c}\text { Temperature } \\
\text { by } 616 \%\end{array}$ & $\Delta$ \\
\hline 4 & 0 & $115 \cdot 33$ & $21 \cdot 25$ & $6 \cdot 147$ & 0 & 0 \\
\hline 4 & $17^{\circ} \cdot 1425$ & $422 \cdot 84$ & $255 \cdot 80$ & $15 \cdot 685$ & $17^{\circ} \cdot 661$ & $\cdot 236$ \\
\hline 4 & $23^{\circ} \cdot 793$ & $534 \cdot 71$ & $341 \cdot 05$ & $19 \cdot 157$ & $24^{\circ} .089$ & $\cdot 296$ \\
\hline 5 & $30^{\circ} \cdot 582$ & $653 \cdot 49$ & $431 \cdot 71$ & $22 \cdot 833$ & $30^{\circ} \cdot 896$ & $\cdot 314$ \\
\hline 2 & $38^{\circ} \cdot 569$ & $793 \cdot 18$ & $\ldots \ldots$ & $\approx 7 \cdot 1 \tau 5$ & $38^{\circ} \cdot 935$ & $\cdot 366$ \\
\hline 2 & $51^{\circ} \cdot 040$ & $\ldots \ldots$ & & $33 \cdot 864$ & $51^{\circ} \cdot 320$ & $\cdot 280$ \\
\hline 4 & $59^{\circ} \cdot 137$ & ..... & $\ldots \ldots$ & $38 \cdot 256$ & $59^{\circ} \cdot 452$ & $\cdot 315$ \\
\hline
\end{tabular}

The first four series, Tables $\mathrm{X}$ to XIII, were made with one bulb to the air thermometer. A new bulb was now made, whose capacity was $192.0 \mathrm{c.cm}$., that of the old being $201.98 \mathrm{c.cm}$. The value of $\frac{v}{V}$ for the new bulb was $\cdot 0058$. The values of $h^{\prime}$ and $\%$ were obtained as follows:-

\begin{tabular}{rcc} 
& $a$ & $h^{\prime}$ \\
June 8th & $.00366 \% 90$ & \%53.8\%6 \\
June 22d & $.003669 \%$ \% & \%53.805 \\
June 25th & $.00366 \% \% 9$ & \%53.83\% \\
\cline { 2 - 2 } Mean & .0036685 & $-753 \cdot 84$
\end{tabular}

This value of $\alpha$ is calculated with the old coefficient for glass. The new would have given $\cdot 003671 \%$.

It now remains to determine from these experiments the most probable values of the constants in the formula, comparing the air with the mercurial thermometer. The formula is, as we have found,

$$
t=T-a t\left(t^{\prime}-t\right)(b-t) \text {; }
$$

but $I$ have generally used it in the following form:

$$
\begin{aligned}
& t=C V-t_{0}-m t(100-t)(1-n(100+t)), \\
& t=C^{\prime} V-t_{0}^{\prime}-m t(40-t)(1-n(40+t)) .
\end{aligned}
$$




\begin{tabular}{|c|c|c|c|c|c|c|c|c|c|c|c|c|c|c|c|c|}
\hline 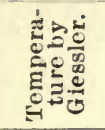 & 0 & $\begin{array}{l}5 \\
\vdots \\
i\end{array}$ & $\begin{array}{l}\infty \\
: 0 \\
\vdots \\
0 \\
0\end{array}$ & 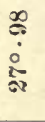 & 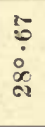 & $\begin{array}{l}0 \\
\stackrel{0}{0} \\
\dot{0} \\
\dot{7}\end{array}$ & $\begin{array}{l}\ddot{8} \\
\dot{0} \\
\dot{\sigma}\end{array}$ & $\begin{array}{l}\mathscr{0} \\
\stackrel{0}{0} \\
\stackrel{\infty}{*}\end{array}$ & $\begin{array}{l}8 \\
\dot{0} \\
\dot{0} \\
\dot{15}\end{array}$ & $\begin{array}{l}\overrightarrow{0} \\
\ddot{0} \\
\dot{0} \\
\dot{H}\end{array}$ & 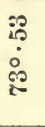 & $\begin{array}{l}\vec{F} \\
\dot{0} \\
\dot{+} \\
\infty\end{array}$ & $\begin{array}{l}\overrightarrow{7} \\
\dot{0} \\
\dot{0} \\
\infty\end{array}$ & $\vdots$ & $\begin{array}{l}\text { ஜ } \\
\stackrel{\circ}{\circ} \\
\stackrel{8}{\circ}\end{array}$ & $\vdots$ \\
\hline 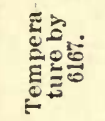 & & $\begin{array}{l}0 \\
0 \\
0 \\
0 \\
0\end{array}$ & $\begin{array}{l}00 \\
0 \\
0 \\
0 \\
0\end{array}$ & $\begin{array}{l}\stackrel{0}{\infty} \\
\infty \\
\stackrel{0}{0} \\
\dot{0} \\
\stackrel{\alpha}{\infty}\end{array}$ & $\begin{array}{l}0 \\
0 \\
0 \\
0 \\
0 \\
0 \\
0\end{array}$ & 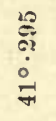 & $\begin{array}{l}\mathscr{g} \\
\dot{g} \\
\dot{0} \\
\dot{\sigma}\end{array}$ & \begin{tabular}{l}
$\overrightarrow{7}$ \\
\multirow{2}{\infty}{} \\
0 \\
0 \\
0 \\
+1
\end{tabular} & $\frac{20}{50}$ & $\begin{array}{l}\stackrel{P}{+} \\
\stackrel{6}{+} \\
\dot{0}\end{array}$ & 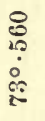 & $\begin{array}{l}\stackrel{2}{*} \\
\stackrel{+}{0} \\
\dot{+}\end{array}$ & 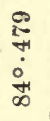 & 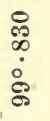 & $\begin{array}{l}: 8 \\
\stackrel{20}{\circ} \\
\dot{8} \\
\dot{8}\end{array}$ & $\vdots$ \\
\hline $\begin{array}{l}\dot{5} \\
\frac{0}{0} \\
\frac{0}{0} \\
0\end{array}$ & $\begin{array}{l}8 \\
\dot{0} \\
+\end{array}$ & $\stackrel{\bar{a}}{\dddot{\infty}}$ & $\begin{array}{l}\infty \\
\dot{\sigma} \\
\dot{\sigma}\end{array}$ & $\begin{array}{l}\overrightarrow{0} \\
\ddot{\infty} \\
\vec{x}\end{array}$ & $\begin{array}{l}\overrightarrow{0} \\
\dot{\vec{\theta}}\end{array}$ & 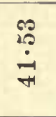 & 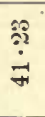 & $\begin{array}{l}\overrightarrow{0} \\
\dot{q} \\
\dot{q}\end{array}$ & 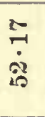 & $\begin{array}{l}\stackrel{5}{4} \\
\dot{+1}\end{array}$ & $\begin{array}{l}\overrightarrow{0} \\
\dot{0}\end{array}$ & $\begin{array}{l}\stackrel{\infty}{\not} \\
\dot{+} \\
\infty\end{array}$ & $\begin{array}{l}\stackrel{\text { † }}{+} \\
\dot{\infty}\end{array}$ & $\vdots$ & $\begin{array}{l}\infty \\
\stackrel{2}{0} \\
\dot{\delta}\end{array}$ & $\begin{array}{l}\mathscr{g} \\
\dot{\delta}\end{array}$ \\
\hline$-1 \frac{5}{6}$ & $\begin{array}{l}\infty \\
\stackrel{2}{*} \\
\stackrel{0}{0}\end{array}$ & $\begin{array}{l}0 \\
0 \\
0 \\
0 \\
0 \\
0\end{array}$ & $\begin{array}{l}\infty \\
\stackrel{8}{0} \\
\dot{ \pm}\end{array}$ & 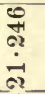 & $\begin{array}{l}\infty \\
\text { al } \\
\vdots \\
\dot{1} \\
\sigma 2\end{array}$ & 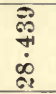 & 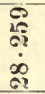 & $\begin{array}{l}\infty \\
\ddot{2} \\
\dot{\alpha} \\
\dot{c}\end{array}$ & $\begin{array}{l}\stackrel{\infty}{0} \\
\stackrel{0}{0} \\
\dot{+}\end{array}$ & $\begin{array}{l}\stackrel{m}{+} \\
\dot{0} \\
\dot{\theta}\end{array}$ & $\begin{array}{l}8 \\
0 \\
\infty \\
\dot{s} \\
\text { in }\end{array}$ & $\begin{array}{l}20 \\
\dot{20}\end{array}$ & 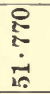 & $\begin{array}{l}\not \\
\dot{8} \\
\dot{8}\end{array}$ & $\vdots$ & $\begin{array}{l}\stackrel{2}{20} \\
\stackrel{10}{0}\end{array}$ \\
\hline$>\frac{80}{0}$ & $\begin{array}{l}\overline{8} \\
\dot{\vec{\theta}} \\
2\end{array}$ & 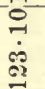 & $\begin{array}{l}0 \\
\dot{0} \\
\dot{0} \\
5\end{array}$ & $\begin{array}{l}8 \\
8 \\
8 \\
8\end{array}$ & 送 & $\vdots$ & $\vdots$ & & $\vdots$ & & $\vdots$ & & $\vdots$ & & & \\
\hline$>\frac{g}{6}$ & $\begin{array}{l}\vec{D} \\
\dot{+} \\
\Xi\end{array}$ & \begin{tabular}{l}
$\overrightarrow{0}$ \\
$\dot{0}$ \\
$\dot{0}$ \\
\hdashline
\end{tabular} & $\begin{array}{l}\hat{i} \\
\dot{8} \\
\dot{8}\end{array}$ & $\begin{array}{l}0 \\
\infty \\
\dot{\alpha} \\
\dot{0}\end{array}$ & $\frac{9}{30}$ & $\begin{array}{l}\vec{b} \\
\dot{d} \\
\dot{3}\end{array}$ & $\begin{array}{l}-5 \\
\infty \\
\infty \\
\infty \\
\infty \\
\infty\end{array}$ & $\begin{array}{l}\vdots \\
\vdots\end{array}$ & $\begin{array}{l}\vdots \\
\vdots \\
\vdots\end{array}$ & & $\vdots$ & & $\begin{array}{l}\vdots \\
\vdots \\
\vdots\end{array}$ & & & \\
\hline 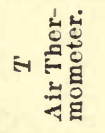 & 0 & 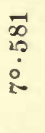 & $\begin{array}{l}\overrightarrow{1} \\
\text { ò } \\
\dot{0} \\
\vdots \\
0\end{array}$ & $\begin{array}{l}20 \\
0 \\
0 \\
0 \\
6\end{array}$ & \begin{tabular}{l}
0 \\
\multirow{7}{*}{} \\
$\dot{0}$ \\
0 \\
0 \\
0
\end{tabular} & $\begin{array}{l}0 \\
0 \\
0 \\
\vdots \\
7\end{array}$ & 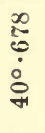 & $\begin{array}{l}\vec{J} \\
0 \\
0 \\
0 \\
\infty \\
+1\end{array}$ & $\begin{array}{l}\stackrel{8}{0} \\
\stackrel{0}{0} \\
i \\
20\end{array}$ & $\begin{array}{l}\mathscr{8} \\
\stackrel{\infty}{0} \\
\dot{0} \\
0\end{array}$ & 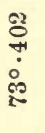 & $\begin{array}{l}\ddot{*} \\
\text { of } \\
\dot{0} \\
\dot{0}\end{array}$ & 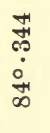 & $\begin{array}{l}\vec{\infty} \\
0 \\
0 \\
\dot{8}\end{array}$ & 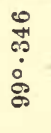 & $\begin{array}{l}8 \\
8 \\
\vdots \\
8\end{array}$ \\
\hline$\cong 0$ & $\vdots$ & $\begin{array}{l}\vec{c} \\
\dot{c} \\
\dot{i s}\end{array}$ & $\underset{\dot{P}}{\stackrel{3}{*}}$ & $\begin{array}{l}\infty \\
\stackrel{0}{0} \\
\dot{1}\end{array}$ & $\begin{array}{l}\stackrel{8}{0} \\
\dot{\text { is }}\end{array}$ & $\stackrel{5}{\dot{s}}$ & $\begin{array}{l}\ddot{8} \\
\dot{\text { is }}\end{array}$ & $\begin{array}{l}\vec{H} \\
\dot{0}\end{array}$ & $\overrightarrow{a ?}$ & $\stackrel{8}{\circ}$ & $\overrightarrow{7}$ & $\stackrel{10}{\stackrel{0}{0}}$ & $\begin{array}{l}\stackrel{2}{\odot} \\
\dot{0}\end{array}$ & $\vec{m}$ & 足 & $\vdots$ \\
\hline $\begin{array}{l}=\underset{ \pm}{ \pm} \\
=2\end{array}$ & $\vdots$ & $\begin{array}{l}\stackrel{8}{0} \\
\dot{H}\end{array}$ & 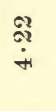 & 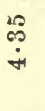 & $\begin{array}{l}\mathscr{\%} \\
\stackrel{+}{+}\end{array}$ & $\underset{\dot{H}}{\stackrel{H}{L}}$ & 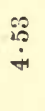 & $\stackrel{\infty}{\stackrel{\infty}{\circ}}$ & $\stackrel{?}{\stackrel{+}{*}}$ & $\begin{array}{l}\mathscr{x} \\
\dot{+1}\end{array}$ & 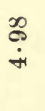 & $\begin{array}{l}\stackrel{62}{2} \\
\stackrel{1}{20}\end{array}$ & $\stackrel{2}{\stackrel{2}{0}}$ & $\begin{array}{l}\stackrel{0}{\circ} \\
\stackrel{0}{0} \\
\text { is }\end{array}$ & $\begin{array}{l}82 \\
\ddot{20} \\
\dot{20}\end{array}$ & $\vdots$ \\
\hline$\stackrel{i}{\beth}$ & $\vdots$ & $\begin{array}{l}\stackrel{9}{*} \\
\dot{0} \\
\dot{\sigma}\end{array}$ & $\begin{array}{l}\mathscr{\mathscr { D }} \\
\stackrel{\infty}{\infty} \\
\dot{\mathscr{D}}\end{array}$ & $\begin{array}{l}\hat{\theta} \\
\dot{0} \\
\dot{x}\end{array}$ & $\stackrel{\mathbb{2}}{\stackrel{2}{2}}$ & $\underset{-1}{\mathscr{8}}$ & $\begin{array}{l}\stackrel{2}{2} \\
\stackrel{2}{0} \\
\stackrel{0}{0}\end{array}$ & $\begin{array}{l}\stackrel{\vartheta}{0} \\
\dot{\alpha} \\
\stackrel{2}{2}\end{array}$ & 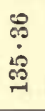 & $\begin{array}{l}\vec{D} \\
\dot{0} \\
\dot{0} \\
-1\end{array}$ & $\begin{array}{l}\stackrel{+}{0} \\
\dot{0} \\
\stackrel{्}{-}\end{array}$ & 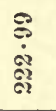 & 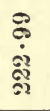 & 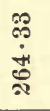 & $\begin{array}{l}\ddot{0} \\
\dot{\theta} \\
\ddot{0}\end{array}$ & $\vdots$ \\
\hline$\stackrel{\beth}{\beth}$ & $\vdots$ & $\begin{array}{l}\overrightarrow{7} \\
\dot{0} \\
\dot{0}\end{array}$ & 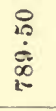 & 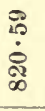 & 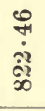 & $\begin{array}{l}\vec{H} \\
\dot{0} \\
\dot{0} \\
\infty \\
\infty \\
\infty\end{array}$ & 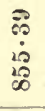 & $\begin{array}{l}\stackrel{0}{0} \\
\dot{0} \\
\infty\end{array}$ & 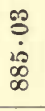 & $\begin{array}{l}\overrightarrow{0} \\
\dot{\infty} \\
\vec{\sigma}\end{array}$ & $\begin{array}{l}\overrightarrow{0} \\
\dot{9} \\
\stackrel{9}{\sigma}\end{array}$ & $\begin{array}{l}0 \\
\dot{0} \\
\dot{i} \\
\stackrel{2}{6}\end{array}$ & \begin{tabular}{l}
$:$ \\
$\stackrel{\leftrightarrow}{\circ}$ \\
\multirow{5}{*}{}
\end{tabular} & $\begin{array}{l}8 \\
\dot{+} \\
\stackrel{5}{0}\end{array}$ & $\begin{array}{l}\mathscr{0} \\
\infty \\
\dot{\sigma 2} \\
\stackrel{0}{0}\end{array}$ & $\vdots$ \\
\hline 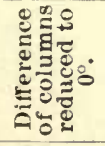 & $\begin{array}{c}\infty \\
62 \\
0 \\
\dot{\alpha} \\
1 \\
1\end{array}$ & $\begin{array}{l}\overrightarrow{1} \\
\dot{0} \\
1\end{array}$ & $\begin{array}{l}\dot{2} \\
\dot{0} \\
+\end{array}$ & 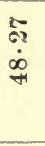 & $\begin{array}{l}10 \\
\ddot{0} \\
\dot{0}\end{array}$ & $\stackrel{\overrightarrow{+}}{\stackrel{5}{+}}$ & $\begin{array}{l}\stackrel{4}{0} \\
\dot{\infty} \\
\infty\end{array}$ & $\begin{array}{l}\infty \\
\stackrel{0}{+} \\
\dot{0}\end{array}$ & 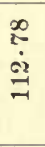 & $\begin{array}{l}\infty \\
\stackrel{0}{0} \\
\dot{\Phi} \\
\stackrel{-}{-1}\end{array}$ & 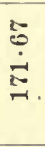 & 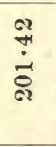 & 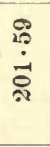 & 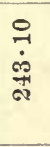 & 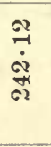 & $\vdots \vdots$ \\
\hline 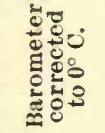 & $\vdots$ & 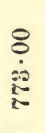 & $\begin{array}{l}\mathfrak{R} \\
\dot{R} \\
i\end{array}$ & 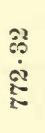 & $\begin{array}{l}\overrightarrow{Q ?} \\
\dot{q 2} \\
\vec{q}\end{array}$ & $\begin{array}{l}\hat{\alpha} \\
\stackrel{\alpha}{\alpha} \\
\dot{\alpha}\end{array}$ & 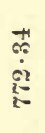 & $\begin{array}{l}\overrightarrow{0} \\
\dot{\alpha} \\
\vec{i}\end{array}$ & $\begin{array}{l}20 \\
\stackrel{20}{2} \\
\stackrel{2}{2} \\
\stackrel{2}{2}\end{array}$ & 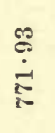 & $\begin{array}{l}\stackrel{+}{0} \\
\stackrel{\vec{t}}{*}\end{array}$ & 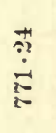 & 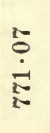 & $\begin{array}{l}\stackrel{8}{0} \\
\dot{0} \\
\stackrel{0}{*}\end{array}$ & $\underset{0}{\square}$ & $\vdots$ \\
\hline 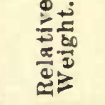 & Q2 & 00 & ar & Q) & 02 & -1 & $\therefore$ & $\Rightarrow$ & 62 & + & $\Leftrightarrow$ & $=0$ & $\propto$ & 0 & 0 & : \\
\hline
\end{tabular}




\begin{tabular}{|c|c|c|c|c|c|c|c|c|c|}
\hline 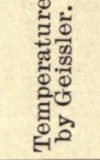 & 0 & & & $\begin{array}{l}\infty \\
20 \\
\dot{0} \\
\stackrel{-}{-1}\end{array}$ & $\begin{array}{l}\infty \\
\infty \\
\dot{0} \\
\infty \\
\infty \\
-1\end{array}$ & 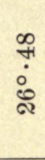 & $\begin{array}{l}\overrightarrow{0} \\
\ddot{0} \\
\stackrel{\circ}{\circ}\end{array}$ & $\begin{array}{l}\mathscr{0} \\
\dot{0} \\
\infty \\
\infty\end{array}$ & $\begin{array}{l}\text { की } \\
\stackrel{0}{0} \\
\infty \\
\infty\end{array}$ \\
\hline 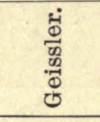 & $\begin{array}{l}\stackrel{0}{0} \\
\dot{0} \\
+\end{array}$ & $\vdots \vdots$ & $\vdots$ & 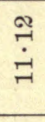 & $\begin{array}{l}0 \\
\stackrel{0}{0} \\
\dot{\sigma}\end{array}$ & $\begin{array}{l}\vec{\sigma} \\
\dot{\theta}\end{array}$ & 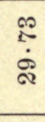 & @̊ & $\begin{array}{l}10 \\
\stackrel{2}{0} \\
\infty \\
0\end{array}$ \\
\hline$\wedge \stackrel{\Leftrightarrow}{0}$ & $\frac{\not{H}}{\dot{\sigma}}$ & $\begin{array}{l}\text { ळூ } \\
\dot{\delta}\end{array}$ & $\begin{array}{l}\stackrel{5}{\infty} \\
\stackrel{8}{\delta}\end{array}$ & 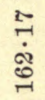 & $\underset{\text { ¿ }}{\stackrel{\infty}{\leftrightarrows}}$ & 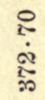 & $\begin{array}{l}\infty \\
\stackrel{0}{+} \\
\stackrel{\leftrightarrow}{+}\end{array}$ & $\vdots \vdots$ & $\vdots$ \\
\hline$\Delta \frac{0}{0}$ & $\begin{array}{l}20 \\
20 \\
\stackrel{20}{7}\end{array}$ & $\begin{array}{l}\text { के } \\
\text { iो } \\
\text { مे }\end{array}$ & $\begin{array}{l}\stackrel{0}{0} \\
\dot{\infty} \\
\vec{\sigma}\end{array}$ & $\begin{array}{l}\stackrel{H}{\circ} \\
\dot{\leftrightarrow} \\
\stackrel{\circ}{\circ}\end{array}$ & $\begin{array}{l}\stackrel{H}{\leftarrow} \\
\dot{\infty} \\
\stackrel{+}{+}\end{array}$ & 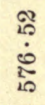 & $\begin{array}{l}20 \\
\dot{0} \\
\dot{0} \\
0\end{array}$ & \begin{tabular}{l}
$\stackrel{\infty}{0}$ \\
$\stackrel{+}{+}$ \\
\multirow{i}{*}{}
\end{tabular} & $\begin{array}{l}\infty \\
\infty \\
i \\
\infty \\
i\end{array}$ \\
\hline 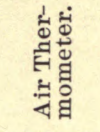 & 0 & $\begin{array}{l}H \\
\overrightarrow{7} \\
\text { in }\end{array}$ & $\begin{array}{l}10 \\
\infty \\
0 \\
0 \\
\text { in }\end{array}$ & $\begin{array}{l}\overrightarrow{20} \\
\stackrel{2}{+} \\
\dot{0}\end{array}$ & \begin{tabular}{l}
$\infty$ \\
\multirow{0}{0}{} \\
0 \\
0 \\
$\infty$ \\
-1
\end{tabular} & 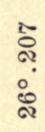 & $\begin{array}{l}20 \\
0 \\
\dot{0} \\
\dot{0}\end{array}$ & $\begin{array}{l}\stackrel{0}{0} \\
\vdots \\
\infty \\
\infty\end{array}$ & $\begin{array}{l}\infty \\
\infty \\
\infty \\
\stackrel{0}{0} \\
\infty \\
\infty\end{array}$ \\
\hline Dit & $\vdots$ & is & $\vec{r}$ & $\overrightarrow{c 0}$ & is & $\begin{array}{l}10 \\
\dot{0}\end{array}$ & $\stackrel{2}{2}$ & $\begin{array}{l}\text { के } \\
\text { is }\end{array}$ & $\begin{array}{l}\stackrel{8}{\circ} \\
\text { is }\end{array}$ \\
\hline $\begin{array}{l}\underset{ \pm}{\mid \vec{\lambda}} \\
2 \|\end{array}$ & $\begin{array}{l}\stackrel{H}{0} \\
\dot{\infty}\end{array}$ & $\begin{array}{l}\stackrel{\leftrightarrow}{\circ} \\
\dot{\infty}\end{array}$ & $\begin{array}{l}8 \\
\dot{\sigma}\end{array}$ & $\stackrel{\ddot{H}}{\dot{H}}$ & $\stackrel{\infty}{\stackrel{\infty}{+}}$ & $\begin{array}{l}\stackrel{P}{2} \\
\stackrel{+}{+}\end{array}$ & ๑̊ & $\stackrel{\Re}{\stackrel{f}{+}}$ & 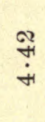 \\
\hline in & $\vdots$ & $\begin{array}{l}\dot{\sigma} \\
\dot{\sigma}\end{array}$ & $\begin{array}{l}\text { मे } \\
\stackrel{-1}{二}\end{array}$ & 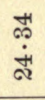 & $\begin{array}{l}\stackrel{\vartheta}{+} \\
\dot{f} \\
\dot{H}\end{array}$ & $\begin{array}{l}\infty \\
\dot{0} \\
\dot{0}\end{array}$ & $\begin{array}{l}\text { is } \\
\text { + } \\
\text { it }\end{array}$ & $\begin{array}{l}0_{0} \\
\dot{\delta} \\
\end{array}$ & $\stackrel{\circ}{\stackrel{\leftrightarrow}{+}}$ \\
\hline \pm & $\begin{array}{l}\text { की } \\
\text { की } \\
\dot{0} \\
\text { सी }\end{array}$ & $\begin{array}{l}\stackrel{+}{+} \\
\dot{0} \\
\stackrel{0}{0}\end{array}$ & $\begin{array}{l}\stackrel{2}{+} \\
\stackrel{\leftrightarrow}{*} \\
\stackrel{0}{*}\end{array}$ & \begin{tabular}{l}
$\infty$ \\
$\infty$ \\
\multirow{+}{*}{}
\end{tabular} & $\begin{array}{l}\vec{\phi} \\
\dot{\delta} \\
\dot{0}\end{array}$ & $\underset{\infty}{\stackrel{\infty}{\infty}}$ & $\begin{array}{l}\text { o } \\
\text { iी } \\
\infty\end{array}$ & $\underset{\infty}{\stackrel{\vec{b}}{\dot{10}}}$ & $\begin{array}{l}\stackrel{\infty}{\circ} \\
\dot{\$} \\
\stackrel{+}{\infty}\end{array}$ \\
\hline 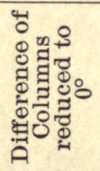 & $\begin{array}{l}\text { H } \\
\vdots \\
\dot{0} \\
1\end{array}$ & $\begin{array}{l}8 \\
\dot{0} \\
\dot{0} \\
1\end{array}$ & $\begin{array}{l}\ddot{0} \\
\dot{0} \\
1\end{array}$ & $\begin{array}{l}\mathscr{8} \\
\dot{-} \\
+\end{array}$ & $\begin{array}{l}\text { के } \\
\dot{+} \\
\text { के }\end{array}$ & $\frac{5}{4}$ & $\begin{array}{l}\stackrel{\leftrightarrow}{+} \\
\dot{8}\end{array}$ & $\begin{array}{l}\text { के } \\
\stackrel{\infty}{\infty} \\
\stackrel{\infty}{\infty}\end{array}$ & $\stackrel{\infty}{\stackrel{\infty}{\infty}}$ \\
\hline 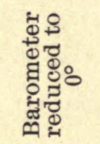 & ڤ̊ & $\begin{array}{l}\stackrel{8}{0} \\
\dot{0} \\
0\end{array}$ & $\begin{array}{l}8 \\
\dot{8} \\
\Leftrightarrow\end{array}$ & $\begin{array}{l}\text { to } \\
\dot{8} \\
0 \\
0\end{array}$ & \begin{tabular}{l}
$\stackrel{0}{\infty}$ \\
$\dot{0}$ \\
$\dot{8}$ \\
\multirow{2}{*}{}
\end{tabular} & $\begin{array}{l}\infty \\
\infty \\
\dot{8} \\
\stackrel{0}{0}\end{array}$ & $\begin{array}{l}\mathscr{D} \\
\dot{0} \\
\dot{0}\end{array}$ & $\begin{array}{l}\infty \\
\infty \\
\dot{8} \\
0 \\
0\end{array}$ & $\begin{array}{l}\stackrel{0}{\infty} \\
\dot{\leftrightarrow} \\
\dot{0}\end{array}$ \\
\hline 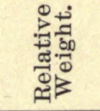 & $r$ & -1 & $r$ & -1 & -1 & - & $\tau$ & $r$ & - \\
\hline
\end{tabular}




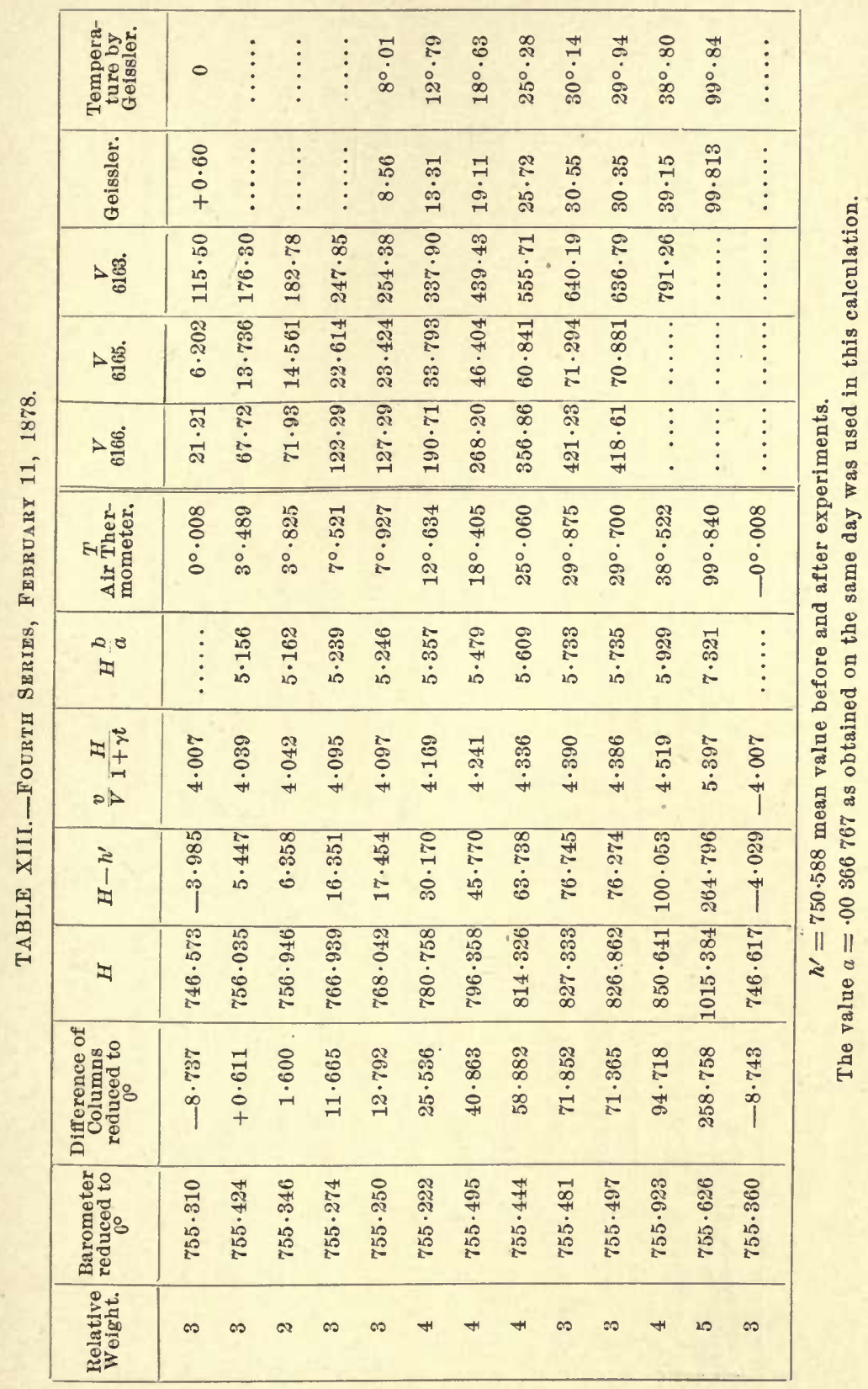




\begin{tabular}{|c|c|c|c|c|c|c|c|c|c|c|c|c|}
\hline$\triangleleft \frac{D}{2}$ & $\bar{c}$ & & : & $\stackrel{23}{5}$ & $\begin{array}{l}\tilde{a} \\
\dot{\sigma} \\
\dot{+}\end{array}$ & $\stackrel{\square}{\check{+}}$ & $\begin{array}{l}\ddot{2} \\
\dot{+}\end{array}$ & $\begin{array}{l}\hat{6} \text { है } \\
\dot{+}\end{array}$ & $\begin{array}{l}\text { कै } \\
\stackrel{9}{+} \\
+\end{array}$ & 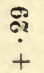 & $\stackrel{0}{+}$ & $\vdots$ \\
\hline$\triangleleft \stackrel{\overline{0}}{0}$ & $\bar{j}$ & $\begin{array}{l}-1 \\
\dot{+}\end{array}$ & $\overline{0}$ & 8 & 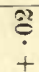 & $\stackrel{5}{0}$ & $\begin{array}{l}t \\
\dot{+} \\
+\end{array}$ & $\begin{array}{l}+ \\
\dot{+} \\
+\end{array}$ & $\begin{array}{l}02 \\
\dot{+} \\
+\end{array}$ & $\begin{array}{l}10 \\
\dot{0} \\
+\end{array}$ & $\overline{0}$ & $\vdots$ \\
\hline 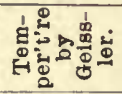 & 0 & & & 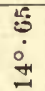 & $\begin{array}{l}\text { : } \\
0 \\
0 \\
0\end{array}$ & : & $\ddot{\infty}$ & $\frac{6}{0}$ & $\begin{array}{l}\overrightarrow{7} \\
\dot{1} \\
\dot{9} \\
c\end{array}$ & $\begin{array}{l}8 \\
0 \\
0\end{array}$ & $\stackrel{10}{10}$ & $\vdots$ \\
\hline 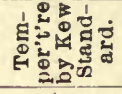 & 0 & $\begin{array}{l}+ \\
0 \\
0 \\
\infty\end{array}$ & $\begin{array}{l}0 \\
\dot{a} \\
\vdots \\
:\end{array}$ & $\begin{array}{l}8 \\
\vdots \\
\vdots \\
-1\end{array}$ & \begin{tabular}{l}
9 \\
\multirow{2}{*}{} \\
$\vdots$ \\
\hdashline
\end{tabular} & के & $\begin{array}{l}0 \\
\dot{20} \\
\dot{2} \\
\text { के }\end{array}$ & $\frac{\pi}{4}$ & $\stackrel{-1}{\circ}$ & $\begin{array}{l}0 \\
\dot{1} \\
0 \\
0\end{array}$ & $\begin{array}{l}0 \\
0 \\
0 \\
0\end{array}$ & $\vdots$ \\
\hline $\begin{array}{l}0 \\
0 \\
0 \\
0\end{array}$ & $\begin{array}{l}\stackrel{0}{0} \\
\dot{0} \\
+\end{array}$ & $\vdots$ & $\vdots$ & 点 & 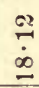 & $\begin{array}{l}8 \\
\dot{1} \\
62\end{array}$ & $\begin{array}{l}4 \\
\dot{5} \\
\dot{5}\end{array}$ & 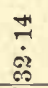 & $\begin{array}{l}\infty \\
\dot{8}\end{array}$ & $\stackrel{8}{8}$ & $\begin{array}{l}\overrightarrow{8} \\
\dot{8} \\
10\end{array}$ & $\vdots$ \\
\hline - & $\vdots$ & $\vdots$ & $\begin{array}{l}\text { के } \\
\dot{8} \\
\dot{8}\end{array}$ & : & $\stackrel{\overrightarrow{0}}{\overrightarrow{+}}$ & $\stackrel{+1}{+1}$ & $\begin{array}{l}\overline{0} \\
\dot{\infty} \\
\dot{0}\end{array}$ & $\stackrel{20}{\ddot{\infty}}$ & $\begin{array}{l}\infty \\
2 \\
2 \\
\text { is } \\
5 \\
5\end{array}$ & $\vdots$ & $\vdots$ & $\vdots$ \\
\hline & $\infty$ & 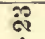 & $\bar{\sigma}$ & के & 8 & in & 26 & 8 & 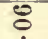 & H & $x_{\infty}^{x-1}$ & $\vdots$ \\
\hline 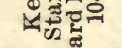 & $\therefore$ & 5 & is & $\infty$ & ti & g. & $\infty$ & $\infty$ & 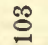 & $\stackrel{\mathscr{q}}{9}$ & $\stackrel{9}{9}$ & : \\
\hline & $g$ & \pm & 25 & $G_{0}$ & $\hat{\dot{\alpha}}$ & ڤె & $\nexists$ & 안 & 0 & $:$ & : & : \\
\hline & $\stackrel{20}{=}$ & 20 & $\frac{92}{60}$ & 옹 & g) & $\stackrel{R}{T}$ & $\begin{array}{l}\infty \\
\infty \\
\infty\end{array}$ & 5 & $\vec{\infty}$ & : & : & : \\
\hline 18 & 25 & D & 7 & î & 8 & $\frac{2}{2}$ & : & $\stackrel{20}{\infty}$ & $\vdots$ & : & $\vdots$ & : \\
\hline & 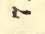 & aे & $\overrightarrow{c 0}$ & $\underset{\infty}{\infty}$ & 23 & 5 & E & 19 & . & : & . & • \\
\hline & $\vec{\sigma}$ & $\frac{20}{8}$ & $\overbrace{8}^{8}$ & ஓ & $\frac{a t}{F}$ & $\sum_{\substack{0 \\
-2}}^{2}$ & 是 & $\stackrel{\text { 赵 }}{\forall}$ & $\begin{array}{l}\infty \\
\infty \\
0\end{array}$ & 움 & $\begin{array}{c}10 \\
0 \\
0\end{array}$ & $\frac{92}{28}$ \\
\hline 들 & $\therefore$ & $\stackrel{\infty}{\infty}$ & $\stackrel{\circ}{=}$ & $\stackrel{\circ}{\exists}$ & 늑 & $\therefore$ & $\stackrel{\circ}{:=2}$ & $\stackrel{0}{\infty}$ & $\stackrel{\circ}{\circ}$ & $\stackrel{0}{\circ}$ & $\stackrel{\circ}{B}$ & 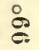 \\
\hline$\infty 0$ & $\begin{array}{l}\vec{\infty} \\
\text { ? } \\
?\end{array}$ & 孚 & \% & $\stackrel{\mathscr{\infty}}{\infty}$ & त्ञ & $\begin{array}{l}0 \\
0 \\
0\end{array}$ & $\stackrel{5}{2}$ & $\stackrel{8}{\circ}$ & ? & 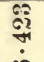 & हैं & $\vdots$ \\
\hline & & $\therefore$ & 20 & & & 20 & 18 & 18 & $\Rightarrow$ & 0 & 5 & : \\
\hline$=7_{+}^{2}$ & $\overline{8}$ & E્త్ర & $\stackrel{8}{\infty}$ & $\begin{array}{l}0 \\
15 \\
\text { al } \\
\text { ? }\end{array}$ & $\overbrace{2}^{2}$ & 产 & $\stackrel{\infty}{7}$ & $\frac{20}{20}$ & 总 & 噌 & 周 & के \\
\hline $2 \bar{p}$ & $\dot{f}$ & $\dot{H}$ & $\dot{+}$ & $\dot{f}$ & + & $\dot{\pi}$ & $\dot{+}$ & $\dot{f}$ & $\dot{H}$ & $\dot{H}$ & $\dot{f}$ & $\therefore$ \\
\hline & के & 눈 & . & $\bar{c}$ & ฉి & 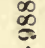 & $\stackrel{0}{0}$ & $\vec{\circ}$ & $\begin{array}{l}\infty \\
\infty \\
0\end{array}$ & $\frac{\infty}{0}$ & $\stackrel{\mathscr{F}}{*}$ & $\overline{8}$ \\
\hline $\mathbb{N}$ & $\dot{i}$ & 5 & \& & 10 & 深 & 官 & $\infty$ & $\infty$ & $\dot{0}$ & eq & 昆 & 9 \\
\hline & $\frac{12}{\infty}$ & $\frac{\pi}{0}$ & 趈 & $\overline{0}$ & 5 & $\begin{array}{l}\infty \\
\overbrace{0}^{2} \\
\infty\end{array}$ & 禹 & F & $\begin{array}{l}0 \\
\infty\end{array}$ & $\begin{array}{l}\infty \\
15 \\
3\end{array}$ & $\begin{array}{l}\mathrm{E}^{2} \\
\mathrm{c}^{2}\end{array}$ & ㅎ․ \\
\hline 7 & $\dot{8}$ & $\dot{E}$ & $\dot{\infty}$ & $\dot{\infty}$ & 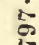 & 递 & $\dot{\vec{C}}$ & : & 20 & $\dot{\infty}_{\infty}$ & $\dot{-}$ & $\stackrel{\infty}{0}$ \\
\hline 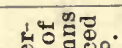 & ث艹 & D & 8 & $\begin{array}{l}\infty \\
\infty\end{array}$ & $\ddot{0}$ & 5 & 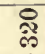 & $\frac{9}{0}$ & 亲 & 臬 & 舟 & 8 \\
\hline 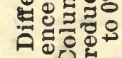 & $\dot{\vec{l}}$ & $G_{2}$ & ๕ & \% & $\dot{\infty}+\underset{+}{+}$ & $i_{i 5}$ & i & $\dot{\infty}$ & $\dot{\infty}$ & $\stackrel{\circ}{g}$ & $\dot{0}$ & $\underset{\leftrightarrow}{\stackrel{\leftrightarrow}{Q}}$ \\
\hline$\$$ & $\stackrel{2}{m}$ & 을 & 52 & $\infty$ & $\mathscr{0}$ & $\underset{\infty}{\pi}$ & : & 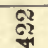 & $\begin{array}{l}02 \\
\infty \\
0\end{array}$ & $8_{8}^{\infty}$ & $\stackrel{0}{\infty}$ & 80 \\
\hline 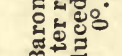 & $\dot{8}$ & $\dot{8}$ & 过 & क्ष & $\stackrel{\infty}{+}$ & $\underset{i}{2}$ & $\dot{i}$ & $\underset{E}{i}$ & 亲 & i- & $\dot{9}$ & : \\
\hline 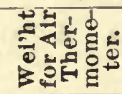 & a) & Q2 & a) & - & Q2 & a) & $\infty$ & $\Leftrightarrow$ & -1 & $\theta 2$ & a) & Q? \\
\hline
\end{tabular}




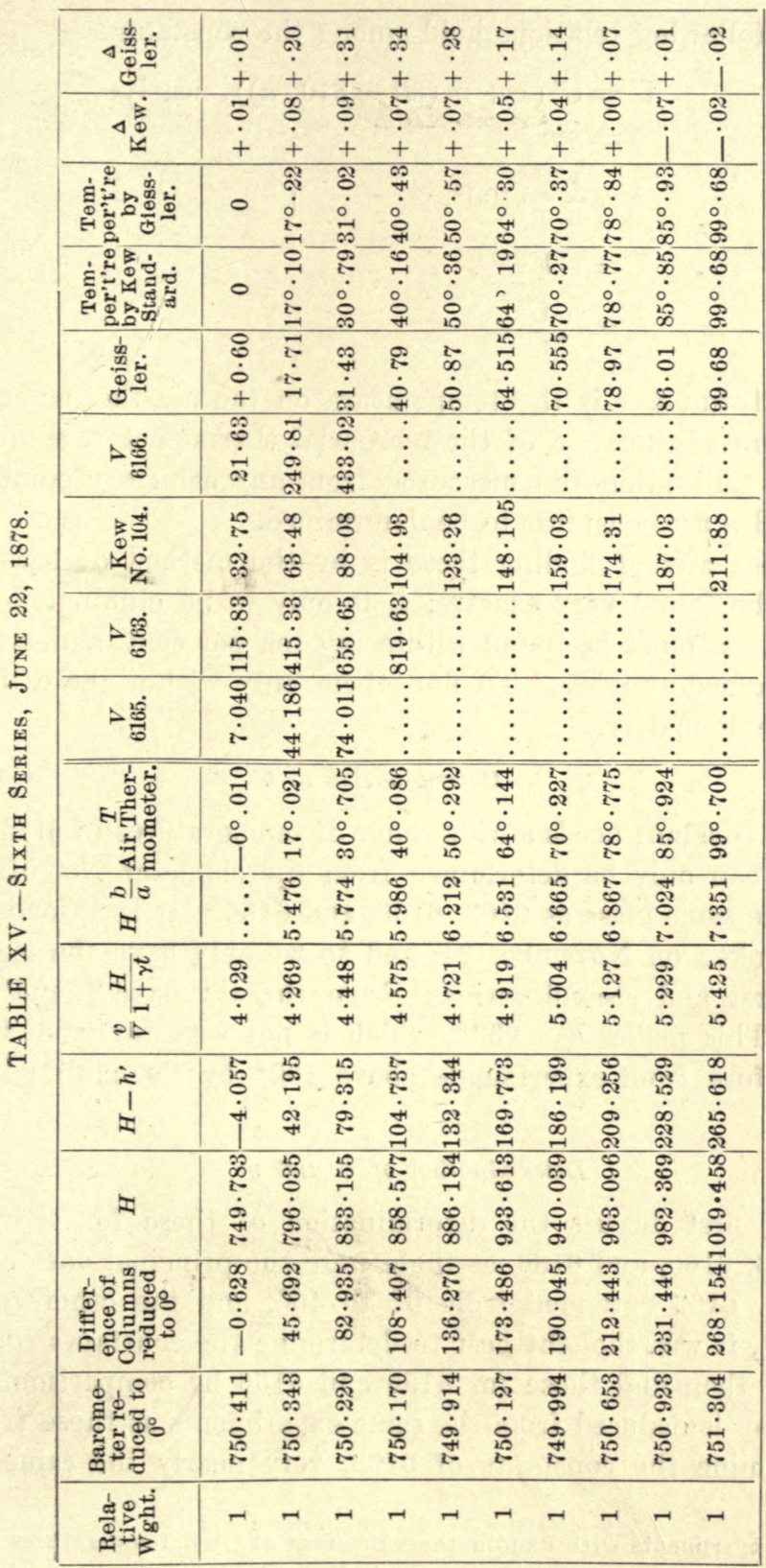


And the following relations hold among the constants:

$$
\begin{aligned}
C & =C^{\prime}(1+m(60-8400 n)), \text { nearly }, \\
a & =m n, \\
b & =\frac{1}{n}-100^{\circ}, \\
T & =C V-t_{0}, \\
t_{0} & =t^{\prime}{ }^{\prime} C^{\prime} .
\end{aligned}
$$

In these formulæ $t$ is the temperature on the air thermometer; $V$ is the volume of the stem of the mercurial thermometer, as determined from the calibration and measured from any arbitrary point; and $C^{\prime}$, $t^{\prime}, m$, and $n$ are constants to be determined.

The best way of finding these is by the method of least squares. $C^{\prime}$ must be found very exactly; $t_{0}$ is only to be eliminated from the equations; $m$ must be found within say ten per cent, and $n$ need only be determined roughly. To find them only within these limits is a very difficult matter.

\section{Determination of $\boldsymbol{n}$}

As this constant needs a wide range of temperatures to produce much effect, it can only be determined from thermometer No. 6167 , which was of the same glass as 6163,6165 , and 6166 . It is unfortunate that it was broken on November 21, and so we only have the experiments of the first and second series. From these I have found $n=.003$ nearly. This makes $b=233^{\circ}$, which is not very far from the values found before from experiments above $100^{\circ}$ by Regnault on ordinary glass. ${ }^{15}$

\section{Determination of $C$ and $m$}

I shall first discuss the determination of these for thermometers Nos. 6163,6165 , and 6166 , as these were the principal ones used.

As No, 6163 extended from $0^{\circ}$ to $40^{\circ}$, and the others only from $0^{\circ}$ to $30^{\circ}$, it was thought best to determine the constants for this one first, and then find those for 6165 and 6166 by comparison. As this comparison is deduced from the same experiments as those from which we determine the constants of 6163 , very nearly the same result is

\footnotetext{
15 Some experiments with Baudin thermometers at high temperatures have given me about $240^{\circ}$, - a remarkable agreement, as the point must be uncertain to $10^{\circ}$ or more.
} 
found as if we obtained the constants directly by comparison with the air thermometer.

The constants of 6163 can be found either by comparison with 6167 , or by direct comparison with the air thermometer. I shall first determine the constants for No. $616 \%$.

The constants $C$ and $t_{0}$ for this thermometer were found directly by observation of the $0^{\circ}$ and $100^{\circ}$ points; and we might assume these, and so seek only for $m$. In other words, we might seek only to express the difference of the thermometers from the air thermometer by a formula. But this is evidently incorrect, seeing that we thus give an infinite weight to the observations at the $0^{\circ}$ and $100^{\circ}$ points. The true way is obviously to form an equation for each temperature, giving each its proper weight. Thus from the first series we find for No. $616 \%$,-

$$
\begin{array}{ccc}
\text { Weight. } & \text { Equations of Condition. } \\
4 & 0 & =6.147 \mathrm{C}-t_{0}, \\
4 & 17^{\circ} \cdot 427=15 \cdot 685 \mathrm{C}-t_{0}-930 \mathrm{~m}, \\
4 & 23^{\circ} \cdot 793=19 \cdot 157 \mathrm{C}-t_{0}-1140 \mathrm{~m}, \\
\& \mathrm{c} . & \& \mathrm{c} . & \& \mathrm{c} . \\
5 & 100^{\circ}=60.156 \mathrm{C}-t_{0},
\end{array}
$$

which can be solved by the method of least squares. As $t_{0}$ is unimportant, we simply eliminate it from the equations. I have thus found,-

$$
\begin{aligned}
& \text { weight. } \\
& 1 \text { Nor. } 14 \quad C=1.85171 \quad m=.000217 \\
& 2 \text { Nov. 20, } 21 \quad C=1.8512 \% \quad m=.000172 \\
& \text { Mean } \quad C=1.85142 \quad m=.000187
\end{aligned}
$$

The difference in the values of $m$ is due to the observations not being so good as were afterwards obtained. However, the difference only signifies about $0^{\circ} .03$ difference from the mean at the $50^{\circ}$ point. After November 20 the errors are seldom half of this, on account of the greater experience gained in observation.

The ratio of $C$ for $616 \%$ and 6163 is found in the same way.

$\begin{array}{ccc}\text { Weight. } & \text { Nov. 14 } & .0310091 \\ 1 & \text { Nov. 20 } & .0309846 \\ 2 & \text { Mean } & -0309928\end{array}$


Hence for 6163 we have in this way

$$
C=.057381 \quad C^{\prime}=.056995 \quad m=.00018 \% .
$$

By direct comparison of No. 6163 with the air thermometer, we find the following:

\begin{tabular}{|c|c|c|c|}
\hline & Weight. & & \\
\hline Nov. 14 & 1 & $\cdot 056920$ & .000239 \\
\hline Nor. 20 & 2 & .056985 & $\cdot 000166$ \\
\hline Jan. 25 & 3 & .056986 & .000226 \\
\hline Feb. 11 & 4 & .056997 & .000155 \\
\hline June 8 & 3 & .056961 & $.0000 \% 1$ \\
\hline June 22 & 2 & $\cdot 056959$ & .000115 \\
\hline
\end{tabular}

The values of $C^{\prime}$ agree with each other with great exactness, and the probable error is only $\pm 0{ }^{\circ} 003 \mathrm{C}$. at the $40^{\circ}$ point.

The great differences in the values of $m$, when we estimate exactly what they mean in degrees, also show great exactness in the experiments. The mean value of $m$ indicates a difference of only $0^{\circ} .05$ between the mercurial and air thermometer at the $20^{\circ}$ point, the $0^{\circ}$ and $40^{\circ}$ points coinciding. The probable error of $m$ in degrees is only $\pm 0^{\circ} .003 \mathrm{C}$.

There is one more method of finding $m$ from these experiments; and that is by comparing the values of $C^{\prime}$ with No. $616 \%$, the glass of $616 \%$ being supposed to be the same as that of 6163 .

We have the formula

$$
C=C^{\prime}(1+34 \cdot 8 m) \text {. }
$$

Hence

$$
m=\frac{C-C^{\prime}}{34 \cdot 8 C^{\prime}} .
$$

\begin{tabular}{|c|c|c|}
\hline $\begin{array}{l}\text { Date. } \\
\text { Nov. } 14\end{array}$ & $\begin{array}{c}\text { Weight. } \\
1\end{array}$ & $\begin{array}{l}\text { Value of } m \\
.000236\end{array}$ \\
\hline Nov. 20 & 2 & .000218 \\
\hline Jan. 25 & 3 & $.00021 \%$ \\
\hline Feb. 11 & 4 & .000197 \\
\hline June 8 & 3 & .000215 \\
\hline June 22 & 2 & .000216 \\
\hline & Mean & .000213 \\
\hline
\end{tabular}

We thus obtain the following results: 
The results for $m$ are then as follows:

From direct comparison of No. 6167 with the air thermometer $\cdot 000187$ From direct comparison of No. 6163 with the air thermometer $\cdot 000154$ From comparison of No. 6163 with No. $616 \%$

.000213

The first and last are undoubtedly the most exact numerically, but they apply to No. 6167 , and are also, especially the first, derived from somewhat higher temperatures than the $20^{\circ}$ point, where the correction is the most important. The ralue of $m$, as determined in either of these ways, depends upon the determination of a difference of temperature amounting to $0^{\circ} \cdot 30$, and hence should be quite exact.

The value of $m$, as obtained from the direct comparison of No. 6163 with the air thermometer, depends upon the determination of a difference of about $0^{\circ} \cdot 05$ between the mercurial and the air thermometer. At the same time, the comparison is direct, the temperatures are the same as we wish to use, and the glass is the same. I have combined the results as follows:

$$
\begin{array}{cc}
m \text { from No. } 6167 & .000200 \\
m \text { from No. } 6163 & .000154 \\
\text { Mean } & .00018^{18}
\end{array}
$$

It now remains to deduce from the tables the ratios of the constants for the different thermometers.

The proper method of forming the equations of condition are as follows, applying the method to the first series:

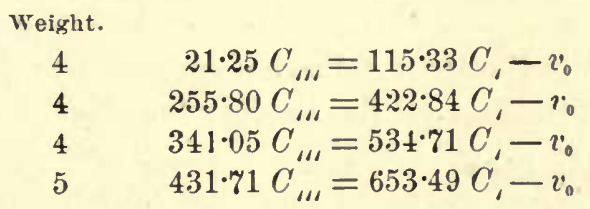

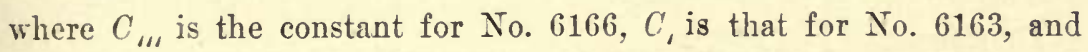
$v_{0}$ is a constant to be eliminated. Dividing by $C_{1}$, the equations can be solved for $\frac{C_{1 u}}{C,}$. The following table gires the results:

\footnotetext{
16 See Appendix to Thermometry, where it is finally thought best to reject the value from No. 6167 altogether.
} 
TABLE XVI.-RATIOS OF Constants.

\begin{tabular}{|c|c|c|c|c|c|c|}
\hline Date. & Weight. & $\frac{6163}{6167}$ & $\frac{6166}{6167}$ & $\frac{6166}{6163}$ & $\frac{6165}{6163}$ & $\frac{6165}{6166}$ \\
\hline $\begin{array}{lr}\text { Nov. } & 14 \\
\text { Nov. } & 20 \\
\text { Jan. } & 25 \\
\text { Feb. } & 11 \\
\text { June } & 8 \\
\text { June } & 22\end{array}$ & $\begin{array}{l}1 \\
2 \\
3 \\
4 \\
3 \\
2\end{array}$ & $\begin{array}{c}.031009 \\
.030985 \\
\ldots \ldots \\
\ldots \ldots \\
\ldots \ldots \\
\ldots \ldots\end{array}$ & $\begin{array}{c}.040658 \\
.040670 \\
\ldots \ldots \\
\ldots \ldots \\
\ldots \ldots \\
\ldots \ldots\end{array}$ & $\begin{array}{l}1 \cdot 3111 \\
1 \cdot 3128 \\
1 \cdot 3122 \\
1 \cdot 3115 \\
1 \cdot 3108 \\
1 \cdot 3122\end{array}$ & $\begin{array}{l}\ldots \ldots \\
\ldots \ldots \\
\ldots \ldots \\
8.0588 \\
8.0605 \\
8 \cdot 0588\end{array}$ & $\begin{array}{l}\cdots \\
\cdots \ldots \\
\cdots \cdot 14 \\
6 \cdot 149 \\
6 \cdot 1428\end{array}$ \\
\hline \multicolumn{2}{|c|}{ Mean } & $\begin{array}{r}.030993 \\
\pm .00005\end{array}$ & $\begin{array}{r}.040666 \\
\pm .000003\end{array}$ & $\begin{array}{r}1.31175 \\
\pm \quad .0004\end{array}$ & $\begin{array}{r}8.0594 \\
\pm \quad .0002\end{array}$ & $\begin{array}{r}6.1451 \\
\pm .0004\end{array}$ \\
\hline
\end{tabular}

From these we have the following, as the final most probable results:

$$
\begin{aligned}
& C_{u}^{\prime \prime}=8.0601 C_{1} \text {, } \\
& C_{\text {III }}=1.31175 C_{\text {, }} \text {, } \\
& C_{1}=031003 C_{\text {iv }} \text {, } \\
& C_{11}={ }^{24991 C_{\mathrm{iv}}} \text {, } \\
& C_{u}={ }^{\circ} 040661 C_{\mathrm{iv}} \text {, }
\end{aligned}
$$

of which the last three are only used to calculate the temperatures on the mercurial thermometer, and hence are of little importance in the remainder of this paper.

The value of $C^{\prime}$ which we have found for the old value of the coefficient of expansion of glass was

$$
C^{\prime}=\cdot 056976
$$

and hence, corrected to the new coefficient, it is, as I have shown,

Hence,

$$
\begin{aligned}
& C_{1}=.056962 . \\
& C_{1}=.45912, \\
& C_{11}=.074720 .
\end{aligned}
$$

And we have finally the three following equations to reduce the thermometers to temperatures on the air thermometer:

Thermometer No. 6163:

$T=.056962 V^{\prime}-t^{\prime}{ }_{0}-.00018 T(40-T)(1-.003(T+40))$.

Thermometer No. 6165:

$T=.45912 \nabla^{\prime \prime}-t_{0}{ }^{\prime \prime}-.00018 T(T-40)(1-.003(T+40))$.

Thermometer No. 6166:

$T=.074720 V^{\prime \prime \prime}-t_{0}^{\prime \prime \prime}-.00018 T(T-40)(1-.003(T+40))$;

where $\nabla^{\prime}, \nabla^{\prime \prime}$, and $\nabla^{\prime \prime \prime}$ are the volumes of the tube obtained by calibration; $t_{0}{ }^{\prime}, t_{0}{ }^{\prime \prime}$, and $t_{0}{ }^{\prime \prime \prime}$ are constants depending on the zero point, and 
of little importance where a difference of temperature is to be measured; and $T$ is the temperature on the air thermometer.

On the mercurial thermometer, using the $0^{\circ}$ and $100^{\circ}$ points as fixed, we have the following by comparison with No. 6167 :

Thermometer No. $6163 ; t=.057400 \nabla-t_{0}$;

Thermometer No. $6165 ; t=.46265 \nabla-t_{0}$;

Thermometer No. $6166 ; t=\cdot 075281 \nabla-t_{0}$.

\section{The Kero Standard}

The Kew standard must be treated separately from the above, as the glass is not the same. This thermometer has been treated as if its scale was arbitrary.

In order to have variety, I have merely plotted all the results with this thermometer, including those given in the Appendix, and drawn a curve through them. Owing to the thermometer being only divided to $\frac{1}{2}^{\circ} \mathrm{F}$., the readings could not be taken with great accuracy, and so the results are not very accordant; but $I$ have done the best I could, and the result probably represents the correction to at least $0^{\circ} .02$ or $0^{\circ} .03$ at every point.

\section{(d) Reduction to the Absolute Scale}

The correction to the air thermometer to reduce to the absolute scale has been given by Joule and Thomson, in the Philosophical Transactions for 1854; but as the formula there used is not correct, I have recalculated a table from the new formula used by them in their paper of 1862.

That equation, which originated with Rankine, can be placed in the form

$$
\frac{p v}{\mu}=C\left(1-m \frac{\mu_{0}}{\mu^{2}} D\right) ;
$$

where $p, v$, and $\mu$ are the pressure, volume, and absolute temperature of a given weight of the air; $D$ is its density referred to air at $0^{\circ} \mathrm{C}$. and $760 \mathrm{~mm}$. pressure; $\mu_{0}$ is the absolute temperature of the freezing point; and $m$ is a constant which for air is $0^{\circ} .33 \mathrm{C}$.

For the air thermometer with constant volume

or, since $D=1$,

$$
\begin{gathered}
T=100 \frac{p_{t}-p_{0}}{p_{100}-p_{0}} ; \\
\therefore T=\left(\mu-\mu_{0}\right)\left(1+m D\left(\frac{1}{\mu}-\frac{1}{\mu_{100}}\right)\right) ;
\end{gathered}
$$

$$
\mu-\mu_{0}=T-\cdot 00088 T \frac{100-T}{273+T}
$$

from which I have calculated the following table of corrections: 
TABLE XVII.-Reduction of Air Thermoneter to Absolute Scale.

\begin{tabular}{|c|c|c|}
\hline$\frac{T}{\text { Air Thermometer. }}$ & $\begin{array}{c}\mu-\mu_{0} \\
\text { Absolute Temperature. }\end{array}$ & $\begin{array}{l}\stackrel{\Delta}{\text { or Correction to Air }} \\
\text { Thermometer. }\end{array}$ \\
\hline $\begin{array}{r}0 \\
10 \\
20 \\
30 \\
40 \\
50 \\
60 \\
70 \\
80 \\
90 \\
100 \\
200 \\
300 \\
400 \\
500\end{array}$ & $\begin{array}{c}0 \\
9 \cdot 9972 \\
19 \cdot 9952 \\
29 \cdot 9939 \\
39 \cdot 9933 \\
49 \cdot 9932 \\
59 \cdot 9937 \\
69 \cdot 9946 \\
79 \cdot 9956 \\
89 \cdot 9978 \\
100 \cdot 000 \\
200 \cdot 037 \\
300 \cdot 092 \\
400 \cdot 157 \\
500 \cdot 228\end{array}$ & $\begin{array}{c}0 \\
-.0029 \\
-.0048 \\
-.0061 \\
-.0067 \\
-.0068 \\
-.0063 \\
-.0054 \\
-.0044 \\
-.0022 \\
0 \\
+.037 \\
+.092 \\
+.157 \\
+.228\end{array}$ \\
\hline
\end{tabular}

It is a curious circumstance, that the point of maximum difference occurs at about the same point as in the comparison of the mercurial and air thermometers.

From the previous formula, and from this table of corrections, the following tables were constructed.

TABLE XVIII.-Thermometer No. 6163.

\begin{tabular}{|c|c|c|c|c|c|c|c|c|c|}
\hline 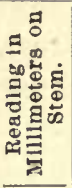 & 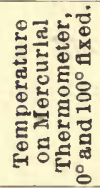 & 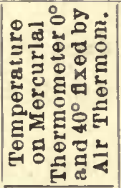 & 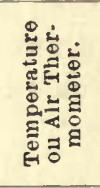 & 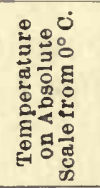 & 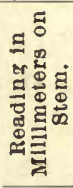 & 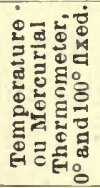 & 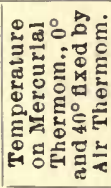 & 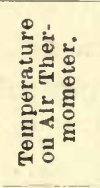 & 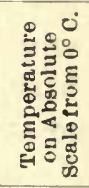 \\
\hline 50 & - & _-9097 & -9911 & _. 911 & 240 & $20^{\circ} \cdot 557$ & $20^{\circ} \cdot 409$ & $20^{\circ} \cdot 350$ & $20^{\circ} \cdot 345$ \\
\hline $58 \cdot 1$ & 0 & 0 & 0 & 0 & 250 & $21 \cdot 6 \pi 0$ & 21.515 & $21 \cdot 457$ & $21 \cdot 452$ \\
\hline 60 & $+\cdot 217$ & $+\cdot 215$ & $+\cdot 214$ & $+\cdot 214$ & 260 & $22 \cdot 776$ & $23 \cdot 616$ & $22-559$ & $22 \cdot 554$ \\
\hline 70 & $1 \cdot 356$ & $1 \cdot 336$ & $1 \cdot 328$ & $1 \cdot 328$ & 270 & $23 \cdot 884$ & $23 \cdot 713$ & $23 \cdot 657$ & 23.652 \\
\hline 80 & $2 \cdot 494$ & $2 \cdot 475$ & $2 \cdot 461$ & $2 \cdot 460$ & 280 & $24 \cdot 989$ & $24 \cdot 810$ & $24 \cdot 755$ & $24 \cdot 750$ \\
\hline 90 & $3 \cdot 631$ & $3 \cdot 604$ & $3 \cdot 584$ & $3 \cdot 583$ & 290 & $26 \cdot 093$ & $25 \cdot 90 \tau$ & $25 \cdot 854$ & $25 \cdot 848$ \\
\hline 100 & $4 \cdot 767$ & $4 \cdot 733$ & $4 \cdot 707$ & $4 \cdot 706$ & 300 & $27 \cdot 200$ & $27 \cdot 006$ & $26 \cdot 956$ & $26 \cdot 950$ \\
\hline 110 & $5 \cdot 903$ & $5 \cdot 860$ & $5 \cdot 829$ & $5 \cdot 827$ & 310 & $28 \cdot 311$ & $28 \cdot 108$ & $28 \cdot 060$ & $28.051 ;$ \\
\hline 120 & 7.036 & $6 \cdot 986$ & 6.950 & $6 \cdot 948$ & 320 & $29 \cdot 425$ & $29 \cdot 214$ & $29 \cdot 169$ & $29 \cdot 163$ \\
\hline 130 & $8 \cdot 170$ & $8 \cdot 111$ & $8 \cdot 071$ & $8 \cdot 069$ & 330 & $30 \cdot 541$ & $30 \cdot 324$ & 82 & $30 \cdot 276$ \\
\hline 140 & $9 \cdot 304$ & $9 \cdot 237$ & $9 \cdot 193$ & $9 \cdot 190$ & 340 & $31 \cdot 662$ & $31 \cdot 436$ & $31 \cdot 398$ & $31 \cdot 392$ \\
\hline 150 & $10 \cdot 436$ & 10.361 & $10 \cdot 314$ & $10 \cdot 311$ & 350 & 32.782 & $32 \cdot 548$ & 32.514 & $32 \cdot 508$ \\
\hline 160 & $11 \cdot 568$ & $11 \cdot 485$ & $11 \cdot 435$ & $11 \cdot 432$ & 360 & $33 \cdot 903$ & $33 \cdot 660$ & 630 & $33 \cdot 624$ \\
\hline 170 & $12 \cdot 700$ & $12 \cdot 608$ & $12 \cdot 556$ & $12 \cdot 553$ & 370 & $35 \cdot 023$ & $34 \cdot 773$ & $34 \cdot 748$ & $34 \cdot 742$ \\
\hline 180 & $13 \cdot 829$ & $13 \cdot 730$ & $13 \cdot 676$ & $13 \cdot 6 \% 2$ & 380 & $36 \cdot 143$ & $35 \cdot 884$ & & $35 \cdot 857$ \\
\hline 190 & $14 \cdot 957$ & $14 \cdot 850$ & $14 \cdot 794$ & $14 \cdot 790$ & 390 & $37 \cdot 261$ & $36 \cdot 994$ & $36 \cdot 979$ & 36.972 \\
\hline 200 & $16 \cdot 0 \$ 1$ & $15 \cdot 966$ & $15 \cdot 909$ & $15 \cdot 905$ & 400 & $38 \cdot 377$ & $38 \cdot 103$ & $38 \cdot 094$ & 38.087 \\
\hline 210 & $17 \cdot 203$ & 17.080 & $17 \cdot 022$ & $17 \cdot 018$ & 410 & $39 \cdot 492$ & $39 \cdot 210$ & $39 \cdot 206$ & $39 \cdot 199$ \\
\hline 220 & $18 \cdot 322$ & $18 \cdot 191$ & $18 \cdot 132$ & $18 \cdot 127$ & 420 & $40 \cdot 604$ & $40 \cdot 314$ & $40 \cdot 316$ & $40 \cdot 309$ \\
\hline 230 & $19 \cdot 440$ & $19 \cdot 301$ & $19 \cdot 242$ & $19 \cdot 237$ & & & & & \\
\hline
\end{tabular}


TABLE XIX.-THERMOMETER No. 6165.

\begin{tabular}{|c|c|c|c|c|c|c|c|c|c|}
\hline 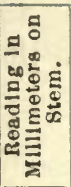 & 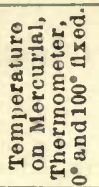 & 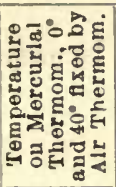 & 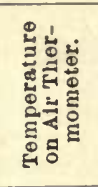 & 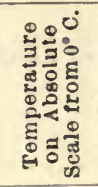 & 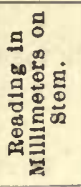 & 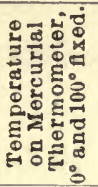 & 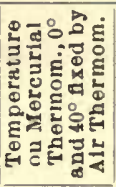 & 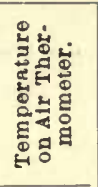 & 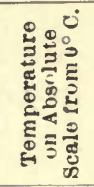 \\
\hline 30 & -.964 &.$- \$ 60$ & -.457 & -.957 & 230 & 17· 198 & 17.0067 & 17.009 & $17 \cdot 005$ \\
\hline 35 & 0 & 0 & 0 & 0 & 240 & $18 \cdot 056$ & $17 \cdot 920$ & $17 \cdot 861$ & $17 \cdot 857$ \\
\hline 40 & $+\cdot 463$ & +.460 & $+\cdot 457$ & +.457 & 250 & $18 \cdot 917$ & $18 \cdot 773$ & $18 \cdot 714$ & $18 \cdot 709$ \\
\hline 50 & $1 \cdot 357$ & $1 \cdot 376$ & $1 \cdot 36 \mathrm{~S}$ & $1 \cdot 365$ & 260 & $19 \cdot 771$ & $19 \cdot 621$ & $19 \cdot 562$ & $19 \cdot 557$ \\
\hline 60 & $2 \cdot 307$ & $2 \cdot 290$ & $2 \cdot 276$ & $2 \cdot 275$ & 270 & $20 \cdot 621$ & $20 \cdot 465$ & $20 \cdot 406$ & $20 \cdot 401$ \\
\hline 70 & $3 \cdot 216$ & $3 \cdot 192$ & $3 \cdot 174$ & $3 \cdot 173$ & 280 & $21 \cdot 469$ & $21 \cdot 306$ & $21 \cdot 247$ & $21 \cdot 242$ \\
\hline 80 & $4 \cdot 122$ & 4.092 & $4 \cdot 069$ & $4 \cdot 068$ & 290 & $22 \cdot 308$ & $22 \cdot 139$ & $22 \cdot 081$ & $22 \cdot 076$ \\
\hline 90 & 5.022 & $4 \cdot 984$ & $4 \cdot 957$ & $4 \cdot 955$ & 300 & $23 \cdot 144$ & $22 \cdot 969$ & $22 \cdot 912$ & 22.907 \\
\hline 100 & $5 \cdot 916$ & $5 \cdot 872$ & $5 \cdot 841$ & $5 \cdot 839$ & 310 & $23 \cdot 974$ & $23 \cdot 792$ & $23 \cdot 736$ & $23 \cdot 731$ \\
\hline 110 & $6 \cdot 804$ & 6.753 & $6 \cdot 714$ & 6.712 & 320 & $24 \cdot 796$ & $24 \cdot 607$ & 24.552 & $24 \cdot 547$ \\
\hline 120 & $7 \cdot 685$ & 7.628 & $7 \cdot 590$ & $7 \cdot 588$ & 330 & $25 \cdot 618$ & $25 \cdot 424$ & $25 \cdot 370$ & $25 \cdot 365$ \\
\hline 130 & S. 564 & $8 \cdot 500$ & $8 \cdot 459$ & $8.456^{\circ}$ & 340 & $26 \cdot 433$ & $26 \cdot 232$ & $26 \cdot 180$ & $26 \cdot 174$ \\
\hline 140 & $9 \cdot 439$ & 9.368 & $9 \cdot 324$ & $9 \cdot 321$ & 350 & $27 \cdot 245$ & $27 \cdot 038$ & $26 \cdot 987$ & $26 \cdot 981$ \\
\hline 150 & $10 \cdot 309$ & $10 \cdot 232$ & $10 \cdot 186$ & $10 \cdot 183$ & 360 & 28.049 & $27 \cdot 837$ & $27 \cdot 788$ & $27 \cdot 782$ \\
\hline 160 & $11 \cdot 174$ & $11 \cdot 091$ & $11 \cdot 042$ & $11 \cdot 039$ & 370 & $28 \cdot 856$ & $28 \cdot 637$ & $28 \cdot 590$ & $28 \cdot 584$ \\
\hline 170 & 12.038 & 11.947 & $11 \cdot 896$ & $11 \cdot 893$ & 390 & $29 \cdot 651$ & $29 \cdot 426$ & $29 \cdot 382$ & $29 \cdot 376$ \\
\hline 180 & $12 \cdot 900$ & $12 \cdot 802$ & 12.749 & 12.746 & 390 & $30 \cdot 449$ & $30 \cdot 218$ & $30 \cdot 176$ & $30 \cdot 170$ \\
\hline 190 & $13 \cdot 760$ & $13 \cdot 655$ & $13 \cdot 601$ & $13 \cdot 598$ & 400 & $31 \cdot 249$ & $31 \cdot 011$ & $30 \cdot 971$ & $30 \cdot 965$ \\
\hline 200 & $14 \cdot 619$ & $14 \cdot 508$ & $14 \cdot 453$ & $14 \cdot 450$ & 410 & $32 \cdot 073$ & $31 \cdot 829$ & $31 \cdot 782$ & $31 \cdot 786$ \\
\hline 210 & $15 \cdot 479$ & $15 \cdot 362$ & $15 \cdot 305$ & $15 \cdot 302$ & 420 & $32 \cdot 861$ & $32 \cdot 611$ & $32 \cdot 577$ & $32 \cdot 581$ \\
\hline 220 & $16 \cdot 340$ & $16 \cdot 215$ & $16 \cdot 157$ & $16 \cdot 153$ & & & & & \\
\hline
\end{tabular}

TABLE XX. - Thervometer No. 6166 .

\begin{tabular}{|c|c|c|c|c|c|c|c|c|c|}
\hline 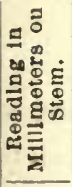 & 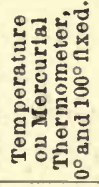 & 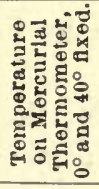 & 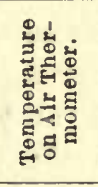 & 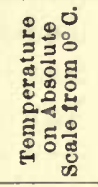 & 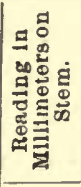 & 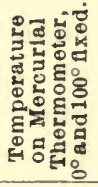 & 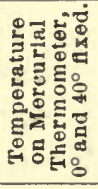 & 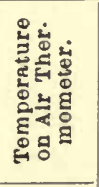 & 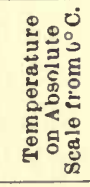 \\
\hline 20 & -.036 & -.036 & -.034 & -.034 & 230 & $16 \cdot 478$ & $16 \cdot 356$ & $16 \cdot 2998$ & $16 \cdot 294$ \\
\hline 30 & $+\cdot 770$ & $+\cdot 764$ & $+\cdot 759$ & $+\cdot 759$ & 240 & $17 \cdot 259$ & $17 \cdot 132$ & $17 \cdot 074$ & $0 \pi 0$ \\
\hline 40 & $1 \cdot 5 \% 4$ & & $1 \cdot 553$ & $1 \cdot 5553$ & 250 & & 17 & & \\
\hline 50 & $2 \cdot 368$ & & $2 \cdot 336$ & $2 \cdot 335$ & 260 & & 86 & 627 & \\
\hline 60 & $3 \cdot 156$ & & & $3 \cdot 114$ & $2 \% 0$ & 309 & 164 & 405 & 100 \\
\hline$\tau 0$ & $3 \cdot 941$ & & $3 \cdot 8$ & $3 \cdot 888$ & 280 & 392 & & 182 & \\
\hline so & $4 \cdot 726$ & & $4 \cdot 665$ & $4 \cdot 664$ & 290 & $21 \cdot 176$ & 21 . & & \\
\hline 90 & & & & & 300 & & & 735 & 730 \\
\hline 100 & & & & & 310 & 11 & $2: 2$ & 511 & 06 \\
\hline 110 & & & $6 \cdot 988$ & & 320 & & 23 & 292 & 287 \\
\hline 120 & $7 \cdot 862$ & & $7 \cdot 765$ & & 330 & 075 & & 075 & \\
\hline 130 & $8 \cdot 649$ & $8 \cdot 5$ & $8 \cdot 544$ & $8 \cdot 542$ & 340 & $24 \cdot 855$ & & $24 \cdot 855$ & \\
\hline 140 & & & & & 350 & $25 \cdot 634$ & & $25 \cdot 634$ & 25 \\
\hline 150 & $10 \cdot 2$ & 10 . & $10 \cdot 105$ & $10 \cdot 1$ & 360 & $26 \cdot 415$ & 26 . & $26 \cdot 412$ & 26 \\
\hline 160 & 11 & 10. & 10.887 & 10 . & 370 & 27. & 27. & $27 \cdot 195$ & 27 \\
\hline $1 \% 0$ & $11 \cdot 805$ & $11 \cdot 717$ & $11 \cdot 667$ & $11 \cdot 664$ & 380 & 28 & 28 & $27 \cdot 982$ & 27 \\
\hline 180 & $12 \cdot 589$ & $12 \cdot 496$ & $12 \cdot 444$ & $12 \cdot 441$ & 390 & $29 \cdot 030$ & 28 . & $28 \cdot 768$ & 28 \\
\hline 190 & $13 \cdot 3 \% 0$ & $13 \cdot 271$ & $13 \cdot 217$ & $13 \cdot 214$ & 400 & $29 \cdot 819$ & 29. & $29 \cdot 550$ & 29 \\
\hline 200 & $14 \cdot 148$ & $14 \cdot 043$ & $13 \cdot 988$ & $13 \cdot 984$ & 410 & $30 \cdot 608$ & $30 \cdot 381$ & $30 \cdot 339$ & 30. \\
\hline 210 & $14 \cdot 923$ & $14 \cdot 8$ & $14 \cdot 756$ & $14 \cdot 752$ & 420 & $31 \cdot 396$ & $31 \cdot 162$ & $31 \cdot 123$ & $31 \cdot 117$ \\
\hline 220 & $15 \cdot 699$ & $15 \cdot 583$ & $15 \cdot 526$ & $15 \cdot 5: 22$ & 430 & $32 \cdot 189$ & $31 \cdot 950$ & $31 \cdot 914$ & $31 \cdot 908$ \\
\hline
\end{tabular}


In using these tabies a correction is of course to be made should the zero point change.

TABLE XXI.-Coprection of Kew Standard to the Absolute Scale.

\begin{tabular}{|c|c|}
\hline Temperature C. & $\begin{array}{c}\text { Correction in } \\
\text { degrees C. }\end{array}$ \\
\hline $0^{\circ}$ & 0 \\
$10^{\circ}$ & -.03 \\
$20^{\circ}$ & $\underline{-.05}$ \\
$30^{\circ}$ & -.06 \\
$40^{\circ}$ & -.07 \\
$50^{\circ}$ & -.07 \\
$60^{\circ}$ & -.06 \\
$70^{\circ}$ & -.04 \\
$80^{\circ}$ & -.02 \\
$90^{\circ}$ & -.01 \\
$100^{\circ}$ & 0 \\
\hline
\end{tabular}

\section{Appendix to Thermometry}

The last of January, 18\%9, Mr. S. W. Holman, of the Massachusetts Institute of Technology, came to Baltimore to compare some thermometers with the air thermometer; and by his kindness I will give here the results of the comparison which we then made together.

As in this comparison some thermometers made by Fastré in 1851 were used, the results are of the greatest interest.

The tables are calculated with the newest value for the coefficient of expansion of glass. The calibration of all the thermometers, except the two by Casella, has been examined, and found good. The Casella thermometers had no reservoir at the top, and could not thus be readily calibrated after being made. The Geissler also had none, but I succeeded in separating a column.

The absence of a reservoir at the top should immediately condemn a standard, for there is no certainty in the work done with it.

From these tables we would draw the inference that No. 6163 represents the air thermometer with considerable accuracy. At the same time, both tables would give a smaller value of $m$ than $I$ have used, and not very far from the value found before by direct comparison, namely, $\cdot 00015$.

The difference from using $m=\cdot 00018$ would be a little over $0^{\circ} \cdot 01 \mathrm{C}$. at the $20^{\circ}$ point.

All the other thermometers stand above the air thermometer, between $0^{\circ}$ and $100^{\circ}$, by amounts ranging between about $0^{\circ} .05$ and $0^{\circ} .35 \mathrm{C}$., 
TABLE XXII.-SEVENTH SeRIES.

\begin{tabular}{|c|c|c|c|c|c|c|c|c|c|c|}
\hline \multirow[b]{2}{*}{$\begin{array}{c}\text { Alr } \\
\text { Ther- } \\
\text { mome- } \\
\text { ter. }\end{array}$} & \multicolumn{5}{|c|}{ Orlglnal Readlngs. } & \multicolumn{5}{|c|}{ Reduced Readings. } \\
\hline & 6163. & $\begin{array}{c}7334 \\
\text { Baudin. }\end{array}$ & $\begin{array}{c}\text { Kew } \\
\text { Stand- } \\
\text { ard } \\
\text { No. 104. }\end{array}$ & $\begin{array}{c}32374 \\
\text { Casella. }\end{array}$ & $\begin{array}{l}\text { Gelss- } \\
\text { ler. }\end{array}$ & $\begin{array}{c}6163 \\
\text { Reduced } \\
\text { to Air } \\
\text { Ther- } \\
\text { mome- } \\
\text { ter. }\end{array}$ & $\begin{array}{c}7334 \\
\text { Baudin. }\end{array}$ & $\begin{array}{c}\text { Kew } \\
\text { Stand- } \\
\text { ard } \\
\text { No. 104. }\end{array}$ & $\begin{array}{c}32374 \\
\text { Casella. }\end{array}$ & $\begin{array}{c}\text { Gelss- } \\
\text { ler. }\end{array}$ \\
\hline 8 & $1758 \cdot 83$ & $-\cdot 11$ & $32 \cdot 68$ & $+\cdot 20$ & $+\cdot 69$ & 8 & 8 & ○ & 8 & 8 \\
\hline $18 \cdot 43$ & $63 \cdot 5$ & & $33 \cdot 60$ & .71 & & .52 & & .52 & .51 & \\
\hline $6 \cdot 08$ & $113 \cdot 0$ & & $43 \cdot 65$ & $6 \cdot 33$ & & $6 \cdot 08$ & & $6 \cdot 11$ & $6 \cdot 13$ & \\
\hline $12 \cdot 68$ & $171 \cdot 55$ & $12 \cdot 59$ & $55 \cdot 47$ & $12 \cdot 91$ & $13 \cdot 42$ & $12 \cdot 65$ & $12 \cdot 73$ & $12 \cdot 68$ & $12 \cdot 70$ & $12 \cdot 82$ \\
\hline $20 \cdot 49$ & $242 \cdot 0$ & $20 \cdot 48$ & $69 \cdot 55$ & 20.77 & $21 \cdot 29$ & 20.49 & $20 \cdot 63$ & $20 \cdot 57$ & $20 \cdot 56$ & $20 \cdot 74$ \\
\hline $24 \cdot 55$ & $278 \cdot 8$ & $24 \cdot 50$ & $76 \cdot 90$ & $24 \cdot 80$ & $25 \cdot 33$ & $24 \cdot 54$ & $24 \cdot 66$ & $24 \cdot 61$ & $24 \cdot 59$ & $24 \cdot 81$ \\
\hline $29 \cdot 51$ & $323 \cdot 9$ & $29 \cdot 49$ & $85 \cdot 88$ & $29 \cdot 80$ & $30 \cdot 32$ & 2952 & $29 \cdot 66$ & $29 \cdot 61$ & 29 & \\
\hline $39 \cdot 45$ & $413 \cdot 1$ & $39 \cdot 43$ & $103 \cdot 72$ & $39 \cdot 76$ & $40 \cdot 22$ & $39 \cdot 47$ & $39 \cdot 62$ & $39 \cdot 53$ & $39 \cdot 54$ & $39 \cdot 80$ \\
\hline $39 \cdot 15$ & $410 \cdot 7$ & $39 \cdot 15$ & $103 \cdot 23$ & $39 \cdot 48$ & $39 \cdot 98$ & $39 \cdot 20$ & $39 \cdot 34$ & $39 \cdot 26$ & $39 \cdot 26$ & $39 \cdot 56$ \\
\hline $51 \cdot 17$ & $\ldots \ldots$ & $51 \cdot 10$ & $124 \cdot 84$ & $51 \cdot 49$ & $51 \cdot 83$ & $\ldots \ldots$ & $51 \cdot 32$ & $51 \cdot 29$ & $51 \cdot 26$ & $51 \cdot 49$ \\
\hline $61 \cdot 12$ & & $61 \cdot 05$ & $142 \cdot 73$ & 61.47 & $61 \cdot 69$ & & $61 \cdot 29$ & $61 \cdot 24$ & $61 \cdot 23$ & $61 \cdot 41$ \\
\hline $70 \cdot 74$ & & $70 \cdot 57$ & $159 \cdot 87$ & 71.00 & $71 \cdot 14$ & & $70 \cdot 83$ & $70 \cdot 78$ & $70 \cdot 76$ & $70 \cdot 92$ \\
\hline $80 \cdot 09$ & $\ldots \ldots$ & $79 \cdot 74$ & $176 \cdot 50$ & $80 \cdot 31$ & $80 \cdot 25$ & $\ldots \ldots$ & $80 \cdot 02$ & $80 \cdot 04$ & 80.06 & $80 \cdot 10$ \\
\hline $80 \cdot 39$ & $\ldots \ldots$ & $80 \cdot 15$ & $177 \cdot 23$ & $80 \cdot 74$ & $80 \cdot 66$ & & $80 \cdot 43$ & $80 \cdot 44$ & $80 \cdot 49$ & $80 \cdot 51$ \\
\hline $89 \cdot 95$ & & $89 \cdot 63$ & $194 \cdot 35$ & $90 \cdot 22$ & $90 \cdot 11$ & & $89 \cdot 93$ & $89 \cdot 97$ & $89 \cdot 97$ & 90.03 \\
\hline $89 \cdot 92$ & & $89 \cdot 59$ & $194 \cdot 22$ & $90 \cdot 18$ & 90.06 & & $89 \cdot 89$ & $89 \cdot 90$ & $89 \cdot 93$ & $89 \cdot 98$ \\
\hline $100 \cdot 00$ & $\ldots \ldots$ & $99 \cdot 69$ & $212 \cdot 37$ & $100 \cdot 06$ & $99 \cdot 32$ & $\ldots \ldots$ & $100 \cdot 00$ & 100.00 & $100 \cdot 00$ & $100 \cdot 00$ \\
\hline
\end{tabular}

TABLE XXIII.-EIGHTH SERIES.

\begin{tabular}{|c|c|c|c|c|c|c|c|c|c|c|}
\hline \multirow[b]{2}{*}{$\begin{array}{l}\text { Alr } \\
\text { Ther- } \\
\text { momo- } \\
\text { ter. }\end{array}$} & \multicolumn{5}{|c|}{ Original Readings. } & \multicolumn{5}{|c|}{ Reduced Readings. } \\
\hline & 6163. & $\begin{array}{c}376 \\
\text { Fastré. }\end{array}$ & $\begin{array}{c}7316 \\
\text { Baudin. }\end{array}$ & $\begin{array}{c}368 \\
\text { Fastré. }\end{array}$ & Casella. & $\begin{array}{c}6163 \\
\text { Reduced } \\
\text { to Alr } \\
\text { Ther- } \\
\text { mome- } \\
\text { ter. } \\
\end{array}$ & $\begin{array}{c}376 \\
\text { Frastré. }\end{array}$ & $\begin{array}{c}7316 \\
\text { Baudin. }\end{array}$ & $\begin{array}{c}368 \\
\text { Fastré. }\end{array}$ & $\begin{array}{c}3235 \\
\text { Casella. }\end{array}$ \\
\hline 8 & $1758^{\circ} \cdot 60$ & $111 \cdot 3$ & $-\cdot 23$ & $87 \cdot 6$ & $32 \cdot 80$ & 8 & ¿ & 8 & 8 & 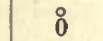 \\
\hline 3.67 & $90 \cdot 7$ & $130 \cdot 0$ & $\ldots \ldots$ & $106 \cdot 25$ & $39 \cdot 35$ & $3 \cdot 61$ & $3 \cdot 64$ & $\ldots \ldots$ & $3 \cdot 64$ & $3 \cdot 65$ \\
\hline $11 \cdot 55$ & $161 \cdot 6$ & $170 \cdot 9$ & $11 \cdot 40$ & $147 \cdot 2$ & $53 \cdot 70$ & $11 \cdot 56$ & $11 \cdot 60$ & $11 \cdot 64$ & $11 \cdot 62$ & $11 \cdot 63$ \\
\hline $20 \cdot 72$ & $243 \cdot 7$ & 217.9 & $20 \cdot 59$ & $194 \cdot 2$ & $70 \cdot 15$ & $20 \cdot 70$ & $20 \cdot 75$ & $20 \cdot 84$ & $20 \cdot 80$ & $20 \cdot 79$ \\
\hline $32 \cdot 19$ & $347 \cdot 4$ & $276 \cdot 9$ & $32 \cdot 09$ & $253 \cdot 2$ & $90 \cdot 80$ & $32 \cdot 17$ & $32 \cdot 24$ & $32 \cdot 34$ & $32 \cdot 28$ & $32 \cdot 29$ \\
\hline $39 \cdot 36$ & $411 \cdot 85$ & $313 \cdot 85$ & $39 \cdot 26$ & $290 \cdot 1$ & $103 \cdot 68$ & $39 \cdot 36$ & $39 \cdot 43$ & $39 \cdot 52$ & $39 \cdot 48$ & $39 \cdot 45$ \\
\hline $50 \cdot 71$ & $\ldots \ldots$ & $372 \cdot 0$ & $50 \cdot 57$ & $2 \pm 8 \cdot 2$ & $123 \cdot 65$ & $\ldots \ldots$ & $50 \cdot 75$ & $50 \cdot 84$ & $50 \cdot 80$ & $50 \cdot 57$ \\
\hline $60 \cdot 10$ & $\ldots \ldots$ & $420 \cdot 0$ & $59 \cdot 92$ & $396 \cdot 45$ & $140 \cdot 80$ & & $60 \cdot 10$ & $60 \cdot 19$ & $60 \cdot 21$ & $60 \cdot 12$ \\
\hline $73 \cdot 82$ & $\ldots \ldots$ & $490 \cdot 6$ & $73 \cdot 59$ & $466 \cdot 85$ & $165 \cdot 68$ & & $73 \cdot 84$ & $73 \cdot 87$ & $73 \cdot 93$ & $73 \cdot 97$ \\
\hline $86 \cdot 50$ & & $555 \cdot 25$ & $86 \cdot 16$ & $531 \cdot 22$ & $188 \cdot 20$ & $\ldots \ldots$ & $86 \cdot 48$ & $86 \cdot 51$ & $86 \cdot 56$ & $86 \cdot 56$ \\
\hline & $\ldots \ldots$ & $550 \cdot 2$ & $85 \cdot 21$ & $5: 25 \cdot 95$ & $186 \cdot 42$ & & $86 \cdot 45$ & $85 \cdot 50$ & $85 \cdot 45$ & 85.51 \\
\hline $100 \cdot 00$ & $\ldots \ldots$ & $624 \cdot 93$ & $99 \cdot 70$ & $600 \cdot 58$ & $3212 \cdot 45$ & $\ldots \ldots$ & $100 \cdot 00$ & $100 \cdot 00$ & $100 \cdot 00$ & $100 \cdot 00$ \\
\hline
\end{tabular}

none standing below. Indeed, no table has ever been published showing any thermometer standing below the air thermometer between $0^{\circ}$

${ }^{17}$ The original readings in ice were 58.68 and 58.45 , to which .15 was added to allow for the pressure of water in the comparator. This, of course, gives the same final result as if $\cdot 15$ were subtracted from each of the other temperatures. No correction was made to the others.

${ }^{18}$ Probably some error of reading. 
and $100^{\circ}$. By inference from experiments above $100^{\circ}$ on crystal glass by Regnault, thermometers of this glass should stand below, but it never seems to have been proved by direct experiment. The Fastré thermometers are probably made of this glass, and my Baudin's certainly contain lead; and yet these stand above, though only to a small amount, in the case of the Fastré's.

The Geissler still seems to retain its pre-eminence as having the greatest error of the lot.

The Baudin thermometers agree well together, but are evidently made from another lot of glass from the No. 6167 used before. These last two depart less from the air thermometer. The explanation is plain, as Baudin had manufactured more than one thousand thermometers between the two, and so had probably used up the first stock of glass. And even glass of the same lot differs, especially as Regnault has shown that the method of working it before the blow-pipe affects it very greatly.

It is very easy to test whether the calorimeter thermometers are of the same glass as any of the others, by testing whether they agree with No. 6163 throughout the whole range of $40^{\circ}$. The difference in the values of $m$ for the two kinds of glass will then be about .003 of the difference between them at $20^{\circ}$, the $0^{\circ}$ and $40^{\circ}$ points agreeing. The only difficulty is in calibrating or reading the $100^{\circ}$ thermometers accurately enough.

The Baudin thermometers were very well calibrated, and were graduated to $\frac{1}{10}^{\circ} \mathrm{C}$., and so were best adapted to this kind of work. Hence I have constructed the following tables, making the $0^{\circ}$ and $40^{\circ}$ points agree.

TABLE XXIV.-COMPARISON OF 6163 AND THE BAUDIN STANDARDS.

\begin{tabular}{|c|c|c|c|c|c|}
\hline $\begin{array}{l}6163 \\
\text { Mercurial } \\
0^{\circ} \text { and } 40^{\circ} \\
\text { fixed. }\end{array}$ & 7334.19 & Difference. & $\begin{array}{c}6163 \\
\text { Mercurial } \\
0^{\circ} \text { and } 40^{\circ} \\
\text { fixed. }\end{array}$ & 7316.19 & Difference. \\
\hline $\begin{array}{c}0 \\
12 \cdot 699 \\
20 \cdot 547 \\
24 \cdot 604 \\
29 \cdot 564 \\
39 \cdot 337\end{array}$ & $\begin{array}{c}0 \\
12 \cdot 673 \\
20 \cdot 553 \\
24 \cdot 567 \\
29 \cdot 550 \\
39 \cdot 337\end{array}$ & $\begin{array}{c}0 \\
+.026 \\
-.006 \\
+.037 \\
+.014 \\
0\end{array}$ & $\begin{array}{c}0 \\
11 \cdot 609 \\
20 \cdot 762 \\
32 \cdot 203 \\
39 \cdot 358\end{array}$ & $\begin{array}{c}0 \\
11 \cdot 584 \\
20 \cdot 746 \\
32 \cdot 211 \\
39 \cdot 358\end{array}$ & $\begin{array}{c}0 \\
+.025 \\
+.016 \\
-.008 \\
0\end{array}$ \\
\hline
\end{tabular}

${ }^{19} \mathrm{~A}$ correction of $0^{\circ} .01$ was made to the zero points of these thermometers on account of the pressure of the water. 
Taking the average of the two, it would seem that No. 6163 stood about 015 higher than the mean of 7334 and 7316 at the $20^{\circ}$ point, or 6163 has a higher value of $m$ by $\cdot 000045$ than the others.

These differ about 17 from the air thermometer at $40^{\circ}$, which gives the value of $m$ about .000104 . Whence $m$ for 6163 is .00015 , as we have found before by direct comparison with the air thermometer.

I am inclined to think that the former value, $\cdot 00018$, is too large, and to take $\cdot 00015$, which is the value found by direct comparison, as the true value. As the change, however, only makes at most a difference of $0^{\circ} .01$ at any one point, and as I have already used the previous value in all calculations, $I$ have not thought it worth while to go over all my work again, but will refer to the matter again in the final results, and then reduce the final results to this value.

\section{III.-CALORIMETRY}

\section{(a) Specific Heat of Water}

The first observers on the specific heat of water, such as De Luc, completed the experiment with a view of testing the thermometer; and it is curious to note that both De Luc and Flaugergues found the temperature of the mixture less than the mean of the two equal portions of which it was composed, and hence the specific heat of cold water higher than that of warm.

The experiments of Flaugergues were apparently the best, and he found as follows: ${ }^{20}$

3 parts of water at $0^{\circ}$ and 1 part at $80^{\circ} \mathrm{R}$. gave $19^{\circ} .86 \mathrm{R}$.

2 parts of water at $0^{\circ}$ and 2 parts at $80^{\circ} \mathrm{R}$. gave $39^{\circ} .81 \mathrm{R}$.

1 part of water at $0^{\circ}$ and 3 parts at $80^{\circ} \mathrm{R}$. gave $59^{\circ} .87 \mathrm{R}$.

But it is not at all certain that any correction was made for the specific heat of the vessel, or whether the loss by evaporation or radiation was guarded against.

The first experiments of any accuracy on this subject seem to have been made by F. E. Neumann in $1831 .^{21}$. He finds that the specific heat of water at the boiling point is 1.0127 times that at about $28^{\circ} \mathrm{C}$. $\left(22^{\circ} \mathrm{R}.\right)$.

The next observer seems to have been Regnault, ${ }^{23}$ who, in 1840 ,

${ }^{20}$ Gehler, Phys. Wörterbuch, i, 641.

${ }^{21}$ Pogg. Ann., xxili, 40.

${ }^{22}$ Ibid., li, 72 . 
found the mean specific heat between $100^{\circ} \mathrm{C}$. and $16^{\circ} \mathrm{C}$. to be 1.00709 and 1.00890 times that at about $14^{\circ}$.

But the principal experiments on the subject were published by Regnault in $1850,{ }^{23}$ and these have been accepted to the present time. It is unfortunate that these experiments were all made by mixing water above $100^{\circ}$ with water at ordinary temperatures, it being assumed that water at ordinary temperatures changes little, if any. An interpolation formula was then found to represent the results; and it was assumed that the same formula held at ordinary temperature, or even as low as $0^{\circ} \mathrm{C}$. It is true that Regnault experimented on the subject at points around $4^{\circ} \mathrm{C}$. by determining the specific heat of lead in water at various temperatures; but the results were not of sufficient accuracy to warrant any conclusions except that the variation was not great.

Boscha has attempted to correct Regnault's results so as to reduce them to the air thermometer; but Regnault, in Comptes Rendus, has not accepted the correction, as the results were already reduced to the air thermometer.

Hirn (Comptes Rendus, lxx, 592, 831) has given the results of some experiments on the specific heat of water at low temperatures, which give the absurd result that the specific heat of water increases about six or seven per cent between zero and $13^{\circ}$ ! The method of experiment was to immerse the bulb of a water thermometer in the water of the calorimeter, until the water had contracted just so much, when it was withdrawn. The idea of thus giving equal quantities of heat to the water was excellent, but could not be carried into execution without a great amount of error. Indeed, experiments so full of error only confuse the physicist, and are worse than useless.

The experiments of Jamin and Amaury, by the heating of water by electricity, were better in principle, and, if carried out with care, would doubtless give good results. But no particular care seems to have been taken to determine the variation of the resistance of the wire with accuracy, and the measurement of the temperature is passed over as if it were a very simple, instead of an immensely difficult matter. Their results are thus to be rejected; and, indeed, Regnault does not accept them, but believes there is very little change between $5^{\circ}$ and $25^{\circ}$.

In Poggendorff's Annalen for 1870 a paper by Pfaundler and Platter appeared, giving the results of experiments around $4^{\circ} \mathrm{C}$., and deducing the remarkable result that water from $0^{\circ}$ to $10^{\circ} \mathrm{C}$. varied as much as

${ }^{23}$ Pogg. Ann., lxxix, 241; also, Rel. d. Exp., i, 729. 
twenty per cent in specific heat, and in a very irregular manner,-first decreasing, then increasing, and again decreasing. But soon after another paper appeared, showing that the results of the previous experiments were entirely erroneous.

The new experiments, which extended up to $13^{\circ} \mathrm{C}$., seemed to give an increase of specific heat up to about $6^{\circ}$, after which there was apparently a decrease. It is to be noted that Geissler's thermometers were used, which I have found to depart more than any other from the air thermometer.

But as the range of temperature is very small, the reduction to the air thermometer will not affect the results very much, though it will somewhat decrease the apparent change of specific heat.

In the Journal de Physique for November, 1878, there is a notice of some experiments of M. von Münchausen on the specific heat of water. The method was that of mixture in an open vessel, where evaporation might interfere very much with the experiment. No reference is made to the thermometer, but it seems not improbable that it was one from Geissler; in which case the error would be very great, as the range was large, and reached even up to $70^{\circ} \mathrm{C}$. The error of the Geissler would be in the direction of making the specific heat increase more rapidly than it should. The formula he gives for the specific heat of water at the temperature $t$ is

$$
1+\cdot 000302 t
$$

Assuming that the thermometer was from Geissler, the formula, reduced to the air thermometer, would become approximately

$$
1-.00009 t+\cdot 0000015 t^{2} \text {. }
$$

Had the thermometer been similar to that of Recknagel, it would have been $1+\cdot 000045 t+\cdot 000001 t^{2}$.

It is to be noted that the first formula would actually give a decrease of specific heat at first, and then an increase.

As all these results vary so very much from each other, we can hardly say that we know anything about the specific heat of water between 0 and $100^{\circ}$, though Regnault's results above that temperature are probably very nearly correct.

It seems to me probable that my results with the mechanical equivalent apparatus give the variation of the specific heat of water with considerable accuracy; indeed, far surpassing any results which we can obtain by the method of mixture. It is a curious result of those experiments, that at low temperatures, or up to about $30^{\circ} \mathrm{C}$., the spe- 
cific heat of water is about constant on the mercurial thermometer made by Baudin, but decreases to a minimum at about $30^{\circ}$ when the reduction is made to the air thermometer or the absolute scale, or, indeed, the Kew standard.

As this curious and interesting result depends upon the accurate comparison of the mercurial with the air thermometer, I have spent the greater part of a year in the study of the comparison, but have not been able to find any error, and am now thoroughly convinced of the truth of this decrease of the specific heat. But to make certain, I have instituted the following independent series of investigations on the specific heat of water, using, however, the same thermometers.

The apparatus is shown in Fig. 4. A copper vessel, $A$, about $20 \mathrm{~cm}$. in diameter and $23 \mathrm{~cm}$. high, rests upon a tripod. In its interior is a three-way stopcock, communicating with the small interior vessel $B$, the vessel $A$, and the vulcanite spout $C$. By turning it, the vessel $B$ could be filled with water, and its temperature measured by the thermometer $D$, after whicn it could be delivered through the spout into the calorimeter. As the vessel $B$, the stopcock, and most of the spout, were within the vessel $A$, and thus surrounded by water, and as the vulcanite tube was very thin, the water could be delivered into the calorimeter without appreciable change of temperature. The proof of this will follow later.

The calorimeter, $E$, was of very thin copper, nickel-plated very thinly. A hole in the back at $F$ allowed the delivery spout to enter, and two openings on top admitted the thermometers. A wire attached to a stirrer also passed through the top. The calorimeter had a capac. ity of about three litres, and weighed complete about 388.3 grammes. Its calorific capacity was estimated at $35 \cdot 4$ grammes. It rested on three vulcanite pieces, to prevent conduction to the jacket. Around the calorimeter on all sides was a water-jacket, nickel-plated on its interior, to make the radiation perfectly definite.

The calorific capacity of the thermometers, including the immersed stem and the mercury of the bulb, was estimated as follows: $14 \mathrm{~cm}$. of stem weighed about $3.8 \mathrm{gr}$., and had a capacity of $\cdot 8 \mathrm{gr} . ; 10 \mathrm{gr}$. of mercury had a capacity of $\cdot 3 \mathrm{gr}$; t total, $1 \cdot 1 \mathrm{gr}$.

Often the vessel $B$ was removed, and the water allowed to flow directly into the calorimeter.

The following is the process followed during one experiment at low temperatures. The vessel $A$ was filled with clean broken ice, the opening into the stopcock being covered with fine gauze to prevent any 
small particles of ice from flowing out. The whole was then covered with cloth, to prevent melting. The vessel was then filled with water, and the two thermometers immersed to get the zero points. The calorimeter being about two-thirds filled with water, and having been weighed, was then put in position, the holes corked up, and one thermometer placed in it, the other being in the melting ice. An observation of its temperature was then taken every minute, it being frequently stirred.

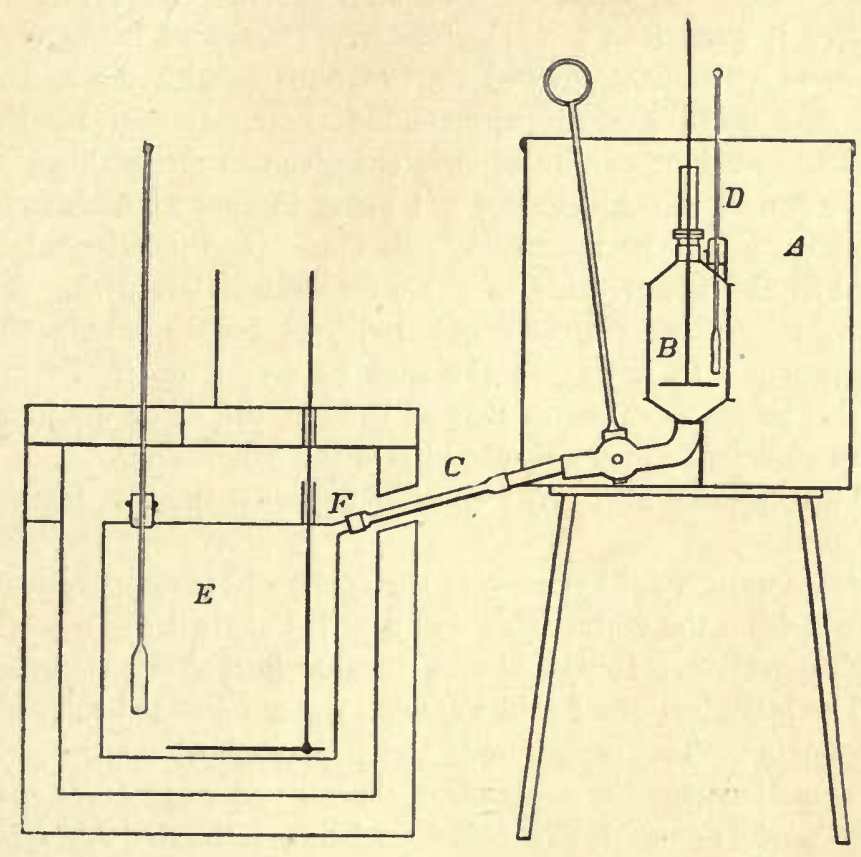

FIG. 4.

When enough observations had been obtained in this way, the cork was taken out of the aperture $F$ and the spout inserted, and the water allowed to run for a given time, or until the calorimeter was full. It was then removed, the cork replaced, and the second thermometer removed from the ice to the calorimeter. Observations were then taken as before, and the ressel again weighed.

Two thermometers were used in the way specified, so that one might approach the final temperature from above and the other from below. But no regular difference was ever observed, and so some experiments 
were made with both thermometers in the calorimeter during the whole experiment.

The principal sources of error are as follows:

1st. Thermometers lag behind their true reading. This was not noticed, and would probably be greater in thermometers with very fine stems like Geissler's. At any rate, it was almost eliminated in the experiment by using two thermometers.

$2 \mathrm{~d}$. The water may be changed in temperature in passing through the spout. This was eliminated by allowing the water to run some time before it went into the calorimeter. The spout being very thin, and made of vulcanite, covered on the outside with cloth, it is not thought that there was any appreciable error. It will be discussed more at length below, and an experiment given to prove this.

$3 \mathrm{~d}$. The top of the calorimeter not being in contact with the water, its temperature may be uncertain. To eliminate this, the calorimeter was often at the temperature of the air to commence with. Also the water was sometimes violently agitated just before taking the final reading, previous to letting in the cold water. Even if the temperature of this part was taken as that of the air, the error would scarcely ever be of sufficient importance to vitiate the conclusions.

4th. The specific heat of copper changes with the temperature. Unimportant.

5 th. Some water might remain in the spout whose temperature might be different from the rest. This was guarded against.

6th. Evaporation. Impossible, as the calorimeter was closed.

7th. The introduction of cold water may cause dew to be deposited on the calorimeter. The experiments were rejected where this occurred.

The corrections for the protruding thermometer stem, for radiation, \&c., were made as usual, the radiation being estimated by a series of observations before and after the experiment, as is usual in determining the specific heat of solids.

June 14, 1878.-First Experiment

\begin{tabular}{clll} 
Time. & Ther. 6163. & Ther. 6166. & \multicolumn{1}{c}{0 Points. } \\
41 & $296 \cdot 75$ & $6163,57 \cdot 9$ & Air, $21^{\circ} \mathrm{C}$. \\
42 & $296 \cdot 7$ & $6165,34 \cdot 8$ & Jacket about $25^{\circ} \mathrm{C}$. \\
43 & $296 \cdot 7$ & $6166,20 \cdot 5^{1}$ & \\
44 & $296 \cdot 65$ & &
\end{tabular}


Time. Ther. 6163. Ther.6166. 0 Points.

$44 \frac{1}{4}-44^{\frac{3}{4}}$ Water running.

Calorimeter before $\quad 2043 \cdot 0$

$46 \frac{1}{2}$

$218 \cdot y \quad 251 \cdot \%$

$47 \frac{1}{2}$

$218 \cdot 8 \quad 251 \cdot 8$

$48 \frac{1}{2}$

$218 \cdot 9$

$252 \cdot 0$

Temperature before $296 \cdot 6$

Correction for $0 \frac{+\cdot 2}{296 \cdot 8}=26^{\circ} \cdot 597$

Correction for stem $+\cdot 019$

Initial temperature of calorimeter

$$
\overline{26^{\circ} \cdot 616}
$$

“ after

$2853 \cdot 3$

Water at $0^{\circ}$ added

$810 \cdot 3$

Thermometer

$1 \cdot 1$

Total at $0^{\circ}$

$811 \cdot 4$

Calorimeter before $\quad 2043.0$

Weight of Vessel

Water

$1654 \cdot 7$

Capacity of calorimeter $\quad 35 \cdot 4$

“ thermometer $1 \cdot 1$

Total capacity

$1691 \cdot 2$

$$
\begin{array}{lr}
218 \cdot 6+\cdot 2=218 \cdot 8=17^{\circ} \cdot 994 & 251 \cdot 6-1=251 \cdot 5=17^{\circ} \cdot 962 \\
\text { Correction for stem } \begin{array}{l}
-.006 \\
17^{\circ} \cdot 988
\end{array} & \text { Correction for stem } \frac{-\cdot 006}{17^{\circ} \cdot 956}
\end{array}
$$

Mean temperature of mixture, $17^{\circ} \cdot 972$.

$\frac{\text { Mean specific heat } 0^{\circ}-18^{\circ}}{\text { Mean specific heat } 18^{\circ}-27^{\circ}}=\frac{1691 \cdot 2 \times 8^{\circ} \cdot 614}{811 \cdot 4 \times 17^{\circ} \cdot 97 \cdot 2}=1.0025$,

\section{June 14.-Second Experiment}

Calorimeter before 2016.3; temperature 361.4 by No. 6163 .

Calorimeter after $3047 \cdot 0$; temperature 244.5 and $288 . \%$.

$$
\text { Air, } 21^{\circ} \mathrm{C} \text {; jacket about } 2 \%^{\circ} \text {. }
$$

$361 \cdot 4+\cdot 2=361 \cdot 6=33^{\circ} \cdot 803$, or $33^{\circ} \cdot 863$ when corrected for stem. $244 \cdot 5+\cdot 2=244 \cdot 7=20^{\circ} \cdot 865$; no correction for stem. $288 \cdot 7-\cdot 1=288 \cdot 6=20^{\circ} \cdot 846$; no correction for stem.

$$
\text { Mean, } 20^{\circ} \cdot 855 \text {. }
$$

$\frac{\text { Mean specific heat between } 0^{\circ} \text { and } 21^{\circ}}{\text { Mean specific heat between } 21^{\circ} \text { and } 34^{\circ}}=1.0062$.

June 14.-Third Experiment

Calorimeter before $1961 \cdot 8$; temperature 293.6 by No. 6166 . Calorimeter after $3044 \cdot 6$; temperature $243 \cdot 7$ and $213 \cdot 0$.

Air and jacket, about $18^{\circ} \mathrm{C}$.

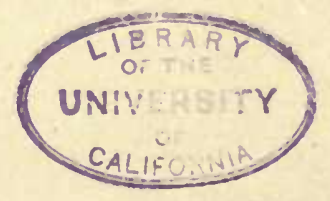


$393 \cdot 6-\cdot 1=393 \cdot 5=29^{\circ} \cdot 036$, or $29^{\circ} \cdot 07 \%$ when corrected for stem. $243 \cdot 7-\cdot 1=243 \cdot 6=17^{\circ} \cdot 349$; no correction for stem. $213 \cdot 0+\cdot 2=213 \cdot 2=17^{\circ} \cdot 374$; no correction for stem.

$$
\text { Mean, } 17^{\circ} \cdot 361 \text {. }
$$

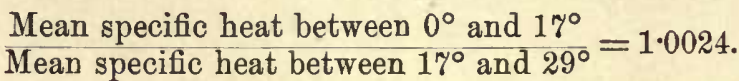

It is to be observed that thermometer No. 6166 in all cases gave temperatures about $0^{\circ} .02$ or $0^{\circ} .03$ below No. 6163 . This difference is undoubtedly in the determination of the zero points, as on June 15 the zero points were found to be 20.4 and $58 \cdot 0$. As one has gone up and the other down, the mean of the temperatures needs no correction.

June 15

Calorimeter before $2068 \cdot 2$; temperature $364 \cdot 6$ by No. 6166 . Calorimeter after $2929 \cdot 2$; temperature $249 \cdot \%$ and $21 \% \cdot \%$.

Air and jacket at about $22^{\circ} \mathrm{C}$.

$264 \cdot 6=26^{\circ} \cdot 766$, or $26^{\circ} \cdot 782$ when corrected for stem. $249 \cdot 7=18^{\circ} \cdot 822$, or $17^{\circ} \cdot 812$ when corrected for stem. $21 \% \cdot 7+\cdot 1=21 \% \cdot 8=17^{\circ} \cdot 884$, or $17^{\circ} \cdot 8 \% 4$ when corrected for stem.

Rejected on account of great difference in final temperatures by the two thermometers, which was probably due to some error in reading.

\section{June 21}

Calorimeter before $2002 \cdot 7$; temperature $330 \cdot 3$ by No. 6163 . Calorimeter after $3075 \cdot 2$; temperature 221.9 and $256 \cdot 6$.

Air and jacket, $21^{\circ} \mathrm{C}$.

$330 \cdot 3+\cdot 1=330 \cdot 4=30^{\circ} \cdot 321$, or $30^{\circ} \cdot 359$ when corrected for stem. $221.9+\cdot 1=222 \cdot 0=18^{\circ} \cdot 349$, or $18^{\circ} \cdot 343$ when corrected for stem. $256 \cdot 6+\cdot 0=256 \cdot 6=18^{\circ} \cdot 358$, or $18^{\circ} \cdot 352$ when corrected for stem.

$$
\text { Mean, } 18^{\circ} \cdot 34 \% \text {. }
$$

$\frac{\text { Specific heat between } 0^{\circ} \text { and } 18^{\circ}}{\text { Specific heat between } 18^{\circ} \text { and } 30^{\circ}}=1.006 \%$.

\section{June 21}

Calorimeter before $2073 \cdot 8$; temperature $347 \cdot 8$ by No. 6166 . Calorimeter after $2986 \cdot 8$; temperature 234.5 and $206 \cdot 6$.

Air and jacket, about $21^{\circ} \mathrm{C}$. 
$347 \cdot 8+\cdot 0=347 \cdot 8=25^{\circ} \cdot 457$, or $25^{\circ} \cdot 471$ when corrected for stem. $234 \cdot 5+\cdot 0=234 \cdot 5=16^{\circ} \cdot 643$, or $16^{\circ} \cdot 636$ when corrected for stem. $206 \cdot 6+\cdot 1=206 \cdot 7=16^{\circ} \cdot 651$, or $16^{\circ} \cdot 644$ when corrected for stem.

Mean, $16^{\circ} \cdot 640$.

$\frac{\text { Specific heat between } 0^{\circ} \text { and } 17^{\circ}}{\text { Specific heat between } 17^{\circ} \text { and } 25^{\circ}}=\cdot 99971$.

Rejected because dew was formed on the calorimeter.

A series was now tried with both thermometers in the calorimeter from the beginning.

\section{June 25}

Calor. before $2220 \cdot 3$; temperat. $325 \cdot 6$ by No. $6166 ; 309 \cdot 9$ by No. 6165 . Calor. after $3031 \cdot 4$; temperat. 233.4 by No. $6166 ; 224 \cdot 6$ by No. 6165 . Air, $24^{\circ} \cdot 2$ C.; jacket, $23^{\circ} \cdot 5$.

$325 \cdot 6+\cdot 0=325 \cdot 6=23^{\circ} \cdot 725$, or $23^{\circ} \cdot 726$ when corrected for stem. $309 \cdot 9+\cdot 2=310 \cdot 1=23^{\circ} \cdot 739$, or $23^{\circ} \cdot 740$ when corrected for stem. $233 \cdot 4+\cdot 0=233 \cdot 4=16^{\circ} \cdot 558$, or $16^{\circ} \cdot 545$ when corrected for stem. $224 \cdot 6+\cdot 2=224 \cdot 8=16^{\circ} \cdot 562$, or $16^{\circ} \cdot 549$ when corrected for stem. Means, $23^{\circ} \cdot 733$ and $16^{\circ} \cdot 54 \%$.

$\frac{\text { Specific heat between } 0^{\circ} \text { and } 11^{\circ}}{\text { Specific heat between } 16^{\circ} \text { and } 24^{\circ}}=1.0010$.

\section{June 25}

Calor. before 2278.6 ; temperat. 340.35 by No. $6166 ; 324.1$ by No. 6163 . Calor. after $3130 \cdot 2$; temperat. 242.5 by No. $6166 ; 232.8$ by No. 6165 .

$$
\text { Air, } 23^{\circ} \cdot 5 \text { C.; jacket, } 22^{\circ} \cdot 5 \text {. }
$$

$340 \cdot 35+\cdot 0=340 \cdot 35=24^{\circ} \cdot 87 \%$, or $24^{\circ} \cdot 881$ when corrected for stem. $324 \cdot 1+\cdot 2=324 \cdot 3=24^{\circ} \cdot 899$, or $24^{\circ} \cdot 903$ when corrected for stem. $242 \cdot 5+\cdot 0=242 \cdot 5=17^{\circ} \cdot 264$, or $17^{\circ} \cdot 253$ when corrected for stem. $232 \cdot 8+\cdot 2=233 \cdot 0=17^{\circ} \cdot 261$, or $17^{\circ} \cdot 250$ when corrected for stem.

$$
\frac{\text { Specific heat between } 0^{\circ} \text { and } 17^{\circ}}{\text { Specific heat between } 17^{\circ} \text { and } 25^{\circ}}=1.002 \%
$$

\section{June 25}

Calor. before $2316 \cdot 35$; temperat. $386 \cdot 1$ by No. $6166 ; 368 \cdot 4$ by No. 6165 . Calor. after 2966.90 ; temperat. $295 \cdot 4$ by No. $6166 ; 281 \cdot 7$ by No. 6165 .

$$
\text { Air, } 23^{\circ} \cdot 5 \mathrm{C} \text {; jacket, } 22^{\circ} \cdot 5 \text {. }
$$


$386 \cdot 1+\cdot 0=386 \cdot 1=28^{\circ} \cdot 455$, or $28^{\circ} \cdot 465$ when corrected for stem. $268 \cdot 4+\cdot 2=368 \cdot 6=28^{\circ} \cdot 472$, or $28^{\circ} .482$ when corrected for stem. $295 \cdot 4+\cdot 0=295 \cdot 4=21^{\circ} \cdot 374$, or $21^{\circ} \cdot 368$ when corrected for stem. $281 \cdot 7+\cdot 2=281 \cdot 9=21^{\circ} \cdot 400$, or $21^{\circ} \cdot 394$ when corrected for stem.

$$
\text { Means, } 28^{\circ} \cdot 473 \text { and } 21^{\circ} \cdot 381 \text {. }
$$

\section{$\frac{\text { Specific heat between } 0^{\circ} \text { and } 21^{\circ}}{\text { Specific heat between } 21^{\circ} \text { and } 28^{\circ}}=1.0045$.}

Two experiments were made on June 23 with warm water in vessel $A$, readings being taken of the temperature of the water, as it flowed out, by one thermometer, which was then transferred to the calorimeter as before.

\section{June 23}

Water in $A$ while running, $314 \cdot 15$ by No. 6163 .

Calor. before $1530 \cdot 9$; temperat. $281 \cdot 1$ by No. 6166 .

Calor. after $2996 \cdot 3$; temperat. $328 \cdot 4$ by No. $6166 ; 272 \cdot 7$ by No. 6163. $314 \cdot 15+\cdot 1=314 \cdot 25=28^{\circ} \cdot 526$, or $28^{\circ} \cdot 552$ when corrected for stem. $281 \cdot 1+\cdot 0=281 \cdot 1=20^{\circ} \cdot 262$, or $20^{\circ} \cdot 258$ when corrected for stem. $328.4+\cdot 0=328.4=23^{\circ} \cdot 945$, or $23^{\circ} \cdot 950$ when corrected for stem. $272 \cdot 7+\cdot 1=272 \cdot 8=23^{\circ} \cdot 960$, or $23^{\circ} \cdot 966$ when corrected for stem.

$$
\frac{\text { Specific heat between } 20^{\circ} \text { and } 24^{\circ}}{\text { Specific heat between } 24^{\circ} \text { and } 29^{\circ}}=\cdot 9983 \text {. }
$$

\section{June 23}

Water in $A$ while running, 383.9 by No. 6163 .

Calor. before $1624 \cdot 9$; temperat. 286.75 by 6166 .

Calor. after $3048 \cdot 2$; temperat. 392.45 by 6166 , and $318 \cdot 1$ by 6163 .

$383.9+\cdot 1=384 \cdot 0=36^{\circ} \cdot 303$, or $36^{\circ} \cdot 35 \%$ when corrected for stem. $286 \cdot 75+\cdot 0=286 \cdot 75=20^{\circ} \cdot 702$, or $20^{\circ} \cdot 700$ when corrected for stem. $392 \cdot 45+\cdot 0=392 \cdot 45=28^{\circ} \cdot 954$, or $28^{\circ} \cdot 980$ when corrected for stem. $318 \cdot 1+\cdot 1=318 \cdot 2=28^{\circ} \cdot 964$, or $28^{\circ} .992$ when corrected for stem.

$$
\frac{\text { Specific heat between } 21^{\circ} \text { and } 29^{\circ}}{\text { Specific heat between } 29^{\circ} \text { and } 36^{\circ}}=\cdot 9954 \text {. }
$$

To test the apparatus, and also to check the estimated specific heat of the calorimeter, the water was almost entirely poured out of the calorimeter, and warm water placed in the vessel $A$, which was then allowed to flow into the calorimeter. 
Water in $A$ while running, 309.0 by No. 6163 .

Calor. before $391 \cdot 3$; temperat. $314 \cdot 5$ by 6166 .

Calor. after $3129 \cdot 0$; temperat. $308 \cdot 3$ by 6166 , and 378.5 by 6163 .

Air about $21^{\circ} \mathrm{C}$.

Therefore, water lost $0^{\circ} \cdot 078$, and calorimeter gained $5^{\circ}$. Hence the capacity of the calorimeter is 39 .

Another experiment, more carefully made, in which the range was greater, gave 35 .

The close agreement of these with the estimated amount is, of course, only accidental, for they depend upon an estimation of only $0^{\circ} .08$ and $0^{\circ} .12$ respectively. But they at least show that the water is delivered into the calorimeter without much change of temperature.

A few experiments were made as follows between ordinary temperatures and $100^{\circ}$, seeing that this has already been determined by Regnault.

Two thermometers were placed in the calorimeter, the temperature of which was about $5^{\circ}$ below that of the atmosphere. The vessel $B$ was then filled, and the water let into the calorimeter, by which the temperature was nearly brought to that of the atmosphere; the operation was then immediately repeated, by which the temperature rose about $5^{\circ}$ above the atmosphere. The temperature of the boiling water was given by a thermometer whose $100^{\circ}$ was taken several times.

As only the rise of temperature is needed, the zero points of the thermometers in the calorimeter are unnecessary, except to know that they are within $0^{\circ} .02$ of correct.

June 18

Temperature of boiling water, $99^{\circ} \cdot 9$.

Calor. before $2684 \cdot \%$; temperat. $259 \cdot 2$ by 6166 , and $248 \cdot 3$ by 6165 .

Calor. after $2993 \cdot 2$; temperat. 381.0 by 6166 , and 363.4 by 6165 .

$259 \cdot 3=18^{\circ} \cdot 568$, or $18^{\circ} \cdot 555$ when corrected for stem.

$248 \cdot 3=18^{\circ} \cdot 564$, or $18^{\circ} \cdot 551$ when corrected for stem.

$381 \cdot 0=28^{\circ} \cdot 054$, or $28^{\circ} \cdot 065$ when corrected for stem.

$363 \cdot 4=28^{\circ} \cdot 055$, or $28^{\circ} \cdot 066$ when corrected for stem.

$$
\frac{\text { Specific heat } 28^{\circ}-100^{\circ}}{\text { Specific heat } 18^{\circ}-28^{\circ}}=1 \cdot 0024 \text {. }
$$

Other experiments gave 1.0015 and 1.0060 , the mean of all of which 
is 1.0033 . Regnault's formula gives 1.005 ; but going directly to his experiments, we get about 1.004 , the other quantity being for $110^{\circ}$.

The agreement is very satisfactory, though one would expect my small apparatus to lose more of the heat of the boiling water than Regnault's. Indeed, for high temperatures my apparatus is much inferior to Regnault's, and so I have not attempted any further experiments at high temperatures.

My only object was to confirm by this method the results deduced from the experiments on the mechanical equivalent; and this I have done, for the experiments nearly all show that the specific heat of water decreases to about $30^{\circ}$, after which it increases. But the mechanical equivalent experiments give by far the most accurate solution of the problem; and, indeed, give it with an accuracy hitherto unattempted in experiments of this nature.

But whether water increases or decreases in specific heat from $0^{\circ}$ to $30^{\circ}$ depends upon the determination of the reduction to the air thermometer. According to the mercurial thermometers Nos. 6163, 6165 and 6166 , treating them only as mercurial thermometers, the specific heat of water up to $30^{\circ}$ is nearly constant, but by the air thermometer, or by the Kew standard or Fastré, it decreases.

Full and complete tables of comparison are published, and from them any one can satisfy himself of the facts in the case.

I am myself satisfied that I have obtained a very near approximation to absolute temperatures, and accept them as the standard. And by this standard the specific heat of water undoubtedly decreases from $0^{\circ}$ to about $30^{\circ}$.

To show that I have not arrived at this result rashly, I may mention that I fought against a conclusion so much at variance with my preconceived notions, but was forced at last to accept it, after studying it for more than a year, and making frequent comparisons of thermometers, and examinations of all other sources of error.

However remarkable this fact may be, being the first instance of the decrease of the specific heat with rise of temperature, it is no more remarkable than the contraction of water to $4^{\circ}$. Indeed, in both cases the water hardly seems to have recovered from freezing. The specific heat of meiting ice is infinite. Why is it necessary that the specific heat should instantly fall, and then recover as the temperature rises? Is it not more natural to suppose that it continues to fall even after the ice is melted, and then to rise again as the specific heat approaches infin- 
ity at the boiling point? And of all the bodies which we should select as probably exhibiting this property, water is certainly the first.

\section{(b.) Heat Capacity of Calorimeter}

During the construction of the calorimeter, pieces of all the material were saved in order to obtain the specific heat. The calorimeter which Joule used was put together with screws, and with little or no solder. But in my calorimeter it was necessary to use solder, as it was of a much more complicated pattern. The total capacity of the solder used was only about $\frac{1}{600}$ of the total capacity including the water; and if we should neglect the whole, and call it copper, the error would be only about $\frac{1}{1200}$. Hence it was considered sufficient to weigh the solder before and after use, being careful to weigh the scraps. The error in the weight of solder could not possibly have been as great as ten per cent, which would affect the capacity only 1 part in 12,000 .

To determine the nickel used in plating, the calorimeter was weighed before and after plating; but it weighed less after than before, owing to the polishing of the copper. But I estimated the amount from the thickness of a loose portion of the plating. I thus found the approximate weight of nickel, but as it was so small, I counted it as copper. The following are the constituents of the calorimeter:-

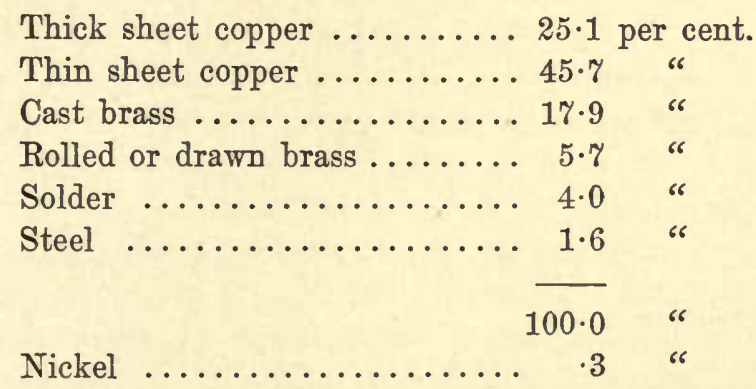

To determine the mean specific heat, the basket of a Regnault's apparatus was filled with the scraps in the above proportion, allowing the basket of brass gauze, which was very light, to count toward the drawn brass. The specific heat was then determined between $20^{\circ}$ and $100^{\circ}$, and between about $10^{\circ}$ and $40^{\circ}$. Between $20^{\circ}$ and $100^{\circ}$ the ordinary steam apparatus was used, but between $10^{\circ}$ and $40^{\circ}$ a special apparatus filled with water was used, the water being around the tube containing the basket, in the same manner as the steam is in the 
original apparatus. In the calorimeter a stirrer was used, so that the basket and water should rapidly attain the same temperature. The water was weighed before and after the experiment, to allow for evaporation. A correction of about 1 part in 1000 was made, on account of the heat lost by the basket in passing from the apparatus to the calorimeter, in the $100^{\circ}$ series, but no correction was made in the other series. The thermometers in the calorimeter were Nos. 6163 and 6166 in the different experiments.

The principal difficulty in the determination is in the correction for radiation, and for the heat which still remains in the basket after some time. After the basket has descended into the water, it commences to give out heat to the water; this, in turn, radiates heat; and the temperature we measure is dependent upon both these quantities.

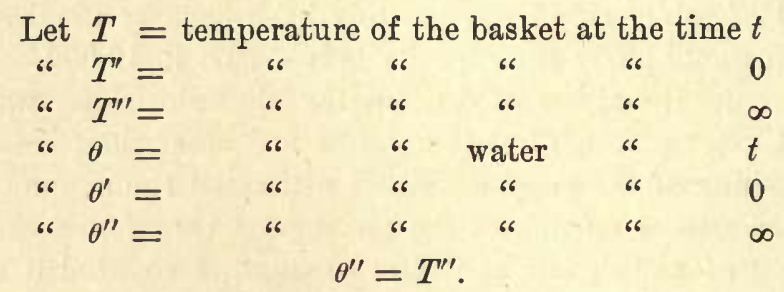

We may then put approximately

$$
T-T^{\prime \prime}=\left(T^{\prime}-T^{\prime \prime}\right) \varepsilon^{-\frac{t}{c}},
$$

where $c$ is a constant. But

$$
\frac{T^{\prime}-T^{\prime \prime}}{\theta^{\prime \prime}-\theta^{\prime}}=\frac{T^{\prime \prime}-T}{\theta-\theta^{\prime}}
$$

hence

To find $c$ we have

$$
\theta-\theta^{\prime}=\left(\theta^{\prime \prime}-\theta^{\prime}\right)\left(1-\varepsilon^{-\frac{i}{c}}\right)
$$

$$
c=\frac{1}{t} \log _{e} \frac{\theta^{\prime \prime}-\theta^{\prime}}{\theta^{\prime \prime}-\theta}
$$

where $\theta^{\prime \prime}$ can be estimated sufficiently accurately to find $C^{\prime}$ approximately.

These formulæ apply when there is no radiation. When radiation takes place, we may write, therefore, when $t$ is not too small,

$$
\theta-\theta^{\prime}=\left(\theta^{\prime \prime}-\theta^{\prime}\right)\left(1-\varepsilon^{-\frac{t}{c}}\right)-C\left(t-t_{0}\right),
$$

where $C$ is a coefficient of radiation, and $t_{0}$ is a quantity which must be subtracted from $t$, as the temperature of the calorimeter does not rise 
instantaneously. To estimate $t_{0}, T_{\alpha}$ being the temperature of the air, we have, according to Newton's law of cooling,

$$
\begin{gathered}
C\left(t-t_{0}\right)=\frac{C}{\theta^{\prime \prime}-T_{a}^{\prime}} \int_{0}^{t}\left(\theta-T_{a}^{\prime}\right) d t \text { nearly, } \\
t_{0}=c \frac{\theta^{\prime \prime}-\theta^{\prime}}{\theta^{\prime \prime}-T_{a}} \text { nearly, }
\end{gathered}
$$

where it is to be noted that $\frac{C}{\theta^{\prime \prime}-T_{a}}$ is nearly a constant for all values of $\theta^{\prime \prime}-T_{\alpha}$ according to Newton's law of cooling.

The temperature reaches a maximum nearly at the time

$$
t_{m}=c \log _{e} \frac{\theta^{\prime \prime}-\theta^{\prime}}{c C^{\prime}}
$$

and if $\theta_{m}$ is the maximum temperature, we have the value of $\theta^{\prime \prime}$ as follows:

$$
\begin{aligned}
\theta^{\prime \prime} & =T^{\prime \prime}=\theta_{m}+C^{\gamma}\left(t_{m}+c-t_{0}\right) ; \\
T^{\prime \prime} & =\theta_{m}+C\left(t_{m}+c \frac{T_{a}-\theta^{\prime}}{\theta^{\prime \prime}-T_{a}^{\prime}}\right) ;
\end{aligned}
$$

and this is the final temperature provided there was no loss of heat.

When the final temperature of the water is nearly equal to that of the air, $C$ will be small, but the time $t_{m}$ of reaching the maximum will be great. If $a$ is a constant, we can put $C=a\left(\theta^{\prime \prime}-T_{a}\right)$, and $C\left(t_{m}+c-t_{0}\right)$ will be a minimum, when

$$
C=\frac{\theta^{\prime \prime}-\theta^{\prime}}{c}, \text { or } T_{a}=\theta^{\prime \prime}-\frac{\theta^{\prime \prime}-\theta^{\prime}}{a c} \text {. }
$$

That is, the temperature of the air must be lower than the temperature of the water, so that $T_{\alpha}=\theta^{\prime \prime}$ as nearly as possible; but the formula shows that this method makes the corrections greater than if we make $T_{a}=\theta^{\prime}$, the reason being that the maximum temperature is not reached until after an infinitc time. It will in practice, however, be found best to make the temperature of the water at the beginning about that of the air. It is by far the best and easiest method to make all the corrections graphically, and I have constructed the following graphical method from the formulæ.

First make a series of measurements of the temperature of the water of the calorimeter, before and after the basket is dipped, together with the times. Then plot them on a piece of paper as in Fig. 5, making the scale sufficiently large to insure accuracy. Five or ten centimeters to a degree are sufficient.

$n a b c d$ is the plot of the temperature of the water of the calori26 
meter, the time being indicated by the horizontal line. Continue the line $d c$ until it meets the line $l a$. Draw a horizontal line through the point $l$. At any point, $b$, of the curve, draw a tangent and also a vertical line $b g$; the distance $e g$ will be nearly the value of the constant $c$ in the formulæ. Lay off $l f$ equal to $c$, and draw the line $f h k$ through the point $h$, which indicates the temperature of the atmosphere or of the vessel surrounding the calorimeter. Draw a vertical line, $j k$, through the point $k$. From the point of maximum, $c$, draw a line, $j c$, parallel to $d m$, and where it meets $k j$ will be the required point, and will give the value of $\theta^{\prime \prime}$. Hence, the rise of temperature, corrected for all errors, will be $k j$.

This method, of course, only applies to cases where the final temperature of the calorimeter is greater than that of the air; otherwise there will be no maximum.

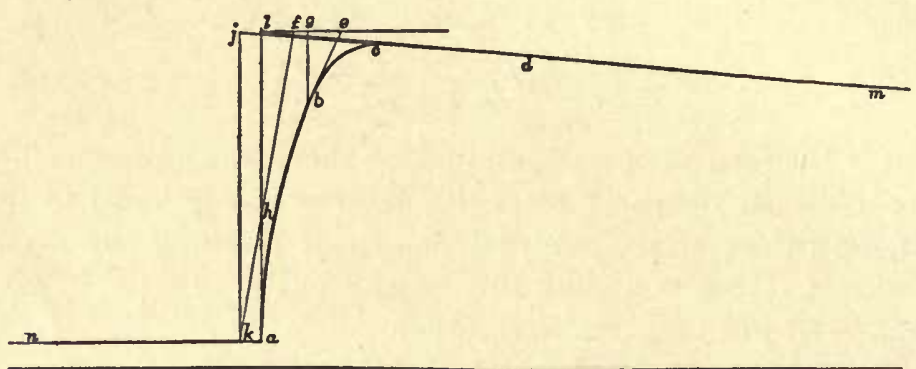

FIG. 5.

In practice, the line $d m$ is not straight, but becomes more and more nearly parallel to the base line. This is partly due to the constant decrease of the difference of temperature between the calorimeter and the air, but is too great for that to account for it. I have traced it to the thin metal jacket surrounding the calorimeter, and I must condemn, in the strongest possible manner, all such arrangements of calorimeters as have such a thin metal jacket around them. The jacket is of an uncertain temperature, between that of the calorimeter and the air. When the calorimeter changes in temperature, the jacket follows it but only after some time; hence, the heat lost in radiation is uncertain. The true method is to have a water jacket of constant temperature, and then the rate of decrease of temperature will be nearly constant for a long time.

The following results have been obtained by Mr. Jacques, Fellow of the University, though the first was obtained by myself. Corrections were, of course, made for the amount of thermometer stem in the air. 
Temperature.

$24^{\circ}$ to $100^{\circ}$
$26^{\circ}$ to $100^{\circ}$
$25^{\circ}$ to $100^{\circ}$
$13^{\circ}$ to $39^{\circ}$
$14^{\circ}$ to $38^{\circ}$
$9^{\circ}$ to $41^{\circ}$

Mean Specific Heat.

.0915

.0915

.0896

.0895

.0885

.0910

To reduce these to the mean temperature of $0^{\circ}$ to $40^{\circ}$, I have used the rate of increase found by Béde for copper. They then become, for the mean from $0^{\circ}$ to $40^{\circ}$,-

\begin{aligned} & .0897 \\ & .0897 \\ & .0878 \\ & .0893 \\ & .0883 \\ & .0906 \\ & \hline$-0892 \pm \cdot 00027\end{aligned}$

As the capacity of the calorimeter is about four per cent of that of the total capacity, including the water, this probable error is about $\frac{1}{8000}$ of the total capacity, and may thus be considered as satisfactory.

I have also computed the mean specific heat as follows, from other observers:-

$$
\begin{aligned}
& \text { Copper between } 20^{\circ} \text { and } 100^{\circ} \text { nearly. } \\
& .0949 \text { Dulong. } \\
& .0935 \text { Regnault. } \\
& .0952 \text { Regnault. } \\
& .0933 \text { Béde. } \\
& .0930 \text { Kopp. } \\
& .0940
\end{aligned}
$$

This reduced to between $0^{\circ}$ and $40^{\circ}$ by Béde's formula gives $\cdot 0922$. Hence we have the following for the calorimeter: ${ }^{24}$ -

- ${ }^{24}$ The cast brass was composed of 28 parts of copper, 2 of tin, 1 of zinc, and 1 of lead. The rolled brass was assumed to have the same composition. The solder was assumed to be made of equal parts of tin and lead. 


\begin{tabular}{|c|c|c|}
\hline & Per cent. & Specific Heat between $0^{\circ}$ and $40^{\circ} \mathrm{C}$. \\
\hline Copper & $91 \cdot 4$ & .0922 \\
\hline Zine & $\cdot 7$ & .0896 \\
\hline Tin & $3 \cdot 6$ & .0550 \\
\hline Lead & $2 \cdot \%$ & .0310 \\
\hline Steel & $1 \cdot 6$ & $\cdot 1110$ \\
\hline
\end{tabular}

The close agreement of this number with the experimental result can only be accidental, as the reduction to the air thermometer would decrease it somewhat, and so make it even lower than mine. However, the difference could not at most amount to more than 0.5 per cent, which is very satisfactory.

The total capacity of the calorimeter is reckoned as follows:-

Weight of calorimeter .......... 3712 kilogrammes.

Weight of screws ..............0016 kilogrammes.

Weight of part of suspending wires. . 0052 kilogrammes.

Total weight....3 8780 kilogrammes.

$$
\text { Capacity }=3.878 \times \cdot 0892=\cdot 3459 \text { kilogrammes. }
$$

To this must be added the capacity of the thermometer bulb and several inches of the stem, and of a tube used as a safety valve, and we must subtract the capacity of a part of the shaft which was joined to the shaft turning the paddles. Hence,

$$
\begin{aligned}
& .3459 \\
& +.0011 \\
& +.0010 \\
& \text {-.0010 } \\
& \text { Capacity }=\cdot 3470
\end{aligned}
$$

As this is only about four per cent of the total capacity, it is not necessary to consider the variation of this quantity with the temperature through the range from $0^{\circ}$ to $40^{\circ}$ which $\mathrm{I}$ have used.

\section{IV.-DETERMINATION OF EQUIVALENT}

\section{(a.) Historical Remarks}

The history of the determination of the mechanical equivalent of heat is that of thermodynamics, and as such it is impossible to give it at length here. 
I shall simply refer to the few experiments which a priori seem to possess the greatest value, and which have been made rather for the determination of the quantity than for the illustration of a method, and shall criticise them to the best of my ability, to find, if possible, the cause of the great discrepancies.

\section{General Review of Methods}

Whenever heat and mechanical energy are converted the one into the other, we are able by measuring the amounts of each to obtain the ratio. Every equation of thermodynamics proper is an equation between mechanical energy and heat, and so should be able to give us the mechanical equivalent. Besides this, we are able to measure a certain amount of electrical energy in both mechanical and heat units, and thus to also get the ratio. Chemical energy can be measured in heat units, and can also be made to produce an electric current of known mechanical energy. Indeed, we may sum up as follows the different kinds of energy whose conversion into one another may furnish us with the mechanical equivalent of heat.' And the problem in general would be the ratio by which each kind of energy may be converted into each of the others, or into mechanical or absolute units.
a. Mechanical energy.
b. Heat.
c. Electrical energy.
d. Magnetic energy.
e. Gravitation energy.
$f$. Radiant energy.
g. Chemical energy.
$h$. Capillary energy.

Of these different kinds of energy, only the first five can be measured other than by their conversion into other forms of energy, although Sir William Thomson, by the introduction of such terms as "cubic mile of sunlight," has made some progress in the case of radiation. Hence for these five only can the ratio be known.

Mechanical energy is measured by the force multiplied by the distance through which the force acts, and also by the mass of a body multiplied by half the square of its velocity. Heat is usually referred to the quantity required to raise a certain amount of water so many degrees, though hitherto the temperature of the water and the reduction to the air thermometer have heen almost neglected. 
The energy of electricity at rest is the quantity multiplied by half the potential; or of a current, it is the strength of current multiplied by the electro-motive force, and by the time; or for all attractive forces varying inversely as the square of the distance, Sir William Thomson has given the expression

$$
\frac{1}{8 \pi} \int R^{2} d v
$$

where $R$ is the resultant force at any point in space, and the integral is taken throughout space.

These last three kinds of energy are already measured in absolute measure and hence their ratios are accurately known. The only ratio, then, that remains is that of heat to one of the others, and this must be determined by experiment alone.

But although we cannot measure $f, g, h$ in general, yet we can often measure off equal amounts of energy of these kinds. Thus, although we cannot predict what quantities of heat are produced when two atoms of different substances unite, yet, when the same quantities of the same substances unite to produce the same compound, we are safe in assuming that the same quantity of chemical energy comes into play.

According to these principles, I have divided the methods into direct and indirect.

Direct methods are those where $b$ is converted directly or indirectly into $a, c, d$, or $e$, or vice versa.

Indirect methods are those where some kind of energy, as $g$, is converted into $b$, and also into $a, c, d$, or $e$.

In this classification $I$ have made the arrangement with respect to the kinds of energy which are measured, and not to the intermediate steps. Thus Joule's method with the magneto-electric machine would be classed as mechanical energy into heat, although it is first converted into electrical energy. The table does not pretend to be complete, but gives, as it were, a bird's-eye view of the subject. It could be extended by including more complicated transformations; and, indeed, the symmetrical form in which it is placed suggests many other transformations. As it stands, however, it includes all methods so far used, besides many more.

In the table of indirect methods, the kind of energy mentioned first is to be eliminated from the result by measuring it both in terms of heat and one of the other kinds of energy, whose value is known in absolute or mechanical units. 
It is to be noted that, although it is theoretically possible to measure magnetic energy in absolute units, yet it cannot be done practically with any great accuracy, and is thus useless in the determination of the equivalent. It could be thus left out from the direct methods without harm, as also out of the next to last term in the indirect methods.

\section{TABLE XXV.-Synopsis OF Methods For OBtaining the}

mechanical Equivalent of Heat.

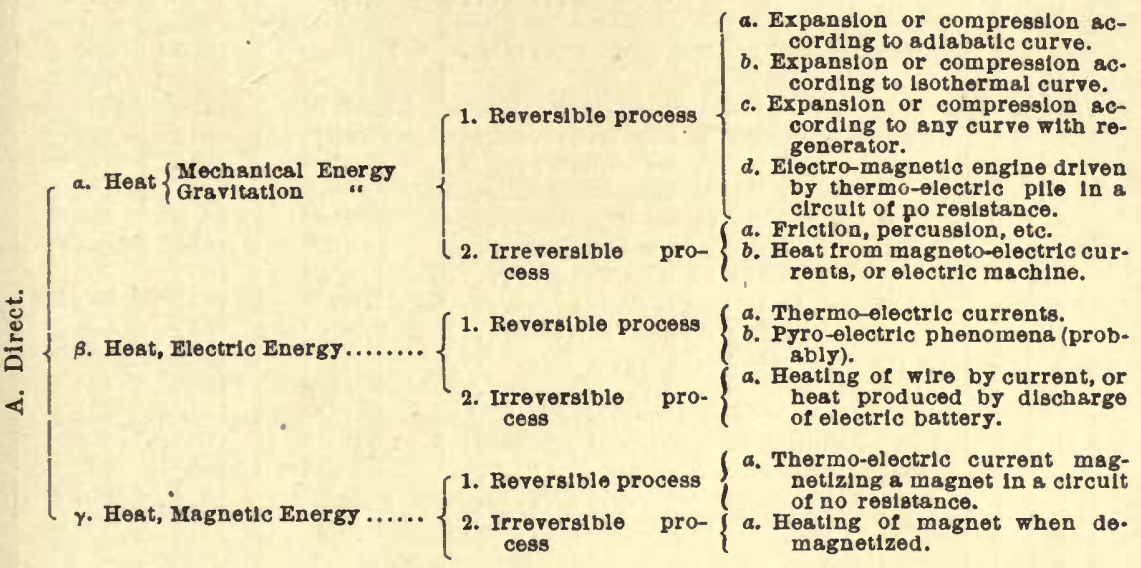

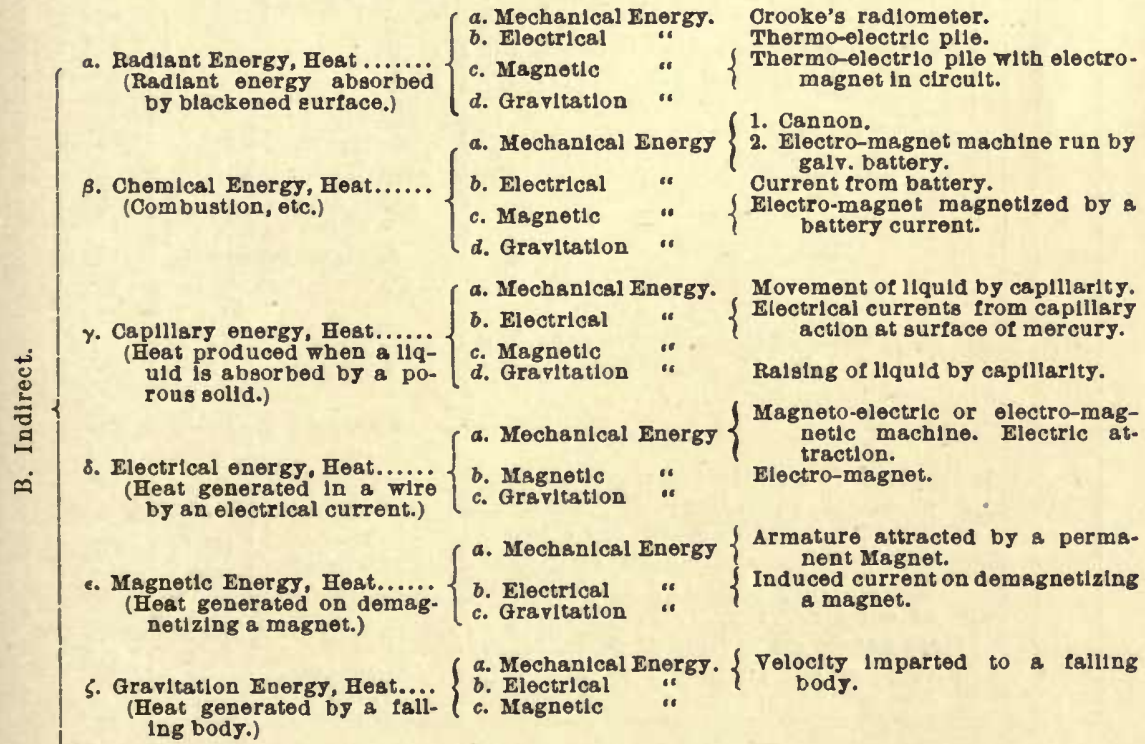


TABLE XXVI.-Historical Table of Experimental Results.

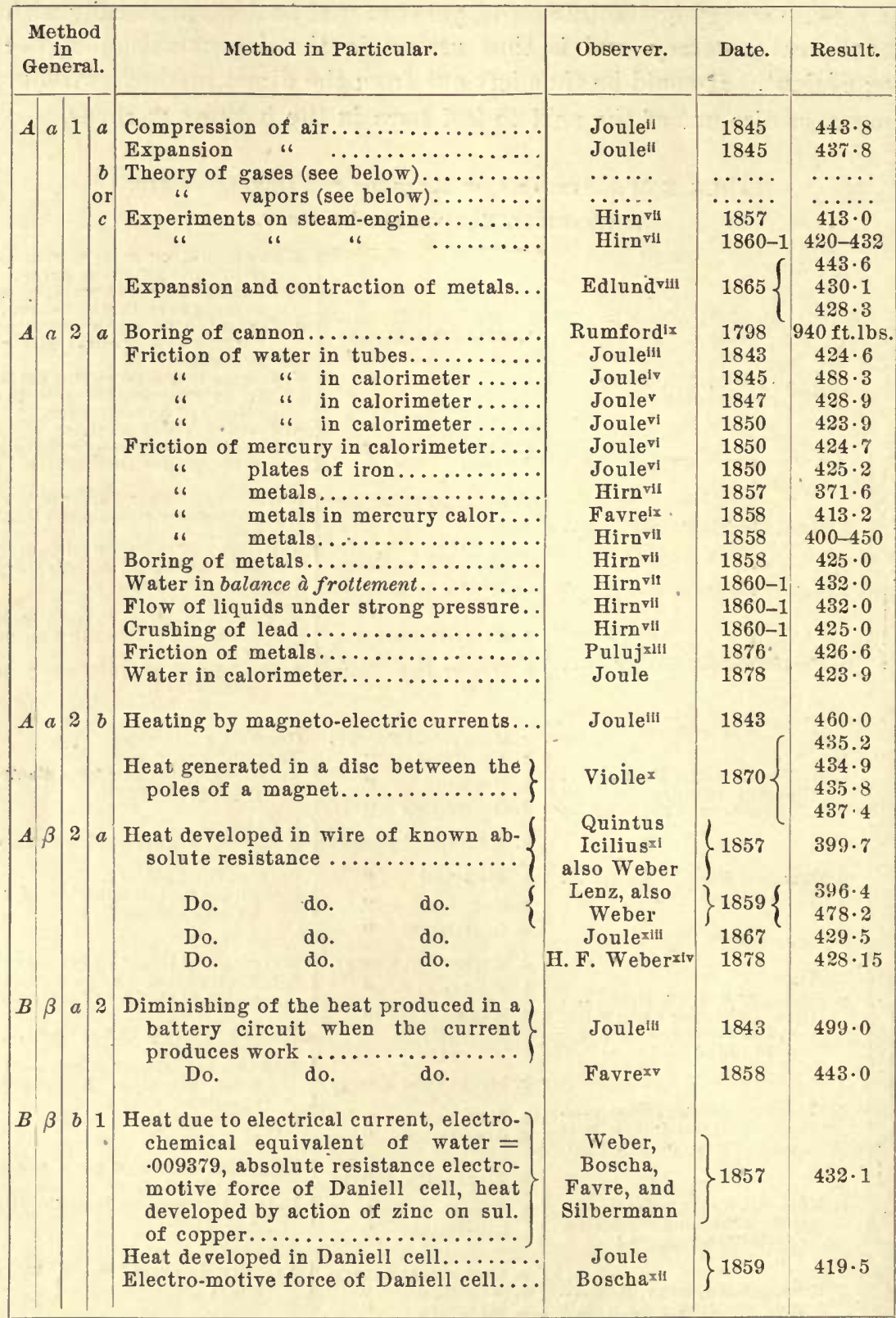




\section{Results of Best Determinations *}

On the basis of this table of methods I have arranged the following table, showing the principal results so far obtained.

In giving the indirect results, many persons have only measured one of the transformations required; and as it would lengthen out the table very much to give the complete calculation of the equivalent from these selected two by two, I have sometimes given tables of these parts. As the labor of looking up and reducing these is very great, it is very possible that there have been some omissions.

I have taken the table published by the Physical Society of Berlin, ${ }^{1}$ as the basis down to $185 \%$, though many changes have been made even within this limit.

I shall now take up some of the principal methods, and discuss them somewhat in detail.

\section{Method from Theory of Gases}

As the different constants used in this method have bren obtained by many observers, I first shall give their results.

TABLE XXVII.-SPECIFIC Heat of Gases.

\begin{tabular}{|c|c|c|c|c|c|}
\hline & $\begin{array}{c}\text { Limit to } \\
\text { Temperature. }\end{array}$ & $\begin{array}{c}\text { Approximate } \\
\text { Temperature } \\
\text { of Water. }\end{array}$ & $\begin{array}{l}\text { Temperature } \\
\text { reduced to }\end{array}$ & Specific Heat. & \\
\hline \multirow[t]{3}{*}{ Air....... } & ....... & $\ldots \ldots q$ & $\begin{array}{c}\text { Mercurial } \\
\text { Thermometer }\end{array}$ & \}$\cdot 2669 \quad\{$ & $\begin{array}{c}\text { Delaroche and } \\
\text { Bérard. }\end{array}$ \\
\hline & $20^{\circ}$ to $210^{\circ}$ & ${ }^{25} 14^{\circ} \cdot 2\{$ & $\begin{array}{c}\text { Air } \\
\text { Thermometer }\end{array}$ & \}$\cdot 23751^{x 71}$ & Regnault. \\
\hline & $20^{\circ}$ to $100^{\circ}$ & $20^{\circ}$ & $\begin{array}{c}\text { Mercurial } \\
\text { Thermometer }\end{array}$ & \}$\cdot 2389 \times \mathrm{vII}$ & E. Wiedemann. \\
\hline \multirow[t]{3}{*}{ Hydrogen... } & & & $\begin{array}{c}\text { Mercurial } \\
\text { Thermometer }\end{array}$ & \}$^{3} 3 \cdot 2936 \quad\{$ & $\begin{array}{l}\text { Delaroche and } \\
\text { Bérard. }\end{array}$ \\
\hline & $15^{\circ}$ to $200^{\circ}$ & $12^{\circ} \cdot 2\{$ & $\begin{array}{c}\text { Air } \\
\text { Thermometer }\end{array}$ & \} $3 \cdot 4090 \times \mathrm{v1}$ & Regnault. \\
\hline & $21^{\circ}$ to $100^{\circ}$ & $21^{\circ}$ & $\begin{array}{c}\text { Mercurial } \\
\text { Thermometer }\end{array}$ & \} $3 \cdot 410^{\mathrm{x} 11}$ & E. Wiedemann. \\
\hline
\end{tabular}

${ }^{25}$ Taking mean results on page 101 of Rel. des Exp., tom. ii., 
TABLE XXVIII.-Coefficient of Expansion of Air under Constant Volome

\begin{tabular}{|c|c|c|}
\hline . & $\begin{array}{l}\text { Taking Expansion of Mercury } \\
\text { according to Regnault. }\end{array}$ & $\begin{array}{l}\text { Taking Expansion of Mercury } \\
\text { according to W üllner's Re- } \\
\text { calculation of Regnault's } \\
\text { Experiments. }\end{array}$ \\
\hline 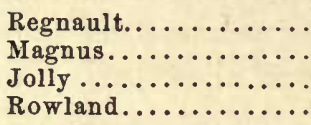 & $\begin{array}{l}.0036655 \\
.0036678 \\
.0036695 \\
.0036675\end{array}$ & $\begin{array}{l}.0036687 \\
.0036710 \\
.0036727 \\
.0036707\end{array}$ \\
\hline Mean.............. & .0036676 & .0036708 \\
\hline
\end{tabular}

TABLE XXIX. - Ratio of Specific Heats of AIR.

\begin{tabular}{|c|c|c|c|}
\hline Method. & Observer. & Date. & $\begin{array}{c}\text { Ratio } \\
\text { of } \\
\text { Hpecific } \\
\text { Heats. }\end{array}$ \\
\hline $\left.\begin{array}{l}\text { Method of Clément \& Désormes, } \\
\text { globe } 20 \text { litres............ }\end{array}\right\}$ & Clément \& $\left.{ }_{\text {Désormes }}{ }^{\mathbf{v} i l i}\right\}$ & $\begin{array}{c}1812 \\
\text { Published in } \\
1819\end{array}$ & $\{1 \cdot 354$ \\
\hline Never fully published.......... & Gay-Lussac et Welter xix. & $\ldots$ & $1 \cdot 3748$ \\
\hline Method of Clément \& Désormes.. & Delaroche et Bérardxii .. & $\ldots$ & $1 \cdot 249$ \\
\hline Using Breguet thermometer..... & Favre \& Silbermann xxil & 1853 & $1 \cdot 421$ \\
\hline $\left.\begin{array}{c}\text { Clément \& Désormes, globe }{ }^{39} \\
\quad \text { litres } \ldots \ldots \ldots \ldots \ldots \ldots \ldots\end{array}\right\}$ & Masson $^{x x} \ldots \ldots \ldots$ & 1858 & $1 \cdot 4196$ \\
\hline Clément \& Désormes............ & Weisbach ${ }^{\mathbf{x x}} \ldots$ & 1859 & $1 \cdot 4025$ \\
\hline $\left.\begin{array}{r}\text { Clément \& Désormes, globe } 10 \\
\text { litres } \ldots \ldots \ldots \ldots \ldots \ldots \ldots \ldots\end{array}\right\}$ & Hirn ${ }^{x \times 11} \ldots \ldots$ & 1861 & $1 \cdot 3845$ \\
\hline $\begin{array}{l}\text { Passage of gas from one vessel } \\
\text { into another, globes } 60 \text { litres }\end{array}$ & Cazln $x$ xiv & 1862 & $1 \cdot 41$ \\
\hline $\begin{array}{l}\text { Pressure in globe changed by } \\
\text { aspirator, globe } 25 \text { litres.... }\end{array}$ & Dupréxxv ... & 1863 & ..... \\
\hline $\left.\begin{array}{l}\text { Heating of gas by electric cur- } \\
\text { rent } \ldots \ldots \ldots \ldots \ldots \ldots \ldots \ldots \ldots \ldots\end{array}\right\}$ & Jamin \& Richardxxvil & 1864 & $1 \cdot 41$ \\
\hline Clément \& Désormes............ & Tresca et Laboulayexxix. & 1864 & .... \\
\hline $\left.\begin{array}{l}\text { Barometer nnder air-pump re- } \\
\quad \text { ceiver of } 6 \text { litres............ }\end{array}\right\}$ & Kohlrauschexvi $\ldots \ldots \ldots$ & 1869 & $1 \cdot 302$ \\
\hline $\left.\begin{array}{c}\text { Compression and expansion of } \\
\text { gas by piston................ }\end{array}\right\}$ & Regnault...... & 1871 & $\begin{array}{l}\text { Results lost } \\
\text { in the siege } \\
\text { of Paris. }\end{array}$ \\
\hline $\left.\begin{array}{l}\text { Clément \& Désormes with metal- } \\
\text { lic manometer, globe } 70 \text { litres }\end{array}\right\}$ & Röntgen ${ }^{\mathbf{x x} \| l} \ldots$ & 1873 & $1 \cdot 4053$ \\
\hline Compression of gas by piston... & Amagatxxx........ & 1874 & $1 \cdot 397$ \\
\hline
\end{tabular}


ON the Mechanical Equivalent of Heat

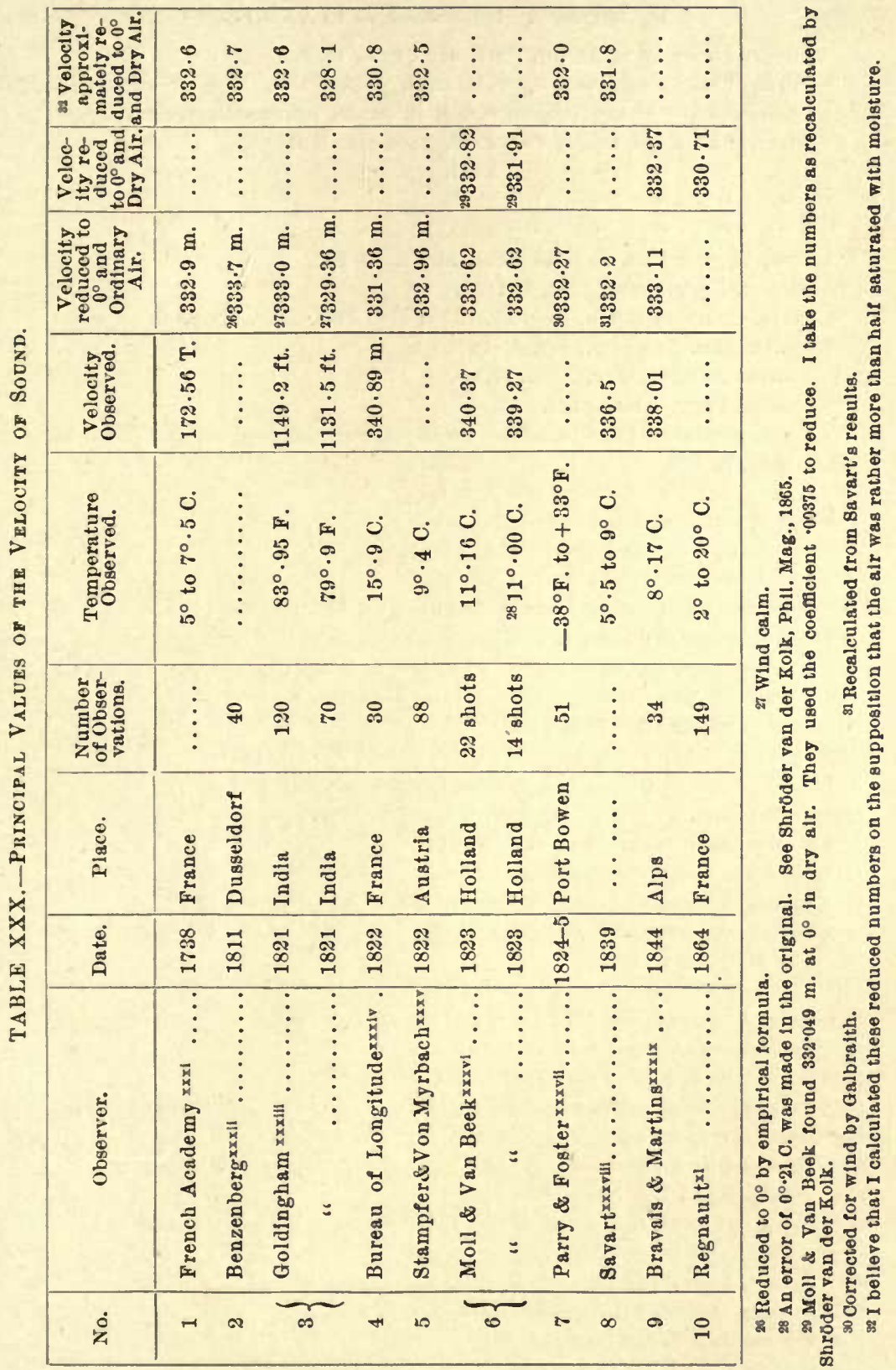




\section{References. (Tables XXVI to XXX.)}

I Physical Society of Berlin, Fort. der Phys., 1858.

it Joule, Phil. Mag., ser. 3, vol. xxvi. See also Mec. Wärmeäquivalent, Gesammelte Abhandlungen von J. P. Joule, Braunschweig, 1872.

iit Joule, Phil. Mag., ser. 3, vol. xxiii. See also 2 above.

iv " " " "

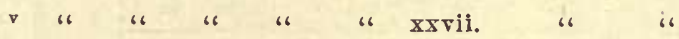

vi " " " "

vil Hirn, Théorie Méc. de la Chaleur, ser. 1, $3^{\text {me }}$ ed.

vill Edlund, Pogg. Ann., cxiv. 1, 1865.

ix Favre, Comptes Rend., Feb. 15, 1858; also Phil. Mag., xv. 406.

× Violle, Ann. de Chim., ser. 4, xxii. 64.

xi Quintus Icilius, Pogg. Ann., ci. 69.

sil Boscha, Pogg. Ann., cviii. 162.

xlil Joule, Report of the Committee on Electrical Standards of the B. A., I.ondon, 1873, p. 175.

xiv H. F. Weber, Phil. Mag., ser. 5, v. 30.

$x$ Favre, Comptes Rend., xlvii. 599.

× ${ }^{*}$ Regnault, Rel. des Expériences, tom. ii.

zvil E. Wiedemann, Pogg. Ann., clvii. 1.

xvill Clément et Désormes, Journal de Physique, Ixxxix. 333, 1819.

xix Laplace, Méc. Céleste, v. 125.

$\mathrm{xx}$ Masson, Ann. de Chim. et de Phys., ser. 3, tom. liii.

×xI Weisbach, Der Civilingenieur, Neue Folge, Bd. v., 1859.

xxil Hirn, Théorie Méc. de la Chaleur, i, 111.

xxill Favre et Silbermann, Ann. de Chim., ser. 3, xxxrii. 1851.

xxlv Cazin, Ann. de Chim., ser. 3, tom. Ixvi.

Ixv Dupré, Ann. de Chim., 3me ser., lxvii. 359, 1863.

xxvi Kohlrausch, Pogg. Ann., cxxxvi. 618.

xxvil Röntgen, Pogg. Ann., cxlviii. 603.

xxvill Jamin et Richard, Comptes Rend., lxxi. 336.

xxix Tresca et Laboulaye, Comptes Rend., Iviii. 358. Ann. du Conserv. des Arts et Métiers, vi. 365 .

xIx Amagat, Comptes Rend., 1xxvii. 1325.

xxx Mém. de l'Acad. des Sci., 1738, p. 128.

rxxil Benzenberg, Gilbert's Annalen, xlii. 1.

xxzll! Goldingham, Phil. Trans., 1823, p. 96.

xzxiv Ann. de Chim., 1822, xx. 210 also, Euvres de Arago, Mém. Sci., ii. 1.

xxxv Stampfer und Von Myrbach, Pogg. Ann., v. 496.

xxxvl Moll and Van Beek, Phil. Trans., 1824, p.424. See also Shröder van der Kolk, Phil. Mag., 1865.

xxxvil Parry and Foster, Journal of the Third Voyage, 1824-5, Appendix, p. 86. Phil. Trans., 1828, p. 97.

xxxvili Savart, Ann. de Chim., ser. 2, Ixxi. 20. Recalculated.

xxxix Bravais et Martins, Ann. de Chim., ser. 3, xiii. 5 .

xs Regnault, Rel. des Exp., iii. 533.

xll Delaroche et Bérard, Ann. de Chim., lxxxv. 72 and 113.

xili Puluj, Pogg. Ann., clvii. 656. 
Estimating the weight rather arbitrarily, I have combined them as follows:

\begin{tabular}{|c|c|c|}
\hline No. & $\begin{array}{l}\text { Velocity at } 0^{\circ} \cdot \mathrm{C} . \\
\text { Dry Air. }\end{array}$ & $\begin{array}{l}\text { Estimated Weight } \\
\text { of Observation. }\end{array}$ \\
\hline 1 & $332 \cdot 6$ & 2 \\
\hline 2 & $332 \cdot 7$ & 2 \\
\hline 3 & $330 \cdot 9$ & .2 \\
\hline 4 & $330 \cdot 8$ & 4 \\
\hline 5 & $332 \cdot 5$ & 3 \\
\hline 6 & $332 \cdot 8$ & 7 \\
\hline 7 & $332 \cdot 0$ & 1 \\
\hline 8 . & $331 \cdot 8$ & 1 \\
\hline 9 & $332 \cdot 4$ & 4 \\
\hline 10 & $330 \cdot 7$ & 10 \\
\hline & $331 \cdot 75$ & \\
\hline
\end{tabular}

Or, corrected for the normal carbonic acid in the atmosphere, it becomes 331.78 metres per second in dry pure air at $0^{\circ} \mathrm{C}$.

From Regnault's experiments on the velocity in pipes I find by graphical means $331.4 \mathrm{~m}$. in free air, which is very similar to the above.

\section{Calculation from Properties of Gases}

$K=$ specific heat of gas at constant pressure.

$k=$ specific heat of gas at constant volume.

$p=$ pressure in absolute units of a unit of mass.

$v=$ volume in absolute units of a unit of mass.

$\mu=$ absolute temperature.

$J=$ Joule's equivalent in absolute measure.

$r=\frac{K}{k}$.

General formula for all bodies:

$$
\begin{aligned}
r & =\frac{1}{1-\frac{\mu}{J K}\left(\frac{d p}{d_{\mu}}\right)_{v}\left(\frac{d v}{d \mu}\right)_{p}} \\
r & =-\frac{V^{2}}{v^{2}}\left(\frac{d v}{d p}\right)_{\mu} \\
\therefore \quad J & =\frac{\mu}{K}\left(\frac{d p}{d^{\mu}}\right)_{v}\left(\frac{d v}{d \mu}\right) \frac{\gamma}{\gamma-1} .
\end{aligned}
$$


Also,

$$
J=-\frac{\mu}{K} \frac{\left(\frac{d v}{d \mu}\right)_{p}^{2}}{\left(\frac{d v}{d p}\right)_{\mu}+\frac{v^{2}}{V^{2}}} .
$$

Application to gases; Ranline's formula is,-

$$
\begin{aligned}
p v & =R\left(\mu-m \frac{\mu_{0}}{\mu} \frac{v_{0}}{v}\right), \\
\left(\frac{d p}{d \mu}\right)_{v} & =\frac{p}{\mu}\left(1+2 m \frac{\mu_{0}}{\mu} \frac{v_{0}}{v}\right), \\
\left(\frac{d v}{d \mu}\right)_{p} & =\frac{v}{\mu}\left(1+3 m \frac{\mu_{0}}{\mu^{2}} \frac{v_{0}}{v}\right), \\
\left(\frac{a v}{d p}\right)_{\mu} & =-R \frac{\mu}{p^{2}}=-\frac{p_{0} v_{0}}{\mu_{0}} \frac{\mu}{p^{2}}\left(1+\frac{m}{\mu_{0}}\right) .
\end{aligned}
$$

If $\alpha_{v}$ is the coefficient of expansion between $0^{\circ}$ and $100^{\circ}$, then

whence

$$
\mu_{0}=\frac{1}{a_{v}}(1+\cdot 00635 m) \text {, }
$$

$$
J=\frac{p v \mu}{K} a_{p}^{\prime} \alpha_{v}^{\prime}\left(\frac{r}{\gamma-1}\right),
$$

where $a_{p}^{\prime}$ and $\alpha_{v}^{\prime}$ are the true coefficients of expansion at the given temperature;

$$
\begin{aligned}
\therefore \quad J & =\frac{p v}{K \mu}\left(1+5 m \frac{\mu_{0}}{\mu^{2}} \frac{v_{0}}{v}\right) \frac{r}{r-1} ; \\
J & =\frac{1}{K \mu} \frac{1+6 m \frac{\mu_{0}}{\mu^{2}} \frac{v_{0}}{v}}{\frac{p_{0} v_{0} \rho_{\mu}}{p^{2} v^{2} \mu_{0}}\left(1+\frac{m}{\mu_{0}}\right)-\frac{1}{V^{2}}\left(\frac{v_{0}}{v}\right)^{2}} .
\end{aligned}
$$

According to Thomson and Joule's experiments $m=0^{\circ} \cdot 33 \mathrm{C}$. for air and about $2^{\circ} \cdot 0$ for $\mathrm{CO}_{2}$. Hence $\mu_{0}=272^{\circ} \cdot 99$.

The equations should be applied to the observations directly at the given temperature, but it will generally be sufficient to use them after reduction to $0^{\circ} \mathrm{C}$. Using $K=\cdot 2375$ according to Regnault for air, we have for the latitude of Baltimore,-

$$
\begin{array}{rlrl}
\text { From Röntgen's value } r=1 \cdot 4053 & \frac{J}{g}=430 \cdot 3 \cdot{ }^{33} \\
\text { “ Amagat's " } & 1 \cdot 397 & \frac{J}{g}=436 \cdot 6 . \\
\text { " velocity of sound } 331 \cdot 78 \mathrm{~m} \text {. per sec. } \frac{J}{g}=429 \cdot 6 .
\end{array}
$$

${ }^{33}$ Röntgen gives the value $428 \cdot 1$ for the latitude of Paris as calculated by a formula of Shröder v. d. Kolk, and $427 \cdot 3$ from the formula for a perfect gas, and these both agree more nearly with my result than that calculated from my own formula. 
Using Wiedemann's value for $K, \cdot 2389$, these become

$$
\frac{J}{g}=427 \cdot 8 ; \quad \frac{J}{g}=434 \cdot 0 ; \quad \frac{J}{g}=427 \cdot 1 .
$$

As Wiedemann, however, used the mercurial thermometer, and as the reduction to the air thermometer would increase these figures from $\cdot 2$ to $\cdot 8$ per cent, it is evident that Regnault's value for $K$ is the more nearly correct. I take the weights rather arbitrarily as follows:

$\begin{array}{lcr} & \text { Weight. } & J . \\ \text { Röntgen } & 3 & 430 \cdot 3 \\ \text { Amagat } & 1 & 436 \cdot 6 \\ \text { Velocity of sound } & 4 & 429 \cdot 6 \\ & & \text { Mean } 430 \cdot 7\end{array}$

And this is of course the value referred to water at $14^{\circ} \mathrm{C}$. and in the latitude of Baltimore. My value at this point is $42 \% \cdot \%$.

This determination of the mechanical equivalent from the properties of air is at most very imperfect, as a very slight change in either $\gamma$ or the velocity of sound will produce a great change in the mechanical equivalent.

\section{From Theory of Vapors}

Another important method of calculating the mechanical equivalent of heat is from the equation for a body at its change of state, as for instance in vaporization. Let $v$ be the volume of the vapor, and $v^{\prime}$ the volume of the liquid, $H$ the heat required to vaporize a unit of mass of the water; also let $p$ be the pressure in absolute units, and $\mu$ the absolute temperature. Then

$$
v-v^{\prime}=\frac{J H}{\mu\left(\frac{d p}{d \mu}\right)} .
$$

The quantity $H$ and the relation of $p$ to $\mu$ have been determined with considerable accuracy by Regnault. To determine $J$ it is only required to measure the volume of saturated steam from a given weight of water; and the principal difficulty of the process lies in this determination, though the other quantities are also difficult of determination.

This volume can be calculated from the density of the vapor, but this is generally taken in the superheated state. 
The experiments of Fairbairn and Tate ${ }^{34}$ are probably the best direct experiments on the density of saturated vapor, but even those do not pretend to a greater accuracy than about 1 in 100. With Regnault's values of the other quantities, they give about Joule's value for the equivalent, namely 425. Hirn, Herwig, and others have also made the determination, but the results do not agree very well. Herwig even nsed a Geissler standard thermometer, which I have shown to depart very much from the air thermometer.

Indeed, the experiments on this subject are so uncertain, that physicists have about concluded to use this method rather for the determination of the volume of saturated vapors than for the mechanical equivalent of heat.

\section{From the Steam-Engine and Expansion of Metals}

The experiments of Hirn on the steam-engine and of Edlund on the expansion and contraction of metals, are very excellent as illustrating the theory of the subject, but cannot have any weight as accurate determinations of the equivalent.

\section{From Friction Experiments}

Experiments of this nature, that is, irreversible processes for converting mechanical energy into heat, give by far the best methods for the determination of the equivalent.

Rumford's experiment of 1798 is only valuable from an historical point of view. Joule's results since 1843 undoubtedly give the best data we yet have for the determination of the equivalent. The mean of all his friction experiments of 1847 and 1850 which are given in the table is $425 \cdot 8$, though he prefers the smallest number, $423 \cdot 9$, of 1850 . This last number is at present accepted throughout the civilized world, though there is at present a tendency to consider the number too small. But this value and his recent result of 1878 have undoubtedly as much weight as all other results put together.

As sources of error in these determinations I would suggest, first, the use of the mercurial instead of the air thermometer. Joule compared his thermometers with one made by Fastré. In the Appendix to Thermometry I give the comparison of two thermometers made by Fastré in 1850, with the air thermometer, as well as of a large number of others. From this it seems that all thermometers as far as measured

${ }^{34}$ Phil. Mag., ser. 4, xxí, 230. 
stand above the air thermometer between $0^{\circ}$ and $100^{\circ}$, and that the average for the Fastre at $40^{\circ}$ is about $0^{\circ} \cdot 1 \mathrm{C}$. Using the formula given in Thermometry this would produce an error of about 3 parts in 1000 at $15^{\circ} \mathrm{C}$., the temperature Joule used.

The specific heat of copper which Joule uses, namely, 09515 , is undoubtedly too large. Using the value deduced from more recent experiments in calculating the capacity of my calorimeter, .0922, Joule's number would again be increased 13 parts in 10,000 , so that we have, -

Joule's value $\ldots \ldots \ldots \ldots \ldots \ldots \ldots+423 \cdot 9$, water at $15^{\circ} \cdot 7 \mathrm{C}$.

Reduction to air thermometer.......+1.3

Correction for specific heat of copper... $\cdot 5$

Correction to latitude of Baltimore.. . $+\cdot 5$

$426 \cdot 2$

It does not seem improbable that this should be still further increased, seeing that the reduction to the air thermometer is the smallest admissible, as most other thermometers which I have measured give greater correction, and some even more than three times as great as the one here used, and would thus bring the value even as high as 429 .

One very serious defect in Joule's experiments is the small range of temperature used, this being only about half a degree Fahrenheit, or about six divisions on his thermometer. It would seem almost impossible to calibrate a thermometer so accurately that six divisions should be accurate to one per cent, and it would certainly need a very skillful observer to read to that degree of accuracy. Further, the same thermometer " $A$ " was used throughout the whole experiment with water, and so the error of calibration was hardly eliminated, the temperature of the water being nearly the same. In the experiment on quicksilver another thermometer was used, and he then finds a higher result, $424 \cdot 7$, which, reduced as above, gives $42 \% \cdot 0$ at Baltimore.

The experiments on the friction of iron should be probably rejected on account of the large and uncertain correction for the energy given out in sound.

The recent experiments of 1878 give a value of 7772.55 , which reduced gives at Baltimore $426 \cdot 2$, the same as the other experiment.

The agreement of these reduced values with my value at the same temperature, namely $42 \% \cdot 3$, is certainly very remarkable, and shows what an accurate experimenter Joule must be to get with his simple 27 
apparatus results so near those from my elaborate apparatus, which almost grinds out accurate results without labor except in reduction. Indeed, the quantity is the same as I find at about $20^{\circ} \mathrm{C}$.

The experiments of Hirn of $1860-61$ seem to point to a value of the equivalent higher than that found by Joule, but the details of the experiment do not seem to have been published, and they certainly were not reduced to the air thermometer.

The method used by Violle in 1870 does not seem capable of accuracy, seeing that the heat lost by a disc in rapid rotation, and while carried to the calorimeter, must have been uncertain.

The experiments of Hirn are of much interest from the methods used, but can hardly have weight as accurate determinations. Some of the methods will be again referred to when I come to the description of apparatus.

\section{Method by Heat Generated by Electric Current}

The old experiments of Quintus Icilius or Lenz do not have any except historical value, seeing that Weber's measure of absolute resistance was certainly incorrect and we now have no means of finding its error.

The theory of the process is as follows. The energy of electricity being the product of the potential by the quantity, the energy expended by forcing the quantity of electricity, $Q$, along a wire of resistance, $R$, in a second of time, must be $Q^{2} R$, and as this must equal the mechanical equivalent of the heat generated, we must have $J H=$ $Q^{2} R t$, where $H$ is the heat generated and $t$ is the time the current $Q$ flows.

The principal difficulty about the determination by this method seems to be that of finding $R$ in absolute measure. A table of the values of the ohm as obtained by different observers, was published by me in my paper on the 'Absolute Unit of Electrical Resistance,' in the American Journal of Science, Vol. XV, and I give it here with some changes.

The ratio of the Siemens unit to the ohm is now generally taken at .9536, though previous to 1864 there seems to have been some doubt as to the value of the Siemens unit.

Since 1863-4, when units of resistance first began to be made with great accuracy, two determinations of the heat generated have been made. The first by Joule with the ohm, and the second by H. F. Weber, of Zurich, with the Siemens unit. 
Each determination of resistance with each of these experiments gives one value of the mechanical equivalent. As Lorenz's result was only in illustration of a method, I have not included it among the exact determinations.

TABLE XXXI.

\begin{tabular}{|c|c|c|c|}
\hline Date. & Observer. & Value of Ohm. & Remarks. \\
\hline 1849 & Kirchhoff & .88 to $\cdot 90$ & Approximately. \\
\hline 1851 & Weber & .95 to $\cdot 97$ & Approximately. \\
\hline 1862 & Weber & $\left\{\begin{array}{l}1.088 \\
1.075\end{array}\right.$ & $\begin{array}{l}\text { From Thomson's unit. } \\
\text { From Weber's value of Stemens unit. }\end{array}$ \\
\hline $1863-4$ & B. A. Committee & $\left\{\begin{array}{c}1 \cdot 0000 \\
\cdot 993\end{array}\right.$ & $\begin{array}{l}\text { Mean of all results. } \\
\text { Corrected by Rowland to zero vel- } \\
\text { ocity of coll. }\end{array}$ \\
\hline 1870 & Kohlrausch & 1.0193 & \\
\hline 1873 & Loren & .975 & Approximately. \\
\hline 1876 & Rowland & $.9911^{35}$ & $\begin{array}{l}\text { From a preliminary comparison with } \\
\text { the B. A. unit. }\end{array}$ \\
\hline 1878 & H. F. Weber & 1.0014 & $\begin{array}{l}\text { Using ratio of Siemens unit to ohm, } \\
\quad .9536 \text {. }\end{array}$ \\
\hline
\end{tabular}

The result found by Joule was $J=2518 \%$ in absolute measure using feet and degrees F., which becomes $429 \cdot 9$ in degrees C. on a mercurial thermometer and in the latitude of Baltimore, compared with water at $18^{\circ} \cdot 6 \mathrm{C}$.

TABLE XXXII.-EXPERIMENTS OF JOULE.

\begin{tabular}{|c|c|c|c|}
\hline Observer. & $\begin{array}{l}\text { Value of } \\
\text { B. A. Unit. }\end{array}$ & $\begin{array}{l}\text { Mechanical equivalent } \\
\text { from Joule's Exp. }\end{array}$ & $\begin{array}{l}\text { Mechanical equivalent } \\
\text { reduced to Air Ther- } \\
\text { mometer and cor- } \\
\text { rected for sp. Ht. of } \\
\text { Copper. }\end{array}$ \\
\hline B. A. Committec ......... & $1 \cdot 0000$ & $429 \cdot 9$ & $431 \cdot 4$ \\
\hline Ditto corrected by Rowland & .993 & $426 \cdot 9$ & $428 \cdot 4$ \\
\hline Kohlrausch............. & $1 \cdot 0193$ & $438 \cdot 2$ & $439 \cdot 7$ \\
\hline Rowland $\ldots \ldots \ldots \ldots \ldots$ & .9911 & $426 \cdot 1$ & $427 \cdot 6$ \\
\hline H. F. Weber........... & $1 \cdot 0014$ & $430 \cdot 5$ & $432 \cdot 0$ \\
\hline
\end{tabular}

The experiments of $\mathrm{H}$. F. Weber ${ }^{36}$ gave 428.15 in the latitude of Zurich and for $1^{\circ} \mathrm{C}$. on the air thermometer and at a temperature of $18^{\circ} \mathrm{C}$. This reduced to the latitude of Baltimore gives $428 \cdot 45$.

My own value at this temperature is $426 \cdot 8$, which agrees almost exactly with the fourth value from my own determination of the absolute unit. ${ }^{37}$

35 Given .9912 by mistake in the other tables.

36 Phil. Mag., 1878, 5th ser., v. 135.

s7 The value of the ohm fonnd by reversing the calculation would be 992 , almost exactly my valne. 
There can be no doubt that Joule's result is most exact, and hence I have given his results twice the weight of Weber's. Weber used a wire of about 14 ohms' resistance, and a small calorimeter holding only 250 grammes of water. This wire was apparently placed in the water without any insulating coating, and yet current enough was sent through it to heat the water $15^{\circ}$ during the experiment. No precaution seems to have been taken as to the current passing into the water, which Joule accurately investigated. Again, the water does not seem to have been continuously stirred, which Joule found necessary. And further, Newton's law of cooling does not apply to so great a range as $15^{\circ}$, though the error from this source was probably small. Further-

TABLE XXXIII.

\begin{tabular}{|c|c|c|c|}
\hline \multicolumn{3}{|c|}{ EXPERIMENTS OF H. F. WEBER. } & \multirow{2}{*}{$\begin{array}{l}\text { Mean of Joule and } \\
\text { Weber, giving Joul } \\
\text { twice the Weight of } \\
\text { Weber. } \\
\begin{array}{l}\text { Mean equivalent re- } \\
\text { duced to Air Ther } \\
\text { mometer in the Lati- } \\
\text { tude of Baltimore. }\end{array}\end{array}$} \\
\hline Observer. & $\begin{array}{l}\text { Value of } \\
\text { B. A. Unit. }\end{array}$ & $\begin{array}{c}\text { Mechanical equivalent } \\
\text { of Heat from Weber's } \\
\text { Experiments. }\end{array}$ & \\
\hline B. A. Committee.......... & $1 \cdot 000$ & $427 \cdot 9$ & $430 \cdot 2$ \\
\hline Ditto corrected by Rowland & .993 & $424 \cdot 9$ & $427 \cdot 2$ \\
\hline Kohlrausch............... & 1.0193 & $436 \cdot 2$ & $439 \cdot 1$ \\
\hline Rowland $\ldots \ldots \ldots \ldots \ldots$ & .9911 & $424 \cdot 1$ & $426 \cdot 4$ \\
\hline H. F. Weber...... & 1.0014 & $428 \cdot 5$ & $431 \cdot 4$ \\
\hline
\end{tabular}

more, I know of no platinum which has an increase of coefficient of .001054 for $1^{\circ} \mathrm{C}$., but it is usually given at about $\cdot 003$.

There can be no doubt that experiments depending on the heating of a wire give too small a value of the equivalent, seeing that the temperature of the wire during the heating must always be higher than that of the water surrounding it, and hence more heat will be generated than there should be. Hence the numbers should be slightly increased. Joule used wire of platinum-silver alloy, and Weber platinum wire, which may account for Weber's finding a smaller value than Joule, and Weber's value would be more in error than Joule's. Undoubtedly this is a serious source of error, and I am about to repeat an experiment of this kind in which it is entirely avoided. Considering this source of error, these experiments confirm both my value of the $\mathrm{ohm}$ and of the mechanical equivalent, and unquestionably show a large error in Kohlrausch's absolute value of the Siemens unit or ohm. 
The experiments of Joule and Favre, where the heat generated by a current, both when it does mechanical work and when it does not, are very interesting, but can hardly have any weight in an estimation of the true value of the equivalent.

The method of calculating the equivalent from the chemical action in a battery, or the electro-motive force required to decompose any substance, such as water, is as follows:

Let $E$ be such electro-motive force and $c$ be the quantity of chemical substance formed in battery or decomposed in voltameter per second. Then total energy of current of energy per second is $E Q$, where $Q$ is the current, or $c Q H J$, where $H$ is the heat generated by unit of $c$, or required to decompose unit of $c$. Hence, if the process is entirely reversible, we must have in either case

$$
C H J=E \text {. }
$$

But the process is not always reversible, seeing that it requires more electro-motive force to decompose water than is given by a gas battery. This is probably due to the formation at first of some unstable compound like ozone. The process with a battery seems to be best, and we can thus apply it to the Daniell cell. The following quantities are mostly taken from Kohlrausch.

The quantity $c$ has been found by various observers, and Kohlrausch ${ }^{38}$ gives the mean value as .009421 for water according to his units (mg., $\mathrm{mm}$., second system). Therefore for hydrogen it is $\cdot 00104 \%$.

The quantity $H$ can be observed directly by short-circuiting the battery, or can be found from experiments like those of Favre and Silbermann.

The electro-motive force $E$ can be made to depend either upon the absolute measure of resistance, or can be determined, as Thomson has done, in electro-static units. In electro-magnetic units it is

$\begin{array}{cccc}\text { After Waltenhofen } & \text { Siemens. } & \text { Ohms. } & \begin{array}{c}\text { Absolute Measure } \\ \text { according to my } \\ \text { Determination. }\end{array} \\ \text { " Kohlrausch } & 11.43 & 10.90 & 10.80 \times 10^{10} \\ & 11.71 & 11.17 & 11.07 \times 10^{10}\end{array}$

After Favre, 1 equivalent of zinc developes in the Daniell cell 23993 heat units;

$$
\therefore \frac{J}{g}=\frac{E}{c H g} \text {. }
$$

${ }^{38}$ Pogg. Ann., cxlix, 179.

${ }^{39}$ Given by Kohlrausch, Pogg. Ann., cxlix, 182. 
On the mg., mm., second system, we have $E=10.935 \times 10^{10}, c=$ $.00104 \%, H=23993, g=9800.5$ at Baltimore.

$$
\therefore \quad \frac{J}{g}=444160 \mathrm{~mm} .=444 \cdot 2 \text { metres. }
$$

Using Kohlrausch's value for absolute resistance, he finds $456 \cdot 5$, which is much more in error than that from my determination. I do not give the calculation from the Grove battery, because the Grove battery is not reversible, and action takes place in it even when no current flows.

Thomson finds the difference of potential between the poles of a Daniell cell in electro-static measure to be 00374 on the $\mathrm{cm}$., grm., second system. ${ }^{40}$ Using the ratio $29,900,000,000 \mathrm{~cm}$. per second, as I have recently found, but not yet published, we have $111,800,000$ on the electro-magnetic system or $11.18 \times 10^{10}$ on the mm., mg., second system. This gives

$$
\frac{J}{g}=474.3 \text { metres. }
$$

\section{General Criticism}

All the results so far obtained, except those of Joule, seem to be of the crudest description; and even when care was apparently taken in the experiment, the method seems to be defective, or the determination is made to rest upon the determination of some other constant whose value is not accurately known. Again, only one or two observers have compared their thermometers with the air thermometer, although I have shown in 'Thermometry' that an error of more than one per cent may be made by this method. The range of temperature is also small as a general rule and the specific heat of water is assumed constant.

Hence a new determination, aroiding these sources of error, seems to be imperatively demanded.

\section{(b.) Description of Apparatus}

1. Premiminary Remarks

As we have seen in the historical portion, the only experiments of a high degree of accuracy to the present time are those of Joule. Looked at from a general point of view, the principal defects of his method were the use of the mercurial instead of the air thermometer, and the small rate at which the temperature of his calorimeter rose.

40 Thomson, Papers on Electrostatics and Magnetism, p. 246. 
In devising a new method a great rise of temperature in a short time was considered to be the great point, combined, of course, with an accurate measurement of the work done. For a great rise of temperature great work must be done, which necessitates the use of a steam-engine or other motive power. For the measurement of the work done, there is only one principle in use at present, which is, that the work transmitted by any shaft in a given time is equal to $2 \pi$ times the product of the moment of the force by the number of revolutions of the shaft in that time.

In mechanics it is common to measure the amount of the force twisting the shaft by breaking it at the given point, and attaching the two ends together by some arrangement of springs whose stretching gives the moment. Morin's dynamometer is an example. Hirn ${ }^{41}$ gives a method which he seems to consider new, but which is immediately recognized as Huyghens's arrangement for winding clocks without stopping them. As cords and pulleys are used which may slip on each other, it cannot possess much accuracy. I have devised a method by cogwheels which is more accurate, but which is better adapted for use in the machine-shop than for scientific experimentation.

But the most accurate method known to engineers for measuring the work of an engine is that of White's friction brake, and on this I have based my apparatus. Hirn was the first to use this principle in determining the mechanical equivalent of heat. In his experiment a horizontal axis was turned by a steam-engine. On the axis was a pulley with a flat surface, on which rested a piece of bronze which was to be heated by the friction. The moment of the force with which the friction tended to turn the piece of bronze was measured, together with the velocity of revolution. This experiment, which Hirn calls a balance de frottement, was first constructed by him to test the quality of oils used in the industrial arts. He experimented by passing a current of water through the apparatus and observing the temperature of the water before and after passing through. He thus obtained a rough approximation to Joule's equivalent.

He afterward constructed an apparatus consisting of two cylinders about $30 \mathrm{~cm}$. in diameter and $100 \mathrm{~cm}$. long, turning one within the other, the annular space between which could be filled with water, or through which a stream of water could be made to flow whose temperature could be measured before and after. The work was measured by the same method as before. 
But in neither of these methods does Hirn seem to have recognized the principle of the work transmitted by a shaft being equal to the moment of the force multiplied by the angle of rotation of the shaft. In designing his apparatus, he evidently had in view the reproduction in circular motion of the case of friction between two planes in linear motion.

Since I designed my apparatus, Puluj ${ }^{42}$ has designed an instrument to be worked by hand, and based on the principle used by Hirn. He places the revolving axis vertical, and the friction part consists of two cones rubbing together. But no new principle is involved in his apparatus further than in that used by Hirn.

In my apparatus one of the new features has been the introduction of the Joule calorimeter in the place of the friction cylinders of Hirn or the cones of Puluj. At first sight the currents and whirlpools in such a calorimeter might be supposed to have some effect; but when the motion is steady, it is readily seen that the torsion of the calorimeter is equal to that of the shaft, and hence the principle must apply.

This change, together with the other new features in the experiments and apparatus, has at once made the method one of extreme accuracy, surpassing all others very many fold.

\section{General Description}

The apparatus was situated in a small building, entirely separate from the other University buildings, and where it was free from disturbances.

Fig. 6 gives a general view of the apparatus. To a movable axis, $a b$, a calorimeter similar to Joule's is attached, and the whole is suspended by a torsion wire, $c$. The shaft of the calorimeter comes out from the bottom, and is attached to a shaft, ef, which receives a uniform motion from the engine by means of the bevel wheels $g$ and $h$. To the axis, $a b$, an accurate turned wheel, $k l$, was attached, and the moment of the force tending to turn the calorimeter was measured by the weights $o$ and $p$, attached to silk tapes passing around the circumference of the wheel in combination with the torsion of the suspending wire. To this axis was also attached a long arm, having two sliding weights, $q$ and $r$, by which the moment of inertia could be varied or determined.

42 Pogg. Ann., clvii, 437.

43 Jonle's latest results were published after this was written, and I was not aware that he had made this improvement until lately. The result of his experiment, however, reached me soon after, and I have referred to it in the paper, but I did not see the complete paper nntil mnch later. 


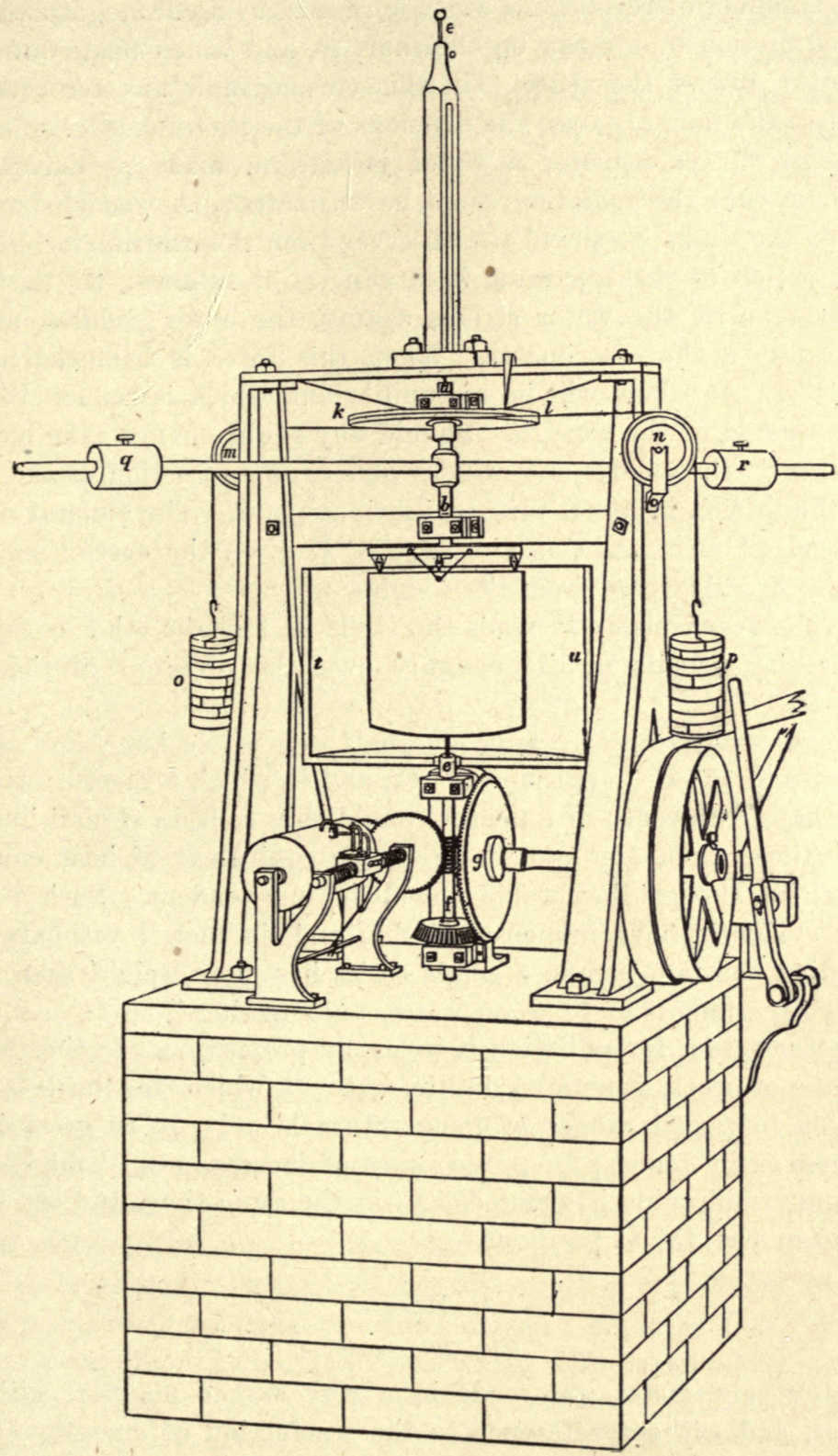

FIG. 6. 
The number of revolutions was determined by a chronograph, which received motion by a screw on the shaft ef, and which made one revolution for 102 of the shaft. On this chronograph was recorded the transit of the mercury over the divisions of the thermometer.

Around the calorimeter a water jacket, $t u$, made in halves, was placed, so that the radiation could be estimated. A wooden box surrounded the whole, to shield the observer from the calorimeter.

The action of the apparatus is in general as follows: As the inner paddles revolve, the water strikes against the outer paddles, and so tends to turn the calorimeter. When this force is balanced by the weights $o p$, the whole will be in equilibrium, which is rendered stable by the torsion of the wire $c d$. Should any slight change take place in the velocity, the calorimeter will revolve in one direction or the other until the torsion brings it into equilibrium again. The amount of torsion read off on a scale on the edge of $k l$ gives the correction to be added to or subtracted from the weights $o p$.

One observer constantly reads the circle $k l$, and the other constantly records the transits of the mercury over the divisions of the thermometer.

A series extending over from one half to a whole hour, and recording a rise of $15^{\circ} \mathrm{C}$. to perhaps $25^{\circ} \mathrm{C}$, and in which a record was made for perhaps each tenth of a degree, would thus contain several hundred observations, from any two of which the equivalent of heat could be determined, though they would not all be independent. Such a series would evidently have immense weight; and, in fact, I estimate that, neglecting constant errors, a single series has more weight than all of Joule's experiments of 1849 , on water, put together. ${ }^{44}$

The correction for radiation is inversely proportional to the ratio of the rate of work generated to the rate at which the heat is lost; and this for equal ranges of temperature is only $\frac{1}{50}$ as great in my measures as in Joule's; for Joule's rate of increase was about $0^{\circ} .62 \mathrm{C}$. per hour, while mine is about $35^{\circ} \mathrm{C}$. in the same time, and can be increased to over $45^{\circ} \mathrm{C}$. per hour.

\section{Details}

The Calorimeter

Joule's calorimeter was made in a very simple manner, with few paddles, and without reference to the production of currents to mix

44 Forty experiments, with an average rise of temperature of $0^{\circ} .56 \mathrm{~F}$., equal to $0^{\circ} .31 \mathrm{C}$., gives a total rise of $12^{\circ} .4 \mathrm{C}$, which is only about two-thirds the average of one of my experiments. As my work is measured with equal accuracy, and my radiation with greater, the statement seems to be correct. 
up the water. Hence the paddles were made without solder, and were screwed together. Indeed, there was no solder about the apparatus.

But, for my purpose, the number of paddles must be multiplied, so that there shall be no jerk in the motion, and that the resistance may be great; they must be stronger, to resist the force from the engine, and they must be light, so as not to add an uncertain quantity to the calorific capacity. Besides this, the shape must be such as to cause the whole of the water to run in a constant stream past the thermometer, and to cause constant exchange between the water at the top and at the bottom.

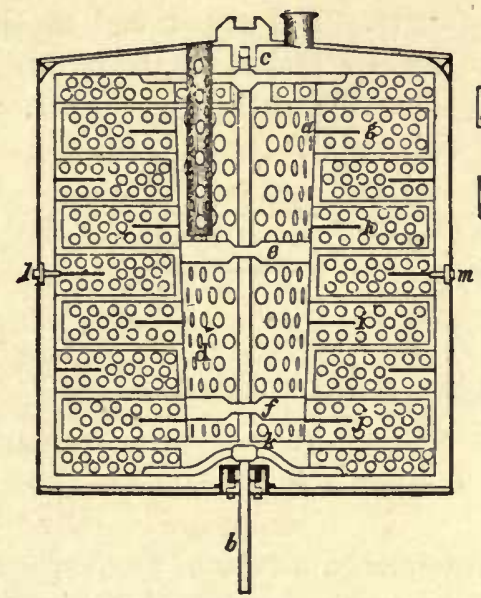

FIG. 7.

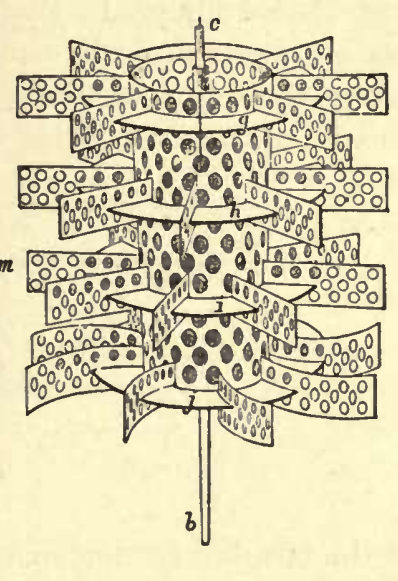

Frg. 8.

Fig. 7 shows a section of the calorimeter, and Fig. 8 a perspective view of the revolving paddles removed from the apparatus, and with the exterior paddles removed from around it; which could not, however, be accomplished physically without destroying them.

To the axis $c b$, Fig. 7 , which was of steel, and $6 \mathrm{~mm}$. in diameter, a copper cylinder, ad, was attached, by means of four stout wires at $e$, and four more at $f$. To this cylinder four rings, $g, h, i, j$, were attached, which supported the paddles. Each one had eight paddles, but each ring was displaced through a small angle with reference to the one below it, so that no one paindle came over another. This was to make the resistance continuous, and not periodical. The lower row of pad-dles were turned backwards, so that they had a tendency to throw the water outwards and make the circulation, as I shall show afterwards. 
Around these movable paddles were the stationary paddles, consisting of five rows of ten each. These were attached to the movable paddles by bearings, at the points $c$ and $k$, of the shaft, and were removed with the latter when this was taken from the calorimeter. When the whole was placed in the calorimeter, these outer paddles were attached to it by means of four screws, $l$ and $m$, so as to be immovable.

The cover of the calorimeter was attached to a brass ring, which was nicely ground to another brass ring on the calorimeter, and which could be made perfectly tight by means of a little white-lead paint. The shaft passed through a stuffing-box at the bottom, which was entirely within the outer surface of the calorimeter, so that the heat generated should all go to the water. The upper end of the shaft rested in a bearing in a piece of brass attached to the cover. In the cover there were two openings, - one for the thermometer, and the other for filling the calorimeter with water.

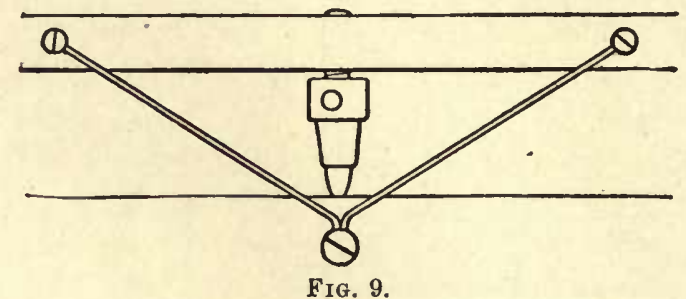

From the opening for the thermometer, a tube of copper, perforated with large holes, descended nearly to the centre of the calorimeter. The thermometer was in this sieve-like tube at only a short distance from the centre of the calorimeter, with the revolving paddles outside of it, and in the stream of water, which circulated as shown by the arrows.

This circulation of water took place as follows. The lower paddles threw the water violently outwards, while the upper paddles were prevented from doing so by a cylinder surrounding the fixed paddles. The consequence was, that the water flowed up in the space between the outer shell and the fixed paddles, and down through the central tube of the revolving paddles. As there was always a little air at the top to allow for expansion, it would also aid in the same direction. -These currents, which were very violent, could be observed through the openings.

The calorimeter was attached to a wheel, fixed to the shaft $a b$, by 
the method shown in Fig. 9. At the edge of the wheel, which was of the exact diameter of the calorimeter, two screws were attached, from which wires descended to a single screw in the edge of the calorimeter. Through the wheel, a screw armed with a vulcanite point pressed upon the calorimeter, and held it firmly. Three of these arrangements, at distances of $120^{\circ}$, were used. To centre the calorimeter, a piece of vulcanite at the centre was used. By this method of suspension very little heat could escape, and the amount could be allowed for by the radiation experiments.

\section{The Torsion System}

The torsion wire was of such strength that one millimeter on the scale at the edge of the wheel signified 11.8 grammes, or about $\frac{1}{200}$ of the weights op generally used. There were stops on the wheel, so that it could not move through more than a small angle. The weights were suspended by very flexible silk tapes, $6 \mathrm{~mm}$. or $8 \mathrm{~mm}$. broad and $0.3 \mathrm{~mm}$. thick. They varied from $4.5 \mathrm{k}$. to $8.5 \mathrm{k}$. taken together. The shaft, $a b$, was of uniform size throughout, so that the wire $c$ suspended the whole system, and no weight rested on the bearings.

The pulleys, $m, n$, Fig. 6 , were very exactly turned and balanced, and the whole suspended system was so free as to vibrate for a considerable time. However, as will be shown hereafter, its freedom is of little consequence.

\section{The Water Jacket}

Around the calorimeter, a water jacket, $t u$, was placed, so that the radiation should be perfectly definite. During the preliminary experiments a simple tin jacket was used, whose temperature was determined by two thermometers, one above and the other below, inserted in tubes attached to the jacket.

\section{The Driving Gear}

The cog-wheels, $g, h$, were made by Messrs. Brown and Sharpe, of Providence, and were so well cut that the motion transmitted to the calorimeter must have been very uniform.

\section{The Chronograph}

The cylinder of the chronograph was turned by a screw on the shaft ef, and received one revolution for 102 of the paddles; 155 revolutions of the cylinder, or 15,810 of the paddles, could be recorded, though, 
when necessary, the paper could be changed without stopping, and the experiment thus continued without interruption.

\section{The Frame and Foundation}

The frame was very massive and strong, so as to prevent oscillation; and the whole instrument weighed about 500 pounds as nearly as could be estimated. It was placed on a solid brick pier, with a firm foundation in the ground. The trembling was barely perceptible to the hand when running the fastest.

\section{The Engine}

The driving power was a petroleum engine, which was very efficient in driving the apparatus with uniformity.

\section{The Balance}

For weighing the calorimeter, a balance capable of showing the presence of less than $\frac{1}{10}$ gramme with 15,000 grammes was used. The weights, however, by Schickert, of Dresden, were accurate among themselves to at least $5 \mathrm{mg}$. for the larger weights, and in proportion for the smaller. A more accurate balance would have been useless, as will be seen further on.

\section{Adjustments}

There are few adjustments, and they were principally made in the construction.

In the first place, the shafts $a b$ and ef must be in line. Secondly, the wheels $m n$ must be so adjusted that their planes are vertical, and that the tapes shall pass over them symmetrically, and that their edges shall be in the plane of the wheel $k l$.

Deviation from these adjustments only produced small error.

\section{(c.) Theory of the Experiment}

\section{Estimation of Work Done}

The calorimeter is constantly receiving heat from the friction, and is giving out heat by radiation and conduction. Now, at any given instant of time, the temperature of the whole of the calorineter is not the same. Owing to the violent stirring, the water is undoubtedly at a very uniform temperature throughout. But the solid parts of the calorimeter cannot be so. The greatest difference of temperature is evidently soon after the commencement of the operation. But after 
some time the apparatus reaches a stationary state, in which, but for the radiation, the rise of temperature at all points would be the same. This steady state will be theoretically reached only after an infinite time; but as most of the metal is copper, and quite thin, and as the whole capacity of the metal work is only about four per cent of the total capacity, I have thought that one or two minutes was enough to allow, though, if others do not think this time sufficient, they can readily reject the first few observations of each series. When there is radiation, the stationary state will never be reached theoretically, though practically there is little difference from the case where there is no radiation.

The measurement of the work done can be computed as follows. Let $M$ be the moment of the force tending to turn the calorimeter, and $d \theta$ the angle moved by the shaft. The work done in the time $t$ will be fMdA. If the moment of the force is constant, the integral is simply $M \theta$; but it is impossible to obtain an engine which runs with perfect steadiness, and although we may be able to calculate the integral, as far as long periods are concerned, by observation of the torsion circle, yet we are not thus able to allow for the irregularity during one revolution of the engine. Hence I have devised the following theory. I have found, by experiments with the instrument, that the moment of the force is very nearly, for high velocities at least, proportional to the square of the velocity. For rapid changes of the velocity, this is not exactly true, but as the paddles are very numerous in the calorimeter, it is probably very nearly true. We have then

$$
M=C\left(\frac{d \theta}{d t}\right)^{2}
$$

where $C$ is a constant. Hence the work done becomes

$$
w=C \int\left(\frac{d \theta}{d t}\right)^{2} d \theta=C \int\left(\frac{d \theta}{d t}\right)^{3} d t .
$$

As we allow for irregularities of long period by readings of the torsion circle, we can assume ir this investigation that the mean velocity is constant, and equal to $v_{0}$. The form of the variation of the velocity must be assumed, and I shall put, without further discussion,

$$
\frac{d \theta}{d t}=v_{0}\left(1+c \cos \frac{2 \pi t}{a}\right) \text {. }
$$

We then find, on integrating from $a$ to 0 ,

$$
v=C v_{0}^{3} a\left(1+\frac{3}{2} c^{2}\right),
$$


which is the work on the calorimeter during one revolution of the engine.

The equation of the motion of the calorimeter, supposing it to be nearly stationary, and neglecting the change of torsion of the suspending wire, is

$$
\frac{m}{g} \frac{d^{2} \psi}{d t^{2}}-\frac{W D}{2}+C v_{0}^{2}\left(1+c \cos \frac{2 \pi t}{a}\right)^{2}=0,
$$

where $m$ is the moment of inertia of the calorimeter and its attachments, $\psi$ is the angular position of the calorimeter, $W$ is the sum of the torsion weights, and $D$ is the diameter of the torsion wheel. Hence,

$$
\begin{aligned}
\psi-\psi_{0}=\frac{g}{m}\left\{\frac { 1 } { 2 } t \left[C v_{0}^{2}\left(1+\frac{1}{2} c^{2}\right)\right.\right. & -W D] \\
& \left.+O v_{0}^{2}\left[\frac{a^{2} c^{2}}{16 \pi^{2}} \sin ^{2} \frac{2 \pi t}{a}-\frac{a^{2} c}{2 \pi^{2}}\left(\cos \frac{2 \pi t}{a}\right)\right]\right\} .
\end{aligned}
$$

When $W D=2 C v_{0}^{2}\left(1+\frac{1}{2} c^{2}\right)$, the calorimeter will merely oscillate around a given position, and will reach its maximum at the times $t=0$, $\frac{1}{2} a, a, \& \mathrm{c}$.

The total amplitude of each oscillation will be very nearly

$$
\psi^{\prime \prime}-\psi^{\prime \prime}=\frac{C v_{0}^{2} g a^{2} c}{\pi^{2} m}=\frac{W D g a^{2} c}{2 \pi^{2} m} .
$$

If $x$ is the amplitude of each oscillation, as measured in millimetres, on the edge of the wheel of diameter $D$, we have $\psi-\psi^{\prime}=\frac{2 x}{D}$.

Hence

$$
c=\frac{1}{2} \frac{m x n^{2}}{C D g}
$$

where $n$ is the number of revolutions of the engine per second.

Having found $c$ in this way, the work will be, during any time,

$$
w=\pi W D N\left(1+c^{2}\right),
$$

where $N$ is the total number of revolutions of the paddles.

A variation of the velocity of ten per cent from the mean, or twenty per cent total, would thus only cause an error of one per cent in the equivalent.

Hence, although the engine was only single acting, yet it ran easily, had great excess of power, and was very constant as far as long periods were concerned. The engine ran very fast, making from 200 to 250 revolutions per minute. The fly-wheel weighed about 220 pounds, and had a radius of $1 \frac{1}{4}$ feet. At four turns per second, this gives an energy of about 3400 foot-pounds stored in the wheel. The calorimeter required about one-half horse-power to drive it; and, assuming the same 
for the engine friction, we have about 140 foot-pounds of work required per revolution. Taking the most unfavorable case, where all the power is given to the engine at one point, the velocity changes during the revolution about four per cent, or $c$ would nearly equal .02, causing an error of 1 part in 2500 nearly. By means of the shaking of the calorimeter, I have estimated $c$ as follows, the value of $m$ being changed by changing the weight on the inertia bar, or taking it off altogether. The estimate of the shaking was made by two persons independently.

$\begin{array}{ll}\text { m. } & \\ 2,200,000 & \text { grms. cm. } \\ 3,100,000 & \text { "6 } \\ 11,800,000 & \text { "6 }\end{array}$

$\begin{array}{cc}x \text { observed. } & c \text { calculated. } \\ .6 \mathrm{~mm} . & 016 \\ .36 \text { 66 } & \cdot 013 \\ .13 \text { ๘6 } & \cdot 017 \\ \text { Mean, } & c=.015\end{array}$

causing a correction of 1 part in 5000 .

Another method of estimating the irregularity of running is to put on or take off weights until the calorimeter rests so firmly against the stops that the vibration ceases. Estimated in this way, I have found a little larger value of $c$, namely, about $\cdot 01 \%$.

But as one cannot be too careful about such sources of error, I have experimented on the equivalent with different velocities and with very different ways of running the engine, by which $c$ was greatly changed, and so have satisfied myself that the correction from this source is inappreciable in the present state of the science of heat.

Hence I shall simply put for the work

$$
w=\pi N W D \text {, }
$$

in gravitation measure at Baltimore. 'To reduce to absolute measure, we must multiply by the force of gravity given by the formula

$$
g=9 \cdot 78009+\cdot 0508 \sin ^{2} \varphi,
$$

which gives $9 \cdot 8005$ metres per second at Baltimore. If the calorimeter moved without friction, no work would be required to cause it to vibrate back and forth, as I have described; but when it moves with friction, some work is required. When I designed the apparatus, I thus had an idea that it would be best to make it as immovable as possible by adding to its moment of inertia by means of the inertia bar and weights. But on considering the subject further, I see that only the excess of energy represented by $c^{2} \pi N W D$ can be used in this way. For, when the calorimeter is rendered nearly immovable by its great moment 
of inertia, the work done on it is, as we have seen, $\pi N W D\left(1+c^{2}\right)$; but if it had no inertia, it is evident that the work would be only $\pi N W D$. If, therefore, the calorimeter is made partially stationary, either by its moment of inertia or by friction, the work, will be somewhere between these two, and the work spent in friction will be only so much taken from the error. Hence in the latter experiments the inertia bar was taken off, and then the calorimeter constantly vibrated through about half a millimeter on the torsion scale.

Besides this quick vibration, the calorimeter is constantly moving to the extent of a few millimetres back and forth, according to the varying velocity of the engine. As frequent readings were taken, these changes were eliminated. In very rare cases the weights had to be changed during the experiment; but this was very seldom.

The vibration and irregular motion of the calorimeter back and forth served a very useful purpose, inasmuch as it caused the friction of the torsion apparatus to act first in one direction and then in the other, so that it was finally eliminated. The torsion apparatus moved very freely when the calorimeter was not in position, and would keep vibrating for some minutes by itself, but with the calorimeter there was necessarily some binding. But the vibration made it so free that it would return quickly to its exact position of equilibrium when drawn aside, and would also quickly show any small addition to the weights. This was tried in each experiment.

To measure the heat generated, we require to know the calorific capacity of the whole calorimeter, and the rise of temperature which would have taken place provided no heat had been lost by radiation. The capacity of the calorimeter alone I have discussed elsewhere, finding the total amount equal to $347 \mathrm{k}$. of water at ordinary temperatures. The total capacity of the calorimeter is then $A+\cdot 347$, where $A$ is the weight of water. Hence Joule's equivalent in absolute measure is

$$
J=\frac{102 \pi n W D}{(A+\cdot 347)\left(t-t^{\prime}\right)}, q,
$$

where $n$ is the number of revolutions of the chronograph, it making one revolution to 102 of the paddles.

The corrections needed are as follows:

1st. Correction for weighing in air. This must be made to $W$, the cast-iron weights, and to $A+\cdot 34 \%$, the water and copper of the calorimeter. If $\lambda$ is the density of the air under the given conditions, the correction is $-.835 \lambda$. 
$2 d$. For the weight of the tape by which the weights are hung. This is $\frac{\cdot 0006}{W}$.

3d. For the expansion of torsion wheel, $D^{\prime}$ being the diameter at $20^{\circ} \mathrm{C}$. This is $000018\left(t^{\prime \prime}-20^{\circ}\right)$. Hence,

$$
J=102 \pi g \frac{n W D^{\prime}}{\left(A+\cdot 34^{\prime}\right)\left(t-t^{\prime}\right)}\left(1+\cdot 000018\left(t^{\prime \prime}-20\right)+\frac{.0006}{W}-\cdot 835 \lambda\right),
$$

where $t-t^{\prime}$ is the rise of the temperature corrected for radiation.

\section{Radiation}

The correction for radiation varies, of course, with the difference of temperature between the calorimeter and jacket; but, owing to the rapid generation of heat, the correction is generally small in proportion. The temperature generated was generally about $0^{\circ} \cdot 6$ per minute. The loss of temperature per minute by radiation was approximately $\cdot 0014 \theta^{\circ}$ per minute, where $\theta$ is the difference of the temperature. This is one per cent for $10^{\circ} \cdot \%$, and four per cent for $14^{\circ} \cdot 2$. Generally, the calorimeter was cooler than the jacket to start with, and so a rise of about $20^{\circ}$ could be accomplished without a rate of correction at any point of more than four per cent, and an average correction of less than two per cent. An error of ten per cent is thus required in the estimation of the radiation to produce an average error of 1 in 500, or 1 in 250 at a single point. The coefficients never differ from the mean more than about two per cent. The observations on the equivalent, being at a great variety of temperatures, check each other as to any error in the radiation.

The losses of heat which I place under the head of radiation include conduction and convection as well. I divide the losses of heat into the following parts: 1st. Conduction down the shaft; 2d. Conduction by means of the suspending wires or vulcanite points to the wheel above; $3 d$. True radiation; 4th. Convection by the air. To get some idea of the relative amounts lost in this way, we can calculate the loss by conduction from the known coefficients of conduction, and we can get some idea of the relative loss from a polished surface from the experiments of Mr. Nichol. In this way I suppose the total coefficient of radiation to be made up approximately as follows:

Conduction along shaft $\ldots \ldots \ldots \ldots \cdot 00011$

Conduction along suspending wires.... $\cdot 00006$

True radiation ................. .00017

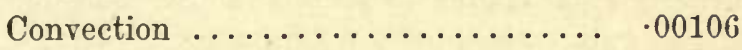

Total $\ldots \ldots \ldots \ldots \ldots \overline{\cdot 00140}$ 
The conduction through the vulcanite only amounts to 0000002 .

From this it would seem that three-fourths of the loss is due to radiation and convection combined.

The last two losses depend upon the difference of temperature between the calorimeter and the jacket, but the first two upon the difference between the calorimeter and frame of the machine and the wheel respectively. The frame was always of very nearly the same temperature as the water jacket, but the wheel was usually slightly above it. 'At first its temperature was noted by a thermometer, and the loss to it computed separately; but it was found to be unnecessary, and finally the whole was assumed to be a function of the temperature of the calorimeter and of the jacket only.

At first sight it might seem that there was a source of error in having a journal so near the bottom of the calorimeter, and joined to it by a shaft. But if we consider it a moment, we shall see that the error is inappreciable; for even if there was friction enough in the journal to heat it as fast as the calorimeter, it would decrease the radiation only seven per cent, or make an average error in the experiment of only 1 in 700 . But, in fact, the journal was very perfectly made, and there was no strain on it to produce friction; besides which, it was connected to a large mass of cast-iron which was attached to the base. Hence, as a matter of fact, the journal was not appreciably warmer after running than before, although tested by a thermometer. The difference could not have been more than a degree or so at most.

The warming of the wheel by conduction and of the journal by friction would tend to neutralize each other, as the wheel would be warmer and the journal cooler during the radiation experiment than the friction experiment.

The usual method of obtaining the coefficient of radiation would be to stop the engine while the calorimeter was hot, and observe the cooling, stirring the water occasionally when the temperature was read. This method I used at first, reading the temperature at intervals of about a half to a whole hour. But on thinking the matter over, it became apparent that the coefficient found in this way would be too small, especially at small differences of temperature; for the layer next to the outside would be cooled lower than the mean temperature, and the heat could only get to the outside by conduction through the water or by convection currents.

Hence I arranged the engine so as to run the paddles very slowly, so as to stir the water constantly, taking account of the number of 
the revolutions and the torsion, so as to compute the work. As I had foreseen, the results in this case were higher than by the other method. At low temperatures the error of the first method was fifteen per cent; but at high, it did not amcunt to more than about three to five per cent, and probably at very high temperatures it would almost vanish.

I do not consider it necessary to give all the details of the radiation experiments, but will merely remark that, as the calorimeter was nickelplated, and as seventy-five per cent of the so-called radiation is due to convection by the air, the coefficients of radiation were found to be very constant under similar conditions, even after long intervals of time.

The experiments were divided into two groups; one when the temperature of the jacket was about $5^{\circ} \mathrm{C}$., and the other when it averaged about $20^{\circ} \mathrm{C}$.

The results were then plotted, and the mean curve drawn through them, from which the following coefficients were obtained. These coefficients are the loss of temperature per minute, and per degree difference of temperature.

TABLE XXXV.*_COEFFICIENTS OF RADIATION.

\begin{tabular}{|c|c|c|}
\hline $\begin{array}{c}\text { Difference be- } \\
\text { tween Jacket and } \\
\text { Calorimeter. }\end{array}$ & Jacket $5^{\circ}$. & Jacket 20 \\
\hline 0 & .00138 & .00134 \\
-5 & .00135 & .00130 \\
0 & .00137 & .00132 \\
+5 & .00142 & .00138 \\
10 & .00148 & .00144 \\
15 & .00154 & .00150 \\
20 & .00158 & .00154 \\
25 & & \\
\hline
\end{tabular}

As the quantity of water in the calorimeter sometimes varied slightly, the numbers should be modified to suit, they being true when the total capacity of the calorimeter was 8.75 kil. The total surface of the calorimeter was about $2350 \mathrm{sq}$. cm., and the unit of time one minute. To compare my results with those of McFarlane and of Nichol given in the Proc. R. S. and Proc. R. S. E., I will reduce my results so that they can be compared with the tables given by Professor Everett in his 'Illustrations of the Centimeter-Gramme-Second System of Units,' pp. 50, 51 . 
The reducing factor is $\cdot 0621$, and hence the last results for the jacket at $20^{\circ} \mathrm{C}$. become:

TABLE XXXVI.

\begin{tabular}{|c|c|c|c|}
\hline $\begin{array}{c}\text { Difference of } \\
\text { Temperature. }\end{array}$ & $\begin{array}{c}\text { Coefficient of Radia- } \\
\text { tion on the C. G. S. } \\
\text { System. }\end{array}$ & $\begin{array}{c}\text { McFarlane's } \\
\text { Value. }\end{array}$ & Ratio. \\
\cline { 2 - 4 } 0 & .000081 & .000168 & $2 \cdot 07$ \\
5 & .000082 & .000178 & $2 \cdot 17$ \\
10 & .000086 & .000186 & $2 \cdot 16$ \\
15 & .000089 & .000193 & $2 \cdot 17$ \\
20 & .000093 & .000201 & $2 \cdot 16$ \\
25 & .000096 & .000207 & 2.15 \\
\hline
\end{tabular}

The variation which I find is almost exactly, that given by McFarlane, as is shown by the constancy of the column of ratios. But my coefficients are less than half those of McFarlane. This may possibly be due to the fact that the walls of McFarlane's enclosure were blackened, and to his surface being of polished copper and mine of polished nickel: his surface may also have been better adapted by its form to the loss of heat by convection. The results of Nichol are also much lower than those of McFarlane.

The fact that the coefficients of radiation are less with increased temperature of jacket is just contrary to what Dulong and Petit found for radiation. But as $I$ have shown that convection is the principal factor, I am at a loss to check my result with any other observer. Dulong and Petit make the loss from convection dependent only upon the difference of temperature, and approximately upon the square root of the pressure of the gas. Theoretically it would seem that the loss should be less as the mean temperature rises, seeing that the air becomes less dense and its viscosity increases. Should we substitute density for pressure in Dulong's law, we should have the loss by convection inversely as the square root of the mean absolute temperature, or approximately the absolute temperature of the jacket. This would give a decrease of one per cent in the radiation for about $6^{\circ}$, which is not far from what I have found.

To estimate the accuracy with which the radiation has been obtained is a very difficult matter, for the circumstances in the experiment are not the same as when the radiation was obtained. In the first place, although the water is stirred during the radiation, yet it is not stirred so violently as during the experiment. Further, the wheel above the calorimeter is warmer during radiation than during the experiment. 
Both these sources of error tend to give too small coefficients of radiation, and this is confirmed by looking over the final tables. But I have not felt at liberty to make any corrections based on the final results, as that would destroy the independence of the observations. But we are able thus to get the limits of the error produced.

During the preliminary experiments a water jacket was not used, but only a tin case, whose temperature was noted by a thermometer above and below. The radiation under these circumstances was larger, as the case was not entirely closed at the bottom, and so permitted more circulation of air.

\section{Corrections to Thermometers, etc.}

Among the other corrections to the temperature as read off from the thermometers, the correction for the stem at the temperature of the air is the greatest. The ordinary formula for the correction is $.000156 n\left(t-t^{\prime \prime}\right)$. But, in applying this correction, it is difficult to estimate $n$, the number of degrees of thermometer outside the calorimeter and at the temperature of the air, seeing that part of the stem is heated by conduction. The uncertainty vanishes as the thermometer becomes longer and longer, or rather as it is more and more sensitive. But even then some of the uncertainty remains. I have sought to avoid this uncertainty by placing a short tube filled with water about the lower part of the thermometer as it comes out of the calorimeter. The temperature of this was indicated by a thermometer, by aid of which also the heat lost to the water by conduction through the thermometer stem could be computed; this, however, was very minute compared with the whole heat generated, say 1 in 10,000 .

The water being very nearly at the temperature of the air, the stem above it could be assumed to be at the temperature of the air indicated by a thermometer hung within an inch or two of it. The correction for stem would thus have to be divided into two parts, and calculated separately. Calculated in this way, I suppose the correction is perfectly certain to much less than one hundredth of a degree: the total amount was seldom over one-tenth of a degree.

Among the uncertain errors to which the measurement of temperature is subjected, I may mention the following:

1. Pressure on bulb. A pressure of $60 \mathrm{~cm}$. of water produced a change of about $0^{\circ} .01$ in the thermometers. When the calorimeter was entirely closed there was soon some pressure generated. Hence the introduction of the safety-tube,-a tube of thin glass about $10 \mathrm{~cm}$. 
long, extending through a cork in the top of the calorimeter. The top of the safety-tube was nearly closed by a cork to prevent evaporation. Had the tube been shorter, water would have been forced out, as well as air.

2. Conduction along stem from outside to thermometer bulb. To avoid this, not only was the bulb immersed, but also quite a length of stem. As this portion of the stem, as also the bulb, was surrounded by water in violent motion, there could have been no large error from this source. The immersed stem to the top of the bulb was generally about $5 \mathrm{~cm}$. or more, and the stem only about $8 \mathrm{~cm}$. in diameter.

3 . The thermometer is never at the temperature of the water, because the latter is constantly rising; but we do not assume that it is so in the experiment. We only assume that it lags behind the water to the same amount at all parts of the experiment, and this is doubtless true.

To see if the amount was appreciable, I suddenly threw the apparatus out of gear, thus stopping it. The temperature was observed to continue rising about $0^{\circ} .02 \mathrm{C}$. Allowing $0^{\circ} .01$ for the rise due to motion after the word "Stop" was given, we have about $0^{\circ} .01 \mathrm{C}$. as the amount the thermometer lagged behind the water.

4. Evaporation. A possible source of error exists in the cooling of the calorimeter by evaporation of water leaking out from it.

The water was always weighed before and after the experiment in a balance giving $\frac{1}{10}$ gramme with accuracy. The normal amount of loss from removal of thermometer, wet corks, \&c., was about 1 gramme. The calorimeter was perfectly tight, and had no leakage at any point in its normal state. Once or twice the screws of the stuffing-box worked loose, but these experiments were rejected.

The evaporation of 1 gramme of water requires about 600 heat units, which is sufficient to depress the temperature of the calorimeter about $0^{\circ} .07 \mathrm{C}$. As the only point at which evaporation could take place was through a hole less than $1 \mathrm{~mm}$. diameter in the safety-tube, I think it is reasonable to assume that the error from this source is inappreciable. But to be doubly certain, I observed the time which drops of water of known weight and area, placed on the warm calorimeter, took to dry. From these experiments it was evident that it would require a considerable area of wet surface to produce an appreciable effect. This wet surface never existed unless the calorimeter was wet by dew deposited on the cool surface. To guard against this error, the calorimeter was never cooled so low that dew formed; it was carefully rubbed with a 
towel, and placed in the apparatus half an hour to an hour before the experiment, exposed freely to the air. The surface being polished, the slightest deposit of dew was readily visible. The greatest care was taken to guard against this source of error, and I think the experiment is free from it.

\section{(d.) Results}

\section{Constant Data}

Joule's equivalent in gravitation measure is of the dimensions of length only, being the height which water would have to fall to be heated one degree. Or let water flow downward with uniform velocity through a capillary tube impervious to heat; assuming the viscosity constant, the rate of variation of height with temperature will be Joule's equivalent.

Hence, besides the force of gravity the only thing required in absolute measure is some length. The length that enters the equation is the diameter of the torsion wheel. This was determined under a microscope comparator by comparison with a standard metre belonging to Professor Rogers of Harvard Observatory, which had been compared at Washington with the Coast Survey standards, as well as by comparison with one of our own metre scales which had also been so compared. The result was $\cdot 26908$ metre at $20^{\circ} \mathrm{C}$.

To this must be added the thickness of the silk tape suspending the weights. This thickness was carefully determined by a micrometer screw while the tape was stretched, the screw having a flat end. The result was $\cdot 00031 \mathrm{~m}$.

So that, finally, $D^{\prime}=\cdot 26939$ metre at $20^{\circ} \mathrm{C}$. Separating the constant from the variable parts, the formula now becomes

$$
\begin{aligned}
\frac{J}{g} & =\frac{86 \cdot 324}{A+\cdot 347}\left(1+\cdot 000018\left(t^{\prime \prime}-20\right)+\frac{.0006}{W}-\cdot 835 \lambda\right) \frac{W n}{t-t^{\prime}} . \\
g & =9 \cdot 8005 \text { at Baltimore. }
\end{aligned}
$$

It is unnecessary to have the weights exact to standard, provided they are relatively correct, or to make double weighings, provided the same scale of the balance is always used. For both numerator and denominator of the fraction contain a weight.

\section{Experimental Data and Tables of Results}

In exhibiting the results of the experiments, it is much more satisfactory to compute at once from the observations the work necessary to raise 1 kil. of the water from the first temperature observed to each suc- 
ceeding temperature. By interpolation in such a table we can then reduce to even degrees. To compare the different results $I$ have then added to each table such a quantity as to bring the result at $20^{\circ}$ about equal to 10,000 kilogramme-metres.

The process for each experiment may be described as follows. The calorimeter was first filled with distilled water a little cooler than the atmosphere, but not so cool as to cause a deposit of dew. It was then placed in the machine and adjusted to its position, though the outer half of the jacket was left off for some time, so that the calorimeter should become perfectly dry; to aid which the calorimeter was polished with a cloth. The thermometer and safety-tube were also inserted at this time.

After half an hour or so, the chronograph was adjusted, the outer half of the jacket put in place, the wooden screen fixed in position, and all was ready to start. The engine, which had been running quietly for some time, was now attached, and the experiment commenced. First the weights had to be adjusted so as to produce equilibrium as nearly as possible.

The observers then took their positions. One observer constantly recorded the transit of the mercury over the divisions of thermometer, making other suitable marks, so that the divisions could be afterwards recognized. He also read the thermometers giving the temperatures of the air, the bottom of the calorimeter thermometer, and of the wheel just above the calorimeter; and sometimes another, giving that of the cast-iron frame of the instrument.

The other observer read the torsion wheel once every revolution of the chronograph cylinder, recording the time by his watch. He also recorded on the chronograph every five minutes by his watch, and likewise stirred the water in the jacket at intervals, and read its temperature.

The recording of the time was for the purpose of giving the connecting link between the readings of the torsion circle and of the thermometer. This, however, as the readings were quite constant, had only to be done roughly, say to half a minute of time, though the records of time on the chronograph were true to about a second.

The thermometers to read the temperature of the water in the jacket were graduated to $0^{\circ} \cdot 2 \mathrm{C}$., but were generally read to $0^{\circ} \cdot 1 \mathrm{C}$., and had been compared with the standards. There was no object in using more delicate thermometers.

After the experiment bad continued long enough, the engine was 
stopped and a radiation experiment begun. The last operation was to weigh the calorimeter again, after removing the thermometer and safety tube, and also the weights which had been used.

The chronograph sheet, having then been removed from the cylinder, had the time records identified and marked, as well as the thermometer records. Each ine of the chronograph record was then numbered arbitrarily, and a table made indicating the stand of the thermometer and the number of the revolutions and fractions of a revolution as recorded on the chronograph sheet. The times at which these temperatures were reached was also found by interpolation, and recorded in another column.

From the column of times the readings of the torsion circle could be identified, and so all the necessary data would be at hand for calculating the work required to raise the temperature of one kilogramme of the water from the first recorded temperature to any succeeding temperature.

As these temperatures usually contained fractions, the amount of work necessary to raise one kilogramme of the water to the even degrees could then be found from this table by interpolation. Joule's equivalent at any point would then be merely the difference of any two succeeding numbers; or, better, one tenth the difference of two numbers situated $10^{\circ}$ apart, or, in general, the difference of the numbers divided by the difference of the temperatures.

It wonld be a perfectly simple matter to make the record of the torsion circle entirely automatic, and I think I shall modify the apparatus in that manner in the future.

It would take too much space to give the details of each experiment; but, to show the process of calculation, I will give the experiment of Dec. $17,18 \% 8$, as a specimen. The chronograph sheet, of course, I cannot give. The computation is at first in gravitation measure, but afterwards reduced to absolute measure.

The calorimeter before the experiment weighed $12 \cdot 2733 \mathrm{kil}$.

The calorimeter after the experiment weighed $12 \cdot 2716$ kil.

Weight of calorimeter alone

Mean $12 \cdot 2720$ kil.

$\therefore$ Water alone weighed

$3 \cdot 8721$ kil.

8.3999 kil.

$\cdot 3470$ kil.

Total capacity $\overline{8 \cdot 7469}$ kil. 
The correction for weighing in air was $\cdot 835 \lambda=\cdot 00106$.

The total term containing the correction is therefore $\cdot 99878$.

$$
\begin{array}{ll}
\log 86.324 & =1 \cdot 9361316 \\
\log \cdot 99878 & =\frac{1.9994698}{1 \cdot 9356014} \\
\log 8 \cdot 7469 & =\frac{.9418542}{.97772}=\log 9.85706 . \\
\log \text { const. factor } & =.993747
\end{array}
$$

Hence the work per kilogramme is $9.85706 \Sigma W n$ in gravitation measure, the term $\Sigma W n$ being used to denote the sum of products similar to $W n$ as obtained by simultaneous readings of torsion circle and records on chronograph sheet.

Zero of torsion wheel, $79 \cdot 3 \mathrm{~mm}$.

Value of $1 \mathrm{~mm}$. on torsion wheel $\cdot 0118$ kil.

The following were the records of time on the chronograph sheet:Time observed. Revolutions of Chronograph. Time calculated.

$\begin{array}{lrl}15 & 8 \cdot 74 & 15 \cdot 2 \\ 20 & 25 \cdot 32 & 20 \cdot 1 \\ 25 & 42 \cdot 10 & 25 \cdot 0 \\ 30 & 59 \cdot 05 & 30 \cdot 0 \\ 35 & 76 \cdot 00 & 35 \cdot 0 \\ 40 & 93 \cdot 03 & 40 \cdot 0 \\ 45 & 109 \cdot 97 & 45 \cdot 0 \\ 50 & 126 \cdot 92 & 50 \cdot 0 \\ 55 & 144.14 & 55 \cdot 0\end{array}$

The times were calculated by the formula

$$
\text { Time }=\cdot 294 \times \text { Revolutions }+12 \cdot 66,
$$

which assumes that the engine moves with uniform velocity. As the principal error in using an incorrect interpolation formula comes from the calculation of the radiation, and as this formula is correct within a few seconds for all the higher temperatures, we can use it in the calculation of the times.

The records of the transits of the mercury over the divisions of the thermometer were nearly always made for each division, but it is useless to calculate for each. I usually select the even centimeters, and take the mean of the records for several divisions on each side.

While the mercury was rising $1 \mathrm{~cm}$. on No. 6163, there would be 
about seven revolutions of the chronograph, and consequently seven readings of the torsion circle, each one of which was the average for a little time as estimated by the eye.

I have obtained more than thirty series of results, but have thus far reduced only fourteen, five of which are preliminary, or were made with the simple jacket instead of the water jacket, the radiation to which was much greater, as there was a hole at the bottom which allowed more circulation of the air. The mean of the preliminary results agrees so closely with the mean of the final results, that I have in the end given them equal weight.

On March 24th, the same thermometer was used for a second experiment directly after the first, seeing that the chronograph failed to work in the first experiment until $8^{\circ}$ was reached. The error from this cause was small, as the first experiment only reached to $26^{\circ} \mathrm{C}$., and hence there could have been no change of zero, as this is very nearly the temperature at which the thermometer was generally kept.

Having thus calculated the work in conjunction with the temperature, I have next interpolated so as to obtain the work at the even degrees. The tables so formed I have combined in two ways: first, I have added to the column of work in each table an arbitrary number, such as to make the work at $20^{\circ}$ about 10,000 , and have then combined them as seen in Table LI, and, secondly, I have subtracted each number from the one $10^{\circ}$ farther down the table, and divided the numbers so found by 10 , thus obtaining the mechanical equivalent of heat.

In these tables four thermometers have been used, and yet they were so accurate that little difference can be observed in the experiments which can be traced to an error of the thermometer, although the Kew standard has some local irregularities. The greatest difference between any column of Table LI and the general mean is only 10 kilogrammemetres, or 0.023 degree, and this includes all errors of calibration of thermometers, radiation, \&c. This seems to me to be a very remarkable result, and demonstrates the surpassing accuracy of the method. Indeed, the limit of accuracy in thermometry is the only limit which we can at present give to this method of experinent. Hence the large proportional time spent on that subject.

The accuracy of the radiation is demonstrated, to some extent, by the agreement oi the results obtained even with different temperatures of the jacket. But on close observation it seems apparent that the coefficients of radiation should be further increased as there is a tendency of the end figures in each series to become too high. This is 
exactly what we should suppose, as we have seen that nearly all sources of error tend in the direction of making the radiation too small. For instance, an error came from not stirring the water during the radiation, and there must be a small residual error from not stirring so fast during radiation as during the experiment. Besides this, some parts around the calorimeter were warm during the radiation which were cool during the experiment. And both of these make the correction for radiation too small. However, the error from this source is small, and cannot possibly affect the general conclusions. In each column of Tables LI and LII a dash is placed at the temperature of the jacket, and for fifteen degrees below this point the error in the radiation must produce only an inappreciable error in the equivalent: taking the observations within this limit as the standards, and rejecting the others, we should still arrive at very nearly the same conclusions as if we accepted the whole.

Most of the experiments are made with a weight of about $\% \cdot 3$ kil., as everything seemed to work best with this weight But for the sake of a test I have run the weight up to 8.6 and down to 4.4 kil., by which the rate of generation of the heat was changed nearly three times. By this the correction for the radiation and the error due to the irregularity of the engine are changed, and yet scarcely an appreciable difference in the results can be observed.

The tables explain themselves very well, but some remarks may be in order. Tables XXXVII to $\mathrm{L}$ inclusive are the results of fourteen experiments selected from the total of about thirty, the others not having been worked up yet, though I propose to do so at my leisure.

Table LI gives the collected results. At the top of each column the date of the experiment and number of the thermometer are given, together with the approximate torsion weight and the rate of rise of temperature per hour. The dash in each column gives approximately the temperature of the jacket, and hence of the air. There are four columns of mean values, but the last, produced from the combination of the table by parts, is the best.

Table LII gives the mechanical equivalent of heat as deduced from intervals of $10^{\circ}$ on Table LI. The selection of intervals of $10^{\circ}$ tends to screen the variation of the specific heat of water from view, but a smaller interval gives too many local irregularities. In taking the mean I have given all the observations equal weight, but as the Kew standard was only graduated to $\frac{1}{2}^{\circ} \mathrm{F}$. it was impossible to calibrate it so accurately as to avoid irregularities of $0^{\circ} .02 \mathrm{C}$. which would affect 
the quantities 1 in 500. Hence, in drawing a curve through the results, as given in the last column, I have almost neglected the Kew, and have otherwise sought to draw a regular curve without points of inflection. The figures in the last column I consider the best.

Table LIII takes the mean values as found in Tables LI and LII, and exhibits them with respect to the temperatures on the different thermometers, to the different parts of the earth, and also gives the reduction to the absolute scale. I am inclined to favor the absolute scale, using $m=\cdot 00015$, as given in the Appendix to Thermometry, rather than $\cdot 00018$, as used throughout the paper.

Table LIV gives what I consider the final result of the experiment. It is based on the result $m=.00015$ for the thermometers, and is corrected for the irregularity of the engine by adding 1 in 4000 .

The minor irregularities are also corrected so that the results signify a smooth curve, without irregularity or points of contrary flexure. But the curve for the work does not differ more than three kilogrammemetres from the actual experiment at any point, and generally coincides with it to about one kilogramme-metre. These differences signify $0^{\circ} .007 \mathrm{C}$. and $0^{\circ} .002 \mathrm{C}$, respectively. The mechanical equivalent is for single degrees rather than for ten degrees, as in the other tables.

TABLE XXXVII.-FIrST SERIES.-Preliminary.

January 16,1878 . Jacket and Air about $14^{\circ} \mathrm{C}$.

\begin{tabular}{|c|c|c|c|c|c|c|c|c|c|c|}
\hline \multirow{2}{*}{ 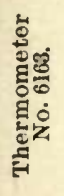 } & \multirow[b]{2}{*}{$\underset{\Xi}{\dot{\Xi}}$} & \multicolumn{2}{|c|}{ Correction. } & \multirow{2}{*}{ 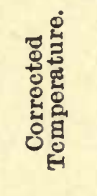 } & \multirow{2}{*}{ 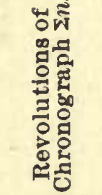 } & \multirow{2}{*}{ 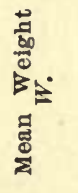 } & \multirow{2}{*}{ 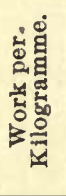 } & \multirow{2}{*}{ 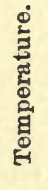 } & \multirow{2}{*}{ 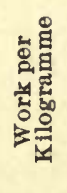 } & \multirow{2}{*}{ 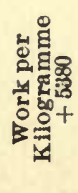 } \\
\hline & & $\begin{array}{l}\text { घ્ } \\
\text { 㟧 }\end{array}$ & 芯 & & & & & & & \\
\hline 140 & $52 \cdot 0$ & .005 & 0 & $9^{\circ} \cdot 185$ & $5 \cdot 485$ & & 0 & & & \\
\hline 160 & & -.0 & -.017 & & $18 \cdot \bar{c}$ & $\begin{array}{l}7.509 \\
7.478\end{array}$ & 951 & 10 & 348 & 5728 \\
\hline 180 & & & -.0 & & 3 & & 1906 & 11 & 77 & 6155 \\
\hline 203 & 63 & & -.01 & & $45 \cdot 3$ & & 3010 & 12 & 120 & 658 \\
\hline 220 & & & -.00 & & & & 38 & 13 & $162 !$ & 700 \\
\hline 240 & 70 & & $+\cdot 0$ & & 6 & & 478 & 14 & 205 & 743 \\
\hline 259 & 74 . & & +.06 & & 81 . & & 570 & 15 & 248 & 786 \\
\hline 289 & $80 \cdot 0$ & & $+\cdot 161$ & & $101 \cdot 214$ & & 7156 & 16 & 2912 & 8292 \\
\hline$\cdots$ & .... & $\cdots$ & ...... & $\cdots \cdot$ & ..... & & .. & 17 & 33 & 8720 \\
\hline$\cdots$ & $\ldots$ & & ...... & & & & & 18 & 37 & 914 \\
\hline .. & $\cdots$ & & ..... & . & - & & $\ldots$ & 19 & 41 & 957 \\
\hline .. & $\ldots$ & & ...... & ...... & $\ldots$. & & $\ldots$ & 2 & & 9999 \\
\hline & $\cdots$ & & & & & & $\cdots$ & 21 & 50 & 10428 \\
\hline & $\ldots$ & ... & ..... & & & & $\cdots$ & 22 & & 10852 \\
\hline & & & & & & & & 23 & 58 & 11279 \\
\hline & .. & & $\cdots \cdot$ & ..... & & & .... & 24 & 6 & 11706 \\
\hline & & & & & & 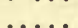 & ... & 25 & & 12133 \\
\hline - & ... & & ..... & $\cdots \cdots$ & $\cdots$ & & ... & 26 & 7180 & 12560 \\
\hline
\end{tabular}


TABLE XXXVIII._SEcond SERIES.-Preliminary.

March 7, 1878. Jacket $18^{\circ} .5$ to $22^{\circ} .5$. Air about $21^{\circ} \mathrm{C}$.

\begin{tabular}{|c|c|c|c|c|c|c|c|c|c|c|}
\hline \multirow{2}{*}{ 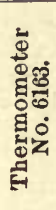 } & \multirow[b]{2}{*}{ 离 } & \multicolumn{2}{|c|}{ Correction. } & \multirow{2}{*}{ 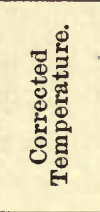 } & \multirow{2}{*}{ 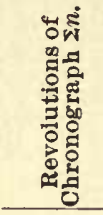 } & \multirow{2}{*}{ 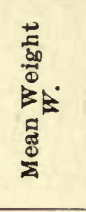 } & \multirow{2}{*}{ 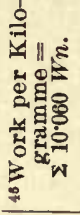 } & \multirow{2}{*}{ 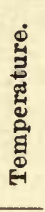 } & \multirow{2}{*}{ 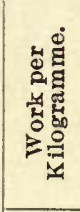 } & \multirow{2}{*}{ 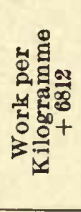 } \\
\hline & & $\frac{\dot{g}}{\infty}$ & 密 & & & & & & & \\
\hline 170 & 19.9 & -.016 & 0 & $12^{\circ} \cdot 537$ & $5 \cdot 03$ & & 0 & 13 & 198 & 7010 \\
\hline 180 & $\ldots$ & $\ldots .$. & $\ldots \ldots$ & $13 \cdot 646$ & $11 \cdot 12$ & & 474 & 14 & 625 & 7437 \\
\hline 190 & & & & $14 \cdot 755$ & $17 \cdot 22$ & $7 \cdot 7$ & 947 & 15 & 1052 & 7864 \\
\hline 200 & & ....... & & $15 \cdot 863$ & $23 \cdot 36$ & $\begin{array}{l}7.666 \\
7.642\end{array}$ & 1421 & 16 & 1480 & 8292 \\
\hline 210 & $26 \cdot 8$ & -.010 & -.036 & $16 \cdot 972$ & 29.55 & & 1897 & 17 & 1909 & 8721 \\
\hline 220 & $\ldots$ & $\ldots \ldots$ & $\ldots \ldots$ & 18.085 & $35 \cdot 70$ & 7.641 & 2369 & 18 & 2333 & 9145 \\
\hline 230 & $\ldots$ & ...... & $\ldots .$. & $19 \cdot 196$ & 41.90 & & 2845 & 19 & 2761 & 9573 \\
\hline 240 & & ...... & & $20 \cdot 305$ & $48 \cdot 09$ & 7.61 & 3319 & 20 & 3189 & 10001 \\
\hline 250 & 33.8 & +.003 & -.036 & 21.419 & $54 \cdot 30$ & & 3794 & 21 & 3615 & 10427 \\
\hline 260 & $\ldots$ & $\ldots \ldots$ & $\ldots \ldots$ & $22 \cdot 533$ & & $\begin{array}{r}7.596 \\
7.582\end{array}$ & & 22 & 4041 & 10853 \\
\hline 270 & $\ldots$ & ...... & $\ldots \ldots$ & $23 \cdot 642$ & $66 \cdot 69$ & 7.552 & 4740 & 23 & 4467 & 11279 \\
\hline 280 & & & & $24 \cdot 754$ & $72 \cdot 92$ & & 5213 & 24 & $489 \%$ & 11704 \\
\hline 290 & $40 \cdot 8$ & $+0 \cdot 20$ & -.001 & $25 \cdot 867$ & $79 \cdot 16$ & 7.0476 & 5687 & 25 & 5318 & 12130 \\
\hline 300 & $\ldots$ & $\ldots \ldots$ & $\ldots \ldots$ & $26 \cdot 990$ & $85 \cdot 4^{2}$ & & 6164 & 26 & 5744 & 12556 \\
\hline 310 & $\ldots$ & & & $28 \cdot 119$ & $91 \cdot 67$ & 7.604 & 6643 & 27 & 6168 & 12980 \\
\hline 320 & & & & $29 \cdot 253$ & 97.98 & & 7125 & 28 & 6593 & 13405 \\
\hline 330 & $47 \cdot 8$ & +.044 & +.073 & $30 \cdot 393$ & $104 \cdot 28$ & 7.011 & 7608 & 29 & 7017 & 13829 \\
\hline 340 & & & $\ldots \ldots$ & $31 \cdot 540$ & $110 \cdot 67$ & 7.602 & 8097 & 30 & 7441 & 14253 \\
\hline 350 & $51 \cdot 4$ & & & $32 \cdot 689$ & $117 \cdot 12$ & 7.592 & 8590 & 31 & 7867 & 14679 \\
\hline 360 & & & & $33 \cdot 842$ & $123 \cdot 54$ & & 9081 & 32 & 8294 & 15106 \\
\hline 370 & $55 \cdot 0$ & $+\cdot 072$ & $+\cdot 184$ & $34 \cdot 998$ & $130 \cdot 04$ & $\begin{array}{l}7.576 \\
7.550\end{array}$ & 9576 & 33 & 8722 & 15534 \\
\hline 380 & & & & $36 \cdot 158$ & $136 \cdot 56$ & $\begin{array}{l}7.020 \\
7.550\end{array}$ & 10071 & 34 & 9149 & 15961 \\
\hline 390 & $58 \cdot 7$ & $+\cdot 588$ & $+\cdot 261$ & $37 \cdot 321$ & $143 \cdot 08$ & & 10567 & 35 & 9577 & 16389 \\
\hline$\cdots$ & ... & ...... & $\cdots \cdots$ & ...... & ...... & & .... & 36 & 10004 & 16816 \\
\hline . & & & & & ...... & & & 37 & 10430 & 17242 \\
\hline
\end{tabular}

TABLE XXXIX.-ThIRD SERIEs. - Preliminary.

March 12,1878 . Jacket $13^{\circ} \cdot 2$ to $16^{\circ} \cdot 6$. Air about $15^{\circ} \mathrm{C}$.

\begin{tabular}{|c|c|c|c|c|c|c|c|c|c|c|}
\hline \multirow{2}{*}{ 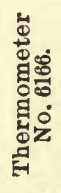 } & \multirow[b]{2}{*}{$\underset{\Xi}{\stackrel{\Xi}{E}}$} & \multicolumn{2}{|c|}{ Correction. } & \multirow{2}{*}{ 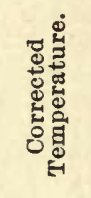 } & \multirow{2}{*}{ 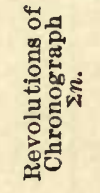 } & \multirow{2}{*}{ 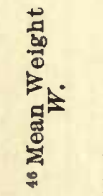 } & \multirow{2}{*}{ 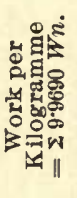 } & \multirow{2}{*}{ 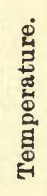 } & \multirow{2}{*}{ 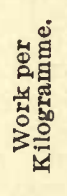 } & \multirow{2}{*}{ 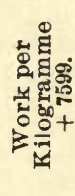 } \\
\hline & & 焉 & జّं & & & & & & & \\
\hline 205 & $28 \cdot 0$ & 0 & 0 & $14^{\circ} \cdot 368$ & $3 \cdot 156$ & & 0 & $\therefore$ & & \\
\hline 210 & $28 \cdot 6$ & 0 & +.002 & $14 \cdot 754$ & $5 \cdot 334$ & 7.51667 & 164 & 15 & 269 & 7868 \\
\hline 220 & 29.9 & & ...... & $15 \cdot 529$ & $9 \cdot 770$ & $7 \cdot 5167$ & 495 & 16 & 696 & 8295 \\
\hline 230 & $31 \cdot 1$ & +.003 & $+\cdot 010$ & $16 \cdot 307$ & $14 \cdot 184$ & & 827 & 17 & 1122 & 8721 \\
\hline
\end{tabular}

45 In the calculation of this colnmn, more exact data were used than given in the other two columns, seeing that the original calculation was made every $5 \mathrm{~mm}$. of the thermometer. Hence the last figure may not always agree with the rest of the data.

${ }^{46}$ As this table was originally calculated for every $5 \mathrm{~mm}$. on the thermometer, I have given the weights which were used to check the more exact calculation. 
TABLE XXXIX.-Continued.

\begin{tabular}{|c|c|c|c|c|c|c|c|c|c|c|}
\hline \multirow{2}{*}{ 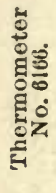 } & \multirow[b]{2}{*}{ 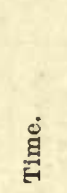 } & \multicolumn{2}{|c|}{ Correction. } & \multirow{2}{*}{ 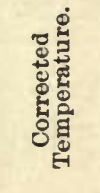 } & \multirow{2}{*}{ 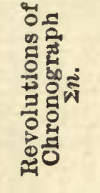 } & \multirow{2}{*}{ 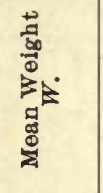 } & \multirow{2}{*}{ 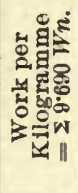 } & \multirow{2}{*}{ 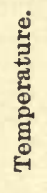 } & \multirow{2}{*}{ 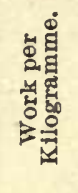 } & \multirow{2}{*}{ 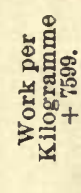 } \\
\hline & & 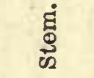 & 芯 & & & & & & & \\
\hline 240 & $32 \cdot 4$ & & & $17^{\circ} \cdot 090$ & $18 \cdot 642$ & & 1160 & 18 & 1548 & 9147 \\
\hline 250 & $33 \cdot 6$ & $+\cdot 009$ & $+\cdot 021$ & $17 \cdot 875$ & $23 \cdot 080$ & $\cdot 7 \cdot 5462$ & 1495 & 19 & 1975 & 9574 \\
\hline 260 & $34 \cdot 9$ & $\ldots \ldots$ & & $18 \cdot 662$ & $27 \cdot 550$ & & 1831 & 20 & 2401 & 10000 \\
\hline 270 & $36 \cdot 2$ & +.014 & $+\cdot 038$ & $19 \cdot 452$ & $32 \cdot 014$ & & 2167 & 21 & 2828 & 10427 \\
\hline 280 & $37 \cdot 4$ & & & $20 \cdot 242$ & $36 \cdot 474$ & $7 \cdot 5668$ & 2504 & 22 & 3253 & 10852 \\
\hline 290 & $38 \cdot 7$ & +.019 & $+\cdot 055$ & $21 \cdot 029$ & $40 \cdot 924$ & & 2840 & 23 & 3676 & 11275 \\
\hline 300 & 399 & & & $21 \cdot 825$ & $45 \cdot 424$ & & 3179 & 24 & 4101 & 11700 \\
\hline 310 & $41 \cdot 2$ & +.024 & +.089 & $22 \cdot 619$ & $49 \cdot 838$ & $7 \cdot 5875$ & 3514 & 25 & 4526 & 12125 \\
\hline 320 & $42 \cdot 5$ & & & $23 \cdot 418$ & $54 \cdot 302$ & & 3853 & 26 & 4951 & 12550 \\
\hline 330 & 43.7 & +.030 & $+\cdot 120$ & $24 \cdot 220$ & $58 \cdot 844$ & & 4194 & 27 & 5378 & 12977 \\
\hline 340 & $45 \cdot 0$ & & & $25 \cdot 023$ & $63 \cdot 366$ & $7 \cdot 5763$ & 4536 & 28 & 5803 & 13402 \\
\hline 350 & $46 \cdot 3$ & $+\cdot 038$ & $+\cdot 159$ & $28 \cdot 825$ & 67.874 & & 4876 & 29 & 6226 & 13825 \\
\hline 360 & $47 \cdot 6$ & & & $26 \cdot 628$ & $72 \cdot 403$ & & 5219 & 30 & 6653 & 14252 \\
\hline 370 & $48 \cdot 9$ & +.047 & $+\cdot 202$ & $27 \cdot 438$ & $76 \cdot 987$ & $7 \cdot 5872$ & 5565 & 31 & 7078 & 14677 \\
\hline 380 & $50 \cdot 1$ & & & $28 \cdot 253$ & $81 \cdot 550$ & & 5910 & & & ..... \\
\hline 390 & $51 \cdot 4$ & +.056 & $+\cdot 251$ & $29 \cdot 069$ & $86 \cdot 100$ & & 6255 & & & \\
\hline 400 & $52 \cdot 7$ & & & 29.884 & $90 \cdot 720$ & $7 \cdot 5801$ & 6604 & .. & & \\
\hline 410 & $54 \cdot 0$ & $+\cdot 066$ & $+\cdot 304$ & $30 \cdot 703$ & $95 \cdot 316$ & & 6951 & $\ldots$ & & \\
\hline 420 & $55 \cdot 3$ & $\ldots .$. & ..... & $31 \cdot 519$ & $99 \cdot 920$ & & 7299 & .. & & $\ldots$. \\
\hline
\end{tabular}

TABLE XL. - Fourth SERIEs.-Preliminary. 47

March 24, 1878. Jacket $5^{\circ} .4$ to $8^{\circ} .2$. Air about $6^{\circ} \mathrm{C}$.

\begin{tabular}{|c|c|c|c|c|c|c|c|c|c|c|}
\hline \multirow{2}{*}{ 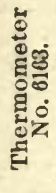 } & \multirow[b]{2}{*}{ 思 } & \multicolumn{2}{|c|}{ Correction. } & \multirow{2}{*}{ 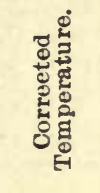 } & \multirow{2}{*}{ 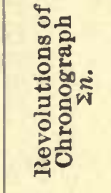 } & \multirow{2}{*}{ 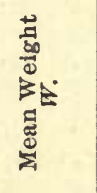 } & \multirow{2}{*}{ 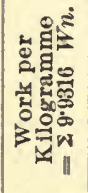 } & \multirow{2}{*}{ 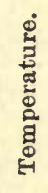 } & \multirow{2}{*}{ 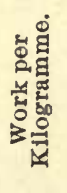 } & \multirow{2}{*}{ 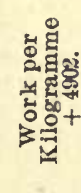 } \\
\hline & & 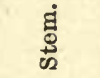 & 芯 & & & & & & & \\
\hline 130 & $27: 4$ & $+\cdot 002$ & 0 & $8^{\circ} \cdot 071$ & $42 \cdot 364$ & & 0 & 8 & -30 & 4872 \\
\hline 140 & $29 \cdot 2$ & & & $9 \cdot 204$ & $48 \cdot 898$ & & 485 & 9 & 398 & 5300 \\
\hline 150 & $31 \cdot 0$ & +.010 & +.019 & $10 \cdot 340$ & $55 \cdot 438$ & & 968 & 10 & 823 & 5725 \\
\hline 160 & $32 \cdot 9$ & & & $11 \cdot 480$ & 62.066 & $\begin{array}{l}7.442 \\
7.405\end{array}$ & 1458 & 11 & 1252 & 6154 \\
\hline 170 & $34 \cdot 7$ & $+\cdot 017$ & $+\cdot 050$ & $12 \cdot 620$ & $68 \cdot 669$ & $7 \cdot 390$ & 1944 & 12 & 1680 & 6582 \\
\hline 180 & $36 \cdot 6$ & & & $13 \cdot 763$ & $75 \cdot 330$ & $\begin{array}{l}7.390 \\
7.398\end{array}$ & 2433 & 13 & 2107 & 7009 \\
\hline 190 & $38 \cdot 4$ & $+\cdot 025$ & +.093 & $14 \cdot 908$ & $81 \cdot 973$ & $\begin{array}{l}7.398 \\
7.431\end{array}$ & 2921 & 14 & 2534 & 7436 \\
\hline 200 & $40 \cdot 3$ & & & $16 \cdot 054$ & $88 \cdot 597$ & $\begin{array}{l}7.431 \\
7.490\end{array}$ & 3410 & 15 & 3960 & 8862 \\
\hline 210 & $42 \cdot 2$ & +.034 & $+\cdot 150$ & $17 \cdot 202$ & $95 \cdot 264$ & & 3902 & 16 & 3387 & 8289 \\
\hline 220 & $44 \cdot 2$ & & & $18 \cdot 350$ & $101 \cdot 941$ & 7.433 & 4395 & 17 & 3815 & 8717 \\
\hline 230 & $46 \cdot 1$ & $+\cdot 046$ & $+\cdot 222$ & $19 \cdot 504$ & $108 \cdot 588$ & & 4886 & 18 & 4245 & 9147 \\
\hline 240 & $\ldots$ & ...... & & ...... & $\ldots$ & & $\ldots$ & 19 & 4672 & 9574 \\
\hline 250 & $\cdots$ & & & & & $7 \cdot 4617$ & & 20 & 5098 & 10000 \\
\hline 260 & & & & & & & & 21 & 5524 & 10426 \\
\hline 270 & $53 \cdot 6$ & +.073 & $+\cdot 399$ & $24 \cdot 124$ & $135 \cdot 158$ & & 6855 & 22 & 5950 & 10852 \\
\hline 280 & $55 \cdot 7$ & & & $25 \cdot 288$ & 141.803 & $\begin{array}{l}.509 \\
7.502\end{array}$ & 7350 & 23 & 6376 & 11278 \\
\hline 290 & $57 \cdot 7$ & +.084 & $+\cdot 524$ & $26 \cdot 456$ & $148 \cdot 427$ & & 7844 & 24 & 6802 & 11704 \\
\hline ... & $\ldots$ & & ...... & ...... & $\ldots \ldots$ & & $\cdots$ & 25 & 7228 & 12130 \\
\hline & $\cdots$ & & & & & & & 26 & 7651 & 12553 \\
\hline
\end{tabular}

${ }^{47}$ The first part of the experiments was lost, as the pen of the chronograph did not work. 
TABLE XLI.-Fifte Series.-Preliminary.

March 24, 1878. Jacket $5^{\circ} \cdot 4$ to $8^{\circ} .4$. Air about $6^{\circ} \mathrm{C}$.

\begin{tabular}{|c|c|c|c|c|c|c|c|c|c|c|}
\hline \multirow{2}{*}{ 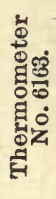 } & & \multicolumn{2}{|c|}{ Correction. } & \multirow{2}{*}{ 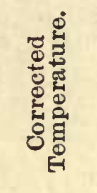 } & \multirow{2}{*}{ 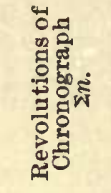 } & \multirow{2}{*}{ 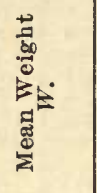 } & \multirow{2}{*}{ 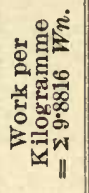 } & \multirow{2}{*}{ 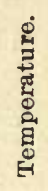 } & \multirow{2}{*}{ 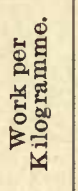 } & \multirow{2}{*}{ 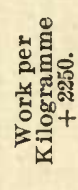 } \\
\hline & & $\begin{array}{l}\text { 苟 } \\
\text { 证 }\end{array}$ & జ్లై & & & & & & & \\
\hline 75 & 0.9 & -.003 & 0 & $1: 891$ & $3 \cdot 154$ & & 0 & $\stackrel{\circ}{2}$ & 46 & 2296 \\
\hline 80 & $1 \cdot 7$ & & & 2.451 & $6 \cdot 118$ & & 239 & 3 & 477 & 2727 \\
\hline 90 & $3 \cdot 4$ & -.002 & -.012 & $3 \cdot 569$ & $12 \cdot 174$ & & 723 & 4 & 906 & 3156 \\
\hline 100 & $5 \cdot 1$ & & & $4 \cdot 690$ & $18 \cdot 172$ & $\begin{array}{l}8.0409 \\
8.0074\end{array}$ & 1200 & 5 & 1332 & 3582 \\
\hline 110 & $6 \cdot 8$ & 0 & -.017 & $5 \cdot 810$ & $24 \cdot 212$ & $\begin{array}{l}8.0074 \\
7.9170\end{array}$ & 1677 & 6 & 1759 & 4009 \\
\hline 120 & $8 \cdot 5$ & $\ldots \ldots$ & & $6 \cdot 936$ & $30 \cdot 397$ & 7.8973 & 2161 & 6 & 2189 & 4439 \\
\hline 130 & $10 \cdot 2$ & +.003 & -.012 & $8 \cdot 060$ & $36 \cdot 621$ & 7.8986 & 2647 & 8 & 2621 & 4871 \\
\hline 140 & $12 \cdot 0$ & & & $9 \cdot 190$ & $42 \cdot 854$ & 7.8512 & 3132 & 9 & 3050 & 5300 \\
\hline 150 & $13 \cdot 7$ & +.007 & $+\cdot 005$ & $10 \cdot 323$ & $49 \cdot 068$ & 7.8012 & 3614 & 10 & 3477 & 5727 \\
\hline 160 & $15 \cdot 5$ & & & $11 \cdot 459$ & $55 \cdot 398$ & 7.8061 & 4103 & 11 & 3905 & 6155 \\
\hline 170 & $17 \cdot 2$ & $+\cdot 015$ & $+\cdot 032$ & $12 \cdot 600$ & $61 \cdot 707$ & $\begin{array}{l}7.7799 \\
7.7622\end{array}$ & 4588 & 12 & 4333 & 6583 \\
\hline 180 & $19 \cdot 0$ & & & $13 \cdot 742$ & $68 \cdot 036$ & 7.7643 & 5073 & 13 & 4759 & 7009 \\
\hline 190 & $20 \cdot 8$ & +.024 & $+\cdot 068$ & $14 \cdot 882$ & $74 \cdot 358$ & & 5558 & 14 & 5183 & 7433 \\
\hline 200 & $22 \cdot 6$ & +.028 & $+\cdot 092$ & $16 \cdot 025$ & $80 \cdot 716$ & 7.8419 & 6047 & 15 & 5608 & 7858 \\
\hline 210 & $24 \cdot 3$ & & ...... & $17 \cdot 170$ & $87 \cdot 064$ & 7.8468 & 6539 & 16 & 6036 & 8286 \\
\hline 220 & $26 \cdot 1$ & +.039 & $+\cdot 150$ & $18 \cdot 316$ & $93 \cdot 402$ & $\begin{array}{l}7.8468 \\
7.8579\end{array}$ & 7030 & 17 & 6466 & 8716 \\
\hline 230 & $27 \cdot 9$ & & ...... & $19 \cdot 467$ & $99 \cdot 677$ & & 7518 & 18 & 6895 & 9145 \\
\hline 240 & $29 \cdot 6$ & $+\cdot 050$ & $+\cdot 270$ & $20 \cdot 615$ & $105 \cdot 950$ & & 8006 & 19 & 7320 & 9570 \\
\hline 250 & $\cdots$ & $\ldots \ldots$ & $\ldots \ldots$ & ...... & ........ & 7.898 & $\ldots$. & 20 & 7745 & 9995 \\
\hline 260 & & & & & & & & 21 & 8170 & 10420 \\
\hline 270 & $34 \cdot 9$ & $+\cdot 069$ & $+\cdot 351$ & $24 \cdot 072$ & $124 \cdot 863$ & $7 \cdot 9038$ & 9482 & 22 & 8597 & 10847 \\
\hline 280 & $36 \cdot 7$ & & & $25 \cdot 231$ & $131 \cdot 181$ & $7 \cdot 9091$ & 9976 & 23 & 9024 & 11274 \\
\hline 290 & $38 \cdot 5$ & $+\cdot 087$ & $+\cdot 450$ & $26 \cdot 395$ & $137 \cdot 560$ & 7.8979 & 10474 & 24 & 9451 & 11701 \\
\hline 300 & $40 \cdot 2$ & & $\cdots$ & $27 \cdot 565$ & $143 \cdot 972$ & 7.8974 & 10974 & 25 & 9878 & 11128 \\
\hline 310 & $42 \cdot 1$ & $+\cdot 109$ & $+\cdot 583$ & $28 \cdot 748$ & $150 \cdot 467$ & 8.8974 & 11481 & 26 & 10305 & 12555 \\
\hline - & .... & ...... & ....... & ...... & ......... & & & 27 & 10733 & 12983 \\
\hline & & & & & ... & & $\ldots$ & 28 & 11160 & 13410 \\
\hline
\end{tabular}

TABLE XLII. - Sixth Series.

May 14,1878 . Jacket $12^{\circ} .1$ to $12^{\circ} .4$. Air about $13^{\circ} \mathrm{C}$.

\begin{tabular}{|c|c|c|c|c|c|c|c|c|c|c|}
\hline \multirow{2}{*}{ 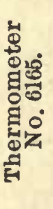 } & \multirow[b]{2}{*}{ 离 } & \multicolumn{2}{|c|}{ Correction. } & \multirow{2}{*}{ 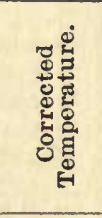 } & \multirow{2}{*}{ 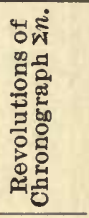 } & \multirow{2}{*}{ 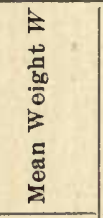 } & \multirow{2}{*}{ 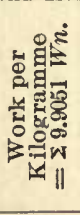 } & \multirow{2}{*}{ 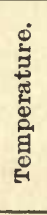 } & \multirow{2}{*}{ 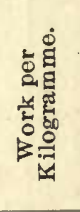 } & \multirow{2}{*}{ 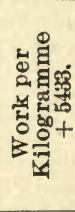 } \\
\hline & & $\begin{array}{l}\text { घi } \\
\text { 峁 }\end{array}$ & تึ & & & & & & & \\
\hline 140 & $46 \cdot 4$ & -.002 & 0 & $9: 319$ & $1 \cdot 93$ & & 0 & $\stackrel{\circ}{9}$ & -137 & 5296 \\
\hline 150 & 47. & & & $10 \cdot 178$ & 7.07 & $7 \cdot 2291$ & 370 & 10 & 293 & 5726 \\
\hline 160 & $49 \cdot 4$ & .000 & -.007 & 11.032 & $12 \cdot 19$ & & 735 & 11 & 721 & 6154 \\
\hline 170 & $50 \cdot 9$ & & ....... & $11 \cdot 8$ & $17 \cdot 37$ & $7 \cdot 1608$ & 1102 & 12 & 1151 & 6584 \\
\hline 180 & $52 \cdot 5$ & +.002 & -.008 & $12 \cdot 740$ & 22.52 & & 1467 & 13 & 1579 & 7012 \\
\hline 190 & $54 \cdot 0$ & & & $13 \cdot 5$ & $27 \cdot ?$ & $7 \cdot 1500$ & 1835 & 14 & 2007 & 7440 \\
\hline 200 & $55 \cdot 5$ & $\because .006$ & -.002 & $14 \cdot 454$ & $32 \cdot 88$ & & 2201 & 15 & 2434 & 7867 \\
\hline 210 & $57 \cdot 0$ & & ....... & $15 \cdot 314$ & $38 \cdot 07$ & $7 \cdot 1512$ & 2568 & 16 & 2863 & 8296 \\
\hline 220 & $58 \cdot 5$ & $+\cdot 010$ & +.011 & $16 \cdot 174$ & $43 \cdot 29$ & & 2938 & 17 & 3290 & 8723 \\
\hline
\end{tabular}


TABLE XLII._Continued.

\begin{tabular}{|c|c|c|c|c|c|c|c|c|c|c|}
\hline \multirow{2}{*}{ 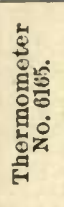 } & \multirow[b]{2}{*}{ 离 } & \multicolumn{2}{|c|}{ Correction. } & \multirow{2}{*}{ 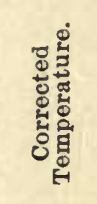 } & \multirow{2}{*}{ 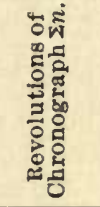 } & \multirow{2}{*}{ 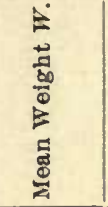 } & \multirow{2}{*}{ 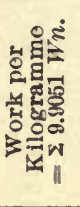 } & \multirow{2}{*}{ 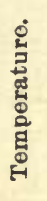 } & \multirow{2}{*}{ 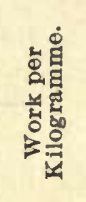 } & \multirow{2}{*}{ 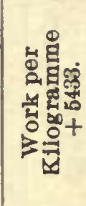 } \\
\hline & & 㷰 & త్ర & & & & & & & \\
\hline 230 & 0.0 & & & :037 & & & & & & \\
\hline 240 & 61 & +.015 & +.031 & & & & 3306 & 18 & 3716 & 9149 \\
\hline 250 & & & & & & & 3675 & & 4142 & 95 \\
\hline & & & & & & & $\cdots$ & & 4567 & 100 \\
\hline 270 & $6 \cdot 2$ & +.024 & +.075 & 2 & $69 \cdot 27$ & & & & & $104:$ \\
\hline 280 & & & & & & & & & & \\
\hline 290 & & +031 & $+\cdot 113$ & & & & & & & \\
\hline 300 & & & & & & & & & & \\
\hline 310 & & +.039 & $+\cdot 158$ & & & & & & & \\
\hline 320 & & & & & & & & & & \\
\hline 330 & & $+\cdot 047$ & $+\cdot 212$ & & & & & & & \\
\hline 340 & & & & & & & & & & \\
\hline & & +.056 & +.272 & & & & & & & \\
\hline 360 & & & & & & & & & & 14 \\
\hline & & +.065 & $+\cdot 341$ & & & & & & & \\
\hline & & & & & & & $83 !$ & 32 & & 151 \\
\hline & & +.076 & +.417 & & & & & & 10096 & 15529 \\
\hline & & & & & & & 911 & . & .... & $\ldots$ \\
\hline 41 & & $+\cdot 087$ & $+\cdot 504$ & & & & & & & \\
\hline 420 & $88 \cdot 7$ & & & $33 \cdot 226$ & $146 \cdot 08$ & & $\begin{array}{r}10193 \\
\end{array}$ & . & & \\
\hline
\end{tabular}

TABLE XLIII.-SEVENTH SERIES.

May 15,1878 . Jacket $11^{\circ} .8$ to $12^{\circ}$. Air about $12^{\circ} \mathrm{C}$.

\begin{tabular}{|c|c|c|c|c|c|c|c|c|c|c|}
\hline \multirow{2}{*}{ 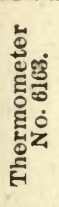 } & \multirow[b]{2}{*}{$\stackrel{\stackrel{\oplus}{E}}{\mathbb{H}}$} & \multicolumn{2}{|c|}{ Correction. } & \multirow{2}{*}{ 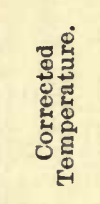 } & \multirow{2}{*}{ 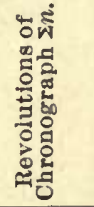 } & \multirow{2}{*}{ 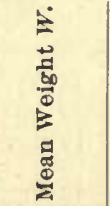 } & \multirow{2}{*}{ 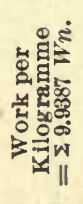 } & \multirow{2}{*}{ 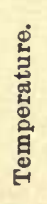 } & \multirow{2}{*}{ 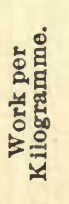 } & \multirow{2}{*}{ 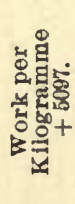 } \\
\hline & & $\frac{8}{8}$ & జّँّ & & & & & & & \\
\hline 130 & 30.9 & -.004 & 0 & $8^{\circ} .538$ & 5.07 & & 0 & 0 & & \\
\hline 140 & 32.2 & & & 9.315 & 9.73 & 7.2350 & 335 & 9 & 199 & 5296 \\
\hline 150 & 33.6 & -.002 & -.006 & 10.094 & 14.36 & & 668 & 10 & 628 & 5725 \\
\hline 160 & 35.0 & & & 10.875 & 18.98 & 7.3011 & 1003 & 11 & 1056 & 6153 \\
\hline 170 & 36.3 & 0 & -.010 & 11.654 & 23.5 & & 1335 & 12 & 1484 & 6581 \\
\hline 180 & 37.6 & & & 12.433 & 28.16 & 7.3165 & 1670 & 13 & 1913 & 7010 \\
\hline 190 & 33.9 & +.003 & -.008 & 13.209 & 32.74 & & 2003 & 14 & 2344 & 7441 \\
\hline 200 & 40.2 & & & 13.984 & 37.31 & 7.3460 & 2337 & 15 & 2770 & 7867 \\
\hline 210 & 41.5 & +.006 & .000 & 14.7 & 41.84 & & 2667 & 16 & 319 & 8293 \\
\hline 220 & 42.8 & ...... & & 15.536 & 46.38 & 7.3094 & 2998 & 17 & 8623 & 8720 \\
\hline 230 & 44.2 & +.010 & +.013 & 16.3 & & & 3332 & 18 & 4052 & 9149 \\
\hline 240 & 45.5 & & & 17.103 & 55.62 & 7.2846 & 3667 & 19 & 4478 & 9575 \\
\hline 250 & 46.9 & +.014 & +.032 & 17.8 & 60.29 & & 4005 & 20 & 4906 & 10003 \\
\hline 260 & 48.3 & & & & & 7.2822 & & 21 & 5324 & 10421 \\
\hline 270 & 49.6 & +.019 & +.056 & 19.4 & 69.63 & & 4681 & 22 & 5754 & 10851 \\
\hline 280 & 50.9 & & & & 74.34 & 7.2610 & 5021 & 23 & 6179 & 11276 \\
\hline 290 & 52.3 & +.025 & +.090 & 21.079 & 79.01 & & 5358 & 24 & 6603 & 11700 \\
\hline
\end{tabular}


TABLE XLIII.-Continued.

\begin{tabular}{|c|c|c|c|c|c|c|c|c|c|c|}
\hline \multirow{2}{*}{ 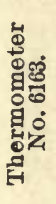 } & \multirow[b]{2}{*}{ 昜 } & \multicolumn{2}{|c|}{ Correction. } & \multirow{2}{*}{ 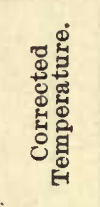 } & \multirow{2}{*}{ 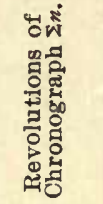 } & \multirow{2}{*}{ 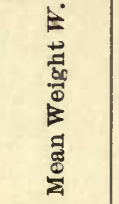 } & \multirow{2}{*}{ 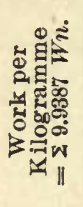 } & \multirow{2}{*}{ 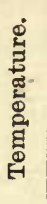 } & \multirow{2}{*}{ 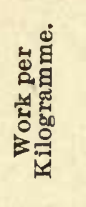 } & \multirow{2}{*}{ 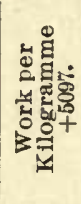 } \\
\hline & & $\frac{\dot{d}}{\Delta}$ & జึં & & & & & & & \\
\hline 300 & 53.6 & & & 21.866 & 83.71 & 7.2504 & 5697 & 25 & 7028 & 12125 \\
\hline 310 & 55.0 & +.032 & +.127 & 22.665 & 88.42 & & 6037 & 26 & 7454 & 12551 \\
\hline 320 & 56.4 & & & 23.471 & 93.14 & 7.2893 & 6379 & 27 & 7883 & 12980 \\
\hline 330 & 57.8 & +.039 & +.172 & 24.281 & 97.88 & & 6722 & 28 & 8307 & 13404 \\
\hline 340 & 59.2 & & & 25.088 & 102.61 & 7.3047 & 7065 & 29 & 8729 & 13826 \\
\hline 350 & 60.5 & +.046 & +.222 & 25.896 & 107.36 & & 7410 & 30 & 9157 & 14254 \\
\hline 360 & 61.9 & ...... & $\ldots .$. & 26.706 & 112.14 & 7.3389 & 7759 & 31 & 9582 & 14679 \\
\hline 370 & 63.2 & +.055 & +.279 & 27.523 & 116.88 & & 8104 & 32 & 10009 & 15106 \\
\hline 380 & 64.6 & & & 28.346 & 121.62 & 7.4109 & 8454 & $\cdots$ & $\ldots \ldots$ & $\cdots$ \\
\hline 390 & 66.0 & +.065 & +.345 & 29.172 & 126.34 & & 8801 & .. & & \\
\hline 400 & 67.4 & & & 29.996 & 131.12 & 7.4356 & 9155 & .. & & \\
\hline 410 & 68.8 & +.075 & +.419 & 30.827 & 135.90 & & 9508 & .. & $\ldots \ldots$ & \\
\hline 420 & 70.1 & +.080 & +.456 & 31.653 & 140.66 & & 9861 & $\ldots$ & & \\
\hline
\end{tabular}

TABLE XLIV.-Erghth Shries.

May 23, 1878 . Jacket $16^{\circ} .2$ to $16^{\circ} .5$. Air about $20^{\circ} \mathrm{C}$.

\begin{tabular}{|c|c|c|c|c|c|c|c|c|c|c|}
\hline \multirow{2}{*}{ 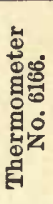 } & \multirow[b]{2}{*}{ 惫 } & \multicolumn{2}{|c|}{ Correction. } & \multirow{2}{*}{ 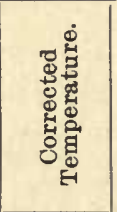 } & \multirow{2}{*}{ 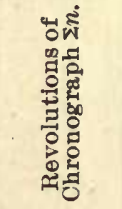 } & \multirow{2}{*}{ 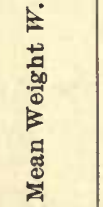 } & \multirow{2}{*}{ 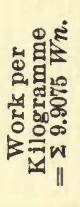 } & \multirow{2}{*}{ 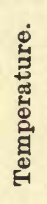 } & \multirow{2}{*}{ 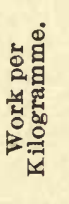 } & \multirow{2}{*}{ 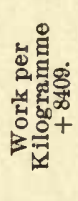 } \\
\hline & & $\begin{array}{l}\text { घू } \\
\text { 怘 }\end{array}$ & జึّ & & & & & & & \\
\hline 230 & 23.9 & -.007 & 0 & $16^{\circ} .287$ & 39.120 & & 0 & $\circ$ & & \\
\hline 240 & 25.4 & ...... & $\ldots \ldots$ & 17.063 & 43.982 & 6.9137 & 333 & i7 & 306 & 8715 \\
\hline 250 & 26.8 & & & $\ldots \ldots$ & & & $\ldots$ & 18 & 735 & 9144 \\
\hline 260 & 28.3 & $\ldots \ldots$ & & & & & & 19 & 1163 & 9572 \\
\hline 270 & 29.7 & .000 & +.005 & 19.405 & 58.602 & & 1338 & 20 & 1592 & 10001 \\
\hline 280 & 31.2 & & & 20.190 & 63.503 & & 1673 & 21 & 2019 & 10428 \\
\hline 290 & 32.7 & $\ldots$ & ..... & 20.978 & 68.428 & & 2010 & 22 & 2446 & 10855 \\
\hline 300 & 34.2 & & $\cdots$ & 21.765 & 73.351 & 6.8878 & 2346 & 23 & 2871 & 11280 \\
\hline 310 & 35.6 & +.008 & +.040 & 22.554 & 78.283 & 6.8866 & 2682 & 24 & 3298 & 11707 \\
\hline 320 & 37.1 & $\ldots$ & $\ldots$ & 23.350 & 83.245 & 6.8594 & 3020 & 25 & 3722 & 12131 \\
\hline 330 & 38.6 & & $\ldots$ & 24.151 & 88.314 & 6.8358 & 3363 & 26 & 4150 & 12559 \\
\hline 340 & 40.1 & +.017 & +.085 & 24.952 & 93.294 & 6.8748 & 3702 & 27 & 4574 & 12983 \\
\hline 350 & 41.6 & $\ldots$ & . & 25.751 & 98.275 & 6.9184 & 4044 & 28 & 4999 & 13408 \\
\hline 360 & 43.1 & & $\ldots$ & 26.552 & 103.232 & 6.9444 & 4385 & 29 & 5423 & 13832 \\
\hline 370 & 44.6 & +.028 & +.144 & 27.361 & 108.216 & 6.9291 & 4727 & 30 & 5851 & 14260 \\
\hline 380 & 46.0 & & $\ldots$ & 28.175 & 113.269 & 6.9338 & 5074 & 31 & 6275 & 14684 \\
\hline 390 & 47.5 & ... & & 28.989 & 118.281 & 6.9385 & 5418 & .. & $\ldots$ & $\ldots$ \\
\hline 400 & 49.0 & +.039 & +.217 & 29.800 & 123.329 & 6.9444 & 5766 & .. & $\ldots$ & $\ldots$ \\
\hline 410 & 50.6 & & & 30.624 & 128.399 & 6.9467 & 6115 & .. & $\ldots$ & $\ldots$ \\
\hline 420 & 52.1 & +.047 & +.281 & 31.445 & 133.480 & 6.9314 & 6464 & .. & $\ldots$ & $\ldots$ \\
\hline
\end{tabular}


TABLE XLV. - Ninth Series.

May 27,1878 . Jacket $19^{\circ} .6$ to $20^{\circ}$. Air about $23^{\circ} \mathrm{C}$.

\begin{tabular}{|c|c|c|c|c|c|c|c|c|c|c|}
\hline \multirow{2}{*}{ 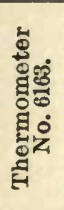 } & \multirow[b]{2}{*}{$\begin{array}{l}\text { छ̊ } \\
\text { है }\end{array}$} & \multicolumn{2}{|c|}{ Correction. } & \multirow{2}{*}{ 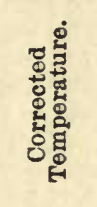 } & \multirow{2}{*}{ 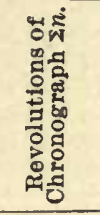 } & \multirow{2}{*}{ 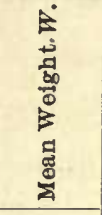 } & \multirow{2}{*}{ 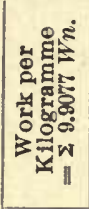 } & \multirow{2}{*}{ 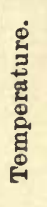 } & \multirow{2}{*}{ 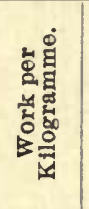 } & \multirow{2}{*}{ 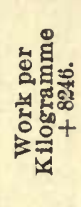 } \\
\hline & & $\begin{array}{l}\text { घू: } \\
\text { \& }\end{array}$ & "్ష & & & & & & & \\
\hline 200 & 38.0 & -.015 & 0 & & 6.33 & & 0 & 16 & 47 & 8293 \\
\hline 210 & & & & & 11. & 8.8108 & 473 & 17 & 473 & 8719 \\
\hline 220 & & 011 & -.010 & & & & 946 & & 901 & 9147 \\
\hline 230 & 42 & & & & 22 . & 8.7341 & 1419 & 19 & 1326 & 9572 \\
\hline & & .005 & -.0 & & & & 1895 & 20 & 1754 & 10000 \\
\hline 250 & 45. & & & & 33. & & 2368 & 21 & 2180 & 10426 \\
\hline 260 & & & -.0 & & & & & 22 & 2606 & 10852 \\
\hline 270 & & & & & & & & 23 & 3031 & 11277 \\
\hline 280 & & & +.012 & & 50 & & 3785 & 24 & 3457 & 11703 \\
\hline 290 & & & & & & 8.4399 & 4263 & 25 & 3883 & 12129 \\
\hline 300 & & & +.0 & & & & 4737 & 26 & 4312 & 12558 \\
\hline & & & & & & 8.4765 & 5215 & 27 & 4734 & 12980 \\
\hline 320 & & & +.0 & & & & 5697 & 28 & 5159 & 13405 \\
\hline 33 & & & & & & 8.4552 & 6182 & 29 & 5584 & 13830 \\
\hline & & & & & & & $66 \mathrm{C}$ & 30 & 6010 & 14256 \\
\hline & & & & & & 8.4015 & 7159 & 31 & 6435 & 14681 \\
\hline & & & & & & & 76 & 3 & 6860 & \\
\hline & & & & & & 8.4222 & 8143 & 33 & 7286 & 15532 \\
\hline & & & +.242 & & & & 8638 & 34 & 7714 & 15960 \\
\hline & & & & & & 4706 & 9128 & 35 & 8138 & 16384 \\
\hline & & & +.32 & & & & 9626 & 36 & 8565 & 16811 \\
\hline 410 & & & & & & 4316 & 10126 & 37 & 8988 & 17234 \\
\hline 420 & 71.8 & +.105 & +.419 & 40.833 & 132.26 & & 10620 & 38 & 9414 & 17660 \\
\hline ... & $\cdots$ & & & & & & & 39 & & \\
\hline & & & & & & & & 40 & 10268 & 18514 \\
\hline & & & & & & & & 41 & 10691 & 18937 \\
\hline
\end{tabular}


TABLE XLVI.-TeNTH SERIES.

June 3,1878 . Jacket $18^{\circ} .1$ to $18^{\circ} .4$. Air about $20^{\circ} \mathrm{C}$.

\begin{tabular}{|c|c|c|c|c|c|c|c|c|c|c|}
\hline \multirow{2}{*}{ 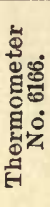 } & \multirow[b]{2}{*}{$\underset{⿱ 乛}{\stackrel{\Xi}{E}}$} & \multicolumn{2}{|c|}{ Correction. } & \multirow{2}{*}{ 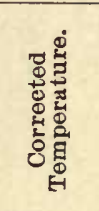 } & \multirow{2}{*}{ 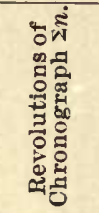 } & \multirow{2}{*}{ 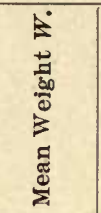 } & \multirow{2}{*}{ 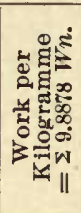 } & \multirow{2}{*}{ 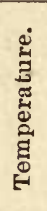 } & \multirow{2}{*}{ 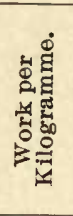 } & \multirow{2}{*}{ 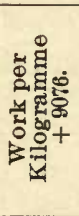 } \\
\hline & & 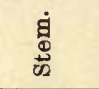 & 苐 & & & & & & & \\
\hline 250 & 4.1 & -.007 & 0 & $17^{\circ} .838$ & 7.82 & & 0 & $1 \stackrel{8}{8}$ & 69 & 9145 \\
\hline 260 & 7.0 & & & 18.617 & $\ldots \ldots$ & 4.3899 & $\ldots$ & 19 & 496 & 9572 \\
\hline 270 & 9.9 & -.003 & +.004 & 19.401 & 23.19 & & 667 & 20 & 925 & 10001 \\
\hline 280 & 12.8 & $\ldots \ldots$ & $\ldots \ldots$ & 20.188 & 30.95 & 4.3919 & 1005 & 21 & 1350 & 10426 \\
\hline 290 & 15.7 & +.003 & +.020 & 20.978 & 38.70 & & 1341 & 22 & 1778 & 10854 \\
\hline 300 & 18.7 & $\ldots \ldots$ & $\ldots \ldots$ & 21.763 & 46.41 & 4.3912 & 1676 & 23 & 2204 & 11280 \\
\hline 310 & 21.6 & +.008 & +0.037 & 22.551 & 54.21 & & 2014 & 24 & 2627 & 11703 \\
\hline 320 & 24.5 & ... & $\ldots \ldots$ & 23.354 & 62.04 & 4.3907 & 2354 & 25 & 3054 & 12130 \\
\hline 330 & 27.5 & +.014 & +.078 & 24.162 & 69.92 & & 2696 & 26 & 3479 & 12555 \\
\hline 340 & 30.5 & $\ldots \ldots$ & $\ldots \ldots$ & 24.970 & 77.92 & 4.3624 & 3041 & 27 & 3904 & 12980 \\
\hline 350 & 33.6 & +.020 & +.132 & 25.780 & 85.89 & & 3385 & 28 & 4332 & 13408 \\
\hline 360 & 36.6 & $\ldots$. & $\ldots \ldots$ & 26.593 & 93.94 & 4.3542 & 3731 & 29 & 4852 & 13828 \\
\hline 370 & 39.6 & +.028 & +.198 & 27.415 & 102.05 & & 4081 & 30 & 5179 & 14255 \\
\hline 380 & 42.7 & & & 28.246 & 110.34 & 4.3362 & 4437 & 31 & 5604 & 14680 \\
\hline 390 & 45.8 & +.036 & +.281 & 29.079 & 118.49 & & 4786 & $\ldots$ & & \\
\hline 400 & 48.9 & & & 29.911 & 126.66 & 4.3978 & 5141 & & & \\
\hline 410 & 52.0 & +.044 & +.377 & 30.754 & 134.89 & & 5499 & & & \\
\hline
\end{tabular}

TABLE XLVII. - Eleventh Series.

June 19,1878 . Jacket $19^{\circ} .6$ to $20^{\circ}$. Air about $23^{\circ} \mathrm{C}$.

\begin{tabular}{|c|c|c|c|c|c|c|c|c|c|c|}
\hline \multirow{2}{*}{ 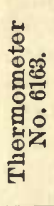 } & \multirow[b]{2}{*}{ 总 } & \multicolumn{2}{|c|}{ Correction. } & \multirow{2}{*}{ 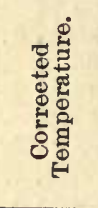 } & \multirow{2}{*}{ 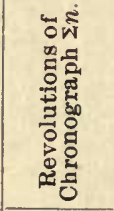 } & \multirow{2}{*}{ 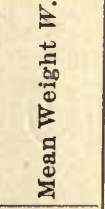 } & \multirow{2}{*}{ 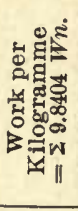 } & \multirow{2}{*}{ 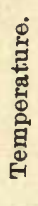 } & \multirow{2}{*}{ 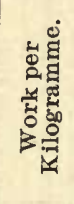 } & \multirow{2}{*}{ 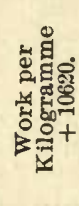 } \\
\hline & & $\frac{\dot{E}}{\dot{0}}$ & శ్ & & & & & & & \\
\hline 250 & $\ldots$ & -.002 & 0 & $21^{\circ} .450$ & 8.933 & 6757 & 0 & 21 & -192 & 10428 \\
\hline 260 & $\ldots$ & +.002 & +.006 & 22.562 & 16.087 & & 476 & 22 & 235 & 10855 \\
\hline 270 & $\ldots$. & $\ldots \ldots$ & $\ldots \ldots$ & & $\ldots \ldots$ & 6.7678 & & 23 & 662 & 11282 \\
\hline 280 & & +.010 & +.029 & 24.789 & $\begin{array}{ll}30 & 281\end{array}$ & & 1421 & 24 & 1087 & 11707 \\
\hline 290 & & $\ldots .$. & $\ldots \ldots$ & 25.907 & 37.439 & 6.7749 & 1899 & 25 & 1511 & 12131 \\
\hline 300 & $\ldots$ & +.019 & +.063 & 27.032 & 44.655 & & 2379 & 26 & 1939 & 12559 \\
\hline 310 & $\ldots$ & $\ldots \ldots$ & $\ldots \ldots$ & 28.168 & 51.848 & 6.7896 & 2860 & 27 & 2365 & 12985 \\
\hline 320 & $\ldots$ & +.031 & +.113 & 29.307 & 59.098 & & 3344 & 28 & 2789 & 13409 \\
\hline 330 & $\ldots$ & $\ldots \ldots$ & $\ldots \ldots$ & 30.456 & 66.390 & 6.7973 & 3832 & 29 & 3214 & 13834 \\
\hline 340 & $\ldots$ & +.043 & +.177 & 31.612 & 73.724 & & 4323 & 30 & 3638 & 14258 \\
\hline 350 & & & & 32.774 & 81.153 & 6.8188 & 4817 & 31 & 4063 & 14683 \\
\hline 360 & $\ldots$ & +.058 & +.257 & 33.939 & 88.462 & & 5311 & 32 & 4488 & 15108 \\
\hline 370 & $\ldots$ & $\ldots \ldots$ & $\ldots \ldots$ & 35.110 & 95.734 & 6.9165 & 5807 & 33 & 4913 & 15533 \\
\hline 380 & $\ldots$ & +.072 & +.351 & 36.280 & 103.093 & & 6307 & 34 & 5337 & 15957 \\
\hline 390 & $\ldots$ & $\ldots \ldots$ & $\ldots \ldots$ & 37.456 & 110.560 & 6.7876 & 6808 & 35 & 5760 & 16380 \\
\hline 400 & $\ldots$ & +.087 & +.463 & 38.637 & 118.121 & & 7311 & 36 & 6187 & 16807 \\
\hline 410 & $\ldots$ & & $\ldots \ldots$ & 39.821 & 125.693 & 6.7808 & 7815 & 37 & 6614 & 17234 \\
\hline 420 & $\ldots$ & +.106 & +.595 & 41.010 & 133.250 & & 8321 & 38 & 7040 & 17660 \\
\hline$\ldots$ & ... & ..... & $\ldots \ldots$ & ..... & $\ldots \ldots$ & ..... & ... & 39 & 7465 & 18085 \\
\hline$\ldots$ & $\ldots$ & ..... & ..... & ..... & ..... & $\ldots \ldots$ & $\ldots$ & 40 & 7891 & 18511 \\
\hline$\ldots$ & . & $\ldots \ldots$ & $\ldots \ldots$ & $\ldots \ldots$ & $\ldots \ldots$ & & $\ldots$ & 41 & 8317 & 18937 \\
\hline
\end{tabular}




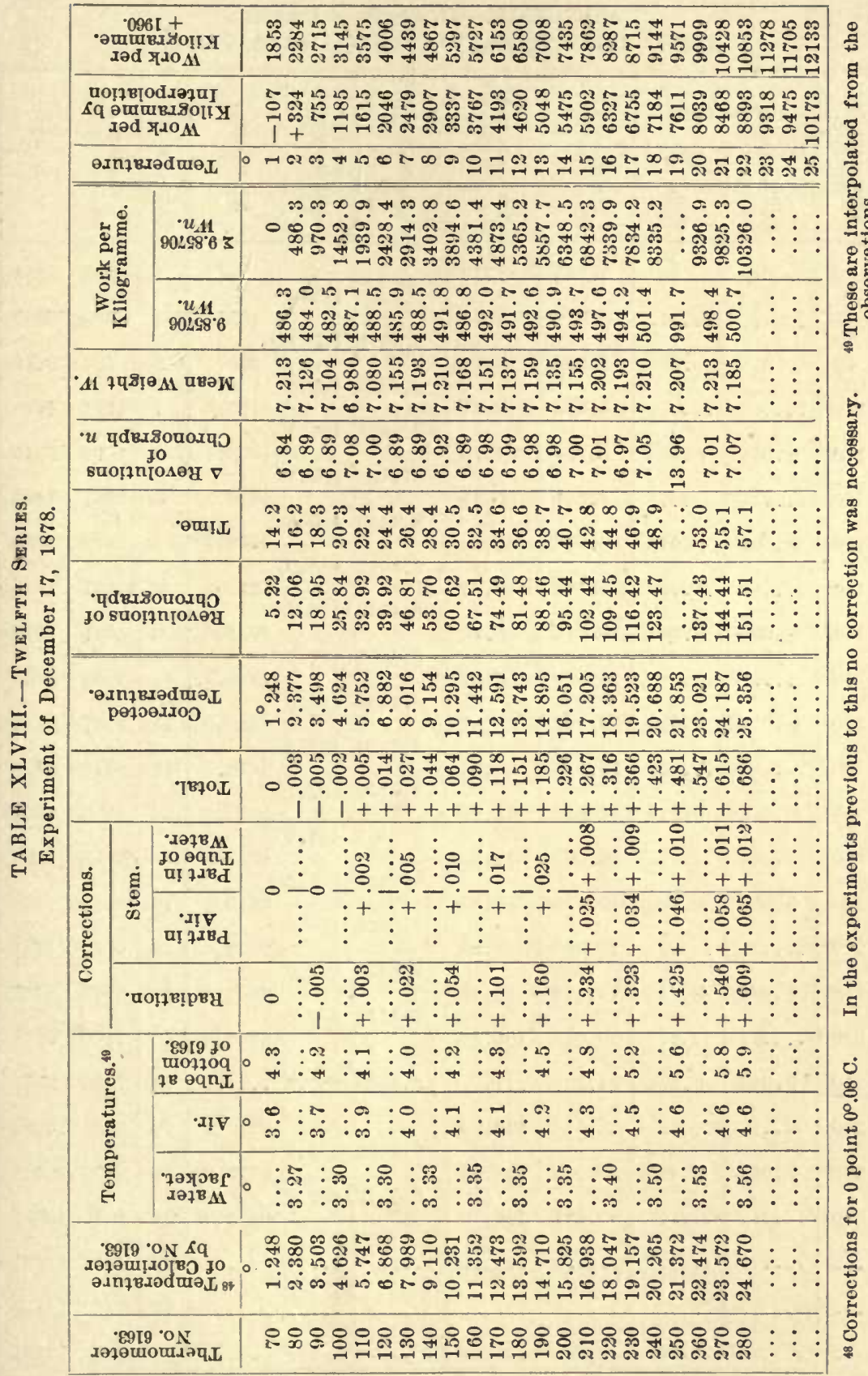


TABLE XLIX. - Thirteenth Series.

Dec. 19,1878 . Jacket $3^{\circ} .2$ to $3^{\circ} .5$. Air $4^{\circ} .2$ to $5.2 \mathrm{C}$.

\begin{tabular}{|c|c|c|c|c|c|c|c|c|c|c|}
\hline \multirow{2}{*}{ 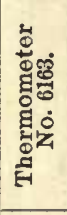 } & \multicolumn{2}{|c|}{ Corrections. } & \multirow{2}{*}{ 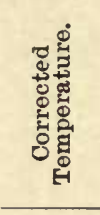 } & \multirow{2}{*}{ 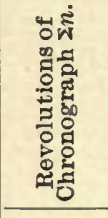 } & \multirow{2}{*}{ 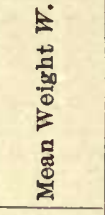 } & \multirow{2}{*}{ 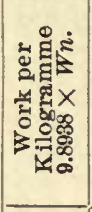 } & \multirow{2}{*}{ 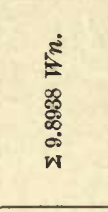 } & \multirow{2}{*}{ 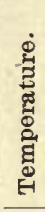 } & \multirow{2}{*}{ 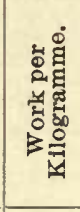 } & \multirow{2}{*}{ 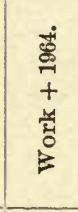 } \\
\hline & $\begin{array}{l}\dot{g} \\
\frac{8}{\infty}\end{array}$ & 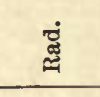 & & & & & & & & \\
\hline 70 & 0 & 0 & 1.248 & 1.72 & 86610 & 4850 & 0 & $\stackrel{\circ}{1}$ & -106 & 1858 \\
\hline 80 & ...... & ..... & 2.378 & 7.38 & .0010 & 100.0 & 485.0 & 2 & +323 & 2287 \\
\hline 90 & 0 & -.003 & 3.500 & 13.11 & . 0081 & 200.2 & 970.1 & 3 & 754 & 2718 \\
\hline 100 & ..... & ...... & 4.626 & 18.89 & 8.3688 & 481.1 & 1452.3 & 4 & 1184 & 3148 \\
\hline 110 & +.001 & +.003 & 5.751 & 24.70 & 8.4155 & 487.1 & 1933.4 & 5 & 1612 & 3576 \\
\hline 120 & $\ldots \ldots$ & $\cdots \cdot$ & 6.881 & 30.55 & 8.4189 & & 2420.5 & 6 & 2041 & 4005 \\
\hline 130 & +.005 & +.019 & 8.013 & 36.38 & 8.3953 & 489.2 & 2906.1 & 7 & 2472 & 4436 \\
\hline 140 & $\ldots \ldots$ & ...... & 9.148 & 42.27 & 8.4366 & 486.6 & 3395.3 & 8 & 2901 & 4865 \\
\hline 150 & +.009 & +.044 & 10.284 & 48.10 & 8,4484 & 486.5 & 3881.9 & 9 & 3331 & 5295 \\
\hline 160 & ..... & ..... & 11.424 & 53.92 & 8.4189 & 490,6 & 4368.4 & 10 & 3760 & 5724 \\
\hline 170 & +.016 & +.080 & 12.569 & 59.81 & 8.3988 & 491.1 & 4859.0 & 11 & 4187 & 6151 \\
\hline 180 & $\cdots \cdots$ & $\cdots \cdots$ & 13.713 & 65.72 & 8.4153 & 487.1 & 5350.1 & 12 & 4615 & 6579 \\
\hline 190 & +.023 & +.126 & 14.859 & 71.57 & 8.3 & 491.7 & 5837.2 & 13 & 5045 & 7009 \\
\hline 200 & $\ldots \ldots$ & ..... & 16.005 & 77.50 & & 489.4 & 6328.9 & 14 & 5472 & 7436 \\
\hline 210 & +.033 & +.183 & 17.154 & 83.40 & 8.39 & 490.2 & 6818.3 & 15 & 5898 & 7862 \\
\hline 220 & ..... & ..... & 18.300 & 89.30 & & & 7308.5 & 16 & 6327 & 8291 \\
\hline 230 & +.044 & +.251 & 19.452 & 95.23 & & & 7801.5 & 17 & 6753 & 8717 \\
\hline 240 & ..... & ...... & 20.604 & 101.17 & & & 8297.9 & 18 & 7180 & 9144 \\
\hline 250 & +.056 & +.332 & 21.760 & $\cdots \cdots$ & 8.4555 & 981.3 & & 19 & 7608 & 9572 \\
\hline 260 & & & 22.912 & 112.90 & & & 9279.2 & 20 & 8038 & 10002 \\
\hline 270 & +.069 & +.424 & 24.065 & 118.81 & & 494.7 & 9773.9 & 21 & 8465 & 10429 \\
\hline 280 & ..... & ...... & 25.221 & 124.70 & 0.7 & & 10267.9 & 22 & 8891 & 10855 \\
\hline$\cdots$ & ..... & ..... & .... & … & 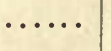 & & ...... & 23 & 9317 & 11281 \\
\hline .. & & ...... & & $\cdots \cdots$ & $\cdots$ & & ...... & 24 & 9746 & 11710 \\
\hline & & & ...... & ...... & ....... & $\ldots$. & . . . . & 25 & 10173 & 12137 \\
\hline
\end{tabular}


TABLE L. - Fourteenth Series.

December 20,1878 . Jacket $1^{\circ} .5$ to $1^{\circ} .9$. Air about $3^{\circ} .4 \mathrm{C}$.

\begin{tabular}{|c|c|c|c|c|c|c|c|c|c|c|c|}
\hline \multirow[b]{2}{*}{ 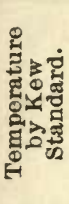 } & \multirow[b]{2}{*}{$\underset{\sharp}{\stackrel{\sharp}{*}}$} & \multicolumn{3}{|c|}{ Corrections. } & \multirow[b]{2}{*}{ 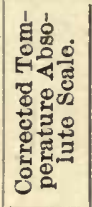 } & \multirow[b]{2}{*}{ 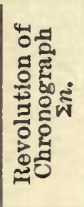 } & \multirow[b]{2}{*}{ 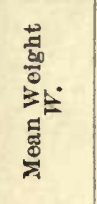 } & \multirow[b]{2}{*}{ 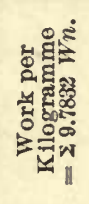 } & \multirow[b]{2}{*}{ 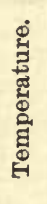 } & \multirow[b]{2}{*}{ 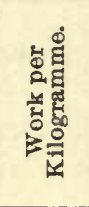 } & \multirow[b]{2}{*}{ 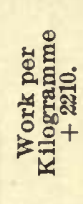 } \\
\hline & & 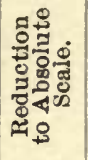 & 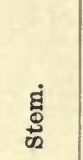 & జึં & & & & & & & \\
\hline 36.0 & 56.0 & .00 & 0 & 0 & 1.82 & 8.03 & 7.3682 & 0 & $\stackrel{8}{2}$ & 77 & 2287 \\
\hline 38.5 & 58.4 & & & & 3.23 & 16.37 & 7.3458 & 601 & 3 & 503 & 2713 \\
\hline 41.0 & .9 & -.01 & .00 & +.01 & 4.62 & 24.78 & 7.3705 & 1206 & 4 & 936 & 3146 \\
\hline 43.5 & 3.3 & & & & 6.02 & 33.19 & 7.4012 & 1812 & 5 & 1370 & 3580 \\
\hline 46.0 & & -.02 & +.01 & +.04 & 7.43 & 41.48 & 7.4142 & 2412 & 6 & 1803 & 4013 \\
\hline 48. & & & & & 84 & 49.81 & 7.4177 & 301 & 7 & 2226 & 4436 \\
\hline 51.0 & & -.03 & +.02 & +.09 & 10.26 & 58.18 & 7.4390 & 3624 & 8 & 2656 & 4866 \\
\hline 53.5 & & & & & 1.68 & 66.56 & 7.4107 & 4234 & 9 & 3084 & 529 \\
\hline 56.0 & & -.04 & +.03 & +.16 & 13.12 & 74.95 & 7.3493 & 4842 & 10 & & 5723 \\
\hline 58.5 & & & & & & 83.56 & 7.3 & 5461 & 11 & & 615 \\
\hline 61.0 & & -.04 & +.05 & +.25 & 16.01 & 92.27 & 7.2335 & 6085 & 12 & 436 & 6579 \\
\hline 63.5 & & & & & 17.46 & & 7.1603 & 6703 & 1 & & 7000 \\
\hline 66.0 & & -.05 & +.06 & +.38 & & 109.95 & 7.2075 & 7330 & 14 & 220 & 7430 \\
\hline 68.5 & & & & & 20.39 & I18.84 & 7.1839 & 7957 & 1 & 550 & 7860 \\
\hline 71.0 & & & +.08 & +.52 & & & & 8589 & 16 & & 8291 \\
\hline 73.5 & & & & & & & & 9218 & 17 & & 8717 \\
\hline 76.0 & & -.05 & +.10 & +.69 & 24 & & & & 18 & & 9145 \\
\hline 78.5 & & & & & & & & & 19 & & 9574 \\
\hline . & & & & & & & & & 20 & 7791 & 10001 \\
\hline & & & & & & & & & 21 & 8219 & 10429 \\
\hline & & & & & & & & & 22 & 8648 & 10858 \\
\hline & & & & & & & & & 23 & 9074 & 11284 \\
\hline & & & & & & & & & 24 & 9499 & 11709 \\
\hline & & & & & & & & ..... & 25 & 9925 & 12135 \\
\hline & & & & & & & & & 26 & 10352 & 12562 \\
\hline
\end{tabular}




\begin{tabular}{|c|c|}
\hline 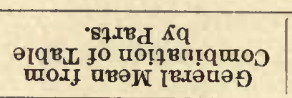 & 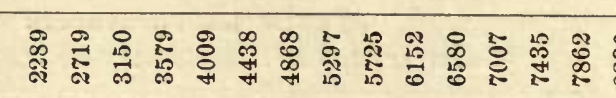 \\
\hline 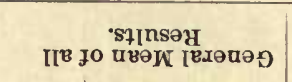 & 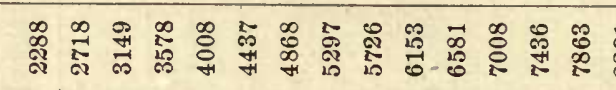 \\
\hline symergy & 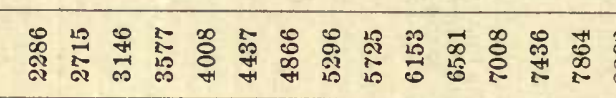 \\
\hline 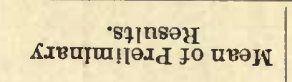 & \\
\hline 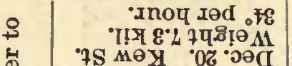 & | \\
\hline 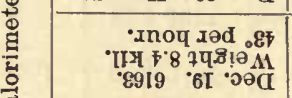 & 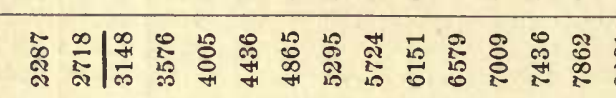 \\
\hline 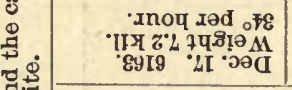 & 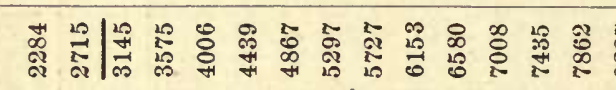 \\
\hline 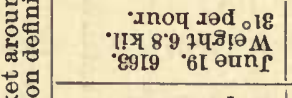 & 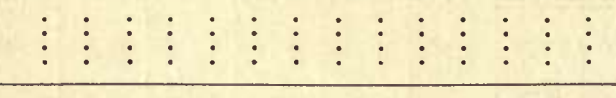 \\
\hline 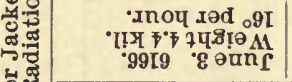 & 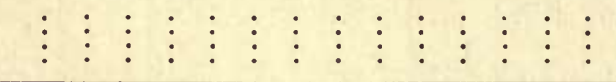 \\
\hline 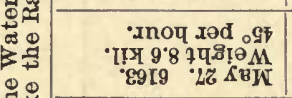 & : \\
\hline 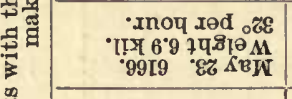 & : : : : : : $: \vdots: \vdots \vdots$ \\
\hline 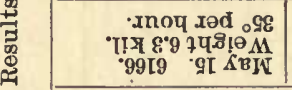 & 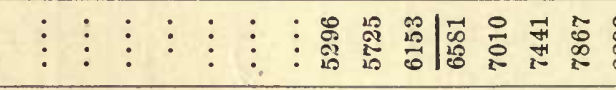 \\
\hline 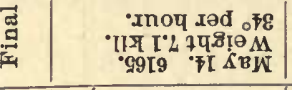 & 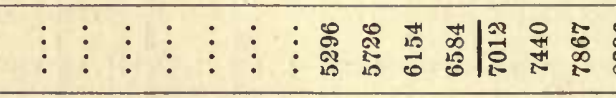 \\
\hline 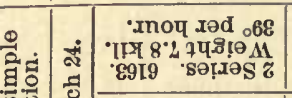 & 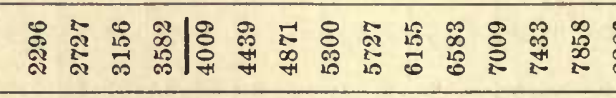 \\
\hline 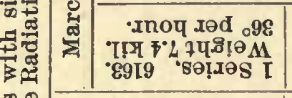 & 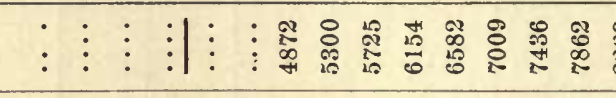 \\
\hline 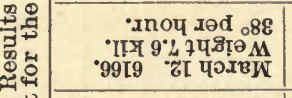 & 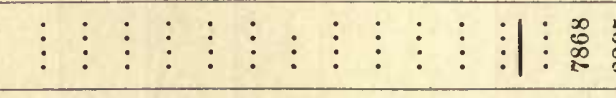 \\
\hline 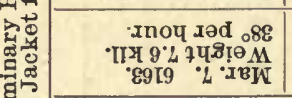 & ¿: $: \vdots \vdots: \vdots \vdots: \vdots \vdots \vdots$ \\
\hline 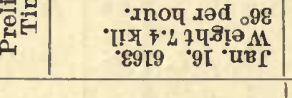 & \\
\hline
\end{tabular}




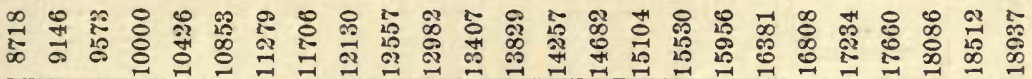

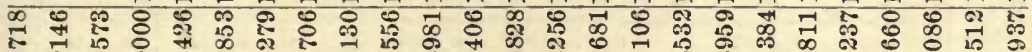

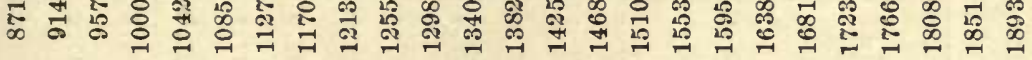

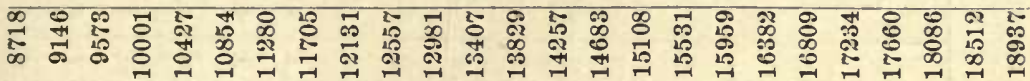

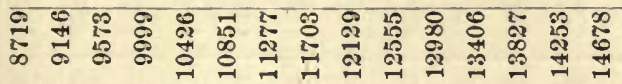

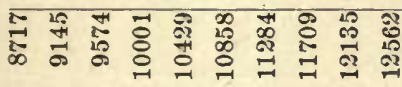

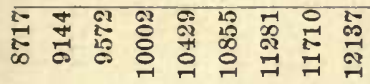

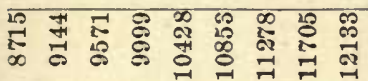

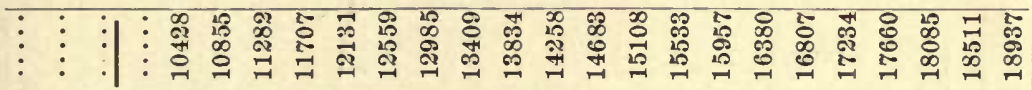

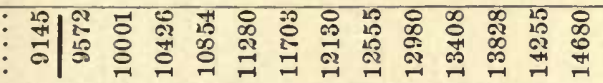

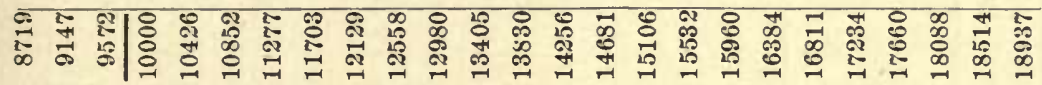

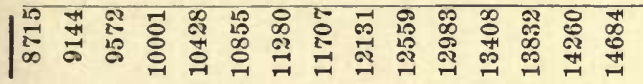

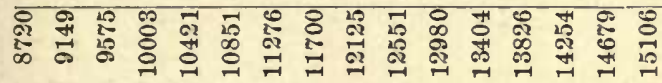

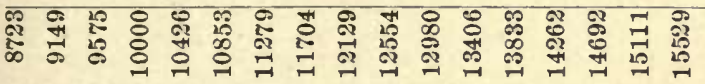

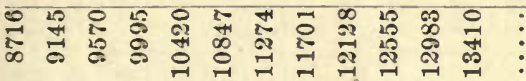

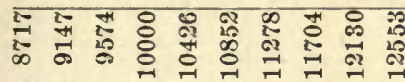

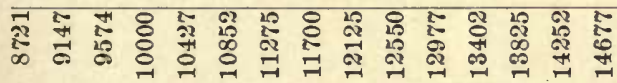

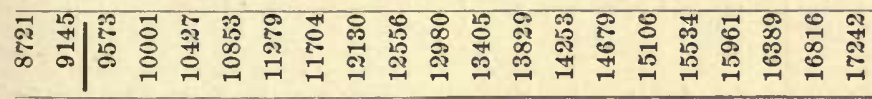

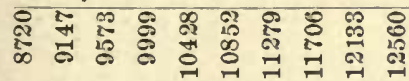

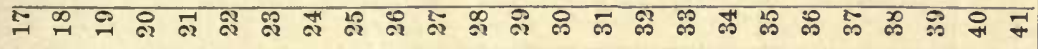




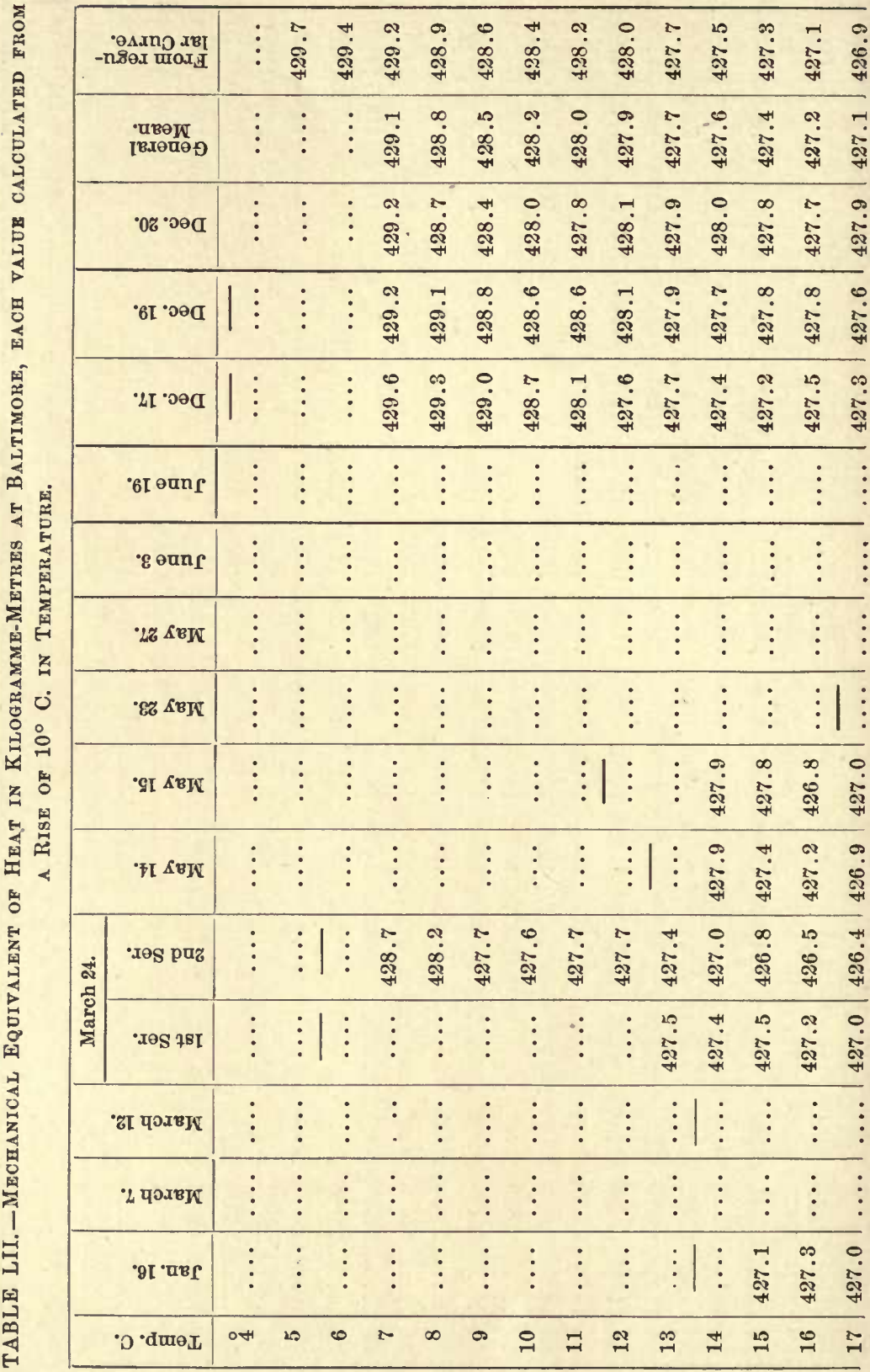




\begin{tabular}{|c|c|c|c|c|c|c|c|c|c|c|c|c|c|c|c|c|c|c|}
\hline \begin{tabular}{l}
5 \\
$\dot{0}$ \\
\multirow{4}{*}{}
\end{tabular} & $\begin{array}{l}\text { थ } \\
\stackrel{0}{*} \\
\text { Fे }\end{array}$ & 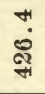 & 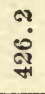 & $\begin{array}{l}\text { † } \\
\text { सै }\end{array}$ & $\begin{array}{l}\text { ?0 } \\
20 \\
\text { से }\end{array}$ & $\begin{array}{l}\infty \\
\text { in } \\
\text { अे }\end{array}$ & $\begin{array}{l}\text { Ta } \\
\text { Fै }\end{array}$ & $\begin{array}{l}0 \\
\dot{0} \\
\text { SO }\end{array}$ & $\begin{array}{l}\text { in } \\
\text { if } \\
\text { Fे }\end{array}$ & $\begin{array}{l}\text { 40 } \\
\text { is } \\
\text { क्ष }\end{array}$ & \begin{tabular}{l}
$\not$ \\
20 \\
\multirow{2}{*}{}
\end{tabular} & $\begin{array}{l}+4 \\
100 \\
\text { अै }\end{array}$ & $\begin{array}{l}\text { H. } \\
\text { ঞ̊ } \\
\text { अ }\end{array}$ & \begin{tabular}{l}
+1 \\
$\stackrel{2}{*}$ \\
\multirow{2}{*}{}
\end{tabular} & 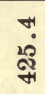 & \begin{tabular}{l}
20 \\
20 \\
\multirow{1}{*}{}
\end{tabular} & $\begin{array}{l}20 \\
20 \\
\text { 20 } \\
\text { Fi }\end{array}$ & यद \\
\hline$\stackrel{0}{\stackrel{0}{2}}$ & $\begin{array}{l}\circ \\
\stackrel{0}{ } \\
\text { ㄱ }\end{array}$ & F্ & 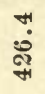 & $\begin{array}{l}\dddot{1} \\
\dot{0} \\
\text { \$ }\end{array}$ & $\begin{array}{l}0 \\
0 \\
\stackrel{0}{*}\end{array}$ & 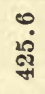 & \begin{tabular}{l}
20 \\
20 \\
20 \\
\multirow{2}{*}{}
\end{tabular} & $\begin{array}{l}0 \\
\text { ap } \\
\text { హิ } \\
\text { Fे }\end{array}$ & $\begin{array}{l}20 \\
20 \\
\text { ₹ै }\end{array}$ & \begin{tabular}{l}
71 \\
\multirow{3}{*}{} \\
\multirow{3}{*}{}
\end{tabular} & $\begin{array}{l}\text { in } \\
\text { 10 } \\
\text { Fे }\end{array}$ & 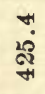 & \begin{tabular}{l}
$\not$ \\
20 \\
\multirow{2}{*}{}
\end{tabular} & \begin{tabular}{l}
$2 \rho$ \\
20 \\
20 \\
\multirow{2}{2}{}
\end{tabular} & \begin{tabular}{l}
$\Re$ \\
20 \\
\multirow{F}{*}{}
\end{tabular} & 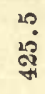 & $\begin{array}{l}0 \\
\text { 官 } \\
\text { ș }\end{array}$ & 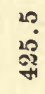 \\
\hline 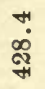 & 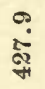 & $\stackrel{\leftrightarrow}{\stackrel{\leftrightarrow}{\circ}}$ & $\underset{+}{\stackrel{1}{x}}$ & $\vdots \vdots$ & $\vdots$ & $\vdots$ & $\vdots$ & $\vdots$ & $\vdots$ & & & & . & . & . & . & : & $\vdots$ \\
\hline$\underset{+}{\stackrel{+}{*}}$ & 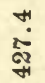 & 於 & $\vdots$ & $\vdots$ & $\vdots$ & $\vdots$ & $\vdots$ & & & & & & & & & . & : & $\vdots$ \\
\hline 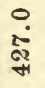 & 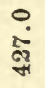 & 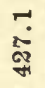 & & $\vdots$ & $\vdots$ & $\vdots$ & $\vdots$ & : & : & : & & : & ${ }^{\circ}$ & & & . & & $\vdots$ \\
\hline : & $\vdots$ & $\vdots$ & $\vdots \vdots$ & $\vdots$ & $\vdots$ & $\vdots \vdots$ & $\vdots$ & $\begin{array}{l}10 \\
20 \\
\text { 20 }\end{array}$ & 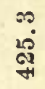 & 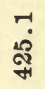 & \begin{tabular}{l}
$\circ$ \\
ip \\
\multirow{2}{*}{}
\end{tabular} & 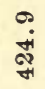 & $\underset{+}{\infty}$ & $\begin{array}{l}0 \\
\text { क्ष } \\
\text { बi }\end{array}$ & $\begin{array}{l}\text { 무 } \\
\text { బิ } \\
\text { హ. }\end{array}$ & †ํ. & \begin{tabular}{l}
$\infty$ \\
20 \\
\multirow{2}{*}{}
\end{tabular} & 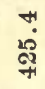 \\
\hline$\vdots$ & $\vdots$ & $\vdots$ & $\vdots \vdots$ & $\vdots \vdots$ & $\begin{array}{l}\text { \& } \\
\text { \& } \\
\text { \& }\end{array}$ & \begin{tabular}{l}
0 \\
10 \\
3 \\
\multirow{2}{*}{+4}
\end{tabular} & $\begin{array}{l}+4 \\
20 \\
\text { *ै }\end{array}$ & \begin{tabular}{l}
$+!$ \\
20 \\
\multirow{3}{*}{}
\end{tabular} & $\vdots$ & $\vdots$ & & & & & & & & \\
\hline$\vdots$ & $\vdots$ & $\vdots$ & $\begin{array}{l}10 \\
\dot{0} \\
\text { ș }\end{array}$ & \begin{tabular}{l} 
İ \\
$\stackrel{0}{*}$ \\
\multirow{F}{*}{}
\end{tabular} & \begin{tabular}{l}
$\infty$ \\
20 \\
\multirow{\sigma}{*}{}
\end{tabular} & $\begin{array}{l}\infty \\
\stackrel{\infty}{20} \\
\stackrel{9}{*}\end{array}$ & \begin{tabular}{l}
0 \\
20 \\
\multirow{2}{*}{}
\end{tabular} & \begin{tabular}{l}
10 \\
20 \\
20 \\
\multirow{2}{*}{}
\end{tabular} & $\begin{array}{l}+4 \\
\text { is } \\
\text { ज्ञ }\end{array}$ & $\begin{array}{l}20 \\
20 \\
29 \\
F\end{array}$ & 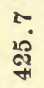 & \begin{tabular}{l}
$2 \rho$ \\
20 \\
\multirow{2}{*}{}
\end{tabular} & $\begin{array}{l}\infty \\
\text { of } \\
\text { of }\end{array}$ & \begin{tabular}{l}
+ \\
Ha \\
\multirow{2}{*}{}
\end{tabular} & $\begin{array}{l}20 \\
10 \\
\text { si } \\
+1\end{array}$ & \begin{tabular}{l}
$\infty$ \\
20 \\
\multirow{2}{*}{} \\
\multirow{2}{*}{}
\end{tabular} & \begin{tabular}{l}
$\infty$ \\
ip \\
\multirow{2}{*}{}
\end{tabular} & \begin{tabular}{l}
$\mathscr{0}$ \\
\multirow{2}{*}{} \\
\multirow{2}{*}{}
\end{tabular} \\
\hline$\vdots$ & $\vdots$ & $\vdots$ & 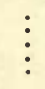 & $\begin{array}{l}\infty \\
\dot{0} \\
\stackrel{\leftrightarrow}{\sigma}\end{array}$ & \begin{tabular}{l}
$\not{0}$ \\
$\stackrel{0}{*}$ \\
\multirow{H}{*}{}
\end{tabular} & $\begin{array}{l}\stackrel{0}{0} \\
\stackrel{\leftrightarrow}{*}\end{array}$ & $\begin{array}{l}\text { o } \\
\text { is } \\
\text { St }\end{array}$ & \begin{tabular}{l}
0 \\
20 \\
\multirow{2}{*}{}
\end{tabular} & $\vdots$ & $\vdots$ & & & & & & & & $\vdots$ \\
\hline 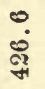 & $\begin{array}{l}0 \\
\text { शै }\end{array}$ & \begin{tabular}{l}
$\infty$ \\
20 \\
\multirow{2}{\alpha}{}
\end{tabular} & 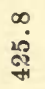 & \begin{tabular}{l}
$\stackrel{0}{0}$ \\
$\dot{0}$ \\
\multirow{2}{*}{}
\end{tabular} & \begin{tabular}{l} 
य? \\
20 \\
\multirow{4}{*}{}
\end{tabular} & $\begin{array}{l}\text { ㄹ. } \\
\text { भ̊ } \\
\text { अे }\end{array}$ & $\begin{array}{l}\text { ㅁ } \\
20 \\
\text { sf } \\
\text { से }\end{array}$ & $\begin{array}{l}\infty \\
\text { is } \\
\text { of } \\
\text { के }\end{array}$ & \begin{tabular}{l}
20 \\
20 \\
\multirow{2}{*}{}
\end{tabular} & : & : & : & . & & & & & $\vdots$ \\
\hline ড. & 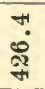 & $\begin{array}{l}\text { ? } \\
\stackrel{0}{2} \\
\text { अै }\end{array}$ & 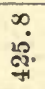 & 역 & 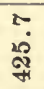 & \begin{tabular}{l}
$\infty$ \\
in \\
\multirow{3}{*}{+}
\end{tabular} & $\begin{array}{l}\text { ণ } \\
\text { \&్ } \\
\text { \& }\end{array}$ & $\begin{array}{l}0 \\
\dot{0} \\
\stackrel{1}{*}\end{array}$ & $\begin{array}{l}\infty \\
\text { a } \\
\text { \$ิ }\end{array}$ & \begin{tabular}{l}
0 \\
20 \\
20 \\
\multirow{7}{*}{+}
\end{tabular} & : & $\vdots$ & $\vdots$ & : & • & $\cdot$ & : & : \\
\hline $\begin{array}{l}20 \\
0 \\
0 \\
\text { sू } \\
4\end{array}$ & $\begin{array}{l}\infty \\
\infty \\
\infty \\
+1\end{array}$ & $\begin{array}{l}\stackrel{0}{0} \\
\stackrel{5}{5}\end{array}$ & \begin{tabular}{l}
$\stackrel{0}{0}$ \\
$\&$ \\
\multirow{\alpha}{*}{}
\end{tabular} & : & $\begin{array}{l}20 \\
80 \\
\text { of }\end{array}$ & : & & $\vdots$ & : & & : & : & : & 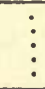 & : & : & : & $\vdots$ \\
\hline 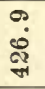 & \begin{tabular}{l}
$\infty$ \\
$\infty$ \\
\multirow{+}{*}{}
\end{tabular} & \begin{tabular}{l}
$\infty$ \\
$\dot{2}$ \\
\multirow{H}{*}{}
\end{tabular} & $\begin{array}{l}\text { H. } \\
\dot{\infty} \\
\text { Na }\end{array}$ & $\vdots \vdots$ & $\vdots$ & $\vdots \vdots$ & $\vdots$ & $\vdots$ & : & $\vdots$ & : & : & : & : & : & . & $\vdots$ & $\vdots$ \\
\hline$\vdots$ & $\vdots$ & $\begin{array}{l}5 \\
\text { ag } \\
\text { G }\end{array}$ & $\begin{array}{l}20 \\
20 \\
\text { कर } \\
\text { Fे }\end{array}$ & $\begin{array}{l}0 \\
20 \\
\text { హै }\end{array}$ & $\begin{array}{l}10 \\
\text { is } \\
\text { is }\end{array}$ & ت. & $\begin{array}{l}\text { ?2 } \\
\text { ig } \\
\text { Fे }\end{array}$ & $\begin{array}{l}0 \\
\text { ฌ़ } \\
\text { హิ }\end{array}$ & $\vdots$ & $\vdots \vdots$ & $\vdots$ & $\vdots$ & • & . & . & • & : & $\vdots$ \\
\hline 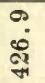 & $\stackrel{8}{\circ}$ & $\begin{array}{l}0 \\
0 \\
02 \\
\text { O1 }\end{array}$ & \begin{tabular}{l}
$\ddot{4}$ \\
$\dot{\sigma}$ \\
\multirow{H}{*}{}
\end{tabular} & \begin{tabular}{l} 
? \\
is \\
\multirow{4}{*}{+}
\end{tabular} & \begin{tabular}{l}
$\stackrel{0}{0}$ \\
$\dot{0}$ \\
\multirow{2}{*}{+}
\end{tabular} & \begin{tabular}{l}
0 \\
$\dot{0}$ \\
\multirow{2}{*}{+}
\end{tabular} & 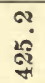 & $\begin{array}{l}\text { ? } \\
20 \\
\text { S? }\end{array}$ & 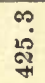 & $\begin{array}{l}29 \\
20 \\
20 \\
\text { 20 }\end{array}$ & 20 & \begin{tabular}{l}
$\stackrel{0}{0}$ \\
aิ \\
\multirow{+}{*}{}
\end{tabular} & $\begin{array}{l}0 \\
0 \\
: 0 \\
*\end{array}$ & \begin{tabular}{l}
$\because$ \\
$\ddot{0}$ \\
\multirow{H}{*}{}
\end{tabular} & - & : & $\begin{array}{l}: \\
:\end{array}$ & $\vdots$ \\
\hline \begin{tabular}{l}
0 \\
\multirow{5}{*}{} \\
\multirow{3}{*}{}
\end{tabular} & $\begin{array}{l}\stackrel{0}{4} \\
\stackrel{5}{*}\end{array}$ & 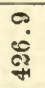 & $\begin{array}{l}\infty \\
\dot{0} \\
\text { of } \\
+\end{array}$ & $\begin{array}{l}\vdots \\
\vdots\end{array}$ & $\begin{array}{l}\vdots \\
\vdots\end{array}$ & $\vdots$ & : & : & : & $\vdots$ & : & $\vdots$ & . & . & : & • & $\vdots$ & $\vdots$ \\
\hline$\stackrel{\infty}{\sim}$ & $\stackrel{\Omega}{-}$ & જે & $\vec{a}$ & 跑 & $\stackrel{\mathscr{c}}{\mathscr{2}}$ & હ゙ & ลิ & है & ลे & $\begin{array}{l}\infty \\
: 2\end{array}$ & 尺̊ & ణి & $\vec{\infty}$ & డึ & $\mathscr{6}$ & लే & लొ & है \\
\hline
\end{tabular}




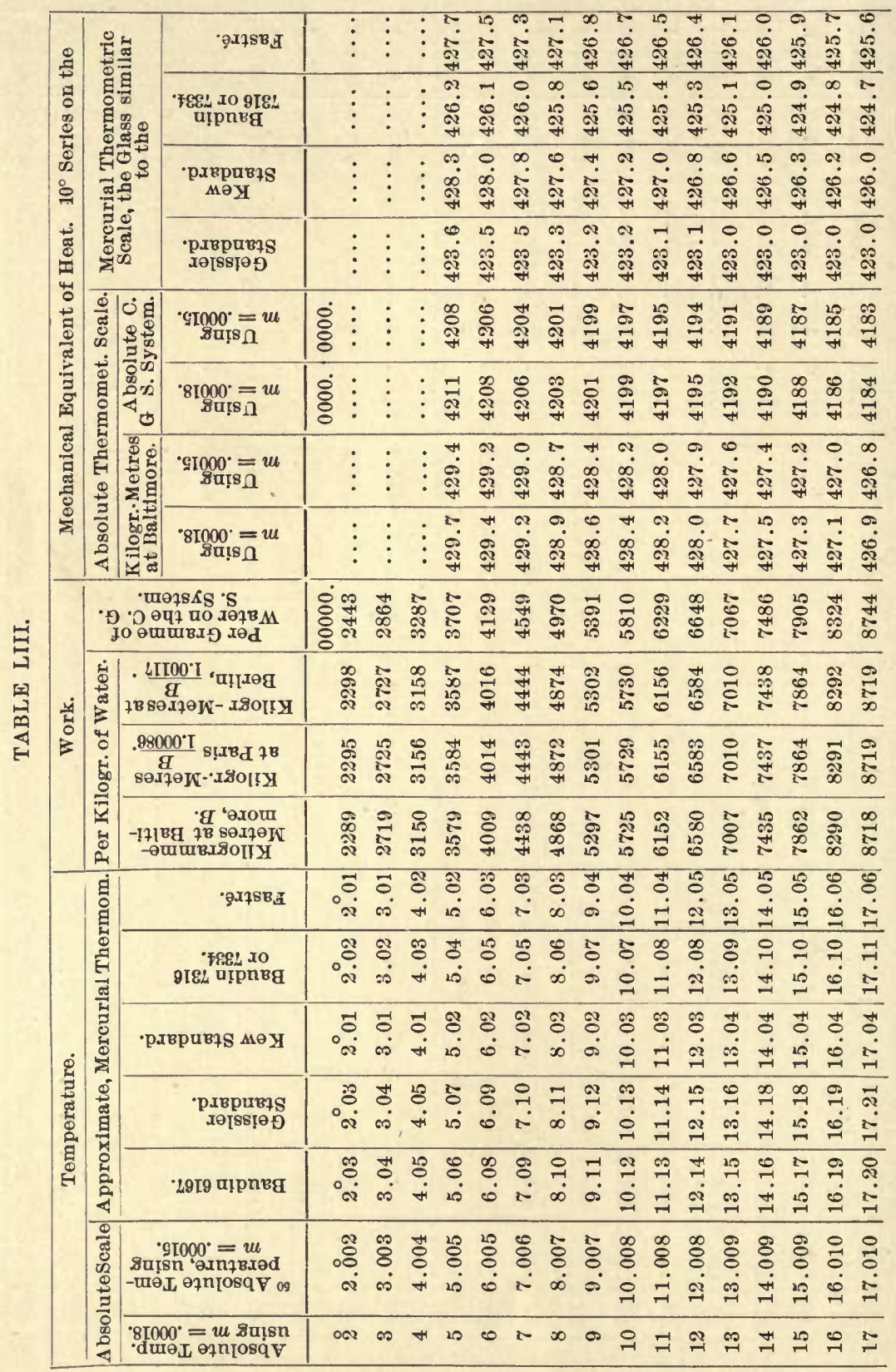




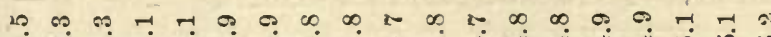

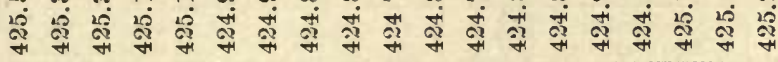

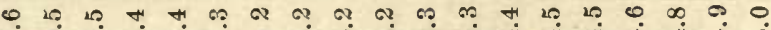

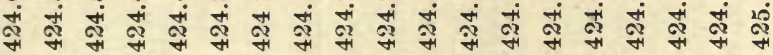

$\infty$ T 0 u

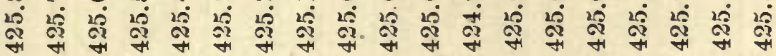

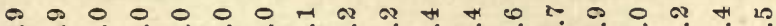

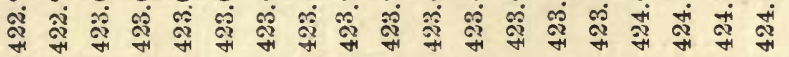

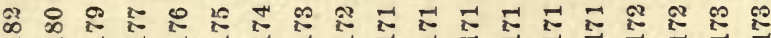

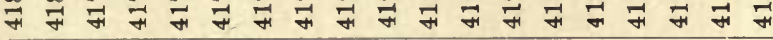

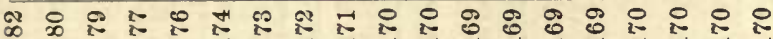

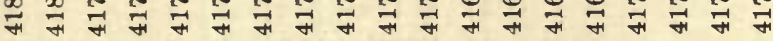

ए य प

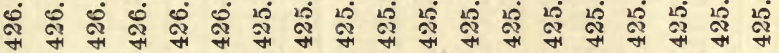

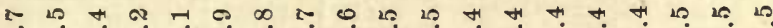

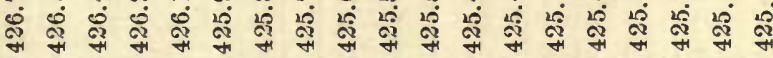

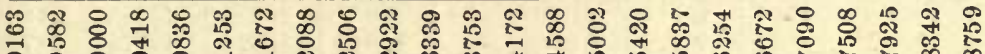

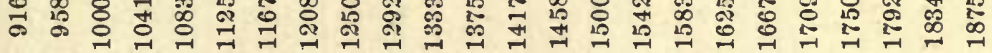

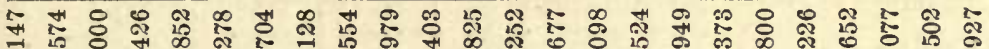

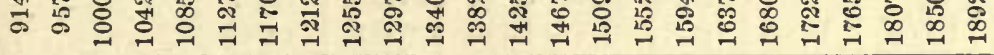

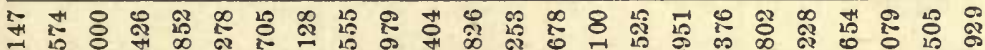

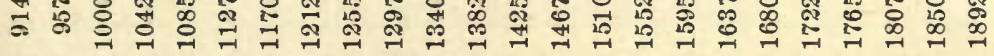
운

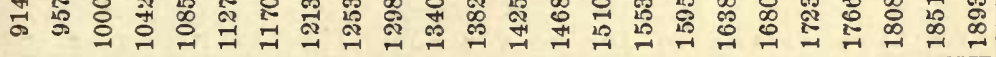

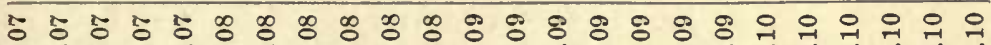

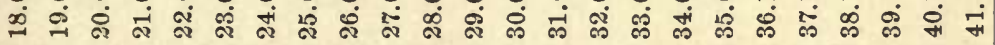

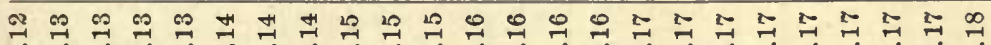

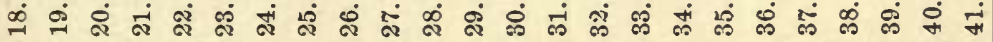

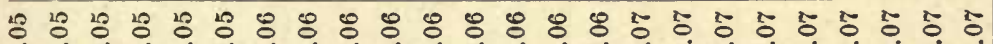

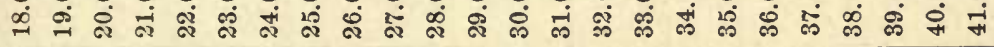

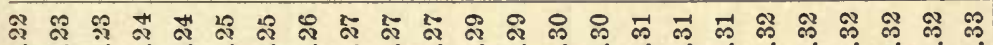

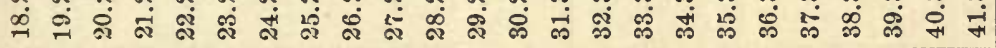

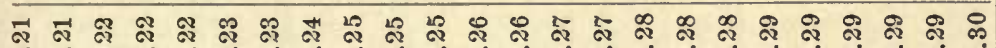

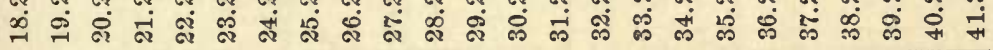

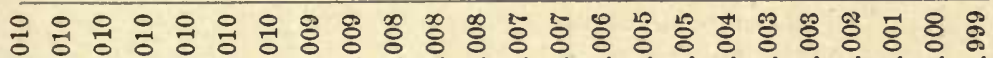

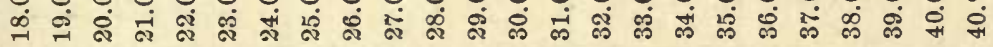

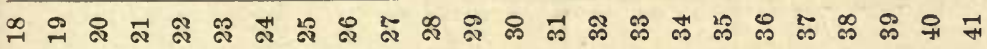


Table LiV.-Final most Probable Results.

\begin{tabular}{|c|c|c|c|c|c|c|c|c|c|}
\hline \multirow{2}{*}{ 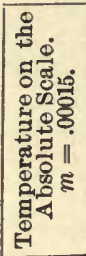 } & \multicolumn{2}{|c|}{ Work. } & \multicolumn{2}{|c|}{$\begin{array}{l}\text { Mechanical } \\
\text { Equivalent. }\end{array}$} & \multirow{2}{*}{ 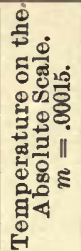 } & \multicolumn{2}{|c|}{ Work. } & \multicolumn{2}{|c|}{$\begin{array}{l}\text { Mechanical } \\
\text { Equivalent. }\end{array}$} \\
\hline & 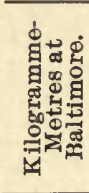 & 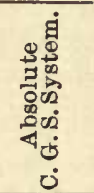 & 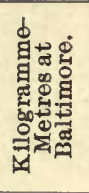 & 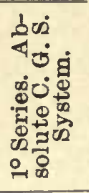 & & 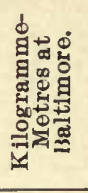 & 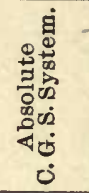 & 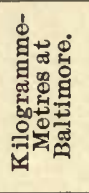 & 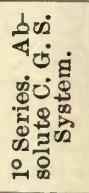 \\
\hline$\AA$ & 2289 & 00000 . & & 0000. & 220 & 10852 & $\begin{array}{l}00000 . \\
10835\end{array}$ & 426.1 & $\begin{array}{l}0000 . \\
4176\end{array}$ \\
\hline 3 & 2720 & 2865 & & . & 23 & 11278 & 11253 & 426.0 & 4175 \\
\hline 4 & 3150 & 3286 & & & 24 & 11704 & 11670 & 425.9 & 4174 \\
\hline 5 & 580 & 3708 & 429.8 & 4212 & 25 & 12130 & 12088 & 425.8 & 4173 \\
\hline 6 & 009 & 4129 & 295 & 4209 & 26 & 12556 & 12505 & 425.7 & 4172 \\
\hline 7 & 439 & 455 & 4 & 4207 & 27 & 12982 & 12922 & 425.6 & 4171 \\
\hline 8 & 368 & 4970 & & 4204 & & 13407 & 13339 & 42 & 4171 \\
\hline 9 & & 5390 & & 4202 & 29 & 13833 & 13756 & 425 & 4170 \\
\hline 10 & 5726 & 5811 & & 4200 & 30 & 14258 & 14173 & 425 & 4171 \\
\hline 11 & & & & & 31 & 146 & & 425 & 4171 \\
\hline 12 & & 6650 & & 4196 & 32 & 15110 & 15008 & 42 & 4171 \\
\hline 13 & & 707 & & & 33 & & & & \\
\hline 14 & & 7489 & & 41 & 34 & 15961 & 158 & & 41 \\
\hline 15 & & & & & 35 & & & & 4173 \\
\hline 16 & & & & & 3 & 16812 & & 425.8 & 4173 \\
\hline 17 & 872 & 8745 & & & & 17238 & 17094 & & \\
\hline 18 & & & & & & & & & \\
\hline 19 & 957 & 95 & & & 39 & & & & $\ldots$ \\
\hline 20 & & & & & 40 & & & & \\
\hline 21 & 10426 & 10418 & 426.2 & 4177 & 41 & 18943 & 18765 & & $\cdots$ \\
\hline
\end{tabular}

TABLE LV.-Quantity to AdD to the Equivalent at Baltimore to REDUCE TO ANY LATITUDE.

\begin{tabular}{|c|c|}
\hline Latitude. & $\begin{array}{c}\text { Addition in } \\
\text { Kilogramme-Metres. }\end{array}$ \\
\cline { 1 - 2 } 0 & +0.89 \\
10 & +0.82 \\
20 & +0.63 \\
30 & +0.34 \\
40 & +0.08 \\
50 & -0.41 \\
60 & -0.77 \\
70 & -1.06 \\
80 & -1.26 \\
90 & -1.33 \\
\hline
\end{tabular}

Manchester-0.5; Paris - 0.4; Berlin -0.5. 


\section{CONCLUDING REMARKS, AND CRITICISM OF RESULTS AND METHODS}

On looking over the last four columns of Table LIII, which gives the results of the experiments as expressed in terms of the different mercurial thermometers, we cannot but be impressed with the unsatisfactory state of the science of thermometry at the present day, when nearly all physicists accept the mercurial thermometer as the standard between $0^{\circ}$ and $100^{\circ}$. The wide discrepancy in the results of calorimetric experiments requires no further explanation, especially when physicists have taken no precaution with respect to the change of zero after the heating of the thermometer. They show that thermometry is an immensely difficult subject, and that the results of all physicists who have not made a special study of their thermometers, and a comparison with the air thermometer, must be greatly in error, and should be rejected in many cases. And this is specially the case where Geissler thermometers have been used.

The comparison of my own thermometers with the air thermometer is undoubtedly by far the best so far made, and I have no improvements to offer beyond those $I$ have already mentioned in the 'Appendix to Thermometry.' And I now believe that, with the improvement to the air thermometer of an artificial atmosphere of constant pressure, we could be reasonably certain of obtaining the temperature at any point up to $50^{\circ} \mathrm{C}$. within $0^{\circ} .01 \mathrm{C}$. from the mean of two or three observations. I believe that my own thermometers scarcely differ much more than that from the absolute scale at any point up to $40^{\circ} \mathrm{C}$., but they represent the mean of eight observations. However, there is an uncertainty of $0^{\circ} .01 \mathrm{C}$. at the $20^{\circ}$ point, owing to the uncertainty of the value of $m$. But taking $m=00015$, I hardly think that the point is uncertain to more than that amount for the thermometers Nos. 6163, 6165, and 6166 .

As to the comparison of the other thermometers, it is evidently unsatisfactory, as they do not read aceurately enough. However, the figures given in Table LIII are probably very nearly correct.

The study of the thermometers from the different makers introduces the question whether there are any thermometers which stand below the air thermometer between $0^{\circ}$ and $100^{\circ}$. As far as I can find, nobody has ever published a table showing such a result, although Bosscha infers that thermometers of "Cristal de Choisy-le-Roi" should stand below, and his inference has been accepted by Regnault. But it does not seem to have been proved by direct experiment. My Baudin thermometers seem to contain lead as far as one can tell from the blackening in a gas 
flame, but they stand very much above the air thermometer at $40^{\circ}$. I have since tried some of the Baudin thermometers up to $300^{\circ}$, and find that they stand below the air thermometer between $100^{\circ}$ and $240^{\circ}$; they coincide at about $240^{\circ}$, and stand above between $240^{\circ}$ and $300^{\circ}$. This is very nearly what Regnault found for "Verre Ordinaire." It is to be noted that the formula obtained from experiments below $100^{\circ}$ makes them coincide at $233^{\circ}$, which is remarkably close to the result of actual experiment, especially as it would require a long series of experiments to determine the point within $10^{\circ}$.

The comparison of thermometers also shows that all thermometers in accurate investigations should be used as thermometers with arbitrary scales, neither the position of the zero point nor the interval between the $0^{\circ}$ and $100^{\circ}$ points being assumed correct. The text books only give the correction for the zero point, but my observations show that the interval between the $0^{\circ}$ and $100^{\circ}$ points is also subject to a secular change as well as to the temporary change due to heating. Of all the thermometers used, the Geissler is the worst in this as in other respects, except accuracy of calibration, in which it is equal to most of the others.

The experiments on the specific heat of water show an undoubted decrease as the temperature rises, a fact which will undoubtedly surprise most physicists as much as it surprised me. Indeed, the discovery of this fact put back the completion of this paper many months, as I wished to make certain of it. There is now no doubt in my mind, and I put the fact forth as proved. The only way in which an error accounting for this decrease could have been made appears to me to be in the determination of $m$ in "Thermometry." The determination of $m$ rests upon the determination of a difference of only $0^{\circ} \cdot 05 \mathrm{C}$. between the air thermometer and the mercurial, the $0^{\circ}$ and $40^{\circ}$ points coinciding, and also upon the comparison of the thermometers with others whose value of $m$ was known, as in the Appendix. Although the quantity to be measured is small, yet there can be no doubt at least that $m$ is larger than zero; and if so, the specific heat of water certainly has a minimum at about $30^{\circ}$.

One point that might be made against the fact is that the Kew standard, Table $\mathrm{L}$, gives less change than the others. But the calibration of the Kew standard, although excellent, could hardly be trusted to $0^{\circ} .02$ or $0^{\circ} .03 \mathrm{C}$., as the graduation was only to $\frac{1}{2}^{\circ} \mathrm{F}$. In drawing the curve for the difference between the Kew standard and the air thermometers, I ignored small jrregularities and drew a regular curve. On 
looking over the observations again, I see that, had I taken account of the small irregularities, it would have made the observations agree more nearly with the other thermometers. Hence the objection vanishes. However, I intend working up some observations which I have with the Kew standard at a higher temperature, and shall publish them at a future time.

There is one other error that might produce an apparent decrease in the specific heat, and that is the slight decrease in the torsion weight from the beginning to the end of most of the experiments, probably due to the slowing of the engine. By this means the torsion circle might lag behind. I made quite an investigation to see if this source of error existed, and came to the conclusion that it produced no perceptible effect. An examination of the different experiments shows this also, for in some of them the weight increases instead of decreasing. See Tables XXXVII to L.

The error from the formation of dew might also cause an apparent decrease; but I have convinced myself by experiment, and others can convince themselves from the tables, that this error is also inappreciable.

The observations seem to settle the point with regard to the specific heat at the $4^{\circ}$ point within reasonable limits. There does not seem to be a change to any great extent at that point, but the specific heat decreases continuously through that point. It would hardly be possible to arrive at this so accurately as I have done by any method of mixture, for Pfaundler and Platter, who examined this point, could not obtain results within one per cent, while mine show the fact within a fraction of one per cent.

The point of minimum cannot be said to be known, though I have placed it provisionally between $30^{\circ}$ and $35^{\circ} \mathrm{C}$, , but it may vary much from that.

The method of obtaining the specific heat of the calorimeter seems to be good. The use of solder introduces an uncertainty, but it is too small to affect the result appreciably. The different determinations of the specific heat of the calorimeter do not agree so well as they might, but the error in the equivalent resulting from this error is very small, and, besides, the mean result agrees well with the calculated result. It may be regarded as satisfactory.

The apparatus for determining the equivalent could scarcely be improved much, although perhaps the record of the torsion might be made automatic and continuous. The experiment, however, might be im- 
proved in two ways; first, by the use of a motive power more regular in its action; and, second, by a more exact determination of the loss due to radiation. The effect of the irregularity of the engine hàs been calculated as about 1 in 4000 , and I suppose that the error due to it cannot be as much as that after applying the correction. The error due to radiation is nearly neutralized, at least between $0^{\circ}$ and $30^{\circ}$, by using the jacket at different temperatures. There may be an error of a small amount at that point $\left(30^{\circ}\right)$ in the direction of making the mechanical equivalent too great, and the specific heat may keep on decreasing to even $40^{\circ}$.

Between the limits of $15^{\circ}$ and $25^{\circ} \mathrm{I}$ feel almost certain that no subsequent experiments will change my values of the equivalent so much as two parts in one thousand, and even outside those limits, say between $10^{\circ}$ and $30^{\circ}$, I doubt whether the figures will ever be changed much more than that amount.

It is my intention to continue the experiments, as well as work up the remainder of the old ones. I shall also use some liquids in the calorimeter other than water, and so have the equivalent in terms of more than one fluid.

Baltimore, 1878-79. Finished May 27, 1879. 
APPENDIX TO PAPER ON THE MECHANICAL EQUIVALENT OF HEAT, CONTAINING THE COMPARISON WITH DR. JOULE'S THERMOMETER

\author{
[Proceedings of the American Academy of Arts and Sciences, $X V I, 38-45,1881$ ] \\ Presented, March, 1880
}

In the body of this paper I have given an estimate of the departure of Dr. Joule's thermometer from the air thermometer, based on the comparison of thermometers of similar glass. But as it seemed important that the classical determinations of this physicist should be reduced to some exact standard, I took to England with me last summer one of my standards,-Baudin, No. 6166,- -and sent it to Dr. Joule with a statement of the circumstances. He very kindly consented to make the comparison, and I now have the results before me. These confirm the estimate that I had previously made, and cause our values for the equivalent to agree with great accuracy. The following is the table of the comparison:-

\begin{tabular}{|c|c|c|c|c|}
\hline \multicolumn{2}{|c|}{ Readings. } & \multicolumn{3}{|c|}{ Temperatures. } \\
\hline Baudin, No. 6166. & Joule. & $\begin{array}{l}\text { By perfect Air } \\
\text { Theermometer } \\
\text { according to } \\
\text { No. } 6168 \text {. }\end{array}$ & $\begin{array}{c}\text { By Joule's } \\
\text { Thermometer. }\end{array}$ & Difference. \\
\hline 21.88 & 22.62 & ○ & 8 & ○̊ \\
\hline 41.930 & 59.410 & 1.590 & 1.578 & -.012 \\
\hline 48.782 & 72.200 & 2.126 & 2.127 & +.001 \\
\hline 53.705 & 81.340 & 2.511 & 2.519 & .008 \\
\hline 58.916 & 90.877 & 2.918 & 2.928 & .010 \\
\hline 64.914 & 101.777 & 3.382 & 3.396 & .014 \\
\hline 73.374 & 117.291 & 4.039 & 4.061 & .022 \\
\hline 80.176 & 129.990 & 4.567 & 4.606 & .039 \\
\hline 85.268 & 139.255 & 4.961 & 5.003 & .042 \\
\hline 90.564 & 148.834 & 5.370 & 5.414 & .044 \\
\hline 94.243 & 155.460 & 5.654 & 5.698 & .044 \\
\hline 99.168 & 164.400 & 6.036 & 6.082 & .046 \\
\hline 104.030 & 173.140 & 6.413 & 6.457 & .044 \\
\hline 108.863 & 182.040 & 6.789 & 6.839 & .050 \\
\hline 113.706 & 190.885 & 7.165 & 7.218 & .053 \\
\hline 114.000 & 191.382 & 7.188 & 7.239 & .051 \\
\hline 1121.507 & 1219.497 & 17.772 & 18.445 & $\ldots$ \\
\hline
\end{tabular}

${ }^{1}$ Evidently a mistake in the readings. 
Continued.

\begin{tabular}{|c|c|c|c|c|}
\hline \multicolumn{2}{|c|}{ Readings. } & \multicolumn{3}{|c|}{ Temperatures. } \\
\hline Baudin, No. 6166. & Joule. & $\begin{array}{c}\text { By perfect Air } \\
\text { Thermometer } \\
\text { according to } \\
\text { No. } 6166 .\end{array}$ & $\begin{array}{c}\text { By Joule's } \\
\text { Thermometer. }\end{array}$ & Difference. \\
\hline 135.858 & 231.115 & 8.890 & $8 . \grave{9} 44$ & .054 \\
\hline 140.467 & 239.939 & 9.249 & 9.309 & .060 \\
\hline 143.405 & 245.006 & 9.479 & 9.540 & .061 \\
\hline 146.445 & 250.566 & 9.717 & 9.778 & .061 \\
\hline 152.360 & 261.481 & 10.180 & 10.246 & .066 \\
\hline 158.770 & 273.239 & 10.681 & 10.751 & 070 \\
\hline 164.635 & 283.957 & 11.138 & 11.211 & .073 \\
\hline 170.485 & 294.739 & 11.595 & 11.670 & .075 \\
\hline 175.436 & 303.682 & 11.979 & 12.057 & .078 \\
\hline 182.795 & 316.968 & 12.550 & 12.627 & .077 \\
\hline 188.705 & 327.746 & 13.008 & 13.089 & .081 \\
\hline 193.954 & 337.220 & 13.412 & 13.495 & .083 \\
\hline 199.558 & 347.294 & 13.844 & 13.928 & .084 \\
\hline 206.054 & 259.060 & 14.343 & 14.432 & .089 \\
\hline 211.528 & 368.953 & 14.764 & 14.857 & .093 \\
\hline 216.440 & 377.826 & 15.142 & 15.237 & .095 \\
\hline 221.858 & 387.562 & 15.560 & 15.655 & .095 \\
\hline 229.601 & 401.419 & 16.158 & 16.249 & .091 \\
\hline 235.598 & 412.367 & 16.623 & 16.719 & .096 \\
\hline 241.028 & 422.258 & 17.045 & 17.143 & .098 \\
\hline 247.436 & 433.800 & 17.541 & 17.638 & .097 \\
\hline 253.704 & 445.267 & 18.028 & 18.130 & .102 \\
\hline 259.786 & 456.286 & 18.500 & 18.603 & .103 \\
\hline 266.086 & 467.817 & 19.991 & 19.097 & .106 \\
\hline 273.143 & 480.643 & 19.539 & 19.648 & .109 \\
\hline 280.176 & 493.442 & 20.086 & 20.197 & .111 \\
\hline 287.634 & 506.906 & 20.666 & 20.774 & .108 \\
\hline 294.927 & 520.052 & 21.232 & 21.338 & .106 \\
\hline 304.148 & 536.832 & 21.947 & 22.058 & .111 \\
\hline 310.397 & 548.152 & 22.432 & 22.544 & .112 \\
\hline 316.596 & 559.336 & 22.916 & 23.023 & .107 \\
\hline 321.271 & 568.051 & 23.282 & 23.397 & .115 \\
\hline 327.148 & 578.528 & 23.742 & 23.846 & .104 \\
\hline 333.661 & 590.661 & 24.251 & 24.367 & .116 \\
\hline 339.664 & 601.596 & 24.719 & 24.836 & .117 \\
\hline 346.557 & 614.004 & 25.254 & 25.369 & .115 \\
\hline 552.878 & 625.510 & 25.746 & 25.862 & .116 \\
\hline 359.986 & 638.526 & 26.299 & 26.421 & .122 \\
\hline 365.080 & 647.833 & 26.697 & 26.820 & .123 \\
\hline 371.811 & 660.071 & 27.225 & 27.345 & .120 \\
\hline 382.770 & 680.149 & 28.087 & 28.206 & .119 \\
\hline
\end{tabular}

We can discuss the comparison of these thermometers in two ways; either by direct comparison at the points we desire, or by the representation of the differences by a formula.

Joule's result in 1850 was referred to water at about $14^{\circ} \mathrm{C}$., and in 1878 to water at $16^{\circ} \cdot 5 \mathrm{C}$. Taking intervals in the above table of from 
$6^{\circ}$ to $12^{\circ}$, so that the mean shall be nearly $14^{\circ}$ and $16^{\circ} \cdot 5$, I find the following for the ratios:-

\begin{tabular}{rl}
1.0044 & 1.0042 \\
1.0042 & 1.0042 \\
1.0049 & 1.0040 \\
1.0047 & 1.0030 \\
1.0047 & 1.0035 \\
1.0052 & 1.0035 \\
\hline Mean, $\quad \frac{1.0047}{1.0037}$
\end{tabular}

So that we have the following for Joule's old and new values:-

\begin{tabular}{|c|c|c|}
\hline & Old. & $\begin{array}{c}\text { New. } \\
423.9\end{array}$ \\
\hline Correction for thermometer & $\begin{array}{r}423 \cdot 9 \\
2 \cdot 0\end{array}$ & $\begin{array}{r}423 \cdot 9 \\
1 \cdot 6\end{array}$ \\
\hline Correction for latitude & $\cdot 5$ & .5 \\
\hline Correction for sp. ht. of copper & $\cdot 7$ & \\
\hline & $427 \cdot 1$ & $426 \cdot 0$ \\
\hline My value & $427 \cdot 7$ & $42 \% \cdot 1$ \\
\hline Difference & $\cdot 6$ & $1 \cdot 1$ \\
\hline
\end{tabular}

or 1 in 700 and 1 in 390 , respectively.

But the correction found in this way is subject to local irregularities, and it is perhaps better in many respects to get the equation giving the temperature of Joule's thermometer on the air thermometer. Let $T$ be the temperature by Joule's thermometer, and $t$ that by the air thermometer. Then I have found

$$
t=0.002+1.00125 T-.00013\{100-T\}\{1-.003(100+T)\}
$$

The factor 1.00125 enters in the formula, probably because the thermometer which Joule used to get the value of the divisions of his thermometer was not of the same kind of glass as his standard. The relative error at any point due to using the mercurial rather than the air thermometer will then be

$$
E=1-\frac{d t}{d T}=-.00125+\cdot 00000039\left\{23300-666 t+3 t^{2}\right\}
$$


From this $I$ have constructed the following table:-

\begin{tabular}{|c|c|c|c|}
\hline \multirow{2}{*}{ Temperature. } & \multirow{2}{*}{ E. } & \multicolumn{2}{|c|}{$\begin{array}{c}\text { A pproximate Addition to Equivalent } \\
\text { as measured on Joule's Thermometer. }\end{array}$} \\
\cline { 2 - 3 } & & Metric System. & English System. \\
\hline 0 & .0078 & 3.3 & 6.0 \\
5 & .0066 & 2.8 & 5.1 \\
10 & .0054 & 2.3 & 4.2 \\
15 & .0042 & 1.8 & 3.2 \\
20 & .0031 & 1.3 & 2.4 \\
25 & .0021 & .9 & 1.6 \\
30 & .0011 & .5 & .8 \\
\hline
\end{tabular}

Corrected in this way we have,-

Joule's value

Reduction to air thermometer

Reduction to latitude of Baltimore

Correction for sp. ht. of copper

$\begin{array}{lll}\text { My value } & \overline{42 \% \cdot 0} & \overline{426 \cdot 1} \\ \text { Difference } & \frac{42 \% \cdot 7}{. \%} & \frac{42 \% \cdot 1}{1 \cdot 0}\end{array}$

or 1 in 600 and 1 in 426 , respectively.

But it is evident that all the other temperatures used in the experiment must also be corrected, and I have done this in the following manner. The principal other correction required is in the capacity of the calorimeter, and this amounts to considerable in the experiments on mercury and cast-iron, where no water is used. Dr. Joule informs me that the thermometer with which he compared mine was made in 1844, but does not give any mark by which to designate it, although it is evidently the thermometer called " $A$ " by him. I shall commence with the experiments of $184 \%$. The calorimeter was composed of the following substances, whose capacities I recompute according to what in my paper I have considered the most probable specific heats.

\begin{tabular}{|c|c|c|c|c|}
\hline & Weight. & $\begin{array}{l}\text { Capacity accord- } \\
\text { ing to Joule. }\end{array}$ & $\begin{array}{l}\text { Most probable } \\
\text { Specific Heat. }\end{array}$ & $\begin{array}{l}\text { Most probable } \\
\text { Capacity. }\end{array}$ \\
\hline Water & 87617 grains & 77617 & $1 \cdot 000$ & 77617 \\
\hline Brass & 24800 grains & 2319 & .0900 & 2232 \\
\hline Copper & 11237 grains & 1056 & .0922 & 1036 \\
\hline Tin & (?) & 363 & ... & 363 \\
\hline & Total capacity & 81355 & & 81248 \\
\hline
\end{tabular}


Equivalent found

Correction for thermometer

Correction for capacity

Correction for latitude

Corrected value
781.5 at about $59^{\circ} \mathrm{F}$.

$3 \cdot 3$

$1 \cdot 3$

$\cdot 9$

$78 \% \cdot 0$

or 442.8 at $15^{\circ} \mathrm{C}$. on the air thermometer.

The other experiment, on sperm oil, made at this time, is probably hardly worth reducing. The experiments of 1850 are of the highest importance and should be accurately reduced.

In the experiments with water the capacity of the calorimeter is corrected as follows:-

\begin{tabular}{|c|c|c|c|c|}
\hline & Weight. & $\begin{array}{c}\text { Capacity used } \\
\text { by Joule. }\end{array}$ & $\begin{array}{l}\text { Most probable } \\
\text { Specific Heat. }\end{array}$ & $\begin{array}{l}\text { Most probable } \\
\text { Capacity. }\end{array}$ \\
\hline Water & $93229 \cdot 7$ & $93229 \cdot 7$ & 1.000 & $93229 \cdot 7$ \\
\hline Copper & 25541 . & $2430 \cdot 2$ & .092 & $2349 \cdot 8$ \\
\hline Brass & 18901 . & $1800 \cdot 0$ & $\cdot 091$ & $1720 \cdot 0$ \\
\hline Brass stopper & $\ldots \ldots$ & $10 \cdot 3$ & $\cdots$ & $10 \cdot 3$ \\
\hline Total capa & city & $97470 \cdot 2$ & & $97309 \cdot 8$ \\
\hline
\end{tabular}

Therefore correction is $\cdot 0016$.

Hence the result with water requires the following corrections:-

Joule's value

Correction for thermometer

Correction for latitude

Correction for capacity

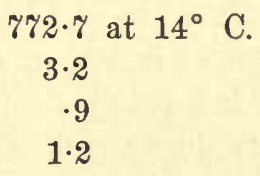

$1 \cdot 2$

$\overline{778 \cdot 0}$

or 426.8 on the air thermometer in the latitude of Baltimore at the temperature of $14^{\circ} \mathrm{C}$., nearly.

In the next experiment, with mercury, Joule determined the capacity of the apparatus by experiment. The mean of the experiments was that the apparatus lost $20^{\circ} \cdot 33155 \mathrm{~F}$. in heating 143430 grains of water $3^{\circ} \cdot 13305 \mathrm{~F}$. To reduce these to the air thermometer we must divide respectively by 1.0042 and 1.0056 . Therefore the capacity must be divided by $1 \cdot 0014$. Therefore the corrected values are:-

Correction for thermometer

$\begin{array}{cc}772.8 \text { at } 9^{\circ} \text { C. } & 775.4 \text { at } 11^{\circ} \mathrm{C} \text {. } \\ 4.4 & 4.0 \\ 1.1 & 1 \cdot 1 \\ \frac{.9}{779 \cdot 2} & \frac{.9}{781 \cdot 4}\end{array}$

Correction for capacity

Correction for latitude 
The reduction to the air thermometer was made for the temperatures of $9^{\circ} \mathrm{C}$. and $11^{\circ} \mathrm{C}$. respectively, but they both refer to the temperature of the water used when the capacity was determined; this was about $9^{\circ} \mathrm{C}$. Hence these experiments gave $427 \cdot 5$ and $428 \cdot 7$ on the air thermometer, with the water at about $9^{\circ} \mathrm{C}$.

The next experiments, with cast-iron, can be corrected in the same manner, and thus become

Correction for thermometer

Correction for capacity

Correction for latitude

\begin{tabular}{rr}
776.0 & 773.9 \\
4.2 & 4.3 \\
1.1 & 1.1 \\
.9 & .9 \\
\hline 782.2 & $780 \cdot 2$
\end{tabular}

and these are as before for water at $9^{\circ}$.

The determination by the heating of a wire, whose resistance was measured in ohms, can be thus reduced. The value found by Joule was 429.9 in the latitude of Baltimore at $18^{\circ} \cdot 6 \mathrm{C}$.

Using the capacity of the copper $\cdot 0922$, as I have done in my paper, this quantity will be increased to $430 \cdot 3$. But I have given reasons in my paper on the "Absolute Unit of Electrical Resistance" to show that there should be a correction to the B. A. Committee's experiments, which would make the ohm .993 earth quadrant $\div$ second, instead of 1.000 as it was meant to be, which nearly agrees with the quantity which I found, namely, $\cdot 991$. Taking my value $\cdot 9911$, Joule's result will reduce as follows:-

Correction for thermometer

Correction for capacity

Corrected for ohm

Corrected value
429.9 at $18^{\circ} \cdot 6 \mathrm{C}$.

$+1 \cdot 5$

$+\cdot 4$

$-3 \cdot 8$

$428 \cdot 0$ at $18^{\circ} \cdot 6 \mathrm{C}$.

The last determinations in the 'Philosophical Transactions' of 1878 can be reduced as follows:

The capacity of the calorimeter was determined by experiment, instead of calculated from the specific heat of copper given by Regnault, as in the older experiments. The value used, 4842.4 grains, corresponded to a specific heat of brass of about $\cdot 090$, which is almost exactly what I have considered right. The reduction to the air thermometer will decrease it somewhat, and the correction for the increase of the 
specific heat of brass and the decrease of the specific heat of water will also change it somewhat. In all, the amount will be about 1 in 200 . Hence the reduction becomes as follows:

\section{Joule's values}

Correction for thermometer

Correction for capacity

Correction for latitude

Correction to vacuum

Corrected values

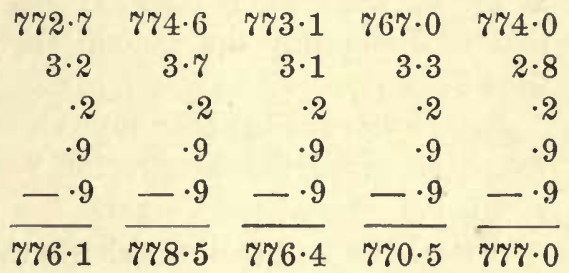

at $14^{\circ .7}$ at $12^{\circ} .7$ at $12^{\circ} .5$ at $14^{\circ} .5$ at $17^{\circ} .3$

To reduce the values in English measure to metres and the Centigrade scale, I have simply taken the reducing factor $1.8 \times \cdot 304794$, although the barometer on the two systems is not exactly the same: for this is taken into account in the comparison of the thermometers. However, a barometer at 30 in. and $60^{\circ} \mathrm{F}$. is equivalent to $759.86 \mathrm{~mm}$. at $0^{\circ} \mathrm{C}$. which hardly makes a difference of $0^{\circ} .01 \mathrm{C}$. in the temperature of the hundred-degree point.

\begin{tabular}{|c|c|c|c|c|c|c|c|c|c|}
\hline \multirow[t]{2}{*}{ No. } & \multirow[t]{2}{*}{ Date. } & \multirow[t]{2}{*}{ Method. } & \multirow{2}{*}{$\begin{array}{l}\text { Tem. } \\
\text { of } \\
\text { water. }\end{array}$} & \multirow{2}{*}{$\begin{array}{c}\text { Joule's } \\
\text { Value. }\end{array}$} & \multicolumn{2}{|c|}{$\begin{array}{l}\text { Joule's Value re- } \\
\text { duced to Air Ther- } \\
\text { mometer and Iati- } \\
\text { tude of Baltimore. }\end{array}$} & \multirow{2}{*}{${ }^{\infty}$} & \multirow{2}{*}{ J. $-\mathrm{R}$. } & \multirow{2}{*}{ 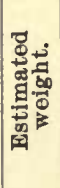 } \\
\hline & & & & & $\begin{array}{c}\text { English } \\
\text { measure. }\end{array}$ & $\begin{array}{l}\text { Metric } \\
\text { system. }\end{array}$ & & & \\
\hline 1 & 1847 & Friction of water & 15 & 781.5 & 787.0 & 442.8 & 427.4 & +15.4 & 0 \\
\hline 2 & 1850 & " water & 14 & 772.7 & 778.0 & 426.8 & 427.7 & $-\quad .9$ & 10 \\
\hline 3 & "1 & mercury & 9 & 772.8 & 779.2 & 427.5 & 428.8 & -1.3 & 2 \\
\hline 4 & " & mercury & 9 & 775.4 & 781.4 & 428.7 & 428.8 & $-\quad .1$ & 2 \\
\hline 5 & "6 & iron & 9 & 776.0 & 782.2 & 429.1 & 428.8 & & 1 \\
\hline 6 & " & " iron & 9 & 773.9 & 780.2 & 428.0 & 428.8 & $-\quad .8$ & 1 \\
\hline 7 & 1867 & Electric heating & 18.6 & $\ldots \ldots$ & $\ldots \ldots$ & 428.0 & 426.7 & +1.3 & 3 \\
\hline 8 & 1878 & Friction of water & 14.7 & 772.7 & 776.1 & 425.8 & 427.6 & -1.8 & 2 \\
\hline 9 & " & "6 6 & 12.7 & 774.6 & 778.5 & 427.1 & 428.0 & $-\quad .9$ & 3 \\
\hline 10 & " & " & 15.5 & 773.1 & 776.4 & 426.0 & 427.3 & -1.3 & 5 \\
\hline 11 & 16 & “ & 14.5 & 767.0 & 770.5 & 422.7 & 427.5 & -4.8 & 1 \\
\hline 12 & "6 & 6 & 17.3 & 774.0 & 777.0 & 426.3 & 426.9 & -.6 & 1 \\
\hline
\end{tabular}

In combining these so as to get at the true difference of Joule's and my result, we must give these different determinations weights according to their respective accuracy, especially as some of the results, as No. 11, have very little weight. Joule rejected quite a number of his results, but I have thought it best to include them, giving them small weights, however. In this way we obtain a value for Joule's experiment 
of $426 \cdot 75$ at $14^{\circ} \cdot 6$, my value at this point being $427 \cdot 52$. The difference amounts to 1 in 550 only. Giving the observations equal weight, this would have been 1 in 430 nearly. The quantity 426.75 is what I find at $18^{\circ} \mathrm{C}$. So that my result at this particular temperature differs from that of Joule only the amount that water changes in specific heat in $3^{\circ} \cdot 4 \mathrm{C}$.

Joule's value is less than my value to the amount given, but the value from the properties of air, 430.7 at $14^{\circ} \mathrm{C}$. is greater, although the method can have little weight.

It might be well to diminish my values by 1 part in 1000 so as to make them represent the mean of Joule's and my own experiments. It is seen that the experiment by the method of electric heating agrees very exactly with the other experiments, because I have reduced it to my value of the ohm. Hence I regard it as a very excellent confirmation of my value of that unit.

Baltimore, February 16, 1880. 


\section{PHYSICAL LABORATORY: COMPARISONS OF STANDARDS}

[Johns Hopkins University Circulars, No. 3, p. 31, 1880]

In order to secure uniformity throughout the country in certain physical standards, and to facilitate the use of the absolute system of heat measurement, it has been thought advisable to organize in the physical department of this University a sub-department, where comparisons of standards can be made.

Comparison of Thermometers.-At present we are only-able to make comparisons of thermometers, and so to reduce their degrees to the absolute scale of the perfect gas thermometer.

As the work is very laborious, it is proposed to make this sub-department self-supporting, by a system of fees sufficient to cover the bare cost of the labor, so that all may avail themselves of the facilities here offered.

In a recent study of standard thermometers by Geissler, Baudin, Fastré, Casella and from Kew, and the comparison of the same with the air thermometer, the differences due to the variety of the glass amounted to $0^{\circ} \cdot 2$ or $0^{\circ} .3 \mathrm{C}$., and the differences from the air thermometer were as high sometimes as $0^{\circ} \cdot 3 \mathrm{C}$. at the $40^{\circ}$ point.

The error from using uncompared mercurial thermometers in calorimetric investigations may amount to one or two per cent. For this reason the air thermometer has been taken as the standard, and all comparisons will be reduced to the final absolute standard of the perfect gas thermometer.

Very complete studies of thermometers have been made between $0^{\circ}$ and $40^{\circ} \mathrm{C}$., and a less complete study between $0^{\circ}$ and $100^{\circ}$, and between $100^{\circ}$ and $250^{\circ}$. Up to $100^{\circ}$ our thermometers have not only been compared with the air thermometer, but also with standards by Fastré, Geissler, Casella, Baudin and from Kew.

The study from $0^{\circ}$ to $40^{\circ}$ has been published by the American Academy of Sciences, at Boston, in a memoir on the Mechanical Equivalent of Heat. One of our thermometers is also now in the hands of Dr. Joule, who has compared it with the original thermometers used by him in the determination of the Mechanical Equivalent of Heat. 
The apparatus for the comparison up to $100^{\circ} \mathrm{C}$. is described in the paper above referred to. The thermometers are totally immersed in the water with their stems very near the bulbs of the air thermometers. From $100^{\circ}$ up to $250^{\circ}$ an oil bath is used, the bulbs only being in the oil, but the stems are heated to the same degree by being in contact with a heavy copper bar, whose temperature is noted by separate thermometers.

The ordinary comparison is made with the stems of the thermometers in a vertical position. Where they are used in a horizontal position a correction will have to be made, and this correction will be determined when it is so desired. When the comparison is made only to $40^{\circ}$, we can compare them in a horizontal position, but we cannot then insure the same accuracy as when they are vertical, and it is never advisable to use them in that position.

Where desired, a study will be made of the changes of the zero point as a function of the temperature to which it has been heated, and of the time, but this study is not advised, as it does not lead to very valuable results.

Thermometers with metal, wooden or paper scales are generally too poor to be worth comparison, and would often be spoiled by the immersion in the water. Thermometers with metal caps of Geissler's form are often injured, especially when heated to $250^{\circ} \mathrm{C}$. Therefore, comparisons of thermometers of these classes will not be undertaken, except in the case of standards long used for some particular purpose, or in that of fine Geissler thermometers.

Three intervals for the comparison have been selected.

A. Between $0^{\circ}$ and $40^{\circ}$ for thermometers used for meteorological observations, determination of the temperature of standards of length, calorimetric determinations, and all purposes where extreme accuracy is desired within that limit. To obtain the full value of such a comparison, thermometers should be graduated at least as fine as $0^{\circ} \cdot 1 \mathrm{C}$. or $0^{\circ} \cdot 2 \mathrm{~F}$ :

B. Between $0^{\circ}$ and $100^{\circ} \mathrm{C}$. It is advised that the thermometers sent be graduated at least as fine as $0^{\circ} \cdot 2 \mathrm{C}$. or $0^{\circ} \cdot 5 \mathrm{~F}$.

C. Between $100^{\circ}$ and $250^{\circ}$ for thermometers used by chemists in the determination of melting or boiling points. Thermometers should be graduated to $1^{\circ} \mathrm{C}$. or $1^{\circ} \mathrm{F}$.

Three kinds of comparison will be made for each of the intervals $0^{\circ}$ to $40^{\circ}, 0^{\circ}$ to $100^{\circ}$, and $100^{\circ}$ to $250^{\circ}$, as follows:

1st. Direct comparison with the air thermometer, and also a primary 
standard. This comparison is very laborious, and is not recommended except in very exceptional cases, as more than one comparison should be made to insure good results.

2nd. Comparison with primary standards which have been compared many times with the air thermometer. This is recommended where an error of $\frac{1}{100}^{\circ}$ is of some importance.

3rd. Comparison with seoondary standards which have been compared many times with the primary standards, and not very often directly with the air thermometer. This is recommended in all ordinary cases, where an error of $\frac{2}{100}^{\circ}$ can be tolerated.

When several comparisons are made, the following intervals will be allowed between the experiments, so that the zero reading may be allowed to return to its primitive value.

Thermometers heated to $40^{\circ} \mathrm{C}$. about 1 week. Thermometers heated to $100^{\circ} \mathrm{C}$. about 6 weeks. Thermometers heated to $250^{\circ} \mathrm{C}$. about 4 months.

The latter interval.is too small for an accurate return.

For the exact details of the method of comparison, I must refer to the above mentioned paper on the Mechanical Equivalent of Heat.

It is advisable in all cases where great accuracy is desired, that a numbers of comparisons be made, seeing that delicate thermometers are constantly varying through slight limits, and the average state can only be determined by repeated experiments.

Reports.-In the report of the comparison, the original readings will be given together with the reduced ones, and the plot of the curve of errors of the thermometer at every point. From this curve, the error of the thermometer at any reading can be found.

It is proposed to publish at the end of the year a complete report of all the comparisons made during the year, together with all new determinations of the errors of the standards, and to send it to any address at a price which we will hereafter announce.

Fees.-The comparators allow five thermometers only to be placed in them, of which two are our own standards in ordinary comparisons, and one in direct comparisons with the air thermometer. Therefore, three thermometers can be compared as easily as one in ordinary cases, and four in direct comparisons. Hence the following system of fees has been made out. 


\section{A. When a number of Thermometers are sent}

Comparison between $0^{\circ}$ and $40^{\circ} \mathrm{C}$. for 3 or 4 thermometers.

Direct, probable error at each point.......... $=\frac{1}{100}^{\circ} \$ 2000$

Primary Standards, probable error at each point $=\frac{-1}{100}{ }^{\circ} \quad 1100$

Secondary Standards, probable error at each point $=\frac{2}{10} \sigma^{\circ} \quad 800$

$0^{\circ}$ and $100^{\circ}$ for 3 or 4 thermometers.

Direct, probable error at each point.......... $=\frac{2}{100} 0^{\circ} \$ 2500$

Primary Standards, probable error at each point $=\frac{2}{100}{ }^{\circ} \quad 1200$

Secondary Standards, probable error at each point $=\frac{4}{100}{ }^{\circ} \quad 900$

$100^{\circ}$ to $250^{\circ}$ for 3 or 4 thermometers.

Direct, probable error at each point.......... $=\frac{1}{10}{ }^{\circ} \$ 2000$

Primary Standards, probable error at each point $=\frac{1}{10}{ }^{\circ} 1200$

Secondary Standards, probable error at each point $=\frac{1}{5}^{\circ} \quad 900$

\section{B. For Single Thermometers}

For single thermometers, the fees for the direct comparisons should be reduced to one-third, and for the ordinary ones to one-half the above figures. But in this case the thermometer will have to remain here until enough accumulate to fill the comparators.

Directions for Sending.-With each thermometer, send the name of maker, the date when made, purpose for which it is used, and the highest temperature to which it has lately been heated, and the date of such heating, together with the kind of comparison desired, and whether the thermometer is generally used in the horizontal or the vertical position.

In packing, the thermometer should be placed in a small box, which should again be packed with straw in a larger box.

The thermometers, both during transit and while here, must be at the owners' risk. Only sufficient fees have been charged to cover the bare cost of the comparison, and we bear the risk of our own standards, which are probably more valuable than any of those which will be sent to us. But every care will be taken, and the probability of an accident is very small.

We expect soon to be able to make other comparisons, and notice will then be given of the fact by the issue of another circular. 
ON GEISSLER THERMOMETERS: REMARKS BY PROFESSOR ROWLAND ON THE PRECEDING LETTER, ${ }^{1}$ IN A COMMUNICATION DATED JOHNS HOPKINS UNIVERSITY, APRIL 29,1881

[American Journal of Science [3], XXI, 451-453, 1881]

Through the kindness of Dr. Waldo, I have been allowed to see the above and would like to give a few words of explanation.

In reading what $I$ had to say with respect to the Geissler thermometer, the reader should remember that I was not writing on general thermometry, but only on that part which should be useful to me in measuring differences of temperature within the limits of $0^{\circ}$ and $45^{\circ} \mathrm{C}$. And so I merely made a study of thermometers, their change of zero and other points, as it affected the problem which I had before me. I am well aware that there are formulæ for giving the changed readings of thermometers due to previous heating, but, according to well known principles in such cases, I preferred to eliminate such error by the proper use of the thermometer rather than trust to an uncertain theory.

In the course of my investigation I discovered the fact that the Geissler thermometers, especially the one I then used, departed more from the air thermometer than any other. Now the Geissler thermometer has been used for many years by physicists, principally German, without any reduction to the air thermometer. And this correction was so great, amounting to over $0^{\circ} \cdot 3 \mathrm{C}$., for the specimen I used, at the $45^{\circ}$ point, that $I$ thought it right to call attention to the point. And I acknowledge that the picture was present in my mind of a physicist reading a thermometer from a distance by a telescope to avoid the heat of the body and parallax, and recording his results to thousandth of a degree, and all this on a thermometer having an error of $0^{\circ} \cdot 3 \mathrm{C}$.! As Dr. Thiesen remarks: If one is to compare his thermometer with the air thermometer, the amount of correction is of little importance: but departure from the air thermometer is certainly not a recommendation and, indeed, must introduce slight errors. The most accurate

${ }^{1}$ [By Dr. M. Thiesen, replying to Rowland's criticisms of the Geissler thermometers, as expressed in his memoir 'On the Mechanical Equivalent of Heat.']

31 
readings which one can make on an air thermometer will vary several hundredths of a degree.

Hence we can never use with accuracy the direct comparison with the air thermometer but must express the difference of the two instruments by some formula of the form:

$$
\Delta=a+b t+c t^{2}+\& c .
$$

Should we take an infinite number of terms this formula would express all the irregularities of our observations. But by limiting the number of terms the curve of differences becomes smoother and smoother and the formula expresses less and less the irregularities of the experiment. The number of terms to be used is a matter of judgment, and this point I sought to determine by the use of the observations of Regnault and others. The rejection of the higher powers of $t$ is more or less of an assumption founded on the fact that we are reasonably certain that the curve of differences between the mercurial and the air thermometer is a smooth curve. It is evident that the less the correction to be introduced the less the rejection of the higher powers of $t$ will affect our results.

We now come to my criticism of the Geissler thermometer for not having a reservoir at the top. Dr. Thiesen has in some way misunderstood my principal reason for its presence. My reason was not that "es vermindert die Schädlichkeit der im Quecksilber zurückgebliebenen Spuren von Luft" but that only by its use can the mercury in the bulb be entirely free from air. Take a thermometer and turn it with the bulb on top. If the thermometer is large, in nine cases out of ten the mercury will separate and fall down: allow it to remain and observe the bubble-like vacuum in the bulb. Turn the bulb in various directions so as to wash the whole interior of the bulb, as it were, and then bring the thermometer into a vertical position, keeping the bubble in sight. As the mercury flows back, the bubble diminishes and finally, in a good thermometer, almost disappears: but in most thermometers a good sized bubble of air, in some cases as large as the wire of a pin, remains. It is the most important function of a reservoir at the top to permit such manipulations as to drive all such air into the top reservoir and to make the mercury and the glass assume such perfect contact that the bulb can be turned uppermost without the mercury separating, even in thermometers of large size and with good generous bulbs. In many Geissler thermometers such a test might succeed, not on account of the freedom from air, but because the capillary tube and bulb are so small 
and the column so short that the capillary action is sufficient to prevent the fall. Now I think that a thermometer in which there is this layer of air around the mercury in the bulb must be uncertain in its action; hence my opinion is unaltered that all thermometers in which we cannot remove this layer or at least make certain of its absence should be rejected.

Furthermore, with respect to calibration, the reservoir is not essential to the calibration of thermometers whose range is $0^{\circ}$ and $100^{\circ} \mathrm{C}$. But my remarks apply better to those whose range is between $0^{\circ}$ and $30^{\circ} \mathrm{C}$. or $40^{\circ} \mathrm{C}$. Here calibration is impossible with a short column at ordinary temperatures unless some of the mercury can be stored up in the reservoir so as to allow the column to move over the whole scale. And it is within this limit that thermometers are of the greatest value in the physical laboratory.

The other defects of the Geissler thermometer, the scale which was always coming loose, the metal cap which was never tight and always allowed water to enter, the small capillary tube which wandered with perfect irregularity from side to side over the scale, all these were so obvious that I confined my remarks to the more obscure errors.

Furthermore, I believe there is some error in most Geissler thermometers from the small size of the bulb and the capillary tube, and this I have mentioned on p. $124^{1}$ of the paper referred to. Pfaundler and Platter, in a paper on the specific heat of water, in Poggendorff's Annalen for $18 \% 0$, found an immense variation within small limits. In a subsequent paper $^{2}$ the authors traced this error to the lagging of the thermometer behind its true reading.

The authors used Geissler thermometers graduated to $\frac{1}{50}{ }^{\circ} \mathrm{C}$ !! in a series of experiments made by plunging the thermometer into water after slightly heating or cooling the thermometer so that in one case the mercury fell and the other rose to the required point. When the thermometer fell about $6^{\circ}$ or $8^{\circ} \mathrm{C}$. it lagged behind $0^{\circ} \cdot 0654$ and when it rose $3^{\circ}$ or $4^{\circ}$ it lagged $0^{\circ} \cdot 022$, making a difference of $0^{\circ} \cdot 087$ C.! Now my thermometers made by Baudin show no effect of this kind. They indicate accurately the temperature whether they rise or fall to the given point, provided the interval is not too great. The fact then remains that a Geissler thermometer graduated to $\frac{1}{50}{ }^{\circ} \mathrm{C}$. may be uncertain to $0^{\circ} .087 \mathrm{C}$., while a Baudin graduated to $\mathrm{mm}$., one $\mathrm{mm}$. being from $\frac{1}{10}^{\circ}$ to $\frac{1}{14}^{\circ} \mathrm{C}$. is not uncertain to $0^{\circ} \cdot 01$ or $0^{\circ} \cdot 02 \mathrm{C}$. May not the 
cause be found in the layer of air around the mercury of the bulb which cannot be removed without a reservoir at the top? Or may we not also look for such an effect from the minute size of the bore of the capillary tube which creates a different pressure in the bulb from a rising or falling meniscus? Possibly the two may be combined. 


\section{PART IV \\ LIGHT}





\section{PRELIMINARY NOTICE OF THE RESULTS ACCOMPLISHED IN THE MANUFACTURE AND THEORY OF GRATINGS FOR OPTICAL PURPOSES}

[Johns Hopkins University Circulars, No. 17, pp. 248, 249, 1882; Philosophical Mragazine [4], XIII, 469-474, 1882 ; Nature, 26, 211-213, 1882 ; Journal de Physique, $I I, 5-11,1883]$

It is not many years since physicists considered that a spectroscope constructed of a large number of prisms was the best and only instrument for viewing the spectrum, where great power was required. These instruments were large and expensive, so that few physicists could possess them. Professor Young was the first to discover that some of the gratings of Mr. Rutherfurd showed more than any prism spectroscope which had then been constructed. But all the gratings which had been made up to that time were quite small, say one inch square, whereas the power of a grating in resolving the lines of the spectrum increases with the size. Mr. Rutherfurd then attempted to make as large gratings as his machine would allow, and produced some which were nearly two inches square, though he was rarely successful above an inch and three-quarters, having about thirty thousand lines. These gratings were on speculum metal and showed more of the spectrum than had ever before been seen, and have, in the hands of Young, Rutherfurd, Lockyer and others, done much good work for science. Many mechanics in this country and in France and Germany, have sought to equal Mr. Rutherfurd's gratings, but without success.

Under these circumstances, I have taken up the subject with the resources at command in the physical laboratory of the Johns Hopkins University.

One of the problems to be solved in making a machine is to make a perfect screw, and this, mechanics of all countries have sought to do for over a hundred years and have failed. On thinking over the matter, I devised a plan whose details I shall soon publish, by which I hope to make a practically perfect screw, and so important did the problem seem that I immediately set Mr. Schneider, the instrument maker of the university, at work at one. The operation seemed so successful that I 
immediately designed the remainder of the machine, and have now had the pleasure since Christmas of trying it. The screw is practically perfect, not by accident, but because of the new process for making it, and I have not yet been able to detect an error so great as one one-hundredthousandth part of an inch at any part. Neither has it any appreciable periodic error. By means of this machine I have been able to make gratings with 43,000 lines to the inch, and have made a ruled surface with 160,000 lines on it, having about 29,000 lines to the inch. The capacity of the machine is to rule a surface $6 \frac{1}{4} \times 4 \frac{1}{4}$ inches with any required number of lines to the inch, the number only being limited by the wear of the diamond. The machine can be set to almost any number of lines to the inch, but I have not hitherto attempted more than 43,000 lines to the inch. It ruled so perfectly at this figure that I see no reason to doubt that at least two or three times that number might be ruled in one inch, though it would be useless for making gratings.

"All gratings hitherto made have been ruled on flat surfaces. Such gratings require a pair of telescopes for viewing the spectrum; these telescopes interfere with many experiments, absorbing the extremities of the spectrum strongly; besides, two telescopes of sufficient size to use with six inch gratings would be very expensive and clumsy affairs. In thinking over what would happen were the grating ruled on a surface not flat, I thought of a new method of attacking the problem, and soon found that if the lines were ruled on a spherical surface the spectrum would be brought to a focus without any telescope. This discovery of concave gratings is important for many physical investigations, such as the photographing of the spectrum both in the ultraviolet and the ultra-red, the determination of the heating effect of the different rays, and the determination of the relative wave lengths of the lines of the spectrum. Furthermore it reduces the spectroscope to its simplest proportions, so that spectroscopes of the highest power may be made at a cost which can place them in the hands of all observers. With one of my new concave gratings I have been able to detect double lines in the spectrum which were never before seen.

The laws of the concave grating are very beautiful on account of their simplicity, especially in the case where it will be used most. Draw the radius of curvature of the mirror to the centre of the mirror, and from its central point with a radius equal to half the radius of curvature draw a circle; this circle thus passes through the centre of curvature of the mirror and touches the mirror at its centre. Now if the source of light is anywhere in this circle, the image of this source and the 
different orders of the spectra are all brought to focus on this circle. The word focus is hardly applicable to the case, however, for if the source of light is a point the light is not brought to a single point on the circle but is drawn out into a straight line with its length parallel to the axis of the circle. As the object is to see lines in the spectrum only, this fact is of little consequence provided the slit which is the source of light is parallel to the axis of the circle. Indeed it adds to the beauty of the spectra, as the horizontal lines due to dust in the slit are never present, as the dust has a different focal length from the lines of the spectrum. This action of the concave grating, however, somewhat impairs the light, especially of the higher orders, but the introduction of a cylindrical lens greatly obviates this inconvenience.

The beautiful simplicity of the fact that the line of foci of the different orders of the spectra are on the circle described above leads immediately to a mechanical contrivance by which we can move from one spectrum to the next and yet have the apparatus always in focus; for we only have to attach the slit, the eye-piece and the grating to three arms of equal length, which are pivoted together at their other ends and the conditions are satisfied. However we move the three arms the spectra are always in focus. The most interesting case of this contrivance is when the bars carrying the eye-piece and grating are attached end to end, thus forming a diameter of the circle with the eye-piece at the centre of curvature of the mirror, and the rod carrying the slit alone movable. In this case the spectrum as viewed by the eye-piece is normal, and when a micrometer is used the value of a division of its head in wave-lengths does not depend on the position of the slit, but is simply proportional to the order of the spectrum, so that it need be determined once only. Furthermore, if the eye-piece is replaced by a photographic camera the photographic spectrum is a normal one. The mechanical means of keeping the focus is especially important when investigating the ultra-violet and ultra-red portions of the solar spectrum.

Another important property of the concave grating is that all the superimposed spectra are in exactly the same focus. When viewing such superimposed spectra it is a most beautiful sight to see the lines appear colored on a nearly white ground. By micrometric measurement of such superimposed spectra we have a most beautiful method of determining the relative wave lengths of the different portions of the spectrum, which far exceeds in accuracy any other method yet devised. In working in the ultra-violet or ultra-red portions of the spectrum we 
can also focus on the superimposed spectrum and so get the focus for the portion experimented on.

The fact that the light has to pass through no glass in the concave grating makes it important in the examination of the extremities of the spectrum where the glass might absorb very much.

There is one important research in which the concave grating in its present form does not seem to be of much use, and that is in the examination of the solar protuberances; an instrument can only be used for this purpose in which the dust in the slit and the lines of the spectrum are in focus at once. It might be possible to introduce a cylindrical lens in such a way as to obviate this difficulty. But for other work on the sun the concave grating will be found very useful. But its principal use will be to get the relative wave lengths of the lines of the spectrum, and so to map the spectrum; to divide lines of the spectrum which are very near together, and so to see as much as possible of the spectrum; to photograph the spectrum so that it shall be normal; to investigate the portions of the spectrum beyond the range of vision; and lastly to put in the hands of any physicist at a moderate cost such a powerful instrument as could only hitherto be purchased by wealthy individuals or institutions.

To give further information of what can be done in the way of gratings I will state the following particulars:

The dividing engine can rule a space $6 \frac{1}{4}$ inches long and $4 \frac{1}{4}$ inches wide. The lines, which can be $4 \frac{1}{4}$ inches long, do not depart from a straight line so much as $\frac{1}{10000}$ inch, and the carriage moves forward in an equally straight line. The screw is practically perfect and has been tested to $\frac{1}{100000}$ inch without showing error. Neither does it have any appreciable periodic error, and the periodic error due to the mounting and graduated head can be entirely eliminated by a suitable attachment. For showing the production of ghosts by a periodic error, such an error can be introduced to any reasonable amount. Every grating made by the machine is a good one, dividing the 1474 line with ease, but some are better than others. Rutherfurd's machine only made one in every four good, and only one in a long time which might be called first-class. One division of the head of the screw makes 14,438 lines to the inch. Any fraction of this number in which the numerator is not greater than say 20 or 30 can be ruled. Some exact numbers to the millimetre, such as $400,800,1200$, etc., can also be ruled. For the finest definition either 14,438 or 28,876 lines to the inch are recommended, the first for ordinary use and the second for examining the extremities of the 
spectrum. Extremely brilliant gratings have been made with 43,314 lines to the inch, and there is little difficulty in ruling more if desired. The following show some results obtained:

Flat grating, 1 inch square, 43,000 lines to the inch. Divides the 1474 line in the first spectrum.

Flat grating, $2 \times 3$ inches, 14,438 lines to the inch, total 43,314 . Divides 1474 in the first spectrum, the $E$ line (Ångström 5269.4) in the second and is good in the fourth and even fifth spectrum.

Flat grating, $2 \times 3$ inches, 1200 lines to one millimetre. Shows very many more lines in the $B$ and $A$ groups than were ever before seen.

Flat grating, $2 \times 3 \frac{1}{4}$ inches, 14,438 lines to the inch. This has most wonderful brilliancy in one of the first spectra, so that $I$ have seen the $Z$ line, wave-length 8240 (see Abney's map of the ultra-red region), and determined its wave-length roughly, and have seen much further below the $A$ line than the $B$ line is above the $A$ line. The same may be said of the violet end of the spectrum. But such gratings are only obtained by accident.

Concave grating, $2 \times 3$ inches, 7 feet radius of curvature, 4818 lines to the inch. The coincidences of the spectra can be observed to the tenth or twelfth spectrum.

Concave grating, $2 \times 3$ inches, 14,438 lines to the inch, radius of curvature 8 feet. Divides the 1474 line in the first spectrum, the $E$ line in the second, and is good in the third or fourth.

Concave grating, $3 \times 5 \frac{1}{2}$ inches, 17 feet radius of curvature, 28,876 lines to the inch, and thus nearly 160,000 lines in all. This shows more in the first spectrum than was ever seen before. Divides 1474 and $E$ very widely and shows the stronger component of Angström 5275 double. Second spectrum not tried.

Concave grating, $4 \times 5 \frac{3}{4}$ inches, 3610 lines to the inch, radius of curvature 5 feet 4 inches. This grating was made for Professor Langley's experiments on the ultra-red portion of the spectrum, and was thus made very bright in the first spectrum. The definition seems to be very fine notwithstanding the short focus and divides the 1474 line with ease. But it is difficult to rule so concave a grating as the diamond marks differently on the different parts of the plate.

These give illustrations of the results accomplished, but of course many other experiments have been made. I have not yet been able to decide whether the definition of the concave grating fully comes up to that of a flat grating, but it evidently does so very nearly. 


\title{
ON CONCAVE GRATINGS FOR OPTICAL PURPOSES ${ }^{1}$
}

\author{
[American Journal of Science [3], XXVI, 87-98, 1883; Philosophical Magazine
} [5], $X V I, 197-210,1883]$

\section{General Theory}

Having recently completed a very successful machine for ruling gratings, my attention was naturally called to the effect of irregularity in the form and position of the lines and the form of the surface on the definition of the grating. Mr. C. S. Peirce has recently shown, in the American Journal of Mathematics, that a periodic error in the ruling produces what have been called ghosts in the spectrum. At first I attempted to calculate the effect of other irregularities by the ordinary method of integration, but the results obtained were not commensurate with the labor. I then sought for a simpler method. Guided by the fact that inverse methods in electrical distribution are simpler than direct methods, I soon found an inverse method for use in this problem.

In the use of the grating in most ordinary spectroscopes, the telescopes are fixed together as nearly parallel as possible, and the grating turned around a vertical axis to bring the different spectra into the field of view. The rays striking on the grating are nearly parallel, but for the sake of generality I shall assume that they radiate from a point in space and shall investigate the proper ruling of the grating to bring the rays back to the point from which they started. The wave fronts will be a series of spherical shells at equal distances apart. If

${ }^{1}$ An abstract of thls paper with some other matter was given at the Physical Society of London in November last, the paper being in my hand in its present shape at that time. As I wished to make some additions, for which I have not yet had time, I did not then publish it. I was much surprised soon after to see an article on this sabject which had been presented to the Physical Society and was published in the Philosophical Magazine. The article contains nothing more than an extension of my remarks at the Physical Society and formulæ similar to those in this paper. As I have not before this published anything except a preliminary notice of the concave gratings, I expected a little time to work up the subject, seeing that the practical work of photographing the spectrum has recently absorbed all my time. But probably I have waited too long. 
these waves strike on a reflecting surface, they will be reflected back provided they can do so all in the same phase. A sphere around the radiant point satisfies the condition for waves of all lengths and thus gives the case of ordinary reflection. Let any surface cut the wave surfaces in any manner and let us remove those portions of the surface which are cut by the wave surfaces; the light of that particular wavelength can then be reflected back along the same path in the same phase and thus, by the above principle, a portion will be sent back. But the solution holds for only one wave-length and so white light will be drawn out into a spectrum. Hence we have the important conclusion that a theoretically perfect grating for one position of the slit and eye-piece can be ruled on any surface, flat or otherwise. This is an extremely important practical conclusion and explains many facts which have been observed in the use of gratings. For we see that errors of the dividing engine can be counterbalanced by errors in the flatness of the plate, so that a bad dividing engine may now and then make a grating which is good in one spectrum but not in all. And so we often find that one spectrum is better than another. Furthermore Professor Young has observed that he could often improve the definition of a grating by slightly bending the plate on which it was ruled.

From the above theorem we see that if a plate is ruled in circles whose radius is $r \sin \mu$ and whose distance apart is $\Delta r / \sin \mu$, where $\Delta r$ is constant, then the ruling will be appropriate to bring the spectrum to a focus at a distance, $r$, and angle of incidence, $\mu$. Thus we should need no telescopes to view the spectrum in that particular position of the grating. Had the wave surfaces been cylindrical instead of spherical the lines would have been straight instead of circular, but at the above distances apart. In this case the spectrum would have been brought to a focus, but would have been diffused in the direction of the lines. In the same way we can conclude that in flat gratings any departure from a straight line has the effect of causing the dust in the slit and the spectrum to have different foci, a fact sometimes observed.

We also see that, if the departure from equal spaces is small, or, in other words, the distance $r$ is great, the lines must be ruled at distances apart represented by

$$
c\left(1-\frac{\cos ^{2} \mu}{r \sin \mu} x+\& c .\right)
$$

in order to bring the light to a focus at the angle $\mu$ and distance $r, c$ being a constant and $x$ the distance from some point on the plate. If $\mu$ changes sign, then $r$ must change in sign. Hence we see that the 
effect of a linear error in the spacing is to make the focus on one side shorter and the other side longer than the normal amount. Professor Peirce has measured some of Mr. Rutherfurd's gratings and found that the spaces increased in passing along the grating, and he also found that the foci of symmetrical spectra were different. But this is the first attempt to connect the two. The definition of a grating may thus be very good even when the error of run of the screw is considerable, provided it is linear.

\section{Concave Gratrings}

Let us now take the special case of lines ruled on a spherical surface; and let us not confine ourselves to light coming back to the same point, but let the light return to another point. Let the co-ordinates of the radiant point and focal point be $y=0, x=-a$ and $y=0, x=+a$, and let the centre of the sphere whose radius is $\rho$ be at $x^{\prime}, y^{\prime}$. Let $r$ be the distance from the radiant point to the point $x, y$, and let $R$ be that from the focal point to $x, y$. Let us then write

$$
2 b=R+r c,
$$

where $c$ is equal to \pm 1 according as the reflected or transmitted ray is used. Should we increase $b$ by equal quantities and draw the ellipsoids or hyperboloids so indicated, we could use these surfaces in the same way as the wave surfaces above. The intersections of these surfaces with any other surface form what are known as Huyghens' zones. By actually drawing these zones on the surface, we form a grating which will diffract the light of a certain wave-length to the given focal point. For the particular problem in hand, we need only work in the plane $x, y$ for the present.

Let $s$ be an element of the curve of intersection of the given surface with the plane $x, y$. Then our present problem is to find the width of Huyghens' zones on the surface, that is $d s$ in terms of $d b$.

The equation of the circle is

$$
\left(x-x^{\prime}\right)^{2}+\left(y-y^{\prime}\right)^{2}=\rho^{2}
$$

and of the ellipse or hyperbola

or

$$
\begin{gathered}
R+r c=2 b \\
\left(b^{2}-a^{2}\right) x^{2}+b^{2} y^{2}=b^{2}\left(b^{2}-a^{2}\right)
\end{gathered}
$$

in which $c$ has disappeared.

$$
d s=\sqrt{d x^{2}+d y^{2}} ; \quad \frac{d x}{d y}=-\frac{y-y^{\prime}}{x-x^{\prime}},
$$




$$
\begin{gathered}
d x\left\{\left(b^{2}-a^{2}\right) x-b^{2} y \frac{x-x^{\prime}}{y-y^{\prime}}\right\}=b\left\{2 b^{2}-\left(x^{2}+y^{2}+a^{2}\right)\right\} d b \\
d y\left\{-\left(b^{2}-a^{2}\right) x \frac{y-y^{\prime}}{x-x^{\prime}}+b^{2} y\right\}=b\left\{2 b^{2}-\left(x^{2}+y^{2}+a^{2}\right)\right\} d b, \\
\therefore \quad \frac{d s}{d b}=\rho b \frac{2 b^{2}-\left(x^{2}+y^{2}+a^{2}\right)}{\left(b^{2}-a^{2}\right)\left(y-y^{\prime}\right) x-b^{2}\left(x-x^{\prime}\right) y} .
\end{gathered}
$$

This equation gives us the proper distance of the rulings on the surface, and if we could get a dividing engine to rule according to this formula the problem of bringing the spectrum to a focus without telescopes would be solved. But an ordinary dividing engine rules equal spaces and so we shall further investigate the question whether there is any part of the circle where the spaces are equal. We can then write

$$
\frac{d s}{d b}=C .
$$

And the differential of this with regard to an arc of the circle must be zero. Differentiating and reducing by the equations

we have

$$
\frac{d x}{d y}=-\frac{y-y^{\prime}}{x-x^{\prime}} ; \quad \frac{d b}{d y}=-\frac{\rho}{C\left(x-x^{\prime}\right)},
$$

$$
\begin{aligned}
& \rho\left\{2 x b\left(y-y^{\prime}\right)-2 y b\left(x-x^{\prime}\right)-\frac{\rho}{C^{\prime}}\left[6 b^{2}-\left(x^{2}+y^{2}+a^{2}\right)\right]\right\} \\
& ++C\left\{\left(y-y^{\prime}\right)\left[\left(b^{2}-a^{2}\right)\left(y-y^{\prime}\right)-b^{2} y\right]-\left(x-x^{\prime}\right)\left[\left(b^{2}-a^{2}\right) x-b^{2}\left(x-x^{\prime}\right)\right]\right. \\
& \left.+\frac{2 b \rho}{C^{\prime}}\left[x\left(y-y^{\prime}\right)-y\left(x-x^{\prime}\right)\right]\right\}=0 .
\end{aligned}
$$

It is more simple to express this result in terms of $R, r, \rho$ and the angles between them.

Let $\mu$ be the angle between $\rho$ and $r$, and $\nu$ that between $\rho$ and $R$. Let us also put

$$
\alpha=\frac{\mu-\nu}{2} \text { and } \varepsilon=\frac{\mu+\nu}{2} \text {. }
$$

Let $\beta, \gamma$ and $\delta$ also represent the angles made by $r, R$ and $\rho$ respectively with the line joining the source of light and focus, and let

$$
\eta=\frac{\beta+\gamma}{2}
$$

Then we have

$$
x=\frac{R \cos \gamma+r \cos \beta}{2} ; \quad y=\frac{R \sin \gamma+r \sin \beta}{2} ; a=\frac{r \cos \beta-R \cos \gamma}{2},
$$




$$
\begin{aligned}
& \left(b^{2}-a^{2}\right)\left(y-y^{\prime}\right)^{2}+b^{2}\left(x-x^{\prime}\right)^{2}=\rho^{2}\left(b^{2}-a^{2} \sin ^{2} \delta\right), \\
& b^{2}-a^{2}=R r \cos ^{2} a, \\
& \sin \eta=\frac{R+r}{2 a} \sin \alpha ; \quad \cos \eta=\frac{r-R}{2 a} \cos a, \\
& R=b-\frac{a}{b} x, \quad r=b+\frac{a}{b} x, \\
& x=b \frac{\cos \eta}{\cos \alpha} ; \quad y=a \frac{\sin \gamma \sin \beta}{\sin \alpha \cos \alpha}=\frac{R r}{b} \sin \eta \cos \alpha, \\
& b^{2} y\left(y-y^{\prime}\right)+x\left(b^{2}-a^{2}\right)\left(x-x^{\prime}\right)=\frac{b R r \rho}{2}(\cos \mu+\cos \nu), \\
& 2 b^{2}-\left(x^{2}+y^{2}+a^{2}\right)=R r, \\
& x\left(b^{2}-a^{2}\right)\left(y-y^{\prime}\right)-b^{2} y\left(x-x^{\prime}\right)=\frac{R r b \rho}{2}(\sin \mu+\sin \nu), \\
& C=\frac{2}{\sin \mu+\sin \nu}=\frac{1}{\cos \alpha \sin \varepsilon}, \\
& 2 a \cos \delta=r \cos \mu-R \cos \nu, \\
& 2 a \sin \delta=r \sin \mu-R \sin \nu . \\
&
\end{aligned}
$$

On substituting these values and reducing, we find

$$
{ }^{2} \rho=\frac{2 R r \cos \alpha \cos \varepsilon}{r \cos ^{2} \nu+R \cos ^{2} \mu} \text {. }
$$

${ }^{2} \mathrm{~A}$ more simple solution is the following: $\frac{d s}{d b}$ must be constant in the direction in which the dividing engine rules. If the dividing engine rules in the direction of the axis $y$, the differential of this with respect to $y$ must be zero. But we can also take the reciprocal of this quantity and so we can write for the equation of condition

$$
\frac{d}{d y} \quad \frac{d(R+r)}{d s}=0 .
$$

Taking a circle as our curve we can write

and

$$
\begin{aligned}
& \left(x-x^{\prime}\right)^{2}+\left(y-y^{\prime}\right)^{2}=\rho^{2} \\
& \left(x-x^{\prime \prime}\right)^{2}+\left(y-y^{\prime \prime}\right)^{2}=R^{2},
\end{aligned}
$$$$
\left(x-x^{\prime \prime \prime}\right)^{2}+\left(y-y^{\prime \prime \prime}\right)^{2}=r^{2},
$$

$\frac{d(R+r)}{d s}=\frac{1}{\rho}\left\{\left(y-y^{\prime}\right)\left(\frac{x-x^{\prime \prime}}{R}+\frac{x-x^{\prime \prime \prime}}{r}\right)-\left(x-x^{\prime}\right)\left(\frac{y-y^{\prime \prime}}{R}+\frac{y-y^{\prime \prime \prime}}{r}\right)\right\}$

$\frac{d}{d y} \frac{d(R+r)}{d s}=\frac{1}{\rho}\left\{\frac{x-x^{\prime \prime}}{R}+\frac{x-x^{\prime \prime \prime}}{r}-\left(y-y^{\prime}\right)\left[\frac{\left.x-x^{\prime \prime}\right)\left(y-y^{\prime \prime}\right)}{R^{3}}\right.\right.$

$$
\left.\left.+\frac{\left(x-x^{\prime \prime \prime}\right)\left(y-y^{\prime \prime \prime}\right)}{r^{3}}\right]-\left(x-x^{\prime}\right)\left[\frac{\left(x-x^{\prime \prime}\right)^{2}}{R^{3}}+\frac{\left(x-x^{\prime \prime \prime}\right)^{2}}{r^{3}}\right]\right\}=0 .
$$

Making

$$
x=0, \quad y=0, \quad y^{\prime}=0, \quad x^{\prime}=\rho,
$$

we have

$$
\frac{x^{\prime \prime}}{R}+\frac{x^{\prime \prime \prime}}{r}-\rho\left(\frac{x^{\prime \prime 2}}{R^{3}}+\frac{x^{\prime \prime \prime 2}}{r^{3}}\right)=0 \text {, }
$$

or

$$
\rho=R r \frac{\cos \mu+\cos \nu}{r \cos ^{2} \nu+R \cos ^{2} \mu}=\frac{2 R r \cos a \cos \varepsilon}{r \cos ^{2} \nu+R \cos ^{2} \mu} \text {. }
$$


Whence the focal length is

$$
r=\frac{\rho R \cos ^{2} \mu}{2 R \cos \alpha \cos \varepsilon-\rho \cos ^{2} \nu} .
$$

For the transmitted beam, change the sign of $R$. Supposing $\rho, R$ and $\nu$ to remain constant and $r$ and $\mu$ to vary, this equation will then give the line on which all the spectra and the central image are brought to a focus.

By far the most interesting case is obtained by making

$$
r=\rho \cos \mu, \quad R=\rho \cos \nu,
$$

since these values satisfy the equation. The line of foci is then a circle with a radius equal to one-half $\rho$. Hence if a source of light

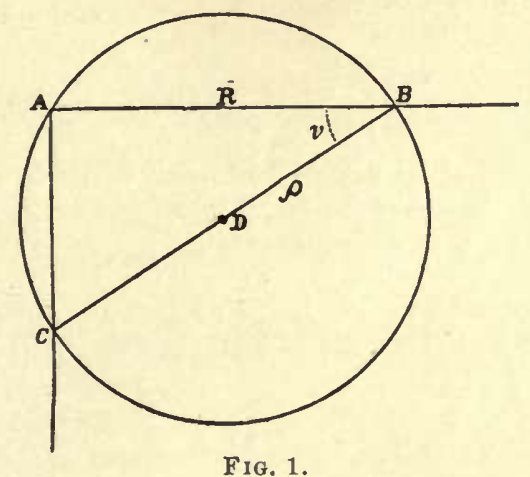

exists on this circle, the reflected image and all the spectra will be brought to a focus on the same circle. Thus if we attach the slit, the eye-piece and the grating to the three radii of the circle, however we move them, we shall always have some spectrum in the focus of the eye-piece. But in some positions the line of foci is so oblique to the direction of the light that only one line of the spectrum can be seen well at any one time. The best position of the eye-piece as far as we consider this fact is thus the one opposite to the grating and at its centre of curvature. In this position the line of foci is perpendicular to the direction of the light, and we shall show presently that the spectrum is normal at this point whatever the position of the slit, provided it is on the circle.

Fig. 1 represents this case; $A$ is the slit, $C$ is the eye-piece, and $B$ is the grating with its centre of curvature at $C$. In this case all the conditions are satisfied by fixing the grating and eye-piece to the bar $B C$ 
whose ends rest on carriages moving on the rails $A B$ and $A C$ at right angles to each other; when desired, the radius $A D$ may be put in to hold everything steady, but this has been found practically unnecessary.

The proper formulæ for this case are as follows: If $\lambda$ is the wavelength and $w$ the distance apart of the lines of the grating from centre to centre, then we have

where $N$ is the order of the spectrum.

$$
\frac{1}{O}=\frac{\lambda N}{2 w}=\frac{\sin \psi}{2},
$$

$$
\therefore \quad \lambda=\frac{w \sin v}{N} \text {. }
$$

Now in the given case $\rho$ is constant and so $N \lambda$ is proportional to the line $A C$. Or, for any given spectrum, the wave-length is proportional to that line.

If a micrometer is fixed at $C$ we can consider the case as follows:

$$
\begin{aligned}
& \frac{1}{C}=\frac{\lambda N}{2 \omega}=\frac{1}{2}(\sin \mu+\sin \nu), \\
& \frac{d \lambda}{d \mu}=\frac{w}{N} \cos \mu .
\end{aligned}
$$

If $D$ is the distance the cross-hairs of the micrometer move forward for one division of the head, we can write for the point $C$

$$
d^{\mu}=\frac{D}{l^{\prime \prime}}
$$

and for the same point $\mu$ is zero. Hence

$$
\Delta \lambda=\frac{w D}{N_{\mu}} \text {. }
$$

But this is independent of $\nu$ and we thus arrive at the important fact that the value of a division of the micrometer is always the same for the same spectrum and can always be determined with sufficient accuracy from the dimensions of the apparatus and number of lines on the grating, as well as by observation of the spectrum.

Furthermore, this proves that the spectrum is normal at this point and to the same scale in the same spectrum. Hence we have only to photograph the spectrum to obtain the normal spectrum and a centimeter for any of the photographs always represents the same increase of wave-length.

It is to be specially noted that this theorem is rigidly true whether the adjustments are correct or not, provided only that the micrometer is on the line drawn perpendicularly frum the centre of the grating, even if it is not the centre of curvature. 
As the radius of curvature of concave gratings is usually great, the distance through which the spectrum remains practically normal is very great. In the instrument which I principally use, the radius of curvature $\rho$, is about 21 feet 4 inches, the width of the ruling being about 5.5 inches. In such an instrument the spectrum thrown on a flat plate is normal within about 1 part in 1,000,000, for 6 inches and less than 1 in 35,000 , for 18 inches. In plotographing the spectrum on a flat plate, the definition is excellent for 12 inches, and by use of a plate bent to 11 feet radius, a plate of 20 inches in length is in perfect focus and the spectrum still so nearly normal as to have its error neglected for most purposes.

Another important property of the concave grating is that all the superimposed spectra are in focus at the same point, and so by micrometric measurements the relative wave-lengths are readily determined. Hence, knowing the absolute wave-length of one line, the whole spectrum can be measured. Professor Peirce has determined the absolute wave-length of one line with great care and I am now measuring the coincidences. This method is greatly more accurate than any hitherto known, as by a mere cye inspection, the relative wave-length can often be judged to 1 part in 20,000 and with a micrometer to 1 in $1,000,000$. Again, in dealing with the invisible portion of the spectrum, the focus can be obtained by examining the superimposed spectrum. Captain Abney, by using a concave mirror in the place of telescopes, has been enabled to use this method for obtaining the focus in photographing the ultra red rays of the spectrum. It is also to be noted that this theorem of the normal spectrum applies also to the flat grating used with telescopes and to either reflecting or transmitting gratings; but in these cases only a small portion of the spectrum can be used, as no lens can be made perfectly achromatic. And so, as the distance of the micrometer has constantly to be changed when one passes along the spectrum, its constant does not remain constant but varies in an irregular manner. But it would be possible to fix the grating, one objective and the camera rigidly on a bar, and then focus by moving the slit or the other objective. In this case the spectrum would be rigidly normal, but would probably be in focus for only a small length and the adjustment of the focus would not be automatic.

But nothing can exceed the beauty and simplicity of the concave grating when mounted on a movable bar such as I have described and illustrated in Fig. 1. Having selected the grating which we wish to use, we mount it in its plate-holder and put the proper collimating ere-piece 
in place. We then carefully adjust the focus by altering the length of $D$ until the cross-hairs are at the exact centre of curvature of the grating. On moving the bar the whole series of spectra are then in exact focus, and the value of a division of the micrometer is a known quantity for that particular grating. The wooden way $A C$, on which the carriage moves, is graduated to equal divisions representing wavelengths, since the wave-length is proportional to the distance $A C$. We can thus set the instrument to any particular wave-length we may wish to study, or even determine the wave-length to at least one part in five thousand by a simple reading. By having a variety of scales, one for each spectrum, we can immediately see what lines are superimposed on each other and identify them accordingly when we are measuring their relative wave-length. On now replacing the eye-piece by a camera, we are in a position to photograph the spectrum with the greatest ease. We put in the sensitive plate, either wet or dry, and move to the part we wish to photograph; having exposed for that part, we move to another part, raise the plate to another position and expose once more. We have no thought for the focus, for that remains perfect, but simply refer to the table giving the proper exposure for that portion of the spectrum and so have a perfect plate. Thus we can photograph the whole spectrum on one plate in a few minutes, from the $F$ line to the extreme violet in several strips, each 20 inches long. Or we may photograph to the red rays by prolonged exposure. Thus the work of days with any other apparatus becomes the work of hours with this. Furthermore, each plate is to scale, an inch on any one of the strips representing exactly so much difference of wave-length. The scale of the different orders of spectra are exactly proportional to the order. Of course the superposition of the spectra gives the relative wave-length. To get the superposition, of course, photography is the best method.

Having so far obtained only the first approximation to the theory of the concave grating, let us now proceed to a second one. The dividing engine rules equal spaces along the chord of the circular arc of the grating: the question is whether any other kind of ruling would be better, for the dividing engine is so constructed that one might readily change it to rule slightly different from equal spaces.

The condition for theoretical perfection is that $C$ shall remain constant for all portions of the mirror. I shall therefore investigate how nearly this is true.

Let $\rho$ be the radius of curvature and let $R$ and $r$ be the true distances to any point of the grating, $R_{0}$ and $r_{0}$ being the distances to the 
centre. Let $\mu$ and $\nu$ be the general values of the angles and $\mu_{0}$ and $\nu_{0}$ the angles referred to the centre of the mirror. The condition is that

$$
\frac{2}{C}=\sin \mu+\sin \nu
$$

shall be a constant for all parts of the surface of the grating. Let us then develope $\sin \mu$ and $\sin \nu$ in terms of $\mu_{0}, \nu_{0}$ and the angle $\delta$ between the radii drawn to the centre of the grating and to the point under consideration. Let $\delta^{\prime}$ be the angle between $R$ and $R_{0}$. Then we can write immediately

$$
\begin{gathered}
\rho \sin \mu=\rho \sin \mu_{0} \cos \delta^{\prime}+R_{0} \sin \delta^{\prime}-\rho \cos \mu_{0} \sin \delta^{\prime}, \\
\sin \mu=\sin \mu_{0} \cos \delta^{\prime}\left\{1+\frac{R_{0}}{\rho \sin \mu_{0}} A \tan \delta^{\prime}\right\},
\end{gathered}
$$

where

$$
A=1-\frac{\rho \cos \mu_{0}}{R_{0}} \text {. }
$$

Developing the value of $\cos \delta^{\prime}$ in terms of $\delta$, we have $\cos \delta^{\prime}=\cos \delta\left\{1+\frac{A}{2}\left[1+\frac{\rho \cos \mu_{0}}{R_{0}}\right] \delta^{2}\right.$

$$
\left.-\frac{\rho \sin \mu_{0}}{2 R_{0}}\left[1+A\left(1+\frac{2 \rho}{R_{0}}\right)\right] \delta^{2}+\& c .\right\} \text {. }
$$

As the cases we are to consider are those where $A$ is small, it will be sufficient to write

Whence we have

$$
\tan \delta^{\prime}=\frac{\rho \cos \mu_{0}}{R_{0}} \delta .
$$

$$
\begin{aligned}
\sin \mu= & \sin \mu_{0} \cos \delta\left\{1+\cot \mu_{0} A \delta+\frac{A}{2}\left[1+\frac{\rho \cos \mu_{0}}{R_{0}}\right] \delta^{2}\right. \\
& \left.+\left[A^{2} \cot \mu_{0}\left(1+\frac{\rho \cos \mu_{0}}{R_{0}}\right)-\frac{\rho \sin \mu_{0}}{2 R_{0}}\left(1+A\left(1+\frac{2 \rho}{R_{0}}\right)\right)\right] \delta^{3}+\& c .\right\}
\end{aligned}
$$

We can write the value of $\sin \nu$ from symmetry. But we have

$$
2 \frac{d b}{d s}=\sin \mu+\sin \nu .
$$

In this formula, $d b$ can be considered as a constant depending on the wave-length of light, etc., and $d s$ as the width apart of the lines on the grating. The dividing engine rules lines on the curved surface according to the formula

$$
2 \frac{d b}{d s}=\cos \delta\left(\sin \mu_{0}+\sin \nu_{0}\right) .
$$

But this is the second approximation to the true theoretical ruling. And this ruling will not only be approximately correct, but exact when 
all the terms of the series except the first vanish. In the case where the slit and focus are on the circle of radius $\frac{1}{2} \rho$, as in the automatic arrangement described above, we have $A=0$ and the second and third terms of the series disappear, and we can write since we have

$$
\begin{gathered}
\frac{R_{0}}{\rho}=\cos \mu_{0} \text { and } \frac{r_{0}}{\rho}=\cos \nu_{0}, \\
2 \frac{d b}{d s}=\cos \delta\left(\sin \nu_{0}+\sin \nu_{0}\right)\left(1-\frac{1}{2} \frac{\sin \mu_{0} \tan \mu_{0}+\sin \nu_{0} \tan \nu_{0}}{\sin \mu_{0}+\sin \nu_{0}} \delta^{3}+\& c .\right)
\end{gathered}
$$

But in the automatic arrangement we also have $\nu_{0}=0$, and so the formula becomes

$$
2 \frac{d b}{d s}=\cos \delta\left(\sin \mu_{0}+\sin \nu_{0}\right)\left\{1-\frac{1}{2} \tan \mu_{0} \delta^{3}+\& c .\right\} .
$$

To find the greatest departure from theoretical perfection, $\delta$ must refer to the edge of the grating. In the gratings which I am now making, $\rho$ is about 260 inches and the width of the grating about $5 \cdot 4$ inches. Hence $\delta=\frac{1}{100}$ approximately and the series becomes

$$
1-\frac{1}{2,000,000} \tan \mu_{0} .
$$

Hence the greatest departure from the theoretical ruling, even when $\tan \mu_{0}=2$, is 1 in $1,000,000$. Now the distance apart of the components of the 1474 line is somewhat nearly one forty-thousandth of the wave-length and I scarcely suppose that any line has been divided by the best spectroscope in the world whose components are less than onethird of this distance apart. Hence we see that the departure of the ruling from theoretical perfection is of little consequence until we are able to divide lines twenty times as fine as the 1474 line. Even in that case, since the error of ruling varies as $\partial^{3}$, the greater portion of the grating would be ruled correctly.

The question now comes up as to whether there is any limit to the resolving power of a spectroscope. This evidently depends upon the magnifying power and the apparent width of the lines. The magnifying power can be varied at pleasure and so we have only to consider the width of the lines of the spectrum. The width of the lines evidently depends, in a perfect grating, upon three circumstances, the width of the slit, the number of lines in the grating and the true physical width of the line. The width of the slit can be varied at pleasure, the number of lines on the grating can be made very great (160,000 in one of mine), and hence we are only limited by the true physical width of the lines. 
We have numerous cases of wide lines, such as the $C$ line, the components of the $D^{3}$ and $H$ lines and numerous others which are perfectly familiar to every spectroscopist. Hence we are free to suppose that all lines have some physical width, and we are limited by that width in the resolving power of our spectroscope. Indeed, from a theoretical standpoint, we should suppose this to be true: for the molecules only vibrate freely while swinging through their free path and in order to have the physical width one one-hundred-thousandth of the wave-length, the molecule must make somewhat nearly one hundred thousand vibrations in its free path: but this would require a free path of about $\frac{1}{200000}$ inch! Hence it would be only the outermost solar atmosphere that could produce such fine lines and we could hardly expect to see much finer ones in the solar spectrum. Again " it is found impossible to obtain interference between two rays whose paths differ by much more than ๖0,000 wave-lengths.

All the methods of determining the limits seem to point to about the 150,000 th of the wave-length as the smallest distance at which the two lines can be separated in the solar spectrum by even a spectroscope of infinite power. As we can now nearly approach this limit I am strongly of the opinion that we have nearly reached the limit of resolving power, and that we can never hope to see very many more lines in the spectrum than can be seen at present, either by means of prisms or gratings.

It is not to be supposed, however, that the average wave-length of the line is not more definite than this, for we can easily point the crosshairs to the centre of the line to perhaps 1 in $1,000,000$ of the wavelength. The most exact method of detecting the coincidences of a line of metal with one in the solar spectrum would thus be to take micrometric measurements first on one and then on the other; but I suppose it would take several readings to make the determination to 1 in $1,000,000$.

Since writing the above I have greatly improved my apparatus and can now photograph 150 lines between the $H$ and $K$ lines, including many whose wave-length does not differ more than 1 in about 80,000 . I have also photographed the 1474 and $b_{3}$ and $b_{4}$, widely double, and also $E$ just perceptibly double. With the eye much more can be seen, but I must say that I have not yet seen many signs of reaching a limit. The

${ }^{3}$ I have recently discovered that each component of the $D$ line is donble probably from the partial reversal of the line as we nearly always see it in the flame spectram.

4This method of determining the limit has been suggested to me by Prof. C. S. Hastings, of this University. 
lines yet appear as fine and sharp as with a lower power. If my grating is assumed to be perfect, in the third spectrum I should be able to divide lines whose wave-lengths differed, in about 150,000 , though not to photograph them.

The $E$ line has components, about $\frac{1}{60000}$ th of the wave-length apart. I believe I can resolve lines much closer than this, say 1 in 100,000 at least. Hence the idea of a limit has not yet been proved.

However, as some of the lines of the spectrum are much wider than others we should not expect any definite limit, but a gradual falling off as we increase our power. At first, in the short wave-lengths at least, the number of lines is nearly proportional to the resolving power, but this law should fail as we approach the limit. 


\title{
ON MR. GLAZEBROOK'S PAPER ON THE ABERRATION OF CONCAVE GRATINGS
}

\author{
[American Journal of Science [3], XXVI, 214, 1883; Philosophical Magazine [5], \\ $X V I, 210,1883]$
}

In the June number of the Philosophical Magazine, Mr. R. T. Glazebrook has considered the aberration of the concave grating and arrives at the conclusion that the ones which I have hitherto made are too wide for their radius of curvature. As I had published nothing but a preliminary notice of the grating at that time, Mr. Glazebrook had not then seen my paper on the subject, of which I gave an abstract at the London Physical Society in November last. In this paper I arrive at the conclusion that there is practically no aberration and that in this respect there is nothing further to be desired.

The reason of this discrepancy is not far to seek. Mr. Glazebrook assumes that the spaces are equal on the arc of the circle. But I do not rule them in this manner; but the equal spaces are equal along the chord of the arc. Again, the surface is not cylindrical, but spherical.

These two errors entirely destroy the value of the paper as far as my gratings are concerned, for it only applies to a theoretical grating, ruled in an entirely different manner from my own, and on a different form of surface.

I am very much surprised to see the method given near the end of the paper for constructing aplanatic gratings on any surface, for this is the method by which I discovered the concave grating originally, and the figure is the same as I put on the blackboard at the meeting of the Physical Society in November last. I say I am surprised, for Mr. Glazebrook's paper was read at the Physical Society, where I had given the same method a few months before, and yet it passed without comment. Indeed, I have given the same method many times at various scientific societies of my own country. However, as Mr. Glazebrook was not present at the meeting referred to, he is entirely without blame in the matter. 


\section{3}

\section{SCREW}

[Encyclopcedia Britannica, Ninth Edition, Volume $X X I$ ]

The screw is the simplest instrument for converting a uniform motion of rotation into a uniform motion of translation (see 'Mechanics,' vol. xv, p. 754). Metal screws requiring no special accuracy are generally cut by taps and dies. A tap is a cylindrical piece of steel having a screw on its exterior with sharp cutting edges; by forcing this with a revolving motion into a hole of the proper size, a screw is cut on its interior forming what is known as a nut or female screw. The die is a nut with sharp cutting edges used to screw upon the outside of round pieces of metal and thus produce male screws. More accurate screws are cut in a lathe by causing the carriage carrying the tool to move uniformly forward, thus a continuous spiral line is cut on the uniformly revolving cylinder fixed between the lathe centres. The cutting tool may be an ordinary form of lathe tool or a revolving saw-like disk (see 'Machine Tools,' vol. xv, p. 153).

Errors of Screws.-For scientific purposes the screw must be so regular that it moves forward in its nut exactly the same distance for each given angular rotation around its axis. As the mountings of a screw introduce many errors, the final and exact test of its accuracy can only be made when it is finished and set up for use. A large screw can, however, be roughly examined in the following manner: (1) See whether the surface of the threads has a perfect polish. The more it departs from this, and approaches the rough, torn surface as cut by the lathe tool, the worse it is. A perfect screw has a perfect polish. (2) Mount upon it between the centres of a lathe and the slip a short nut which fits perfectly. If the nut moves from end to end with equal friction, the screw is uniform in diameter. If the nut is long, unequal resistance may be due to either an error of run or a bend in the screw. (3) Fix a microscope on the lathe carriage and focus its single crosshair on the edge of the screw and parallel to its axis. If the screw runs true at every point, its axis is straight. (4) Observe whether the short nut runs from end to end of the screw without a wabbling motion when the screw is turned and the nut kept from revolving. If it wabbles the 
screw is said to be drunk. One can see this error better by fixing a long pointer to the nut, or by attaching to it a mirror and observing an image in it with a telescope. The following experiment will also detect this error: (5) Put upon the screw two well-fitting and rather short nuts, which are kept from revolving by arms bearing against a straight edge parallel to the axis of the screw. Let one nut carry an arm which supports a microscope focused on a line ruled on the other nut. Screw this combination to different parts of the screw. If during one revolution the microscope remains in focus, the screw is not drunk; and if the cross-hairs bisect the lines in every position, there is no error of run.

Making Accurate Screws.-To produce a screw of a foot or even a yard long with errors not exceeding $\frac{1}{1000}$ th of an inch is not difficult. Prof. Wm. A. Rogers, of Harvard Observatory, has invented a process in which the tool of the lathe while eutting the screw is moved so as to counteract the errors of the lathe screw. The screw is then partly ground to get rid of local errors. But, where the highest accuracy is needed, we must resort in the case of screws, as in all other cases, to grinding. A long, solid nut, tightly fitting the screw in one position, cannot be moved freely to another position unless the screw is very accurate. If grinding material is applied and the nut is constantly tightened, it will grind out all errors of run, drunkenness, crookedness, and irregularity of size. The condition is that the nut must be long, rigid and capable of being tightened as the grinding proceeds; also the screw must be ground longer than it will finally be needed so that the imperfect ends may be removed.

The following process will produce a screw suitable for ruling gratings for optical purposes. Suppose it is our purpose to produce a screw which is finally to be 9 inches long, not including bearings, and $1 \frac{1}{3}$ in. in diameter. Select a bar of soft Bessemer steel, which has not the hard spots usually found in cast steel, and about $1 \frac{3}{8}$ inches in diameter and 30 long. Put it between lathe centres and turn it down to one inch diameter everywhere, except about 12 inches in the centre, where it is left a little over $1 \frac{1}{8}$ inches in diameter for cutting the screw. Now cut the screw with a triangular thread a little sharper than $60^{\circ}$. Above all, avoid a fine screw, using about 20 threads to the inch.

The grinding nut, about 11 inches long, has now to be made. Fig. 1 represents a section of the nut, which is made of brass, or better, of Bessemer steel. It consists of four segments, $-a, a$, which can be drawn about the screw by two collars, $b, b$, and the screw $c$. Wedges between 
the segments prevent too great pressure on the screw. The final clamping is effected by the rings and screws, $d$, $d$, which enclose the flanges, $e$, of the segments. The screw is now placed in a lathe and surrounded by water whose temperature can be kept constant to $1^{\circ} \mathrm{C}$., and the nut placed on it. In order that the weight of the nut may not make the ends too small, it must either be counterbalanced by weights hung from a rope passing over pulleys in the ceiling, or the screw must be vertical during the whole process. Emery and oil seem to be the only available grinding materials, though a softer silica powder might be used towards the end of the operation to clean off the emery and prevent future wear. Now grind the screw in the nut, making the nut pass backwards and forwards over the screw, its whole range being nearly 20 inches at first.

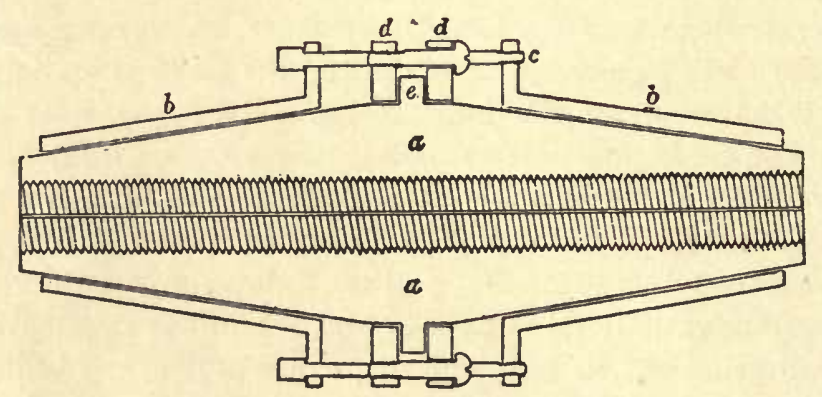

FIG. 1.-Section of Grinding Nut.

Turn the nut end for end every ten minutes and continue for two weeks, finally making the range of the nut only about 10 inches, using finer washed emery and moving the lathe slower to avoid heating. Finish with a fine silica powder or rouge. During the process, if the thread becomes too blunt, recut the nut by a short tap so as not to change the pitch at any point. This must, of course, not be done less than five days before the finish. Now cut to the proper length; centre again in the lathe under a microscope, and turn the bearings. A screw so ground has less errors than from any other system of mounting. The periodic error especially will be too small to be discoveref, though the mountings and graduation and centering of the head will introduce it; it must therefore finally be corrected.

Mounting of Screws.-The mounting must be devised most carefully, and is, indeed, more difficult to make without error than the screw itself. The principle which should be adopted is that no workmanship is perfect; the design must make up for its imperfections. Thus the screw 
can never be made to run true on its bearings, and hence the device of resting one end of the carriage on the nut must be rejected. Also all rigid connection between the nut and the carriage must be avoided, as the screw can never be adjusted parallel to the ways on which the car, riage rests. For many purposes, such as ruling optical gratings, the carriage must move accurately forward in a straight line as far as the horizontal plane is conserned, while a little curvature in the vertical plane produces very little effect. These conditions can be satisfied by making the ways $\mathrm{V}$-shaped and grinding with a grinder somewhat shorter than the ways. By constant reversals and by lengthening or shortening the stroke, they. will finally become nearly perfect. The vertical curvature can be sufficiently tested by a short carriage carrying a delicate spirit level. Another and very efficient form of ways is V-shaped with a flat top and nearly vertical sides. The carriage rests on the flat top and is held by springs against one of the nearly vertical sides. To determine with accuracy whether the ways are straight, fix a flat piece of glass on the carriage and rule a line on it by moving it under a diamond; reverse and rule another line near the first, and measure the distance apart at the centre and at the two ends by a micrometer. If the centre measurement is equal to the mean of the two end ones, the line is straight. This is better than the method with a mirror mounted on the carriage and a telescope. The screw itself must rest in bearings, and the end motion be prevented by a point bearing against its flat end, which is protected by hardened steel or a flat diamond. Collar bearings introduce periodic errors. The secret of success is so to design the nut and its connections as to eliminate all adjustments of the screw and indeed all imperfect workmanship. The connection must also be such as to give means of correcting any residual periodic errors or errors of run which may be introduced in the mountings or by the wear of the machine.

The nut is shown in Fig 2. It is made in two halves, of wrought iron filled with boxwood or lignum vitae plugs, on which the screw is cut. To each half a long piece of sheet steel is fixed which bears against a guiding edge, to be described presently. The two halves are held to the screw by springs, so that each moves forward almost independently of the other. To join the nut to the carriage, a ring is attached to the latter, whose plane is vertical and which can turn round a vertical axis. The bars fixed midway on the two halves of the nut bear against this ring at points $90^{\circ}$ distant from its axis. Hence each half does its share independently of the other in moving the carriage forward. Any want 
of parallelism between the screws and the ways or eccentricity in the screw mountings thus scarcely affects the forward motion of the carriage. The guide against which the steel pieces of the nut rest can be made of such form as to correct any small error of run due to wear of the screw. Also, by causing it to move backwards and forwards periodically, the periodic error of the head and mountings can be corrected.

In making gratings for optical purposes the periodic error must be very perfectly eliminated, since the periodic displacement of the lines only one-millionth of an inch from their mean position will produce

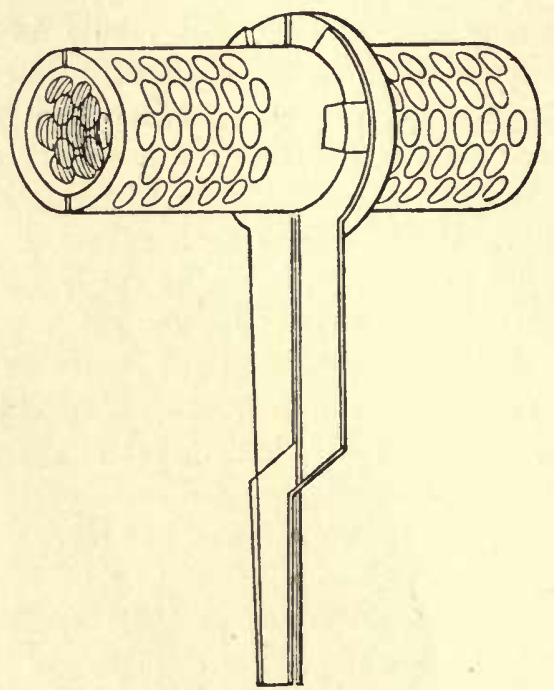

FIG. 2.

"ghosts" in the spectrum. ${ }^{1}$ Indeed, this is the most sensitive method of detecting the existence of this error, and it is practically impossible to mount the most perfect of screws without introducing it. A very practical method of determining this error is to rule a short grating with very long lines on a piece of common thin plate glass; cut it in two with a diamond and superimpose the two halves with the rulings together and displaced sideways over each other one-half the pitch of the screw. On now looking at the plates in a proper light so as to have the spec-

1 In a machine made by the present writer for ruling gratings the periodic error is entirely due to the graduation and centering of the bead. The uncorrected periodic error from this cause displaces the lines $\frac{1}{300000}$ th of an inch, which is sufficient to entirely ruin all gratings made without correcting it. 
tral colors show through it, dark lines will appear, which are wavy if there is a periodic error and straight if there is none. By measuring the comparative amplitude of the waves and the distance apart of the two lines, the amount of the periodic error can be determined. The phase of the periodic error is best found by a series of trials after setting the corrector at the proper amplitude as determined above.

A machine properly made as above and kept at a constant temperature should be able to make a scale of 6 inches in length, with errors at no point exceeding $\frac{1}{100000}$ th of an inch. When, however, a grating of that length is attempted at the rate of 14,000 lines to the inch, four days and nights are required, and the result is seldom perfect, possibly on account of the wear of the machine or changes of temperature. Gratings, however, less than 3 inches long are easy to make. 


\title{
ON THE RELATIVE WAVE-LENGTH OF THE LINES OF THE SOLAR SPECTRUM
}

\author{
[American Journal of Science [3], XXXIII, 182-190, 1887; Philosophical Magazine \\ [5], XXIII, 257-265, 1887]
}

For several years past $I$ have been engaged in making a photographic map of the solar spectrum to replace the ordinary engraved maps and I have now finished the map from the extreme ultra violet, wave-length 3200 , down to wave-length 5790 . In order to place the scale correctly on this map, I have found it necessary to measure the relative wavelengths of the spectrum and to reduce it to absolute wave-lengths by some more modern determination. I have not yet entirely finished the work, but as my map of the spectrum is now being published and as all observers so far seem to accept the measures of Ångström, I have decided that a table of my results would be of value. For as they stand now they have at least ten times the accuracy of any other determination. This great accuracy arises from the use of the concave grating which reduces the problem of relative wave-lengths to the measure of the coincidences of the lines in the different spectra by a micrometer.

The instrument which I have employed has concave gratings 5 or $6 \mathrm{in}$. diameter, having either 7200 or 14,400 lines to the inch and a radius of $21 \mathrm{ft} .6$ in. By my method of mounting, the spectrum is normal where measured, and thus it is possible to use a micrometer with a range of 5 inches. The spectrum keeps in focus everywhere and the constant of the micrometer remains unchanged except for slight variations due to imperfections in the workmanship. The micrometer has no errors of run or period exceeding the $\frac{1}{20000}$ inch. The probable error of a single setting on a good clear line is about $\frac{1}{200000}$ of the wave-length. 1 " of are is about $\cdot 0012$ inch. The $D$ line in the second spectrum is $\cdot 17$ inch or $4.4 \mathrm{~mm}$. wide. Determinations of relative wave-length of good lines seldom differ 1 in 500,000 from each other and never exceed 1 in 100,000 , even with different gratings. This is, of course, for the principal standard lines, and the chance of error is greater at the extremities of the spectrum. The interpolation of lines was made by running the micrometer over the whole spectrum, 5 inches at a time, and adding the 
readings together so as to include any distance, even the whole spectrum. The wave-length is calculated for a fixed micrometer constant and then corrected so as to coincide everywhere very nearly with the standards. I suppose the probable error of the relative determinations with the weight 1 in my table to be not far from 1 in 500,000. Angström thinks his standard lines have an accuracy of about 1 in 50,000 and ordinary lines much less.

As to the absolute measure, it is now well determined that Angström's figures are too small by about 1 part in 6000 . This rests: 1st, on the determination of Peirce made for the U. S. Coast Survey with Rutherfurd's gratings and not yet completely published; $2 \mathrm{~d}$, on an error made by Tresca in the length of the standard metre used by Angström ${ }^{1}$ which increases his value by about 1 in $7700 ; 3 \mathrm{~d}$, on a result obtained in my laboratory with two of my gratings by Mr. Bell, which is published with this paper. Mr. C. S. Peirce has kindly placed his grating at our disposal and we have detected an error of ruling which affects his result and makes it nearly coincide with our own. The wave-length of the mean of the two $E$ lines is

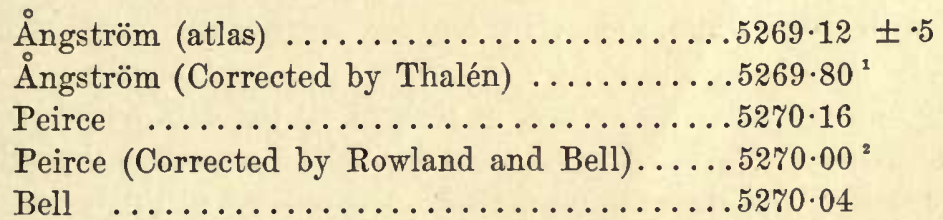

These results are for air at ordinary pressures and temperatures. The last is reduced to $20^{\circ} \mathrm{C}$. and $760 \mathrm{~mm}$. pressure. To reduce to a vacuum multiply by the following:

$\begin{array}{lllllll}\text { Fraunhofer line } & A & C & E & G & H\end{array}$ Correction factor..1.000291 $1.000292 \quad 1.000294 \quad 1.000297 \quad 1.000298$

The relation between my wave-lengths and those of Angström are given by the following, Angström's value being from p. 31 of his memoir :

\begin{tabular}{|c|c|c|c|c|c|}
\hline & A (edge) & B (edge) & & $C$ & \\
\hline Angström & $7597 \cdot 5$ & $686 \% \cdot 10$ & $6717 \cdot 16$ & $6562 \cdot 10$ & $6264 \cdot 31$ \\
\hline Rowland. & 7593.97 & $6867 \cdot 38$ & $6717 \cdot 83$ & $6562 \cdot 96$ & $6265 \cdot 27$ \\
\hline Difference & -3.5 & $\cdot 28$ & .67 & $\cdot 86$ & .96 \\
\hline
\end{tabular}

\footnotetext{
1 Thalén, Sur Spectre du Fer, Société Royale des Sciences d'Upsal, September, 1884, p. 25.

${ }^{2}$ From one grating only.
} 


\begin{tabular}{|c|c|c|c|c|c|}
\hline $\begin{array}{l}\text { Angström } \ldots \ldots \ldots \ldots \\
\text { Rowland } \ldots \ldots \ldots \ldots\end{array}$ & $\begin{array}{c}D_{2} \\
5895 \cdot 13 \\
5896 \cdot 08\end{array}$ & $\begin{array}{c}D_{1} \\
5889 \cdot 12 \\
5890 \cdot 12\end{array}$ & $\begin{array}{l}5708 \cdot 45 \\
5709 \cdot 56\end{array}$ & $\begin{array}{l}\text { Peirce's line } \\
5623 \cdot 36 \\
5624 \cdot 70\end{array}$ & $\begin{array}{l}5454 \cdot 84 \\
5455 \cdot 68\end{array}$ \\
\hline Difference....... . & .95 & $1 \cdot 00$ & $1 \cdot 11$ & $1 \cdot 34$ & $\cdot 84$ \\
\hline ̊nngström & $\begin{array}{c}E \\
5269 \cdot 59\end{array}$ & $\begin{array}{c}E \\
5268 \cdot 67\end{array}$ & $\begin{array}{c}b_{1} \\
5183 \cdot 10\end{array}$ & $5138 \cdot 78$ & $\begin{array}{c}F \\
4860 \cdot 74\end{array}$ \\
\hline Rowland . & $5270 \cdot 43$ & $5269 \cdot 65$ & $5183 \cdot 73$ & $5139 \cdot 47$ & $4861 \cdot 43$ \\
\hline Difference & $\cdot 84$ & .98 & $\cdot 63$ & $\cdot 69$ & $\cdot 69$ \\
\hline $\begin{array}{l}\text { Ångström . } \\
\text { Rowland .. }\end{array}$ & $\begin{array}{l}4702 \cdot 44 \\
4703 \cdot 11\end{array}$ & $\begin{array}{c}G \\
430 \% \cdot 25 \\
430 \% \cdot 96\end{array}$ & & & \\
\hline Difference ......... & .67 & .71 & + & & \\
\hline
\end{tabular}

The greatest variation in these differences is evidently due to the poor definition of Angström's grating by which the numbers refer to groups of lines rather than to single ones. Selecting the best figures, we find that Ångström's wave-lengths must be multiplied by 1.00016 to agree with Bell, while the correction for Ångström's error of scale would be 1.000110 .

It is impossible for me to give at present all the data on which my determinations rest, but I have given in Table I many of the coincidences as observed with sereral gratings, the number of single readings being given in the parenthesis over each set.

Table II gives the wave-lengths as interpolated by the micrometer. It is scarcely possible that any error will be found (except accidental errors) of more than 02 , and from the agreement of the observations I scarcely expect to make any changes in the final table of more than .01 , except in the extremities of the spectrum, where it may amount to .03 in the region of $A$ and $H$ lines. The wave-lengths of weight greater than 1 will probably be found more exact than this. The lines can be identified on my new photograph of the spectrum down to 5790 . Below this there is little trouble in finding the right ones. All maps of the spectrum, especially above $F$, are so imperfect that it is almost impossible to identify my lines upon them. The lines can only be properly identified by a power sufficient to clearly divide $b_{3}$ and $b_{4}$. Some of them are double and most of these have been marked, but as the table has been made for my own use, I have not been very careful to examine each line. This will, however, be finally done. Micrometric measures 
have now been made of nearly all the lines below $b$ with a view of making a map of this region.

Table I gives the coincidences of the different orders of the spectra as observed with several concave gratings on both sides of the normal, the numbers in the brackets indicating the number of observations. The observations have been reduced as nearly as possible to what I consider the true wave-length, the small difference from the numbers given in Table II being the variation of the observations from the mean value. The true way of reducing these observations would be to form a linear equation for each series and reduce by the method of least squares. A simpler way was, however, used and the relative wave-length of the standard lines, marked $S$ in Table II, was obtained; however, some other observations were also included.

Table II gives the wave-lengths reduced to Bell's value for the absolute wave-length of the $D$ line. These were obtained by micrometric measurement from the standards as described before. The weights are given in the first column and some of the lines, which were measured double, have also been marked. But the series has not yet been carefully examined for doubles.

The method is so much more accurate than by means of angular measurement that the latter has little or no weight in comparison.

This table is to be used in connection with my photographic map of the normal spectrum to determine the error of the latter at any point. The map was made by placing the photograph in contact with the scale, which was the same for each order of spectrum, and enlarging the two together. In this way the map has no local irregularities, although the scale may be displaced slightly from its true position, and may be a little too long or short, although as far as I have tested it, it seems to have very little error of the latter sort. The scale was meant in all cases, except the ultra violet, to apply to Peirce's absolute value and so the correction is generally negative, as follows:

\section{Approximate correction to the photographic map of the normal spectrum to reduce to latest absolute value.}

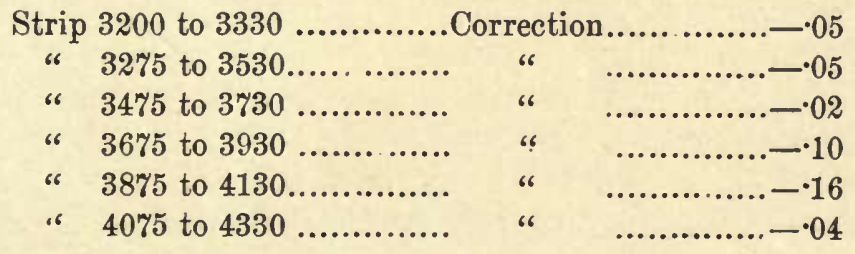




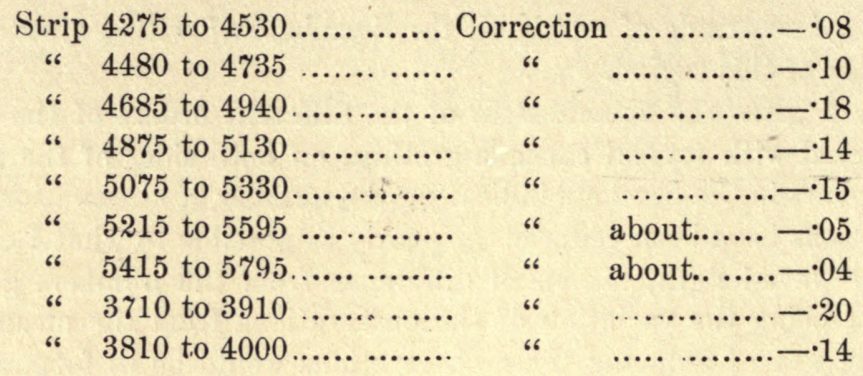

It is to be noted that the third spectrum of the map runs into the second, so that it must not be used beyond wave-length 3200 , as it is mixed with the second in that region.

[The tables are omitted.] 


\section{TABLE OF STANDARD WAVE-LENGTHS}

[Johns Hopkins University Circulars, No. 73, p. 69, 1889; Philosophical Magazine [5], XXVII, 479-484, 1889]

In the 'American Journal of Science' for March, 1887, and the 'London, Dublin and Edinburgh Philosophical Magazine' for the same month, I have published a preliminary list of standards as far as could be observed with the eye, with a few imperfectly observed by photography, the whole being reduced to Bell's and Peirce's values for absolute wave-lengths. Mr. Bell has continued his measurements and found a slightly greater value for the absolute wave-length of the $D$ line, and I have reduced my standards to the new values.

Nearly the whole list has been gone over again, especially at the ends around the $A$ line and in the ultra violet. The wave-lengths of the ultra violet were obtained by photographing the coincidence with the lower wave-lengths, a method which gives them nearly equal weight with those of the visible spectrum.

The full set of observations will be published hereafter, but the present series of standards can be relied on for relative wave-lengths to .02 division of Angström in most cases, though it is possible some of them may be out more than this amount, especially in the extreme red.

As to the absolute wave-length, no further change will be necessary, provided spectroscopists can agree to use that of my table, as has been done by many of them.

By the method of coincidences with the concave grating the wavelengths have been interwoven with each other throughout the whole table so that no single figure could be changed without affecting many others in entirely different portions of the spectrum. The principal difference from the preliminary table is in the reduction to the new absolute wave-length by which the wave-lengths are about 1 in 80,000 larger than the preliminary table. I hope this difference will not be felt by those who have used the old table because measurements to less than $\frac{1}{10}$ division of Angström are rare, the position of the lines of many metals being unknown to a whole division of Angström. As the new map of the spectrum has been made according to this new table, I see no further reason for changing the table in the future. 
No attempt has been made to reduce the figures to a vacuum as the index of refraction of air is imperfectly known, but this should be done where numerical relations of time period are desired.

In the column giving the weight, the primary standards are marked $S$ and the other numbers give the number of separate determination of the wave-length and thus, to some extent, the weight.

Many of these standards are double lines and some of them have faint components near them, which makes the accuracy of setting smaller. This is specially the case when this component is an atmospheric line whose intensity changes with the altitude of the sun. The principal doubles are marked with $d$, but the examination has not been completed yet, especially at the red end of the spectrum.

[A table of the standard wave-lengths is given on p. $78 \mathrm{~J}$. H. U. Circ., but is omitted in this volume.] 


\section{A FEW NOTES ON THE USE OF GRATINGS}

[Johns Hopkins University Circulars, No. 73, pp. 73, 74, 1889]

The ghosts are very weak in most of my gratings. They are scarcely visible in the lower orders of spectra, but increase in intensity as compared with the principal line as the square of the order of the spectrum. Hence, to avoid them, obtain magnification by increasing the focal distances instead of going to the higher orders. The distances from the - principal line in my gratings are the same as the distances of the spectra from the image of the slit when using a grating of 20 lines to the inch. They are always symmetrical on the two sides, and about $\frac{1}{12}$ inch for the violet and $\frac{1}{8}$ inch for the red in a grating of $21 \mathrm{ft}$. 6 in. radius in all orders of spectra. When the given line has the proper exposure on the photographic plate, the ghosts will not show, but over-exposure brings them out faintly in the third spectrum of a 20,000 grating or the 6 th of a 10,000 one. They never cause any trouble, as they are easily recognized and never appear in the solar spectrum. In some cases the higher orders of ghosts are quite as apparent as those of the first order.

The gratings with 10,000 lines to the inch often have better definition than those of 20,000, as they take half the time to rule, and they are quite as good for eye observation. They can also be used for photographing the spectrum by absorbing the overlying spectra, but there are very few materials which let through the ultra violet and absorb the longer wave-lengths. The 10,000 gratings have the advantage, however, in the measurement of wave-lengths by the overlapping spectra, although this method is unnecessary since the completion of my map of the spectrum. By far the best is to use a 20,000 grating and observe down to the $D$ line by photography, using erythrosin plates from the $F$ line down to $D$. Below $D$, cyanine plates can be used, although the time of exposure is from 10 to 60 minutes with a narrow slit. The solar spectrum extends to wave-lengths 3000 , and the map has been continued to this point. Beyond this, the coincidence with the solar spectrum cannot be used, but those of the 1 st and $2 \mathrm{~d}$ or $2 \mathrm{~d}$ and $3 \mathrm{~d}$ spectra can be.

Some complaints have been made to me that one of my gratings has no spectrum beyond 3400 , even of the electric arc. I have never found this the case, as the one I use gives w. 1.2200 , readily with 30 minutes exposure on slow plates, requiring 5 minutes for the most sensitive 
part and using the electric arc. With sensitive plates, the time can be diminished to one-fifth of this.

For eye observations, a very low power eye-piece of 1 or 2 in. focus is best. This, with a focus of $21 \mathrm{ft} .6 \mathrm{in}$. is equivalent to a plane grating with a telescope of a power of 100 or 200 .

In measuring the spectra, an ordinary dividing engine with errors not greater than $\frac{1}{1000}$ inch can be used, going over the measurements twice with the plate reversed between the separate series. The plates are on so very large a scale that the microscope must have a very low power. The one I use has a 1 inch objective and a 2 inch eye-piece. The measured part of the plate is about a foot long, the plates being 19 in. long.

All the spectrum photographs taken at different times coincide perfectly, and this can be used for such problems as the determination of the atmospheric lines. For this purpose, negatives at high and low sun are compared by scraping the emulsion off from half the plates and clamping them together with the edges of the spectra in coincidence. The two spectra coincide exactly line for line except where the atmospheric lines occur.

This method is specially valuable for picking out impurities in metallic spectra, using some standard impurity in all the substances to give a set of fiducial lines; or better, obtaining the coincidence of all the metals with some one metal, such as iron. Making the iron spectrum coincide on the two plates, the other spectra can be compared. This is specially possible because the focus of a properly set up concave grating need not be altered in years of use, for, when necessary, it can be adjusted at the slit, keeping the distance of the grating from the slit constant.

The spectrum of the carbon poles is generally too complicated for use with anything except the more pronounced lines of metals, there being, at a rough guess, 10,000 lines in its spectrum. However, in photographing metallic spectra but few of these show on the plate, as they are mostly faint. The spark discharge gives very nebulous lines for the metals.

Most gratings are ruled bright in the higher orders, but this is more or less difficult, as most diamond points give the first spectrum the brightest. Indeed, it is very easy to obtain ruling which is immensely bright in the first spectrum. Such gratings might be used for gaseous spectra. Short focus gratings of $5 \mathrm{ft}$. radius of curvature, very bright in the first order, require only a fraction of a second exposure for the solar spectrum and the spectrum of a gas can be obtained in less than an hour. 


\section{REPORT OF PROGRESS IN SPECTRUM WORK}

[Johns Hopkins University Circulars, No. 55, pp. 41, 42, 1891; American Journal of Science [3], XLI, 243, 244, 1891; The Chemical News, LXIII, 133, 1891]

During the past year or two a great deal of work has been done in the photography of the spectra of elements and the identification of the lines in the solar spectrum, which it will take a long time to work up, ready for publication. Hence, I have thought that a short account of what has been done up to the present time might be of interest to workers in the subject. In the prosecution of the work financial assistance has been received from the Rumford Fund of the American Academy of Arts and Sciences, as well as from the fund given by Miss Bruce to the Harvard Astronomical Observatory for the promotion of research in astronomical physics, and the advanced state of the work is due to such assistance.

The work may be summed up under the following heads:

1st. The spectra of all known elements, with the exception of a few gaseous ones, or those too rare to be yet obtained, have been photographed in connection with the solar spectrum, from the extreme ultra violet down to the $D$ line, and eye observations have been made on many to the limit of the solar spectrum.

2d. A measuring engine has been constructed with a screw to fit the above photographs, which, being taken with the concave grating, are all normal spectra and to the same scale. This engine measures wavelengths direct, so that no multiplication is necessary, but only a slight correction to get figures correct to $\frac{1}{100}$ of a division of Angström.

3d. A table of standard wave-lengths of the impurities in the carbons, extending to wave-length 2000 , has been constructed to measure wave-lengths beyond the limits of the solar spectrum.

4th. Maps of the spectra of some of the elements have been drawn on a large scale ready for publication.

5th. The greater part of the lines in the map of the solar spectrum have been identified and the substance producing them noted.

6 th. The following rough table of the solar elements has been constructed entirely according to my own observations, although, of course, most of them have been given by others. 
I do not know which are the new ones, but call attention to Silicon, Vanadium, Scandium, Yttrium, Zirconium, Glucinum, Germanium and Erbium, as being possibly new.

Silicon has lines on my map at wave-lengths $3905 \cdot 7,4103 \cdot 1,5708 \cdot \%$, 5772.3 and $5948 . \%$. That at $3905 . \%$ is the largest and most certain. That at $4103 \cdot 1$ is also claimed by Manganese.

ELEMENTS IN THE SUN, ARRANGED ACCORDING TO THE INTENSITY AND THE NUMBER OF LINES IN THE SOLAR SPECTRUM. ACCORDING TO INTENSITY.

Calcium.

Iron.

Hydrogen.

Sodium.

Nickel.

Magnesium.

Cobalt.

Silicon.

Aluminium.

Titanium.

Chromium.

Manganese.

Strontium.

Vanadium.

Barium.

Carbon.

Scandium.

Yttrium.

Zirconium.

Molybdenum.

Lanthanum.

Niobium.

Palladium.

Neodymium.

Copper.

Zinc.

Cadmium.

Cerium.

Glucinum.

Germanium.
ACCORDING TO NUMBER.

Iron (2000 or more).

Nickel.

Titanium.

Manganese.

Chromium.

Cobalt.

Carbon (200 or more).

Vanadium.

Zirconium.

Cerium.

Calcium (75 or more).

Scandium.

Neodymium.

Lanthanum.

Yttrium.

Niobium.

Molybdenum.

Palladium.

Magnesium (20 or more). .

Sodium (11).

Silicon.

Strontium.

Barium.

Aluminium (4).

Cadmium.

Rhodium.

Erbium.

Zinc.

Copper (2).

Silver (2). 
ACCORDING TO INTENSITY.

Rhodium.

Silver.

Tin.

Lead.

Erbium.

Potassium.
ACCORDING TO NUMBER.

Glucinum (2).

Germanium.

Tin.

Lead (1).

Potassium (1).

\section{Hefium}

DOUBTFUL ELEMENTS.

Iridium.

Osmium.

Platinum.

Ruthenium.

Tantalum.

Thorium.

NOT IV SOLAR SPECTRUM.

Antimony.

Arsenic.

Bismuth.

Boron.

Nitrogen (vacuum tube).
Caesium.

Gold.

Indium.

Mercury.

Phosphorus.
Tungsten.

Uranium.

\section{SUBSTANCES NOT YET TRIED.}

Bromine.

Chlorine.

Iodine.

Fluorine.
Oxygen.

Tellurium.

Gallium.
Rubidium.

Selenium.

Sulphur.

Thallium.

Praeseodymium.

These tables are to be accepted as preliminary only, especially the order in the first portion. However, being made with such a powerful instrument, and with such care in the determination of impurities, they must still have a weight superior to most others published.

The substances under the head of "Not in Solar Spectrum" are often placed there because the elements have few strong lines or none at all in the limit of the solar spectrum when the arc spectrum, which I have used, is employed. Thus boron has only two strong lines at $249 \%$. Again, the lines of bismuth are all compound and so too diffuse to appear in the solar spectrum. Indeed, some good reason generally appears for their absence from the solar spectrum. Of course, this is little evidence of their absence from the sun itself.

Indeed, were the whole earth heated to the temperature of the sun, its spectrum would probably resemble that of the sun very closely. 
With the high dispersion here used the "basic lines" of Lockyer are widely broken up and cease to exist. Indeed, it would be difficult to prove anything except accidental coincidences among the lines of the different elements. Accurate investigation generally reveals some slight difference of wave-length or a common impurity.

Furthermore, the strength of the lines in the solar spectrum is generally very nearly the same as that in the electric arc, with only a few exceptions, as for instance calcium. The cases mentioned by Lockyer are generally those where he mistakes groups of lines for single lines or even mistakes the character of the line entirely. Altogether there seems to be very little evidence of the breaking up of the elements in the sun as far as my experiments go.

Even after comparing the solar spectrum with all known elements, there are still many important lines not accounted for. Some of these I have accounted for by silicon and there are probably many more. Of all known substances this is the most difficult to bring out the lines in the visible spectrum although it has a fine ultra-violet one. Possibly iron may account for many more, and all the elements at a higher temperature might develope more. Then, again, very rare elements like scandium, vanadium, etc., when they have a strong spectrum, may cause strong solar lines and thus we may look for new and even rare elements to account for very many more. Indeed, I find many lines accounted for by the rare elements in gadolinite, samarskite and fergusonite other than yttrium, erbium, scandium, praeseodymium, neodymium, lanthanum and cerium, which I cannot identify yet and which may be without a name. For this reason, and to discover rare elements, I intend finally to try unknown minerals, as my process gives me an easy method of detecting any new substance or analyzing minerals however many elements they may contain.

The research is much indebted to the faithful and careful work of Mr. L. E. Jewell who has acted as my assistant for several years. Preliminary publications of results will be made in the 'University Circulars.'

Among the lastest results I may mention the spectroscopic separation of yttrium into three components, and the actual separation into two. 


\section{GRATINGS IN THEORY AND PRACTICE ${ }^{2}$}

[Philosophical Magazine [5], XXXV, 397-419, 1893; Astronomy and Astro-Physics, $X I I, 129-149,1893]$

\section{PART I ${ }^{2}$}

It is not my object to treat the theory of diffraction in general but only to apply the simplest ordinary theory to gratings made by ruling grooves with a diamond on glass or metal. This study I at first made with a view of guiding me in the construction of the dividing engine for the manufacture of gratings, and I have given the present theory for years in my lectures. As the subject is not generally understood in all its bearings I have written it for publication.

Let $p$ be the virtual distance reduced to vacuo through which a ray moves. Then the effect at any point will be found by the summation of the quantity

$$
A \cos b(p-\nabla t)+B \sin b(p-\nabla t),
$$

in which $b=\frac{2 \pi}{l}, l$ being the wave-length. $V$ is the velocity reduced to vacuo, and $t$ is the time. Making $\theta=\tan ^{-1} A$-we can write this

$$
\sqrt{A^{2}+B^{2}} \sin [\theta+b(p-V t)] \text {. }
$$

The energy or intensity is proportional to $\left(A^{2}+B^{2}\right)$.

Taking the expression

$$
(A+i B) e^{-i b\langle p-\nabla t)}
$$

when $i=\sqrt{-1}$, its real part will be the previous expression for the displacement. Should we use the exponential expression instead of the circular function in our summation we see that we can always obtain

1 I am much indebted to Dr. Ames for looking over the proofs of this paper and correcting some errors. In the paper I have, in order to make it complete, given some resnlts obtained previously by others, especially by Lord Raylelgb. The treatment is, however, new, as well as many of the results. My object was originally to obtain some gnide to the effect of errors in gratings so that in constructing my dividing engine I might prevent their appearance if possible.

2 [Part II was never written.] 
the intensity of the light by multiplying the final result by itself with $-i$ in place of $+i$, because we have

$$
(A+i B) e^{-i b(p-v t)} \times(A-i B) e^{i b(p-v t)}=A^{2}+B^{2},
$$

In cases where a ray of light falls on a surface where it is broken up, it is not necessary to take account of the change of phase at the surface but only to sum up the displacement as given above.

In all our problems let the grating be rather small compared with the distance of the screen receiving the light so that the displacements need not be divided into their components before summation.

Let the point $x^{\prime}, y^{\prime}, z^{\prime}$ be the source of light, and at the point $x, y, z$ let it be broken up and at the same time pass from a medium of index of refraction $I^{\prime}$ to one of $I$. Consider the disturbance at a point $x^{\prime \prime}, y^{\prime \prime}$, $z^{\prime \prime}$ in the new medium. It will be

where

$$
e^{-i b\left(I p+I^{p} p-\nabla t\right)},
$$

$$
\begin{aligned}
& \rho^{2}=x^{\prime 2}+y^{\prime \prime 2}+z^{\prime \prime 2}+x^{2}+y^{2}+z^{2}-2\left(x x^{\prime \prime}+y y^{\prime \prime}+z z^{\prime \prime}\right), \\
& p^{2}=x^{\prime 2}+y^{\prime 2}+z^{\prime 2}+x^{2}+y^{2}+z^{2}-2\left(x x^{\prime}+y y^{\prime}+z z^{\prime}\right) .
\end{aligned}
$$

Let the point $x, y, z$ be near the origin of co-ordinates as compared with $x^{\prime}, y^{\prime}, z^{\prime}$ or $x^{\prime \prime}, y^{\prime \prime}, z^{\prime \prime}$ and let $\alpha, \beta, \gamma$ and $\alpha^{\prime}, \beta^{\prime}, \gamma^{\prime}$ be the direction cosines of $\rho$ and $p$. Then, writing

$$
\begin{aligned}
& R=I^{\prime} \sqrt{x^{\prime 2}+y^{\prime 2}+z^{\prime 2}}+I \sqrt{x^{\prime 2}+y^{\prime / 2}+z^{1 / 8}}, \\
& \lambda=I \alpha+I^{\prime} \alpha^{\prime}, \\
& \mu=I \beta+I^{\prime} \beta^{\prime}, \\
& \nu=I \gamma+I^{\prime} \gamma^{\prime},
\end{aligned}
$$

we have, for the elementary displacement,

where

$$
e^{-i b\left[R-V t-\lambda x-\mu y-\nu z+\kappa r^{2}\right]},
$$

and

$$
\kappa=\frac{1}{2}\left[\frac{I^{\prime}}{\sqrt{x^{\prime 2}+y^{\prime 2}+z^{\prime 2}}}+\frac{I}{\sqrt{x^{1 / 2}+y^{1 / 2}+z^{1 / 2}}}\right]
$$

$$
r^{2}=x^{2}+y^{2}+z^{2} \text {. }
$$

This equation applies to light in any direction. In the special case of parallel light, for which $\kappa=0$, falling on a plane grating with lines in the direction of $z$, one condition will be that this expression must be the same for all values of $z$.

Hence

$$
\nu=0 \text {. }
$$

If $N$ is the order of the spectrum and $a$ the grating space we shall see further on that we also have the condition

$$
b a \mu=2 \pi N=\frac{2 \pi a}{l} \mu \text {. }
$$


The direction of the diffracted light will then be defined by the equations

$$
\begin{aligned}
\alpha^{\prime 2}+\beta^{\prime 2}+\gamma^{\prime 2} & =0, \\
l \gamma+I^{\prime} \gamma^{\prime} & =0, \\
I \beta+I^{\prime} \beta^{\prime} & =\frac{l}{a} N,
\end{aligned}
$$

whence,

$$
\begin{aligned}
& I^{\prime} \alpha^{\prime}=I \sqrt{\alpha^{2}+2 \frac{l}{I a} N \beta-\frac{l^{2} N^{2}}{I^{2} a^{2}}}, \\
& I^{\prime} \beta^{\prime}=\frac{l}{a} N-I \beta, \\
& I^{\prime} \gamma^{\prime}=-I \gamma .
\end{aligned}
$$

In the ordinary case where the incident and diffracted rays are perpendicular to the lines of the grating, we can simplify the equations somewhat.

Let $\varphi$ be the angle of incidence and $\psi$ of diffraction as measured from the positive direction of $X$.

$$
\begin{aligned}
& \lambda=I^{\prime} \cos \varphi+I \cos \psi, \\
& \frac{l}{a} N=\mu=I^{\prime} \sin \varphi+I \sin \psi, \\
& b=\frac{2 \pi}{l},
\end{aligned}
$$

where $l$ is the wave-length in vacuo.

In case of the reflecting grating $I=I^{\prime}$ and we can write

$$
\begin{gathered}
\lambda=I\{\cos \varphi+\cos \psi\}, \\
\frac{l}{a} N=\mu=I\{\sin \varphi+\sin \psi\} .
\end{gathered}
$$

This is only a very elementary expression as the real value would depend on the nature of the obstacle, the angles, etc., but it will be sufficient for our purpose.

The disturbance due to any grating or similar body will then be very nearly

$$
\iint e^{-i b\left[R-V t-\lambda x-\mu y-\nu z+\kappa\left(x^{2}+y^{2}+z^{2}\right)\right]} d s,
$$

where $d s$ is a differential of the surface. For parallel rays, $\kappa=0$.

\section{Plane Gratings}

In this case the integration can often be neglected in the direction of $z$ and we can write for the disturbance in case of parallel rays,

$$
e^{-i b(R-V t)} \iint e^{-i b[-\lambda x-\mu y]} d s .
$$




\section{Case I.-Simple Periodic Ruling}

Let the surface be divided up into equal parts in each of which one or more lines or grooves are ruled parallel to the axis of $z$.

The integration over the surface will then resolve itself into an integration over one space and a summation with respect to the number of spaces. For in this case we can replace $y$ by $n a+y$ where $a$ is the width of a space and the displacement becomes

but

$$
e^{-i b(R-V t) \Sigma_{e}+i b \mu a n} \iint e^{+i b(\lambda x+\mu y)} d s,
$$

$$
\sum_{0}^{n-1} e+i b \mu a n=e^{+i \frac{n-1}{2} b a \mu} \frac{\sin n \frac{b \mu_{\mu}}{2}}{\sin \frac{b a a^{\mu}}{2}} .
$$

Multiplying the disturbance by itself with $-i$ in place of $+i$ we have for the light intensity

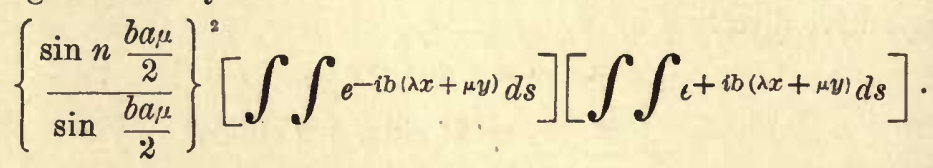

The first term indicates spectral lines in positions given by the equation

$$
\sin \frac{b a \mu}{2}=0
$$

with intensities given by the last integral. The intensity of the spectral lines then depends on the form of the groove as given by the equation $x=f(y)$ and upon the angles of incidence and diffraction. The first factor has been often discussed and it is only necessary to call attention to a few of its properties.

When $b a \mu=2 \pi N, N$ being any whole number, the expression becomes $n^{2}$. On either side of this value the intensity decreases until $n b a \mu^{\prime}=2 \pi N$, when'it becomes 0 .

The spectral line then has a width represented by $\mu^{\prime}-\mu^{\prime \prime}=2 \frac{\mu}{n}$ nearly; on either side of this line smaller maxima exist too faintly to be observed. When two spectral lines are nearer together than half their width, they blend and form one line. The defining power of the spectroscope can be expressed in terms of the quotient of the wave-length by the difference of wave-length of two lines that can just be seen as divided. The defining power is, then,

$$
{ }^{3} n N=n n \frac{\mu}{l} \text {. }
$$

${ }^{3}$ An expression of Lord Rayleigh's. 
Now $n a$ is the width of the grating. Hence, using a grating at a given angle, the defining power is independent of the number of lines to the inch and only depends on the width of the grating and the wavelength. According to this, the only object of ruling many lines to the inch in a grating is to separate the spectra so that, with a given angle, the order of spectrum shall be less.

Practically the gratings with few lines to the inch are much better than those with many, and hence have better definition at a given angle than the latter except that the spectra are more mixed up and more difficult to see.

It is also to be observed that the defining power increases with shorter wave-lengths, so that it is three times as great in the ultra violet as in the red of the spectrum. This is of course the same with all optical instruments such as telescopes and microscopes.

The second term which determines the strength of the spectral lines will, however, give us much that is new.

First let us study the effect of the shape of the groove on the brightness. If $N$ is the order of the spectrum and $a$ the grating space we have

since

$$
\begin{gathered}
\mu=I\left(\sin \varphi+\sin \psi^{\prime}\right)=\frac{N l}{a} \\
\sin \frac{b a \mu}{2}=0
\end{gathered}
$$

and the intensity of the light becomes proportional to

$$
\left[\iint e^{i 2 \pi}\left(\frac{\lambda}{l} x+\frac{N}{a} y\right) d s\right]\left[\iint e^{-i 2 \pi}\left(\frac{\lambda}{l} x+\frac{N}{a} y\right) d s\right] .
$$

It is to be noted that this expression is not only a function of $N$ but also of $l$, the wave-length. This shows that the intensity in general may vary throughout the spectrum according to the wave-length and that the sum of the light in any one spectrum is not always white light.

This is a peculiarity often noticed in gratings. Thus one spectrum may be almost wanting in the green, while another may contain an excess of this color; again there may be very little blue in one spectrum while very often the similar spectrum on the other side may have its own share and that of the other one also. For this reason I have found it almost impossible to predict what the ultra red spectrum may be, for it is often weak even where the visible spectrum is strong.

The integral may have almost any form although it will naturally tend to be such as to make the lower orders the brightest when the diamond rules a single and simple groove. When it rules several lines 
or a compound groove, the higher orders may exceed the lower in brightness and it is mathematically possible to have the grooves of such a shape that, for given angles, all the light may be thrown into one spectrum.

It is not uncommon, indeed, very easy, to rule gratings with immensely bright first spectra, and I have one grating where it seems as if half the light were in the first spectrum on one side. In this case there is no reflection of any account from the grating held perpendicularly: indeed to see one's face, the plate must be held at an angle, in which case the various features of the face are seen reflected almost as brightly as in a mirror but drawn out into spectra. In this case all the other spectra and the central image itself are very weak.

In general it would be easy to prove from the equation that want of symmetry in the grooves produces want of symmetry in the spectra, a fact universally observed in all gratings and one which I generally utilize so that the light may be concentrated in a few spectra only.

\section{Example 1.-Square Grooves}

When the light falls nearly perpendicularly on the plate, we need not take the sides into account but only sum up the surface of the plate and the bottom of the groove. Let the depth be $X$ and the width equal to $\frac{a}{m}$.

The intensity then becomes proportional to

$$
\frac{1}{N^{2}} \sin ^{2} \pi \frac{N}{m} \sin ^{2} \pi \frac{\lambda}{l} X
$$

This vanishes when

or

$$
\begin{gathered}
N=m, 2 m, 3 m, \text { etc. } \\
\frac{\lambda X}{l}=0,1,2,3, \text { etc. }
\end{gathered}
$$

The intensity of the central light, for which $N=0$, will be

$$
\frac{\pi^{2}}{m^{2}} \sin ^{2}\left(\pi \frac{\lambda}{l} X\right) \text {. }
$$

This can be made to vanish for only one angle for a given wavelength. Therefore, the central image will be colored and the color will change with the angle, an effect often observed in actual gratings. The color ought to change, also, on placing the grating in a liquid of different index of refraction since $\lambda$ contains $I$, the index of refraction.

It will be instructive to take a special case, such as light falling perpendicularly on the plate. For this case 


$$
\varphi=0, \lambda=I\left(1+\cos \psi^{\prime}\right) \text { and } \mu=I \sin \psi=\frac{N l}{a} .
$$

Hence $\lambda=I\left\{1+\sqrt{1-\left(\frac{N l}{a I}\right)^{2}}\right\}$.

The last term in the intensity will then be

$$
\sin ^{2}\left\{\pi X I\left[\frac{1}{l}+\sqrt{\frac{1}{l^{2}}}-\left(\frac{N}{a I}\right)^{2}\right]\right\}
$$

As an example, let the green of the second order vanish. In this case, $l=.00005$. $\quad N=2$. Let $a=.0002 \mathrm{~cm}$. and $I=1$.

Then,

Whence,

$$
\begin{gathered}
X\left[20000+\sqrt{(20000)^{2}-(10000)^{2}}\right]=n . \\
X=\frac{n}{37300 .},
\end{gathered}
$$

where $n$ is any whole number. Make it 1 .

Then the intensity, as far as this term is concerned, will be as follows:

\begin{tabular}{lcr} 
& \multicolumn{2}{c}{$\begin{array}{c}\text { Minima where Intensity is } 0 . \\
\text { Wave-lengths. }\end{array}$} \\
1st spec. & .0000526 & .0000268 \\
2nd " & .0000500 & .0000266 \\
3rd " & .0000462 & .0000263 \\
4tb " & .0000416 & .0000259 \\
5th " & etc. &
\end{tabular}

\begin{tabular}{ccc}
\multicolumn{4}{c}{ Maxima where Intensity is 1.} \\
.0001000 & .00003544 & .00002137 \\
.0000833 & .00003463 & .00002119 \\
.0000651 & .00003333 & .00002089 \\
.0000499 & .00003169 & .00002050 \\
etc. & etc. &
\end{tabular}

The central light will contain the following wave-lengths as a maximum:

$$
.0001072 \quad .00003575 \quad .0000214 \text {, etc. }
$$

Of course it would be impossible to find a diamond to rule a rectangular groove as above and the calculations can only be looked upon as a specimen of innumerable light distributions according to the shape of groove.

Every change in position of the diamond gives a different light distribution and hundreds of changes may be made every day and yet the same distribution will never return, although one may try for years.

\section{Example 2.-Triangular Groove}

Let the space $a$ be cut into a triangular groove, the equations of the sides being $x=-c y$, and $x=c^{\prime}(y-a)$, the two cuttings coming together at the point $y=u$. Hence we have $-c u=c^{\prime}(u-a)$, and $d s=d y \sqrt{1+c^{2}}$ or $d y \sqrt{1+c^{\prime 2}}$. Hence the intensity is proportional to 


$$
\begin{aligned}
l^{2}\left\{\frac{1+c^{2}}{(\mu-c \lambda)^{2}} \sin ^{2}\right. & \frac{\pi(\mu-c \lambda)}{l}+\frac{1+c^{\prime 2}}{\left(\mu+c^{\prime} \lambda\right)^{2}} \sin ^{2} \frac{\pi(a-u)\left(\mu+c^{\prime} \lambda\right)}{l} \\
+ & \frac{\sqrt{\left(1+c^{2}\right)\left(1+c^{\prime 2}\right)}}{(\mu-c y)\left(\mu+c^{\prime} \lambda\right)} \sin \frac{\pi u(\mu-c \lambda)}{l} \sin \frac{\pi(a-u)\left(\mu+c^{\prime} \lambda\right.}{l} \\
& \left.\cos \frac{\pi}{l}\left[\left(\mu+c^{\prime} \lambda\right)(a-u)-n(\mu-c \lambda)\right]\right\} .
\end{aligned}
$$

This expression is not symmetrical with respect to the normal to the grating, unless the groove is symmetrical, in which case $c=c^{\prime}$ and $u=\frac{a}{2}$.

In this case, as in the other, the colors of the spectrum are of variable intensity, and some of them may vanish as in the first example, but the distribution of intensity is in other respects quite different.

\section{Case II.-Multiple Periodic Ruling}

Instead of having only one groove ruled on the plate in this space $a$, let us now suppose that a series of similar lines are ruled.

We have, then, to obtain the displacement by the same expression as before, that is

$$
\frac{\sin n \frac{b a \mu}{2}}{\sin \frac{b a a^{\mu}}{2}} \iint e^{i b(\lambda x+\mu y)} d s
$$

except that the last integral will extend over the whole number of lines ruled within the space $a$.

In the spaces $a$ let a number of equal grooves be ruled commencing at the points $y=0, y_{1}, y_{2}, y_{3}$, etc., and extending to the points $w, y_{1}+w$, $y_{2}+w$, etc. The surface integral will then be divided into portions from $w$ to $y_{1}$, from $y_{1}+w$, to $y_{2}$, etc., on the original surface of the plate for which $x=0$, and from $w$ to 0 , from $y_{1}+w$ to $y_{1}$, etc., for the grooves.

The first series of integrals will be

$$
\begin{aligned}
\int e^{i b \mu y} d y= & \frac{1}{i b \mu}\left\{-e^{i b \mu w}+t^{i b \mu y_{1}}-t^{i b \mu\left(y_{1}+w\right)}+e^{i b \mu y_{2}}-\text { etc. }\right\} \\
& =\frac{1}{i b \mu}\left\{-e^{i b \mu w}+\left(1-e^{i b \mu w}\right)\left(e^{i b \mu y_{1}}+e^{i b \mu y_{2}}+\text { etc. }\right)+e^{i b \mu a}\right\}
\end{aligned}
$$

But, $e^{i b \mu a}=1$ since $b \mu a=2 \pi N$ for any maximum, and thus the integral becomes

$$
\frac{1-e^{i b \mu w}}{i b_{\mu} \mu}\left\{1+e^{i b \mu y_{1}}+e^{i b \mu y_{2}}+\text { etc. }\right\}
$$


The second series of integrals will be

$$
\int_{0}^{w} e^{i b(\lambda x+\mu y)} d s\left\{1+e^{i b \mu y_{1}}+\text { etc. }\right\}
$$

The total integral will then be

$$
\frac{\sin n \frac{b a \mu}{2}}{\sin \frac{b a_{\mu}}{2}}\left[\frac{1-e^{i b \mu w}}{i b \mu}+\int_{0}^{w} e^{i b(\lambda x+\mu y)} d s\right]\left[1+e^{i b \mu y_{1}}+e^{i b \mu y_{2}}+\text { etc. }\right] .
$$

As before, multiply this by the same with the sign of $i$ changed to get the intensity.

\section{Example 1.-Equal Distances}

The space, $a$, contains $n^{\prime}-1$ equidistant grooves, so that $y_{1}=y_{2}-y_{1}$ $=$ etc., $=\frac{a}{n^{\prime}}$

metals with some one metal, such as iron. Making the iron spectrum

Hence the displacement becomes

$$
\sum_{0}^{n^{\prime}-1} e^{i b \mu \frac{a}{n^{\prime}} n}=e^{i b a \mu} \frac{\sin \frac{b a \mu}{2}}{\sin \frac{b a \mu}{2 n^{\prime}}} .
$$

$$
\frac{\sin n \frac{b a_{\mu}}{2}}{\sin \frac{b a \mu}{2 n^{\prime}}}\left[\frac{1-e^{i b \mu w}}{i b^{\prime}}+\int_{0}^{w} e^{i b(\lambda x+\mu y)} d s\right] .
$$

As the last term is simply the integral over the space $\frac{a}{n^{\prime}}$ in a different form from before, this is a return to the form we previously had except that it is for a grating of $n n^{\prime}$ lines instead of $n$ lines, the grating space being $\frac{a}{n^{\prime}}$.

$$
\begin{aligned}
& \text { Example 2.-Two Grooves } \\
& 1+e^{i b \mu y_{1}}=2 e^{\frac{i b \mu y_{1}}{2}} \cos \frac{b \mu y_{1}}{2} .
\end{aligned}
$$

But $b a \mu=2 N \pi$. Hence this becomes

$$
2 e^{i \pi N} \frac{y_{1}}{2} \cos \pi N \frac{y_{1}}{a} .
$$

The square of the last term is a factor in the intensity. Hence the spectrum will vanish when we have

$$
N \frac{y_{1}}{a}=\frac{1}{2}, \frac{3}{2}, \frac{5}{2}, \text { etc. }^{4}
$$

A theorem of Lord Rayleigh's. 
or

$$
N=\frac{1}{2} \frac{a}{y_{1}}, \frac{3}{2} \frac{a}{y_{1}}, \frac{5}{2} \frac{a}{y_{1}}, \text { etc. }
$$

Thus when $\frac{a}{y_{1}}=2$, the 1st, $3 \mathrm{~d}$, etc., spectra will disappear, making a grating of twice the number of lines to the $\mathrm{cm}$.

When $\frac{a}{y_{1}}=4$, the $2 \mathrm{~d}, 6 \mathrm{th}, 10 \mathrm{th}$, etc., spectra disappear. When $\frac{a}{y_{1}}=6$, the $3 \mathrm{~d}, 9$ th, etc., spectra disappear.

The case in which $\frac{a}{y_{1}}=4$, as Lord Rayleigh has shown, would be very useful as the second spectrum disappears leaving the red of the first and the ultra violet of the third without contamination by the second. In this case two lines are ruled and two left out. This would be easy to do but the advantages would hardly pay for the trouble owing to the following reasons: Suppose the machine was ruling 20,000 lines to the inch. Leaving out two lines and ruling two would reduce the dispersion down to a grating with 5000 lines to the inch. Again, the above theory assumes that the grooves do not overlap. Now I believe that in nearly, if not all, gratings with 20,000 lines to the inch the whole surface is cut away and the grooves overlap. This would cause the second spectrum to appear again after all our trouble.

Let the grooves be nearly equidistant, one being slightly displaced. In this case $y_{1}=\frac{a}{2}+v$.

$$
\cos ^{2} \pi \frac{N y_{1}}{a}=\left(\cos \frac{\pi N}{2} \cos \frac{\pi N v}{a}-\sin \frac{\pi N}{2} \sin \frac{\pi N v}{a}\right)^{2}
$$

For the even spectra this is very nearly unity, but for the odd it becomes

$$
\left(\pi N \frac{v}{a}\right)^{2}
$$

Hence the grating has its principal spectra like a grating of space $\frac{a}{2}$ but there are still the intermediate spectra due to the space $a$, and of intensities depending on the squares of the order of spectrum, and the squares of the relative displacement, a law which I shall show applies to the effect of all errors of the ruling.

This particular effect was brought to my attention by trying to use a tangent screw on the head of my dividing engine to rule a grating with say 28,872 lines to the inch, when a single tooth gave only 14,436 to the inch. However carefully I ground the tangent screw I never was 
able to entirely eliminate the intermediate spectra due to 14,436 lines, and make a pure spectrum due to 28,872 lines to the inch, although I could nearly succeed.

\section{Example 3.-One Groove in $m$ Misplaced}

Let the space $a$ contain $m$ grooves equidistant except one which is displaced a distance $v$. The displacement is now proportional to $1+e^{i b \mu \frac{a}{m}}+e^{2 i b \mu \frac{a}{m}}+$ etc. $+e^{i b \mu\left(p \frac{a}{m}+v\right)}+$ etc. $+e^{i b \mu \frac{m-1}{m} a}$

$$
=e^{i b \mu \frac{m-1}{2 m} a}\left\{\frac{\sin \frac{b \mu a}{2}}{\sin \frac{b \mu a}{2 m}}+i b \mu v e^{i b \mu a} \frac{2 p-m+1}{2 m}\right\}
$$

Multiplying this by itself with $-i$ in place of $+i$, and adding the factors in the intensity, we have the whole expression for the intensity. One of the terms. entering the expression will be

$$
\frac{\sin n \frac{b a_{\mu}}{2}}{\sin \frac{b a_{\mu}}{2 m}} \frac{\sin n \frac{b a_{\mu}}{2}}{\sin \frac{b a_{\mu}}{2}} \sin \frac{b a_{\mu}}{2} \frac{2 p-m+1}{m} .
$$

Now the first two terms have finite values only around the points $\frac{b a \mu}{2}=m N \pi$, where $m N$ is a whole number. But $2 p-m+1$ is also a whole number, and hence the last term is zero at these points. Hence the term vanishes and leaves the intensity, omitting the groove factor,

$$
\frac{\sin ^{2} n \frac{b a \mu}{2}}{\sin \frac{b a \mu}{2 m}}+(b \mu v)^{2} \frac{\sin ^{2} n \frac{b a \mu}{2}}{\sin ^{2} \frac{b u \mu}{2}} .
$$

The first term gives the principal spectra as due to a grating space of $\frac{a}{m}$ and number of lines $n m$ as if the grating were perfect. The last term gives entirely new spectra due to the grating space, $a$, and with lines of breadth due to a grating of $n$ lines and intensities equal to $(b \mu v)^{2}$.

Hence, when the tangent screw is used on my machine for 14,436 lines to the inch, there will still be present weak spectra due to the 14,436 spacing although I should rule say 400 lines to the mm. This I have practically observed also.

The same law holds as before that the relative intensity in these 
subsidiary spectra varies as the square of the order of the spectrum and the square of the deviation of the line, or lines from their true position.

So sensitive is a ditiding engine to periodic disturbances that all the belts driving the machine must never revolve in periods containing an aliquot number of lines of the grating; otherwise they are sure to make spectra due to their period.

As a particular case of this section we have also to consider

\section{Periodic Errors of Ruling.-Theory of "Ghosts"}

In all dividing engines the errors are apt to be periodic due to "drunken" screws, eccentric heads, imperfect bearings, or other causes. We can then write

$$
y=a_{0} n+a_{1} \sin \left(e_{1} n\right)+a_{2} \sin \left(e_{2} n\right),+ \text { etc. }
$$

The quantities $e_{1}, e_{2}$, etc., give the periods, and $a_{1}, a_{2}$, etc., the amplitudes of the errors. We can then divide the integral into two parts as before, an integral over the groove and spaces and a summation with respect to the numbers.

$$
\Sigma \int_{y^{\prime}}^{y^{\prime \prime}} e^{-i b(\lambda x+\mu y)} d s=\Sigma e^{-i b \mu y^{\prime}} \int_{0}^{y^{\prime \prime}-y^{\prime}}{ }^{-i b\left(\lambda x+\mu_{y}\right) d s .}
$$

It is possible to perform these operations exactly, but it is less complicated to make an approximation, and take $y^{\prime \prime}-y^{\prime}=a$, a constant as it is very nearly in all gratings. Indeed the error introduced is vanishingly small. The integral which depends on the shape of the groove, will then go outside the summation sign and we have to perform the summation

$$
\Sigma e^{-i b \mu}\left\{a_{0} n+a_{1} \sin e_{1} n+a_{2} \sin e_{2} n+\text { etc. }\right\} .
$$

Let $J_{n}$ be a Bessel's function. Then

$$
\begin{aligned}
& \cos (u \sin \varphi)=J_{0}(u)+2\left[J_{2}(u) \cos ^{2} \varphi+J_{4}(u) \cos ^{4} \varphi+\text { etc. }\right] \\
& \sin (u \sin \varphi)=\quad 2\left[J_{1}(u) \sin \varphi+J_{3}(u) \sin ^{3} \varphi+\text { etc. }\right]
\end{aligned}
$$

But $e^{-i u \sin \varphi}=\cos (u \sin \varphi)-i \sin (u \sin \varphi)$.

Hence the summation becomes

$$
\Sigma\left\{\begin{aligned}
& e^{-i b \mu a_{0} n} \\
& \times\left[J_{0}\left(b_{\mu} a_{1}\right)+2\left(-i J_{1}\left(b_{\mu a_{1}}\right) \sin e_{1} n+J_{2}\left(b_{\mu a_{1}}\right) \cos 2 e_{1} n-\text { etc. }\right)\right] \\
& \times\left[J_{0}\left(b_{\mu} a_{2}\right)+2\left(-i J_{1}\left(b_{\mu a_{2}}\right) \sin e_{2} n+J_{2}\left(b \mu a_{2}\right) \cos 2 e_{1} n-\text { etc. }\right)\right] \\
& \times\left[J_{0}\left(b_{\mu} \mu a_{3}\right)+\text { etc. }\right] \\
& \times[\text { etc. }]
\end{aligned}\right.
$$




\section{Case I.-Single Periodic Error}

In this case only $a_{0}$ and $a_{1}$ exist. We have the formula

$$
\Sigma_{0}^{n-1} e^{-i p n}=e^{-i \frac{n-1}{2} p} \frac{\sin \frac{p n}{2}}{\sin \frac{p}{2}} .
$$

Hence the expression for the intensity becomes

$$
\begin{aligned}
\left\{J_{0}\left(b_{\mu} a_{1}\right) \frac{\sin n \frac{b_{\mu} a_{0}}{2}}{\sin \frac{b \mu a_{0}}{2}}\right\}^{2}+J_{1}^{2}\left(b_{\left.\mu a_{1}\right)}\{\right. & \left\{\frac{\left.\sin n \frac{b_{\mu a_{0}+e_{1}}}{2}\right\}^{2}}{\left.\sin \frac{b_{\mu a_{0}+e_{1}}}{2}\right\}^{2}}\right. \\
& \left.+\left\{\frac{\sin n \frac{b \mu a_{0}-e_{1}}{2}}{\sin \frac{b_{\mu-a_{0}-\rho_{1}}}{2}}\right\}^{2}\right\}+ \text { etc. }
\end{aligned}
$$

As $n$ is large, this represents various very narrow spectral lines whose light does not overlap and thus the different terms are independent of each other. Indeed in obtaining this expression the products of quantities have been neglected for this reason because one or the other is zero at all points. These lines are all alike in relative distribution of light and their intensities and positions are given by the following table:

$$
\begin{array}{ccc}
\begin{array}{c}
\text { Places. } \\
\mu=\frac{2 \pi N}{b a_{0}}
\end{array} & \begin{array}{c}
\text { Intensities. } \\
J_{0}^{2}\left(b \mu a_{1}\right)
\end{array} & \begin{array}{c}
\text { Designations. } \\
\text { Primary line }
\end{array} \\
\mu_{1}=\mu \pm \frac{e_{1}}{b a_{0}} & J_{1}^{2}\left(b \mu_{1} a_{1}\right) & \text { Ghosts of } 1 \text { st order. } \\
\mu_{2}=\mu \pm \frac{2 e_{1}}{b a_{0}} & J_{2}^{2}\left(b \mu_{2} a_{1}\right) & \text { Ghosts of } 2 \mathrm{~d} \text { order. } \\
\mu_{3}=\mu \pm \frac{3 e_{1}}{b a_{0}} & J_{3}^{2}\left(b \mu_{3} a_{1}\right) & \text { Ghosts of } 3 \mathrm{~d} \text { order. } \\
\text { etc. } & \text { etc. } & \text { etc. }
\end{array}
$$

Hence the light which would have gone into the primary line now goes to making the ghosts, so that the total light in the line and its ghosts is the same as in the original without ghosts.

The relative intensities of the ghosts as compared with the primary line is

$$
\frac{J_{n}^{2}\left(b_{\mu} a_{1}\right)}{J_{0}^{2}\left(b_{\mu-a_{1}}\right)}
$$


This for very weak ghosts of the first, second, third, etc., order, becomes

$$
\left(\pi N \frac{a_{1}}{a_{0}}\right)^{2}, \quad \frac{1}{2}\left(\pi N \frac{a_{1}}{a_{0}}\right)^{4}, \quad \frac{1}{6}\left(\pi N \frac{a_{1}}{a_{0}}\right)^{6} \text {, etc. }
$$

The intensity of the ghosts of the first order varies as the square of the order of the spectrum and as the square of the relative displacement as compared with the grating space $a_{0}$. This is the same law as we before found for other errors of ruling, and it is easy to prove that it is general. Hence

The effect of small errors of ruling is to produce diffused light around the spectral lines. This diffused light is subtracted from the light of the primary line, and its comparative amount varies as the square of the relative error of ruling and the square of the order of the spectrum.

Thus the effect of the periodic error is to diminish the intensity of the ordinary spectral lines (primary lines) from the intensity 1 to $J_{0}^{2}\left(b \mu a_{1}\right)$, and surround it with a symmetrical system of lines called ghosts, whose intensities are given above.

When the ghosts are very near the primary line, as they nearly always are in ordinary gratings ruled on a dividing engine with a large number of teeth in the head of the screw, we shall have

$$
J_{1}^{2} b a_{1}\left(\mu+\frac{e_{1}}{b a_{0}}\right)+J_{1}^{2} b a_{1}\left(\mu-\frac{e_{1}}{b a_{0}}\right)=2 J_{1}^{2} b a_{1} \mu \text { nearly. }
$$

Hence the total light is by a known theorem,

$$
J_{0}^{2}+2\left[J_{1}^{2}+J_{2}^{2}+\text { etc. }\right]=1 \text {. }
$$

Thus, in all gratings, the intensity of the ghosts as well as the diffused light increases rapidly with the order of the spectrum. This is often marked in gratings showing too much crystalline structure. For the ruling brings out the structure and causes local difference of ruling which is equivalent to error of ruling as far as diffused light is concerned.

For these reasons it is best to get defining power by using broad gratings and a low order of spectra although the increased perfection of the smaller gratings makes up for this defect in some respects.

There is seldom advantage in making both the angle of incidence and diffraction more than $45^{\circ}$, but, if the angle of incidence is 0 , the other angle may be $60^{\circ}$, or even $70^{\circ}$, as in concave gratings. Both theory and practice agree in these statements.

Ghosts are particularly objectionable in photographic plates, especi- 
ally when they are exposed very long. In this case ghosts may be brought out which would be scarcely visible to the eye.

As a special case, take the following numerical results:

$$
\begin{aligned}
& \begin{array}{llll}
N= & 1 & 2 & 3
\end{array} \\
& \frac{a_{1}}{a_{0}}=\frac{1}{25}, \frac{1}{50}, \frac{1}{100} \quad \frac{1}{25}, \frac{1}{50}, \frac{1}{100}, \frac{1}{25}, \frac{1}{50}, \frac{1}{100}, \\
& \left(\pi N \frac{a_{1}}{a_{0}}\right)^{2}=\frac{1}{63}, \frac{1}{252}, \frac{1}{1008} \quad \frac{1}{16}, \frac{1}{63}, \frac{1}{252} \frac{1}{7}, \frac{1}{28}, \frac{1}{102} .
\end{aligned}
$$

In a grating with 20,000 lines to the inch, using the third spectrum, we may suppose that the ghosts corresponding to $\frac{a_{1}}{a_{0}}=\frac{1}{50}$ will be visible and those for $\frac{a_{1}}{a_{0}}=\frac{1}{25}$ very troublesome. The first error is $a_{1}=\frac{1}{1000000}$ in. and the second $a_{1}=\frac{1}{500000}$ in. Hence a periodic displacement of one millionth of an inch will produce visible ghosts and one five hundred thousandth of an inch will produce ghosts which are seen in the second spectrum and are troublesome in the third. With very bright spectra these might even be seen in the first spectrum. Indeed an over exposed photographic plate would readily bring them out.

When the error is very great, the primary line may be very faint or disappear altogether, the ghosts to the number of twenty or fifty or more being often more prominent than the original line. Thus, when

$$
b_{\mu a_{1}}=2 \cdot 405,5 \cdot 52,8 \cdot 65, \text { etc. }=2 \pi N \frac{a_{1}}{a_{0}},
$$

the primary line disappears. When

$$
b_{\mu} a_{1}=0,3 \cdot 83,7 \cdot 02, \text { etc. }=2 \pi N \frac{a_{1}}{a_{0}},
$$

the ghosts of the first order will disappear. Indeed we can make any ghost disappear by the proper amount of error.

Of course, in general

$$
J_{n}=\frac{2(n-1)}{v} J_{n-1}-J_{n-2}
$$

Thus a table of ghosts can be formed readily and we may always tell when the calculation is complete by taking the sum of the light and finding unity. 


\begin{tabular}{|c|c|c|c|c|c|c|c|c|c|c|c|c|c|c|c|}
\hline $27: N$ & $\frac{a_{1}}{a_{0}} J_{0}^{2}$ & $J_{1}^{2}$ & $J_{2}^{2}$ & $J_{3}^{2}$ & $J_{4}^{2}$ & $J_{5}^{2}$ & $J_{6}^{2}$ & $J_{7}^{2}$ & $J_{8}^{2}$ & $J_{9}^{2}$ & $J_{10}^{2}$ & $J_{11}^{2}$ & $J_{12}^{2}$ & $J_{13}^{2}$ & $J_{14}^{2}$ \\
\hline 0. & 1.000 & $\ldots$ & .. & & & & $\cdots$ & $\cdots$ & & $\cdots$ & $\cdots$ & & & & \\
\hline$\cdot 2$ & .980 & .010 & ... & ... & .. & ... & .. & .. & $\cdots$ & .. & $\cdots$ & $\cdots$ & ... & $\cdots$ & \\
\hline$\cdot 4$ & .922 & .038 & $\ldots$ & .. & $\ldots$ & ... & .. & .. & .. & $\ldots$ & ... & $\cdots$ & $\cdots$ & $\cdots$ & \\
\hline 6 & .832 & .082 & .002 & .. & ... & ... & .. & ... & .. & .. & $\cdots$ & $\cdots$ & $\cdots$ & $\cdots$ & \\
\hline$\cdot 8$ & .716 & .136 & .005 & ... & ... & $\ldots$ & ... & $\cdots$ & .. & $\cdots$ & .. & .. & $\cdots$ & - & $\cdots$ \\
\hline $1 \cdot 0$ & .586 & $\cdot 194$ & .012 & $\ldots$ & $\ldots$ & $\ldots$ & $\ldots$ & ... & $\cdots$ & $\cdots$ & $\ldots$ & ... & .. & $\cdots$ & \\
\hline $2 \cdot 0$ & .050 & $\cdot 333$ & $\cdot 124$ & .017 & .001 & ... & .. & .. & $\cdots$ & $\cdots$ & .. & $\cdots$ & .. & .. & \\
\hline $2 \cdot 605$ & .000 & $\cdot 269$ & $\cdot 186$ & .040 & .003 & $\ldots$ & .. & .. & .. & .. & ... & . & .. & $\cdots$ & \\
\hline $3 \cdot$ & .068 & $\cdot 115$ & $\cdot 236$ & .095 & .017 & .002 & . & .. & ... & ... & ... & ... & ... & ... & \\
\hline $3 \cdot 832$ & $\cdot 162$ & .000 & $\cdot 162$ & $\cdot 176$ & .065 & .013 & .002 & ... & ... & $\cdots$ & $\ldots$ & ... & .. & ... & \\
\hline $4 \cdot 0$ & $\cdot 158$ & .004 & $\cdot 133$ & $\cdot 185$ & .079 & .018 & .002 & ... & ... & .. & $\cdots$ & . & $\cdots$ & $\cdots$ & \\
\hline $5 \cdot 0$ & .031 & $\cdot 107$ & .002 & $\cdot 133$ & $\cdot 153$ & .068 & .017 & .003 & $\ldots$ & $\ldots$ & $\cdots$ & $\cdots$ & $\cdots$ & $\ldots$ & \\
\hline $5 \cdot 520$ & .000 & $\cdot 116$ & etc. & & & & & & & & & & , & & \\
\hline $6 \cdot 0$ & .022 & .077 & .059 & .013 & $\cdot 128$ & $\cdot 131$ & .061 & .017 & .003 & $\ldots$ & ... & & ... & ... & \\
\hline $7 \cdot 016$ & .090 & .000 & .090 & tc & & & & & & & & & & & \\
\hline 8. & .029 & .055 & .013 & .085 & .011 & .035 & $\cdot 114$ & $\cdot 103$ & .050 & .016 & .003 & .001 & ... & $\ldots$ & \\
\hline $8 \cdot 654$ & .000 & .075 & etc. & & & & & & & & & & & & \\
\hline 0. & .060 & .002 & .065 & .003 & .048 & .055 & $\cdot 002$ & .047 & $\cdot 101$ & .091 & .051 & .022 & .011 & .009 & \\
\hline
\end{tabular}

This table shows how the primary line weakens and the ghosts strengthen as the periodic error increases, becoming 0 at $2 \pi N_{\frac{a^{\prime}}{a}}=2 \cdot 405$. It then strengthens and weakens periodically, the greatest strength being transferred to one of the ghosts of higher and higher order as the error increases.

Thus one may obtain an estimate of the error from the appearance of the ghost.

Some of these wonderful effects with 20 to 50 ghosts stronger than the primary line I have actually observed in a grating ruled on one of my machines before the bearing end of the screw had been smoothed. The effect was very similar to these calculated results.

\section{Double Periodic Error}

Supposing as before that there is no overlapping of the lines, we have the following:

Places.

$$
\begin{aligned}
& \mu=\frac{2 \pi N}{b a_{0}} \\
& \mu_{1}=\mu \pm \frac{e_{1}}{b a_{0}} \\
& \mu_{2}=\mu \pm \frac{e_{2}}{b a_{0}}
\end{aligned}
$$

Intensities.

$\left.\left[J_{0}\left(b a_{1} \mu\right) J_{0}\left(b a_{2} \mu\right)\right]^{2}\right\}$ Primary line.

$\left[J_{1}\left(b a_{1} \mu_{1}\right) J_{0}\left(b a_{2} \mu_{1}\right)\right]^{2}$

Ghosts of 1st order. 
Places.

Intensities.

$$
\begin{aligned}
& \left.\mu_{3}=\mu \pm \frac{e_{1} \pm e_{2}}{b a_{0}}\left[J_{1}\left(b a_{1} \mu_{3}\right) J_{1}\left(b a_{2} \mu_{3}\right)\right]^{2}\right) \\
& \left.\mu_{4}=\mu \pm \frac{2 e_{1}}{b a_{0}} \quad\left[J_{2}\left(b a_{1} \mu_{4}\right) J_{0}\left(b a_{2} \mu_{4}\right)\right]^{2}\right\} \text { Ghosts of } 2 d \text { order. } \\
& \mu_{5}=\mu \pm \frac{2 e_{2}}{b a_{0}} \quad\left[J_{0}\left(b a_{1} \mu_{5}\right) J_{2}\left(b a_{2} \mu_{5}\right)\right]^{2} \\
& \mu_{6}=\mu \pm \frac{e_{1} \pm 2 e_{6}}{b n_{0}}\left[J_{1}\left(b a_{1} \mu_{6}\right) J_{3}\left(b a_{2} \mu_{6}\right)\right]^{2} \\
& \mu_{7}=\mu \pm \frac{2 e_{1} \pm e_{2}}{b a_{0}}\left[J_{2}\left(b a_{1} \mu_{7}\right) J_{1}\left(b a_{2} \mu_{7}\right)\right]^{2} \\
& \mu_{8}=\mu \pm \frac{3 e_{1}}{b a_{0}} \quad\left[J_{0}\left(b a_{1} \mu_{8}\right) J_{3}\left(b a_{2} \mu_{8}\right)\right]^{2} \\
& \mu_{9}=\mu \pm \frac{3 e_{2}}{b a_{0}} \quad\left[J_{3}\left(b a_{2} \mu_{9}\right) J_{0}\left(b a_{2} \mu_{9}\right)\right]^{2} \\
& \text { Ghosts of } 3 \mathrm{~d} \text { order. }
\end{aligned}
$$

Each term in this table of ghosts simply expresses the fact that each periodic error produces the same ghosts in the same place as if it were the only error, while others are added which are the ghosts of ghosts. The intensities, however, are modified in the presence of these others.

Writing $c_{1}=b a_{1} \mu$ and $c_{2}=b a_{\mu_{2}}$.

The total light. is

$$
J_{0}^{2}\left(c_{1}\right) J_{0}^{2}\left(c_{2}\right)+\left\{\begin{array}{r}
2 J_{1}^{2}\left(c_{1}\right) J_{0}^{2}\left(c_{2}\right) \\
+2 J_{0}^{2}\left(c_{1}\right) J_{1}^{2}\left(c_{2}\right)
\end{array}\right\}+\left\{\begin{array}{r}
2 J_{2}^{2}\left(c_{1}\right) J_{0}^{2}\left(c_{2}\right) \\
+4 J_{1}^{2}\left(c_{1}\right) J_{1}^{2}\left(c_{2}\right) \\
+2 J_{0}^{2}\left(c_{1}\right) J_{2}^{2}\left(c_{2}\right)
\end{array}\right\}+\text { etc. }
$$

which we can prove to be equal to 1 .

Hence the sum of all the light is still unity, a general proposition which applies to any number of errors.

The positions of the lines when there is any number of periodic errors can always be found by calculating first the ghosts due to each error separately; then the ghosts due to these primary ghosts for it as if it were the primary line, and so on ad infinitum.

In case the ghosts fall on top of each other the expression for the intensity fails. Thus when $e_{2}=2 e_{1}, e_{3}=3 e_{1}$, etc., the formula will need modification. The positions are in this case only those due to a single periodic error, but the intensities are very different.

$$
\mu=\frac{2 \pi N}{b a_{0}} \quad\left[\begin{array}{cc}
\text { Places. } & \text { Intensities. } \\
& {\left[J_{0}\left(b a_{1} \mu\right) J_{0}\left(b a_{2} \mu\right)\right]^{2},}
\end{array}\right.
$$




$$
\begin{array}{cc}
\text { Places. } & \text { Intensities. } \\
\mu_{1}=\mu \pm \frac{e_{1}}{b a_{0}} & {\left[J_{1}\left(b a_{1} \mu_{1}\right) J_{0}\left(b a_{2} \mu_{1}\right)-J_{3}\left(b a_{1} \mu_{1}\right) J_{2}\left(b a_{2} \mu_{1}\right)+\text { etc. }\right]^{2}} \\
\text { etc. } & +\left[J_{1}\left(b a_{1} \mu_{1}\right) J_{1}\left(b a_{2} \mu_{1}\right)-J_{8}\left(b a_{1} \mu_{1}\right) J_{1}\left(b a_{2} \mu_{2}\right)+\text { etc. }\right]^{2},
\end{array}
$$

We have hitherto considered cases in which the error could not be corrected by any change of focus in the objective. It is to be noted, however, that for any given angle and focus, every error of ruling can be neutralized by a proper error of the surface, and that all the results we have hitherto obtained for errors of ruling can be produced by errors of surface, and many of them by errors in size of groove cut by the diamond. Thus ghosts are produced not only by periodic errors of ruling but by periodic waves in the surface, or even by a periodic variation in the depth of ruling. In general, however, a given solution will apply only to one angle and, consequently, the several results will not be identical; in some cases, however, they are perfectly so.

Let us now take up some cases in which change of focus can occur. The term $\kappa r^{2}$ in the original formula must now be retained.

Let the lines of the grating be parallel to each other. We can then neglect the terms in $z$ and can write $r^{2}=y^{2}$ very nearly. Hence the general expression becomes

$$
\int e^{i b\left(\lambda x+\mu y-\kappa y^{2}\right)} d s,
$$

where $\kappa$ depends on the focal length. This is supposed to be very large, and hence $\kappa$ is small.

This integral can be divided into two parts, an integral over the groove and the intervening space, and a summation for all the grooves. The first integral will slightly vary with change in the distance of the grooves apart, but this effect is vanishingly small compared with the effect on the summation, and can thus be neglected. The displacement is thus proportional to

$$
\Sigma e^{\imath b\left(\mu y-\kappa y^{2}\right)}
$$

\section{Case I.-Lines at Variable Distances}

In this case we can write in general

$$
y=a n+a_{1} n^{2}+a_{2} n^{3}+\text { etc. }
$$

As $\kappa, a_{1}, a_{2}$, etc., are small, we have for the displacement, neglecting the products of small quantities,

$$
\mathcal{L}_{\epsilon} \boldsymbol{t}^{i b}\left[\mu\left(a n+a_{1} n^{2}+a_{2} n^{2}+\text { etc. }\right)-\kappa a^{2} n^{2}\right] \text {. }
$$


Hence the term $a_{1} n^{2}$ can be neutralized by a change of forms expressed by $\mu a_{1}=\kappa a^{2}$. Thus a grating having such an error will have a different focus according to the angle $n$, and the change will be + on one side and - on the other.

This error often appears in gratings and, in fact, few are without it.

A similar error is produced by the plate being concave, but it can be distinguished from the above error by its having the focus at the same angle on the two sides the same instead of different.

According to this error, $a_{1} n^{2}$, the spaces between the lines from one side to the other of the grating, increase uniformly in the same manner as the lines in the $B$ group of the solar spectrum are distributed. Fortunately it is the easiest error to make in ruling, and produces the least damage.

The expression to be summed can be put in the form

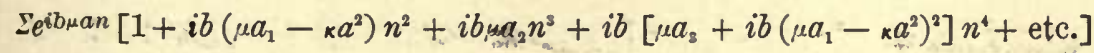

The summation of the different terms can be obtained as shown below, but, in general, the best result is usually sought by changing the focus. This amounts to the same as varying $\kappa$ until $\mu a_{1}-\kappa a^{2}=0$ as before. For the summation we can obtain the following formula from the one already given. Thus

$$
\Sigma_{0}^{n-1} e^{2 i p n}=\frac{\sin n p}{\sin p} e^{i p(n-1)} .
$$

Hence

$$
\sum_{0}^{n-1} n^{m} e^{2 i p n}=\frac{1}{(2 i)^{m}} e^{i p(n-1)}\left(\frac{d}{d p}+i(n-1)\right)^{m} \frac{\sin n p}{\sin p} .
$$

When $n$ is very large, writing $\frac{b \mu a n}{2}=p n=\pi N n+q$, we have

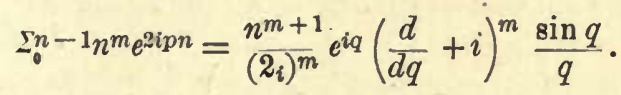

Whence writing

$$
\begin{aligned}
& c=b\left(\mu a_{1}-\kappa a^{2}\right), \\
& c^{\prime}=b_{\mu} a_{2}, \\
& c^{\prime \prime}=b\left[\mu a_{3}+i b\left(\mu a_{1}-\kappa a^{2}\right)^{2}\right], \\
& c^{\prime \prime \prime}=\text { etc., }
\end{aligned}
$$


the summation is

$$
\begin{aligned}
& e^{i q}\left\{\begin{array}{c}
n+i\left(c \frac{n^{3}}{4}+c^{\prime} \frac{n^{4}}{8}+c^{\prime \prime} \frac{n^{5}}{16}+\right) \\
+\left(2 c \frac{n^{3}}{4}+3 c^{\prime} \frac{n^{4}}{8}+4 c^{\prime \prime} \frac{n^{5}}{16}+\right) \frac{d}{d q} \\
-i\left(c \frac{n^{3}}{4}+3 c^{\prime} \frac{n^{4}}{8}+6 c^{\prime \prime} \frac{n^{5}}{16}+\right) \frac{d^{2}}{d q^{2}} \\
-\left(c^{\prime} \frac{n^{4}}{8}+4 c^{\prime \prime} \frac{n^{5}}{16}+\right) \frac{d^{3}}{d q^{3}} \\
+i\left(c^{\prime \prime} \frac{n^{5}}{16}+\right) \frac{d^{4}}{d q^{4}} \\
+ \text { etc. }
\end{array}\right\} \frac{\sin q}{q}, \\
& \frac{d}{d q} \frac{\sin q}{q}=\frac{q \cos q-\sin q}{q^{2}}, \\
& \frac{d^{2}}{d q^{2}} \frac{\sin q}{q}=\frac{-2 q \cos q+\left(2-q^{2}\right) \sin q}{q^{3}} \text {, } \\
& \frac{d^{3}}{d q^{3}} \frac{\sin q}{q}=\frac{q\left(6-q^{2}\right) \cos q-\left(6-3 q^{2}\right) \sin q}{q^{4}} \text {, } \\
& \text { etc. } \\
& \text { etc. }
\end{aligned}
$$

These equations serve to calculate the distribution of light intensity in a grating with any error of line distribution suitable to this method of expansion and at any focal length. For this purpose the above summation must be multiplied by itself with $+i$ in place of $-i$.

The result is for the light intensity

$$
\begin{aligned}
\left\{n \frac{\sin q}{q}+\left(2 c \frac{n^{3}}{4}+2 c \frac{n^{4}}{8}\right.\right. & + \text { etc. }) \frac{d}{d q} \frac{\sin q}{q} \\
& \left.-\left(c^{\prime} \frac{n^{4}}{8}+4 c^{\prime \prime} \frac{n^{5}}{16}+\text { etc. }\right) \frac{d^{3}}{d q^{3}} \frac{\sin q}{q}+\text { etc. }\right\}^{2} \\
& +\left\{\left(c \frac{n^{3}}{4}+3 c^{\prime} \frac{n^{4}}{8}+\text { etc. }\right) \frac{d^{2}}{d q^{2}} \frac{\sin q}{q}\right. \\
& \left.-\left(c^{\prime \prime} \frac{n^{5}}{16}+\text { etc. }\right) \frac{d^{4}}{d q^{4}} \frac{\sin q}{q}+\text { etc. }\right\}^{2} .
\end{aligned}
$$

As might have been anticipated, the effect of the additional terms is to broaden out the line and convert it into a rather complicated group of lines, as can sometimes be observed with a bad grating. At any given angle the same effect can be produced by variation of the plate from a perfect plane. Likewise the effect of errors in the ruling may be neutralized for a given angle by errors of the ruled surface, as noted in the earlier portions of the paper. 


\section{A NEW TABLE OF STANDARD WAVE-LENGTHS}

[Johns Hopkins University Circulars, No. 106, p. 110, 1893; Philosophical Magazine [5], $X X X V I, 49-75,1893$; Astronomy and Astro-Physics, XII, 321-347, 1893]

\section{Prefatory Note}

During the last ten years I have made many observations of wavelengths, and have published a preliminary and a final table of the wavelengths of several hundred lines in the solar spectrum.

For the purpose of a new table I have worked over all my old observations, besides many thousand new ones, principally made on photographs, and have added measurements of metallic lines so as to make the number of standards nearly one thousand.

Nearly all the new neasurements have been made on a new measuring machine whose screw was specially made by my process ${ }^{1}$ to correspond with the plates and to measure wave-lengths direct with only a small correction.

The new measures were made by Mr. L. E. Jewell, who has now become so expert as to have the probable error of one setting about $\frac{1}{1000}$ division of Angström, or 1 part in 5,000,000 of the wave-length. Many of these observations, however, being made with different measuring instruments, and before such experience had been obtained, have a greater probable error. This is especially true of those measurements made with eye observations on the spectrum direct. The reductions of the reading were made by myself.

Many gratings of $6 \mathrm{in}$. diameter and $21 \frac{1}{2}$ feet radius were used; and the observations were extended over about ten years.

The standard wave-length was obtained as follows: Dr. Bell's value of $D_{1}$ was first slightly corrected and became 5896-20. C. S. Peirce's value of the same line was corrected as the result of some measurements made on his grating and became $5896 \cdot 20$. The values of the wave-length then become

1 See Encyc. Brit., art. Screw. 


\begin{tabular}{|c|c|c|}
\hline Weight. & Observer. & $D$. \\
\hline 1 & Ångström, corrected by Thalén & $.5895 \cdot 81$ \\
\hline 2 & Müller \& Kempf $\quad \ldots \ldots \ldots \ldots$ & $.5896 \cdot 25$ \\
\hline 2 & Kurlbaum ..... & $.5895 \cdot 90$ \\
\hline 5 & Peirce ... & $.5896 \cdot 20$ \\
\hline 10 & Bell ..... & $.5896 \cdot 20$ \\
\hline
\end{tabular}

As the relative values are more important for spectroscopic work than the absolute, I take this value without further remark. It was utilized as follows:

1st. By the method of coincidences with the concave grating, the wave-lengths of 14 more lines throughout the visible spectrum were determined from this with great accuracy for primary standards.

$2 \mathrm{~d}$. The solar standards were measured from one end of the spectrum to the other many times; and a curve of error drawn to correct to these primary standards.

3d. Flat gratings were also used.

4th. Measurements of photographic plates from 10 to 19 inches long were made. These plates had upon them two portions of the solar spectrum of different orders. Thus the blue, violet and ultra violet spectra were compared with the visible spectrum, giving many checks on the first series of standards.

5th. Measurements were made of photographic plates having the solar spectrum in coincidence with metallic spectra, often of three orders, thus giving the relative wave-lengths of three points in the spectrum.

Often the same line in the ultra violet had its wave-length determined by two different routes back to two different lines of the visible spectrum. The agreement of these to $\frac{1}{100}$ division of Angström in nearly every case showed the accuracy of the work.

6 th. Finally, the important lines had from 10 to 20 measurements on them, connecting them with their neighbors and many points in the spectrum, both visible and invisible; and the mean values bound the whole system together so intimately that no changes could be made in any part without changing the whole.

This unique way of working has resulted in a table of wave-lengths from 2100 to 7700 whose accuracy might be estimated as follows:

Distribute less than $\frac{1}{100}$ division of Ângström properly throughout 
the table as a correction, and it will become perfect within the limits 2400 and 7000 .

The above is only a sketch of the methods used. The complete details of the work are ready for publication but I have not yet found any journal or society willing to undertake it. ${ }^{2}$

[The tables of wave-lengths are omitted.]

${ }^{2}$ [These details were finally published in the Memoirs of the American Academy of Arts and Sciences, XII, 101-186, 1896, under the title, 'On a Table of Standard WaveLengths of the Spectral Lines.'] 


\section{ON A TABLE OF STANDARD WAVE-LENGTHS OF THE SPECTRAL LINES ${ }^{1}$}

[Memoirs of the American Academy of Arts and Sciences, XII, 101-186, 1896]

Presented May 10,1893

Investigations on Light and Heat, made and published wholly or in part with appropriation from the Rumford Fund

Some years since, having made a machine for ruling gratings and discovered the concave grating, which placed in my hands an excellent process for photographing spectra, I applied myself to photograph the solar spectrum. The property of the concave grating, mounted in the method which I use, of producing a normal spectrum gave me the means of adding a scale of wave-lengths, and so producing a photographic map of the solar spectrum on a very large scale and of great accuracy. I soon after constructed a very much better ruling engine, which is kept at a uniform temperature in the vault of the new physical laboratory of the Johns Hopkins University, with which I have made very much better gratings. I therefore went over the whole process once more, extending the map to include $B$, and making new negatives of the whole spectrum very much better than the old. This set of ten photographic plates is now familiar to most spectroscopists.

In order to place the scale on the negatives, it was necessary to know the wave-lengths of certain standard lines. Of course my first thought was of Angström, whose measurements were the wonder of his time. On trying to place my scale according to his figures, I found it impossible to make them and my photographs agree; and I finally was forced to the conclusion that a new series of standards was needed before I could go further. Here again the concave grating came to my rescuc. All the spectra are in focus at once, and relative measures can thus be made at once by micrometric measures of the overlapping spectra. Again, the spectrum is normal, and so a micrometer of very long range could be used. To obtain the primary standards by means of overlapping spectra, I have used gratings with from 3000 up to 20,000 lines to

'An abstract of this paper bas recently appeared in 'Astronomy and Astro-Physics,' and in the 'London Philosophical Magazine.' 
the inch, and from 13 to $21 \frac{1}{2}$ feet focus. The first series made with the 13-foot grating by Mr. Koyl in 1882 was not found quite accurate enough, and I have since made personally a long series with gratings of $21 \frac{1}{2}$ feet focus which is mnch more accurate. These long focus gratings had from 7000 to 20,000 lines to the inch, and were ruled on two dividing engines, while the 13 -foot one had a less number, possibly 3000. There are two principal errors to guard against in this method, the first peculiar to the method of coincidences, and the second to any method where gratings are used. ${ }^{2}$ The first is that, where spectra are over each other and the lines therefore often on top of each other, the line of one spectrum may be apparently slightly displaced by the presence of one from another spectrum, although the latter may be almost invisible. The use of proper absorbents obviates this difficulty. The second source of error is more subtle, and arises from the diamond ruling differently on different parts of the grating. It is more apt to occur in concave gratings than plane ones, although few are perfectly free from the error, as it is very difficult to get a diamond to rule a concave grating uniformly. Looking at the grating in spectra of different orders, the grating may appear uniform from end to end in one, and possibly brighter at one end than the other in another spectrum. This gives a chance for any imperfection in the form of the surface of the grating, or any errors in its ruling, or indeed the spherical aberration of the lenses or concave grating, to affect the measure-

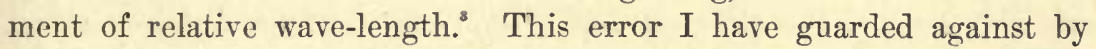
using only uniformly ruled gratings, reversing them, and using a great number of them. I have also used the coincidence of only the lower orders of spectra, such as the $2 \mathrm{~d}, 3 \mathrm{~d}, 4 \mathrm{th}, 5 \mathrm{th}$, and 6 th. Coincidences up to the 12th were, however, observed by Mr. Koyl with the 13-foot concave, and probably have some errors of this nature.

In this way I established about fifteen points in the visible spectrum which served as primary standards. These were so interwoven by the coincidences that I have great confidence in the value of most of them.

${ }^{2}$ The variation of the dispersion of the air with the thermometer and barometer is probably not worth considering for the visible part of the spectrum, although it might be worth investigating for the two extremities of the spectrum.

${ }^{3}$ The error of using gratings of variable brightness in different parts, or those wlth imperfect ruling of any kind, I have constantly guarded against. Such I believe to be the principal causes of the great errors in relative and absolnte wavelengths in Vogel's tables, as the gratings he used, made by Wandschaft, were full of errors of all kinds. 
Indeed, no process of angular measurement could approach the accuracy of this one.

Thus, using a line $P$ to start with, I determine other groups of lines, $a^{\prime}, b^{\prime}, c^{\prime}, d^{\prime}$, ete. From these again I find groups, some of which may be the same as the first; then again from these, other groups. The process can be continued further, but we are apt to come back to the same lines again, and we are further limited by the visibility of the lines. Thus the limit of great accuracy by eye observation in either direction is practically 4200 and 7000 ; although in a dark room, especially in the first spectrum, one ean see much further, even beyond the $A$ group, although it is difficult to set on the lines, and one is apt to mistake groups of lines for single lines." When one uses a group as a standard, and one or more of the group is an atmospheric line which varies, the measures will of course vary also, unless the atmospheric line is in the centre of the group. This is a very common source of error, and has caused me much trouble. In a grating with a very bright second spectrum, I have, however, obtained the coincidence of $A$ with the region whose wave-length is about 5080, and have thus confirmed the value given in my preliminary table, which was obtained by a very long interpolation passing from the first into the second spectrum.

The accuracy of these primary standards can be estimated from the equations given in Table VII. It is there seen that there is scarcely any difference in the different measures as derived from different lines.

It is to be specially noted that the wave-length of $P$ and the lines directly determined from it have no more weight than any of the others. The table might just as well have been arranged with the $D$ line, or any other, first. The true way of discussing the results is to form a series of linear equations, about twenty-six in all, and solve them. This is the method I have used, although I have not discussed them by the method of least squares.

Some miscellaneous observations not included in the table allowed me to add a few more lines to these primary standards.

Having completed these primary standards, I then observed several

4 In a very bright grating I have faintly seen, and even measured, lines down to wave-length 8500. My assistant, Mr. L. E. Jewell, can see far into what is called the ultra violet, even to wave-length 3500 or beyond.

${ }^{5}$ The calculations of this paper have involved about a million figures, of which I have personally written more than half. Hence I am not anxions for more labor of this kind. 
hundred standard lines in the visible spectrum, including these primary standards, with a micrometer having a range of five inches, and very accurately made. The spectrum being strictly normal, the readings so made were proportional to the wave-length. They could have been used simply to interpolate between the primary standards, but I preferred another method. The readings of the micrometer were made to overlap, so that, by adding a constant to each set; a continuous series could be formed for the whole spectrum which would be proportional to the wave-length except for some slight errors due to the working of the apparatus for keeping the focus constant. Making this series coincide with two standards at the ends, the wave-lengths of all could be obtained by simply multiplying the whole series by one number and adding a constant. This usually gave the wave-lengths of the whole spectrum within 0.1 or 0.2 divisions of Angström. The differences of this series from the primary standards were then plotted, and a smooth curve drawn through the points thus found. The ordinates of this curve then gave the correction to be applied at any point.

It is to be noted that the departure from the normal spectrum was very small, and the correction thus found was very certain. The cause of the departure was not apparent, but may have been the slight tilting of the spectrum, by which it was measured somewhat obliquely at places.

The visible spectrum was thus gone over five or more times in this manner, with several different gratings and in different orders of spectra. The results are given in Table $\mathrm{X}$, Columns $C, R, p, q, m, O, e, h$, i, etc. The spectrum from the green down to and including $A$ was also observed on a large instrument for flat gratings, having lenses six and one-half inches in diameter and of eight feet focus. These latter observations are marked $C^{\prime}$. This region I intend at some future time to observe further.

It was now required to observe the ultra violet to complete the series. For this purpose the coincidences of the $2 \mathrm{~d}, 3 \mathrm{~d}, 4 \mathrm{th}, 5$ th, and 6 th spectra of a $7000,21 \frac{1}{2}$ feet radius, grating were photographed. My instrument will take in photographic plates twenty inches long, but there will be a slight departure from a normal spectrum in so long a plate. Hence plates ten inches long were mostly used for this special series. Before the camera was placed a revolving plate of metal about threesixteenths of an inch thick, and having a slit in it of the same width."

6 This is described in the Johns Hopkins Circular of May, 1889, by Dr. Ames. 
When the flat side was parallel to the camera plate, a strip of the spectrum three-sixteenths of an inch wide fell on the plate. When turned ninety degrees, the plate shielded this portion and exposed the rest. Using absorbents, it was thus possible to photograph a strip of say the 4th spectrum between two strips of the 5th. This arrangement is better than having only two edges come together. To correct any movement of the apparatus during the time of exposure, I expose on one spectrum, then on the other, and back again on the first.

- Placing the negatives so obtained on a dividing engine with a microscope of very low power and a tightly stretched cross-hair, the coincidence of the two spectra can be measured. Owing to the large scale of the photographs, - about that of Angström,-an ordinary dividing engine having errors not greater than $\frac{1}{1000}$ inch can be used, but the negatives should be gone over at least twice, reversing them end for end. Two screws were used in the engine and finally another complete machine was constructed, giving wave-lengths direct with only a slight correction. For determining the wave-length of metallic lines, the same process can be used with wonderful accuracy.

The results are given in the columns marked $P l$. with the number of the plates. The accuracy is very remarkable, and I think the figures establish the assertion that the coincidence of solar and metallic lines can be determined with a probable error of one part in 500,000 by only one observation.

This process not only gave me measures of the ultra violet, but also new observations of the visible spectrum. So far in my work on these coincidences, I have only used erythrosin plates going a little below $D$; but cyanine plates might be used to $B$, or even in the ultra red, as Trowbridge has recently shown. One plate, No. 20, however, connects wavelengths 6400 and 3200 .

Thus I have constructed a table of about one thousand lines, more or less, which are intertwined with each other in an immense number of ways. They have been tested in every way I can think of during eight or nine years, and have stood all the tests; and I think I can present the results to the world with confidence that the results of the relative measures will never be altered very much. I believe that no systematic error in the relative wave-lengths of more than about $\pm \cdot 01$ exists anywhere except in the red end as we approach $A$. Possibly $\pm \cdot 03$, or even less, might cover that region.

The relative measures having thus been obtained, we have means in the concave grating of obtaining the wave-lengths of the lines of metals 
to a degree of accuracy hitherto unknown, and thus of solving the great problem of the mathematical distribution of these lines.

But for the comparison of spectra, as measured by different observers, some absolute scale is needed. Hitherto Angström has been used. But it is now very well known that his standard measure was wrong. As his relative measures are also very wrong, I have concluded that the time has come to change not only the relative measures, but the absolute also. To this end Dr. Louis Bell worked in my laboratory for several years with the best apparatus of modern science, using two glass and two speculum metal gratings, ruled on two dividing engines with four varieties of spacing, three of which were incommensurable or nearly so, with two spectrometers of entirely different form, with a variety of standard bars compared in this country and in Europe, and with a special comparator made for the measure of gratings. His result agrees very well with the next best determination, that of Mr. C. S. Peirce of the U. S. Coast Survey. His final result agrees within 1 in 50,000 with his preliminary value." This most recent value, combined with those of Peirce, Müller and Kempf, Kurlbaum and Angström, I have adopted to reduce my final results to, although the calculations are made according to Bell's preliminary value. See Appendix A.

But it rests with scientific men at large oto adopt some absolute standard. The absolute standard is, of course, not so important as the relative, and possibly the average of Angström might be adopted. But for myself I do not believe in continuing an error of this sort indefinitely. All the results obtained before the concave grating came into use were so imperfect, that they must be replaced by others very soon. With a good concave grating one man in a few years could obtain the wave-lengths of the elements with far greater accuracy than now known.

As an aid to this work, I have constructed the table of wave-lengths given in this paper, which have already been adopted by the British Association and by the most noted writers of Germany and other countries, and sincerely hope that it will aid in the work of making the wave-length of a spectrum line a definite quantity within a few hundredths of a division of Angström.

\section{Absoldte Wave-Length of $D$}

The following is an estimate of the absolute wave-length of the $D$ line from the best determinations. First, I shall recalculate the portion of

7 American Journal of Science, 1887. 
Dr. Bell's paper ${ }^{8}$ in which the calibration of the grating space is taken into account. The method of correction is founded on the principle that a linear error in the spaces only affects the focal length, and not the angle, and that small portions which have an error, and thus throw the light far to one side, should be rejected. The corrections Dr. Bell has used seem to me very proper, except to grating III, which appears to me to be twice too great. I find the following:

\begin{tabular}{cccc} 
Grating. & $D$. & Correction. & Final Values. \\
I. & $5896 \cdot 20$ & $-\cdot 02$ & $5896 \cdot 18$ \\
II. & $5896 \cdot 14$ & $+\cdot 09$ & $5896 \cdot 23$ \\
III. & $5896 \cdot 28$ & $-\cdot 06$ & $5896 \cdot 22$ \\
IV. & $5896 \cdot 14$ & $+\cdot 03$ & $5896 \cdot 17$ \\
\multicolumn{4}{c}{ Mean value, $5896 \cdot 20}$.
\end{tabular}

This is very nearly the value given by Dr. Bell.

The determination of Mr. C. S. Peirce of the U. S. Coast Survey is certainly a very accurate one. Dr. Bell and myself have made some attempts to calibrate his gratings, which he sent to us for the purpose, and to correct for the scale used by him. There is great uncertainty in this process, as we had only a portion of the necessary data. The correction of his scale was also uncertain, because the glass scales used by him may have changed since he used them, in the manner thermometer bulbs are known to change. Correcting, then, only for the error of ruling in the gratings, we have:

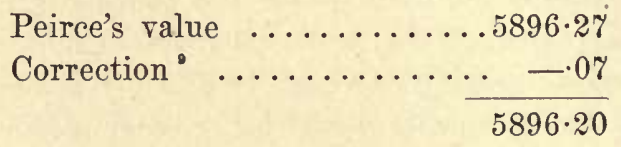

The correction for the scale would be about as much more in the same direction, provided the glass scales had not changed. But it is too uncertain to be used, although I have applied it in my preliminary paper.

Kurlbaum's result, made with two good modern gratings, has the defect that the gratings were 42 and $43 \mathrm{~mm}$. broad, quantities which it is impossible to compare accurately with a metre. His small objectives, one inch in diameter, could not take in light from the whole grating, and so the grating space was not determined from the portion

${ }^{8}$ American Journal of Science, 1888.

${ }^{9}$ Bell, American Journal of Science, May, 1888, p. 365. 
of the grating used. The spectrometer was poor, and the errors of the grating undetermined.

Müller and Kempf used four gratings, evidently of very poor quality, as they give results which differ 1 in 10,000 .

The result of Angström was a marvel at the time, but the Nobert gratings used by him would now be considered very poor. Taking Thalén's correction for error of scale, we have for the mean of the $E$ lines $5269 \cdot 80$, which gives, by my table of relative wave-lengths, $D=$ $5895 \cdot 81$. It is rather disagreeable to estimate the relative accuracy of observations made by different observers and in different countries, but in the interest of scientific progress I have attempted it, as follows:

$\begin{array}{lr}\text { Ångström } \ldots \ldots \ldots \ldots .5895 \cdot 81 & 1 \\ \text { Müller and Kempf } \ldots .5896 \cdot 25 & 2 \\ \text { Kurlbaum } \ldots \ldots \ldots .5895 \cdot 90 & 2 \\ \text { Peirce } \ldots \ldots \ldots \ldots .5896 \cdot 20 & 5 \\ \text { Bell } \ldots \ldots \ldots \ldots \ldots .5896 \cdot 20 & 10\end{array}$

Mean, 5896.156 in air at $20^{\circ}$ and $760 \mathrm{~mm}$. pressure.

This must be very nearly right, and I believe the wave-length to be as well determined as the length of most standard bars. Indeed, further discussion of the question would involve a very elaborate discussion of standard metres, a question involving endless dispute. I think we may say that the above result is within 1 in 100,000 of the correct value, which is very nearly the limit of accuracy of linear measurements. This should be so, as the probable error of the angular measures affects the wave-length only to 1 in $2,000,000,{ }^{10}$ and hence nearly the whole accuracy rests on the linear measures.

\section{Résumé of Process for Obtaining Relative Wave-Lfingths}

1. Determination of about 20 lines in the visible spectrum by coincidences by Koyl. ${ }^{11}$

\footnotetext{
${ }^{10}$ Is not a grating and spectrometer thus the best standard of length, and almost independent of the temperature? Gratings of $10 \mathrm{~cm}$. length can now be ruled on my new engine with almost perfect accuracy, as seen in the calibration of Grating IV in Dr. Bell's paper, and it seems to me the time has come for their practical use.

11 These observations of Mr. Koyl were finally given no weight, on account of the inferior apparatus used. They serre a useful purpose, however, as checks on the other work.
} 
2. Determination of about 15 lines in the visible spectrum by coincidences by Rowland, using several gratings of $21 \frac{1}{2}$ feet focus.

3. Interpolation by direct eye observations with concave gratings of $21 \frac{1}{2}$ feet focus and micrometer of 5 inches range and of almost perfect accuracy.

4. Interpolation by means of flat gratings.

5. Measurement of photographic plates from 10 to 19 inches long, having two or three portions of the spectrum in different orders on them, thus connecting the ultra violet and blue with the visible spectrum. The fact that nearly the same values are obtained for the violet and ultra violet by use of different parts of the visible spectrum proves the accuracy of the latter.

6. Measurement of photographic plates having the solar visible spectrum in coincidence with the metal lines of different orders of spectra. The fact that the wave-lengths of the metal lines are very nearly the same as obtained from any portion of the visible or ultra violet spectrum proves the accuracy of the latter, as well as that of the metallic wave-lengths.

\%. Measurement of plates having metallic spectra of different orders.

\section{Advantages of the Process}

The only other process of obtaining relative wave-lengths is by means of angular measures. Supposing the angle to be about $45^{\circ}$, an error of $1^{\prime \prime}$ will make an error of about 1 in 200,000 in the sine of the angle. When one considers the changes of temperature and barometer measuring on one line and then another, together with the errors of graduation, it would be a difficult matter to measure this angle to $2^{\prime \prime}$, making an error of 1 in 100,000, or about $\frac{1}{20}$ division of Angström.

Looking over the observations of principal standards made under the direction of Professor Vogel in Potsdam, with very poor gratings but an excellent spectrometer, we find the average probable error to be about $\pm \frac{1}{150000}$ of the wave-length, which is not far from the other estimate. This does not include constant errors, and I believe the probable error to be really greater than this.

The method of coincidences by the concave grating gives far superior results. The distance to be measured is very small, and the equivalent

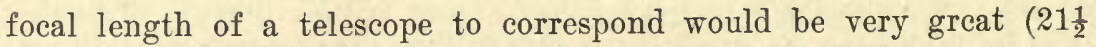
feet). Furthermore, all changes of barometer and thermometer are eliminated at once, except the small effect on the dispersion of the air, which, when known, can be corrected for. It is not to be wondered at 
that this method is far superior to the former. The probable error is, indeed, reduced to $土_{1000000}$, or even less for the best lines. Where the interpolation can be made on photographs, this probable error is scarcely increased at all; but even taking it at twice the above estimate, the method even then remains from three to five times as accurate as that of angular measurement. Indeed, the impression made on my mind in looking over Vogel's Potsdam observations is, that my tables and process are ten times as accurate as theirs; and I think any careful student of both processes will come to a similar conclusion.

The wonderful result that can be obtained by the measurement of photographs on the new micrometer, which can measure plates over twenty inches long, is partly seen in the table. Where the distance is only a few inches, the wave-length of a series of lines can be measured with a probable error of less than $\frac{1}{10 \sigma}$ of a division of Angström. Indeed, a series would determine any line so that the probable error would be even \pm .0000001 of the whole. This would detect a motion in the line of sight of \pm 140 feet per second!

From the tests I have made on my standards, I am led to believe that down to wave-length 7000 , a correction not exceeding \pm .01 division of Ångström (1 part in 500,000), properly distributed, would reduce every part to perfect relative accuracy.

To ascend to the next degree of accuracy would need many small corrections which would scarcely pay. It is reasonable to assume that a higher degree of accuracy will not be needed for twenty-five years, as the present degree is sufficient to distinguish the lines of the different elements from one another in all cases that $\mathrm{I}$ have yet tried.

\section{DetaILS OF WORK}

To reduce all the observations in a given region to one line, relative observations extending a short distance either side of the standard region are necessary. Thus the mean of 4215 and 4222 can be taken as the standard, and, if only one is observed, it can be reduced to the standard by a correction +3.258 or -3.358 . But it is not necessary to take the mean of the lines as a standard, as any one of them may be so taken, or even any other point where there is no line, as the point is only to be used in the mathematical work, and finally disappears altogether.

Table II gives results of this nature. The letters at the top of each series, $e, g, h, j$, etc., are the arbitrary names of the standards. The first columns refer to the series of observations, "Co." being observa- 
tions made at the time of measuring the coincidences; Plates 9,10 , etc., refer to photographic plates; $C, R$, etc., refer to the series as given in the final table, although they may differ very slightly from the latter, as the final table contains slight corrections. Figures in parentheses are the number of readings. The photographs were usually measured from two to six times.

Table III gives the first series of observations made in 1884 with a $21 \frac{1}{2}$ foot concave, 14,436 lines to the inch. The numbers taken for the standards are only preliminary, and agree as nearly as practicable with my Table of Preliminary Standards. As only differences are finally used, they are sufficiently near. The fractions give the order of the spectra observed.

Thus, the first observation on $h$ and $t$ is worked up as follows:

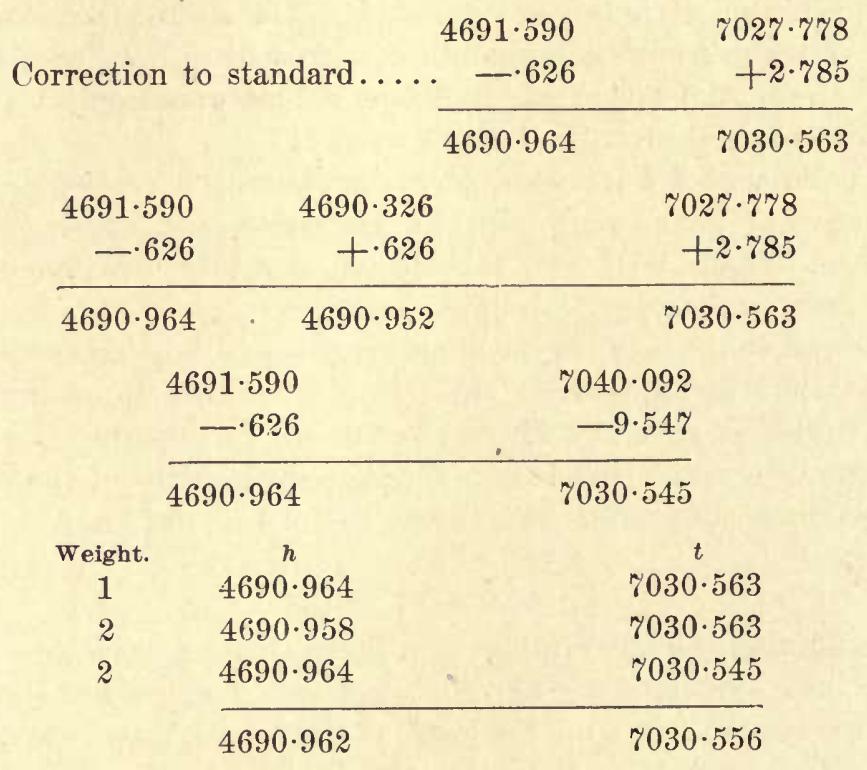

The equation $3 h-2 t=11 \cdot 7 \% 4$ then readily follows.

Tables IV and V are from a $21 \frac{1}{2}$ foot concave with 7218 lines to the inch, used on both sidés, and thus equivalent to two gratings used on one side only. I have not yet determined theoretically whether the minor errors are perfectly neutralized in this manner, but it would evidently have a tendency in this direction.

The photographic coincidences are given in the main table $(\mathrm{X})$, as not only the standards are compared by this process, but whole regions 
are photographed side by side. - Both a 10,000 and a 20,000 concave were used for this work.

Table VI gives the collection of the equations relating to the visible spectrum, the final results being given in Table VII.

The proper method of treating these twenty-six equations would be by the method of least squares. But it would be so long and tedious, and so liable to mistake, that I have adopted the method of starting at one point and going forward until all the equations are reached. Thus (T'able VII), starting with an assumed value of $e$, we can calculate $p, n$, $l, k, j, o, t$.

Using the eight values thus found once more, from $p$ we have $g, k, l$; from $n$ we have $h, t, g$; with similar results for the others. Collecting, we then have $e, f, g, h, j, k, l, n, o, p, q, t$. Using these once more, we have values of all the standards. We could do this any number of times, keeping the proper weights, but I thought this number was sufficient. The second calculation is done in the same manner, starting from $o$, however, and is given in Table VIII.

The results of the two calculations are given in Table IX. Taking the mean and adding the results of local micrometer measurements, we obtain the column marked "Relative Wave-Lengths."

Reducing these values by 1 part in 200,000, we make them agree with the absolute value of the standard as before agreed upon. Thus the column of standards is obtained for use in the visible spectrum.

For ordinary interpolation with the short and imperfect micrometers generally used, and working with a flat grating and a spectrum not normal, the standards would be too far apart. But with such a long and perfect micrometer as I use, and working with the normal spectrum of a concave grating, they are entirely sufficient. However, I have filled in the interval from 7030 to 7621 by some extra substandards at 7230 .

The micrometer for eye observations has a range of five inches, and the machine for measuring photographs of more than twenty inches, both with practically perfect screws made by my process. The eye observations are not an interpolation, in the ordinary sense, between the standards, but the whole series is continuous, the micrometer observations overlapping so that they join together to any length desired. By measuring from the $D$ line in one spectrum to the $D$ line in the next, and including the overlapping spectra, no further standards would be necessary, as all the lines of the spectrum would be determined at once, knowing the wave-length of the $D$ line. But I usually plotted the difference of the standards from the micrometer determination, usually 
amounting to less than one- or two-tenths of a division of Angström, and so corrected the whole series to the standards. Sometimes two, or even three, overlapping spectra were measured at once.

To make Table $\mathrm{X}$, the following process was used:

1st. From all the observations at my disposal, I determined a few more lines around the main standards, and put them in the second column, marked St., so that I should have a greater number of points to draw my curve through.

2d. I then put down a few observations which were made by measuring overlapping spectra.

$3 \mathrm{~d}$. Then the main eye observations were put down as follows:-

\begin{tabular}{|c|c|c|c|c|c|c|c|c|c|}
\hline$p$ & extending & from & 4071 & to 7040 , & $2 \mathrm{~d} s$ & spectrum, & \multicolumn{3}{|c|}{6 grating. } \\
\hline$q$ & " & " & 4999 & to 7035 , & & " 6 & " & "، & " \\
\hline 0 & "6 & " & 4859 & to 7040 , & & “ & “ & 6 & "6 \\
\hline$c$ & "، & 66 & 4859 & to 6079 , & (fra & agmentary). & & & \\
\hline$C$ & “ & " & 6855 & to 6909 , & $2 \mathrm{~d} s$ & spectrum, & 14,436 & $\mathrm{gr}$ & ting. \\
\hline$R$ & ، & $"$ " & 5162 & to 7201 , & & " " & " & “ & " \\
\hline$h$ & "6 & "، & $\mathbf{5 7 4 2}$ & to 7628 , & $1 \mathrm{st}$ & spectrum, & 14,436 & & ting. \\
\hline$i$ & "6 & " & 6065 & to 7671 , & & “ & “ & "، & " \\
\hline$C^{\prime}$ & " & " & 6855 & to 7714 , & plan & ne grating. & & & \\
\hline$R^{\prime}$ & “ & " & 5139 & to 5296 , & $2 \mathrm{~d} s$ & spectrum, & 14,436 & $\mathrm{gl}$ & ting. \\
\hline$t$ & " & " & 6499 & to 6929 , & & "، & " & "6 & " \\
\hline $\boldsymbol{\alpha}$ & " & "، & 6278 & to 6322 , & & "6 & " & ، & "6 \\
\hline$E$ & "، & " & 4048 & to 4824 , & & “ & " & ، & " \\
\hline
\end{tabular}

4th. The series of photographs containing coincident spectra, mostly on plates so short as to make the spectra nearly normal, were now introduced. The plates were numbered from 1 to 20 , Nos. 7 and 19 being rejected because imperfect.

This series of plates was obtained by photographing a narrow strip of one spectrum between two strips of another, the overlapping spectra being separated by absorption. In order to eliminate any change in the apparatus during the exposure, the latter was divided into three parts, the first and third being given to the same spectrum.

This series of plates gives me a continuous series of photographs from wave-length 7200 to the extremity of the ultra violet spectrum, each part being interwoven with one or two other parts of the spectrum. Thus, wave-length 3900 comes from 5200 and 5850 with only a slight difference in values. There is scarcely any difference in any wavelength as derived from any portion of the spectrum; thus proving the accuracy of the whole table. The description of the plates is as follows: 
Table of Standard Wále-Lengths of the Spectral Lines 561

\section{Photographic Coincidences}

CONCAVE, GRATING 10,000 LINES TO THE INCH

\begin{tabular}{|c|c|c|c|c|c|c|c|c|c|}
\hline Spectra & Plato & & & & & & & & Standard \\
\hline$\frac{3}{4}$ & 1 & 4407 & to & 4643 & and & 3331 & to & $3486^{\circ}$ & $f, g$ \\
\hline 4 & 2 & 4637 & " & 4890 & “ & 3478 & " & 3667 & $h, j$ \\
\hline “ & 3 & 4823 & "6 & 5068 & “ & 3612 & "، & 3805 & $j, k$ \\
\hline “ & 4 & 4919 & “ & 5133 & “ & 3683 & " & 3875 & $j, k$ \\
\hline “ & 5 & 5050 & “" & 5288 & “ & 3780 & “ & 4005 & $k, l$ \\
\hline “ & 6 & 5097 & “ & 5333 & “" & 3821 & " & 4157 & $k, l$ \\
\hline “ & 7 & & 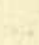 & & 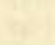 & & & & \\
\hline " & 8 & 5242 & "، & 5477 & “" & 3937 & " & 4121 & $l, m$ \\
\hline “" & 9 & 5405 & " & 5662 & “ & 4073 & “ & 4222 & $m, n, e$ \\
\hline “ & 10 & 5582 & “ & 5816 & “ & 4293 & “ & 4376 & $n, f$ \\
\hline “ & 11 & 5782 & “" & 5934 & “ & 4343 & "، & 4447 & $o, f$ \\
\hline “" & 12 & 4157 & " & 4267 & “ & 3129 & " & 3218 & $e^{2}$ \\
\hline ، & 13 & 4157 & " & 4325 & “ & 3094 & “" & 3246 & $e$ \\
\hline “ & 14 & & & & & 3218 & "6 & 3318 & \\
\hline " & 15 & 4391 & " & 4643 & " & 3292 & “ & 3478 & $f, g$ \\
\hline$\frac{4}{6}$ & 16 & 5788 & “ & 5977 & “" & 3864 & " & 3977 & 0 \\
\hline “6 & 17 & 5788 & " & 5977 & “ & 3864 & " & 3984 & o \\
\hline “ & 18 & 5715 & ، & 5977 & "، & 3875 & " & 3977 & 0 \\
\hline " & 19 & & & & & & & & \\
\hline$\frac{1}{2}$ & 20 & 5853 & 16 & 6569 & " & 3024 & “ & 3267 & $o, p, q$ \\
\hline
\end{tabular}

Plates 7, 14 and 19 were imperfect, owing to clouds passing over the sun, although a part (3218 to 3318) of Plate 14 was used for interpolation, as observations were scanty in that region.

It is seen that some of the plates have only one standard upon them. With a plane grating it would be impossible to work them up, but with the normal spectrum produced by the concave grating only one is necessary, as the multiplier to reduce readings to wave-lengths is nearly a constant. In working up a whole series of plates, there is no trouble in giving a proper value to the constant for any plate in the series which has only one standard.

Plate 17 was measured twice by two dividing engines, and as it was a specially good plate, each measure was given a weight equal to one of the other plates. The principal error to be feared in these plates is a displacement of the instrument between the time of the exposure on the two spectra. This was guarded against by the method above described. In Plates 17 and 20 there was a portion of the plate on which both the spectra fell all the time, and thus gave a test of the displacement. This was found to be zero. The other plates overlap so much that there are generally two or more determinations of each line. A 36 
comparison of these values shows little or no systematic variation in the different plates exceeding $\frac{1}{100}$ division of Angström. Plates 16, 17, 18, and $5,6,8$, all give the region 3900 as derived from 5200 and 5850, and thus give a test of the relative accuracy of these latter regions. It is seen that the two results of the region 3900 differ by about $\cdot 015$ division of Angström. Were the wave-lengths of the region 5170 to 5270 to be increased by 020 the discrepancy would cease. The amount of this quantity seems rather large to be accounted for by any displacement of the spectra on the plates, but still this may be the cause. Again, it is possible that different gratings may give this difference of wave-length from the cause I have mentioned above. This cause, as I have shown, exists in the same degree in plane gratings as in concave. I have not attempted to correct it in this case, but have simply taken the mean of the two values for the region 3900 , and so distributed the error. This is the greatest discrepancy I have found in the results except in the extreme red.

Thus the region 3100 to 3200 , a portion for which Plate 20 is to be relied upon, gives the wave-length of the ultra violet .01 division of Angström higher from the region 4200 than from 6300 . As the discrepancies in this region before the invention of the concave grating were often a whole division of Angström, I have regarded this result as satisfactory. Indeed, until we are able to make all sorts of corrections due to the change in the index of refraction of the air with the barometer and thermometer, it seems to me useless to attempt further accuracy.

With the advent of photographic plates into the table, especially the longer ones required for metallic spectra, it becomes necessary to correct them for the departure from the normal spectrum due to the use of long plates. The plates in the box are bent to the arc of a circle of radius $r$. When afterwards straightened we measure the distance by a linear dividing engine. Hence, what we measure is the arc with radius $r$. Let $\alpha$ and $\beta$ be the angles of incidence and diffraction from the grating. We have then to express $\beta$ in terms of $\delta$. Let $\lambda$ be the wave-length, and $n$ and $N$ the number of lines on the grating to $1 \mathrm{~mm}$. and the order of the spectrum respectively. Then

$$
\begin{aligned}
\lambda & =\frac{1}{n N}(\sin \alpha+\sin \beta) ; \\
\sin \beta & =\frac{2 r}{R} \sin \frac{\delta}{2} \cos \left(\gamma+\beta-\frac{\delta}{2}\right) .
\end{aligned}
$$

In these formulæ $\alpha$ is the angle to the centre of the photographic plate, and $\beta$ and $\delta$ are also measured from the centre. $\gamma$ is the angle 
between the radius from the centre of the photographic plate and the line drawn from that point to the centre of the grating. When properly adjusted, $\gamma$ will be zero. Also, we make $2 r=R$, to obtain perfect focus throughout. So that

$$
\lambda=\frac{1}{n N}\left(\sin \alpha+\sin \frac{\delta}{2}\right) \text {. }
$$

Calling $\lambda_{0}$ the wave-length at the centre of the plate, we have approximately

$$
\lambda-\lambda_{0}=\frac{\delta}{2 n N}-\frac{n^{2} N^{2}}{6}\left(\lambda-\lambda_{0}\right)^{3}+\text { etc. }
$$

The first quantity, $\frac{\delta}{2 n} N$, is the value of $\lambda$ - $\lambda_{0}$, assuming the spectrum to be normal. The last term is the required correction expressed in terms of the provisional ware-length. The correction in actual practice has been made from a plot of the correction on a large scale, and never amounted to more than a few hundredths of a division of Angström, even for the longest plate.

In two or three plates the camera was displaced, so that $\gamma$ had a value. In such cases no attempt was made to measure $\gamma$, but the plates were only used for local interpolation by drawing a curve through certain points used as substandards.

These substandards were principally used for working up the last set of photographic plates containing the solar spectrum and the metal spectra of the same or higher orders, or both. Some of them contained three metallic spectra.

Thus the region 3900 in the solar spectrum has been obtained from both wave-lengths 5200 and 5850 . The mean of these gave values of the substandards for working up the plates taken at this point, and containing also metallic lines at $2 \% 00$.

Again, the boron lines 2496 and 2497 have been obtained from the regions 4800,3200 and 3600 . The mean values give substandards for working up the metallic spectra of that region. Also the near coincidence in the values of the wave-lengths of these lines indicate the relative accuracy of the regions $2496,3200,3600$, and 4800 .

The use of these substandards is as follows: The photographic plates, mostly 19 inches long, were measured mostly on a machine giving wavelengths direct. The differences of the results from the substandards were then plotted on a paper having the curve of correction for length upon it in such a way that the final marks should theoretically be a straight line. This was actually the case in all but a few plates, in 
which the camera was displaced. A straight line was then passed through all the marks as nearly as may be, and the correction taken off. This correction could thus be obtained to $\frac{1}{1000}$ division of Angström, and amounted to only a few hundredths of a division at most. Possibly $\frac{1}{10}$ division of Angström was the greatest correction required for length.

In this way each plate represents the average of all the wave-length determinations throughout its extent, and will not admit of any correction save a linear one, should such ever be required in working over the table again.

In every plate having a solar and metallic spectrum upon it, there is often-indeed always - a slight displacement. This is due either to some slight displacement of the apparatus in changing from one spectrum to the other, or to the fact that the solar and the electric light pass through the slit and fall on the grating differently. In all cases an attempt was made to eliminate it by exposing on the solar spectrum, both before and after the arc, but there still remained a displacement of $\frac{1}{100}$ to $\frac{2}{100}$ division of Angström, which was determined and corrected for by measuring the difference between the metallic and coinciding solar lines, selecting a great number of them, if possible.

The changes from sun to arc light are much more extensive than from one order of solar spectrum to another. In two cases I have tested the latter and found no displacement, and have no fear that it exists in the others.

In working up the plates, I have started at the plates whose centre is at wave-length 4600 , and proceeded either way from that point. For this purpose I have used the plates originally obtained for metallic spectra, generally using the lines due to the impurities. The method, I believe, is obvious from the table. For a long region no substandards are necessary, but are used whenever they become so.

[The tables are omitted.] 


\section{THE SEPARATION OF THE RARE EARTHS}

[Johns Hopkins University Circulars, No. 112, pp. 73, 74, 1894]

In the course of several years' investigations of the so-called "rare earths," such as yttrium, erbium, holmium, cerium, etc., I have devised several methods for their separation. I wish to give an account of these now, and hope soon to be able to publish a complete description of my work and its results.

It was evident very early in the work that cerium, lanthanium, praseodymium, neodymium and thorium differed from the yttrium group, and I have seen no reason to suppose that they can be divided any further. All of these "earths" appear, in varying proportions, in such minerals as gadolinite, samarskite, yttrialite, cerite, etc. Besides the elements of the cerium group here present there are at least seven other substances. For the present I shall speak of them as

$$
a, b, i, d, h, n, c, k \text {. }
$$

Their properties are as follows:

\section{Properties of Elements}

\section{Substance a}

This is the principal element of yttrium and may possibly be divided into two in the future, as I have observed a variation in the are spectrum on adding potash or soda. However, this is no more evidence than occurs in the case of iron or zirconium. I give a process below for producing this pure.

Properties.-No absorption bands. Oxalate and oxide pure white. It occurs in the sun. Its properties are those of yttrium as hitherto obtained, but I am the first to obtain it with any approach to purity.

\section{Mixture of $b, i$ and $d$}

These seem to be the principal ingredients in so-called "erbium."

Oxalate is red. Oxide is pure white. Absorption band is that of "erbium." It colors the electric arc green, and shows the "erbium" emission bands on heating white hot. The substance $b$ is strong in gado- 
linite and weak in samarskite. The solution has the absorption bands of " erbium" and most of these seem to belong to $b$ rather than $i$. However, we can readily prove that the absorption bands of erbium belong to two substances, as we can produce a decided variation in it.

I cannot reconcile this with my spectrum work without assuming a fourth ingredient in " erbium."

Substance $b$ is in the sun, but not $i$. With $b$ and $i$ the substance $d$ always occurs.

\section{Substance d}

This is the principal impurity of a sample of yttrium, kindly furnished me by Dr. Krüss, which my process of making yttrium separates out. It has not been obtained pure, but occurs strongly in the yellow part of the oxides. It is in the sun.

By aid of ferrocyanide of potassium the substance $a$ can be obtained pure from $d$. With this exception $d$ occurs in all the preparations of the yttrium group and cannot be separated from $b, i, c, n$, $h$, or any of the other substances. Indeed, I have found it in some specimens of cerium and lanthanium, although in traces only.

On account of the trouble caused by it and its universal presence, I propose the name demonium for it.

Its principal spectrum line is at w. 1.4000 .6 nearly.

\section{Substance $h$}

This occurs mainly in samarskite. Hints toward its separation will be given below, but I have otherwise obtained none of its properties.

\section{Substances $n, k$ and $c$}

These always occur with $d$ and form a group intermediate between the yttrium and cerium groups. They can be separated from these by sulphate of potassium or sodium by always taking in intermediate portions of the precipitate. They seem to have a weak absorption spectrum in the visible spectrum and strong in the ultra violet, especially $k$.

\section{Chemical Separation}

The first process that suggests itself is that by the sulphates of soda or potash. This is the usual method for separating the cerium from the yttrium groups. When the solution of earth and the sulphate solution are both hot and concentrated, everything except some scandium comes down. When ione in the cold with weaker solutions, there is more or less complete separation of the cerium group. Let the mixed 
earths be dissolved in a very slight excess of nitric acid and diluted somewhat (possibly $1 \mathrm{k}$. to 2 or 3 litres). Place in a warm place, add lumps of sulphate of soda, and stir until no more will dissolve. Continue to add and stir for a day or two until the absorption lines of neodymium disappear from the solution. Filter off and call the solution No. 1. Add caustic potash to the precipitated sulphates and wash so as to leave the oxides once more. Dissolve in nitric acid and precipitate again with sulphate of soda, calling the filtrate No. 2. Proceed in this way possibly 10 or more times. The filtrates contain less and less earths; and the precipitate is more and more the pure cerium group; but a dozen precipitations still leave some impurity.

The portions 1, 2, 3, etc., show decreasing "erbium" absorption bands, and the spectrum shows that the substances $a, b, d, i$ are gradually separated out with parts 1,2 , etc., while the numerous fine lines belonging to $d, n, c$, etc., with the cerium group, fill the spectrum of the portions $8,9,10$, etc. This intermediate group has only very weak absorption bands and evidently has three or four elements in it, as I have produced, at least that number of variations in its spectrum. The group can be obtained fairly free from $a, b$, and $i$, but the substance $d$ persists in all the filtrates and in the precipitated cerium group also. This intermediate group $d$, $n$, etc., seems to be in greater proportion in samarskite than in gadolinite, and there seem to be more elements in samarskite than in gadolinite. One of these I have called $h$.

The oxides, especially for samarskite, are very yellow and dark.

Sulphate of potash has a decided action in separating $a$ and $i$ from $b$, $a$ and $i$ coming down first. After two months, the solution gradually drying, the proportion of $b$ to $a$ in the filtrate increased many times. Sulphate of soda has an action of the same kind, but much weaker. After leaving two months over sulphate of potash and soda, the following was the result of analysis of the soluble part as compared with the original mixture:

Ce., La., etc.
$a$
$b$
$c$
$d$
$i$
$o$

Sulphate of Potash.
$o$
Weak
Mach stronger
$o$
Unchanged
Wcaker
Stronger

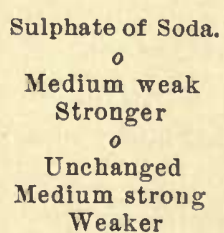

The oxide of the members of this group which are only slightly precipitated by the sulphates of soda and potash is pure snow-white, and hence those of $b$ and $i$ must be so. 
The substance $d$ comes down slightly sooner than $a$ by sulphate of soda, but slightly slower by sulphate of potash. Hence, in purifying yttrium (substance $a$ ) for the last time from the $c$. group, sulphate of potash will increase $d$ in the filtrate and sulphate of soda will decrease it.

\section{Action of oxalic acid}

When the oxalates of the mixed earths, free from the ce. group, are boiled in water to which nitric acid is added, they are nore or less dissolved, leaving a coarse, heavy, red oxalate yielding a pale yellow oxide. The filtrate, set aside to cool, deposits more of the oxalates and leaves a filtrate which contains several of the unknown elements, as also what remains of the ce. group. On separating the ce. group the remainder is quite different from the heary red oxalate, but there is far from complete separation. The analysis showed the following:

$$
a, b, c, d, h, i, n \text {. }
$$

I have not found the separation particularly useful, and it seems to be more apparent than real as tested by the spectroscope.

\section{Ferrocyanide of potassium}

This is the most useful process and easily separates the element $a$, pure and free from all others. To obtain pure $a$ from the mineral gadolinite, Fergusonite or Samarskite:

First obtain the crude mixed earths in the usual manner. Then separate the cerium group as usual until the absorption bands of neodymium no longer appear. For the complete separation without loss this must be done several times, as much of the yttrium group is carried down with the first precipitate, as we have before seen.

The separation of the yttrium (a) from the other elements is effected by precipitating the latter from a weak acid solution by ferrocyanide of potassium. For this purpose the filtrate, after separating the cerium group, can be used at once by slightly acidulating with nitric acid, diluting and adding a weak solution of ferrocyanide of potassium. No precipitate should appear at once, but by standing for an hour or so some will come down. Add more ferrocyanide of potassium and repeat until the filtrate no longer shows the bands of so-called erbium. After this it is best to precipitate with oxalic acid or oxalate of potassium and ignite the precipitate so as to get the earth. Dissolve this in nitric acid and add only water enough to make a very concentrated syrupy solution. 
Place in a beaker at least three inches in diameter and examine with a spectroscope of low power for absorption bands. Probably the bands of neodymium and "erbium" will appear. Separate the first by sulphate of sodium as usual, and the last by ferrocyanide of potassium from an acid solution as above. The filtrate will then contain the pure yttrium $a$, whose calcined oxalate will be pure white without trace of yellow. After separation of iron, calcium, and possibly manganese, the earth will be a pure element as far as I can tell spectroscopically. However, like $\mathrm{Zr}, \mathrm{Fe}$ and many other substances, the addition of $\mathrm{Na}$ or $\mathrm{K}$ to the electric arc while obtaining the spectrum will change the intensity of certain lines of the spectrum, while others are unchanged. If this is considered as evidence of the existence of two elements, then the same evidence will apply to $F e$ and $Z r$. The reason for believing that the substance thus found is an element is based on the fact that its spectrum remains unaltered in all minerals and after all chemical operations that I have been able to devise. Furthermore, I believe that the new process is not only more easy than any other, but also that it has given a single element for the first time, as it eliminates the element $d$. The yield will of course depend on the amount of purity required. From the earths of gadolinite about one-tenth of quite pure yttrium $(a)$ can be obtained and about one-twentieth of very pure.

I have determined spectroscopically that when, by the above process, the absorption band of "erbium" at last disappears from 3 in. of strong solution, all the other elements have also disappeared.

By taking the first precipitate several times by ferrocyanide of potassium from an acid solution, a mixture of many elements is obtained which contains much of that element to which the so-called "erbium" band is due. By dissolving a weighed quantity of this mixture in nitric acid and water and examining the band spectrum, I have determined the limit when the band can no longer be seen. Thus I have proved that when the band vanishes from 3 inches of concentrated syrupy solution of yttrium there cannot exist in it more than $\frac{1}{2}$ per cent of the mixed element as compared with the yttrium, and there is probably less.

I have not found ferrocyanide of potassium useful in the further separation of the elements, but only in separating out $a$ from the others.

When the neodymium band has disappeared by use of sulphate of sodium, all the other elements of the cerium group have disappeared. The element thorium is sometimes present in the crude earths, but disappears after a while fron the purified earths. The conditions for its disappearance I have not determined. 
The elements which persist to the last by the ferrocyanide process are $b$ and $i$, while by Krüss' process the element $d$ persists the longest. As $b+i$ has an absorption spectrum and $d$ probably not, the test of purity by absorption bands is very complete in the new process:

Note.-For help in this investigation my thanks are due to a large number of gentlemen. Professor Schapleigh has sent me a large collection of substances, Mr. Hidden, Professor Wolcott Gibbs, and Professor F. W. Clarke many minerals, Professor Krüss several specimens, and Professor Barker and others have belped me in many ways. 


\title{
57
}

\section{NOTES OF OBSERVATION ON THE RÖNTGEN RAYS}

\author{
By Henry a/ Rowland, N. R. Carmichael and L. J. Briggs
}

[American Journal of Science [4], I, 247, 248, 1896 ; Philosophical Magazine 15], XLI, $381,382,1896]$

The discovery of Hertz some years since that the cathode rays penetrated some opaque bodies like aluminium, has opened up a wonderful field of research, which has now culminated in the discovery by Röntgen of still other rays having even more remarkable properties. We have confirmed, in many respects, the researches of the latter on these rays, and have repeated his experiment in photographing through wood, aluminium, cardboard, hard rubber, and even the larger part of a millimeter of sheet copper.

Some of these photographs have been indistinct, indicating a source of these rays of considerable extent, while others have been so sharp and clear cut that the shadow of a coin at the distance of $2 \mathrm{~cm}$. from the photographic plate has no penumbra whatever, but appears perfectly sharp even with a low power miscroscope.

So far as yet observed the rays proceed in straight lines and all efforts to deflect them by a strong magnet either within or without the tube have failed. Likewise prisms of wood and vulcanite have no action whatever so far as seen, and, contrary to Röntgen, no trace of reflection from a steel mirror at a large angle of incidence could be observed. In this latter experiment the mirror was on the side of the photographic plate next to the source of the rays, and not behind it, as in Röntgen's method.

We have, in the short time we have been at work, principally devoted ourselves to finding the source of the rays. For this purpose one of our tubes made for showing that electricity will not pass through a vacuum was found to give remarkable results. This tube had the aluminium poles within $1 \mathrm{~mm}$. of each other and had such a perfect vacuum that sparks generally preferred $10 \mathrm{~cm}$. in air to passage through the tube. By using potential enough, however, the discharge from an ordinary Ruhmkorff coil could be forced through. The resistance being 
so high the discharge was not oscillatory as in ordinary tubes but only went in one direction.

In this tube we demonstrated conclusively that the main source of the rays was a minute point on the anode nearest to the cathode. At times a minute point of light appeared at this point, but not always.

Added to this source the whole of the anode gave out a few rays. From the cathode no rays whatever came, neither were there any from the glass of the tube where the cathode rays struck it as Röntgen thought. This tube as a source of rays far exceeded all our other collection of Crookes' tubes and gave the plate a full exposure at 5 or $10 \mathrm{~cm}$. in about 5 or 10 minutes with a slow-acting coil giving only about 4 sparks per second.

The next most satisfactory tube had aluminium poles with ends about $3 \mathrm{~cm}$. apart. It was not straight, but had three bulbs, the poles being in the end bulbs and the passage between them being rather wide. In this case the discharge was slightly oscillatory, but more electricity went one way than the other. Here the source of rays was two points in the tube, a little on the cathode side of the narrow parts.

In the other tubes there seemed to be diffuse sources, probably due in part to the oscillatory discharge, but in no case did the cathode rays seem to have anything to do with the Röntgen rays. Judging from the first two most definite tubes the source of the rays seems to be more connected with the anode than the cathode, and in both of the tubes the rays came from where the discharge from the anode expanded itself toward the cathode, if we may roughly use such language.

As to what these rays are it is too early to even guess. That they and the cathode rays are destined to give us a far deeper insight into nature nobody can doubt.

Baltimore, Feb. 20, 1896. 


\section{NOTES ON RÖNTGEN RAYS}

By H. A. Row'land, N. R. Carmichael and L. J. Briggs

[Electrical World, XXVII, 452, 1896]

In the 'American Journal of Science' for March we made a few notes of our researches on the Röntgen rays, reaching the provisiongl conclusion that the main source of the rays was at the anode, and that the cathode rays seemed to have nothing to do with the phenomena presented. A further study of the source of the rays in many other tubes has led us to modify this conclusion somewhat, for, while we still think the anode or its equivalent is the main source of the rays, yet we now have evidence in some of the tubes that it is necessary for the cathode rays to fall on the anode in order that the Röntgen rays may be formed.

In our tubes with a very high vacuum the other sources of rays are very faint indeed. We have never obtained any rays from the cathode except in one case, where undoubtedly there were electrical oscillations which made the cathode momentarily an anode. It can be readily proved that these oscillations always exist in the case of low resistance tubes, and these are probably the cause of many errors in estimating the source of the rays.

In some cases we have found very faint sources of rays as Röntgen found them, where the cathode rays struck the glass, but not where they struck a piece of platinum kept at nearly zero potential. On the anode theory, this might be explained by the fact that the bombarding cathode rays, coming in periodical electrified showers, alternately raise and lower the potential of the glass, thus making it alternately an anode and cathode. In the case of the platinum, this could not occur to the same extent.

That feeble Röntgen rays emanate from some bodies when bombarded by the cathode rays, we are wiling to admit, and, in fact, had long ago come to that conclusion. But we do not agree with Prof. Elihu 'Thomson's general conclusion that these rays are always given out from bombarded surfaces, as we have a tube, with platinum in the focus of a concave electrode, which emits no rays whatever from the platinum, even 
when the platinum is red hot from the l,ombardment, the concave electrode being the cathode and a third wire the anode.

The same tube, with the platinum made an anode and the concave electrode a cathode, produces a profuse radiation of Röntgen rays in all directions on the side of the platinum bombarded by the cathode rays, and none on the other side. In the first case we obtained no rays from the cathode, no rays from the bombarded surface, and only a very weak effect from the anode, indeed almost nothing. Hence the condition for the production of the rays seems to be neither the one or the other but a combination of the two, and we now believe as far as we can yet see that the necessary condition for their production is an anode bombarded by the cathode discharge. The anode may be, howerer, an induced anode formed on the glass, and the cathode rays may vary a great deal and cease to present the usual appearance of cathode rays.

Thus, in the best tube that we have, originally made for showing that electricity will not pass through a vacuum, the main source is a point on the end of the anode, where a little point of light appears. Sometimes, across the little interval of $1 \mathrm{~mm}$. between the electrodes, a faint spark or arc crosses from one electrode to the other, and we think that the rays come out especially well under these conditions. Here the action of the bombarding cathode discharge is rather obscure. This little point of light also sometimes appears on the red hot platinum anode mentioned above, and we have seen it in other tubes, always at the place where Röntgen rays are apparently found.

Prof. Elihu Thomson has kindly sent us some sketches of tubes having the anode bombarded by the cathode, and we had previously designed some tubes of similar shape, but have not yet found anybody in this country capable of making a sufficiently good vacuum. In many of our best tubes the vacuum is so perfect as to cause a resistance equal to a five or six inch spark in the air. The better the vacuum the greater the number of rays sent out.

However, for sharpness of detail, nothing equals the perfect vacuum tube, having its electrodes one $\mathrm{mm}$. apart. Such a tube has been designed by one of us, but we have not been able to get the proper exhaustion.

As to other sources of Röntgen rays, we have tried a torrent of electric sparks in air, from a large battery, and have obtained none. Of course, coins laid on or near the plate under these circumstances, produce impressions, but these are, of course, induction phenomena.

As to sunlight, Tyndall, Abney, Graham Bell and others, have 
shown that some of the rays penetrate vulcanite and other opaque bodies, and we have only to look at an unpainted door, on the other side of which the sun is shining, to convince ourselves that sunlight penetrates wood to a considerable depth.

As to the theory of the Röntgen rays we know little. If the rays are vibrations we can readily determine a rough limit to their length, from the sharpness of the shadows.

Thus our photographs have such sharpness that the complete waves cannot be more than $.0005 \mathrm{~cm}$. long, but are probably much shorter. This is independent of whether the waves are longitudinal like sound or transverse like light, and of course only applies to that portion of them which affects the photographic plate. There may be others of larger size that do not affect the plate.

All efforts to bend the rays from their course, either within or without the tube, by means of a strong magnetic field, have failed, both in our hands and in those of others, and thus, if the rays are radiant particles of matter, they cannot be highly charged particles like the cathode rays. The rays are not refracted by any solid bodies so far tried, and this seems to be against their being waves either in air or ether. They pass through solid bodies, and thus their wave-lengths cannot be very small. We have before seen that it cannot be very great. They cannot be sound waves as they proceed for some distance through a very perfect vacuum.

Altogether we are at a loss for a theory. If we have not yet got a satisfactory theory of light after more than a hundred years of labor, how can we hope to have a theory of the Röntgen rays after knowing of them for only a few months? Let us suspend our judgment for a while, and let us, above all things, be willing to alter our opinions at any moment when fresh light appears. 


\section{9}

\section{THE RÖNTGEN RAY, AND ITS RELATION TO PHYSICS}

\section{(A Topical Discussion)}

[Transactions of the American Institute of Electrical Engineers, XIII, 403-410, 430, 431, 1896]

\section{Opening Remarks by Prof. Henry A. Rowland}

Mr. President and Gentlemen: A gentleman asked me a few moments ago if $I$ knew anything about the X-ray. I told him no; that what I was going to tell to-night was what I did not know about the X-ray. I do not suppose anybody can do any more than that, because all of us know very little about it. We were very much surprised, something like a year ago, by this very great discovery. But I cannot say that we know very much more about it now than we did then. The whole world seems to have been working on it for all this time without having discovered very much more with respect to it.

Now, I suppose it is not necessary for me to go into the history of the thing. We all know it; how Lenard first, probably, discovered these rays, or discovered something very similar to them; how Röntgen afterwards found their particular use, their penetrating power, and so on, although Lenard had found something similar to that before. It is thus not necessary for me to go into the history of the matter, but simply to go over, to some extent, what we know with regard to these rays at the present time. First, there was some discussion, some time ago, as to the source of these rays. Röntgen found that their source was any point that the cathode rays struck upon; and you will remember that when we first knew about these rays they were often called cathode rays. Many persons thought that the cathode rays came through the glass, and Lenard first thought that they did come through his little window, and it is probable that they do at the present time. But the kind of rays that we are considering are very different from the cathode rays. Six months ago there was quite a discussion in regard to the source, and I believe it was finally determined that they came from points where the cathode rays strike. At the same time I was rather opposed to that. In one of my tubes $I$ found that the rays came from 
the anode. I had only the ordinary assortment of Crookes' tubes, and one of the tubes had aluminum wires which were a millimeter apart. In one of these the source of the rays was a point upon the anodenot upon the cathode at all. It was a very small point. The photographs which I obtained by that tube were sharper than any I had seen before. They are so very sharp that in estimating the shadow of an object I determined that the point could not have been a thousandth of an inch in diameter. Therefore the source in this case was a very minute point upon the anode, and that point was nearer the cathode, and I suppose some of the cathode rays might have struck upon it, and it might have obeyed the law that the point where these $\mathrm{X}$-rays are formed is the point on the anode where the cathode rays strike.

I had another very interesting tube, and I was going to bring some of the photographs here to-night; but I thought they were so small that it would be almost impossible to see them. I tried the three cases in this tube: First, the case where the cathode rays strike upon the anode. In that case I got very many Röntgen rays. Then I tried the case where the cathode rays strike upon an object-a piece of platinum. I did not get any rays whatever then. Now, some people say that they, come from the point where the cathode ray strikes. I did not get any whatever in that case. In this case the cathode ray struck upon a piece of platinum in the centre of a bulb, and no rays: were given out by the anode either. Therefore I seemed to have a crucial experiment in each; I seemed to have the case where the cathode ray strikes upon the anode, and I got lots of rays. Then I had the case where the cathode rays strike on a piece of platinum, and I did not get anything at all. Then where the anode itself was free and no cathode rays struck it, I did not get anything from it. It seemed to me as if the source was most abundant when the cathode ray struck upon the anode; and that is the theory, we know, upon which nearly all tubes are formed at the present time. You have the focus tubes in which you focus the cathode rays upon the anode, and in that case you have a very abundant source of rays; but I do not believe you ever could get as small a source of rays as I got with that first tube, where I had a source of a thousandth of an inch diameter. Having such a small source of rays, it gave me a limit to the wave-length, if there were waves at all; it would give me a limit to the wave-length of which I will speak in a moment. As to whether there are any rays where the cathode rays strike on any other objects, we know that there are very feeble ones. It seems to be almost necessary in order to get an abundant source that you should have cathode 
rays strike on the anode. However, that is a point of discussion. Now, as to the source of electricity, we have generally the Ruhmkorff coil. There is one source of which I saw a little note in 'Nature,' where a man had used a large Holtz machine with very good effects. Now it is very much easicr for many persons to use a Holtz machine than to use a Ruhmkorff coil. There are many cases where one cannot have a large battery; and this man said that with the Holtz machine he got as great an effect as with the Ruhmkorff coil. Then we have the Tesla coil, etc. By the way, speaking of the Tesla coil, I am not sure but that you might look back and find that it is very similar to the Henry coil. Henry originally experimented on the induction of electricity, transmitting a spark of electricity from one coil and getting a spark from another, and the Tesla coil is something like that, except that it is made so as to produce a much more voluminous spark.

We all know the properties of the Röntgen rays - they go in a straight line. Every effort to deviate them from a straight line, by any means whatever, has failed, except that when they strike upon an object they are reflected. Now, it is a question for discussion as to whether there is any regular reflection. They strike upon an object, and you get something from that object which will affect a photographic plate. Are those rays which you get from the object Röntgen rays still, or do the Röntgen rays strike upon this object and generate in it some sort of rays which come out, different from the Röntgen rays, and affect the plate? We do not know that. Neither are we quite positive whether there is any reflection of the rays. We know there is turbid reflectionyou may call it-rays strike on the object, and the object becomes a source of rays of some kind. Nobody has ever found out what sort of rays come from the object. Something comes from it, and we generally imagine, and indeed we often state, that they are Röntgen rays that come off the object. But we have good reason to suppose that they may be something else; and they may or may not be regular reflections; some persons say they are and some that they are not. I have seen some photographs made in this city which indicated regular reflections. At the same time I would not be positive as to whether there was any regular reflection. It is rather doubtful. It is a point to be determined.

Then the fluorescence-that is the way Röntgen originally found the ray. You know the way they produce fluorescence-the photographic effect-you all know that. You all know that the magnet does not affect them-does not turn these rays from a straight line.

The polarization of the rays: We have no evidence whatever as to 
the polarization. If they were very small waves, transverse waves, like light, we ought to be able to polarize them. Becquerel, by exposing certain phosphorescent substances to the sun, obtained from them certain rays which penetrated objects like aluminium,.etc. But these rays were evidently small rays of light, because he could polarize them, and he could refract them, and they were probably very short waves of ultra violet light. But we never have been able to discover that there was any such effect in a Röntgen ray. Some persons have claimed that they got polarization; but if there ever was any polarization, it is very small, indeed. One of the principal advances in respect to these rays is that made by J. J. Thomson, in considering the electric discharge of bodies. $\mathrm{He}$ has published most valuable results with regard to the effect of these rays upon gases. When the rays fall upon a gas, they affect the gas in some way so that it becomes a conductor. Now, you can subject the gas to these rays and allow the gas to go through a tube off into another vessel, so that it will discharge an electrified body in that vessel. But he has found the most interesting result that it will not continue long to affect these bodies. After one has allowed a certain amount of electricity to pass through it, it then becomes an insulator again. It only allows a certain amount of electricity to go through it. That is easily explained-or you can explain it-by the Röntgen rays liberating the ions, and only a certain amount of them. Just as soon as these are used up in the conduction of the gas, then it ceases to conduct. So that a certain amount of gas will conduct a certain amount of electricity, and then it stops conducting. That is a most interesting result. It is one of the great advances we have made since Röntgen's discovery. Röntgen knew nearly all we know now about these rays. We have discovered very little indeed; but that point I think we have at least discovered.

Then it is said that these rays affect a sclenite cell in the same way that light affects it-it changes the resistance of the selenite cell.

Of course, we are only considering the theory to-night; at least I am, and we do not have to consider the bones, and so on. I have had some students at work in my laboratory, and it was with the utmost difficulty that I kept them from photographing bones. Bones seemed to be the principal object to be photographed by the Röntgen rays when they were first discovered, and I suppose it is the same now. Most people connect Röntgen rays with bones; but I do not intend to say very much about them.

Now, one important point with respect to these rays is as to whether 
they are homogeneous. Are they like light which can be divided up into a large number of different wave-lengths, or are they homogeneous? There seems to be a great deal of evidence that they are not all the same; that one ought to get a spectrum of them in some way. We can filter them a little bit through objects. After they are filtered through an object, they are probably a little different from what they were before, and some objects probably let through different rays from others. In 'Nature' Mr. Porter, I believe, has shown experiments upon that. $\mathrm{He}$ divides rays into three kinds. At least he finds that under certain circumstances the rays will penetrate bones better than in other casesbones or any other object-they have more penetrating power, and they go through many of those objects that ordinarily stop them. By heating up the tube, and by various arrangements of his spark-gaps, etc., and putting little wires around his tubes, and so on, he can cause them to generate different kinds of rays. That is a very important point, if it is substantiated, and there seems to be little reason to doubt that a number of rays really do exist; that whatever they are that come from the object, they are not all the same; some of them penetrate bodies better than others, and very likely some one will get up some sort of filter that will filter them out, and allow us to use them and to find if they have different properties. At the present we are rather in the dark with regard to this point.

Now I come to the theory of these rays. What is the cause of all these phenomena? There was a time when we were rather selfsatisfied, I' think, with regard to theories of light. We thought that Fresnel and others had discovered what light was-some sort of vibration in the ether; we called it ether; if it had these, waves going through it, then it would produce light, and we were pretty well convinced that the waves were transverse, because we would polarize them; so that we began to be satisfied that we knew something about light. Then Maxwell was born, and he proved that these rays were electromagneticvery nearly proved it. Then Hertz came along and actually showed us how to experiment with these Maxwell waves, most of which were longer than those of light. At the same time they were of the same nature. Well, we got a rather complicated sort of ether by that time. The ether had to do lots of things. One must put upon the ether all the communication between bodies. For instance, what communication is there between this earth and the sun? Why, you have light coming from it and heat. Radiation you might call it all. We have radiation. Then some people thought they discovered electromagnetic disturbance 
from the sun. Sometimes they have seen a sun spot and noted a deflection of the magnetic needle on the earth. Very likely that is true. I don't know that they have discovered any electrostatic effect. But we know that electrostatic effects will be carried on through as perfect a vacuum as you can get. Then we have gravitation action too. Now, you have got all those things-electromagnetic action, light which would be an electromagnetic phenomenon, and then we have gravitation, and we have got to load the ether with all those things. Then we have got to put matter in the ether and have got to get some connection between the matter and the ether. By that time one's mind is in a whirl, and we give it up.

Now we have got something worse yet-we have got Röntgen rays on top of all that. Here is something that goes through the ether, and it not only goes through the ether but shoots in a straight line right through a body. Now, what sort of earthly thing can that be? A body will stop light or do something to it as it goes through; but what on earth can it be that goes through matter in a straight line? Why, our imagination doesn't give us any chance to do anything with that problem. It is a most wonderful phenomenon. Now, we can suppose that they are ultra violet light. Indeed, we can get a limit to the wavelength to some extent. Nobody, however, has ever proved that the Röntgen rays are waves. But we can get a limit of the wave-length if they are waves, because when I have a tube that gives me a shadow which is only a thousandth of an inch broad, or rather from the greatest intensity out to clear glass a thousandth of an inch broad, I can calculate the wave-length of the thing that would produce such a shadow. It has got to be very small indeed; one knows that right away, because any ordinary light would make a few waves at the edge of the shadow, and by measuring those waves you could get the wave-lengths of the light. But there was no appearance whatever on any of my photographs of any such phenomenon as that. I did not have any of these waves at the edge of the shadow whatever. It went directly from blackness to light. But putting it under the microscope and measuring from almost imaginary points, from lightness to darkness, I could get a limit to the wavelength. Now, as to that limit, I published it in one of the journals six months ago, or more, and it came at about one-seventh, I think, that of yellow light. Others have determined the wave-length and got even below one-seventh that of yellow light. Some have got onethirtieth that of yellow light, and so on. Some of them I am rather doubtful about, because they say they have bands. If they have bands 
and diffraction bands, that would prove instantly that the Röntgen rays are waves. But $I$ have never seen the slightest phenomenon of that sort. It is very doubtful that it exists, and those persons who have had it will have to show their photographs very clearly to make us believe it. And therefore we have no evidence whatever that the rays are waves. At the same time we have no evidence that they are not waves. They might be very short waves-infinitely short waves. Let us see what would happen if they were infinitely short waves. They might be so very short as to be too fine-grained for any of our methods of polarization or reflection. Waves are reflected from a solid bodyregularly reflected, because they interfere after they come from the body. You can get the direction-the angle of incidence equals the angle of reflection; you can get that by means of considering them as waves and as interfering after they come from the object. Well, if the object, however, is a very rough sort of thing compared with the wavelength, you will not get a regular reflection. That is what might happen in the case of Röntgen rays. And then again, with regard to refraction of the light, the theory of refraction which comes from considering molecules imbedded in the ether will give you some limit. When we go beyond that limit, we get no refraction. The bending of the violet rays increases up to a certain point and then goes back. We have a case of anomalous refraction very often in some substances like fuchsine, aniline dyes, and so on. Therefore the action of refraction can be accounted for by having very short waves. But when we treat of the theory of the case we have the little molecules of a gas knocking against each other, and they can only go a little distance. We call that the free path of the gas-a very small distance in the ordinary air. Those molecules cannot go more than this very small distance before they stop. Well, now, why should little, short waves of light pass through the gas and not be stopped too? When the waves are very short indeed, it seems to me that the object would be entirely opaque to them, because they would strike upon those molecules, unless they could pass directly throngh the molecules. You would therefore necessarily have these little short waves going directly through the molecules, which we generally think is almost impossible in case of light. And that is one very great objection that I have to that theory.

Then we have another theory-that these are not transverse waves at all; that they are waves like sound, and very short indeed. Well, what would happen then? If they are very short indeed, you have the same objection: They would all strike against the molecules, and they 
would be dispersed very quickly. The shorter the wave-lengths, the more they are dispersed. Take, for instance, short waves that bob against a boat and are reflected back. Thus, if you have a big, long ocean wave, it sweeps around a boat and goes on without being troubled by the boat at all. The shorter the waves, the more they are bothered by the boat, and so it is with respect to other waves - the short waves would probably be stopped by the molecules. So I do not see what we can do with regard to it in that respect. Aceording to Maxwell's law, waves like sound do not exist in the kind of ether that he suggested. But that is all based upon a certain theory that the lines of force were always closed.' He introduced into his equation an expression which indicated that every line of force was a closed path coming back upon itself or ending in electricity, one or the other. Now, if we throw out that, then we can get this kind of compressional waves in the ether. Now, it is not at all impossible that they exist, and as to whether they would go through molecules any better than light waves do, nobody can tell; but it is possible that they might. But if there are waves at all, they must be very short waves. You cannot get over that fact-if they are waves at all, they must be short.

Then, of course, you have the other theory-of little particles of matter flying out from the body, passing through the glass and all other bodies, until they reach a photographic plate or any other place where we are notified of their presence, and these little particles make their way through the air or any other substance. Now, why should not the little particles be stopped very quickly by bodies as well as if the rays were waves? You see we are in trouble here too. Why are not the waves stopped? Why are not the little particles stopped? Stokes has given some sort of a theory with regard to this-that, instead of having a wave motion in the ether, the rays are impulses-a sudden impulseone wave, for instance-not a series of waves at all, but one impulse coming out from the tube. I think if he had seen any very sharp shadows obtained from the Röntgen rays he would not have given that theory. He probably has seen only those very hazy outlines that very many persons take for Röntgen photographs. But if he had seen any very defined ones-very sharp ones-he probably would not have given that theory, because if the Röntgen rays are waves at all, they must be short, and there must be a long series of them to make sharp shadows. This is why Newton gave up the wave theory of light. You remember he gave up this theory because he found that light went straight past an object instead of curving around into the shadow as much as sound 
does. But he was not quite up to his usual pitch when he made that statement, because if he had thought a moment he would have seen that very short waves will go more nearly in a straight line than long ones. But any single impulse, such as Stokes suggests, would go into the shadow. The only wave motion that would go in a straight line is a series of waves, one after another. Therefore, these rays cannot be single impulses coming irregularly.

Prof. Michelson has suggested a theory of rays based on something like vortex rings in the ether. Now, if we have an ether that can carry on light waves and electromagnetic waves, it cannot be a perfect fluid; it has got to be something else. You cannot very well imagine vortex rings in such an ether. So that we are met at every point by some objection. We have been studying light for hundreds of years; we are not anywhere near satisfied with the theory yet, and we cannot very well be expected to be satisfied with the theory of Röntgen rays in one year.

Well, I think that is all I can say with regard to the subject, and I hope the other gentlemen who are to carry on the discussion will satisfy you on all these points that I have brought up and left unanswered.

[There followed a discussion by Professor Elihu Thomson, Professor M. I. Pupin, and others.]

Prof. Rowland:- I made a few notes with regard to what has been said, but they are made in such a way that I do not believe that I can interpret them myself, especially as the hour seems to be getting rather late. One or two remarks, however, I would like to make. When Prof. Thomson said that he got such a large amount of rays from an insulated piece of platinum by letting the cathode rays fall upon it, he made a sketch. With the exception of this end, which was flat, that is the kind of thing that I used. Now, there was absolutely no effect when this was made an anode and this a cathode, so that all the cathode rays were striking on the platinum. I have the photograph; I got no effect whatever. Now, if Prof. Thomson got an effect in this case and I did not get an effect in that case, I have got a case, at least, where none of these rays were produced by the falling of the cathode rays upon the object. It doesn't make any difference how many other persons have something in which they do get an effect. If I did not get an affect, that is one case, understand. That is the case where the cathode ray fell on an object and I got no Röntgen ray. 
If other people got them in other ways, why, there is something else coming in. I don't know what it is.

Prof. Thomson:-I should like to say just there, Professor, if you would allow me, that I used exactly that arrangement first, and got rays with the concave cathode. The anode at this end and the interposed plate of platinum between, with that wire extending outward, is the standard form of Crookes' tube-the first tube, in fact, that I used. I got not only sharp effects but rays.

The Chairman:- Was the platinum red?

Prof. Thomson:-The platinum was red-yes, of course, and it was a vigorous source of rays. I got rays with the same tube that Professor Rowland does not get them.

Prof. RowlaND:-Well, that has nothing to do with the point. The point that I raise is this, that there was certainly no doubt that I did not get any, and the cathode rays were falling from the object. That is the thing. Now, one thing that I wish to remark is that most people draw a tube like that. They don't say where the wires go. Mine generally went out, so that they were very far away from this object. By curving wires around in different ways I can get an inductive action. I don't doubt that I could fix up a tube so that I could get lots of rays out of any part. However, the time is passing, and I will just say one word with regard to the point Prof. Thomson raised with regard to the fluorescence over the surface of the glass. He thought something was stopped by the glass. I must say that Lenard, when he first experimented upon this subject--and I regard his experiments as quite as valuable as Röntgen's, probably-, he got several kinds of rays coming out through an aluminium window. He got rays which were deflected by the magnet, as well as others. He had not separated them, however. When the Lenard paper came to the laboratory I remarked to my students: "That is the best discovery that has been made in many a day." I immediately set somebody to work experimenting. He tried to get some results and would probably have discovered the Röntgen rays at that time if it had not been that the University of Chicago called him off, and Johns Hopkins University was very poor and could not call him back, and he had to stop in the midst of his work. They always say in Baltimore that no man in that city should die without leaving something to Johns Hopkins. Now, Dr. Pupin mentioned a means of showing whether the rays were reflected-a little reflector in which he had them brought to a focus, as I recollect it. I have read an account in which an experimenter did find the rays were brought to a 
focus, showing, provisionally at least, that there was some regular reflection. But these experiments should all be repeated many times before one actually believes them. We don't always believe what we read.

Now, as to Helmholtz's theory of the motion of ether and so onwell, as I said before, what is the motion of the ether? What is motion of the whole ether? You cannot move the ether in the whole universe all at once, and if you do not move the ether in the whole universe all at once but only move a part, then it is a wave, so it amounts to the theory that I gave-an impulse, such as Stokes had. Now, an impulse such as Stokes had does not go in a straight line-it goes around corners-and it does not go in a straight line unless there are lots of waves coming out. We can readily prove that an ordinary molecule, vibrating to ordinary light, must give out a hundred thousand waves without much diminution of amplitude, or else you cannot have the sharp lines in the spectrum that we do. The molecule must vibrate a long time-longer than any bell that we can make. We cannot find a bell that will give out a hundred thousand vibrations without much diminution. For ethereal waves something must vibrate to produce them. What it is I don't know that there is any necessity for discussing, because you can discuss it forever and never get any nearer to it. Something vibrates. Now, the thing that vibrates we don't know. We don't know whether it is electricity or whether it is mechanical motion. We know nothing about it. I have often said to my students, when I showed them the spectrum of some substance like uranium, in which we were taking photographs which would be perhaps ten feet long-so fine in grain that you could not put the point of a pencil on it without finding a line. There were thousands of lines. I said to them: "A molecule of matter is more complicated a great deal than a piano. Counting the overtones and everything, you would not probably get up anywhere near the number of tones you get out of a single molecule of uranium. Therefore it rather looks as if the uranium molecule was very complicated." Of course, all those spectrum lines do not indicate fundamental tones-many are harmonics. - Still it is rather a complicated thing to get a spectrum in which there are many thousands of lines. So when I come to think what a molecule is and try to get up some theory of it, I quite agree with Dr. Pupin that we don't know anything about it. 


\section{4}

\section{DIFFRACTION GRATINGS}

[Encyclopoctía Britannica, New Volumes, III, 458, 459, 1902]

The grating is an optical instrument for the production of the spectrum; it now generally replaces the prism in a spectroscope where large dispersion is needed, or when the ultra-violet portion of the spectrum is to be examined, or when the spectrum is to be photographed. The transparent grating consists of a plate of glass covered with lampblack, gold leaf, opaque collodion or gelatine, the coating being scratched through in parallel lines ruled as nearly equidistant as possible. When the lines are to be ruled very close together, a diamond ruling directly on glass is used. Other transparent materials, such as fluor spar, are sometimes substituted for glass. For certain researches on long waves the grating is made by winding a very fine wire, 1-1000th inch in diameter, in the threads of two fine screws placed parallel to each other, soldering the wire to the screws and then cutting it away on one side of the screws. As the value of a grating is dependent upon the number of lines ruled, it is very desirable to have their number great. Glass is so hard that the diamond employed for the ruling wears away rapidly; and hence the modern grating is generally a reflecting grating, which is made by ruling on a speculum metal surface finely ground and polished. On such a surface it is possible to rule 100,000 lines without damaging the diamond, although its point even then often wears away or breaks down. The lines are generally so close together as 15,000 or 20,000 to the inch, although it is feasible to rule them even closersay 40,000 to 50,000 to the inch. There is little advantage, however, in the higher number and many disadvantages.

The grating produces a variety of spectra from a single source of light, and these are designated as spectra of the first, second, etc., order, the numbering commencing from the central or reflected image and proceeding in either direction from it. The dispersion depends upon the number of lines ruled in a unit of length, upon the order of the spectrum, and upon the angle at which the grating is held to the source of light. The defining power depends upon its width and the angles 
made by the incident and diffracted rays, and is independent of the number of lines per unit of length ruled on the grating. If this number is too small, however, the different order of the spectra will be too much mixed up with each other for easy vision. A convenient number is 15,000 to 20,000 lines to the inch, or from 6000 to 8000 to the centimetre. The defining power is defined as the ratio of the wavelength to the distance apart of the two spectral lines which can be just seen separate in the instrument. Thus the sodium or $D$ lines have wave-lengths which differ from each other by $\cdot 597 \mu \mu$, and their average wave-length is 589.3 $\mu$. A spectroscope to divide them would thus require a defining power of 988 . The most powerful gratings have defining powers from 100,000 to 200,000. Lord Rayleigh's formula for the defining power is

$$
D=N n \text {. }
$$

When $D$ is the defining power, $N$ is the order of the spectrum, and $n$ is the total number of lines ruled on the grating. As the defining power increases with $N$, and since we can observe in a higher order as the number of lines ruled in a unit of length decreases, it is best to express the defining power in terms of the width of the grating, $w$. In this case we have for the maximum defining power $D^{\prime}=20,000 w$ for small gratings, or $D^{\prime}=15,000 w$ for extra fine large gratings, $w$ being the width of the gratings in centimetres. It is seldom that very large gratings are perfect enough to have a defining power of more than $10,000 w$, owing to imperfection of surface or ruling. The relative brightness of the different orders of spectra depend upon the shape of the groove as ruled by the diamond. No two gratings are ever alike in this respect, but exhibit an infinite variety of distributions of brightness. Copies of glass gratings can be made by photography, contact prints being taken on collodiochloride of silver or other dry plates. Reflecting gratings can be copied by pouring collodion or gelatine over the grating and stripping off the films thus formed. The latter warps, however, and destroys the definition to a great extent. The grating always produces a brighter spectrum in the violet than a prism. In the green the reflecting speculum metal grating may be brighter than a prism spectroscope of five prisms, and for higher dispersion surpasses the prism spectroscope both in definition and brightness in all portions of the spectrum.

To produce the pure spectrum from flat gratings, two telescopes are generally used, as in Fig. 1. 
The telescopes are fixed, and the grating is turned on its axis to pass to different portions of the spectrum. As the glass of the telescopes absorbs the ultra-violet light, this portion of the spectrum is cut off

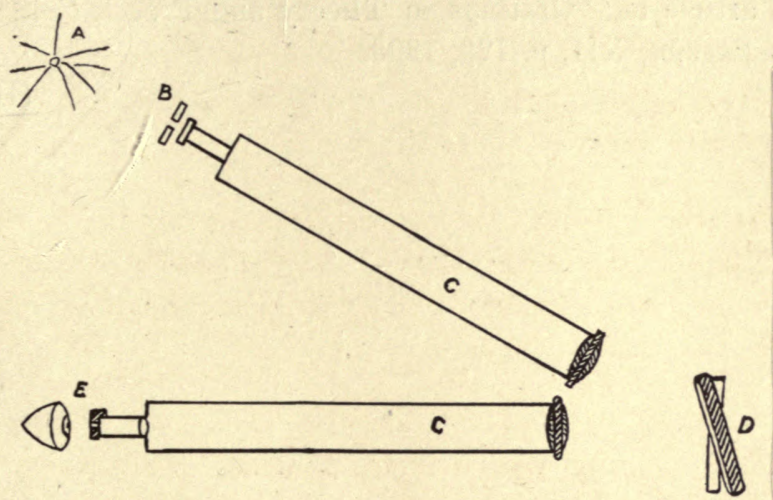

FIG. 1. - Method of using Flat Grating. $A$, source of light; $B$, slit; $C, C$, two telescopes, movable or fixed; $D$, grating, movable about its centre; $E$, eye-piece.

entirely, unless quartz lenses are used. The concave grating avoids this trouble, and produces a spectrum without the aid of lenses, the lines being ruled on a concave surface instead of on a flat one. Such a

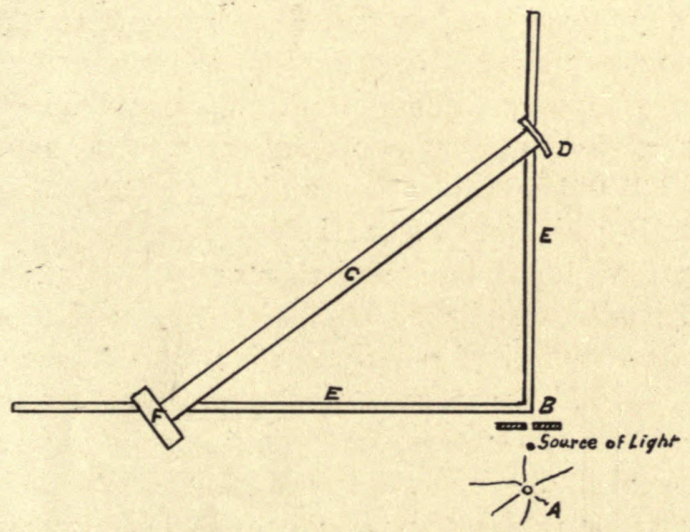

FiG. 2.-Method of using Concave Grating. $A$, source of light; $B$, slit ; $D$, grating mounted in beam $C$, movable along the ways $E, E ; F$, camera-box or eye-piece.

grating, properly mounted, produces what has been called a normal spectrum, and is specially adapted to photographic purposes (Fig. 2). 
A special form of grating of great defining power has been invented by Professor Michelson of the University of Chicago, called the 'echelon' spectroscope (see SPECTROSCOPY). It is, however, of very limited application.

See an article on 'Gratings in Theory and Practice' in Astronomy and Astro-Physics, XII, p. 129, 1893.

(H. A. R.) 
ADDRESSES 



\section{A PLEA FOR PURE SCIENCE ${ }^{1}$}

ADDRESS AS VICE-PRESIDENT OF SECTION B OF THE AMERICAN ASSOCIATION FOR THE advancement of science, minneapolis, minnesota, august 15, 1883

[Proceedings of the American Association for the Advancement of Science, $X X X I I, 105-126$, 1883; Science, II, 242-250, 1883; Journal of Franklin Institute, CXVI, 279-299, 1883]

The question is sometimes asked us as to the time of year we like the best. To my mind, the spring is the most delightful; for Nature then recovers from the apathy of winter, and stirs herself to renewed life. The leaves grow, and the buds open, with a suggestion of vigor delightful to behold; and we revel in this ever-renewed life. But this cannot always last. The leaves reach their limit; the buds open to the full and pass away. Then we begin to ask ourselves whether all this display has been in vain, or whether it has led to a bountiful harvest.

So this magnificent country of ours has rivalled the vigor of spring in its growth. Forests have been leveled, and cities built and a large and powerful nation has been created on the face of the earth. We are proud of our advancement. We are proud of such cities as this, founded in a day upon a spot over which but a few years since, the red man hunted the buffalo. But we must remember that this is only the spring of our country. Our glance must not be backward; for, however beautiful leaves and blossoms are, and however marvelous their rapid increase, they are but leaves and blossoms after all. Rather should we look forward to discover what will be the outcome of all this and what the chance of harvest. For if we do this in time, we may discover the worm which threatens the ripe fruit, or the barren spot where the harvest is withering for want of water.

I am required to address the so-called physical section of this asso-

IIn using the word "science," I refer to physical science, as I know nothing of natural science. Probably my remarks will, however, apply to both, but I do not know. 
ciation. Fain would I speak pleasant words to you on this subject; fain would I recount to you the progress made in this subject by my countrymen, and their noble efforts to understand the order of the universe. But I go out to gather the grain ripe to the harvest, and I find only tares. Here and there a noble head of grain rises above the weeds; but so few are they, that I find the majority of my countrymen know them not, but think that they have a waving harvest, while it is only one of weeds after all. American science is a thing of the future, and not of the present or past; and the proper course of one in my position is to consider what must be done to create a science of physics in this country, rather than to call telegraphs, electric lights, and such conveniences, by the name of science. I do not wish to underrate the value of all these things; the progress of the world depends on them, and he is to be honored who cultivates them successfully. So also the cook who invents a new and palatable dish for the table benefits the world to a certain degree; yet we do not dignify him by the name of a chemist. And yet it is not an uncommon thing, especially in American newspapers, to have the applications of science confounded with pure science; and some obscure American who steals the ideas of some great mind of the past, and enriches himself by the application of the same to domestic uses, is often lauded above the great originator of the idea, who might have worked out hundreds of such applications, had his mind possessed the necessary element of vulgarity. I have often been asked, which was the more important to the world, pure or applied science. To have the applications of a science, the science itself must exist. Should we stop its progress, and attend only to its applications, we should soon degenerate into a people like the Chinese, who have made no progress for generations, because they have been satisfied with the applications of science, and have never sought for reasons in what they have done. The reasons constitute pure science. They have known the application of gunpowder for centuries; and yet the reasons for its peculiar action, if sought in the proper manner, would have developed the science of chemistry, and even of physics, with all their numerous applications. By contenting themselves with the fact that gunpowder will explode, and seeking no farther, they have fallen behind in the progress of the world; and we now regard this oldest and most numerous of nations as only barbarians, and yet our own country is in this same state. But we have done better, for we have taken the science of the old world, and applied it to all our uses, accepting it like the rain of heaven, without asking whence it came, or even acknowledging the 
debt of gratitude we owe to the great and unselfish workers who have given it to us; and, like the rain of heaven, this pure science has fallen upon our country, and made it great and rich and strong.

To a civilized nation of the present day, the applications of science are a necessity, and our country has hitherto succeeded in this line only for the reason that there are certain countries in the world where pure science has been and is cultivated, and where the study of nature is cousidered a noble prrsuit; but such countries are rare, and those who wish to pursue pure science in our own country must be prepared to face public opinion in a manner which requires much moral courage. They must be prepared to be looked down upon by every successful inventor whose shallow mind imagines that the only pursuit of mankind is wealth, and that he who obtains most has best succeeded in this world. Everybody can comprehend a million of money; but how few can comprehend any advance in scientific theory, especially in its more abstruse portions! And this, I believe, is one of the causes of the small number of persons who have ever devoted themselves to work of the higher order in any human pursuit. Man is a gregarious animal, and depends very much, for his happiness, on the sympathy of those around him; and it is rare to find one with the courage to pursue his own ideas in spite of his surroundings. In times past, men were more isolated than at present, and each came in contact with a fewer number of people. Hence that time constitutes the period when the great sculptures, paintings and poems were produced. Each man's mind was comparatively free to follow its own ideals, and the results were the great and unique works of the ancient masters. To-day the railroad and the telegraph, the books and newspapers, have united each individual man with the rest of the world; instead of his mind being an individual, a thing apart by itself, and unique, it has become so influenced by the outer world, and so dependent upon it, that it has lost its originality to a great extent. The man who in times past would naturally have been in the lowest depths of poverty, mentally and physically, to-day measures tape behind a counter, and with lordly air advises the naturally born genius how he may best bring his outward appearance down to a level with his own. A new idea he never had, but he can at least cover his mental nakedness with ideas imbibed from others. So the genius of the past soon perceives that his higher ideas are too high to be appreciated by the world; his mind is clipped down to the standard form; every natural offshoot upwards is repressed, until the man is no higher than his fellows. Hence the world, through the abundance of 
its intercourse, is reduced to a level. What was formerly a grand and magnificent landscape, with mountains ascending above the clouds, and depths whose gloom we cannot now appreciate, has become serene and peaceful. The depths have been filled, and the heights levelled, and the wavy harvests and smoky factories cover the landscape.

As far as the average man is concerned, the change is for the better. The average life of man is far pleasanter, and his mental condition better, than before. But we miss the vigor imparted by the mountains, We are tired of mediocrity, the curse of our country; we are tired of seeing our artists reduced to hirelings, and imploring Congress to protect them against foreign competition; we are tired of seeing our countrymen take their science from abroad, and boast that they here convert it into wealth; we are tired of seeing our professors degrading their chairs by the pursuit of applied science instead of pure science, or sitting inactive while the whole world is open to investigation; lingering by the wayside while the problem of the universe remains unsolved. We wish for something higher and nobler in this country of mediocrity, for a mountain to relieve the landscape of its monotony. We are surrounded with mysteries, and have been created with minds to enjoy and reason to aid in the unfolding of such mysteries. Nature calls to us to study her, and our better feelings urge us in the same direction.

For generations there have been some few students of science who have esteemed the study of nature the most noble of pursuits. Some have been wealthy, and some poor; but they have all had one thing in common, - the love of nature and its laws. To these few men the world owes all the progress due to applied science, and yet very few ever received any payment in this world for their labors.

Faraday, the great discoverer of the principle on which all machines for electric lighting, electric railways, and the transmission of power, must rest, died a poor man, although others and the whole world have been enriched by his discoveries; and such must be the fate of the followers in his footsteps for some time to come.

But there will be those in the future who will study nature from pure love, and for them higher prizes than any yet obtained are waiting. We have but yet commenced our pursuit of science, and stand upon the threshold wondering what there is within. We explain the motion of the planets by the law of gravitation; but who will explain how two bodies, millions of miles apart, tend to go toward each other with a certain force? We now weigh and measure electricity and electric currents with as much ease as ordinary matter, yet have we made any 
approach to an explanation of the phenomenon of electricity? Light is an undulatory motion, and yet do we know what it is that undulates? Heat is motion, yet do we know what it is that moves? Ordinary matter is a common substance, and yet who shall fathom the mystery of its internal constitution?

There is room for all in the work, and the race has but commenced. The problems are not to be solved in a moment, but need the best work of the best minds, for an indefinite time.

Shall our country be contented to stand by, while other countries lead in the race? Shall we always grovel in the dust, and pick up the crumbs which fall from the rich man's table, considering ourselves richer than he because we have more crumbs, while we forget that he has the cake, which is the source of all crumbs? Shall we be swine, to whom the corn and husks are of more value than the pearls? If I read aright the signs of the times, I think we shall not always be contented with our inferior position. From looking down we have almost become blind, but may recover. In a new country, the necessities of life must be attended to first. The curse of Adam is upon us all, and we must earn our bread.

But it is the mission of applied science to render this easier for the whole world. There is a story which I once read, which will illustrate the true position of applied science in the world. A boy, more fond of reading than of work, was employed, in the early days of the steamengine, to turn the valve at every stroke. Necessity was the mother of invention in his case: his reading was disturbed by his work, and he soon discovered that he might become free from his work by so tying the valve to some movable portion of the engine, as to make it move its own valve. So I consider that the true pursuit of mankind is intellectual. The scientific study of nature in all its branches, of mathematics, of mankind in its past and present, the pursuit of art, and the cultivation of all that is great and noble in the world,- - these are the highest occupation of mankind. Commerce, the applications of science, the accumulation of wealth, are necessities which are a curse to those with high ideals, but a blessing to that portion of the world which has neither the ability nor the taste for higher pursuits.

As the applications of science multiply, living becomes easier, the wealth necessary for the purchase of apparatus can be obtained, and the pursuit of other things besides the necessities of life becomes possible.

But the moral qualities must also be cultivated in proportion to the 
wealth of the country, before much can be done in pure science. The successful sculptor or painter naturally attains to wealth through the legitimate work of his profession. The novelist, the poet, the musician, all have wealth before them as the end of a successful career. But the scientist and the mathematician have no such incentive to work: they must earn their living by other gursuits, usually teaching, and only devote their surplus time to the true pursuit of their science. And frequently, by the small salary which they receive, by the lack of instrumental and literary facilities, by the mental atmosphere in which they exist, and, most of all, by their low ideals of life, they are led to devote their surplus time to applied science or to other means of increasing their fortune. How shall we, then, honor the few, the very few, who, in spite of all difficulties, have kept their eyes fixed on the goal, and have steadily worked for pure science, giving to the world a most precious. donation, which has borne fruit in our greater knowledge of the universe and in the applications to our physical life which have enriched thousands and benefited each one of us? There are also those who have every facility for the pursuit of science, who have an ample salary and every appliance for work, yet who devote themselves to commercial work, to testifying in courts of law, and to any other work to increase their present large income. Such men would be respectable if they gave up the name of professor, and took that of consulting chemist or physicist. And such men are needed in the community. But for a man to occupy the professor's chair in a prominent college, and, by his energy and ability in the commercial applications of his science, stand before the local community as a newspaper exponent of his science, is a disgrace both to him and his college. It is the death-blow to science in that region. Call him by his proper name, and he becomes at once a useful member of the community. Put in his place a man who shall by precept and example cultivate his science, and how different is the result! Young men, looking forward into the world for something to do, see before them this high and noble life, and they see that there is something more honorable than the accumulation of wealth. They are this led to devote their lives to similar pursuits, and they honor the professor who has drawn them to something higher than they might otherwise have aspired to reach.

I do not wish to be misunderstood in this matter. It is no disgrace to make money by an invention, or otherwise, or to do commercial scientific work under some circumstances; but let pure science be the aim of those in the chairs of professors, and so prominently the aim that 
there can be no mistake. If our aim in life is wealth, let us honestly engage in commercial pursuits, and compete with others for its possession; but if we choose a life which we consider higher, let us live up to it, taking wealth or poverty as it may chance to come to us, but letting neither turn us aside from our pursuit.

The work of teaching may absorb the energies of many; and, indeed, this is the excuse given by most for not doing any scientific work. But there is an old saying, that where there is a will there is a way. Few professors do as much teaching or lecturing as the German professors, who are also noted for their elaborate papers in the scientific journals. I myself have been burdened down with work, and know what it is; and yet I here assert that all can find time for scientific research if they desire it. But here, again, that curse of our country, mediocrity, is upon us. Our colleges and universities seldom call for first-class men of reputation, and I have even heard the trustee of a well-known college assert that no professor should engage in research because of the time wasted. I was glad to see, soon after, by the call of a prominent scientist to that college, that the majority of the trustees did not agree with him.

That teaching is important goes without saying. A successful teacher is to be respected; but if he does not lead his scholars to that which is highest, is he not blameworthy? We are, then, to look to the colleges and universities of the land for most of the work in pure science which is done. Let us therefore examine these latter, and see what the prospect is.

One, whom perhaps we may here style a practical follower of Ruskin, has stated that while in this country he was variously designated by the title of captain, colonel, and professor. The story may or may not be true, but we all know enough of the customs of our countrymen not to dispute it on general principles. All men are born equal: some men title of captain, colonel, and professor. The story may or may not be The logic is conclusive; and the same kind of logic seems to have been applied to our schools, colleges, and universities. I have before me the report of the commissioner of education for 1880. According to that report, there were $389,{ }^{2}$ or say, in round numbers, 400 institutions, calling themselves colleges or universities, in our country! We may well exclaim that ours is a great country, having more than the whole world beside. The fact is sufficient. The whole earth could hardly support such a number of first-class institutions. The curse of mediocrity must

3364 reported on, and 25 not reported. 
be upon them, to swarm in such numbers. They must be a cloud of mosquitoes, instead of eagles as they profess; and this becomes evident on further analysis. About one-third aspire to the name of university; and I note one called by that name which has two professors and eighteen students, and another having three teachers and twelve students! These instances are not unique, for the number of small institutions and schools which call themselves universities is very great. It is difficult to decide from the statistics alone the exact standing of these institutions. The extremes are easy to manage. Who can doubt that an institution with over eight hundred students, and a faculty of seventy is of a higher grade than those above cited having ten or twenty students and two or three in the faculty? Yet this is not always true; for I note one institution with over five hundred students which is known to me personally as of the grade of a high school. The statistics are more or less defective, and it would much weaken the force of my remarks if I went too much into detail. I append the following tables, however, of 330 so-called colleges and universities:

$$
\begin{aligned}
& 218 \text { had from } 0 \text { to } 100 \text { students. } \\
& 88 \text { had from } 100 \text { to } 200 \text { students. } \\
& 12 \text { had from } 200 \text { to } 300 \text { students. } \\
& 6 \text { had from } 300 \text { to } 500 \text { students. } \\
& 6 \text { had over } 500 \text { students. }
\end{aligned}
$$

Of 322 so-called colleges and universities:

$$
\begin{aligned}
& 206 \text { had } 0 \text { to } 10 \text { in the faculty. } \\
& 99 \text { had } 10 \text { to } 20 \text { in the faculty. } \\
& 1 \% \text { had } 20 \text { or over in the faculty. }
\end{aligned}
$$

If the statistics were forthcoming,--and possibly they may exist,we might also get an idea of the standing of these institutions and their approach to the true university idea, by the average age of the scholars. Possibly also the ratio of number of scholars to teachers might be of some help. All these methods give an approximation to the present standing of the institutions. But there is another method of attacking the problem, which is very exact, yet it only gives us the possibilities in the case of the institutions. I refer to the wealth of the institution. In estimating the wealth, I have not included the value of grounds and buildings, for this is of little importance, either to the present or future standing of the institution, as good work can be done in a hovel as in a 
palace. I have taken the productive funds of the institution as the basis of estimate. I find:

234 have below $\$ 500,000$.

8 have between $\$ 500,000$ and $\$ 1,000,000$.

8 have over $\$ 1,000,000$.

There is no fact more firmly established, all over the world, than that the higher education can never be made to pay for itself. Usually the cost to a college, of educating a young man, very much exceeds what he pays for it, and is often three or four times as much. The higher the education, the greater this proportion will be; and a university of the highest class should anticipate only a small accession to its income from the fees of students. Hence the test I have applied must give a true representation of the possibilities in every case. According to the figures, only sixteen colleges and universities have $\$ 500,000$ or over of invested funds, and only one-half of these have $\$ 1,000,000$ and over. Now, even the latter sum is a very small endowment for a college; and to call any institution a university which has less than $\$ 1,000,000$ is to render it absurd in the face of the world. And yet more than 100 of our institutions, many of them very respectable colleges, have abused the word "university" in this manner. It is to be hoped that the endowment of the more respectable of these institutions may be increased, as many of them deserve it; and their unfortunate appellation has probably been repented of long since.

But what shall we think of a community that gives the charter of a university to an institution with a total of $\$ 20,000$ endowment, two so-called professors, and eighteen students! or another with three professors, twelve students, and a total of $\$ 2 \%, 000$ endowment, mostly invested in buildings! And yet there are very many similar institulions; there being sixteen with three professors or less, and very many indeed with only four or five.

Such facts as these could only exist in a democratic country, where pride is taken in reducing everything to a level. And I may also say, that it can only exist in the early days of such a democracy; for an intelligent public will soon perceive that calling a thing by a wrong name does not change its character, and that truth, above all things, should be taught to the youth of the nation.

It may be urged, that all these institutions are doing good work in education; and that many young men are thus taught, who could not afford to go to a true college or university. But I do not object to the 
education,- - though I have no doubt an investigation would disclose equal absurdities here,- - for it is aside from my object. But I do object to lowering the ideals of the youth of the country. Let them know that they are attending a sehool, and not a university; and let them know that above them comes the college, and above that the university. Iuet them be taught that they are only half educated, and that there are persons in the world by whose side they are but atoms. In other words, let them be taught the truth.

It may be that some small institutions are of high grade, especially those which are new; but who can doubt that more than two-thirds of our institutions calling themselves colleges and universities are unworthy of the name? Each one of these institutions has so-called professors, but it is evident that they can be only of the grade of teachers. Why should they not be so called? The position of teacher is an honored one, but is not made more honorable by the assumption of a false title. Furthermore, the multiplication of the title, and the ease with which it can be obtained, render it scarcely worth striving for. When the man of energy, ability, and perhaps genius is rewarded by the same title and emoluments as the commonplace man with the modicum of knowledge, who takes to teaching, not because of any aptitude for his work, but possibly because he has not the energy to compete with his fellow-men in business, then I say one of the inducements for the first-class men to become professors is gone.

When work and ability are required for the position, and when the professor is expected to keep up with the progress of his subject, and to do all in his power to advance it, and when he is selected for these reasons, then the position will be worth working for, and the successful competitor will be honored accordingly. The chivalric spirit which prompted Faraday to devote his life to the study of nature may actuate a few noble men to give their lives to scientific work; but if we wish to cultivate this highest class of men in science, we must open a career for them worthy of their efforts.

Jenny Lind, with her beautiful voice, would have cultivated it to some extent in her native village; yet who would expect her to travel over the world, and give concerts for nothing? and how would she have been able to do so if she had wished? And so the scientific man, whatever his natural talents, must have instruments and a library, and a suitable and respectable salary to live upon, before he is able to exert himself to his full capacity. This is true of advance in all the higher departments of human learning, and yet something more is necessary. 
It is not those in this country who receive the largest salary, and have positions in the richest colleges, who have advanced their subject the most: men receiving the highest salaries, and occupying the professor's chair, are to-day doing absolutely nothing in pure science, but are striving by the commercial applications of their science to increase their already large salary. Such pursuits, as I have said before, are honorable in their proper place; but the duty of a professor is to advance his science, and to set an example of pure and true devotion to it which shall demonstrate to his students and the world that there is something high and noble worth living for. Money-changers are often respectable men, and yet they were once severely rebuked for carrying on their trade in the court of the temple.

Wealth does not constitute a university, buildings do not: it is the men who constitute its faculty, and the students who learn from them. It is the last and highest step which the mere student takes. He goes forth into the world, and the height to which he rises has been influenced by the ideals which he has consciously or unconsciously imbibed in his university. If the professors under whom he has studied have been high in their profession, and have themselves had high ideals; if they have considered the advance of their particular subject their highest work in life, and are themselves honored for their intellect throughout the world, - the student is drawn toward that which is highest, and ever after in life has high ideals. But if the student is taught by what are sometimes called good teachers, and teachers only, who know little more than the student, and who are often surpassed and even despised by him, no one can doubt the lowered tone of his mind. He finds that hy his feeble efforts he can surpass one to whom a university has given its highest honor; and he begins to think that he himself is a born genius, and the incentive to work is gone. He is great by the side of the molehill, and does not know any mountain to compare himself with.

A university should have not only great men in its faculty, but have numerous minor professors and assistants of all kinds, and should encourage the highest work, if for no other reason than to encourage the student to his highest efforts.

But, assuming that the professor has high ideals, wealth such as only a large and high university can command is necessary to allow him the fullest development.

And this is specially so in our science of physics. In the early days of physics and chemistry, many of the fundamental experiments could be performed with the simplest apparatus. And so we often find the 
names of Wollaston and Faraday mentioned as needing scarcely anything for their researches. Much can even now be done with the simplest apparatus, and nobody, except the utterly incompetent, need stop for want of it; but the fact remains, that one can only be free to investigate in all departments of chemistry and physics, when he not only has a complete laboratory at his command, but a fund to draw on for the expenses of each experiment. That simplest of the departments of physics, namely, astronomy, has now reached such perfection that nobody can expect to do much more in it without a perfectly equipped observatory; and even this would be useless without an income sufficient to employ a corps of assistants to make the observations and computations. But even in this simplest of physical subjects, there is great misunderstanding. Our country has very many excellent observatories, and yet little work is done in comparison, because no provision has been made for maintaining the work of the observatory; and the wealth which, if concentrated, might have made one effective observatory which would prove a benefit to astronomical science, when scattered among a half-dozen merely furnishes telescopes for the people in the surrounding region to view the moon with. And here I strike the keynote of at least one need of our country, if she would stand well in science; and the following item which I clip from a newspaper will illustrate the matter: "The eccentric old Canadian, Arunah Huntington, who left $\$ 200,000$ to be divided among the public schools of Vermont, has done soniething which will be of little practical value to the schools. Each district will be entitled to the insignificant sum of $\$ 10$, which will not advance much the cause of education." •

Nobody will dispute the folly of such a bequest, or the folly of filling the country with telescopes to look at the moon, and calling them observatories. How much better to concentrate the wealth into a few parcels, and make first-class observatories and institutions with it!

Is it possible that any of our four hundred colleges and universities have love enough of learning to unite with each other and form larger institutions? Is it possible that any have such a love of truth that they are willing to be called by their right name? I fear not; for the spirit of expectation, which is analogous to the spirit of gambling, is strong in the American breast, and each institution which now, except in name, slumbers in obscurity, expects in time to bloom out into full prosperity. Although many of them are under religious influence, where truth is inculcated, and where men are taught to take a low seat at the table in order that they may be honored by being called up higher, and not 
dishonored by being thrust down lower, yet these institutions have thrust themselves into the highest seats, and cannot probably be dislodged.

But would it not be possible so to change public opinion that no college could be founded with a less endowment than say $\$ 1,000,000$, or no university with less than three or four times that amount. From the report of the commissioner of education, I learn that such a thing is taking place; that the tendency towards large institutions is increasing, and that it is principally in the west and southwest that the multiplication of small institutions with big names is to be feared most, and that the east is almost ready for the great coming university.

The total wealth of the four hundred colleges and universities in 1880 was about $\$ 40,000,000$ in buildings, and $\$ 43,000,000$ in productive funds. This would be sufficient for one great university of $\$ 10,000,000$, four of $\$ 5,000,000$, and twenty-six colleges of $\$ 2,000,000$ each. But such an idea can of course never be carried out. Government appropriations are out of the question, because no political trickery must be allowed around the ideal institution.

In the year 1880 the private bequests to all schools and colleges amounted to about $\$ 5,500,000$; and, although there was one bequest of $\$ 1,250,000$, yet the amount does not appear to be phenomenal. It would thus seem that the total amount was about five million dollars in one year, of which more than half is given to so-called colleges and universities. It would be very difficult to regulate these bequests so that they might be concentrated sufficiently to produce an immediate result. But the figures show that generosity is a prominent feature of the American people, and that the needs of the country only have to be appreciated to have the funds forthcoming. We must make the need of research and of pure science felt in the country. We must live such lives of pure devotion to our science, that all shall see that we ask for money, not that we may live in indolent ease at the expense of charity, but that we may work for that which has advanced and will advance the world more than any other subject, both intellectually and physically. We must live such lives as to neutralize the influence of those who in high places have degraded their profession. or have given themselves over to ease, and do nothing for the science which they represent. Let us do what we can with the present means at our disposal. There is not one of us who is situated in the position best adapted to bring out all his powers, and to allow him to do most for his science. All have their difficulties, and I do not think that circumstances will ever radically change a man. If a man has the instinct of 
research in him, it will always show itself in some form. But circumstances may direct it into new paths, or may foster it so that what would otherwise have died as a bud now blossoms and ripens into the perfect fruit.

Americans have shown no lack of invention in small things; and the same spirit when united to knowledge and love of science, becomes the spirit of research. The telegraph-operator, with his limited knowledge of electricity and its laws, naturally turns his attention to the improvement of the only electrical instrument he knows anything about; and his researches would be confined to the limited sphere of his knowledge, and to the simple laws with which he is acquainted. But as his knowledge increases, and the field broadens before him, as he studies the mathematical theory of the subject, and the electromagnetic theory of light loses the dim haze due to distance, and becomes his constant companion, the telegraph instrument becomes to him a toy, and his effort to discover something new becomes research in pure science.

It is useless to attempt to advance science until one has mastered the science: he must step to the front before his blows can tell in the strife. Furthermore, I do not believe anybody can be thorough in any department of science, without wishing to advance it. In the study of what is known, in the reading of the scientific journals, and the discussions therein contained of the current scientific questions, one would obtain an impulse to work, even though it did not before exist; and the same spirit which prompted him to seek what was already known would make him wish to know the unknown. And I may say that I never met a case of thorough knowledge in my own science, except in the case of well-known investigators. I have met men who talked well, and I have sometimes asked myself why they did not do something; but further knowledge of their character has shown the superficiality of their knowledge. I am no longer a believer in men who could do something if they would, or would do something if they had a chance. They are impostors. If the spirit is there, it will show itself in spite of circumstances.

As I remarked before, the investigator in pure science is usually a professor. He must teach as well as investigate. It is a question which has been discussed in late years, as to whether these two functions had better be combined in the same individual, or separated. It seems to be the opinion of most, that a certain amount of teaching is conducive, rather than otherwise, to the spirit of research. I myself think that this is true, and I should myself not like to give up my daily lecture; but 
one must not be overburdened. I suppose that the true solution, in many cases, would be found in the multiplication of assistants, not only for the work of teaching but of research. Some men are gifted with more ideas than they can work out with their own hands, and the world is losing much by not supplying them with extra hands. Life is short: old age comes quickly, and the amount one pair of hands can do is very limited. What sort of shop would that be, or what sort of factory, where one man had to do all the work with his own hands? It is a fact in nature, which no democracy can change, that men are not equal,- that some have brains, and some hands; and no idle talk about equality can ever subvert the order of the universe.

I know of no institution in this country where assistants are supplied to aid directly in research; yet why should it not be so? Even the absence of assistant professors and assistants of all kinds, to aid in teaching, is very noticeable, and must be remedied before we can expect much.

There are many physical problems, especially those requiring exact measurements, which cannot be carried out by one man, and can only be successfully attacked by the most elaborate apparatus, and with a full corps of assistants. Such are Regnault's experiments on the fundamental laws of gases and vapors, made thirty or forty years ago by aid from the French government, and which are the standards to this day. Although these experiments were made with a view to the practical calculation of the steam-engine, yet they were carried out in such a broad spirit that they have been of the greatest theoretical use. Again, what would astronomy have done without the endowment of observatories? By their means, that science has become the most perfect of all branches of physics, as it should be from its simplicity. There is no doubt, in my mind, that similar institutions for other branches of physics, or, better, to include the whole of physics, would be equally successful. A large and perfectly equipped physical laboratory with its large revenues, its corps of professors and assistants, and its machineshop for the construction of new apparatus, would be able to advance our science quite as much as endowed observatories have advanced astronomy. But such a laboratory should not be founded rashly. The value will depend entirely on the physicist at its head, who has to devise the plan, and to start it into practical working. Such a man will always be rare, and cannot always be obtained. After one had been successfully started, others could follow; for imitation requires little brains. 
One could not be certain of getting the proper man every time, but the means of appointment should be most carefully studied so as to secure a good average. There can be no doubt that the appointment should rest with a scientific body capable of judging the highest work of each candidate.

Should any popular element enter, the person chosen would be of the literary-scientific order, or the dabbler on the outskirts who presents his small discoveries in the most theatrical manner. What is required is a man of depth, who has such an insight into physical science that he can tell when blows will best tell for its advancement.

Such a grand laboratory as I describe does not exist in the world, at present, for the study of physics. But no trouble has ever been found in obtaining means to endow astronomical science. Everybody can appreciate, to some extent, the value of an observatory; as astronomy is the simplest of scientific subjects, and has very quickly reached a position where elaborate instruments and costly computations are necessary to further advance. The whole domain of physics is so wide that workers have hitherto found enough to do. But it cannot always be so, and the time has even now arrived when such a grand laboratory should be founded. Shall our country take the lead in this matter, or shall we wait for foreign countries to go before? They will be built in the future, but when and how is the question.

Several institutions are now putting up laboratories for physics. They are mostly for teaching, and we can expect only a comparatively small amount of work from most of them. But they show progress; and, if the progress be as quick in this direction as in others, we should be able to see a great change before the end of our lives.

As stated before, men are influenced by the sympathy of those with whom they come in contact. It is impossible to change public opinion in our favor immediately; and, indeed, we must always seek to lead it, and not be guided by it. For pure science is the pioneer who must not hover about cities and civilized countries, but must strike into unknown forests, and climb the hitherto inaccessible mountains which lead to and command a view of the promised land,- the land which science promises us in the future; which shall not only flow with milk and honey, but shall give us a better and more glorious idea of this wonderful universe. We must create a public opinion in our favor, but it need not at first be the general public. We must be contented to stand aside, and see the honors of the world for a time given to our inferiors; and 
must be better contented with the approval of our own consciences, and of the very few who are capable of judging our work, than of the whole world beside. Let us look to the other physicists, not in our own town, not in our own country, but in the whole world, for the words of praise which are to encourage us, or the words of blame which are to stimulate us to renewed effort. For what to us is the praise of the ignorant? Let us join together in the bonds of our scientific societies, and encourage each other, as we are now doing, in the pursuit of our favorite study; knowing that the world will some time recognize our services, and knowing, also, that we constitute the most important element in human progress.

But danger is also near, even in our societies. When the average tone of the society is low, when the highest honors are given to the mediocre, when third-class men are held up as examples, and when trifling inventions are magnified into scientific discoveries, then the influence of such societies is prejudicial. A young scientist attending the meetings of such a society soon gets perverted ideas. To his mind, a molehill is a mountain, and the mountain a molehill. The small inventor or the local celebrity rises to a greater height, in his mind, than the great leader of science in some foreign land. He gauges himself by the molehill, and is satisfied with his stature; not knowing that he is but an atom in comparison with the mountain, until, perhaps, in old age, when it is too late. But, if the size of the mountain had been seen at first, the young scientist would at least have been stimulated in his endeavor to grow.

We cannot all be men of genius; but we can, at least, point them out to those around us. We may not be able to benefit science much ourselves; but we can have high ideals on the subject, and instil them into those with whom we come in contact. For the good of ourselves, for the good of our country, for the good to the world, it is incumbent on us to form a true estimate of the worth and standing of persons and things, and to set before our own minds all that is great and good and noble, all that is most important for scientific advance, above the mean and low and unimportant.

It is very often said, that a man has a right to his opinion. This might be true for a man on a desert island, whose error would influence only himself; but when he opens his lips to instruct others, or even when he signifies his opinions by his daily life, then he is directly responsible for all his errors of judgment or fact. He has no right to 
think a molehill as big as a mountain, nor to teach it, any more than he has to think the world is flat, and teach that it is so. The facts and laws of our science have not equal importance, neither have the men who cultivate the science achieved equal results. One thing is greater than another, and we have no right to neglect the order. Thus shall our minds be guided aright, and our efforts be toward that which is the highest.

Then shall we see that no physicist of the first class has ever existed in this country, that we must look to other countries for our leaders in that subject, and that the few excellent workers in our country must receive many accessions from without before they can constitute an American science, or do their share in the world's work.

But let me return to the subject of scientific societies. Here American science has its hardest problem to contend with. There are very many local societies dignified by high-sounding names, each having its local celebrity, to whom the privilege of describing some crab with an extra claw, which he found in his morning ramble, is inestimable. And there are some academies of science, situated at our seats of learning, which are doing good work in their localities. But distances are so great that it is difficult to collect men together at any one point. The American Association, which we are now attending, is not a scientific academy, and does not profess to be more than a gathering of all who are interested in science, to read papers and enjoy social intercourse. The National Academy of Sciences contains eminent men from the whole country, but then it is only for the purpose of advising the government freely on scientific matters. It has no building, it has no library; and it publishes nothing except the information which it freely gives to the government, which does nothing for it in return. It has not had much effect directly on American science; but the liberality of the government in the way of scientific expeditions, publications, etc., is at least partly due to its influence, and in this way it has done much good. But it in no way takes the place of the great Royal society, or the great academies of science at Paris, Berlin, Vienna, St. Petersburg, Munich, and, indeed, all the European capitals and large cities. These, by their publications, give to the young student, as well as to the more advanced physicist, models of all that is considered excellent; and to become a member is one of the highest honors to which he can aspire, while to write a memoir which the academy considers worthy to be published in its transactions excites each one to his highest effort. 
The American Academy of Sciences in Boston is perhaps our nearest representation of this class of academies, but its limitation of membership to the state deprives it of a national character.

But there is another matter which influences the growth of our science.

As it is necessary for us still to look abroad for our highest inspiration in pure science, and as science is not an affair of onc town or one country, but of the whole world, it becomes us all to read the current journals of science and the great transactions of foreign societies, as well as those of our own countries. These great transactions and journals should be in the library of every institution of learning in the country, where science is taught. How can teachers and professors be expected to know what has been discovered in the past, or is being discovered now, if these are not provided? Has any institution a right to starve mentally the teachers whom it employs, or the students who come to it? There can be but one answer to this; and an institution calling itself a university, and not having the current scientific journals upon its table or the transactions of societies upon its library shelves, is certainly not doing its best to cultivate all that is best in this world.

We call this a free country, and yet it is the only one where there is a direct tax upon the pursuit of science. The low state of pure science in our country may possibly be attributed to the youth of the country; but a direct tax, to prevent the growth of our country in that subject, cannot be looked upon as other than a deep disgrace. I refer to the duty upon foreign books and periodicals. In our science, no books above elementary ones have ever been published, or are likely to be published in this country; and yet every teacher in physics must have them, not only in the college library, but on his own shelves, and must pay the government of this country to allow him to use a portion of his small salary to buy that which is to do good to the whole country. All freedom of intercourse which is necessary to foster our growing science is thus broken off, and that which might, in time, relieve our country of its mediocrity, is nipped in the bud by our government, which is most liberal when appealed to directly on scientific subjects.

One would think that books in foreign languages might be admitted free; but to please the half-dozen or so workmen who reprint German books, not scientific, our free intercourse with that country is cut off. Our scientific associations and societies must make themselves heard in this matter, and show those in authority how the matter stands. 
In conclusion, let me say once more that I do not believe that our country is to remain long in its present position. The science of physics, in whose applications our country glories, is to arise among us, and make us respected by the nations of the world. Such a prophecy may seem rash with regard to a nation which does not yet do enough physical work to support a physical journal. But we know the speed with which we advance in this country: we see cities springing up in a night, and other wonders performed at an unprecedented rate. And now we see physical laboratories being built, we see a great demand for thoroughly trained physicists, who have not shirked their mathematics, both as professors and in so-called practical life; and perhaps we have the feeling, common to all true Americans, that our country is going forward to a glorious future, when we shall lead the world in the strife for intellectual prizes as we now do in the strife for wealth.

But if this is to be so, we must not aim low. The problems of the universe cannot be solved without labor; they cannot be attacked without the proper intellectual as well as physical tools; and no physicist need expect to go far without his mathematics. No one expects a horse to win in a great and long race who has not been properly trained; and it would be folly to attempt to win with one, however pure his blood and high his pedigree, without it. The problems we solve are more diffcult than any race; the highest intellect cannot hope to succeed without proper preparation. The great prizes are reserved for the greatest efforts of the greatest intellects, who have kept their mental eye bright and flesh hard by constant exercise. Apparatus can be bought with money, talents may come to us at birth; but our mental tools, our mathematics, our experimental ability, our knowledge of what others have done before us, all have to be obtained by work. The time is almost past, even in our own country, when third-rate men can find a place as teachers, because they are unfit for everything else. We wish to see brains and learning, combined with energy and immense working power, in the professor's chair; but, above all, we wish to see that high and chivalrous spirit which causes one to pursue his idea in spite of all difficulties, to work at the problems of nature with the approval of his own conscience, and not of men before him. Let him fit himself for the struggle with all the weapons which mathematics and the experience of those gone before him can furnish, and let him enter the arena with the fixed and stern purpose to conquer. Let him not be contented to stand back with the crowd of mediocrity, but let him press forward for a front place in the strife. 


\section{A Plea for Pure Science}

The whole universe is before us to study. The greatest labor of the greatest minds has only given us a few pearls; and yet the limitless ocean, with its hidden depths filled with diamonds and precious stones, is before us. The problem of the universe is yet unsolved, and the mystery involved in one single atom yet eludes us. The field of research only opens wider and wider as we advance, and our minds are lost in wonder and astonishment at the grandeur and beauty unfolded before us. Shall we help in this grand work, or not? Shall our country do its share, or shall it still live in the almshouse of the world? 


\title{
THE PHYSICAL LABORATORY IN MODERN EDUCATION
}

\author{
ADDRESS FOR COMMEMORATION DAY OF THE JOHNS HOPKINS UNIVERSITY, \\ FEBRUARY 2\%, 1886
}

[Johns Hopkins University Circulars, No. 50, pp. 103-105, 1886]

From the moment we are born into this world down to the day when we leave it, we are called upon every moment to exercise our judgment with respect to matters pertaining to our welfare. While nature has supplied us with instincts which take the place of reason in our infancy, and which form the basis of action in very many persons through life, yet, more and more as the world progresses and as we depart from the age of childhood, we are forced to discriminate between right and wrong, between truth and falsehood. No longer can we shelter ourselves behind those in authority over us, but we must come to the front and each one decide for himself what to believe and how to act in the daily routine and the emergencies of life. This is not given to us as a duty which we can neglect if we please, but it is that which every man or woman, consciously or unconsciously, must go through with.

Most persons cut this Gordian knot, which they cannot untangle, by accepting the opinions which have been taught them and which appear correct to their particular circle of friends and associates: others take the opposite extreme and, with intellectual arrogance, seek to build up their opinions and beliefs from the very foundation, individually and alone, without help from others. Intermediate between these two extremes comes the man with full respect for the opinions of those around him, and yet with such discrimination that he sees a chance of error in all and most of all in himself. He has a longing for the truth and is willing to test himself, to test others and to test nature until he finds it. He has the courage of his opinions when thus carefully formed, and is then, but not till then, willing to stand before the world and proclaim what he considers the truth. Like Galileo and Copernicus, he inaugurates a new era in science, or like Luther, in the religious belief of mankind. He neither shrinks within himself at the thought of having an opinion of his own, nor yet believes it to be the only one worth considering in the world; he is neither crushed with intellectual humility, nor yet exalted with intellectual pride; he sees that the problems of nature and society can be solved, and yet he knows that this can only come 
about by the combined intellect of the world acting through ages of time and that he, though his intellect were that of Newton, can, at best, do very little toward it. Knowing this he seeks all the aids in his power to ascertain the truth, and if he, through either ambition or love of truth, wishes to impress his opinions on the world, he first takes care to have them correct. Above all, he is willing to abstain from having opinions on subjects of which he knows nothing.

It is the province of modern education to form such a mind while at the same time giving to it enough knowledge to have a broad outlook over the world of science, art and letters. Time will not permit me to discuss the subject of education in general, and, indeed, I would be transgressing the principles above laid down if I shonld attempt it. I shall only call attention at this present time to the place of the laboratory in modern education. I have often had a great desire to know the state of mind of the more eminent of mankind before modern science changed the world to its present condition and exercised its influence on all departments of knowledge and speculation. But I have failed to picture to myself clearly such a mind while, at the same time, the study of human nature, as it exists at present, shows me much that I suppose to be in common with it. As far as I can see, the unscientific mind differs from the scientific in this, that it is willing to accept and make statements of which it has no clear conception to begin with and of whose truth it is not assured. It is an irresponsible state of mind without clearness of conception, where the connection between the thought and its object is of the vaguest description. It is the state of mind where opinions are given and accepted without ever being subjected to rigid tests, and it may have some connection with that state of mind where everything has a personal aspect and we are guided by feelings rather than reason.

When, by education, we attempt to correct these faults, it is necessary that we have some standard of absolute truth: that we bring the mind in direct contact with it and let it be convinced of its errors again and again. We may state, like the philosophers who lived before Galileo, that large bodies fall faster than small ones, but when we see them strike the ground together we know that our previous opinion was false and we learn that even the intellect of an Aristotle may be mistaken. Thus we are taught care in the formation of our opinions and find that the unguided human mind goes astray almost without fail. We must correct it constantly and convince it of error over and over again until it discovers the proper method of reasoning, which will surely accord with the truth in whatever conclusions it may reach. There is, however, 
danger in this process that the mind may become over cautious and thus present a weakness when brought in contact with an unscrupulous person who cares little for truth and a great deal for effect. But if we believe in the maxim that truth will prevail and consider it the duty of all educated men to aid its progress, the kind of mind which I describe is the proper one to foster by education. Let the student be brought face to face with nature: let him exercise his reason with respect to the simplest physical phenomenon and then, in the laboratory, put his opinions to the test; the result is invariably humility, for he finds that nature has laws which must be discovered by labor and toil and not by wild flights of the imagination and scintillations of so-called genius.

Those who have studied the present state of education in the schools and colleges tell us that most subjects, including the sciences, are taught as an exercise to the memory. I myself have witnessed the melancholy sight in a fashionable school for young ladies of those who were born to be intellectual beings reciting page after page from memory, without any effort being made to discover whether they understood the subject or not. There are even many schools, so-called, where the subject of physics or natural philosophy itself is taught, without even a class experiment to illustrate the subject and connect the words with ideas. Words, mere words, are taught and a state of mind far different from that above described is produced. If one were required to find a system of education which would the most surely and certainly disgust the student with any subject, I can conceive of none which would do this more quickly than this method, where he is forced to learn what he does not understand. It is said of the Faraday that he never could understand any scientific experiment thoroughly until he had not only seen it performed by others, but had performed it himself. Shall we then expect children and youth to do what Faraday could not do? A thousand times better never teach the subject at all.

Tastes differ, but we may safely say that every subject of study which is thoroughly understood is a pleasure to the student. The healtliy mind as well as the healthy body craves exercise, and the school room or the lecture room should be a source of positive enjoyment to those who enter it. Above all. the study of nature, from the magnificent universe, across which light itself, at the rate of 186,000 miles per second, cannot go in less than hundreds of years, down to the atom of which millions are required to build up the smallest microscopic object, should be the most interesting subject brought to the notice of the student.

Some are born blind to the beauties of the world around them, some 
have their tastes better developed in other directions, and some have minds incapable of ever understanding the simplest natural phenomenon; but there is also a large class of students who have at least ordinary powers and ordinary tastes for scientific pursuits: to train the powers of observation and classification let them study natural history, not only from books, but from prepared specimens or directly from nature: to give eare in experiment and convince them that nature forgives no error, let them enter the chemical laboratory: to train them in exact and logical powers of reasoning, let them study mathematics: but to combine all this training in one and exhibit to their minds the most perfect and systematic method of discovering the exact laws of nature, let them study physics and astronomy, where observation, common sense and mathematies go hand in hand. The object of education is not only to produce a man who knows, but one who does; who makes his mark in the struggle of life and succeeds well in whatever he undertakes: who can solve the problems of nature and of humanity as they arise, and who, when he knows he is right, can boldly convince the world of the fact. Men of action are needed as well as men of thought.

There is no doubt in my mind that this is the point in which much of our modern education fails. Why is it? I answer that the memory alone is trained and the reason and judgment are used merely to refer matters to some authority who is considered final, and worse than all, they are not trained to apply their knowledge constantly. To produce men of action they must be trained in action. If the languages be studied, they must be made to translate from one language to the other until they have perfect facility in the process. If mathematics be studied, they must work problems, more problems and problems again, until they have the use of what they know. If they study the sciences, they must enter the laboratory and stand face to face with nature; they must learn to test their knowledge constantly and thus see for themselves the sad results of vague speculation; they must learn by direct experiment that there is such a thing in the world as truth and that their own mind is most liable to error. They must try experiment after experiment and work problem after problem until they become men of action and not of theory.

This, then, is the use of the laboratory in general education, to train the mind in right modes of thought by constantly bringing it in contact with absolute truth and to give it a pleasant and profitable method of exercise which will call all its powers of reason and imagination into play. Its use in the special training of scientists needs no remark, for it 
is well known that it is absolutely essential. The only question is whether the education of specialists in science is worth undertaking at all, and of these I have only to consider natural philosophers or physicists. I might point to the world around me, to the steam engine, to labor-saving machinery, to the telegraph, to all those inventions which make the present age the "Age of Electricity," and let that be my answer. Nobody could gainsay that the answer would be complete, for all are benefited by these applications of science, and he would be considered absurd who did not recognize their value. These follow in the train of physics, but they are not physics; the cultivation of physics brings them and always will bring them, for the selfishness of mankind can always be relied upon to turn all things to profit. But in the education pertaining to a university we look for other results. The special physicist trained there must be taught to cultivate his science for its own sake. He must go forth into the world with enthusiasm for it and try to draw others into an appreciation of it, doing his part to convince the world that the study of nature is one of the most noble of pursuits, that there are other things worthy of the attention of mankind besides the pursuit of wealth. He must push forward and do what he can, according to his ability, to further the progress of his science.

Thus does the university, from its physical laboratory, send forth into the world the trained physicist to advance his science and to carry to other colleges and technical schools his enthusiasm and knowledge. Thus the whole country is educated in the subject and others are taught to devote their lives to its pursuit, while some make the applications to the ordinary pursuits of life that are appreciated by all.

But for myself, I value in a scientific mind most of all that love of truth, that care in its pursuit and that humility of mind which makes the possibility of error always present more than any other quality. This is the mind which has built up modern science to its present perfection, which has laid one stone upon the other with such care that it to-day offers to the world the most complete monument to human reason. This is the mind which is destined to govern the world in the future and to solve problems pertaining to politics and humanity as well as to inanimate nature.

It is the only mind which appreciates the imperfections of the human reason and is thus careful to guard against them. It is the only mind that values the truth as it should be valued and ignores all personal feeling in its pursuit. And this is the mind the physical laboratory is built to cultivate. 


\section{ADDRESS AS PRESIDENT OF THE ELECTRICAL CONFER- ENCE AT PHILADELPHIA, SEPTEMBER 8, 1884}

[Report of the Conference, pp. 12-28, Washington, 1886]

To the student of science who has a disposition to look into the pages of history, no life has greater interest than that of Archimedes, and yet there are few men about whom so little is known. Living more than two thousand years ago, the accounts of him which have come to us are little short of fabulous, and yet they are of such a nature that we can say without any doubt that he was a genius such as the world has seldom seen. To him we owe some of the fundamental facts of mechanics, such as the principle of the lever and the pulley, and the fact that a body immersed in a liquid loses in weight as much as an equal volume of the liquid weighs. And in military engineering his success was so great that he prolonged the siege of Syracuse by the Romans from what would probably have been a few days to three years. His engines shot against the enemy immense numbers of darts and huge stones, which mowed them down in columns, and falling on their ships destroyed them. He thrust out huge beams from the walls over the ships and drew them into the air, where they swung to and fro to the amazement and terror of the Romans and were finally dropped and sunk to the bottom of the sea. He is even said to have set them on fire by means of the reflected light of the sun. But his principal work was in geometry, and of this I only need to quote the words of Professor De Morgan referring to those geometrical works of Archimedes which have come down to us. "Here," says Professor De Morgan, "he finds all that relates to the surface and solidity of the sphere, cone and cylinder and their segments. A modern work on the differential calculus would not give more results than are found here." As to the quality of the individual, the impression which his writings give us is that of a power which has never been surpassed. No one has a right to say that Newton himself, in the place of Archimedes, could have done more.

Thus before the birth of modern science, in the dim ages of the past when the light of history begins to fade and the mist of legend to cover 
our view, there lived a man of almost superhuman intellect whose mind seemed equally adapted to either pure or applied science. And yet Plutarch says of him: "Archimedes possessed so high a spirit, so profound a soul, and such treasures of scientific knowledge, that, though the inventions (referring to his military engines) had now obtained for him the renown of more than human sagacity, he yet would not deign to leave behind him any commentary or writing on such subjects, but, repudiating as sordid and ignoble the whole trade of engineering, and every sort of art that lends itself to mere use and profit, he placed his whole affection and ambition in those purer speculations where there can be no reference to the vulgar needs of life; studies, the superiority of which to all others is unquestioned, and in which the only doubt can be, whether the beauty and grandeur of the subjects examined, or the precision and cogency of the methods and means of proof, most deserve our admiration."

Here, then, at the dawn of science the question of the relative value of pure and applied science had been brought up. To the people of Syracuse, who had to defend themselves against an overwhelming enemy, the military engines of Archimedes were of far more interest than the whole of geometry, for the knowledge of the ratio of the solid contents of a sphere and its circumscribed cylinder cannot bring a dead man to life or restore wealth to a plundered city. And yet, from a point of view distant more than two thousand years, we are forced to admit that Archimedes was right. Archimedes' engines of destruction have passed away, but the geometrical and mechanical truths which he discovered are to-day almost the axioms of the mathematician and the worker in physical science, and the ratio of the circumference of a circle to its radius is to-day the most important of our physical constants.

But this is only a meager part of the influence of this man. The truths which he discovered have formed a part of the education of every student of mathematics to the present time, and have given pure intellectual enjoyment to all. They have helped to form the minds of all those whom we consider great in our science, and they have done their share in that march of progress which is gradually transforming the world.

Great should be the honor in which we hold the intellect of Archimedes, but greater should be our reverence when we approach that noble spirit which could ignore all worldly considerations and prefer the truths of geometry to the vast physical power given him by his other inventions, which were his amusements for a moment. We now see that he 
was right, but we cannot for a moment suppose that he foresaw, except dimly, any so-called practical advantages from his discoveries. A thousand times no! He preferred his geometrical labors because of a subtle quality of his mind, an instinct toward that which was highest and noblest and a faith that the pursuit of what is noble is the surest road to the final happiness of the individual and of the world. Our highest moral qualities are of this nature, and we despise as the lowest of the low one who is honest because "honesty is the best policy," but esteem him whose instincts lead him to honesty whatever the consequences.

So we reverence the noble and lofty spirit of Archimedes, and yet we do not at the present day quite agree with his estimate of the relative value of his works. His military inventions were far from worthy of being despised, even though the only reason were that they gave the world three more years of Archimedes' life. The world is not formed of disembodied spirits, but of men, in whom there is a wonderful combination of mind and matter, and a sound mind in a sound body is the highest type of manhood. But we also know that the mind is hampered by many considerations connected with the body. Archimedes recognized this, and his noble spirit revolted at it. But to-day we see that no progress can come from this method of treatment; the body still remains, however much we may despise it, and the buzzing of a fly can disturb the most profound thought of the philosopher.

We now study the laws of nature and seek thus to assist our bodies in obeying the thoughts of our minds. Our railroads carry us hither and thither on the earth with somewhat the facility of spirits, and our thoughts pass with almost the speed of light to the uttermost portion of the earth. The steam engine does our work, and labor-saving machinery takes the place of our hands. With a minimum amount of labor we can to-day possess luxuries unknown even to kings in ancient times, and our minds are free to study the order of nature or engage in any intellectual pursuit we may desire. Instead of being the slaves of nature and groveling in the dust before her to find the food which we crave, we have now assumed the command, and find her a willing servant to those who know her language.

But here we reach the keystone of the problem. To command her we must know her language. Knowledge, then, is the price of her service, and she obeys not the ignorant or degraded, but grinds them into dust beneath her heel.

Knowledge, then, is power, and it is more than power; it is that which the intellect most eraves and is the object of many of our highest 
aspirations. What truth is, is the goal of intellectual mankind in all ages, and its pursuit leads not only to intellectual but also to physical satisfaction.

The pursuit of the one leads to the other, and we shall see as we proceed that the only way for the world to progress in practical science is by the cultivation of the theoretical science.

Pure science must exist before its applications, and the truths of pure science are far more reaching in their effects than any of its applications; and yet the applications of science often have a much more immediate interest for the world at large than many discoveries in pure science, which will finally revolutionize it, both physically and mentally. They both have their importance and both are at work in causing that intellectual and material progress in which the world is now pushing forward with giant steps. But there is this difference-the names of the great inventors are seen in every paper and their deeds are recounted to the rising youth of the country as examples to be followed. And yet the discoveries of the principles on which their inventions are based may have died in comparative obscurity, with poverty knocking at the door. We are in no danger of forgetting those who have been successful in those applications of science which are in daily use, and it is useless to repeat the story of the telegraph or telephone, but it will be of more interest for me to recall to your minds a few of the landmarks in our science and then to consider the present state of our science, with a possible glance into the future.

Thus we shall obtain a clearer view of how our science has been built up and of the means which are necessary for its further progress. We shall also see the relations between pure and applied science, and the relative importance of the two in the progress of the world.

It is impossible for one here to discuss the reasons why the ancients followed their science to so short a distance and the world had to wait more than two thousand years before the light of modern science commenced to shine. It must be left to the psychologists and historians. But this I may say, modern progress is cumulative. By the study of the science of the past, the minds of men are trained for its further advance in the future, and so when there was no science to study there could be but little training of the mind in the true methods of thought.

The average intellect of mankind has improved, and what could only have been comprehended in past times by a few is to-day understood by the majority of educated persons. And this increase has been most apparent in the reason and moral sense of mankind, the two qualities of 
the mind which come most into play in the study of science. To the mind of the ancients, where the imagination ran riot without the guide of reason or a warming from their moral sense to speak the truth, it was easier to attribute the attraction of rubbed amber to an inherent soul or essence, which, awakened by friction, went forth and brought back the small particles floating around, than to examine and find out the truth.

The simple experiment of the amber remained without investigation for 2200 years. Had the reasoning of many modern persons been followed, we should never have had a science of electricity. Why should anybody investigate this phenomenon, this feeble force, which could only attract a few particles of dust? The world could eat, drink, and take its ease without doing anything in the matter, and it did so for more than two thousand years of intellectual, moral and physical degradation. Then the awakening came, and men began to feel that they were reasoning beings. 'They began to see that there were other pleasures in the world besides animal pleasures, and that they had been placed in this wonderful universe that they might exalt their intelligence by its proper study. No question of gain entered into the minds of these early investigators, but they were led by that instinct toward truth which indicates the highest type of man. And yet their researches have transformed the world, not only intellectually, but physically. Some would say that science had been degraded by its applications, but who that looks over the world at the present time can think so? There is no danger of this view becoming general; the danger is in the other direction, and that science shall be degraded in the estimate of the world by the idea that its principal use is to be applied to the cornmon purposes of life. A thousand times no! Its use is in the intellectual training of mankind and the high and noble pleasure it gives to those who are born to understand it; to lift mankind above the level of the brute and to make him appreciate the beauties and wonders of nature; to cause him to stand in humiliation and awe before that universe which the intellect of ages has attempted to understand and yet has failed; to make even Newton say, "I know not what the world may think of my labors, but to myself it seems to me that I have been but as a child playing on the seashore; now finding some pebble rather more polished, and now some shell rather more agreeably variegated than another, while the immense ocean of truth extended itself unexplored before me."

But the great moral law of the universe here enters. If the world 
would only pursue those things which are high and right and noble its reward would not be confined to the minds of men. Physical rewards await it as well, and discase, that principal cause of human misery, would almost pass away when the effect of inheritance from the present generation had passed. So the pursuit of pure science brings not only the rewards I have mentioned, but the physical rewards of applied science and the pursuit of applied science gives wealth which may be again employed to further pure science. So the two react on each other to produce that perfect whole, modern science, pure and applied.

This moral law of the universe is well illustrated by the well-known story of Solomon:

"The Lord appeared to Solomon in a dream by night; and God said, Ask what I shall give thee.

"And Solomon said, Thou hast made thy servant king instead of David my father, and I am but a little child; I know not how to go out or come in. Give therefore thy servant an understanding heart to judge thy people, that I may discern between good and bad; for who is able to judge this thy so great a people?

"And God said unto him, Because thou hast asked this thing, and hast not asked for thyself long life, neither hast asked riches for thyself, nor hast asked the life of thine enemies, but hast asked for thyself understanding to discern judgment, behold, I have done according to thy words; lo, I have given thee a wise and an understanding heart, so that there was none like thee before thee, neither after thee shall any arise like unto thee. And I have also given thee that which thou hast not asked, both riches and honor; so that there shall not be any among the kings like unto thee all thy days.",

So the world, when it chose knowledge and truth above all things, acquired not only the treasures of pure theoretical science, but also the wealth and riches and honor which come from applied science such as the world has never seen before and could see in no other way.

It is to William Gilbert, an English physician, that we owe the commencement of the modern science of electricity. His book on the magnet was published in 1600 , and contained his electrical experiments. Thus, at this early date, the similarity of electrical to magnetic attraction was recognized. But how slowly did the subject advance! The difference between conductors and non-conductors was discovered by Gray. But not until 1746, 150 years after Gilbert, was the Leyden jar invented. Then the remarkable nature of the phenomenon became apparent, and the world was startled by it. The subtle spirit which went 
forth from the amber, which was so feeble as only to attract dust, now flashed forth with light and sound and heat, and could cause the strength of the giant to vanish. To the world at large there was now something worth looking into. But do we think that the spark from the Leyden jar is more wonderful than the gentle attraction of the amber? By no means, for, to the scientist, they are both equally remarkable, and beyond our powers of explanation. It is only to the vulgar and uneducated taste that the tinsel and gewgaws of an electric spark appeal more strongly than the subtle spirit of the amber. Nevertheless, despicable as the means, the spark of the Leyden jar acted as a trumpet call to Europe and even America to come to the study of the wonderful science of electricity. At no other time has there been such excitement over any electrical discovery, and electrical experiments became general.

It was only after the discovery of the Leyden jar that the idea of an electric current occurred to mankind, and this current was even transmitted to a distance by a wire and a shock given to a person across the Thames, the water forming the return circuit. And the English experimenters even went so far as to form a circuit with the two observers two miles apart, using the earth as the return circuit. Thus the fundamental fact which forms the basis of the telegraph was early observed.

But isolated facts are of little value unless connected together by something which we call a theory, and in this line we owe much to Franklin, whose letters upon this subject appeared between 1747 and 1754. To him we owe the theory of positive and negative electricity, and the fact that they are always generated in equal amounts, a law whose importance can scarcely be estimated. He investigated the Leyden jar, and showed that the coatings had equal positive and negative charges, and explained the fact that the jar cannot be charged when the outside coating is insulated. He invented the charge and discharge by cascade and showed that it was the glass of the jar and not the coatings which contained the charge. He discovered the property of points in discharging an electrified body, and the identity of lightning with electricity. He also made the first experiments upon atmospheric electricity.

To Canton is due the honor of giving the first experiments on induction, but Franklin is the first who gave the general law of this species of action. Truly our country and this city should honor the memory of this man.

But it is not my purpose to repeat to you in detail the familiar history 40 
of our science. Thus far no important applications of electricity had been discovered; there was nothing but pure science to attract investigators, and thus the science remained for many years after.

But no science is complete unless it is quantitative as well as qualitative. It is now very nearly one hundred years since Coulomb laid the foundation of electrostatics and Aepinus and Cavendish commenced to lay the foundation of mathematical electricity, and they were followed by Laplace, Biot, Poisson, and Murphy.

The discoveries by Galvani and Volta in 1790 and 1800 , and by Oersted in 1820, gave us the galvanic battery and electro-magnetism, and it was not until the latter date that any useful practical application was possible. Then, so complete was the science that no factor of other than minor importance was necessary to transmit intelligence from one extremity of the earth to the other.

By the labors of the immortal Faraday, electro-magnetic induction was discovered and the modern dynamo-electric machine became a certainty.

To his other labors, both experimental and theoretical, the modern science of electricity owes much, but it is familiar to all. The name of Faraday needs no eulogy from me, for it stands where it can never be hidden, and the spark which Faraday first kindled now dazzles us at every street corner. No wealth came to him, though he had only to hold out his hand for it. But the holding out of one's hand takes time, which Faraday could not spare from his labors, and so the wealth which was rightly his went to others. Who will follow in his footsteps and live such a life that the thought of it almost fills one with reverence? It is not only his intellect which we admire; it is his moral qualities which fill us with awe-his noble and unselfish spirit.

The name of Faraday brings us down to modern times, whose history it is unnecessary to repeat in detail, especially as there are some now present who have contributed largely to bring the science to its present perfection.

One of the principal features which we remark in our modern science of electricity is the perfection of our means of measuring both electrical and magnetic quantities. In this connection the great names of Gauss and Weber appear, the fathers of the modern absolute system of electrical and magnetic measurement, and that of Sir William 'Thomson, in no less degree distinguished. On the laws of electric attraction we base our electrostatic system of measurement, and on the magnetic action of the current, the great discovery of Oersted, we base our electro- 
magnetic system, and we connect these two systems by that great physical constant, the ratio of electro-magnetic to the electrostatic system of units.

What can be simpler in theory than the electrostatic system, based, as it is, on the law that electric attraction varies inversely as the square of the distance? We only have to know how the electricity is distributed and its attraction is known. Hence we must select the simplest possible case, such as two parallel disks, and to render the problem calculable, we add a guard ring to the movable disk. We then have the absolute electrometer of Thomson. This gives us a measure of the electric potential. Knowing the capacity and difference of potential of the surface of a condenser, we know its charge. But all these quantities, the calculation of the electrometer and the capacity of the condenser, depend upon the mathematical theory of electric distribution. Are we able to calculate the capacity of condensers of all forms? I am sorry to say we are not. The modern method of treatment is due to George Green, an English investigator, whose name should be held in honor by all electricians. But this method is what is called an inverse one. It is not a method by which we can calculate the distribution on any body at random, but the shape of the body and the electrical distribution on it are both found at once by a species, as it were, of exploration and discovery. So that we cannot make our electrometers and condensers of any shape and then calculate them, but we are forced to make them of some simple geometrical form whose solution is known. We fit our apparatus to the mathematics rather than the mathematics to the apparatus.

But when we have satisfied all the conditions we measure out our static charges as easily as a quantity of matter. The manufacturer sells the oxygen and hydrogen in iron cylinders and determines the amount by the product of the capacity of the cylinders by the pressure. Were there any buyers of electricity we might sell them a Leyden jar full and determine the amount by the product of the capacity of the jar by the electric potential. According to this analogy, then, the electricity is similar to matter and the potential fluid pressure, while the word capacity has a similar meaning in both.

In the electro-magnetic method of electrical measurement we make use of the magnetic action of the current, either on a neighboring magnet or another current or portion of the same current. The laws of the action of a current on a magnet were discovered by Biot and Savart, and of two currents on each other by Ampère, and the results applied to 
practical measurement to-day give us galvanometers of all kinds and the electro-dynamometer of Weber. By the galvanometer we can measure the quantity of electricity passing at any moment, but by the electro-dynamometer we measure the integral square of the current, a quantity on which the heating of the circuit and the energy expended depend.

Thus the electro-dynamometer measures the energy from an alternating current dynamo-electric machine as easily as from one giving a continuous current, but to know this energy we must know something else besides the integral square of the current, and this is either the resistance of the circuit or the electromotive force. But the measurement of electromotive force depends on a resistance. The question then comes up as to what unit of resistance is the proper one. Here we have to refer to the mathematical theory of the subject, and the great law of the conservation of energy tells us that what is known as the absolute unit of electrical resistance is the proper one for use in this case. Hence the great practical use of determining this unit. The experiments of Kirchhoff, Weber, Kohlrausch, and the British Association found a value from 1 to 3 per cent too large.

Many years ago I myself experimented on the subject, and obtained a result about 4 per cent too high. Recently Lord Rayleigh has taken up the matter and made a series of experiments of unparalleled accuracy in this line. The International Commission, determined on by the Electrical Congress in Paris in 1881, met in April of this year at Paris, and has now given us a legal ohm defined as being the resistance of a column of mercury 106 centimetres long and 1 millimetre in section at $0^{\circ} \mathrm{C}$. The length best satisfying the experiments is about $106 \cdot 25$, but it was considered best to use the round number. The experiments which I have been making under an appropriation from the Government are now barely completed, but they will probably agree very well with the latter figure. Hence, we can say that we now know this unit of resistance to one part in one thousand, at least. And so we are in a position to measure the energy of a current to the same degree of accuracy, as far as this quantity is concerned.

But to measure a current by the tangent galvanometer one requires to know the intensity of the earth's magnetism, a quantity difficult to determine and constantly varying with time and place. The electrodynamometer, when made with care, is excellent, but a good one is immensely expensive. Our methods, then, of current measurement are bad, unless carried out in a completely equipped physical laboratory. 
With a practical standard of electromotive force, such as a Clark's standard cell or a thermo-electric battery, this difficulty partially vanishes. Better, perhaps, we might make simple electro-dynamometers with constants determined by comparisons with a more costly instrument.

But where shall these standards be kept? Evidently the Government, which decides on our standards of weights and measures, should take in charge the electrical standards, and possibly also the thermometric standards. The formation of such a Bureau of Physical Standards will be brought to the attention of this Conference.

Having given certain standards then, the measurement of currents and current energy becomes easy. The amount of heat generated in a wire of known resistance by a known current is also easily found from the absolute system of electrical measurement.

Besides the two so-called absolute systems of measurement of electricity and electric currents, we have also one based on the chemical action of the current whose laws were discovered by Faraday. Knowing the electro-chemical equivalent of some substance, we are able to measure the time integral of the current or the total quantity of the current which has passed.

The absolute measurement of magnetism is equally simple with that of electricity, and it is a common observation to find the earth's magnetic force. But Faraday has put in our hands a very simple method of measuring a magnetic field, and to-day all are familiar with his beautiful laws with respect to magnetic lines of force. We know the laws of electro-magnetism, and just how many lines of force (better induction) can pass through a piece of iron of given cross-section, and what is their relative resistance when passing through air or iron. In fact, we have all that is necessary for a complete theory of the dynamo-electric machine, and consequently we find that the latter agrees perfectly with theory, and no fact has becn observed with reference to it which could not have been foreseen from theory by a person of proper intelligence.

This part of electrical science, the measurement of electrical and magnetic quantities, is thus in a very forward state, based, as it is, on the mathematical theory of the subject. But, in reality, this forms but a very small portion of our science. Shall we be contented with a simple measurement of that of which we know nothing? I think nobody would care to stop at this point, although he might be forced to do so. The mind of man is of a nobler cast, and seeks knowledge for itself alone. We are not so base as to be honest because "Honesty is the best policy," 
neither are we so ignoble as to seek knowledge because " Knowledge is power"-two sayings which are certainly true, but low and sordid in their tone.

We have, then, the beautiful fabric of mathematical electricity given to the world by Poisson, Green, Helmholtz, Thomson, Maxwell, and others whose names are immortal. No hypothesis as to the nature of electricity rests at its base. Starting from the most simple laws of electricity and magnetism, it rises from a stable foundation and rears its form high in the air, never to be overturned, whatever the fate of the so-called electric fluid or the ultimate theory of magnetism. On the simple fact that there is no electric force inside a closed conductor, it is proved that the electric attraction and repulsion varies inversely as the square of the distance. The fact is sufficient to give us the whole theory of electrostatic distribution on conductors.

From the simple fact that we can break a magnet up into parts which are similar to each other, and that these parts attract and repel each other in a certain manner, we derive many important facts with regard to magnetism.

From the magnetic action of the current we find, by an application of the great law of conservation of energy, all the laws of induced currents, either from magnets or other currents. By an almost superhuman effort of the intellect we detach our electric currents from matter, and suppose them to take place in the ether of space, and we have the grand electro-magnetic theory of light given to us by Maxwell.

But the subject is too vast to be treated in a moment. Suffice it to say that no person at the present day has the right to express an opinion on any theoretical question connected with electricity without a knowledge of its mathematics.

This study has led us to alter our ideas on many questions. What is the mechanism of electric or magnetic attraction? Faraday has given us his idea of lines of force, and has made them play an important part in the theory of magnetic induction. When treated mathematically, Maxwell has shown that all electric and magnetic attractions can be explained by a tension along the lines of force and pressure at right angles to them-an idea due to Faraday.

The mathematical theory of these lines shows that all electrostatic forces between either conductors or non-conductors can be explained in this manner. As the laws of magnetic attraction are the same in every way as electrostatic attraction, if we should do away with electric conduction, it follows that magnetic attraction is to be explained in exactly 
the same manner. In obtaining this result Maxwell calculated the forces acting on the medium at every point, and compares these with imaginary stresses in a medium at the given point. Hence, the energy stored up can be represented either as due to the mutual attraction of the electricity at a distance, or to the stresses in the medium at every point, and thus, as Thomson has shown, by a volume integral of the square of the force at every point. Hence, we are at liberty to deny the existence of all kction at a distance, and attribute it to the intervening medium, which, to be logical, we must assume to be continuous and not molecular in constitution.

Thomson has pointed out that magnetism must be of the nature of rotation, such as possibly vortex motion in a fluid, and Maxwell has done something toward making a mechanical model of such a medium. Thomson's wonderful address at Montreal has also given us much to think of in the same direction.

But here we have reached the limit of our science, and even that servant of our reason, imagination, fails us. We are yet unable to picture to ourselves what takes place in a medium subject to electrostatic action. We are face to face with the great problem of nature, and the questions, What is matter? What is electricity? evoke no answer from the wisest among us. Our mathematics has guided us safely up to a certain point and will guide us still further; science will advance and we shall know more. But, for the present, this is the limit which we have yet attained in this direction. However, the idea of a medium is still serviceable in other portions of our science.

We have seen that the medium explains the electrical and magnetic attraction of bodies at rest. The question then comes up as to what happens in the medium when these bodies move. Are the imaginary stresses in the medium transmitted from place to place instantaneously or do they require time? Mathematics in the hands of the immortal Maxwell has answered this question, and we now know that any magnetic or electric distrubance is propagated through space with a velocity equal to the ratio of the electro-magnetic to the electrostatic unit of electricity. This great physical constant has now been found by experiment to be equal to the velocity of light, and thus has arisen that great modern theory, Maxwell's electro-magnetic theory of light. Indeed, at the present day, so perfectly does this theory agree with experiment that we can almost regard it as a certainty. The velocity of light and the ratio of the units agree far within the limits of experimental error. The fact that bodies having a true (not electrolytic) electric conduction are 
always more or less opaque, the refraction and dispersion of light, double refraction, and diffraction, all are explained on this theory with an ease and simplicity wanting in all other theories; and, lastly, an electro-magnetic phenomenon has been discovered, which, when applied to this theory of light, explains the rotation of the plane of polarization produced by a magnet. There is no fact in nature seriously in disagreement with this theory, and it serves to connect two of our most important branches of physics, light and electricity.

But some physicists say that it is not a true theory, because it is not mechanical, the object of these physicists being to reduce every phenomenon of nature to matter and motion. Whether this is necessary or not I leave to the philosophers. But it is to be noted that the old mechanical theory that light is a vibration in a medium having the properties of an elastic solid is not entirely at variance with the new theory. The medium we call ether. The electro-magnetic theory says that the waves of light are waves of electric displacement, while the old theory says they are waves of ether. Make electricity and the ether equal to each other and the two theories become one. We have arrived at that hazy and unsatisfactory theory of Edlund that ether and electricity are one, except that by this theory electricity is presented to us as an elastic solid!

But the ground trembles beneath us, and we shall soon be plunged in the mire of vague speculation if we do not draw back.

Among the other questions which depend for their solution on the presence of a medium may be mentioned the mutual action of two electrified bodies moving in space. It has been found that electricity carried through space on a charged body has exactly the same magnetic effect on a stationary magnetic needle as if it had been conducted.

But when electrified bodies move uniformly forward in space, we can conceive of no mutual effect from such motion unless it is relative to a medium, for we cannot even conceive of absolute motion.

Assuming the medium to exist, we then know that a positively and a negatively charged body flying through space with the velocity of light would have their electric attraction just balanced by their magnetic repulsion, and so would exert no force on each other.

But it is a most wonderful fact that we have never been able to discover anything on the earth by which our motion through a medium can be directly proved. Carried, as we suppose, by the earth with immense velocity through regions of space filled with ether, we have never yet been able to prove any direct influence from this ethereal wind. 
The assumption of a medium allows us to solve in some cases that prolylem so long under discussion by electricians-namely, the true velocity of an electric current. We now know that the term velocity hardly applies to this case, and that the current arrives at different points so gradually that we know not when to say it has arrived. But there is certainly a minimum time when even an infinitesimal current can reach a distant point. Suppose two wires stretched in space with their ends near together at one end and a Leyden jar be discharged from one to the other at the near end. The minimum possible time of obtaining a spark at the distant end will evidently be the time required by light to pass from the Leyden jar to the distant point, not around the wire, but in a straight line. In this case the greatest maximum velocity is thus twice that of light reckoned around the wire, and may be any amount greater when we bend the wire. For all ordinary distances this velocity may be considered infinite, and the retardation to depend only on the electrostatic capacity and magnetic self-induction of the wire. Treated in this way, we have Thomson's mathematical theory of the propagation of an electric wave along a telegraph wire or cable, a theory of great practical use in telegraphy and telephony. But until the action in the external medium is also taken into account, it can only be considered an approximation. For we can never move a magnet, discharge a Leyden jar, or complete the circuit of a battery, without causing a wave of electro-magnetic disturbance in the ether, and every signal which is sent along a telegraph line is accompanied by a wave in the ether, which travels outward into space with the velocity of light. Truly the idea of a medium is to-day the keystone of electrical theory, but we can hardly suppose that it has even yet attained a fraction of the importance to which it is destined to rise.

Let me now call your attention to one of the most wonderful facts connected with electrical science. When we are dealing with the electrostatic action of electricity, we find that it is the so-called electric fluid which attracts the opposite. Not only do we observe the attraction of bodies oppositely charged, but the electricity itself on the two bodies is displaced by its mutual action. But when we come to investigate the mutual attraction or repulsion of electric currents on each other, we find an entirely different law. In this case the conductors carrying the currents attract or repel each other, but the currents within those conductors have no influence of attraction or repulsion to displace themselves within the body of the conductor. In other words, the current is not displaced by the action of a neighboring magnet, but flows on calmly as if it were not present. 
This to me is one of the most wonderful facts in electrical science, and lies at the foundation of our science. It cannot be ignored in any further progress we may make in electrical theory, but points out a radical difference between electrostatic and electro-magnetic action.

I have said there is no action of a magnet in displacing an electric current, and have thus stated the broad general fact, and which is perfectly true in some metals. But in others there is a small action which changes in direction with the material. The elements of the electric current within the material are rotated around the lines of magnetic force, sometimes in one direction and sometimes in the other, according to the material. But the action is, in all cases, very weak. When applied to the electro-magnetic theory of light, this action leads to the magnetic rotation of the plane of polarization of light. As to the explanation of both these actions, Thomson has remarked in the case of light, from dynamical considerations, the rotation can only come from a true rotation of something in the magnetic field, and leads us to think of all magnetic action as of the nature of vortex motion in a fluid. But here our theory ends for the present. We have obtained a clew, but it is not yet worked up.

I have now taken a rapid glance at some of the modern advances of electrical science, and we have not yet had to give up the old idea that electricity is liquid. To the profound thinker this idea is very vague, and there are some facts at variance with it, but it is still useful. We often hear persons say that this old idea is gone, and that electricity is "force," whatever they may mean by that. But let us see. The work or energy of an electric current between any two points is the quantity of electricity passed multiplied by the potential; this work goes to heating the wire. Let a current of water be passing in a pipe, and the quantity of water multiplied by the difference of pressure between two points gives us the work which has been done in the intervening space, and which has produced heat. The analogy is complete. No electricity has been destroyed in the one case, or water in the other, but the work has come from the fall of potential in the one case, and the fall of pressure in the other; the resultant is the same in both-heat. Again, we can obtain work from the mutual attraction and repulsion of electrified bodies, and the work in this case always comes from the change of potential between the bodies while the electric charges remain undisturbed in quantity. Electricity, then, is not energy, but is more of the nature of matter.

So far for electricity in the state of rest or steady flow. But when it 
changes from rest to motion, all known liquids have a property known as inertia; furthermore, they have weight. But the electric fluid has neither inertia nor weight as far as we have yet experimented, and in this respect differs from all known matter. Furthermore, we have never yet been able to separate clectricity from ordinary matter. When we pass electricity through a vacuum, the resistance becomes less and less, and one may have hopes of finally having àn electric current through a vacuum. But, as the exhaustion proceeds, we observe that the resistance begins to increase until it reaches such a point that no discharge can take place. Electricity cannot exist, then, without matter, a fact fatal to the idea of a fluid, however useful that may be. We have but. one conclusion from this, and that is that electricity is a property of matter. Do with it what we may, it can never be separated from matter, and when we have an electrical separation the lines of force must always begin and end in matter.

The theory of matter, then, includes electricity and magnetism, and hence light; it includes gravitation, heat, and chemical action; it forms the great problem of the universe. When we know what matter is, then the theories of light and heat will also be perfect; then and only then, shall we know what is electricity and what is magnetism.

It is the problem of the universe which looms up before us and before which we stand in awe. The intellect of the greatest among us appears but feeble and we all, like Newton, appear but as children on the seashore. But how few of us find the shells which Newton did. and how few of us try. The problem is vast and the means for its solution must be of corresponding magnitude. Our progress so far has been but small. When we push our inquiry in any direction we soon reach a limit; the region of the unknown is infinitely greater than the known, and there is no fear of there not being work for the whole world for centuries to come. As to the practical applications which await us, the telegraph, the telephone, and electric lighting are but child's play to what the world will see in the future.

But what is necessary to attain these results? We have seen how the feeble spirit, which was waked up by friction in the amber and went forth to draw in light bodies, has grown until it now dazzles the world by its brilliancy, and carries our thoughts from one extremity of the world to the other. It is the genius of Aladdin's lamp which, when thoroughly roused, goes forth into the world to do us service, and returns bearing us wealth and honor and riches. But it can never be the servant of an ignorant or lazy world. Like the genius of Aladdin's lamp it appeared 
to the world when the amber was rubbed, but the world knew not the language in which to give it orders, and was too lazy to learn it. The spirit of the amber appeared before them to receive its orders, but was only gazed at in silly wonder, and retired in disgust. They had but to order it and it would have gone to the uttermost parts of the earth with almost the velocity of light to do their bidding. But in their ignorance they knew not its language. For two thousand years they did not study it, and when they then began to do so it took them two hundred and fifty years to learn the language sufficiently to make a messenger of it. And even now we are but children studying its A B C. It is knowledge, more knowlédge, that we want.

I have briefly recounted the advances which we have now made in one science, and, however heautiful it may appear, we have soon reached the limit of the known, and have stood in wonder before the vast unknown. For very much of our science we see no practical applications, but we value it no less on that account. We study it because we have been gifted with minds whose exercise delights us, and because it seems to us one of the highest and noblest of employments. And we know by the history of the past that the progress of the world depends on our pursuit, and that practical applications, such as the world has never even conceived of, await us. It is necessary that some should go before to clear the way for the world's advance.

This is the work of the pure scientist; to him the problem of the universe is worth devoting his life, and he looks upon wealth as only adding to his means of research. He hopes not to solve the problem himself, but is contented if he may add some small portion to human knowledge; if he may but do his part in the march of human progress. He looks not for practical applications, but he knows full well that his most abstruse discoveries will finally be made useful to mankind at large, and so troubles himself no further about it.

The science which he crcates is studied by others. Their minds are educated by it and their hearts entranced by its beauties. And some are led to devote their lives to its further advancement. But the whole world benefits by it intellectually. The wayward spirit of the amber has vanished forever, and prosaic, law-abiding electricity has taken its place even in the estimation of the most ignorant. The world has advanced, and in great part from the study of science.

Then comes the practical man, who sees that other benefits can be reaped besides those of pure intellectual enjoyment. While the investigator toils to understand the problem of the universe, the practical 
man seeks to make a servant of our knowledge. He seeks to increase the power of our bodies and to make the bonds by which the mind is united to it less irksome. It is he that increases the wealth of the world, and thus allows those so disposed to cultivate their tastes and to elevate themselves above the savages. The progress of the world depends upon his inventions.

Let not, then, the devotee of pure science despise practical science, nor the inventor look apon the scientific discoverer as a mere visionary person. They are both necessary to the world's progress and they are necessary to each other.

To-day our conntry, by its liberal patent laws, encourages applied science. We point to our inventions with pride, and our machinery in many of the arts is not surpassed. But in the cultivation of the pure sciences we are but children in the eyes of the world. Our country has now attained wealth, and this wealth should partly go in this direction. We have attained an honorable position in applied science, and now let us give back to the world what we have received in the shape of pure science. Thus shall we no longer be dependent, but shall earn our own science as well as inventions.

Let physical laboratories arise; let men of genius be placed at their head, and, best of all, let them be encouraged to pursue their work by the sympathy of those around them. Let the professors be given a liberal salary, so that men of talent may be contented. Let technical schools also be founded, and let them train men to carry forward the great work of applied science.

Let them not be machines to grind out graduates by the thousand, irrespective of quality. But let each one be trained in theoretical science, leaving most of his practical science to be learned afterward, avoiding, however, overtraining. Life is too short for one man to know everything, but it is not too short to know more than is taught in most of our technical schools. It is not telegraph operators, but electrical engineers that the future deniands.

Such a day has almost come to our country and we welcome its approach.

Then, and not till then, should our country be proud and point with satisfaction to her discoveries in science, pure and applied, while she has knowledge enough to stand in humiliation before that great undiscovered ocean of truth on whose shores Newton thought he had but played. 


\section{THE ELECTRICAL AND MAGNETIC DISCOVERIES OF FARADAY}

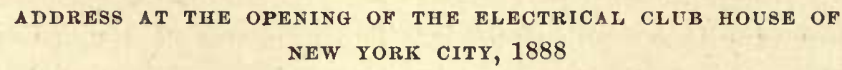

[Electrical Review, New York, Feb. 4, 1888]

In the progress of all sciences there are epochs when men, thoroughly fitted by nature, if not by education also, for the most successful study and advancement of their science, are born into the world, and by their natural talent, perseverance and love of their science, give it an impetus which stamps their name forever on its history. But, however great they may be, we know enough of the nature of scientific progress to be sure that there never was one of such grcatness as to be absolutely necessary to human progress. The world would never have stood still on account of the absence of any name from its annals, and even the place of the immortal Newton would sooner or later have been filled by others, and all the discoveries of his "Principia" have been known to us now, even had he never existed.

Discoveries, then, have their origin not only in the presence of men of exceptional genius in the world, but in a true and overwhelming progress of science which marches forward to the understanding of the universe, irrespective of the efforts of any single individual to promote or retard it. It is a great fact, whose explanation we find in the craving of mankind for knowledge of nature and power over her.

As men of genius are born, they find the discoveries of those who have gone before them awaiting them. They join in the good work, and add their efforts toward the advancement of knowledge. But in all cases they start at the point where those who have gone before them have left off; if their work is good they continue it; if it is bad they replace it by better, that the structure of science may be reared on solid foundations, and grow surely and steadily toward a perfect whole.

To understand, then, the place of any man like Faraday in the history of science, we must also inderstand the state of that science at the time when he did his work.

Michael Faraday, the son of a smith, was born in 1791, and was apprenticed to a bookseller and bookbinder in 1804. He educated himself by reading, and became the assistant of the great chemist, Sir Humphry 
Davy, when he was twenty-two years old. His attention was first given to chemistry, but was finally attracted to electricity by the discovery of electro-magnetism by Oersted, in 1820. At this period the subject of electrostatics was very far advanced even as compared with modern times.

More than 200 years before, Gilbert had commenced the study of electricity, and divided bodies into electrics and non-electrics, according as they produced or did not produce electricity by friction. Nearly 100 years before, Stiphen Gray had discovered the difference between conductors and non-conductors, and had shown the means of carrying electrical effects to a distance of several hundred feet by means of a conducting thread or wire suspended by non-conducting threads of silk. Otto von Guericke, du Fay and Wilke had shown that there were two kinds of electricity-resinous and vitreous. The Leyden jar had been discovered by the Dutch philosophers. Franklin had written his celebrated series of letters on electricity, explaining the phenomenon of the Leyden jar and induction as clearly as we can do it at present, giving his theory of positive and negative electricity to the world, and demonstrating in the most perfect manner the electrical nature of thunder and lightning.

Aepinus and Cavendish had applied mathematics to the subject, and the latter had discovered the law of inverse squares, and made for himself a series of graduated condensers, by which he measured the capacity of differently shaped bodies. They had been followed by Laplace, Poisson and Biot in mathematical electricity. Conlomb had introduced his torsion balance, the first accurate instrument for electrical measurement.

Galvani and Volta had shown how to produce a current of electricity by the galvanic battery. The chemical action of electricity had long been known, and had been forcibly brought before the world by the immortal experiments of Davy only a short time before, and Ritter had discovered polarization and the storage battery.

But, although many persons had suspected that there was some connection between electricity and magnetism it was not until Oersted, in 1820, discovered the nature of this connection, and Ampère had given the laws of the attraction of currents, that the science of electro-magnetism became a subject of investigation. This new discovery aroused the attention of the scientific world to another field of research, and especially awakened in Faraday that sublime curiosity with respect to its laws, which finally led him to his first discovery in this subject. 
The new fact of electro-magnetism interested him. Soon he found that the turning of the needle, as found by Oersted, could be accounted for by the attempt of the north pole to revolve around the wire in one direction and the south pole in the other. Not content with demonstrating the theory, he invented some pieces of apparatus by which this revolution could be realized, and every collection of physical apparatus now has them. The little wires or magnets hanging in the cups of mercury are familiar to all, and form the first notable instance of a continuous rotary motion produced by the electric current; it was the first form of electro-magnetic motor so common in our day. But we can not call this a great discovery, as the principles were very apparent.

Eight or nine years now passed before Faraday gave anything of importance to the world in the subject of electricity and magnetism.

Seebeck discovered thermo-electricity. Ohm discovered the law connecting electro-motive force, resistance and current, and the whole scientific world was alert to discover new facts. Faraday brooded on the subject: the electric current produced magnetism, why should not magnetism produce an electric current? At the present age of the world we could answer this question at once, by aid of the great law of the conservation of energy. But fifty-seven years ago it was unknown, except in a very vague manner; the foreshadowing of this great law soon came into the mind of Faraday, but at this period he could only grope blindly in the dark. He knew that a piece of soft iron became magnetic in the presence of a magnet, and that a conductor was electrified by induction when near a charged body. Reasoning by analogy, why should not a conducting circuit have a current generated in it in the presence of a wire carrying a current? This was Faraday's reasolling, and he proceeded to test it by experiment. Winding two wires side by side, on a cylinder of wood, he passed strong currents of electricity through one of them, and attached the other at its two ends to a galvanometer.Not he slightest permanent deflection was observed, and many a man would have pronounced the experiment a failure. But Faraday was not of that nature; he tried again and again, and while bending over the galvanometer in a vain effort to see a slight permanent deflection, he noticed a little jerk of the needle, almost too small to be noticed. His attention was arrested by this curious action, and he proceeded to investigate it.

He found that this slight movement of the needle was in one direction on making the current, and the opposite direction on breaking it. He substituted a helix, enclosing an unmagnetized needle for the gal- 
vanometer, and he found that it was magnetized by this electrical wave, at the moment of making or breaking the main circuit.

But Faraday was not content until he had discovered all the laws of this new action; he placed two wires on boards, so that, when near together, they were parallel to each other. He now found that the action took place, not only when the current was interrupted, but also when one wire was moved with respect to the other.

So far, the new effect had only been obtained near an electric current. But Faraday did not forget the connection between electricity and magnetism, but now proceeded to give a new aspect to his discovery.

For this purpose he chose a ring of wrought iron on which he wound two coils of wire which he attached to a battery, and to a galvanometer, as before. From the presence of the iron, however, he obtained an immensely greater effect than at first, so that, instead of an almost microscopical deflection, the needle of the galvanometer whirled around three or four times, and on attaching two points of charcoal to the ends of the secondary wire, he observed a minute spark between them on completing the main current. The same increased effects occurred on placing bars of iron in straight coils of wire, and Faraday had now proved that the new effect was dependent on the magnetic action of the current.

He now made one step further, and showed that these induced currents could be obtained from permanent magnets without the aid of other currents, by the simple motion of a wire near a magnet, and that they were specially intense when the wire was wound on a soft iron cylinder, which was then moved near the poles of a magnet. Not content with observing these currents by a galvanometer, he obtained a powerful permanent magnet and allowed his bar of iron, wound with the coil, to come in contact with the poles, the circuit being broken at the same instant. A spark was observed at this broken junction every time the bar came down on the poles. Tyndall tells a very curious story of this experiment which we can well recall. Faraday was attending a meeting of the British Association in 0xford, in 1832, and was requested to show some of his wonderful results to the scientists there gathered. While he was thus occupied a dignitary of the University entered and inquired what was going on. Prof. Daniell, who was standing near, explained the matter in popular language. The Dean listened with attention, and looked earnestly at the brilliant spark, but a moment afterwards he assumed a serious countenance, and shook his head: "I am sorry for it," said he, as he walked away; "I am sorry for 41 
it; indeed I am sorry for it; it is putting new arms into the hands of the incendiary." This occurred a short time after the papers had been filled with the doings of the hayrick burners.

Now, after more than fifty years, the spark of Faraday blazes at every street corner, but it has never been found more efficient than an ordinary lucifer match in the burning of hayricks.

Faraday's attention was now called to the explanation of a curious action discovered by Arago, who found that a rotating disk of copper carried a magnetic needle with it when the latter was suspended over it. The explanation had never been obtained, but Faraday now saw that it was but an instance of his newly discovered action. In order to show that currents were induced in the revolving plate, he mounted it between the poles of a magnet and connected the centre with one pole of a galvanometer; on pressing a wire from the other pole to the edge, Faraday obtained a continuous current of electricity. This was the first continuous current dynamo ever constructed.

But he rested not until he had obtained the laws of induced currents and expressed them in such simple language that they have ever since been the admiration of the scientific world.

In giving the law of the production of these induced currents, Faraday for the first time made use of his famous "lines of force," although he here calls them magnetic curves.

He showed that a wire must cut these lines in order to have a current induced in it. In order to account for the induction in neighboring wires on making and breaking an electric current, he pictured in his mind the lines of force moving. The current could only start gradually after contact was made, and while it was increasing the lines of force always closed on themselves in rings, were expanding outwards cutting any wires near it, and inducing currents in them. When the current was broken, the lines contracted and produced contrary induced currents.

In after years he made his law quantitative, and proved that the integral induced current was in proportion to the number of lines of force cut by the wire.

In his papers of 1831-2 I find these lines always called magnetic curves, and his laws of induced currents are given in terms of these curves. This idea of lines of force was ever after one of the principal - points around which the mind of Faraday revolved. He applied it to electrical action as well as to magnetic, and we see him in after years striving to do away with action at a distance, and substitute for it a medium filled with these lines of force. 
The medium subjected to electrical or magnetic forces is, according to Faraday's idea, polarized in the direction of these lines of force, so that each particle only has to act upon the one next to it in order that the force may be transmitted to any distance. In Faraday's mind these lines had not only an imaginary existence as being the direction in which the north pole of a needle or an electrified particle tended to move in space, but also a real existence. He imagined them as elastic bands repelling each other laterally, and binding the north and south poles of a magnet, or the positive and negative electricities, together.

It was only in after years that he discovered all the properties of these lines, and I shall therefore return to them again.

Guided by these lines of force, he investigated the subject of induced electric currents in so complete a manner that nothing of fundamental importance has ever been added to the subject. True, to-day we understand the subject much better than Faraday ever did. The mathematical researches of Helmholtz, Thomson, Maxwell and others have thrown a flood of light upon the induction of electric currents, and the law of the conservation of energy gives us means of proving all its laws, and indeed of showing that magneto-electric induction is the consequence of the magnetic action of the current as discovered by Oersted.

But fifty years ago this law of the conservation of energy was too little known to be used in this way. It required the support of just such experiments as those of Faraday to bring into existence and to prove it. Hence, Faraday had but little to guide hin to the discovery, except that subtle reasoning of a man of genius which almost amounts to instinct.

The difference of common and voltaic electricity next engaged his attention. A Leyden jar highly charged might have large sparks and a loud sound; it might ignite alcohol and produce a strong shock when passed through the human body, but it was almost incapable of decomposing water, and could scarcely affect a magnetic needle. The voltaic battery, on the other hand, could produce the latter effects, but not the former.

How did these two kinds of electricity differ?

Faraday answered this by producing all the effects with one kind of clectricity that could be obtained from the other. He showed that the difference was caused by there being great tension, or, as we call it, potential in one case, with very little quantity, while in the other there was great quantity with low tension. By charging Leyden jar batteries 
of different sizes with the same number of turns of his machine, and discharging them through a galvanometer, he proved that the sudden deflection of the instrument depended on the quantity, and not the tension, of the electricity. He then arranged a little voltaic battery out of zinc and platinum wires, so that, when joined to the galvanometer for three seconds, it gave the same swing to the needle as the Leyden jar battery charged with thirty turns of his machine. By this means he was able to estimate that a small battery which decompased a grain of water, furnished as much electricity as 800,000 discharges of his large Leyden battery, and would form a powerful stroke of lightning, if discharged at once.

The investigation gives us the first rough idea of the magnitude of the quantities involved in frictional and voltaic electricity, and it may be considered as the first rough approximation to the ratio of the electromagnetic to the electrostatic units of electricity.

But Faraday was a chemist. His associations with Davy had made him familiar from the first with the chemical action of the battery, and it is but natural that his attention should be directed to its investigation. In the progress of these researches he noted the curious fact that all bodies which could be decomposed by electricity when a fluid, could neither conduct the current nor be decomposed by it when they were solidified by the cold. The conduction and decomposition went together. Rising from this to a general law, he finally proved, by immense labor, that, for a given quantity of electricity, whatever the decomposing conductor may be, the amount of chemical action is the same. The current, the size of the electrodes and the strength of the solution might vary, but the amount decomposed by a given quantity of electricity remained the same. Furthermore, the amount of different substances separated was in proportion to their chemical equivalents. Hence, the voltameter for measuring the electric currents which, in the form of the silver voltameter, is to-day one of our most accurate instruments.

As I have mentioned before, the leading idea in Faraday's mind was the replacing of all action at a distance by curved lines of force which had a definite physical existence. So, in attacking this subject of electrolysis, he very quickly showed that Davy's idea that the poles separated an electrolyte, by actually attracting its components, was false, and that the theory, according to which decomposition and recomposition took place throughout the whole course of the current in the electrolyte, was correct. 
Faraday now took up an analogous subject-the source of the electricity in the voltaic battery. He showed that the current from the battery was proportional to the amount of zinc dissolved, and that the direction of the current depended on the direction of the chemical action.

The theory of Volta, that the contact of two metals was the source of electricity, was thus effectually disposed of, so that even the recent attempt to revive that ancient theory could only have met with the disaster which befell it.

It is impossible for me, in a few minutes, to give account of all that Faraday did on these subjects of electrolysis and the theory of the voltaic battery. His work is a perfect mine of results-not haphazard and disconnected, but each designed to elucidate some point in theory or demonstrate some law, and his name must forever be associated with this subject. His law of the definite chemical action of the current will always form an enduring monument to his fame.

Every discovery that Faraday made only served as a guide to him in making fresh ones.

We have seen that Faraday found that when an electrolyte was in the solid state it no longer conducted the current. To most observers this would only have been an interesting, but disconnected, fact. But the far-sighted mind of Faraday perceived in this an explanation of no less a subject than that of electric induction. As in the electrolyte, he conceived the particles to be arranged in certain directions, decomposing and recomposing along lines in the direction of the electric currents, so in the solidified electrolyte there was some arrangement along the lines in which the current wished to pass, that is, of electric force. Hence his theory of the nature of electric induction and of electric force. It was not action at a distance, but the action of contiguous particles on each other. As in magnetism, so in electricity, the action was carried to a distance by a medium.

Not content with merely giving the theory, he proceeded to prove it. If it were true, then the nature of the medium should affect the amount of the induction. We all know his beautiful apparatus for testing this-the two globular Leyden jars which could be filled with air, glass, oil of turpentine, gases, etc., how he divided the charge of one between the two and measured it on a Coulomb electrometer, and thus discovered that his inference was correct, that each substance had a specific inductive capacity, and that the charge of a condenser depended not only on the area of the surface and the thickness of the 
dielectric, but also on the nature of the latter, air or vacuum producing the least condensing effect, and glass, sulphur, etc., a greater one.

To complete his mental vision of an electrified system, it was necessary for him to test in a very complete manner the idea that positive and negative electricities are generated in equal quantities. To accomplish this, he erected a room of twelve feet on a side out of a framework covered with tinfoil, and the whole insulated. By generating electricity inside of it, he was able to prove in a more complete manner than had been done before that we never generate positive electricity or negative electricity by itself, but always in equal quantities together. Every complete electrostatic system contains equal quantities of positive and negative electricity, which are separated by a dielectric, through which they are connected by the lines of electric induction, whose tension produced electric attraction.

To-day, when the mathematics of Maxwell have added clearness to the subject, we see every electrostatic system made up of minute and equal portions of positive and negative electricity, connected together by tubes of induction as by elastic bands, these tubes repelling each other laterally, so as to be held in position, we know that the attraction of all electrified bodies is accounted for by such a system, which was roughly conceived by Faraday, but in which the positions and form of every line can now be calculated.

It is impossible, on the present occasion, to follow Faraday through all his researches on the different forms of electric discharge, and his continued researches on electrolysis; but I will pass immediately to two of his greatest discoveries, the action of magnetism on light and diamagnetism. In his researches on optical glass he had discovered a variety of heavy glass, called silicated borate of lead. On placing this between the poles of a magnet, and looking through it along the lines of force, he found that the plane of polarization was rotated.

Using other substances, he found that most of them had some effect of this kind in the magnetic field. The laws of the magnetic rotation he found very different from those of the ordinary rotation of turpentine or sugar, and altogether it forms a most interesting and important experiment when considering the theory of magnetism.

Not content with discovering this law with his piece of optical glass, he now sought to discover whether there was any force of attraction or repulsion between it and the magnet. Hanging it up between the poles, he discovered that as iron was attracted by a magnet, so the heavy glass was repelled. He called this property diamagnetism, and showed 
that all bodies were acted upon by magnetism and could be classified as magnetic or dianagnetic. Magnetism now had a universal signifieance as applying to all bodies. It was universal in its action, and all bodies responded to it to some extent at least. Even gases were acted on by it, and the oxygen of the air was found quite strongly magnetic.

Quickly his mind seized another idea.

As the intense magnetism of iron, nickel and cobalt was destroyed by heat, might it not be possible that all bodies should become magnetic when cold? He carefully tried the experiment, but never was able to find any effect with the means of producing cold at his command.

In reading Faraday's papers we are surprised at the elearness with which his laws are expressed. Although he naturally wished to bring his lines of force into use in this case of diamagnetism, yet we now find him making no use of them. His law says that magnetie substances in the field of a magnet tend to the stronger part of the field, and the diamagnetic to the weaker, irrespective of the direction of the lines of force.

Bismuth he found the most strongly diamagnetic of all bodies. In using a crystal of this substance instead of a bar, he found that it would set itself in a magnetie field, even if this was uniform. On using other substances he proved the general law that all crystals possessed this property and he called it magne-crystallie force.

The researches on diamagnetism and magne-crystallic force oceupied Faraday's time for five years, from 1845 to 1850 , and he was now in the sixtieth year of his age. No more great discoveries fell to his lot, but his mind turned more and more to brooding over the consequences of his past discoveries and following ont their results.

The idea of lines of force was still on his mind, and the diseovery of diamagnetism had now given him a further insight into their nature. He saw that the magnetic and diamagnetic nature of bodies could be explained by considering them as good or bad conductors of these lines of force. Iron was a good eonductor and bismuth a bad one. When soft iron was placed in a magnetic field, the lines of force, or, as we now more exactly term them, the lines of induction, were more easily conducted by it than by the air, and they were defleeted toward and through it; but a piece of bismuth was a poorer conductor and these lines of force tended to pass around it rather than through it. By surrounding a weak magnetic body by a strong magnetie fluid he found that it possessed all the properties of a diamagnetic one. Pursuing the subject, he showed how the lines of induction were distributed around and within 
a magnet, and how we are able to measure them by the induced current in moving wires. The method of exploring the magnetic field is the only exact method which has ever been devised for use in such cases as the field of modern dynamo-electric machines, or in most of the problems of modern electrical engineering. He also proved that the lines of induction are always closed circuits, whether they are due to permanent magnets or electric currents, thus forever destroying our hope of obtaining a continuous current by induction without the use of a commutator.

When a soft iron bar was approached to the magnet, it drew the lines in upon itself; they proceeded down the bar until they were forced into the badly conducting air and the number which went further down the bar to those which passed out into the air at any point was in proportion to the conductivity of the two. A steel magnet was, in his eyes, like a voltaic pile in water. As the current of electricity was forced forward by the electromotive force of the pile and diffused itself in currents through the water, so the lines of magnetic induction were formed by the coercive power of the steel. It is now known to be a fact that the distribution of magnetism on a steel magnet, or indeed in any case, can be calculated by these principles Faraday laid down. The idea of a magnetic circuit is familiar now to all electrical engineers.

To Faraday's eye, a magnet not only consisted of a piece of steel or loadstone which is apparent to our ordinary vision, but included all the space around which was filled with lines of force; it was bounded only by the limits of the universe. The steel served merely to bind together the ring-like lines of induction which passed from the magnet to every point of space.

Faraday was not a mathematician, and could not thus follow out the consequences of his great ideas. This has been done for him by the immortal Maxwell. He has taken up the idea that electrical and mag- netic forces only proceed to a distance by aid of the intervening particles of matter, or ether, as the case may be, and has given it a mathematical basis.

To-day a body charged with electricity, a magnet or a wire carrying an electrical current, all are incomplete without the space around them.

When we attach a battery to a wire and the current apparently flows through it as if it were a current of water, Faraday's idea shows us that we are only looking at the matter superficially; around that wire and permeating space in every part are lines of magnetic force, and lines of electrostatic force. At the moment of joining the battery to the wire this whole complicated system of lines of force must be formed. At the 
moment of breaking circuit, the system must vanish, and we obtain the energy stored up in this space surrounding the wire in the bright spark known as the extra current.

What a flood of light this throws on many experiments such as those of Wheatstone, on the velocity of electricity. With his wire arranged in parallel loops around an ordinary room, he discharged a Leyden jar through it, and assumed that the electricity passed through the whole wire before a spark could form at the distant end. But we know that whole room was instantly filled with moving lines of magnetic force, which induced currents in every wire they crossed, and hence what Wheatstone measured was merely the current induced from one wire or those near it.

Thomson and Maxwell have shown that the medium around a wire carrying an electric current is in motion, and that the vortex filaments form Faraday's lines of magnetic force; for Faraday's discovery of the magnetic rotation of the plane of polarization of light can be explained in no other way.

Thus the discoveries of Faraday have been engrafted on our science, and form one of its most essential features. They are among the foundation stones of the edifice of our science.

We know far more than the electricians of that day, in the details of the subject, and mathematics has given us a broad view of electricity and magnetism, such as never before was obtained. In its practical use and measurement we have made immense strides in devising methods and instruments, and we now carry out our experiments on a scale which Faraday could not attempt, seeing that subject, which has hitherto been best adapted to the contemplation of a few philosophers, has become of use to all, and electricity bids fair to become our most important servant.

The spark, which Faraday more than fifty years ago observed in a darkened room, now blazes out almost with the power of the sun, but it is still the spark of Faraday. Though it is a thousand times as large, it is still made on the prineiples which Faraday laid down, and nothing except mechanical details has ever been added to its process.

How suitable, then, that we should remember his name on this occasion, since his discoveries have served as the basis of all progress in electrical engineering. Had Faraday not lived we should not have been here to-night. True, as I have shown before, the progress of science could only have been delayed by the absence of any one man, but how long, in this case, we cannot tell. We can only receive with gratitude 
what Faraday has given freely to us, and speak his name with the reverence due, not only to his intellectual eminence, but to his character. Too noble to leave science for the wealth held out to him, he persevered in it to the end, and gave to the world the fruits of his labor in his 'Experimental Researches in Electricity.'

He never obtained from the world the material reward for his labor, but died a poor man, who had enriched the world.

We stand at an important epoch in the history of our science. We have gone far enough into its practical applications to see some distance into the future. The are light, which Davy brought into prominence at the beginning of this century, fed by the machines of Faraday, blazes throughout the night in all cities of the world. The incandescent light, known long to scientists, has been improved and bids fair to rival gas in cheapness, as it surpasses it in beauty. The secondary battery discovered by Ritter eighty years or more ago, improved by Planté and Faure in recent times, still struggles to fill the place assigned to it, to be replaced by one before long which shall not waste fifty per cent of the power given to it, and weigh tons for a few foot-pounds of energy stored up. We see it in its new form replacing the laboring horses in the streets, and serving in many cases where small power is needed. But the transmission of energy seems to me to open one of the widest fields, and the time is not very distant when a few large engines will replace the numerous small ones in our cities; when also the power of waterfalls may be made available at a distance.

The principle of the telephone also is destined to bear unseen fruit.

There is work for all, the practical and theoretical man alike.

The philosopher, studying the problems of the universe, deems himself rewarded by some new fact discovered, some new law demonstrated. To him the universe is a problem to solve, and his motto is, "Science is knowledge."

He sees before him the time when man's insight into nature shall be vastly increased, and esteems the science of to-day as but an atom to what we shall know in the future. While not despising the wealth, he seldom has time for its accumulation, as he considers other things of vastly more importance; the truth is what he seeks; the truth as to this wonderful universe in which we live. What is matter? what is electricity, what is the medium which transmits light from one point to another, how comes it that the earth is magnetic? These are some of the problems he is trying to solve. He knows that one man can do but little toward it, even though he should surpass what Faraday has done, but 
he trusts to the combined efforts of mankind, shown in the steady progress of science, to finally arrive at a solution.

The devotee of applied science, the so-called practical man, looks upon the forces of nature as his servants, and strives to become their master. The world must move, its work must be accomplished. We are not satisfied to live as our fathers have done, and we must have luxuries unknown to them. Our thoughts must fly to the farthest parts of the earth in an instant, at our bidding, and we must pass from point to point on the wings of the wind, for flesh and blood is too slow for us. To accomplish this, the engineer harnesses the forces of nature and compels them to work for him. He takes the discoveries of the philosopher and uses them for the practical needs of daily life. His motto is, "Science is power." As he ministers more directly to the present generation of mankind than to the generations to come, as does the philosopher, so he often reaps his reward in the present, and retains some of that wealth which his inventions bring into the world. For the source of the wealth of the world is labor, and the labor of the forces of nature, in our behalf, surpasses very many fold that of human flesh and blood. He who adds but the slightest to our power over these forces enriches the world, and is entitled to its practical, as well as its sentimental gratitude, be he philosopher or engineer. The great question which we should ask ourselves is how our science can best be furthered. The philosopher must precede the engineer. To have the applications of electricity, there must be a science of electricity. This science cannot depend for its existence on practical men whose minds are engrossed with other than theoretical problems. It must exist in minds like Faraday, which are specially adapted to its reception and advancement-men who are willing to devote their lives to it, and who have the ability to further it. We cannot create such men, but we can give them our practical as well as our sentimental sympathy, when found. The philosopher is made of flesh and blood as well as other men. He must live and have his tastes gratified as well as others. His place in the world as at present constituted is usually that of a professor in our universities and colleges. Are only men like Faraday chosen for these positions? Of the four hundred or more, how many choose their professors on account of their eminence in theoretical science? Are there a dozen? I doubt it. Furthermore, what facilities and encouragement would they have in these institutions to do work? Too far away from each other to be a mutual help, they have but an incomplete scientific life. Faraday could not have been himself in 
Africa and would have languished in our own country. In London, in contact with the science of Europe and encouraged by its atmosphere, with the Royal Society at which to announce his discoveries and the Royal Institution in which to make them, Faraday, in spite of poor education, was stimulated to his best efforts. Alone in one of our isolated colleges, cut off from intercourse with the rest of the world by a so-called protective duty on his very life, books, with no journal specially devoted to theoretic physics, and no society like the Royal Society, who can say whether his discoveries would have been made or not? The endowment of research seems to me to offer the best means out of the difficulty. Let professorships be endowed and funds to pay the expenses of apparatus and assistants be formed in our universities, with the understanding that the research is to be the principal work; work, while teaching is not to be neglected. The result will be the formation of a scientific atmosphere in which men like Faraday can live and labor, and the dry bones of the pedagogue be replaced by the fire and life of the original investigator. And let not practical science be neglected. Let us have scientific schools of the highest grade, where modern science is taught, so that fifty years shall not again pass, as it has done, before a discovery like that of Faraday is utilized.

Furthermore, let us have scientific societies and clubs like the present, where men of like tastes can meet and interchange ideas.

Thus we meet together to-night, electricians all, practical and theoretical, at a time in the history of our science and of the world which will in future be called the beginning of the age of electricity.

The feeble attraction of the amber has become a mighty force, which is destined to make itself felt, and it is to be hoped that our mutual intercourse in this Club may aid us all in our efforts to make an impress on its future history. 


\section{ON MODERN VIEWS WITH RESPECT TO ELECTRIC CURRENTS}

ADDRESS BEFORE THE AMERICAN INSTITUTE OF ELECTRICAL ENGINEERS, NEW YORK, MAY 22,1889

[Transactions of the American Institute of Electrical Engineers, VI, 342-357, 1889]

As, a short time since, I stood in a library of scientific books and glanced around me at the works of the great masters in physics, my mind wandered back to the time when the apparatus for a complete course of lectures on the subject cf electricity consisted of a piece of amber and a few light bodies to be attracted by it. From that tune until now, when we stand in a magnificent laboratory with elaborate and costly apparatus in great part devoted to its study, how greatly has the world changed and how our science of electricity has expanded both in theory and practice until, in the one case, it threatens to include within itself nearly the whole of plyysies, and in the other to make this the age of electricity.

Were I to trace the history of the views of physicists with respect to electric currents it would include the whole history of electricity. The date when the conception of an electric current was possible was when Stephen Gray, about 170 years ago, first divided bodies into conductors and non-conductors, and showed that the first possessed the property of transmitting electrical attractions to a distance. But it was only when the Leyden jar was discovered that the idea of a current became very definite. The notion that electricity was a subtle fluid which could flow along metal wires as water flows along a tube, was then prevalent, and, indeed, remains in force to-day among all except the leaders in scientific thought. It is not my intention to depreciate this notion, which has served and still serves a very important purpose in science. But, for many years, it has been recognized that it includes only a very small portion of the truth and that the mechanism by which energy is transmitted from one point of space to another by means of an electric current is a very complicated one.

Here for instance, on the table before me are two rubber tubes filled with water, in one of which the water is in motion, in the other at rest. It is impossible, by any means now known to us, to find out, without moving the tubes, which one has the current of water flowing in it and 
which has the water at rest. Again, I have here two wires, alike in all respects, except that one has a current of electricity flowing in it and the other has not. But in this case I have only to bring a magnetic needle near the two to find out in which one the current is flowing. On our ordinary sense the passage of the current has little effect; the air around it does not turn green or the wire change in appearance. But we have only to change our medium from air to one containing magnetic particles to perceive the commotion which the presence of a current may cause. Thus this other wire passes through the air near a large number of small suspended magnets, and, as I pass the current through it, every magnet is affected and tends to turn at right angles to the wire and even to move toward it and wrap itself around it. If we suppose the number of these magnets to become very great and their size small, or if we imagine a medium, every atom of which is a magnet, we see that no wire carrying a current of electricity can pass through it without creating the greatest commotion. Possibly this is a feeble picture of what takes place in a mass of iron near an electric current.

Again, coil the wire around a piece of glass, or indeed, almost any transparent substance, and pass a strong current through the wire. With our naked eye alone we can see no effect whatever, as the glass is apparently unaltered by the presence of the current; but, examined in the proper way, by means of polarized light, we see that the structure of the glass has been altered throughout in a manner which can only be explained by the rotation of something within the glass many millions of times every second.

Once more, bring a wire in which no current exists nearer and nearer to the one carrying the current, and we shall find that its motion in such a neighborhood causes or tends to cause an electric current in it. Or, if we move a large solid mass of metal in the neighborhood of such a current we find a peculiar resistance unfelt before, and if we force it into motion we shall perceive that it becomes warmer and warmer as if there was great friction in moving the metal through space.

Thus, by these tests, we find that the region around an electric current has very peculiar properties which it did not have before, and which, although stronger in the neighborhood of the current, still extend to indefinite distances in all directions, becoming weaker as the distances increase.

How great, then, the difference between a current of water and a current of electricity. The action of the former is confined to the interior of the tube, while that of the latter extends to great distances on all 
sides, the whole of space being agitated by the formation of an electric current in any part. To show this agitation, I have here two large frames with coils of wire around them. They hang face to face about 6 feet apart. Through one I discharge this Leyden jar, and immediately you see a spark at a break in the wire of the other coil, and yet there is no apparent connection between the two. I can carry the coils 50 feet or more apart, and yet by suitable means I can observe the disturbances due to the current in the first coil.

The question is forced upon us as to how this action takes place. How is it possible to transmit so much power to such a distance across apparently unoccupied space? According to our modern theory of physics there must be some medium engaged in this transmission. We know that it is not the air, because the same effects take place in a vacuum, and, therefore, we must fall back on that medium which transmits light and which we have named the ether. That medium which is supposed to extend unaltered throughout the whole of space, whose existence is very certain but whose properties we have yet but vaguely conceived.

I cannot, in the course of one short hour, give even an idea of the process by which the minds of physicists have been led to this conclusion or the means by which we have finally completely identified the ether which transmits light with the medium which transmits electrical and magnetic disturbances. The great genius who first identified the two is Maxwell, whose electro-magnetic theory of light is the centre around which much scientific thought is to-day revolving, and which we regard as one of the greatest steps by which we advance nearer to the understanding of matter and its laws. It is this great discovery of Maxwell which allows me, at the present time, to attempt to explain to you the wonderful events which happen everywhere in space when one establishes an electric current in any other portion.

In the first place, we discover that the disturbance does not take place in all portions of space at once, but proceeds outwards from the centre of the disturbance with a velocity exactly equal to the velocity of light. So that, when I touch these wires together so as to complete the circuit of yonder battery, I start a wave of ethereal disturbance which passes outwards with a velocity of 185,000 miles per second, thus reaching the sun in about eight minutes, and continues to pass onwards forever or until it reaches the bounds of the universe. And yet none of our senses inform us of what has taken place unless we sharpen them by the use of suitable instruments. Thus, in the case of these two coils of wire, suspended near each other, which we have already used, when the wave 
from the primary disturbance reaches the second coil, we perceive the disturbance by means of the spark formed at the break of the coil. Should I move the coils further apart, the spark in the second coil would be somewhat delayed, but the distance of 185,000 miles would be necessary before this delay could amount to as much as one second. Hence the effects we observe on the earth take place so nearly instantaneously that the interval of time is very difficult to measure, amounting, in the present case, to only $\frac{1}{150000000}$ of a second.

It is impossible for me to prove the existence of this interval, but I can at least show you that waves have something to do with the action here observed. For instance, I have here two tuning forks mounted on sounding boxes and tuned to exact unison. I sound one and then stop its vibrations with my hand, instantly you hear that the other is in vibration, caused by the waves of sound in the air between the two. When, however, I destroyed the unison by fixing this piece of wax on one of the forks, the action ceases.

Now, this combination of a coil of wire and a Leyden jar is a vibrating system for electricity and its time of vibration is about $10,000,000$ times a second. This second system is the same as the first, and therefore its time of vibration is the same. You see how well the experiment works now because the two are in unison. But let me take away this second Leyden jar, thus destroying the unison, and you see that the sparks instantly cease. Replacing it, the sparks reappear. Adding another on one side and they disappear again, only to reappear when the system is made symmetrical by placing two on each side.

This experiment and that of the tuning forks have an exact analogy to one another. In each we have two vibrating systems connected by a medium capable of transmitting vibrations, and they both come under the head of what we know as sympathetic vibrations. In the one case, we have two mechanical tuning forks connected by the air; in the other, two pieces of apparatus which we might call electrical tuning forks, connected by the luminiferous ether. The vibrations in one case can be seen by the eye or heard by the ear, but in the other case they can only be perceived when we destroy them by making them produce a spark. The fact that we are tble tr. increase the effect by proper tuning demonstrates that vibrations are concerned in the phenomenon. This can, however, be separately demonstrated by examining the spark by means of a revolving mirror, when we find that it is made up of many successive sparks corresponding to the successive backward and forward movements of the current. 
The fact of the oscillatory character of the Leyden jar discharge was first demonstrated by our own countryman, Henry, in 1832, but he pursued the subject only a short distance, and it remained for Sir William Thomson to give the mathematical theory and prove the laws according to which the phenomenon takes place.

Thus, in the case of a charged Leyden jar whose inner and outer coatings have been suddenly joined by a wire, the electricity flows back and forth along the wire unitil all the energy originally stored up in the jar has expended itself in heating the wire or the air where the spark takes place and in generating waves of disturbance in the ether which move outward into space with the velocity of light. These ethereal waves we have demonstrated by letting them fall on this coil of wire and causing the electrical disturbance to manifest itself by electric sparks.

I have here another more powerful arrangement for producing electromagnetic waves of very long wave-length, each one being about 500 miles long. It consists of a coil, within which is a bundle of iron wires. On passing a powerful alternating current through the coil, the iron wires are rapidly magnetized and demagnetized, and send forth into space a system of electro-magnetic waves at the rate of 360 in a second.

Here, also, I have another piece of apparatus [a lamp] for sending out the same kind of electro-magnetic waves; on applying a match, we start it into action. But the last apparatus is tuned to so high a piteh that the waves are only $\frac{1}{50000}$ inch long, and 55,000,000,000,000 are given out in one second. These short waves are known by the name of light and radiant heat, though the name radiation is more exact. Placing any body near the lamp so that the radiation can fall on it, we observe that when the body absorbs the rays it is heated by them; the well-known property of so-called radiant heat and light. Is it not possible for us to get some substance to absorb the long waves of disturbance, and so obtain a heating effect? I have here such a substance in the shape of a sheet of copper, which I fasten on the face of a thermopile, and I hold it where the waves are the strongest [near the coil while the alternating current is passing through it]. As I have anticipated, great heat is generated by their absorption, and soon the plate of copper becomes very warm, as we see by this thermometer, by feeling it with the hand, or even by the steam from water thrown upon it. In this experiment the copper has not touched the coil or the iron wire core, although if it did they are very much cooler than itself. The heat has been produced by the absorption of the waves in the same way as a 
blackened body absorbs the rays of shorter wave-length from the lamp; and, in both cases, heat is the result. ${ }^{1}$

But in this experiment, as in the first one, the wave-like nature of the disturbance has not been proved experimentally. We have caused electric sparks, and have heated the copper plate across an interval of space, but have not in either of these cases proved experimentally the progressive nature of the disturbance; for a ready means of experimenting on the waves, obtaining their wave-length and showing their interferences, has hitherto been wanting. This deficiency has been recently overcome by Professor Hertz, of Carlsruhe, who has made a study of the action of the coil, and has shown us how to use it for experiments on the ethereal waves, whose existence had before been made certain by the mathematics of Maxwell.

I scarcely know how to present this subject to a non-technical audience and make it clear how a coil of wire with a break in it can be used to measure the velocity and wave-lengths of ethereal waves. However, I can but try. If the waves moved very slowly, we could readily measure the time the first coil took to affect the second, and show that this time was longer as the distance was greater. But it is absolutely inappreciable by any of our instruments, and another method must be found. To obtain the wave-length Professor Hertz used several methods, but that by the formation of stationary waves is the most easily grasped. Mr. Ames holds in his hand one end of a spiral spring, which makes a very heary and flexible rope. As he sends a wave down it, you see that it is reflected at the further end, and returns again to his hand. If, however, he sends a succession of waves down the rope, the reflected waves interfere with the direct ones, and divide the rope into a succession of nodes and loops, which you now observe. So a series of sound waves, striking on a wall, form a system of stationary waves in front of the wall. With this in view, Professor Hertz established his apparatus in front of a reflecting wall, and observed the nodes and loops by the sparks produced in a ring of wire. It is impossible for me to repeat this experiment before you, as it is a very delicate one, and the sparks produced are almost microscopic. Indeed, I should have to erect an entirely different apparatus, as the waves from the one before me are nearly $\frac{1}{4}$ mile long, the time of vibration of the system being very great, that is $\frac{1}{1000000}$ of a second. To produce shorter waves we must use appa-

1 The thermopile was connected with a delicate mirror galvanometer, the deflections of which were shown on a screen. 
ratus tuned, as it were, to a higher pitch, in which the same principle is, however, employed, but the ethereal waves are shorter, and thus several stationary waves can be contained in one room.

The testing coil is then moved to different portions of the room, and the nodes are indicated by the disappearance of the sparks, and the loops by the greater brightness of them. The presence of stationary waves is thus proved, and their half wave-length found from the distance from node to nolle, for stationary waves can always be considered as produced by the interference of two progressive waves advancing in opposite directions.

However interesting a further description of Professor Hertz's experiments may be, we have gone as far in that direction as our subject carries us, for we have demonstrated that the production of a current in a wire is accompanied by a disturbance in the surrounding space; and, although $I$ have not experimentally demonstrated the ethereal waves, yet I have proved the existence of electric oscillations in the coils of wire and the ether surrounding it.

Our mathematics has demonstrated, and experiments like those of Professor Hertz have confirmed the demonstration, that the wave disturbance in the ether is an actual fact.

The closing of a battery circuit, then, and the establishment of a current of electricity in a wire is a very different process from the formation of a current of water in a pipe, though, after the first shock, the laws of the flow of the two are very much alike. But even then, the medium around the current of electricity has very strange properties, showing that it is accompanied by a disturbance throughout space. The wire is but the core of the disturbance, which latter extends indefinitely in all directions.

One of the strangest things about it is that we can calculate with perfect exactness the velocity of the wave propagation and the amount of the disturbance at every point and at any instant of time; but as yet we cannot conceive of the details of the mechanism which is concerned in the propagation of an electric current. In this respect our subject resembles all other branches of physics in the partial knowledge we have of it. We know that light is the undulation of the luminiferous ether, and yet the constitution of the latter is unknown. We know that the atoms of matter can vibrate with purer tones than the most perfect piano, and yet we cannot even conceive of their constitution. We know that the sun attracts the planets with a force whose law is known, and yet we fail to picture to ourselves the process by which it takes our earth within 
its grasp at the distance of many millions of miles and prevents it from departing forever from its life-giving rays. Science is full of this half knowledge, and the proper attitude of the mind is one of resignation toward that which it is impossible for us to know at present and of earnest striving to help in the advance of our science, which shall finally allow us to answer all these questions.

The electric current is an unsolved mystery, but we have made a very great advance in understanding it when we know that we must look outside of the wire at the disturbance in the medium before we can understand it: a view which Faraday dimly held fifty years ago, which was given in detail in the great work of Maxwell, published sixteen years since, and has been the guide to most of the work done in electricity for a very long time. A view which has wrought the greatest changes in the ideas which we have conceived with respect to all electrical phenomena.

So far, we have considered the case of alternating electric current in a wire connecting the inner and outer coatings of a Leyden jar. The invention of the telephone, by which sound is carried from one point to another by means of electrical waves, has forced into prominence the subject of these waves. Furthermore, the use of alternating currents for electric lighting brings into play the same phenomenon. Here, again, the difference between a current of water and a current of electricity is very marked. A sound wave, traversing the water in the tube, produces a to and fro current of water at any given point. So, in the electrical vibration along a wire, the electricity moves to and fro along it in a manner somewhat similar to the water, but with this difference:the disturbance from the water motion is confined to the tube and the oscillation of the water is greatest in the centre of the tube, while, in the case of the electric current, the ether around the wire is disturbed and the oscillation of the current is greatest at the surface of the wire and least in its centre. The oscillations in the water take place in the tube without reference to the matter outside the tube, whereas the electric oscillations in the wire are entirely dependent on the surrounding space, and the velocity of the propagation is nearly independent of the nature of the wire, provided only that it is a good conductor.

We have, then, in the case of electrical waves along a wire, a disturbance ontside the wire and a current within it, and the equations of Maxwell allow us to calculate these with perfect accuracy and give all the laws with respect to them.

We thus find that the velocity of propagation of the waves along a 
wire, hung far away from other bodies and made of good conducting material, is that of light, or 185,000 miles per second; but when it is hung near any conducting matter, like the earth, or inclosed in a cable and sunk into the sea, the velocity becomes much less. When hung in space, away from other bodies, it forms, as it were, the core of a system of waves in the ether, the amplitude of the disturbance becoming less and less as we move away from the wire. But the most curious fact is that the electric currer t penetrates only a short distance into the wire,

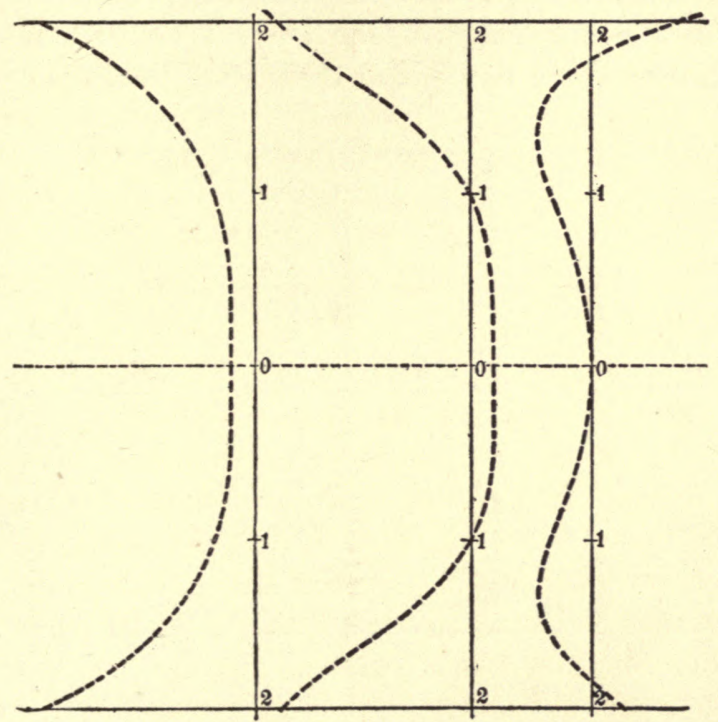

DiAgRAM 1.

being mostly confined to the surface, especially where the number of oscillations per second is very great.

The electrical waves at the surface of a conductor are thus, in some respects, very similar to the waves on the surface of the water. The greatest motion in the latter case is at the surface, while it diminishes as we pass downward and soon becomes inappreciable. Furthermore, the depth to which the disturbance penetrates into the water increases with increase of the length of the wave, being confined to very near the surface for very short waves. So the disturbance in the copper penetrates deeper as the waves and the time of oscillation are longer, and the disturbance is more nearly confined to the surface as the waves become shorter. I have recently made the complete calculations with respect 
to these waves, and have drawn some diagrams to illustrate the penctration of the alternating current into metal cylinders. The first diagram represents the current at different depths in a copper cylinder, $45 \mathrm{~cm}$. diameter, or an iron one $14 \frac{1}{2} \mathrm{~cm}$. diameter, traversed by an alternating. current with 200 reversals per second. The first and second curves show us the current at two different instants of time, and show us how the phase changes as we pass downward into the cylinder. By reference to the third curve we see that it may be even in the opposite direction in the centre of the cylinder from what it is at the surface. The third curve gives us the amplitude of the current oscillations at different depths irrespective of the phase, and it shows us that the current at the

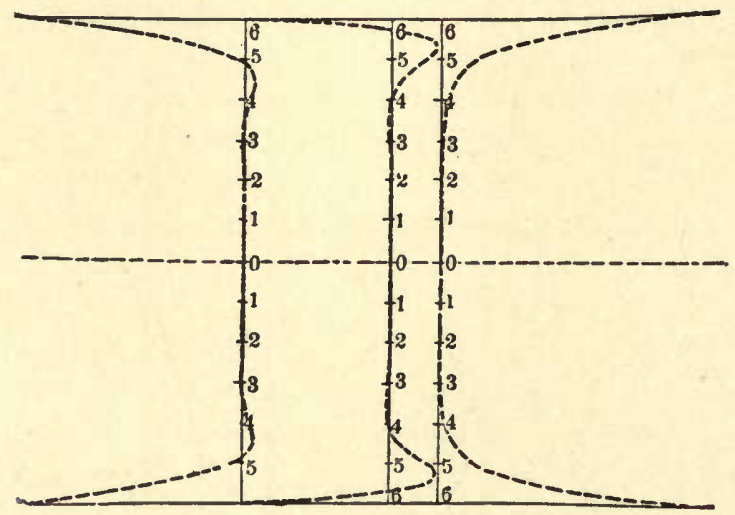

DIAGRAM 2.

centre is only about 10 per cent of that at the surface in this case. The second diagram shows us the distribution in the same cylinders when the number of reversals of the current is increased to 1800 per second. Here we see that the disturbance is almost entirely confined to the surface, for at a depth of only $7 \mathrm{~mm}$. the disturbance almost entirely vanishes.

There are very many practical applications of these theoretical results for electric currents. The most obvious one is to the case of conductors for the alternating currents used in producing the electric light. We find that when these are larger than about half an inch diameter they should be replaced by a number of conductors less than half an inch diameter, or by strips about a quarter of an inch thick, and of any convenient width. But this is a matter to be attended to by the electric light companies.

Prof. Oliver J. Lodge has recently, in the British Association, drawn 
attention to the application of these results to lightning rods. Almost since the time of Franklin there have been those who advocated the making of lightning rods hollow, to increase the surface for a given amount of copper. We now know that these persons had no reason for their belief, as they simply drew the inference from the fact that electricity at best is on the surface. Neither were the advocates of the solid rods quite correct, for they reasoned from the fact that electricity in a state of steady flow cccupies the whole area of the conductor equally. The true theory, we now know, indicates that neither party was entirely correct and that the surface is a very important factor in the case of a current of electricity so sudden as that from a lightning discharge. But increase of surface can best be obtained by multiplying the number of conductors, rather than making them flat or hollow; and, at the same time, Maxwell's principle of enclosing the building within a cage can be carried out. Theory indicates that the current penetrates only onetenth the distance into iron that it does into copper. As the iron has seven times the resistance of copper, we should need 70 times the surface of iron that we should of copper. Hence I prefer copper wire about a quarter of an inch diameter and nailed directly to the house without insulators, and passing down the four corners, around the eaves and over the roof, for giving protection from lightning in all cases where a metal roof and metal down spouts do not accomplish the same purpose.

Whether the discharge of lightning is ascillatory or not does not enter into the question, provided it is only sufficiently sudden. I have recently solved the mathematical problem of the electric oscillations along a perfectly conducting wire joining two infinite and perfectly conducting planes parallel to each other, and find that there is no definite time of oscillation, but that the system is capable of vibrating in any time in which it is originally started. The case of lightning between a cloud of limited extent and the earth along a path through the air of great resistance is a very different problem. Both the cloud and the path of the electricity are poor conductors, which tends to lengthen the time. If I were called on to estimate as nearly as possible what took place in a flash of lightning, I would say that I did not believe that the discharge was always oscillating, but more often consisted of one or more streams of electricity at intervals of a small fraction of a second, each one continuing for not less than $\frac{1}{10000}$ second. An oscillating current with 100,000 reversals per'second would pentetrate about $\frac{1}{60}$ inch into copper and $\frac{1}{600}$ inch into iron. The depth for copper would constitute a considerable portion of a wire $\frac{1}{4}$ inch diameter, and, as there are other considerations 
to be taken into account, I helieve it is scarcely worth while making tubes, or flat strips, for such small sizes.

It is almost impossible to draw proper conclusions from experiments on this subject in the laboratory such as those of Prof. Oliver J. Lodge. The time of oscillation of the current in most pieces of laboratory apparatus is so very small, being often the $\frac{1}{100000000}$ of a second, that entirely wrong inferences may be drawn from them. As the size of the apparatus increases, the time of oscillation increases in the same proportion, and changes the whole aspect of the case. I have given $\frac{1}{100000}$ of a second as the shortest time a lightning flash could probably occupy. I strongly suspect it is often much greater, and thus departs even further from the laboratory experiments of Professor Lodge, who has, however, done very much toward drawing attention to this matter and showing the importance of surface in this case. All shapes of the rod with equal surface are not, however, equally efficient. Thus, the inside surface of a tube does not count at all. Neither do the corrugations on a rod count for the full value of the surface they expose, for the current is not distributed uniformly over the surface; but I have recently proved that rapidly alternating currents are distributed over the surface of very good conductors in the same manner as electricity at rest would be distributed over them, so that the exterior angles and corners possess much more than their share of the current, and corrugations on the wire concentrate the current on the outer angles and diminish it in the hollows. Even a flat strip has more current on the edges than in the centre.

For these reasons, shape, as well as extent of surface, must be taken into account, and strips have not always an advantage over wires for quick discharges.

The fact that the lightning rod is not melted on being struck by lightning is not now considered as any proof that it has done its work properly. It must, as it were, seize upon the discharge and offer it an easier passage to the earth than any other. Such sudden currents of electricity we have seen to obey very different laws from continuous ones, and their tendency to stick to a conductor and not fly off to other objects depends not only on having them of small resistance, but also on having what we call the self-induction as small as possible. This latter can be diminished by having the lightning rod spread sideways as much as possible, either by rolling it into strips, or better, by making a network of rods over the roof, with several connections to the earth at the corners, as I have before described. 
Thus we see that the theory of lightning rods, which appeared so simple in the time of Franklin, is to-day a very complicated one, and requires for its solution a very complete knowledge of the dynamics of electric currents. In the light of our present knowledge the frequent failure of the old system of rods is no mystery, for I doubt if there are a hundred buildings in the country properly protected from lightning. With our modern advances, perfect protection might be guaranteed in all cases, if expense were no object.

So much for the rod itself, and now let us turn to other portions of the electrical system, for we have seen that, in any case, the conductor is only the core of a disturbance which extends to great distances on all sides. Were the clouds, the earth and the streak of heated air called the lightning flash all perfect conductors we could calculate the entire disturbance. It might then consist of a series of stationary waves between the two planes, extending indefinitely on all sides but with gradually decreasing amplitude as we pass away from the centre. The oscillations, once set up, would go on forever, as there would be no poor conductors to damp them. But when the clouds and the path of the lightning both have very great resistance, the energy is very soon converted into heat and the oscillations destroyed. I have given it as my opinion that this is generally the case and that the oscillations seldom take place, but I may be wrong, as there is little to guide me except guesswork. If they take place, however, we have a ready explanation of what is sometimes called a back stroke of lightning. That is, a man at the other end of the cloud a mile or more distant from the lightning stroke sometimes receives a shock, or a new lightning flash may form at that point and kill him. This may be caused, according to our present theory, by the arrival of the waves of electrical disturbance which might themselves cause a slight shock or even overturn the equilibrium then existing and cause a new electric discharge.

We have now considered the case of oscillations of electricity in a few instances and can turn to that of steady currents. The closing of an electric current sends ethereal waves throughout space, but after the first shock the current flows steadily without producing any more waves. However, the properties of the space around the wire have been permanently altered, as we have already seen. Let us now study these properties more in detail. I have before me a wire in which I can produce a powerful current of electricity, and we have seen that the space around it has been so altered that a delicately suspended magnetic needle cannot remain quiet in all positions but stretches itself at right angles to 
the wire, the north pole tending to revolve around it in one direction and the south pole in the other. This is a very old experiment, but we now regard it as evidence that the properties of the space around the wire have been altered rather than that the wire acts on the magnet from a distance.

Put, now, a plate of glass around the wire, the latter being vertical and the former with its plane horizontal, and pass a powerful current through the wire. On now sprinkling iron filings on the plate, they arrange themselves in circles around the wire and thus point out to us the celebrated lines of magnetic force of Faraday. Using two wires with currents in the same direction we get these other curves, and, testing the forces acting on the wire, we find that they are trying to move towards each other.

Again, pass the currents in the opposite directions and we get these other curves and the currents repel each other. If we assume that the lines of force are like rubber bands, which tend to shorten in the direction of their length and repel each other sideways, Faraday and Maxwell have shown that all magnetic attraction and repulsions are explained. The property which the presence of the electric current has conferred on the luminiferous ether is then one by which it tends to shorten in one direction and spread out in the other two directions.

We have thus done away with action at a distance, and have accounted for magnetic attraction by a change in the intervening medium as Faraday partly did almost fifty years ago. For this change in the surrounding medium is as much a part of the electric current as any thing that goes on within the wire.

To illustrate this tension along the lines of force, I have constructed this model, which represents the section of a coil of wire with a bar of iron within it. The rubber bands represent the lines of force which pass around the coil and through the iron bar, as they have an easier passage through the iron than the air. As we draw the bar down and let it go, you see that it is drawn upward and oscillates around its position of equilibrium until friction brings it to rest. Here, again, I have a coil of wire with an iron bar within it with one end resting on the floor. As we pass the current and the lines of magnetic force form around the coil and pass through the iron, it is lifted upwards although if weighs 24 pounds and oscillates around its position of equilibrium exactly the same as though it were sustained by rubber bands as in the model. The rubber bands in this case are invisible to our eye, but our mental vision pictures them to us as lines of magnetic 
force in the luminiferous ether drawing the bar upward by their contractile force. This contractile force is no small quantity, as it may amount, in some cases, to one or even two hundred pounds to the square inch, and thus rivals the greatest pressure which we use in our steam engines.

Thus the luminiferous ether is, to-day, a much more important factor in science than the air we breathe. We are constantly surrounded by the two, and the presance of the air is manifest to us all; we feel it, he hear by its aid, and we even see it, under favorable circumstances, and the velocity of its motion as well as the amount of moisture it carries is a constant topic of conversation with mankind at large. The luminiferous ether, on the other hand, eludes all our senses and it is only with imagination, the eye of the mind, that its presence can be perceived. By its aid in conveying the vibrations we call light, we are enabled to see the world around us, and by its other motions which cause magnetism, the mariner steers his ship through the darkest night when the heavenly bodies are hid from view. When we speak in a telephone, the vibrations of the voice are carried forward to the distant point by waves in the luminiferous ether, there again to be resolved into the sound waves of the air. When we use the electric light to illuminate our streets, it is the luminiferous ether which conveys the energy along the wires as well as transmits it to our eye after it has assumed the form of light. We step upon an electric street car and feel it driven forward with the power of many horses, and again it is the luminiferous ether, whose immense force we have brought under our control and made to serve our purpose. No longer a feeble, uncertain sort of medium, but a mighty power, extending throughout all space and binding the whole universe together, so that it becomes a living unit in which no one portion can be changed without ultimately involving every other portion.

To this, ladies and gentlemen, we have been led by the study of electrical phenomena, and the ideas which I have set forth constitute the most modern views held by physicists with respect to electric currents. 


\section{THE HIGHEST AIM OF THE PHYSICIST}

ADDRESS DELIVERED AS PRESIDENT OF THE AMERICAN PHYSICAL SOCIETY, AT ITS MEETING IN NEW YORK, OCTOBER 28, 1899

[American Journal of Science [4] VIII, 401-411, 1899; Science, X, 825-833, 1899; Johns Hopkins University Circulars, No. 143, pp. 17-20, 19001

Gentlemen and Fellow Physicists of America:-We meet to-day on an occasion which marks an epoch in the history of physics in America; may the future show that it also marks an epoch in the history of the science which this society is organized to cultivate! For we meet here in the interest of a science above all sciences which deals with the foundation of the universe, with the constitution of matter from which everything in the universe is made and with the ether of space by which alone the various portions of matter forming the universe affect each other even at such distances as we may never expect to traverse, whatever the progress of our science in the future.

We, who have devoted our lives to the solution of problems connected with physics, now meet together to help each other and to forward the interests of the subject which we love,-a subject which appeals most strongly to the better instincts of our nature and the problems which tax our minds to the limit of their capacity and suggest the grandest and noblest ideas of which they are capable.

In a country where the doctrine of the equal rights of man has been distorted to mean the equality of man in other respects, we form a small and unique body of men, a new variety of the human race, as one of our greatest scientists calls it, whose views of what constitutes the greatest achievement in life are very different from those around us. In this respect we form an aristocracy, not of wealth, not of pedigree, but of intellect and of ideals, holding him in the highest respect who adds the most to our. knowledge or who strives after it as the highest good.

Thus we meet together for mutual sympathy and the interchange of knowledge, and may we do so ever with appreciation of the benefits to ourselves and possibly to our science. Above all, let us cultivate the idea of the dignity of our pursuit so that this feeling may sustain us in the midst of a world which gives its highest praise, not to the investiga- 
tion in the pure ethereal physics which our society is formed to cultivate, but to the one who uses it for satisfying the physical rather than the intellectual needs of mankind. He who makes two blades of grass grow where one grew before is the benefactor of mankind; but he who obscurely worked to find the laws of such growth is the intellectual superior as well as the greater benefactor of the two.

How stands our country, then, in this respect? My answer must still be now, as it was fifteen years ago, that much of the intellect of the country is still wasted in the pursuit of so-called practical science which ministers to our physical needs and but little thought and money is given to the grander portion of the subject which appeals to our intellect alone. But your presence here gives evidence that such a condition is not to last forever.

Even in the past we have a few names whom scientists throughout the world delight to honor: Franklin, who almost revolutionized the science of electricity by a few simple but profound experiments; Count Rumford, whose experiments almost demonstrated the nature of heat; Henry, who might have done much for the progress of physics had he published more fully the results of his investigations; Mayer, whose simple and ingenious experiments have been a source of pleasure and profit to many. This is the meager list of those whom death allows me to speak of and who have earned mention here by doing something for the progress of our science. And yet the record has been searched for more than a liundred years. How different had I started to record those who have made useful and beneficial inventions!

But I know, when I look in the faces of those before me, where the eager intellect and high purpose sit enthroned on bodies possessing the vigor and strength of youth, that the writer of a hundred years hence can no longer throw such a reproach upon our country. Nor can we blame those who have gone before us. The progress of every science shows us the condition of its growth. Very few persons, if isolated in a semi-civilized land, have either the desire or the opportunity of pursuing the higher branches of science. Even if they should be able to do so, their influence on their science depends upon what they publish and make known to the world. A hermit philosopher we can imagine might make many useful discoveries. Yet, if he keeps them to himself, he can never claim to have benefited the world in any degree. His unpublished results are his private gain, but the world is no better off until he has made them known in language strong enough to call attention to them and to convince the world of their truth. Thus, to encourage the growth of any science, the best thing we can do is to meet 
together in its interest, to discuss its problems, to criticise each other's work and, best of all, to provide means by which the better portion of it may be made known to the world. Furthermore, let us encourage discrimination in our thoughts and work. Let us recognize the eras when great thoughts have been introduced into our subject and let us honor the great men who introduced and proved them correct. Let us forever reject such foolish ideas as the equality of mankind and carefully give the greater credit to the greater man. So, in choosing the subjects for our investigation, let us, if possible, work upon those subjects which will finally give us an advanced knowledge of some great subject. I am aware that we cannot always do this: our ideas will often flow in side channels: but, with the great problems of the universe before us, we may some time be able to do our share toward the greater end.

What is matter; what is gravitation; what is ether and the radiation through it; what is electricity and magnetism; how are these connected together and what is their relation to heat? These are the greater problems of the universe. But many infinitely smaller problems we must attack and solve before we can even guess at the solution of the greater ones.

In our attitude toward these greater problems how do we stand and what is the foundation of our knowledge?

Newton and the great array of astronomers who have succeeded him have proved that, within planetary distances, matter attracts all others with a force varying inversely as the square of the distance. But what sort of proof have we of this law? It is derived from astronomical observations on the planetary orbits. It agrees very well within these immense spaces; but where is the evidence that the law holds for smaller distances? We measure the lunar distance and the size of the earth and compare the force at that distance with the force of gravitation on the earth's surface. But to do this we must compare the matter in the earth with that in the sun. This we can only do by assuming the law to be proved. Again, in descending from the earth's gravitation to that of two small bodies, as in the Cavendish experiment, we assume the law to hold and deduce the mass of the earth in terms of our unit of mass. Hence, when we say that the mass of the earth is $5 \frac{1}{2}$ times that of an equal volume of water we assume the law of gravitation to be that of Newton. Thus a proof of the law from planetary down to terrestrial distances is physically impossible.

Again, that portion of the law which says that gravitational attrac- 
tion is proportional to the quantity of matter, which is the same as saying that the attraction of one body by another is not affected by the presence of a third, the feeble proof that we give by weighing bodies in a balance in different positions with respect to each cannot be accepted on a larger scale. When we can tear the sun into two portions and prove that either of the two halves attracts half as much as the whole, then we shall have a proof worth mentioning.

Then as to the relation of gravitation and time what can we say? Can we for a moment suppose that two bodies moving through space with great velocities have their gravitation unaltered? I think not. Neither can we accept Laplace's proof that the force of gravitation acts instantaneously through space, for we can readily imagine some compensating features unthought of by Laplace.

How little we know then of this law which has been under observation for two hundred years!

Then as to matter itself how have our views changed and how are they constantly changing. The round hard atom of Newton which God alone could break into pieces has become a molecule composed of many atoms, and each of these smaller atoms has become so elastic that after vibrating 100,000 times its amplitude of vibration is scarcely diminished. It has become so complicated that it can vibrate with as many thousand notes. We cover the atom with patches of electricity here and there and make of it a system compared with which the planetary system, nay the universe itself, is simplicity. Nay more: some of us even claim the power, which Newton attributed to God alone, of breaking the atom into smaller pieces whose size is left to the imagination. Where, then, is that person who ignorantly sneers at the study of matter as a material and gross study? Where, again, is that man with gifts so God-like and mind so elevated that he can attack and solve its problem?

To all matter we attribute two properties, gravitation and inertia. Without these two matter cannot exist. The greatest of the natural laws states that the power of gravitational attraction is proportional to the mass of the body. This law of Newton, almost neglected in the thoughts of physicists, undoubtedly has vast import of the very deepest meaning. Shall it mean that all matter is finally constructed of uniform and similar primordial atoms or can we find some other explanation?

That the molecules of matter are not round, we know from the facts of crystallography and the action of matter in rotating the plane of polarization of light. 
That portions of the molecules and even of the atoms are electrically charged, we know from electrolysis, the action of gases in a vacuum tube and from the Zeeman effect.

That some of them act like little magnets, we know from the magnetic action of iron, nickel and cobalt.

That they are elastic, the spectrum shows, and that the vibrating portion carries the electrified charge with it is shown by the Zeeman effect.

Here, then, we have made quite a start in our problem: but how far are we from the complete solution? How can we imagine the material of which ordinary or primordial atoms are made, dealing as we do only with aggregation of atoms alone? Forever beyond our sight, vibrating an almost infinite number of times in a second, moving hither and yon with restless energy at all temperatures beyond the absolute zero of temperature, it is certainly a wonderful feat of human reason and imagination that we know as much as we do at present. Encouraged by these results, let us not linger too long in their contemplation but press forward to the new discoveries which await us in the future.

Then as to electricity, the subtile spirit of the amber, the demon who reached out his glutinous arms to draw in the light bodies within his reach, the fluid which could run through metals with the greatest ease but could be stopped by a frail piece of glass! Where is it now? Vanished, thrown on the waste heap of our discarded theories to be replaced by a far nobler and exalted one of action in the ether of space.

And so we are brought to consider that other great entity-the ether: filling all space without limit, we imagine the ether to be the only means by which two portions of matter distant from each other can have any mutual action. By its means we imagine every atom in the universe to be bound to every other atom by the force of gravitation and often by the force of magnetic and electric action, and we conceive that it alone conveys the vibratory motion of each atom or molecule out into space to be ever lost in endless radiation, passing out into infinite space or absorbed by some other atoms which happen to be in its path. By it all electromagnetic energy is conveyed from the feeble attraction of the rubbed amber through the many thousand horse-power conveyed by the electric wires from Niagara to the mighty rush of energy always flowing from the sun in a flood of radiation. Actions feeble and actions mighty from inter-molecular distances through interplanetary and inter-stellar distances until we reach the mighty distances which bound the universe-all have their being in this wondrous ether. 
And yet, however wonderful it may be, its laws are far more simple than those of matter. Every wave in it, whatever its length or intensity, proceeds onwards in it according to well known laws, all with the same speed, unaltered in direction from its source in electrified matter, to the confines of the universe unimpaired in energy unless it is disturbed by the presence of matter. However the waves may cross each other, each proceeds by itself without interference with the others.

So with regard to gravitation, we have no evidence that the presence of a third body affects the mutual attraction of two other bodies or that the presence of a third quantity of electricity affects the mutual attraction of two other quantities. The same for magnetism.

For this reason the laws of gravitation and of electric and magnetic action including radiation are the simplest of all laws when we confine them to a so-called vacuum, but become more and more complicated when we treat of them in space containing matter.

Subject the ether to immense electrostatic, magnetic or gravitational forces and we find absolutely no signs of its breaking down or even change of properties. Set it into vibration by means of an intensely hot body like that of the sun and it conveys many thousand horse-power for each square foot of surface as quietly and with apparently unchanged laws as if it were conveying the energy of a tallow dip.

Again, subject a millimeter of ether to the stress of many thousand, nay even a million, volts and yet we see no signs of breaking down.

Hence the properties of the ether are of ideal simplicity and lead to the simplest of natural laws. All forces which act at a distance, always obey the law of the inverse square of the distance and we have also the attraction of any number of parts placed near each other equal to the arithmetical sum of the attractions when those parts are separated. So also the simple law of ethereal waves which has been mentioned above.

At the present time, through the labors of Maxwell supplemented by those of Hertz and others, we have arrived at the great generalization that all wave disturbances in the ether are electromagnetic in their nature. We know of little or no ethereal disturbance which can be set up by the motion of matter alone: the matter must be electrified in order to have sufficient hold on the ether to communicate its motion to the ether. The Zeeman effect even shows this to be the case where molecules are concerned and when the period of vibration is immensely great. Indeed the experiment on the magnetic action of electric convection shows the same thing. By electrifying a disc in motion it appears as if the disc holds fast to the ether and drags it with it, thus setting up the peculiar ethereal motion known as magnetism. 
Have we not another case of a similar nature when a huge gravitational mass like that of the earth revolves on its axis? Has not matter a feeble hold on the ether sufficient to produce the earth's magnetism?

But the experiment of Lodge to detect such an action apparently showed that it must be very feeble. Might not his experiment have succeeded had he used an electrified revolving disc?

To detect something dependent on the relative motion of the ether and matter has been and is the great desire of physicists. But we always find that, with one possible exception, there is always some compensating feature which renders our efforts useless. This one experiment is the aberration of light, but even here Stokes has shown that it may be explained in either of two ways: first, that the earth moves through the ether of space without disturbing it, and second, that it carries the ether with it by a kind of motion called irrotational. Even here, however, the amount of action probably depends upon relative motion of the luminous source to the recipient telescope.

So the principle of Doppler depends also on this relative motion and is independent of the ether.

The result of the experiments of Foucault on the passage of light through moving water can no longer be interpreted as due to the partial movement of the ether with the moving water, an inference due to imperfect theory alone. The experiment of Lodge, who attempted to set the ether in motion by a rapidly rotating disc, showed no such result.

The experiment of Michelson to detect the ethereal wind, although carried to the extreme of accuracy, also failed to detect any relative motion of the matter and the ether.

But matter with an electrical charge holds fast to the ether and moves it in the manner required for magnetic action.

When electrified bodies move together through space or with reference to each other we can only follow their mutual actions through very slow and uniform velocities. When they move with velocities comparable with that of light, equal to it or even beyond it, we calculate their mutual actions or action on the ether only by the light of our imagination unguided by experiment. The conclusions of J. J. Thomson, Heaviside and Hertz are all results of the imagination and they all rest upon assumptions more or less reasonable but always assumptions. A mathematical investigation always obeys the law of the conservation of knowledge: we never get out more from it than we put in. The knowledge may be changed in form, it may be clearer and more exactly stated, but the total amount of the knowledge of nature given out by 
the investigation is the same as we started with. Hence we can never predict the result in the case of velocities beyond our reach, and such calculations as the velocity of the cathode rays from their electromagnetic action has a great element of uncertainty which we should do well to remember.

Indeed, when it comes to exact knowledge, the limits are far more circumscribed.

How is it, then, that we hear physicists and others constantly stating what will happen beyond these limits? Take velocities, for instance, such as that of a material body moving with the velocity of light. There is no known process by which such a velocity can be obtained even though the body fell from an infinite distance upon the largest aggregation of matter in the universe. If we electrify it, as in the cathode rays, its properties are so changed that the matter properties are completely masked by the electromagnetic.

It is a common error which young physicists are apt to fall into to obtain a law, a curve or a mathematical expression for given experimental limits and then to apply it to points outside those limits. This is sometimes called extrapolation. Such a process, unless carefully guarded, ceases to be a reasoning process and becomes one of pure imagination specially liable to error when the distance is too great.

But it is not my purpose to enter into detail. What I have given suffices to show how little we know of the profounder questions involved in our subject.

It is a curious fact that, having minds tending to the infinite, with imaginations unlimited by time and space, the limits of our exact knowledge are very small indeed. In time we are limited by a few hundred or possibly thousand years: indeed the limit in our science is far less than the smaller of these periods. In space we have exact knowledge limited to portions of our earth's surface and a mile or so below the surface, together with what little we can learn from looking through powerful telescopes into the space beyond. In temperature our knowledge extends from near the absolute zero to that of the sun but exact knowledge is far more limited. In pressures we go from the Crookes vacuum still containing myriads of flying atoms to pressures limited by the strength of steel but still very minute compared with the pressures at the centre of the earth and sun, where the hardest steel would flow like the most limpid water. In velocities we are limited to a few miles per second; in forces, to possibly 100 tons to the square inch; in mechanical rotations, to a few hundred times per second. 
All the facts which we have considered, the liability to error in whatever direction we go, the infirmity of our minds in their reasoning power, the fallibility of witnesses and experimenters, lead the scientist to be specially skeptical with reference to any statement made to him or any so-called knowledge which may be brought to his attention. The facts and theories of our science are so much more certain than those of history, of the testimony of ordinary people on which the facts of ordinary history or of legal evidence rest, or of the value of medicines to which we trust when we are ill, indeed to the whole fabric of supposed truth by which an ordinary person guides his belief and the actions of his life, that it may seem ominous and strange if what I have said of the imperfections of the knowledge of physics is correct. How shall we regulate our minds with respect to it: there is only one way that I know of and that is to avoid the discontinuity of the ordinary, indeed the so-called cultivated legal mind. There is no such thing as absolute truth and absolute falsehood. The scientific mind should never recognize the perfect truth or the perfect falsehood of any supposed theory or observation. It should carefully weigh the chances of truth and error and grade each in its proper position along the line joining absolute truth and absolute error.

The ordinary crude mind has only two compartments, one for truth and one for error; indeed the contents of the two compartments are sadly mixed in most cases: the ideal scientific mind, however, has an infinite number. Each theory or law is in its proper compartment indicating the probability of its truth. As a new fact arrives the scientist changes it from one compartment to another so as, if possible, to always keep it in its proper relation to truth and error. Thus the fluid nature of electricity was once in a compartment near the truth. Faraday's and Maxwell's researches have now caused us to move it to a compartment nearly up to that of absolute error.

So the law of gravitation within planetary distances is far toward absolute truth, but may still need amending before it is advanced farther in that direction.

The ideal scientific mind, therefore, must always be held in a state of balance which the slightest new evidence may change in one direction or another. It is in a constant state of skepticism, knowing full well that nothing is certain. It is above all an agnostic with respect to all facts and theories of science as well as to all other so-called beliefs and theories.

Yet it would be folly to reason from this that we need not guide our 
life according to the approach to knowledge that we possess. . Nature is inexorable; it punishes the child who unknowingly steps off a precipice quite as severely as the grown scientist who steps over, with full knowledge of all the laws of falling bodies and the chances of their being correct. Both fall to the bottom and in their fall obey the gravitational laws of inorganic matter, slightly modified by the muscular contortions of the falling object but not in any degree changed by the previous belief of the person. Natural laws there probably are, rigid and unchanging ones at that. Understand them and they are beneficent: we can use them for our purposes and make them the slaves of our desires. Misunderstand them and they are monsters who may grind us to powder or crush us in the dust. Nothing is asked of us as to our belief: they act unswervingly and we must understand them or suffer the consequences. Our only course, then, is to act according to the chances of our knowing the right laws. If we act correctly, right; if we act incorrectly, we suffer. If we are ignorant we die. What greater fool, then, than he who states that belief is of no consequence provided it is sincere.

An only child, a beloved wife, lies on a bed of illness. The physician says that the disease is mortal; a minute plant called a microbe has obtained entrance into the body and is growing at the expense of its tissues, forming deadly poisons in the blood or destroying some vital organ. The physician looks on without being able to do anything. Daily he comes and notes the failing strength of his patient and daily the patient goes downward until he rests in his grave. But why has the physician allowed this? Can we doubt that there is a remedy which shall kill the microbe or neutralize its poison? Why, then, has he not used it? He is employed to cure but has failed. His bill we cheerfully pay because he has done his best and given a chance of cure. The answer is ignorance. The renedy is yet unknown. The physician is waiting for others to discover it or perhaps is experimenting in a crude and unscientific manner to find it. Is not the inference correct, then, that the world has been paying the wrong class of men? Would not this ignorance have been dispelled had the proper money been used in the past to dispel it? Such deaths some people consider an act of God. What blasphemy to attribute to God that which is due to our own and our ancestors' selfishness in not founding institutions for medical research in sufficient number and with sufficient means to discover the truth. Such deaths are murder. Thus the present generation suffers for the sins of the past and we die because our ancestors dissipated their wealth in armies and navies, in the foolish pomp and circumstance of 
society, and neglected to provide us with a knowledge of natural laws. In this sense they were the murderers and robbers of future generations of unborn millions and have made the world a charnel house and place of mourning where peace and happiness might have been. Only their ignorance of what they were doing can be their excuse, but this excuse puts them in the class of boors and savages who act according to selfish desire and not to reason and to the calls of duty. Let the present generation take warning that this reproach be not cast on it, for it cannot plead ignorance in this respect.

This illustration from the department of medicine I have given because it appeals to all. But all the sciences are linked together and must advance in concert. The human body is a chemical and physical problem, and these sciences must advance before we can conquer disease.

But the true lover of physics needs no such spur to his actions. The cure of disease is a very important object and nothing can be nobler than a life devoted to its cure.

The aims of the physicist, however, are in part purely intellectual: he strives to understand the universe on account of the intellectual pleasure derived from the pursuit, but he is upheld in it by the knowledge that the study of nature's secrets is the ordained method by which the greatest good and happiness shall finally come to the human race.

Where, then, are the great laboratories of research in this city, in this country, nay, in the world? We see a few miserable structures here and there occupied by a few starving professors who are nobly striving to do the best with the feeble means at their disposal. But where in the world is the institute of pure research in any department of science with an income of $\$ 100,000,000$ per year? Where can the discoverer in pure science earn more than the wages of a day laborer or cook? But $\$ 100,000,000$ per year is but the price of an army or of a navy designed to kill other people. Just think of it, that one per cent of this sum seems to most people too great to save our children and descendants from misery and even death!

But the twentieth century is near-may we not hope for better things before its end? May we not hope to influence the public in this direction?

Let us go forward, then, with confidence in the dignity of our pursuit. Let us hold our heads high with a pure conscience while we seek the truth, and may the American Physical Society do its share now and in generations yet to come in trying to unravel the great problem of the constitution and laws of the universe. 


\section{BIBLIOGRAPHY}





\section{BIBLIOGRAPHY}

1. The Vortex Problem.

Scientific American XIII, 308, 1865.

2. Paine's Electro-magnetic Engine.

Scientific American XXV, 21, 1871.

3. Illustration of Resonances and Actions of a Similar Nature. Journal of the Franklin Institute XCIV, 275-278, 1872.

4. On the Auroral Spectrum.

American Journal of Science (3), V, 320, 1873.

5. On Magnetic Permeability, and the Maximum of Magnetism of Iron, Steel and Nickel.

Philosophical Magazine (4), XLVI, 140-159, 1873.

American Journal of Science (3), VI, 416-425, 1873 (abstract).

6. On the Magnetic Permeability and Maximum of Magnetism of Nickel and Cobalt.

Philosophical Magazine (4), XLVIII, 321-340, 1874.

\%. On a new Diamagnetic Attachment to the Lantern, with a Note on the Theory of the Oscillations of Inductively Magnetized Bodies. American Journal of Science (3), IX, 357-361, 1875.

8. Notes on Magnetic Distribution:

Proceedings of the American Academy of Arts and Sciences, XI, 191, 192, 1876. (Presented June 9, 1875.)

9. Note on Kohlrausch's Determination of the Absolute Value of the Siemens Mercury Unit of Electrical Resistance.

Philosophical Magazine (4), L, 161-163, 1875.

10. Preliminary Note on a Magnetic Proof Plane.

American Journal of Science (3), X, 14-17, 1875.

11. Studies on Magnetic Distribution.

American Journal of Science (3), X, 325-335, 451-459, 1875.

Ibid., XI, 17-29, 103-108, 1876.

Philosophical Magazine (4), L, 257-277, 348-367, 1875.

12. On the Magnetic Effeet of Electric Convection.

American Journal of Science (3), XV, 30-38, 1878.

See also Monatsberichte Akad. Berlin, pp. 211-216, 1876.

American Journal of Science (3), XII, 390-394, 1876.

Philosophical Magazine (5), II, 233-237, 1876.

Annales de Chimie et de Physique (5), XII, 119-125, 1877. 
13. Note on the Magnetic Effect of Electric Convection. Philosophical Magazine (5), VII, 442-443, 1879.

14. Note on the Theory of Electric Absorption. American Journal of Mathematics, I, 53-58, 1878.

15. Research on the Absolute Unit of Electrical Resistance. American Journal of Science (3), XV, 281-291, 325-336, 430-439, 1878.

16. On the Mechanical Equivalent of Heat, with Subsidiary Researches on the Variation of the Mercurial from the Air-Thermometer and on the Variation of the Specific Heat of Water.

Proceedings of the American Academy of Arts and Sciences, XV, 75-200, 1880.

Atti del R. Instituto, Venezia, VII, 1436, 1881. (Appendix.)

17. On Professors Ayrton and Perry's New Theory of the Earth's Magnetism, with a Note on a New Theory of the Aurora.

Philosophical Magazine (5), VIII, 102-106, 1879.

Proceedings of the Physical Society, III, 93-98, 18 i9.

18. On the Diamagnetic Constants of Bismuth and Calc-spar in Absolute Measure. By H. A. Rowland and W. W. Jacques. American Journal of Science (3), XVIII, 360-371, 1879.

19. Preliminary Notes on Mr. Hall's Recent Discovery. American Journal of Mathematics, II, 354-356, 1879. Philosophical Magazine (5), IX, 432-434, 1880. Proceedings of the Physical Society, IV, 10-13, 1880.

20. Physical Laboratory; Comparison of Standards. Johns Hopkins University Circulars No. 3, p. 31, 1880.

21. Appendix to Paper on the Mechanical Equivalent of Heat, Containing the Comparison with Dr. Joule's Thermometer.

Proceedings of the American Academy of Arts and Sciences, XVI, $38-45,1881$.

Johns Hopkins University Circulars No. 3, p. 30, 1880 (abstract).

22. On the Efficiency of Edison's Electric Light. By H. A. Rowland and G. F. Barker.

American Journal of Science (3), XIX, 337-339, 1880.

23. On the Motion of a Perfect Incompressible Fluid when no Solid Bodies are Present.

American Journal of Mathematics, III, 226-268, 1880.

24. On the General Equations of Electro-magnetic Action, with Application to a New Theory of Magnetic Attraction and to the Theory of the Magnetic Rotation of the Plane of Polarization of Light. American Journal of Mathematics, III, 89-113, 1881. 
25. On the New Theory of Magnetic Attractions, and the Magnetic Rotation of Polarized Light.

Philosophical Magazine (5), XI, 254-261, 1881.

26. On Geissler Thermometers.

American Journal of Science (3), XXI, 451-453, 1881.

2\%. Electric Absorption of Crystals. By H. A. Rowland and E. L. Nichols.

Philosophical Magazine (5), XI, 414-419, 1881.

Proceedings of the Physical Society, IV, 215-221, 1881.

28. On Atmospheric Electricity.

Johns Hopkins University Circulars No. 19, pp. 4, 5, 1882.

29. Preliminary Notice of the Results Accomplished in the Manufacture and Theory of Gratings for Optical Purposes.

Johns Hopkins University Circulars, No. 17, pp. 248, 249, 1882.

Philosophical Magazine (4), XIII, 469-474, 1882.

Nature, 26, 211-213, 1882.

Journal de Physique, II, 5-11, 1883.

30. On Concave Gratings for Optical Purposes.

American Journal of Science (3), XXVI, 87-98, 1883.

Philosophical Magazine (5), XVI, 197-210, 1883.

31. On Mr. Glazebrook's Paper on the Aberration of Concave Gratings. American Journal of Science (3), XXVI, 214, 1883.

Philosophical Magazine (5), XVI, 210, 1883.

32. On the Propagation of an Arbitrary Electro-magnetic Disturbance, on Spherical Waves of Light and the Dynamical Theory of Diffraction.

American Journal of Mathematics, VI, 359-381, $18>4$.

Philosophical Magazine (5), XVII, 413-437, 1884.

33. Screw.

Encyclopaedia Britannica, Ninth Edition, Vol. 21.

34. The Determination of the Ohm. Extract from a letter to the International Congress at Paris, 1884.

Procès-Verbaux, Deuxième Session, p. 37, Paris 1884.

35. The Theory of the Dynamo.

Report of the Electrical Conference at Philadelphia in November, 1884, pp. 72-83, 90, 91, 104, 107. Washington, 1886.

Electrical Review (N. Y.), Nov. 1, 8, 15, 22, 1884.

36. On Lightning Protection.

Report of the Electrical Conference at Philadelphia in November, 1884, pp. 172-174. 
37. On the Value of the $\mathrm{Ohm}$.

La Lumière Électrique, XXVI, pp. 188, 477, 1887.

38. On a Simple and Convenient Form of Water-battery. American Journal of Science (3), XXXIII, 147, 1887. Philosophical Magazine (5), XXIII, 303, 1887. Johns Hopkins University Circulars No. 57, p. 80, 1887.

39. On the Relative Wave-lengths of the Lines of the Solar Spectrum. American Journal of Science (3), XXXIII, 182-190, 1887. Philosophical Magazine (5), XXIII, 257-265, 1887.

40. On an Explanation of the Action of a Magnet on Chemical Action. By H. A. Rowland and Louis Bell. American Journal of Science (3), XXXVI, 39-47, 1888. Philosophical Magazine (5), XXVI, 105-114, 1888.

41. Table of Standard Wave-lengths. Philosophical Magazine (5), XXVII, 479-484, 1889. Johns Hopkins University Circulars No. 73, p. 69, 1889.

42. A Few Notes on the Use of Gratings.

Johns Hopkins University Circulars No. 73, pp. 73, 74, 1889.

43. On the Electro-magnetic Effect of Convection-Currents. By H. A. Rowland and C. T. IIutchinson.

Philosophical Magazine (5), XXVII, 445-460, 1889.

44. On the Ratio of the Electro-static to the Electro-magnetic Unit of Electricity. By H. A. Rowland, E. H. Hall, and L. B. Fletcher. American Journal of Science (3), XXXVIII, 289-298, 1889.

Philosophical Magazine (5), XXVIII, 304-315, 1889.

45. Electro-magnetic Waves and Oscillations at the Surface of Conductors.

American Journal of Mathematics, XI, 373-387, 1889.

46. Report of Progress in Spectrum Work.

The Chemical News, LXIII, 133, 1891.

Johns Hopkins University Circulars No. 85, pp. 41, 42, 1891.

American Journal of Science (3), XLI, 243, 244, 1891.

47. Notes on the Theory of the Transformer.

Philosophical Magazine (5), XXXIV, 54-57, 1892.

Electrical World XX, 20, 1892.

Johns Hopkins University Circulars No. 99, pp. 104, 105, 1892.

48. Notes on the Effect of Harmonics in the Transmission of Power by Alternating Currents.

Electrical World, XX, 368, 1892.

La Lumière Électrique, XLVII, 42-44, 1893. 
49. Gratings in 'Theory and Practice.

Philosophical Magazine (5), XXXV, 397-419, 1893.

Astronomy and Astro-Physies, XII, 129-149, 1893.

50. A New Table of Standard Wave-lengths.

Philosophical Magazine (5), XXXVI, 49-75, 1893.

Astronomy and Astro-Physics, XII, 321-347, 1893.

Johns Hopkins University Circulars No. 106, p. 110, 1893.

51. On a Table of Standard Wave-lengths of the Spectral Lines.

Memoirs of the American Academy of Arts and Sciences, XII, 101$186,1896$.

52. The Separation of the Rare Earths.

Johns Hopkins University Circulars No. 112, pp. 73, 74, 1894.

53. Modern Theories as to Electricity.

The Engineering Magazine, VIII, 589-596, 1895.

54. A Preliminary Table of Solar Spectrum Wave-lengths.

Astrophysical Journal, I-V, 1895-1897.

55. Corrections and Additions to Professor H. A. Rowland's Table of

Solar Spectrum Wave-lengths.

Astrophysical Journal VI, 384-392, 1897.

56. The Arc-Spectra of the Elements. I. Boron and Beryllium. II.

Germanium. III. Platinum and Osmium. IV. Rhodium, Ruthenium and Palladium. By H. A. Rowland and R. R. Tatnall. Astrophysical Journal, I, 14-17, 149-153, 1895; II, 184-187, 1895; III, 286-291, 1896.

5\%. Notes of Observations on the Röntgen Rays. By H. A. Rowland, N. R. Carmichael and L. J. Briggs.

American Journal of Science (4), I, 247, 248, 1896.

Philosophical Magazine (5), XLI, 381-382, 1896.

58. Notes on Röntgen Rays. By H. A. Rowland, N. R. Carmichael and L. J. Briggs.

Electrical World, XxVII, 452, 1896.

59. The Röntgen Ray and its Relation to Physics.

Transactions of the American Institute of Electrical Engineers, XIII, 403-410, 430, 431, 1896.

60. Electrical Measurement by Alternating Currents.

American Journal of Science (4), IV, 429-448, 1897.

Philosophical Magazine (5), XLV, 66-85, 1898.

61. Are--Spectra of Vanadium, Zirconium and Lanthanum. By H. A.

Rowland and C. N. Harrison.

Astrophysical Journal, VII, 273-294, 373-389, 1898. 
62. Electrical Measurements. By H. A. Rowland and T. D. Penniman. American Journal of Science (4), VIII, 35-57, 1899.

Johns Hopkins University Circulars No. 135, pp. 51, 52, 1898 (abstract).

63. Resistance to Ethereal Motion. By H. A. Rowland, N. E. Gilbert and P. C. McJunckin.

Johns Hopkins University Circulars No. 146, p. 60, 1900.

64. Diffraction Gratings.

Encyclopaedia Britannica, New Volumes, III, 458, 459, 1902.

\section{ADDRESSES}

1. A Plea for Pure Science. Address as Vice-President of Section B of the American Association for the Advancement of Science, Minneapolis, August 15, 1883.

Proceedings of the American Association for the Advancement of Science, XXXII, 105-126, 1883.

Science, II, 242-250, 1883.

Journal of the Franklin Institute, CXVI, 279-299, 1883.

2. The Physical Laboratory in Modern Education. Address for Commemoration Day of the Johns Hopkins University, February 22, 1886.

Johns Hopkins University Circulars No. 50, pp. 103-105, 1886.

3. Address as President of the Electrical Conference at Philadelphia, September 8, 1884.

Report of the Electrical Conference at Philadelphia in September, 1884, Washington, 1886.

4. The Electrical and Magnetic Discoveries of Faraday. Address at the Opening of the Electrical Club House of New York City, 1888.

Electrical Review, Feb. 4, 1888.

5. On Modern Views with Respect to Electric Currents. Address before the American Institute of Electrical Engineers, New York, May 22, 1889.

Transactions of the American Institute of Electrical Engineers, VI, 342-357, 1889.

Electrical World, XIII, p. 319, 1889.

See also Electrical World, XIII, p. 142, 1889. 
6. The Highest Aim of the Physicist. Address as President of the American Physical Society, New York, October 28, 1899.

Science, X, 825-833, 1899.

American Journal of Science (4), VIII, 401-411, 1899.

Johns Hopkins University Circulars No. 143, pp. 17-20, 1900.

\section{REPORTS AND BOOKS}

1. Report of the Electrical Commission Appointed to Consider the Location, Arrangement and Operation of Electric Wires in the District of Columbia. Washington, 1892.

By Andrew Rosewater, H. A. Rowland, and Francis R. Shunk.

2. Elements of Physics.

By H. A. Rowland and J. S. Ames, New York, 1900, XIII + 263. pp. 



\section{DESCRIPTION \\ OF \\ DIVIDING ENGINES}




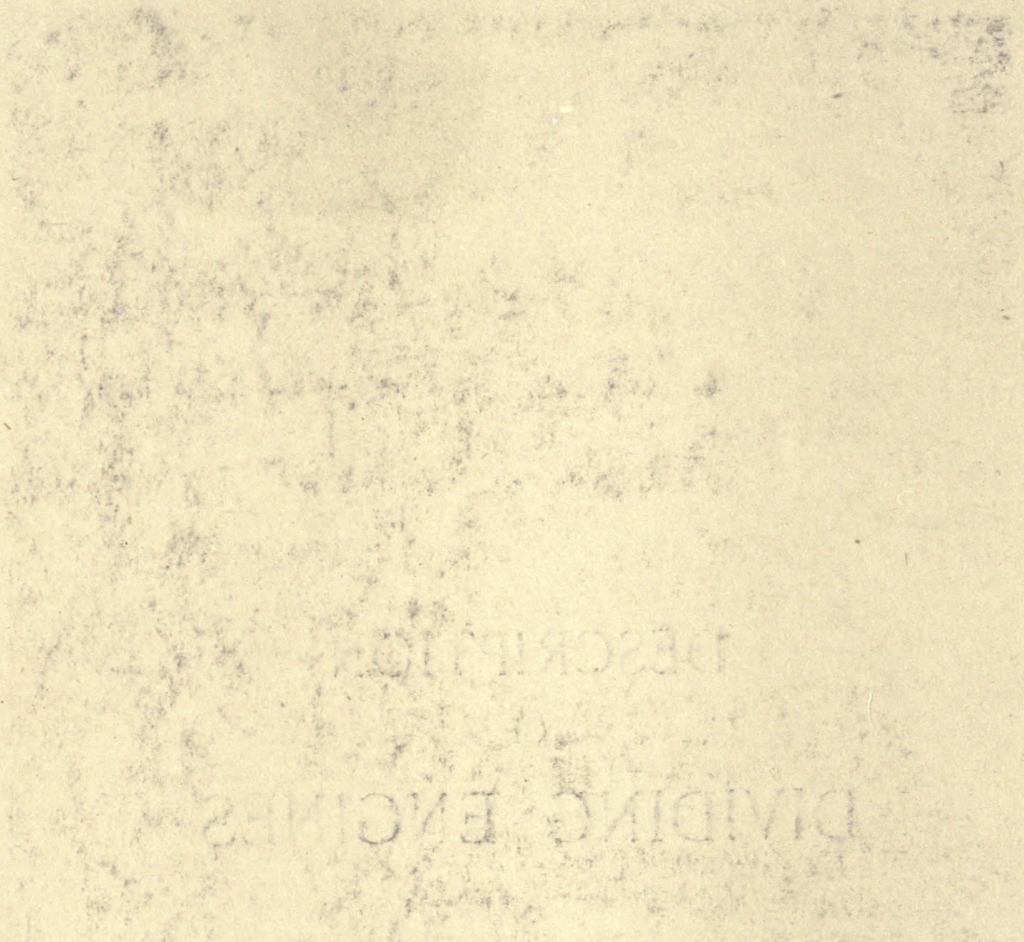

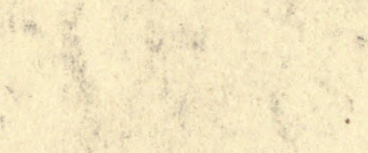




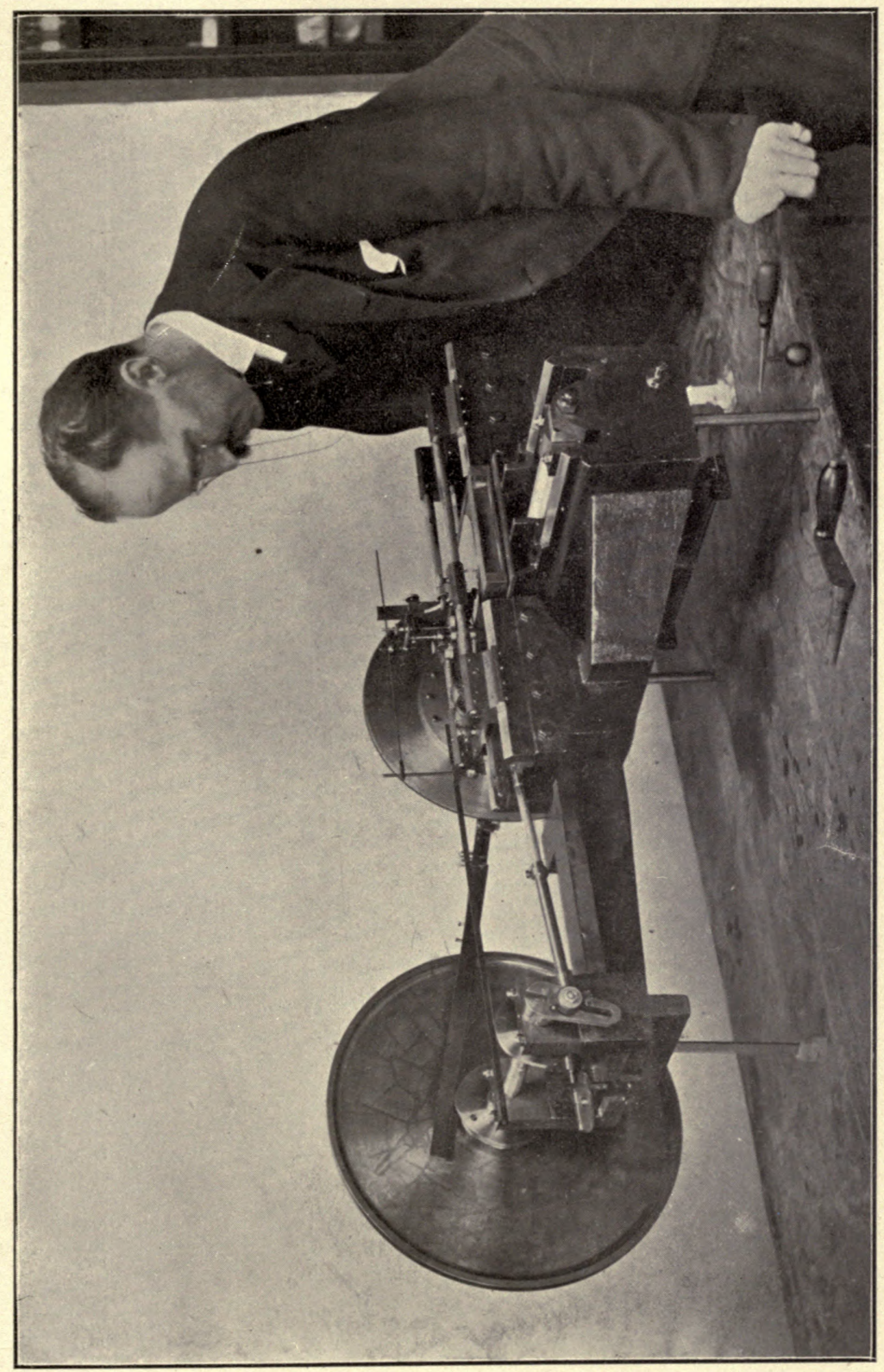


9 


\section{A DESCRIPTION OF THE DIVIDING ENGINES DESIGNED BY PROFESSOR ROWLAND. ${ }^{*}$}

Three dividing engines were made under Professor Rowland's direction, all embodying the same general principles as given in his article on the "Screw" in the Encyclopedia Britannica (this volume p. 506). The screws of all three have approximately twenty threads to the inch; and the number of teeth in the ratchet wheels of the first, second and third machines is such that they rule $14,438,15,020$ and 20,000 lines in an inch. The three machines are kept in the sub-basement of the Physical Laboratory of the Johns Hopkins University under such conditions as will secure a practically constant temperature for long intervals of time. Each machine is driven by a separate water-motor whose speed can be regulated at will.

The machines have been used almost exclusively for the ruling of diffraction gratings, although a few centimetre scales have been made. The gratings have been, with only four or five exceptions, made of "speculum metal," having the composition, copper 126 lbs. 4 oz., tin 58 lbs. 9 oz., and as homogeneous as possible. The rough metal plates were cast under Professor Rowland's direction, and were then figured and polished. After the ruling was completed, the gratings were carefully tested in order to see if they were free from "ghosts," diffused light and defective definition.

To test the screw, ratchet-head and thrust screw for periodic errors, Professor Rowland used the following method: he ruled a space of about one centimetre on a polished surface, then pushed the carriage bos $\mathrm{k}$ this distance, turned the grating-holder through a minute angle ar: again ruled a surface of about the same width as before. There is th produced a cross-ruling, the lines being slightly inclined to each other; and when examined by reflected light, a series of undulations is

\footnotetext{
${ }^{1}$ Unfortanately Professor Rowland never published a description of these machines ; anc the Committee has failed to find any information concerning the inception of the idea or the history of the construction of the first machine. It bas been thought best, therefore, to give, first, a general description of the design of the engines with varions necessary details of some of the working parts and, second, drawings made to scale, showing all the connections of the intricate mechanism; both of these have been prepared nnder the direction of J. S. Ames, Secretary of the Committee, and have been approved by the Committee.
} 
seen to cross the lines at right angles, corresponding to the points of intersection of the two sets of rulings. This pattern resembles closely in appearance that of watered silk. The corrector of the machine is adjusted until this undulatory pattern is as regular and has as small an amplitude as possible.

Any description of Rowland's dividing engines, however brief, would be incomplete without some mention of Mr. Theodore Schneider who for twenty-five years was Professor Rowland's mechanician and assistant and who died only a few weeks before him. It was he who made the screws and most of the working parts of the machines, and it was he who superintended the ruling of every grating that has left the Physical Laboratory of the Johns Hopkins University for use elsewhere in the world.

\section{General Design of Dividing Engine ${ }^{2}$}

The object of this machine is to rule straight lines on metal or glass surfaces, exactly parallel and at exactly equal distances apart. The surface to be ruled is attached to a frame which is moved forward by a nut as it is advanced by a screw; the ruling edge is generally a diamond mounted in such a manner as to be drawn to and fro across the surface to be ruled, but to be in contact with it during only one of these motions.

Rotary motion is imparted to the main shaft ( 48 A) by means of a driving pulley, operated by a belt attached to a water motor (not shown in the cuts). Mounted on the main shaft are the cams $(46,47)$ for operating the pawl-levers, which turn the screw and advance the nut; the cam (55) controlling the mechanism for raising the diamond; and the crank (50) which by means of the comnecting rod and cross-head impart a reciprocating motion to the ruling carriage and its diamond. By means of adjustments in the crank and connecting rod, the length of stroke of the diamond may be varied, and rulings of different lengths are thus obtained.

In each revolution of the main shaft, the cycle of operations that occur is as follows: Let the diamond be on the plate in a position to begin ruling. It is moved forward, i. e. toward the shaft, by means of the ruling frame and parts described, and a line is ruled. The stroke of the engine being now about to reverse, the cam controlling the mechanism for lifting the diamond performs its duty; and, while the engine is on its return stroke, with the diamond off the plate, the latter is

\footnotetext{
2 The fignres in the text refer to the numbered parts in the cuts which follow the
} article. 
advanced a space equal to the desired distance between the rulings. This is done by the cams operating the pawl-levers ( 26 and 40 ), which cause the pawl (41) to rise to a pre-determined position corresponding to one or more teeth of the graduated ratchet head, then to engage this wheel and, being now forced down to its normal position, to cause the wheel and the feed-screw, to which it is attached, to turn through a small definite angle. The rotation of the screw causes the nut to advance towards the ratchet head; and the nut pushes forward the plate-carriage to which the plate to be ruled is secured. The engine being now at the end of its return stroke, the diamond is lowered into contact with the plate, and is ready for ruling the next line. These operations are repeated until the requisite number of lines is ruled. During each cycle of operations a slight additional motion is imparted to the nut and thus to the plate-carriage by means of the corrector mechanism, in order that any periodic errors of the screw, screw-head, etc., may be eliminated.

The ruling-carriage with its diamond holder moves along truncated $\mathrm{V}$-ways, as shown in the cuts, the surfaces in contact being the steel ways and the box-wood linings to the grooves on the carriage. These box-wood linings press against both the sides and the top of the ways and are adjustable. The plate-carriage moves along $\mathrm{V}$-ways, the surfaces in contact being the steel ways and the cast-iron carriage. These two pairs of ways are accurately at right angles to each other.

\section{Detatliad Descriptions}

I. Mechanism connecting the plate-carriage and the nut. See Fig. 5. The plate-carriage carries a thrust collar (20) through which the feed-screw passes freely. It is held in position by pins engaging in the top and bottom of the plate-carriage. The thrust of the nut in advancing is communicated by two lugs, one on each side of the nut casings (21), to two correspondingly located screw-heads in the thrust collar; and, finally, screw-heads in the top and bottom of the thrust-collar transfer the thrust to correspondingly located lings (22) in the platccarriage.

II. Pawl mechanism. See Fig. 4.

The degree of rotation imparted to the graduated ratchet-head depends upon the number of teeth the pawl engages in each revolution of the main-shaft and may be varied by altering the size of the cams (46 and 47) on which the pawl-levers 26 and 40 rest. The pawl-lever 
(26), to which the bell-crank (42) is piroted, causes the pawl to rise to a height corresponding to the number of teeth to be engaged on the graduated ratchet-head., The other lever (40) has the function of engaging and disengaging the pawl. The cycle of operations that occurs in one revolution of the main shaft is as follows: The pawl-lever (26) is raised by the cam (47), and in so doing gauges the degree of revolution to be imparted to the graduated ratchet-head and feedscrew. The other lever (40), which is pivoted on the pawl-lever (26), is raised further, and thereby permits cam (46) and the bell-crank (42) to carry the pawl (41) forward into engagement with the graduated ratchet-head. The weight (45) attached to the bell-crank arm insures a positive engagement of the pawl. The depth to which the pawl enters between the teeth of the graduated ratchet-head is governed by the adjusting screw (43) and a stop on pawl-lever (26). The pawl being engaged, both levers (26 and 40) now descend, causing the graduated ratchet-head and feed-screw to turn to an extent governed by the number of teeth engaged. Lever (40) now descends to a position coincident with that of the lever (26), and in so doing causes the stop on lever (40) to press against the adjusting screw (44) on the bell-crank, and thereby withdraws the pawl from the teeth of the graduated ratchethead.

\section{Diamond and ruling head. See Figs. 1 and 5 .}

The diamond (1) is firmly secured by means of solder in a holder (2), which may be adjusted to different inclinations. The frame carrving the diamond, holder and dash-pot has an axle, centering in bearing screws (3) and contained in an adjustable support (4). This support may be raised or lowered to meet the requirements of plates of different thickness. Normally, the end of the frame carrying the diamond and holder, owing to its predominance of weight, would cause the diamond to be in contact with the plate continuously. In order to raise it on the return stroke of the engine, a weighted lift rod (5\%) is caused to press on the end of the frame near the dash-pot. The height to which the diamond is thus lifted off the plate is governed by nuts, which may be adjusted on the stem of the lift-rod and which on their descent come to rest on the plate $56 \mathrm{~A}$.

The raising of the weighted lift-rod is primarily caused by cam (55) on the main shaft; the intermediate mechanism consists of the lever (54), vertical oscillating rod (53), reciprocating rod (35), rocking stem (34), and lifting lever (56). The action of the dashers and dash-pot 
filled with oil is to dampen any vibrations of the frame which carries the diamond, and to check its descent on the plate.

IV. Corrector mechanism. See Figs. 1, 4 and 5.

The wear of the threads contained in the lignum vitæ plugs of the split nut-casing is taken up by the screws in the adjusting rings (17), bringing the two parts of tine nut closer to the feed-screw. Each side of the nut is provided with a wing-shaped lever, the lower ends of which are confined in guides forming part of the lower corrector frame (39); but they are free to travel in the direction that the nut moves. When the screw is turning and the nut advancing, these wings are pressed tight against the guide-plate (39 A) of the corrector frame; and thus the nut will receive additional motions from any displacement of the corrector. In this manner periodic errors of the screw may be neutralized by the action of the corrector. The precise amount of correction is controlled by the adjustments of the eccentric (25). This gives the requisite amount of movement at the proper instants to the corrector lever (28), which in turn moves the rocking shaft, corrector frame, crank, lower frame and, finally, the wings of the nut. The disc (24) may be adjusted and clamped, as shown in Fig. 4, in different positions in the plane of the graduated ratchet-head; and the position of the corrector eccentric (25) with respect to a fixed radius of the graduated ratchet-head must be such as to make the phase of the correction opposite that of the periodic crror. The amount of eccentricity of the eccentric can be varied by means of set-screws, as is evident from the cut; and this must be regulated so that the amplitude of the correction equals that of the periodic error.

\section{Deschiptive Drawings of Dividing Engine No. 3}

At the end of this article are five cuts of dividing engine No. 3, drawn to scale, one quarter of the actual size, showing different views and operations. They may be described as follows:

Fig. 1. Side elevation, showing the engine in a ruling position.

Fig. 2. Plan view of the foregoing.

Fig. 3. Plan view, showing the plate-carriage. The plate, plateholder and ruling-head are omitted.

Fig. 4. Side elevation opposite to Fig. 1, showing the engine in the return stroke position.

Fig. 5. Transverse sectional elevation, showing the feed-screw, nut, etc. The mechanism actuating the corrector-frame is shown as an end-view. 


\section{Explanation of Numbers in the Cuts}

(Similar numerals refer to like parts throughout the different views.)

1. Ruling diamond.

2. Adjustable diamond holder.

3. Adjustable support for the axis of the diamond-frame.

4. Ruling-head, carrying ruling mechanism.

5. Rods of the ruling carriage.

6. Plate to be ruled.

\%. Adjustable box-wood slides of ruling carriage. (N. B.-There are box-wood slides pressed against the sides as well as the top of the ways of the frame.)

8. Plate-holder.

9. Clamps for plate-holder.

10. Bed-plate.

11. Plate-carriage, which is moved by the nut and which rests on ways. (N. B.- The plate-carriage has a cross-beam below the feedscrew. See Fig. 5.)

12. Feed-screw.

13. Hardened steel step in end of feed-screw.

14. Hardened steel thrust-screw.

15. Casing of the split nut, holding the plugs 16 .

16. Lignum vitæ plugs, tapped for engaging feed-screw.

17. Adjusting rings for nut, with their adjusting screws.

18 and 19. Wings of the nut, controlled by the corrector, $39 \mathrm{~A}$.

20. Thrust collar, loosely attached to plate-carriage, 11 .

21. Abutting lugs, rigidly attached to nut-casing 15 , and in contact with collar 20 .

22. Abutting lugs of plate-carriage, in contact with screw-heads in collar 20.

23. Graduated ratchet-head attached to the feed-screw.

24. Disc for phase-adjustment of corrector, being movable around the axis of the screw in the plane of the ratchet wheel.

25. Eccentric for adjusting amplitude of corrector, being movable around an axis near one end so as to vary the eccentricity.

26. Pawl-lever, which raises or lowers the pawl, when it is disengaged or engaged, respectively, in the ratchet wheel by means of lever 40.

27. Hollow arbor, serving as pivot for pawl-lever.

28. Corrector lever, resting on 25 , and pivoted at 31 .

29. Corrector frame. 
30. Hardened steel centres for corrector frame.

31. Rocking shaft, rotated by means of lever 28 .

32. Bearing for wrist-pin of lower corrector frame.

33. Crank for rocking corrector; a slight rotation of the shaft 31, thus giving a slight sidewise motion to the frame 39.

34. Rocking stem, which moves the lifting-lever 56, of ruling head.

35 . Rod to communicate reciprocating motion to 34 .

36. Base-frame of engine.

$3 \%$ Casings of ruling carriage, holding the adjustable box-wood slides, 7 .

38. Adjustable weight for corrector lever.

39. Lower corrector frame, moved by the crank 33.

39 A. Corrector guide-plate, along which the wings of the nut move.

40. Lever for engaging and disengaging pawl, by means of bell-crank 42 .

41. Pawl, driving ratchet wheel.

42. Bell-crank which is piroted on 26 ; to one end the pawl is attached, and the other is raised by the lever 40 and lowered by the weight 45 .

43 and 44. Adjusting screws attached to 42 , for regulating the pawl engagement. The stops are attached to 26 and 40 .

45. Weight hanging from bell-crank.

46. Cam operating lever, 40 ; attached to main shaft.

47. Cam operating pawl-lever, 26; attached to main shaft.

(These two cams regulate the number of teeth of ratchet wheel which the pawl clears each revolution of the main-shaft.)

48. Driving pulley, attached to main shaft.

48 A. Main shaft.

49. Connecting rod to give reciprocating motion to diamond-holder by means of 52 and $3 \%$.

50. Crank arm, designed to vary the length of stroke of the diamond.

51. Bar connecting cross-liead 52, and ruling frame $3 \%$.

52. Cross-head, driven by connecting rod 49.

53. Oscillating rod, connecting 35 and 54 .

54. Lever operating stop mechanism for lifting diamond, resting on $\mathbf{5 5}$.

55. Cam attached to main shaft and operating the lever 54 .

56. Lever for lifting rod 57; it is operated by the rocking-stem 34 .

56 A. Stop-plate regulating drop of rod $5 \%$.

5\%. Rod for lifting diamond.

58. Dash-pot attached to the lever which carries the diamond-holder 2, and which is piroted at 3 .

59. Adjustments for holding and regulating the dashers.

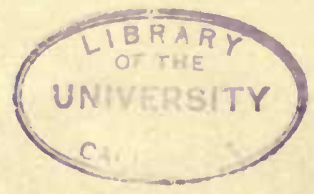



FIG. I

Side elevation, showing the engine in a ruling position

LIBRARY
OF THE
UNIVERSITY
OAT 


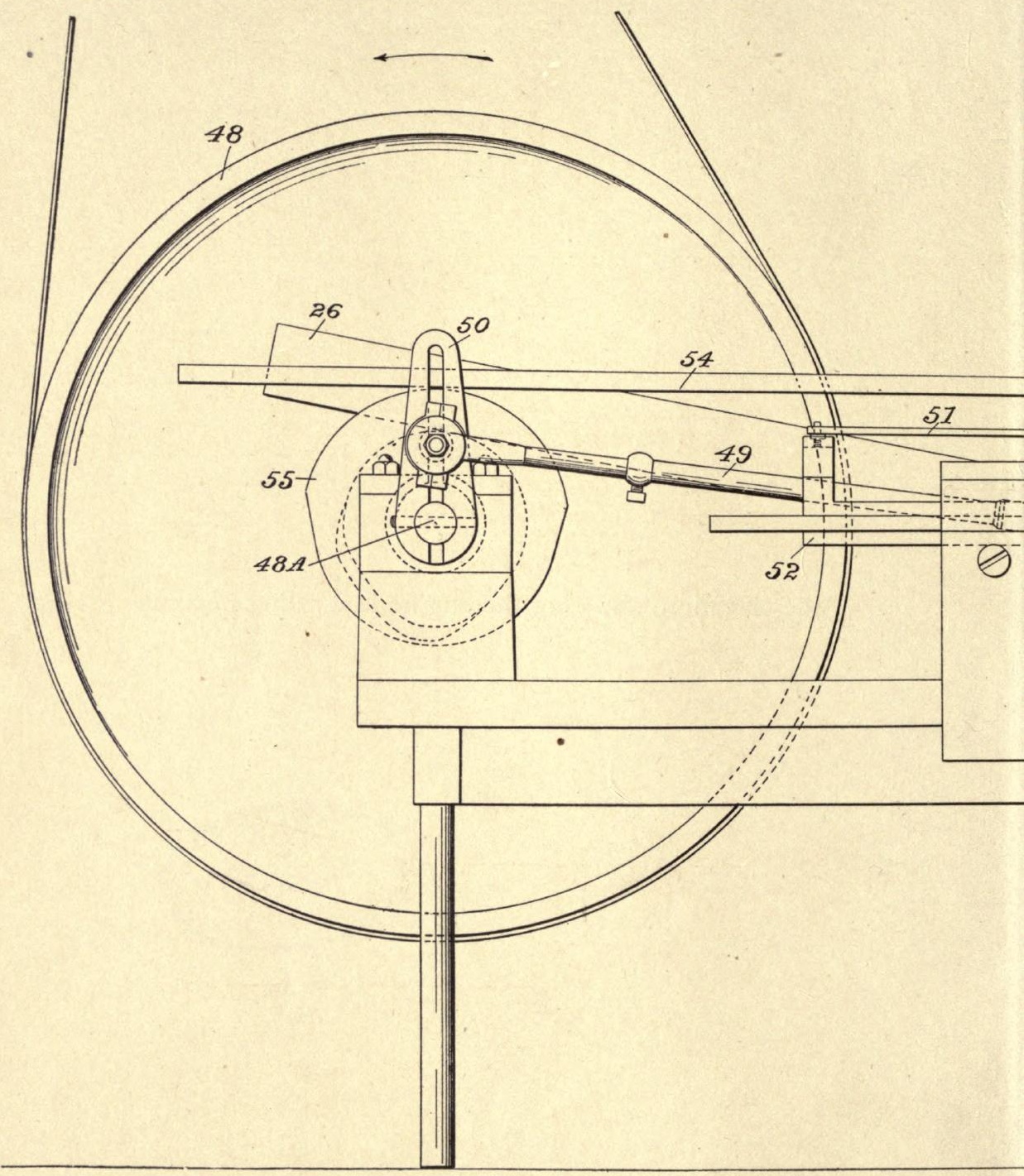

SIDE ELEVATION, SHOWING T 


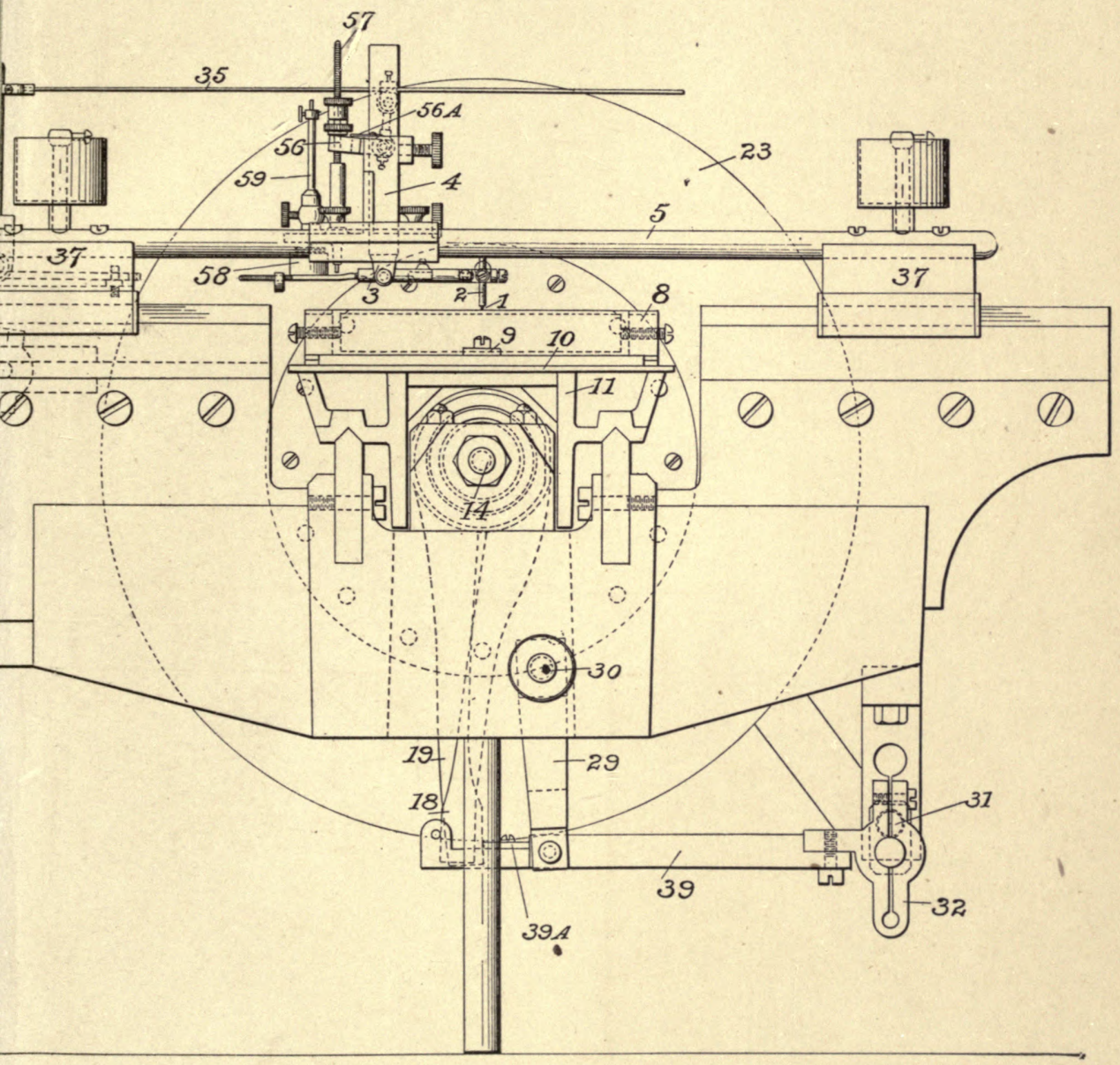

1.

ENGINE IN A RULING POSITION. 


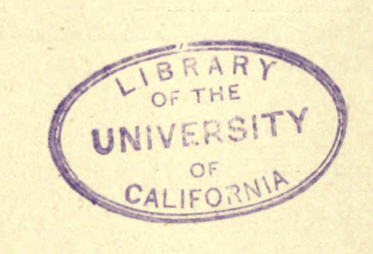


FIG. 2

Plan view of the foregoing
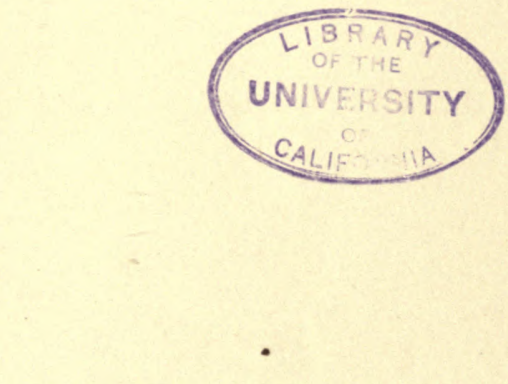


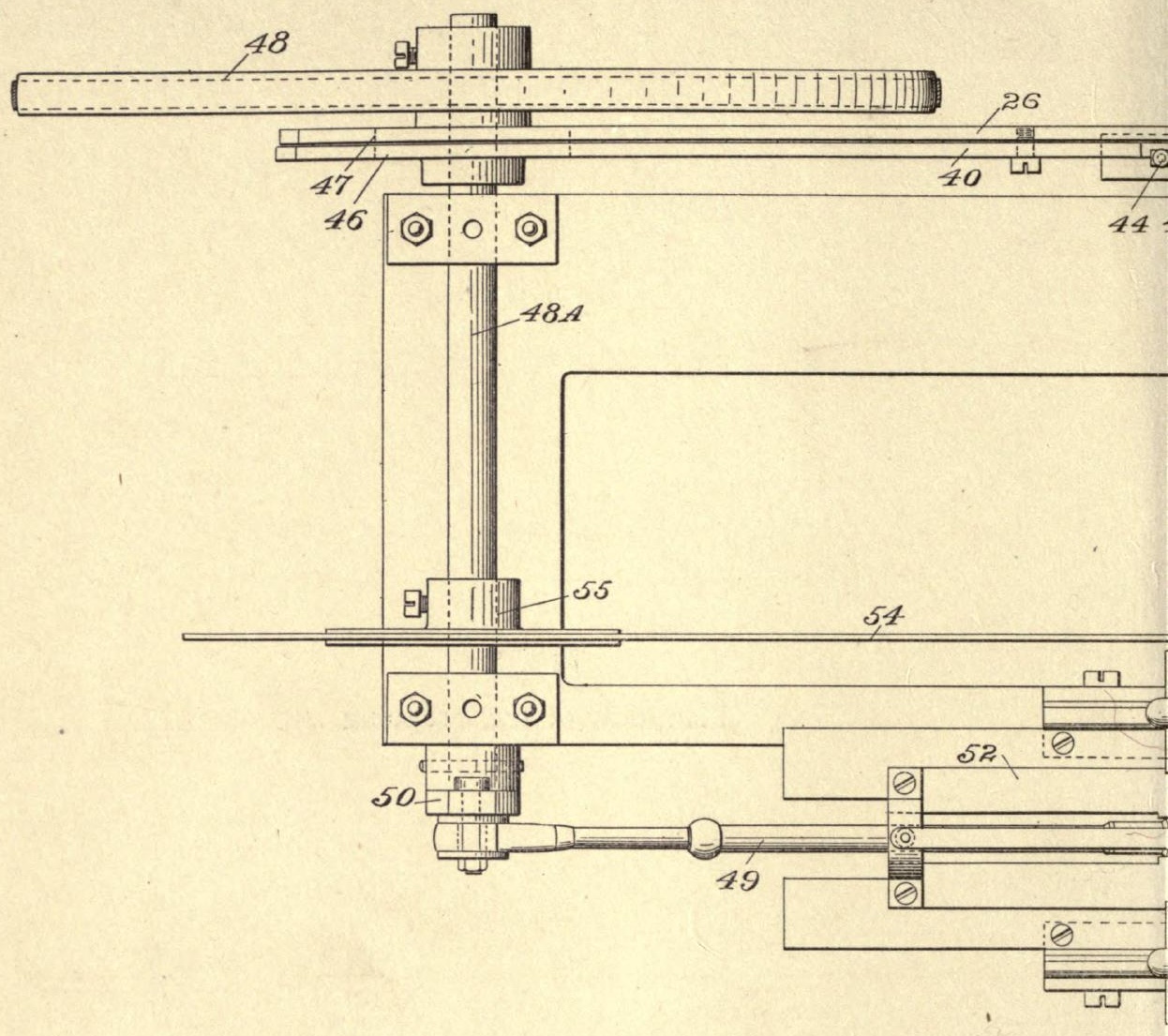

FIG. 2.

PLAN VIEW OF THE FOREGOING. 


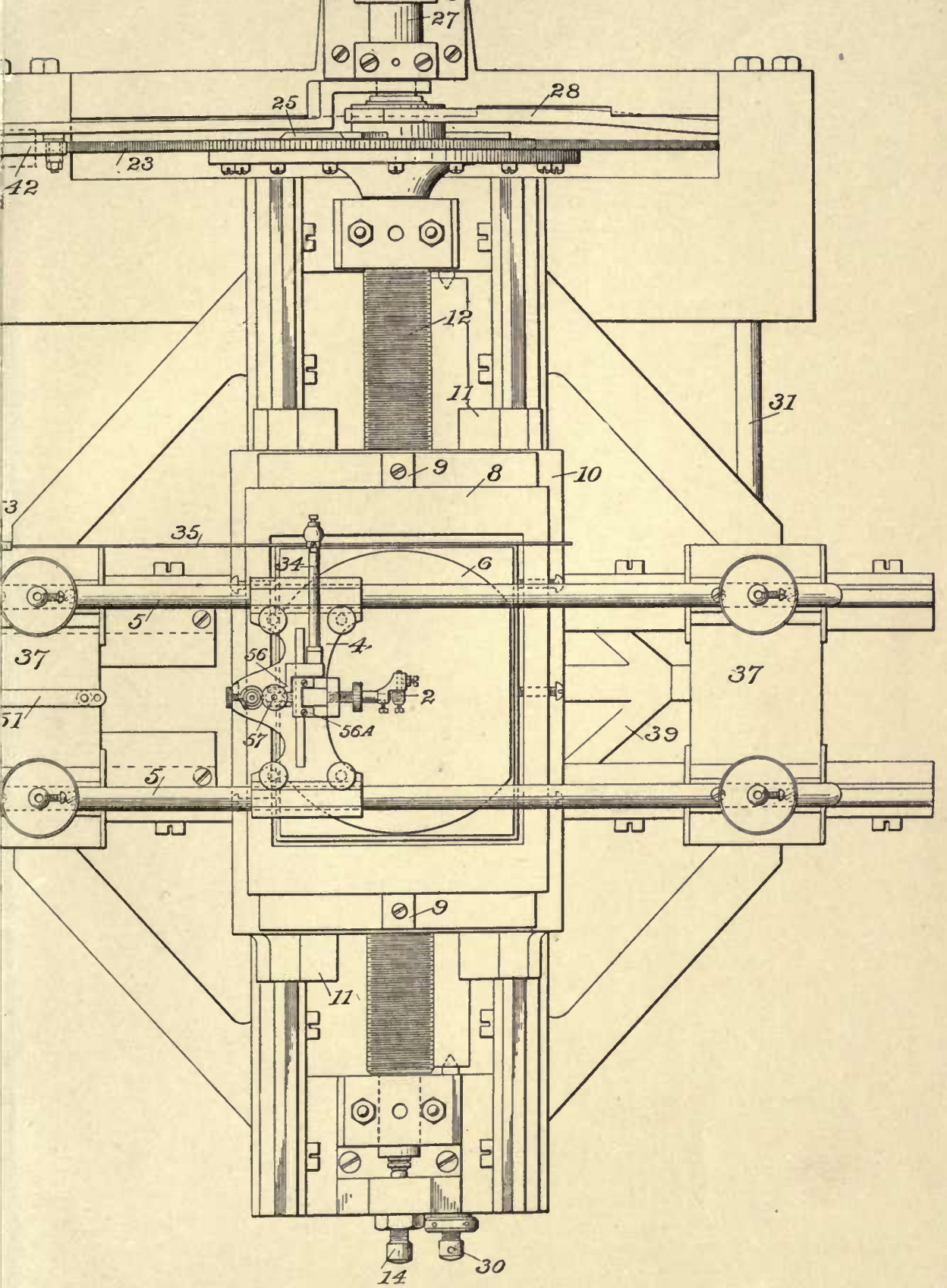


$\Theta$ 
FIG. 3

Plan view, showing the plate-carriage. The plate, plate-holder and ruling-head are omitted

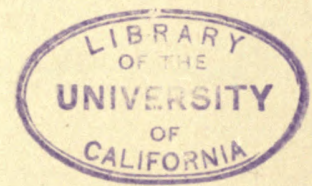




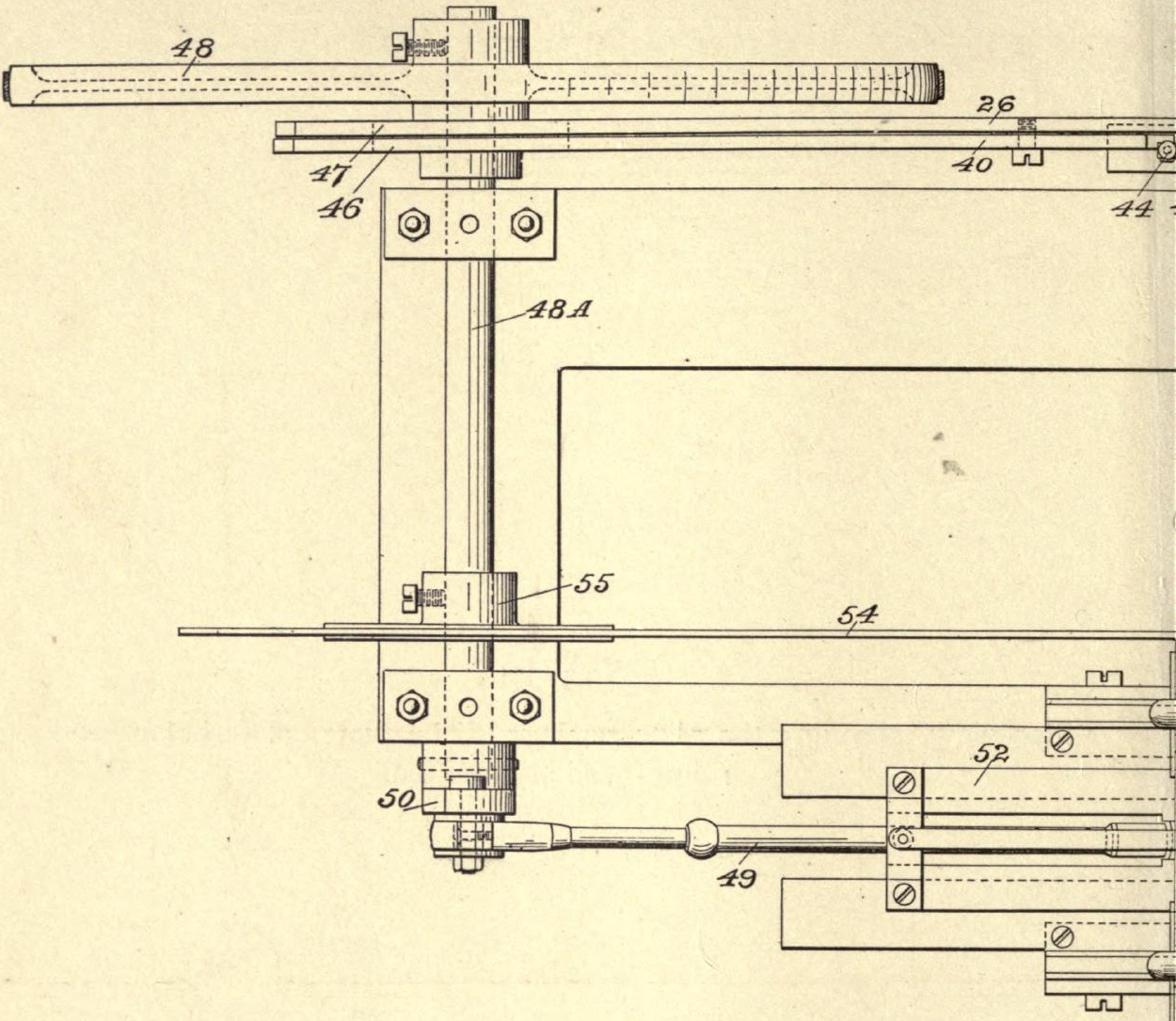

FIG. 3.

PLAN VIEW, SHOWING THE PLATE-CARRIAGE.

THE PLATE, PLATE-HOLDER AND RULING-HEAD ARE OMITTED. 


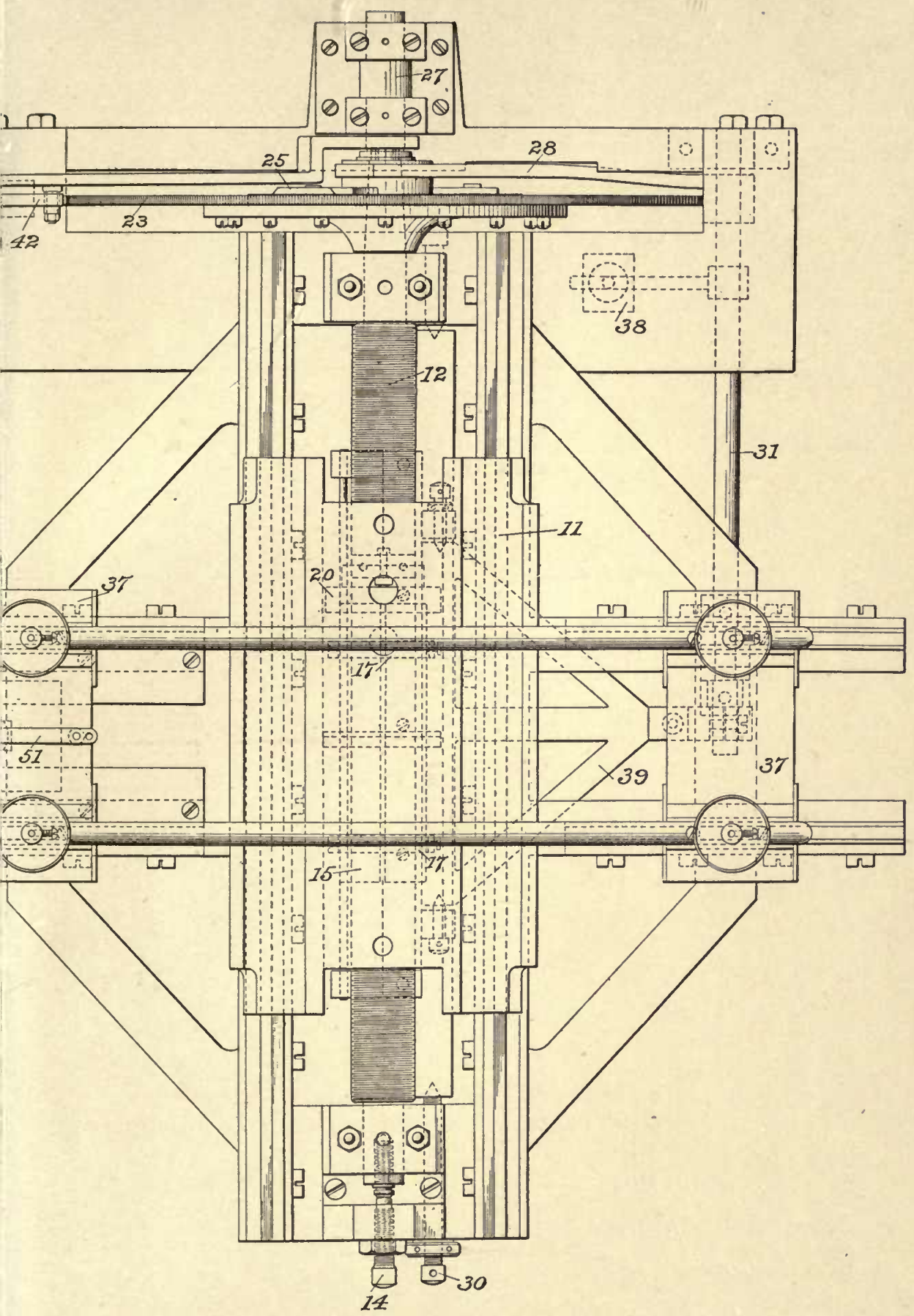




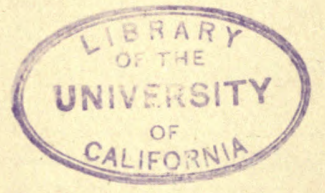


FIG. 4

Side elevation opposite to Fig. I, showing the engine in the return stroke position

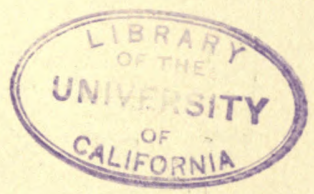




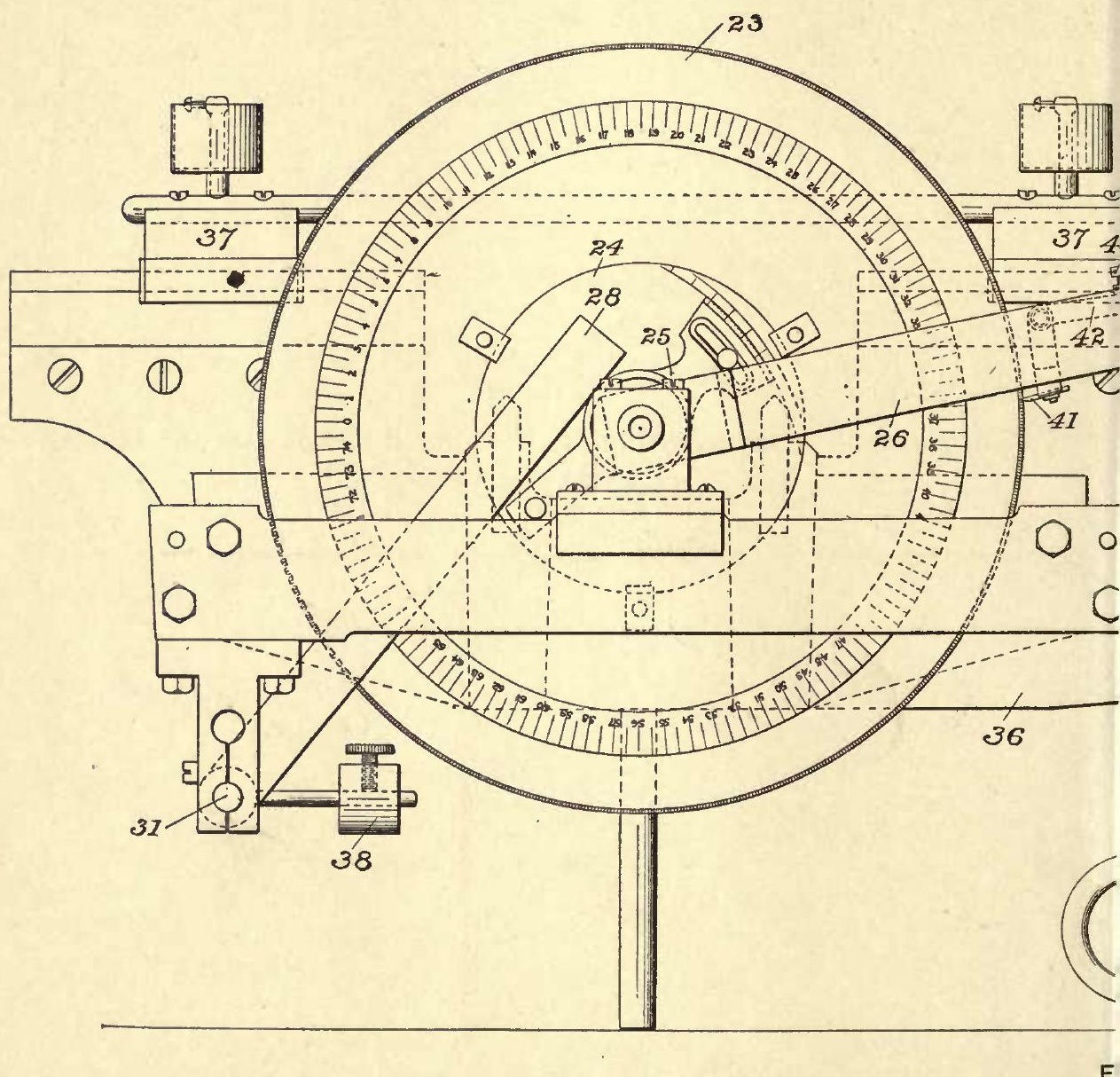

SIDE ELEVATION OPPOSITE TO FIG. 1, SHOWINI 


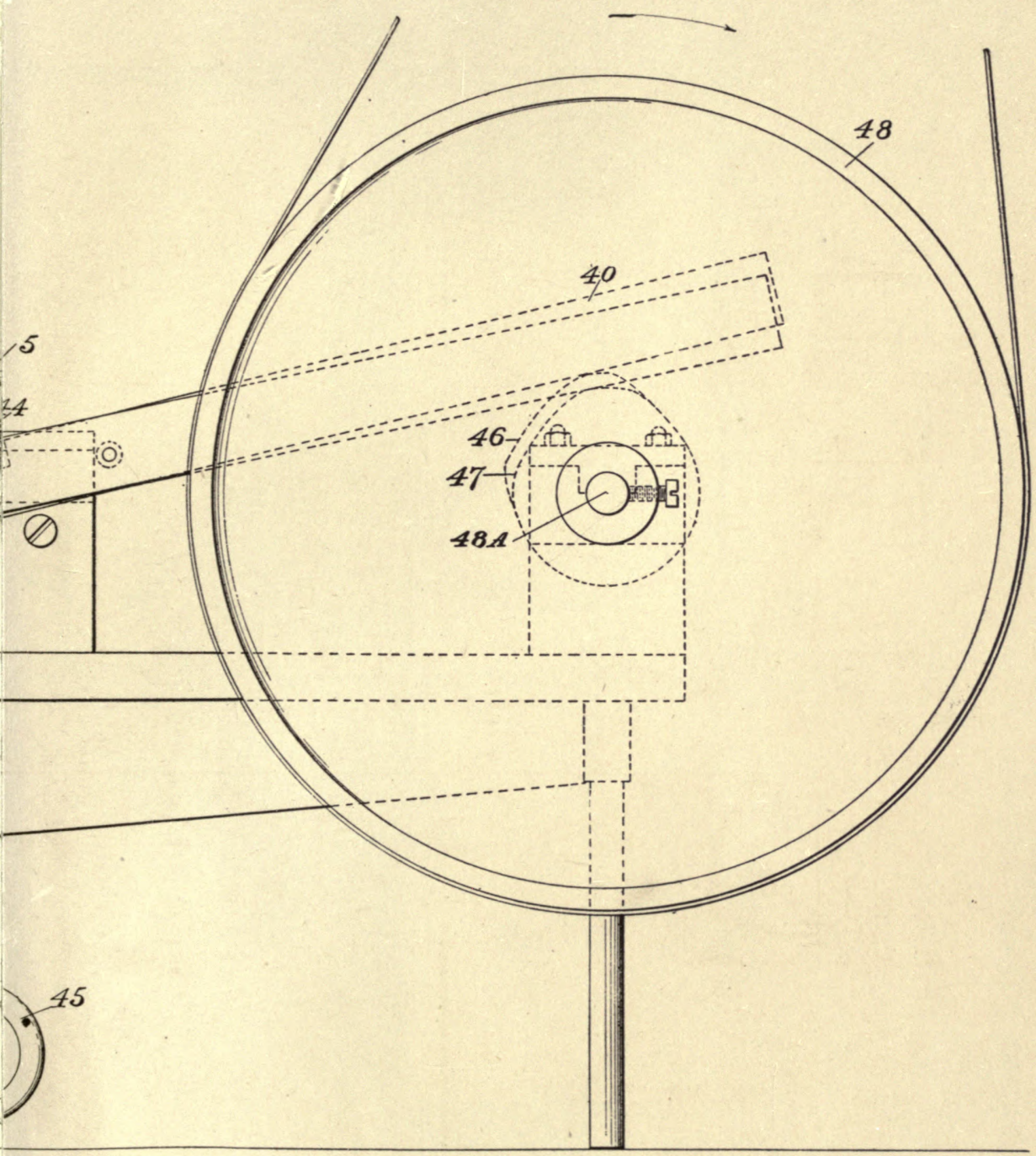

HE ENGINE IN THE RETURN STROKE POSITION. 



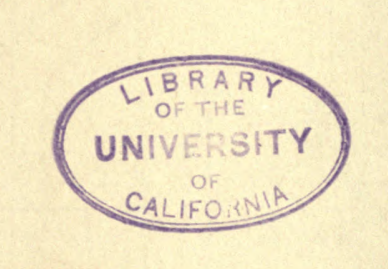




\section{INDEX}

Numbers refer to pages.

A.

Aberration Problems, 674.

Abney, Sir William de W., 491, 499, 574.

Absorption, Electric, 139, 297, 319, 321.

Absorption, Electric, of Crystals, 204.

Academy of Arts and Sciences, American, 7, 343, 611.

Academy of Sciences, National, 1, $15,610$.

Academy, French, 411.

Aepinus, 626, 639.

Air-thermometer, 358, 366.

Alternating Currents, 276, 280, 294, $314,661$.

Amáat, E. H., 410.

Amaury (see Jamin), 344, 388.

Ames, J. S., 525, 551, 691.

Ampère, 627, 639.

Anderson's Method of Measuring Resistance, 308.

Ångström, A. J., 512, 513, 546, 553, 555.

Ångström's Scale, 517, 553.

Arago, 642.

Archimedes, 619, 620, 621.

Atmospheric Electricity, 183, 212.

Aurora, Spectrum of the, 2, 31 .

Aurora, Theory of the, 179.

Ayrton, W. E., 179, 182, 183, 213, 278.

B.

Barker, George F., 3, 200, 364, 570.

Barometer, 362.

Basic Lines of Lockyer, 524.

Battery, Water, 241.

Baudin's Thermometers, 364, 386, 465.
Becquerel, A. C., 184, 214.

Beek, A. van, 411.

Bell, Graham, 574.

Bell, Louis, 242,513 et seq., 545,546 , 553 et seq.

Benzenberg, J. F., 411.

Bérard, J. E., 409, 410.

Berlin, University of, 4, 128.

Berlin Academy, 4.

Biot, J. B., 39, 90, 114, 116, 626, 627, 639.

Bosscha, J., 408, 465.

Boyle, Robert, 7.

Brashear, J. A., 9.

Bravais, A., 411.

Briggs, L. J., 571, 573.

B. A. Unit, $82,84,145,146,156,217$, 239.

Bruce, Miss, 521.

c.

Calorimetry, 387 .

Canton, John, 625.

Capacity, Electric, 297 et seq., 314 et seq.

Carmichael, N. R., 571, 573.

Cathetometer, 361.

Cavendish, Henry, 626, 639.

Cazin, A. A., 36, 48, 410.

Chapman (see Rutherfurd), 8.

Chemical Reaction, Action of Magnet on, 242.

Clarke, F. W., 570.

Clausius, R. J. E., 204, 205, 210.

Clément (see Désormes), 410.

Colardeau, 249.

Concave Gratings, 488, 492, 505.

Condenser, Standard, 267.

Convection, Electric, 128, 138, 179, 251.

Copernicus, 614. 
Coulomb, C. A., 95, 96, 103, 119, 120, $123,626,639,645$.

Crémieu, V., 5.

Crystals, Electric Absorption of, 204.

Crystals, Magnetic Properties of, 187.

\section{D.}

Daniell, J. F., 641.

Davy, Sir Humphry, 638, 639, 644, 650.

De Morgan, 619.

Delaroche, F., 409, 410.

Deluc, J. A., 387.

Désormes, C. B., 410.

Diamagnetism, 75, 184.

Distribution, Magnetic, 80, 85, 89 .

Dividing Engines, 487, 508, 693.

Doppler's Principle, 674.

Dub, C. J., 36.

Du Fay, 639.

Dulong, P. L., 438.

Duncan, Louis, 283.

Dupré, Athanase, 410.

E.

Earths, The Rare, 565.

Echelon Spectroscope, 590.

Edelmann, M. T., 266.

Edison's Electric Light, 200.

Edlund, E., 408, 416, 132.

Electric Absorption, 139, 297, 319, 321.

Electric Absorption of Crystals, 204.

Electric Convection, 128, 138, 179, 251.

Electric Currents, Theory of, 653.

Electric Light, Edison's, 200.

Electric Units, 10.

Electric Units, Ratio of, 266.

Electrical Congresses, 10, 212, 217.

Electricity, Theories of, 285, 635 .

Electricity, Atmospheric, 183, 212.

Electrodynamometer, 268, 284, 294, 314.

Electrometer, Absolute, 266.

Elements in the Sun, 522.

Ellis, Wm., 357.

Energy, Conservation of, 2, 6, 24.

Energy of Alternating Currents, 283.
Ether, Properties of the, 290 et seq., 338, 580, 586, 632, 667, 673.

Ethereal Motion, Resistance to, 338.

Expansion of Air under Constant Volume, 410.

\section{F.}

Fairbairn, Sir Wm., 416.

Faraday, M., 24, 26, 40, 43, 56, 89, $155,184,224,242,251,286,288$, $289,596,604 ; 616,626,629,630$, 638 et seq., 660,666 .

Faraday's Lines of Force, 37, 127, 286.

Farrand, 12.

Fastré's Thermometers, 365, 386, 416.

Faure, 650.

Favre, P. A., 408, 410, 421.

Fiske, Lieut., 238.

Fitzgerald, G. F., 229, 231.

Flaugergues, H., 387.

Fleming, J. A., 278.

Fletcher, L. B., 266.

Fortin-barometer, 362.

Foster, Henry, 411.

Foucault, J. B. L., 674.

Foucault-currents, 219, 234.

Frankfort-Lauffen Experiments, 284.

Franklin, Benjamin, 625, 639, 663, $665,669$.

Fraunhofer, J., 7.

Fresnel, A., 580.

Friction Brake, 423.

G.

Galileo, 614.

Galvani, 626, 639.

Galvanometers, 40, 159, 165, 268.

Gaugain, J. M., 42.

Gauss, 97, 148, 181, 626.

Gay Lussac, 410.

Geissler Thermometers, 465, 478, 481.

Ghosts in Spectra, 490, 492, 510, 519, 536.

Gibbs, O. Walcott, 364, 570.

Gilbert, N. E., 338.

Gilbert, William, 624, 639.

Gilman, D. C., 14, 15.

Glazebrook, R. T., 240, 505. 
Goldingham, John, 411.

Gramme Armature, 222, 224, 227, 228.

Gratings, 7, 487, 492, 587, 525.

Gratings, Concave, 488, 492, 505.

Gratings, Nobert, 8, 555.

Gratings, Manufacture of, 487, 508, 693.

Gratings, Use of, 519.

Gratings, Wandschaft's, 549.

Grating-spectroscope, 489, 499, 512, 551, 588.

Gravitation, Cause of, 292.

Gravitation, Law of, 670 .

Gray, Stephen, 624, 639, 653.

Green, George, 39, 90, 108, 114, 115, $116,627,630$.

Green, James, 362.

Grooves in Gratings, Theory of, 529 et seq.

Guerricke, Otto v., 639.

\section{H.}

Hall, E. H., 197, 266.

Hall Effect, The, 197.

Harmonics in Alternating Currents, 276, 280, 300, 301 .

Harris Unit Jar, 208, 209.

Harrison, C. N., 685.

Harvard University, 5.

Hastings, C. S., 7, 503.

Heat, Mechanical Equivalent of, 5, 343 et seq., 469.

Heaviside, Oliver, 674.

Helmholtz, H. von, 4, 29, 83, 128, $131,138,159,167,179,251,314$, $586,630,643$.

Henry, Joseph, 53, 578, 657, 669.

Hertz, H., 289, 580, 658, 659, 673, 674.

Herwig, H. A. B., 416.

Hidden, 570.

Himstedt, F., 5.

Hirn, G. A., 344, 388, 408, 410, 416, $418,423,424$.

Holman, S. W., 364, 384. .

Hutchinson, C. T., 4, 251.

Hysteresis, 276 et seq., 281.

\section{I.}

Icilius, Quintus, 36, 408, 418.

Inductance, Measurement of, 294 et seq., 314,325 .

Ionization of Gases, 579.

\section{J.}

Jacobi, M. H. ., 36.

Jacobi Unit, 147.

Jacques, W. W., 80, 81, 145, 174, 184, 193.

Jamin, J. C., 71, 80, 81, 89, 90, 96, 97, 122 et seq., 344, 388, 410.

Jenkin, Fleming, 150, 169.

Jewell, L. E., 524, 545, 550.

Johns Hopkins University, 4.

Jolly, P. G., 410.

Joule, 6, 7, 24, 27, 36, 52, 53, 146, 344, $381,408,414,416,417,419,421$, 469.

Joule's Thermometers, 417, 469.

\section{K.}

Kelvin, Lord (see Thomson, Sir William).

Kempf, P., 546, 553, 555.

Kew Thermometers, 363, 366, 381, 466.

Kimball, A. L., 239.

Kirchhoff, G. R., 145, 156, 239, 419, 628.

Koenig, Rudolph, 29, 217.

Kohlrausch, F. W., 4, 82, 83, 84, 146 et seq., 410, 421, 628 .

Koyl, C. A., 549, 555.

Krüss, Dr., 566, 570 .

Kurlbaum, F., 546, 553, 554, 555.

\section{I.}

Laboratories, Physical, 614.

Laboulaye, C. P. L. de, 410.

Langley, S. P., 491.

Laplace, 626, 639, 671.

Lecher, E., 4, 252.

Lenard, P., 576, 585.

Lenz, H. F. E., 36, 408, 418.

Lightning, 236.

Lightning-rods, 237, 663.

Lippmann, G., 5.

Lockyer, Sir J. Norman, 487, 524.

Lodge, Sir O. J., 662, 664, 674.

Lorenz, L. V., 146, 155, 156, 217, 239, 419.

\section{M.}

Magnetic Circuit, 3, 38, 89, 225, 276. Magnetic Distribution, $80,85,89$. 
Magnetic Induction, Measurement of, 98.

Magnetic Permeability, 35, 56.

Magnetic Proof Plane, 85.

Magnetism of Earth, 179, 213.

Magnetism, Cause of, 673.

Magnets, Lifting Power of, 52.

Magnets and Chemical Reactions, 242.

Magnetization, Maximum, 35, 56.

Magnetization, Temporary, 49.

Magnus, H. G., 410.

Marcou, P. B., 216.

Marianini, S. G., 71.

Martins, C. F., 411.

Mascart, E., 240.

Masson, A. P., 410.

Matthiessen, A., 147.

"Maxwell, J. C., 3, 52, 57, 71, 83, 89, $114,128,139,149,170,198,199$, $224,251,289,580,660,673$.

Maxwell's Electromagnetic Theory, $7,198,199,289,630,631$.

Mayer, Alfred M., 669.

Mayer, J. R., 24.

McFarlane, D., 437, 438.

McJunckin, P. C., 338.

Mechanical Equivalent of Heat, $343,469$.

Mendenhall, T. C., 1.

Michelson, A. A., 584, 590, 674.

Michie, Professor, 15.

Moll, G., 411.

Motors, Electric, 280, 281.

Müller, G., 546, 553, 555.

Müller, J. H. J., 35, 36, 48.

Münchausen, v., 344, 389.

Murphy, Robert 626.

Myrback, v., 411.

\section{N.}

National Academy of Sciences, 1, 15,610 .

Nesbit, 53.

Neumann, F. E., 146, 387.

Neumann's Coefficient, 35, 57, 73, 116.

Newton, Sir Isaac, 56, 286, 293, 615, $623,638,671$.

Nichol, J. P., 435, 437, 438.

Nichols, E. L., 204, 249, 250.
Niven's Method of Measuring Inductance, 309.

Nobert Gratings, 8, 555.

0.

Oersted, 626, 639, 640 .

Ohm, Determination of the, 217, 239, 419, 628.

Ohm's Law for Currents, 139, 141, 238, 640.

Ohm's Law for Magnetic Induction, 3, 38, 89, 90 .

\section{P.}

Paine's Electromagnetic Engine, 24.

Parry, E., Capt., 411.

Peirce, C. S., 492, 494, 513 et seq., 545 et seq.

Penniman, T. D., 297, 298, 314.

Permeability, Magnetic, 35, 56, 73.

Perry, John, 179, 182, 183, 213.

Petit, P., 438.

Pfaundler, L., 344, 351, 388, 467.

Phillips Academy, Andover, 11.

Pickering, E. C., 364 .

Pickering, W., 343.

Planté, G., 650.

Platter (see Pfaundler), 344, 351, $388,467$.

Plücker, J., 184.

Poggendorff, J. C., 348.

Poisson, 626, 630, 639.

Porous Plug Experiment, 346.

Porter, A. W., 580.

Power, Transmission of, 280.

Proof Plane, Magnetic, 85.

Puluj, J., 408, 424.

Pupin, M. I., 584, 585, 586.

\section{R.}

Radiation of Heat, 435.

Rankine, W. J. M., 381.

Rayleigh, Lord, 240, 294, 525, 528, $534,588,628$.

Rays, Röntgen, 571, 573, 576.

Recknagel, G. F., 356, 358, 389.

Regnault, V., 344,353 et seq., 365 , $368,376,388$ et seq., 409 et seq., $466,607$.

Remsen, Ira, 242 et seq.

* References to Maxwell are so numerous that only the more important ones are noted here. 
Rensselaer Polytechnic Institute, $2,12$.

Resistance, Electrical; Effect of Magnetic field on, 338 .

Resistance, Electrical; Measurement of, 313.

Resolving Power, 502, 528, 588.

Resonances, 2, 28.

Richard (see Jamin), 410.

Riecke, E., 36 .

Ritter, J. W., 639, 650.

Röntgen, W. C.2 4, 252, 410, 414.

Röntgen-rays, $571, \overline{573}, 576$.

Rogers, W. A., 441, 507.

Rosa, E. B., 266.

Royal Society of London, 3.

Ruling Engines, 8, 487, 508, 691.

Rumford, Count, 6, 408, 416, 669.

Rumford-fund, $7,343,521,548$.

Rutherfurd, L. W., 8, 487, 494, 513.

\section{s.}

Savart, F., 411, 627.

Schiller, N. N., 252.

Schneider, Theodore, 9, 487, 692.

Scott, C. F., 237.

Screws, Perfect, 8, 487, 506.

Sears, David, 80, 98.

Seebeck, L. F. W. A., 134, 640.

Shröder v. d. Kolk, 411, 414.

Siemens Armature, 219, 221,. 222, 228.

Siemens Unit, 4, 147, 152, 155, 156.

Silbermann, J. T., 408, 410, 421.

"Skin-Effect" of Alternating Currents, 283, 661 .

Solar Spectrum, 9, 512, 521.

Sound, Velocity of, 411.

Specific Heats of Air, Ratio of, 410.

Specific Heats of Gases, 409, 410.

Specific Heat of Water, 387.

Spectroscope, Concave Grating, 489, $499,512,551,589$.

Spectroscope, Plane Grating, 588.

Spectrum of the Aurora, 2, 31.

Spectrum, Solar, 512, 521.

Stampfer, S., 411.

Stefan, J., 69.

Steinheil, 166, 168.

Steinmetz, C. P., 278.

Stokes, Sir G. G., 674.
Stoletow, A. G., 36, 48, 50, 71, 73, 91, $105,154$.

Sturgeon, William, 53.

T.

Tate, T., 416.

Tatnall, R. R., 685.

Telegraph, Multiplex Printing, 10.

Temperature, Absolute Scale of, 381.

Temperature, Effect of, on Magnetization, $58,65,74$.

Tesla, Nicola, 578 .

Thalén, T. R., 513, 546, 555.

Thermometers, Air, 358, 366 .

Thermometers, Mercurial, 346, 363.

Thermometers, Mercurial and air, 352.

Thermometers, Comparisons of, 477.

Thermometers, Standard, 363.

Thermometry, 345, 439.

Thiessen, M. F., 481.

Thompson, S. P., 233, 234, 235.

Thomson, Elihu, 232, 235, 573, 574, $584,585$.

Thomson, J. J., 579, 674.

*Thomson, Sir William, 37, 77, 78. $79,148,213,346,381,414,421$, $626,649,657$.

Thunderstorms, Theory of, 183, 213.

Transformers, Theory of, 276, 280.

Tresca, H. E., 410, 513.

Trowbridge, John, 215, 364.

Tyndall, John, 26, 27, 97, 574, 641.

$\nabla$.

Venetian Institute; Prize Essay, 7.

Verdet, M. E., 58, 79.

Violle, J. I. G., 408, 418.

Vogel, H. C., 549, 556, 557.

Volta, 626, 639, 645 .

Vortex in Outlet of Water, 23.

\section{W.}

Waldo, L., 481.

Waltenhofen, A. K., 421.

Wandschaft's Gratings, 549.

Water, Specific Heat of, 387.

Water Battery, 241.

* The references to Lord Kelvin are so numerous that only the important ones are noted here. 
Wave-lengths, Standard, 512, 517, $521,545,548$.

Webb, F. C., 38,

Weber, 36, 48, 49, 125, 137, 147, 148, $152,153,156,160,170.184,240$, 408, 418, 419, 626, 628.

Weber, H. F., 155, 408, 418, 419, 420.

Weisbach, J., 410.

Welsh's Thermometers, 365 .

Welter, J. J., 410.

West Point Military Academy, 14.

Wheatstone, C., 649 .

Wiedemann, E., 409, 415.
Wiedemann, G., 240.

Wilke, J. K., 639.

Wollaston, W. H., 604.

Wüllner, A., 368, 410.

Y.

Yale University, 11.

Young, C. A., 487, 493.

Young, Thomas, 7.

Z.

Zieman Effect, 672, 673.

\section{IBRAR}

UNIVERSITY 


r 





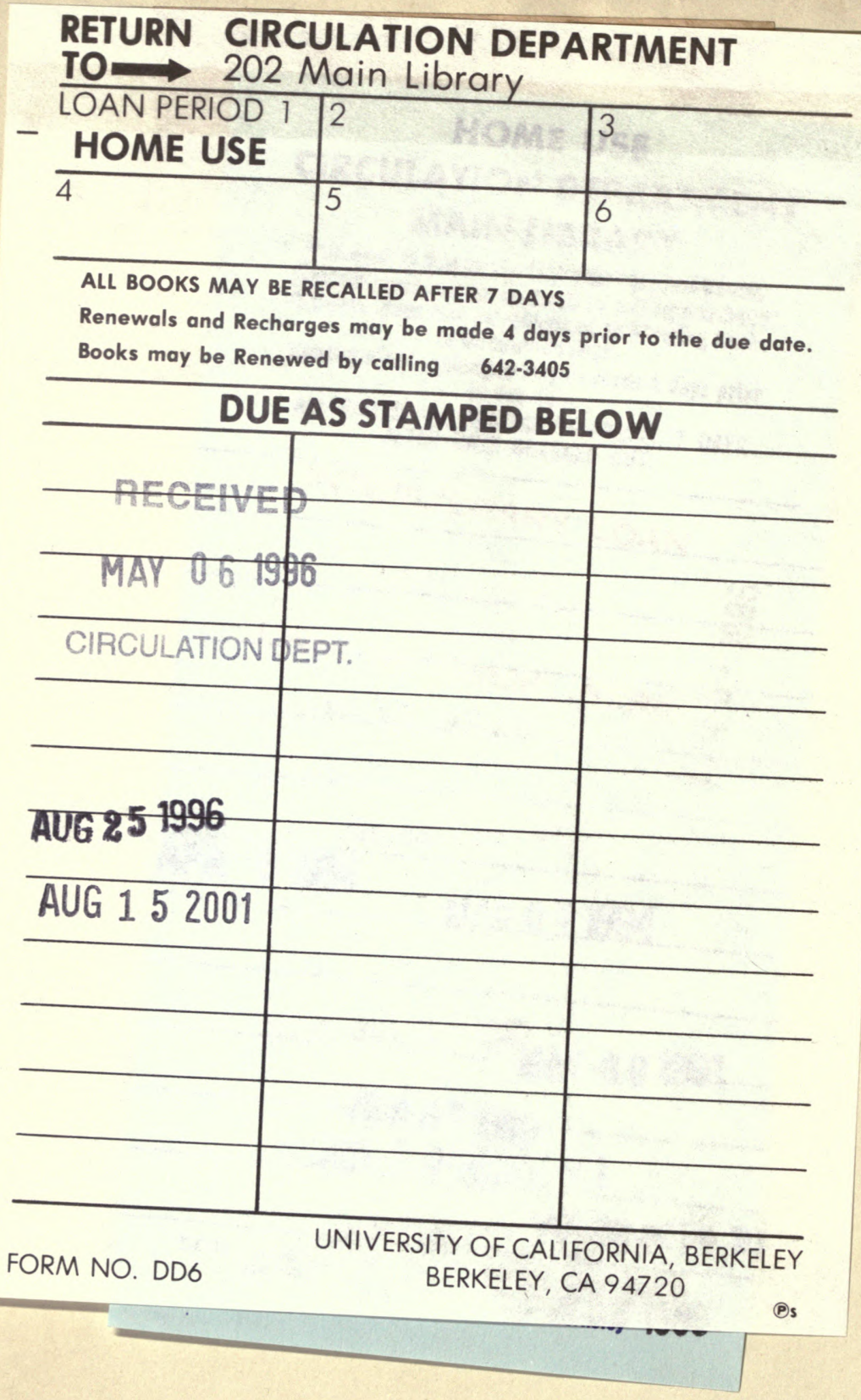


YD 02320

\section{U.C. BERKELEY LIBRARIES} |||||||||||||||||||||||||||||||||||||||| B003017143 
